\title{
CONEXIÓN DE CONVERTIDORES CON CONMUTACIONES SUAVES CON ENTRADA Y SALIDA PARALELO: APLICACIÓN A CONVERTIDORES DE ALTA RELACIÓN DE TRANSFORMACIÓN
}

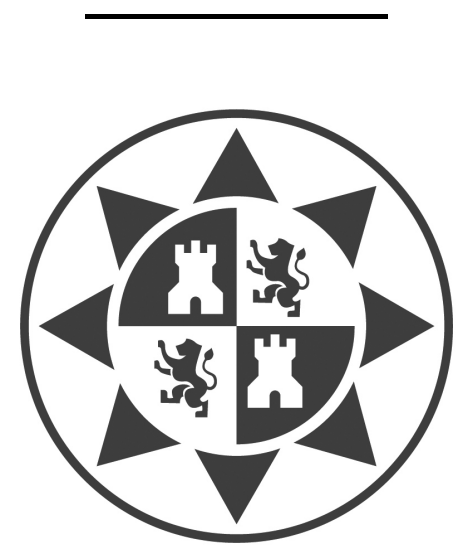

TESIS DOCTORAL

Jacinto María Jiménez Martínez

Escuela Internacional de Doctorado Universidad Politécnica de Cartagena

Diciembre 2018 



\section{CONEXIÓN DE \\ CONVERTIDORES CON \\ CONMUTACIONES SUAVES \\ CON ENTRADA Y SALIDA \\ PARALELO: APLICACIÓN A \\ CONVERTIDORES DE ALTA \\ RELACIÓN DE \\ TRANSFORMACIÓN}

Doctorando:

Jacinto María Jiménez Martínez

Directores:

José Antonio Villarejo Mañas

María Esther de Jódar Bonilla

Escuela Internacional de Doctorado

Universidad Politécnica de Cartagena

Diciembre 2018 



\section{CONFORMIDAD DE SOLICITUD DEAUTORIZACIÓN DE DEPÓSITO DE} TESIS DOCTORAL POR EL/LA DIRECTOR/A DE LA TESIS

D. JOSE ANTONIO VILLAREJO MAÑAS, y $\mathrm{D}^{\mathrm{a}}$. MARIA ESTHER DE JODAR BONILLA, Directores de la Tesis doctoral CONEXIÓN DE CONVERTIDORES CON CONMUTACIONES SUAVES CON ENTRADA Y SALIDA PARALELO: APLICACIÓN A CONVERTIDORES DE ALTA RELACIÓN DE TRANSFORMACIÓN

\section{INFORMAN:}

Que la referida Tesis Doctoral, ha sido realizada por D/D ${ }^{\mathrm{a}}$. JACINTO MARÍA JIMÉNEZ MARTÍNEZ_, dentro del Programa de Doctorado ENĒERGÍAS RENOVABLES Y EFICIENCIA ENERGÉTICA_, dando la conformidad para que sea presentada ante el Comité de Dirección de la Escuela Internacional de Doctorado para ser autorizado su depósito.

La rama de conocimiento en la que esta tesis ha sido desarrollada es:

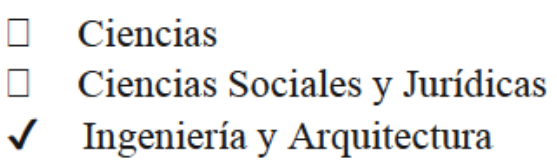

En Cartagena, a 5 de Noviembre de 2018

\section{EL/LA DIRECTOR/A DE LA TESIS}

Firmado digitalmente por JOSE ANTONIO|VILLAREJO|MAÑAS Nombre de reconocimiento (DN):CD=IOSE ANTONIOIVIIL AREIO| MAÑAS, serialNumber

Sn=VILLAREO MAÑAS

Fecha: 2018.11.08 12:07:57 +01' $00^{\prime}$

Fdo.: Jose Antonio Villarejo Mañas
EL/LA CODIRECTOR/A DE LA TESIS

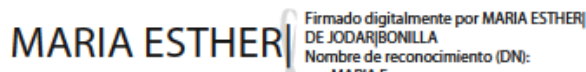

DE JODAR| BONILLA $\mathrm{Cn}=$ MARIA
serialNumb serialNumb
givenName givenName
JODARBONILLA, ou $=$ Giudadanos, Fecha: $2018.11 .081036: 29+01^{\circ} 00$ Fdo.: María Esther de Jódar Bonilla 



\title{
CONFORMIDAD DE DEPÓSITO DE TESIS DOCTORAL
}

\section{POR LA COMISIÓN ACADÉMICA DEL PROGRAMA}

D. ÁNGEL MOLINA GARCÍA, Presidente/a de la Comisión Académica del Programa ENERGÍAS RENOVABLES Y EFICIENCIA ENERGÉTICA.

\section{INFORMA:}

Que la Tesis Doctoral titulada, "CONEXIÓN DE CONVERTIDORES CON CONMUTACIONES SUAVES CON ENTRADA Y SALIDA PARALELO: APLICACIÓN A CONVERTIDORES DE ALTA RELACIÓN DE TRANSFORMACIÓN”, ha sido realizada, dentro del mencionado Programa de Doctorado, por D. JACINTO MARÍA JIMÉNEZ MARTÍNEZ, bajo la dirección y supervisión del Dr. JOSE ANTONIO VILLAREJO MAÑAS - y la Dra. MARIA ESTHER DE JÓDAR BONILLA.

En reunión de la Comisión Académica, visto que en la misma se acreditan los indicios de calidad correspondientes y la autorización del Director/es de la misma, se acordó dar la conformidad, con la finalidad de que sea autorizado su depósito por el Comité de Dirección de la Escuela Internacional de Doctorado.

La Rama de conocimiento por la que esta tesis ha sido desarrollada es:

\author{
Ciencias \\ Ciencias Sociales y Jurídicas \\ $\checkmark$ Ingeniería y Arquitectura
}

En Cartagena, a 8 de Noviembre de 2018

EL PRESIDENTE DE LA COMISIÓN ACADÉMICA

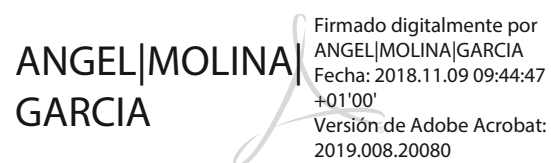

Fdo: ÁNGEL MOLINA GARCÍA 

A Juana María.

A Pedro, Marta, Juan e Isabel. 

Dicebat Bernardus Carnotensis nos esse quasi nanos, gigantium humeris insidentes, ut possimus plura eis et remotiora videre, non utique proprii visus acumine, aut eminentia corporis, sed quia in altum subvenimur et extollimur magnitudine gigantea.

Juan de Salisbury, Metalogicon (1159) 



\section{Agradecimientos}

He pensado más de mil y una veces cómo escribir esta página, y ninguna de las que he pensado durante el desarrollo de este trabajo me parecía la más adecuada. Por tanto, que vaya por delante mi agradecimiento a todas aquellas personas que considerando que debieran estar mencionadas expresamente no lo están. Les pido mis más sinceras disculpas por tal motivo.

A las tres personas, que me han mostrado una paciencia sin límites y un ánimo inagotable hasta extremos insospechados para empujarme a seguir y darme su apoyo cuando se me antojaba complicado continuar, o imposible terminar el trabajo. Cada uno lo ha hecho como Dios le ha hado a entender, por tanto, bien hecho está. Estas personas, son mi mujer, Juana $\mathrm{M}^{\mathrm{a}}$, y mis directores de tesis, José Antonio y Esther, a los que les agradezco todos sus esfuerzos por ayudarme a llegar hasta aquí, y que reconozco que no han sido pocos.

Hay, por supuesto, más personas que incluso no estaban en un principio y que también son y seguirán siendo una motivación para mi en muchos aspectos de mi vida. Gracias a Pedro, Marta, Juan e Isabel por aguantar, por «sufrir» de forma indirecta y por animarme también a concluir la tesis.

A los miembros del Departamento de Tecnología Electrónica, por sus comentarios y su ayuda cuando había dudas y tareas que solucionar durante este camino. Entre ellos a Antonio por su soporte en el laboratorio.

A Javier, Joaquín y Jose Antonio. Siempre los he querido mencionar, por su confianza puesta en mí y que no pudo dar los frutos esperados en su momento. El primero es un entusiasta donde los haya y que me contagió su entusiasmo en mi primer contacto con la investigación. Mirando atrás, me ayudan a entender un poco más la frase del filósofo Ortega y Gasset indicando que: «Yo soy yo y mi circunstancia, y si no la salvo a ella no me salvo yo.»

Me haría falta mucho espacio para incluir los nombres de todos los que no están o los que también me han apoyado de forma silenciosa, que me conocen incluso mejor que yo mismo, y aún así, lo han hecho desinteresadamente. Gracias. 



\section{Resumen}

La asociación de etapas de potencia con entrada paralelo y salida paralelo ha sido ampliamente estudiada durante los últimos años. Las topologías con conmutaciones suaves sin aislamiento se encuentran incluidas dentro de esos trabajos, en los que se ha comprobado el correcto funcionamiento de varios módulos conectados cuando comparten las mismas señales de disparo, a la vez que hay presente un buen reparto dinámico y estático de corrientes.

Las topologías con aislamiento también están descritas en la literatura. Por ejemplo, el convertidor flyback con enclavamiento activo se ha utilizado ampliamente en convertidores para aplicaciones de energía solar fotovoltaica y en convertidores con alta relación de transformación. Las bobinas acopladas (o transformadores) permiten el aislamiento galvánico. Además, es una solución cuando se necesitan combinar niveles altos y bajos de tensión, como podría ser el caso de convertidores conectados a red, donde los niveles de entrada y de salida deben adecuarse.

Por otro lado, los convertidores con enclavamiento activo tienen una impedancia de salida elevada, lo que sugiere que puede conseguirse un buen reparto de carga empleando el mismo ciclo de trabajo para todas las etapas del convertidor.

Este trabajo ayuda a profundizar en el reparto de corriente entre etapas con conmutaciones suaves, tomando el convertidor mencionado como un ejemplo de aplicación en el que se requiere aislamiento y alta relación de transformación.

Un breve estado del arte relacionado con la conexión de etapas de potencia se ha incluido al principio de este trabajo. También se enumeran los principales métodos de reparto de corriente. Otros aspectos, como por ejemplo, la diferencia entre convertidor de potencia y etapa de potencia, y el efecto de la impedancia de salida también se mencionan. De igual forma se hace con los convertidores con conmutaciones a tensión cero (ZVS).

A continuación, las topologías básicas con conmutaciones suaves y sin aislamiento, esto es, reductor, elevador y reductor-elevador, son ampliamente analizadas cuando operan en régimen permanente de forma individual o en multietapa. Su respuesta en régimen dinámico también es objeto de estudio.

La validación del funcionamiento en paralelo de topologías con aislamien- 
to con alta relación de transformación es otro objetivo de este trabajo. El empleo de las bobinas acopladas podría influir en el reparto de corriente de los módulos conectados en paralelo. Por tanto, tanto la relación de transformación como los elementos parásitos deberían ser tenidos en cuenta en el análisis. Los resultados obtenidos con estas consideraciones, y mostrados en la parte final de este documento, se han conseguido a partir de un prototipo de laboratorio que emplea un simple lazo de control implementado digitalmente. 


\section{Abstract}

Connection of input-parallel output-parallel power stages has been investigated for last years. Non-isolated soft-switching topologies are included in those studies, showing and testing their correct operation when the same gate signals are shared betwen connected modules. A good dynamic and steady-state current sharing is also verified.

Isolated soft switching topologies are also described in literature. For example, the Active-Clamp Flyback converter is widely used in photovoltaic module-integrated converters and high step-up ratio converters. Coupled inductors (or transformers) provide galvanic isolation. Besides, it's a solution when low and high-voltage levels are needed, e.g., in grid-connected converters, where input and output-voltage levels must be adapted.

On the other hand, active-clamp converters have high output impedance, which suggests that a good load sharing could be easily achieved using the same duty cycle for all of the stages in the converter.

This works helps to understand more deeply the current sharing between soft-switching stages, taking the Active-Clalmp Flyback converter as an application example where isolation and high step-up ratio is required.

A brief state-of-art related to power stages connection has been included in the introductory part of this work. Current sharing methods are also added in that summary. Another issues, e.g., difference between power converter and power stage and the effect of output impedance are cited too. ZeroVoltage-Switching converters are also described.

After that, basic non-isolated soft swiching topologies, i.e., buck, boost and buck-boost power stages, are broadly analized under stead-state operation, in both, single and multiphase-connected operation. Dynamic response is also showed.

Testing of isolated soft-switching topologies with high step-up ratio operating in parallel is another objective in this work. The use of coupled inductors could influence current sharing in paralled-connected modules. So, the transform ratio and parasitic elements should be taken into account. Results under these assumptions are showed in the last part of this document, where they are collected from an experimental prototype using a simple and digitally-implemented closed-loop control technique. 



\section{Índice}

Agradecimientos xiii

Resumen $\quad$ xv

Abstract xvii

1. ESTADO DEL ARTE 1

1.1. Introducción . . . . . . . . . . . . . . . 1

1.2. ¿Convertidor de potencia o etapa de potencia? . . . . . . 6

1.3. Característica de salida . . . . . . . . . . . . 8

1.4. Reparto de corriente en la conexión paralelo. Métodos pasivos. 9

1.4.1. Conexión directa. . . . . . . . . . . . . . . . 10

1.4.2. Efecto de la impedancia. . . . . . . . . . . . . . 13

1.4.3. Resistencias ecualizadoras . . . . . . . . . . . . 17

1.4.4. Convertidores con propiedades de reparto inherentes . 17

1.4.5. Realimentación dependiente de la corriente. . . . . . . 19

1.4.6. Baja ganancia en continua. . . . . . . . . . . 20

1.4.7. Otros métodos pasivos . . . . . . . . . . . . . 22

1.5. Convertidores PWM con conmutaciones suaves. . . . . . . . 23

1.5.1. Snubbers como ayuda a la conmutación. . . . . . . . 24

1.5.2. Del enclavamiento activo a las conmutaciones suaves. . 27

1.5.3. La impedancia de salida. . . . . . . . . . . . . . 30

1.6. Planteamiento de la tesis . . . . . . . . . . . . . . 31

2. REPARTO ESTÁTICO 33

2.1. Funcionamiento de un convertidor ZVS. . . . . . . . . . 33

2.2. Análisis en régimen permamente. . . . . . . . . . . 38

2.2.1. Ejemplo para un elevador ZVS. . . . . . . . . . 41

2.2.2. Ejemplo para un reductor ZVS. . . . . . . . . . . . . 43

2.2.3. Ejemplo para un red.elev. ZVS. . . . . . . . . . . . . . . 43

2.2.4. Ejemplo para un flyback ZVS. . . . . . . . . . . . . 44

2.3. Característica de salida. . . . . . . . . . . . . . 45

xix 
2.3.1. Ejemplo de reparto en paralelo. . . . . . . . . . . . . 49

2.4. Parámetros que afectan al reparto de carga. . . . . . . . . . . 52

2.5. Impedancia de salida en régimen permanente. . . . . . . . . . 56

2.5.1. Ejemplo. . . . . . . . . . . . . . . 60

3. REPARTO DINÁMICO

3.1. Impedancia de salida. . . . . . . . . . . . . . . . . . . . . . 63

3.2. Influencia del condensador de clamp. . . . . . . . . . . . . . . 69

3.3. Reclutamiento de etapas. . . . . . . . . . . . . . . . 78

3.3.1. Reductor ZVS. . . . . . . . . . . . . . . . . . . 80

3.3.2. Elevador ZVS. . . . . . . . . . . . . . . . . . . 83

3.3.3. Reductor elevador ZVS. . . . . . . . . . . . . . 85

4. APLICACIÓN $\quad 89$

4.1. Introducción. . . . . . . . . . . . . . . . . . . . . . . . . . . 89

4.2. Topología flyback ZVS con capacidades parásitas. . . . . . . . 92

4.2.1. Etapas. . . . . . . . . . . . . . . . . . . . 94

4.2.2. Análisis en régimen permanente. . . . . . . . . . . . . 102

4.3. Topología flyback ZVS modificada. . . . . . . . . . . . . . . . 109

4.3.1. Etapas. . . . . . . . . . . . . . . . . . . . 111

4.3.2. Análisis en régimen permanente. . . . . . . . . . . . . 117

4.3.3. Modelo promediado. . . . . . . . . . . . . . . . . . . . 121

4.3.4. Modelo en régimen permanente . . . . . . . . . . . . . 122

4.4. Prototipo y validación experimental . . . . . . . . . . 126

5. CONCLUSIONES 133

5.1. Conclusiones. . . . . . . . . . . . . . . . . . 133

5.2. Aportaciones. . . . . . . . . . . . . . . . . . . . 134

5.3. Futuros trabajos. . . . . . . . . . . . . . . . . . 134

A. REDUCTOR ZVS 137

A.1. Funcionamiento del Reductor ZVS . . . . . . . . . . . . . 137

A.1.1. Etapa 1. $\left(t_{0}<t<t_{1}\right)$. . . . . . . . . . . . 138

A.1.2. Etapa 2. $\left(t_{1}<t<t_{2}\right)$. . . . . . . . . . . . . . 139

A.1.3. Etapa 3. $\left(t_{2}<t<t_{3}\right)$. . . . . . . . . . . . . . . . 142

A.1.4. Etapa 4. $\left(t_{3}<t<t_{4}\right)$. . . . . . . . . . . . . . . . 144

A.1.5. Etapa 5. $\left(t_{4}<t<t_{5}\right)$. . . . . . . . . . . . . . . . 148

A.1.6. Etapa 6. $\left(t_{5}<t<t_{6}\right)$. . . . . . . . . . . . . . . 150

A.2. Análisis en régimen permanente. . . . . . . . . . . . . . . 150

A.2.1. Sin considerar rizado. . . . . . . . . . . . . . . . . 150

A.2.2. Considerando rizado. . . . . . . . . . . . . . . . . . 154

A.2.3. Comparativa. . . . . . . . . . . . . . . . 158 
A.3. Modelo promediado . . . . . . . . . . . . . . . . 159

A.3.1. Método aplicado . . . . . . . . . . . . . . . 160

A.3.2. Modelo promediado para el reductor ZVS . . . . . . 163

A.3.3. Balance energético . . . . . . . . . . . . . . 167

A.3.4. Validación del modelo promediado . . . . . . . . . 168

A.4. Modelo en régimen permanente . . . . . . . . . . . . . 169

A.4.1. Balance energético . . . . . . . . . . . . . 171

A.5. Modelo en pequeña señal . . . . . . . . . . . . . . . . . 172

A.6. Funciones de transferencia . . . . . . . . . . . . . . . . 178

A.6.1. Desde el modelo o el circuito de pequeña señal . . . . 178

A.6.2. Desde el modelo matricial . . . . . . . . . . . . . . 183

A.7. Impedancia de salida . . . . . . . . . . . . . . . . . . 184

A.7.1. Cálculo de $Z_{t h}$. . . . . . . . . . . . . . . . . . . 184

A.7.2. Cálculo de $Z_{\text {out }}$. . . . . . . . . . . . . . . . . 186

A.7.3. Validación de las expresiones obtenidas. . . . . . . . 189

B. ELEVADOR ZVS 191

B.1. Funcionamiento del Elevador ZVS . . . . . . . . . . . . . 191

B.1.1. Etapa 1. $\left(t_{0}<t<t_{1}\right) \ldots \ldots \ldots 192$

B.1.2. Etapa 2. $\left(t_{1}<t<t_{2}\right) \ldots \ldots \ldots 3$

B.1.3. Etapa 3. $\left(t_{2}<t<t_{3}\right) \ldots \ldots \ldots 196$

B.1.4. Etapa 4. $\left(t_{3}<t<t_{4}\right) \ldots \ldots \ldots 198$

B.1.5. Etapa 5. $\left(t_{4}<t<t_{5}\right) \ldots \ldots \ldots 202$

B.1.6. Etapa 6. $\left(t_{5}<t<t_{6}\right) \ldots \ldots \ldots 204$

B.2. Análisis en régimen permanente. . . . . . . . . . . 204

B.2.1. Sin considerar rizado. . . . . . . . . . . . . 204

B.2.2. Considerando rizado. . . . . . . . . . . . . . . 208

B.2.3. Comparativa. . . . . . . . . . . . . . . 213

B.3. Modelo promediado . . . . . . . . . . . . . . . . . 214

B.3.1. Modelo promediado para el elevador ZVS . . . . . . . 215

B.3.2. Balance energético . . . . . . . . . . . . . . . . . 219

B.3.3. Validación del modelo promediado . . . . . . . . . 220

B.4. Modelo en régimen permanente . . . . . . . . . . . . . . 221

B.4.1. Balance energético . . . . . . . . . . . . . . 224

B.5. Modelo en pequeña señal . . . . . . . . . . . . . . . . . . . 224

B.6. Funciones de transferencia . . . . . . . . . . . . . . . . . 229

B.6.1. Desde el modelo o el circuito de pequeña señal . . . . 230

B.6.2. Desde el modelo matricial . . . . . . . . . . . . . . . 237

B.7. Impedancia de salida. . . . . . . . . . . . . . . 238

B.7.1. Cálculo de $Z_{t h} \ldots \ldots \ldots$. . . . . . . . . . . . 239

B.7.2. Cálculo de $Z_{\text {out }}$. . . . . . . . . . . . . . . . . . . 242 
B.7.3. Validación de las expresiones obtenidas.

\section{REDUCTOR ELEVADOR ZVS}

C.1. Funcionamiento del Reductor-Elevador ZVS . . . . . . . . . . 247

C.1.1. Etapa 1. $\left(t_{0}<t<t_{1}\right) \ldots \ldots \ldots$. . . . . . 248

C.1.2. Etapa 2. $\left(t_{1}<t<t_{2}\right) \quad \ldots \ldots \ldots$. . . . . . . 249

C.1.3. Etapa 3. $\left(t_{2}<t<t_{3}\right) \ldots \ldots \ldots \ldots 252$

C.1.4. Etapa 4. $\left(t_{3}<t<t_{4}\right) \quad \ldots \ldots \ldots . \ldots \ldots 254$

C.1.5. Etapa 5. $\left(t_{4}<t<t_{5}\right) \ldots \ldots$. . . . . . . . 259

C.1.6. Etapa 6. $\left(t_{5}<t<t_{6}\right) \ldots \ldots . \ldots 260$

C.2. Análisis en régimen permanente. . . . . . . . . . . 261

C.2.1. Sin considerar rizado. . . . . . . . . . . . . . 261

C.2.2. Considerando rizado. . . . . . . . . . . . . . 265

C.2.3. Comparativa. . . . . . . . . . . . . . . . . 270

C.3. Modelo promediado . . . . . . . . . . . . . . . . . . . 271

C.3.1. Modelo promediado para el reductor-elevador ZVS . . 272

C.3.2. Balance energético . . . . . . . . . . . . . . . . 277

C.3.3. Validación del modelo promediado . . . . . . . . . . . 277

C.4. Modelo en régimen permanente . . . . . . . . . . . 278

C.4.1. Balance energético . . . . . . . . . . . . . . . 281

C.5. Modelo en pequeña señal . . . . . . . . . . . . . . . . . . . . 282

C.6. Funciones de transferencia . . . . . . . . . . . . . . . . . 288

C.6.1. Desde el modelo o el circuito de pequeña señal . . . . 288

C.6.2. Desde el modelo matricial . . . . . . . . . . . . . . . . 294

C.7. Impedancia de salida . . . . . . . . . . . . . . . . . . 295

C.7.1. Cálculo de $Z_{t h} \ldots \ldots$. . . . . . . . . . . . . . . 295

C.7.2. Cálculo de $Z_{\text {out }}$. . . . . . . . . . . . . . . . . . 298

C.7.3. Validación de las expresiones obtenidas. . . . . . . . . 300

D. FLYBACK ZVS TIPO BUCK-BOOST 305

D.1. Descripción y funcionamiento. . . . . . . . . . . . 305

D.1.1. Etapa 1. $\left(t_{0}<t<t_{1}\right) \ldots \ldots 307$

D.1.2. Etapa 2. $\left(t_{1}<t<t_{2}\right) \ldots \ldots \ldots 308$

D.1.3. Etapa 3. $\left(t_{2}<t<t_{3}\right) \ldots \ldots \ldots 311$

D.1.4. Etapa 4. $\left(t_{3}<t<t_{4}\right) \ldots \ldots \ldots 314$

D.1.5. Etapa 5. $\left(t_{4}<t<t_{5}\right) \ldots \ldots \ldots 319$

D.1.6. Etapa 6. $\left(t_{5}<t<t_{6}\right)$. . . . . . . . . . 320

D.2. Análisis en régimen permanente. . . . . . . . . . . . 321

D.2.1. Sin considerar rizado. . . . . . . . . . . . . 321

D.2.2. Considerando rizado. . . . . . . . . . . . . . . 325

D.2.3. Comparativa. . . . . . . . . . . . . . 330 
D.3. Modelo promediado . . . . . . . . . . . . . . . . . 332

D.3.1. Modelo promediado para el flyback ZVS . . . . . . . 332

D.3.2. Balance energético . . . . . . . . . . . . 337

D.3.3. Validación del modelo promediado . . . . . . . . . . 338

D.4. Modelo en régimen permanente . . . . . . . . . . . . . 340

D.4.1. Balance energético . . . . . . . . . . . . . . 342

D.5. Modelo en pequeña señal . . . . . . . . . . . . . . . . 343

D.6. Funciones de transferencia . . . . . . . . . . . . . . . 351

D.6.1. Desde el modelo o el circuito de pequeña señal . . . . 351

D.6.2. Desde el modelo matricial . . . . . . . . . . . . 358

D.7. Impedancia de salida . . . . . . . . . . . . . . . . . . 359

D.7.1. Cálculo de $Z_{t h} \ldots \ldots$. . . . . . . . . . . . . 360

D.7.2. Cálculo de $Z_{\text {out }}$. . . . . . . . . . . . . . . . . 362

D.7.3. Validación de las expresiones obtenidas. . . . . . . . 365

E. FLYBACK ZVS TIPO BOOST 369

E.1. Descripción y funcionamiento. . . . . . . . . . . . . . . 369

E.1.1. Etapas. . . . . . . . . . . . . . . . 370

E.2. Análisis en régimen permanente. . . . . . . . . . . . . 374

E.2.1. Sin considerar rizado. . . . . . . . . . . . . 374

E.2.2. Considerando rizado. . . . . . . . . . . . . . 378

E.2.3. Comparativa. . . . . . . . . . . . . . . 383

E.3. Modelo promediado. . . . . . . . . . . . . . . . 385

E.3.1. Balance energético . . . . . . . . . . . . . . 391

E.3.2. Validación del modelo promediado . . . . . . . . . . 392

E.4. Modelo en régimen permanente . . . . . . . . . . . . 393

E.4.1. Balance energético . . . . . . . . . . . . . . . 396

E.5. Modelo en pequeña señal . . . . . . . . . . . . . . . . 397

E.6. Impedancia de salida . . . . . . . . . . . . . . . 405

E.6.1. Cálculo de $Z_{t h} \ldots \ldots \ldots$. . . . . . . . 406

E.6.2. Cálculo de $Z_{\text {out }}$. . . . . . . . . . . . . . . . . 407

E.6.3. Validación de las expresiones obtenidas. . . . . . . . . 411

F. FLYBACK ZVS MODIFICADO

F.1. Descripción y funcionamiento. . . . . . . . . . . . . . 415

F.1.1. Etapas. . . . . . . . . . . . . . . . . 417

F.2. Análisis en régimen permanente. . . . . . . . . . . . . 422

F.2.1. Sin considerar rizado. . . . . . . . . . . . . . 422

F.2.2. Considerando rizado. . . . . . . . . . . . . . . . . 429

F.2.3. Comparativa. . . . . . . . . . . . . . 446

F.3. Modelo promediado. . . . . . . . . . . . . . . . . 448 
F.3.1. Balance energético . . . . . . . . . . . . . . 458

F.3.2. Validación del modelo promediado . . . . . . . . . . 460

F.4. Modelo en régimen permanente . . . . . . . . . . . . . . 461

F.4.1. Balance energético . . . . . . . . . . . . . . . 464 


\section{Índice de figuras}

1.1. Tipos de arquitectura para un sistema de alimentación.(LuO y BAtarseh, 2005, Fig.1): a) centralizada; b) modular; y c) distribuida. . . . . . . . . . . . . 3

1.2. Ejemplo típico de un sistema distribuido.(Sun, 2007, Fig.1.1). 4

1.3. Conexión en paralelo de: a) dos etapas; b) dos convertidores. . 7

1.4. Ejemplo de controlador para los convertidores de la Figura 1.3. 7

1.5. Característica de salida de un convertidor de potencia regulado en tensión y con limitación de corriente. . . . . . . . . 9

1.6. Métodos de reparto de corriente según (LuO y otros, 1999). . 11

1.7. Convertidores regulados en tensión y con limitación de corriente: a) característica V-I; b) reparto de carga en paralelo. 12

1.8. Característica V-I para dos convertidores con tensión de vacío y resistencia de salida diferentes. . . . . . . . . . . . . 13

1.9. Reparto de corriente vs. regulación de tensión. $R_{d r o o p, a} \geq$ $R_{\text {droop }, b \ldots \ldots \ldots \ldots \ldots \ldots \ldots \ldots \ldots}$

1.10. Uso de resistencias ecualizadoras en reparto pasivo. . . . . . . 17

1.11. Medida de la corriente para producir droop. . . . . . . . . . 19

1.12. Control en modo tensión con baja ganancia. . . . . . . . . . . 21

1.13. Control en modo corriente con baja ganancia. . . . . . . . . . 21

1.14. Curvas droop en función de $I_{o, r e f} \ldots \ldots \ldots \ldots 23$

1.15. Redes de protección de transistores contra sobretensiones: a) snubber RC; b) snubber RCD; c) snubber LC; y d) snubber

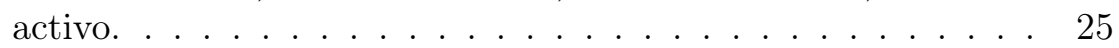

1.16. Red de apagado con otra posible conexión del snubber. Fig. 24.12 de (Kassakian y otros, 1991) . . . . . . . . . 26

1.17. Red de sobretensión o voltage clamp. . . . . . . . . . . . 26

1.18. Empleo de redes de enclavamiento activo en diferentes convertidores: a) en un forward; y b) en un flyback. . . . . . . . 28

1.19. Convertidores con red de enclavamiento activo: a) reductor; b) elevador; c) reductor elevador. . . . . . . . . . . . . . 29 
2.1. Convertidores con red de enclavamiento activo: a) reductor; b) elevador; c) reductor elevador; y d) Flyback. . . . . . . . . 34

2.2. Principales curvas en un convertidor ZVS. . . . . . . . . . . 36

2.3. Principales curvas en un convertidor ZVS al considerar rizado. 37

2.4. Simulación de un elevador ZVS: a) Tensión de salida; b) Intensidad en el diodo; y c) Intensidad en L . . . . . . . . . . . 42

2.5. Simulación de un reductor ZVS: a) Tensión de salida; b) Intensidad en el diodo principal; y c) Intensidad en L . . . . . . 43

2.6. Simulación de un Red-Elev. ZVS: a) Tensión de salida; b) Intensidad en el diodo principal; y c) Intensidad en L . . . . . 44

2.7. Simulación de un flyback ZVS: a) Tensión de salida; b) Intensidad en el diodo principal; y c) Intensidad en L . . . . . . . . 45

2.8. Caract.de salida para reductor ZVS: a) según $L_{r} ;$ y b) según $L .47$

2.9. Caract.de salida en función de $L_{r}$ (izqda.) y de $L$ (dcha.) para: a) elev.ZVS ; b) red.elev.ZVS; y c) Flyb.ZVS. . . . . . . . . . 48

2.10. Corriente de salida para tres etapas reductor ZVS en paralelo: a) con variación en $L_{r} ; \mathrm{y}$ b) con variación en $L \ldots$. . . . . . . 50

2.11. Corrientes en diodo y bobina principal de tres etapas elevador ZVS en paralelo con variación en $L$. . . . . . . . . . . . . . . 51

2.12. Corrientes en diodo y bobina principal de tres etapas flyback ZVS en paralelo con variación en $L_{m} \ldots \ldots$. . . . . . . . . . 52

2.13. Circuito promediado para convertidores ZVS: a) reductor; b) elevador; c) red-elev.; y d) flyback. . . . . . . . . . . . . . . 57

2.14. Circuitos en régimen permanente para: a) reductor ZVS; b) elevador ZVS; d) red-elev. ZVS, y d) flyback ZVS. . . . . . . . 58

3.1. Circuitos en pequeña señal para convertidores ZVS. . . . . . . 64

3.2. Circuitos empleados para obtener la impedancia de salida para convertidores ZVS. . . . . . . . . . . . . . . 65

3.3. $\left|Z_{\text {out }}(s)\right|$ para convertidores ZVS. . . . . . . . . . 67

3.4. $\left|Z_{m e}(s)\right|$ para convertidores ZVS. . . . . . . . . . . . 68

3.5. Efecto de $C_{c}$ en $\left|Z_{m e}(s)\right|$ para convertidores ZVS. . . . . . . 69

3.6. Efecto del ciclo de trabajo en $\left|Z_{m e}(s)\right|$ para convertidores ZVS. 70

3.7. Efecto del ciclo de trabajo en $\left|Z_{m e}(s)\right|$ cuando $V_{o}$ es constante. 71

3.8. Término $\left|Z_{t h}(s)\right|$ con sus asíntotas. Determinación de $f_{1}$ y $f_{2}$. 72

3.9. Evolución de $i_{L}(t)$ para variación del $10 \%$ en carga resistiva para un convertidor formado por tres etapas en paralelo. . . . 74

3.10. Evolución de $i_{L}(t)$ para $\Delta D=0,01$ en ciclo de trabajo. . . . . 75

3.11. Evolución de $i_{L}(t)$ ante perturbaciones para elevador(izqda.) y red.elev.(dcha.) y $\left|Z_{t h}(s)\right|$, con diferente condensador de clamp. 76

3.12. Evolución de $i_{L}(t)$ cuando cambia el ciclo de trabajo de una de las etapas, para reductor (izquierda) y flyback (derecha). . 77 
3.13. Tensión de clamp, $V_{c}$, en función de $L_{r}$ para mismo régimen de carga. . . . . . . . . . . . . . . . . 79

3.14. Reductor ZVS: a) modelo promediado; y b) circuito empleado para obtener $i_{L}$ con tensión constante a la salida. . . . . . . . 81

3.15. Reductor ZVS de tres etapas al conectar una de ellas. . . . . 81

3.16. Evolución de $i_{L}(t)$ al reclutar una etapa. . . . . . . . . . 81

3.17. a) Ganancia y b) fase de $G_{i d}(s)$ para reductor normal y ZVS. 82

3.18. Elevador ZVS: a) modelo promediado; y b) circuito empleado para obtener $i_{L}$ con tensión constante a la salida. . . . . . . . 83

3.19. Elevador ZVS de tres etapas al conectar una de ellas. . . . . . 83

3.20. Evolución de $i_{L}(t)$ al reclutar una etapa. . . . . . . . . . 84

3.21. a) Ganancia y b) fase de $G_{i d}(s)$ para elevador normal y ZVS. 84

3.22. Red.Elev.ZVS: a) modelo promediado; y b) circuito empleado para obtener $i_{L}$ con tensión constante a la salida. . . . . . 85

3.23. Evolución de $i_{L}(t)$ al reclutar una etapa. . . . . . . . 86

3.24. a) Ganancia y b) fase de $G_{i d}(s)$ para red.elev. normal y ZVS. $\quad 86$

4.1. Flyback ZVS con red de enclavamiento activo: a) tipo buckboost; b) tipo boost. . . . . . . . . . . . . . . . . 91

4.2. Convertidor flyback ZVS-boost con capacidades parásitas. . . 93

4.3. El mismo convertidor tras agrupar las capacidades parásitas. . 94

4.4. Etapas por las que evoluciona el flyback ZVS-real en un periodo: (a) Etapa 1. (b) Etapa 2. (c) Etapa 3. (d) Etapa 4. (e) Etapa 5. (f) Etapa 6. . . . . . . . . . . . . . . . . . . . 95

4.6. Etapa 1: (a) Circuito equivalente; (b) y (c) Mallas obtenidas tras superposición; y (d) Simplificación de (a) cuando $v_{C_{e q}}(t)$

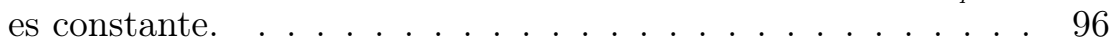

4.5. Circuitos equivalentes a los de la Figura 4.4: (a) Etapa 1. (b) Etapa 2. (c) Etapa 3. (d) Etapa 4. (e) Etapa 5. (f) Etapa 6. . 97

4.7. Etapa 6: (a) Circuito equivalente; (b) y (c) mallas obtenidas al aplicar superposición. . . . . . . . . . . . . . . . 98

4.8. Etapa 2: (a) Circuito equivalente con condiciones iniciales; b) y (c) mallas obtenidas tras superposición. . . . . . . . . . 98

4.9. Curvas principales de un flyback ZVS-real. . . . . . . . . . . . 101

4.10. Característica de salida de un flyback ZVS-real. . . . . . . . . 109

4.11. Característica de salida ante variaciones de $C_{t}$. . . . . . . 109

4.12. Convertidor flyback ZVS-real con diodo $D_{c l}$. . . . . . . . . 110

4.13. Etapas por las que evoluciona el flyback ZVS-modif. durante un ciclo: (a) Etapa 1. (b) Etapa 2. (c) Etapa 3. (d) Etapa 4.

(e) Etapa 5. (f) Etapa 6. (g) Etapa 7. (h) Etapa 8. . . . . . . 113 
4.14. Circuitos empleados para el análisis del flyback ZVS-modif. durante un periodo completo: (a) Etapa 1. (b) Etapa 2. (c) Etapa 3. (d) Etapa 4. (e) Etapa 5. (f) Etapa 6. (g) Etapa 7. (h) Etapa 8. . . . . . . . . . . . . . . . . . . . . . 114

4.15. Curvas ideales para el flyback ZVS-modificado. . . . . . . . . 116

4.16. Característica de salida de un flyback ZVS-modificado. . . . . 120

4.17. Característica de salida de un flyback ZVS-modificado para diferentes valores de $C_{t}$. . . . . . . . . . . . . . 120

4.18. Circuito promediado del flyback ZVS-modificado. . . . . . . . 122

4.19. Simulación de la respuesta a un escalón en el ciclo de trabajo de un flyback ZVS-modif. y su modelo promediado. . . . . . . 123

4.20. Circuito en régimen permanente de un flyback ZVS-modificado.124

4.21. Conexión de las dos etapas que forman el prototipo. . . . . . 127

4.22. Prototipo de laboratorio:dos etapas flyback ZVS-modificadas conectadas en paralelo y bloque de control. . . . . . . . . 127

4.23. Corriente en bobina resonante y en transistor principal: a) sin diodo clamp; y b) con diodo de clamp(derecha). . . . . . . . . 128

4.24. Característica de salida para las dos etapas del convertidor con diodo de clamp (línea continua) y sin diodo (línea de trazos) con diferentes bobinas resonantes. . . . . . . . . . . . . . 128

4.25. Tensión de salida y corriente en $L_{r}$ para las dos etapas cuando entra en funcionamiento la etapa 2 (izqda.) y detalle (dcha.). 131

4.26. Tensión de salida y corriente en $L_{r}$ para las dos etapas cuando se desconecta la etapa 2 (izqda.) y detalle (dcha.). . . . . . . 132

4.27. Rendimiento del prototipo. Entrada de segunda etapa con 70 W.132

A.1. Reductor ZVS. . . . . . . . . . . . . . . . . 137

A.2. Reductor ZVS durante la etapa 1. Circuito equivalente. . . . . 138

A.3. Reductor ZVS durante la etapa 2. Circuito equivalente. . . . . 139

A.4. Malla izquierda del circuito equivalente: a) en el dominio del tiempo; y b) en el dominio de Laplace. . . . . . . . . . . . . . 141

A.5. Equivalente Thévenin del conjunto bobina-fuente de corriente. 141

A.6. Reductor ZVS durante la etapa 3. Circuito equivalente. . . . . 142

A.7. Reductor ZVS durante la etapa 4. Circuito equivalente. . . . . 144

A.8. Malla izquierda del circuito equivalente: a) en el dominio del tiempo; y b) en el dominio de Laplace. . . . . . . . . . . . . 147

A.9. Equivalente Thévenin del conjunto bobina-fuente de corriente. 147

A.10.Reductor ZVS durante la etapa 5. Circuito equivalente. . . . . 149

A.11.Reductor ZVS durante la etapa 6 . . . . . . . . . . . 150

A.12.Curvas principales de un reductor ZVS. . . . . . . . . . 151 
A.13. Circuitos empleados para el análisis del reductor ZVS considerando rizado durante un ciclo completo: (a) Etapa 1. (b) Etapa 2. (c) Etapa 3. (d) Etapa 4. (e) Etapa 5. (f) Etapa 6. . 154

A.14.Curvas principales de un reductor ZVS considerando rizado. . 155

A.15.Característica de salida de un Reductor ZVS. . . . . . . . . . 160

A.16.Modelo promediado de un reductor ZVS. . . . . . . . . . . 167

A.17.Reductor ZVS. Respuesta a escalón del ciclo de trabajo: a) Tensión de salida; y b) Intensidad en la bobina. . . . . . . . . 168

A.18.Reductor ZVS. Respuesta a escalón en el ciclo de trabajo incluyendo corrección: a) Tensión de salida; y b) Intensidad en la bobina. . . . . . . . . . . . . . . . . . . . . 169

A.19.Reductor ZVS en régimen permanente. Comparación entre el modelo promediado y los valores medios con y sin corrección en el ciclo de trabajo del circuito conmutado: a) Tensión de salida; y b) Intensidad en la bobina. . . . . . . . . . . . . . . 170

A.20.Circuito en régimen permanente de un Reductor ZVS en MCC: (a) con fuentes dependientes; (b) con transformador equivalente ideal. . . . . . . . . . . . . . . . . . . . . 171

A.21.Modelo en pequeña señal de un reductor ZVS. . . . . . . . . . 177

A.22. Circuito equivalente de un reductor cuando $\hat{d}=0 \ldots \ldots . .180$

A.23.Reductor ZVS. Func. de transferencia en función de la tensión de entrada para: a) Tensión de salida, $G_{v g}(s)$; y b) Intensidad en la bobina, $G_{i g}(s) \ldots \ldots \ldots \ldots$

A.24.Circuito equivalente de un reductor ZVS cuando $\hat{v}_{g}=0 \quad \ldots 182$

A.25.Reductor ZVS. Func. de transferencia en función del ciclo de trabajo para: a) Tensión de salida, $G_{v d}(s)$; y b) Intensidad en la bobina, $G_{i d}(s) \ldots \ldots \ldots \ldots$. . . . . . . . . 182

A.26. Circuito para obtener la impedancia de salida de un reductor ZVS. . . . . . . . . . . . . . . . . 184

A.27.Circuito empleado para obtener $Z_{t h}(s) \ldots \ldots \ldots \ldots . . \ldots 185$

A.28.Representación gráfica de $\left|Z_{t h}(s)\right|$ y los elementos que la forman.186

A.29. Circuito empleado para el cálculo de $R_{t h}$ de un reductor ZVS. 186

A.30.Circuito empleado para el cálculo de $Z_{\text {out }}(s)$ de un reductor

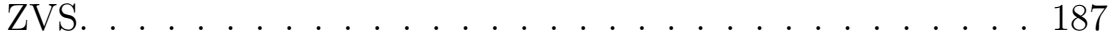

A.31.Representación gráfica de $\left|Z_{\text {out }}(s)\right|$ y los elementos que la forman. . . . . . . . . . . . . . . . . . . 188

A.32.Representación gráfica de $\left|Z_{\text {out }}(s)\right|$ y resultados de simulación. 189

B.1. Elevador ZVS. . . . . . . . . . . . . . . . . . . . 191

B.2. Elevador ZVS: a)durante la etapa 1; b)circuito equivalente. . 192

B.3. Elevador ZVS a)durante la etapa 2; b)circuito equivalente. . . 193 
B.4. Circuito equivalente de la Etapa 2: a) en el dominio del tiempo; y b) en el dominio de Laplace.

B.5. Equivalente Thévenin del conjunto bobina-fuente de corriente. 195

B.6. Elevador ZVS a)durante la etapa 3; b)circuito equivalente. . . 196

B.7. Elevador ZVS a)durante la etapa 4; b)circuito equivalente. . . 198

B.8. Circuito equivalente en la Etapa 4: a) en el dominio del tiempo; y b) en el dominio de Laplace. . . . . . . . . . . . . . . 201

B.9. Equivalente Thévenin del conjunto bobina-fuente de corriente. 201

B.10.Elevador ZVS a)durante la etapa 5; b)circuito equivalente. . . 203

B.11.Elevador ZVS durante la etapa 6. . . . . . . . . . . . . . 204

B.12.Curvas principales de un elevador ZVS. . . . . . . . . . 205

B.13. Circuitos empleados para el análisis del elevador ZVS considerando rizado durante un ciclo completo: (a) Etapa 1. (b)

Etapa 2. (c) Etapa 3. (d) Etapa 4. (e) Etapa 5. (f) Etapa 6. . 209

B.14.Curvas principales de un elevador ZVS considerando rizado. . 210

B.15. Característica de salida de un Elevador ZVS. . . . . . . . . . 215

B.16. Modelo promediado de un elevador ZVS. . . . . . . . . . . . 220

B.17.Elevador ZVS. Respuesta a escalón del ciclo de trabajo: a) Tensión de salida; y b) Intensidad en la bobina. . . . . . . . . 221

B.18.Elevador ZVS. Respuesta a escalón en el ciclo de trabajo incluyendo corrección: a) Tensión de salida; y b) Intensidad en la bobina. . . . . . . . . . . . . . . . . . 222

B.19.Elevador ZVS en régimen permanente. Comparación entre el modelo promediado y los valores medios con y sin corrección en el ciclo de trabajo del circuito conmutado: a) Tensión de salida; y b) Intensidad en la bobina. . . . . . . . . . . . . . . 222

B.20. Circuito en régimen permanente de un elevador ZVS en MCC: (a) con fuentes dependientes; (b) con transformador equivalente ideal. . . . . . . . . . . . . . . . . . . . . . . 223

B.21.Modelo en pequeña señal de un elevador ZVS. . . . . . . . . . 229

B.22. Circuito equivalente de un elevador ZVS cuando $\hat{d}=0 \ldots 231$

B.23. Circuito resultante al simplificar la malla inferior derecha. . . 232

B.24. Circuito resultante al pasar todo a la izquierda. . . . . . . . 232

B.25.Elevador ZVS. Func. de transferencia en función de la tensión de entrada para: a) Tensión de salida, $G_{v g}(s)$; y b) Intensidad en la bobina, $G_{i g}(s) \ldots \ldots \ldots \ldots \ldots \ldots$

B.26. Circuito equivalente de un elevador ZVS cuando $\hat{v}_{g}=0 \ldots 235$

B.27. Circuito resultante al simplificar la malla inferior derecha. . . 236

B.28. Circuito resultante al pasar todo al lado derecho. . . . . . . . 237

B.29. Elevador ZVS. Func. de transferencia en función del ciclo de trabajo para: a) Tensión de salida, $G_{v d}(s)$; y b) Intensidad en la bobina, $G_{i g}(s) \ldots \ldots \ldots \ldots \ldots$. . . . . . . . . . . . . . . . 
B.30.Circuito para obtener la impedancia de salida de un elevador ZVS. . . . . . . . . . . . . . . . . 239

B.31.Circuito empleado para obtener $Z_{t h}(s) \ldots \ldots \ldots \ldots$

B.32.Representación gráfica de $\left|Z_{t h}(s)\right|$ y los elementos que la forman.241

B.33. Circuito empleado para el cálculo de $R_{t h}$ de un elevador ZVS. 241

B.34. Circuito empleado para el cálculo de $Z_{\text {out }}(s)$ de un elevador ZVS: a) desplazando elementos; y b) agrupando elementos. . . 242

B.35.Representación gráfica de $\left|Z_{\text {out }}(s)\right|$ y los elementos que la forman. . . . . . . . . . . . . . . . . . . . 244

B.36. Representación gráfica de $\left|Z_{\text {out }}(s)\right|$, y resultados de simulación. 245

B.37.Representación gráfica de $\left|Z_{m e}(s)\right| \ldots \ldots \ldots . \ldots . . \ldots 246$

C.1. Reductor-Elevador ZVS. . . . . . . . . . . . . . . . . 247

C.2. Red-Elevador ZVS: a)durante la etapa $1 ;$ b)circuito equivalente; c) circuito para cálculos. . . . . . . . . . . . . . . . . . . 248

C.3. Red-Elevador ZVS a)durante la etapa 2 ; b)circuito equivalente; c) circuito para cálculos. . . . . . . . . . . . . . . . . . . 249

C.4. Circuito equivalente de la Etapa 2: a) en el dominio del tiempo; y b) en el dominio de Laplace. . . . . . . . . . . . . . . . 251

C.5. Equivalente Thévenin del conjunto bobina-fuente de corriente. 251

C.6. Red-Elevador ZVS a)durante la etapa 3; b)circuito equivalente; c) circuito para cálculos. . . . . . . . . . . . . . . 253

C.7. Red-Elevador ZVS a)durante la etapa 4 ; b)circuito equivalente; c) circuito para cálculos. . . . . . . . . . . . . . . . 255

C.8. Circuito equivalente en la Etapa 4: a) en el dominio del tiempo; y b) en el dominio de Laplace. . . . . . . . . . . . . . 257

C.9. Equivalente Thévenin del conjunto bobina-fuente de corriente. 257

C.10.Red-Elevador ZVS a)durante la etapa 5; b)circuito equivalente; c) circuito para cálculos. . . . . . . . . . . . . . . . . . 259

C.11.Red-Elevador ZVS durante la etapa 6. . . . . . . . . . . . 261

C.12.Curvas principales de un reductor-elevador ZVS. . . . . . . . 262

C.13.Circuitos empleados para el análisis del reductor-elevador ZVS considerando rizado durante un ciclo completo: (a) Etapa 1.

(b) Etapa 2. (c) Etapa 3. (d) Etapa 4. (e) Etapa 5. (f) Etapa 6.266

C.14.Curvas principales de un reductor-elevador ZVS considerando rizado. . . . . . . . . . . . . . . 267

C.15.Característica de salida de un Red.-Elev. ZVS. . . . . . . . . 272

C.16. Modelo promediado de un reductor elevador ZVS. . . . . . . . 276

C.17.Red.-Elevador ZVS. Respuesta a escalón del ciclo de trabajo:

a) Tensión de salida; y b) Intensidad en la bobina. . . . . . . 278 
C.18.Red.-Elevador ZVS. Respuesta a escalón en el ciclo de trabajo incluyendo corrección: a) Tensión de salida; y b) Intensidad en la bobina. . . . . . . . . . . . . . . . . . . . . 279

C.19.Red.-Elevador ZVS en régimen permanente. Comparación entre el modelo promediado y los valores medios con y sin corrección en el ciclo de trabajo del circuito conmutado: a) Tensión de salida; y b) Intensidad en la bobina. . . . . . . . . . . . . . 279

C.20.Circuito en régimen permanente de un reductor elevador ZVS en MCC: (a) con fuentes dependientes; (b) con transformadores ideales. . . . . . . . . . . . . . . . . . . 280

C.21.Modelo en pequeña señal de un reductor elevador ZVS. . . . . 287

C.22.Reductor-Elevador ZVS. Func. de transferencia en función de la tensión de entrada para: a) Tensión de salida, $G_{v g}(s) ;$ y b) Intensidad en la bobina, $G_{i g}(s) \ldots \ldots . \ldots . . . . .291$

C.23.Red.-Elev. ZVS. Func. de transferencia en función del ciclo de trabajo para: a) Tensión de salida, $G_{v d}(s)$; y b) Intensidad en la bobina, $G_{i g}(s) \ldots \ldots \ldots \ldots$. . . . . . . . . 294

C.24.Circuito para el cálculo de la impedancia de salida de un red.elev. ZVS. . . . . . . . . . . . . . . . . 296

C.25. Circuito empleado para el cálculo de $Z_{t h}(s)$ de un reductorelevador ZVS. . . . . . . . . . . . . . . . . . . 296

C.26. Representación gráfica de $\left|Z_{t h}(s)\right|$ y los elementos que la forman.297

C.27. Circuito empleado para el cálculo de $R_{t h}$ de un reductorelevador ZVS. . . . . . . . . . . . . . . . . 298

C.28. Circuito de la Figura C.24 a) tras cambiar L de posición; y b) agrupando elementos. . . . . . . . . . . . . . . . 299

C.29.Representación gráfica de $\left|Z_{\text {out }}(s)\right|$ y los elementos que la forman. . . . . . . . . . . . . . . . 301

C.30.Representación gráfica de $\left|Z_{\text {out }}(s)\right|$ y resultados de simulación. 302

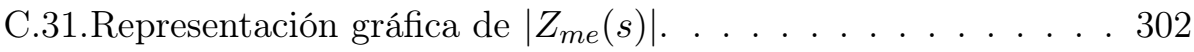

D.1. Convertidor flyback ZVS. . . . . . . . . . . . . 305

D.2. Convertidor flyback ZVS: a)durante la etapa 1; b)circuito equivalente; c) circuito para cálculos. . . . . . . . . . . . . . 307

D.3. Convertidor flyback ZVS a)durante la etapa 2 ; b)circuito equivalente; c) circuito para cálculos. . . . . . . . . . . . . 308

D.4. Circuito equivalente de la Etapa 2: a) en el dominio del tiempo; y b) en el dominio de Laplace. . . . . . . . . . . . . . . 310

D.5. Equivalente Thévenin del conjunto bobina-fuente de corriente. 310

D.6. Convertidor flyback ZVS a)durante la etapa 3; b)circuito equivalente; c) circuito para cálculos. . . . . . . . . . . . . . . 312 
D.7. Convertidor flyback ZVS a)durante la etapa 4; b)circuito equivalente; c) circuito para cálculos. . . . . . . . . . . . . . . 315

D.8. Circuito equivalente en la Etapa 4: a) en el dominio del tiempo; y b) en el dominio de Laplace. . . . . . . . . . . . . 317

D.9. Equivalente Thévenin del conjunto bobina-fuente de corriente. 317

D.10.Convertidor flyback ZVS a)durante la etapa 5; b)circuito equivalente; c) circuito para cálculos. . . . . . . . . . . . . . . . 319

D.11.Convertidor flyback ZVS durante la etapa 6. . . . . . . . . . . 321

D.12.Curvas principales de un flyback ZVS. . . . . . . . . . . . 322

D.13.Circuitos empleados para el análisis del flyback ZVS considerando rizado durante un ciclo completo: (a) Etapa 1. (b)

Etapa 2. (c) Etapa 3. (d) Etapa 4. (e) Etapa 5. (f) Etapa 6. . 326

D.14.Curvas principales de un flyback ZVS considerando rizado. . . 327

D.15.Característica de salida de un flyback ZVS. . . . . . . . . . 332

D.16.Modelo promediado de un flyback ZVS. . . . . . . . . . . . 337

D.17.Flyback ZVS. Respuesta a escalón del ciclo de trabajo: a) Tensión de salida; y b) Intensidad en la bobina. . . . . . . . . 338

D.18.Flyback ZVS. Respuesta a escalón en el ciclo de trabajo incluyendo corrección: a) Tensión de salida; y b) Intensidad en la bobina. . . . . . . . . . . . . . . . . . 339

D.19.Flyback ZVS en régimen permanente. Curvas de tensión e intensidad con y sin corrección en el ciclo de trabajo del circuito conmutado: a) Tensión de salida sin corrección e; b) Idem aplicando corrección; y c) Intensidad en la bobina. . . . . . . . . . 340

D.20.Circuito en régimen permanente de un flyback ZVS en MCC: (a) con fuentes dependientes; (b) con transformadores ideales. 341

D.21.Modelo en pequeña señal de un flyback ZVS. . . . . . . . . . 350

D.22.Circuito equivalente de un flyback ZVS cuando $\hat{d}=0$. . . . . 354

D.23.Flyback ZVS. Func. de transferencia en función de la tensión de entrada para: a) Tensión de salida, $G_{v g}(s)$; y b) Intensidad en la bobina, $G_{i g}(s) \ldots \ldots \ldots \ldots$

D.24.Circuito equivalente de un flyback ZVS cuando $\hat{v}_{g}=0 \ldots 357$

D.25.Flyback ZVS. Func. de transferencia en función del ciclo de trabajo para: a) Tensión de salida, $G_{v d}(s)$; y b) Intensidad en

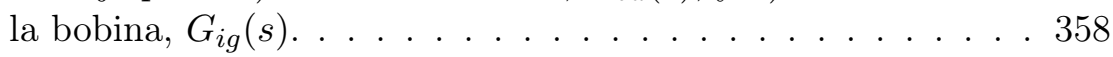

D.26.Circuito para obtener $Z_{\text {out }}(s)$ de un flyback ZVS. . . . . . . . 360

D.27.Circuito empleado para el cálculo de $Z_{t h}(s)$ de un flyback ZVS.360

D.28.Representación gráfica de $\left|Z_{t h}(s)\right|$ y los elementos que la forman.361

D.29.Circuito empleado para el cálculo de $R_{t h}$ de un flyback ZVS. . 362

D.30.Circuito de la Figura D.26 a) tras cambiar $L_{m}$ de posición; b) y agrupando elementos. . . . . . . . . . . . . 363 
D.31.Representación gráfica de $\left|Z_{\text {out }}(s)\right|$ y los elementos que la forman. . . . . . . . . . . . . . . 365

D.32.Representación gráfica de $\left|Z_{\text {tot }}(s)\right|,\left|Z_{\text {out }}(s)\right|$ y resultados de simulación. . . . . . . . . . . . . 366

D.33.Representación gráfica de $\left|Z_{m e}(s)\right| \ldots \ldots \ldots$. . . . . 367

E.1. Convertidor flyback con enclavamiento activo-boost. . . . . . 369

E.2. Etapas por las que evoluciona el convertidor en un periodo: (a) Etapa 1; (b) Etapa 2; (c) Etapa 3; (d) Etapa 4; (e) Etapa $5 ;$ y (f) Etapa 6. . . . . . . . . . . . . . . . . .

E.3. Circuitos equivalentes a los de la Figura E.2 empleados para el análisis: (a) Etapa 1; (b) Etapa 2; (c) Etapa 3; (d) Etapa 4; (e) Etapa 5; y (f) Etapa 6. (La corriente $i_{x}$ indica a $i_{r}$ durante

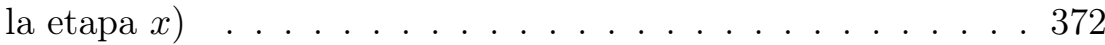

E.4. Curvas principales de un flyback ZVS-boost. . . . . . . . . . 375

E.5. Circuitos empleados para el análisis del flyback ZVS considerando rizado durante un ciclo completo: (a) Etapa 1. (b) Etapa 2. (c) Etapa 3. (d) Etapa 4. (e) Etapa 5. (f) Etapa 6. . 379

E.6. Curvas principales de un flyback ZVS-boost considerando rizado. 380

E.7. Característica de salida de un flyback ZVS-boost. . . . . . . . 385

E.8. Modelo promediado de un flyback ZVS ideal: a) obtenido a partir de las ecuaciones; b) después de simplificar. Son magnitudes promediadas. . . . . . . . . . . . . . . . 391

E.9. Flyback ZVS. Respuesta a escalón del ciclo de trabajo: a) Tensión de salida; y b) Intensidad en la bobina. . . . . . . . . 392

E.10. Respuesta a escalón en el ciclo de trabajo incluyendo corrección: a) Tensión de salida; y b) Intensidad en la bobina. . . . 393

E.11. Curvas de tensión e intensidad con y sin corrección en el ciclo de trabajo del circuito conmutado: a) Tensión de salida sin corrección e; b) Ídem aplicando corrección; y c) Intensidad en la bobina. . . . . . . . . . . . . . . . . . . . 394

E.12. Circuito en régimen permanente de un flyback ZVS en MCC:

(a) con fuentes dependientes; (b) con transformadores ideales. 395

E.13. Modelo en pequeña señal de un flyback ZVS-boost. . . . . . . 404

E.14. Circuito para obtener $Z_{\text {out }}(s)$ en un flyback ZVS-boost. . . . . 406

E.15. Circuito para calcular $Z_{t h}(s)$ en un flyback ZVS-boost. . . . . 406

E.16. Representación gráfica de $\left|Z_{t h}(s)\right|$ y los elementos que la forman.408

E.17. Circuito empleado para el cálculo de $R_{t h}$ de un flyback ZVS. . 408

E.18. Circuito de la Figura E.14 a) tras cambiar $L_{m}$ de posición; b) y agrupando elementos. . . . . . . . . . . . . . . . 409

E.19. Representación gráfica de $\left|Z_{\text {out }}(s)\right|$ y los elementos que la forman. . . . . . . . . . . . . . . . . 411 
E.20.Representación gráfica de $\left|Z_{\text {tot }}(s)\right|,\left|Z_{\text {out }}(s)\right|$ y resultados de simulación. . . . . . . . . . . . . . . 4 412

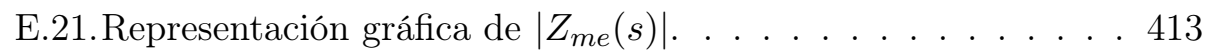

F.1. Flyback ZVS-modificado. Se añade un diodo $D_{c}$ entre $\mathrm{A}$ y masa al flyback ZVS-real. . . . . . . . . . . . . . . 4 416

F.2. Flyback ZVS-modificado tras agrupar las capacidades parásitas. 416

F.3. Etapas por las que evoluciona el flyback ZVS modificado durante un periodo: (a) Etapa 1. (b) Etapa 2. (c) Etapa 3. (d) Etapa 4. (e) Etapa 5. (f) Etapa 6. (g) Etapa 7. (h) Etapa 8. . 418

F.4. Circuitos empleados para el análisis del flyback ZVS-modif. durante un periodo completo: (a) Etapa 1. (b) Etapa 2. (c) Etapa 3. (d) Etapa 4. (e) Etapa 5. (f) Etapa 6. (g) Etapa 7.

(h) Etapa 8. . . . . . . . . . . . . . . . . . . 4 420

F.5. Principales curvas para el flyback ZVS-modif. . . . . . . . . . 423

F.6. Circuitos empleados para el análisis del flyback ZVS-modificado, durante un periodo completo considerando rizado: (a) Etapa 1. (b) Etapa 2. (c) Etapa 3. (d) Etapa 4. (e) Etapa 5. (f) Etapa 6. (g) Etapa 7. (h) Etapa 8. . . . . . . . . . . . . . . . . . . . 430

F.7. Principales curvas de un flyback ZVS-modif. considerando rizado. . . . . . . . . . . . . . . . . 4 432

F.8. Característica de salida de un flyback ZVS-modif. . . . . . . . 448

F.9. Modelo promediado de un flyback ZVS-modificado. Son magnitudes promediadas. La fuente dependiente $i_{e q, 2+3}$ equivale a la suma de las fuentes $i_{e q, 2}$ con $i_{e q, 3} \ldots \ldots$. . . . . . . 458

F.10.Flyback ZVS-modif. Respuesta a escalón del ciclo de trabajo: a) Tensión de salida; y b) Intensidad en la bobina. . . . . . . 460

F.11. Respuesta a escalón en el ciclo de trabajo incluyendo corrección: a) Tensión de salida; y b) Intensidad en la bobina. . . . 461

F.12. Curvas de tensión e intensidad con y sin corrección en el ciclo de trabajo del circuito conmutado: a) Tensión de salida sin corrección e; b) Ídem aplicando corrección; y c) Intensidad en la bobina. . . . . . . . . . . . . . . . 462

F.13. Circuito en régimen permanente de un flyback ZVS-modificado. La fuente dependiente $I_{e q, 2+3}$ equivale a la suma de las fuentes $I_{e q, 2}$ con $I_{e q, 3} \ldots \ldots \ldots \ldots$. . . . . . . . . . . 464 



\section{Índice de Tablas}

2.1. Tensiones en un convertidor ZVS cuando no hay rizado. . . . 38

2.2. Tensiones en un convertidor ZVS al considerar rizado. . . . . 38

2.3. Intensidad media en la bobina principal. . . . . . . . . . . . 39

2.4. Ciclo efectivo y rizado de corriente en la bobina. . . . . . . . 40

2.5. Tensiones en función de la duración de los intervalos. . . . . . 40

2.6. Tensiones en función de la corriente en la bobina. . . . . . . . 40

2.7. Parámetros de un elevador ZVS. . . . . . . . . . . . . . . 41

2.8. Elevador ZVS: cálculos teóricos y simulados . . . . . . . . . . 42

2.9. Parámetros de un reductor ZVS. . . . . . . . . . . . 43

2.10. Reductor ZVS: cálculos teóricos y simulados . . . . . . . . . . 44

2.11. Parámetros de un red-elev. ZVS. . . . . . . . . . . . . . 44

2.12. Red-Elev ZVS: cálculos teóricos y simulados . . . . . . . . . . 44

2.13. Parámetros de un flyback ZVS. . . . . . . . . . . . . . 45

2.14. Flyback ZVS: cálculos teóricos y simulados . . . . . . . . . 45

2.15. Característica de salida. . . . . . . . . . . . . . 46

2.16. Reparto de corriente para tres etapas reductor ZVS en paralelo. Resultados teóricos (y simulados). . . . . . . . . . . . . 49

2.17. Reparto de corriente para tres etapas elevador ZVS en paralelo. Resultado teórico (y de simulación). . . . . . . . . . . . 51

2.18. Reparto de corriente para tres etapas flyback ZVS en paralelo. Resultado teórico (y de simulación). . . . . . . . . . . 52

2.19. Intensidad media de salida cuando no se considera rizado en $L .54$

2.20. Dependencia de $I_{o}$ con diferentes parámetros. . . . . . . . . . 54

2.21. Reductor ZVS. Error en el reparto para tres etapas en paralelo. 55

2.22. Elevador ZVS. Reparto de corriente para tres etapas en paralelo. 55

2.23. Red.Elev. ZVS. Error en el reparto para tres etapas en paralelo. 55

2.24. Error en reparto de corriente para dos etapas flyback ZVS en paralelo. . . . . . . . . . . . . . 56

2.25. Expresiones para resistencia y fuente de intensidad equivalentes del circuito promediado (minúscula) y de régimen permanente (Mayúscula) . . . . . . . . . . . . . . . . . . 58 
2.26. Característica de salida y su derivada. . . . . . . . . . . . 59

2.27. Expresiones del equivalente Thévenin en régimen permanente. 61

2.28. Reparto estático de carga para Elevador ZVS. Valor medio de la corriente a la salida en cada etapa cuando el circuito es conmutado, promediado y eq. Thévenin. $\left(L_{r}=70 \mu \mathrm{H}\right) \ldots$. . .

2.29. Reparto estático de carga para Reductor ZVS. Valor medio de la corriente a la salida en cada etapa cuando el circuito es conmutado, promediado y eq. Thévenin. $\left(L_{r}=2 \mu \mathrm{H}\right)$. . . . . .

2.30. Reparto estático de carga para Reductor-Elevador ZVS. Valor medio de la corriente a la salida en cada etapa cuando el circuito es conmutado, promediado y eq. Thévenin. $\left(L_{r}=70 \mu \mathrm{H}\right) .62$

2.31. Reparto estático de carga para flyback ZVS. Valor medio de la corriente a la salida en cada etapa cuando el circuito es conmutado, promediado y eq. Thévenin. $\left(L_{r}=2 \mu \mathrm{H}\right) . \quad \ldots$. . 62

3.1. Expresiones para $r_{x}$ y $g_{x}$ en el modelo en pequeña señal. . . . 63

3.2. Términos que forman $Z_{\text {out }}(s) \ldots \ldots \ldots \ldots 6$

3.3. Convertidores ZVS. Parámetros de funcionamiento. . . . . . . 66

3.4. Relación de energías para conmutaciones sin pérdidas. . . . . 78

4.1. Condiciones de trabajo de un flyback ZVS-real. . . . . . . . . 108

4.2. Condiciones de trabajo de un flyback ZVS-modificado. . . . . 119

4.3. Principales componentes empleados en el prototipo. . . . . . . 126

4.4. Datos registrados para dos etapas flyback ZVS con diferentes bobinas resonantes. . . . . . . . . . . . . . . . . 129

4.5. Datos registrados para dos etapas flyback ZVS-modif. con diferentes bobinas resonantes. . . . . . . . . . . 130

A.1. Condiciones de trabajo de un reductor ZVS. . . . . . . . . 158

A.2. Parámetros calculados cuando se considera o no el rizado. . . 159

A.3. Fracción de tiempo para cada etapa por las que evoluciona el reductor ZVS. . . . . . . . . . . . . . . . . . . . 159

A.4. Parámetros del modelo en pequeña señal del reductor ZVS. . 177

B.1. Condiciones de trabajo de un elevador ZVS. . . . . . . . . . 213

B.2. Parámetros calculados cuando se considera o no el rizado. . . 214

B.3. Fracción de tiempo para cada etapa por las que evoluciona el elevador ZVS. . . . . . . . . . . . . . . . . . . . . . 214

B.4. Parámetros del modelo en pequeña señal del reductor ZVS. 228

C.1. Condiciones de trabajo de un Red-Elev. ZVS. . . . . . . . . . 270

C.2. Parámetros calculados cuando se considera o no el rizado. . . 271 
C.3. Fracción de tiempo para cada etapa por las que evoluciona el Red-Elev. ZVS. . . . . . . . . . . . . . . . . . . . 271

C.4. Parámetros del modelo en pequeña señal del Red-Elev.ZVS. . 287

D.1. Condiciones de trabajo de un flyback. ZVS. . . . . . . . . . 331

D.2. Parámetros calculados cuando se considera o no el rizado. . . 331

D.3. Fracción de tiempo para cada etapa por las que evoluciona el flyback ZVS. . . . . . . . . . . . . . . . 331

D.4. Parámetros del modelo en pequeña señal del flyback ZVS. . . 349

E.1. Condiciones de trabajo de un flyback ZVS-boost. . . . . . . . 384

E.2. Parámetros calculados cuando se considera o no el rizado. . . 384

E.3. Fracción de tiempo para cada etapa por las que evoluciona el convertidor. . . . . . . . . . . . . . . . . 384

E.4. Parámetros del modelo en pequeña señal del flyback ZVS-boost.403

F.1. Condiciones de trabajo de un flyback ZVS-modif. . . . . . . . 447

F.2. Parámetros calculados cuando se considera o no el rizado. . . 447

F.3. Fracción de tiempo para cada etapa por las que evoluciona el convertidor. . . . . . . . . . . . . . . . 447 



\section{Capítulo 1}

\section{Estado del arte}

En este capítulo se introducen los elementos básicos sobre los que trata el reparto de la carga que gestionan varias etapas conectadas en paralelo. La literatura es amplia al respecto. Básicamente hay dos métodos que facilitan la gestión de la carga: los métodos pasivos y los métodos activos. Este trabajo se centra en los primeros. Mas concretamente, en los convertidores con conmutaciones a tensión nula, también llamadas ZVS (Zero Voltage Switching).

Finalmente se enuncian los objetivos a desarrollar en el resto de capítulos.

\subsection{Introducción}

Las aplicaciones de convertidores $\mathrm{CC} / \mathrm{CC}$ de altas prestaciones demandan principalmente velocidad de respuesta junto a una alta densidad de energía y un buen rendimiento. Una alta densidad de energía suele implicar un convertidor de pequeñas dimensiones. La disminución de tamaño se puede conseguir aumentando la frecuencia de conmutación. Así los magnéticos y condensadores reducirán su tamaño. Como efecto positivo, la respuesta dinámica del convertidor también aumenta. Sin embargo, un aumento de la frecuencia de conmutación implica elevar las pérdidas por conmutación en los dispositivos semiconductores (y disminuir el rendimiento), además del consiguiente aumento de las interferencias electromagnéticas (MOHAN y otros, 2003).

La capacidad de proceso de energía eléctrica de un convertidor continuacontinua $(\mathrm{CC} / \mathrm{CC})$ está limitada, dado que depende de los valores máximos de tensión y corriente de los dispositivos semiconductores que lo forman. Esto es, aunque existan semiconductores para trabajar con altas frecuencias de conmutación, pueden no ser los que soportan los mayores esfuerzos eléctri- 
cos. Y los que soportan un mayor número de voltios y/o amperios, (además de ser los de mayores dimensiones), se suelen caracterizar por un valor significativo de resistencia durante la conducción, lo que significa un aumento de las pérdidas por conducción y, de nuevo, la degradación del rendimiento del convertidor. Incluso se complica el diseño del convertidor al usar esos componentes electrónicos dado que hay que añadir elementos adicionales por lo que no está tan clara la ventaja del aumento de la capacidad de la energía gestionada.

Se podría pensar que la conexión en paralelo de varios semiconductores para repartir la potencia es una solución factible dado que el reparto de corriente está bien resuelto de forma estática (MOSFETs y nuevas generaciones de IGBTs). Sin embargo, éste aumenta las capacidades en los componentes reduciendo el rendimiento a baja potencia.

Una solución muy frecuente en aplicaciones que requieran alta tensión y/o alta corriente, consiste en asociar varios convertidores de menor potencia para distribuir la gestión de energía y aliviar en parte los problemas relacionados con el estrés de los componentes a la vez que aumentar la eficiencia del conjunto. También se facilita el diseño térmico dado que cada convertidor maneja solo una parte de la energía total. El proceso de diseño y los costes de desarrollo se acortan al ser unidades fabricadas de la misma forma, y como consecuencia se puede hacer escalable la capacidad energética del sistema y mejorar su fiabilidad con la sencilla inclusión de una unidad redundante que solo funcionaría cuando una de las principales tuviera algún fallo (SHORTT y otros, 1987; TABisz y otros, 1992; MiWA, 1992; Choi, 1992; MANias y Kostakis, 1993; Mammano, 1993; Watson, 1998; Balogh, 2002; Luo y Batarseh, 2005; Sun, 2007; Chen y otros, 2009).

Claramente, las ventajas que supone tener un sistema de varias unidades frente a otro con una sola son los motivos de su gran aceptación en aplicaciones de los sistemas electrónicos complejos. Por tanto, a la hora de diseñar el sistema de alimentación hay que valorar el número de unidades teniendo en cuenta la potencia a procesar (gestionar) por este equipo y donde se va a situar esa gestión. De esta forma, quedará definida la arquitectura o estructura del sistema desde el punto de vista del procesado de la energía.

Básicamente, se consideran tres tipos de arquitectura que son (LUO y BATARSEH, 2005) la arquitectura centralizada, la arquitectura modular, y la arquitectura distribuida. Se han representado en la Figura 1.1.

- Un convertidor con arquitectura centralizada tiene una única unidad de conversión de energía, que está localizada en un solo punto del sistema. Puede presentar una o varias salidas según sea la carga alimentada.

- Un convertidor con arquitectura modular incluye varias unidades localizadas en el mismo punto, que se pueden combinar entre si para 


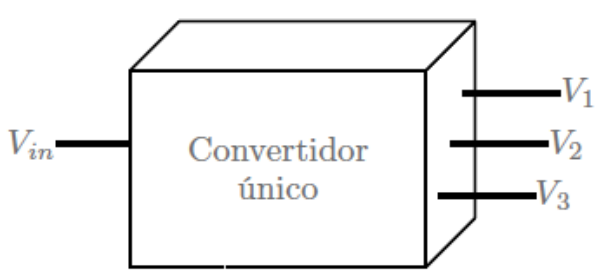

(a)

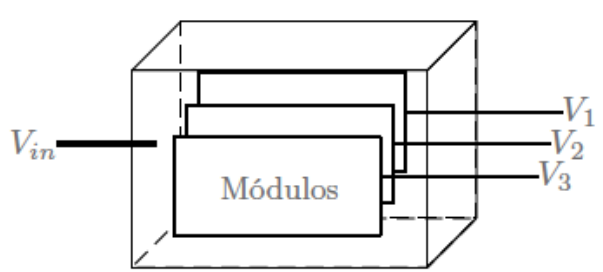

(b)

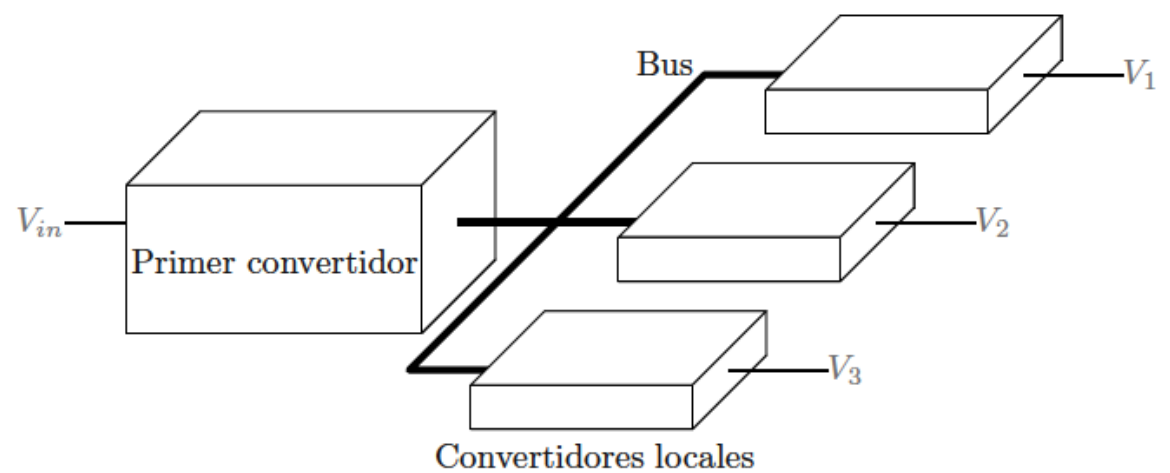

(c)

Figura 1.1: Tipos de arquitectura para un sistema de alimentación.(LuO y BAtARSEH, 2005, Fig.1): a) centralizada; b) modular; y c) distribuida.

cumplir los requisitos eléctricos (tensión, corriente y potencia) que demanda la carga. A este tipo de unidades estandarizadas se les denomina módulos. Esta arquitectura es particularmente útil para diseños de potencia elevada, porque con la conexión de varios módulos de menor potencia en un conjunto se consigue el funcionamiento equivalente a una unidad de mayor potencia además de las ventajas presentadas anteriormente.

- Por último, un convertidor con arquitectura distribuida, normalmente reparte la gestión de energía en varios puntos diferenciados. La fuente de energía alimenta a varias unidades, cuyas salidas se conectan a un bus intermedio del cual se alimentan otras unidades que están situadas más cerca de la carga. Tanto éstas como aquellas, pueden ser del tipo centralizado o del tipo modular. Un ejemplo de un sistema distribuido está reflejado en la Figura 1.2

En cuanto a la forma de combinar varias unidades de conversión de energía para formar un sistema $\mathrm{CC} / \mathrm{CC}$ completo, se habla de cuatro posibles asociaciones: entrada paralelo y salida paralelo (EPSP), entrada paralelo y salida serie (EPSS), entrada serie y salida serie (ESSS) y entrada serie y salida paralelo (ESSP). 


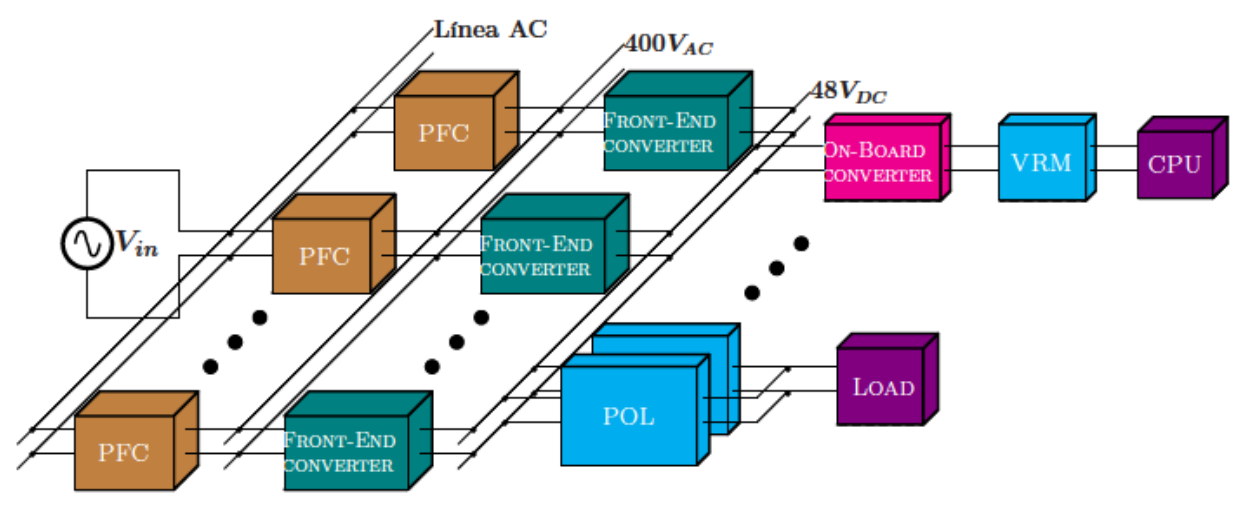

Figura 1.2: Ejemplo típico de un sistema distribuido.(Sun, 2007, Fig.1.1).

Cada esquema tiene su propia área de aplicación (CHEN y otros, 2009). Por ejemplo, los equipos relacionados con telecomunicaciones y computación, incluyen sistemas distribuidos formados por asociaciones EPSP que suministran baja tensión y altas corriente a partir de una misma fuente de energía (ChOI, 1992; PANOv y JovanOvic, 2002). Las asociaciones ESSP se emplean en las aplicaciones donde la tensión de entrada es alta y la tensión de salida es baja, como sistemas eléctricos de tracción y accionadores industriales, o fuentes auxiliares de energía (KIm y otros, 2001; GrbOviC, 2009). Otras aplicaciones como los rayos X y sistemas fotovoltaicos incorporan conexiones del tipo EPSS porque requieren altas tensiones a la salida (MANIAS y KOSTAKIS, 1993), mientras que la conexión ESSS también es utilizada en otras aplicaciones que necesitan tensiones altas tanto a la entrada como a la salida, por ejemplo (InOue y AkAGi, 2007).

Hay que indicar que el sistema CC/CC construido en base a alguno de los esquemas antes mencionado también deberá incluir algún mecanismo adicional al que incorpora el módulo para conseguir una salida regulada, bien en tensión, bien en corriente, que consiga el equilibrio entre los módulos que lo forman desde el punto de vista de la potencia. Esto es, un reparto adecuado de la tensión para los módulos conectados en serie o adecuado de la corriente entre los convertidores conectados en paralelo. Otra vez, serán necesarios elementos adicionales en prácticamente todos los casos. De forma que este es el precio que hay que pagar por todos los beneficios derivados de la interconexión de equipos.

La asociación EPSP es la más frecuente y ha sido tratada ampliamente en la literatura, así como los aspectos relacionados con su estabilidad y el mecanismo de reparto de corriente (KISLOVSKI, 1983; HirShBERG, 1985; SMALL, 1988a; MAmmano y Jordan, 1991; Siri y otros, 1992a,b; JAmerSon y otros, 1993; GlaSer y Witulski, 1994; PANOV y otros, 1997; ThOtTUVE- 
Lil y Verghese, 1998; Choi, 1998; Luo y otros, 1999; Lin y Chen, 2000; LeE y otros, 2001; BAlogh, 2002; Kim y otros, 2002; Huang y Tse, 2007; Sun, 2007; MaO y otros, 2007; De $\sim$ Jodar y otros, 2008; Huang y Tse, 2009; VillareJo y otros, 2009; DE $\sim$ JodAR, 2010; DE $\sim$ JodAR y otros, 2010, 2013), porque el diseño se podrá optimizar buscando la mayor fiabilidad y el menor coste posible cuando se aseguren las mismas variaciones térmicas y los mismos esfuerzos en los componentes de cada unidad. Los objetivos y beneficios conseguidos de esta forma se podrían resumir en los siguientes (TABISZ y otros, 1992; BALOGH, 2002):

- Modularidad. El sistema es fácilmente ajustable a diferentes combinaciones de cargas. Basta con añadir o eliminar módulos para aumentar la potencia de salida.

- Redundancia. En un sistema redundante hay al menos una unidad de reserva que puede dar un pico de corriente por encima de la corriente que demanda la carga. Así, ante un fallo en una de las unidades, se puede eliminar ésta sin interrumpir el funcionamiento del sistema. Incluso se puede hacer trabajar a cada unidad por debajo de su límite, reduciendo la energía disipada y la temperatura máxima alcanzada.

- Estandarización. El diseño de un sistema de gran potencia y altas prestaciones no es fácil. La fabricación mediante sistemas modulares de potencia estándar facilita el diseño y la construcción, por ejemplo, al aumentar el abanico de posibles componentes que pueden ser seleccionados para la fabricación de cada módulo individual.

- Minimización de estrés en componentes y mejor disipación. Las tensiones y corrientes que deben soportar los componentes son proporcionales a la potencia del convertidor, cuando se reparte la potencia entre las distintas unidades, disminuyen estos requisitos. Además, al procesar una menor potencia y teniendo un adecuado reparto de cargas, la potencia disipada es la misma y será menor que si fuera un único elemento el que maneje toda las potencia. También se tiene una mayor superficie de disipación. Esto último alargará también la vida de los componentes.

Empleando una técnica de interleaving, consistente en generar secuencias de disparo desplazadas en el tiempo para cada una de los módulos involucrados, se podrán reducir los rizados de la corriente en la entrada y salida y en la tensión (Miwa y otros, 1992; Perreault y Kassakian, 1997; MaO y otros, 2007). Por otro lado, la interconexión entre las diferentes unidades también podrá ser utilizada para asegurar la estabilidad, fiabilidad y una buena respuesta dinámica

En resumen, el estudio del reparto de la carga entre varios elementos conectados en paralelo no solo trata el equilibro o igualdad en las corrientes 
individuales, sino también la búsqueda de la máxima eficiencia del conjunto en todo el rango posible de cargas. También incluye la minimización de los posibles puntos de fallo, delimitando y/o eliminando los elementos que son únicos, para conseguir la máxima modularidad posible y facilitar, por tanto, la redundancia al sistema. La mínima o nula interconexión entre elementos también simplifica el diseño. Tampoco hay que olvidar que se debe ofrecer una buena regulación de tensión y mantener una buena dinámica ante posibles perturbaciones, a las que hay que añadir la conexión y desconexión de elementos según sea necesario o por fallo de alguno de ellos. El uso de técnicas de control que mejoren el funcionamiento del conjunto, sobre todo con la incorporación de la tecnología digital, así como la exploración de nuevos métodos avanzados de control junto al empleo de nuevas topologías, o modificación de las ya conocidas, para su uso en paralelo también importante.

\section{2. ¿Convertidor de potencia o etapa de potencia?}

Ha llegado el momento de hacer notar la diferencia entre dos términos aparentemente similares para referirse a las unidades, elementos o módulos citados anteriormente. Estos dos términos son convertidor de potencia y etapa de potencia. No se distinguen cuando se habla de un único convertidor o una única etapa de potencia, pero si cabe diferenciarlos en el caso de la asociación en paralelo.

Una etapa de potencia está constituida por los componentes por los que pasa la energía en su camino desde la fuente origen hacia la carga. El convertidor de potencia incluye la etapa de potencia y además, todos los elementos de control, sistemas de protección, etc. que son necesarios para un correcto funcionamiento.

En la Figura 1.3, se ha representado la asociación paralelo de dos convertidores y la asociación paralelo de dos etapas de potencia. La diferencia radica en la existencia de elementos duplicados en el caso de convertidores mientras que en caso de varias etapas, los elementos de control son los mínimos necesarios para asegurar el correcto funcionamiento del conjunto.

Para poder tener las mayores ventajas posibles es necesario duplicar elementos, mientras que si se busca un menor número de elementos empeora la modularidad. En el primer caso, esta claro que cada convertidor puede funcionar tanto en paralelo como de forma independiente. En el segundo caso, al existir un único controlador para todas las etapas (MAO y otros, 2007), éstas no podrán funcionar de forma independiente.

La configuración formada por de etapas en paralelo con un único controlador, sin embargo, es muy utilizada para diseñar un sólo convertidor. El nombre que recibe en la literatura es el de convertidor multifase o multietapa.

Un ejemplo de controlador analógico empleado está representado en la Figura 1.4, donde se compara una señal de entrada, $V_{o}$, por ejemplo, una medi- 


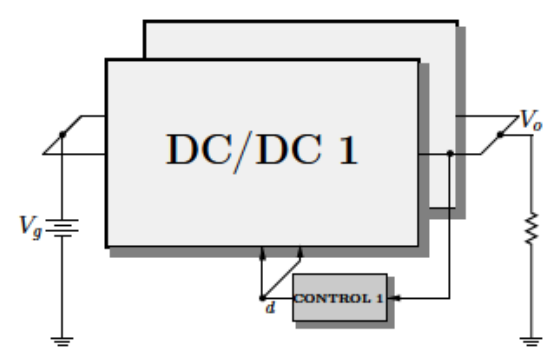

(a)

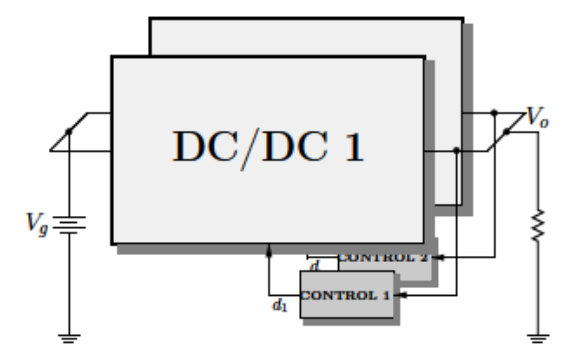

(b)

Figura 1.3: Conexión en paralelo de: a) dos etapas; b) dos convertidores.

da de la tensión de salida del convertidor realizada con un sensor de tensión, con un nivel de referencia propio.

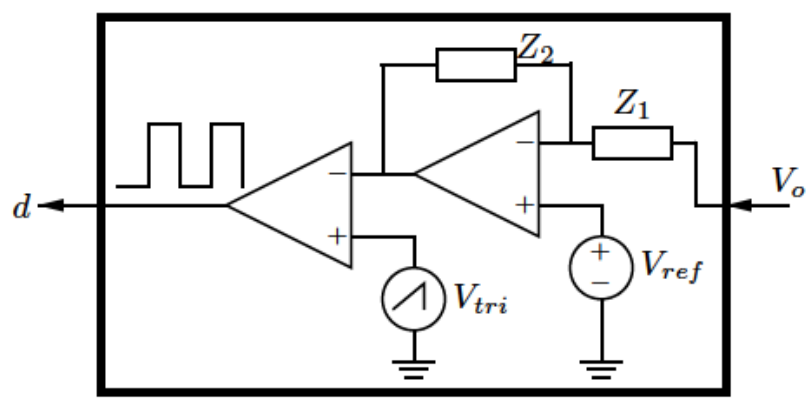

Figura 1.4: Ejemplo de controlador para los convertidores de la Figura 1.3.

El error obtenido con la diferencia de valores, pasa por un comparador, denominado amplificador de error, y sirve para generar una actuación que modifica las señales de disparo con el objetivo de minimizar el error inicial. En el caso más sencillo de varias etapas de potencia, el lazo de control genera una actuación común para todas las etapas, y basada en un mismo error, mientras que en el caso de varios convertidores, se generan sendas actuaciones del control, basadas en errores obtenidos internamente a cada convertidor.

$$
\begin{aligned}
& \text { Para el controlador de la Figura 1.4, se sabe que la salida del comparador es } \\
& \text { igual a: } \\
& \qquad V_{\text {comp }}=V_{\text {ref }}\left(1+\frac{Z_{2}}{Z_{1}}\right)-V_{o} \frac{Z_{2}}{Z_{1}} H_{V} \\
& \text { donde } H_{V} \text { es la ganancia del sensor de tensión. En régimen permanente, debe } \\
& \text { ser igual a } V_{\text {ref }} \text { por lo que, despejando } V_{o} \text { se tiene que: } \\
& \qquad V_{\text {comp }}=V_{\text {ref }} \Rightarrow V_{o}=\frac{V_{\text {ref }}}{H_{V}}
\end{aligned}
$$

En el caso de usar tecnología digital, hay un solo procesador para todas las etapas que forman el convertidor, por lo que pueden aparecer determinadas restricciones si se desean aprovechar todas las ventajas que ofrecen 
los convertidores multietapa, especialmente cuando trabajan en interleaving. Por ejemplo, al implementar todos los controladores sobre un único procesador y se necesita un cálculo individual para la actuación sobre cada etapa (suponiendo que todas las señales de disparo se generen con ese elemento) aparece una limitación en el ancho de banda disponible cuando el número de etapas es elevado porque el tiempo total disponible estará cubierto. El cálculo de una sola actuación que sea la misma para todas las etapas sería una alternativa para no llegar a este límite.

Si el lazo de control es para la tensión de salida, su actuación esta orientada a conseguir una tensión prácticamente constante a lo largo de todo el rango de trabajo, es decir, que para cualquier régimen de carga, el controlador modifica los disparos para que el convertidor ofrezca la misma tensión de salida.

En el caso de la conexión en paralelo de dos o mas convertidores, el reparto de corriente puede no existir, porque aunque sean de idéntica tensión nominal, no significa que se repartan la carga. Es más, pueden aparecer grandes desigualdades entre las corrientes de cada uno de ellos. La causa última es que, en la práctica, los convertidores presentan leves diferencias en sus características debido a varios motivos: tolerancias en la referencia interna de tensión o en las impedancias conectadas, diferencias entre los componentes que forman el amplificador de error, etc., sin mencionar los elementos parásitos o incluso el cableado. El caso de conexión directa de varios convertidores conectados en paralelo está detallado en la sección 1.4.1.

\subsection{Característica de salida}

La representación gráfica en un plano V-I de la dependencia de la tensión de salida con la corriente de salida se conoce como la característica de salida del convertidor y sirve para poner de manifiesto la relación entre ambas magnitudes. Por ejemplo, la característica de salida de un convertidor de potencia regulado/controlado en tensión está representada en la Figura 1.5. La tensión en vacío está representada por $V_{\emptyset}$, y la corriente máxima suministrada es $I_{\text {o.max }}$. Además, $I_{C L}$ es el valor a partir del cual el convertidor empieza a limitar la corriente de salida.

Como ya se ha mencionado anteriormente, la tensión de salida no depende de la corriente, y se corresponde con una línea horizontal en todo el posible rango de trabajo ${ }^{1}$. Al final del rango de trabajo, entra en juego la protección/limitación por intensidad que hace caer bruscamente la tensión hasta cero, si fuera necesario, sin un aumento apreciable de corriente. En cualquier punto de esta curva, la pendiente se corresponde con la impedancia de salida, siendo muy baja cuando el tramo es horizontal y casi infinita

\footnotetext{
${ }^{1}$ En la práctica, siempre habrá una caída de la tensión con la corriente, pero será tan leve que prácticamente se considera inexistente.
} 
al final de la zona límite en la que la curva es prácticamente vertical. Se dice

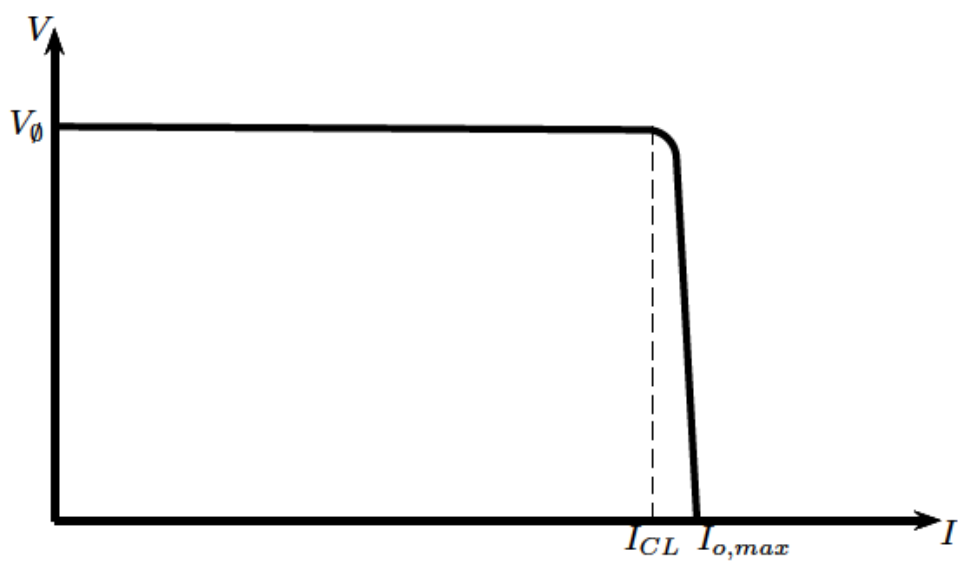

Figura 1.5: Característica de salida de un convertidor de potencia regulado en tensión y con limitación de corriente.

entonces, que, en su zona normal de funcionamiento, el convertidor tiene una baja impedancia de salida. Por el contrario, se considera cierta impedancia de salida cuando la característica de salida tiene una pendiente apreciable no nula.

\subsection{Reparto de corriente en la conexión paralelo. Métodos pasivos.}

Con el fin de asegurar un correcto funcionamiento del conjunto, a la vez que un rendimiento óptimo, es necesario un método que garantice el reparto de corrientes. Se deben prevenir las consecuencias de un mal reparto, que acortarían la vida del sistema y obligaría a desiguales esfuerzos eléctricos a los componentes de los convertidores. Una gran variedad de posibilidades han sido propuestas y estudiadas durante los últimos veinticinco años, con sus características particulares que dificultan su clasificación/selección, como: tipo de implementación (analógica o digital), medición directa o indirecta, cantidad de sensores empleados ( $\mathrm{N}, \mathrm{N}-1$, sensorless $)$, efectividad de acuerdo al número de fases, controlador (lineal o no lineal), etc.

El trabajo de Luo (LuO y otros, 1999) recogía una clasificación de los métodos existentes hasta esa fecha,según el mecanismo de operación para el reparto. Están indicados en la Figura 1.6. Básicamente, están agrupados en dos categorías: los denominados métodos pasivos, y los citados como métodos activos.Propone hasta cuatro posibles métodos pasivos y otros tantos métodos activos, en los que la actuación propuesta podría estar insertada en 
tres tipos de estructuras de control diferentes.

Los métodos pasivos, están basados en la caída de tensión que se produce en la salida de un convertidor cuando aumenta la corriente a la salida. Este comportamiento es el mismo que presentaría un convertidor ideal con una impedancia a su salida, donde a mayor valor de ésta o de la corriente, mayor es la caída de tensión. De aquí, que también se emplee el término droop para referirse a este tipo de métodos. En el punto de funcionamiento hay un reparto de corriente, mejor o peor, entre los elementos conectados. Sin embargo, ni el error en el reparto ni otro tipo de información es compartida por los elementos que forman el sistema. Por tanto, son las conexiones en paralelo más simples porque solo hay que conectar las tensiones de entrada y de salida. La regulación de tensión empeora cuando se emplean estos métodos.

Los métodos activos, al contrario que los pasivos, incluyen un mecanismo de control para el reparto de corriente, con el que se intenta conseguir un reparto casi idéntico de corrientes entre las etapas o convertidores que forman el sistema completo, a la vez que una mejor regulación de la tensión de salida frente a la de los métodos pasivos (ThotTuvelil y Verghese, 1998). Para implementar este mecanismo es necesario emplear información de otros elementos, además de estar insertado en algún punto del sistema de control propio del convertidor. Como resultado, tanto los esquemas de control como las conexiones son más complejas, y la fiabilidad del sistema ante fallos puede disminuir(SHI y otros, 2012).

En 2008, en el trabajo de Huang (HuAng, 2008) se presentó una clasificación de los tipos de conexión en paralelo desde el punto de vista de la teoría de circuitos, resultando tres posibles conexiones en paralelo. Para ello consideró que un convertidor podría funcionar como fuente de tensión o de corriente. La inclusión o no de un mecanismo de reparto de corriente añade un segundo nivel de clasificación.

A continuación se describe la problemática asociada a la conexión directa de dos convertidores cuando están fuertemente regulados en tensión y presentan cierta dependencia de la tensión con la carga. Después se presentarán los mecanismos pasivos que hacen variar la tensión de salida con la corriente y por tanto permiten cierto reparto de carga entre los elementos en paralelo. Los métodos activos están fuera del objeto de este trabajo y no serán tratados.

\subsubsection{Conexión directa.}

En un convertidor con una fuerte regulación de tensión, su característica de salida ya se ha indicado en la Figura 1.5. Al conectar dos convertidores 


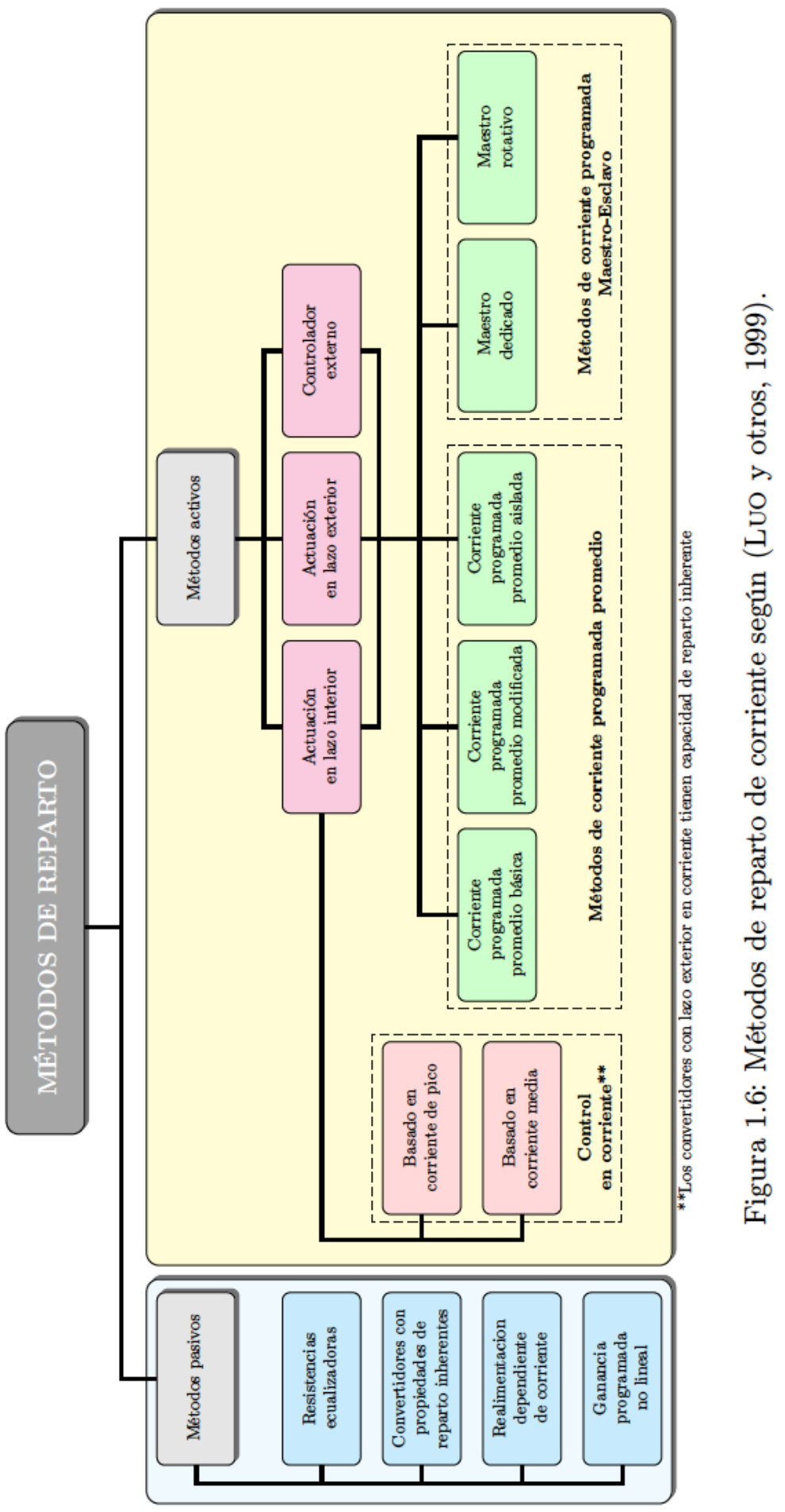


de este tipo, sus características de salida generalmente no coinciden aunque tengan la misma forma. En la Figura 1.7a se ha representado la característica de salida de dos convertidores similares.

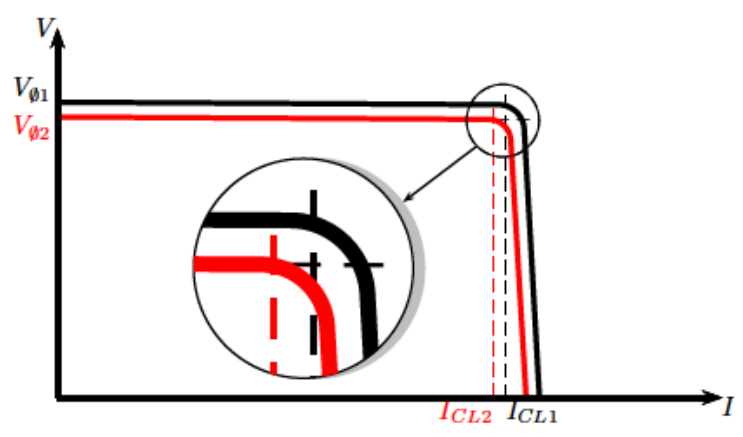

(a)

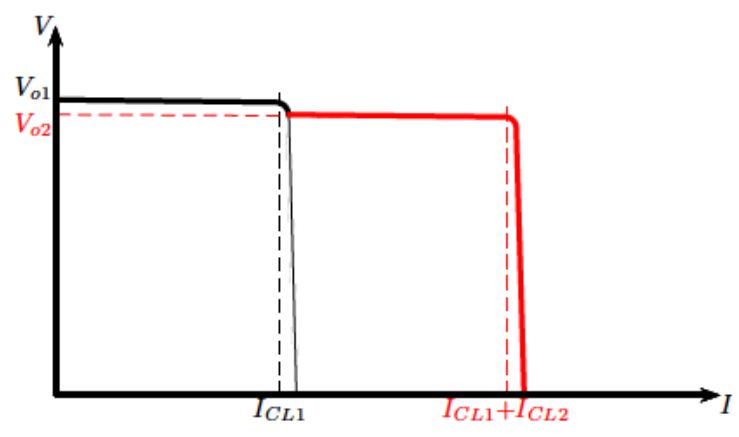

(b)

Figura 1.7: Convertidores regulados en tensión y con limitación de corriente: a) característica V-I; b) reparto de carga en paralelo.

Es prácticamente imposible que los dos tengan la misma característica, bien por diferencias entre sus controladores o entre las referencias de tensión, por lo que cada uno tendrá una curva V-I diferente tanto en las tensiones de salida en vacío, $V_{\emptyset 1}$ y $V_{\emptyset 2}$ respectivamente, como en los límites de corriente, $I_{C L 1}$ e $I_{C L 2}$ respectivamente.

A la vista de esas curvas, ya se puede intuir que el reparto de corriente no va a existir (o si existe va a ser muy malo) cuando se conectan en paralelo $^{2}$. Inicialmente, la tensión de salida, $V_{o}$, será la del convertidor con mayor tensión de salida, $V_{\emptyset 1}$, que entregará toda la corriente dado que el otro convertidor no entrará en funcionamiento. Por tanto, toda la corrien-

\footnotetext{
${ }^{2}$ Se considera cada convertidor con un flujo unidireccional de corriente, por ejemplo, con sendos diodos a la salida.
} 
te la suministra el primero. En consecuencia no existirá ningún reparto de carga.

A medida que aumenta la carga, el primer convertidor llegará a su límite de corriente, $I_{C L 1}$, y su tensión de salida se reducirá hasta igualar a la del segundo convertidor de forma que entrará en funcionamiento. Entonces, el primero pasará a trabajar como una fuente de corriente mientras que éste hará de regulador de tensión. Si hubiera mas convertidores, a medida que aumentara la carga, empezarían a trabajar sucesivamente y el orden de entrada será de mayor a menor tensión de salida.

La curva V-I conjunta para el caso de dos convertidores está indicada en la Figura 1.7b. Nótese que hay reparto de corriente cuando se sobrepasa el límite de corriente del primer convertidor. Uno dará la corriente máxima, y el otro suministrará la diferencia hasta llegar al total del valor demandado. En ese caso, más que un método de reparto de cargas es un método de reclutamiento, en el que los convertidores son reclutados según sea la demanda de carga.

Además el funcionamiento en las zonas donde entren las limitaciones puede penalizar el comportamiento dinámico de los convertidores.

\subsubsection{Efecto de la impedancia.}

En el caso de que la tensión de salida presente una dependencia con la corriente suministrada a la carga antes de la limitación de carga, las características de salida en la zona donde no hay limitación de corriente podrían ser como las de la Figura 1.8 donde se han considerado diferente tensión de salida en vacio, $V_{\emptyset 1}$ y $V_{\emptyset 2}$, y diferente resistencia de salida, $R_{1}$ y $R_{2}$, lo que se corresponde con dos curvas de diferente inclinación.

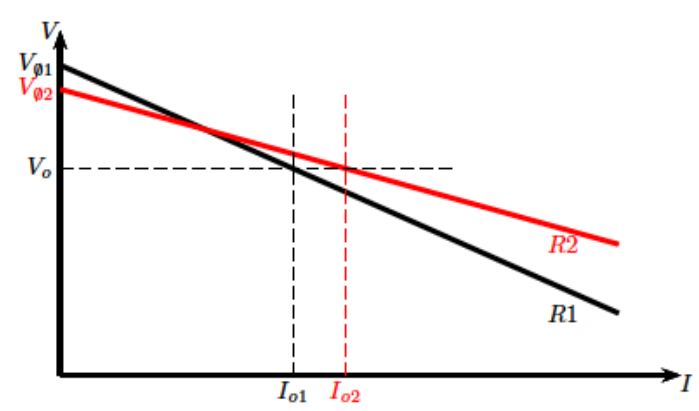

Figura 1.8: Característica V-I para dos convertidores con tensión de vacío y resistencia de salida diferentes.

La expresión de la característica de salida se corresponde con:

$$
\begin{aligned}
& V_{1}=V_{\emptyset 1}-I_{1} R_{1} \\
& V_{2}=V_{\emptyset 2}-I_{2} R_{2}
\end{aligned}
$$


$\mathrm{Al}$ estar conectados en paralelo, la tensión de salida, $V_{o}$, será la misma para los dos convertidores, por lo que a cada convertidor le corresponderá una corriente diferente de salida, $I_{o, 1}$ e $I_{o, 2}$, como se observa en la misma figura. El reparto sería idéntico únicamente si las características V-I fueran iguales. Pero, si hay alguna diferencia entre los convertidores, por leve que sea, se traducirá en diferencia en las curvas y por tanto, también en el reparto, que no será equitativo.

La diferencia de corrientes dependerá de otros parámetros como indica la expresión (1.4) donde $R_{L}$ es la resistencia de carga:

$$
\Delta I \equiv I_{o, 1}-I_{o, 2}=\frac{R_{2} V_{\emptyset 1}-R_{1} V_{\emptyset 2}+2\left(V_{\emptyset 1}-V_{\emptyset 2}\right) R_{L}}{R_{1} R_{2}+R_{1} R_{L}+R_{2} R_{L}}
$$

DEMOSTRACIÓN DE (1.4): Se despeja $V_{o}$ a partir de las dos ecuaciones de cada convertidor y de la tensión de salida como:

$$
\left.\begin{array}{l}
I_{o, 1}=\frac{V_{\emptyset 1}-V_{o}}{R_{1}} \\
I_{o, 2}=\frac{V_{\emptyset 2}-V_{o}}{R_{2}} \\
I_{o}=\left(I_{o, 1}+I_{o, 2}\right)=\frac{V_{o}}{R_{L}}
\end{array}\right\} \Rightarrow V_{o}=\frac{\left(V_{\emptyset 1} R_{2}+V_{\emptyset 2} R_{1}\right) R_{L}}{R_{1} R_{2}+R_{L}\left(R_{1}+R_{2}\right)}
$$

Entonces, la corriente que corresponde a cada convertidor es:

$$
\begin{aligned}
& I_{o, 1}=\frac{V_{\emptyset 1}-\frac{\left(V_{\emptyset 1} R_{2}+V_{\emptyset 2} R_{1}\right) R_{L}}{R_{1} R_{2}+R_{L}\left(R_{1}+R_{2}\right)}}{R_{1}}=\frac{V_{\emptyset 1} R_{2}+R_{L}\left(V_{\emptyset 1}-V_{\emptyset 2}\right)}{R_{1} R_{2}+R_{1} R_{L}+R_{2} R_{L}} \\
& I_{o, 2}=\ldots=\frac{V_{\emptyset 2} R_{1}+R_{L}\left(V_{\emptyset 2}-V_{\emptyset 1}\right)}{R_{1} R_{2}+R_{1} R_{L}+R_{2} R_{L}}
\end{aligned}
$$

Y como $\Delta I=I_{o, 1}-I_{o, 2}$, al sustituir(1.6) se obtiene el resultado de (1.4).

Con estas condiciones, se observa que, aumentando $R_{1}$ y $R_{2}$ en la misma proporción, disminuye la diferencia de corrientes, puesto que significa un aumento de la pendiente de las curvas V-I, en cuyo caso, las corrientes asignadas a cada etapa tienden a aproximarse.

Puede particularizarse la diferencia de corrientes indicada en (1.4) para el caso en el que la tensión de vacío sea la misma, o cuando las dos etapas tengan idéntica impedancia de salida. 


\section{MISMA TENSIÓN DE SALIDA EN VACIO.}

En este caso, suponiendo $V_{\emptyset}=V_{\emptyset 1}=V_{\emptyset 2}$, la diferencia (1.4) queda como:

$$
\Delta I=I_{o, 1}-I_{o, 2}=I_{o} \frac{R_{2}-R_{1}}{R_{1}+R_{2}}
$$

$$
\begin{aligned}
& \text { DEMOSTRACIÓN DE (1.7): Se obtiene } I_{o} \text { a partir de las dos ecuaciones de } \\
& \text { cada convertidor como: } \\
& \left.\begin{array}{l}
I_{o, 1}=\frac{V_{\emptyset}-V_{o}}{R_{1}} \\
I_{o, 2}=\frac{V_{\emptyset}-V_{o}}{R_{2}} \\
I_{o}=\left(I_{o, 1}+I_{o, 2}\right)=\frac{V_{o}}{R_{L}}
\end{array}\right\} \Rightarrow I_{o}=\left(V_{\emptyset}-V_{o}\right)\left[\frac{1}{R_{1}}+\frac{1}{R_{2}}\right] \\
& \text { Entonces, se despeja } V_{\emptyset} \text { de aquí como: } \\
& \qquad V_{\emptyset}=V_{o}+\frac{V_{o}}{R_{L}} \times \frac{R_{1} R_{2}}{R_{1}+R_{2}}=V_{o} \frac{R_{1} R_{2}+R_{1} R_{L}+R_{2} R_{L}}{R_{1} R_{L}+R_{2} R_{L}} \\
& \text { Cuando se sustituye (1.9) en (1.4) se obtiene el resultado indicado en (1.7). }
\end{aligned}
$$

De esta forma, se concluye que la tensión de vacío no influye en la diferencia de corrientes. Solo afectaría la relación entre las resistencias de salida de las etapas, además de la corriente de salida.

Como la tensión de salida en vacío es la misma, la conexión en paralelo de las etapas equivaldría a una única etapa con una resistencia de salida igual al paralelo de las resistencias individuales de cada una de las etapas, que estarían alimentadas del mismo punto.

Aplicada esta idea al reparto de carga, se podría analizar como si la corriente total a la salida, $I_{o}$, se repartiera entre las diferentes resistencias en paralelo, obteniendo una diferencia de corrientes que solo dependería de las resistencias individuales.

\section{MISMA RESISTENCIA DE SALIDA.}

Suponiendo $R_{1}=R_{2}=R_{\text {droop }}$, (1.4) queda como:

$$
\Delta I=I_{o 1}-I_{o 2}=\frac{\Delta V_{\emptyset}}{R_{\text {droop }}}
$$

donde $\Delta V_{0}=V_{\emptyset 1}-V_{\emptyset 2}$, es la diferencia entre las tensiones de vacío. Resulta que el reparto depende unicamente de esa diferencia y de la resistencia de salida. A mayor diferencia de tensiones, suponiendo resistencia de salida constante, se obtiene un peor reparto de corrientes, puesto que $\Delta I$ aumentaría.

Por otro lado, la mejora del reparto de corriente, aumentado el valor de $R_{\text {droop }}$, implicará una mayor diferencia de tensión dentro del rango de 
trabajo, y por tanto, una mala regulación de tensión. La situación contraria también es cierta: una buena regulación de tensión supone un mal reparto de corrientes. Cualquier diseño tendrá que considerar ambos aspectos enfrentados y llegar a un compromiso entre ellos. Esta idea se comprueba fácilmente en la Figura 1.9 donde se han elegido dos valores diferentes para $R_{\text {droop }}$, y un valor no nulo para $\Delta V_{0}$.

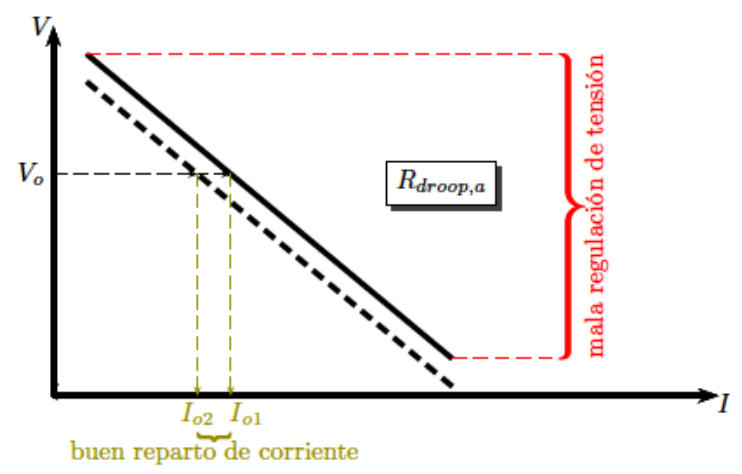

(a)

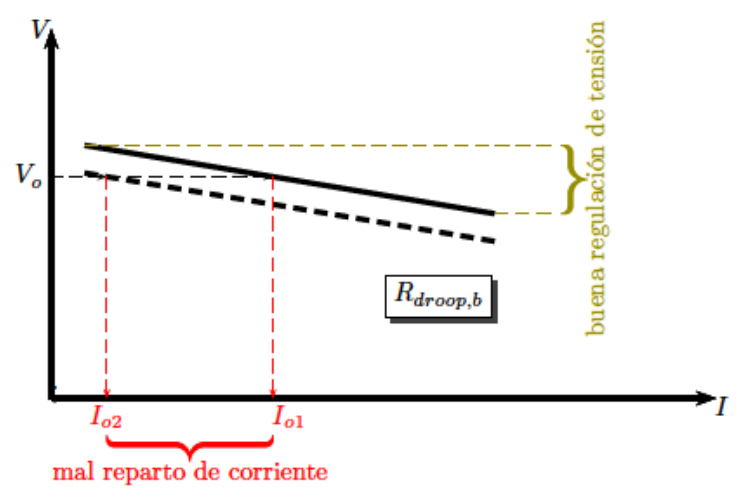

(b)

Figura 1.9: Reparto de corriente vs. regulación de tensión. $R_{\text {droop }, a} \geq R_{d r o o p, b}$

En el caso de baja carga, tampoco hay un reparto de corriente aunque las curvas tengan una pendiente similar. En vacío, la tensión de salida corresponderá al convertidor de mayor tensión. Al aumentar la carga, este convertidor seguirá suministrando energía hasta que la corriente demandada produzca una caída de tensión igual a la diferencia de las tensiones en vacío de ambos convertidores. El valor de corriente en ese punto es:

$$
\Delta I_{L L}=\frac{\Delta \emptyset}{R_{1}}
$$

A partir de este punto habría un reparto de la carga entre ambos convertidores con la diferencia de corrientes indicada en (1.4). 


\subsubsection{Resistencias ecualizadoras}

Un método sencillo para conseguir la dependencia de la tensión de salida con la corriente suministrada consiste en añadir a la salida del convertidor una resistencia serie. De esta forma, la impedancia de salida del conjunto convertidor-resistencia es mayor que sin ella y se facilita el reparto de corrientes sin tener que actuar sobre el lazo de control del convertidor.

En la Figura 1.10 se ha representado un esquema de dos convertidores que utilizan resistencias ecualizadoras para conseguir este reparto. La resistencia debe añadirse fuera del lazo de control para que la regulación de tensión no elimine esta dependencia. Nótese el uso de diodos para indicar el flujo unidireccional de corriente en cada convertidor.

La principal desventaja de este método es la degradación de la eficiencia del convertidor debido a las perdidas en esas resistencias. A mayor carga mayores pérdidas.

Por otro lado, a baja carga no se produciría un reparto de corriente porque habría un único convertidor funcionando, que correspondería al de mayor tensión sin carga.

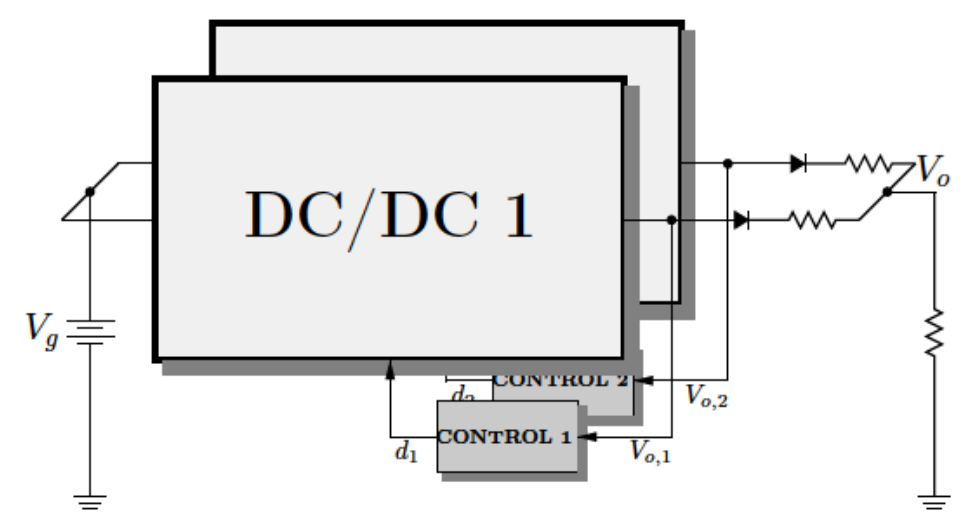

Figura 1.10: Uso de resistencias ecualizadoras en reparto pasivo.

En estas condiciones, el convertidor de mayor tensión suministrará toda la corriente mientras que la tensión no disminuya hasta la tensión en vacío del segundo convertidor. Algo similar al caso de conexión directa, mencionado en apartados anteriores en el que el segundo convertidor empezaba a funcionar cuando la tensión alcanzaba su punto de regulación.

\subsubsection{Convertidores con propiedades de reparto inherentes}

Otra forma de conseguir el reparto de corriente en un sistema en paralelo consiste en elegir convertidores que puedan presentar una dependencia de tensión con la corriente a la salida (LUO y otros, 1999; GLASER y WITULSKI, 
1994). Por ejemplo, la tensión de salida de convertidores operando en modo de conducción discontinuo (MCD), presenta una dependencia con la carga. Es posible cierto reparto sin ningún mecanismo extra. Sólo se necesitan ciclos de trabajo iguales o lo más parecidos posible y también componentes similares.

Un caso particular es la rectificación síncrona. Por ejemplo, la impedancia de salida de un reductor en modo de conducción continuo no es elevada. De hecho, está formada por la impedancia de los transistores y la resistencia de la bobina. En este caso, la diferencia de corrientes en función de la variación del ciclo de trabajo será función de la impedancia del convertidor (PETERCHEV y otros, 2003). Para $N$ etapas en paralelo:

$$
\Delta I_{i}=\frac{N-1}{N} \frac{V_{g}}{R_{e q}} \Delta d
$$

donde $R_{e q}$ representa la impedancia equivalente de cada etapa de potencia. Con un controlador digital es posible conseguir ciclos de trabajo muy similares, o con una diferencia mínima, que sea suficiente para asegurar el reparto entre las corrientes incluso con baja $R_{e q}$.

La rectificación síncrona tiene aplicación en reductores para baja tensión. Hay que indicar que la temperatura también tiene un efecto favorable en este caso. Si hubiera una etapa con una impedancia menor del resto, por ella circularía una mayor corriente, por lo que aumentaría su temperatura. El incremento térmico producirá un aumento de la resistencia del transistor, que produciría una reducción de la corriente en esa etapa.

Para mayores tensiones, se emplearían diodos, y la diferencia de corrientes no es predominantemente resistiva sino que habrá que tener en cuenta la caída de tensión del diodo. En este caso, la temperatura tendría un efecto contrario, porque a mayor corriente, mayor temperatura en la etapa, y la caída de tensión en el diodo disminuiría. La corriente aumentaría de nuevo, con el consiguiente aumento térmico. El reparto en este caso sería peor.

Los convertidores con enclavamiento activo son convertidores cuya tensión de salida también depende de la corriente. Por tanto, presentan un buen reparto de corriente cuando se conectan en paralelo, dado que tienen una impedancia de salida elevada que facilita su conexión en paralelo como (DE $\sim$ Jodar y otros, 2008; De $\sim$ JodAR, 2010; De $\sim$ JodAr y otros, 2012). Este tipo de convertidores también son interesantes desde el punto de vista de las conmutaciones sin pérdidas de los semiconductores, y son tratados mas adelante de forma mas detallada. 


\subsubsection{Realimentación dependiente de la corriente.}

Para producir el mismo efecto que con resistencias ecualizadoras, y evitar las pérdidas en ellas, se puede actuar sobre el lazo de control para implementar una impedancia de salida pero sin la limitación debida a la disipación de potencia. Uno de estos métodos es el que utiliza una medida de la corriente a la salida para actuar sobre el control. Se mide la corriente de salida y se emplea para producir una caída de la tensión de referencia del lazo de tensión del convertidor. De esta forma, a mayor corriente a la salida, la tensión de referencia disminuirá y la tensión de salida, que sigue a la referencia, tendrá la misma tendencia. Esta idea es la representada en la Figura 1.11.

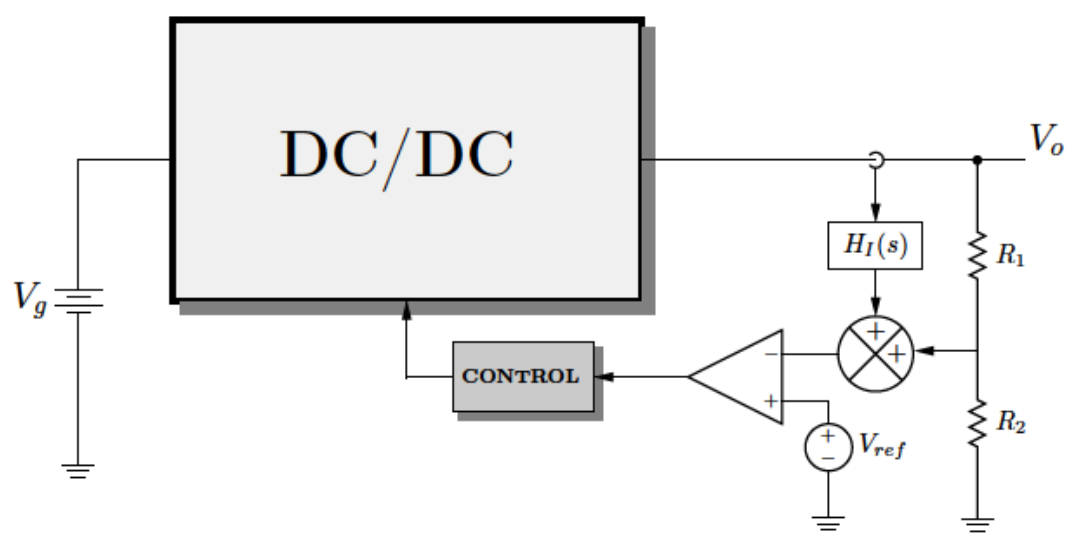

Figura 1.11: Medida de la corriente para producir droop.

En este caso, para crear una caída de tensión proporcional a la corriente de carga se puede medir la corriente de salida y restar a la referencia de tensión del lazo, una cantidad proporcional a la carga. La tensión de referencia así generada se modificará linealmente con la corriente del convertidor(BATARSEH y otros, 1994; JAMERSON y otros, 1993; IRVING y JovANOVIC, 2000). En la Figura 1.11 se representa este efecto. Ahora la tensión de salida regulada podrá calcularse como

$$
V_{o}=V_{o, \max }-R_{e q} I
$$

donde $V_{o . \max }$ y $R_{e q}$ son la tensión inicial de salida y la resistencia equivalente del sistema respectivamente. Sus expresiones son:

$$
\begin{aligned}
V_{o, \max } & =V_{\text {ref }} \frac{1}{H_{V}}=V_{\text {ref }} \frac{R_{1}+R_{2}}{R_{2}} \\
R_{e q} & =\frac{H_{I}}{H_{V}}
\end{aligned}
$$

siendo $H_{I}$ la red de medida de la corriente (que bien puede ser una resistencia) y $H_{V}$ es la red de medida de la tensión, formada por $R_{1}$ y $R_{2}$. 
DEMOSTRACIÓN DE (1.13):

La señal obtenida en el amplificador de error es igual a:

$$
V_{A E, \text { out }}=V_{\text {ref }}\left(1+\frac{Z_{2}}{Z_{1}}\right)-\left(H_{I} I+H_{V} V_{o}\right) \frac{Z_{2}}{Z_{1}}
$$

$\mathrm{Y}$ en régimen permanente, esta señal debe ser igual a $V_{\text {ref }}$ por lo que, despejando $V_{o}$ se tiene que:

$$
V_{A E, \text { out }}=V_{\text {ref }} \Rightarrow V_{o}=\frac{V_{\text {ref }}}{H_{V}}-\frac{H_{I}}{H_{V}} I
$$

Cuando no circula corriente, $I=0$ y la tensión de salida tendrá el mayor valor posible, $V_{o, \max }$. De nuevo:

$$
\left.V_{A E, \text { out }}\right|_{I=0}=V_{o, \max } \Rightarrow V_{o, \max }=\frac{V_{\text {ref }}}{H_{V}}
$$

Y la resistencia equivalente coincide con la expresión (1.14).

Con este método, se mejora la eficiencia respecto al que utiliza resistencias ecualizadoras, pero no desaparece el compromiso entre el reparto y la regulación de tensión.

\subsubsection{Baja ganancia en continua.}

Para poder implementar este tipo de control la característica en continua del convertidor debe tener dependencia de la carga, condición que se da en casi todos los convertidores, ya que todos tienen una mayor o menor impedancia de salida debida a las resistencias de los componentes, cableado, etc.(BALOGH, 2002). En lazo abierto y régimen permanente (o continua), la tensión de salida, $V_{\text {out }, O L}$ es igual a:

$$
V_{\text {out }, O L}=V_{o}-R_{o, O L} I
$$

donde $R_{o, O L}$ corresponde a la impedancia de salida en lazo abierto, que es una resistencia en continua.

Cuando se cierre el lazo de control de tensión, el valor de la impedancia de salida se reduce por un factor que es función de la ganancia del lazo, de la siguiente forma(ERICKSON y MAKSIMOVIC, 2005, Ec.(9.6)):

$$
Z_{\text {out }, C L}(s)=\frac{Z_{\text {out }, O L}(s)}{1+G_{E A}(s)}
$$

Y su valor de continua, también se verá afectado de la misma forma:

$$
R_{\text {out }, C L}=\frac{R_{\text {out }, O L}}{1+G_{E A}(0)}
$$

Si la ganancia de realimentación es grande, la impedancia de salida será muy pequeña y la dependencia de $V_{o}$ con la corriente no será apreciable 
y el reparto de corriente ya no será posible. Entonces, la estrategia a seguir es limitar la ganancia del lazo en continua, y ajustarla para conseguir la característica V-I con la inclinación deseada. Si el controlador incorpora una elemento integrador, la ganancia en continua será bastante grande. $\mathrm{Al}$ eliminar este elemento del esquema de control, la ganancia disminuye y se producirá la caída de tensión con la corriente. Así está reflejado este caso en la Figura 1.12 para un control en modo tensión.

El efecto de la baja ganancia en continua va a suponer, como efecto negativo, una mala regulación estática y dinámica.

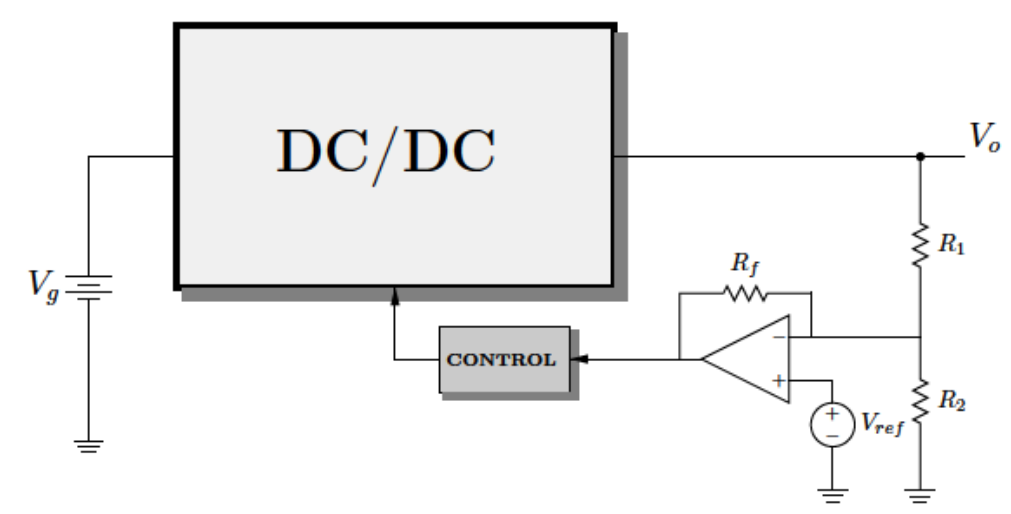

Figura 1.12: Control en modo tensión con baja ganancia.

Esta idea se puede aplicar en un lazo de corriente. Ver la Figura 1.13. En este caso, la tensión de salida presenta una ecuación como la expresión

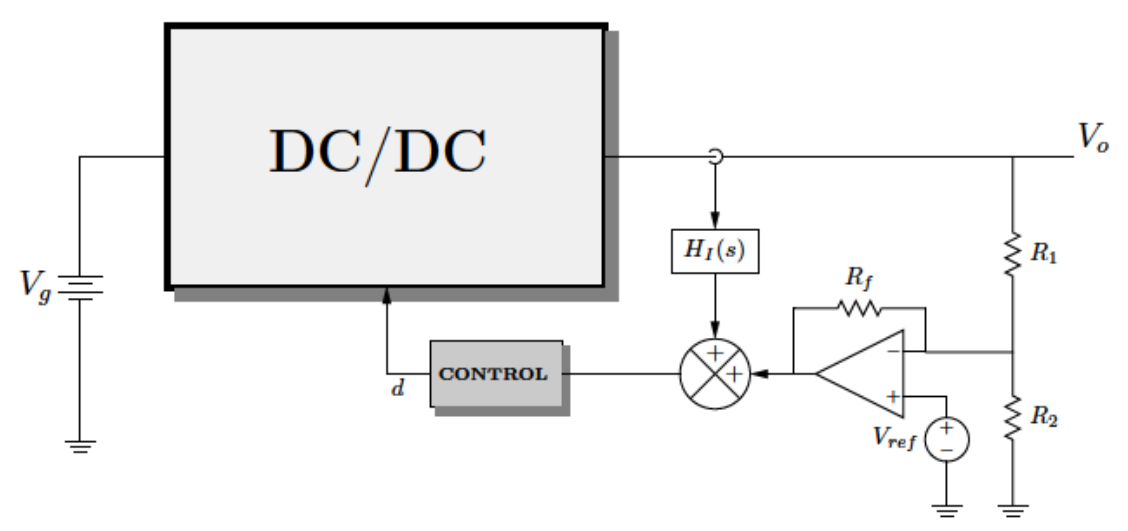

Figura 1.13: Control en modo corriente con baja ganancia.

(1.13), siendo $V_{o, \max }$ y $R_{e q}$ de la siguiente forma:

$$
\begin{aligned}
V_{o, \text { max }} & =V_{\text {ref }}\left(1+\frac{R_{1}}{R_{2}}+\frac{R_{1}}{R_{f}}\right) \\
R_{e q} & =H_{I} \frac{R_{1}}{R_{f}}
\end{aligned}
$$


donde $H_{I}$ la ganancia de la red que mide la corriente.

$$
\begin{aligned}
& \text { DEMOSTRACIÓN DE (1.21): } \\
& \text { La tensión } V_{\text {ref }} \text { aparece reflejada en el terminal } V^{-} \text {del amplif. de error. } \\
& \text { Entonces, se deduce fácilmente que la salida de este es igual a: } \\
& \qquad V_{A E, \text { out }}=V_{\text {ref }}\left(1+\frac{R_{f}}{R_{2}}+\frac{R_{f}}{R_{1}}\right)-V_{o} \frac{R_{f}}{R_{1}} \\
& \text { Y en régimen permanente,debe ser igual a } H_{I} I \text { de forma que a la salida haya } \\
& \text { una tensión igual a } V_{o} \text {, obligado por el lazo de corriente. } \\
& \text { Entonces: } \\
& \qquad V_{A E, \text { out }}=H_{I} I \Rightarrow V_{o}=V_{\text {ref }}\left(1+\frac{R_{1}}{R_{2}}+\frac{R_{1}}{R_{f}}\right)-H_{I} \frac{R_{1}}{R_{f}} I \\
& \text { Cuando no circula corriente, } I=0 \text { y la tensión de salida tendrá el mayor } \\
& \text { valor posible, } V_{o, \text { max }} \text {. De nuevo: } \\
& \qquad\left.V_{A E, \text { out }}\right|_{I=0}=V_{o, \text { max }} \Rightarrow V_{o, \text { max }}=V_{\text {ref }}\left(1+\frac{R_{1}}{R_{2}}+\frac{R_{1}}{R_{f}}\right) \\
& \text { Y la resistencia equivalente coincide con la indicada en }(1.21) .
\end{aligned}
$$

\subsubsection{Otros métodos pasivos}

Otro método consiste en implementar una ganancia no lineal programada. Se implementa un mecanismo que varia la ganancia en función de la carga, de forma que se pueda mejorar la regulación de tensión y conseguir un buen reparto de corriente, sobre todo con una carga elevada. Así se asegura el reparto en todo el rango de trabajo.

En otro trabajo, (KIM y otros, 2002), se presenta un un método que actúa sobre la referencia de tensión, modificándola según se alcance o no un valor de carga (corriente) previamente fijado. Basándose en el método descrito en 1.4.5, incorpora una modificación de la pendiente de caída de la tensión a partir de una determinada corriente circulante fijada de antemano, $I_{o, r e f}$ por esa etapa.

La ecuación que sigue la característica V-I es del tipo indicado en (1.13), pero si la corriente máxima supera un determinado valor de referencia, pasa a ser de la forma (1.25), donde $k_{1}=R_{e q}$ :

$$
V_{o}=V_{o, \max }-k_{1} I-k_{2}\left(I_{\max }-I_{o, r e f}\right)
$$

En la Figura 1.14 hay un ejemplo de dos etapas conectadas en paralelo. Nótese el cambio de pendiente de las curvas a partir de un valor de corriente determinado, $I_{o, r e f}$. Si la corriente máxima vuelve a estar por debajo del valor de referencia, las curvas pasan a ser las marcadas con ("), de forma que se mejora considerablemente el reparto de la carga. 


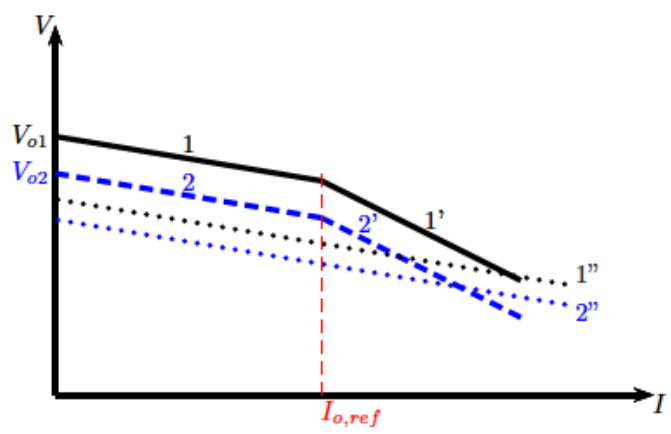

Figura 1.14: Curvas droop en función de $I_{o, r e f}$.

La modificación de la pendiente permite un reparto de cargas mas equilibrado a la vez que se consigue mantener la regulación de tensión dentro de los márgenes de trabajo.

\subsection{Convertidores PWM con conmutaciones sua- ves.}

Cuando un dispositivo semiconductor trabaja en conmutación, se mueve entre dos posibles estados: estado OFF (corte) y estado ON (saturación). En ambos casos su disipación de potencia es relativamente pequeña. Sin embargo no sucede así en las transiciones de un estado a otro puesto que coinciden en el tiempo valores elevados de tensión y de corriente. La potencia (o energía) disipada no es pequeña a pesar de que la transición es de pequeña duración. Esta es una de las razones por las que es necesario controlar o limitar los valores de tensión y/o de corriente durante las transiciones, ya que la energía disipada se convierte en calor y puede llegar a provocar la destrucción del dispositivo por temperatura excesiva. Además, el dispositivo debe permanecer dentro de una zona segura de operación (SOA, de Safety Operation Area), sin superar las tensiones y corrientes máximas y potencia máxima admisible. Para algunos dispositivos, incluso no se deben superar las tasas de variación de tensión y corriente que garantizan una operación fiable del mismo.

El efecto que ejercen otros elementos del circuito donde se encuentra insertado el dispositivo también debe ser tenido en cuenta. Por ejemplo, en las transiciones ON-OFF y OFF-ON de un transistor, algún diodo del circuito también cambiará su estado. Habrá que realizar la transición lo suficientemente lenta para evitar que el efecto de recuperación inversa del diodo afecte al transistor. También los elementos parásitos (inductancias debidas a las conexiones de terminales, pistas, etc... y capacidades parásitas) tie- 
nen gran influencia en los esfuerzos eléctricos de los dispositivos (Ver cap.24 de (KASSAKIAN y otros, 1991) y cap.27 de (Mohan y otros, 2003)) a la vez que se produce el aumento de la energía disipada

\subsubsection{Snubbers como ayuda a la conmutación.}

En la situación mencionada se recurre a redes de ayuda a la conmutación (también llamadas redes de protección, redes amortiguadoras o simplemente snubbers $^{3}$ ) para ayudar a absorber la energía procedente de los elementos reactivos durante la conmutación. Estas redes están formadas por varios elementos dispuestos de una forma determinada en torno al dispositivo que protegen. En general, incorporan, bien un condensador en paralelo junto a otros elementos para controlar la evolución de la tensión en el interruptor, bien una bobina en serie para el control de la corriente. Hay que indicar que este tipo de redes no son parte fundamental del circuito principal (por ejemplo, un convertidor de potencia), sino una adición para disminuir los esfuerzos eléctricos y y asegurar un régimen de trabajo seguro de los semiconductores. La complejidad adicional y el coste añadido sobre el convertidor básico debe sopesarse frente a los beneficios conseguidos por su uso. Además, siempre quedará la opción de sustituir el dispositivo por otro que soporte mayores tensiones y corrientes. El diseñador debe ser capaz de resolver este compromiso.

Una primera clasificación podría estar referida a la función que realizan. Se distingue entre snubber de apagado (para el control de la pendiente de subida de la tensión en el dispositivo), snubber de enclavamiento o clamp (para fijar la tensión a un valor máximo), y snubber de encendido (para el control de la pendiente de subida de la corriente). Por otro lado, si la energía almacenada se disipa en una resistencia se habla de snubber disipativo y si no se elimina en el el mismo, sino que se transfiere a la fuente o a la carga se dice que es un snubber no disipativo.

Si la red de protección incorpora algún diodo se dice que es un snubber polarizado, mientras que si incorpora algún elemento activo auxiliar la red de protección se denomina activa, por oposición a las redes denominadas pasivas en las que no hay elementos susceptibles de ser controlados.

En la Figura 1.15 se han representado algunas de las redes clásicas de protección para aliviar los esfuerzos eléctricos en un transistor, empleando un condensador en paralelo.

La red de la Figura 1.15a es un snubber no polarizado, mientras que el de tipo polarizado corresponde a la Figura 1.15b. Ambos son de tipo disipativo mientras que la Figura 1.15c y 1.15d no lo són. Éste último snubber es activo porque incorpora un transistor auxiliar.

\footnotetext{
${ }^{3}$ Trad.: Amortiguadores
} 


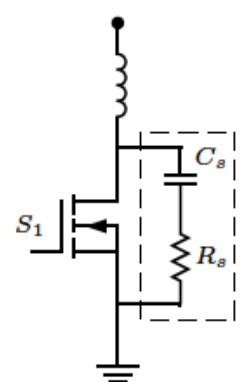

(a)

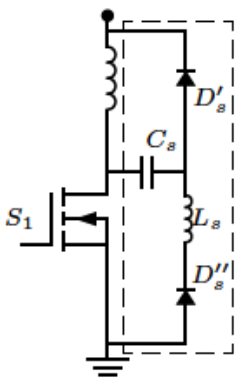

(c)

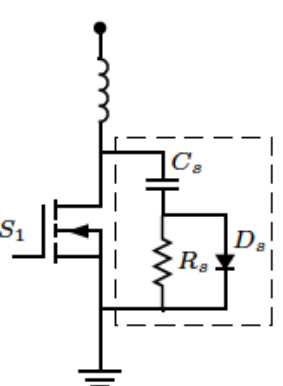

(b)

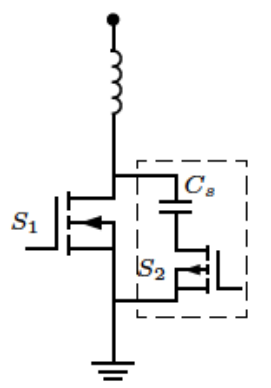

(d)

Figura 1.15: Redes de protección de transistores contra sobretensiones: a) snubber RC; b) snubber RCD; c) snubber LC; y d) snubber activo.

El snubber de la Figura $1.15 \mathrm{~b}$, está formado por $C_{s}, R_{s}$ y $D_{s}$, y es un snubber de apagado que se diseña para controlar la pendiente de subida de la tensión durante el apagado y reducir la potencia que disipa el transistor en esta transición.Aunque, con el condensador ya se podría limitar la variación de tensión y no sería necesario ningún elemento adicional, sucederá que cuando el transistor conmutar de nuevo al ON, el condensador se descarga sobre aquel y primero y la corriente podría alcanzar valores elevados que provocarían la destrucción del transistor. Para evitar esto se añade la resistencia $R_{s}$ que limita la corriente de descarga y que, adicionalmente, sirve de elemento de disipación de la energía almacenada en el condensador (snubber disipativo). La función del diodo, $D_{s}$ es para que la carga del condensador se produzca únicamente en el momento del apagado (snubber polarizado).

Por supuesto, para seleccionar un condensador apropiado, se debe conseguir un compromiso entre la energía disipada por el transistor en el apagado y las pérdidas de la resistencia del snubber (ver figura 27.15 de (MOHAN y otros, 2003), que es muy similar a la que aparece en (CALKIn y HAMILTON, 1976)). Otras referencia clásica en la literatura es (MCMurraY, 1972), que junto a (MCMurray, 1980) incluyen criterios para el diseño de snubbers.

La conexión de esta red se hace generalmente en paralelo al transistor principal, pero podría conectarse en otros puntos del circuito siempre que 
sea posible el control de la tensión del transistor al que se protege. Por ejemplo, en la disposición mostrada en la Figura 1.16 el condensador $C_{s}$ se ha conectado entre masa y el emisor, dado que el colector está fijado a un punto de tensión constante. De esta forma también se puede controlar la tensión del transistor con el condensador.

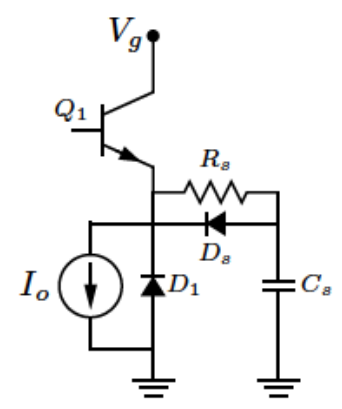

Figura 1.16: Red de apagado con otra posible conexión del snubber. Fig. 24.12 de (KASSAKIAN y otros, 1991)

Al considerar las inductancias parásitas en el circuito, aparecen sobretensiones cuando se produce el apagado de un transistor. Si hay un snubber de apagado también es posible limitar el valor de la tensión, pero el condensador puede no ser el óptimo para la protección por sobretensión y las pérdidas en el conjunto snubber-transistor serían mayores. Incluso, puede no ser necesario el uso de un snubber de apagado (por ejemplo con transistores MOSFET), pero, debido a los elementos parásitos, puede que si sea necesario un snubber de sobretensión. Este último también recibe el nombre de snubber de enclavamiento de tensión (voltage clamp) y está formado por los mismos elementos que el de de apagado pero con otra disposición. En la figura 1.17 se ha incluido una red de este tipo, formada por $C_{c}, R_{c}$ y $D_{c}$.

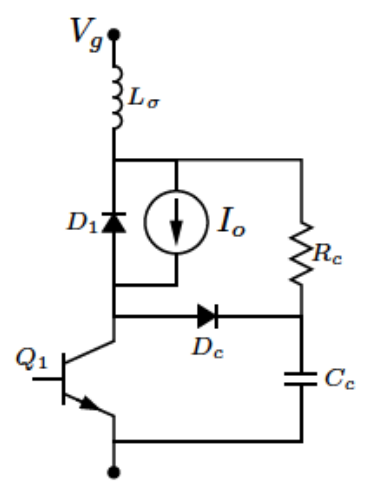

Figura 1.17: Red de sobretensión o voltage clamp.

El condensador debe ser lo suficientemente grande para absorber la energía que cede la inductancia parásita. Desde el punto de la energía, se debe 
cumplir la igualdad (1.26)

$$
\frac{1}{2} C_{c} \Delta V_{c}^{2}=\frac{1}{2} L_{\sigma} I_{o}^{2}
$$

donde $\Delta V_{c}$ es el incremento que sufre la tensión del clamp cuando absorbe la energía de la inductancia parásita, $L_{\sigma}$. Dicha energía es posteriormente devuelta hacia la resistencia. El condensador varía su tensión en torno a la tensión de enclavamiento durante la carga y descarga de la energía de la inductancia.

En estas y otras redes disipativas, la energía que se almacena en el condensador durante un tiempo, pasa a disiparse en la resistencia de la propia red. Dicho de otra forma, aunque se consiguen reducir los esfuerzos eléctricos de los semiconductores, existe energía que se pierde en la resistencia del snubber. Incluso, si la frecuencia de trabajo llega a ser muy elevada, estas pérdidas pueden ser inaceptables. En un snubber no disipativo, no hay resistencia, y la energía almacenada se recicla, devolviéndola bien a la carga, bien a la fuente de entrada, o incluso en circulación hasta el comienzo del siguiente ciclo. Un ejemplo podría ser la red de la Figura 1.15c.

$\mathrm{Si}$ en la red incluye algún elemento activo auxiliar, por ejemplo un transistor, como en la Figura 1.15d, ésta se denomina red activa, por oposición a las denominadas redes pasivas (como las presentadas hasta ahora) en la que no hay elementos susceptibles de ser controlados.

\subsubsection{Del enclavamiento activo a las conmutaciones suaves.}

Las redes activas han sido empleadas como red de enclavamiento de tensión, (HARADA y SAKAMOTO, 1990), como ayuda a la descarga de la energía del transformador de un convertidor tipo forward (VINCIARELLI, 1984), como ayuda a la conmutación a tensión nula (ZVS de Zero Voltage Switching) en convertidores tipo flyback (HENZE y otros, 1988; Yoshida y otros, 1992; WATSON y otros, 1994, 1996a).

En (HARAda y SAKAmoto, 1990), para fijar la máxima tensión en el interruptor principal de un convertidor CC/CC tipo reductor. El interruptor auxiliar se activaba de forma complementaria al transistor principal. Quedaba comprobado de forma experimental la desaparición de la sobretensión debida a las inductancias parásitas, cuya energía era empleada de forma efectiva para la conmutación a tensión nula del transistor principal. Se proponía su uso en otros convertidores $\mathrm{CC} / \mathrm{CC}$, comprobando en un flyback no solo la ausencia de oscilaciones sino también la mejora del rendimiento al usar este snubber.

Para la descarga de la energía acumulada en el primario del transformador de un forward, hay varias soluciones, (CARSTEn, 1978; Vinciarelli, 1984; Carsten, 1990; Xi y Jain, 2003; Basso, 2008, cap.8). Una de ellas es, por ejemplo, la adición de un tercer devanado acoplado al primario y conectado 
a la fuente. En el primario aparece una tensión de polaridad opuesta a la inicial que es un inconveniente a tener en cuenta, dada la elevada tensión que soporta el transistor principal. La otra solución emplea una red activa, donde la tensión que soporta el interruptor es menor.

Antes de continuar, hay que indicar que existen otras técnicas distintas a estas redes para conseguir conmutaciones suaves tanto en tensión como en corriente, por ejemplo en (HuA, 1994), pero quedan fuera de este trabajo.

En la Figura 1.18 se muestra la situación de la red de enclavamiento activo en un forward y en un flyback.

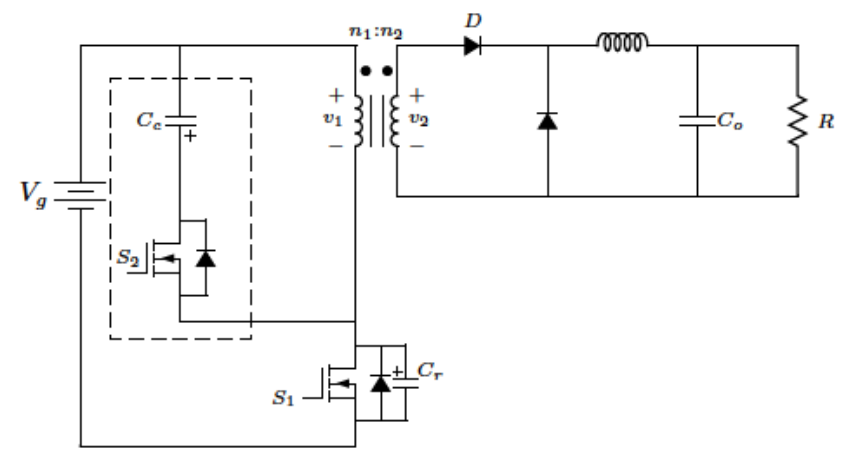

(a)

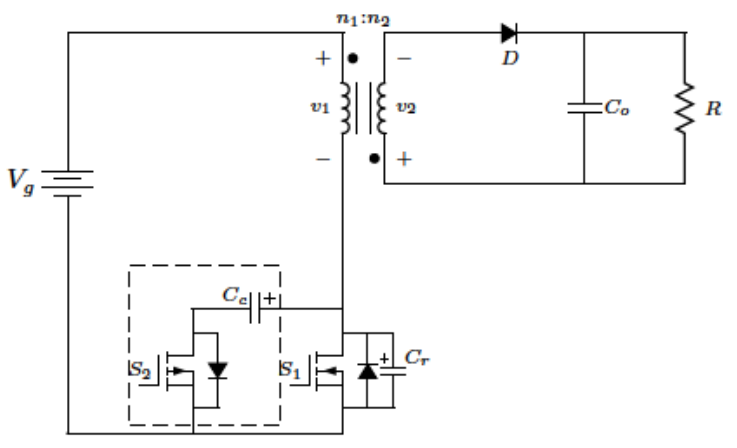

(b)

Figura 1.18: Empleo de redes de enclavamiento activo en diferentes convertidores: a) en un forward; y b) en un flyback.

Como se observa, la situación es diferente para cada caso. En la Figura 1.18a el clamp se aplica al devanado primario mientras que en la $\mathrm{Fi}$ gura 1.18b, se aplica al interruptor principal. En realidad, son similares y podrían intercambiarse. Su principal diferencia es la tensión del condensador del clamp, cuya relación con la tensión de entrada recuerda a un reductorelevador o a la de un elevador. Para la primera disposición:

$$
V_{C_{c, \text { redelev }}}=V_{g} \frac{D}{(1-D)}
$$


Y para la disposición en el flyback:

$$
V_{C_{c, \text { elev }}}=V_{g} \frac{1}{(1-D)}=V_{g} \frac{D}{(1-D)}+V_{g}
$$

Dada esta diferencia en la tensión del condensador, (de $V_{g}$ voltios para el segundo caso) hace menos habitual el segundo caso. También provoca una mayor corriente a través del condensador del filtro de entrada. Una posible ventaja, sin embargo, es que puede emplearse un MOSFET de canal $\mathrm{p}$ y más sencillo de disparar en algunas aplicaciones. Respecto a otras consideraciones (comportamiento en estado estacionario, esfuerzos de tensión en los interruptores, mecanismo de «reset», etc.) ambos tipos de clamp son similares (DALAL, 1996).

El uso de la red activa, ademas de fijar la tensión máxima del interruptor, también permite las conmutaciones suaves del interruptor que protege y por tanto, la reducción de las pérdidas de conmutación y la consiguiente mejora del rendimiento.

Para conseguir las conmutaciones suaves en un amplio rango de carga, sería necesario añadir una pequeña bobina resonante que interactúe con el circuito activo (que funcione de la misma forma que las inductancias parásitas del circuito) en función de las condiciones del circuito. El objetivo sería mantener la cantidad de energía que es reciclada y así favorecer las conmutaciones suaves en condiciones de baja carga. En la Figura 1.19 se muestran los tres convertidores básicos con una red de enclavamiento activo y con conmutaciones suaves ZVS, que incorporan una pequeña bobina, $L_{r}$.

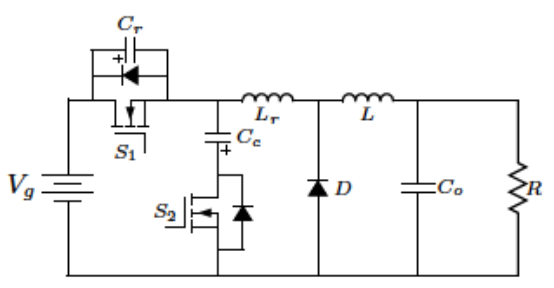

(a)

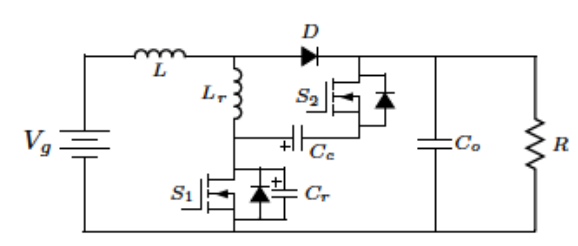

(b)

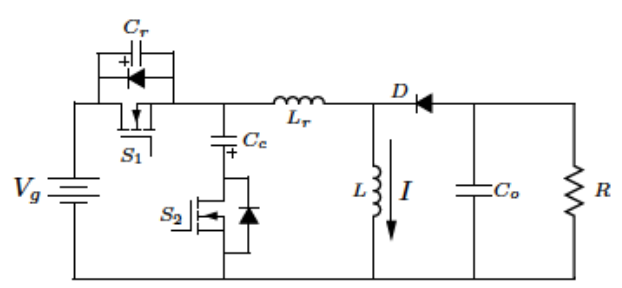

(c)

Figura 1.19: Convertidores con red de enclavamiento activo: a) reductor; b) elevador; c) reductor elevador. 
Puesto que existen diferentes posibilidades de conexión de esta red dentro del circuito principal, se ha dado lugar a una familia de convertidores $\mathrm{CC} / \mathrm{CC}$ con conmutaciones suaves denominada convertidores Zero-Voltage-Swithed Pulse-Width-Modulated (ZVS-PWM), que conjugan las conmutaciones suaves con la reducción del esfuerzo eléctrico de los semiconductores (HENG y Oruganti, 1994; Duarte y Barbi, 1995, 1997a,b).

Partiendo de la idea de Heng de 1994 (Heng y Oruganti, 1994), basada en el planteamiento de un marco común para todos los convertidores convencionales de un interruptor que incorporaban una red activa que permitiera conmutaciones ZVS, aparecieron varios trabajos de Duarte et al. en los años 1995 y 1997 (DUARTE y BARBI, 1995, 1997b,a) en la misma línea de agrupar, según un criterio determinado, los convertidores con conmutaciones suaves en una misma familia. Estos trabajos trataban de la síntesis, análisis e implementación de un conjunto de convertidores $\mathrm{CC} / \mathrm{CC}$ convencionales, a los que se les añadía el circuito de enclavamiento activo, en sus diferentes configuraciones. De esta forma era sencillo derivar nuevas topologías a partir de la combinación de ambos elementos.

$\mathrm{Al}$ experimentar con un convertidor con active-clamp, se consiguen las conmutaciones suaves tanto del transistor principal como del auxiliar. El diodo principal no tiene conmutaciones suaves sino que presenta una resonancia entre su capacidad parásita y la inductancia resonante del clamp. La solución propuesta por Jovanonic en 1998 (JovanOVIC, 1998) consistente en añadir un diodo auxiliar para fijar la tensión, consigue eliminar esta oscilación reduciendo las pérdidas del diodo principal. Incluso se produce la conmutación sin pérdidas de todos los elementos si a este diodo auxiliar se le añade un condensador en paralelo como propone Duarte en su trabajo de 2002 (DuARTE y BARBI, 2002).

\subsubsection{La impedancia de salida.}

Las topologías con enclavamiento activo han sido objeto de estudio desde el punto de vista de conmutaciones suaves ZVS, estabilidad, comportamiento dinámico, etc. Entre otros trabajos, además de los ya citados previamente, están (Jitaru, 1992; Jitaru y Birca-Galateanu, 1998; Li y otros, 2002; Papanikolaou y Tatakis, 2004; King y Strasser, 2010; Masihuzzaman y otros, 2010). También se pueden encontrar trabajos en los que se obtenían modelos simplificados de estos convertidores, como el de Lakshminarasamma et al. en 2004 (LAKShminarasamma y otros, 2004) pero no incluían el condensador auxiliar en ese modelo.

En el trabajo de Lakshminarasamma de 2004 se comprobó experimentalmente la alta impedancia de salida así como la dependencia de la tensión con la corriente de salida. Los trabajos de Duarte et al., citados anterior- 
mente estaban centrados principalmente en las conmutaciones suaves. Aún así comprobaron en el laboratorio la dependencia de la tensión de salida y su dependencia con la corriente en la carga para diferentes topologías con enclavamiento activo.

En el trabajo de De Jódar et al. de 2008 (DE ${ }^{\sim}$ JodAr y otros, 2008) se comprueba que un convertidor reductor con enclavamiento activo presenta una impedancia de salida elevada, y es aprovechada esta característica para su conexión en paralelo con etapas similares. De hecho, y como se mencionó en apartados anteriores, si la impedancia de salida es grande, es posible un reparto de corriente a la salida. Esta impedancia depende de la frecuencia de conmutación y de los elementos resonantes que se añaden al circuito para conseguir las conmutaciones suaves. Hay que indicar que también es posible el reparto si la impedancia de entrada es alta como se comprueba para otra topología elevadora con transición a tensión nula en el trabajo de De Jódar et al. de 2010 (DE $\sim$ JODAR, 2010) en determinadas condiciones. Si consideramos el convertidor con un transformador de continua, la impedancia puede verse «desde el primario o el secundario».

\subsection{Planteamiento de la tesis}

El planteamiento de esta tesis está centrado en el reparto de carga en la conexión en paralelo de topologías de un interruptor con aislamiento y alta relación de transformación que incorporan enclavamiento activo. Ya ha sido comprobado que con las topologías sin aislamiento es posible el reparto de carga cuando se añade un circuito de enclavamiento activo. De igual forma que las topologías convencionales con aislamiento son derivadas de las topologías sin aislamiento, así se puede hacer con las topologías con enclavamiento activo. De esta forma, se obtendrían topologías idénticas a las presentadas hace mas de 20 años con la intención originaria de hacer un reset del transformador o bobinas empleadas.

A la vista de la literatura presentada, la intuición nos dice que si tendrán alta impedancia de salida, lo que facilitará su conexión en paralelo, de forma que sean estables en régimen permanente y en régimen dinámico. Sin embargo, el uso de transformadores para conseguir la alta relación de transformación a la vez que aislamiento, incluye un elemento adicional que es la inductancia y capacidad distribuida que afectará a este comportamiento, por lo que habrá que estudiar de qué forma influye en el reparto de carga.

Como objetivos específicos se indican los siguientes:

- Valorar la viabilidad de la conexión en paralelo de los convertidores con aislamiento que incorporan enclavamiento activo. 
- Modelado de la topología seleccionada y obtención de las funciones de transferencia mas importantes para diseñar el control de la conexión en paralelo en régimen permanente y en régimen dinámico.

- Estudiar la influencia de los parámetros mas importantes de diseño en el reparto.

- Construcción de un prototipo controlado digitalmente, que sirva de comprobación de los estudios teóricos y simulaciones previas y validación en una aplicación determinada.

- Comprobación del correcto funcionamiento del convertidor durante la entrada y salida de etapas en la conexión en paralelo. 


\section{Capítulo 2}

\section{Reparto Estático}

Este capítulo recoge los detalles de funcionamiento de los convertidores ZVS, tabulando los parámetros mas notables para las topologías básicas ZVS: reductor, elevador, red.-elev. y flyback. Los Anexos añadidos al final del trabajo incluyen un análisis más detallado. La conexión en paralelo es factible, dada la relación existente entre la tensión y la intensidad a la salida, que muestra una característica decreciente a medida de aumenta la carga. Esta dependencia, está relacionada con la impedancia en régimen permanente.

También se ha incluido un sencillo análisis, con ejemplo numérico incluido, sobre el efecto de la tolerancia en el ciclo de trabajo, en bobina principal y en bobina resonante en el reparto de carga.

\subsection{Funcionamiento de un convertidor ZVS.}

Un convertidor con enclavamiento activo como el indicado la Figura 2.1 (o convertidor ZVS), es un convertidor formado por un transistor principal, $S_{1}$, la bobina, $L$ (o de magnetización $L_{m}$ si se incluye un transformador o dos bobinas acopladas) junto con el diodo $D$ y el condensador de salida, $C_{o}$. El transistor $S_{1}$, se encarga de regular el flujo de energía hacia la carga. En la bobina principal (o bobinas acopladas), se acumula la energía mientras que $S_{1}$ está ON. Cuando pasa a corte, la bobina devuelve la energía hacia la carga a través del diodo de salida, $D$, y el condensador $C_{o}$.

El condensador que representa la capacidad del transistor principal aparece como $C_{r}$. La red de enclavamiento activo incluye el condensador de clamp $C_{c}$ y el transistor $S_{2}$ además de la bobina auxiliar, $L_{r}$, también denominada bobina resonante. Las inductancias parásitas del circuito se incluyen en esta bobina. La bobina intercambia energía con los condensadores mencionados durante determinados instantes del periodo de conmutación, T. Por último, los diodos en antiparalelo del transistor principal y auxiliar también están incluidos, y son $D_{1}$ y $D_{2}$ respectivamente.

El análisis de este tipo de convertidor ya se ha realizado en la literatu- 


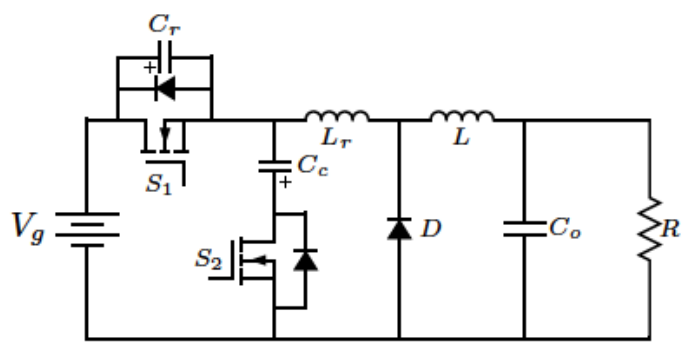

(a)

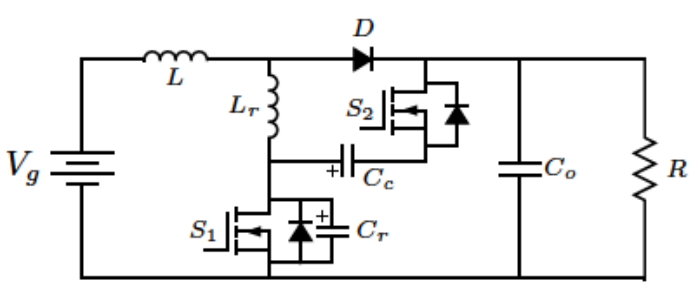

(b)

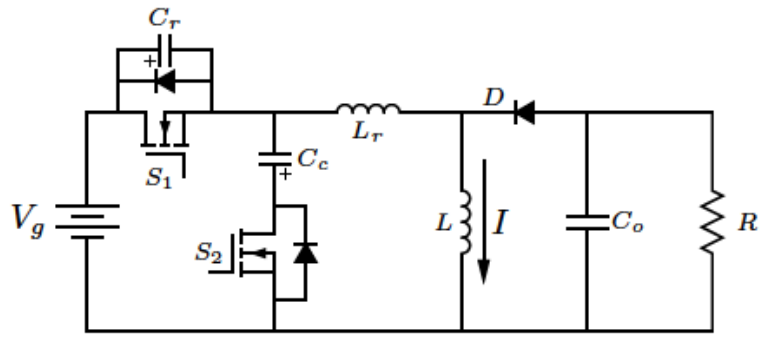

(c)

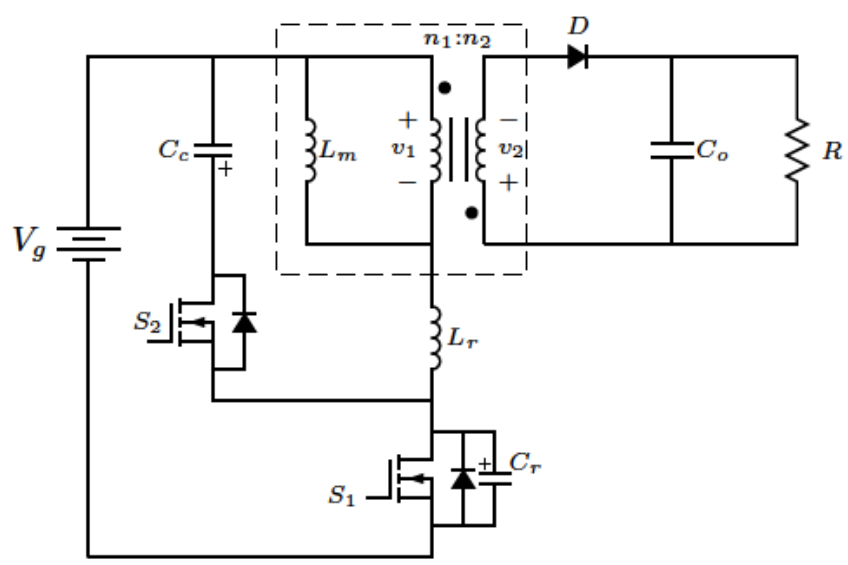

(d)

Figura 2.1: Convertidores con red de enclavamiento activo: a) reductor; b) elevador; c) reductor elevador; y d) Flyback.

ra, (DuARTe y BARBi, 1995; WAtson y otros, 1996b,a; DuArte y BARBi, 
1997a,b; LiN y otros, 2005), partiendo de ciertas simplificaciones. La bobina $L$ se supone lo suficientemente grande como para considerar una corriente sin rizado por ella, y de un valor constante $I$, mientras que el valor de la bobina $L_{r}$ es bastante inferior al de la principal. El valor de los condensadores $C_{c}$ y $C_{o}$ se supone lo suficientemente grande como para considerar que la tensión en sus extremos, $v_{C_{c}}$ y $v_{C_{o}}$ respectivamente, se mantiene constante durante todo el periodo. Los interruptores $S_{1}$ y $S_{2}$ conmutan de forma complementaria, dejando un pequeño tiempo muerto para permitir las conmutaciones suaves. Se consideran componentes ideales sin pérdidas de forma que $P_{\text {entrada }}=P_{\text {salida }}$.

Con las consideraciones mencionadas, el convertidor evoluciona en seis intervalos o etapas a lo largo de un periodo completo. Previamente a $t_{0}$, el interruptor principal $S_{1}$ esta cerrado y el interruptor auxiliar $S_{2}$ está abierto. Con $d$ se representa el ciclo de trabajo. En el primer intervalo $\left(\left[t_{0}-t_{1}\right]\right)$ se abre $S_{1}$ y lo hace con pérdidas reducidas por la existencia de $C_{r}$. Entonces, se produce un intercambio de energía entre $L_{r}$ y el condensador $C_{r}$ hasta que la tensón sea $v_{C_{r}}(t 1)$ en $t_{1}$. Desde aquí, comienza a conducir el diodo principal, $D$ a la vez que se produce una resonancia entre $L_{r}$ y $C_{r}$ hasta que su tensión llega a $v_{C_{r}}\left(t_{2}\right)$. Esto sucede en $t_{2}$. A partir de ahora la corriente resonante circula por el diodo del clamp, $D_{2}$, lo que permite la conmutación ZVS de $S_{2}$. El condensador $C_{c}$ intercambia energía con $L_{r}$ a tensión constante, de forma que la corriente evoluciona hasta un valor de polaridad opuesta a la que tenía en $t_{2}$. La duración de este tercer intervalo es el tiempo que está $S_{2}$ conduciendo y es igual a $(1-d) T$. Ahora, la bobina de nuevo resuena con $C_{r}$ para descargarlo totalmente. Este instante se considera como $t_{4}$. Entonces, la corriente circula por el diodo $D_{1}$, lo que permite el encendido ZVS de $S_{1}$ un tiempo después de $t_{4}$, pero antes de que la corriente cambie de polaridad de nuevo ${ }^{1}$. Una vez que lo ha hecho, crece hasta hacerse igual que el valor que tenía en $t_{0}$. Desde este momento, $t_{5}$, el diodo principal deja de conducir y la corriente circula por $S_{1}$ hasta que pasa a OFF para dar comienzo al siguiente periodo.

Las curvas obtenidas durante un ciclo completo de trabajo están representadas en la Figura 2.2, donde se han representado los disparos de los dos transistores y las curvas de tensión y corriente de los elementos principales. La apertura del transistor principal es en $t_{0}$. La duración de cada intervalo está indicada como $d_{x} T$, siendo $d_{x}$ la fracción del periodo que corresponde con la duración del intervalo $x$.

Cuando la corriente en la bobina principal presenta cierto rizado, entre $I_{\min }$ e $I_{\max }$, el funcionamiento sigue siendo similar con la única diferencia en

\footnotetext{
${ }^{1}$ El tiempo muerto entre el apagado de $S_{2}$ y el encendido de $S_{1}$ debe ser mayor que $t_{4}-t_{3}$ pero inferior al tiempo que tarda en cambiar de polaridad la intensidad resonante para conseguir conmutación suave al encender $S_{1}$.
} 


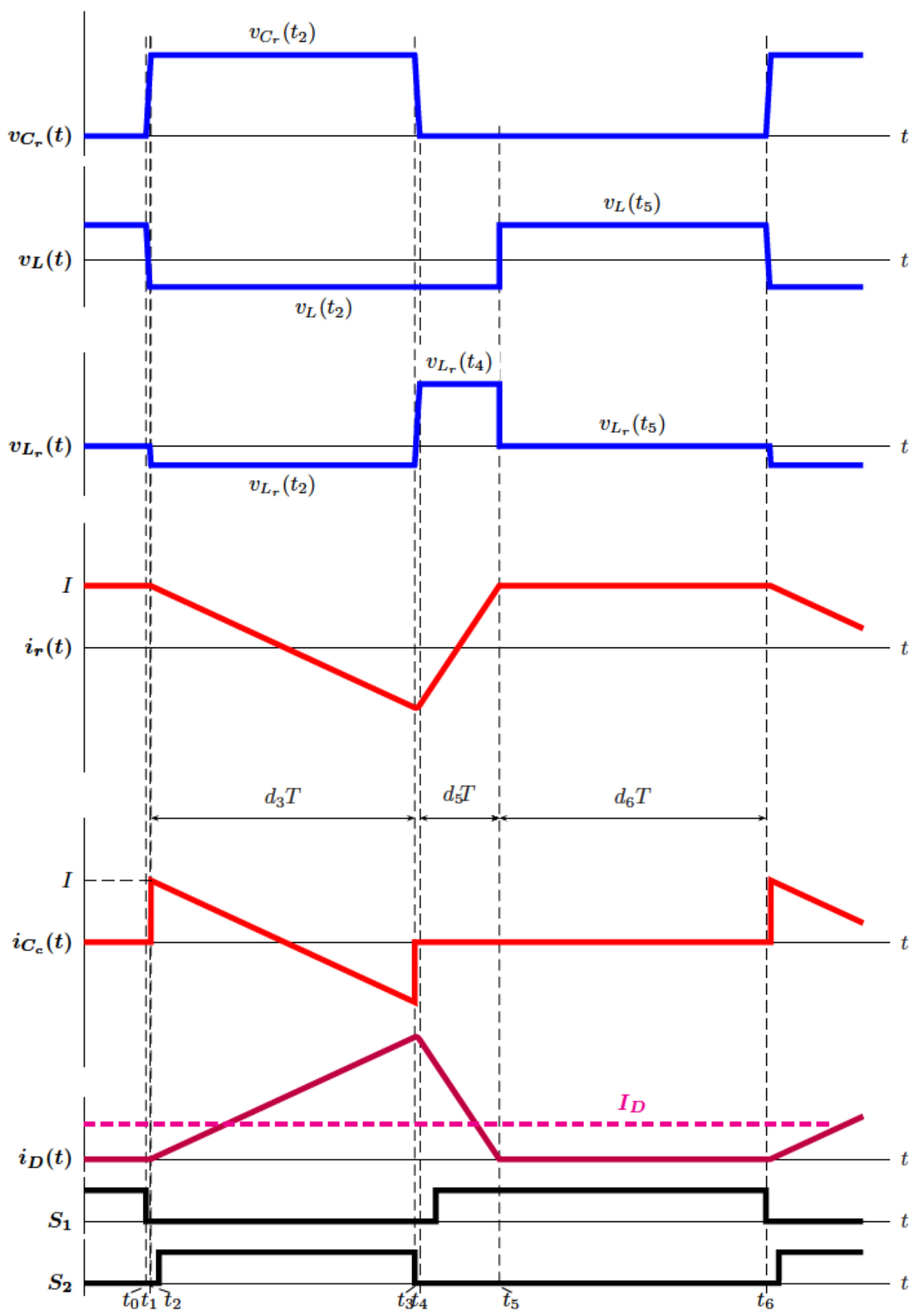

Figura 2.2: Principales curvas en un convertidor ZVS.

los instantes que delimitan los intervalos de tiempo por los que evoluciona el convertidor. Ahora, las curvas de corriente incluyen el rizado y se encuentran indicadas en la Figura 2.3. 


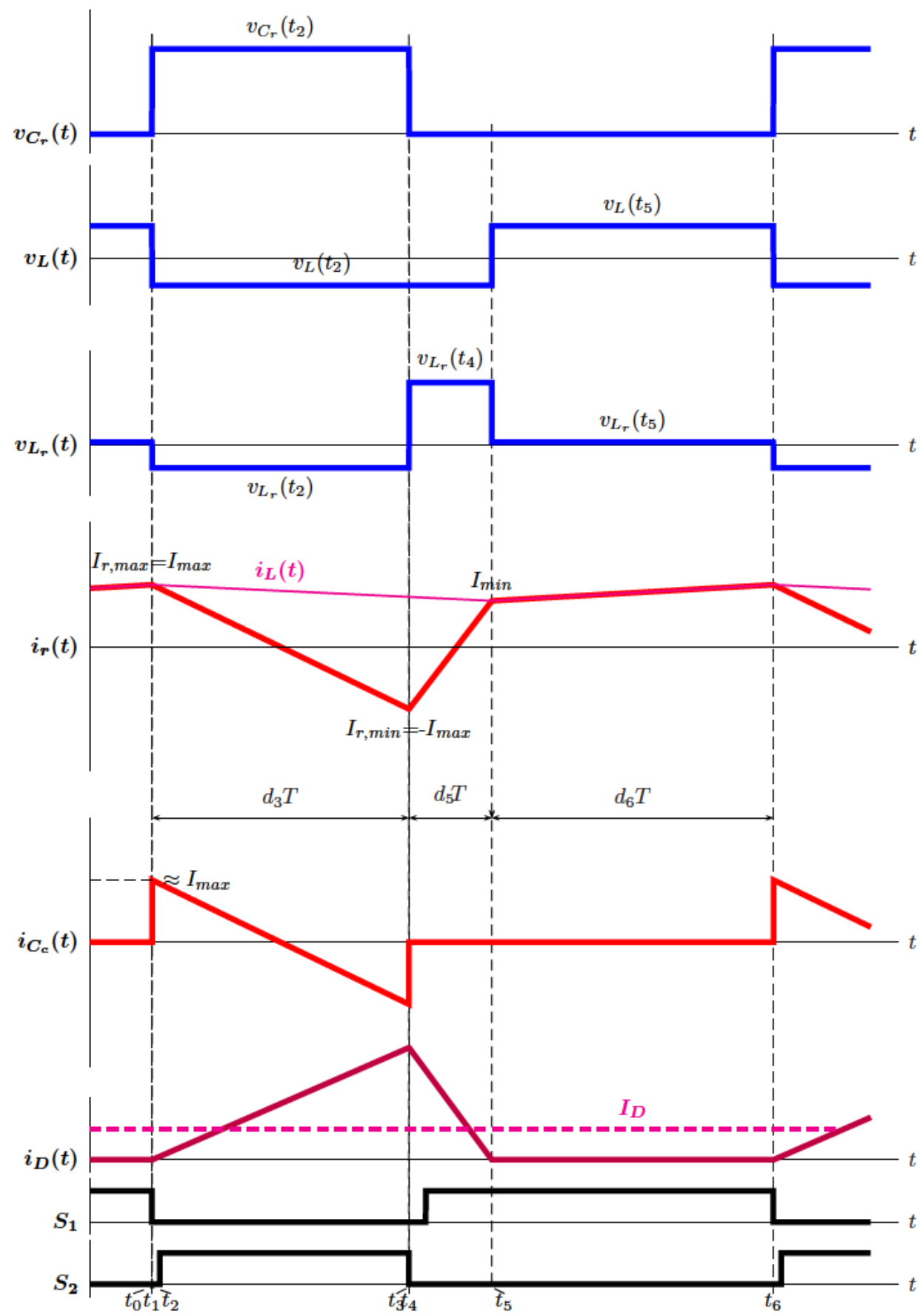

Figura 2.3: Principales curvas en un convertidor ZVS al considerar rizado.

En la Tabla 2.1 se indican las expresiones de las tensiones alcanzadas en los diferentes intervalos cuando no hay rizado, mientras que la Tabla 2.2 recoge los valores de tensión considerando el rizado. En este último caso, 
aparece un factor en las expresiones de la tensión en la bobina principal en forma de ratio entre bobinas. Hay que notar que la tensión de salida del reductor-elevador se ha considerado con valor positivo; y que $L$ en el caso del flyback, (incluido porque es caso de estudio mas adelante), corresponde $\mathrm{a} \cdot L_{m}$.

\begin{tabular}{ccccccc}
\hline Convertidor & $v_{C_{r}}\left(t_{2}\right)$ & $v_{L}\left(t_{2}\right)$ & $v_{L}\left(t_{5}\right)$ & $v_{L_{r}}\left(t_{2}\right)$ & $v_{L_{r}}\left(t_{4}\right)$ & $v_{L_{r}}\left(t_{5}\right)$ \\
\hline Reductor & $V_{c}+V_{g}$ & $-V_{o}$ & $\approx V_{g}-V_{o}$ & $-V_{c}$ & $V_{g}$ & $\approx 0$ \\
Elevador & $V_{c}+V_{o}$ & $V_{g}-V_{o}$ & $\approx V_{g}$ & $-V_{c}$ & $V_{o}$ & $\approx 0$ \\
Red-Elev & $V_{c}+V_{g}$ & $-V_{o}$ & $\approx V_{g}$ & $-V_{c}+V_{o}$ & $V_{g}+V_{o}$ & $\approx 0$ \\
Flyback & $V_{c}+V_{g}$ & $-n V_{o}$ & $\approx V_{g}$ & $-V_{c}+n V_{o}$ & $V_{g}+n V_{o}$ & $\approx 0$ \\
\hline
\end{tabular}

Tabla 2.1: Tensiones en un convertidor ZVS cuando no hay rizado.

\begin{tabular}{ccccccc}
\hline Convertidor & $v_{C_{r}}\left(t_{2}\right)$ & $v_{L}\left(t_{2}\right)$ & $v_{L}\left(t_{5}\right)$ & $v_{L_{r}}\left(t_{2}\right)$ & $v_{L_{r}}\left(t_{4}\right)$ & $v_{L_{r}}\left(t_{5}\right)$ \\
\hline Reductor & $V_{c}+V_{g}$ & $-V_{o}$ & $\left(V_{g}-V_{o}\right) \frac{L}{L+L_{r}}$ & $-V_{c}$ & $V_{g}$ & $\left(V_{g}-V_{o}\right) \frac{L_{r}}{L+L_{r}}$ \\
Elevador & $V_{c}+V_{o}$ & $V_{g}-V_{o}$ & $V_{g} \frac{L}{L+L_{r}}$ & $-V_{c}$ & $V_{o}$ & $V_{g} \frac{L_{r}}{L+L_{r}}$ \\
Red-Elev & $V_{c}+V_{g}$ & $-V_{o}$ & $V_{g} \frac{L}{L+L_{r}}$ & $-V_{c}+V_{o}$ & $V_{g}+V_{o}$ & $V_{g} \frac{L_{r}}{L+L_{r}}$ \\
Flyback & $V_{c}+V_{g}$ & $-n V_{o}$ & $V_{g} \frac{L_{m}}{L+L_{r}}$ & $-V_{c}+n V_{o}$ & $V_{g}+n V_{o}$ & $V_{g} \frac{L_{m}}{L_{m}+L_{r}}$ \\
\hline
\end{tabular}

Tabla 2.2: Tensiones en un convertidor ZVS al considerar rizado.

\subsection{Análisis en régimen permamente.}

Para el análisis se desprecian los intervalos $\left[t_{0}-t_{1}\right],\left[t_{1}-t_{2}\right]$ y $\left[t_{3}-t_{4}\right]$ por ser de escasa duración en relación con el resto. De esta forma, el ciclo de trabajo en régimen permanente se representa por $D$, y se puede considerar que:

$$
\begin{aligned}
D T & =d_{5} T+d_{6} T \\
(1-D) T & =d_{3} T
\end{aligned}
$$

En un convertidor convencional, la bobina principal acumula energía durante $D T$, mientras que en un convertidor ZVS, la bobina principal presenta una corriente creciente solo durante una parte de ese tiempo, que coincide con el sexto intervalo, de duración $d_{6} T$. A $d_{6}$ se le llama ciclo efectivo, $d_{e f}$, porque es la parte del ciclo de trabajo en el que la bobina, efectivamente, acumula energía.

La intensidad en la bobina principal, $I$, está relacionada con la intensidad de entrada, $I_{g}$ y de salida, $I_{o}$, como indica en la Tabla 2.3. También se ha incluido la intensidad en el diodo principal, $I_{D}$. 


\begin{tabular}{c|c|c|c|c}
\hline & Reductor & Elevador & Red-Elev & Flyback \\
\hline$I$ & $I_{o}$ & $I_{g}$ & $I_{g}+I_{o}$ & $I_{g}+I_{o} / n$ \\
$I_{D}$ & $I-I_{g}$ & $I_{o}$ & $I_{o}$ & $I_{o}$ \\
\hline
\end{tabular}

Tabla 2.3: Intensidad media en la bobina principal.

El cálculo de $d_{5}$ se obtiene a partir de la variación de la corriente en la bobina resonante, $i_{r}(t)$, durante el intervalo $\left[t_{4}-t_{5}\right]$ :

$$
d_{5}=\frac{1}{T} L_{r} \frac{I_{\min }+I_{\max }}{v_{L_{r}}\left(t_{4}\right)}=\frac{2 L_{r} f_{s}}{v_{L_{r}}\left(t_{4}\right)} I
$$

al considerar $I_{\max }$ e $I_{\min }$ como:

$$
\begin{aligned}
& I_{\text {max }}=I+\frac{1}{2} \Delta i_{L} \\
& I_{\text {min }}=I-\frac{1}{2} \Delta i_{L}
\end{aligned}
$$

donde $I$ es la intensidad media en $L$ y $\Delta i_{L}$ es el rizado de corriente en la bobina principal, obtenido como:

$$
\begin{aligned}
\Delta i_{L} & =\frac{1}{L}\left|v_{L}\left(t_{2}\right)\right|\left(d_{3}+d_{5}\right) T \\
\Delta i_{L} & =\frac{1}{L}\left|v_{L}\left(t_{5}\right)\right| d_{6} T
\end{aligned}
$$

Otra expresión para $d_{5}$, que depende la intensidad de salida, se consigue al emplear las relaciones de la Tabla 2.3 en (2.2), de forma que:

$$
d_{5}=\frac{2 L_{r} f_{s}}{n V_{g}} I_{o}
$$

siendo $n$ la relación de transformación para el flyback ZVS y $n=1$ para los otros tres convertidores ZVS.

Con el balance voltios-segundo en la bobina principal, se obtiene la expresión del ciclo efectivo, $d_{6}$, con la que se puede obtener el rizado de corriente en la bobina principal. Ambas expresiones, particularizadas para cada convertidor están recogidas en la Tabla 2.4

Por otro lado, se puede obtener una expresión de la tensión de salida en función de la duración los intervalos, a partir del balance voltios-segundo en la bobina principal. Cuando se hace este balance en la bobina resonante se obtiene una expresión para la tensión del clamp en función de la duración de los intervalos. Estas expresiones están indicadas en la Tabla 2.5, donde se han particularizado para cada convertidor ZVS. 


\begin{tabular}{ccc}
\hline Convertidor & $d_{e f} \equiv d_{6}$ & $\Delta I_{L}$ \\
\hline Reductor & $\frac{\left(L+L_{r}\right) V_{o}}{L_{r} V_{o}+L V_{g}}$ & $\frac{\left(V_{g}-V_{o}\right) V_{o} T}{L_{r} V_{o}+L V_{g}}$ \\
Elevador & $\frac{\left(L+L_{r}\right)\left(V_{g}-V_{o}\right)}{L_{r} V_{g}-\left(L+L_{r}\right) V_{o}}$ & $\frac{\left(V_{g}-V_{o}\right) V_{g} T}{L_{r} V_{g}-\left(L+L_{r}\right) V_{o}}$ \\
Red-Elev & $\frac{\left(L+L_{r}\right) V_{o}}{L V_{g}+\left(L+L_{r}\right) V_{o}}$ & $\frac{V_{o} V_{g} T}{L V_{g}+\left(L+L_{r}\right) V_{o}}$ \\
Flyback & $\frac{\left(L_{m}+L_{r}\right) n V_{o}}{L_{m} V_{g}+\left(L_{m}+L_{r}\right) n V_{o}}$ & $\frac{n V_{o} V_{g} T}{L_{m} V_{g}+\left(L_{m}+L_{r}\right) n V_{o}}$ \\
\hline
\end{tabular}

Tabla 2.4: Ciclo efectivo y rizado de corriente en la bobina.

\begin{tabular}{ccc}
\hline Convertidor & $V_{o}$ & $V_{c}$ \\
\hline Reductor & $V_{g} \frac{L}{L+L_{r}} \frac{d_{6}}{1-d_{6} \frac{L_{r}}{L+L_{r}}}$ & $V_{g} \frac{d_{5}+d_{6}}{d_{3}}-V_{o} \frac{1}{d_{3}}$ \\
Elevador & $V_{g} \frac{\left(1-d_{6} \frac{L_{r}}{L+L_{r}}\right)}{\left(1-d_{6}\right)}$ & $V_{g} \frac{1}{d_{3}}-V_{o}$ \\
Red-Elev & $V_{g} \frac{L}{L+L_{r}} \frac{d_{6}}{\left(1-d_{6}\right)}$ & $V_{g} \frac{d_{5}+d_{6}}{d_{3}}$ \\
Flyback & $V_{g} \frac{L_{m}}{L_{m}+L_{r}} \frac{d_{6}}{\left(1-d_{6}\right) n}$ & $V_{g} \frac{d_{5}+d_{6}}{d_{3}}$ \\
\hline
\end{tabular}

Tabla 2.5: Tensiones en función de la duración de los intervalos.

Otra expresión equivalente para la tensión de clamp se obtiene al hacer el balance neto de carga en $C_{c}$. Y cuando se igualan ambas expresiones de $V_{c}$ se consigue una nueva expresión para $V_{o}$, en función de la intensidad, que está recogida en la Tabla 2.6, donde se ha hecho uso de las equivalencias indicadas en (2.1).

\begin{tabular}{ccc}
\hline Convertidor & \multicolumn{1}{c}{$V_{c}$} & $V_{o}$ \\
\hline Reductor & $\frac{I_{\max }}{(1-D)} 2 L_{r} f$ & $V_{g} D-2 L_{r} f I_{\max }$ \\
Elevador & $\frac{I_{\max }}{(1-D)} 2 L_{r} f$ & $\frac{V_{g}}{(1-D)}-\frac{I_{\max }}{(1-D)} 2 L_{r} f$ \\
Red-Elev & $\frac{I_{\max }}{(1-D)} 2 L_{r} f+V_{o}$ & {$\left[\frac{V_{g} D}{(1-D)}-\frac{I_{\max }}{(1-D)} 2 L_{r} f\right]$} \\
Flyback & $\frac{I_{\max }}{(1-D)} 2 L_{r} f+n V_{o}$ & $\frac{V_{g} D}{(1-D) n}-\frac{I_{\max }}{(1-D) n} 2 L_{r} f$ \\
\hline
\end{tabular}

Tabla 2.6: Tensiones en función de la corriente en la bobina.

La expresión de la tensión de salida en régimen permanente recogida en la Tabla 2.5 es similar a la de un convertidor convencional, donde el término $d_{6}$ equivale al ciclo de trabajo, que anteriormente se había definido como ciclo efectivo, $d_{e f}$. La expresión equivalente de la misma tensión de salida 
recogida en la Tabla 2.6, representa la dependencia de dicha tensión con el valor máximo de la intensidad en la bobina principal. A medida que la bobina principal aumenta, el rizado disminuye, de forma que cuando la bobina es lo suficientemente grande como para despreciar el rizado de corriente, el valor máximo de corriente, $I_{\max }$ se sustituye por el valor medio $I$.

Esa última expresión también se puede ver como la tensión de salida que se obtendría si se colocara una resistencia de valor $2 L_{r} f /(1-D)^{2}$ entre la salida del convertidor y la carga. Por tanto, pensando en el reparto de corriente, se podrían conectar dos convertidores convencionales en paralelo, como está indicado en la Figura 1.10 y ecualizar el reparto. Respecto al término que aparece multiplicando a esa intensidad, $2 L_{r} f$, es un término que se puede interpretar de la misma manera que la resistencia propia de la bobina de un convertidor convencional, aunque sin pérdidas, de forma que la tensión de salida se vería afectada por la caída de tensión producida en esa resistencia. En los dos casos citados la resistencia incluiría pérdidas, mientras que para un convertidor ZVS, corresponde a un término equivalente a una resistencia pero sin incluir las pérdidas de una resistencia.

Las expresiones obtenidas anteriormente se pueden emplear, para obtener de forma teórica las principales magnitudes involucradas en la selección de componentes de alguno de los convertidores ZVS tratados, partiendo de unas especificaciones o parámetros de funcionamiento, así como en la simulación del mismo convertidor conmutado para su validación posterior.

\subsubsection{Ejemplo para un elevador ZVS.}

Por ejemplo, partiendo de las especificaciones de un elevador ZVS recogidas en la Tabla 2.7 se obtienen otros parámetros. Así, con $V_{g}$ y $V_{\text {out }}$ junto con la potencia suministrada, se consigue el valor medio de la intensidad en la entrada y en la salida, $I_{g}$ e $I_{\text {out }}$, y el valor de la carga resistiva a la salida. Empleando $(2.4)$ y $(2.5)$ se obtiene $d_{5}$ y los valores máximos y mínimos de corriente, mientras que $d_{6}$ se obtiene de la Tabla 2.6, de forma que el ciclo de trabajo necesario para conseguir las especificaciones iniciales es la suma de éste con $d_{5}$.

\begin{tabular}{llllllll}
\hline \hline$V_{g}$ & $160 \mathrm{~V}$ & $P$ & $400 \mathrm{~W}$ & $C_{o}$ & $5 \mu \mathrm{F}$ & $C_{c}$ & $0,47 \mu \mathrm{F}$ \\
$V_{o}$ & $400 \mathrm{~V}$ & $f$ & $100 \mathrm{kHz}$ & $L$ & $600 \mathrm{\mu H}$ & $L_{r}$ & $70 \mu \mathrm{H}$ \\
\hline
\end{tabular}

Tabla 2.7: Parámetros de un elevador ZVS.

$$
C_{c} \geq\left[\frac{1-D}{\pi f}\right]^{2} \frac{1}{L_{r}}
$$

Con estos datos se simula un elevador ZVS conmutado con componentes ideales, y se mide la tensión de salida, la intensidad en el diodo y en la bobina bobina principal. Las curvas están representadas en la Figura 2.4, 
mientras que en la Tabla 2.8 se han confrontado los resultados teóricos y los medidos en la simulación para intensidades y tensiones, junto con el error relativo entre ambos valores. Los parámetros que se comparan son el ciclo de trabajo empleado, la tensión media del condensador de clamp, la tensión media en el condensador a la salida, y la corriente media del diodo de salida. El tiempo muerto entre el apagado del interruptor principal y el encendido del interruptor auxiliar debe ser un valor tal que permita la descarga completa del condensador resonante $C_{r}$, y a su vez no supere el tiempo que la corriente $i_{r}$ emplea en invertir su sentido. En el modelo teórico se considera que este tiempo ya está incluido en $d_{5}$.

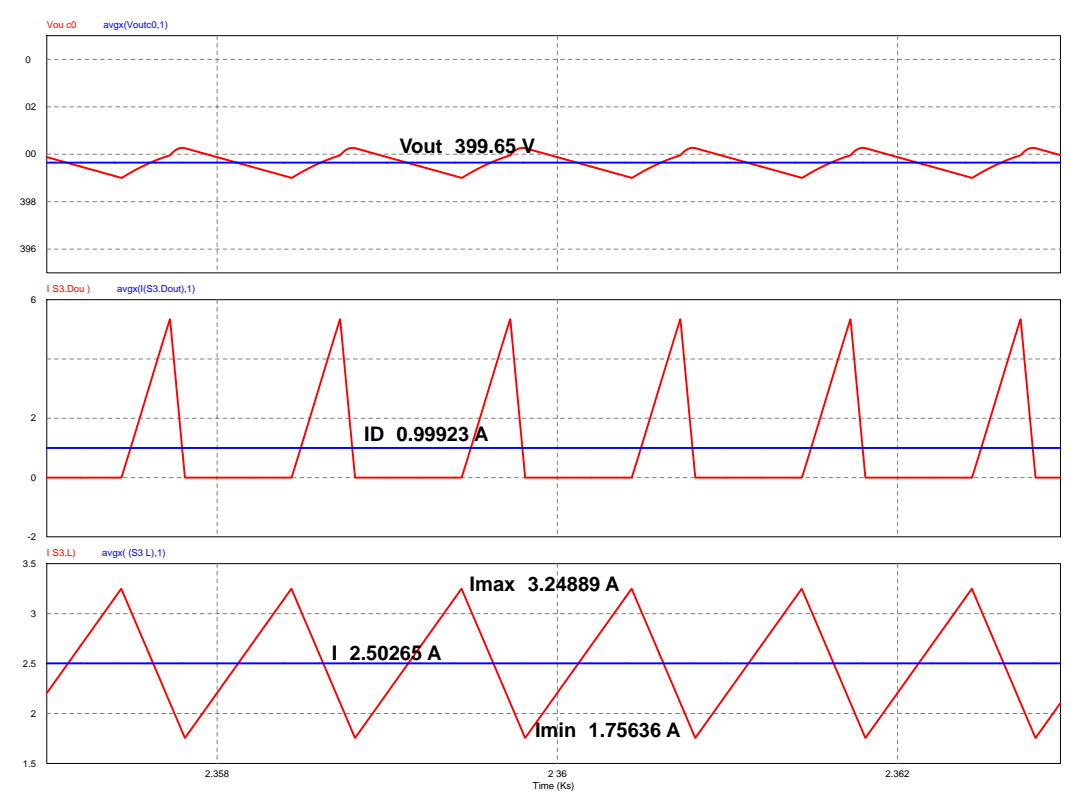

Figura 2.4: Simulación de un elevador ZVS: a) Tensión de salida; b) Intensidad en el diodo; y c) Intensidad en L

\begin{tabular}{|c|c|c|c|c|c|c|c|c|c|c|}
\hline & $\begin{array}{c}d_{5} \\
-\end{array}$ & $\begin{array}{c}d_{6} \\
-\end{array}$ & $\begin{array}{l}D \\
-\end{array}$ & $\begin{array}{c}V_{c} \\
(\mathrm{~V})\end{array}$ & $\begin{array}{c}V_{o} \\
(\mathrm{~V})\end{array}$ & $\begin{array}{l}I_{D} \\
(\mathrm{~A})\end{array}$ & $\begin{array}{c}I \\
(\mathrm{~A})\end{array}$ & $\begin{array}{l}\Delta_{i_{L}} \\
(\mathrm{~A})\end{array}$ & $\begin{array}{c}I_{\max } \\
(\mathrm{A})\end{array}$ & $\begin{array}{c}I_{\min } \\
(\mathrm{A})\end{array}$ \\
\hline TEO. & 0,0875 & 0,6262 & 0,7137 & 158,85 & 400 & 1 & 2,5 & 1,495 & 3,247 & 1,752 \\
\hline SIM. & 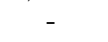 & - & - & 156,87 & 399,65 & 0,999 & 2,503 & 1,493 & 3,249 & 1,756 \\
\hline ERROR (\%) & & & & 1,26 & 0,087 & 0,1 & 0,12 & 0,13 & 0,062 & 0,23 \\
\hline
\end{tabular}

Tabla 2.8: Elevador ZVS: cálculos teóricos y simulados

Los resultados de la simulación son similares a los obtenidos teóricamente, presentando un error muy pequeño. El valor del condensador de clamp se ha obtenido para asegurar que durante el intervalo que aparece la resonancia del conjunto $C_{c}-L_{r}$, la corriente siempre sea decreciente. Este comportamiento 
tiene una duración igual a la mitad del periodo de resonancia de ambos elementos. A partir de aquí se obtiene un valor mínimo del condensador. Si aumentamos el tamaño del condensador, la corriente resonante se acercará a un comportamiento rectilíneo, la variación de tensión experimentada será menor para la misma energía intercambiada y la tensión medida será mas cercana a la propuesta por el modelo.

\subsubsection{Ejemplo para un reductor ZVS.}

Para un reductor ZVS, con las especificaciones indicadas en la Tabla 2.9, se obtienen los resultados teóricos y simulados que están recogidos en la Figura 2.5 y la Tabla 2.10 .

\begin{tabular}{llllllll}
\hline \hline$V_{g}$ & $30 \mathrm{~V}$ & $P$ & $130 \mathrm{~W}$ & $C_{o}$ & $5 \mu \mathrm{F}$ & $C_{c}$ & $5,2 \mu \mathrm{F}$ \\
$V_{o}$ & $13 \mathrm{~V}$ & $f$ & $100 \mathrm{kHz}$ & $L$ & $24 \mu \mathrm{H}$ & $L_{r}$ & $2 \mu \mathrm{H}$ \\
\hline
\end{tabular}

Tabla 2.9: Parámetros de un reductor ZVS.

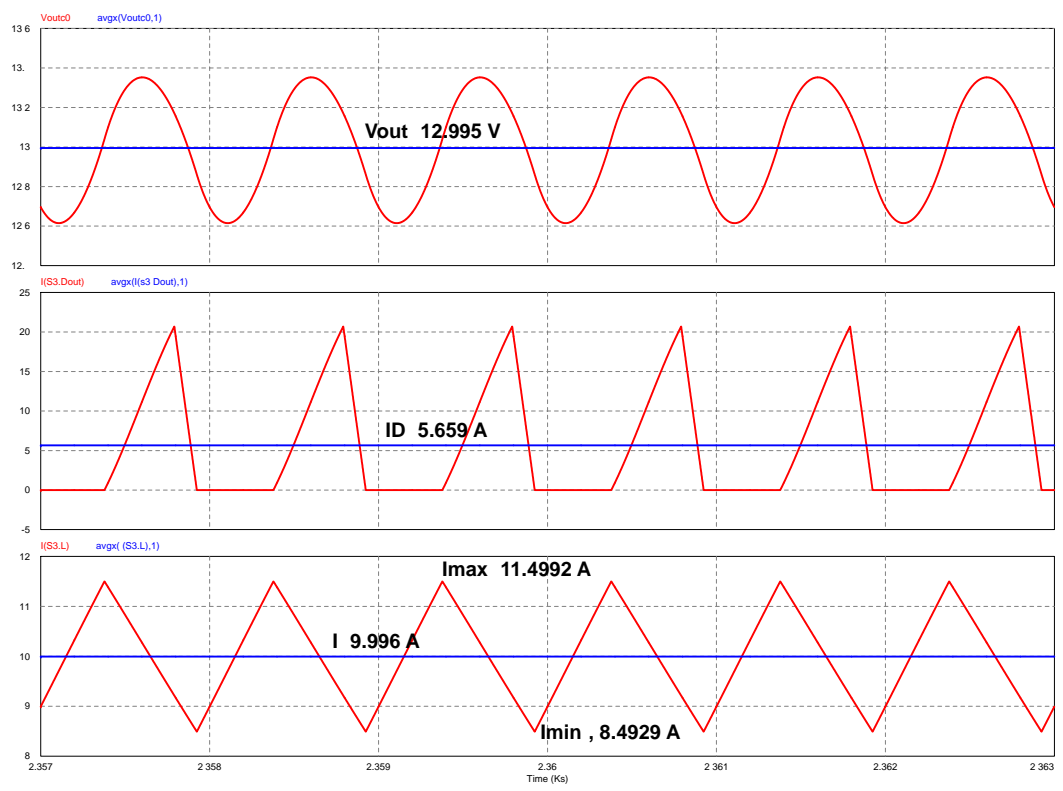

Figura 2.5: Simulación de un reductor ZVS: a) Tensión de salida; b) Intensidad en el diodo principal; y c) Intensidad en L

\subsubsection{Ejemplo para un red.elev. ZVS.}

En el caso del reductor elevador ZVS con los parámetros de diseño de la Tabla 2.11, se obtienen los resultados indicados en la Figura 2.6 y la Tabla 2.12 donde la similitud numérica se pone de nuevo de manifiesto. 


\begin{tabular}{ccccccccccc}
\hline & $d_{5}$ & $d_{6}$ & $D$ & $V_{c}$ & $V_{o}$ & $I_{D}$ & $I$ & $\Delta_{i_{L}}$ & $I_{\max }$ & $I_{\min }$ \\
& - & - & - & $(\mathrm{V})$ & $(\mathrm{V})$ & $(\mathrm{A})$ & $(\mathrm{A})$ & $(\mathrm{A})$ & $(\mathrm{A})$ & $(\mathrm{A})$ \\
\hline TEO. & 0,1333 & 0,4531 & 0,5864 & 11,103 & 13 & 5,667 & 10 & 2,9626 & 11,481 & 8,519 \\
SIM. & - & - & - & 10,199 & 12,996 & 5,659 & 9,996 & 3,006 & 11,499 & 8,493 \\
\hline ERROR (\%) & & & & 8,86 & 0,03 & 0,14 & 0,04 & 1,44 & 0,16 & 0,31 \\
\hline
\end{tabular}

Tabla 2.10: Reductor ZVS: cálculos teóricos y simulados

\begin{tabular}{llllllll}
\hline \hline$V_{g}$ & $160 \mathrm{~V}$ & $P$ & $400 \mathrm{~W}$ & $C_{o}$ & $5 \mu \mathrm{F}$ & $C_{c}$ & $0,47 \mu \mathrm{F}$ \\
$V_{o}$ & $400 \mathrm{~V}$ & $f$ & $100 \mathrm{kHz}$ & $L$ & $800 \mu \mathrm{H}$ & $L_{r}$ & $70 \mu \mathrm{H}$ \\
\hline
\end{tabular}

Tabla 2.11: Parámetros de un red-elev. ZVS.

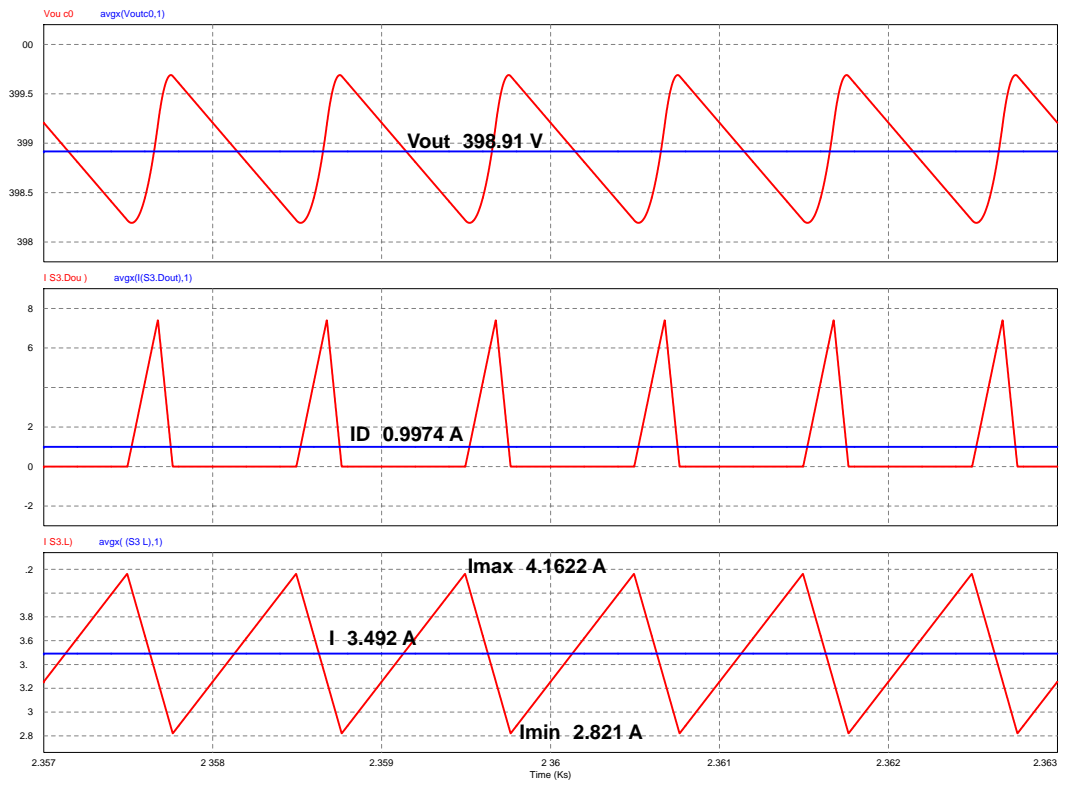

Figura 2.6: Simulación de un Red-Elev. ZVS: a) Tensión de salida; b) Intensidad en el diodo principal; y c) Intensidad en L

\begin{tabular}{ccccccccccc}
\hline & $d_{5}$ & $d_{6}$ & $D$ & $V_{c}$ & $V_{o}$ & $I_{D}$ & $\begin{array}{c}I \\
(\mathrm{~V})\end{array}$ & $\begin{array}{c}\Delta_{i_{L}} \\
(\mathrm{~V})\end{array}$ & $\begin{array}{c}I_{\max } \\
(\mathrm{A})\end{array}$ & $\begin{array}{c}I_{\min } \\
(\mathrm{A})\end{array}$ \\
\hline TEO. & - & - & - & $(\mathrm{A})$ & $(\mathrm{A})$ & $(\mathrm{A})$ \\
SIM. & -0875 & 0,7311 & 0,8186 & 721,98 & 400 & 1 & 3,5 & 1,345 & 4,172 & 2,827 \\
\hline ERROR (\%) & & - & - & 719,07 & 398,91 & 0,997 & 3,492 & 1,341 & 4,162 & 2,821 \\
\hline
\end{tabular}

Tabla 2.12: Red-Elev ZVS: cálculos teóricos y simulados

\subsubsection{Ejemplo para un flyback ZVS.}

Para el flyback ZVS con los parámetros de diseño de la Tabla 2.13, se obtienen los resultados de la Figura 2.6 y de la Tabla 2.14. 


\begin{tabular}{llllllll}
\hline \hline$V_{g}$ & $20 \mathrm{~V}$ & $P$ & $100 \mathrm{~W}$ & $C_{o}$ & $5 \mu \mathrm{F}$ & $C_{c}$ & $2,2 \mu \mathrm{F}$ \\
$V_{o}$ & $400 \mathrm{~V}$ & $f$ & $100 \mathrm{kHz}$ & $L_{m}$ & $24 \mu \mathrm{H}$ & $L_{r}$ & $2 \mu \mathrm{H}$ \\
$n_{1}: n_{2}$ & $1: 12$ & & & & & & \\
\hline
\end{tabular}

Tabla 2.13: Parámetros de un flyback ZVS.

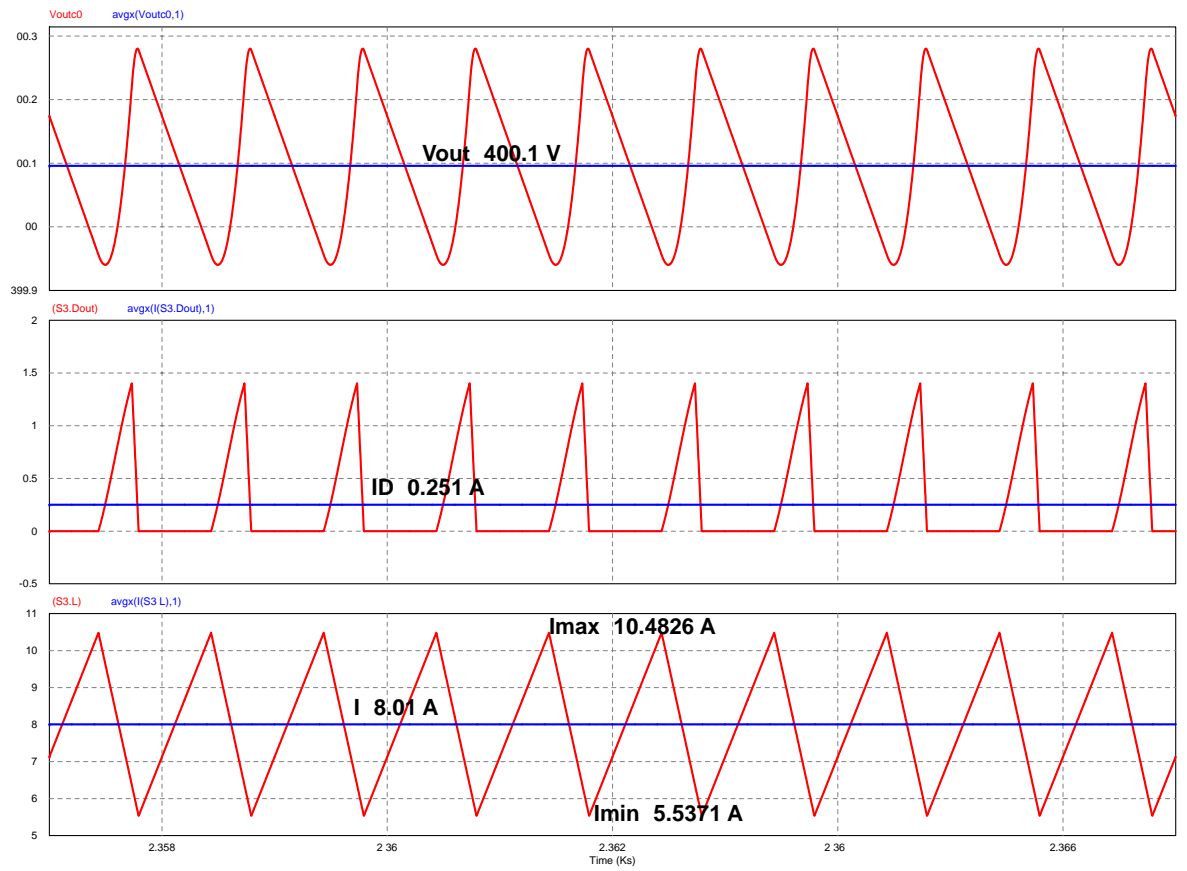

Figura 2.7: Simulación de un flyback ZVS: a) Tensión de salida; b) Intensidad en el diodo principal; y c) Intensidad en L

\begin{tabular}{|c|c|c|c|c|c|c|c|c|c|c|}
\hline & $\begin{array}{c}d_{5} \\
-\end{array}$ & $\begin{array}{c}d_{6} \\
-\end{array}$ & $\begin{array}{l}D \\
-\end{array}$ & $\begin{array}{c}V_{c} \\
(\mathrm{~V})\end{array}$ & $\begin{array}{c}V_{o} \\
(\mathrm{~V})\end{array}$ & $\begin{array}{l}I_{D} \\
(\mathrm{~A})\end{array}$ & $\begin{array}{c}I \\
(\mathrm{~A})\end{array}$ & $\begin{array}{c}\Delta_{i_{L}} \\
(\mathrm{~A})\end{array}$ & $\begin{array}{c}I_{\max } \\
(\mathrm{A})\end{array}$ & $\begin{array}{c}I_{\min } \\
(\mathrm{A})\end{array}$ \\
\hline TEO. & 0,06 & 0,6436 & 0,7036 & 47,47 & 400 & 0,250 & 8 & 4,951 & 10,475 & 5,525 \\
\hline SIM. & - & - & - & 45,76 & 400,1 & 0,251 & 8,01 & 4,946 & 10,483 & 5,537 \\
\hline ERROR (\%) & & & & 3,74 & 0,024 & 0,40 & 0,12 & 0,10 & 0,076 & 0,22 \\
\hline
\end{tabular}

Tabla 2.14: Flyback ZVS: cálculos teóricos y simulados

\subsection{Característica de salida.}

La característica de salida, $V_{o}=f\left(I_{o}\right)$, para las diferentes topologías analizadas se obtiene al hacer uso de la relación entre la variación de corriente y la duración del intervalo $d_{5}$ indicada en (2.2). Esta relación se emplea en la expresión de la tensión de salida recogida en la Tabla 2.6, sabiendo que hay 
una relación entre la intensidad en la bobina y la intensidad en la salida, que está indicada en la Tabla 2.3. La igualdad de potencias $V_{o} I_{o}=V_{g} I_{g}$ puede ser necesaria en algún caso.

Si se quiere obtener $I_{o}=g\left(V_{o}\right)$, se despeja, bien de la característica de salida obtenida anteriormente, bien de (2.5), y sabiendo que $d_{5}=D-d_{6}$. Finalmente, se llega a las expresiones de la Tabla 2.15, que indican la característica de salida tomando $V_{o}$ o $I_{o}$ como variable independiente.

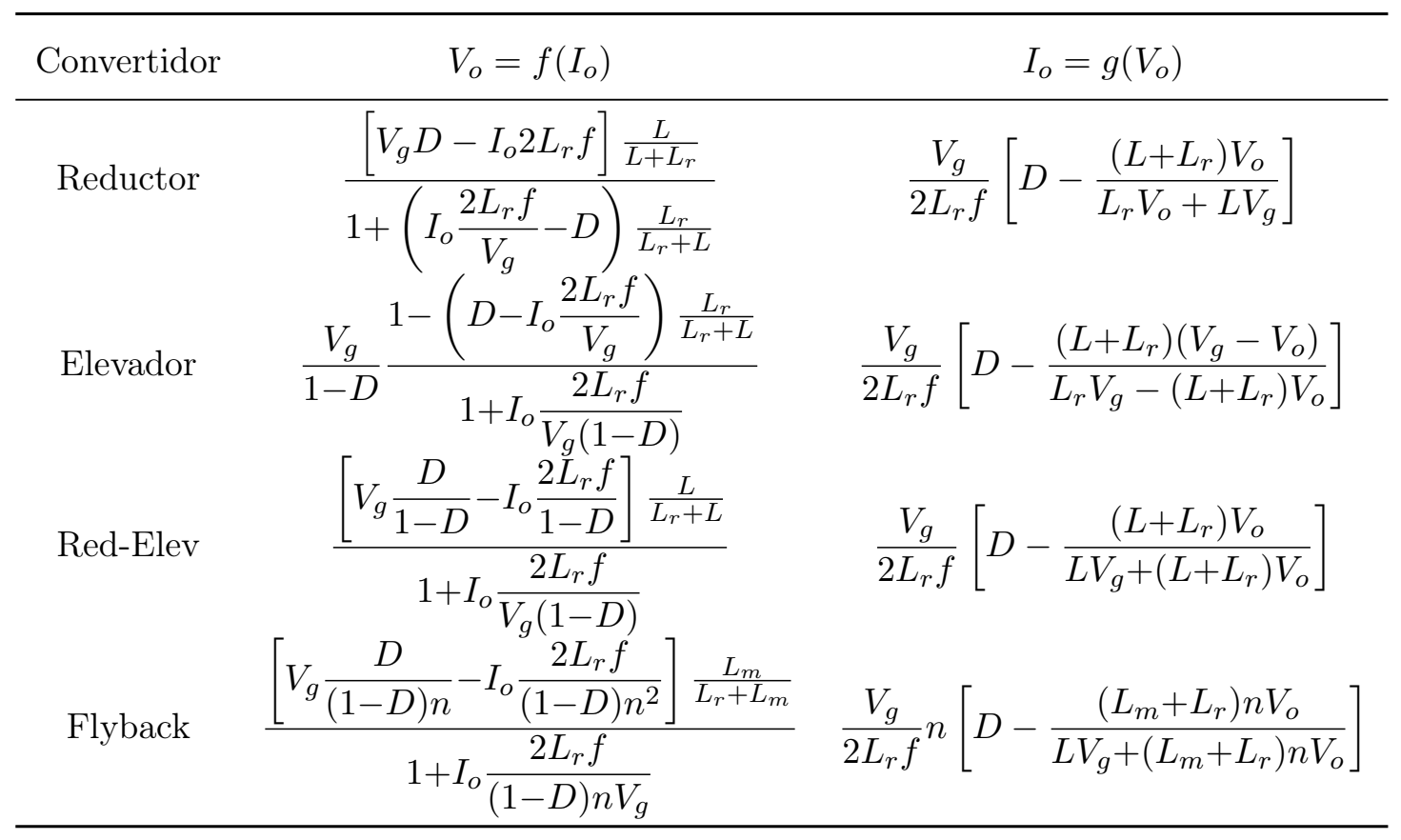

Tabla 2.15: Característica de salida.

Una representación gráfica de la característica de salida permite observar la evolución de la tensión de salida en función de la intensidad y anticipar el reparto de corriente si se conectaran en paralelo varios convertidores de este tipo.

A partir de los parámetros de funcionamiento de un reductor ZVS indicados en el apartado anterior, se ha representado su característica de salida en la Figura 2.8 empleando el mismo ciclo de trabajo y tomando diferentes valores de bobina resonante en torno a un valor considerado. De la misma forma se ha hecho con la bobina principal. 


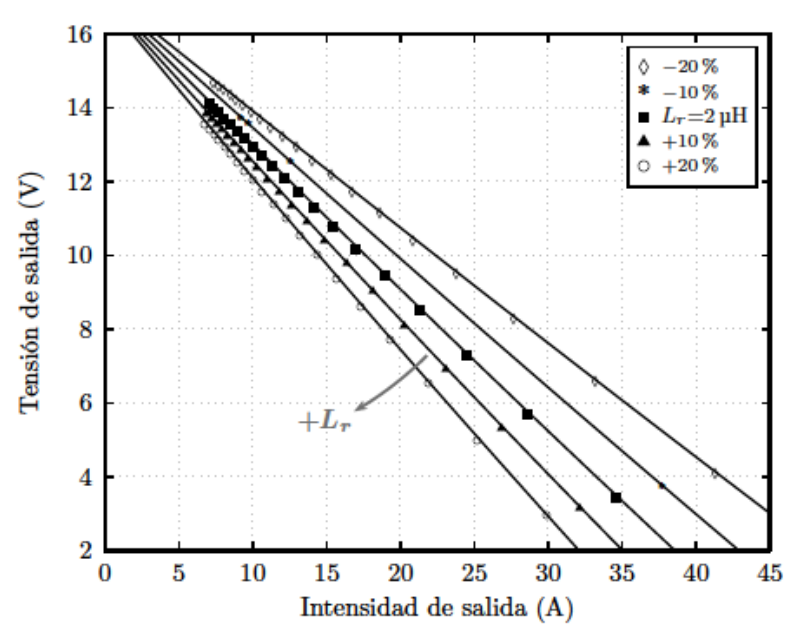

(a)

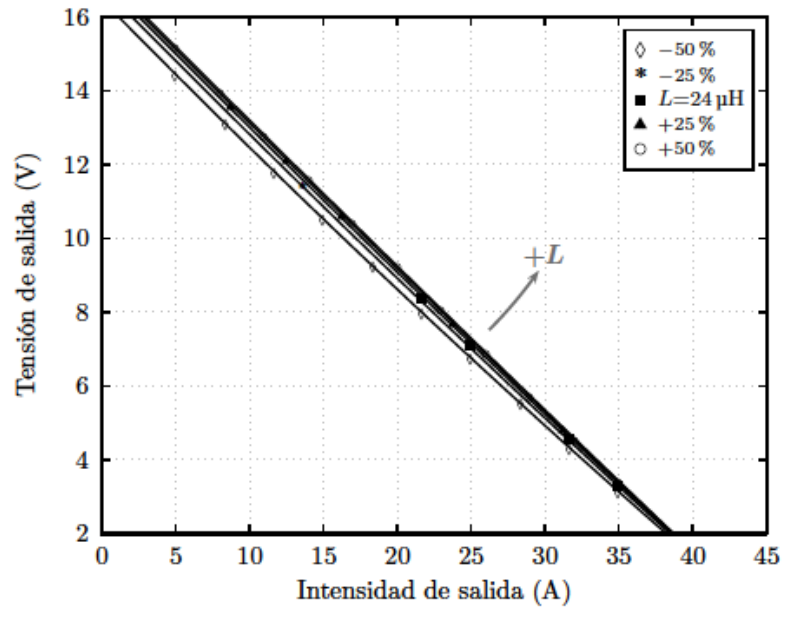

(b)

Figura 2.8: Caract.de salida para reductor ZVS: a) según $L_{r} ; \mathrm{y}$ b) según $L$.

Independientemente del valor elegido de bobina, se puede observar que en cada una de las curvas representadas, la tensión de salida decrece con la intensidad. Esto es por la inclusión de la bobina resonante, que aporta al comportamiento del convertidor una alta impedancia de salida, que facilita el reparto de corriente en una conexión en paralelo de etapas similares, como ya se ha indicado en el apartado 1.4.2 del capítulo anterior.

También se puede comprobar, que la característica experimenta un mayor cambio frente a las variaciones de la bobina resonante que frente a las variaciones de la bobina principal, puesto que las curvas según $L_{r}$ sufren una mayor dispersión que las curvas obtenidas según $L$. La prueba es que siendo el rango de variación de la bobina principal del $\pm 50 \%$, sus curvas están mas concentradas que las representadas para la bobina resonante (considerando los mismos ejes I-V) a pesar de que tiene un rango de variación inferior, que es del $\pm 20 \%$. Esto significa que la característica de salida es mucho mas sensible a las variaciones de $L_{r}$ que a las variaciones de $L$.

Por último, indicar que un aumento en la bobina resonante, considerando la misma carga, significa una disminución de la tensión de salida, es decir, un aumento de la impedancia de salida. En cambio, un aumento de la bobina principal, con la misma carga, produce el efecto contrario dado que la tensión aumenta respecto a otra etapa con menor bobina. Ahora, la impedancia de salida disminuye.

Este comportamiento se extiende al resto de topologías analizadas, como se observa si se analizan de la misma forma cada una de las características de salida representadas en la Figura 2.9. En este caso, se han empleado los parámetros de funcionamiento del apartado anterior. 

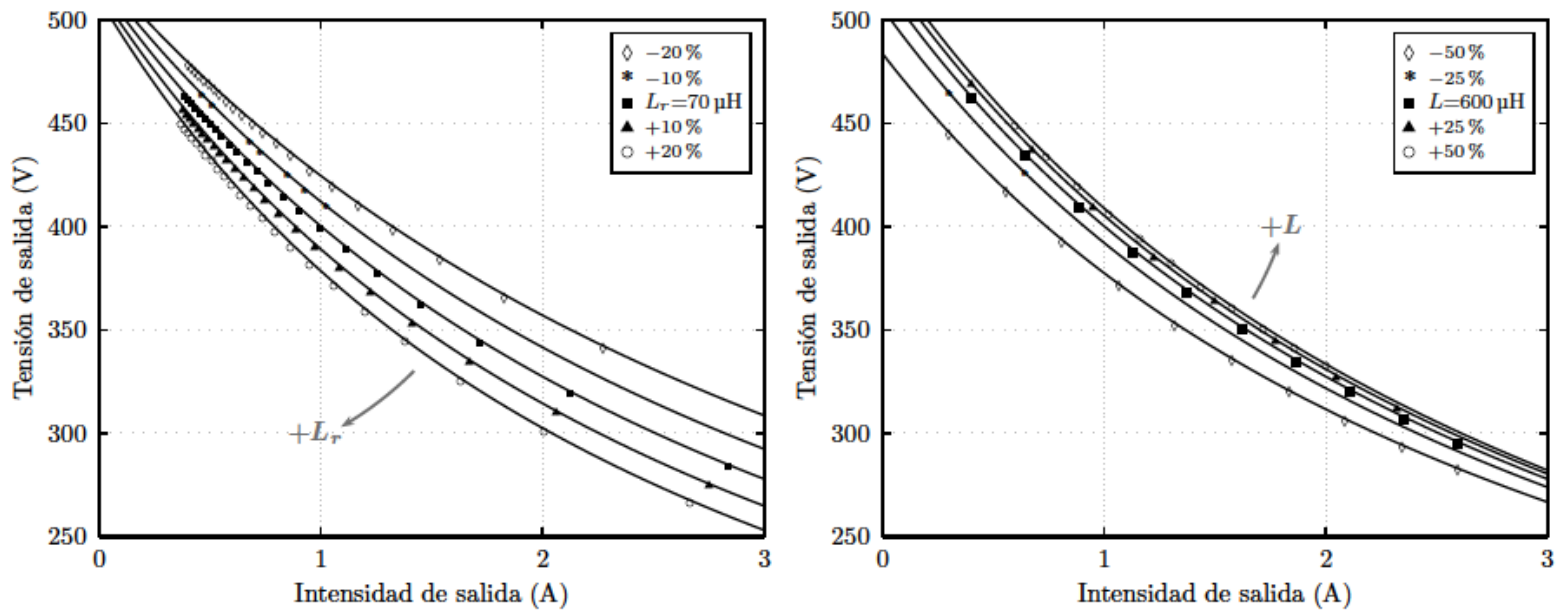

(a)
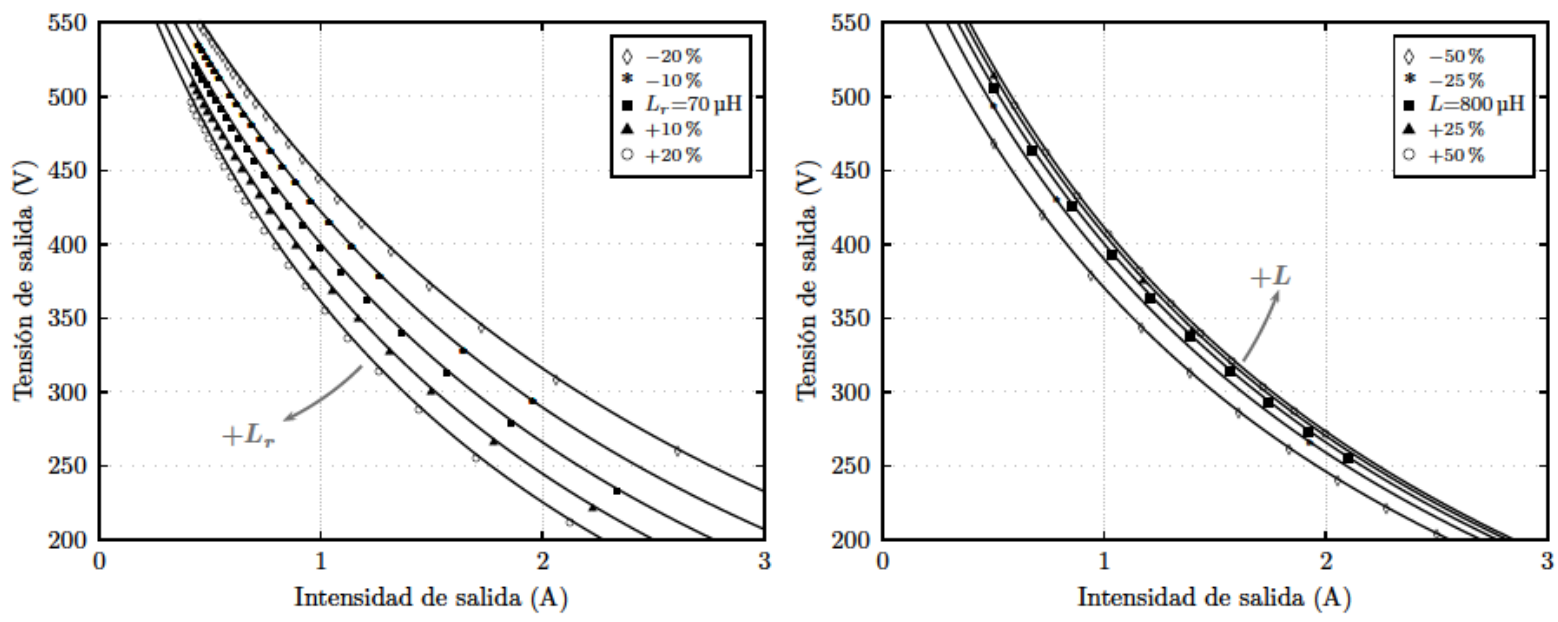

(b)
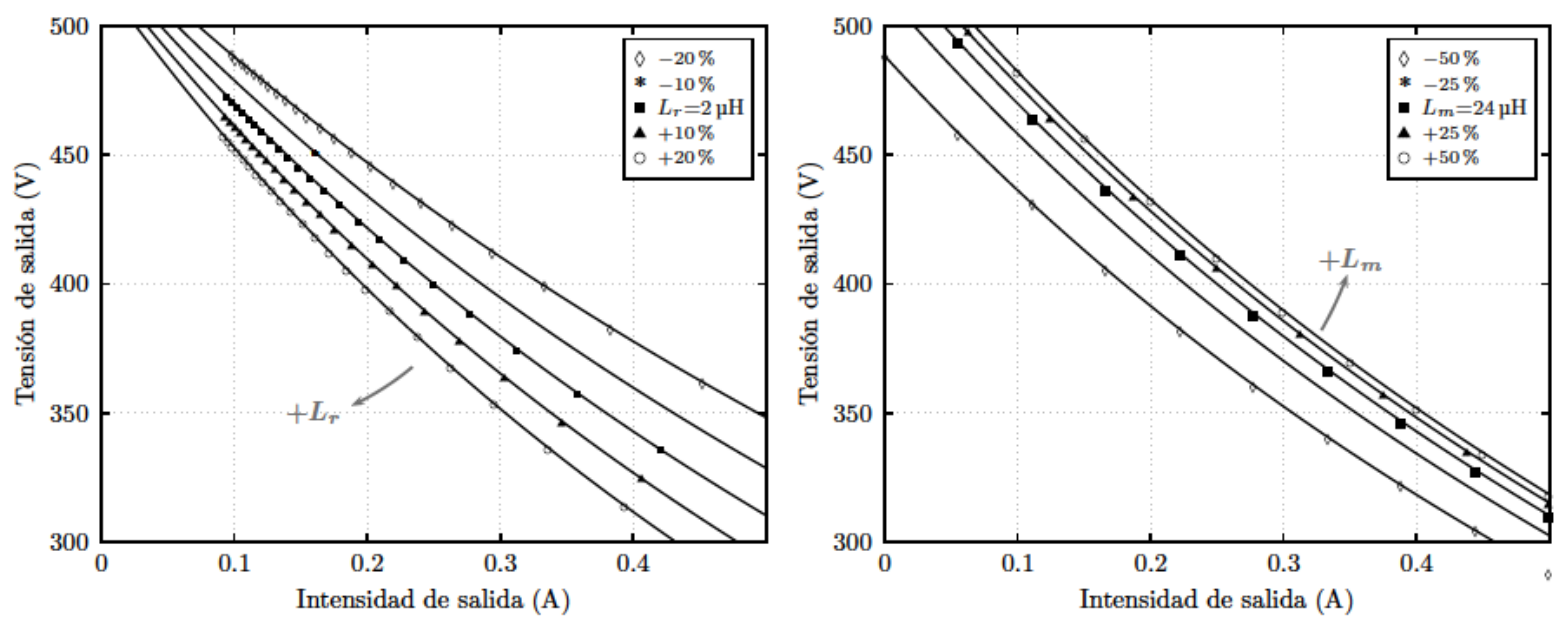

(c)

Figura 2.9: Caract.de salida en función de $L_{r}$ (izqda.) y de $L$ (dcha.) para: a) elev.ZVS ; b) red.elev.ZVS; y c) Flyb.ZVS. 


\subsubsection{Ejemplo de reparto en paralelo.}

Con la característica de salida representada anteriormente se puede predecir como será el reparto de carga entre varias etapas conectadas en paralelo. Por supuesto, éste será equitativo si las etapas son idénticas, mientras que no lo será cuando aparezcan diferencias entre ellas. Cuando la diferencia aparece en la bobina resonante, la etapa que gestiona mayor carga es aquella etapa con menor bobina resonante. Si la diferencia es únicamente sobre la bobina principal, sucederá que gestionará mayor carga aquella etapa que tenga un mayor valor de bobina principal.

Cuando se realiza la conexión en paralelo de varias etapas, el ciclo de trabajo, $D$, es el mismo para todas. Y su valor se puede predecir de forma teórica a partir de la expresión de la característica de salida. Si no hay ninguna diferencia entre etapas, el ciclo de trabajo se obtiene considerando que cada etapa gestiona una fracción idéntica de la carga.

En el caso de que haya diferencias entre las etapas, no se sabe que parte de la potencia total gestiona cada etapa ni tampoco se conoce el ciclo de trabajo. Este se obtiene resolviendo un sistema de ecuaciones formado por la ecuación de la característica de salida de cada etapa donde se conocen todos los datos excepto $D$, mas una última ecuación donde la suma de las corrientes de salida de cada etapa se iguala a la corriente total que se necesita para conseguir la potencia de salida deseada.

Considérese el ejemplo de tres etapas en paralelo tipo reductor ZVS, con los parámetros de la Tabla 2.9, que gestionan entre las tres etapas el triple de lo que gestionaría una sola etapa, es decir, un total de $390 \mathrm{~W}$. Tras plantear el sistema de ecuaciones, se obtiene el valor del ciclo de trabajo y las tres corrientes de salida, $I_{o, 1}, I_{o, 2}$ e $I_{o, 3}$. Además se introducen diferencias bien en $L_{r}$, bien en la bobina principal $L$.

En la Tabla 2.16 se ha indicado el valor del ciclo de trabajo y los valores teóricos de las corrientes de salida, así como el valor obtenido en la simulación del sistema completo, que se ha indicado entre paréntesis. Los resultados teóricos y de simulación son muy similares.

\begin{tabular}{|c|c|c|c|c|c|c|}
\hline & \multicolumn{3}{|c|}{ VARIACIÓN EN $L_{r}=2 \mu \mathrm{H}$} & \multicolumn{3}{|c|}{ VARIACIÓN EN $L=24 \mu \mathrm{H}$} \\
\hline & $\Delta L_{r, i}$ & $D$ & $I_{o, i}(\mathrm{~A})$ & $\Delta L_{i}$ & $D$ & $I_{o, i}(\mathrm{~A})$ \\
\hline ETAPA 1 & 0 & & $10,553(10,552)$ & 0 & & $9,743(9.729)$ \\
\hline ETAPA 2 & $+10 \%$ & 0,5938 & $9,464(9,453)$ & $+25 \%$ & 0,5830 & $10,031(10.025)$ \\
\hline ETAPA 3 & $+5 \%$ & & $9,983(9,976)$ & $+50 \%$ & & $10,226(10.226)$ \\
\hline$\sum_{i} I_{o, i}$ & & & $30(29,981)$ & & & $30(29.980)$ \\
\hline
\end{tabular}

Tabla 2.16: Reparto de corriente para tres etapas reductor ZVS en paralelo. Resultados teóricos (y simulados). 
Con este ejemplo, se pone de manifiesto de forma numérica el efecto que produce la diferencia entre los valores de las bobinas de cada etapa sobre el reparto de carga, y que se había indicado anteriormente en el apartado anterior sobre la representación gráfica de la característica de salida. Como la etapa 1 tiene una bobina resonante de $2 \mu \mathrm{H}$, y las otras dos etapas tienen bobinas de mayor valor, la etapa 1 es la que gestiona mas carga. Cuando se considera solo el efecto de la bobina principal, la etapa 1 es la que presenta el valor mas pequeño y es la etapa que gestiona menor carga de las tres etapas. En este caso, la etapa 3 la que gestiona mayor carga por tener mayor valor de $L$.

En la Figura 2.10 se ha representado la corriente de salida de cada etapa obtenida en la simulación, y su valor medio. Se comprueba el mismo comportamiento en el reparto no equitativo de las corrientes en función del valor de la bobina principal o de la bobina resonante, gestionando mayor corriente la etapa con menor bobina resonante o con mayor bobina principal.

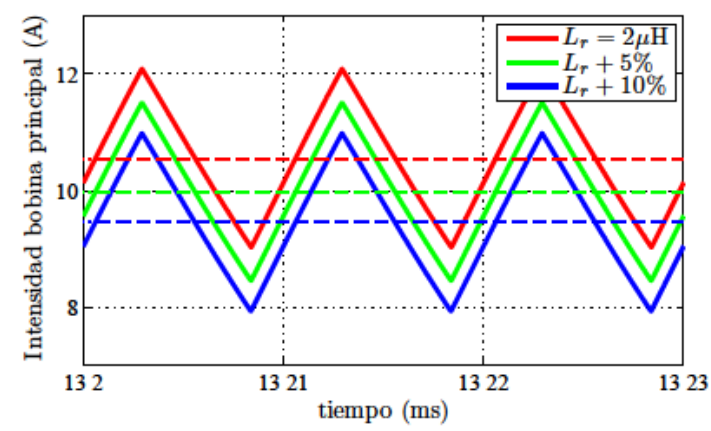

(a)

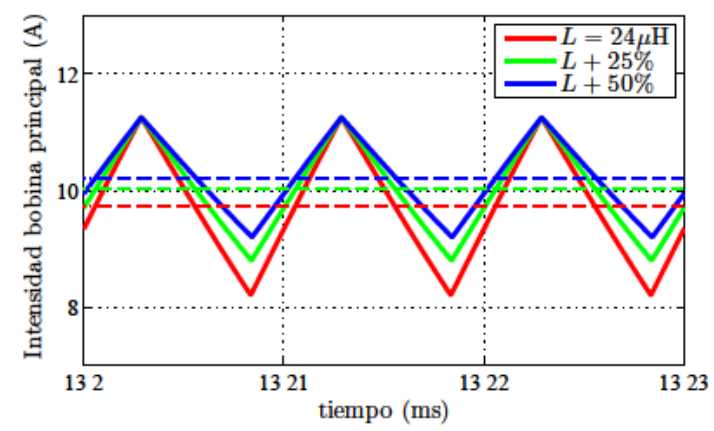

(b)

Figura 2.10: Corriente de salida para tres etapas reductor ZVS en paralelo: a) con variación en $L_{r} ; \mathrm{y}$ b) con variación en $L$.

Como se muestra en Figura 2.10b, la modificación del valor de la bobina principal no produce un cambio en el valor máximo de la corriente en esa bobina. La justificación se puede obtener de la expresión de $V_{o}=f\left(I_{o}\right)$ de la Tabla 2.6 donde $I_{\max }$ debe permanecer constante cuando el resto de términos de la expresión también lo son. $\mathrm{Al}$ no aparecer $L$ en esa expresión, su valor no afecta al valor máximo. También se muestra la disminución el rizado de corriente a medida que aumenta el valor de $L$, despareciendo éste cuando se hace muy grande dicho valor, coincidiendo entonces el valor medio y el valor máximo.

De aquí se deduce que, con los mismos parámetros de funcionamiento, 
el rizado implica una corriente de salida inferior a la que habría sin rizado, que significa una impedancia de salida mayor, que mejoraría el reparto. Por tanto, desde el punto de vista del reparto en paralelo basado en la impedancia de salida, el peor caso sería el contrario, es decir, la inexistencia de rizado, dado que la intensidad de salida sería mayor (lo que significaría una menor impedancia de salida).

Dado que la aproximación sin rizado de corriente (o de pequeño rizado) es la condición empleada al obtener el modelo promediado de un convertidor, resulta que considerar el modelo promediado para analizar el reparto significa considerar el caso mas desfavorable.

En el caso de tres etapas tipo elevador ZVS que gestionan entre todas el triple de potencia que una única etapa, los resultados obtenidos considerando los parámetros de funcionamiento utilizados anteriormente, están recogidos en la Tabla 2.17. En la Figura 2.11 se ha representado la corriente en el diodo de salida y en la bobina principal cuando cada etapa tiene un valor diferente de $L$.

\begin{tabular}{|c|c|c|c|c|c|c|}
\hline & \multicolumn{3}{|c|}{ VARIACIÓN EN $L_{r}=70 \mu \mathrm{H}$} & \multicolumn{3}{|c|}{ VARIACIÓN EN $L=600 \mu \mathrm{H}$} \\
\hline & $\Delta L_{r, i}$ & $D$ & $I_{o, i}(\mathrm{~A})$ & $\Delta L_{i}$ & $D$ & $I_{o, i}(\mathrm{~A})$ \\
\hline ETA & 0 & & 1,062 & 0 & & $0,949(0.949)$ \\
\hline ETAPA 2 & $+10 \%$ & 0,7191 & $0,940(0,9$ & $+25 \%$ & 0,7092 & $1,006(1.006)$ \\
\hline ETAPA 3 & $+5 \%$ & & $0,998(0,997)$ & $+50 \%$ & & $1,045(1.045)$ \\
\hline$\sum_{i} I_{o, i}$ & & & $3(2,998)$ & & & $3(3)$ \\
\hline
\end{tabular}

Tabla 2.17: Reparto de corriente para tres etapas elevador ZVS en paralelo. Resultado teórico (y de simulación).

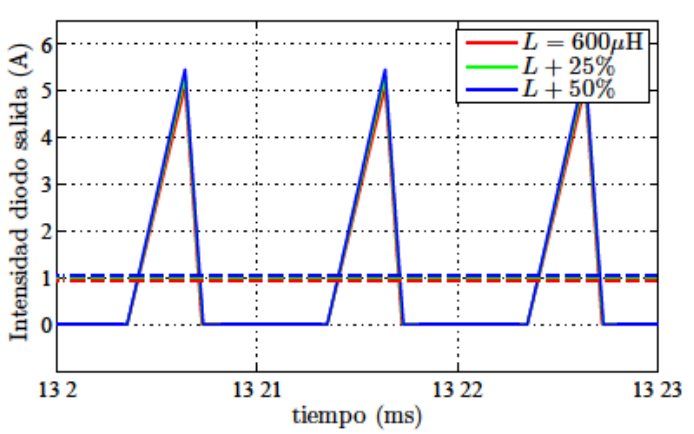

(a)

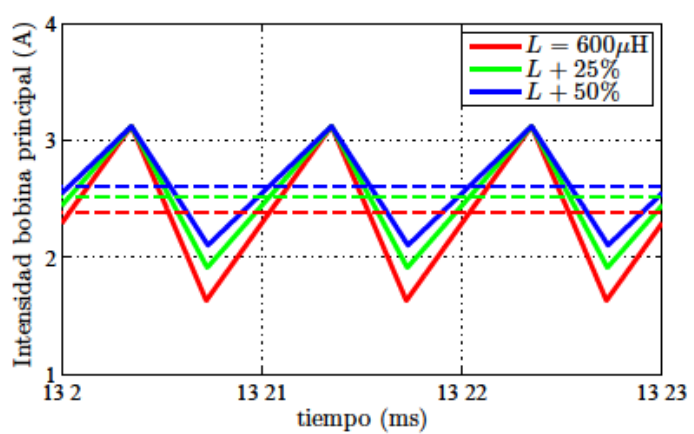

(b)

Figura 2.11: Corrientes en diodo y bobina principal de tres etapas elevador ZVS en paralelo con variación en $L$. 
Otra vez se cumple que la etapa con mayor bobina resonante tiene la menor carga y la que tiene mayor bobina principal es la que gestiona mas energía de las tres. También resulta que el valor máximo de la corriente es el mismo para las tres etapas.

En el caso de tres etapas flyback ZVS en paralelo también sucede el mismo comportamiento, como se pone de manifiesto en los resultados de la Tabla 2.18 y en la Figura 2.12.

\begin{tabular}{|c|c|c|c|c|c|c|}
\hline & \multicolumn{3}{|c|}{ VARIACIÓN EN $L_{r}=2 \mu \mathrm{H}$} & \multicolumn{3}{|c|}{ VARIACIÓN EN $L_{m}=24 \mu \mathrm{H}$} \\
\hline & $\Delta L_{r, i}$ & $D$ & $I_{o, i}(\mathrm{~A})$ & $\Delta L_{i}$ & $D$ & $I_{o, i}(\mathrm{~A})$ \\
\hline ETAPA 1 & 0 & & $0,266(0,266)$ & 0 & & $0,237(0.237)$ \\
\hline ETAPA 2 & $+10 \%$ & 0,7073 & $0,235(0,235)$ & $+25 \%$ & 0,7004 & $0,252(0.252)$ \\
\hline ETAPA 3 & $+5 \%$ & & $0,249(0,249)$ & $+50 \%$ & & $0,261(0.261)$ \\
\hline$\sum_{i} I_{o, i}$ & & & $0,750(0,750)$ & & & $0,750(0.750)$ \\
\hline
\end{tabular}

Tabla 2.18: Reparto de corriente para tres etapas flyback ZVS en paralelo. Resultado teórico (y de simulación).

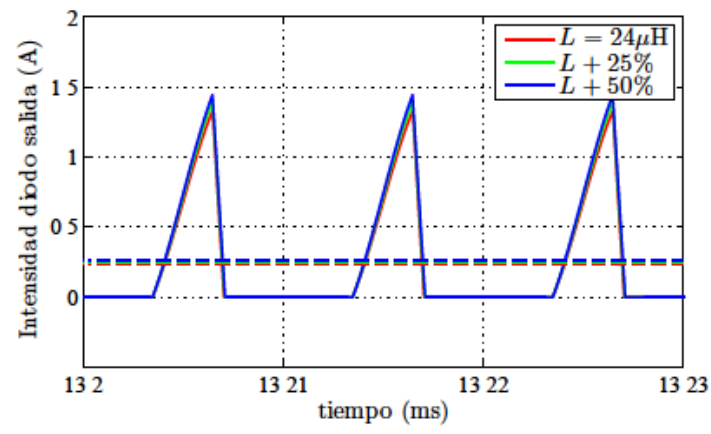

(a)

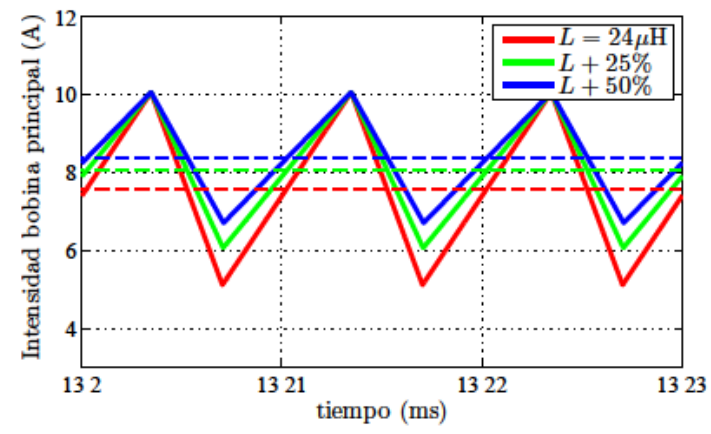

(b)

Figura 2.12: Corrientes en diodo y bobina principal de tres etapas flyback ZVS en paralelo con variación en $L_{m}$.

\subsection{Parámetros que afectan al reparto de carga.}

$\mathrm{Al}$ conectar varias etapas idénticas en paralelo, como se ha visto en el apartado anterior, la corriente se repartirá por igual entre todas. En el caso de existir diferencias entre las etapas, la corriente que circula por cada una de las etapas será diferente. Estas diferencias pueden ser debidas a varios factores: 
- Ciclo de trabajo,

- Tolerancia en las bobinas,

- Diferencias entre caídas de tensión en los semiconductores, especialmente los diodos, por la dependencia negativa que tiene la tensión con la temperatura.

En lo que respecta a las tensiones de entrada y salida, no se tendrán en cuenta en el caso de la conexión paralelo-paralelo. Respecto a las tolerancias en las bobina principal, producen rizados diferentes, y por tanto diferentes corrientes medias. Cuando se considera la bobina resonante, ésta se diseña para que todas las etapas tengan la misma impedancia, por lo que no tiene sentido plantear su efecto en el reparto para tolerancias grandes. Respecto a las caídas de tensión en los diodos, su variación con la temperatura puede agravar esta diferencia, aunque se puede considerar despreciable, sobre todo para tensiones de salida grandes.

Para estudiar como influyen las bobinas y el ciclo de trabajo, hay que estudiar como afecta cada parámetro a la corriente media de salida. La variación de la intensidad de salida con los diferentes parámetros se obtiene haciendo la derivada parcial respecto a cada parámetro. De forma que la variación en el reparto cuando existe una determinada tolerancia en un parámetro queda descrita en (2.6), donde $\Delta x$ representa la variación en términos absolutos del parámetro $x$ :

$$
\Delta I_{o}=\left|\frac{\partial I_{o}}{\partial D} \Delta D\right|+\left|\frac{\partial I_{o}}{\partial L_{r}} \Delta L_{r}\right|+\left|\frac{\partial I_{o}}{\partial L} \Delta L\right|
$$

El peor caso posible respecto al reparto, sucederá cuando la impedancia sea la mas pequeña posible, porque es cuando existe mayor diferencia de corrientes para cualquier parámetro. La menor impedancia se presenta cuando no hay rizado de corriente, es decir, cuando $L$ se hace muy grande. Por tanto, habrá que utilizar las expresiones cuando la bobina $L$ es muy grande, o lo que es lo mismo, hacer el límite de esas expresiones cuando $L \rightarrow \infty$.

Hacer $L \rightarrow \infty$ significa anular el tercer término de (2.6), con lo que no se podrá estudiar la influencia de la bobina principal en el reparto. Por tanto, hay que que tratar la expresión de la derivada parcial sin ninguna simplificación excepto la consideración de $L$ con un mayor valor que la bobina resonante.

La expresión de $I_{\text {out }}$ para el peor caso está indicada en la Tabla 2.19, mientras que en la Tabla 2.20 están recogidas las variaciones de la corriente de salida con el ciclo de trabajo, $D$, y con la bobina resonante. También se ha incluido la variación con la bobina principal con el supuesto de que $L$ es mucho mayor que $L_{r}$. 


\begin{tabular}{cc}
\hline Convertidor & $\lim _{L \rightarrow \infty} I_{o}$ \\
\hline Reductor & $\frac{V_{g} D-V_{o}}{2 L_{r} f}$ \\
Elevador & $\frac{V_{g}}{V_{o}}\left[\frac{V_{g}-V_{o}(1-D)}{2 L_{r} f}\right]$ \\
Red-Elev & $\frac{V_{g}}{V_{g}+V_{o}}\left[\frac{V_{g} D-V_{o}(1-D)}{2 L_{r} f}\right]$ \\
Flyback & $\frac{n V_{g}}{V_{g}+n V_{o}}\left[\frac{V_{g} D-n V_{o}(1-D)}{2 L_{r} f}\right]$ \\
\hline
\end{tabular}

Tabla 2.19: Intensidad media de salida cuando no se considera rizado en $L$.

\begin{tabular}{cccc}
\hline Convertidor & $\lim _{L \rightarrow \infty} \frac{\partial I_{o}}{\partial D}$ & $\lim _{L \rightarrow \infty} \frac{\partial I_{o}}{\partial L_{r}}$ & $\left.\frac{\partial I_{o}}{\partial L}\right|_{L \gg L_{r}}$ \\
\hline \multirow{2}{*}{ Reductor } & $\frac{V_{g}}{2 L_{r} f}$ & $\frac{V_{o}-D V_{g}}{2 L_{r}{ }^{2} f}$ & $\frac{V_{o}\left(V_{g}-V_{o}\right)}{2 f L^{2} V_{g}}$ \\
Elevador & $\frac{V_{g}}{2 L_{r} f}$ & $-\frac{V_{g}\left(V_{g}-V_{o}+D V_{o}\right)}{2 L_{r}{ }^{2} V_{o} f}$ & $-\frac{V_{g}^{2}\left(V_{g}-V_{o}\right)}{2 f L^{2} V_{o}^{2}}$ \\
Red-Elev & $\frac{V_{g}}{2 L_{r} f}$ & $-\frac{V_{g}(D-1)}{2 L_{r}{ }^{2} f}-\frac{V_{g}{ }^{2}}{2 L_{r}{ }^{2} f\left(V_{g}+V_{o}\right)}$ & $\frac{V_{g} V_{o}}{2 f L^{2}\left(V_{g}+V_{o}\right)^{2}}$ \\
Flyback & $\frac{V_{g} n}{2 L_{r} f}$ & $-\frac{V_{g} n\left(D V_{g}-V_{o} n+D V_{o} n\right)}{2 L_{r}^{2} f\left(V_{g}+V_{o} n\right)}$ & $\frac{V_{g}{ }^{2} V_{o} n^{2}}{2 f L_{m}^{2}\left(V_{g}+n V_{o}\right)^{2}}$ \\
\hline
\end{tabular}

Tabla 2.20: Dependencia de $I_{o}$ con diferentes parámetros.

Si hay $N$ etapas ZVS conectadas en paralelo con idéntica característica de salida, la corriente que circulará por cada uno de ellos será la misma e igual a la N-ésima parte de la intensidad $I_{o}$, que es la intensidad suministrada a la carga.

Las variaciones en algunos parámetros afectarán al reparto de corriente, de forma que la diferencia de corriente entre ellas se corresponderá con la expresión (2.6) mientras que la intensidad en la carga no sufrirá variación. Se considera la corriente real de la etapa $k$ con el término $I_{o, k}$, siendo su valor igual a la corriente de salida total, $I_{o}$, dividida entre el numero de etapas.

Suponiendo todas las etapas idénticas, menos la etapa $k$, en la que se incluyen variaciones de todos los parámetros, el error que se puede cometer respecto al valor nominal, será la diferencia entre la corriente real de esa etapa menos la corriente nominal que debería tener. Este error se obtiene de la siguiente forma:

$$
\begin{aligned}
\operatorname{error}_{k} & \equiv I_{o, k}-\frac{I_{o}}{N}=\left(I_{o}+\Delta I_{o}\right)-\frac{(N-1) I_{o}+\left(I_{o}+\Delta I_{o}\right)}{N} \\
& =\frac{N-1}{N} \Delta I_{o}
\end{aligned}
$$


Conociendo las tolerancias unitarias, $\delta x$, de los diferentes parámetros, se puede estimar la variación en el reparto cuando hay varias etapas conectadas dado que la relación entre la tolerancia unitaria y la variación en términos absolutos de un parámetro es igual a $\Delta x=x \times \delta x$.

En el caso de tres convertidores del tipo reductor ZVS en paralelo, con las especificaciones indicadas en la Tabla 2.9, y una potencia de salida de $P_{\text {out }}=390 \mathrm{~W}$, siendo la corriente nominal de salida por etapa de $10 \mathrm{~A}$; considerando tolerancias del 0,5\% para el ciclo de trabajo, del $10 \%$ para la bobina principal y del $10 \%$ para la bobina resonante, se espera una variación máxima de corriente como la indicada en la Tabla 2.21.

\begin{tabular}{|c|c|c|c|c|}
\hline$x$ & $\left|\frac{N-1}{N} \frac{\partial I_{o}}{\partial x} x\right|$ & $\delta x$ & $\frac{N-1}{N} \frac{\partial I_{o}}{\partial x} x$ & $\times \delta x$ \\
\hline $\mathrm{d}=0,5667$ & 28,333 & 0,005 & 0,1417 & \\
\hline $\mathrm{L}=24 \mu \mathrm{H}$ & 0,9531 & 0,1 & 0,0953 & \\
\hline$L_{r}=2 \mu \mathrm{H}$ & 6,6667 & 0,1 & 0,6667 & \\
\hline \multicolumn{5}{|c|}{ error $_{k}=0,9036 \quad(\approx 9,04 \%)$} \\
\hline
\end{tabular}

Tabla 2.21: Reductor ZVS. Error en el reparto para tres etapas en paralelo.

Para tres elevadores ZVS con las especificaciones ya mecionadas se espera una variación máxima de corriente igual a la que se indica en la Tabla 2.22. En este caso, la potencia de salida total es de $1200 \mathrm{~W}$ y la corriente de salida nominal por etapa es de $1 \mathrm{~A}$.

\begin{tabular}{|c|c|c|c|c|}
\hline$x$ & $\left|\frac{N-1}{N} \frac{\partial I_{o}}{\partial x} x\right|$ & $\delta x$ & $\frac{N-1}{N} \frac{\partial I_{O}}{\partial x} x$ & $\times \delta x$ \\
\hline $\mathrm{d}=0,6875$ & 5,238 & 0,005 & 0,0262 & \\
\hline $\mathrm{L}=600 \mu \mathrm{H}$ & 0,1863 & 0,1 & 0,0186 & \\
\hline$L_{r}=70 \mu \mathrm{H}$ & 0,667 & 0,1 & 0,0667 & \\
\hline & $\operatorname{ror}_{k}=0,11$ & \multicolumn{2}{|c|}{$(\approx 11,15 \%)$} & \\
\hline
\end{tabular}

Tabla 2.22: Elevador ZVS. Reparto de corriente para tres etapas en paralelo.

Cuando son tres convertidores reductor-elevador ZVS con las especificaciones empleadas anteriormente y una potencia de salida total de $1200 \mathrm{~W}$ con una corriente de salida nominal por etapa es de $1 \mathrm{~A}$, se esperan los resultados de la Tabla 2.23 .

\begin{tabular}{|c|c|c|c|c|}
\hline$x$ & $\frac{N-1}{N} \frac{\partial I_{o}}{\partial x} x$ & $\delta x$ & $\left|\frac{N-1}{N} \frac{\partial I_{o}}{\partial x} x\right|$ & $\times \delta x$ \\
\hline $\mathrm{d}=0,8018$ & 6,1088 & 0,005 & 0,0305 & \\
\hline $\mathrm{L}=800 \mu \mathrm{H}$ & 0,1205 & 0,1 & 0,0121 & \\
\hline$L_{r}=70 \mu \mathrm{H}$ & 0,6667 & 0,1 & 0,0667 & \\
\hline
\end{tabular}

Tabla 2.23: Red.Elev. ZVS. Error en el reparto para tres etapas en paralelo. 
En el caso de tres flyback ZVS con las especificaciones de la Tabla 2.13 se esperan los resultados de la Tabla 2.24.La potencia de salida total es de $300 \mathrm{~W}$ con una corriente de salida nominal por etapa es de $0,25 \mathrm{~A}$

\begin{tabular}{ccc|c}
\hline$x$ & $\left|\frac{N-1}{N} \frac{\partial I_{o}}{\partial x} x\right|$ & $\delta x$ & $\left|\frac{N-1}{N} \frac{\partial I_{o}}{\partial x} x\right| \times \delta x$ \\
\hline $\mathrm{d}=0,6850$ & 1,9028 & 0,005 & 0,0095 \\
$\mathrm{~L}=24 \mu \mathrm{H}$ & 0,0490 & 0,1 & 0,0049 \\
$L_{r}=2 \mu \mathrm{H}$ & 0,1667 & 0,1 & 0,0167 \\
\hline \multicolumn{3}{c}{ error $_{k}=0,0311$} & $(\approx 12,44 \%)$ \\
\hline
\end{tabular}

Tabla 2.24: Error en reparto de corriente para dos etapas flyback ZVS en paralelo.

De todos los resultados anteriores, se deduce que la variación de corriente debida a la tolerancia del ciclo de trabajo es muy sensible. A pesar de ello, su efecto queda minimizado cuando el sistema es controlado digitalmente puesto que la tolerancia suele ser inferior a la inicialmente prevista. Entonces, la desviación de corriente se debe principalmente a las tolerancias en las bobinas. Si la bobina principal se toma de un valor mas grande, se minora su efecto y solo queda la tolerancia de la bobina resonante, que permite minimizar la diferencia de corriente si se mantiene en un valor pequeño.

\subsection{Impedancia de salida en régimen permanente.}

La impedancia de salida en régimen permanente se obtiene a partir del modelo en régimen permanente del convertidor, que a su vez es una particularización de su modelo promediado. Una vez determinada la impedancia puede estudiarse el reparto estático de carga entre varias etapas conectadas en paralelo basado en esa impedancia.

En la Figura 2.13 se incluye el circuito promediado que se refleja de las ecuaciones del modelo promediado de cada convertidor ZVS, obtenido según el método indicado en (CHEN y Sun, 2006) y que se encuentra desarrollado en el anexo correspondiente a cada topología (Anexos 1 a 4 ).

En cada uno de los circuitos representados no aparece $L_{r}$ de forma explicita, sino que está incluida dentro del término $r_{e q}$, denominado resistencia equivalente, porque presenta dimensiones de impedancia. El elemento en forma de fuente equivalente de corriente, $i_{e q}$, es dependiente de la energía que es consumida por la mencionada resistencia equivalente, a la vez que no consume ninguna energía de la fuente externa. Por tanto no influye en el balance energético del circuito y se comporta como una resistencia sin pérdidas. 


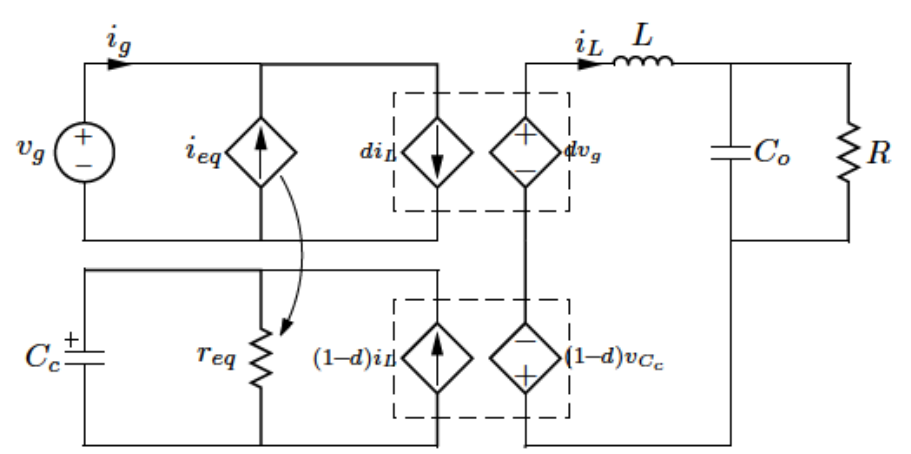

(a)

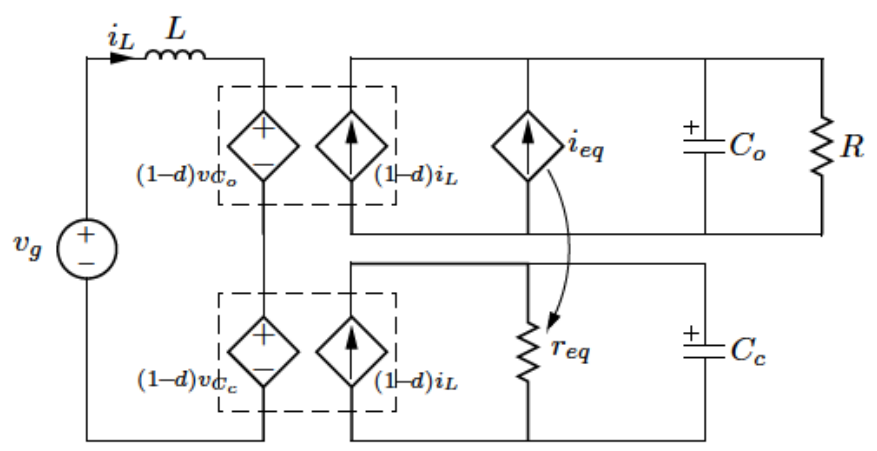

(b)

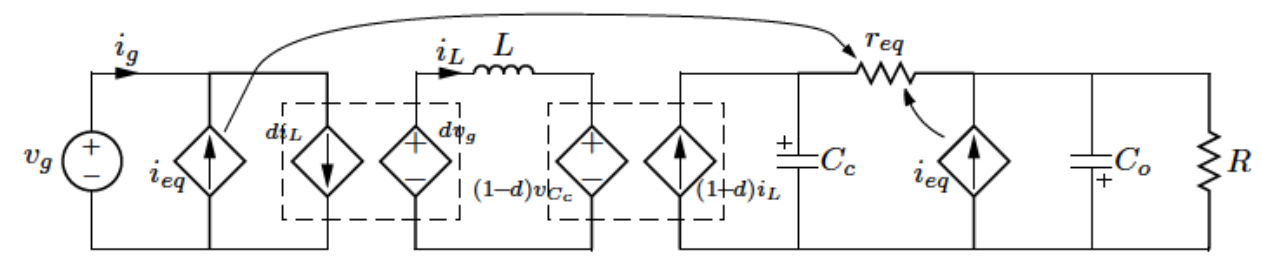

(c)

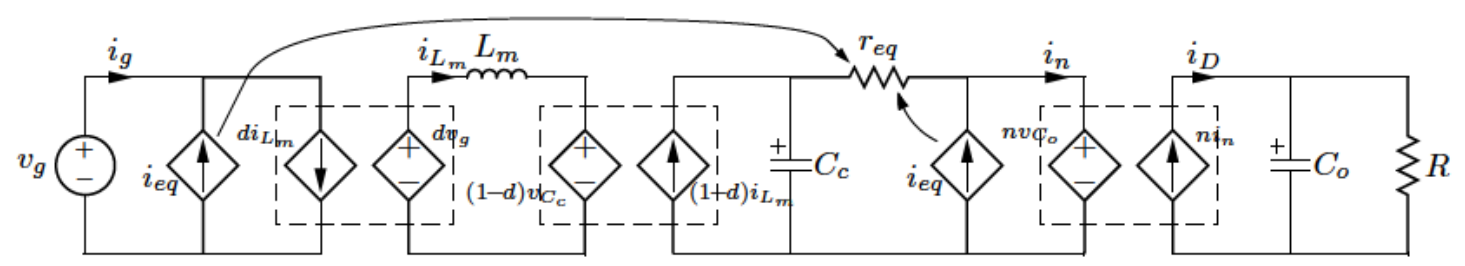

(d)

Figura 2.13: Circuito promediado para convertidores ZVS: a) reductor; b) elevador; c) red-elev.; y d) flyback.

En la Figura 2.14 están representados los circuitos en régimen permanente para los mismos convertidores anteriores, obtenidos a partir de los circuitos promediados. En el modelo en régimen permanente, $R_{e q}$ e $I_{e q}$ son $r_{e q}$ e $i_{e q}$, con términos constantes. Sus expresiones se recogen en la Tabla 2.25. 


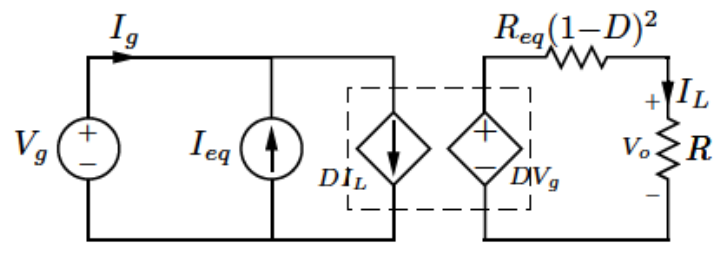

(a)

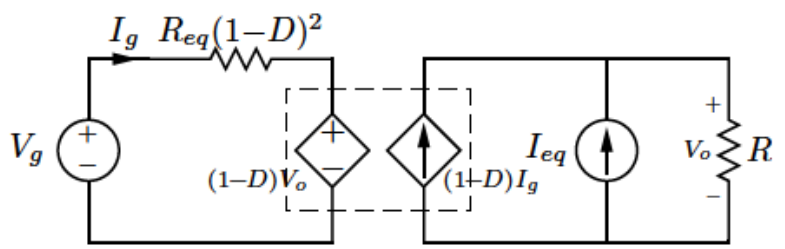

(b)

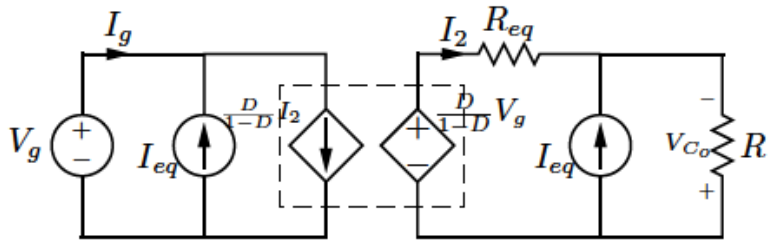

(c)

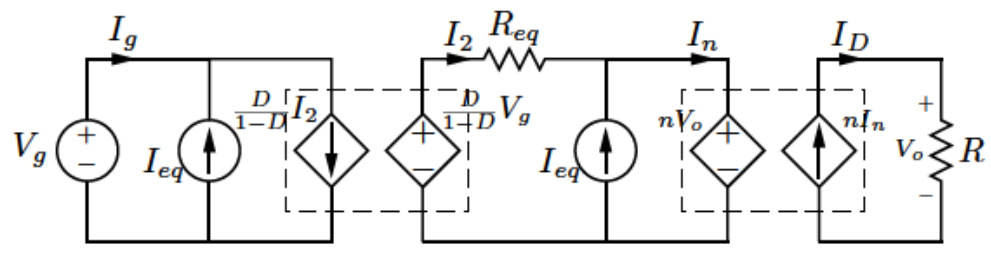

(d)

Figura 2.14: Circuitos en régimen permanente para: a) reductor ZVS; b) elevador ZVS; d) red-elev. ZVS, y d) flyback ZVS.

\begin{tabular}{lcccc}
\hline & Reductor & Elevador & Red-Elev. & Flyback \\
\hline$i_{e q}$ & $\frac{v_{C_{c}}^{2}}{v_{g} r_{e q}}$ & $\frac{v_{C_{c}}^{2}}{v_{C_{o}} r_{e q}}$ & $\frac{\left(v_{C_{c}}-v_{C_{o}}\right)^{2}}{\left(v_{g}+v_{C_{o}} r_{e q}\right.}$ & $\frac{\left(v_{C_{c}}-n v_{C_{o}}\right)^{2}}{\left(v_{g}+n v_{C_{o}}\right) r_{e q}}$ \\
\hline$r_{e q}$ & \multicolumn{5}{c}{$\frac{2 L_{r} f}{(1-d)^{2}}$} \\
\hline$R_{e q}$ & \multicolumn{2}{c}{$\frac{}{(1-D)^{2}}$} \\
\hline$I_{e q}$ & $\frac{V_{c}^{2}}{V_{g} R_{e q}}$ & $\frac{V_{c}^{2}}{V_{o} R_{e q}}$ & $\frac{\left(V_{c}-V_{o}\right)^{2}}{\left(V_{g}+V_{o}\right) R_{e q}}$ & $\frac{\left(V_{c}-n V_{o}\right)^{2}}{\left(V_{g}+n V_{o}\right) R_{e q}}$ \\
\hline
\end{tabular}

Tabla 2.25: Expresiones para resistencia y fuente de intensidad equivalentes del circuito promediado (minúscula) y de régimen permanente (Mayúscula). 
De cada uno de estos circuitos se obtiene la relación entre la tensión y la intensidad de salida, esto es, la característica de salida. Sus expresiones están indicadas en la Tabla 2.26. Otra forma de llegar a ellas es hacer $L \rightarrow \infty$ en las expresiones de la Tabla 2.15. La explicación es sencilla: el modelo promediado se obtiene con la aproximación de pequeño rizado, que es lo mismo que decir que la bobina es muy grande, que es similar a hacer $L \rightarrow \infty$.

\begin{tabular}{|c|c|c|}
\hline Convertidor & $V_{o}=f\left(I_{o}\right)$ & $d V_{o} / d I_{o}$ \\
\hline Red. & $\begin{array}{c}V_{g} D-I_{o} 2 L_{r} f \\
V_{g}\end{array}$ & $-2 L_{r} f$ \\
\hline Elev. & $\frac{\frac{1-D}{1+I_{o} \frac{2 L_{r} f}{V_{g}(1-D)}}}{D}$ & $-2 L_{r} f\left[\frac{V_{o}}{V_{g}}\right]^{2}$ \\
\hline Red-Elev & $\frac{V_{g} \frac{D}{1-D}-I_{o} \frac{2 L_{r} f}{1-D}}{1+I_{o} \frac{2 L_{r} f}{V_{g}(1-D)}}$ & $-2 L_{r} f\left[\frac{V_{g}+V_{o}}{V_{g}}\right]^{2}$ \\
\hline Flyback & $V_{g} \frac{D}{(1-D) n}-I_{o} \frac{2 L_{r} f}{(1-D) n^{2}}$ & $\underline{2 L_{r} f}\left[\underline{V_{g}+n V_{o}}\right]^{2}$ \\
\hline 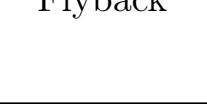 & $1+I_{o} \frac{2 L_{r} f}{(1-D) n V_{g}}$ & $\frac{n^{2}}{V_{g}}$ \\
\hline
\end{tabular}

Tabla 2.26: Característica de salida y su derivada.

Aproximando la característica de salida en un punto de funcionamiento por un modelo lineal que pasa por ese punto, se distinguen dos parámetros, que dependen del punto de funcionamiento donde esté trabajando el convertidor: el primero de ellos equivale a una resistencia, de valor igual a la derivada de la característica de salida (puesto que tiene dimensiones de impedancia); el otro equivale a una fuente de tensión, $V_{0}$, cuyo valor es igual a la intersección del eje de ordenadas con una recta que se prolonga desde el punto de trabajo y tangente a la característica de salida. Dicha tensión se obtiene como:

$$
V_{0}=V_{o}-\frac{d V_{o}}{d I_{o}} \times I_{o}=\left\{\begin{array}{cc}
V_{g} D & \text { (Reductor) } \\
V_{o}+\frac{V_{o}}{V_{g}}\left[V_{g}-V_{o}(1-D)\right] & \text { (Elev.) } \\
\frac{D\left(V_{g}+V_{o}\right)^{2}-V_{o}^{2}}{V_{g}} & \text { (Red.Elev.) } \\
\frac{D\left(V_{g}+n V_{o}\right)^{2}-\left(n V_{o}\right)^{2}}{n V_{g}} & \text { (Flyback) }
\end{array}\right.
$$

y no depende de $L_{r}$. La expresión de la derivada de la característica de salida, evaluada en un punto de funcionamiento, aparece en la misma tabla que ésta, 
y muestra una dependencia con la bobina resonante, $L_{r}$, y con las tensiones de entrada y de salida.

En el caso de conexión en paralelo, como la tensión de entrada y de salida y el ciclo de trabajo son los mismos para todas las etapas conectadas, resulta que en la aproximación lineal, la tensión $V_{0}$ siempre es la misma para todas las etapas (aunque su valor se diferente para cada punto de funcionamiento). $\mathrm{Y}$ se puede concluir que el reparto solo depende de una relación entre las diferentes resistencias de cada etapa, como se indica en el apartado 1.4.2 del capítulo dedicado al estado del arte.

Ahora bien, como, en este caso, el término de resistencia de cada etapa es proporcional a $L_{r}$, la diferencia de reparto entre las etapas va a depender, en última instancia, de la diferencia entre las bobinas resonantes.

Por ejemplo, para $N$ etapas conectadas en paralelo funcionando en un punto concreto, cada una con una bobina resonante $L_{r, k}$ y realizando la aproximación lineal mencionada, la corriente de salida en la etapa $k$ tiene la expresión:

$$
I_{o, k}=\frac{I_{o}}{\sum_{i=1}^{N}\left(\frac{L_{r, k}}{L_{r, i}}\right)}
$$

A esta expresión se llega cuando se determina la corriente que circula por cada una de las $N$ resistencias conectadas en paralelo, $R_{k}$ alimentadas desde una fuente de tensión constante y que están conectadas en el otro extremo a una carga resistiva $R_{L}$ por la que circula una intensidad $I_{o}$. Si se considera que el valor de cada una de esas resistencias es directamente proporcional al término $L_{r}$, resulta que $R_{k}=K \times L_{r}$. Tras simplificar, se obtiene la expresión indicada.

\subsubsection{Ejemplo.}

Se realiza la simulación de tres etapas, con diferentes bobinas resonantes, que están conectadas en paralelo. Cada etapa está representada por su modelo promediado en un punto de funcionamiento. También se realiza otra simulación cuando cada etapa se representa con el equivalente Thévenin del circuito en régimen permanente, formado por una resistencia, $R_{t h}$, y una fuente de tensión, $V_{t h}$. Las expresiones de estos dos términos para los convertidores tratados, se encuentran recogidas en la Tabla 2.27 .

La tensión Thévenin incluye un primer término, que recuerda a la tensión de salida de un convertidor convencional, que se suma al producto $I_{e q} R_{e q}$, para mostrar un valor dependiente del punto de funcionamiento, pero independiente de $L_{r}$. Por otro lado, el término que hace de resistencia Thévenin, $R_{t h}$, incluye a la resistencia $R_{e q}$ multiplicada por un factor que aparece 


\begin{tabular}{ccccc}
\hline & Reductor & Elevador & Red-Elev. & Flyback \\
\hline$V_{t h}$ & $V_{g} D$ & $\frac{V_{g}}{1-D}+I_{e q} R_{e q}$ & $\frac{V_{g} D}{1-D}+I_{e q} R_{e q}$ & $\frac{1}{n}\left[\frac{V_{g} D}{1-D}+I_{e q} R_{e q}\right]$ \\
$R_{t h}$ & $R_{e q}(1-D)^{2}$ & $R_{e q}$ & $R_{e q}$ & $R_{e q} / n^{2}$ \\
\hline
\end{tabular}

Tabla 2.27: Expresiones del equivalente Thévenin en régimen permanente.

cuando se toma su valor visto desde la salida del convertidor, y por tanto, dependiente de la bobina resonante.

Los resultados obtenidos en la medida de la corriente del terminal de salida de cada etapa para los dos tipos de simulaciones citadas son casi idénticas. Por ejemplo, para el elevador ZVS de tres etapas, se obtienen los resultados de la Tabla 2.28 estando el convertidor en el punto de funcionamiento ya usado anteriormente. $\bar{I}_{o, \text { prom }}$ representa la corriente medida sobre el modelo promediado mientras que $\bar{I}_{o, t h}$ es la corriente medida en el equivalente Thévenin. En la misma tabla se ha incluido la columna citada como $\bar{I}_{o, \text { conm }}$, que corresponde al valor medio de la corriente medida a la salida de una etapa cuando el convertidor se considera con su circuito conmutado.

\begin{tabular}{cccccc}
\hline & $\Delta L_{r, i}$ & & $\bar{I}_{o, \text { conm }}(\mathrm{A})$ & $\bar{I}_{o, \text { prom }}(\mathrm{A})$ & $\bar{I}_{o, t h}(\mathrm{~A})$ \\
\cline { 5 - 6 } \cline { 5 - 6 } ETAPA 1 & 0 & & 1,061 & 1,048 & 1,048 \\
ETAPA 2 & $+5 \%$ & & 0,998 & 0,998 & 0,9989 \\
ETAPA 3 & $+10 \%$ & & 0,940 & 0,953 & 0,953 \\
\hline & & $D$ & 0,7191 & 0,6917 & 0,6917 \\
& & $V_{o}(\mathrm{~V})$ & 399,89 & 399,97 & 399,56 \\
\hline
\end{tabular}

Tabla 2.28: Reparto estático de carga para Elevador ZVS. Valor medio de la corriente a la salida en cada etapa cuando el circuito es conmutado, promediado y eq. Thévenin. $\left(L_{r}=70 \mu \mathrm{H}\right)$.

Hay que indicar que el ciclo de trabajo empleado para el circuito conmutado es diferente del que se indica para el modelo promediado, porque en éste último no se considera el rizado. Por este mismo motivo, no coinciden los valores de corriente del conmutado con el circuito promediado.

Por otro lado, también se comprueba que el equivalente Thévenin sirve para modelar el reparto en régimen permanente y que la expresión (2.9), deducida en el apartado anterior para la corriente en una etapa predice el reparto estático de carga del modelo promediado bajo las consideraciones analizadas.

Las mismas simulaciones se han hecho para los otros convertidores, como se muestra en las Tablas 2.30, Tabla 2.30 y Tabla 2.31 . 


\begin{tabular}{cccccc}
\hline & $\Delta L_{r, i}$ & & $\bar{I}_{o, \text { conm }}(\mathrm{A})$ & $\bar{I}_{o, \text { prom }}(\mathrm{A})$ & $\bar{I}_{o, t h}(\mathrm{~A})$ \\
\cline { 5 - 6 } \cline { 5 - 6 } ETAPA 1 & 0 & & 10,541 & 10,483 & 10,484 \\
ETAPA 2 & $+5 \%$ & & 9,975 & 9,984 & 9,984 \\
ETAPA 3 & $+10 \%$ & & 9,454 & 9,531 & 9,530 \\
\hline & & $D$ & 0,5938 & 0,5731 & 0,5731 \\
& & $V_{o}(\mathrm{~V})$ & 12,990 & 12,999 & 12,999 \\
\hline
\end{tabular}

Tabla 2.29: Reparto estático de carga para Reductor ZVS. Valor medio de la corriente a la salida en cada etapa cuando el circuito es conmutado, promediado y eq. Thévenin. $\left(L_{r}=2 \mu \mathrm{H}\right)$.

\begin{tabular}{cccccc}
\hline & $\Delta L_{r, i}$ & & $\bar{I}_{o, \text { conm }}(\mathrm{A})$ & $\bar{I}_{o, \text { prom }}(\mathrm{A})$ & $\bar{I}_{o, t h}(\mathrm{~A})$ \\
\cline { 1 - 1 } \cline { 5 - 6 } ETAPA 1 & 0 & & 1,057 & 1,048 & 1,048 \\
ETAPA 2 & $+5 \%$ & & 0,998 & 0,998 & 0,998 \\
ETAPA 3 & $+10 \%$ & & 0,944 & 0,953 & 0,953 \\
\hline & & $D$ & 0,8236 & 0,8060 & 0,5731 \\
& & $V_{o}(\mathrm{~V})$ & 399,75 & 399,97 & 399,96 \\
\hline
\end{tabular}

Tabla 2.30: Reparto estático de carga para Reductor-Elevador ZVS. Valor medio de la corriente a la salida en cada etapa cuando el circuito es conmutado, promediado y eq. Thévenin. $\left(L_{r}=70 \mu \mathrm{H}\right)$.

\begin{tabular}{cccccc}
\hline & $\Delta L_{r, i}$ & & $\bar{I}_{o, \text { conm }}(\mathrm{A})$ & $\bar{I}_{o, \text { prom }}(\mathrm{A})$ & $\bar{I}_{o, t h}(\mathrm{~A})$ \\
\cline { 1 - 3 } \cline { 5 - 6 } ETAPA 1 & 0 & & 0,266 & 0,262 & 0,262 \\
ETAPA 2 & $+5 \%$ & & 0,250 & 0,250 & 0,250 \\
ETAPA 3 & $+10 \%$ & & 0,235 & 0,238 & 0,238 \\
\hline & & $D$ & 0,7073 & 0,6879 & 0,6879 \\
& & $V_{o}(\mathrm{~V})$ & 400,16 & 399,99 & 399,99 \\
\hline
\end{tabular}

Tabla 2.31: Reparto estático de carga para flyback ZVS. Valor medio de la corriente a la salida en cada etapa cuando el circuito es conmutado, promediado y eq. Thévenin. $\left(L_{r}=2 \mu \mathrm{H}\right)$. 


\section{Capítulo 3}

\section{Reparto Dinámico}

En este capítulo se muestra la influencia de ciertos elementos pasivos incorporados en la topología ZVS, que si bien, no afectan al reparto en régimen permanente, tienen influencia en parte del rango de frecuencias de trabajo, y pueden llevar a un mal reparto de carga ante escalones de la misma. Se incide en el efecto del condensador de clamp sobre la dinámica de cada convertidor. Para ello, el primer paso es obtener la impedancia de salida.

\subsection{Impedancia de salida.}

La impedancia de salida de un convertidor ZVS se obtiene a partir de su modelo en pequeña señal, que a su vez, se deriva de su modelo promediado. Para obtener el modelo promediado se ha seguido el método indicado en (Chen y Sun, 2006). Tras la linealización, se consigue el modelo en pequeña señal. En la Figura 3.1. se ha representado el circuito en pequeña señal para varios convertidores ZVS El procedimiento seguido para la obtención de los dos tipos de modelos, como sus ecuaciones junto con las expresiones de los diferentes parámetros incluidos en ellos, están detalladas en el anexo correspondiente a cada convertidor ZVS.

Algunos de los parámetros del modelo en pequeña señal se han indicado en la Tabla 3.1. Estos parámetros, que se consideran constantes, son dependientes del punto de funcionamiento del convertidor.

\begin{tabular}{|c|c|c|c|c|c|c|c|}
\hline$r_{1}$ & $g_{1}$ & $r_{2}$ & $g_{2}$ & $r_{3}$ & $g_{c}$ & $r_{5}$ & $g_{f}$ \\
\hline$\frac{V_{g}}{I_{e q}}$ & $\frac{2 I_{e q}}{V_{c}}$ & $\frac{V_{o}}{I_{e q}}$ & $\frac{2 I_{e q}}{V_{c}}$ & $\frac{V_{g}+V_{o}}{2(1-D) I+I_{e q}}$ & $\frac{2 I_{e q}}{V_{c}-V_{o}}$ & $\frac{V_{g}+n V_{o}}{2(1-D) I+I_{e q}}$ & $\frac{2 I_{e q}}{V_{c}-n V_{o}}$ \\
\hline \multicolumn{2}{|c|}{ (Reductor ZVS) } & \multicolumn{2}{|c|}{ (Elevador ZVS) } & \multicolumn{2}{|c|}{ (Red-Elev. ZVS) } & \multicolumn{2}{|c|}{ (Flyback ZVS) } \\
\hline
\end{tabular}

Tabla 3.1: Expresiones para $r_{x}$ y $g_{x}$ en el modelo en pequeña señal.

Para medir la impedancia en un punto del circuito, se conecta una fuente 


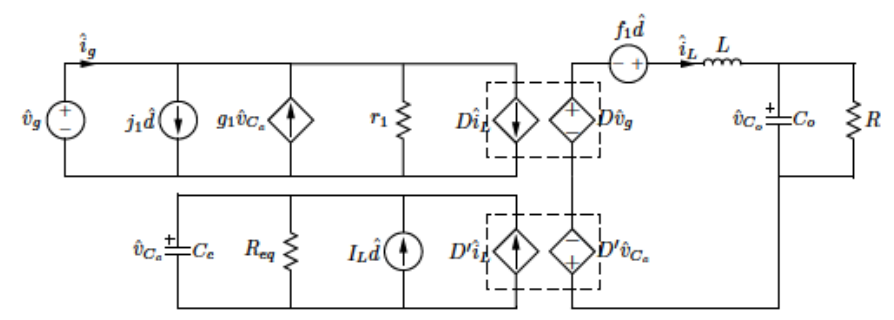

(a) Reductor.

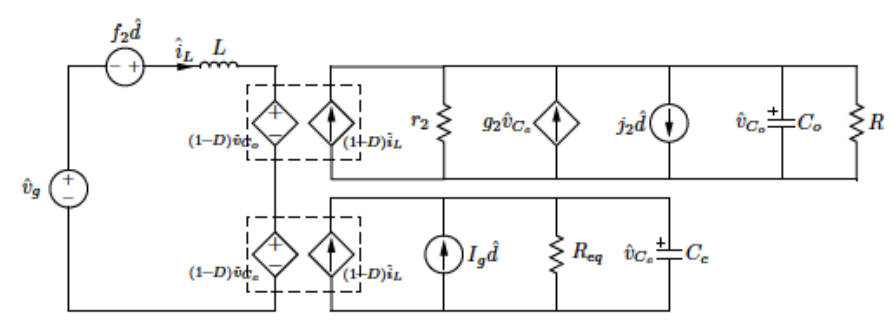

(b) Elevador.

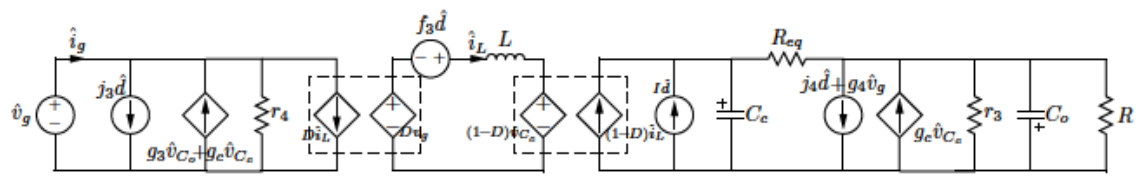

(c) Red.Elev.

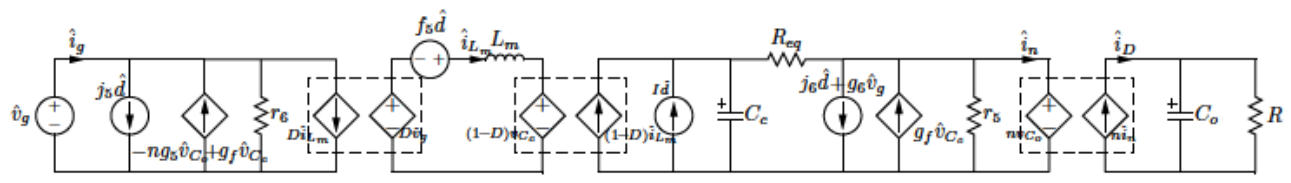

(d) Flyback.

Figura 3.1: Circuitos en pequeña señal para convertidores ZVS.

independiente en ese punto y se anulan las entradas independientes, (la tensión de entrada y el ciclo de trabajo en este caso). La impedancia de salida, $Z_{\text {out }}(s)$, se obtiene conectando una fuente de test, $\hat{i}_{t}$, a la salida del circuito. A partir de la tensión que aparece en sus terminales, $\hat{v}_{t}$, se determina esta impedancia como:

$$
Z_{\text {out }}(s)=\left.\frac{\hat{v}_{t}(s)}{\hat{i}_{t}(s)}\right|_{\begin{array}{l}
\hat{v}_{g}=0 \\
\hat{d}=0
\end{array}}
$$

En el caso de la conexión en paralelo de varias etapas, la carga resistiva es común a todas ellas, por lo que, en su lugar, se conecta la fuente de test como se indica en la Figura 3.2. El condensador de salida $C_{o}$ puede ser un elemento propio de cada etapa o ser un elemento común a todas ellas, en cuyo caso también se excluye. 


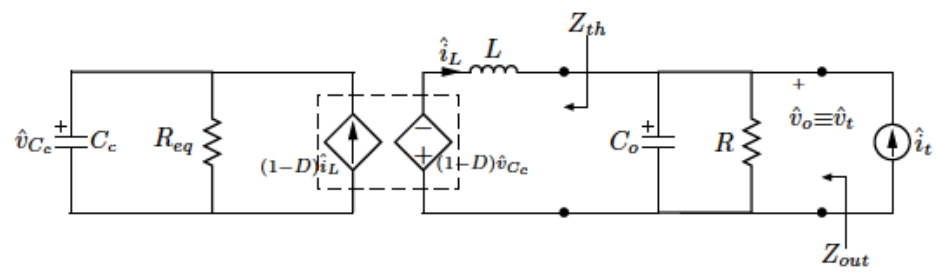

(a) Reductor.

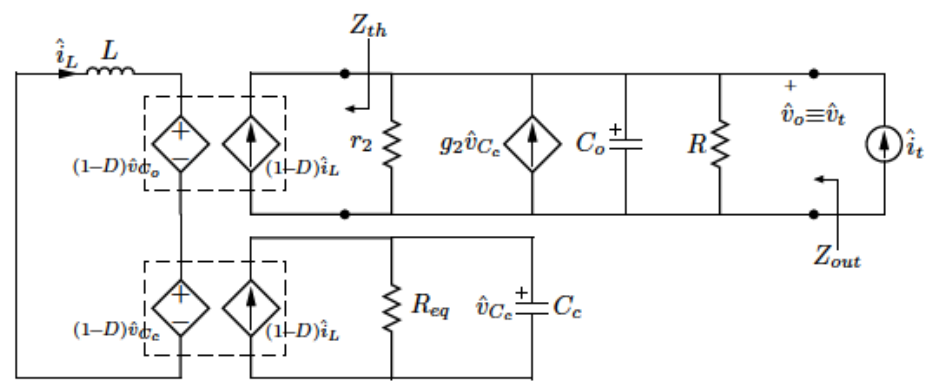

(b) Elevador.

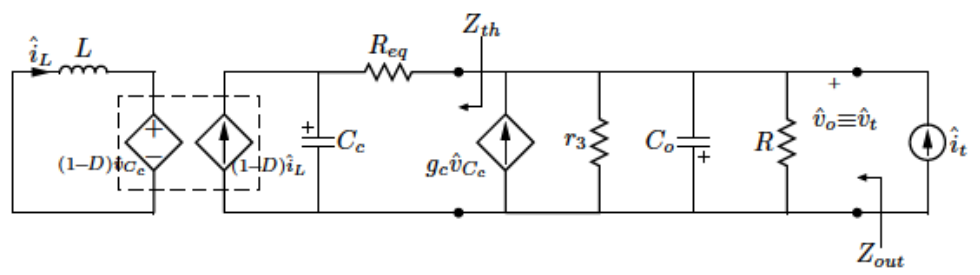

(c) Red.Elev.

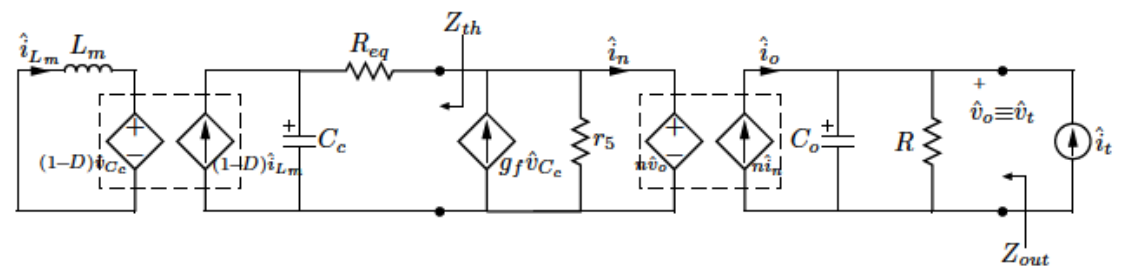

(d) Flyback.

Figura 3.2: Circuitos empleados para obtener la impedancia de salida para convertidores ZVS.

La impedancia de salida resultante está formada por varios elementos en paralelo: un primer grupo formado por la bobina principal, $L$ (о $L_{m}$ en el caso del flyback ZVS), el condensador del clamp, $C_{c}$, y la resistencia equivalente, $R_{e q}$, incluidos en una impedancia denominada $Z_{t h}(s)$; otro elemento que es una fuente de corriente dependiente de $\hat{v}_{C_{c}}$, y es función del punto de trabajo en el que se encuentre el convertidor, y que, después del análisis de su posición, equivale a una impedancia en paralelo que se denota como $Z_{c}(s)$; el último grupo se asigna al término $Z_{o}(s)$, y está formado por el paralelo de 
una resistencia en pequeña señal, $r_{x}$, y los elementos propios de una etapa a la salida, esto es, el condensador, $C_{o}$, (y la carga resistiva si tuviera que considerarse en el cálculo de la impedancia de salida).

De esta forma, cada uno de los circuitos representados se reducen al paralelo de los tres elementos mencionados, como indica la expresión (3.2), donde $n$ es la relación de transformación para el flyback, y 1 para los otros convertidores.La expresión de cada término se indica en la Tabla 3.2, donde se ha incorporado una columna para agrupar el condensador de clamp, $C_{c}$, con otro elemento, y compactar las expresiones representadas.

$$
Z_{\text {out }}(s)=\frac{1}{n^{2}}\left[Z_{\text {th }}(s) \| Z_{c}(s)\right] \| Z_{o}(s)
$$

\begin{tabular}{ccccc}
\hline & $Z_{l}(s)$ & $Z_{t h}(s)$ & $Z_{c}(s)$ & $Z_{o}(s)$ \\
\hline Red. & $R_{e q} \| s C_{c}$ & $L s+(1-D)^{2} Z_{l}(s)$ & - & $s C_{o} \| R$ \\
Elev. & $R_{e q} \| s C_{c}$ & $s L^{\prime}+Z_{l}(s)$ & $\frac{Z_{t h}(s)}{g_{2} Z_{l}(s)}$ & $r_{2}\left\|s C_{o}\right\| R$ \\
RedElev. & $s L^{\prime} \| C_{c}$ & $R_{e q}+Z_{l}(s)$ & $-\frac{Z_{t h}(s)}{g_{c} Z_{l}(s)}$ & $r_{3}\left\|s C_{o}\right\| R$ \\
Flyback & $s L_{m}^{\prime} \| s C_{c}$ & $R_{e q}+Z_{l}(s)$ & $-\frac{Z_{t h}(s)}{g_{f} Z_{l}(s)}$ & $r_{5} / n^{2}\left\|s C_{o}\right\| R$ \\
\hline \multicolumn{5}{c}{$L^{\prime}=L /(1-D)^{2}$ y $L_{m}^{\prime}=L_{m} /(1-D)^{2}}$. \\
\hline
\end{tabular}

Tabla 3.2: Términos que forman $Z_{\text {out }}(s)$.

La validez de la expresión de $Z_{\text {out }}(s)$ se comprueba con la simulación del convertidor conmutado trabajando en un punto de funcionamiento para medir la impedancia. Se elige el mismo punto de funcionamiento que en capítulos anteriores. Los parámetros se indican en la Tabla 3.3. El ciclo de trabajo, $D$, corresponde al modelo promediado.

\begin{tabular}{lllllllllll}
\hline \hline \multirow{2}{*}{ Reductor } & $V_{g}$ & $30 \mathrm{~V}$ & $P$ & $130 \mathrm{~W}$ & $C_{o}$ & $5 \mu \mathrm{F}$ & $C_{c}$ & $5,2 \mu \mathrm{F}$ & $D$ & 0,5667 \\
& $V_{o}$ & $13 \mathrm{~V}$ & $f$ & $100 \mathrm{kHz}$ & $L$ & $24 \mu \mathrm{H}$ & $L_{r}$ & $2 \mu \mathrm{H}$ & & \\
\hline \multirow{2}{*}{ Elevador } & $V_{g}$ & $160 \mathrm{~V}$ & $P$ & $400 \mathrm{~W}$ & $C_{o}$ & $5 \mu \mathrm{F}$ & $C_{c}$ & $0,47 \mu \mathrm{F}$ & $D$ & 0,6875 \\
& $V_{o}$ & $400 \mathrm{~V}$ & $f$ & $100 \mathrm{kHz}$ & $L$ & $600 \mu \mathrm{H}$ & $L_{r}$ & $70 \mu \mathrm{H}$ & & \\
\hline \multirow{2}{*}{ Red.Elev. } & $V_{g}$ & $160 \mathrm{~V}$ & $P$ & $400 \mathrm{~W}$ & $C_{o}$ & $5 \mu \mathrm{F}$ & $C_{c}$ & $0,47 \mu \mathrm{F}$ & $D$ & 0,8018 \\
& $V_{o}$ & $400 \mathrm{~V}$ & $f$ & $100 \mathrm{kHz}$ & $L$ & $800 \mu \mathrm{H}$ & $L_{r}$ & $70 \mu \mathrm{H}$ & & \\
\hline \multirow{2}{*}{ Flyback } & $V_{g}$ & $20 \mathrm{~V}$ & $P$ & $100 \mathrm{~W}$ & $C_{o}$ & $5 \mu \mathrm{F}$ & $C_{c}$ & $2,2 \mu \mathrm{F}$ & $D$ & 0,685 \\
& $V_{o}$ & $400 \mathrm{~V}$ & $f$ & $100 \mathrm{kHz}$ & $L_{m}$ & $24 \mu \mathrm{H}$ & $L_{r}$ & $2 \mu \mathrm{H}$ & $n$ & $1: 12$ \\
\hline
\end{tabular}

Tabla 3.3: Convertidores ZVS. Parámetros de funcionamiento. 
En la Figura 3.3 se ha representado gráficamente la expresión analítica (modelo promediado) de la impedancia de salida en módulo y su equivalente en la simulación del convertidor conmutado. La similitud obtenida para los cuatro convertidores confirma la validez de la expresión analítica anterior.

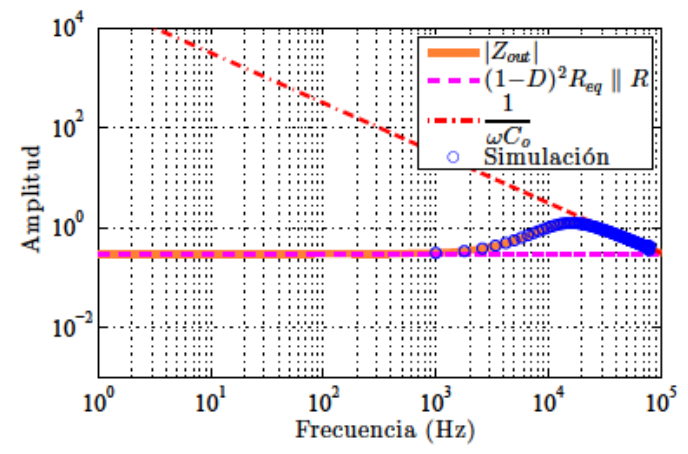

(a) Reductor.

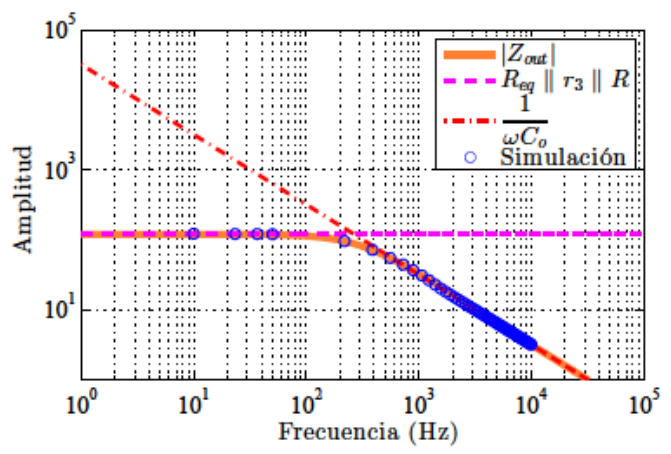

(c) Red.Elev.

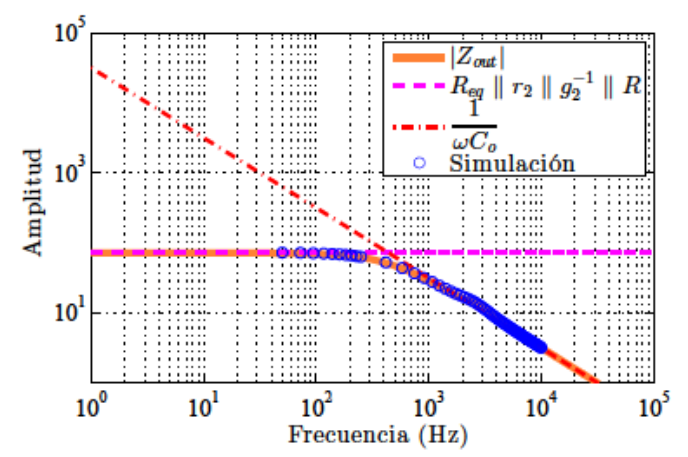

(b) Elevador.

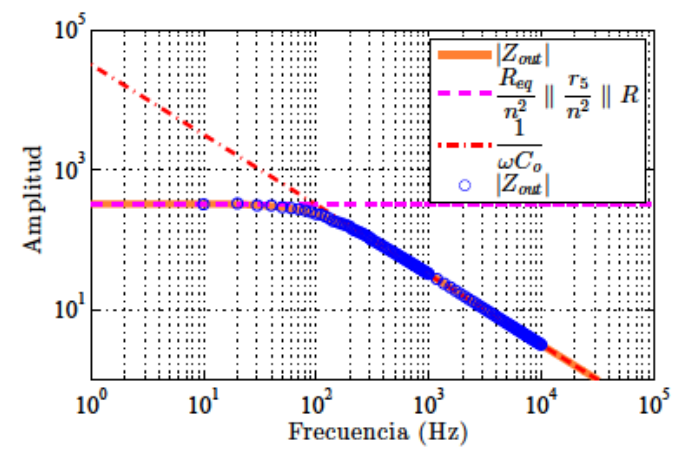

(d) Flyback.

Figura 3.3: $\left|Z_{\text {out }}(s)\right|$ para convertidores ZVS.

En las mismas gráficas, se han incluido las asíntotas que presenta dicha impedancia. Como se observa, hay una asíntota horizontal a baja frecuencia, que se corresponde con el paralelo de $R_{e q}$ y la impedancia de pequeña señal, $r_{x}$ que aparece en ese modelo. En el caso del elevador se incluye la conductancia $g_{2}$. La carga resistiva conectada a la salida forma parte de esta asíntota dado que la impedancia se ha obtenido con ella, esto es, en un punto de funcionamiento del convertidor. En algún caso aparece en esta asíntota, el factor $(1-D)^{2}$, o el término $n$, según el tipo de convertidor de que se trate. Respecto a las altas frecuencias, predomina el comportamiento del condensador de salida, $C_{o}$, que se manifiesta con la forma a la que tiende la curva $Z_{\text {out }}(s)$ en esa zona, que tiende a una asíntota debida a la impedancia de dicho condensador.

En el caso de conexión en paralelo de varias etapas en las que el condensador de salida y a la carga resistiva, son comunes a todas ellas, la impedancia 
que se considera no debe incluir estos dos elementos en paralelo.

Esta impedancia, que se va a denominar impedancia multietapa, $Z_{m e}(s)$, se puede obtener su expresión como (3.3), y se ha representado en la Figura 3.4. El término $Z_{o, m e}(s)$ equivale al término $Z_{o}(s) \sin$ considerar ni $R \mathrm{ni}$ $C_{o}$.

$$
Z_{m e}(s)=\frac{1}{n^{2}}\left[Z_{t h}(s) \| Z_{c}(s)\right] \| Z_{o, m e}(s)
$$

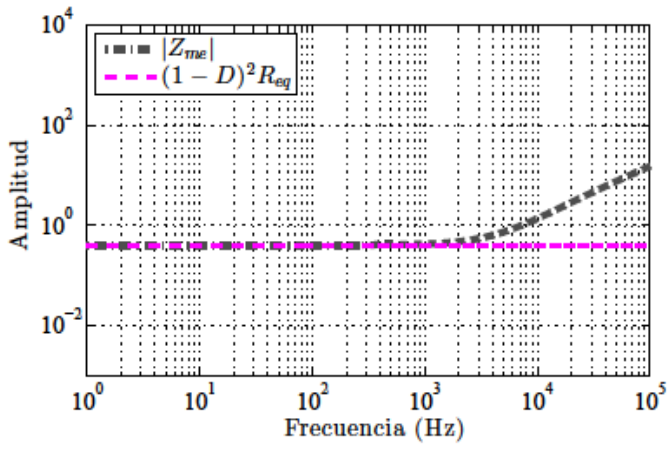

(a) Reductor.

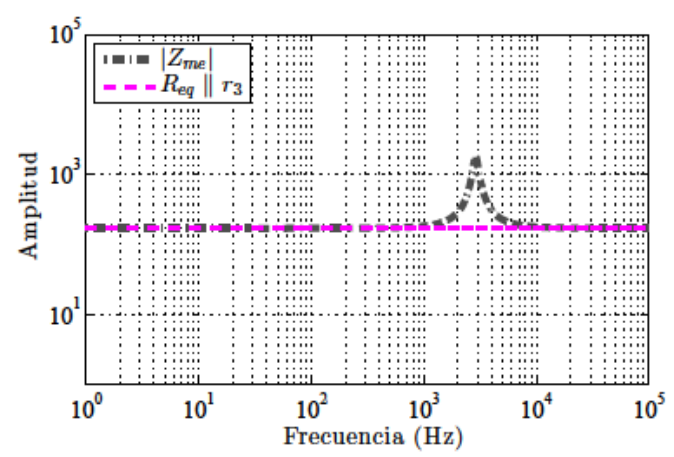

(c) Red.Elev.

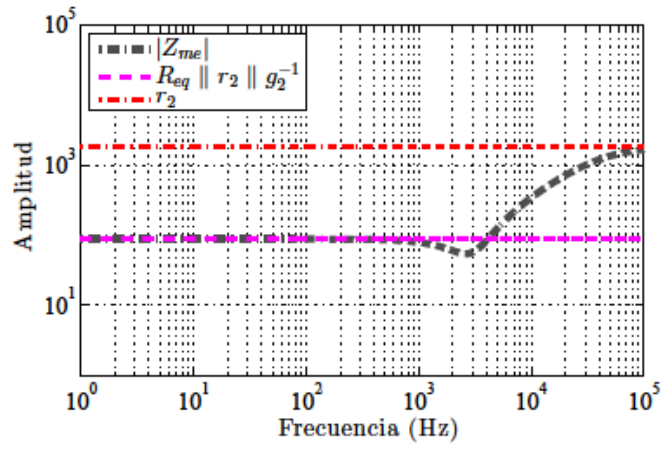

(b) Elevador.

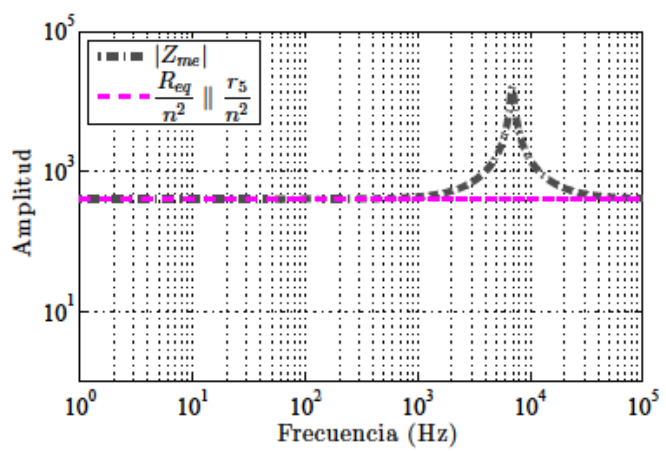

(d) Flyback.

Figura 3.4: $\left|Z_{m e}(s)\right|$ para convertidores ZVS.

Se observa que a bajas frecuencias se repite el mismo comportamiento que con la impedancia de salida $Z_{\text {out }}$, es decir, aparece una asíntota horizontal a pesar de que no está incluida la carga resistiva a la salida. El valor de esta asíntota se corresponde con la impedancia de salida en régimen permanente (o el módulo de la derivada de la característica de salida) obtenida en el capítulo anterior.

También se observa que para todo el rango de frecuencias, el módulo de la impedancia $Z_{m e}(s)$ es de un valor igual o superior a su valor de continua, por lo que parece lógico que habrá también reparto a altas frecuencias si se ha garantizado previamente el reparto de régimen permanente. 
El efecto de los elementos añadidos al convertidor para conseguir las conmutaciones suaves, esto es, $L_{r}$ y $C_{c}$, sobre $Z_{m e}(s)$ sirve para matizar la validez de la afirmación indicada en el párrafo anterior. En el caso de la bobina resonante, ésta no aparece de forma explicita como elemento inductivo en el modelo en pequeña señal, sino que aparece como parte del valor de un término resistivo, que es $R_{e q}$. Como este factor se diseña para conseguir un buen reparto en continua, el efecto de $L_{r}$, solo aparece en la asíntota horizontal de la impedancia multietapa.

\subsection{Influencia del condensador de clamp.}

El condensador del clamp aparece de forma explicita en el modelo de pequeña señal, y se incluye en la impedancia $Z_{m e}(s)$. Su efecto se observa en la Figura 3.5 donde se ha representado el módulo de la impedancia considerado diferentes valores de $C_{c}$.

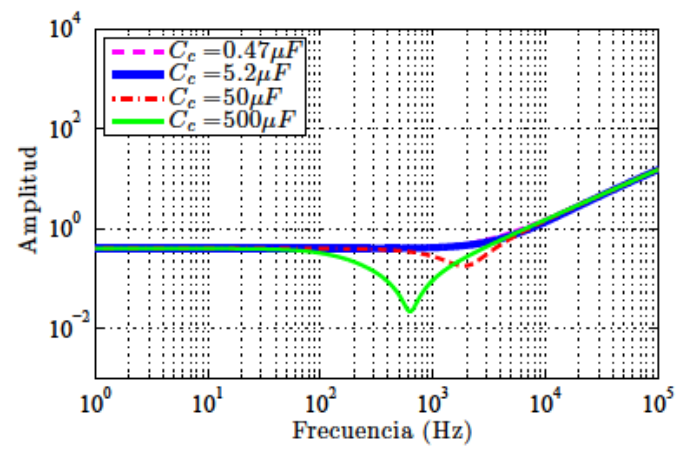

(a) Reductor.

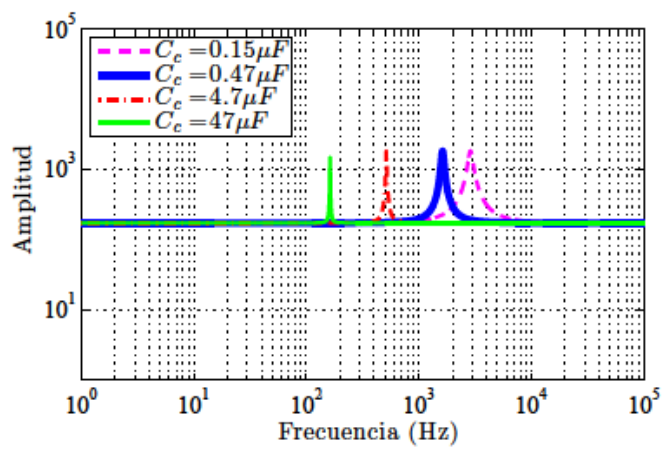

(c) Red.Elev.

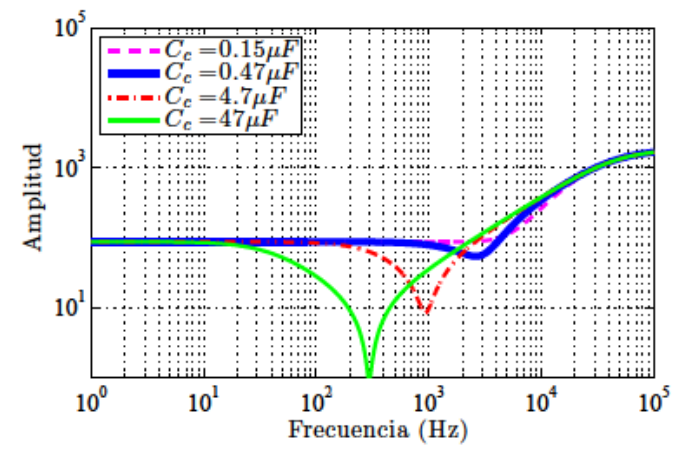

(b) Elevador.

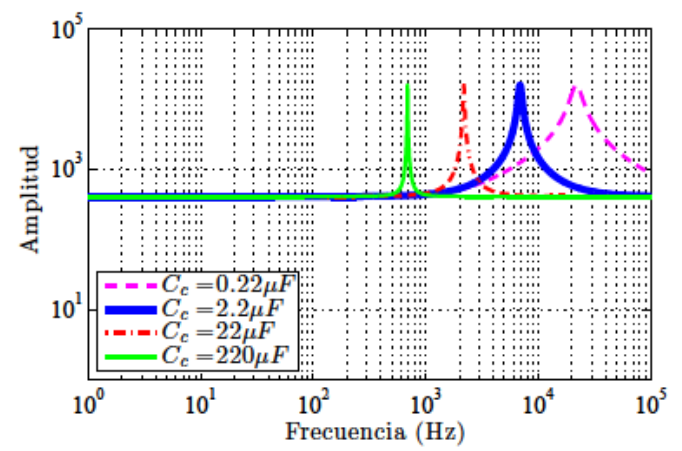

(d) Flyback.

Figura 3.5: Efecto de $C_{c}$ en $\left|Z_{m e}(s)\right|$ para convertidores ZVS.

Este condensador modifica el comportamiento de la impedancia con la frecuencia. En el caso del reductor y elevador ZVS, se produce una disminución de su módulo, por lo que puede llegar a alterar el reparto en deter- 
minadas frecuencias por la menor impedancia puesta en juego. Este efecto adquiere mayor importancia a medida que aumenta el valor del condensador ya que el efecto se extiende a frecuencias cada vez mas bajas. Para el flyback ZVS (y el reductor elevador) el comportamiento es el contrario. Aumenta la impedancia en determinadas frecuencias por lo que el reparto dinámico de carga se considera garantizado si también lo está en régimen permanente, dado que el módulo de la impedancia es el mismo en todas las frecuencias, excepto en torno aquellas en las que se produce cierta resonancia entre los elementos que forman esta impedancia. Esta frecuencia de resonancia depende del valor del condensador del clamp, de la bobina principal del convertidor, y del punto de funcionamiento del convertidor a través del ciclo de trabajo.

Por otro lado, su efecto sobre el reparto en régimen permanente es nulo dado que el módulo de la impedancia en continua no modifica su valor. Algo que era de esperar al tratarse de un condensador. Otra cosa es que cambie el punto de funcionamiento del convertidor al variar alguno de los parámetros de funcionamiento. Por ejemplo, si se varía únicamente el ciclo de trabajo, se produce una variación en la impedancia como está representada en la Figura 3.6.

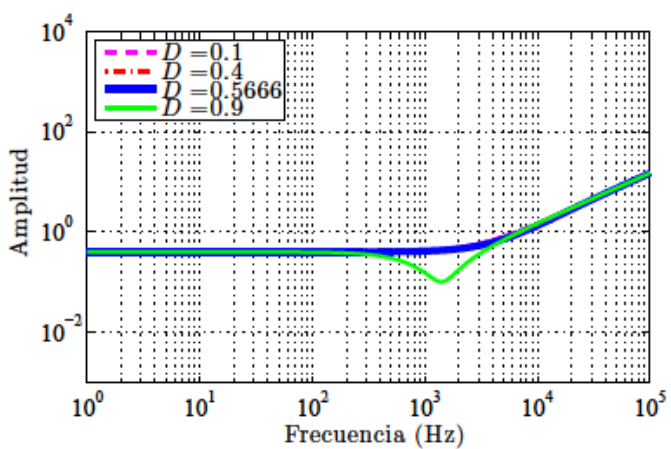

(a) Reductor.

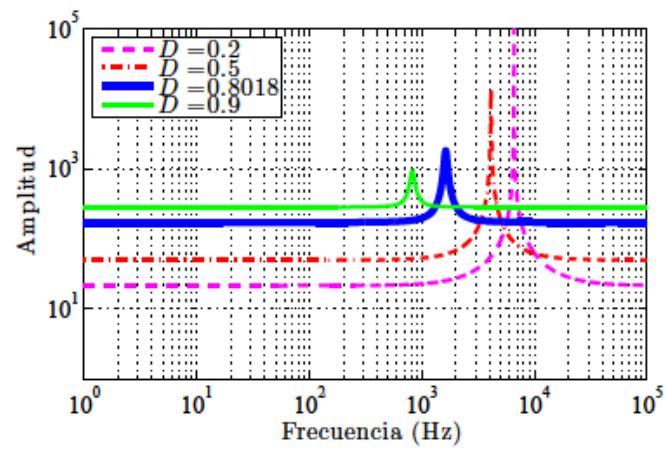

(c) Red.elev.

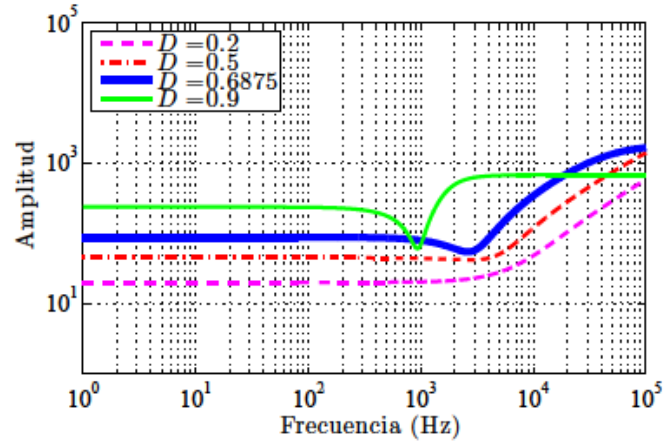

(b) Elevador.

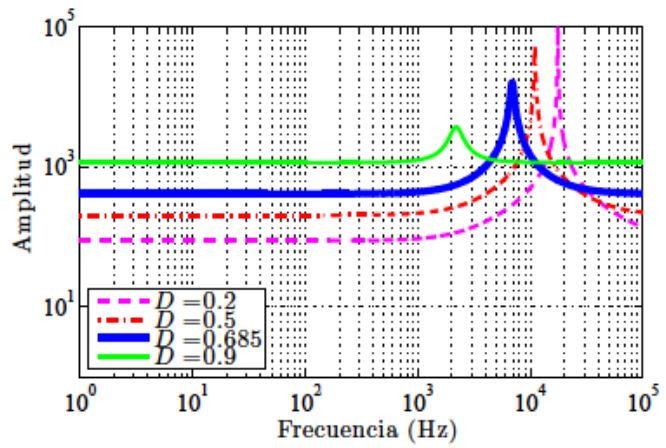

(d) Flyback.

Figura 3.6: Efecto del ciclo de trabajo en $\left|Z_{m e}(s)\right|$ para convertidores ZVS. 
En este caso se ha representado el módulo de esa impedancia para diferentes ciclos de trabajo y manteniendo la misma carga resistiva a la salida. En este supuesto, la tensión de salida es diferente para cada valor de ciclo de trabajo. El primer efecto apreciado es la modificación de la impedancia en régimen permanente para diferentes valores de ciclo de trabajo. De hecho, la asíntota horizontal de esta impedancia está formada por el término $R_{e q}$ donde está incluido el ciclo de trabajo.

Cabe notar el caso del reductor ZVS, donde el factor $(1-D)^{2}$ que multiplica a $R_{e q}$ elimina la dependencia con el ciclo de trabajo de esta asíntota, y por tanto, la asíntota no cambia. A la misma vez, el ciclo de trabajo produce el efecto de considerar un condensador de clamp de valor igual a $C_{c} /(1-D)^{2}$. Por eso, el efecto observado es similar cuando el valor del condensador $C_{c}$ es excesivamente elevado.

Suponiendo constante la tensión de salida, (por ejemplo, con un condensador muy grande) la impedancia en continua no cambia aunque varíe el ciclo de trabajo. En la Figura 3.7 se ha representado la impedancia considerando este supuesto, comprobando que su valor en continua es constante en cada uno de los convertidores tratados.

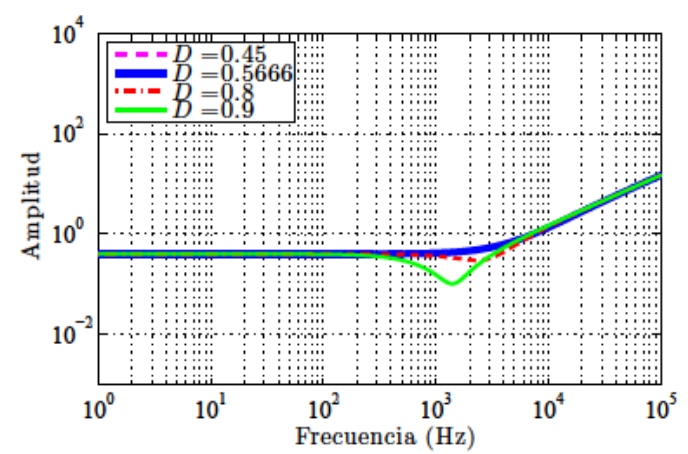

(a) Reductor.

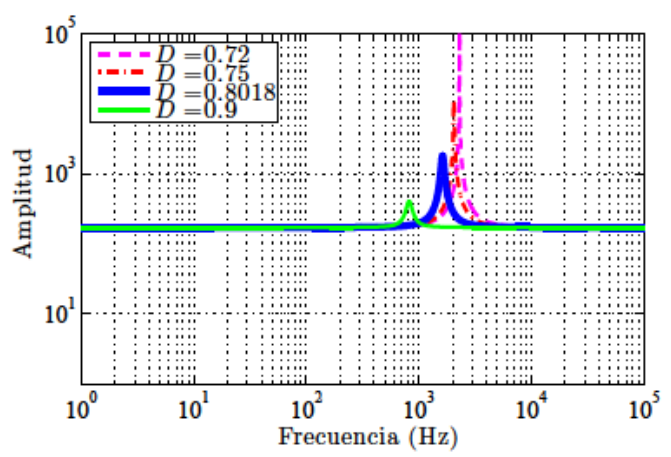

(c) Red.elev.

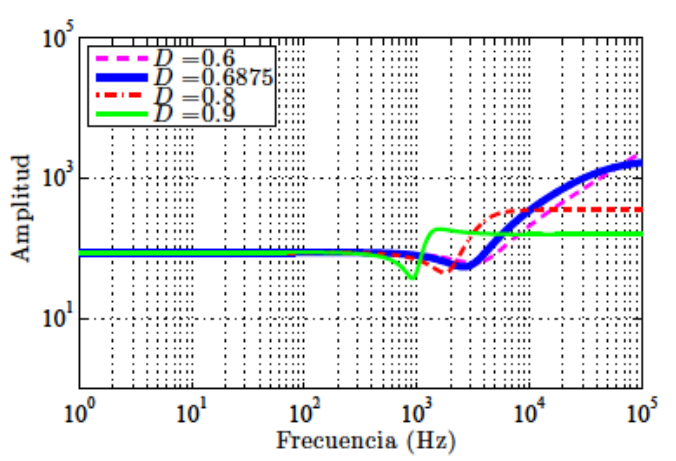

(b) Elevador.

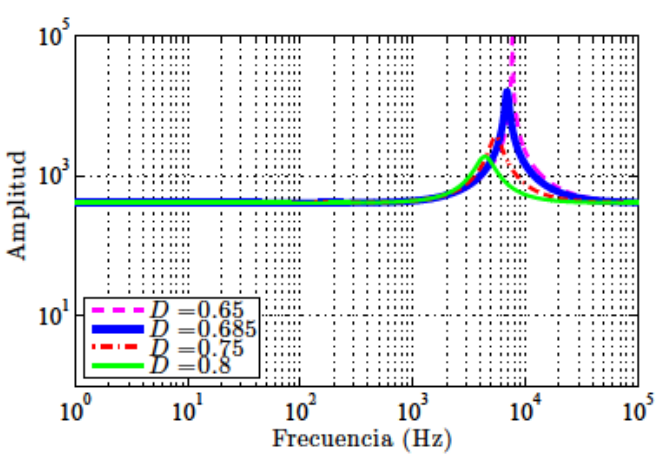

(d) Flyback.

Figura 3.7: Efecto del ciclo de trabajo en $\left|Z_{m e}(s)\right|$ cuando $V_{o}$ es constante. 
En el caso de altas frecuencias se produce un efecto similar al obtenido cuando aumenta el valor de $C_{c}$. La explicación pasa por observar la expresión $Z_{m e}(0)$, desarrollada en el anexo correspondiente de cada convertidor, y que es idéntica a la variación de $V_{o}$ con $I_{o}$ indicada en la Tabla 2.26 del capítulo anterior. En esa expresión se incluye $V_{o}$ junto a otros términos, que no dependen de $D$. Por tanto, un cambio en éste no altera el valor de continua.

La variación del módulo de la impedancia a un valor mayor o menor para diferentes frecuencias no debería ser un problema para el reparto siempre que todas las etapas presentaran el mismo valor de impedancia. En el momento que aparecieran diferencias, el desequilibrio estaría presente, y sería mayor cuanto mas pequeño fuera el valor de la impedancia, tal y como se ha puesto de manifiesto en el capítulo de reparto estático. En el caso de conexiones o desconexiones de carga producidos a determinadas frecuencias, puede no ser el adecuado, debido a la disminución de la impedancia.

Un valor límite para el condensador de clamp, de forma que el módulo de la impedancia no decrezca, puede relacionarse con las frecuencias donde se produce esa variación. Para ello se empieza por la representación gráfica de $Z_{t h}(s)$ en la Figura 3.8.

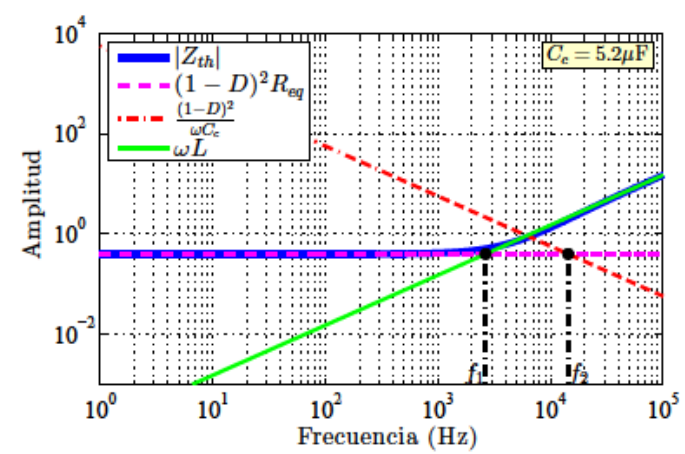

(a) Reductor.

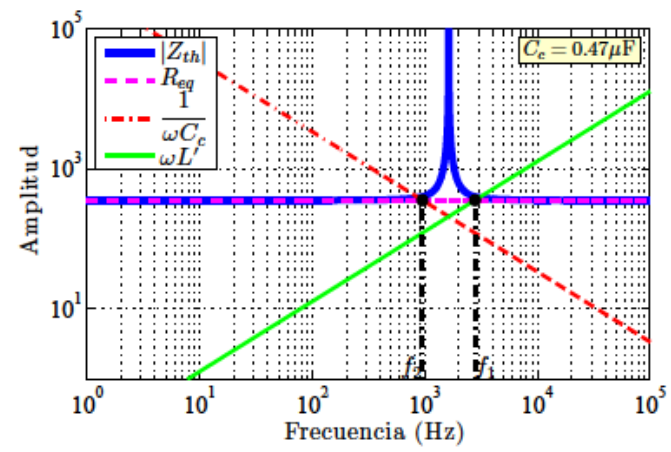

(c) Red.elev.

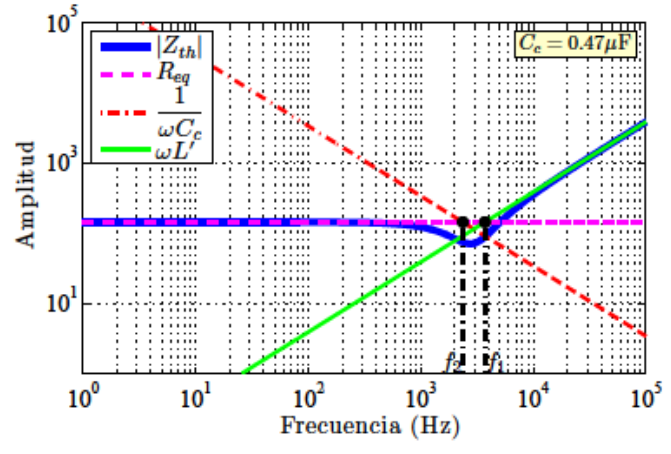

(b) Elevador.

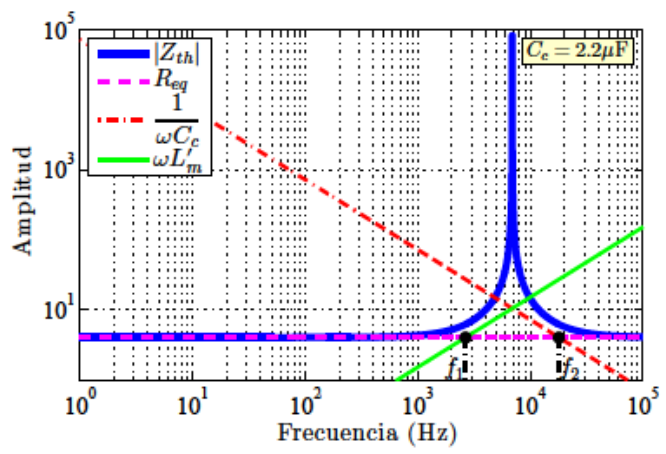

(d) Flyback.

Figura 3.8: Término $\left|Z_{t h}(s)\right|$ con sus asíntotas. Determinación de $f_{1}$ y $f_{2}$. 
Dicha representación, que incluye las impedancias de los elementos que la forman, muestra que la variación de la impedancia se produce alrededor de frecuencia donde se cruzan la impedancia del condensador del clamp y de la bobina principal.

Se acota un intervalo dado entre $f_{1}$, frecuencia de cruce de la asíntota horizontal con la impedancia bobina principal, y $f_{2}$, el cruce con la del condensador. A medida que aumenta el valor de $C_{c}$, la frecuencia $f_{2}$ disminuiría y podría ser inferior a $f_{1}$, momento en el que la disminución de $\left|Z_{m e}(s)\right|$ empieza a ser apreciable. Hay que hacer notar que emplear la asíntota horizontal de $Z_{t h}(s)$ conduce a un criterio mas restrictivo que si se empleara la asíntota de $Z_{m e}(s)$, puesto que esta última es inferior a aquella, y por tanto, $f_{1}$ sería un valor mas pequeño, y por tanto, mas exigente con $f_{2}$.

La relación entre frecuencias tiene la misma expresión para los convertidores tratados y se indica en (3.4), donde $L$ se identifica con $L_{m}$ en el caso del flyback ZVS.

$$
\left.\begin{array}{l}
f_{1}=\frac{L_{r} f}{\pi L} \\
f_{2}=\frac{(1-D)^{2}}{4 \pi C_{c} L_{r} f}
\end{array}\right\} \Rightarrow \frac{f_{2}}{f_{1}}=\frac{L}{C_{c}}\left[\frac{(1-D)}{2 L_{r} f}\right]^{2}
$$

Por otro lado, dando un valor de rizado admisible, se puede limitar el valor del condensador de clamp, que no debe ser necesariamente pequeño puesto que este condensador es un elemento de almacenamiento de energía, que no está conectado a la salida como el caso del condensador $C_{o}$.

El rizado de tensión en $C_{c}$ se obtiene como el cociente entre la carga involucrada y su valor de capacidad. Considerando la forma que presenta la corriente del condensador, $i_{C_{c}}(t)$, anteriormente representada en la Figura 2.3 , y tomando de la tabla 2.6 el valor de la tensión del clamp en régimen permanente, se obtiene un valor relativo del rizado de tensión como:

$$
\left.\begin{array}{l}
\Delta V_{c}=\frac{\Delta Q}{C_{c}}=\frac{1}{C_{c}} \frac{(1-D) T}{4} I_{\max } \\
V_{c} \geq \frac{I_{\max }}{(1-D)} 2 L_{r} f
\end{array}\right\} \Rightarrow\left(\frac{\Delta V_{c}}{V_{c}}\right) \leq \frac{(1-D)^{2}}{8 C_{c} L_{r} f^{2}}
$$

Combinando este resultado con la relación de frecuencias, se obtiene que:

$$
\frac{f_{2}}{f_{1}}=2 \frac{L}{L_{r}}\left(\frac{\Delta V_{c}}{V_{c}}\right)
$$

Y esta expresión se cumplirá fácilmente dado que el valor de la bobina principal suele ser bastante mayor que el de la bobina resonante y el condensador con un cierto valor de rizado admisible. Así se asegura un valor de impedancia suficiente para conseguir el reparto en todo el rango de frecuencias. Se comprueba de forma numérica con la expresión (3.4), o de forma gráfica, 
que $f_{2}>f_{1}$ para el reductor y para el flyback con los parámetros de funcionamiento empleados, mientras que el elevador y el reductor-elevador no cumplen esta condición. En ese caso, la dinámica del sistema ante perturbaciones será mas oscilante.

Un cambio en la carga que está conectada a la salida un convertidor compuesto por varias etapas en paralelo, provocará también cambio en el punto de funcionamiento de cada una de las etapas, que pasarán de un régimen permanente inicial hasta llegar a un régimen permanente final. $\mathrm{Y}$ durante esa transición, entra en juego la impedancia de cada etapa. Para ilustrar este efecto se ha recogido en la Figura 3.9 la evolución temporal de la corriente en la bobina principal de cada una de las tres etapas conectadas en paralelo que forman el convertidor ZVS. Otra vez se considera que hay cierta tolerancia en la bobina resonante $L_{r}$ de cada etapa, similar al empleado para el análisis del reparto en régimen permanente del capítulo anterior, que añade una mayor claridad a la representación gráfica.

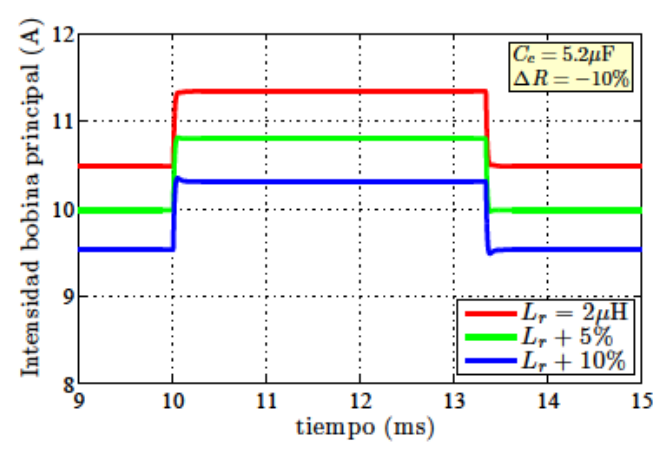

(a) Reductor.

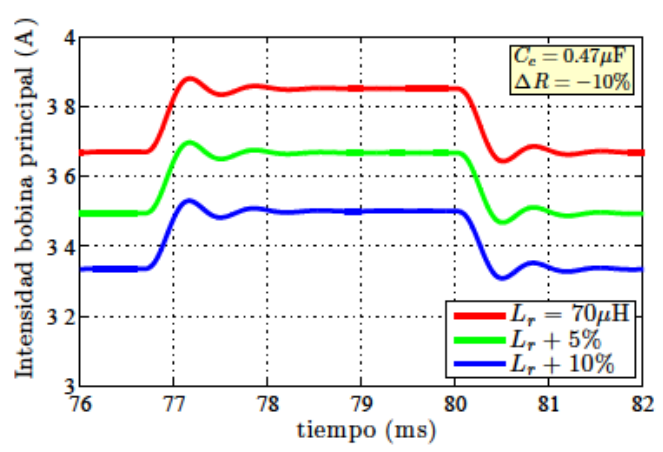

(c) Red.elev.

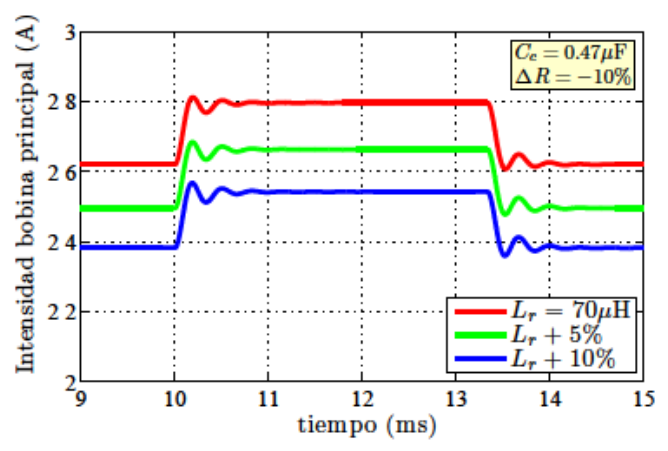

(b) Elevador.

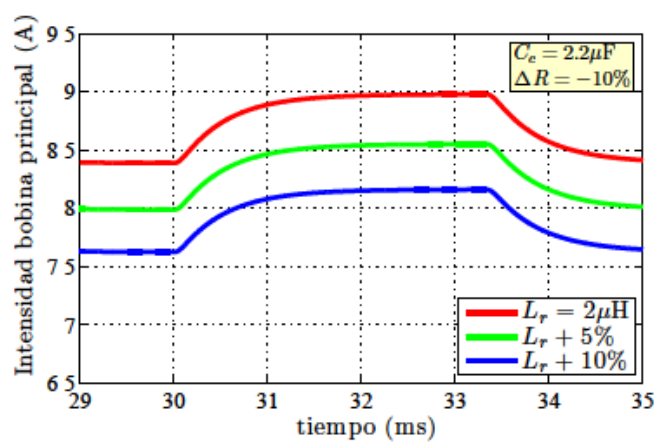

(d) Flyback.

Figura 3.9: Evolución de $i_{L}(t)$ para variación del $10 \%$ en carga resistiva para un convertidor formado por tres etapas en paralelo.

La perturbación consiste en un cambio del $-10 \%$ del valor nominal de la la resistencia a la salida. El ciclo de trabajo se mantiene constante. Las 
curvas se han simulado con el modelo promediado validado en el anexo correspondiente. Para cada tipo de convertidor se ha considerado el mismo valor de $C_{c}$ que el empleado en la Figura 3.8.

Como se puede observar en la Figura 3.9, el reparto en régimen permanente se mantiene después del escalón de carga. También se mantiene el reparto durante el transitorio puesto que se observan evoluciones similares en cada una de las tres etapas. La diferencia mas palpable es para el elevador y el reductor elevador, donde la oscilación es mayor. Resulta que no se cumple el criterio de (3.6).

Lo mismo sucede ante un cambio en el ciclo de trabajo, como aparece reflejado en las curvas de la Figura 3.10. Ahora se ha representado la variación de la corriente en la bobina principal ante un incremento de 0,01 sobre el valor nominal del ciclo de trabajo: La carga a la salida es constante de forma que si aumenta el ciclo de trabajo, la tensión de salida también lo hace, y por tanto, también la intensidad.

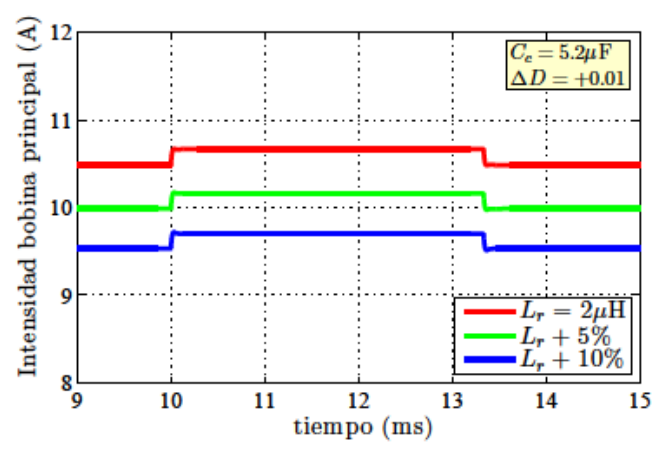

(a) Reductor.

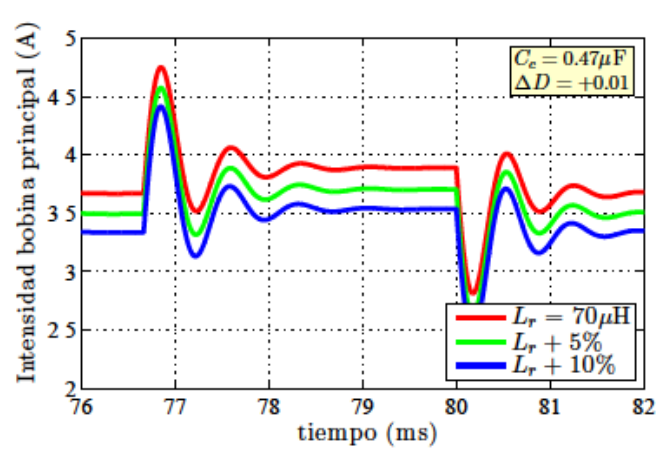

(c) Red.elev.

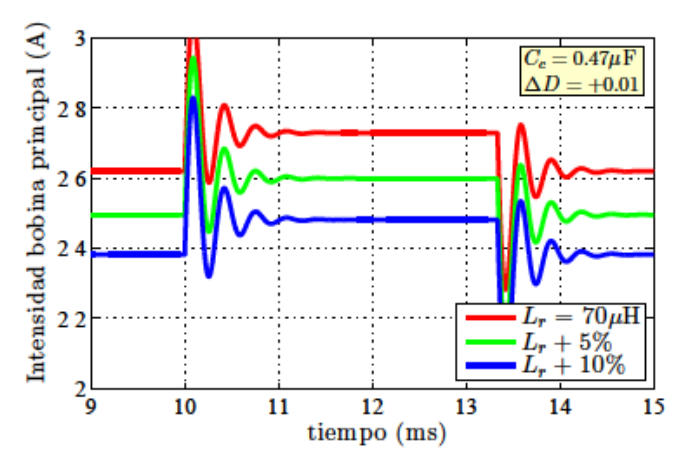

(b) Elevador.

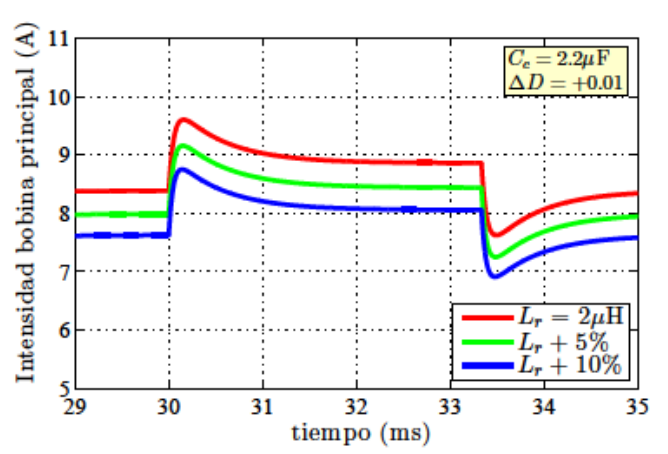

(d) Flyback.

Figura 3.10: Evolución de $i_{L}(t)$ para $\Delta D=0,01$ en ciclo de trabajo.

Se vuelve a mantener el reparto tras el escalón y una oscilación similar en cada etapa durante el transitorio. La oscilación es mas amortiguada para el reductor y el flyback que para los otros dos convertidores. 
Cuando se escoge un condensador que si cumple la condición impuesta anteriormente para las frecuencias $f_{1}$ y $f_{2}$ la oscilación queda atenuada. Así se comprueba para el elevador y el reductor elevador en las curvas de la Figura 3.11 ante las mismas perturbaciones y con un condensador tres veces menor que el empleado anteriormente.
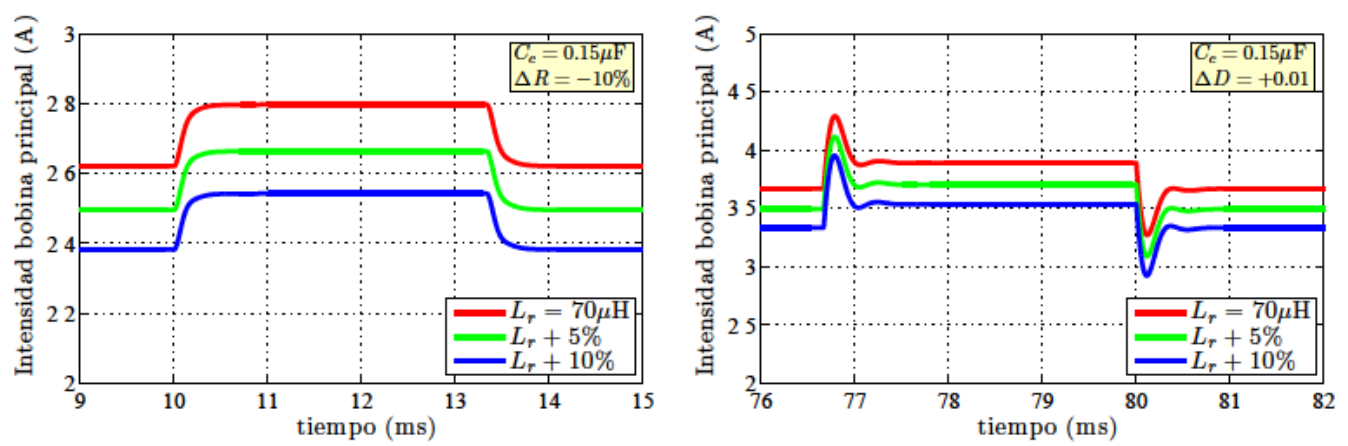

(a) Variación de carga.
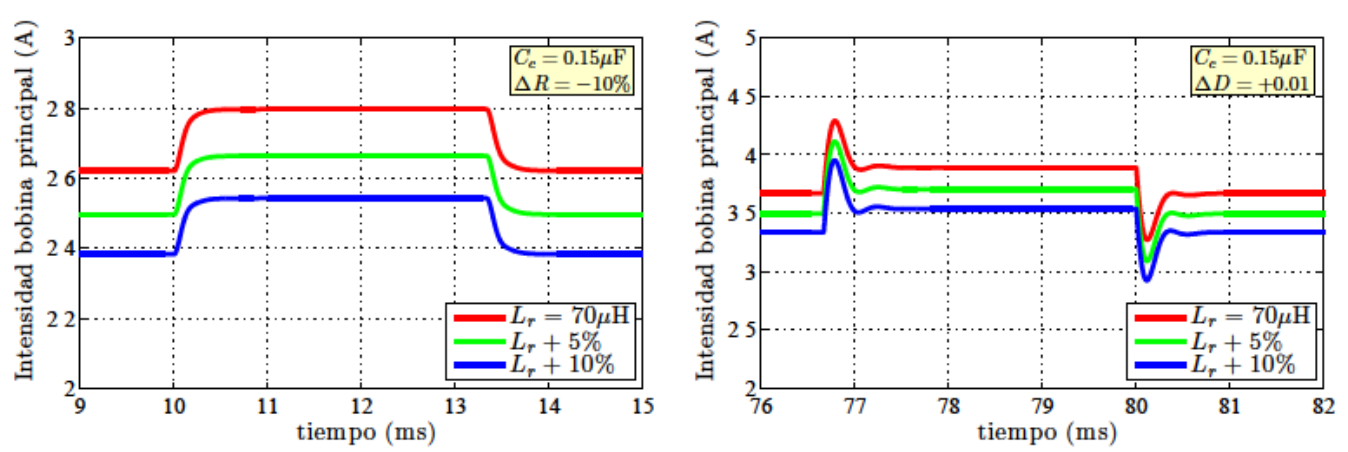

(b) Variación en ciclo de trabajo.
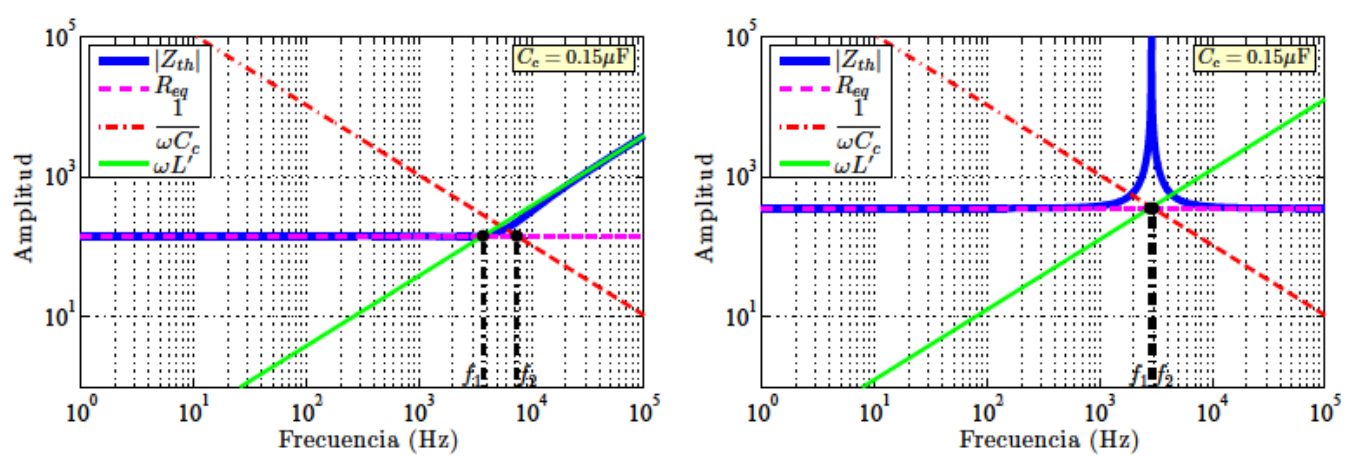

(c) $\left|Z_{t h}(s)\right|$.

Figura 3.11: Evolución de $i_{L}(t)$ ante perturbaciones para elevador(izqda.) y red.elev.(dcha.) y $\left|Z_{t h}(s)\right|$, con diferente condensador de clamp.

Sobre la curva de la impedancia $Z_{t h}(s)$ del elevador y del red.elevador 
con el nuevo condensador de clamp se comprueba que la nueva frecuencia de corte es mayor que $f_{1}$.

También se ha estudiado como afecta el valor del condensador de clamp ante un escalón del ciclo de trabajo en una de las etapas, manteniendo el resto de etapas con el ciclo de trabajo nominal. El incremento del ciclo de trabajo en una de las etapas implica una mayor tensión y corriente en esa etapa. Entonces, el resto de etapas se adaptan a las nuevas condiciones modificando su corriente. El transitorio presenta una evolución mas oscilante a medida que el condensador de clamp aumenta su valor. Tras llegar al nuevo régimen permanente se mantiene el reparto entre las etapas, y sigue siendo no equitativo, aunque limitado como era de esperar.

En la Figura 3.12 se ha representado la evolución de la corriente en la bobina de una de las tres etapas de un reductor y de un flyback cuando cambia el ciclo de trabajo en una de ellas, donde se comprueba la evolución de la corriente en cada etapa y también el efecto de aumentar el condensador de clamp a diez veces el valor nominal.
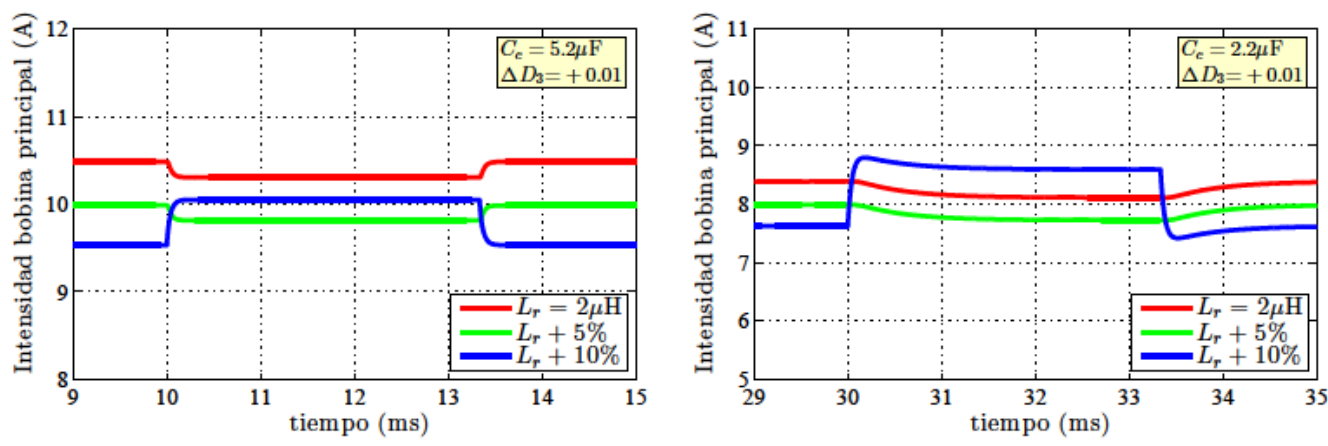

(a) $C_{c}$ nominal.
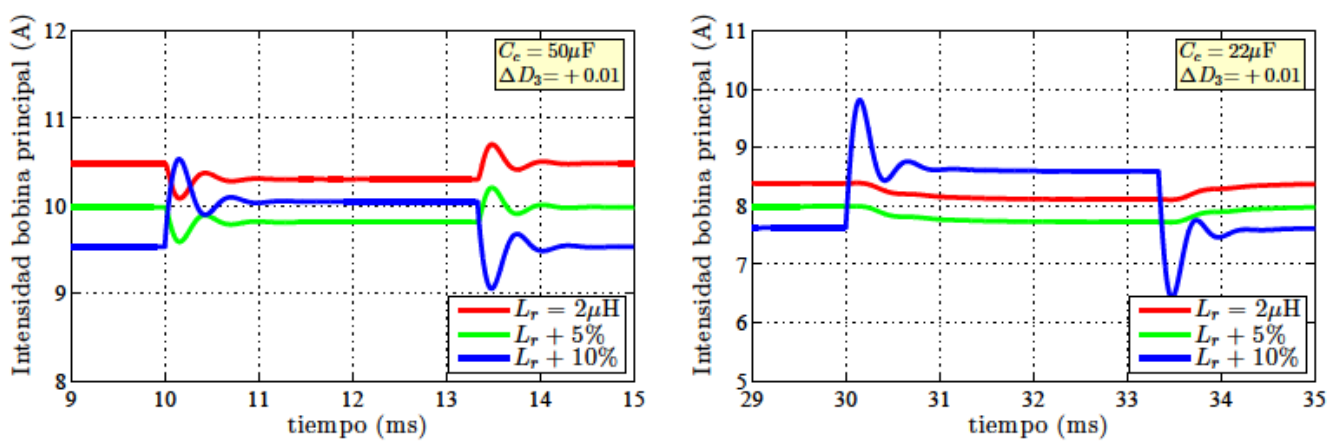

(b) $C_{c}$ diez veces el nominal.

Figura 3.12: Evolución de $i_{L}(t)$ cuando cambia el ciclo de trabajo de una de las etapas, para reductor (izquierda) y flyback (derecha). 
Como conclusión de este apartado, se puede decir que el condensador de clamp afecta, no solo a la impedancia, sino también a la dinámica del convertidor, modificando su respuesta ante perturbaciones. Y es cierto que esta influencia es obvia, pero en la literatura se han encontrado algún modelo que no incluyen esta capacidad (LAKSHMINARASAMMA y otros, 2004), y por norma general se desprecia su efecto, considerándose la dinámica la misma que un convertidor sin el circuito de clamp.

\subsection{Reclutamiento de etapas.}

La importancia del reparto dinámico cobra gran relevancia en el reclutamiento de etapas dentro de un convertidor multietapa, porque cada una de ellas tratará de responder ante perturbaciones provocadas por la conexión y desconexión, bien de ella misma o de las otras etapas

El reclutamiento de etapas permite ganar prestaciones si es posible elegir el numero de etapas que intervienen en función de la potencia total. Mas aún en el caso de convertidores ZVS, en los que el rendimiento mejora respecto a su homólogo convencional a partir de un cierto rango de potencias en el que se consiguen las conmutaciones a tensión nula. La inclusión de una bobina resonante, $L_{r}$ facilita la descarga del condensador del interruptor principal del convertidor, $C_{r}$. Con baja carga la bobina resonante no acumula la energía suficiente para descargar a $C_{r}$ durante la conmutación y por tanto, no es posible conseguir la conmutación sin pérdidas, siendo mas que discutible la mejora del rendimiento.

Para conseguir las conmutaciones sin pérdidas, debe cumplirse una relación de energías, cuya obtención se describe en cada uno de los anexos correspondientes a los convertidores ZVS tratados. En esa relación de energías se incluyen los dos elementos mencionados anteriormente, junto con el condensador de clamp, $C_{c}$, y su tensión, además de la carga que gestiona el convertidor en forma de intensidad en la bobina principal, I. En la Tabla 3.4 se indica la relación para los cuatro convertidores estudiados.

\begin{tabular}{cc}
\hline \hline Red. & $C_{r} V_{c}^{2}+L_{r} I^{2} \geq C_{r} V_{g}^{2}$ \\
Elev. & $C_{r} V_{c}^{2}+L_{r} I^{2} \geq C_{r} V_{o}^{2}$ \\
RedElev. & $C_{r}\left(V_{c}-V_{o}\right)^{2}+L_{r} I^{2} \geq C_{r}\left(V_{g}+V_{o}\right)^{2}$ \\
Flyback & $C_{r}\left(V_{c}-n V_{o}\right)^{2}+L_{r} I^{2} \geq C_{r}\left(V_{g}+n V_{o}\right)^{2}$ \\
\hline \hline
\end{tabular}

Tabla 3.4: Relación de energías para conmutaciones sin pérdidas.

A la vista de estas relaciones, se deduce que no se consiguen conmutaciones sin pérdidas a cualquier carga. Por ello, para aumentar el rango de 
potencia para el que se consiguen conmutaciones suaves, se sobredimensionará la bobina resonante. El aumento de la bobina resonante producirá un mayor estrés en los componentes porque aumenta la tensión media del condensador de clamp y por tanto, la tensión en el transistor principal. Además de perder margen dinámico en el condensador, ya que la tensión perdida en $R_{e q}$ no puede utilizarse para cargar la bobina de filtro.

Considerando el mismo régimen de carga, si aumenta $L_{r}$, la tensión de salida disminuirá, y se deberá compensar con un mayor ciclo de trabajo, para mantener la misma tensión y corriente a la salida. Al aumentar $L_{r}$, la tensión media del condensador de clamp también aumentará. En este incremento también influye el ciclo de trabajo. Como ejemplo, se ha obtenido la tensión del clamp para diferentes bobinas resonantes, en una etapa que trabaja a plena carga con los parámetros de funcionamiento de los ejemplos anteriores.

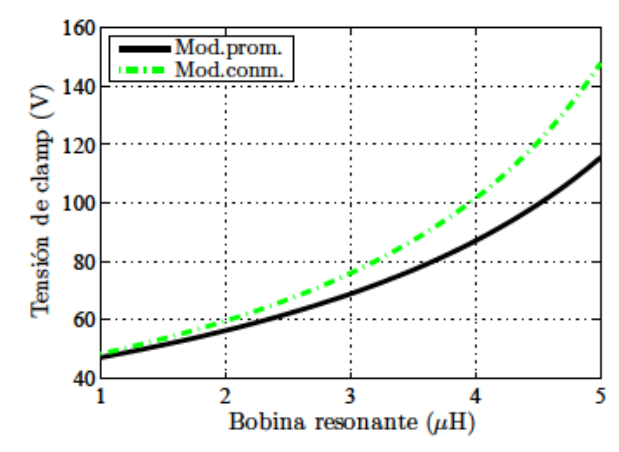

(a) Reductor.

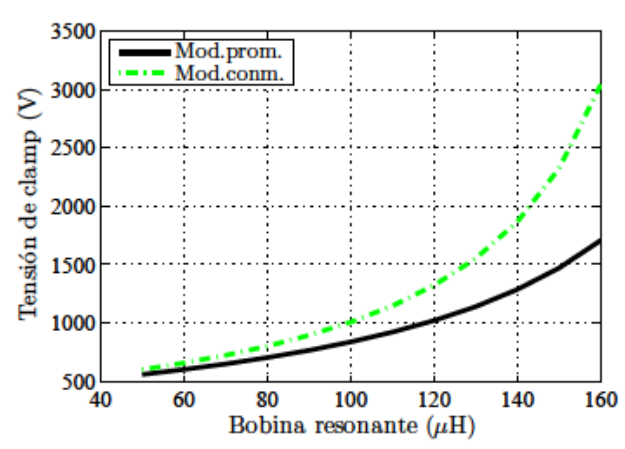

(c) Red.elev.

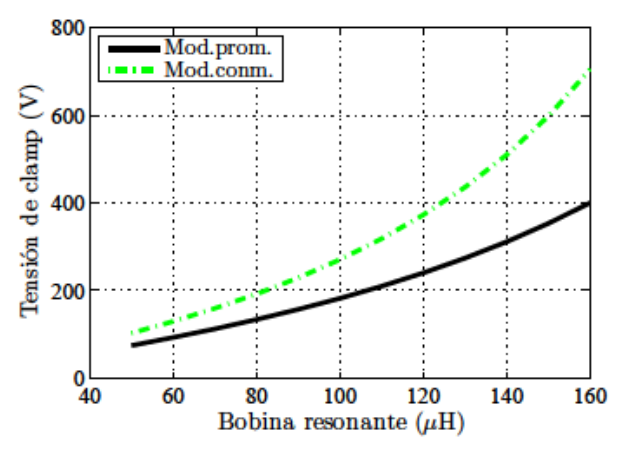

(b) Elevador.

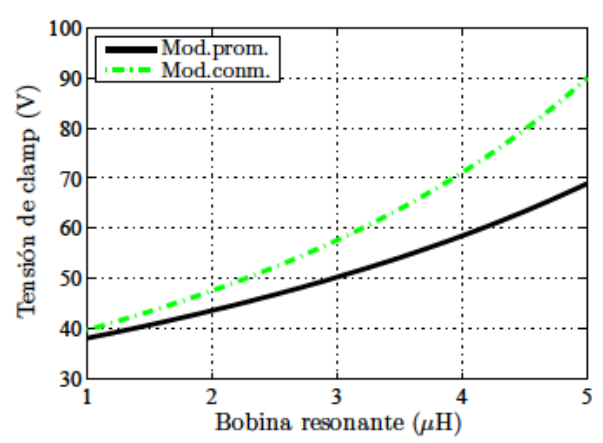

(d) Flyback.

Figura 3.13: Tensión de $c l a m p, V_{c}$, en función de $L_{r}$ para mismo régimen de carga. 
Se puede comprobar en la 3.13 que a medida que aumenta el valor de la bobina resonante, aumenta la tensión de clamp.

En la misma representación se ha incluido la evolución de $V_{c}$ para el modelo promediado y para el modelo conmutado. Para ambas curvas se ha utilizado la expresión de la Tabla 2.6, que incluye la corriente máxima y el ciclo trabajo. Ambos parámetros son diferentes según el modelo considerado para el cálculo, y por eso hay dos curvas diferentes. En el modelo promediado la corriente máxima coincide con la corriente media, y el ciclo de trabajo es menor que en el modelo conmutado. Este ciclo de trabajo se obtiene a partir de la expresión de la característica de salida descrita en el capitulo anterior (ver Tabla 2.26 o Tabla 2.15).

Otro método para asegurar conmutaciones sin pérdidas en un mayor rango de potencias en el convertidor ZVS formado por múltiples etapas consiste en activar y desactivar etapas en función de la carga total, así, para baja carga, solo funcionarán un número mínimo de etapas, mientras que cuando esté a plena carga, estarán funcionando todas. De esta forma no se necesita un valor de bobina resonante tan elevado como si fuera un único convertidor, puesto que las etapas activas estarás trabajando con una carga que les permita tener conmutaciones sin pérdidas y la bobina resonante estará ajustada a esa carga y no para la carga total.

El problema del reparto estático cuando hay varias etapas en paralelo ya está tratado en el capítulo anterior, de forma que la existencia de mas de una etapa funcionando no es un problema para el reparto estático. Habrá que tener cuidado en el momento en el que una etapa sea reclutada para la gestión de la energía, porque su incorporación al conjunto provoca un cambio en el estado energético, y la dinámica generada estará influenciada también por el condensador de clamp, como ya se ha puesto de manifiesto anteriormente.

Para mostrar este comportamiento se toma como ejemplo un convertidor de tres etapas, donde una de ellas se conecta o desconecta según la demanda de carga. Se considera tensión de salida constante para hacer el caso mas sencillo. En este caso, la corriente de salida de las etapas que ya estaban en uso no cambian, dado que las tensiones permanecen constantes. La etapa que entra en funcionamiento experimenta un cambio que puede estudiarse a partir del modelo promediado.

\subsubsection{Reductor ZVS.}

En la Figura 3.14 se ha indicado el modelo promediado del reductor y la modificación del mismo cuando se considera tensión constante a la salida. El ciclo de trabajo y la tensión de entrada se consideran constantes. 


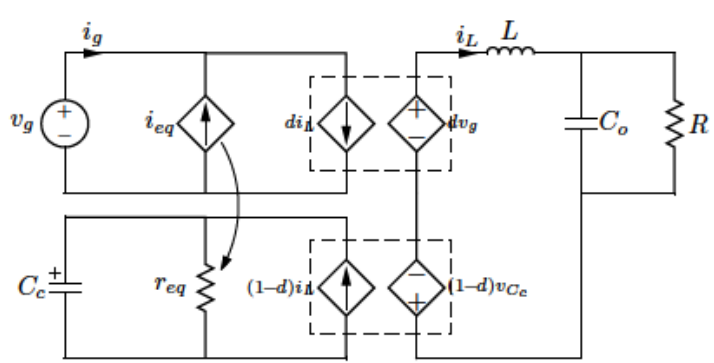

(a)

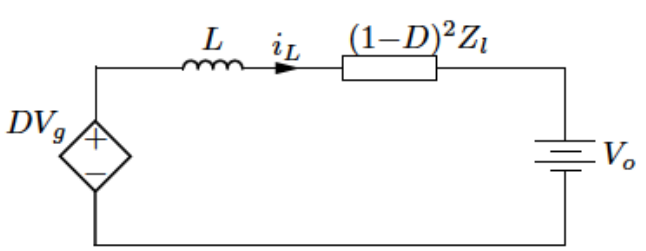

(b)

Figura 3.14: Reductor ZVS: a) modelo promediado; y b) circuito empleado para obtener $i_{L}$ con tensión constante a la salida.

Tras reagrupar términos, se comprueba que $Z_{t h}(s)$ es la impedancia que interviene en esta dinámica. A la vista del circuito, el hecho de conectar la tercera etapa, es equivalente a conectar su impedancia, $Z_{t h, 3}$ entre las dos tensiones, como se indica en la Figura 3.15. La evolución temporal de la corriente en la bobina de cada etapa se ha representado en la Figura 3.16

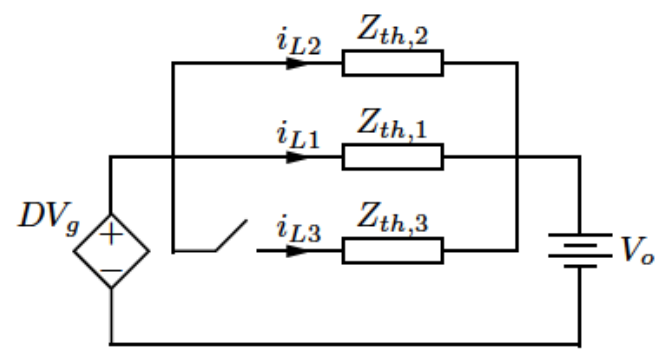

Figura 3.15: Reductor ZVS de tres etapas al conectar una de ellas.

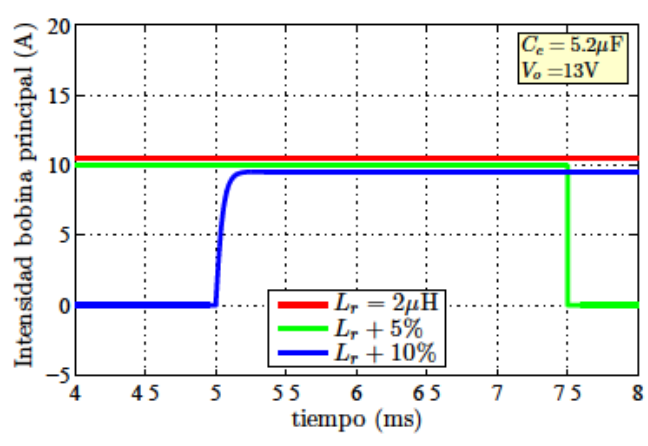

(a) $C_{c}$ nominal.

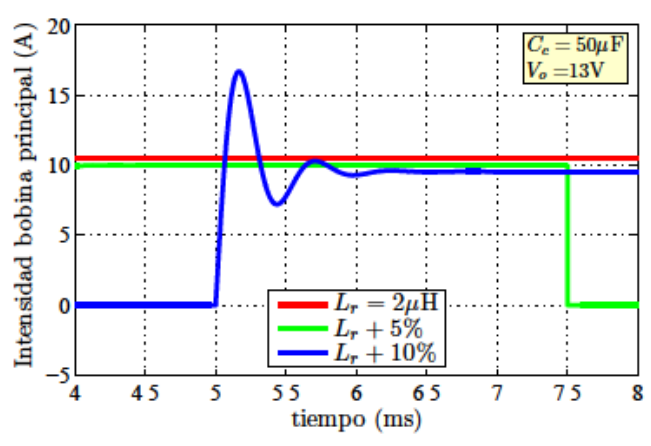

(b) $C_{c}$ diez veces mayor.

Figura 3.16: Evolución de $i_{L}(t)$ al reclutar una etapa.

Antes de conectar la tercera etapa, hay otras dos etapas conectadas que 
están en régimen permanente, por las que circula una corriente de 10,484 A y $9,984 \mathrm{~A}$ respectivamente. Hay que indicar que cada etapa tiene una bobina resonante de diferente valor, en torno al nominal de $L_{r}=2 \mu \mathrm{H}$. Al conectar la tercera etapa en paralelo con las otras dos, la impedancia experimenta un escalón de tensión que genera una corriente con la evolución representada. Tras esa transición, la corriente se estabiliza en un valor de 9,531 A. El transitorio depende de los elementos que forman la impedancia puesta en juego. Y entre ellos se encuentra el condensador $C_{c}$. Se ha representado la evolución de esa corriente para diferentes valores del condensador, siendo mas oscilante para el caso de mayor valor.

La conexión o desconexión de una etapa no afecta al resto, al no experimentan ningún cambio sus condiciones de funcionamiento.

También hay que tener en cuenta si esa etapa se conecta por primera vez, o si ya ha intervenido con anterioridad. Inicialmente está descargado, mientras que en ciclos posteriores tendría una energía no nula y la variación de la corriente sería menor que inicialmente. Por tanto, el peor caso corresponderá a un estado inicial de energía nula, que es el considerado en la representación gráfica.

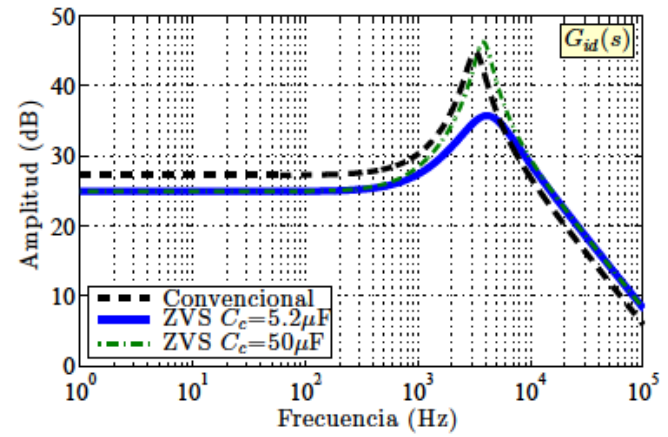

(a)

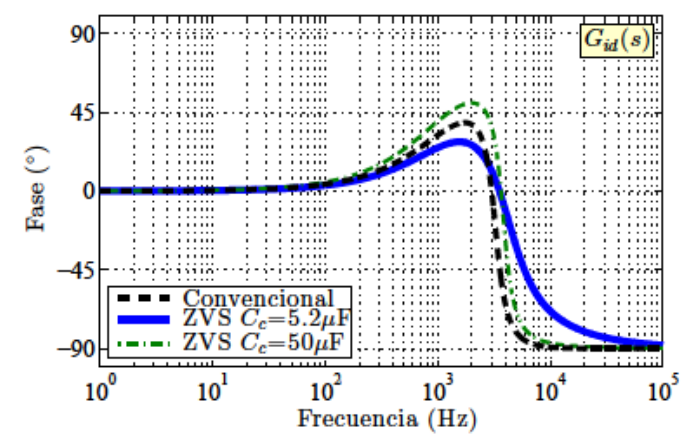

(b)

Figura 3.17: a) Ganancia y b) fase de $G_{i d}(s)$ para reductor normal y ZVS.

En la Figura 3.17Se ha representado la función de transferencia de la corriente en la bobina de un reductor ZVS en función del ciclo de trabajo, $G_{i d}(s)$, para dos valores diferentes de clamp. Su obtención se detalla en el Anexo correspondiente. Se comprueba que un mayor valor de $C_{c}$ produce un incremento del pico de resonancia de la ganancia. Este pico se aproxima al de un reductor convencional, cuya función también se ha representado en la misma Figura. Desde el punto de vista de la fase, el convertidor ZVS con un menor condensador de clamp, tiene mayor margen de fase en las frecuencias interesantes para el control. A medida que aumenta su valor, el margen va disminuyendo hasta alcanzar el de un reductor convencional. 
De la comparación de ambos convertidores, considerando el diseño para las mismas tensiones de entrada y salida e idéntico régimen de carga, un reductor ZVS no tendrá mayor problema para ser controlado que un convertidor normal aunque aumente el condensador de clamp. Esto es, un controlador diseñado para un reductor convencional seguirá funcionando en un reductor ZVS.

\subsubsection{Elevador ZVS.}

En la Figura 3.18 se ha indicado el modelo promediado del elevador y la modificación del mismo cuando se considera tensión constante a la salida. El ciclo de trabajo y la tensión de entrada se consideran constantes.

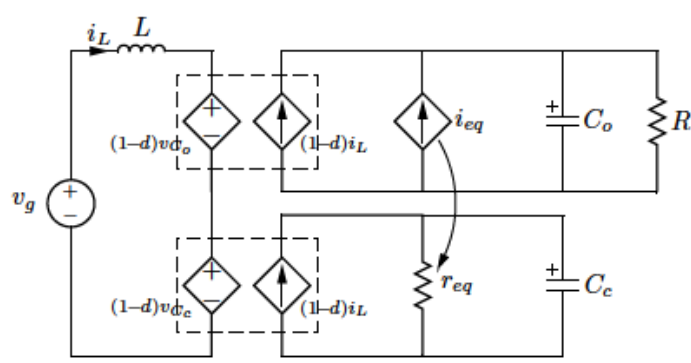

(a)

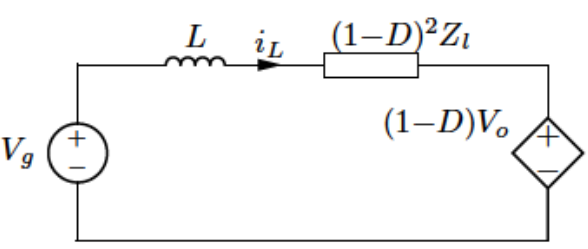

(b)

Figura 3.18: Elevador ZVS: a) modelo promediado; y b) circuito empleado para obtener $i_{L}$ con tensión constante a la salida.

Tras reagrupar términos, se comprueba que $Z_{t h}^{\prime}(s)=(1-D)^{2} Z_{t h}(s)$ es la impedancia que interviene en esta dinámica. A la vista del circuito, el hecho de conectar la tercera etapa, es equivalente a conectar su impedancia, entre las dos tensiones, como se indica en la Figura 3.19. La evolución temporal de la corriente en la bobina de cada etapa se ha representado en la Figura 3.20

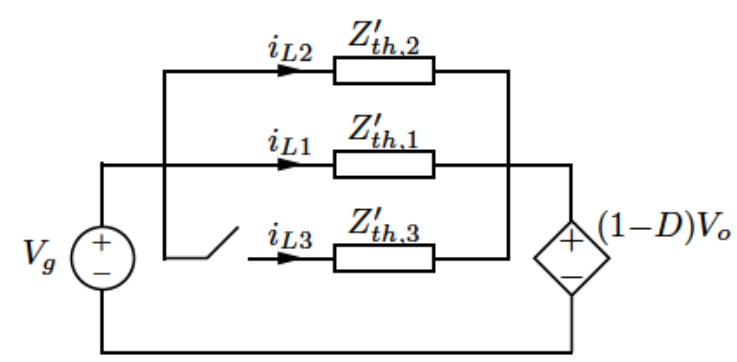

Figura 3.19: Elevador ZVS de tres etapas al conectar una de ellas.

Antes de conectar la tercera etapa, hay otras dos etapas conectadas que están en régimen permanente, donde hay un reparto de carga, siendo 2,62 A y $2,46 \mathrm{~A}$ la corriente en cada etapa. El valor nominal es de $L_{r}=70 \mu \mathrm{H}$. Al 


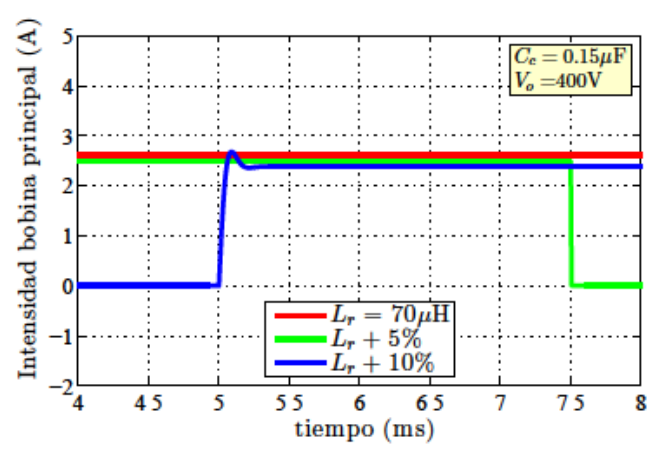

(a) $C_{c}$ nominal.

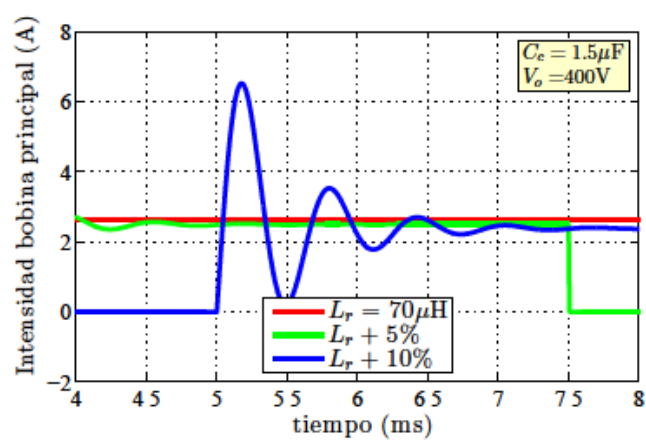

(b) $C_{c}$ diez veces mayor.

Figura 3.20: Evolución de $i_{L}(t)$ al reclutar una etapa.

conectar la tercera etapa la impedancia experimenta un escalón de tensión que genera una corriente con la evolución representada. Tras esa transición, la corriente se estabiliza en un valor de 2,38 A. El transitorio depende del condensador de clamp puesto que forma parte de la impedancia involucrada. Se observa una evolución mas resonante para un condensador mas grande. Otra vez, igual que con el reductor ZVS, la conexión o desconexión de una etapa no afecta al resto.

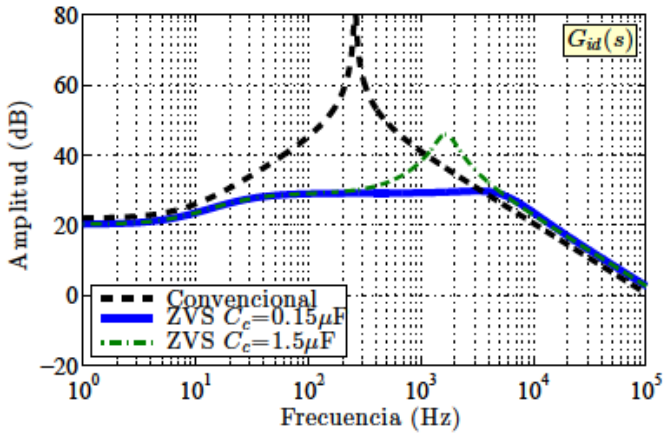

(a)

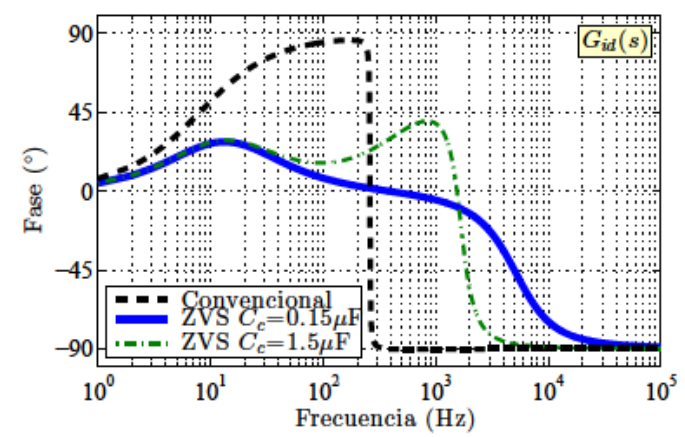

(b)

Figura 3.21: a) Ganancia y b) fase de $G_{i d}(s)$ para elevador normal y ZVS.

La obtención de la función de transferencia $G_{i d}(s)$ para un elevador ZVS está detallada en el anexo correspondiente y se ha comparado con la de un elevador convencional para las mismas características de funcionamiento. Observando su representación gráfica en la Figura 3.21 tanto en amplitud como en fase para dos valores diferentes de clamp, se comprueba que un mayor valor de este produce un incremento del pico de resonancia de la ganancia. Este pico se aproxima al que presenta un elevador normal. Desde 
el punto de vista de la fase, el convertidor ZVS con un menor condensador de clamp, también tiene mayor margen de fase en las frecuencias de control. A medida que aumenta su valor, el margen va disminuyendo hasta alcanzar el de un elevador convencional. Por lo tanto, es mas fácil de control el sistema con un condensador pequeño, pero no será mas difícil de controlar que un elevador normal.

\subsubsection{Reductor elevador ZVS.}

En la Figura 3.22 se ha indicado el modelo promediado del red.elevador y la modificación del mismo cuando se considera tensión constante a la salida. El ciclo de trabajo y la tensión de entrada se consideran constantes. Se ha añadido un diodo a la salida del circuito modificado para mantener el mismo sentido de la corriente a la salida que el circuito conmutado al que está representando este circuito modificado.

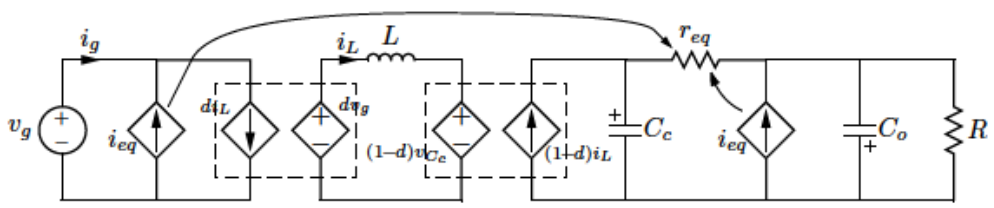

(a)

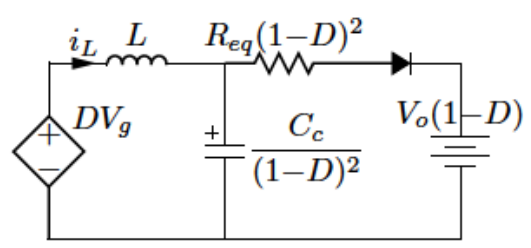

(b)

Figura 3.22: Red.Elev.ZVS: a) modelo promediado; y b) circuito empleado para obtener $i_{L}$ con tensión constante a la salida.

La dinámica durante el reclutamiento tiene dos etapas. Una primera etapa cuando el condensador de clamp está descargado, en la que solo interviene $L$ y el mismo condensador. Este tramo dura hasta que la tensión en el condensador alcanza la tensión de la fuente de la salida. A partir de aquí, se obtiene otra expresión para la corriente en la bobina. Ya no hay una expresión conocida para la impedancia que interviene en este transitorio, aunque no sería difícil de obtener una expresión de la corriente para los dos tramos mencionados.

El hecho de conectar la tercera etapa, es equivalente a conectar esa impedancia, entre las dos tensiones, y la corriente en la bobina de cada etapa tendría una evolución temporal similar a la representada en la Figura 3.23 


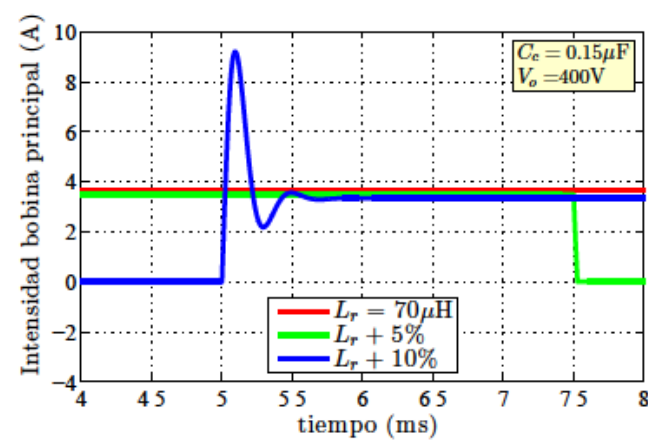

(a) $C_{c}$ nominal.

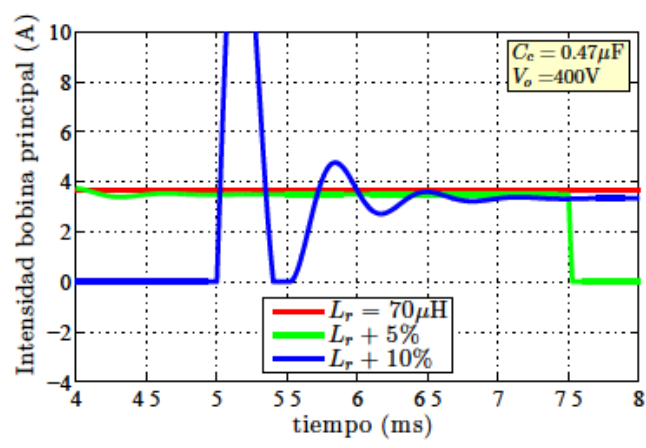

(b) $C_{c}$ tres veces mayor.

Figura 3.23: Evolución de $i_{L}(t)$ al reclutar una etapa.

Antes de conectar la tercera etapa, hay otras dos etapas conectadas que están en régimen permanente, donde hay un reparto de carga, siendo 3,67 A y $3,49 \mathrm{~A}$ la corriente en cada etapa. El valor nominal es de $L_{r}=70 \mu \mathrm{H}$. Al conectar la tercera etapa la impedancia experimenta un escalón de tensión que genera una corriente con la evolución representada. Tras esa transición, la corriente se estabiliza en un valor de 3,34 A. El transitorio depende del condensador de clamp puesto que forma parte de la impedancia involucrada. Se observa una evolución más resonante para un condensador más grande. Otra vez, igual en los casos anteriores, la conexión o desconexión de una etapa no afecta al resto.

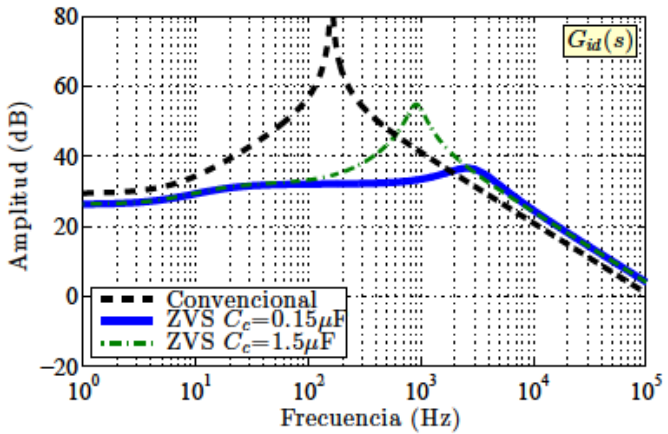

(a)

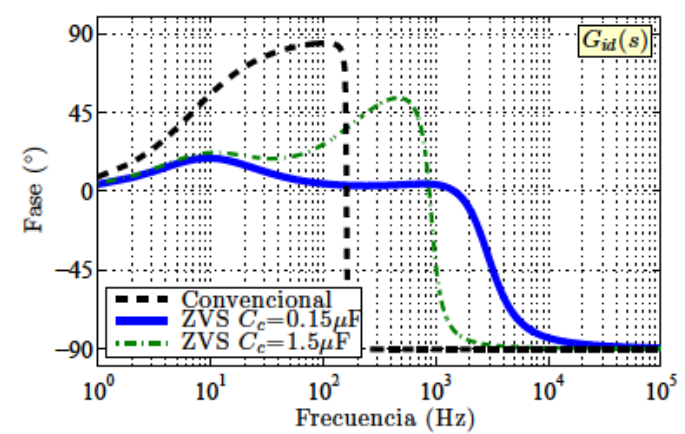

(b)

Figura 3.24: a) Ganancia y b) fase de $G_{i d}(s)$ para red.elev. normal y ZVS.

La obtención de la función de transferencia $G_{i d}(s)$ para un red.elev.ZVS está detallada en el anexo correspondiente y se ha comparado con la de un red.elev. convencional para las mismas características de funcionamiento. Observando su representación gráfica en la Figura 3.24 tanto en amplitud 
como en fase para dos valores diferentes de clamp, se comprueba que un mayor valor de este produce un incremento del pico de resonancia de la ganancia. Desde el punto de vista de la fase, el convertidor ZVS con un menor condensador de clamp, también tiene mayor margen de fase en las frecuencias de control. A medida que aumenta su valor, el margen va disminuyendo hasta alcanzar el de un reductor elevador convencional. Por lo tanto, es mas fácil de controlar el sistema con un condensador pequeño, pero no será mas difícil que controlar uno normal.

Estas afirmaciones se pueden extrapolar al flyback ZVS dada la similitud entre ambos convertidores. 



\section{Capítulo 4}

\section{Aplicación a convertidores con alta relación de transformación}

El desarrollo de un prototipo de laboratorio basado en un convertidor ZVS formado en dos etapas tipo flyback con enclavamiento activo conectadas en paralelo permite realizar una validación de los resultados teóricos presentados en los capítulos anteriores.

En un primer montaje, los resultados experimentales no son coincidentes con los esperados teóricamente. La causa es la no consideración en el desarrollo teórico de los capacidad parásita asociada a las bobinas acopladas. Estos elementos, se desprecian inicialmente porque su efecto en el circuito no es significativo en la frecuencia de trabajo. Lo que sucede ahora es que la alta relación de transformación cambia las frecuencias de resonancia y la aproxima a la frecuencia de trabajo. El resultado es una resonancia apreciable en el rango de frecuencias de trabajo. Dando por supuesto que los elementos parásitos no son exactamente iguales en dos bobinas similares, el reparto de la carga entre las etapas también será diferente.

La aplicación de un diodo de enclavamiento para fijar la tensión de un punto del circuito minimiza el efecto de estas diferencias y el reparto de carga se aproxima de nuevo al reparto equitativo entre las etapas.

\subsection{Introducción.}

Las aplicaciones industriales, comerciales y otras orientadas al consumo en áreas como telecomunicaciones, computación, vehículos eléctricos terrestres y aéreos, requieren de convertidores continua continua de altas prestaciones, con alta velocidad de respuesta y una elevada densidad de energía a la vez que un buen rendimiento y fiabilidad. En otros casos, también se requiere una ganancia elevada para pasar de tensiones pequeñas a otras de mayor valor, por ejemplo en aplicaciones de energía renovables para conexión 
a red (Li y otros, 2012). Aumentar la frecuencia de conmutación supone una reducción de tamaño de los magnéticos y una velocidad de respuesta, pero también implica el aumento de pérdidas por conmutación en los dispositivos semiconductores y de las interferencias electromagnéticas (EMI).

El aumento de la densidad de energía se puede conseguir con la asociación de varias unidades o etapas de menor potencia para distribuir la gestión y aliviar en parte los problemas relacionados con el estrés de los componentes a la vez que aumentar la eficiencia del conjunto (LUO y BATARSEH, 2005; Chen y otros, 2009). Además, se consiguen otras ventajas: un diseño térmico más sencillo, dado que cada convertidor maneja solo una parte de la energía total; un proceso de diseño más corto, con el consiguiente ahorro en los costes de fabricación, al ser módulos idénticos; un aumento de la fiabilidad del sistema con la sencilla inclusión de una unidad redundante para su uso en caso de fallo; y una capacidad energética escalable y adaptable en función de las necesidades (CHEN y otros, 2009).

Siendo la asociación mas frecuente es la asociación con entrada paralelo y salida paralelo (EPSP), surge la necesidad inmediata de incorporar algún tipo de mecanismo que distribuya la carga de forma equitativa entre las unidades que la forman. El trabajo desarrollado en los capítulos anteriores concluye que las topologías con enclavamiento activo, presentan una capacidad de reparto inherente debida a su impedancia de salida. De esta forma es factible un reparto de la carga en la conexión en paralelo.

Este tipo de reparto es un método pasivo, frente a los métodos de reparto activos que incluyen un mecanismo adicional para conseguir un reparto equilibrado entre las diferentes etapas conectadas en paralelo. La literatura es muy numerosa en lo que respecta a los dos grupos citados (HEDEL, 1980; Kislovski, 1983; HirshberG, 1985; Thorsell y Lindman, 1988; Small, 1988b; Tanaka y otros, 1988; Petruzziello y otros, 1990; MamMANO y JORDAN, 1991; JORDAN, 1991; SiRI y otros, 1992a,b; JAMERSON y otros, 1993; Glaser y Witulski, 1994; Batarseh y otros, 1994; JovanoVIC y otros, 1996; PANov y otros, 1997; ThotTuVelil y Verghese, 1998; Choi, 1998; Lin y Chen, 2000; Irving y Jovanovic, 2000; Kim y otros, 2002; Zhang y otros, 2004; Abu-QAhouq y otros, 2004; Chen y otros, 2004; Cheng y otros, 2005; Sun, 2007; Mao y otros, 2007; Huang y Tse, 2007; Panov y Jovanovic, 2008; Erdogan y Aydemir, 2009b,a; Anand y FERnANDEs, 2012). Otros trabajos se centran en: la búsqueda de la máxima eficiencia del conjunto en todo el rango posible de carga (ABU-QAHOUQ y otros, 2008a,b; AbU-QAhouQ, 2010; HAMAR y Toth, 2009; SePsi y otros, 2010; Hamar, 2009; Su y Liu, 2013; Renaudineau y otros, 2014); el uso de la tecnología digital, lo cual permite implementar otras técnicas de control, (Huth, 1996; Peterchev y otros, 2003; Saggini y otros, 2004; Zhang y otros, 2005; MAZumder y otros, 2005, 2008; BAE y otros, 2008; Zhang y otros, 2009; LAI y otros, 2009; BeHJATi y otros, 2014); y el empleo de otras 
topologías o modificación de las «tradicionales» (TSENG y LiANG, 2004; WANG, 2011, 2012; SHI y otros, 2012, 2014) para mejorar la eficiencia del sistema a la vez que aumentar la energía gestionada.

En concreto, uno de estos convertidores, el convertidor flyback con enclavamiento activo es una topología capaz de combinar aislamiento con conmutaciones suaves. También es posible obtener una alta ganancia, gracias a la relación de transformación. La red de enclavamiento activo, formada por el condensador de clamp, $C_{4}$, y el interruptor $S_{2}$, puede disponerse en dos configuraciones para este convertidor, como se indica en la 4.1

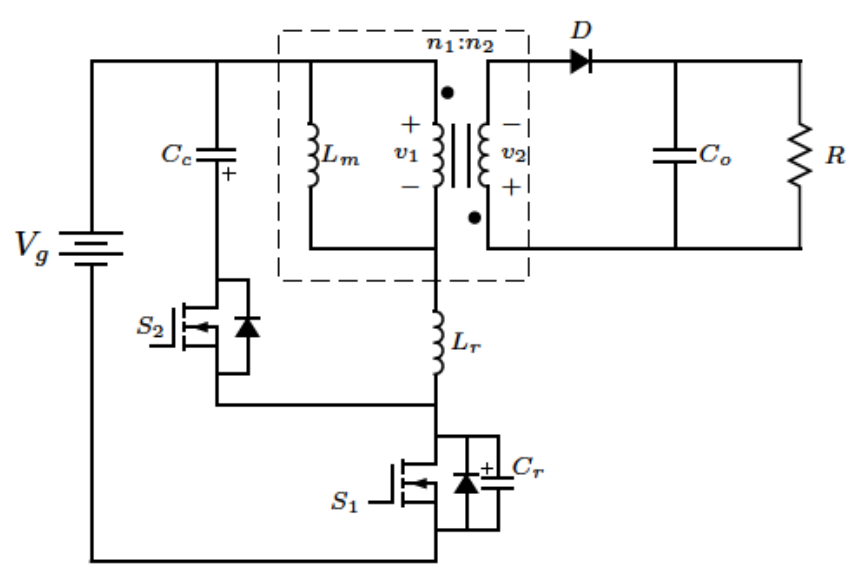

(a)

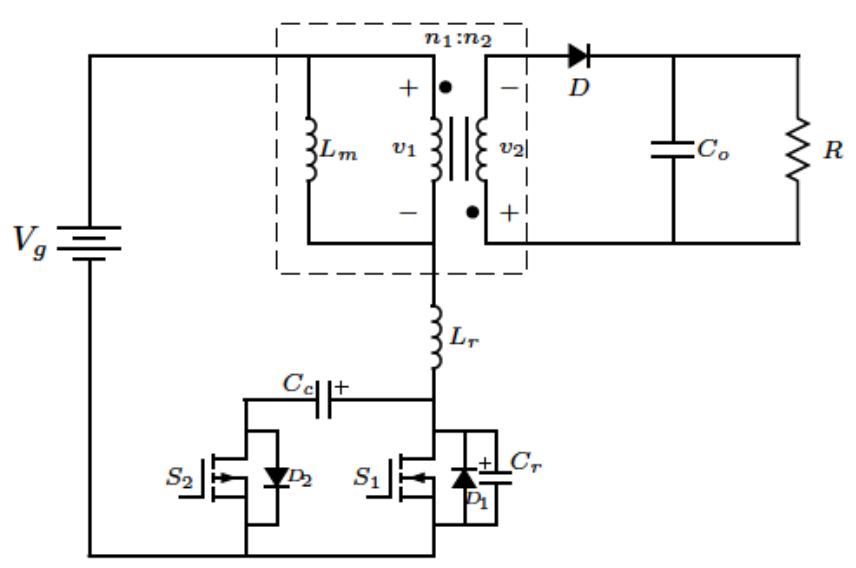

(b)

Figura 4.1: Flyback ZVS con red de enclavamiento activo: a) tipo buck-boost; b) tipo boost.

La principal diferencia es la tensión del condensador $C_{c}$, que tiene una expresión respecto a la tensión de entrada, $V_{g}$, que recuerda a la de un reductor elevador en el primer caso, y a la de un elevador en el segundo. 
Esto es, para el primer caso:

$$
V_{c, a)}=V_{g} \frac{D}{(1-D)}
$$

Y para el segundo caso:

$$
V_{c, b)}=V_{g} \frac{1}{(1-D)}=V_{g} \frac{D}{(1-D)}+V_{g}
$$

En (MAppus, 2004) se establece una comparativa sobre la aplicación de una u otra topología. Además del valor de la tensión alcanzada en el condensador del clamp, que afecta a algunas de las tensiones que van a soportar el resto de elementos del circuito, y de que para determinadas tensiones de entrada, no se podría emplear el tipo boost por un aspecto técnico (dado que el abanico de MOSFET tipo P se reduce bastante mas allá de 200V), el uso de cualquiera de las topologías no añade una ventaja clara. Previamente, en (DALAL, 1996) ya se establecía que ambas disposiciones tienen un comportamiento similar en el comportamiento estático, excepto por la mayor tensión de clamp ya indicada.

En el caso del reparto de la carga gestionada por varias etapas conectadas en paralelo, donde la impedancia de salida de las topologías ZVS juega un papel importante, los resultados teóricos obtenidos son idénticos, de forma que no será necesario mencionar la topología empleada al hablar de la impedancia. Los Anexos D y E entran en mayor detalle en el análisis de esta magnitud, para las dos topologías flyback con enclavamiento activo mencionadas. En este capítulo se ha optado por el uso de un MOSFET de canal P, por tener cierta facilidad en el driver de disparo empleado.

\subsection{Topología flyback ZVS con capacidades pará- sitas.}

En el análisis teórico realizado en los anexos de los convertidores ZVS, se ha considerado siempre que el convertidor tratado es ideal, es decir, no se ha considerado ningún elemento parásito. En el caso concreto del flyback ZVS no se ha considerado tampoco ninguna capacidad parásita de las bobinas acopladas. En la realidad, estas capacidades interaccionan durante determinados instantes de funcionamiento, con otros elementos presentes en el circuito en determinados y se generan resonancias, que alteran el comportamiento del circuito en mayor o menor medida.

El efecto de las oscilaciones debidas a estos elementos parásitos ya ha sido mencionado anteriormente en la literatura para convertidores con enclavamiento activo en (Jovanovic, 1998; DuARTE y BARBI, 2002); o para 
convertidores que además incluyen algún tipo de aislamiento en (Hren y otros, 2006; SPIAZZi y otros, 2009; MASIHUZZAMAN y LAKSHMINARASAMMA, 2010; SpIAZZI y otros, 2010); etcétera. Uno de los efectos el aumento de la tensión inversa que soporta el diodo de salida al doble del valor teórico sin considerar estas resonancias.

En la Figura 4.2 ha representado un convertidor flyback con enclavamiento activo tipo boost como el estudiado en el Anexo E, al que se han añadido dos capacidades, $C_{t}$ y $C_{f}$. Este circuito se referirá, de ahora en adelante, como flyback ZVS-real. Las dos capacidades incorporadas corresponden a la capacidad parásita del secundario de las bobinas acopladas y a la capacidad parásita del diodo de salida, respectivamente. Durante el funcionamiento del circuito, estos elementos interaccionan con otros elementos del mismo, principalmente con la bobina $L_{r}$, introduciendo comportamientos resonantes que modifican algunos de sus parámetros.

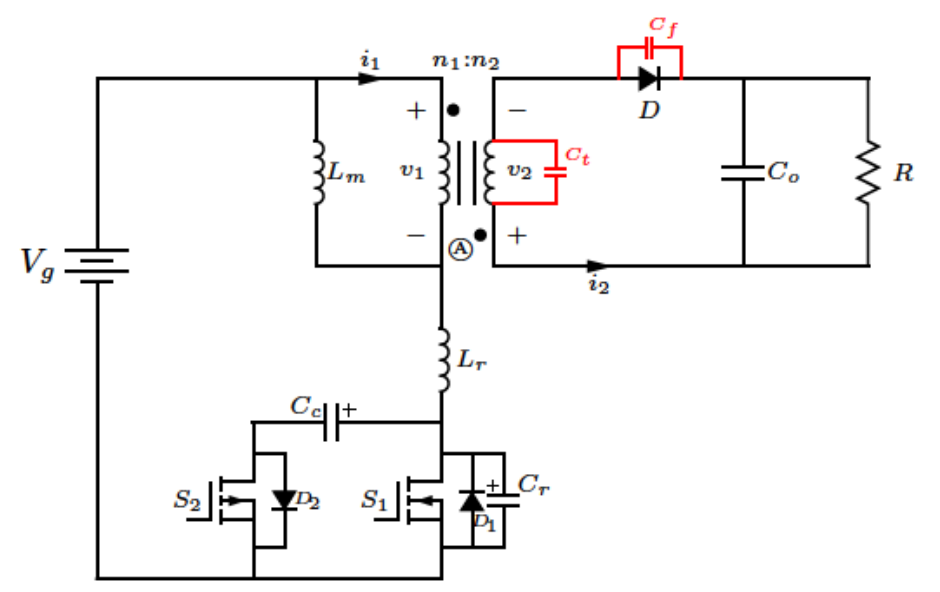

Figura 4.2: Convertidor flyback ZVS-boost con capacidades parásitas.

Desde el punto de vista del análisis del circuito, ambas capacidades parásitas se pueden agrupar en una única capacidad, $C_{e q}$, vista desde el primario del transformador tal y como está indicado en la Figura 4.3. Su valor es:

$$
C_{e q}=\left(\frac{n_{2}}{n_{1}}\right)^{2}\left(C_{t}+C_{f}\right)
$$

En las bobinas acopladas, se puede comprobar que la corriente del diodo de salida, $i_{D}(t)$, está relacionada con la corriente de primario, $i_{1}(t)$ de la siguiente forma:

$$
n_{1} i_{1}(t)=n_{2} i_{2}(t)=-n_{2} i_{D}(t)
$$

donde se ha considerado el sentido positivo de $i_{1}(t)$ entrando por el punto de primario y de $i_{2}(t)$ saliente por el punto de secundario.

Cuando se aplica la ley de nodos de Kirchhoff en (A), se comprueba que:

$$
i_{m}(t)+i_{1}(t)+i_{C_{e q}}(t)=i_{r}(t)
$$




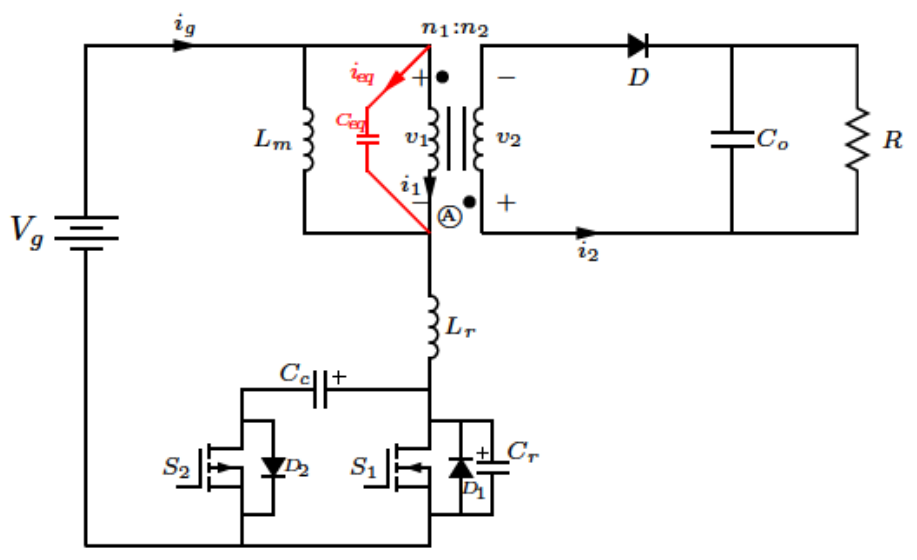

Figura 4.3: El mismo convertidor tras agrupar las capacidades parásitas.

Siendo $i_{m}(t)$ la corriente que circula por la bobina de magnetización $L_{m}, i_{r}(t)$ la corriente circulante por la bobina resonante $L_{r}$, e $i_{C_{e q}}(t)$ la corriente que circula por la capacidad parásita equivalente. Combinando las expresiones (4.4) y (4.5) se consigue:

$$
i_{m}(t)+i_{C_{e q}}(t)=i_{r}(t)+\frac{1}{n} i_{D}(t)
$$

siendo $n=n_{1} / n_{2}$.

Por otro lado la corriente de entrada, $i_{g}(t)$ es igual a la corriente $i_{r}(t)$, y puede relacionarse con la corriente de salida $i_{D}$ de la siguiente forma:

$$
i_{g}(t)+\frac{1}{n} i_{D}(t)=i_{m}(t)+i_{C_{e q}}(t)
$$

\subsubsection{Etapas.}

El convertidor evoluciona por varias etapas a lo largo de un periodo de conmutación. En concreto son seis etapas a lo largo de un periodo, y se describen a continuación. Se considera $L_{m}$ lo suficientemente grande como para considerar una corriente constante de valor $I$. También se considera $L_{r}$ pequeña en comparación con $L_{m}$. Las seis etapas están representadas en la Figura 4.4.

Etapa $1\left[t_{0}-t_{1}\right]$ : Antes de $t_{0}$, el interruptor principal $S_{1}$ está conduciendo, y el interruptor auxiliar $S_{2}$ está abierto. $L_{r}$ y $C_{e q}$ están intercambiando energía de forma resonante. En $t=t_{0}, S_{1}$ pasa a $\mathrm{OFF}$, y se añade $C_{r}$ al intercambio de energía. La corriente $i_{r}$ a través de éste último lo carga de forma resonante hasta una tensión $V_{c}$ que es cuando acaba esta etapa.

Etapa $2\left[t_{1}-t_{2}\right]$ : Cuando $v_{C_{r}}$ alcanza la tensión $V_{c}$, el diodo $D_{2}$ empieza a conducir y la corriente en $L_{r}$ tiene una componente constante, $I$, debida 


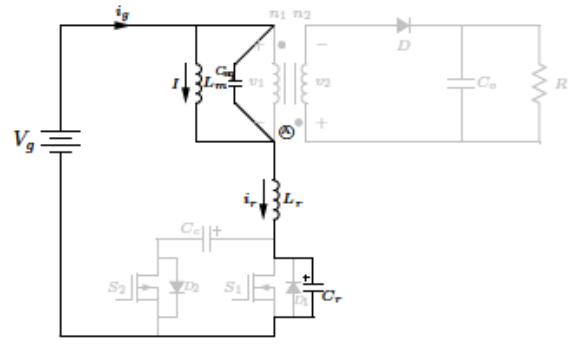

(a)

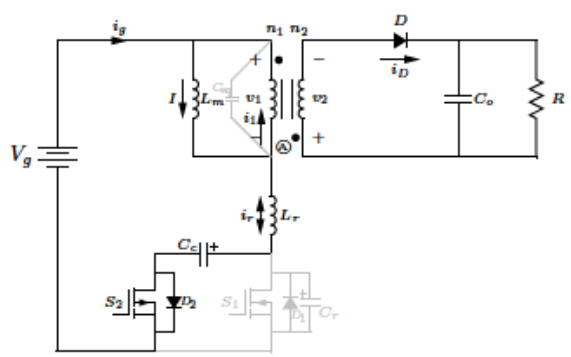

(c)

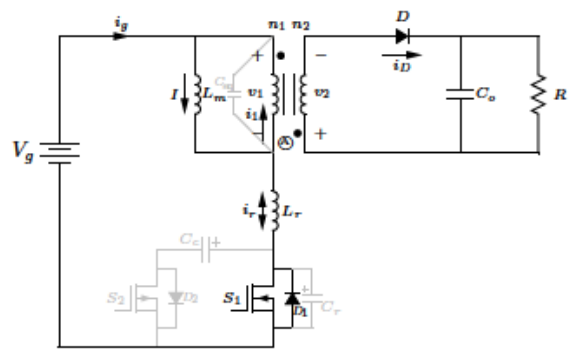

(e)

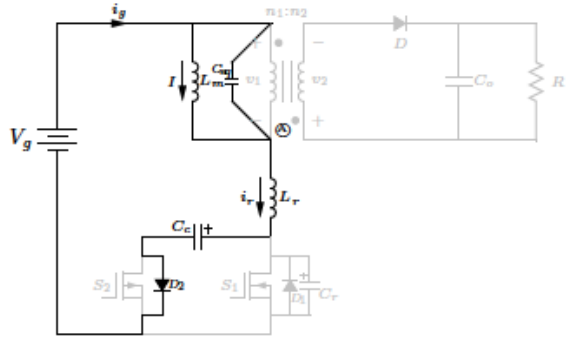

(b)

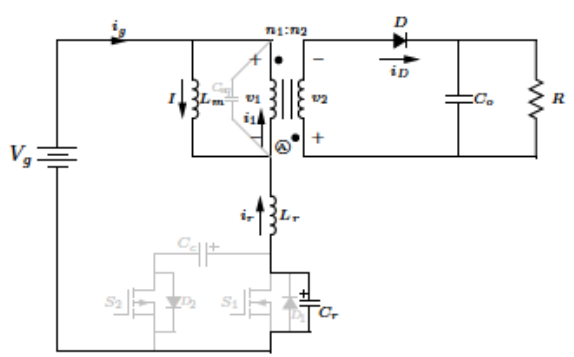

(d)

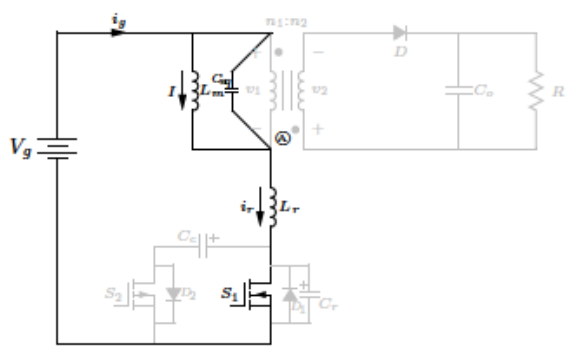

(f)

Figura 4.4: Etapas por las que evoluciona el flyback ZVS-real en un periodo: (a) Etapa 1. (b) Etapa 2. (c) Etapa 3. (d) Etapa 4. (e) Etapa 5. (f) Etapa 6.

a $L_{m}$, y una componente resonante debida a $C_{e q}$. Ambos elementos intercambian energía hasta que acaba esta etapa cuando la tensión $v_{C_{e q}}$ llega a $-n V_{o}$.

Etapa $3\left[t_{2}-t_{3}\right]$ : Cuando $v_{C_{e q}}=-n V_{o}$ en $t_{2}$, el diodo de salida $D$ empieza a conducir al quedar polarizado en directa. Nótese que $C_{e q}$ mantiene una tensión constante en sus extremos mientras $D$ esté en conducción, por lo que efecto capacitivo no se tiene en cuenta. La bobina $L_{r}$ está sometida a una tensión constante de valor $V_{g}+n V_{o}-V_{c}$, y la corriente $i_{r}$ evoluciona de forma lineal. En algún momento de este intervalo, y antes de que $i_{r}$ cambie su sentido, se puede conmutar $S_{2}$ sin pérdidas porque no tiene tensión en su extremos al estar conduciendo $D_{2}$. Esta etapa acaba cuando $S_{2}$ pase a OFF.

Etapa $4\left[t_{3}-t_{4}\right]$ : Ahora la corriente de la bobina resonante se emplea en 
descargar la energía almacenada en $C_{r}$ hasta hacer su tensión nula. En $t_{5}$ se hace $v_{C_{r}}=0$.

Etapa $5\left[t_{4}-t_{5}\right]$ : En el instante que la tensión de $C_{r}$ es nula, la corriente pasa a circular por el diodo $D_{1}$, y es posible la conmutación de $S_{1}$ con tensión nula. La conmutación debería realizarse antes de que la corriente $i_{r}$ cambie de nuevo su sentido de circulación al inicial. La evolución es lineal porque $L_{r}$ está sometida a una tensión $V_{g}+n V_{o}$. También evoluciona de forma lineal la corriente en $D$. Al final de esta etapa, ambas corrientes alcanzan los valores de $I$ y 0 respectivamente. En este momento, $D$ deja de conducir.

Etapa $6\left[t_{5}-t_{6}\right]$ : Esta etapa comienza cuando el diodo $D$ ha dejado de conducir, y la tensión de la capacidad $C_{e q}$, que estaba fijada a un valor constante, ya no permanece así, sino que intercambia energía de forma resonante con $L_{r}$. Esta etapa termina cuando $S_{1}$ pasa a OFF y volvería a la primera etapa. El valor de $i_{r}(t)$ y de $v_{C_{e q}}$ depende del instante en el que se abra el interruptor principal.

Cada etapa tiene su circuito equivalente, que está recogido en la Figura 4.5. Se ha considerado $L_{m}$ lo suficientemente grande como para suponer corriente constante, de valor $I$, y mucho mayor que la bobina resonante $L_{r}$. El valor de los condensadores $C_{o}$ y $C_{c}$, es tal que la tensión en sus extremos se mantiene constante, de valores $V_{o}$ y $V_{c}$, en todo el periodo $T$. La duración de una etapa se indica con el término $d$ con un subíndice con el número. Por ejemplo, $d_{1} T=\left(t_{0}-t_{1}\right)$ representa la duración de la etapa 1 .

En la primera etapa, intervienen $C_{e q}$ y $C_{r}$. El circuito empleado para su resolución está representado en la Figura 4.5a. Las condiciones iniciales en los elementos reactivos se indican como $V_{e q 0} \mathrm{e} I_{r 0}$. Cuando $C_{e q}$ es mucho mayor que la capacidad resonante, se podría considerar como una fuente de tensión constante, y quedaría una malla como la indicada en la Figura 4.6d.

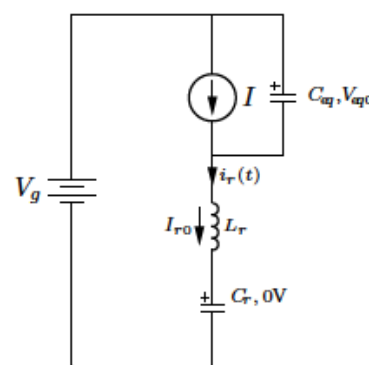

(a)

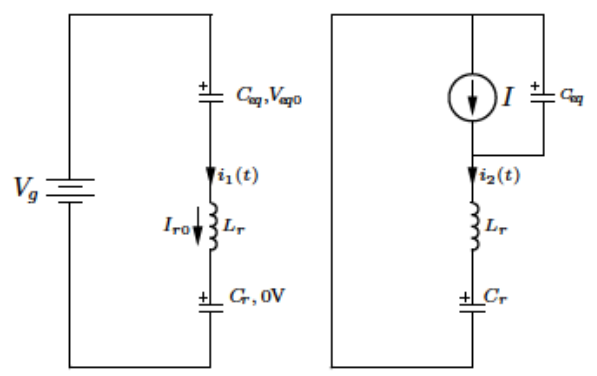

(b) (c)

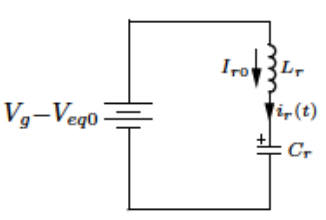

(d)

Figura 4.6: Etapa 1: (a) Circuito equivalente; (b) y (c) Mallas obtenidas tras superposición; y (d) Simplificación de (a) cuando $v_{C_{e q}}(t)$ es constante.

En el instante $t_{0}$, las condiciones iniciales, $I_{r 0}$ y $V_{e q 0}$, tienen las expresio- 


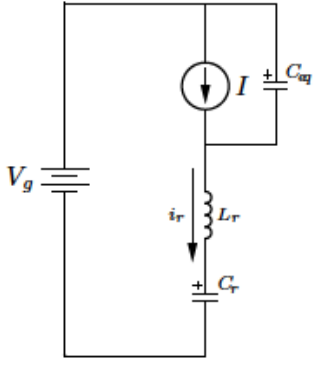

(a)

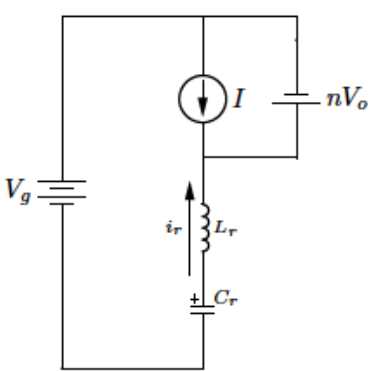

(d)

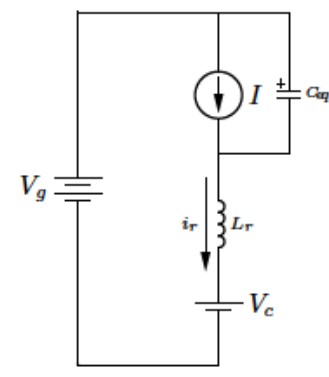

(b)

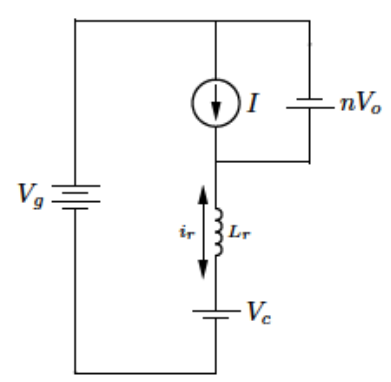

(c)

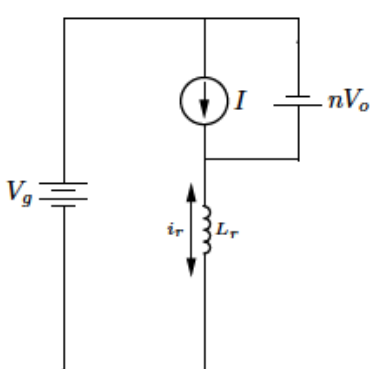

(e)

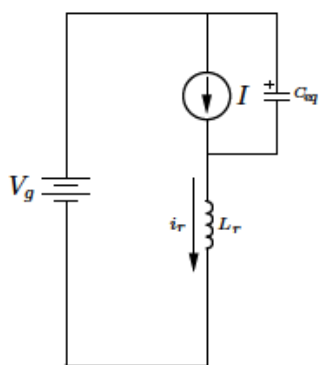

(f)

Figura 4.5: Circuitos equivalentes a los de la Figura 4.4: (a) Etapa 1. (b) Etapa 2. (c) Etapa 3. (d) Etapa 4. (e) Etapa 5. (f) Etapa 6.

nes indicadas en 4.8 .

$$
\begin{aligned}
V_{e q 0} & \equiv v_{C_{e q}}\left(t_{0}\right)=V_{g}-\left(V_{g}+n V_{o}\right) \cos \left(\omega_{e q} d_{6} T\right) \approx v_{C_{e q}}\left(t_{1}\right) \equiv V_{e q 1} \\
I_{r 0} & \equiv i_{r}\left(t_{0}\right)=I+\frac{\left(V_{g}+n V_{o}\right)}{Z_{e q}} \sin \left(\omega_{e q} d_{6} T\right)
\end{aligned}
$$

donde $Z_{e q}=\sqrt{L_{r} / C_{e q}}, \mathrm{y} \omega_{e q}=1 / \sqrt{L_{r} C_{e q}}$.

Estas expresiones se han obtenido al analizar la etapa 6, puesto que los instantes $t_{6}$ y $t_{0}$ son equivalentes. Su circuito equivalente está indicado en la Figura 4.7.

El condensador equivalente interviene también en la etapa 2. La Figura 4.8 muestra las mallas empleadas para analizar esta etapa, en la que se ha supuesto que $V_{e q 1}=v_{C_{e q}}\left(t_{1}\right) \approx v_{C_{e q}}\left(t_{0}\right) \equiv V_{e q 0}$.

Tras analizar todas las etapas, se obtienen las expresiones de las principales magnitudes, indicadas a continuación. Para la tensión en $C_{r}$ y la 


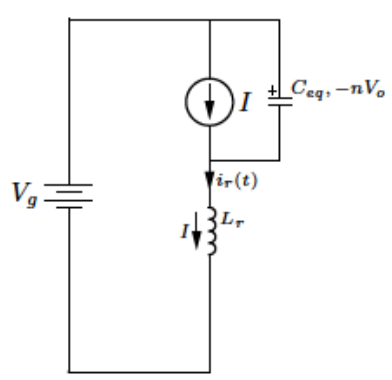

(a)

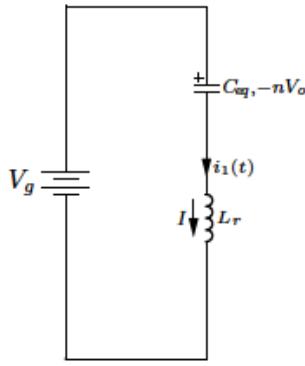

(b)

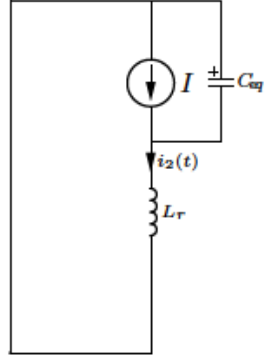

(c)

Figura 4.7: Etapa 6: (a) Circuito equivalente; (b) y (c) mallas obtenidas al aplicar superposición.

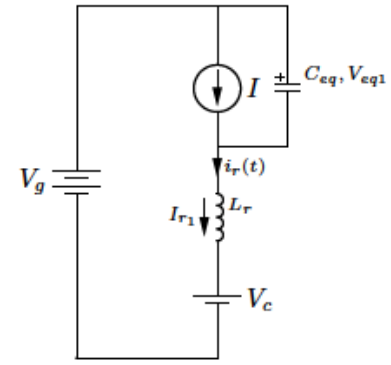

(a)

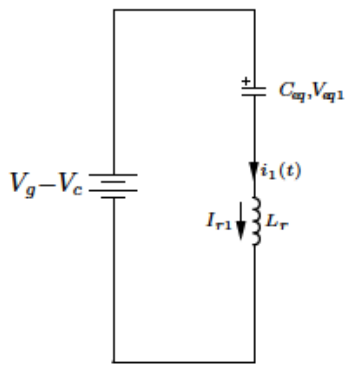

(b)

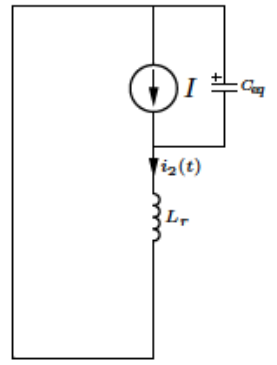

(c)

Figura 4.8: Etapa 2: (a) Circuito equivalente con condiciones iniciales; b) y (c) mallas obtenidas tras superposición.

intensidad en $L_{r}$ :

$$
v_{C_{r}}(t)= \begin{cases}\left(V_{g}-V_{e q 0}\right)\left(1-\cos \left[\omega_{r}\left(t-t_{0}\right)\right]\right) & t \in\left[t_{0}, t_{1}\right] \\ \quad+I_{r 0} Z_{r} \sin \left[\omega_{r}\left(t-t_{0}\right)\right] & t \in\left[t_{1}, t_{3}\right] \\ V_{c} & t \in\left[t_{3}, t_{4}\right] \\ \left.\quad+V_{g}+n V_{o}\right)\left(1-\cos \left[\omega_{r}\left(t-t_{3}\right)\right]\right) & \text { resto }\left[\omega_{r}\left(t-t_{3}\right)\right]+I_{r 3} Z_{r} \sin \left[\omega_{r}\left(t-t_{3}\right)\right]\end{cases}
$$




$$
i_{r}(t)= \begin{cases}I_{r 0} \cos \left[\omega_{r}\left(t-t_{0}\right)\right]+\frac{V_{g}-V_{e q 0}}{Z_{r}} \sin \left[\omega_{r}\left(t-t_{0}\right)\right] & t \in\left[t_{0}, t_{1}\right] \\ I+\left(I_{r 1}-I\right) \cos \left[\omega_{e q}\left(t-t_{1}\right)\right] & t \in\left[t_{1}, t_{2}\right] \\ \quad+\frac{\left(V_{g}-V_{e q 1}-V_{c}\right)}{Z_{e q}} \sin \left[\omega_{e q}\left(t-t_{1}\right)\right] & t \in\left[t_{2}, t_{3}\right] \\ I_{r 2}+\frac{\left(V_{g}+n V_{o}-V_{c}\right)}{L_{r}}\left(t-t_{2}\right) & t \in\left[t_{3}, t_{4}\right] \\ I_{r 3} \cos \left[\omega_{r}\left(t-t_{3}\right)\right]+\frac{\left(V_{g}+n V_{o}-V_{c}\right)}{Z_{r}} \sin \left[\omega_{r}\left(t-t_{3}\right)\right] & t \in\left[t_{4}, t_{5}\right] \\ I_{r 4}+\frac{\left(V_{g}+n V_{o}\right)}{L_{r}}\left(t-t_{4}\right) & t \in\left[t_{5}, t_{6}\right] \\ I+\frac{\left(V_{g}+n V_{o}\right)}{Z_{e q}} \sin \left[\omega_{e q}\left(t-t_{5}\right)\right] & \end{cases}
$$

donde se han considerado los siguientes términos:

$$
\begin{aligned}
& Z_{r}=\sqrt{L_{r} / C_{r}} \\
& \omega_{r}=1 / \sqrt{L_{r} C_{r}} \\
& I_{r 1} \equiv i_{r}\left(t_{1}\right)=I_{r 0} \cos \omega_{r} d_{1} T+\frac{V_{g}-V_{e q 0}}{Z_{r}} \sin \omega_{r} d_{1} T \\
& I_{r 2} \equiv i_{r}\left(t_{2}\right)=I+\left(I_{r 1}-I\right) \cos \omega_{e q} d_{2} T+\frac{\left(V_{g}-V_{e q 1}-V_{c}\right)}{Z_{e q}} \sin \omega_{e q} d_{2} T \\
& I_{r 3} \equiv i_{r}\left(t_{3}\right)=I_{r 2}+\frac{\left(V_{g}+n V_{o}-V_{c}\right)}{L_{r}} d_{3} T \\
& I_{r 4} \equiv i_{r}\left(t_{4}\right)=I_{r 3} \cos \omega_{r} d_{4} T+\frac{\left(V_{g}+n V_{o}-V_{c}\right)}{Z_{r}} \sin \omega_{r} d_{4} T
\end{aligned}
$$

La tensión en el condensador equivalente, $v_{C_{e q}}(t)$ está indicada a continuación, mientras que la intensidad que pasa por él, $i_{C_{e q}}(t)$, es igual a $i_{r}(t)-I$ durante la etapa 2 y 6 . Durante el resto de etapas no circula ninguna intensidad.

$$
v_{C_{e q}}(t)=\left\{\begin{array}{lc}
V_{e q 0} & t \in\left[t_{0}, t_{1}\right] \\
V_{g}-V_{c}-\left(V_{g}-V_{e q 1}-V_{c}\right) \cos \left[\omega_{e q}\left(t-t_{1}\right)\right] & t \in\left[t_{1}, t_{2}\right] \\
\quad+n V_{o} \quad+\left(I_{r 1}-I\right) Z_{e q} \sin \left[\omega_{e q}\left(t-t_{1}\right)\right] & t \in\left[t_{2}, t_{5}\right] \\
V_{g}-\left(V_{g}+n V_{o}\right) \cos \left[\omega_{e q}\left(t-t_{5}\right)\right] & t \in\left[t_{5}, t_{6}\right]
\end{array}\right.
$$




$$
i_{C_{e q}}(t)= \begin{cases}\frac{\left(V_{g}+n V_{o}\right) \sin \left(w_{e q} d_{6} T\right)}{Z_{e q}} \cos \left[\omega_{e q}\left(t-t_{1}\right)\right] & t \in\left[t_{1}, t_{2}\right] \\ +\frac{\left.\left(V_{g}+n V_{o}\right) \cos \left(w_{e q} d_{6} T\right)-V_{c}\right)}{Z_{e q}} \sin \left[\omega_{e q}\left(t-t_{1}\right)\right] & \\ \frac{\left(V_{g}+n V_{o}\right)}{Z_{e q}} \sin \left[\omega_{e q}\left(t-t_{5}\right)\right] & t \in\left[t_{5}, t_{6}\right] \\ 0 & \text { resto }\end{cases}
$$

La intensidad en el condensador $C_{c}$ es igual a $i_{r}(t)$ durante la etapa 2 y 3. En el resto es nula.

$i_{C_{c}}(t)= \begin{cases}I-\left(I-I_{r 1}\right) \cos \left[\omega_{e q}\left(t-t_{1}\right)\right]+\frac{\left(V_{g}-V_{e q 1}-V_{c}\right)}{Z_{e q}} \sin \left[\omega_{r}\left(t-t_{1}\right)\right] & t \in\left[t_{1}, t_{2}\right] \\ I_{r 2}+\frac{\left(V_{g}+n V_{o}-V_{c}\right)}{L_{r}}\left(t-t_{2}\right) & t \in\left[t_{2}, t_{3}\right] \\ 0 & \text { resto }\end{cases}$

Las expresiones de tensión en las bobinas de magnetización y resonante también están calculadas como:

$$
\begin{gathered}
v_{L_{m}}(t)=v_{C_{e q}}(t) \\
v_{L_{r}}(t)= \begin{cases}\left(V_{g}-V_{e q 0}\right) \cos \left[\omega_{r}\left(t-t_{0}\right)\right]-I_{r 0} Z_{r} \sin \left[\omega_{r}\left(t-t_{0}\right)\right] & t \in\left[t_{0}, t_{1}\right] \\
V_{g}-V_{c}-v_{C_{e q}}(t) & t \in\left[t_{1}, t_{2}\right] \\
V_{g}+n V_{o}-V_{c} & t \in\left[t_{2}, t_{3}\right] \\
\left(V_{g}+n V_{o}\right) \cos \left[\omega_{r}\left(t-t_{3}\right)\right]-V_{c} \cos \left[\omega_{r}\left(t-t_{3}\right)\right]-I_{r 3} Z_{r} \sin \left[\omega_{r}\left(t-t_{3}\right)\right] & t \in\left[t_{3}, t_{4}\right] \\
V_{g}+n V_{o} & t \in\left[t_{4}, t_{5}\right] \\
V_{g}-v_{C_{e q}}(t) & t \in\left[t_{5}, t_{6}\right]\end{cases}
\end{gathered}
$$

Esta última se puede reescribir como (4.17) cuando se sustituyen la expresión de $v_{C_{e q}}$ en (4.16):

$$
v_{L_{r}}(t)= \begin{cases}\left(V_{g}-V_{e q 0}\right) \cos \left[\omega_{r}\left(t-t_{0}\right)\right]-I_{r 0} Z_{r} \sin \left[\omega_{r}\left(t-t_{0}\right)\right] & t \in\left[t_{0}, t_{1}\right] \\ \left(V_{g}-V_{e q 1}-V_{c}\right) \cos \left[\omega_{e q}\left(t-t_{1}\right)\right]-\left(I_{r 1}-I\right) Z_{e q} \sin \left[\omega_{e q}\left(t-t_{1}\right)\right] & t \in\left[t_{1}, t_{2}\right] \\ V_{g}+n V_{o}-V_{c} & t \in\left[t_{2}, t_{3}\right] \\ \left(V_{g}+n V_{o}\right) \cos \left[\omega_{r}\left(t-t_{3}\right)\right]-V_{c} \cos \left[\omega_{r}\left(t-t_{3}\right)\right]-I_{r 3} Z_{r} \sin \left[\omega_{r}\left(t-t_{3}\right)\right] & t \in\left[t_{3}, t_{4}\right] \\ V_{g}+n V_{o} & t \in\left[t_{4}, t_{5}\right] \\ \left(V_{g}+n V_{o}\right) \cos \left[\omega_{e q}\left(t-t_{5}\right)\right] & t \in\left[t_{5}, t_{6}\right]\end{cases}
$$


En la Figura 4.9 se han representado las principales magnitudes a lo largo de las seis etapas por las que evoluciona esta topología durante un ciclo completo.

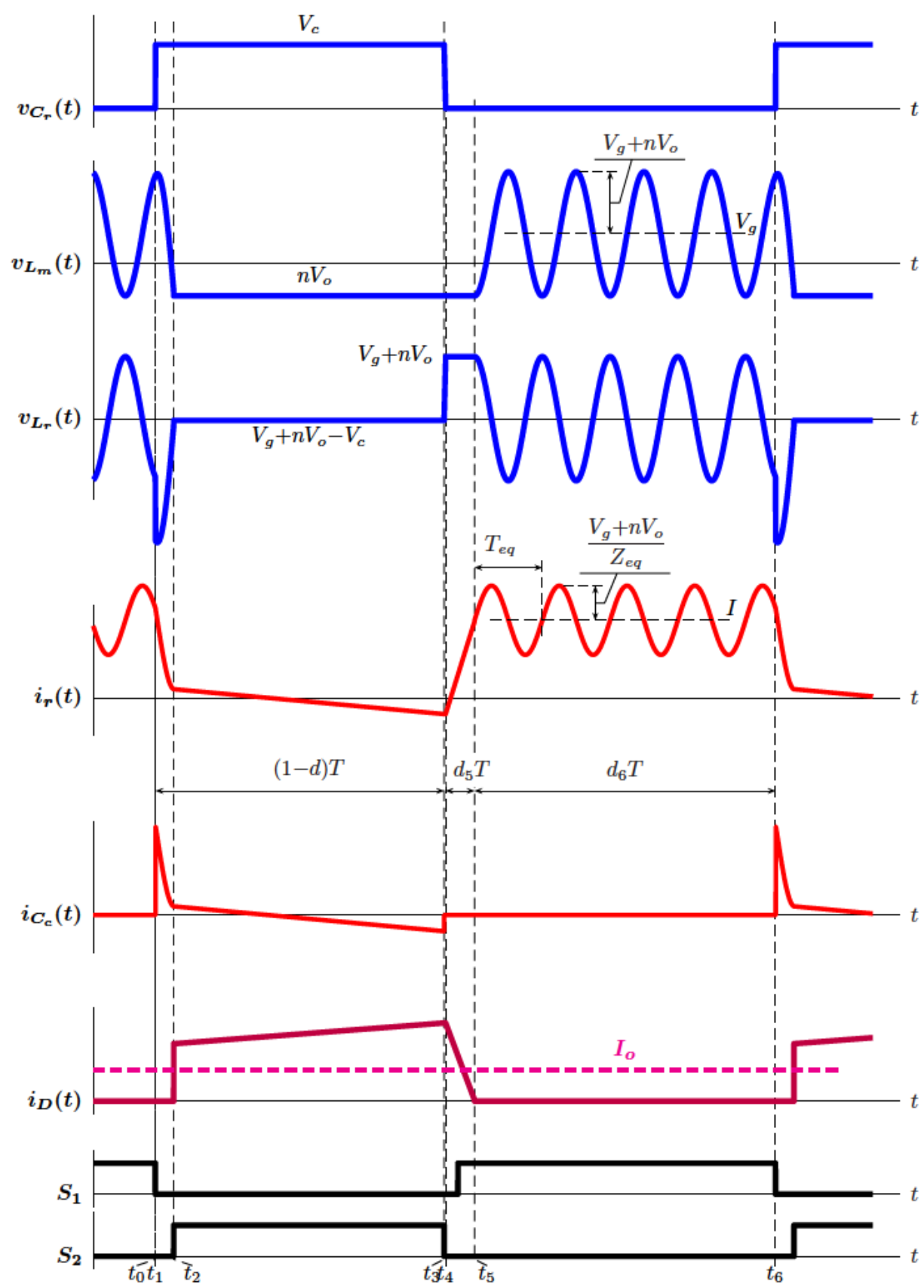

Figura 4.9: Curvas principales de un flyback ZVS-real. 


\subsubsection{Análisis en régimen permanente.}

En esta sección se realizará el análisis en régimen permanente sin considerar el rizado. Este es el análisis que se empleará para comprobar que en el instante $t_{6}$, justo cuando transistor $S_{1}$ pasa a corte, el valor de la corriente resonante $i_{r}(t)$ tiene una gran variabilidad, dado que depende de los elementos parásitos a través de $w_{e q}$. Pequeñas variaciones en dichos elementos conducirán a grandes variaciones en el valor de la corriente en este instante.

Para este análisis se mantienen las consideraciones mencionadas anteriormente, respecto a $L_{m}$ para suponer corriente constante, de valor $I$, respecto a $L_{r}$ que es mucho mas pequeña qe $L_{m}$. También se mantiene constante la tensión en $C_{o}$ y en $C_{c}$, de valores $V_{o}$ y $V_{c}$ respectivamente. Recuérdese, que la duración de cualquiera de las etapas es igual a una fracción del periodo total $T$, indicada como $d_{x} T$, donde $x$ indica la etapa.

También se va a suponer despreciable la etapa 1 y la etapa 4 puesto que la frecuencia característica del conjunto $L_{r}-C_{r}$ es mucho mas grande que la frecuencia de conmutación por lo que la carga-descarga de $C_{r}$ es prácticamente instantánea. Entonces, $d_{1} \approx 0$ y $d_{4} \approx 0$. De esta forma solo tendrán en cuenta cuatro etapas.

\section{TENSION MEDIA EN BOBINA DE MAGNETIZACIÓN}

La tensión media en la bobina $L_{m}$ es nula. Se puede escribir la siguiente ecuación:

$$
\underbrace{\frac{1}{T} \int_{0}^{d_{2} T} v_{C_{e q}}(t) d t}_{I_{1}}-n V_{o}\left(d_{3}+d_{5}\right)+\underbrace{\frac{1}{T} \int_{0}^{d_{6} T} v_{C_{e q}}(t) d t}_{I_{2}}=0
$$

\section{TENSION MEDIA EN BOBINA RESONANTE}

De igual manera, la tensión media en la bobina $L_{r}$ es nula. Se puede escribir la siguiente ecuación:

$$
\begin{aligned}
& \underbrace{\frac{1}{T} \int_{0}^{d_{2} T}\left(V_{g}-V_{c}-v_{C_{e q}}(t)\right) d t}_{I_{3}}+\left(V_{g}+n V_{o}-V_{c}\right) d_{3} \\
& +\left(V_{g}+n V_{o}\right) d_{5}+\underbrace{\frac{1}{T} \int_{0}^{d_{6} T}\left(V_{g}-v_{C_{e q}}(t)\right) d t}_{I_{4}}=0
\end{aligned}
$$

Cuando se desarrollan las integrales $I_{3}$ e $I_{4}$, se pueden dejar en función de $I_{1}$ e $I_{2}$ y se llega a la siguiente ecuación:

$$
\left.V_{g}\left(d_{2}+d_{3}+d_{5}+d_{6}\right)-V_{c}\left(d_{2}+d_{3}\right)+n V_{o}\right)\left(d_{3}+d_{5}\right)-I_{1}-I_{2}=0
$$


Sumando las ecuaciones (4.18) y (4.20) desaparecen las integrales y se llega a la ecuación (4.21)

$$
V_{g}\left(d_{2}+d_{3}+d_{5}+d_{6}\right)-V_{c}\left(d_{2}+d_{3}\right)+\underline{n V_{0}\left(d_{3}+d_{5}\right)}-\underline{n V_{0}\left(d_{3}+d_{5}\right)}=0
$$

en donde se sabe que $d_{2}+d_{3}+d_{5}+d_{6}=1$. Entonces se despeja $V_{c}$ que queda como:

$$
V_{c}=\frac{V_{g}}{d_{2}+d_{3}}=\frac{V_{g}}{(1-D)}
$$

siendo $d_{2}+d_{3}=(1-D)$. Es necesario calcular la duración de los intervalos para obtener una expresión aplicable a partir de la tensión entrada y salida y potencia.

\section{CARGA DE $C_{e q}$. CALCULO DE $d_{2}$}

La duración de la etapa 2 está relacionada con la tensión del condensador $C_{e q}$. El condensador previamente está intercambiando energía con $L_{r}$ de forma resonante. En $t_{0}$ se abre el interruptor $S_{1}$ y el intercambio de energía ahora se hace de forma resonante hasta que el condensador equivalente se carga a una tensión negativa igual a $-n V_{o}$. Instante en el que el diodo de salida se polariza en directa. Como no se considera la etapa 1, la tensión del condensador en $t_{1}$ se considera igual a la que tenía en $t_{0}$, luego:

$$
V_{e q 1} \equiv v_{C_{e q}}\left(t_{1}\right) \approx v_{C_{e q}}\left(t_{0}\right) \equiv V_{e q 0}=V_{g}-\left(V_{g}+n V_{o}\right) \cos \left(\omega_{e q} d_{6} T\right)
$$

Lo mismo sucede con la intensidad resonante, puesto que al no considerar la etapa 1 , resulta que:

$$
I_{r 1} \equiv i_{r}\left(t_{1}\right) \approx i_{r}\left(t_{0}\right) \equiv I_{r 0}=I+\frac{\left(V_{g}+n V_{o}\right)}{Z_{e q}} \sin \left(\omega_{e q} d_{6} T\right)
$$

Se puede obtener una expresión para la tensión al final de esta etapa sabiendo que $v_{C_{e q}}\left(t_{2}\right)=-n V_{o}$. Entonces, de (4.12):

$$
V_{g}-V_{c}-\left(V_{g}-V_{e q 1}-V_{c}\right) \cos \left(\omega_{e q} d_{2} T\right)+\left(I_{r 1}-I\right) Z_{e q} \sin \left(\omega_{e q} d_{2} T\right)=-n V_{o}
$$

Sustituyendo (4.23) y (4.24), da lugar a:

$$
\begin{aligned}
V_{g}-V_{c}-\left(\left(V_{g}+n V_{o}\right) \cos \left(\omega_{e q} d_{6} T\right)-V_{c}\right) \cos \left(\omega_{e q} d_{2} T\right) \\
+\left(V_{g}+n V_{o}\right) \sin \left(\omega_{e q} d_{6} T\right) \sin \left(\omega_{e q} d_{2} T\right)=-n V_{o}
\end{aligned}
$$

Tras reordenar, queda:

$$
V_{g}+n V_{o}-V_{c}+V_{c} \cos \left(\omega_{e q} d_{2} T\right)=\left(\left(V_{g}+n V_{o}\right) \cos \left[\omega_{e q}\left(d_{2}+d_{6}\right) T\right]\right.
$$

O de otra forma:

$$
\cos \left[\omega_{e q}\left(d_{2}+d_{6}\right) T\right]=1+\frac{V_{c}}{V_{g}+n V_{o}}\left(\cos \left(\omega_{e q} d_{2} T\right)-1\right)
$$


INCREMENTO DE $i_{r}(t)$ EN ETAPA 5. CALCULO DE $d_{5}$

La corriente resonante, $i_{r}(t)$ evoluciona linealmente desde $i_{r}\left(t_{4}\right)$ hasta $i_{r}\left(t_{5}\right)=I$ durante la etapa 5 , en la que la bobina $L_{r}$ está sometida a una tensión $V_{g}+n V_{o}$. La duración de esta etapa es el tiempo que se tarda en llegar a ese valor, pero para su obtención es necesario conocer el valor de la intensidad resonante en $t_{4}, I_{r 4}$. Y para ello hay que obtener también su valor en $t_{3}$, y su valor en $t_{2}$ y su valor en $t_{1}$. Como las etapas 1 y 4 se desprecian por ser de muy corta duración, resultan los siguientes valores para $I_{r 1}, I_{r 2}$, $I_{r 3}, I_{r 4}$ e $I_{5}$ :

$$
\begin{aligned}
& I_{r 1} \equiv i_{r}\left(t_{1}\right) \approx i_{r}\left(t_{0}\right) \equiv I_{r 0}=I+\frac{\left(V_{g}+n V_{o}\right)}{Z_{e q}} \sin \left(\omega_{e q} d_{6} T\right) \\
& I_{r 2} \equiv i_{r}\left(t_{2}\right)=I+\frac{\left(V_{g}+n V_{o}\right)}{Z_{e q}} \sin \left[\omega_{e q}\left(d_{2}+d_{6}\right) T\right]-\frac{V_{c}}{Z_{e q}} \sin \left(\omega_{e q} d_{2} T\right) \\
& I_{r 4} \equiv i_{r}\left(t_{4}\right) \approx i_{r}\left(t_{3}\right) \equiv I_{r 3}=I_{r 2}+\frac{\left(V_{g}+n V_{o}-V_{c}\right)}{L_{r}} d_{3} T \\
& I_{r 5} \equiv i_{r}\left(t_{5}\right)=I_{r 4}+\frac{\left(V_{g}+n V_{o}\right)}{L_{r}} d_{5} T
\end{aligned}
$$

La duración de la etapa 5 se obtiene haciendo $I_{r 5}=I$. Entonces:

$d_{5} T=-\frac{\left(V_{g}+n V_{o}-V_{c}\right)}{\left(V_{g}+n V_{o}\right)} d_{3} T-\frac{L_{r}}{Z_{e q}} \sin \left[\left(w_{e q}\left(d_{2}+d_{6}\right) T\right]+\frac{V_{c} L_{r}}{\left(V_{g}+n V_{o}\right) Z_{e q}} \sin \left(w_{e q} d_{2} T\right)\right.$

CORRIENTE MEDIA EN EL CONDENSADOR EQUIVALENTE $C_{e q}$.

La corriente en el condensador $C_{e q}$ está indicada en (4.13). La corriente media es nula, por lo que:

$$
\underbrace{\frac{1}{T} \int_{0}^{d_{2} T} i_{C_{e q}}(t) d t}_{I_{1}}+\underbrace{\frac{1}{T} \int_{0}^{d_{6} T} i_{C_{e q}}(t) d t}_{I_{2}}=0
$$

Desarrollando ambas integrales, $I_{1} I_{2}$, se llega a la siguiente igualdad:

$$
\begin{aligned}
& \frac{1}{T}\left(\frac{\left(V_{g}+n V_{o}\right) \sin \left(w_{e q} d_{6} T\right)}{Z_{e q}{ }^{2 W_{e q}}} \sin \left(\omega_{e q} d_{2} T\right)-\right. \\
& \left.-\frac{\left(\left(V_{g}+n V_{o}\right) \cos \left(w_{e q} d_{6} T\right)-V_{c}\right)}{Z_{e q}^{2 w_{e q}}}\left[\cos \left(\omega_{e q} d_{2} T\right)-1\right]\right)- \\
& -\frac{1}{T}\left(\frac{\left(V_{g}+n V_{o}\right)}{Z_{e q} w_{e q}}\left[\cos \left(\omega_{e q} d_{6} T\right)-1\right]\right)=0
\end{aligned}
$$


Simplificando se llega a la expresión:

$$
\begin{aligned}
& \left(V_{g}+n V_{o}\right) \sin \left(w_{e q} d_{6} T\right) \sin \left(\omega_{e q}\left(d_{2} T\right)\right)- \\
& -\left(V_{g}+n V_{o}\right) \cos \left(w_{e q} d_{6} T\right) \cos \left(\omega_{e q} d_{2} T\right)+V_{c}\left[\cos \left(\omega_{e q} d_{2} T\right)-1\right]+ \\
& +\left(V_{g}+n V_{o}\right)=0
\end{aligned}
$$

De donde se obtiene la misma expresión que está indicada en (4.28)

CORRIENTE MEDIA EN EL CONDENSADOR DE CLAMP $C_{c}$.

Como se sabe que $I_{c, \text { med }}=0$, resulta que:

$$
\underbrace{\frac{1}{T} \int_{0}^{d_{2} T} i_{C_{c}}(t) d t}_{I_{3}}+\underbrace{\frac{1}{T} \int_{0}^{d_{3} T} i_{C_{c}}(t) d t}_{I_{4}}=0
$$

durante la etapa $2, i_{C_{c}}(t)=i_{r}(t)=I+i_{C_{e q}}(t)$, y con la igualdad de la ecuación (4.31) se desarrolla $I_{3}$ como:

$$
\begin{aligned}
I_{3} & =\frac{1}{T} \int_{0}^{d_{2} T} i_{C_{c}}(t) d t \\
& =I d_{2}+\frac{1}{T} \int_{0}^{d_{2} T}{ }^{{ }} i_{e q}(t) d t \\
& =I d_{2}-\frac{1}{T} \int_{0}^{d_{6} T} i_{C_{e q}}(t) d t \\
& =I d_{2}+\frac{\left(V_{g}+n V_{o}\right)}{Z_{e q} w_{e q} T}\left[\cos \left(\omega_{e q} d_{6} T\right)-1\right]
\end{aligned}
$$

La integral $I_{4}$ es:

$$
\begin{aligned}
I_{4} & =\frac{1}{T} \int_{0}^{d_{3} T} i_{C_{c}}(t) d t \\
& =\frac{1}{T} \int_{0}^{d_{3} T} I_{r 2}+\frac{\left(V_{g}+n V_{o}-V_{c}\right)}{L_{r}} t d t \\
& =I_{r 2} d_{3}+\frac{\left(V_{g}+n V_{o}-V_{c}\right)}{L_{r}} \frac{d_{3}^{2} T}{2}
\end{aligned}
$$

donde la expresión de $I_{r 2}$ está indicada en (4.29). Al sustituirla, se obtiene:

$$
\begin{aligned}
& I\left(d_{2}+d_{3}\right)+\frac{\left(V_{g}+n V_{o}\right)}{Z_{e q} w_{e q} T}\left[\cos \left(\omega_{e q} d_{6} T\right)-1\right]+ \\
& +\left[\frac{\left(V_{g}+n V_{o}\right)}{Z_{e q}} \sin \left[\omega_{e q}\left(d_{2}+d_{6}\right) T\right]-\frac{V_{c}}{Z_{e q}} \sin \left(\omega_{e q} d_{2} T\right)\right] d_{3}+ \\
& +\left(V_{g}+n V_{o}-V_{c}\right) \frac{d_{3}^{2} T}{2 L_{r}}=0
\end{aligned}
$$


La expresión encerrada entre corchetes y que multiplica $d_{3}$ se puede sustituir por la igualdad (4.38), que se ha obtenido del cálculo de $d_{5} T$.

$\left[\frac{\left(V_{g}+n V_{o}\right)}{Z_{e q}} \sin \left[\omega_{e q}\left(d_{2}+d_{6}\right) T\right]-\frac{V_{c}}{Z_{e q}} \sin \left(\omega_{e q} d_{2} T\right)\right]=-\frac{T}{L_{r}}\left[\left(V_{g}+n V_{o}-V_{c}\right) d_{3}+\left(V_{g}+n V_{o}\right) d_{5}\right]$

Entonces, (4.37) queda simplificada como:

$$
\begin{aligned}
& I\left(d_{2}+d_{3}\right)+\frac{\left(V_{g}+n V_{o}\right)}{Z_{e q} w_{e q} T}\left[\cos \left(\omega_{e q} d_{6} T\right)-1\right] \\
& -\left(V_{g}+n V_{o}\right) \frac{d_{5} d_{3} T}{L_{r}}-\left(V_{g}+n V_{o}-V_{c}\right) \frac{d_{3}^{2} T}{2 L_{r}}=0
\end{aligned}
$$

\section{CORRIENTE MEDIA EN LA BOBINA RESONANTE, $I_{r}$ Y EN}

\section{LA ENTRADA $I_{g}$.}

A la vista del circuito de la figura La corriente en la bobina resonante, $i_{r}(t)$, coincide con la corriente en la entrada, $i_{g}(t)$ El término $I_{n}$ se puede obtener haciendo el valor medio a la expresión (4.7), que se ha reescrito a continuación.

$$
i_{g}(t)+\frac{1}{n} i_{D}(t)=i_{m}(t)+i_{C_{e q}}(t)
$$

Haciendo el valor medio, ysabiendo que $I_{C_{e q}, \text { med }}=0$, resulta que:

$$
I_{g}+\frac{I_{o}}{n}=I
$$

Al aplicar la igualdad de potencias, $P_{g}=P_{o}$, se pueden deducir sendas expresiones que relacionan la corriente media en la bobina, con la corriente media a la salida y a la entrada:

$$
\begin{gathered}
I_{o} \frac{V_{o}}{V_{g}}+\frac{I_{o}}{n}=I \Rightarrow I=I_{o}\left[\frac{1}{n}+\frac{V_{o}}{V_{g}}\right] \\
I_{g}+\frac{I_{g} V_{g}}{n V_{o}}=I \Rightarrow I=I_{g}\left[\frac{V_{g}}{n V_{o}}+1\right]
\end{gathered}
$$

Por otro lado, la corriente media en la entrada (o en la bobina resonante) es igual a:

$$
\begin{aligned}
I_{g} & =I_{r} \\
& =\underbrace{\frac{1}{T} \int_{0}^{d_{2} T} i_{r}(t) d t+\frac{1}{T} \int_{0}^{d_{3} T} i_{r}(t) d t}_{0 \text { porque } I_{c, \text { med }}=0}+\underbrace{\frac{1}{T} \int_{0}^{d_{5} T} i_{r}(t) d t}_{I_{5}}+\underbrace{\frac{1}{T} \int_{0}^{d_{6} T} i_{r}(t) d t}_{I_{6}}
\end{aligned}
$$


La integral $I_{5}$ es igual a:

$$
\begin{aligned}
I_{5}= & \frac{1}{T} \int_{0}^{d_{5} T} i_{r}(t) d t \\
= & I_{r, 4} d_{5}+\frac{1}{T} \int_{0}^{d_{2} T} \frac{\left(V_{g}+n V_{o}\right)}{L_{r}} t d t \\
= & {\left[I_{r, 2}+\frac{\left(V_{g}+n V_{o}-V_{c}\right)}{L_{r}} d_{3} T\right] d_{5}+\frac{\left(V_{g}+n V_{o}\right)}{L_{r}} \frac{d_{5}^{2} T}{2} } \\
= & {\left[I+\frac{\left(V_{g}+n V_{o}\right)}{Z_{e q}} \sin \left[\omega_{e q}\left(d_{2}+d_{6}\right) T\right]-\frac{V_{c}}{Z_{e q}} \sin \left(\omega_{e q} d_{2} T\right)\right] d_{5}+} \\
& +\frac{\left(V_{g}+n V_{o}-V_{c}\right)}{L_{r}} d_{3} T d_{5}+\frac{\left(V_{g}+n V_{o}\right)}{L_{r}} \frac{d_{5}^{2} T}{2}
\end{aligned}
$$

Empleando la igualdad (4.38), obtenida del cálculo de $d_{5} T$, la integral $I_{5}$ queda finalmente así:

$$
\begin{aligned}
I_{5}= & I d_{5}-\frac{T}{L_{r}}\left[\left(V_{g}+n V_{o}-V_{c}\right) d_{3}+\left(V_{g}+n V_{o}\right) d_{5}\right] d_{5} \\
& +\frac{\left(V_{g}+n V_{o}-V_{c}\right)}{L_{r}} d_{3} d_{5} T+\frac{\left(V_{g}+n V_{o}\right)}{L_{r}} \frac{d_{5}^{2} T}{2} \\
= & I d_{5}-\frac{\left(V_{g}+n V_{o}\right)}{L_{r}} \frac{d_{5}^{2} T}{2}
\end{aligned}
$$

La integral $I_{6}$ es igual a:

$$
\begin{aligned}
I_{6} & =\frac{1}{T} \int_{0}^{d_{6} T} i_{r}(t) d t \\
& =\frac{1}{T} \int_{0}^{d_{6} T} I+\frac{\left(V_{g}+n V_{o}\right)}{Z_{e q}} \sin \left(\omega_{e q} t\right) d t \\
& =I d_{6}-\frac{\left(V_{g}+n V_{o}\right)}{Z_{e q} w_{e q} T}\left[\cos \left(\omega_{e q} d_{6} T\right)-1\right]
\end{aligned}
$$

Sumando el resultado de las dos integrales se llega a la siguiente expresión:

$$
I_{g}=I\left(d_{5}+d_{6}\right)-\frac{\left(V_{g}+n V_{o}\right)}{L_{r}} \frac{d_{5}^{2} T}{2}-\frac{\left(V_{g}+n V_{o}\right)}{Z_{e q} w_{e q} T}\left[\cos \left(\omega_{e q} d_{6} T\right)-1\right]
$$

\section{CARACTERÍSTICA DE SALIDA.}

La expresión de la tensión de salida frente a la intensidad de salida se puede obtener a partir de la suma de (4.47) y (4.39), haciendo uso de la relación entre corrientes indicada en (4.41). De esta forma:

$$
\frac{I_{o}}{n}=\frac{\left(V_{g}+n V_{o}\right)}{2 L_{r} f}\left(d_{5}+d_{3}\right)^{2}-V_{g} \frac{d_{3}^{2}}{\left(d_{2}+d_{3}\right) 2 L_{r} f}
$$


donde se ha sustituido $V_{c}$ por su expresión, $V_{g} /\left(d_{2}+d_{3}\right)$ deducida anteriormente.

De esta última expresión se obtiene la relación entre corriente y tensión de salida. No ha sido posible obtener una expresión para los intervalos $d_{2} T$, $d_{3} T$ y $d_{5} T$, y por tanto, no se puede deducir una expresión analítica para la característica de salida de forma parecida las obtenida en los anexos anteriores.

En este caso, se propone un procedimiento para obtener la relación buscada entre tensión e intensidad, de forma numérica a partir de unas especificaciones dadas.

1. Se parte de $V_{g}, D$, y los elementos pasivos $L_{r}, C_{r}, C_{f}$ y $C_{t}$.

2. Se elige una tensión de salida $V_{o}$ para la que hay que obtener $I_{o}$.

3. Se obtienen $d_{2} \mathrm{y} d_{6}$ a partir del sistema de ecuaciones formado por (4.28) y (4.30), con la condición $d_{2} \leq\left(w_{e q} 2 T\right)^{-1}$.

4. Como $d_{3}=1-D-d_{2}$ y $d_{5}=D-d_{6}$, se calcula el valor de $I_{o}$ según (4.48).

5. Se repite este proceso para tantos puntos $\left(I_{o}, V_{o}\right)$ como se desee.

Como ejemplo de este procedimiento, se ha obtenido la característica de salida a partir de unas especificaciones de un flyback ZVS dadas en la Tabla 4.1. La curva obtenida está representada en la Figura 4.10.

\begin{tabular}{llll}
\hline \hline$V_{g}$ & $20 \mathrm{~V}$ & $C_{o}$ & $5 \mu \mathrm{F}$ \\
$P$ & $100 \mathrm{~W}$ & $C_{c}$ & $2,2 \mu \mathrm{F}$ \\
$V_{\text {out }}$ & $400 \mathrm{~V}$ & $L_{m}$ & $24 \mu \mathrm{H}$ \\
$f$ & $100 \mathrm{kHz}$ & $L_{r}$ & $2 \mu \mathrm{H}$ \\
$n_{1}: n_{2}$ & $1: 12$ & & \\
$t_{m}$ & $100 \mathrm{~ns}$ & $C_{r}$ & $1 \mathrm{nF}$ \\
$C t$ & $60 \mathrm{pF}$ & $C_{f}$ & $45 \mathrm{pF}$ \\
\hline
\end{tabular}

Tabla 4.1: Condiciones de trabajo de un flyback ZVS-real.

El ciclo de trabajo se ha calculado a partir de esas especificaciones, pero sin considerar los elementos parásitos, es decir, como si fuera un flyback ZVS ideal sin rizado, siendo $D=0,685$. También se ha realizado la simulación de un convertidor flyback ZVS real, en la que el valor de la bobina de magnetización se ha tomado muy grande, para conseguir que no haya rizado en dicha bobina. De esta forma, se puede emplear el mismo ciclo de trabajo que para los cálculos teóricos.

Los resultados representados ponen de manifiesto la validez del procedimiento planteado.

Si se introduce una variación del $5 \%$ en una de las capacidades parásitas, por ejemplo, $C_{t}$, la característica V-I de la salida será significativamente 


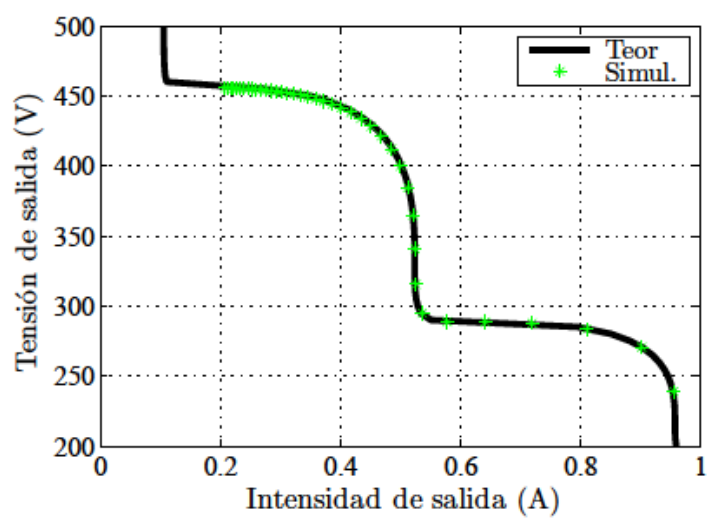

Figura 4.10: Característica de salida de un flyback ZVS-real.

diferente y su aplicación al reparto de carga de carga de dos etapas no será adecuada para un reparto equitativo. En la Figura 4.11 se ha representado la curva V-I cuando varía en un cinco por ciento la capacidad parásita $C_{t}$.

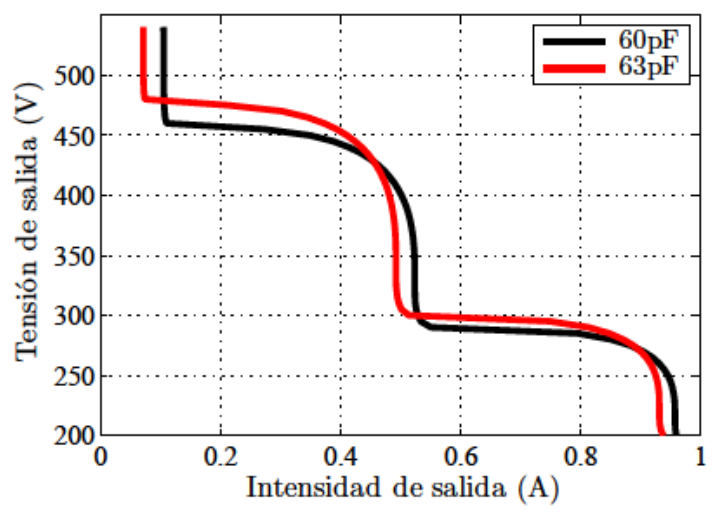

Figura 4.11: Característica de salida ante variaciones de $C_{t}$.

Como se puede observar, una pequeña variación en la capacidad parásita puede producir una gran diferencia en el reparto de corriente en los tramos de poca pendiente.

\subsection{Topología flyback ZVS modificada.}

La solución que se propone, con el fin de obtener un reparto mucho más equilibrado, consiste en colocar un diodo en el flyback ZVS-real, que fije la 
tensión en un punto de la malla en la aparece la resonancia. El convertidor resultante está representado en la Figura4.12. De ahora en adelante, se citará esta topología como flyback ZVS-modificado.

Como se ha visto anteriormente, la resonancia es debida a $L_{r}$ y a $C_{e q}$, luego, el nodo (A) es el que deberá fijarse a una tensión constante a través del diodo $D_{c l}$, donde se ha incluido su capacidad de unión, $C_{d}$. De esta forma, las capacidades parásitas estarán a tensión constante y se anulará la resonancia. Finalmente, en la apertura de $S_{1}$, la corriente resonante tendrá un valor similar incluso con diferentes capacidades parásitas.

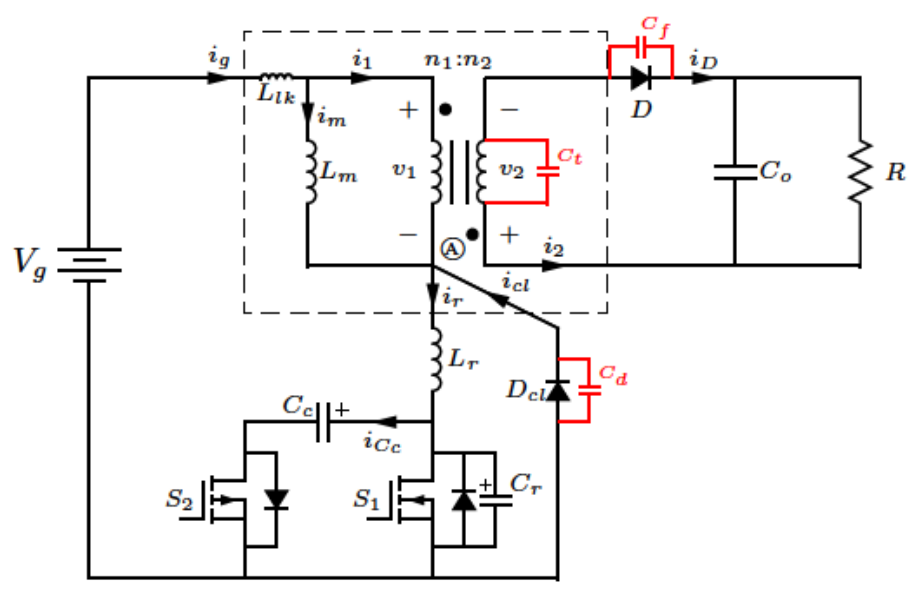

Figura 4.12: Convertidor flyback ZVS-real con diodo $D_{c l}$.

Desde el punto de vista del análisis del circuito, se han agrupado las capacidades $C_{d}, C_{t}$ y $C_{f}$ en un único condensador, $C_{c l}$ en paralelo con el nuevo diodo, y que incluye su propia capacidad $C_{d}$. Su valor es:

$$
C_{c l}=C_{d}+\left(\frac{n_{2}}{n_{1}}\right)^{2}\left(C_{t}+C_{f}\right)
$$

En las bobinas acopladas, se puede comprobar la corriente del diodo de salida, $i_{D}(t)$, está relacionada con la corriente de primario, $i_{1}(t)$ de la siguiente forma:

$$
n_{1} i_{1}(t)=n_{2} i_{2}(t)=-n_{2} i_{D}(t)
$$

donde se ha considerado el sentido positivo de $i_{1}(t)$ entrando por el punto de primario y de $i_{2}(t)$ saliente por el punto de secundario.

Considerando $i_{c l}(t)$ como la corriente que circula por la rama del diodo de clamp, se aplica la ley de nodos de Kirchhoff en (A):

$$
i_{m}(t)+i_{1}(t)=i_{r}(t)+i_{c l}(t)=i_{r}(t)+\left(i_{D_{c l}}(t)-i_{C_{c l}}(t)\right)
$$

Siendo $i_{m}(t)$ la corriente que circula por la bobina de magnetización, $i_{r}(t)$ la corriente circulante por la bobina resonante. Las intensidades en el diodo $D_{c}$ 
y en el condensador $C_{c l}$ se denotan como $i_{D_{c l}}(t)$ e $i_{C_{c l}}(t)$ respectivamente. Combinando las expresiones (F.2) y (F.3) se consigue:

$$
i_{m}(t)+i_{c l}(t)=i_{r}(t)+\frac{1}{n} i_{D}(t)
$$

siendo $n=n_{1} / n_{2}$.

Por otro lado la corriente de entrada, $i_{g}(t)$ cumple la relación (F.5):

$$
i_{g}(t)=i_{1}+i_{m}(t)
$$

que puede relacionarse con la corriente de salida $i_{D}$ de la siguiente forma:

$$
i_{g}(t)+\frac{1}{n} i_{D}(t)=i_{m}(t)
$$

También se puede relacionar con $i_{c l}(t)$ a partir de (F.3) como:

$$
i_{g}(t)+i_{c l}(t)=i_{r}(t)
$$

\subsubsection{Etapas.}

Esta topología evoluciona por ocho etapas a lo largo de un periodo de conmutación, y se describen a continuación. Se considera una bobina $L_{m}$ lo suficientemente grande como para suponer corriente constante, de valor $I$. De igual forma, el valor de los condensadores $C_{o}$ y $C_{c}$, es tal que la tensión en sus extremos se mantiene constante, de valores $V_{o}$ y $V_{c}$, durante todo el periodo $T$.

Intervalo $1\left[t_{0}-t_{1}\right]$ : Antes de $t_{0}$, el diodo de clamp $D_{c l}$ y el interruptor principal $S_{1}$ están conduciendo con corrientes $I_{D_{c l}}$ e $I+I_{D_{c l}}$ respectivamente, mientras que el interruptor auxiliar $S_{2}$ está abierto. En $t=t_{0}, S_{1}$ pasa a OFF y la corriente que está pasando por la bobina $L_{r}$ disminuye a la vez que carga el condensador $C_{r}$ de forma resonante. La corriente por $D_{c l}$ disminuye de la misma forma. Finaliza cuando el condensador alcanza la tensión $V_{c}$.

Intervalo $2\left[t_{1}-t_{2}\right]$ : El diodo $D_{2}$ empieza a conducir y la corriente en $L_{r}$ evoluciona linealmente porque queda sometida a una tensión constante de valor $-V_{c}$. La corriente de $D_{c l}$ disminuye linealmente hasta anularse en $t_{2}$ porque $i_{r}$ alcanza el valor $I$.

Intervalo $3\left[t_{2}-t_{3}\right]: D_{c l}$ deja de conducir e interviene su capacidad, $C_{c l}$. Se carga hasta $V_{g}+n V_{o}$ en $t_{3}$, cuando resuena con $L_{r}$. La tensión del primario es tal, que $D$ queda polarizado en directa y empieza a conducir.

Intervalo $4\left[t_{3}-t_{4}\right]$ : A partir de $t_{3}$, la corriente resonante evoluciona de forma lineal, sometida a una tensión constante de valor $V_{g}+n V_{o}-V_{c}$. En algún momento de este intervalo, y antes de que $i_{r}$ se haga negativa, se puede conmutar $S_{2}$ sin pérdidas porque $D_{2}$ está conduciendo. Este intervalo acaba en el instante en que $S_{2}$ pase a OFF. 
Intervalo $5\left[t_{4}-t_{5}\right]$ : La bobina resonante descarga la energía almacenada en $C_{r}$ hasta que su tensión es nula en $t_{5}$.

Intervalo $6\left[t_{5}-t_{6}\right]$ : Ahora la corriente de $L_{r}$ circula por el diodo $D_{1}$, lo cual hace posible la conmutación de $S_{1}$ a tensión nula. Ésta debe realizarse antes de que esta corriente cambie de polaridad. Durante este intervalo, la corriente del diodo $D$ disminuye linealmente hasta anularse en el mismo instante en que $i_{r}$ alcanza el valor de $I$ que es $t_{6}$. En este momento, $D$ deja de conducir.

Intervalo $7\left[t_{6}-t_{7}\right]$ : La capacidad $C_{c l}$ se descarga de forma resonante sobre $L_{r}$, lo que aumentan su corriente desde $I$ hasta un valor $I+I_{D_{c l}}$ cuando se ha descargado totalmente dicha capacidad.

Intervalo $8\left[t_{7}-t_{8}\right]$ : La corriente por $L_{r}$ es constante y este intervalo dura hasta que $S_{1}$ pasa a OFF al final del periodo.

En la Figura 4.13 se indican las ocho etapas por las que evoluciona el convertidor durante un ciclo completo.

Para analizar el funcionamiento del circuito con estas consideraciones se recurre a los circuitos equivalentes que están representados en la Figura 4.14.

Tras analizar todas las etapas se obtienen las expresiones de las principales magnitudes, indicadas a continuación, y que están representadas en la Figura 4.15. La duración de cualquiera de las ocho etapas está indicada con la letra $d$ y un subíndice que indica su número.

Para la tensión en $C_{r}$ y la intensidad en $L_{r}$ :

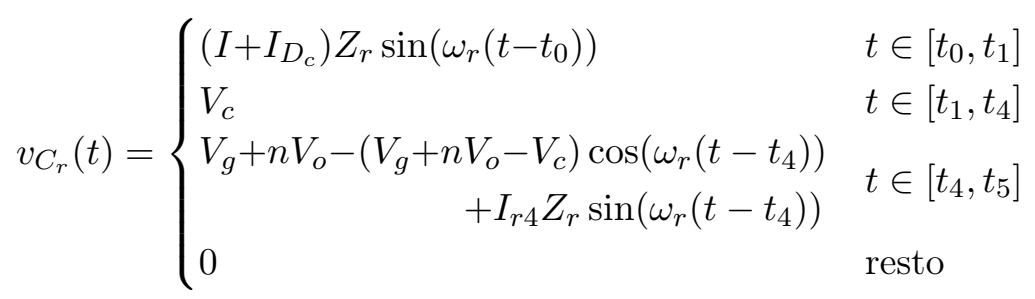




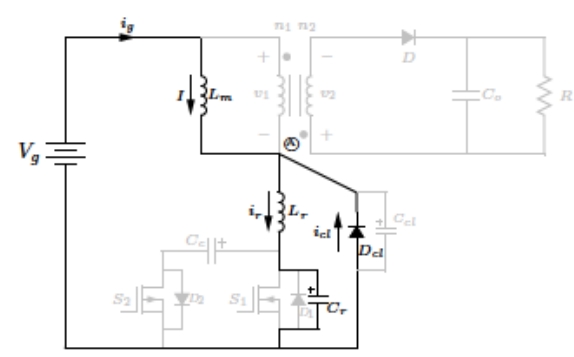

(a)

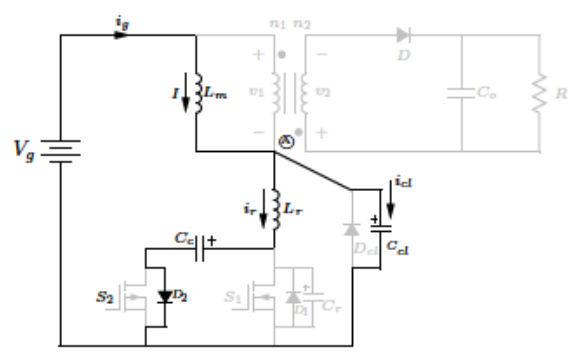

(c)

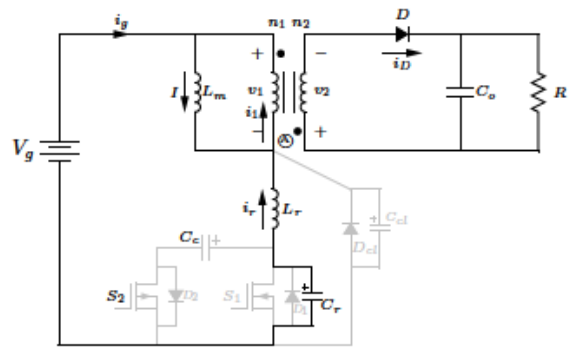

(e)

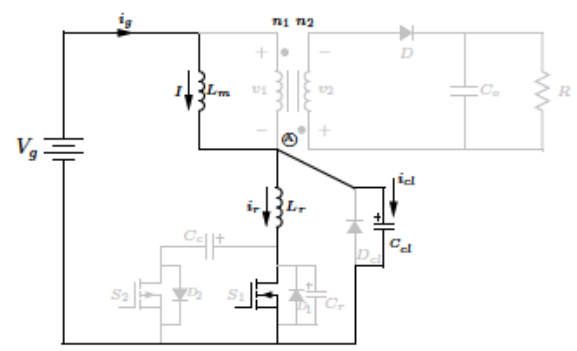

(g)

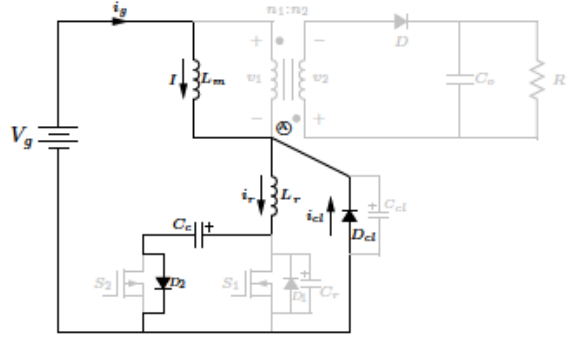

(b)

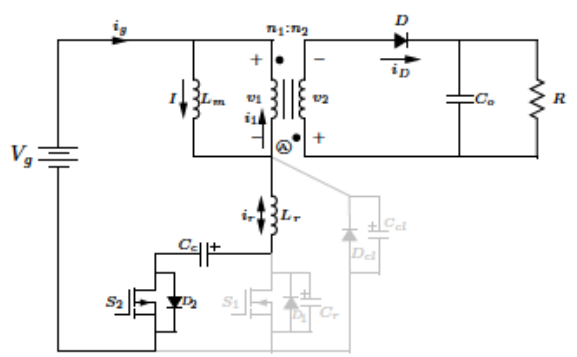

(d)

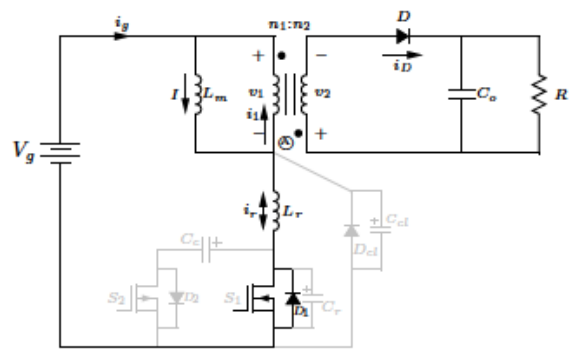

(f)

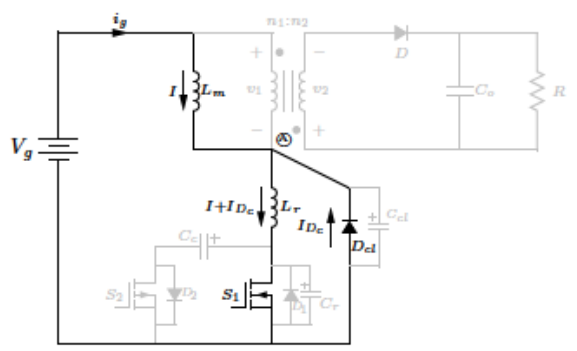

(h)

Figura 4.13: Etapas por las que evoluciona el flyback ZVS-modif. durante un ciclo: (a) Etapa 1. (b) Etapa 2. (c) Etapa 3. (d) Etapa 4. (e) Etapa 5. (f) Etapa 6. (g) Etapa 7. (h) Etapa 8. 


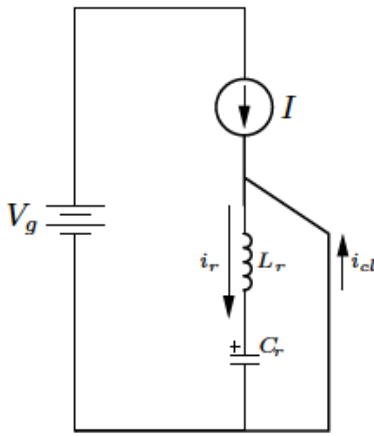

(a)

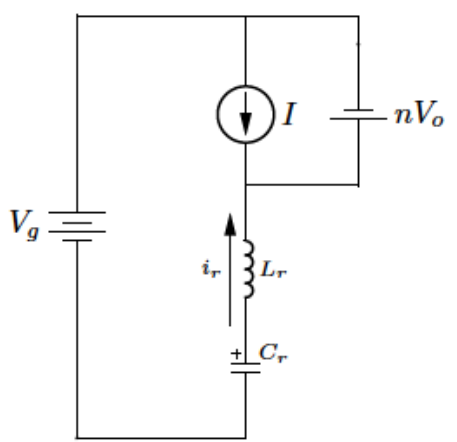

(e)

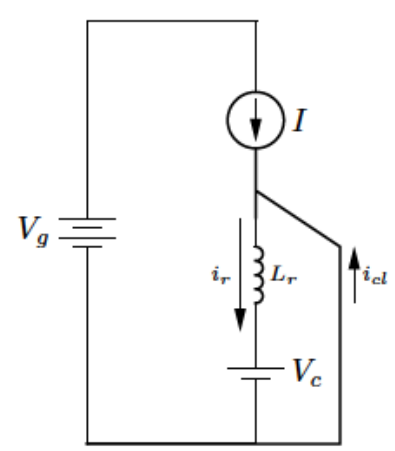

(b)

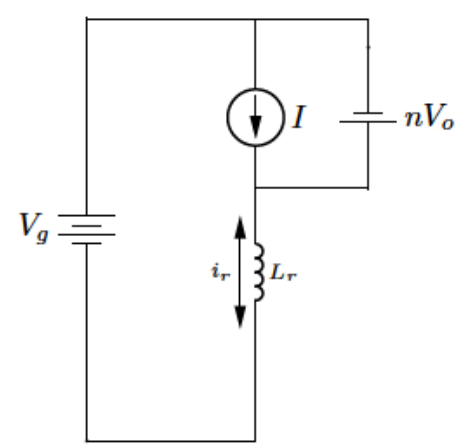

(f)

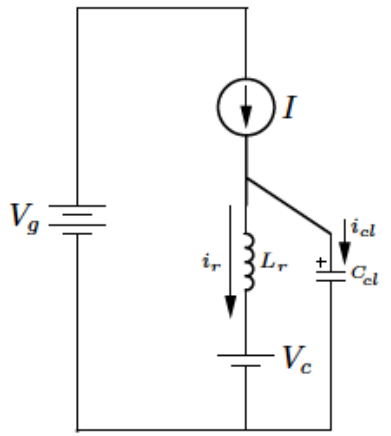

(c)

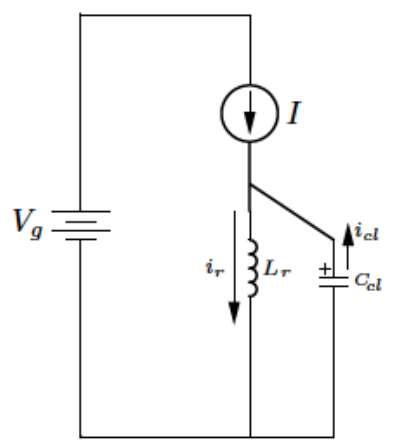

(g)

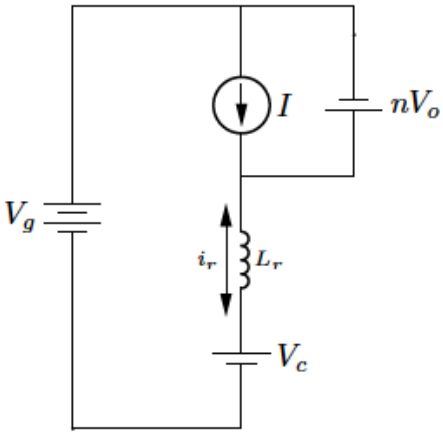

(d)

Figura 4.14: Circuitos empleados para el análisis del flyback ZVS-modif. durante un periodo completo: (a) Etapa 1. (b) Etapa 2. (c) Etapa 3. (d) Etapa 4. (e) Etapa 5. (f) Etapa 6. (g) Etapa 7. (h) Etapa 8.

$$
i_{r}(t)=\left\{\begin{array}{l}
\left(I+I_{D_{c}}\right) \cos \left(\omega_{r}(t-t 0)\right) \\
I_{r 1}-\frac{V_{c}}{L_{r}}\left(t-t_{1}\right) \\
I-\frac{V_{c}}{Z_{D_{c}}} \sin \left(\omega_{D_{c}}(t-t 2)\right) \\
I_{r 3}+\frac{\left(V_{g}+n V_{o}-V_{c}\right)}{L_{r}}\left(t-t_{3}\right) \\
I_{r 4} \cos \left(\omega_{r}\left(t-t_{4}\right)\right)+\frac{\left(V_{g}+n V_{o}-V_{c}\right)}{Z_{r}} \sin \left(\omega_{r}\left(t-t_{4}\right)\right) \\
I_{r 5}+\frac{\left(V_{g}+n V_{o}\right)}{L_{r}}\left(t-t_{5}\right) \\
I+\frac{\left(V_{g}+n V_{o}\right)}{Z_{D_{c}}} \sin \left(\omega_{D_{c}}\left(t-t_{6}\right)\right) \\
\left(I+I_{D_{c}}\right)
\end{array}\right.
$$$$
t \in\left[t_{0}, t_{1}\right]
$$$$
t \in\left[t_{1}, t_{2}\right]
$$$$
t \in\left[t_{2}, t 3\right]
$$$$
t \in\left[t_{3}, t_{4}\right]
$$$$
t \in\left[t_{4}, t_{5}\right]
$$$$
t \in\left[t_{5}, t_{6}\right]
$$$$
t \in\left[t_{6}, t_{7}\right]
$$$$
t \in\left[t_{7}, T_{8}\right]
$$ 
donde se han utilizado los siguientes términos:

$$
\begin{aligned}
I_{D_{c}} & =\frac{V_{g}+n V_{o}}{Z_{D_{c}}} \\
I_{r 1} & \equiv i_{r}\left(t_{1}\right)=\left(I+I_{D_{c}}\right) \cos \omega_{r} d_{1} T \\
I_{r 3} & \equiv i_{r}\left(t_{3}\right)=I-\frac{V_{c}}{Z_{D_{c}} \sin \omega_{D_{c}} d_{3} T} \\
I_{r 4} & \equiv i_{r}\left(t_{4}\right)=I_{r 3}+\frac{\left(V_{g}+n V_{o}-V_{c}\right)}{L_{r}} d_{4} T \\
I_{r 5} & \equiv i_{r}\left(t_{5}\right)=I_{r 4} \cos \omega_{r} d_{4} T+\frac{\left(V_{g}+n V_{o}-V_{c}\right)}{Z_{r}} \sin \omega_{r} d_{4} T \\
Z_{r} & =\sqrt{L_{r} / C_{r}} \quad Z_{D_{c}}=\sqrt{L_{r} / C_{c l}} \\
\omega_{r} & =1 / \sqrt{L_{r} C_{r}} \quad \omega_{D_{c}}=1 / \sqrt{L_{r} C_{c l}}
\end{aligned}
$$

La tensión en el condensador que agrupa a todas las capacidades parásitas, $v_{C_{c l}}(t)$ está indicada a continuación, mientras que la intensidad que circula por él, $i_{C_{c l}}(t)$, durante las etapas 3 y 7 es igual a $\left(I-i_{r}(t)\right)$. En el resto de etapas es nula.

$$
\begin{gathered}
v_{C_{c l}}(t)= \begin{cases}V_{c}-V_{c} \cos \left(\omega_{D_{c}}(t-t 2)\right) & t \in\left[t_{2}, t_{3}\right] \\
\left(V_{g}+n V_{o}\right) & t \in\left[t_{3}, t_{6}\right] \\
\left(V_{g}+n V_{o}\right) \cos \left(\omega_{D_{c}}\left(t-t_{6}\right)\right) & t \in\left[t_{6}, t_{7}\right] \\
0 & \text { resto }\end{cases} \\
i_{C_{c l}}= \begin{cases}\frac{V_{c}}{Z_{D_{c}} \sin \left(\omega_{D_{c}}(t-t 2)\right)} & t \in\left[t_{2}, t_{3}\right] \\
-\frac{\left(V_{g}+n V_{o}\right)}{Z_{D_{c}}} \sin \left(\omega_{D_{c}}\left(t-t_{6}\right)\right) & t \in\left[t_{6}, t_{7}\right] \\
0 & \text { resto }\end{cases}
\end{gathered}
$$

La tensión en el diodo $D_{c l}$ coincide con $-v_{C_{c l}}(t)$ mientras que su intensidad es igual a $i_{r}(t)-I$ durante las etapas 1 y 2 :

$$
i_{D_{c l}}= \begin{cases}\left(I+I_{D_{c}}\right) \cos \left(\omega_{r}(t-t 0)\right)-I & t \in\left[t_{0}, t_{1}\right] \\ I_{r 1}-\frac{V_{c}}{L_{r}}\left(t-t_{1}\right)-I & t \in\left[t_{1}, t_{2}\right] \\ I_{D_{c}} & t \in\left[t_{7}, t_{8}\right] \\ 0 & \text { resto }\end{cases}
$$

La intensidad en el condensador $C_{c}$ es igual a $i_{r}(t)$ durante las etapas, 2 , 


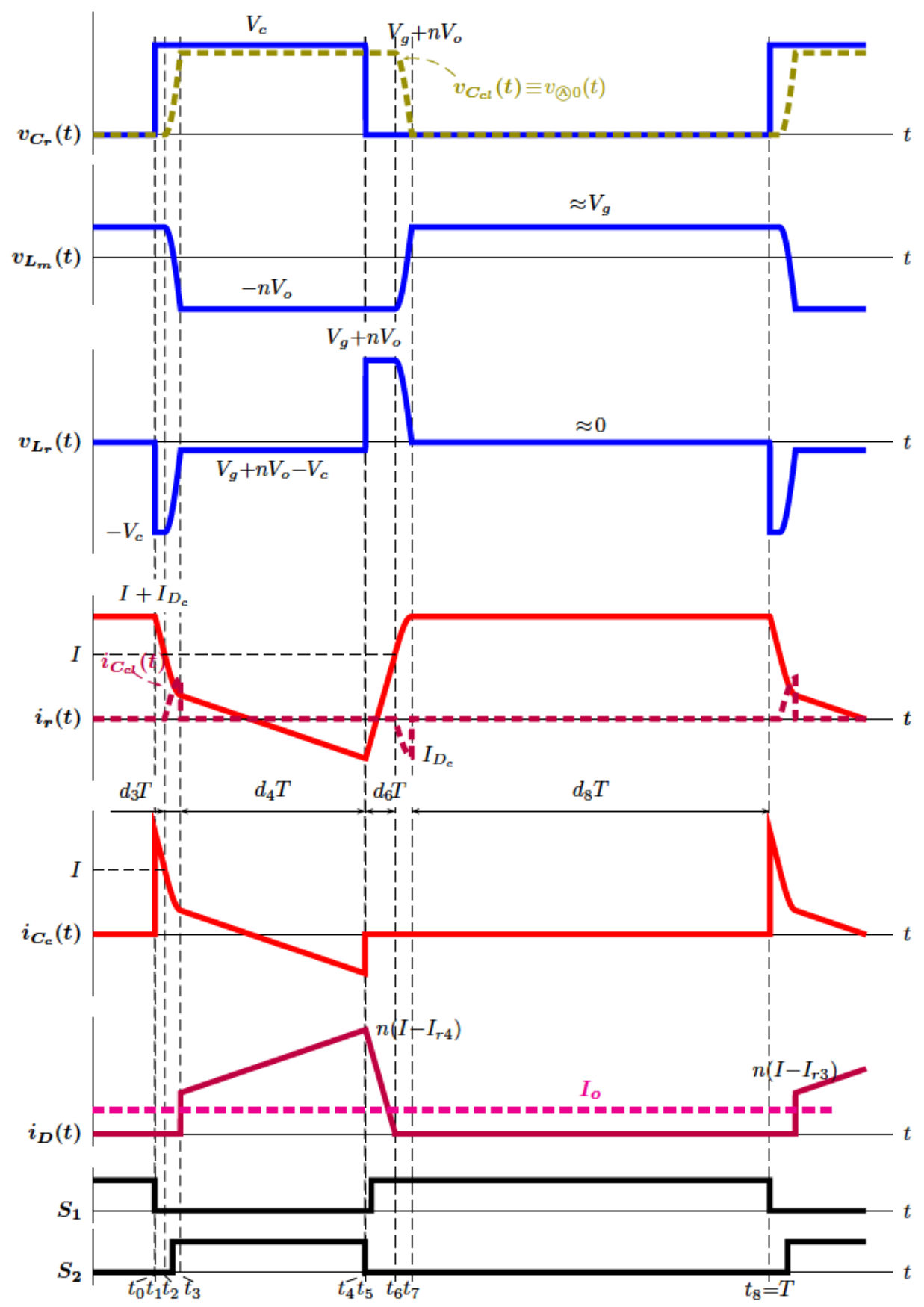

Figura 4.15: Curvas ideales para el flyback ZVS-modificado. 
3 y 4 . En el resto es nula.

$$
i_{C_{c}}(t)= \begin{cases}I_{r 1}-\frac{V_{c}}{L_{r}}\left(t-t_{1}\right) & t \in\left[t_{1}, t_{2}\right] \\ I-\frac{V_{c}}{Z_{D_{c}}} \sin \left(\omega_{D_{c}}\left(t-t_{2}\right)\right) & t \in\left[t_{2}, t 3\right] \\ I_{r 3}+\frac{\left(V_{g}+n V_{o}-V_{c}\right)}{L_{r}}\left(t-t_{3}\right) & t \in\left[t_{3}, t_{4}\right] \\ 0 & \text { resto }\end{cases}
$$

Las expresiones para la tensión en la bobina de magnetización y bobina resonante se calculan como $V_{g}-v_{C_{c l}}(t)$ y $v_{C_{c l}}(t)-v_{C_{r}}(t)$ respectivamente. Entonces:

$$
\begin{array}{r}
v_{L_{m}}(t)= \begin{cases}V_{g} & t \in\left[t_{0}, t_{2}\right] \\
V_{g}-V_{c}+V_{c} \cos \left(\omega_{D_{c}}\left(t-t_{2}\right)\right) & t \in\left[t_{2}, t_{3}\right] \\
-n V_{o} & t \in\left[t_{3}, t_{6}\right] \\
V_{g}-\left(V_{g}+n V_{o}\right) \cos \left(\omega_{D_{c}}\left(t-t_{6}\right)\right) & t \in\left[t_{6}, t_{7}\right] \\
V_{g} & t \in\left[t_{7}, T_{8}\right]\end{cases} \\
v_{L_{r}}(t)= \begin{cases}-\left(I+I_{D_{c}}\right) Z_{r} \sin \left(\omega_{r}(t-t 0)\right) & t \in\left[t_{0}, t_{1}\right] \\
-V_{c} & t \in\left[t_{1}, t_{2}\right] \\
-V_{c} \cos \left(\omega_{D_{c}}\left(t-t_{2}\right)\right) & t \in\left[t_{2}, t_{3}\right] \\
\left(V_{g}+n V_{o}-V_{c}\right) & t \in\left[t_{3}, t_{4}\right] \\
\left(V_{g}+n V_{o}-V_{c}\right) \cos \left(\omega_{r}\left(t-t_{4}\right)\right) & t \in\left[t_{4}, t_{5}\right] \\
-I_{r 4} Z_{r} \sin \left(\omega_{r}\left(t-t_{4}\right)\right) & t \in\left[t_{5}, t_{6}\right] \\
\left(V_{g}+n V_{o}\right) & t \in\left[t_{6}, t_{7}\right] \\
\left(V_{g}+n V_{o}\right) \cos \left(\omega_{D_{c}}\left(t-t_{6}\right)\right) & t \in\left[t_{7}, T_{8}\right] \\
0 & \end{cases}
\end{array}
$$

\subsubsection{Análisis en régimen permanente.}

El análisis en régimen permanente se basa en el balance voltios-segundo en las bobinas y de la carga neta en los condensadores, que es nulo. A partir de aquí se obtendrá las expresiones para la determinación de ciertos parámetros de funcionamiento del convertidor suponiendo unos valores iniciales de diseño. Se comprobará que la influencia de los elementos parásitos se ha minimizado.

Para realizar este análisis, se supone despreciable tanto la etapa 1 como la etapa 5 puesto que el intercambio resonante de energía del conjunto $L_{r}-C_{r}$ se realiza a una frecuencia mucho mas grande que la frecuencia de conmutación, y la carga-descarga de $C_{r}$ será prácticamente instantánea. Por tanto, $d_{1} \approx 0 \mathrm{y}$ 
$d_{5} \approx 0$, empleando únicamente seis etapas. Las expresiones de las principales magnitudes están relacionadas a continuación y su obtención se encuentra detallada en el Anexo F.

$$
\begin{aligned}
& V_{c}=\frac{V_{g}}{d_{2}+d_{3}+d_{4}}=\frac{V_{g}}{(1-D)} \\
& I_{D_{c}}=\frac{\left(V_{g}+n V_{o}\right)}{Z_{D_{c}}} \\
& d_{2} T=\frac{I_{D_{c}}}{V_{c}} L_{r}=\frac{\left(V_{g}+n V_{o}\right)}{Z_{D_{c}} V_{c}} L_{r} \\
& \cos w_{D_{c}} d_{3} T=1-\frac{V_{g}+n V_{o}}{V_{c}} \\
& \frac{V_{c}}{Z_{D_{c}}} \sin \omega_{D_{c}} d_{3} T=\frac{V_{g}+n V_{o}-V_{c}}{L_{r}} d_{4} T+\frac{V_{g}+n V_{o}}{L_{r}} d_{6} T \\
& d_{7} T=\frac{\pi}{2 T \omega_{D_{c}}}
\end{aligned}
$$

También hay que considerar las relaciones:

$$
\begin{aligned}
& \left(d_{2}+d_{3}+d_{4}\right) T=(1-D) T \\
& \left(d_{6}+d_{7}+d_{8}\right) T=D T \\
& I(1-D)=-\frac{V_{c}}{L_{r}} \frac{d_{2}^{2} T}{2}+\frac{V_{g}+n V_{o}}{\omega_{D_{c}} Z_{D_{c}} T}+d_{4} \frac{V_{c}}{Z_{D_{c}}} \sin \omega_{D_{c}} d_{3} T-\frac{\left(V_{g}+n V_{o}-V_{c}\right)}{L_{r}} \frac{d_{4}^{2} T}{2} \\
& I=I_{o}\left(\frac{V_{o}}{V_{g}}+\frac{1}{n}\right)
\end{aligned}
$$

\section{CARACTERÍSTICA DE SALIDA}

La expresión que relaciona la tensión de salida con la intensidad en la salida se obtiene combinando las dos últimas expresiones de (4.66), de forma que:

$I_{o}=\frac{n V_{g}\left[-\frac{V_{c}}{L_{r}} \frac{d_{2}^{2} T}{2}+\frac{V_{g}+n V_{o}}{\omega_{D_{c}} Z_{D_{c}} T}+d_{4} \frac{V_{c}}{Z_{D_{c}}} \sin \omega_{D_{c}} d_{3} T-\frac{\left(V_{g}+n V_{o}-V_{c}\right)}{L_{r}} \frac{d_{4}^{2} T}{2}\right]}{(1-D)\left(V_{g}+n V_{o}\right)}$

En esta expresión es necesario obtener la duración de los intervalos, $d_{2}$, $d_{3}$ y $d_{4}$ que a su vez dependen del punto de funcionamiento del convertidor, es decir, de $V_{g}, V_{o}$ y $D$, por lo que conseguir despejar $V_{o}$ no es sencillo.

En este caso, se propone un procedimiento parecido al empleado para el flyback ZVS con capacidades parásitas, pero que pasa por calcular previamente el ciclo de trabajo $D$.

1. Se parte del punto de funcionamiento dado por $V_{g}, V_{o}$, e $I_{o}$ y los elementos pasivos $L_{r}, C_{r}, C_{f}, C_{t}$ y $C_{d}$. 
2. Se expresan, $V_{c}, d_{2}, d_{3}$ como funciones de $D$ empleando sus expresiones dadas en (4.65). Junto con $d_{4}$ calculado como[(1-D)- $\left.d_{2}-d_{3}\right]$, se sustituyen en (4.67).

3. Se resuelve la ecuación resultante por algún método conocido para obtener el ciclo de trabajo, que debe estar en el intervalo $[0,1]$.

4. Considerando el ciclo de trabajo constante, se toma un valor distinto para $V_{o}$ para el que se desea obtener la intensidad de salida, $I_{o}$.

5. Se recalcula $d_{2}, d_{3}$ y $d_{4}$ con el nuevo valor de $V_{o}$ y se obtiene el valor de intensidad deseado a partir de (4.67)

6. Se repite los dos pasos anteriores para tantos puntos $\left(I_{o}, V_{o}\right)$ como se desee.

Como ejemplo de este procedimiento, se ha obtenido la característica de salida a partir de las mismas especificaciones de un flyback ZVS del apartado anterior, al que se incorpora el diodo de clamp,con una capacidad del idéntico valor que $C_{f}$. Estos valores están dados en la Tabla 4.2. La curva obtenida está representada en la Figura 4.16.

\begin{tabular}{llll}
\hline \hline$V_{g}$ & $20 \mathrm{~V}$ & $C_{o}$ & $5 \mu \mathrm{F}$ \\
$P$ & $100 \mathrm{~W}$ & $C_{c}$ & $2,2 \mu \mathrm{F}$ \\
$V_{\text {out }}$ & $400 \mathrm{~V}$ & $L_{m}$ & $24 \mu \mathrm{H}$ \\
$f$ & $100 \mathrm{kHz}$ & $L_{r}$ & $2 \mu \mathrm{H}$ \\
$n_{1}: n_{2}$ & $1: 12$ & & \\
$t_{m}$ & $100 \mathrm{~ns}$ & $C_{r}$ & $0,1 \mathrm{nF}$ \\
$C t$ & $60 \mathrm{pF}$ & $C_{d}=C_{f}$ & $45 \mathrm{pF}$ \\
\hline
\end{tabular}

Tabla 4.2: Condiciones de trabajo de un flyback ZVS-modificado.

El ciclo de trabajo se ha obtenido según el procedimiento indicado empleando un método numérico para obtener el ciclo de trabajo. El valor obtenido es $D=0,658$. Con ese ciclo de trabajo se ha simulado un convertidor flyback ZVS-modificado, donde se ha empleado un valor de bobina de magnetización lo suficientemente grande como para que su rizado de corriente sea despreciable, y así los resultados simulados puedan ser comparables con los analíticos. Manteniendo constante el ciclo de trabajo se mide la tensión y la intensidad a la salida para diferentes cargas.

La curva obtenida está representada en la Figura 4.16, donde se comprueba la similitud de los resultados obtenidos de forma teórica y en simulación, y permite validar el procedimiento presentado.

La característica de salida de la topología modificada ya no presenta zonas con tramos horizontales como los que aparecían para la topología que no incluía el diodo. La presencia del diodo $D_{c}$ minimiza la distorsión que introducen las capacidades parásitas sobre la curva $I_{o}-V_{o}$. 


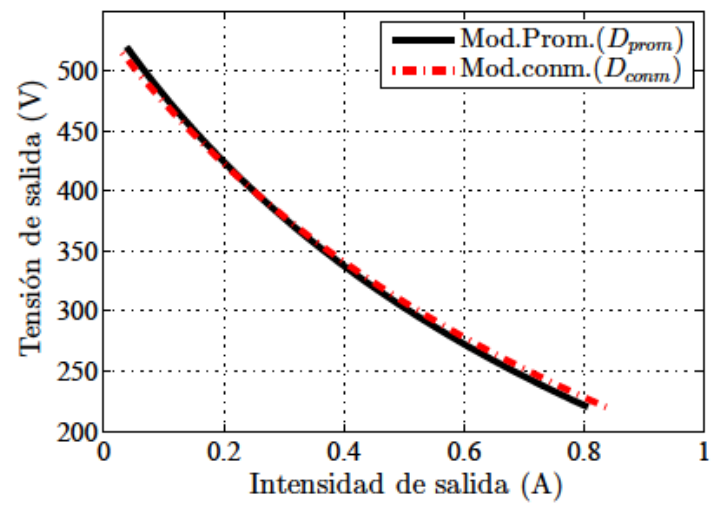

Figura 4.16: Característica de salida de un flyback ZVS-modificado.

Si se introduce una variación de alguna de las capacidades parásitas, por ejemplo, $C_{t}$, la característica V-I de la salida tampoco cambiará significativamente. En la Figura 4.11 se ha representado la curva V-I considerando una variación en torno al $50 \%$ para la capacidad parásita $C_{t}$.

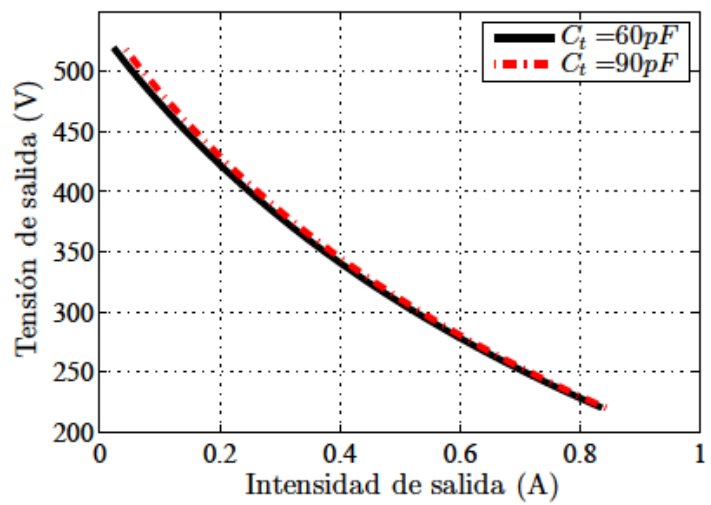

Figura 4.17: Característica de salida de un flyback ZVS-modificado para diferentes valores de $C_{t}$.

Se observa que las curvas V-I son muy similares, lo que indica que el reparto ante variaciones de las capacidades parásitas apenas se verá alterado, por lo que el reparto de carga de carga de dos etapas será muy próximo a un reparto equitativo. 


\subsubsection{Modelo promediado.}

Para hacer el modelo promediado se toman como variables de estado las intensidades en bobinas y las tensiones en condensadores. En concreto, las intensidades $i_{r}$ e $i_{m}$ en las bobinas resonante $L_{r}$ y de magnetización $L_{m}$ respectivamente, y las tensiones $v_{C_{r}}, v_{C_{c}}, v_{C_{o}}$ y $v_{C_{c l}}$ en los condensadores resonante, $C_{r}$, de clamp $C_{c}$, de salida $C_{o}$ y el del diodo del clamp $C_{c l}$.

Se desprecian los tiempos muertos entre las conmutaciones de $S_{1}$ y $S_{2}$ y los intervalos resonantes, y se supone que el condensador de clamp mantiene su tensión constante. Después de estas simplificaciones, desaparecerán las etapas 1 y 5 y los tiempos muertos, de forma que un periodo se puede escribir como:

$$
T \approx d_{2} T+d_{3} T+d_{4} T+d_{6} T+d_{7} T+d_{8} T
$$

El análisis realizado para obtener el modelo promediado se ha incluido en el Anexo F. En este análisis se han agrupado determinadas expresiones incluidas dentro de las ecuaciones de estado, que funcionan como fuentes de corriente equivalentes, denotadas como $i_{e q, x}$. Otra expresión equivale a una resistencia equivalente sin pérdidas, denominada $r_{e q}$. La relación completa está en (4.69).

$$
\begin{aligned}
r_{e q} & =\frac{2 L_{r} f_{s}}{d_{4}^{2}} \\
i_{e q} & =\frac{\left(\bar{v}_{g}+n \bar{v}_{C_{o}}\right)}{2 L_{r} f_{s}} d_{6}^{2} \\
i_{e q, 2} & =\frac{\bar{v}_{C_{c}}}{L_{r}} \frac{d_{2}^{2} T}{2} \\
i_{e q, 3} & =\frac{\bar{v}_{C_{c}}}{Z_{D_{c}} \omega_{D_{c}} T}\left[\cos \left(\omega_{D_{c}} d_{3} T\right)-1\right] \\
i_{e q, 4} & =\frac{\bar{v}_{C_{c}}}{Z_{D_{c}}} \sin \left(\omega_{D_{c}} d_{3} T\right) d_{4}
\end{aligned}
$$

En estos términos agrupados se incluyen ciertos parámetros que están relacionados con las variables e estado y los elementos pasivos del circuito, y son:

$$
\begin{aligned}
& d_{2} T=\frac{\left(\bar{v}_{g}+n \bar{v}_{C_{o}}\right)}{\bar{v}_{C_{c}} \omega_{D_{c}}} \\
& \cos \left(\omega_{D c} d_{3} T\right)=\frac{\bar{v}_{C_{c}}-\bar{v}_{g}-n \bar{v}_{C_{o}}}{\bar{v}_{C_{c}}} \\
& d_{4} T=(1-d) T-d_{2} T-d_{3} T \\
& \frac{\bar{v}_{C_{c}}}{Z_{D c}} \sin \left(\omega_{D_{c}} d_{3} T\right)=\frac{\left(\bar{v}_{g}+n \bar{v}_{C_{o}}-\bar{v}_{C_{c}}\right)}{L_{r}} d_{4} T+\frac{\bar{v}_{g}+n \bar{v}_{C_{o}}}{L_{r}} d_{6} T \\
& d_{7} T=\frac{\pi}{2 \omega_{D_{c}}} \quad d_{8} T=d T-d_{6} T-d_{7} T
\end{aligned}
$$


Con todos estos términos,las ecuaciones de estado promediadas quedan descritas en las expresiones (4.71) a (4.75), y el circuito que se consigue a partir de ellas está representado en la Figura 4.18.

$$
\begin{gathered}
L_{m} \frac{d \bar{i}_{m}}{d t}=\bar{v}_{g}-\bar{v}_{C_{c}}(1-d) \\
C_{c} \frac{d \bar{v}_{C_{c}}}{d t}=\bar{i}_{m}(1-d)+i_{e q, 2}+i_{e q, 3}-i_{e q, 4}-\frac{\bar{v}_{C_{c}}-\left(\bar{v}_{g}+n \bar{v}_{C_{o}}\right)}{r_{e q}} \\
C_{o} \frac{d \bar{v}_{C_{o}}}{d t}=n\left[i_{e q, 4}+\frac{\bar{v}_{C_{c}}-\left(\bar{v}_{g}+n \bar{v}_{C_{o}}\right)}{r_{e q}}+i_{e q}\right]-\frac{\bar{v}_{C_{o}}}{R} \\
\bar{i}_{g}=\bar{i}_{m}-i_{e q, 4}-\frac{\bar{v}_{C_{c}}-\left(\bar{v}_{g}+n \bar{v}_{C_{o}}\right)}{r_{e q}}-i_{e q} \\
\bar{i}_{D}=n\left[i_{e q, 4}+\frac{\bar{v}_{C_{c}}-\left(\bar{v}_{g}+n \bar{v}_{C_{o}}\right)}{r_{e q}}+i_{e q}\right]
\end{gathered}
$$

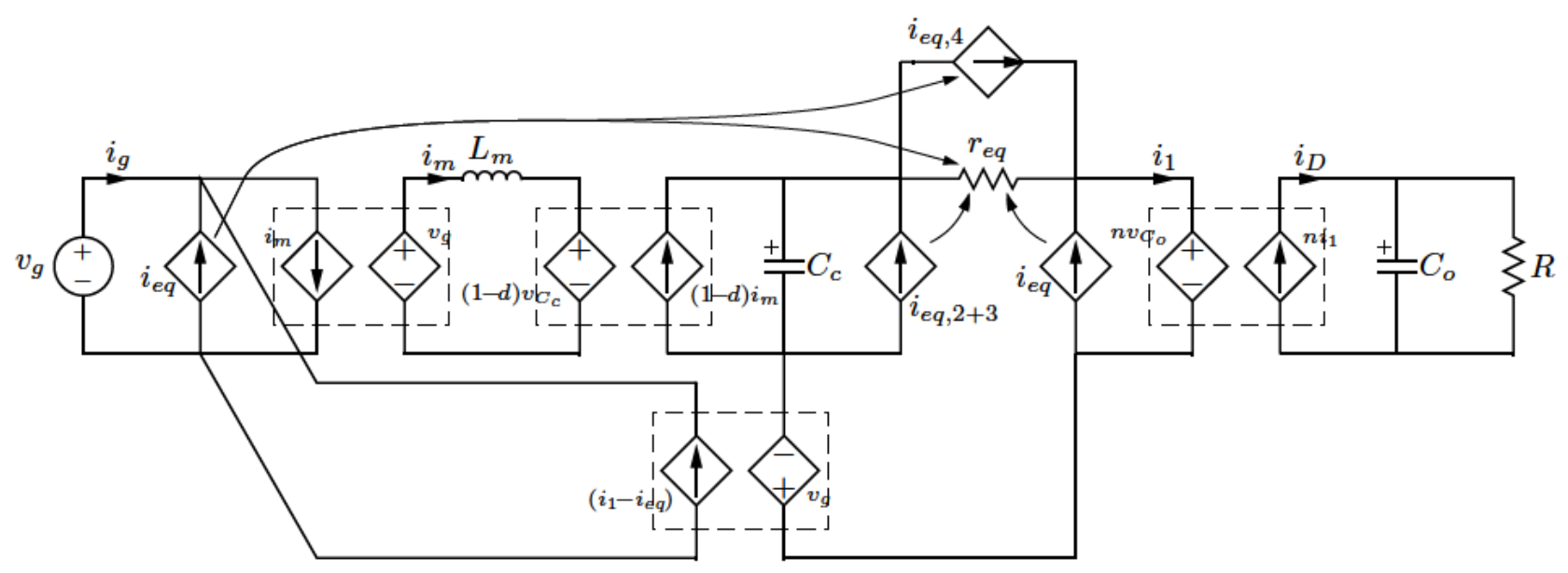

(a)

Figura 4.18: Circuito promediado del flyback ZVS-modificado.

La Figura 4.19 muestra la validez del modelo comparando los resultados de la simulación cuando se produce un cambio en el ciclo de trabajo. Durante el transitorio, el comportamiento de ambas simulaciones también es muy parecida.

\subsubsection{Modelo en régimen permanente}

El modelo en régimen permanente se puede obtener a partir del modelo promediado sustituyendo los valores promedio por valores constantes (por 


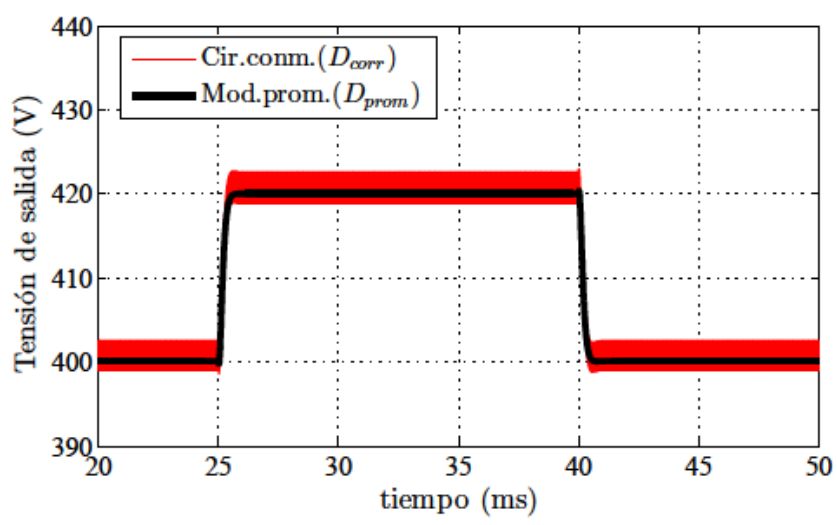

Figura 4.19: Simulación de la respuesta a un escalón en el ciclo de trabajo de un flyback ZVS-modif. y su modelo promediado.

ejemplo, $\bar{i}_{m}$ se sustituye por $I$ ). De esta forma, las ecuaciones del modelo en régimen permanente serán las indicadas en (F.160), en las que nótese que hay ecuaciones con un cero a la izquierda de la igualdad, porque la derivada de una constante es nula.

$$
\begin{aligned}
0 & =V_{g}-(1-D) V_{c} \\
0 & =n I_{e q, 4}+n \frac{V_{c}-\left(V_{g}+n V_{o}\right)}{R_{e q}}+n I_{e q}-\frac{V_{o}}{R} \\
0 & =I(1-D)+I_{e q, 2}+I_{e q, 3}-I_{e q, 4}-\frac{V_{c}-\left(V_{g}+n V_{o}\right)}{R_{e q}} \\
I_{g} & =I-I_{e q, 4}-\frac{V_{c}-\left(V_{g}+n V_{o}\right)}{R_{e q}}-I_{e q} \\
I_{D} & =n\left[I_{e q, 4}+\frac{V_{c}-\left(V_{g}+n V_{o}\right)}{R_{e q}}+I_{e q}\right]
\end{aligned}
$$

donde

$$
\begin{aligned}
R_{e q} & =\frac{2 L_{r} f_{s}}{D_{4}^{2}} \\
I_{e q} & =\frac{\left(V_{g}+n V_{o}\right)}{2 L_{r} f_{s}} D_{6}^{2} \\
I_{e q, 2} & =\frac{V_{c}}{L_{r}} \frac{D_{2}^{2} T}{2} \\
I_{e q, 3} & =\frac{V_{c}}{Z_{D_{c}} \omega_{D_{c}} T}\left[\cos \left(\omega_{D_{c}} D_{3} T\right)-1\right] \\
I_{e q, 4} & =\frac{V_{c}}{Z_{D_{c}}} D_{4} \sin \left(\omega_{D_{c}} D_{3} T\right)
\end{aligned}
$$


y:

$$
\begin{aligned}
& D_{2} T=\frac{\left(V_{g}+n V_{o}\right)}{V_{c} \omega_{D_{c}}} \\
& \cos \left(\omega_{D c} D_{3} T\right)=\frac{V_{c}-V_{g}-n V_{o}}{V_{c}} \\
& D_{4} T=(1-D) T-D_{2} T-D_{3} T \\
& \frac{V_{c}}{Z_{D c}} \sin \left(\omega_{D_{c}} D_{3} T\right)=\frac{\left(V_{g}+n V_{o}-V_{c}\right)}{L_{r}} D_{4} T+\frac{V_{g}+n V_{o}}{L_{r}} D_{6} T \\
& D_{7} T=\frac{\pi}{2 \omega_{D_{c}}} \\
& D_{8} T=D T-D_{6} T-D_{7} T
\end{aligned}
$$

A estas ecuaciones se puede llegar también a partir del circuito promediado de la Figura 4.18. Basta con sustituir los condensadores por un circuito abierto y las bobinas con un cortocircuito. De esta forma se obtienen los circuitos de la Figura 4.20.

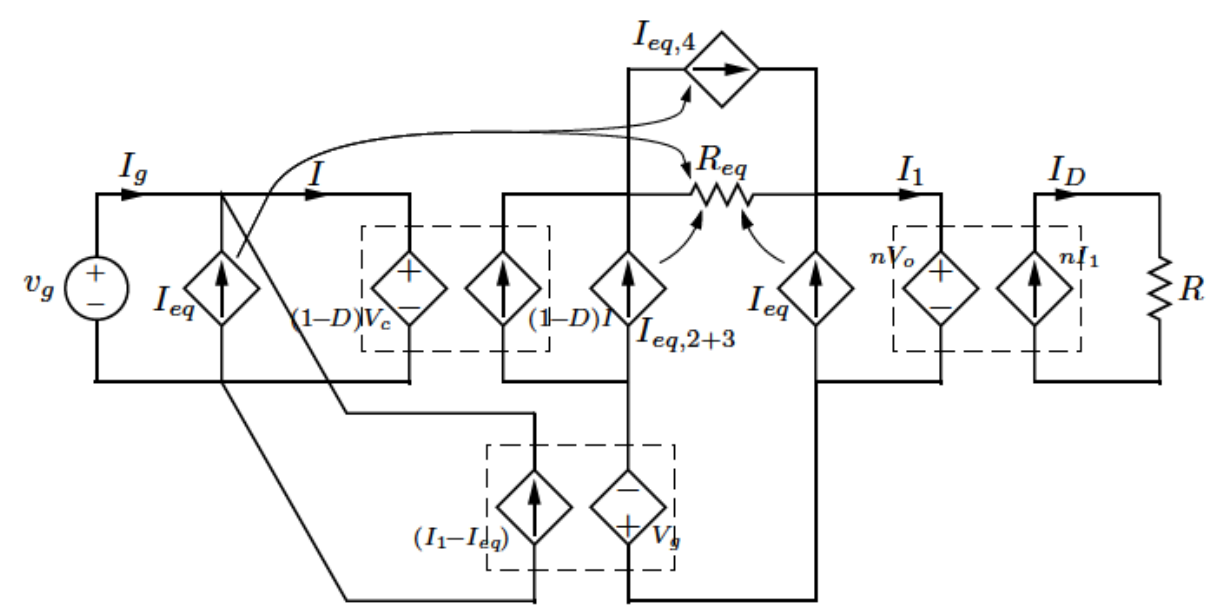

(a)

Figura 4.20: Circuito en régimen permanente de un flyback ZVS-modificado.

Se obtienen las mismas expresiones para las principales magnitudes en régimen permanente cuando se deducen de (F.160) que a partir de los circuitos de la Figura 4.20. El término $V_{o} / R$ es la intensidad a la salida y coincide 
con la intensidad del diodo $I_{D}$.

$$
\begin{aligned}
V_{c} & =\frac{V_{g}}{(1-D)} \\
\frac{V_{o}}{R} & =n I_{e q, 4}+n \frac{V_{c}-\left(V_{g}+n V_{o}\right)}{R_{e q}}+n I_{e q} \equiv I_{o} \\
V_{o} & =\frac{1}{n}\left(V_{c}-V_{g}-(1-D) I R_{e q}\right)-\frac{1}{n}\left[I_{e q, 2}+I_{e q, 3}-I_{e q, 4}\right] R_{e q} \\
I_{g} & =I-I_{e q, 4}-\frac{V_{c}-\left(V_{g}+n V_{o}\right)}{R_{e q}}-I_{e q} \\
I_{D} & =n\left[I_{e q, 4}+\frac{V_{c}-\left(V_{g}+n V_{o}\right)}{R_{e q}}+I_{e q}\right] \equiv I_{o}
\end{aligned}
$$

La expresión que relaciona la tensión de salida con la intensidad en la carga se obtiene fácilmente a partir de la tercera expresión de (F.163) donde se sustituye $V_{c}$.

$$
V_{o}=\frac{1}{n} \frac{V_{g} D}{1-D}-\frac{1}{n}\left[(1-D) I+I_{e q, 2}+I_{e q, 3}-I_{e q, 4}\right] R_{e q}
$$

Se puede observar que la tensión de salida de flyback ZVS modificado se asimila a la de un flyback convencional que tiene a la salida una resistencia de valor $R_{e q} / n^{2}$ (con la particularidad de que es una resistencia equivalente sin pérdidas), y depende con la corriente demandada por la carga (que en un flyback convencional es igual a $n I(1-D))$ y de otros términos que funcionan como fuentes de corriente equivalentes.

La relación entre intensidades de entrada y de salida y en la bobina se obtiene empleando la cuarta y la quinta expresión de (F.163), sabiendo que $I_{D}=I_{o}$. Entonces:

$$
I_{g}+\frac{I_{o}}{n}=I
$$

Por último, empleando la última ecuación se puede conseguir la intensidad de salida en función de la tensión de salida, esto es, la característica de salida. Se indica en (4.82):

$$
I_{o}=\frac{n V_{g}\left[\frac{V_{c}-\left(V_{g}+n V_{o}\right)}{2 L_{r}} d_{4}^{2} T-I_{e q, 2}-I_{e q, 3}+I_{e q, 4}\right]}{(1-d)\left(V_{g}+n V_{o}\right)}
$$

Esta expresión es equivalente a la indicada en (4.67), que es también la característica de salida que se obtiene al realizar el análisis en régimen permanente. 


\subsection{Prototipo y validación experimental}

Se ha construido un prototipo de $200 \mathrm{~W}$, para elevar lat tensión desde $20 \mathrm{~V}$ hasta un máximo de $400 \mathrm{~V}$, formado por dos etapas flyback ZVS conectadas en paralelo, que gestionan hasta $100 \mathrm{~W}$ cada una de ellas. El diseño del circuito impreso es diferente para cada etapa, ni se han seleccionado los componentes buscando los más parecidos entre sí. Los principales elementos están indicados en la Tabla 4.3.

\begin{tabular}{c|c|c}
\hline \hline Componentes & Valor $/$ Referencia & Descripción \\
\hline \hline MOSFET $S_{1}$ & IRFS4321 & canal N $150 \mathrm{~V}, 11,7 \mathrm{~m} \Omega @ V_{G S}=10 \mathrm{~V}$ \\
MOSFET $S_{2}$ & IRF6218S & canal P $-150 \mathrm{~V}, 150 \mathrm{~m} \Omega$ @ $V_{G S}=-10 \mathrm{~V}$ \\
Driver MOSFETs & MCP14E4 & $4.0 \mathrm{~A}$ Dual H-Speed MOSFET Driver \\
Diodo $D$ & C4D05120E & SiC Schottky, 1200 V, 9 A \\
Diodo clamp $D_{c l}$ & MUR420 & Ultrarrápido, 200 V, 4A \\
bobina flyback & Coilcraft KA-4823CL & $28 \mu \mathrm{H}, 8 \mathrm{~m} \Omega \mathrm{DCR}, 1: 12$ \\
Bobina resonante $L_{r}$ & Coilcraft SER2010-102L & $1 \mu \mathrm{H}, 0,9 \mathrm{~m} \Omega \mathrm{DCR}$ \\
Condensador clamp $C_{c}$ & Coilcraft SER2010-202L & $2 \mu \mathrm{H}, 0,9 \mathrm{~m} \Omega \mathrm{DCR}$ \\
Condensador salida $C_{o}$ & $2,2 \mu \mathrm{F}$ & $250 \mathrm{~V}$ \\
condensador entrada $C_{i n}$ & $0,1 \mu \mathrm{F}$ & $630 \mathrm{~V}$ \\
\hline \hline
\end{tabular}

Tabla 4.3: Principales componentes empleados en el prototipo.

Las capacidades parásitas consideradas son de $C_{f}=27 \mathrm{pF}, C_{d}=37 \mathrm{pF}$ y $C_{t}=48,5 \mathrm{pF}$ para el diodo a la salida, el diodo de clamp y para las bobinas acopladas, respectivamente.

El prototipo desarrollado incluye dos etapas del convertidor flyback ZVSmodificado (véase la Figura 4.12), conectadas en paralelo como está indicada en la 4.21. En la Figura 4.22 se incluye una fotografía del mismo.

En la zona derecha de la imagen se encuentran las dos etapas flyback ZVS conectadas en paralelo. El elemento que mas destaca por su tamaño es el conjunto de bobinas acopladas de cada etapa. En la zona izquierda, dedicada a los elementos de control del convertidor se ha dispuesto la placa microcontroladora, que genera los disparos con una frecuencia de conmutación de $100 \mathrm{kHz}$, teniendo en cuenta los tiempos muertos necesarios con el fin de facilitar las conmutaciones suaves. Es un microcontrolador TMSF27027 de Texas Instruments.

En la Figura 4.23 se observa la resonancia con las capacidades parásitas tanto en la intensidad en la bobina resonante como en el transistor principal de una de las etapas del flyback ZVS. En este caso se han tomado las medidas antes de conectar el diodo de clamp. A la derecha se han registrado las mismas magnitudes cuando se emplea el diodo de clamp. Claramente el diodo atenúa las resonancias mencionadas.

Para comprobar el efecto de las resonancias sobre la tensión de salida de una etapa flyback ZVS, se ha registrado su tensión de salida a medida que varía la carga demandada, manteniendo el resto de parámetros constantes. 


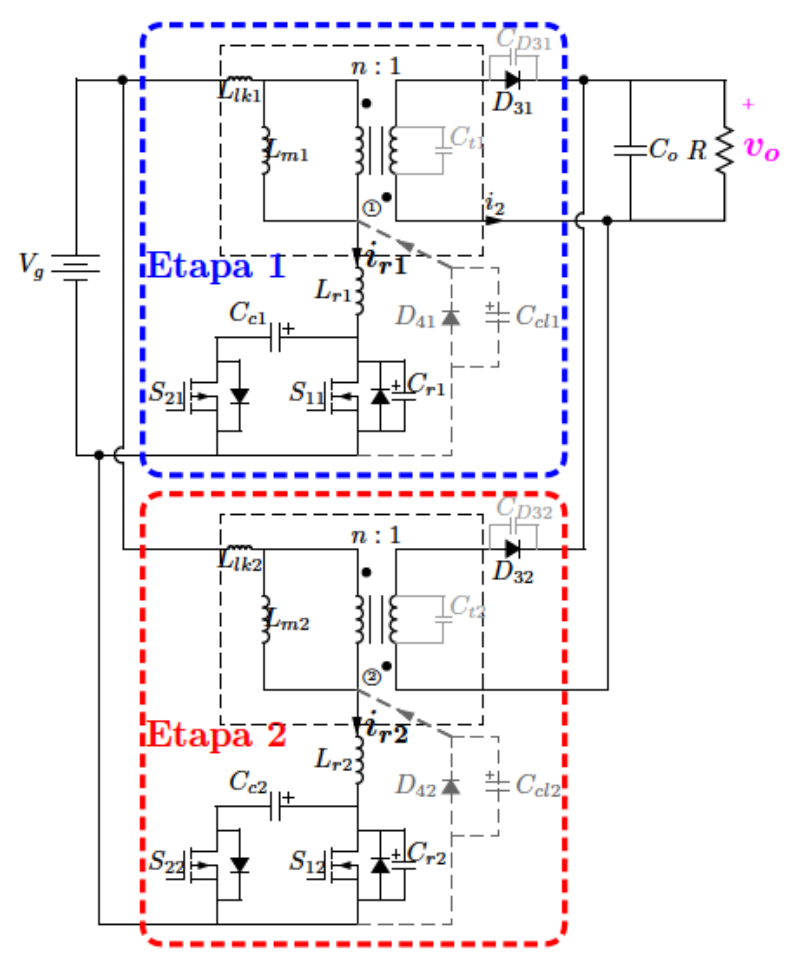

Figura 4.21: Conexión de las dos etapas que forman el prototipo.

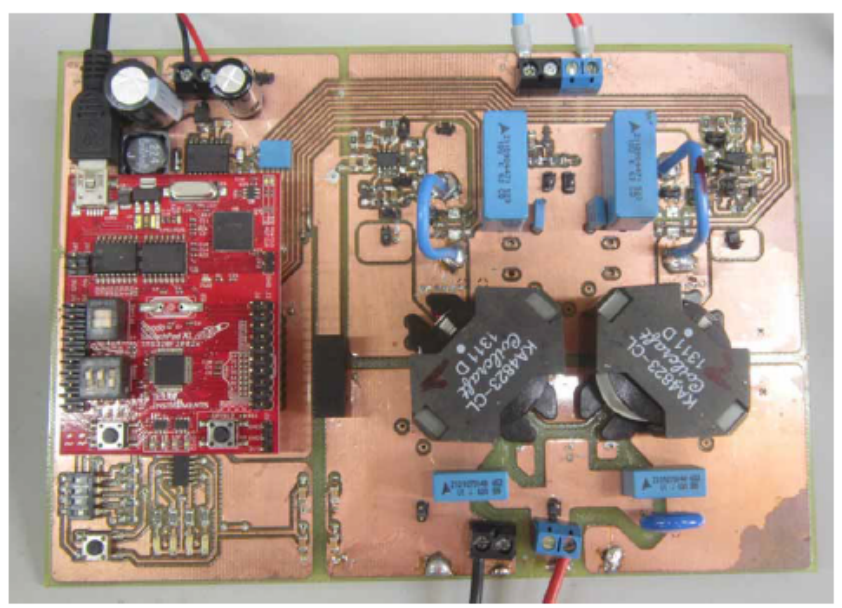

Figura 4.22: Prototipo de laboratorio:dos etapas flyback ZVS-modificadas conectadas en paralelo y bloque de control. 


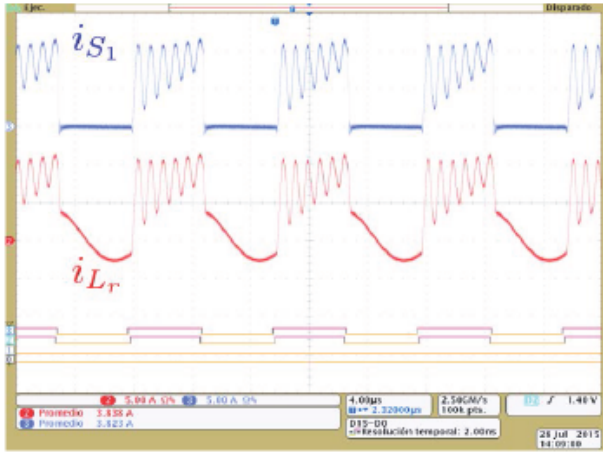

(a)

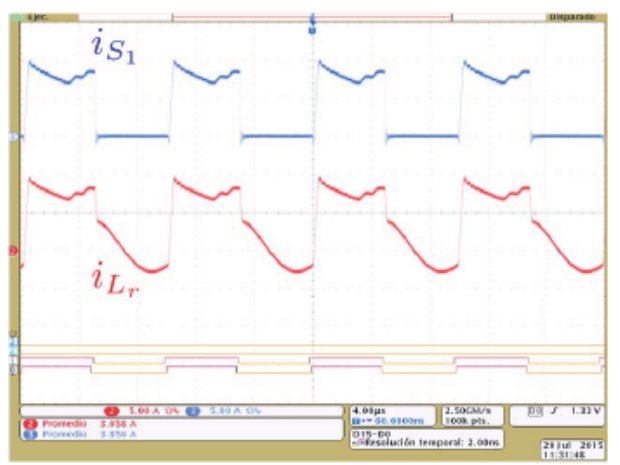

(b)

Figura 4.23: Corriente en bobina resonante y en transistor principal: a) $\sin$ diodo clamp; y b) con diodo de clamp(derecha).

Este experimento se ha realizado para cada etapa flyback ZVS por separado. Los datos numéricos están recogidos en la Tabla 4.4 para las dos etapas flyback ZVS. Las magnitudes registradas son la corriente de entrada, la corriente de salida y la tensión de salida. El mismo experimento se ha realizado para distintos valores de $L_{r}$.

El mismo experimento se ha realizado también cuando se incluye el diodo de clamp. Los datos registrados se encuentran en la Tabla 4.5.

Los datos registrados se han representado gráficamente eun unos ejes $V_{o^{-}}$ $I_{o}$, para observar la característica de salida que tiene cada etapa. Las curvas obtenidas están incluidas en la Figura 4.24. En la mismas figuras se han incluido los datos para las etapas flyback ZVS modificadas.

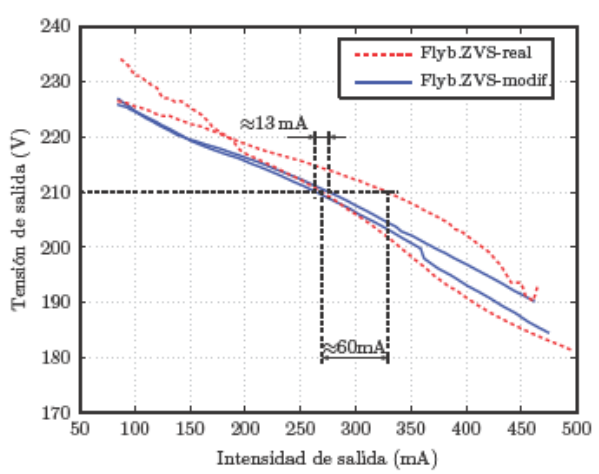

(a) $L_{r}=1 \mu \mathrm{H}$

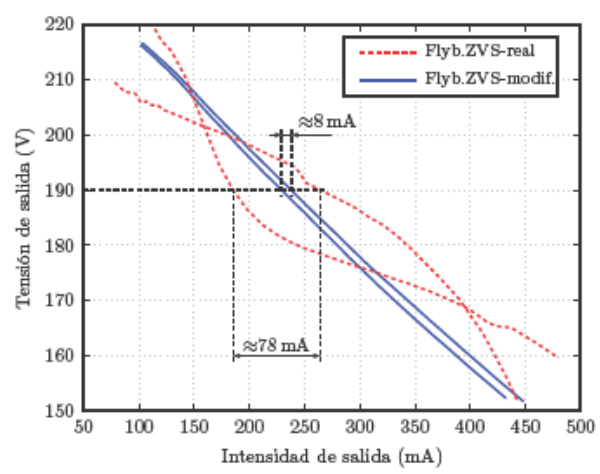

(b) $L_{r}=2 \mu \mathrm{H}$

Figura 4.24: Característica de salida para las dos etapas del convertidor con diodo de clamp (línea continua) y sin diodo (línea de trazos) con diferentes bobinas resonantes. 


\begin{tabular}{|c|c|c|c|c|c|c|c|c|c|c|c|}
\hline \multicolumn{6}{|c|}{$\left(L_{r}=1 \mu \mathrm{H}, D=0,488, V_{g}=20 \mathrm{~V}\right)$} & \multicolumn{6}{|c|}{$\left(L_{r}=2 \mu \mathrm{H}, D=0,488, V_{g}=20 \mathrm{~V}\right)$} \\
\hline$I_{g}(\mathrm{~A})$ & $\begin{array}{c}\text { ETAPA } 1 \\
I_{o}(\mathrm{~mA}) \\
\end{array}$ & $V_{o}(\mathrm{~V})$ & $I_{g}(\mathrm{~A})$ & $\begin{array}{c}\text { ETAPA } 2 \\
I_{o}(\mathrm{~mA}) \\
\end{array}$ & $V_{o}(\mathrm{~V})$ & $I_{g}(\mathrm{~A})$ & $\begin{array}{c}\text { ETAPA } 1 \\
I_{O}(\mathrm{~mA}) \\
\end{array}$ & $V_{o}(\mathrm{~V})$ & $I_{g}(\mathrm{~A})$ & $\begin{array}{c}\text { ETAPA } 2 \\
I_{O}(\mathrm{~mA}) \\
\end{array}$ & $V_{o}(\mathrm{~V})$ \\
\hline 1,14 & 87,2 & 234,1 & 1,04 & 84,4 & 226,5 & 0,9 & 78 & 209,5 & 1,06 & 84,6 & 227,2 \\
\hline 1,18 & 90 & 233,7 & 1,12 & 90,1 & 226,3 & 0,93 & 81 & 209 & 1,1 & 89,2 & 225,2 \\
\hline 1,23 & 95,2 & 232,7 & 1,18 & 95 & 226 & 0,95 & 83,5 & 208,5 & 1,14 & 94,9 & 222,5 \\
\hline 1,29 & 100 & 231,1 & 1,23 & 100 & 225,5 & 1 & 87,7 & 207,8 & 1,21 & 100,7 & 221,2 \\
\hline 1,35 & 104,9 & 231,1 & 1,29 & 105,2 & 225,3 & 1,05 & 92,3 & 207,7 & 1,25 & 104,6 & 220,8 \\
\hline 1,4 & 110,1 & 230,1 & 1,35 & 110,3 & 225 & 1,09 & 96,2 & 207,6 & 1,29 & 109 & 220,2 \\
\hline 1,46 & 115,4 & 229,4 & 1,4 & 115,2 & 224,6 & 1,15 & 102,1 & 205,8 & 1,35 & 114,4 & 219,2 \\
\hline 1,52 & 120 & 228,9 & 1,45 & 120 & 224,1 & 1,2 & 106,6 & 206,1 & 1,4 & 120 & 217,2 \\
\hline 1,57 & 125 & 227,1 & 1,51 & 125,5 & 223,8 & 1,25 & 111,3 & 205,4 & 1,49 & 129,4 & 215,2 \\
\hline 1,63 & 130,3 & 227 & 1,57 & 130,5 & 223,9 & 1,3 & 116,8 & 205,4 & 1,6 & 142 & 210,5 \\
\hline 1,7 & 136,2 & 226,4 & 1,61 & 134,5 & 223,6 & 1,36 & 123 & 204,5 & 1,7 & 156,3 & 203 \\
\hline 1,77 & 142 & 226,5 & 1,68 & 140,2 & 223,1 & 1,4 & 127 & 204,2 & 1,81 & 172,4 & 194,7 \\
\hline 1,87 & 151,9 & 225,1 & 1,74 & 146 & 222,7 & 1,49 & 136,8 & 203,4 & 1,9 & 184,7 & 190,2 \\
\hline 1,96 & 159,9 & 224,1 & 1,81 & 151,7 & 222,3 & 1,61 & 148,3 & 202,4 & 2 & 197,9 & 186,5 \\
\hline 2,01 & 165,6 & 223 & 1,9 & 160,1 & 221,9 & 1,7 & 157 & 201,7 & 2,11 & 212 & 183,7 \\
\hline 2,06 & 171 & 221,7 & 2,01 & 170 & 221,12 & 1,8 & 167,9 & 200,9 & 2,21 & 224,5 & 182 \\
\hline 2,13 & 178 & 220,8 & 2,06 & 175 & 220,8 & 1,91 & 177,9 & 200,1 & 2,3 & 235,2 & 180,8 \\
\hline 2,23 & 187,7 & 219,6 & 2,14 & 182,3 & 220,1 & 2,02 & 190,1 & 199,1 & 2,41 & 246,9 & 179,7 \\
\hline 2,29 & 195,4 & 217,6 & 2,24 & 191,6 & 219,4 & 2,13 & 200,5 & 198,1 & 2,5 & 256,7 & 179 \\
\hline 2,36 & 202 & 216,8 & 2,29 & 196,5 & 219 & 2,2 & 208,7 & 197,3 & 2,62 & 271,1 & 178 \\
\hline 2,46 & 211,2 & 216 & 2,34 & 200,8 & 218,7 & 2,31 & 219,8 & 196,2 & 2,69 & 279,5 & 177,4 \\
\hline 2,58 & 222,8 & 214,9 & 2,39 & 206,3 & 218,3 & 2,42 & 230,8 & 195,2 & 2,8 & 292,2 & 176,5 \\
\hline 2,65 & 229,4 & 214,3 & 2,47 & 213,3 & 217,8 & 2,52 & 241,9 & 194,1 & 2,89 & 302,4 & 175,9 \\
\hline 2,72 & 236 & 213,6 & 2,55 & 221,3 & 217,3 & 2,61 & 252,3 & 191,2 & 3 & 315 & 175,1 \\
\hline 2,78 & 242,4 & 213 & 2,61 & 226,6 & 217 & 2,71 & 262,1 & 190,3 & 3,06 & 321,7 & 174,6 \\
\hline 2,87 & 251,3 & 212,1 & 2,71 & 235,7 & 216,5 & 2,82 & 274,4 & 189 & 3,16 & 334,5 & 173,7 \\
\hline 2,97 & 260,5 & 211,1 & 2,82 & 244,7 & 216 & 2,9 & 283,7 & 188,1 & 3,23 & 342,7 & 173,2 \\
\hline 3,07 & 270,4 & 209,9 & 2,89 & 251,7 & 215,6 & 3 & 295,3 & 186,9 & 3,36 & 357,4 & 172,1 \\
\hline 3,18 & 282,6 & 208,4 & 2,97 & 259,3 & 215,1 & 3,12 & 308,4 & 185,1 & 3,41 & 363,1 & 171,7 \\
\hline 3,27 & 291,3 & 207,1 & 3,04 & 265,2 & 214,7 & 3,21 & 320,2 & 183,8 & 3,51 & 376,1 & 170,6 \\
\hline 3,36 & 300 & 206 & 3,09 & 270,8 & 214,3 & 3,31 & 332,6 & 181,9 & 3,58 & 384 & 169,9 \\
\hline 3,46 & 311,4 & 204,5 & 3,19 & 280,3 & 213,7 & 3,4 & 344,5 & 179,8 & 3,7 & 398,8 & 168,5 \\
\hline 3,54 & 320,2 & 203,2 & 3,25 & 285,9 & 213,3 & 3,5 & 358,2 & 177 & 3,81 & 414 & 166,3 \\
\hline 3,66 & 332,6 & 201,3 & 3,34 & 293,4 & 212,7 & 3,59 & 370,7 & 174,5 & 3,89 & 423 & 165,4 \\
\hline 3,77 & 344,6 & 199,3 & 3,44 & 303 & 212,1 & 3,7 & 390 & 170,3 & 3,97 & 437 & 165,1 \\
\hline 3,85 & 353,7 & 197,8 & 3,57 & 315,16 & 211,1 & 3,8 & 411 & 164,2 & 4,14 & 460 & 162,3 \\
\hline 3,93 & 363,2 & 196,3 & 3,66 & 323,6 & 210,5 & 3,84 & 422 & 160,2 & 4,26 & 480 & 159,6 \\
\hline 4,01 & 371,7 & 194,8 & 3,75 & 332,1 & 209,7 & 3,87 & 431,6 & 156,6 & 4,41 & 505 & 155,5 \\
\hline 4,11 & 383,2 & 193,1 & 3,83 & 339,8 & 209 & 3,86 & 442,1 & 152,1 & 4,5 & 532 & 148,6 \\
\hline 4,18 & 392 & 192,1 & 3,88 & 345,2 & 208,5 & 3,85 & 450 & 147,9 & 4,56 & 549 & 144,4 \\
\hline 4,25 & 399,5 & 191 & 3,95 & 351,5 & 207,9 & 3,85 & 460 & 143,9 & 4,57 & 558 & 141,6 \\
\hline 4,35 & 410 & 189,6 & 3,99 & 356,2 & 207,4 & 3,81 & 471,7 & 137,6 & 4,56 & 566 & 138,3 \\
\hline 4,44 & 420,8 & 188,3 & 4,05 & 362,5 & 206,7 & 3,78 & 481,9 & 132,9 & 4,5 & 575 & 133 \\
\hline 4,53 & 430,1 & 187,3 & 4,14 & 370,5 & 205,9 & 3,78 & 491,3 & 129,4 & 4,521 & 583 & 129,9 \\
\hline 4,63 & 441 & 186,2 & 4,19 & 375,5 & 205,4 & 3,79 & 500,5 & 126,9 & 4,44 & 592 & 125,9 \\
\hline 4,72 & 451,2 & 185,2 & 4,28 & 384,2 & 204,4 & 3,84 & 510 & 125 & 4,4 & 600 & 121,3 \\
\hline 4,82 & 462 & 184,2 & 4,33 & 390,1 & 203,3 & 3,88 & 521,1 & 123,4 & 4,37 & 610 & 117,2 \\
\hline 4,9 & 470,3 & 183,5 & 4,38 & 395,8 & 202,9 & 3,9 & 533 & 122,6 & 4,35 & 626 & 112,1 \\
\hline 5,04 & 487,5 & 182,1 & 4,45 & 402,3 & 201,9 & 4 & 548 & 121,3 & 4,38 & 634 & 110,8 \\
\hline 5,15 & 498 & 181,2 & 4,51 & 409,7 & 200,8 & 4,01 & 556,7 & 121,1 & 4,42 & 651 & 108,5 \\
\hline \multirow[t]{9}{*}{5,29} & 513 & 180 & 4,61 & 420,5 & 199,2 & 4,1 & 562,8 & 120,2 & 4,52 & 674 & 106,6 \\
\hline & & & 4,68 & 431,2 & 196,8 & 4,2 & 579,6 & 119,2 & 4,7 & 711 & 104,4 \\
\hline & & & 4,73 & 434 & 195,9 & 4,3 & 598,2 & 118,3 & 4,89 & 739 & 104,7 \\
\hline & & & 4,81 & 441 & 193,6 & 4,39 & 612 & 117,9 & 5 & 762 & 103,4 \\
\hline & & & 4,86 & 450 & 193,6 & 4,5 & 633,5 & 116,5 & & & \\
\hline & & & 4,91 & 454 & 191,2 & 4,6 & 651 & 115,5 & & & \\
\hline & & & 4,97 & 461 & 190,3 & 4,72 & 676 & 115,3 & & & \\
\hline & & & 4,99 & 464,5 & 192,8 & 4,82 & 693 & 113,3 & & & \\
\hline & & & & & & 5 & 733 & 110,5 & & & \\
\hline
\end{tabular}

Tabla 4.4: Datos registrados para dos etapas flyback ZVS con diferentes bobinas resonantes.

Las curvas obtenidas muestran la similitud en el caso de la topología modificada, de forma que para la misma tensión de salida, se obtendrían valores muy cercanos de intensidad. En cambio, las curvas de las mismas etapas sin considerar el diodo, presentan tendencias diferentes y el reparto de corriente empeora. Por ejemplo, para dos etapas en paralelo con una tensión de salida de $210 \mathrm{~V}$, empleando una bobina $L_{r}=1 \mu \mathrm{H}$, la diferencia de corrientes a la 


\begin{tabular}{|c|c|c|c|c|c|c|c|c|c|c|c|}
\hline \multicolumn{6}{|c|}{$\left(L_{r}=1 \mu \mathrm{H}, D=0,488, V_{g}=20 \mathrm{~V}\right)$} & \multicolumn{6}{|c|}{$\left(L_{r}=2 \mu \mathrm{H}, D=0,488, V_{g}=20 \mathrm{~V}\right)$} \\
\hline$I_{g}(\mathrm{~A})$ & $\begin{array}{c}\text { ETAPA } 1 \\
I_{o}(\mathrm{~mA}) \\
\end{array}$ & $V_{o}(\mathrm{~V})$ & $I_{g}(\mathrm{~A})$ & $\begin{array}{c}\text { ETAPA } 2 \\
I_{o}(\mathrm{~mA}) \\
\end{array}$ & $V_{o}(\mathrm{~V})$ & $I_{g}(\mathrm{~A})$ & $\begin{array}{c}\text { ETAPA } 1 \\
I_{o}(\mathrm{~mA}) \\
\end{array}$ & $V_{o}(\mathrm{~V})$ & $I_{g}(\mathrm{~A})$ & $\begin{array}{c}\text { ETAPA } 2 \\
I_{o}(\mathrm{~mA}) \\
\end{array}$ & $V_{o}(\mathrm{~V})$ \\
\hline 1,034 & 84,7 & 227,3 & 1,02 & 84,3 & 226,2 & 1,16 & 101,5 & 216,3 & 1,17 & 102,5 & 216,7 \\
\hline 1,12 & 91,9 & 226,3 & 1,09 & 90 & 225,8 & 1,2 & 105,6 & 215,6 & 1,2 & 105,1 & 216,3 \\
\hline 1,21 & 99,1 & 225,2 & 1,15 & 95,1 & 225,4 & 1,29 & 114,5 & 214 & 1,25 & 109,7 & 215,5 \\
\hline 1,31 & 108,1 & 224,2 & 1,21 & 99,9 & 224,9 & 1,35 & 120 & 212,9 & 1,3 & 114,7 & 214,6 \\
\hline 1,42 & 118,8 & 223,1 & 1,28 & 107 & 224,1 & 1,4 & 124,6 & 212,1 & 1,35 & 119,7 & 213,7 \\
\hline 1,5 & 125,7 & 222,4 & 1,36 & 114,4 & 223,4 & 1,5 & 135 & 210 & 1,4 & 124,7 & 212,8 \\
\hline 1,63 & 138,4 & 221,1 & 1,4 & 118 & 223 & 1,59 & 143,7 & 208,1 & 1,49 & 133,8 & 211,1 \\
\hline 1,7 & 144,7 & 220,4 & 1,47 & 124,7 & 222,3 & 1,69 & 154,4 & 205,7 & 1,55 & 139,8 & 209,8 \\
\hline 1,82 & 156,5 & 219,3 & 1,55 & 131,8 & 221,5 & 1,8 & 166,7 & 203 & 1,6 & 145,1 & 208,8 \\
\hline 2 & 172,8 & 218,1 & 1,63 & 140,1 & 220,7 & 1,9 & 177,8 & 200,7 & 1,69 & 154,8 & 206,7 \\
\hline 2,17 & 189,3 & 217,1 & 1,77 & 152,7 & 219,8 & 2 & 189,9 & 198 & 1,8 & 166,2 & 204,3 \\
\hline 2,28 & 198,8 & 216,4 & 1,87 & 161,8 & 219,2 & 2,11 & 201,9 & 195,5 & 1,9 & 177,4 & 202 \\
\hline 2,4 & 210,7 & 215,5 & 2,07 & 180 & 218,1 & 2,21 & 214 & 193 & 1,95 & 182,9 & 200,9 \\
\hline 2,51 & 219,6 & 214,8 & 2,16 & 188 & 217,6 & 2,3 & 225,4 & 190,8 & 2 & 188,7 & 199,8 \\
\hline 2,6 & 228,6 & 214,1 & 2,29 & 199,9 & 216,9 & 2,41 & 239,3 & 188 & 2,1 & 199,7 & 197,5 \\
\hline 2,77 & 244,5 & 212,8 & 2,41 & 211,3 & 216,1 & 2,5 & 249,6 & 186 & 2,21 & 212,4 & 195 \\
\hline 2,95 & 261,5 & 211,3 & 2,53 & 221,9 & 215,3 & 2,6 & 262,8 & 183,3 & 2,3 & 223,6 & 192,8 \\
\hline 3,18 & 285,1 & 209,1 & 2,64 & 231,7 & 214,6 & 2,7 & 276,3 & 180,7 & 2,41 & 238 & 190 \\
\hline 3,37 & 303,6 & 207,3 & 2,75 & 242,2 & 213,7 & 2,8 & 290,4 & 177,8 & 2,5 & 248,5 & 187,9 \\
\hline 3,62 & 328,2 & 204,7 & 2,88 & 255,3 & 212,6 & 2,9 & 304,2 & 175,2 & 2,6 & 261,2 & 185,4 \\
\hline 3,78 & 344,1 & 203 & 3,04 & 271,1 & 211,2 & 2,99 & 317,6 & 172,6 & 2,7 & 273,9 & 183 \\
\hline 3,94 & 360,9 & 201,2 & 3,18 & 283,5 & 210,1 & 3,1 & 332,7 & 169,8 & 2,8 & 286,7 & 180,5 \\
\hline 4,14 & 381,8 & 198,9 & 3,32 & 298,7 & 208,6 & 3,2 & 349,1 & 166,8 & 2,91 & 301,5 & 177,6 \\
\hline 4,34 & 402,6 & 196,6 & 3,45 & 311,1 & 207,3 & 3,32 & 366,5 & 163,7 & 3 & 314,8 & 175,1 \\
\hline 4,57 & 426,9 & 194 & 3,56 & 322 & 206,2 & 3,4 & 379,8 & 161,4 & 3,1 & 329,1 & 172,6 \\
\hline 4,83 & 457,2 & 190,8 & 3,69 & 334,5 & 204,9 & 3,51 & 398,3 & 158,1 & 3,22 & 345,8 & 169,6 \\
\hline 4,94 & 469,4 & 189,5 & 3,78 & 344 & 203,9 & 3,6 & 414,9 & 155,3 & 3,33 & 363,6 & 166,4 \\
\hline \multirow[t]{10}{*}{5,12} & 487,9 & 187,4 & 3,88 & 354,8 & 202,7 & 3,69 & 432,4 & 152,3 & 3,4 & 375 & 164,4 \\
\hline & & & 3,96 & 363,2 & 201,8 & 3,78 & 448,2 & 149,5 & 3,5 & 391,9 & 161,4 \\
\hline & & & 4,07 & 373,5 & 200,7 & 3,89 & 469,2 & 146 & 3,6 & 408,6 & 158,5 \\
\hline & & & 4,2 & 388 & 199,4 & 3,99 & 489,7 & 142,7 & 3,7 & 427 & 155,3 \\
\hline & & & 4,31 & 398,7 & 198 & & & & 3,81 & 448,4 & 151,7 \\
\hline & & & 4,47 & 416,5 & 196,2 & & & & 3,9 & 465,2 & 149 \\
\hline & & & 4,56 & 426,7 & 195,2 & & & & 4,01 & 484,1 & 146 \\
\hline & & & 4,72 & 442,8 & 193,6 & & & & & & \\
\hline & & & 4,94 & 465,6 & 191,3 & & & & & & \\
\hline & & & 5,12 & 485,6 & 189,2 & & & & & & \\
\hline
\end{tabular}

Tabla 4.5: Datos registrados para dos etapas flyback ZVS-modif. con diferentes bobinas resonantes.

salida es de $\approx 60 \mathrm{~mA}$, sobre una corriente total de $590 \mathrm{~mA}$, mientras que en el caso del flyback ZVS-modificado, la diferencia es de $\approx 13 \mathrm{~mA}$ sobre un total de $534 \mathrm{~mA}$. En el caso de una bobina resonante mayor, aumenta la impedancia, y la diferencia en el reparto de corrientes es menor. Así se comprueba en las curvas de la derecha de la misma figura.

Respecto al control del convertidor, para los experimentos se ha diseñado un control digital que implementa un lazo de tensión sin ningún mecanismo adicional de ecualizado de corrientes, puesto que el reparto se consigue gracias a la impedancia de salida. La medida de la tensión de salida se ha escalado con un divisor resistivo, y se muestrea la señal acondicionada después de pasar por un amplificador de aislamiento AMC1200 que incorpora los filtros recomendados por el fabricante. El microcontrolador se encarga de evaluar el error que tiene respecto a una referencia de tensión y proporcionar el ciclo de trabajo necesario para compensar el error. Él diseño del controlador digital se ha realizado a partir de un controlador analógico, calculado según (Venable, 1983), al que se le aplica la transformada bilineal.

Para obtener un buen reparto de carga ambas etapas tendrán el mismo 
ciclo de trabajo que propone el controlador digital de tensión.

El reparto de carga pasivo se consigue tanto en régimen permanente como durante la entrada y salida de etapas. En la Figura 4.25 se muestran los registros de las corrientes en la bobina resonante de cada etapa la tensión de salida cuando estando una etapa en funcionamiento, se produce la entrada de una de ellas.

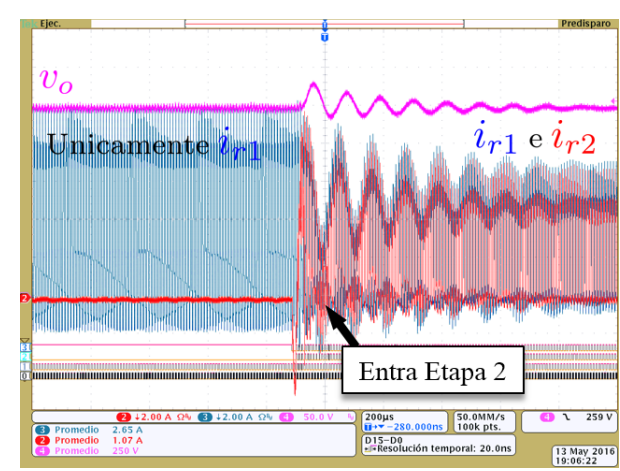

(a)

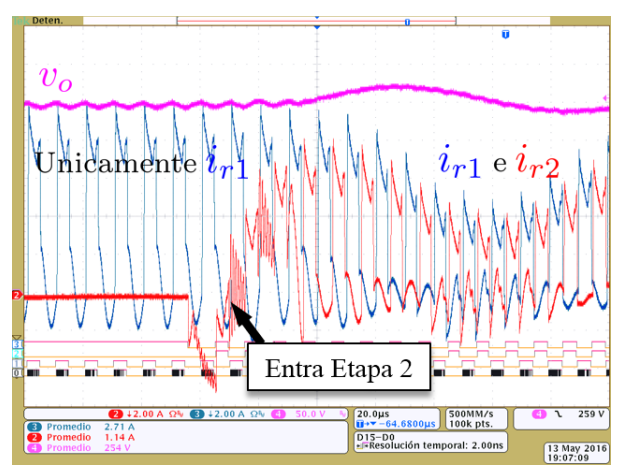

(b)

Figura 4.25: Tensión de salida y corriente en $L_{r}$ para las dos etapas cuando entra en funcionamiento la etapa 2 (izqda.) y detalle (dcha.).

En dicha figura se comprueba que desde el mismo instante en el que se produce la entrada de la segunda etapa, las dos etapas se reparten la corriente hasta alcanzar el nuevo régimen permanente unos ciclos después. El control en tensión mantiene la tensión en el valor indicado inicialmente.

El resultado de la salida de una de las etapas en funcionamiento produce un transitorio para la etapa que queda en funcionamiento, hasta alcanzar el régimen permanente. Así se observa en la Figura 4.26.

Respecto al rendimiento, se ha medido sobre el prototipo, al entrar en funcionamiento una de las etapas.. La Figura 4.27 muestra la entrada de la segunda etapa entra a partir de $70 \mathrm{~W}$. El rendimiento del conjunto se mantiene en un amplio rango de carga una vez que entre la segunda etapa. El valor máximo alcanza el $94 \%$. 


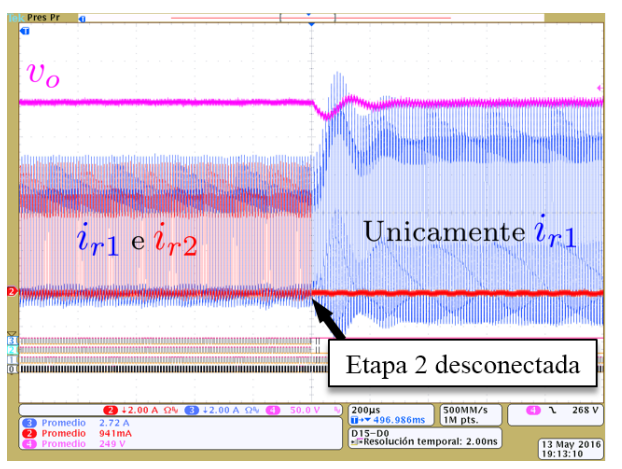

(a)

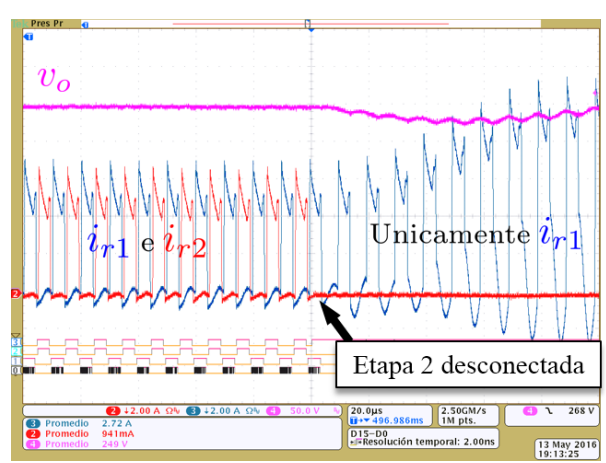

(b)

Figura 4.26: Tensión de salida y corriente en $L_{r}$ para las dos etapas cuando se desconecta la etapa 2 (izqda.) y detalle (dcha.).

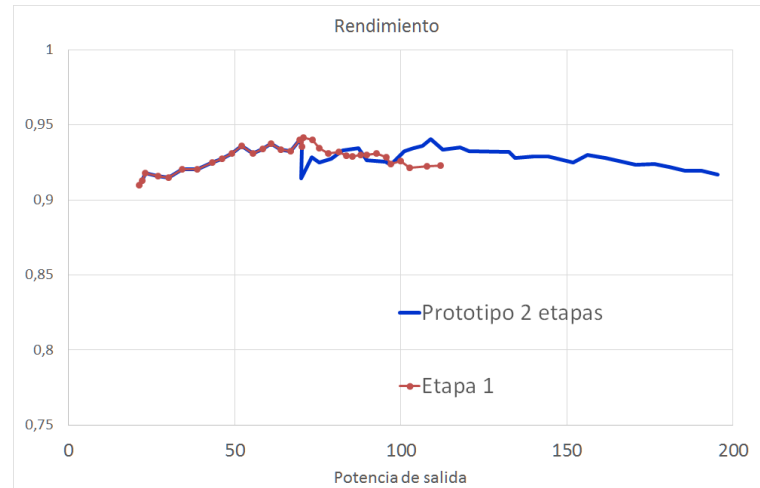

Figura 4.27: Rendimiento del prototipo. Entrada de segunda etapa con $70 \mathrm{~W}$. 


\section{Capítulo 5}

\section{Conclusiones, aportaciones y futuros trabajos}

\subsection{Conclusiones.}

Aunque existen trabajos previos donde se analiza como los convertidores con conmutaciones suaves pueden ser utilizados para mejorar el reparto de potencia entre etapas conectadas en paralelo, en este trabajo se ha realizado un gran esfuerzo para analizar de forma conjunta las topologías básicas buscando semejanzas y particularidades entre ellas. Este estudio se ha realizado tanto para circuitos promediados como para los circuitos en pequeña señal donde se ha puesto especial atención en la definición de la impedancia de salida del convertidor. Esta impedancia de salida no estaba claramente definida en trabajos anteriores.

Se ha analizado el reparto de corrientes entre convertidores conectados en paralelo tanto en funcionamiento estático como dinámico. Se ha comprobado el funcionamiento en lazo abierto y el funcionamiento en lazo cerrado. Mediante las pruebas en lazo abierto se ha podido demostrar la no necesidad de lazos de control adicionales para el control de la corriente. El control en lazo cerrado se ha realizado para demostrar como un microprocesador de bajo coste puede, de forma sencilla, utilizarse en el control de varias etapas en paralelo. El control digital tiene dos ventajas principales: por una parte, los disparos se pueden realizar en interleaving permitiendo reducir el rizado de corrientes y tensiones de forma muy sencilla; por otra parte, la semejanza entre todos los disparos es muy grande, por lo que se elimina una de las principales fuentes de "error" en el reparto de cargas.

Las capacidades parásitas de los componentes pueden ocasionar oscilaciones que afecten al reparto en régimen permanente. Esto es debido a que la impedancia adicional utilizada para el reparto de corrientes depende 
fuertemente de la corriente al inicio de la conducción del transistor. En convertidores de baja potencia no es siempre apreciable este efecto, que queda camuflado por la utilización de snubbers o incluso el uso de topologías con diodos de clamp como las propuestas por Ivo Barbi.

Una forma de acentuar estas oscilaciones es la introducción de un transformador con alta relación de transformación. El transformador añadirá su propia capacidad y a la vez hará que la capacidad del diodo de salida vista en el primario tenga un valor nada despreciable. Para demostrar este efecto se ha diseñado un convertidor flyback con alta relación de transformación y se ha podido evaluar como queda el reparto estático de potencia como consecuencia de estas oscilaciones.

Finalmente se ha podido demostrar que un circuito de clamp permite que el reparto sea muy aproximado al teórico. Este circuito de clamp no afecta apreciablemente al rendimiento del convertidor y sí mejora el reparto de corrientes.

\subsection{Aportaciones.}

Como principal aportación de este trabajo debe destacarse el estudio del reparto de corrientes incluyendo las oscilaciones introducidas por los condensadores parásitos de los componentes. No solo se ha detectado que estas capacidades pueden alejar el reparto de corrientes del deducido mediante modelos con interruptores ideales y modelos promediados, sino que se ha determinado un procedimiento para reducir su efecto mediante circuitos de clamp, especialmente en el caso de un convertidor flyback donde la solución no es obvia.

Se ha demostrado como controladores muy sencillos pueden trabajar con múltiples etapas que utilicen este método de reparto de corriente. Pudiendo incluso adaptar su dinámica según el número de etapas seleccionado.

Por último se han obtenido y tabulado los parámetros para los convertidores con conmutaciones suaves más habituales incluyendo sus modelos tanto en gran señal como en pequeña señal.

\subsection{Futuros trabajos.}

Diseño de un microinversor para energía solar fotovoltaica basado en la conexión en paralelo de etapas flyback similares a la diseñada, donde seleccionando el número de etapas activas se aumente el rango de conmutaciones suaves.

Evaluar cómo un enfoque modular, afecta al tiempo de diseño y desarrollo de un convertidor de "gran potencia". Los módulos estarán formados por 
convertidores con conmutaciones suaves como los estudiados en este trabajo ya que se ha comprobado su fácil conexión en paralelo. 



\section{Apéndice A}

\section{Reductor ZVS}

\section{A.1. Funcionamiento del Reductor ZVS}

El reductor ZVS es un reductor convencional al que se le ha añadido una red de enclavamiento activo que fija la tensión máxima en los extremos del transistor principal. Esta red facilita las conmutaciones a tensión cero, reduciendo las pérdidas que aparecen. Por contra, aumenta la complejidad del circuito y el número de componentes. En la figura A.1 está representado el circuito de este convertidor.

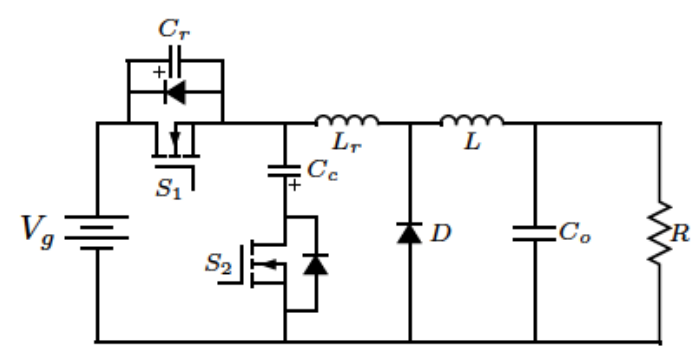

Figura A.1: Reductor ZVS.

La red de enclavamiento activo está formada por el condensador de clamp $C_{c}$ y el interruptor $S_{2}$ junto con su diodo en antiparalelo, $D_{2}$. Además se añade una pequeña inductancia, $L_{r}$, que incluye las inductancias parásitas y que actúa en resonancia con el condensador $C_{c}$ en determinados instantes del periodo. Se ha incluido el diodo $D_{1}$ al interruptor $S_{1}$ y un condensador $C_{r}$ que puede ser el propio del transistor o uno añadido que mejore sus características.

Para explicar el funcionamiento de este convertidor se plantea el estado de los diferentes semiconductores presentes en el circuito, que son los dos interruptores y los tres diodos. En el instante inicial, $t_{0}$, está cerrado $S_{1}$ y el condensador $C_{r}$ está descargado. Se considera el condensador de salida, 
$C_{o}$, de un valor grande para mantener la tensión $v_{C_{o}}$ constante, por lo que se sustituirá el conjunto $\mathrm{C}-\mathrm{R}$ por una fuente de tensión constante de valor $V_{o}$. La bobina, $L$, es lo suficientemente grande para considerar una corriente constante, I. Para un reductor, la corriente de la bobina coincide con la corriente de salida. De esta forma el conjunto formado por $L$ y $C_{o}$ junto con la carga se puede sustituir por una fuente de corriente constante de valor $I_{o}=I$. El condensador de clamp, $C_{c}$ presenta una tensión con la polaridad indicada en figura, de un valor lo suficientemente grande como para suponer una tensión constante, $V_{c}$, no considerando los tramos donde éste se cargue y se descargue y su tensión pueda variar en torno a ese valor. Por último, indicar que el valor de $L_{r}$, aunque comparativamente mas pequeño que $L$, es lo suficientemente grande como para cumplir el criterio de energía que permite conmutaciones suaves en estas condiciones de carga.

\section{A.1.1. Etapa 1. $\left(t_{0}<t<t_{1}\right)$}

Se abre el interruptor $S_{1}$. Esta conmutación se produce a tensión cero puesto que el condensador $C_{r}$ está descargado. Este condensador se carga a corriente constante igual a $I_{o}$, hasta que alcanza el valor de $V_{g}$. La figura B.2 muestra los elementos que intervienen en esta etapa y el circuito equivalente, a partir de las suposiciones iniciales, donde la etapa de salida se ha modelado como una fuente de corriente constante $I_{o}$.

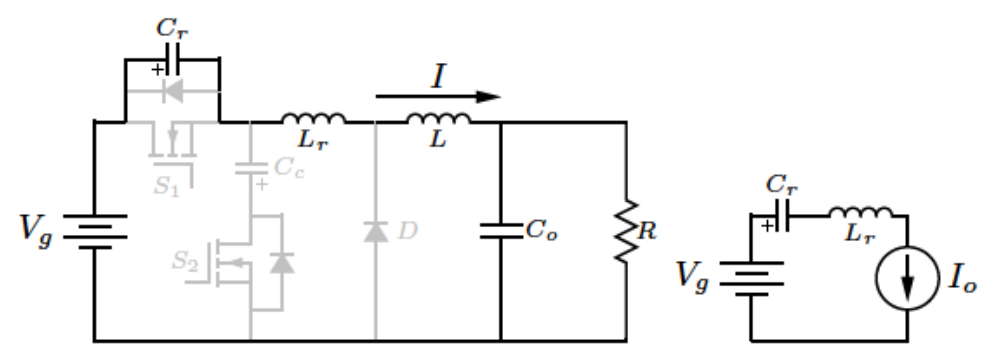

Figura A.2: Reductor ZVS durante la etapa 1. Circuito equivalente.

La tensión en el condensador crece de forma lineal, como se indica en (A.1), mientras que la tensión en la bobina $L_{r}$ es cero ya que la corriente que circula es constante.

$$
\begin{aligned}
i_{C_{r}}(t) & =I_{o} \\
v_{C_{r}}(t) & =\frac{1}{C_{r}} \int_{o}^{t} i_{C_{r}}(t) d t=\frac{I_{o}}{C_{r}} t
\end{aligned}
$$

Esta etapa terminará cuando $v_{C_{r}}(t)=V_{g}$, porque al alcanzar este valor, el diodo $D$ se polarizará en directa y empezará a conducir. La duración de esta etapa es igual $d_{1} T$ cuya expresión está indicada en (A.2). El periodo 
de conmutación es $T$, y $d_{1}$ es la fracción de un periodo correspondiente a la duración de este intervalo.

$$
d_{1} T=\left(t_{1}-t_{0}\right)=\frac{V_{g} C_{r}}{I_{o}}
$$

\section{A.1.2. Etapa 2. $\left(t_{1}<t<t_{2}\right)$}

El diodo $D$ empieza a conducir. La carga sigue demandando una corriente igual a $I_{o}$. En la figura A.3 se muestran los elementos que intervienen y el circuito equivalente. El condensador $C_{r}$ sigue cargándose, pero ahora no es a corriente constante sino a una corriente variable, $i_{1}(t)$, obtenida por la resonancia del condensador y la bobina.

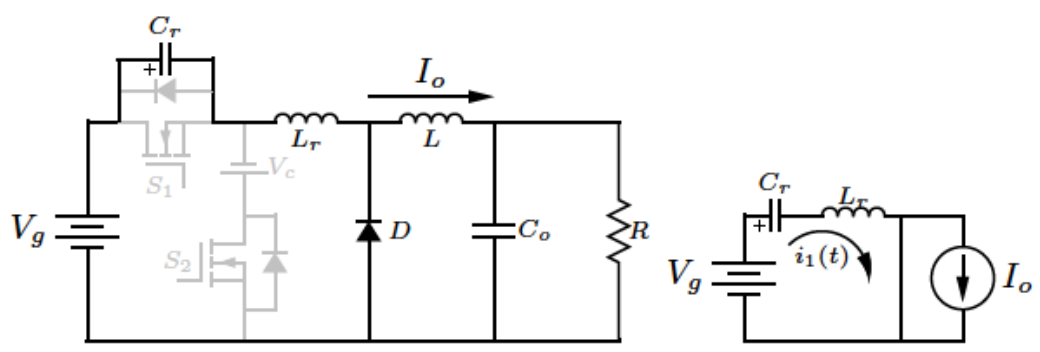

Figura A.3: Reductor ZVS durante la etapa 2. Circuito equivalente.

Obteniendo la corriente $i_{1}(t)$, se conseguirá la evolución de la tensión en $C_{r}$, y cuando alcance el valor de $V_{g}+V_{c}$ terminará esta etapa, ya que se polarizará en directa el diodo del interruptor $S_{2}$, dando lugar a otro subcircuito. la ecuación para esta corriente está dada en (A.3), que es una ecuación integro-diferencial con coeficientes constantes.

$$
V_{g}=v_{C_{r}}(t)+v_{L_{r}}(t)=v_{C_{r}}(0)+\frac{1}{C_{r}} \int_{0}^{t} i_{1}(t) d t+L_{r} \frac{d i_{1}(t)}{d t}
$$

Las condiciones iniciales están indicada en (A.4),

$$
\begin{aligned}
& i_{1}(0)=I_{o} \\
& i_{1}^{\prime}(0)=\frac{1}{L_{r}} v_{L_{r}}(0)=\frac{1}{L_{r}}\left[V_{g}-v_{C_{r}}(0)\right]=0
\end{aligned}
$$

donde la intensidad debe cumplir la condición de continuidad, pero no la derivabilidad. La resolución de esta ecuación diferencial se puede obtener de varias formas. Método A) derivar de nuevo la ecuación, y resolverla como una ecuación diferencial ordinaria de segundo orden con coeficientes constantes teniendo en cuenta las condiciones iniciales. Método B) Aplicar Laplace a la ecuación (A.3) teniendo en cuenta las condiciones iniciales al aplicar Laplace y después obtener la transformada inversa. Método C): Aplicar Laplace 
al circuito y resolverlo en el dominio de Laplace, y una vez obtenida una expresión de la corriente hacer la transformada inversa.

METODO A) para obtener $i_{1}(t)$.

Derivando la ecuación integro-diferencial, se obtiene (A.5), y se mantienen las condiciones iniciales de (A.4):

$$
0=\frac{1}{C_{r}} i_{1}(t)+L_{r} \frac{d^{2} i_{1}(t)}{d t^{2}}
$$

Esta ecuación se resuelve a partir de la ecuación característica, cuya resolución ofrece dos raíces complejas conjugadas, dadas en (A.6):

$$
L_{r} D^{2}+\frac{1}{C_{r}}=0 \Rightarrow D= \pm \sqrt{\frac{-1}{L_{r} C_{r}}}= \pm j \omega_{r}
$$

donde $\omega_{r}=\left(\sqrt{L_{r} C_{r}}\right)^{-1}$ es la frecuencia de resonancia de la bobina y el condensador. Al ser dos raíces complejas conjugadas se prueba con una solución de la forma indicada en (A.7), a la que se le aplican las condiciones (A.4).

$$
i_{1}(t)=K_{1} \cos \left(\omega_{r} t\right)+K_{2} \sin \left(\omega_{r} t\right)
$$

$\mathrm{Al}$ aplicar la primera condición, se deduce que $K_{1}=I_{o}$. Con la segunda condición se deduce que $K_{2}=0$.

$$
\begin{aligned}
i_{1}(0)=I_{o} & \Rightarrow I_{o}=K_{1} * 1+K_{2} * 0 \Rightarrow K_{1}=I_{o} \\
i_{1}^{\prime}(0)=0 & \Rightarrow K_{1} \sin \left(\omega_{r} t\right)\left(-\omega_{r}\right)+\left.K_{2} \cos \left(\omega_{r} t\right) \omega_{r}\right|_{t=0}=0 \Rightarrow K_{2}=0
\end{aligned}
$$

De esta forma la solución para la ecuación diferencial es la indicada en (A.9):

$$
i_{1}(t)=I_{o} \cos \left(\omega_{r} t\right)
$$

METODO B) para obtener $i_{1}(t)$.

Aplicando Laplace a la ecuación integro-diferencial (A.3), se obtiene una expresión como la indicada en (A.10) donde ahora entran en juego los valores iniciales de las variables implicadas, donde $I_{1}(s)=\mathscr{L}\left[i_{1}(t)\right]$.

$$
\begin{aligned}
\mathscr{L}\left[V_{g}\right] & =\mathscr{L}\left[v_{C_{r}}(0)+\frac{1}{C_{r}} \int_{0}^{t} i_{1}(t) d t+L_{r} \frac{d i_{1}(t)}{d t}\right] \\
\frac{V_{g}}{s} & =\frac{v_{C_{r}}(0)}{s}+\frac{1}{C_{r} s} I_{1}(s)+L_{r} s I_{1}(s)-L_{r} i_{1}(0)
\end{aligned}
$$

Recuerde que $v_{C_{r}}(0)=V_{g}$, y que $i_{1}(0)=I_{o}$. Una vez despejada $I_{1}(s)$, se obtiene, tras aplicar Laplace, el resultado (A.11):

$$
I_{1}(s)=I_{o} \frac{s}{s^{2}+\frac{1}{L_{r} C_{r}}} \Rightarrow i_{1}(t)=\mathscr{L}^{-}\left[I_{1}(s)\right]=I_{o} \cos \left(\omega_{r} t\right)
$$


METODO C) para obtener $i_{1}(t)$.

Este método consiste en transformar el circuito al dominio de Laplace y después resolver la corriente transformada, es decir, $I_{1}(s)$ en el circuito resultante. Si se aplica Laplace a la malla izquierda del circuito equivalente de la figura A.3, se obtiene el circuito transformado de la figura A.4b, donde previamente se ha sustituido el condensador cargado a una tensión por un condensador vacío y una fuente de tensión en serie del mismo valor y polaridad que la que tenía inicialmente (figura A.4a). Igualmente, la bobina se ha sustituido por una bobina descargada y una fuente de corriente en paralelo y del mismo valor y sentido que la que estaba circulando inicialmente. En este caso, la tensión inicial del condensador es $V_{g}$ y la corriente inicial en la bobina es $I_{o}$.

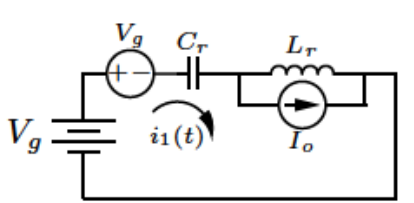

(a)

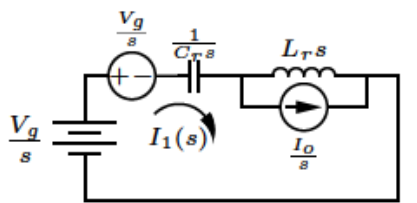

(b)

Figura A.4: Malla izquierda del circuito equivalente: a) en el dominio del tiempo; y b) en el dominio de Laplace.

Para facilitar las operaciones, se sustituye el conjunto bobina-fuente de corriente en el circuito transformado por su equivalente Thévenin. en la figura A.5 se ha representado el circuito equivalente.

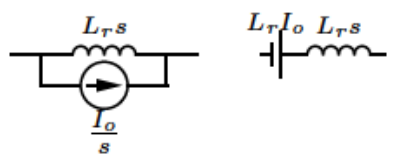

Figura A.5: Equivalente Thévenin del conjunto bobina-fuente de corriente.

De esta forma, se obtiene fácilmente la expresión para $I_{1}(s)$, como se indica en (A.12), a partir de la ecuación de la malla:

$$
\begin{aligned}
\frac{V_{g}}{s} & =\frac{V_{g}}{s}+I_{1}(s) \frac{1}{C_{r} s}-L_{r} I_{o}+I_{1}(s) L_{r} s \\
L_{r} I_{o} & =I_{1}(s)\left(\frac{1}{C_{r} s}+L_{r} s\right) \\
I_{1}(s) & =I_{o} \frac{s}{\frac{1}{L_{r} C_{r} s}+s^{2}} \Rightarrow i_{1}(t)=\mathscr{L}^{-}\left[I_{1}(s)\right]=I_{o} \cos \left(\omega_{r} t\right)
\end{aligned}
$$

Por cualquiera de los métodos se obtiene la misma expresión para la corriente $i_{1}(t)$. Una vez calculada, se obtiene la tensión en el condensador 
como (A.13) :

$$
\begin{aligned}
v_{C_{r}}(t) & =v_{C_{r}}(0)+\frac{1}{C_{r}} \int_{0}^{t} i_{1}(\lambda) d \lambda \\
& =V_{g}+\left[\frac{1}{C_{r}} I_{o} \sin \left(\omega_{r} \lambda\right) \omega_{r}^{-1}\right]_{0}^{t} \\
& =V_{g}+\frac{I_{o}}{\omega_{r} C_{r}} \sin \left(\omega_{r} t\right)
\end{aligned}
$$

la duración de esta etapa será el tiempo que tarda el condensador en alcanzar la tensión $V_{g}+V_{c}$, porque el diodo de $S 2$ se polariza en directa. Este tiempo se deduce de (A.13), y es igual $d_{2} T$, cuya expresión es (A.14).

$$
d_{2} T=\left(t_{2}-t_{1}\right)=\omega_{r}^{-1} \arcsin \left(\frac{V_{c} \omega_{r} C_{r}}{I_{o}}\right)
$$

la corriente por el diodo principal, es igual a (A.15), mientras que la corriente $i_{1}(t)$ al final de esta etapa es $I^{\prime}$, y tiene un valor ligeramente inferior a $I_{o}$.

$$
i_{D}(t)=I_{o}-i_{1}(t)=I_{o}\left(1-\cos \left(\omega_{r} t\right)\right)
$$

\section{A.1.3. Etapa 3. $\left(t_{2}<t<t_{3}\right)$}

El diodo del interruptor auxiliar $S_{2}$ empieza a conducir. A partir de este instante, se puede cerrar este interruptor a tensión cero porque está circulando corriente por su diodo. En la figura A.6 se muestran los elementos que intervienen y el circuito equivalente, donde $i_{c}(t)$ representa la corriente que circula por el condensador de clamp.

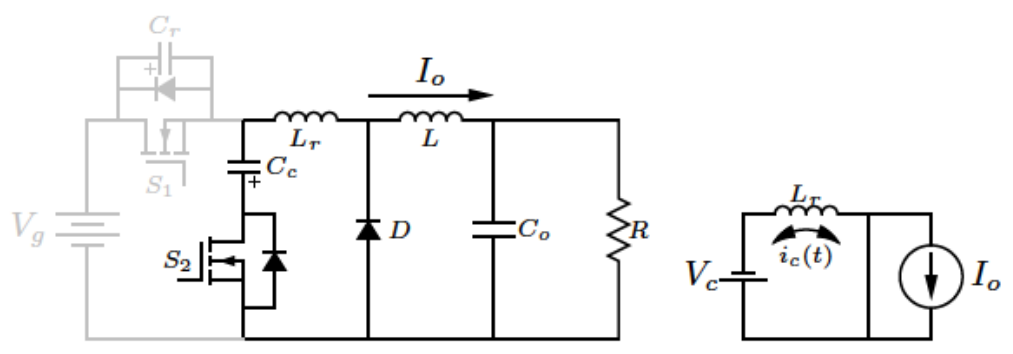

Figura A.6: Reductor ZVS durante la etapa 3. Circuito equivalente.

Nótese que el condensador de clamp es grande para la energía que absorbe por lo que su tensión se mantiene prácticamente constante durante todo este intervalo y de ahí que se represente como una fuente de tensión con la polaridad indicada. Además la corriente $i_{c}(t)$ circula inicialmente en un sentido y luego se invierte cuando la bobina se ha descargado completamente sobre $C_{c}$. Pero como el interruptor $S_{2}$ permanece cerrado, el condensador cederá esta energía extra de nuevo a la bobina, haciendo que la corriente 
circule en el sentido opuesto al inicial. En este caso, la corriente circulará por $S_{2}$ y no por su diodo.

La relación entre la tensión de clamp, y la duración de este intervalo se obtiene resolviendo el circuito equivalente. Para ello hay que resolver la malla izquierda de este circuito para obtener $i_{c}(t)$. En esta malla, la tensión es constante e igual a $V_{c}$. La bobina resonante se descarga a tensión constante y su expresión se obtiene resolviendo la ec.diferencial de primer orden con condiciones iniciales de (A.16):

$$
\begin{aligned}
-V_{c} & =v_{L_{r}}=L_{r} \frac{d i_{c}(t)}{d t} \\
i_{c}(0) & =I^{\prime}
\end{aligned}
$$

Haciendo la separación de variables en cada miembro de (A.16), e integrando se obtiene una solución general, en la que se despeja la constante de integración aplicando la condición inicial.

$$
\begin{aligned}
\frac{-V_{c}}{L_{r}} d t & =d i_{c}(t) \\
\frac{-V_{c}}{L_{r}} \int d t & =\int d i_{c}(t) \\
i_{c}(t) & =\frac{-V_{c}}{L_{r}} t+K \\
i_{c}(0) & =I^{\prime} \Rightarrow K=I^{\prime} \Rightarrow i_{c}(t)=I^{\prime}-\frac{V_{c}}{L_{r}} t
\end{aligned}
$$

En la solución dada en (A.17) se comprueba que $i_{c}(t)$ es decreciente y llega a ser negativa. La duración de esta etapaes $d_{3} T=\left(t_{3}-t_{2}\right)$.

Al final de este periodo, la corriente alcanza un valor igual a $I^{\prime}$ pero en sentido contrario. De esta relación se deduce el valor de la tensión de clamp en función de la bobina resonante y el ciclo de trabajo. Véase (A.18).

$$
i_{c}\left(t_{3}\right)=-I^{\prime} \Rightarrow-I^{\prime}=I^{\prime}-\frac{V_{c}}{L_{r}} d_{3} T \Rightarrow V_{c}=\frac{2 I^{\prime} L_{r}}{d_{3} T}
$$

También se puede razonar de otra forma para llegar a la misma conclusión: Si se habla de régimen permanente, la energía neta en el condensador de clamp durante un ciclo de trabajo, es decir, la energía que toma y que devuelve durante ese tiempo, debe ser nula. Como se ha supuesto un valor de $C_{c}$ suficientemente grande para que $V_{c}$ sea constante, al aplicar esta suposición se llega a que el valor medio de (A.17) es nulo. Dicho de otra forma, el balance de carga en $C_{c}$ deber se nulo. Siendo así, resulta que

$$
\begin{gathered}
\Delta E=0 \Rightarrow \int_{t_{0}}^{t_{0}+T} i_{c}(t) d t=\int_{t_{2}}^{t_{3}} i_{c}(t) d t=\int_{0}^{d_{3} T}\left[I^{\prime}-\frac{V_{c}}{L_{r}} t\right] d t=0 \\
{\left[I^{\prime} t-\frac{V_{c}}{2 L_{r}} t^{2}\right]_{0}^{d_{3} T}=0 \Rightarrow V_{c}=\frac{2 I^{\prime} L_{r}}{d_{3} T}}
\end{gathered}
$$


Este resultado sustituido en (A.17) permite obtener el valor de $i_{c}\left(t_{3}\right)$ para el régimen permanente. Así se indica en (A.20).

$$
i_{c}\left(t_{3}\right)=I^{\prime}-\frac{2 I^{\prime} L_{r}}{L_{r} d_{3} T} d_{3} T=-I^{\prime}
$$

En el caso de un transitorio, la tensión de clamp evolucionará en cada periodo que dure el transitorio de un valor a otro, y el balance de carga no será nulo, y por tanto, no se puede saber a priori el valor de $i_{c}\left(t_{3}\right)$ al final de cada periodo.

Ahora se abre el interruptor, y lo hace a tensión cero porque e condensador $C_{r}$ se había quedado cargado a una tensión $V_{g}+V_{c}$, de forma que el drenador de $S_{2}$ está a tensión cero.

La corriente que pasa por el diodo principal alcanza un valor máximo de (A.21).

$$
i_{D}(t)=I_{o}-i_{c}(t) \Rightarrow I_{D, \max }=i_{D}\left(t_{3}\right)=I_{o}-i_{c}\left(t_{3}\right)=I_{o}+I^{\prime}
$$

\section{A.1.4. Etapa 4. $\left(t_{3}<t<t_{4}\right)$}

Este intervalo se inicia con la apertura de $S_{2}$ a tensión nula. La corriente en $L_{r}$ tiene un sentido inicial opuesto al inicial de la etapa anterior, y ésta ya no puede circular ni por $S_{2}$ ni por su diodo, luego deberá circular a través de $C_{r}$. Dicho de otra forma, la bobina $L_{r}$ acumulará energía, pero esta vez de $C_{r}$ que se descarga de forma resonante sobre aquella. La corriente $i_{4}(t)$ experimenta durante este intervalo una evolución resonante, a la vez que la tensión $V_{C_{r}}(t)$ disminuye hasta hacerse cero, que es donde concluye esta etapa. A partir de este instante, la conducción de la corriente se realiza sobre el diodo $D_{1}$. En el circuito de la figura A.7 se muestran los elementos que están involucrados en esta etapa.
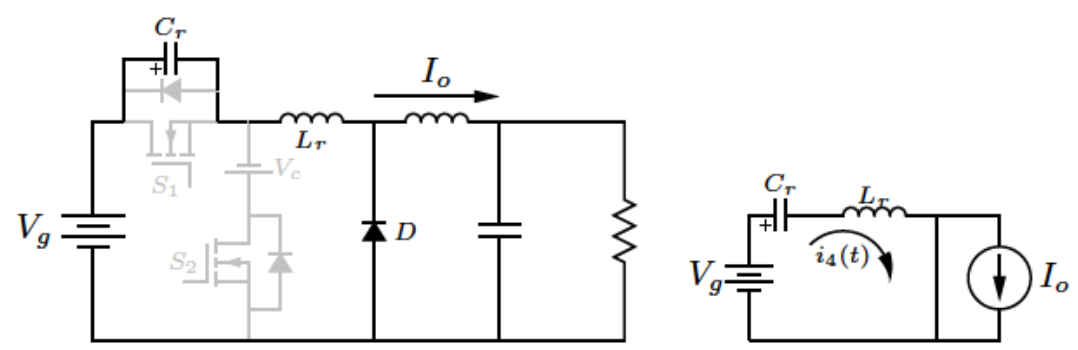

Figura A.7: Reductor ZVS durante la etapa 4. Circuito equivalente.

Una condición necesaria, sobre la que volveremos mas adelante, es que la energía acumulada en $L_{r}$ debe ser como poco igual a la energía que acumula $C_{r}$ para que la bobina consiga descargar por completo al condensador antes de que se elimine. Para la obtención de $i_{4}(t)$ (donde se ha mantenido 
el mismo sentido que en los casos de las etapas anteriores), hay que plantear le ecuación de la malla izquierda del circuito equivalente y resolver la ecuación diferencial resultante utilizando las condiciones iniciales conocidas. La ecuación resultante es una ecuación integro-diferencial con coeficientes constantes dada por (A.22):

$$
V_{g}=v_{C_{r}}(t)+v_{L_{r}}(t)=v_{C_{r}}(0)+\frac{1}{C_{r}} \int_{0}^{t} i_{4}(t) d t+L_{r} \frac{d i_{4}(t)}{d t}
$$

Las condiciones iniciales son (A.23):

$$
\begin{aligned}
& i_{4}(0)=-I^{\prime} \\
& i_{4}^{\prime}(0)=\frac{1}{L_{r}} v_{L_{r}}(0)=\frac{1}{L_{r}}\left[V_{g}-v_{C_{r}}(0)\right]=-\frac{V_{c}}{L_{r}}
\end{aligned}
$$

donde la intensidad debe cumplir la condición de continuidad, pero no la derivabilidad.

La resolución de esta ecuación diferencial, tal y como se ha hecho en la ETAPA 2, se puede obtener de varias formas. Método A) derivar de nuevo la ecuación, y resolverla como una ecuación diferencial ordinaria de segundo orden con coeficientes constantes teniendo en cuenta las condiciones iniciales. Método B) Aplicar Laplace a la ecuación (A.22) teniendo en cuenta las condiciones iniciales al aplicar Laplace y después obtener la transformada inversa. Método C): Aplicar Laplace al circuito y resolverlo en el dominio de Laplace, y una vez obtenida una expresión de la corriente hacer la transformada inversa.

METODO A) para obtener $i_{4}(t)$.

Derivando la ecuación integro-diferencial, se obtiene (A.24), y se mantienen las condiciones iniciales de (A.23):

$$
0=\frac{1}{C_{r}} i_{4}(t)+L_{r} \frac{d^{2} i_{4}(t)}{d t^{2}}
$$

Esta ecuación se resuelve a partir de la ecuación característica, cuya resolución ofrece dos raíces complejas conjugadas, dadas en (A.25):

$$
0=\frac{1}{C_{r}}+L_{r} D^{2} \Rightarrow D= \pm \sqrt{\frac{-1}{L_{r} C_{r}}}= \pm j \omega_{r}
$$

donde $\omega_{r}$ ya se había definido durante la ETAPA 2 como la frecuencia de resonancia bobina-condensador, y es igual a $\left(\sqrt{L_{r} C_{r}}\right)^{-1}$. Al ser dos raíces complejas conjugadas se prueba con una solución de la forma indicada en (A.26), a la que se le aplican las condiciones (A.23).

$$
i_{4}(t)=K_{3} \cos \left(\omega_{r} t\right)+K_{4} \sin \left(\omega_{r} t\right)
$$


$\mathrm{Al}$ aplicar la primera condición, se deduce que $K_{3}=-I^{\prime}$. Con la segunda condición se deduce el valor de $K_{4}$.

$$
\begin{aligned}
i_{4}(0) & =-I^{\prime} \Rightarrow I^{\prime}=K_{3} * 1+K_{4} * 0 \Rightarrow K_{3}=-I^{\prime} . \\
i_{4}^{\prime}(0) & =-\frac{V_{c}}{L_{r}} \Rightarrow K_{3} \sin \left(\omega_{r} t\right)\left(-\omega_{r}\right)+\left.K_{4} \cos \left(\omega_{r} t\right) \omega_{r}\right|_{t=0}=0 \Rightarrow \\
& \Rightarrow K_{4}=-\frac{V_{c}}{\omega_{r} L_{r}}
\end{aligned}
$$

De esta forma la solución para la ecuación diferencial es la indicada en (A.28):

$$
i_{4}(t)=-I^{\prime} \cos \left(\omega_{r} t\right)-\frac{V_{c}}{\omega_{r} L_{r}} \sin \omega_{r} t
$$

METODO B) para obtener $\left.i_{4} t\right)$.

Aplicando Laplace a la ecuación integro-diferencial (A.22), se obtiene una expresión como la indicada en (A.29) donde ahora entran en juego los valores iniciales de las variables implicadas.

$$
\begin{aligned}
\mathscr{L}\left[V_{g}\right] & =\mathscr{L}\left[v_{C_{r}}(0)+\frac{1}{C_{r}} \int_{0}^{t} i_{4}(t) d t+L_{r} \frac{d i_{4}(t)}{d t}\right] \\
\frac{V_{g}}{s} & =\frac{V_{C_{r}}(0)}{s}+\frac{1}{C_{r} s} I_{4}(s)+L_{r} s I_{4}(s)-L_{r} i_{4}(0)
\end{aligned}
$$

Recuerde que $V_{C_{r}}(0)=V_{g}+V_{c}$, y que $i_{4}(0)=-I^{\prime}$. Una vez despejada $I_{4}(s)$, se obtiene, tras aplicar Laplace, el resultado (A.30):

$$
\begin{aligned}
& I_{4}(s)=-I^{\prime} \frac{s}{s^{2}+\frac{1}{L_{r} C_{r}}}-\frac{V_{c}}{L_{r}} \frac{1}{s^{2}+\frac{1}{L_{r} C_{r}}} \\
& i_{4}(t)=\mathscr{L}^{-}\left[I_{4}(s)\right]=-I^{\prime} \cos \left(\omega_{r} t\right)-\frac{V_{c}}{\omega_{r} L_{r}} \sin \omega_{r} t
\end{aligned}
$$

METODO C) para obtener $i_{4}(t)$.

Este método consiste en transformar el circuito al dominio de Laplace y después resolver la corriente transformada, es decir, $I_{4}(s)$ en el circuito resultante. Si se aplica Laplace a la malla izquierda del circuito equivalente de la figura A.7, se obtiene el circuito transformado de la figura A.8b, donde previamente se ha sustituido el condensador cargado a una tensión por un condensador vacío y una fuente de tensión en serie del mismo valor y polaridad que la que tenía inicialmente (figura A.8a). Igualmente, la bobina se ha sustituido por una bobina descargada y una fuente de corriente en paralelo y del mismo valor y sentido que la que estaba circulando inicialmente. En este caso, la tensión inicial del condensador es $V_{g}+V_{c}$ y la corriente incial en la bobina es $-I^{\prime}$. 


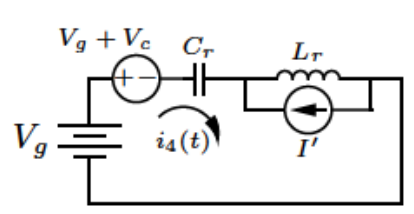

(a)

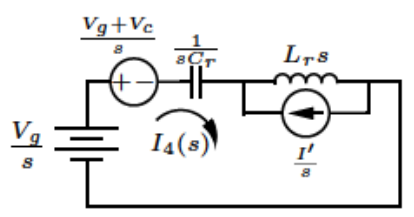

(b)

Figura A.8: Malla izquierda del circuito equivalente: a) en el dominio del tiempo; y b) en el dominio de Laplace.

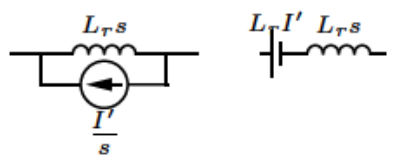

Figura A.9: Equivalente Thévenin del conjunto bobina-fuente de corriente.

Para facilitar las operaciones, se sustituye el conjunto bobina-fuente de corriente en el circuito transformado por su equivalente Thévenin. en la figura A.9 se ha representado el circuito equivalente.

De esta forma, se obtiene fácilmente la expresión para $I_{4}(s)$, como se indica en (A.31), a partir de la ecuación de la malla:

$$
\begin{aligned}
\frac{V_{g}}{s}-\frac{V_{g}+V_{c}}{s}-L_{r} I^{\prime} & =I_{4}(s) \frac{1}{C_{r} s}+I_{4}(s) L_{r} s \\
-\frac{V_{c}}{s}-L_{r} I^{\prime} & =I_{4}(s)\left(\frac{1+L_{r} C_{r} s^{2}}{C_{r} s}\right) \\
I_{4}(s) & =\left(-\frac{V_{c}}{s}-L_{r} I^{\prime}\right)\left(\frac{C_{r} s}{1+L_{r} C_{r} s^{2}}\right) \Rightarrow \\
i_{4}(t) & =\mathscr{L}^{-}\left[I_{4}(s)\right]=-\frac{V_{c}}{\omega_{r} L_{r}} \sin \omega_{r} t-I^{\prime} \cos \left(\omega_{r} t\right)
\end{aligned}
$$

Por cualquiera de los métodos se obtiene la misma expresión para la corriente $i_{4}(t)$. Una vez calculada, se obtiene la tensión en el condensador como (A.32) :

$$
\begin{aligned}
v_{C_{r}}(t) & =v_{C_{r}}(0)+\frac{1}{C_{r}} \int_{0}^{t} i_{4}(\lambda) d \lambda \\
& =V_{g}+V_{c}-\left[\frac{1}{C_{r}} I^{\prime} \sin \left(\omega_{r} \lambda\right) \omega_{r}^{-1}\right]_{0}^{t}-\left[\frac{V_{c}}{C_{r} L_{r} \omega_{r}} \cos \left(\omega_{r} \lambda\right)\left(-\omega_{r}\right)^{-1}\right]_{0}^{t} \\
& =V_{g}+V_{c} \cos \omega_{r} t-\frac{I^{\prime}}{\omega_{r} C_{r}} \sin \left(\omega_{r} t\right)
\end{aligned}
$$

Esta expresión se puede simplificar, agrupando los dos términos de $\sin ()$ y $\cos ()$ en uno solo con amplitud y desfase. De esta forma la tensión en el 
condensador es (A.33):

$$
v_{C_{r}}(t)=V_{g}+V_{r} \cos \left(\omega_{r} t+\phi_{r}\right)
$$

siendo

$$
\begin{aligned}
& V_{r}=\sqrt{V_{c}^{2}+\left(\frac{I^{\prime}}{\omega_{r} C_{r}}\right)^{2}}=\sqrt{V_{c}^{2}+\frac{L_{r}}{C_{r}} I^{\prime 2}} \\
& \phi_{r}=\arctan \left(\frac{I^{\prime}}{V_{c} \omega_{r} C_{r}}\right)
\end{aligned}
$$

La duración de esta etapa, $d_{4} T=\left(t_{4}-t_{3}\right)$, será el tiempo que tarda el condensador en descargase por completo y su tensión es nula, instante en el cual la energía de $L_{r}$ empezará a circular a través del diodo de $S 1$. En este instante, la bobina se ha cargado a una corriente de valor negativo, $I^{\prime \prime}$, y mas pequeño que $-I^{\prime}$, puesto que la bobina toma la carga que le cede el condensador.

Para que la tensión pueda llegar a anularse se debe cumplir que la amplitud $V_{r}$ sea mayor, o al menos igual, que $V_{g}$, luego:

$$
\begin{aligned}
\sqrt{V_{c}^{2}+\frac{L_{r}}{C_{r}} I^{\prime 2}} & \geq V_{g} \\
V_{c}^{2}+\frac{L_{r}}{C_{r}} I^{\prime 2} & \geq V_{g}^{2} \\
C_{r} V_{c}^{2}+L_{r} I^{\prime 2} & \geq C_{r} V_{g}^{2}
\end{aligned}
$$

que indica que la bobina descarga al condensador con la energía que tiene acumulada. Lo que sucede es que antes de empezar a descargarse la bobina, esta aumenta su energía por una parte que ya le cede el propio condensador. Ésta, junto con la que tiene acumulada sirven para descargar el condensador. Se deberá cumplir por tanto la condición indicada enA.35.

La corriente por el diodo principal, es igual a (A.36).

$$
i_{D}(t)=I_{o}-i_{4}(t)
$$

\section{A.1.5. Etapa 5. $\left(t_{4}<t<t_{5}\right)$}

Esta etapa comienza en el instante en que $C_{r}$ se descarga totalmente y su tensión es nula. La corriente circulante por $L_{r}$ necesita un camino para seguir circulando, y lo hace a través del diodo de $S_{1}$, que pasa a estar polarizado en directa. Debido a esto, el interruptor $S_{1}$ puede entrar en conducción a tensión cero en cualquier instante de tiempo en el que circule corriente por su diodo, que será mientras la corriente por $L_{r}$ mantenga el mismo sentido de circulación. Una vez cerrado $S_{1}$, la bobina cede la energía a la fuente, y cuando está descargada, se carga de nuevo con otra polaridad, por lo que la corriente $i_{5}(t)$ terminará circulando en un sentido contrario al que tenía 


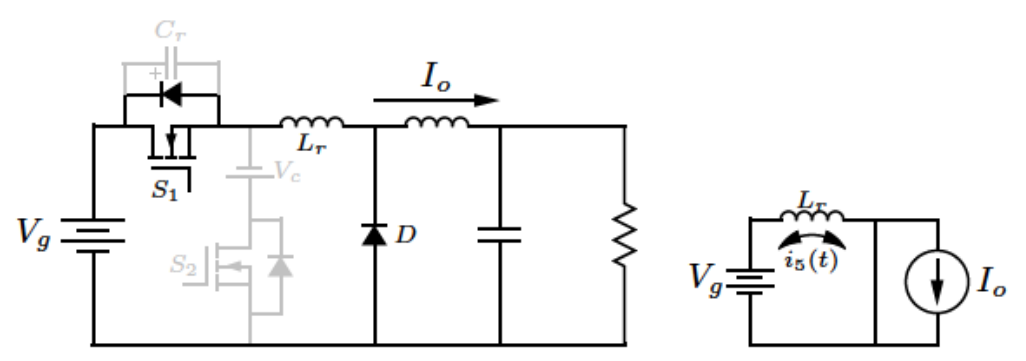

Figura A.10: Reductor ZVS durante la etapa 5. Circuito equivalente.

al iniciar esta etapa. En la figura A.10 se muestran los elementos que están involucrados en esta etapa y el circuito equivalente.

Esta etapa terminará cuando el valor de $i_{5}(t)$ alcance el valor de $I_{o}$, instante en el cual, la corriente por el diodo principal, $i_{D}(t)$ se anulará y pasará a la siguiente etapa. Observando la malla izquierda del circuito equivalente, la corriente circulante evoluciona en función de una tensión constante. La relación entre esta tensión y la duración de este intervalo se obtiene resolviendo dicho circuito. En esta malla, la tensión es igual a $V_{g}$. La bobina resonante se descarga a tensión constante y su expresión se obtiene resolviendo la ec.diferencial de primer orden con condiciones iniciales de (A.37):

$$
\begin{aligned}
V_{g} & =v_{L_{r}}=L_{r} \frac{d i_{5}(t)}{d t} \\
i_{5}(0) & =I^{\prime \prime}
\end{aligned}
$$

Haciendo la separación de variables en cada miembro de (A.37), e integrando se obtiene una solución general, en la que se despeja la constante de integración aplicando la condición inicial.

$$
\begin{aligned}
\frac{V_{g}}{L_{r}} d t & =d i_{5}(t) \\
\frac{V_{g}}{L_{r}} \int d t & =\int d i_{5}(t) \\
i_{5}(t) & =\frac{V_{g}}{L_{r}} t+K_{5} \\
i_{5}(0) & =I^{\prime \prime} \Rightarrow K_{5}=I^{\prime \prime} \Rightarrow i_{5}(t)=I^{\prime \prime}+\frac{V_{g}}{L_{r}} t
\end{aligned}
$$

De la expresión (A.38) se puede deducir la duración de este intervalo, que es el tiempo que tarda en alcanzarse el valor de $I_{o}$, que es igual a

$$
d_{5} T=t_{5}-t_{4}=\left(I_{o}-I^{\prime \prime}\right) \frac{L_{r}}{V_{g}}
$$




\section{A.1.6. Etapa 6. $\left(t_{5}<t<t_{6}\right)$}

Esta etapa comienza cuando el diodo $D$ deja de conducir y concluye cuando se abre el interruptor $S_{1}$, que conmuta a tensión cero puesto que el condensador $C_{r}$ está descargado. A partir de aquí comienza un nuevo ciclo, es decir, $t_{6}=t_{0}+T$. Los elementos que intervienen el circuito pasan a ser los indicados en la figura A.11.

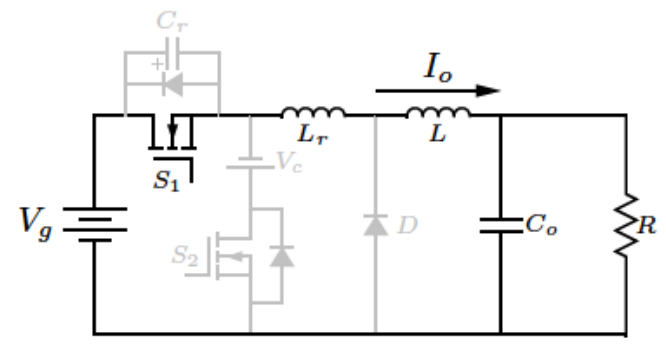

Figura A.11: Reductor ZVS durante la etapa 6.

Uno de los objetivos de la red ZVS es reducir las pérdidas, y se consigue puesto que todas las conmutaciones son a tensión cero. $S_{1}$ pasa a OFF al principio de la ETAPA 1 y lo hace a tensión cero puesto que el condensador $C_{r}$ está descargado. $S_{2}$ pasa a $\mathrm{ON}$ al principio de la ETAPA 3 y lo hace a tensión cero puesto que está circulando corriente por su diodo. $S_{2}$ pasa a $\mathrm{OFF}$ al final de la ETAPA 3 y lo hace a tensión cero puesto que el drenador está a $0 \mathrm{~V}$. $S_{1}$ pasa a $\mathrm{ON}$ en la ETAPA 5 mientras circula corriente por su diodo.

Nótese que se supone cumplida la ecuación (A.35) relativa que la energía almacenada en $L_{r}$ debida a la intensidad circulante, debe ser mayor que la energía del condensador del MOSFET, $C_{r}$, para descargarlo desde la tensión $V_{g}+V_{c}$ hasta cero. En esta ecuación hay que hacer notar que $I^{\prime}$ representa el valor medio de la corriente en ese instante. Utilizando los valores instantáneos, habría que sustituir ese valor por el valor de pico.

\section{A.2. Análisis en régimen permanente.}

\section{A.2.1. Sin considerar rizado.}

Se ha considerado una bobina $L$ lo suficientemente grande como para suponer corriente constante, de valor $I=I_{o}$, que es mucho mayor que la bobina resonante $L_{r}$. De forma similar, el valor de los condensadores $C_{o} \mathrm{y}$ $C_{c}$, es tal que la tensión en sus extremos se mantiene constante, de valores $V_{o}$ y $V_{c}$, durante todo el periodo $T$.

Después de estudiar el funcionamiento del circuito con estas mismas consideraciones, se ha representado en la Figura A.12 las principales magnitudes 
a lo largo de las seis etapas por las que evoluciona esta topología durante un ciclo completo. La duración de cualquiera de estas etapas se indica con la letra $d$ y un subíndice que hace referencia al número de etapa. Por ejemplo, la duración de la etapa 5 es igual a $t_{5}-t_{4}=d_{5} T$.

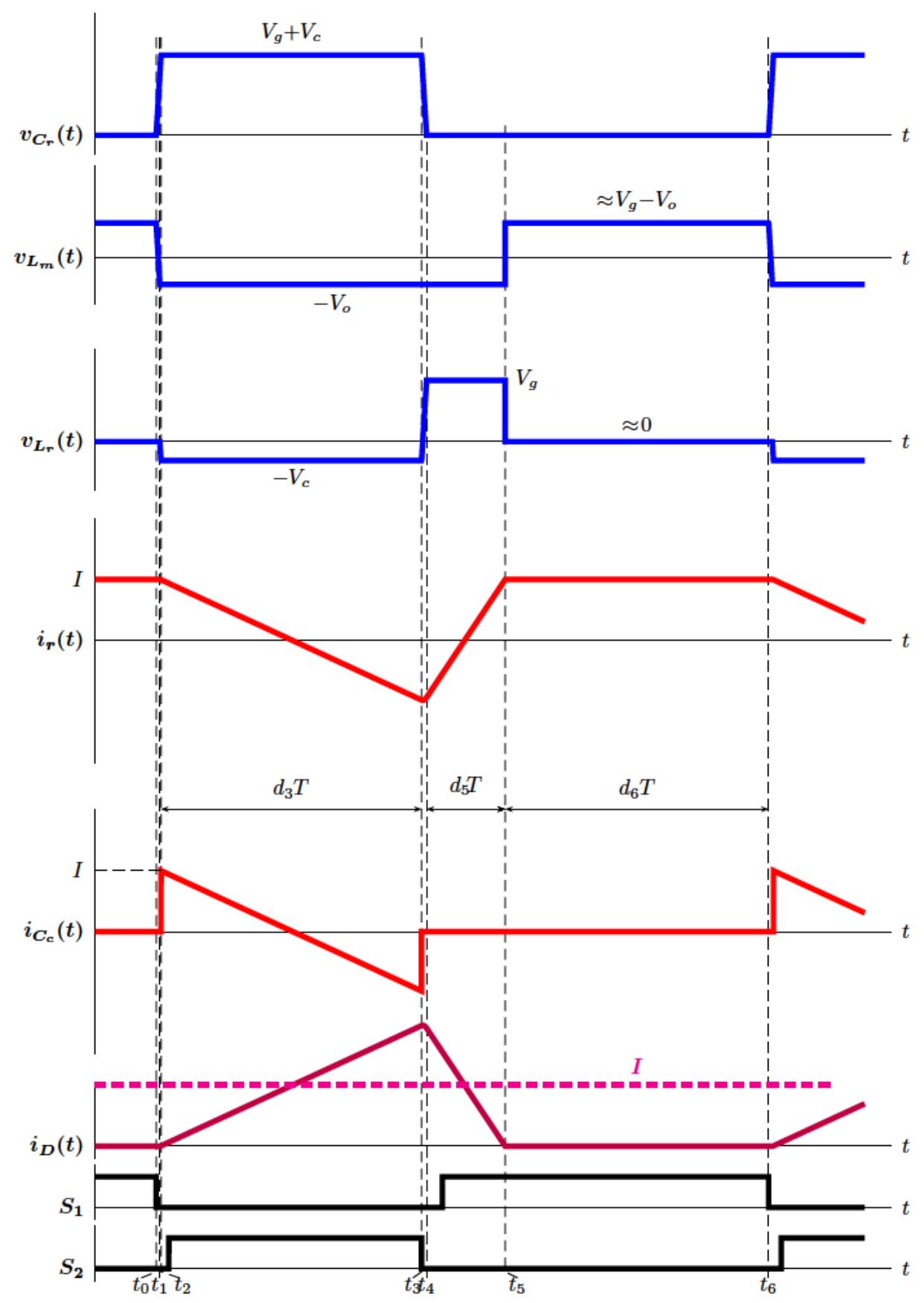

Figura A.12: Curvas principales de un reductor ZVS. 
Para el análisis se desprecian las etapas 1, 2 y 4, puesto que la frecuencia característica del conjunto $L_{r}-C_{r}$ es mucho mas grande que la frecuencia de conmutación y la carga-descarga de $C_{r}$ es prácticamente instantánea. De esta forma solo se consideran tres etapas en el análisis. En este caso se considera que $I^{\prime} \approx I$ y $I^{\prime \prime} \approx-I$. Y como la duración de la etapa 3 es el tiempo que no está cerrado el interruptor principal, resulta que

$$
\begin{aligned}
d_{3} T & =(1-D) T \\
d_{5} T+d_{6} T & =D T
\end{aligned}
$$

Las ecuaciones del régimen permanente se obtienen al considerar nulo tanto el balance voltios-segundo en las bobinas $L$ y $L_{r}$ como la carga neta en los condensadores $C_{o}$ y $C_{c}$.

\section{TENSION MEDIA EN BOBINA PRINCIPAL.}

La tensión media en la bobina $L$ es nula. De aquí se obtiene una expresión para la tensión de salida en función de la entrada:

$$
\frac{1}{\not{X}}\left[-V_{o}\left(d_{3}+d_{5}\right) \not{X}+\left(V_{g}-V_{o}\right) d_{6} \not{X}\right]=0 \Rightarrow V_{o}=V_{g} d_{6}
$$

A $d_{6}$ se le puede llamar ciclo efectivo de trabajo, $D_{\text {ef }}$, puesto que al identificar la expresión que relaciona la tensión de salida con la entrada en un reductor, el factor multiplicador es el ciclo de trabajo. También se puede identificar con el tiempo en el que la bobina principal del convertidor está absorbiendo energía. En el caso del reductor ZVS, el valor del ciclo efectivo es menor que el valor del ciclo de trabajo empleado en las señales de disparo.

\section{TENSION MEDIA EN BOBINA RESONANTE.}

La tensión media en la bobina $L_{r}$ es nula. De aquí se obtiene una expresión para la tensión de $C_{c}$ en función de la tensión de entrada:

$$
\frac{1}{\not{X}}\left[-V_{c} d_{3} \not{X}+V_{g} d_{5} \not{T}\right]=0 \Rightarrow V_{c}=V_{g} \frac{d_{5}}{d_{3}}
$$

Sumando A.41 y A.42 también se puede despejar $V_{c}$ como:

$$
V_{c}=V_{g} \frac{d_{5}+d_{6}}{d_{3}}-V_{o} \frac{1}{d_{3}}
$$

CORRIENTE MEDIA EN EL CONDENSADOR DE CLAMP.

La intensidad media en el condensador de clamp es nula. Entonces:

$$
\begin{aligned}
& \int_{t_{0}}^{t_{0}+T} i_{c}(t) d t=\int_{t_{2}}^{t_{3}} i_{c}(t) d t=\int_{0}^{d_{3} T}\left[I_{o}-\frac{V_{c}}{L_{r}} t\right] d t=0 \\
& {\left[I_{o} t-\frac{V_{c}}{2 L_{r}} t^{2}\right]_{0}^{d_{3} T}=0 \Rightarrow V_{c}=\frac{2 I_{o} L_{r}}{d_{3} T}}
\end{aligned}
$$


Sustituyendo este valor en la expresión empleada para $i_{c}(t)$ se puede obtener el valor de la intensidad del condensador transcurrido el tiempo $d_{3} T$. Entonces:

$$
i_{c}\left(d_{3} T\right)=I_{o}-\frac{V_{c}}{L_{r}} d_{3} T=I_{o}-\frac{\frac{2 I_{o} \mathscr{L}_{r}}{d_{3} T}}{L_{r}} d_{3} T=-I_{o}
$$

\section{DURACIÓN DEL INTERVALO $d_{5}$.}

Se puede obtener a partir de igualar las expresiones de $V_{c}$ dadas en (A.42) y en (A.44). De esta forma:

$$
V_{c}=\frac{2 I_{o} L_{r}}{d_{3} T}=V_{g} \frac{d_{5}}{d_{3}} \Rightarrow d_{5}=\frac{2 I_{o} L_{r}}{V_{g} T}
$$

Expresión a la que se puede llegar también a partir de la tensión en la bobina $L r$ durante la etapa 5, puesto que es conocida la variación que experimenta la intensidad circulante por esa bobina y la tensión a la que está sometida.

$$
\begin{aligned}
\Delta i_{r}(t) & =i_{r}\left(t_{5}\right)-i_{r}\left(t_{4}\right) \approx i_{r}\left(t_{5}\right)-i_{r}\left(t_{3}\right)=I-(-I)=2 I \\
\Delta i_{r}(t) & =\frac{1}{L_{r}} \Delta v_{L_{r}}(t) \Delta t \equiv \frac{1}{L_{r}} V_{g} d_{5} T \\
\Rightarrow d_{5} & =\frac{2 I L_{r}}{V_{g} T}
\end{aligned}
$$

\section{TENSION DE SALIDA.}

Utilizando (A.46) junto con (A.40) en (A.41) se obtiene una expresión para la tensión de salida en régimen permanente:

$$
V_{o}=V_{g} d_{6}=V_{g}\left(D-d_{5}\right)=V_{g} D-Y_{g} \frac{2 I_{o} L_{r}}{V_{g} T}=V_{g} D-I_{o} 2 L_{r} f
$$

A esta expresión también se llega igualando (A.43) con (A.44) y despejando $V_{o}$. Como se puede observar, la tensión de salida de un reductor ZVS depende de la intensidad a la salida.

Esta expresión pone de manifiesto un elemento, equivalente a una resistencia, por el que pasa la intensidad $I_{o}$, que se puede considerar en serie con la bobina principal puesto que, en un reductor, esta intensidad es la misma que pasa por dicha bobina. Su expresión sería (A.49):

$$
R_{e s L}=2 L_{r} f
$$

\section{CARACTERÍSTICA DE SALIDA.}


Es fácil obtener la relación entre la tensión de salida y la intensidad de salida a partir de (A.48), puesto que esa expresión ya es de la forma $V_{o}=f\left(I_{o}\right)$. Por tanto:

$$
V_{o}\left(I_{o}\right)=V_{g} D-I_{o} 2 L_{r} f
$$

La derivada de esta expresión respecto a la intensidad indica como varía la tensión de salida ante las variaciones de la intensidad y tiene dimensiones de resistencia. La expresión de esta derivada es:

$$
\frac{d V_{o}}{d I_{o}}=-2 L_{r} f
$$

que tiene el mismo valor independientemente del punto donde se evalúe.

\section{A.2.2. Considerando rizado.}

Si se considera que la bobina $L$ no es lo suficientemente grande como para suponer corriente constante, $I$, los circuitos equivalentes empleados para el análisis del circuito son diferentes y la relación entre la bobina principal y resonante aparece en las nuevas expresiones de las principales magnitudes.

Los circuitos equivalentes están representados en la Figura A.13 donde se ha considerado la tensión constante en los condensadores $C_{o}$ y $C_{c}$, mientras que las principales magnitudes aparecen en la Figura A.14.

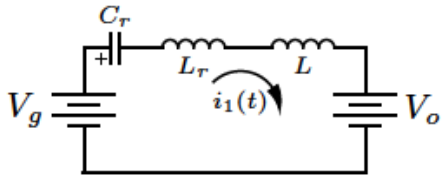

(a)

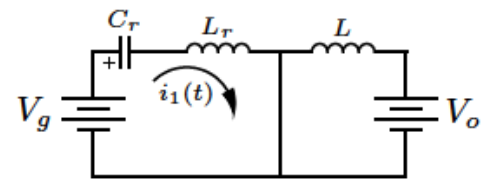

(b)

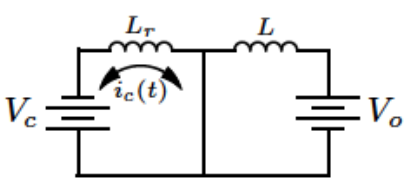

(c)

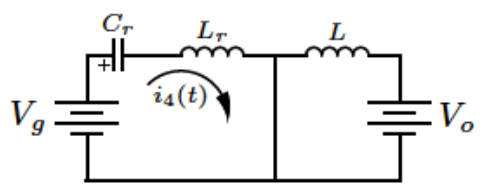

(d)

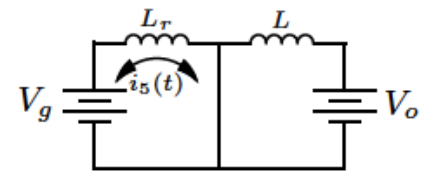

(e)

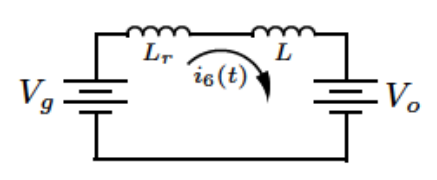

(f)

Figura A.13: Circuitos empleados para el análisis del reductor ZVS considerando rizado durante un ciclo completo: (a) Etapa 1. (b) Etapa 2. (c) Etapa 3. (d) Etapa 4. (e) Etapa 5. (f) Etapa 6.

Planteando el balance voltios-segundo en las bobinas $L$ y $L_{r}$ y la carga neta nula en los condensadores $C_{o}$ y $C_{c}$ con las nuevas curvas, se obtienen otras ecuaciones del régimen permanente. Se desprecian las etapas 1,2 y 4 , y solo intervienen tres etapas.

TENSION MEDIA EN BOBINA PRINCIPAL CON RIZADO. 


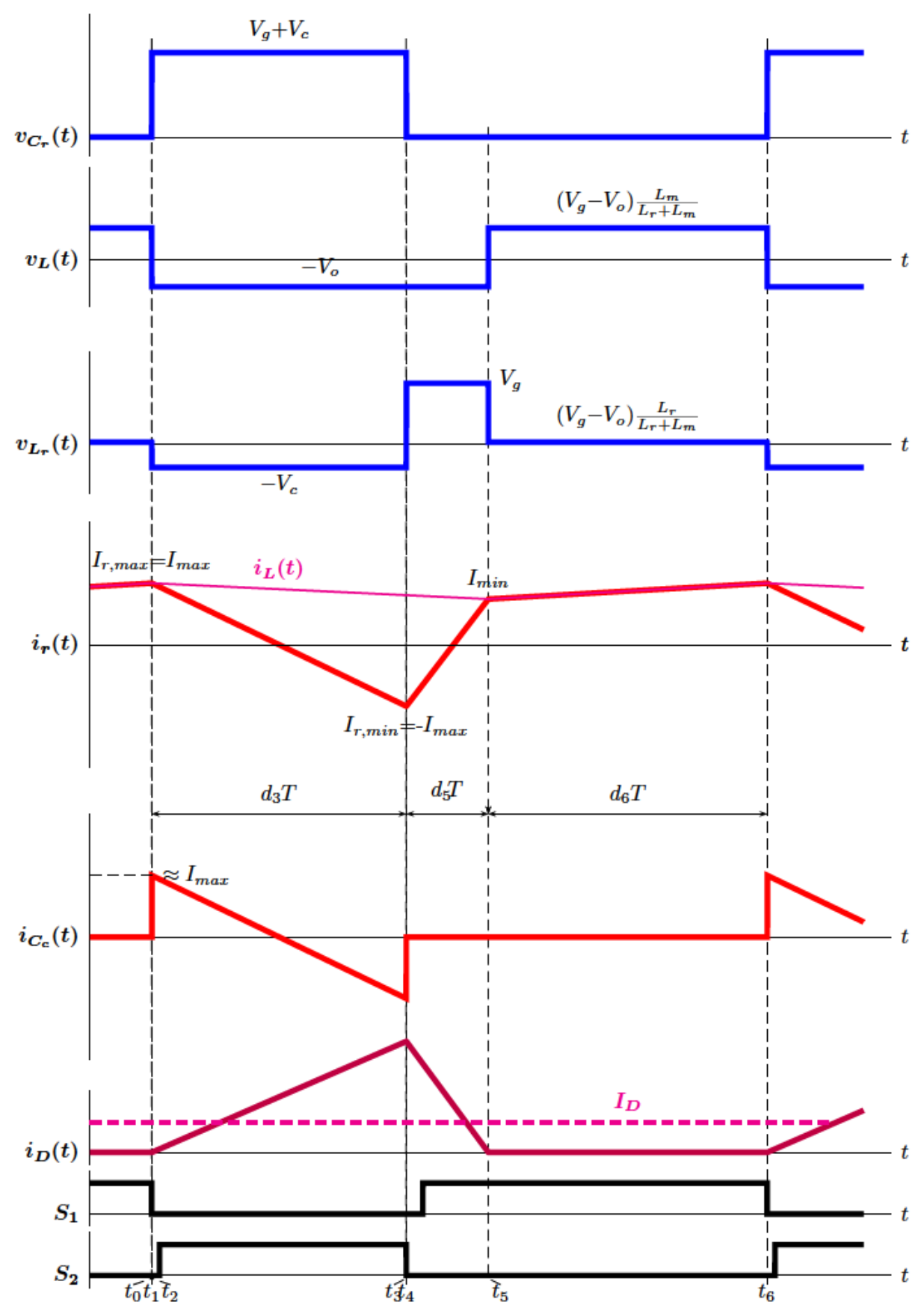

Figura A.14: Curvas principales de un reductor ZVS considerando rizado.

Como la tensión media en la bobina $L$ es nula, resulta que:

$$
\begin{aligned}
& \frac{1}{\mathscr{T}}\left[-V_{o}\left(d_{3}+d_{5}\right) \not{T}+\left(V_{g}-V_{o}\right) \frac{L}{L+L_{r}} d_{6} X\right]=0 \\
& \Rightarrow V_{o}=V_{g} \frac{d_{6} \frac{L}{L+L_{r}}}{1-d_{6} \frac{L_{r}}{L+L_{r}}}
\end{aligned}
$$


TENSION MEDIA EN BOBINA RESONANTE CON RIZADO.

Como la tensión media en la bobina $L_{r}$ es nula, entonces:

$$
\begin{aligned}
& \frac{1}{\not{X}}\left[-V_{c} d_{3} \not{X}+V_{g} d_{5} \not{X}+\left(V_{g}-V_{o}\right) d_{6} \frac{L_{r}}{L+L_{r}}\right]=0 \\
& \Rightarrow V_{c}=V_{g} \frac{d_{5}}{d_{3}}+\left(V_{g}-V_{o}\right) \frac{d_{6}}{d_{3}} \frac{L_{r}}{L+L_{r}}
\end{aligned}
$$

Sumando A.52 y A.53 también se puede despejar $V_{c}$ como:

$$
V_{c}=V_{g} \frac{d_{5}+d_{6}}{d_{3}}-V_{o} \frac{1}{d_{3}}
$$

\section{CORRIENTE MEDIA EN EL CONDENSADOR DE CLAMP CON RIZADO.}

La intensidad media en el condensador de clamp es nula. Entonces:

$$
\begin{aligned}
& \int_{t_{0}}^{t_{0}+T} i_{c}(t) d t=\int_{t_{2}}^{t_{3}} i_{c}(t) d t=\int_{0}^{d_{3} T}\left[I_{\max }-\frac{V_{c}}{L_{r}} t\right] d t=0 \\
& {\left[I_{\text {max }} t-\frac{V_{c}}{2 L_{r}} t^{2}\right]_{0}^{d_{3} T}=0 \Rightarrow V_{c}=\frac{2 I_{\text {max }} L_{r}}{d_{3} T}}
\end{aligned}
$$

Sustituyendo este valor en la expresión empleada para $i_{c}(t)$ se puede obtener el valor de la intensidad del condensador transcurrido el tiempo $d_{3} T$. Entonces:

$$
i_{c}\left(d_{3} T\right)=I_{\max }-\frac{V_{c}}{L_{r}} d_{3} T=I_{\max }-\frac{\frac{2 I_{\max } \not_{r}}{d_{3} T}}{L_{r}} d_{3} T=-I_{\max }
$$

\section{DURACIÓN DEL INTERVALO $d_{5}$ CON RIZADO.}

Se puede calcular a partir de la tensión en la bobina $L r$ durante la etapa 5 , puesto que es conocida la variación que experimenta la intensidad circulante por esa bobina y la tensión a la que está sometida.

$$
\begin{aligned}
\Delta i_{r}(t) & =i_{r}\left(t_{5}\right)-i_{r}\left(t_{4}\right) \approx i_{r}\left(t_{5}\right)-i_{r}\left(t_{3}\right)=I_{\min }-\left(-I_{\max }\right)=2 I \\
\Delta i_{r}(t) & =\frac{1}{L_{r}} \Delta v_{L_{r}}(t) \Delta t \equiv \frac{1}{L_{r}} V_{g} d_{5} T \\
\Rightarrow d_{5} & =\frac{2 I L_{r}}{V_{g} T}
\end{aligned}
$$

Una expresión diferente se obtiene al igualar (A.53) con (A.55) y empleando (A.40) para despejar $d_{5}$ :

$$
\begin{aligned}
& V_{g} \frac{d_{5}}{d_{3}}+\left(V_{g}-V_{o}\right) \frac{\left(D-d_{5}\right)}{\phi_{3}} \frac{L_{r}}{L+L_{r}}=\frac{2 I_{\max } L_{r} f}{\phi_{3}} \\
& \Rightarrow d_{5}=\frac{\left(V_{g}-V_{o}\right) D \frac{L_{r}}{L+L_{r}}-2 I_{\max } L_{r} f}{\left(V_{g}-V_{o}\right) \frac{L_{r}}{L+L_{r}}-V_{g}}
\end{aligned}
$$




\section{TENSION DE SALIDA CON RIZADO.}

Como $d_{6}=\left(D-d_{5}\right)$, resulta que:

$$
\begin{aligned}
d_{6} & =D-\frac{2 I_{\max } L_{r} f-\left(V_{g}-V_{o}\right) D \frac{L_{r}}{L+L_{r}}}{V_{g}-\left(V_{g}-V_{o}\right) \frac{L_{r}}{L+L_{r}}} \\
& =\frac{V_{g} D-2 I_{\max } L_{r} f}{V_{g}-\left(V_{g}-V_{o}\right) \frac{L_{r}}{L+L_{r}}}=\frac{V_{g} D-2 I_{\max } L_{r} f}{V_{g} \frac{L}{L+L_{r}}+V_{o} \frac{L_{r}}{L+L_{r}}}
\end{aligned}
$$

Por otro lado, despejando $d_{6}$ de (A.52) se obtiene:

$$
d_{6}=\frac{V_{o}}{V_{g} \frac{L}{L+L_{r}}+V_{o} \frac{L_{r}}{L+L_{r}}}
$$

Igualando (A.59) y (A.60) se puede despejar $V_{o}$ como:

$$
V_{o}=V_{g} D-I_{o, \max } 2 L_{r} f
$$

También se podría conseguir si se igualan (A.54) con (A.55) y se despeja $V_{o}$. En ambos casos, la expresión obtenida es parecida a (A.48) que se ha obtenido sin considerar rizado. Solo se ha cambiado $I$ por $I_{\max }$. Y el término que multiplica a la intensidad se puede considerar como una resistencia en serie con L, $R_{e s L}$, ya definida anteriormente en (A.49).

\section{RIZADO DE CORRIENTE EN $L$ CON RIZADO.}

Aplicando la definición de rizado de corriente en una bobina, se puede obtener el rizado de corriente en $L$ durante las etapas 3 a 5 o durante la etapa 6 siendo expresiones equivalentes en régimen permanente. Entonces:

$$
\begin{aligned}
\Delta i_{L, 3-5} & =\frac{1}{L}\left|-V_{o}\right|\left(d_{3}+d_{5}\right) T=\frac{1}{L} V_{o}\left(1-d_{6}\right) T \\
\Delta i_{L, 6} & =\frac{1}{L}\left|\left(V_{g}-V_{o}\right)\right| \frac{L}{L+L_{r}} d_{6} T=\frac{\left(V_{g}-V_{o}\right)}{L+L_{r}} d_{6} T
\end{aligned}
$$

Empleando la ecuación (A.60) se obtiene la expresión para el rizado de corriente en $L$ como:

$$
\Delta i_{L}=\frac{\left(V_{g}-V_{o}\right) V_{o} T}{L_{r} V_{o}+L V_{g}}
$$

\section{CARACTERÍSTICA DE SALIDA CON RIZADO.}

La relación entre la tensión de salida y la intensidad de salida se obtiene a partir de la expresión (A.61). Primero se expresa $I_{\max }$ en función del valor medio y del rizado. Para el rizado de corriente se emplea la segunda expresión de (A.62) y se considera $d_{6}=\left(D-d_{5}\right)$, junto con la relación $I=I_{o}$ :

$$
\Delta i_{L}=\frac{\left(V_{g}-V_{o}\right)}{L+L_{r}}\left(D-d_{5}\right) T=\frac{\left(V_{g}-V_{o}\right)}{L+L_{r}}\left(D-\frac{2 I_{o} L_{r}}{V_{g} T}\right) T
$$


Sólo queda sustituir (A.64) en (A.61) y despejar $V_{o}$ para obtener una función de la forma $V_{o}=f\left(I_{o}\right)$. Por tanto:

$$
\begin{aligned}
V_{o} & =V_{g} D-I_{o, \max } 2 L_{r} f=V_{g} D-\left(I_{o}+\frac{1}{2} \Delta i_{L}\right) 2 L_{r} f \\
& =V_{g} D-I_{o} 2 L_{r} f-\frac{1}{\not 2} \frac{\left(V_{g}-V_{o}\right)}{L+L_{r}}\left(D-\frac{2 I_{o} L_{r}}{V_{g} T}\right) \not 2 \not L_{r} f \\
& =V_{g} D-I_{o} 2 L_{r} f-\left(V_{g}-V_{o}\right) \frac{L_{r}}{L+L_{r}}\left(D-\frac{2 I_{o} L_{r} f}{V_{g}}\right) \\
& =\left(V_{g} D-I_{o} 2 L_{r} f\right)\left(1-\frac{L_{r}}{L+L_{r}}\right)+V_{o} \frac{L_{r}}{L+L_{r}}\left(D-\frac{2 I_{o} L_{r} f}{V_{g}}\right) \\
& \Rightarrow V_{o}\left(I_{o}\right)=\frac{\left[V_{g} D-I_{o} 2 L_{r} f\right] \frac{L}{L+L_{r}}}{1+\left(I_{o} \frac{2 L_{r} f}{V_{g}}-D\right) \frac{L_{r}}{L_{r}+L}}
\end{aligned}
$$

\section{A.2.3. Comparativa.}

Está claro que el análisis cuando se considera el rizado será de aplicación al circuito conmutado, donde se tienen en cuenta los valores de las bobinas, mientras que el análisis al no considerar rizado servirá para las expresiones derivadas del modelo promediado, dado que éste parte de la consideración de pequeño rizado.

Los resultados obtenidos para magnitudes tales como el ciclo de trabajo, la tensión de clamp y las variaciones de corriente, a partir de unos valores de diseño ponen de manifiesto estas diferencias. Por ejemplo, con los parámetros de funcionamiento recogidos en la Tabla A.1 se obtienen los valores indicados en la Tabla A.2, en el caso de no considerar el rizado de la corriente en la bobina principal y se compara con el obtenido cuando si se ha considerado.

\begin{tabular}{llll}
\hline \hline$V_{g}$ & $30 \mathrm{~V}$ & $C_{o}$ & $5 \mu \mathrm{F}$ \\
$P$ & $130 \mathrm{~W}$ & $C_{c}$ & $5,2 \mu \mathrm{F}$ \\
$V_{\text {out }}$ & $13 \mathrm{~V}$ & $L$ & $24 \mu \mathrm{H}$ \\
$f$ & $100 \mathrm{kHz}$ & $L_{r}$ & $2 \mu \mathrm{H}$ \\
$t_{m}$ & $75 \mathrm{~ns}$ & $C_{r}$ & $1,0 \mathrm{nF}$ \\
\hline
\end{tabular}

Tabla A.1: Condiciones de trabajo de un reductor ZVS.

En el reductor ZVS la intensidad en la bobina principal es igual a la intensidad en la salida, $I_{o}$. Al considerar el rizado, el ciclo de trabajo obtenido es mayor. La tensión de clamp, $V_{c}$ también es diferente. Es lógico dado que depende del ciclo de trabajo. Si se calcula su valor a partir de la expresión que incluye la intensidad, también se obtendrá un valor diferente según se considere valor medio (sin rizado) o valor máximo (cuando hay rizado). 


\begin{tabular}{ccccccccc}
\hline & $I_{o}$ & $d_{5}$ & $d_{6}$ & $D$ & $\begin{array}{c}V_{c} \\
(\mathrm{~V})\end{array}$ & $\begin{array}{c}\Delta_{i_{L}} \\
(\mathrm{~A})\end{array}$ & $\begin{array}{c}I_{\max } \\
(\mathrm{A})\end{array}$ & $\begin{array}{c}I_{\min } \\
(\mathrm{A})\end{array}$ \\
\hline SIN RIZADO & \multirow{2}{*}{10} & \multirow{2}{*}{0,1333} & 0,4333 & 0,5667 & 9,231 & 0 & - & - \\
CON RIZADO & & & 0,4531 & 0,5864 & 11,104 & 2,9624 & 11,481 & 8,519 \\
\hline \hline
\end{tabular}

Tabla A.2: Parámetros calculados cuando se considera o no el rizado.

En ambos análisis se han considerado despreciables algunas etapas por su pequeña duración en relación a las otras. Esta hipótesis se ha validado midiendo la duración de las seis etapas para los parámetros de funcionamiento de las dos tablas anteriores. Con ayuda de un programa de cálculo simbólico se han planteado las ecuaciones de estado durante las seis etapas y se han resuelto las ecuaciones y obtenido la fracción que ocupa cada etapa dentro de un periodo completo. Se han indicado en la Tabla A.3

\begin{tabular}{ccccccc}
\hline & $d_{1}$ & $d_{2}$ & $d_{3}$ & $d_{4}$ & $d_{5}$ & $d_{6}$ \\
\hline SIN RIZADO & $300 \times 10^{-6}$ & $92 \times 10^{-6}$ & 0,4332 & $392 \times 10^{-6}$ & 0,1332 & 0,4328 \\
CON RIZADO & $270 \times 10^{-6}$ & $87 \times 10^{-6}$ & 0,4135 & $358 \times 10^{-6}$ & 0,1332 & 0,4526 \\
\hline \hline
\end{tabular}

Tabla A.3: Fracción de tiempo para cada etapa por las que evoluciona el reductor ZVS.

A la vista de estos resultados, se puede comprobar que las etapas 1, 2 y 4 son las de menor duración por lo que, como se había previsto, se pueden despreciar.

En cada uno de los dos análisis realizados, la característica de salida obtenida muestra una relación diferente entre tensión e intensidad de salida. Cuando ambas se representan gráficamente se obtiene prácticamente la misma curva, como se pone de manifiesto al observar la Figura A.15 que contiene la característica de salida de un reductor ZVS con los datos de la Tabla A.1. En este caso, la curva representada sin considerar rizado emplea el valor de ciclo de trabajo obtenido con este mismo análisis, mientras que la curva representada al considerar el rizado está obtenida con el ciclo de trabajo calculado con rizado no despreciable.

\section{A.3. Modelo promediado}

La obtención del modelo promediado se puede conseguir mediante el promediado de las formas de onda, mediante el promediado de circuitos o mediante el promediado en el espacio de estados.

En el promediado de espacio de estados se construye la matriz del espacio de estados y se hace el promediado en un periodo. Puede suceder que haya variables de estado lentas y variables de estado rápidas. Lo que se hace es 


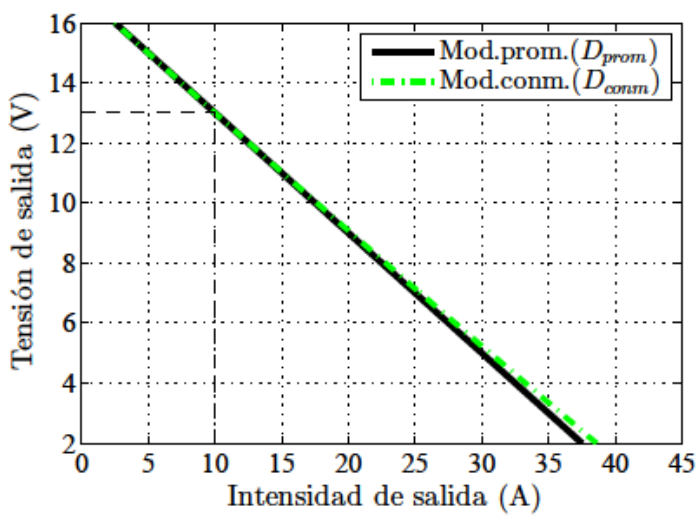

Figura A.15: Característica de salida de un Reductor ZVS.

simplificar el promediado en el espacio de estados separando las variables lentas de las rápidas, en lo que se denomina la fase de relajación. Después se desacoplan estos subsistemas y posteriormente se hace el promedio de la ecuación mediante la integración en un periodo. De aquí se obtiene el modelo promediado en el espacio de estados. Este sistema se puede perturbar y linealizar y se obtiene una expresión del modelo de pequeña señal en el espacio de estados que se puede transformar en un circuito en pequeña señal.

En el promediado de formas de onda se desprecian los intervalos resonantes porque son de muy alta frecuencia y se trabaja con formas de onda en los que no hay resonancias. Se escriben las ecuaciones de las variables de estado en función de los promedios de las formas de onda y sale el modelo promediado, que se perturba y linealiza y se consigue el modelo en pequeña señal, que se transforma en un circuito en pequeña señal.

En el promediado de circuitos, se busca un bloque de dispositivos semiconductores con tantas entradas y salidas como elementos. Se consideran la mitad de las formas de onda como independientes y la otra mitad como dependientes. Se busca que éstas sean una combinación lineal de aquéllas. Aparecerán fuentes dependientes de tensión y corriente que se sustituyen en el circuito y el circuito obtenido será el circuito promediado o de gran señal. A partir de éste, se obtiene el circuito en pequeña señal.

\section{A.3.1. Método aplicado}

Las variables de estado independientes están relacionadas con los elementos que almacenan energía, que suelen ser bobinas y condensadores. En el caso del reductor ZVS, comparado con el reductor convencional, se incluyen tres variables adicionales a la intensidad de la bobina de salida, $L$ y a la tensión del condensador de salida, $C_{o}$, que son la intensidad en la bobina re- 
sonante, $L_{r}$, y la tensión en el condensador resonante, $C_{r}$, y la tensión en el condensador del clamp, $C_{c}$. Es decir, tenemos cinco variables de estado, susceptibles de formar parte de un modelo promediado. Con todas ellas se puede construir un modelo completo que es muy preciso a la vez que complicado dado que incorpora todas las variables de estado. La metodología seguida en (Chen y Sun, 2006) es una metodología general para obtener modelos promediados de convertidores con enclavamiento activo, con un orden menor que el modelo completo pero considerando el efecto de las variables resonantes. Este método es un método general que también sirve para muchos convertidores y permite de forma sistemática el análisis y modelado de convertidores con enclavamiento activo que facilita el diseño de su control y su aplicación práctica.

Un convertidor con enclavamiento activo, comparado con el convertidor convencional, incorpora tres variables de estado adicionales ya mencionadas anteriormente. Algunos modelos (Li y otros, 2002; Athalye y otros, 2003; Jitaru y Birca-Galateanu, 1998; Hakoda y otros, 1998) consideran únicamente la tensión en $C_{c}$ y no tienen en cuenta la intensidad y tensión resonante así como los intervalos resonantes. El convertidor está modelado considerando unicamente la tensión en el condensador de clamp como una variable más, consiguiendo uno modelo de orden reducido (orden menor que el completo). Al no tener en cuenta el efecto de las resonancias, el resultado no incluye los efectos que éstas pueden tener sobre la dinámica del convertidor, y por tanto sobre los requisitos de diseño del control.

El método de promediado de circuitos aplicado a estos convertidores dado en (Athalye y otros, 2001) considera las variables resonantes para conseguir un modelo completo. El modelo se da en forma de circuito equivalente donde se sustituyen los transistores y diodos por fuentes controladas de tensión y de corriente. Comparado con los modelos anteriores es más preciso pero mas complicado debido a la presencia de otras dos variables de estado adicionales.

El método ya mencionado de (CHen y Sun, 2006) considera el efecto de las variables resonantes sobre la dinámica del convertidor y estudia los intervalos asociados a las resonancias, pero sin incluirlas como variables de estado en el modelo final. Los modelos resultantes tienen tanta precisión como el modelo completo pero el orden del modelo resultantes es inferior al completo. El procedimiento de modelado se basa en la separación de variables a escala temporal presentado en (Sun y Grotstollen, 1997) donde se aplica como ejemplo a un reductor cuasiresonante y a un elevador ZVT. Básicamente consiste en hacer una partición del convertidor en dos circuitos o subsistemas: un subsistema lento y otro rápido (Sun y Grotstollen, 1992). El subsistema lento incorpora los principales elementos de almacenamiento de energía y las secciones de entrada y salida del convertidor mientras que el subsistema rápido incluye los elementos resonantes y los dispositivos semiconductores que trabajan en conmutación. 
De esta forma, suponiendo el subsistema lento, descrito por (A.66), y el subsistema rápido descrito por (A.67):

$$
\begin{aligned}
\frac{d x_{s}}{d t} & =f\left(x_{s}, u_{s}, v_{s}\right) \\
\frac{d x_{f}}{d t} & =g_{i}\left(x_{f}, v_{f}\right)
\end{aligned}
$$

donde $u_{s}$ es la entrada al subsistema lento. El acoplamiento entre ambos subsistemas está representado por

$$
\begin{aligned}
& v_{f}=p\left(x_{s}, u_{s}\right) \\
& v_{s}=q_{i}\left(x_{f}, v_{f}\right)
\end{aligned}
$$

Nótese que las variables y funciones de estas ecuaciones son vectores de las dimensiones apropiadas. El índice $i$ de las funciones $g_{i}$ y $q_{i}$ va desde uno hasta $N$, indicando que el sistema rápido incluye los dispositivos semiconductores y se suponen $N$ estados topológicos diferentes durante cada periodo de conmutación.

Con el circuito particionado de esta forma el modelo promediado se deriva en tres pasos:

1. Relajación. Se analiza primero el subsistema rápido suponiendo la entrada $v_{f}$ de valor constante, dado que proviene del subsistema lento. De esta forma, la respuesta de las variables de estado rápidas a lo largo de un periodo de conmutación se obtiene como:

$$
\tilde{x}_{f}=h\left(v_{f}, t\right)
$$

2. Desacoplamiento. El subsistema rápido afecta al subsistema lento a través de su salida, $v_{s}$, que se obtiene con (A.68) y (A.69):

$$
\tilde{v}_{s}=q_{i}\left(h\left(v_{f}, t\right), v_{f}\right)=z\left(v_{f}, t\right)
$$

Por tanto, la dinámica lenta del convertidor, que es el objetivo del modelado promediado puede ser analizada con las ecuaciones de subsistema lento (A.66), considerando $v_{s}$ como la solución de la fase de relajación (A.70). Hacer esto significa sustituir la red dinámica del subsistema rápido por una red resistiva que varía con el tiempo.

3. Promediado. Puesto que la entrada $\tilde{v}_{s}$ es variable con el tiempo se puede expandir en una serie de Fourier. Dado que la frecuencia de conmutación es muy grande y el subsistema lento no es muy sensible a las entradas con variaciones muy rápidas, los términos variables en la serie de Fourier se pueden despreciar, y solo quedaría el término de continua dado por (A.71):

$$
\tilde{v}_{s} \approx \bar{z}\left(v_{f}\right)=\frac{1}{T} \int_{0}^{T} z\left(v_{f}, \tau\right) d \tau
$$


El modelo promediado, independiente del tiempo, que describe la dinámica del convertidor se consigue utilizando (A.71) en (A.66):

$$
\begin{aligned}
\frac{d x_{s}}{d t} & =f\left(x_{s}, u_{s}, \tilde{z}\left(v_{f}\right)\right)=f\left(x_{s}, u_{s}, \bar{z}\left(p\left(x_{s}, u_{s}\right)\right)\right) \\
& =f_{0}\left(x_{s}, u_{s}\right)
\end{aligned}
$$

Con este método general de promediado, basado en la división del convertidor en dos subcircuitos, se trata de dar una interpretación física a dicho método. Sin embargo, no es necesario realizar explícitamente esta partición puesto que el mismo modelo promediado se puede obtener a partir de la descripción completa del convertidor en el espacio de estados. Basta con sustituir (A.68) en (A.66) y en (A.67) de forma que no haya variables intermedias de acoplamiento. Así se consigue la expresión genérica para un sistema descrito en el espacio de estados como

$$
\begin{aligned}
& \frac{d x_{s}}{d t}=f_{i}\left(x_{s}, x_{f}, u_{s}\right) \\
& \frac{d x_{f}}{d t}=g_{i}\left(x_{s}, x_{f}, u_{s}\right)
\end{aligned}
$$

donde se han indicado de forma explicita las ecuaciones relacionadas con las variables lentas y con las rápidas. El subíndice $i$ indica los diferentes estados topológicos por los que pasa el convertidor en un ciclo de conmutación. La obtención del modelo promediado se consigue con el siguiente procedimiento:

1. Resolver (A.74) considerando las variables lentas como constantes y obtener $\tilde{x}_{f}=h\left(x_{s}, u_{s}, t\right)$ (Relajación).

2. Sustituir $x_{f}$ por $\tilde{x}_{f}$ en (A.73) (Desacoplamiento).

3. Promediar el lado derecho de las igualdades (A.73) y (A.74) en un periodo de conmutación (Promediado).

\section{A.3.2. Modelo promediado para el reductor ZVS}

En la sección A.1 se ha explicado el funcionamiento en régimen permanente del reductor ZVS. Del análisis se deduce que la corriente en la bobina resonante $L_{r}$ y la tensión en el condensador resonante $C_{r}$ son las variables de estado rápidas y el resto son las variables de estado lentas. El procedimiento a seguir es obtener las expresiones para las variables rápidas $i_{L_{r}}(t)$ y $v_{C_{r}}(t)$, en un periodo de conmutación (comprobar que son periódicas). La variables lentas se suponen de valor constante para este cálculo. Las expresiones obtenidas, dependerán, por tanto, de las variables lentas y también del tiempo. Éstas se sustituyen en las ecuaciones de estado de las variables lentas de forma que queden desacopladas respecto a las variables rápidas. Se aplica 
ahora un promedio estándar en un periodo de conmutación para eliminar la dependencia con el tiempo, consiguiéndose un modelo promediado de tercer orden (orden reducido) para las tres variables de estado lentas, $v_{C_{c}}(t), v_{o}(t)$ y $i_{L}(t)$.

Aunque matemáticamente no es necesario, se suelen realizar algunas aproximaciones antes de empezar para simplificar los cálculos a realizar. En primer lugar, se ignoran los tiempos muertos entre las conmutaciones de $S_{1}$ y $S_{2}$ que normalmente son muy pequeños en comparación con el periodo. También se desprecian los intervalos resonantes, como se hace en (ATHALYE y otros, 2001) para obtener el modelo completo con el método del interruptor promediado. En este punto cabe indicar que el efecto debido a tener en cuenta los intervalos resonantes sería tan solo a frecuencias muy altas y fuera del rango de interés (que, generalmente, suele ser una fracción de la frecuencia de conmutación porque es donde se aplica el control en lazo cerrado). Además se supone que el condensador de clamp mantiene su tensión constante, lo que quiere decir que la frecuencia de resonancia entre $L_{r}$ y $C_{c}$ es bastante menor que la frecuencia de conmutación.

Considerando estas simplificaciones desaparecen los intervalos resonantes (Etapa 1, 2 y 4) y el tiempo muerto de la etapa 5, de forma que un periodo se puede escribir como:

$$
\begin{aligned}
T & \approx d_{3} T+d_{5} T+d_{6} T \\
(1-d) T & =d_{3} T \\
d T & =d_{5} T+d_{6} T
\end{aligned}
$$

puesto que $d_{1} T=d_{2} T=d_{4} T=0$.

Las curvas principales están representadas en la figura A.12. Suponiendo valores constantes para las variables lentas, la tensión $v_{C_{r}}(t)$, tiene la siguiente expresión ${ }^{1}$ :

$$
v_{C_{r}}(t)= \begin{cases}v_{g}+v_{C_{c}} & t \in\left[t_{2}, t_{3}\right] \\ 0 & t \in\left[t_{4}, t_{5}\right] \\ 0 & t \in\left[t_{5}, t_{6}\right]\end{cases}
$$

mientras que la intensidad en la bobina, $i_{L_{r}}(t)$ se expresa como

$$
i_{L_{r}}(t)= \begin{cases}i_{L}-\frac{v_{C_{c}}}{L_{r}}\left(t-t_{2}\right) & t \in\left[t_{2}, t_{3}\right] \\ i_{L}+\frac{v_{g}}{L_{r}}\left(t-t_{5}\right) & t \in\left[t_{4}, t_{5}\right] \\ i_{L} & t \in\left[t_{5}, t_{6}\right]\end{cases}
$$

Se obtiene $d_{5}$ a partir de la igualdad de valores de $i_{L_{r}}$ en $t_{3}=t_{4}$. Es decir:

$$
i_{L_{r}}\left(t_{3}\right)=i_{L_{r}}\left(t_{4}\right)
$$

\footnotetext{
${ }^{1}$ Se mantiene la numeración de los intervalos, considerando que $t_{0}=t_{1}=t_{2}$ y que $t_{3}=t_{4}$.
} 
Entonces, con (A.77) y (A.78) se obtiene una solución para $d_{5}$ :

$$
d_{5}=\frac{v_{C_{c}}}{v_{g}}(1-d)
$$

Para el resto de variables rápidas si es necesario escribir las ecuaciones de estado. Necesitamos una ecuación para la intensidad en la bobina de salida, $i_{L}(t)$; otra ecuación para la tensión en el condensador de salida, $v_{C_{o}}(t)$; y otra ecuación para la tensión en el condensador de $\operatorname{clamp}, v_{C_{c}}(t)$. Estas ecuaciones son:

$$
\begin{aligned}
& v_{L}(t)=L \frac{d i_{L}(t)}{d t}= \begin{cases}-v_{C_{o}}(t) & t \in\left[t_{2}, t_{3}\right] \\
-v_{C_{o}}(t) & t \in\left[t_{4}, t_{5}\right] \\
v_{g}(t)-v_{C_{o}}(t) & t \in\left[t_{5}, t_{6}\right]\end{cases} \\
& i_{C_{o}}(t)=C_{o} \frac{d v_{C_{o}}(t)}{d t}=i_{L}(t)-\frac{v_{C_{o}}(t)}{R} \\
& i_{C_{c}}(t)=C_{c} \frac{d v_{C_{c}}(t)}{d t}= \begin{cases}i_{L_{r}}(t) & t \in\left[t_{2}, t_{3}\right] \\
0 & t \in\left[t_{4}, t_{5}\right] \\
0 & t \in\left[t_{5}, t_{6}\right]\end{cases}
\end{aligned}
$$

Mientras que las ecuaciones (A.80) y (A.81) son las ecuaciones de estado de un convertidor reductor sin enclavamiento activo, la ecuación adicional (A.82) es la ecuación del condensador de esta red añadida. Al igual que en el reductor convencional, se debe añadir una ecuación relacionada con la intensidad de entrada al convertidor, que es (A.83):

$$
i_{g}(t)= \begin{cases}0 & t \in\left[t_{2}, t_{3}\right] \\ i_{L_{r}}(t) & t \in\left[t_{4}, t_{5}\right] \\ i_{L}(t) & t \in\left[t_{5}, t_{6}\right]\end{cases}
$$

Cuando se sustituyen las variables rápidas en las ecuaciones anteriores, se consigue tener un conjunto de ecuaciones de estado donde solo intervienen las variables de estado lentas, es decir, se han desacoplado de las variables de estado rápidas, que son

$$
\begin{aligned}
& L \frac{d i_{L}(t)}{d t}= \begin{cases}-v_{C_{o}}(t) & t \in\left[t_{2}, t_{3}\right] \\
-v_{C_{o}}(t) & t \in\left[t_{4}, t_{5}\right] \\
v_{g}(t)-v_{C_{o}}(t) & t \in\left[t_{5}, t_{6}\right]\end{cases} \\
& C_{o} \frac{d v_{C_{o}}(t)}{d t}=\left[i_{L}(t)-\frac{v_{C_{o}}(t)}{R}\right] \\
& C_{c} \frac{v_{C_{c}}(t)}{d t}= \begin{cases}i_{L}(t)-\frac{v_{C_{c}}(t)}{L_{r}}\left(t-t_{2}\right) & t \in\left[t_{2}, t_{3}\right] \\
0 & t \in\left[t_{4}, t_{5}\right] \\
0 & t \in\left[t_{5}, t_{6}\right]\end{cases}
\end{aligned}
$$


junto con

$$
i_{g}(t)= \begin{cases}0 & t \in\left[t_{2}, t_{3}\right] \\ i_{L}(t)-\frac{v_{g}(t)}{L_{r}}\left(t-t_{5}\right) & t \in\left[t_{4}, t_{5}\right] \\ i_{L}(t) & t \in\left[t_{5}, t_{6}\right]\end{cases}
$$

Considerando la aproximación de pequeño rizado, es posible sustituir los valores instantáneos de las variables de estado por sus valores promedio, (por ejemplo, $i_{L}(t)$ se sustituye por $\bar{i}_{L}\left\langle i_{L}(t)\right\rangle_{T}=$ y así con el resto). De esta forma, las ecuaciones desacopladas se transforman en

$$
\begin{aligned}
& L \frac{d i_{L}(t)}{d t} \approx \begin{cases}-\bar{v}_{C_{o}} & t \in\left[t_{2}, t_{3}\right] \\
-\bar{v}_{C_{o}} & t \in\left[t_{4}, t_{5}\right] \\
\bar{v}_{g}-\bar{v}_{C_{o}} & t \in\left[t_{5}, t_{6}\right]\end{cases} \\
& C_{o} \frac{d v_{C_{o}}(t)}{d t} \approx\left[\bar{i}_{L}-\frac{\bar{v}_{C_{o}}}{R}\right] \\
& C_{c} \frac{d v_{C_{c}}(t)}{d t} \approx \begin{cases}\bar{i}_{L}-\frac{\bar{v}_{C_{c}}}{L_{r}}\left(t-t_{2}\right) & t \in\left[t_{2}, t_{3}\right] \\
0 & t \in\left[t_{4}, t_{5}\right] \\
0 & t \in\left[t_{5}, t_{6}\right]\end{cases} \\
& i_{g}(t) \approx \begin{cases}0 & t \in\left[t_{2}, t_{3}\right] \\
\bar{i}_{L}+\frac{\bar{v}_{g}}{L_{r}}\left(t-t_{5}\right) & t \in\left[t_{4}, t_{5}\right] \\
\bar{i}_{L} & t \in\left[t_{5}, t_{6}\right]\end{cases}
\end{aligned}
$$

con

$$
d_{5} \approx \bar{v}_{C_{c}}(1-d) / \bar{v}_{g}
$$

Por último hay que eliminar la dependencia del tiempo realizando el promedio de las ecuaciones en un intervalo, de donde se obtiene el modelo promediado para el reductor ZVS, que es un modelo de orden tres, (es un modelo de orden reducido frente al modelo de orden completo que sería de orden cinco).

$$
\begin{aligned}
L \frac{d \bar{i}_{L}}{d t} & =\bar{v}_{g}\left(d-d_{5}\right)-\bar{v}_{C_{o}} \\
C_{o} \frac{d \bar{v}_{C_{o}}}{d t} & =\bar{i}_{L}-\frac{\bar{v}_{C_{o}}}{R} \\
C_{c} \frac{d \bar{v}_{C_{c}}}{d t} & =\bar{i}_{L}(1-d)-\frac{\bar{v}_{g}}{2 L_{r} f_{s}} d_{5}(1-d) \\
\bar{i}_{g} & =\left[\bar{i}_{L} d-\frac{\bar{v}_{g}}{2 L_{r} f_{s}} d_{5}^{2}\right]
\end{aligned}
$$

Con las ecuaciones anteriores se consigue obtener un circuito promediado para este convertidor. Previamente hay que sustituir el parámetro $d_{5}$ por su 
expresión, y se obtienen las ecuaciones indicadas en (A.97).

$$
\begin{aligned}
L \frac{d \bar{i}_{L}}{d t} & =d \bar{v}_{g}-(1-d) \bar{v}_{C_{c}}-\bar{v}_{C_{o}} \\
C_{o} \frac{d \bar{v}_{C_{o}}}{d t} & =\bar{i}_{L}-\frac{\bar{v}_{C_{o}}}{R} \\
C_{c} \frac{d \bar{v}_{C_{c}}}{d t} & =(1-d) \bar{i}_{L}-\frac{(1-d)^{2}}{2 L_{r} f_{s}} \bar{v}_{C_{c}} \\
\bar{i}_{g} & =d \bar{i}_{L}-\frac{\bar{v}_{C_{c}}^{2}}{\bar{v}_{g}} \frac{(1-d)^{2}}{2 L_{r} f_{s}}
\end{aligned}
$$

El circuito obtenido es el indicado en la figura A.16, donde $r_{e q}$ es una resistencia equivalente sin pérdidas que consume la energía generada por la fuente dependiente de corriente de la etapa de entrada dada por $i_{e q}$.

$$
\begin{gathered}
r_{e q}=\frac{2 L_{r} f_{s}}{(1-d)^{2}} \\
i_{e q}=\frac{v_{C_{c}}^{2}}{v_{g} r_{e q}}
\end{gathered}
$$

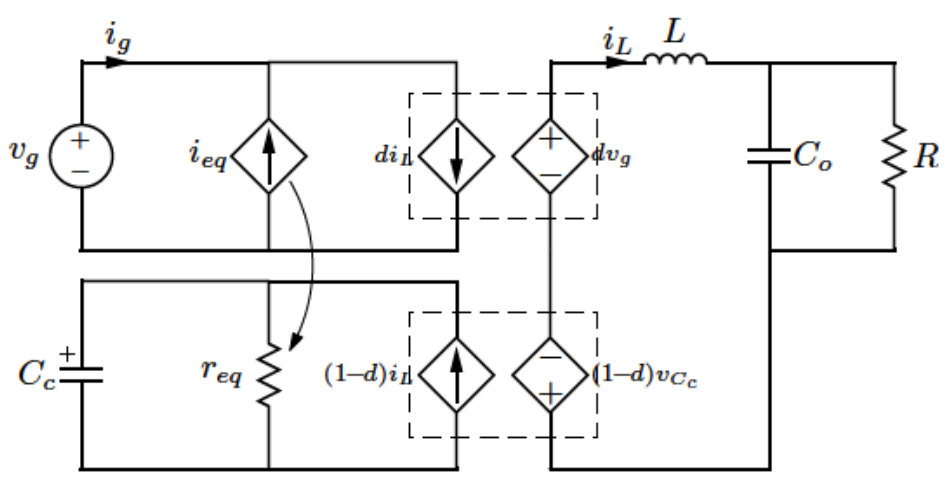

Figura A.16: Modelo promediado de un reductor ZVS.

\section{A.3.3. Balance energético}

En el modelo promediadohay dos elementos que generan energía, la fuente de tensión y la fuente de corriente equivalente, ambas en la entrada. La energía de la fuente de tensión se consume en la carga y se queda almacenada se devuelve en los elementos reactivos, la energía generada en la fuente de corriente se consume toda en $r_{e q}$. Veámoslo.

En $r_{e q}$, la potencia consumida es igual a

$$
P_{r_{e q}}=\frac{v_{C_{c}}^{2}}{r_{e q}}
$$


Multiplicando arriba y abajo por $v_{g}$, se obtiene una expresión

$$
P_{r_{e q}}=\frac{v_{C_{c}}^{2}}{r_{e q}} \frac{v_{g}}{v_{g}}
$$

en la que, agrupando, se obtiene el producto de una fuente de tensión por otra de corriente cuyo valor es igual a $i_{e q}$

$$
P_{r_{e q}}=i_{e q} v_{g}
$$

Esta potencia coincide con la potencia generada por la fuente de corriente $i_{e q}$ en la entrada.

\section{A.3.4. Validación del modelo promediado}

La validación del modelo promediado se realiza mediante simulación, y se compara su comportamiento, tanto en régimen permanente como en régimen dinámico, con un convertidor conmutado. Para ello se eligen de nuevo los parámetros de funcionamiento recogidos en la Tabla A.1, empleando en el convertidor conmutado el ciclo de trabajo calculado al considerar rizado. En el modelo promediado se ha considerado el ciclo de trabajo calculado cuando no se ha considerado el rizado.

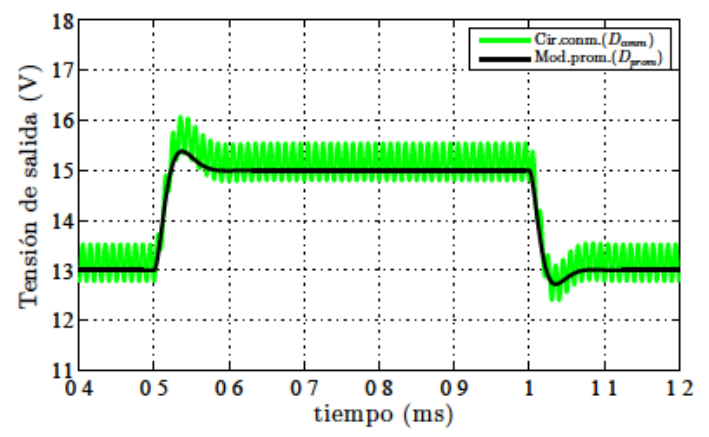

(a)

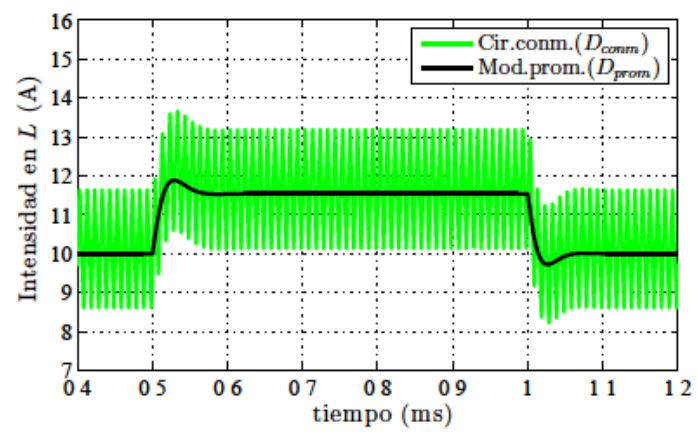

(b)

Figura A.17: Reductor ZVS. Respuesta a escalón del ciclo de trabajo: a) Tensión de salida; y b) Intensidad en la bobina.

Los resultados muestran una dinámica similar para el convertidor conmutado y el modelo promediado. En cuanto al régimen permanente, hay un error puesto que la tensión de salida no coincide. El motivo de esta diferencia tiene su causa en que en el modelo promediado no está incluido el tiempo 
muerto, $t_{m}$, que hay que dejar entre disparos de los transistores principal y de clamp, entre otras cosas, para facilitar las conmutaciones suaves. Además, parte de este tiempo muerto se emplea en descargar el condensador resonante que corresponde a una de las tres etapas que se desprecian para obtener el modelo promediado. En concreto la etapa 4, siendo su duración igual a $d_{4} T$.

Por tanto, hay que realizar una corrección en el ciclo de trabajo empleado para el circuito conmutado, en base a los tiempos mencionados, puesto que ya está incluido de forma implicita en la generación de disparos del propio circuito conmutado. De esta forma, se rehacen las simulaciones del circuito conmutado con el ciclo de trabajo corregido, $D_{\text {corr }}$, como:

$$
D_{\text {corr }}=D_{\text {conm }}-\frac{t_{m}-d_{4} T}{T}
$$

y se obtienen los resultados de la Figura A.18. La corrección equivalente pero para el modelo promediado consistiría en añadir este tiempo extra al ciclo de trabajo empleado en el modelo promediado.

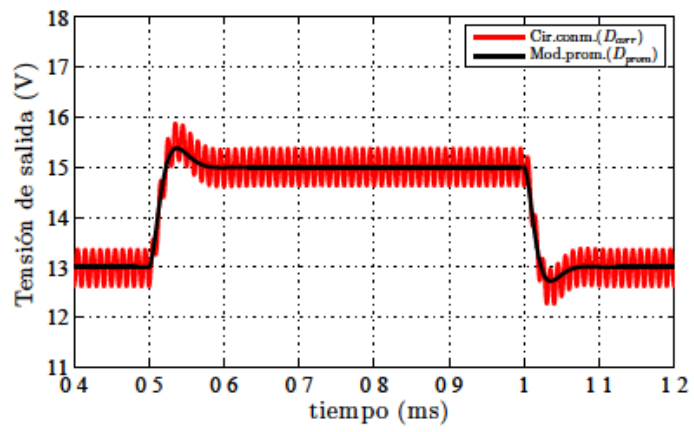

(a)

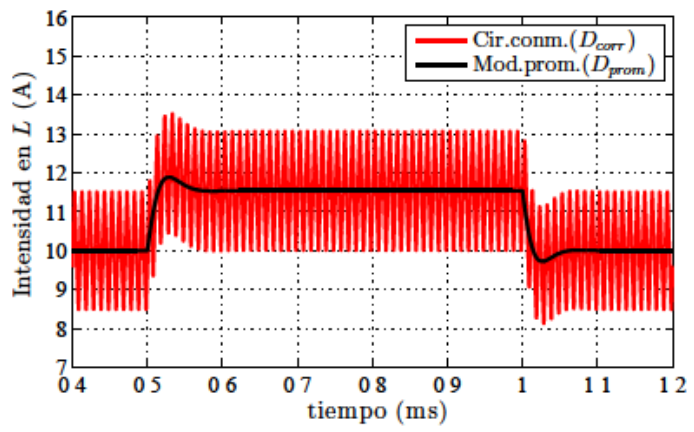

(b)

Figura A.18: Reductor ZVS. Respuesta a escalón en el ciclo de trabajo incluyendo corrección: a) Tensión de salida; y b) Intensidad en la bobina.

Para apreciar la mejora en los resultados se representa el valor medio de la tensión de salida y la intensidad en la bobina para el circuito conmutado antes y después de la corrección y se comparan con el modelo promediado. En la Figura A.19 se pueden comprobar estas curvas.

\section{A.4. Modelo en régimen permanente}

El modelo en régimen permanente se puede obtener a partir del modelo promediado (o de gran señal) sustituyendo los valores promedio por valores 


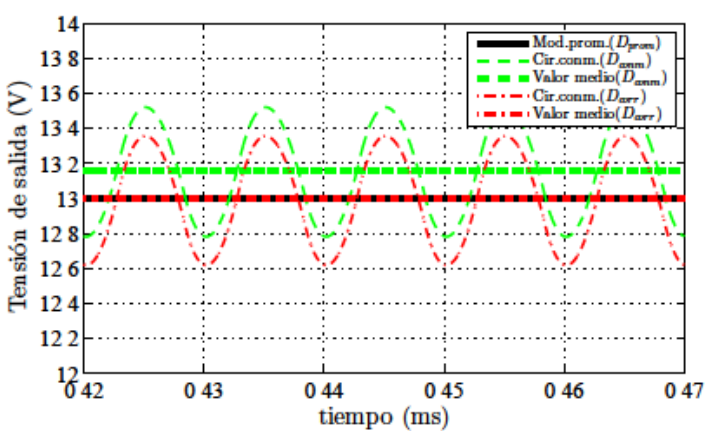

(a)

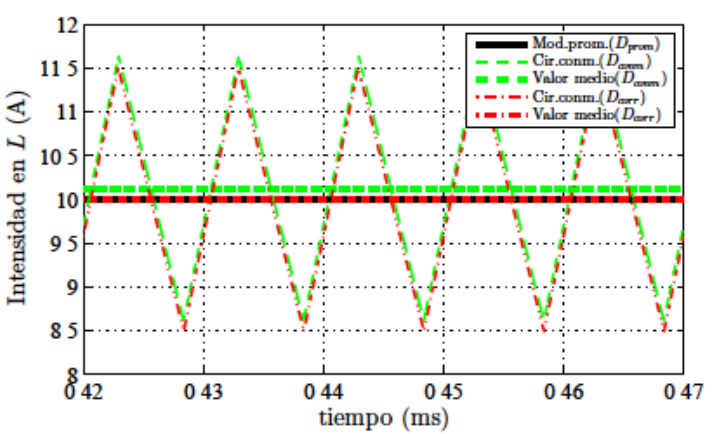

(b)

Figura A.19: Reductor ZVS en régimen permanente. Comparación entre el modelo promediado y los valores medios con y sin corrección en el ciclo de trabajo del circuito conmutado: a) Tensión de salida; y b) Intensidad en la bobina.

constantes (por ejemplo, $\bar{i}_{L}$ se sustituye por $I_{L}$ ). De esta forma, las ecuaciones del modelo en régimen permanente serán las indicadas a continuación. Nótese que la derivada de una constante es cero, de ahí, que haya ecuaciones con un cero a la izquierda de la igualdad:

$$
\begin{aligned}
0 & =V_{g} D-V_{o}-V_{c}(1-D) \\
0 & =I_{L}-\frac{V_{o}}{R} \\
0 & =I_{L}(1-D)-\frac{V_{c}}{R_{e q}} \\
I_{g} & =I_{L} D-I_{e q}
\end{aligned}
$$

donde

$$
R_{e q}=\frac{2 L_{r} f_{s}}{(1-D)^{2}} \quad I_{e q}=\frac{V_{C_{c}}^{2}}{V_{g} R_{e q}}
$$

A estas ecuaciones se puede llegar también a partir del circuito promediado de la figura A.16. Basta con sustituir los condensadores por un circuito abierto y las bobinas con un cortocircuito. De esta forma se obtienen los circuitos de las figuras A.20a y A.20b.

Se obtienen las mismas expresiones para las principales magnitudes en régimen permanente cuando se deducen de (A.104) que a partir de los circuitos 


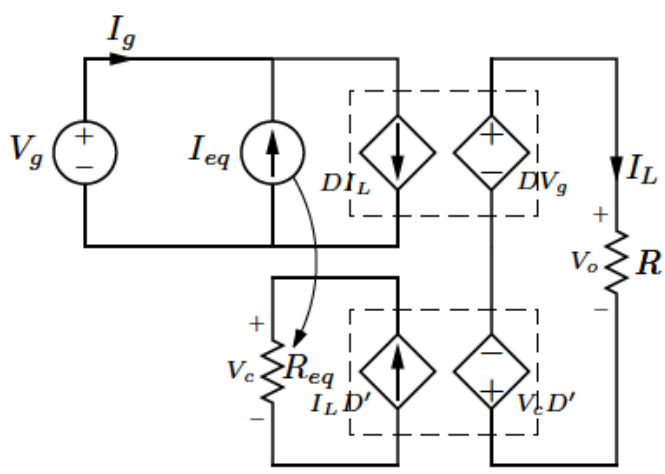

(a)

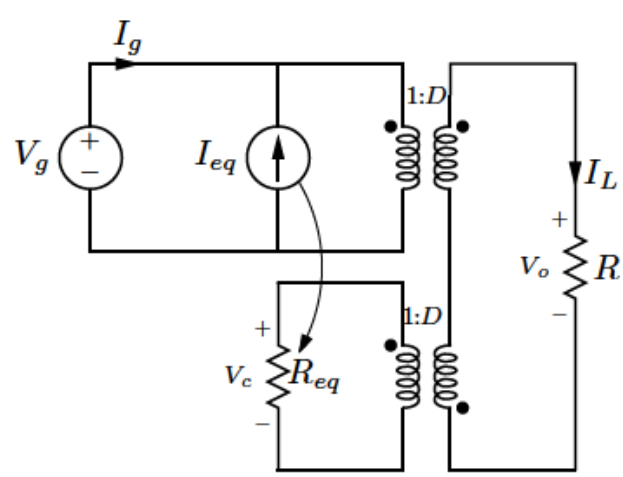

(b)

Figura A.20: Circuito en régimen permanente de un Reductor ZVS en MCC: (a) con fuentes dependientes; (b) con transformador equivalente ideal.

de la figura A.20.

$$
\begin{aligned}
& V_{o}=V_{g} D-V_{c}(1-D) \\
& I_{L}=\frac{V_{o}}{R} \\
& V_{c}=I_{L}(1-D) R_{e q} \\
& I_{g}=I_{L} D-I_{e q}
\end{aligned}
$$

$\mathrm{Si}$ en estas expresiones se elimina $L_{r}$, (que significa que desaparece el circuito de enclavamiento activo), se obtienen las expresiones en régimen permanente para un reductor convencional y lo mismo sucede con los circuitos en régimen permanente de ambas topologías.

La expresión que relaciona la tensión de salida con la intensidad en la carga se obtiene fácilmente a partir de (A.106) como

$$
V_{o}=V_{g} D-I_{L}(1-D)^{2} R_{e q}
$$

donde se observa que la tensión de salida de un reductor ZVS se puede asimilar a un reductor convencional que tiene a la salida una resistencia de valor $(1-D)^{2} R_{e q}$ (con la particularidad de que es una resistencia equivalente sin pérdidas), y evoluciona con la corriente demandada por la carga (que en un reductor convencional es igual a $I_{L}$ ).

\section{A.4.1. Balance energético}

En el modelo en régimen permanente hay dos elementos que generan energía, la fuente de tensión y la fuente de corriente, ambas en la entrada. La energía generada en la fuente de corriente se consume toda en $r_{e q}$. Veámoslo. 
En $R_{e q}$, la potencia consumida es igual a

$$
P_{R_{e q}}=\frac{V_{C_{c}}^{2}}{R_{e q}}
$$

Multiplicando arriba y abajo por $V_{g}$, se obtiene una expresión

$$
P_{R_{e q}}=\frac{V_{C_{c}}^{2}}{R_{e q}} \frac{V_{g}}{V_{g}}
$$

en la que, agrupando, se obtiene el producto de una fuente de tensión por otra de corriente cuyo valor es igual a $I_{e q}$

$$
P_{R e q}=I_{e q} V_{g}
$$

Esta potencia coincide con la potencia generada por la fuente de corriente $i_{e q}$ en la entrada.

En $R$ la potencia consumida es igual a:

$$
\begin{aligned}
P_{R} & =I_{L}^{2} R=V_{o} I_{L}=\left(D V_{g}-(1-D) V_{c}\right) I_{L} \\
& =D V_{g} I_{L}-(1-D) V_{c} I_{L}=V_{g}\left(I_{g}+I_{e q}\right)-V_{c} \frac{V_{c}}{R_{e q}} \\
& =V_{g} I_{g}+V_{g} \frac{V_{C_{c}}^{2}}{V_{g} R_{e g}}-\frac{V_{C_{c}}^{2}}{R_{e g}}=V_{g} I_{g}
\end{aligned}
$$

que coincide, con la potencia generada en la fuente de tensión de la entrada. Esto es, la potencia que suministra la fuente de entrada se entrega a la carga en su totalidad.

\section{A.5. Modelo en pequeña señal}

El modelo en pequeña señal (modelo lineal) se consigue tras realizar el proceso de perturbación y linealización del modelo promediado (modelo no lineal) en torno a un punto de funcionamiento. Se asume que las entradas al modelo, $v_{g}$ y $d$ son iguales a un valor fijo, $V_{g}$ y $D$, mas una pequeña variación de alterna, $\hat{v}_{g}$ y $\hat{d}$. Es decir:

$$
\begin{gathered}
\bar{v}_{g}=V_{g}+\hat{v}_{g} \\
d=D+\hat{d}
\end{gathered}
$$

En respuesta a estas entradas, los valores promediados de las variables de estado sufrirán una variación similar, es decir, presentarán un término constante mas una pequeña variación de alterna. También sucede lo mismo con 
el resto de magnitudes estudiadas, de forma que:

$$
\begin{aligned}
\bar{i}_{L} & =I_{L}+\hat{i}_{L} \\
\bar{v}_{C_{o}} & =V_{o}+\hat{v}_{C_{o}} \\
\bar{v}_{C_{c}} & =V_{c}+\hat{v}_{C_{c}} \\
\bar{i}_{g} & =I_{g}+\hat{i}_{g}
\end{aligned}
$$

Por supuesto, para que el modelo linealizado sea preciso debe cumplirse que las variaciones son pequeñas en comparación con el valor constante que indica el punto de operación del convertidor, es decir:

$$
\begin{aligned}
\left|\hat{v}_{g}\right| & \ll\left|V_{g}\right| \\
|\hat{d}| & \ll|D| \\
\left|\hat{i}_{L}\right| & \ll\left|I_{L}\right| \\
\left|\hat{v}_{C_{o}}\right| & \ll\left|V_{o}\right| \\
\left|\hat{v}_{C_{c}}\right| & \ll\left|V_{c}\right| \\
\left|\hat{i}_{g}\right| & \ll\left|I_{g}\right|
\end{aligned}
$$

Por lo que, introduciendo (A.112) y (A.113) en las ecuaciones del modelo promediado de orden reducido del reductor ZVS, reescritas de nuevo en (A.115) y desarrollando y despreciando los términos de segundo orden se consigue linealizar el modelo promediado.

$$
\begin{aligned}
L \frac{d \bar{i}_{L}}{d t} & =d \bar{v}_{g}-(1-d) \bar{v}_{C_{c}}-\bar{v}_{C_{o}} \\
C_{o} \frac{d \bar{v}_{C_{o}}}{d t} & =\bar{i}_{L}-\frac{\bar{v}_{C_{o}}}{R} \\
C_{c} \frac{d \bar{v}_{C_{c}}}{d t} & =(1-d) \bar{i}_{L}-\frac{(1-d)^{2}}{2 L_{r} f_{s}} \bar{v}_{C_{c}} \\
\bar{i}_{g} & =d \bar{i}_{L}-\frac{\bar{v}_{C_{c}}^{2}}{\bar{v}_{g}} \frac{(1-d)^{2}}{2 L_{r} f_{s}}
\end{aligned}
$$

El proceso de linealización no es mas que un desarrollo de Taylor de una función, $f(x, y, z, \ldots)$ en torno a un punto determinado $\left(x_{0}, y_{0}, z_{0}, \ldots\right)$, considerando únicamente los términos hasta primer orden y despreciando el resto. Por tanto, aplicando el desarrollo de Taylor hasta primer orden a la parte derecha de cada una de las igualdades de las ecuaciones del modelo promediado se consigue llegar a una expresión que permite la obtención del modelo en pequeña señal y también, otra vez, el modelo en régimen permanente. Por ejemplo, la ecuación de la corriente en la bobina de salida del modelo promediado dada en (A.115) se puede escribir como:

$$
L \frac{d \bar{i}_{L}}{d t}=f_{1}\left(\bar{v}_{g}, d, \bar{v}_{C_{o}}, \bar{v}_{C_{c}}\right)
$$


Linealizando la función $f_{1}$ con el desarrollo de Taylor en un punto de funcionamiento dado por $\left(V_{g}, D, I_{L}, V_{o} V_{c}\right)$ se obtiene (A.117). Por supuesto, las ecuación obtenida no será una aproximación muy buena de la función no lineal cuando se trate de un punto alejado del punto de linealización.

$$
\begin{aligned}
& L\left(\frac{d I_{L}}{d t}+\frac{d \hat{i}_{L}}{d t}\right)=f_{1}\left(V_{g}, D, V_{o}, V_{c}\right) \\
& +\left.\hat{v}_{g}(t) \frac{\partial f_{1}\left(\bar{v}_{g}, D, V_{o}, V_{c}\right)}{\partial \bar{v}_{g}}\right|_{\bar{v}_{g}=V_{g}}+\left.\hat{v}_{C_{o}} \frac{\partial f_{1}\left(V_{g}, D, \bar{v}_{C_{o}}, V_{c}\right)}{\partial \bar{v}_{C_{o}}}\right|_{\bar{v}_{C_{o}}=V_{o}} \\
& +\left.\hat{v}_{C_{c}} \frac{\partial f_{1}\left(V_{g}, D, V_{o}, \bar{v}_{C_{c}}\right)}{\partial \bar{v}_{C_{c}}}\right|_{\bar{v}_{C_{c}}=V_{c}}+\left.\hat{d}(t) \frac{\partial f_{1}\left(V_{g}, d, V_{o}, V_{c}\right)}{\partial d}\right|_{d=D}
\end{aligned}
$$

+ términos de orden superior

A continuación se evalúan las derivadas parciales para la ecuación de la corriente en la bobina de salida, $\bar{i}_{L}$. Las derivadas parciales son:

$$
\begin{gathered}
\left.\frac{\partial f_{1}\left(\bar{v}_{g}, D, V_{o}, V_{c}\right)}{\partial \bar{v}_{g}}\right|_{\bar{v}_{g}=V_{g}}=D \\
\left.\frac{\partial f_{1}\left(V_{g}, D, \bar{v}_{C_{o}}, V_{c}\right)}{\partial \bar{v}_{C_{o}}}\right|_{\bar{v}_{C_{o}}=V_{o}}=-1 \\
\left.\frac{\partial f_{1}\left(V_{g}, D, V_{o}, \bar{v}_{C_{c}}\right)}{\partial \bar{v}_{C_{c}}}\right|_{\bar{v}_{C_{c}=V_{c}}}=-(1-D) \\
\left.\frac{\partial f_{1}\left(V_{g}, d, V_{o}, V_{c}\right)}{\partial d}\right|_{d=D}=V_{g}+V_{c}
\end{gathered}
$$

La ecuación (A.117) queda ahora así:

$$
\begin{aligned}
L\left(0+\frac{d \hat{i}_{L}}{d t}\right) & =\left[V_{g} D-V_{o}-V_{c}(1-D)\right] \\
& +D \hat{v}_{g}-\hat{v}_{C_{o}}-(1-D) \hat{v}_{C_{c}}+\left(V_{g}+V_{c}\right) \hat{d} \\
& + \text { términos de orden superior }
\end{aligned}
$$

Los términos constantes de ambos miembros se igualan para conseguir una ecuación para el modelo en régimen permanente que coincide con la primera expresión de (A.104). Los términos de primer orden generan otra ecuación que forma parte del modelo en pequeña señal. El resto de términos de orden superior se desprecian. 
Si se hace de forma similar para el restos de ecuaciones del modelo promediado, vistas como

$$
\begin{aligned}
C_{o} \frac{d \bar{v}_{C_{o}}}{d t} & =f_{2}\left(\bar{i}_{L}, \bar{v}_{C_{o}}\right) \\
C_{c} \frac{d \bar{v}_{C_{c}}}{d t} & =f_{3}\left(d, \bar{i}_{L}, \bar{v}_{C_{c}}\right) \\
\bar{i}_{g} & =f_{4}\left(\bar{v}_{g}, d, \bar{i}_{L}, \bar{v}_{C_{c}}\right)
\end{aligned}
$$

será necesario evaluar las funciones $f_{2}, f_{3}$ y $f_{4}$ y sus derivadas parciales en torno al punto de funcionamiento elegido. Las funciones $f_{2}, f_{3}$ y $f_{4}$ evaluadas en el punto de funcionamiento son:

$$
\begin{aligned}
f_{2}\left(I_{L}, V_{o}\right) & =I_{L}-\frac{V_{o}}{R} \\
f_{3}\left(D, I_{L}, V_{c}\right) & =I_{L}(1-D)-\frac{V_{c}}{R_{e q}} \\
f 4\left(V_{g}, D, I_{L}, V_{c}\right) & =I_{L} D-I_{e q}
\end{aligned}
$$

Las derivadas parciales respecto a las variables de estado de las funciones anteriores, y evaluadas en torno al punto de funcionamiento están indicadas a continuación.

$$
\begin{gathered}
\left.\frac{\partial f_{2}(\ldots)}{\partial \bar{i}_{L}}\right|_{\bar{i}_{L}=I_{L}}=1 \\
\left.\frac{\partial f_{2}(\ldots)}{\partial \bar{v}_{C_{o}}}\right|_{\bar{v}_{C_{o}}=V_{o}}=-\frac{1}{R} \\
\left.\frac{\partial f_{3}(\ldots)}{\partial d}\right|_{d=D}=I_{L} \\
\left.\frac{\partial f_{3}(\ldots)}{\partial \bar{i}_{L}}\right|_{\bar{i}_{L}=I_{L}}=(1-D) \\
\left.\frac{\partial f_{3}(\ldots)}{\partial \bar{v}_{C_{c}}}\right|_{\bar{v}_{C_{c}}=V_{c}}=-\frac{(1-D)^{2}}{2 L_{r} f_{s}}=-\frac{1}{R_{e q}}
\end{gathered}
$$




$$
\begin{aligned}
& \left.\frac{\partial f_{4}(\ldots)}{\partial \bar{v}_{g}}\right|_{\bar{v}_{g}=V_{g}}=-V_{c} \frac{(1-D)^{2}}{2 L_{r} f s}\left(-\frac{1}{V_{g}^{2}}\right)=\frac{I_{e q}}{V_{g}} \\
& \left.\frac{\partial f_{4}(\ldots)}{\partial d}\right|_{d=D}=I_{L}-\frac{V_{C_{c}}^{2}}{V_{g}} \frac{2(1-D)}{2 L_{r} f_{s}}(-1)=I_{L}+\frac{2}{1-D} I_{e q} \\
& \left.\frac{\partial f_{4}(\ldots)}{\partial \bar{i}_{L}}\right|_{\bar{i}_{L}=I_{L}}=D \\
& \left.\frac{\partial f_{4}(\ldots)}{\partial \bar{v}_{C_{c}}}\right|_{\bar{v}_{C_{c}}=V_{c}}=-\frac{2 V_{c}(1-D)^{2}}{V_{g} 2 L_{r} f_{s}}=-2 \frac{I_{e q}}{V_{c}}
\end{aligned}
$$

Entonces, cada una de las ecuaciones (A.120) quedan de la forma indicada en (A.125), (A.126) y(A.127).

$$
\begin{aligned}
C_{o}\left(0+\frac{d \hat{v}_{C_{o}}}{d t}\right) & =\left[I_{L}-\frac{V_{o}}{R}\right] \\
& +\hat{i}_{L}-\frac{1}{R} \hat{v}_{C_{o}} \\
& + \text { términos de orden superior } \\
C_{c}\left(0+\frac{d \hat{v}_{C_{c}}}{d t}\right) & =\left[I_{L}(1-D)-\frac{V_{c}}{R_{e q}}\right] \\
& +I_{L} \hat{d}+(1-D) \hat{i}_{L}-\frac{1}{R_{e q}} \hat{v}_{C_{c}}
\end{aligned}
$$

+ términos de orden superior

$$
\begin{aligned}
I_{g}+\hat{i}_{g} & =\left[I_{L} D-I_{e q}\right] \\
& +\frac{I_{e q}}{V_{g}} \hat{v}_{g}+\left(I_{L}+\frac{2}{1-D} I_{e q}\right) \hat{d}+D \hat{i}_{L}-2 \frac{I_{e q}}{V_{c}} \hat{v}_{C_{c}} \\
& + \text { términos de orden superior }
\end{aligned}
$$

Agrupando todos los términos constantes de las ecuaciones (A.119), (A.125), (A.126) y (A.127) se obtiene el modelo en régimen permanente que coincide con el descrito en (A.106).

Tomando todos los términos de primer orden y despreciando los términos de orden superior, se consigue el modelo en pequeña señal para el reductor 
ZVS dado en (A.128).

$$
\begin{aligned}
L \frac{\hat{i}_{L}}{d t} & =D \hat{v}_{g}-\hat{v}_{C_{o}}-(1-D) \hat{v}_{C_{c}}+\left(V_{g}+V_{c}\right) \hat{d} \\
C_{o} \frac{d \hat{v}_{C_{o}}}{d t} & =\hat{i}_{L}-\frac{1}{R} \hat{v}_{C_{o}} \\
C_{c} \frac{d \hat{v}_{C_{c}}}{d t} & =I_{L} \hat{d}+(1-D) \hat{i}_{L}-\frac{1}{R_{e q}} \hat{v}_{C_{c}} \\
\hat{i}_{g}(t) & =\frac{I_{e q}}{V_{g}} \hat{v}_{g}+\left(I_{L}+\frac{2}{1-D} I_{e q}\right) \hat{d}+D \hat{i}_{L}-2 \frac{I_{e q}}{V_{c}} \hat{v}_{C_{c}}
\end{aligned}
$$

Con estas ecuaciones se construye el circuito en pequeña señal de la figura A.21. Los parámetros $j_{1}, r_{1}, g_{1}$ y $f_{1}$ están indicados en la tabla A.4.

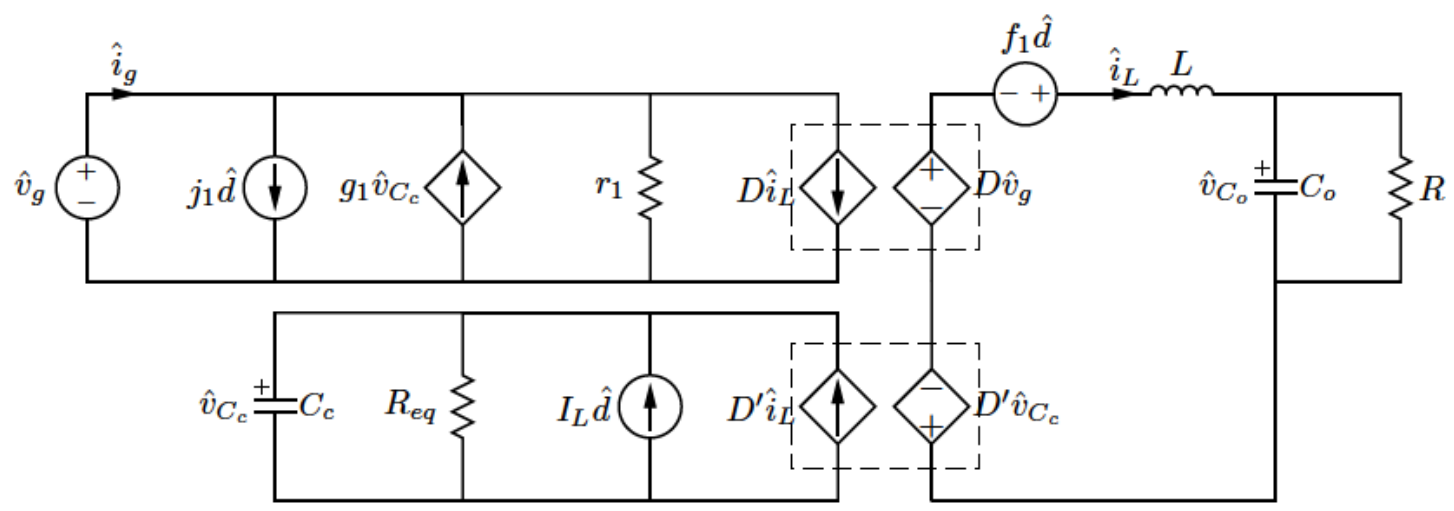

Figura A.21: Modelo en pequeña señal de un reductor ZVS.

\begin{tabular}{ccccc}
\hline Convertidor & $j_{1}$ & $r_{1}$ & $g_{1}$ & $f_{1}$ \\
\hline Reductor & $I_{L}+\frac{2 I_{e q}}{1-D}$ & $\frac{V_{g}}{I_{e q}}$ & $2 \frac{I_{e q}}{V_{c}}$ & $V_{c}+V_{g}$ \\
\hline
\end{tabular}

Tabla A.4: Parámetros del modelo en pequeña señal del reductor ZVS.

El modelo en pequeña señal, obtenido a partir de la linealización del modelo promediado de orden reducido, también se puede escribir en forma matricial. Así, se definen tres vectores, el vector $\hat{\mathbf{x}}$ para las variables de estado, el vector $\hat{\mathbf{u}}$ para las entradas externas e independientes, y el vector ŷ para las salidas del modelo, y serán en este caso:

$$
\hat{\mathbf{x}}=\left[\begin{array}{c}
\hat{i}_{L} \\
\hat{v}_{C_{o}} \\
\hat{v}_{C_{c}}
\end{array}\right], \quad \hat{\mathbf{u}}=\left[\begin{array}{c}
\hat{v}_{g} \\
\hat{d}
\end{array}\right], \quad \hat{\mathbf{y}}=\left[\hat{i}_{g}\right]
$$


de forma que el modelo en pequeña señal de (A.128) se puede escribir, de la forma siguiente:

$$
\begin{array}{r}
\mathbf{K} \frac{d}{d t}\left[\begin{array}{c}
\hat{i}_{L} \\
\hat{v}_{C_{o}} \\
\hat{v}_{C_{c}}
\end{array}\right]=\mathbf{A}\left[\begin{array}{c}
\hat{i}_{L} \\
\hat{v}_{C_{o}} \\
\hat{v}_{C_{c}}
\end{array}\right]+\mathbf{B}\left[\begin{array}{c}
\hat{v}_{g} \\
\hat{d}
\end{array}\right] \\
{\left[\hat{i}_{g}\right]=\mathbf{C}\left[\begin{array}{c}
\hat{i}_{L} \\
\hat{v}_{C_{o}} \\
\hat{v}_{C_{c}}
\end{array}\right]+\mathbf{E}\left[\begin{array}{c}
\hat{v}_{g} \\
\hat{d}
\end{array}\right]}
\end{array}
$$

donde

$$
\begin{aligned}
& \mathbf{K}=\left[\begin{array}{ccc}
L & 0 & 0 \\
0 & C_{o} & 0 \\
0 & 0 & C_{c}
\end{array}\right], \quad \mathbf{A}=\left[\begin{array}{ccc}
1 & -1 & D-1 \\
1 & -\frac{1}{R} & 0 \\
1-D & 0 & \frac{1}{R_{e q}}
\end{array}\right], \\
& \mathbf{B}=\left[\begin{array}{cc}
0 & f_{1} \\
0 & 0 \\
0 & I_{L}
\end{array}\right], \quad \mathbf{C}=\left[\begin{array}{lll}
D & 0 & g_{1}
\end{array}\right], \quad \mathbf{E}=\left[\begin{array}{ll}
r_{1} & j_{1}
\end{array}\right]
\end{aligned}
$$

\section{A.6. Funciones de transferencia}

Las diferentes funciones de transferencia se pueden obtener bien a partir del modelo en pequeña señal dado en las ecuaciones (A.128), bien a partir del circuito en pequeña señal de la figura A.21. Por ambos métodos hay que anular la variable de estado correspondiente y aplicar Laplace para obtener la relación deseada. Otra posibilidad es trabajar con el modelo matricial en pequeña señal, que se verá mas adelante.

\section{A.6.1. Desde el modelo o el circuito de pequeña señal}

Para conseguir $G_{v g}(s)$, se hace nula la variación de la entrada independiente que no interviene en la función de transferencia, en este caso, $\hat{d}(t)=0$, y se aplica Laplace. De esta forma queda que:

$$
\begin{aligned}
s L \hat{i}_{L}(s) & =D \hat{v}_{g}(s)-\hat{v}_{C_{o}}(s)-(1-D) \hat{v}_{C_{c}}(s) \\
s C_{o} \hat{v}_{C_{o}}(s) & =\hat{i}_{L}(s)-\frac{1}{R} \hat{v}_{C_{o}}(s) \\
s C_{c} \hat{v}_{C_{c}}(s) & =(1-D) \hat{i}_{L}(s)-\frac{1}{R_{e q}} \hat{v}_{C_{c}}(s)
\end{aligned}
$$

De la segunda ecuación se despeja $\hat{i}_{L}(s)$ para sustituirla en la tercera 
ecuación y obtener $\hat{v}_{C_{c}}(s)$.

$$
\begin{aligned}
\hat{i}_{L}(s) & =\left[s C_{o}+\frac{1}{R}\right] \hat{v}_{C_{o}}(s)=\frac{1}{Z_{o}(s)} \hat{v}_{C_{o}}(s) \\
\hat{v}_{C_{c}}(s) & =\left[s C_{o}+\frac{1}{R}\right] \frac{1-D}{s C_{c}+\frac{1}{R_{e q}}} \hat{v}_{C_{o}}(s)=(1-D) \frac{Z_{e q}(s)}{Z_{o}(s)} \hat{v}_{C_{o}}(s)
\end{aligned}
$$

en donde se ha definido

$$
\begin{aligned}
& Z_{o}(s)=\left(R \| C_{o}\right)=\left[s C_{o}+\frac{1}{R}\right]^{-1} \\
& Z_{e q}(s)=\left(R_{e q} \| C_{c}\right)=\left[s C_{c}+\frac{1}{R_{e q}}\right]^{-1}
\end{aligned}
$$

Introduciendo (A.133) en (A.132) se obtiene fácilmente la función de transferencia deseada.

$$
G_{v g}(s)=\left.\frac{\hat{v}_{C_{o}}(s)}{\hat{v}_{g}(s)}\right|_{\hat{d}=0}=\frac{D}{1+L s / Z_{o}(s)+(1-D)^{2} Z_{e q}(s) / Z_{o}(s)}
$$

Para conseguir $G_{i g}(s)$, en (A.132) se despejan $\hat{v}_{C_{o}}(s)$ y $\hat{v}_{C_{c}}(s)$ en función de $\hat{i}_{L}(s)$.

$$
\begin{aligned}
& \hat{v}_{C_{o}}(s)=\frac{1}{s C_{o}+\frac{1}{R}} \hat{i}_{L}(s)=Z_{o}(s) \hat{i}_{L}(s) \\
& \hat{v}_{C_{c}}(s)=(1-D) \hat{i}_{L}(s) \frac{1}{s C_{c}+\frac{1}{\text { Rreq }}}=(1-D) Z_{e q}(s) \hat{i}_{L}(s)
\end{aligned}
$$

Introduciendo (A.136) en la primera ecuación se obtiene la función de transferencia deseada.

$$
G_{i g}(s)=\left.\frac{\hat{i}_{L}(s)}{\hat{v}_{g}(s)}\right|_{\hat{d}=0}=\frac{D / Z_{o}(s)}{1+L s / Z_{o}(s)+(1-D)^{2} Z_{e q}(s) / Z_{o}(s)}
$$

La mismas expresiones se obtienen si se utiliza el circuito equivalente en pequeña señal del reductor de la figura A.21. Incialmente se anula la o las fuentes correspondientes a la entrada independiente que no entra en la función de transferencia y se hace Laplace. El circuito resultante para el cálculo de $G_{v g}(s)$ y $G_{i g}(s)$ está representado en la figura A.22 una vez anulado $\hat{d}$.

En la malla de la derecha de este circuito se cumple que

$$
D \hat{v}_{g}(s)=L s \hat{i}_{L}(s)+\hat{v}_{C_{o}}(s)+(1-D) \hat{v}_{C_{c}}(s)
$$




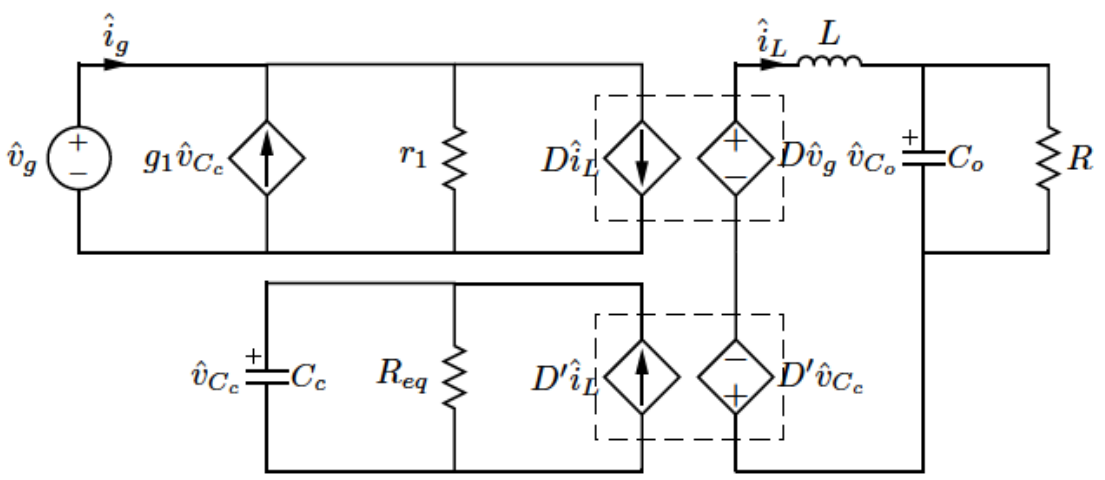

Figura A.22: Circuito equivalente de un reductor cuando $\hat{d}=0$

y la ley de Ohm aplicada a las cargas del circuito implica que

$$
\hat{i}_{L}(s)=\frac{\hat{v}_{C_{o}}(s)}{Z_{o}(s)}, \quad \hat{v}_{C_{c}}(s)=(1-D) Z_{e q}(s) \hat{i}_{L}(s)
$$

Es sencillo comprobar que, utilizando (A.139) en (A.138) y despejando, bien $G_{v g}(s)$, bien $G_{i g}(s)$ se obtienen de nuevo (A.135) y (A.137). Estas funciones de transferencia coinciden con las de un reductor convencional cuando los términos relacionados con el enclavamiento activo, $C_{c}$ y $L_{r}$, desaparecen puesto que $V_{c}, R_{e q}$ y $Z_{e} q$ se anulan.

En la FiguraA.25 están representadas ambas funciones de transferencia con los parámetros de funcionamiento de la Tabla A.1, comparadas con las que tendría un reductor convencional con los mismos parámetros.

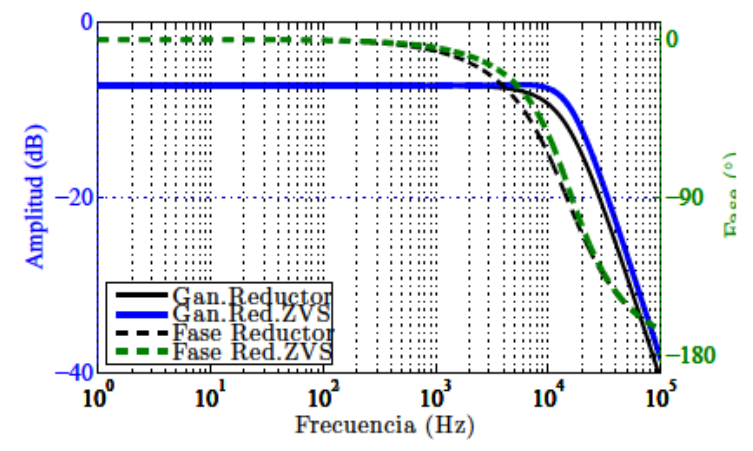

(a)

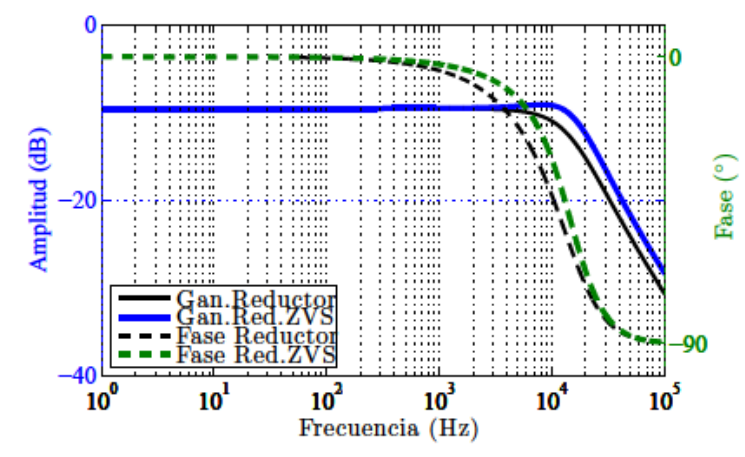

(b)

Figura A.23: Reductor ZVS. Func. de transferencia en función de la tensión de entrada para: a) Tensión de salida, $G_{v g}(s)$; y b) Intensidad en la bobina, $G_{i g}(s)$. 
Igualmente, para conseguir $G_{v d}(s)$, se hace nula la variación de la entrada independiente que no interviene en la función de transferencia, en este caso, $\hat{v}_{g}(t)=0$, y se aplica Laplace. De esta forma queda que:

$$
\begin{aligned}
s L \hat{i}_{L}(s) & =-\hat{v}_{C_{o}}(s)-(1-D) \hat{v}_{C_{c}}(s)+f_{1} \hat{d} \\
s C_{o} \hat{v}_{C_{o}}(s) & =\hat{i}_{L}(s)-\frac{1}{R} \hat{v}_{C_{o}}(s) \\
s C_{c} \hat{v}_{C_{c}}(s) & =I_{L} \hat{d}+(1-D) \hat{i}_{L}(s)-\frac{1}{R_{e q}} \hat{v}_{C_{c}}(s)
\end{aligned}
$$

Es fácil despejar ahora $\hat{i}_{L}(s)$, para sustituirla en la tercera ecuación y obtener $\hat{v}_{C_{c}}(s)$.

$$
\begin{aligned}
\hat{i}_{L}(s) & =\frac{1}{Z_{o}(s)} \hat{v}_{C_{o}}(s) \\
\hat{v}_{C_{c}}(s) & =I_{L} Z_{e q}(s) \hat{d}(s)+(1-D) \frac{Z_{e q}(s)}{Z_{o}(s)} \hat{v}_{C_{o}}(s)
\end{aligned}
$$

Introduciendo (A.141) en (A.140) se obtiene fácilmente la función de transferencia deseada.

$$
G_{v d}(s)=\left.\frac{\hat{v}_{C_{o}}(s)}{\hat{d}(s)}\right|_{\hat{v}_{g}=0}=\frac{f_{1}-(1-D) I_{L} Z_{e q}(s)}{1+L s / Z_{o}(s)+(1-D)^{2} Z_{e q}(s) / Z_{o}(s)}
$$

Para conseguir $G_{i d}(s)$, en (A.140) se despejan $\hat{v}_{C_{o}}(s)$ y $\hat{v}_{C_{c}}(s)$ en función de $\hat{i}_{L}(s)$.

$$
\begin{aligned}
& \hat{v}_{C_{o}}(s)=Z_{o}(s) \hat{i}_{L}(s) \\
& \hat{v}_{C_{c}}(s)=I_{L} Z_{e q}(s) \hat{d}(s)+(1-D) Z_{e q}(s) \hat{i}_{L}(s)
\end{aligned}
$$

Introduciendo (A.143) en la primera ecuación se obtiene la función de transferencia deseada.

$$
G_{i d}(s)=\left.\frac{\hat{i}_{L}(s)}{\hat{d}(s)}\right|_{\hat{v}_{g}=0}=\frac{1}{Z_{o}(s)} \frac{f_{1}-(1-D) I_{L} Z_{e q}(s)}{1+L s / Z_{o}(s)+(1-D)^{2} Z_{e q}(s) / Z_{o}(s)}
$$

Las mismas expresiones se obtienen si se utiliza el circuito equivalente en pequeña señal del reductor de la figura A.21. El circuito resultante para el cálculo de $G_{v d}(s)$ y $G_{i d}(s)$ y está representado en la figura A.24 una vez anulado $\hat{v}_{g}$.

En la malla de la derecha de este circuito se cumple que

$$
f_{1} \hat{d}(s)=L s \hat{i}_{L}(s)+\hat{v}_{C_{o}}(s)+(1-D) \hat{v}_{C_{c}}(s)
$$

y la ley de Ohm aplicada a las cargas del circuito implica que

$$
\hat{i}_{L}(s)=\frac{1}{Z_{o}(s)} \hat{v}_{C_{o}}(s), \quad \hat{v}_{C_{c}}(s)=(1-D) Z_{e q}(s) \hat{i}_{L}(s)+I_{L} Z_{e q}(s) \hat{d}(s)
$$




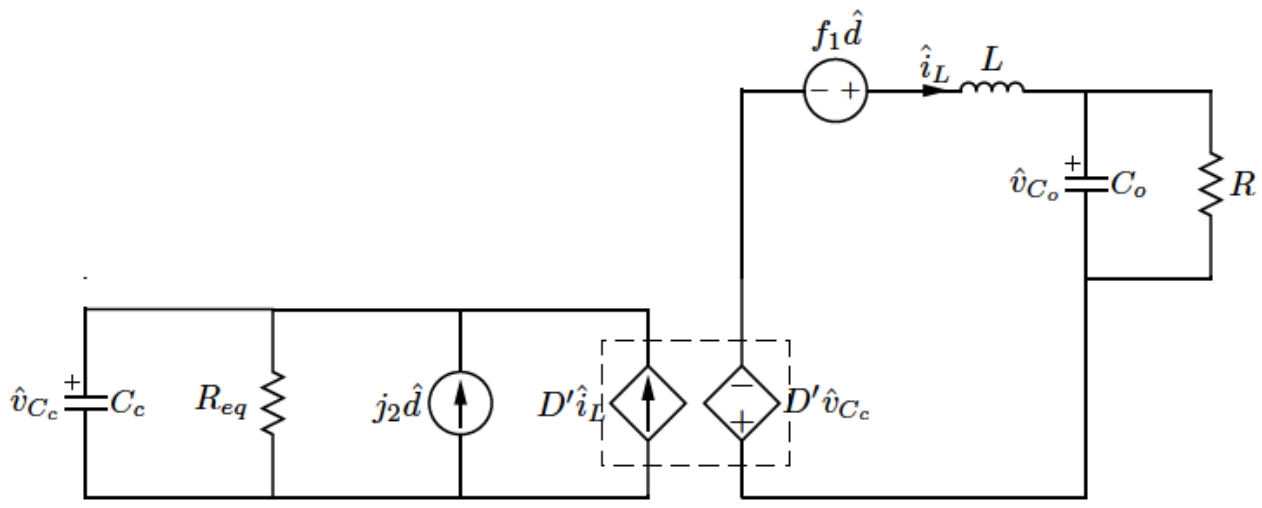

Figura A.24: Circuito equivalente de un reductor ZVS cuando $\hat{v}_{g}=0$

Es sencillo comprobar que, utilizando (A.146) en (A.145) y despejando, bien $G_{v d}(s)$, bien $G_{i d}(s)$, se obtiene de nuevo (A.142) y (A.144). Estas funciones de transferencia coinciden con las de un reductor convencional cuando los términos relacionados con el enclavamiento activo, $C_{c}$ y $L_{r}$, desaparecen puesto que $V_{c}, R_{e q}$ y $Z_{e} q$ se anulan.

En la FiguraA.23 están representadas ambas funciones de transferencia con los parámetros de funcionamiento de la Tabla A.1, comparadas con las que tendría un reductor convencional con los mismos parámetros.

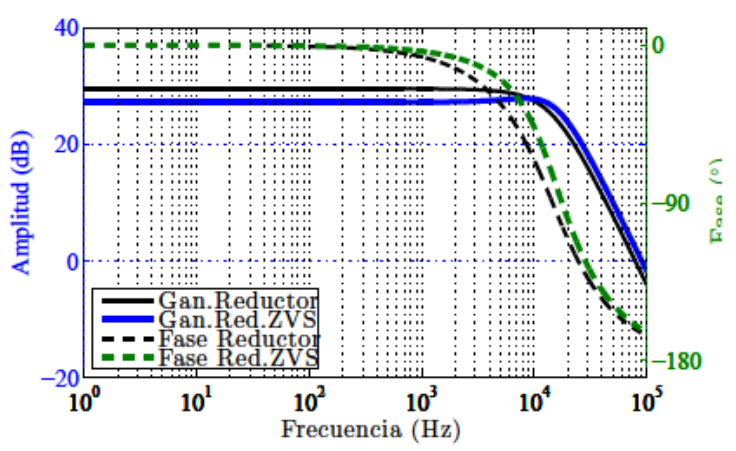

(a)

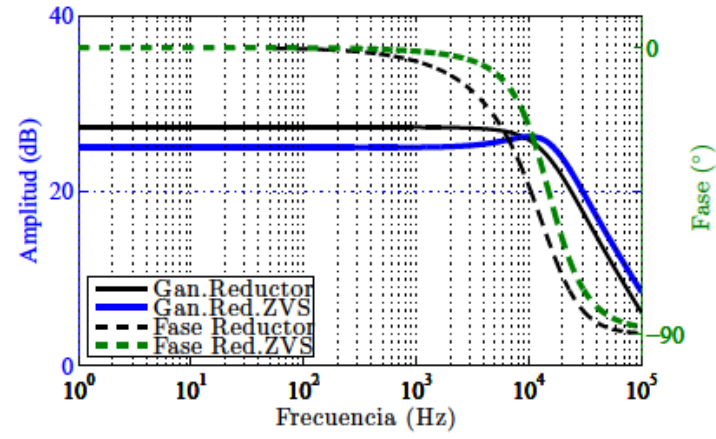

(b)

Figura A.25: Reductor ZVS. Func. de transferencia en función del ciclo de trabajo para: a) Tensión de salida, $G_{v d}(s)$; y b) Intensidad en la bobina, $G_{i d}(s)$. 


\section{A.6.2. Desde el modelo matricial}

Para obtener las funciones de transferencia anteriores ${ }^{2}$, se aplica Laplace a las ecuaciones de estado en forma matricial (suponiendo que las condiciones iniciales con nulas).

$$
\begin{aligned}
\mathbf{K} s \hat{\mathbf{X}}(s) & =\mathbf{A} \hat{\mathbf{X}}(s)+\mathbf{B} \hat{\mathbf{U}}(s) \\
\hat{\mathbf{Y}}(s) & =\mathbf{C} \hat{\mathbf{X}}(s)+\mathbf{E} \hat{\mathbf{U}}(s)
\end{aligned}
$$

En la primera ecuación de (A.147) se despeja el vector de las variables de estado. Por tanto, se tiene que

$$
\mathbf{K} s \hat{\mathbf{X}}(s)-\mathbf{A} \hat{\mathbf{X}}(s)=\mathbf{B} \hat{\mathbf{U}}(s)
$$

o bien

$$
(\mathbf{K} s-\mathbf{A}) \hat{\mathbf{X}}(s)=\mathbf{B} \hat{\mathbf{U}}(s)
$$

Premultiplicando ambos miembros por $(\mathbf{K} s-\mathbf{A})^{-1}$ se obtiene:

$$
\hat{\mathbf{X}}(s)=(\mathbf{K} s-\mathbf{A})^{-1} \mathbf{B} \hat{\mathbf{U}}(s)
$$

Sustituyendo la ecuacion (A.148) en la segunda ecuación de (A.147) se llega a

$$
\hat{\mathbf{Y}}(s)=\left[\mathbf{C}(\mathbf{K} s-\mathbf{A})^{-1} \mathbf{B}+\mathbf{D}\right] \hat{\mathbf{U}}(s)
$$

Puesto, que la relación entre la entrada y la salida de un sistema es la función de transferencia, resulta que la matriz $\mathbf{G}(s)$ definida en (A.150) es la denominada matriz de transferencia que relaciona las diferentes salidas del sistema con las diferentes entradas. Tiene tantas filas como salidas y tantas columnas como entradas.

$$
\mathbf{G}(s)=\mathbf{C}(\mathbf{K} s-\mathbf{A})^{-1} \mathbf{B}+\mathbf{D}
$$

En el caso del reductor ZVS, se redefine el vector de salidas y las matrices C y $\mathbf{E}$, de la siguiente forma:

$$
\hat{\mathbf{y}}=\left[\begin{array}{c}
\hat{i}_{L} \\
\hat{v}_{o}
\end{array}\right], \quad \mathbf{C}=\left[\begin{array}{lll}
1 & 0 & 0 \\
0 & 1 & 0
\end{array}\right], \quad \mathbf{E}=\left[\begin{array}{ll}
0 & 0 \\
0 & 0
\end{array}\right]
$$

De esta forma, la matriz de transferencia, queda como:

$$
\mathbf{G}(s)=\left[\begin{array}{ll}
G_{i g}(s) & G_{i d}(s) \\
G_{v g}(s) & G_{v d}(s)
\end{array}\right]
$$

que es una matriz que recoge las funciones de transferencias obtenidas en el apartado anterior.

\footnotetext{
${ }^{2}$ Ver (OGATA, 2003)
} 


\section{A.7. Impedancia de salida}

La impedancia de salida en pequeña señal se obtiene a partir del modelo en pequeña señal de la Figura A.21. Para ello hay que anular los términos independientes, $\hat{v}_{g}$ y $\hat{d}$ y conectar una fuente independiente, $\hat{i}_{t}$, en los puntos donde que quiere medir la impedancia. La tensión que genera esta fuente sobre el sistema, $\hat{v}_{t}$, se emplea para obtener $Z_{\text {out }}(s)$ como:

$$
Z_{\text {out }}(s)=\left.\frac{\hat{v}_{t}(s)}{\hat{i}_{t}(s)}\right|_{\begin{array}{l}
\hat{v}_{g}=0 \\
\hat{d}=0
\end{array}}
$$

considerando que $\hat{i}_{t}(s)$ es la intensidad que entra por el terminal de mayor tensión de los dos puntos de medida considerados anteriormente. En el circuito de la Figura A.26 se indica gráficamente la conexión de la fuente independiente al modelo en pequeña señal una vez anuladas las fuentes independientes. Además, se ha indicado la impedancia $Z_{t h}$ como la impedancia formada por el condensador del clamp, la resistencia equivalente y la bobina principal.

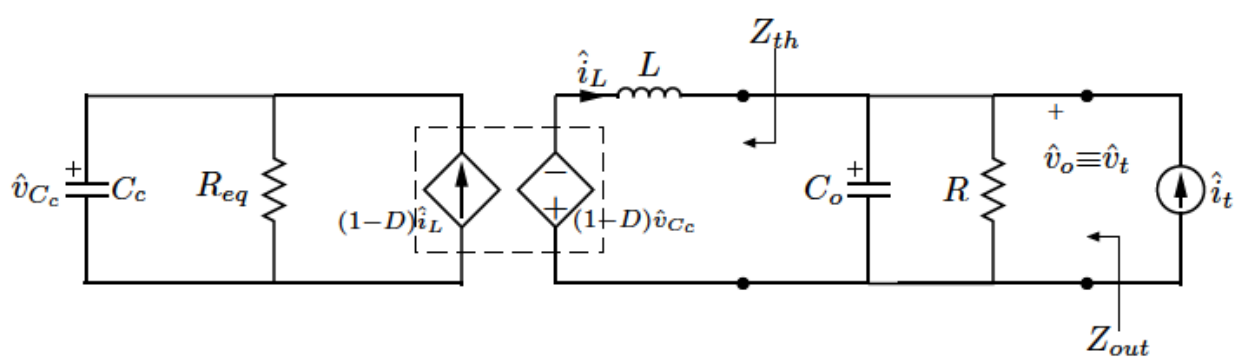

Figura A.26: Circuito para obtener la impedancia de salida de un reductor ZVS.

\section{A.7.1. Cálculo de $Z_{t h}$.}

Para obtener esta impedancia,se utiliza la parte del circuito anterior que concierne al punto de medida de $Z_{t h}$, en el que se conecta la fuente $\hat{i}_{t}$. Queda el circuito de la Figura A.27. Pasando el conjunto $R_{e q}-C_{c}$, al otro lado de las fuentes dependientes, se encontrará en serie con la bobina $L$. La nueva impedancia se pasa hacia la salida, obteniéndose la expresión:

$$
Z_{t h}(s)=L s+(1-D)^{2} Z_{e q}(s)
$$

A esta expresión también se puede llegar a partir del modelo en pequeña señal de (A.128). Basta con anular los términos independientes, $\hat{v}_{g}(t)$ y $\hat{d}(t)$ y cambiar la ecuación relativa a la tensión del condensador $C_{o}$, puesto que éste, 


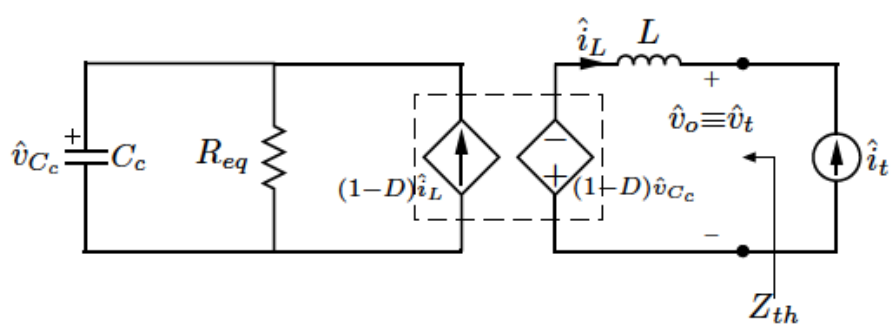

Figura A.27: Circuito empleado para obtener $Z_{t h}(s)$.

junto con la carga $R$, queda fuera y en su lugar se ha conectado una fuente de intensidad, $\hat{i}_{t}(t)$. La nueva ecuación relaciona la intensidad en la bobina, $\hat{i}_{L}(t)$, con esa intensidad. También hay que sustituir el término $\hat{v}_{C_{o}}(t)$ por $\hat{v}_{t}(t)$ en el resto de ecuaciones. Finalmente se aplica Laplace a las ecuaciones resultantes y quedan las ecuaciones indicadas en (A.155):

$$
\begin{aligned}
s L \hat{i}_{L}(s) & =-\hat{v}_{t}(s)-(1-D) \hat{v}_{C_{c}}(s) \\
\hat{i}_{t}(s) & =-\hat{i}_{L}(s) \\
s C_{c} \hat{v}_{C_{c}}(s) & =(1-D) \hat{i}_{L}(s)-\frac{1}{R_{e q}} \hat{v}_{C_{c}}(s)
\end{aligned}
$$

Empleando la igualdad de la segunda ecuación, se despeja $\hat{v}_{C_{c}}$ de la tercera ecuación y se sustituye en en la primera, para obtener una expresión de la impedancia que es equivalente a la mostrada en (A.154):

$$
\begin{aligned}
\hat{v}_{C_{c}}(s) & =-\frac{(1-D)}{s C_{c}+\frac{1}{R_{e q}}} \hat{i}_{t}(s)=-(1-D) Z_{e q}(s) \hat{i}_{t}(s) \\
\hat{v}_{t}(s) & =\left[L s+(1-D)^{2} Z_{e q}(s)\right] \hat{i}_{t}(s) \\
Z_{t h}(s) & =\frac{\hat{v}_{t}(s)}{\hat{i}_{t}(s)}=L s+(1-D)^{2} Z_{e q}(s)
\end{aligned}
$$

En la Figura A.28 se ha representado $\left|Z_{t h}(s)\right|$ para un Reductor ZVS con los parámetros indicados en la Tabla A.1. También están representados los módulos de lss impedancias debidas a los elementos que forman parte de $Z_{t h}(s)$, esto es, la bobina $L$, el condensador $C_{c}$ y el término de resistencia equivalente $R_{e q}$, afectados por el factor correspondiente a su posición respecto al punto donde se obtiene la impedancia.

Al hacer $s=0$ se obtiene la impedancia en continua, que es igual al término $R_{e q}(1-D)^{2}$, y se corresponde con la asíntota horizontal de la impedancia $Z_{t h}(s)$ a baja frecuencia.

$$
Z_{t h}(0)=(1-D)^{2} Z_{e q}(0)=(1-D)^{2} R_{e q}
$$

Este mismo resultado se obtiene analizando el circuito de la Figura A.27 cuando la bobina y el condensador se convierten en un cortocircuito y en 


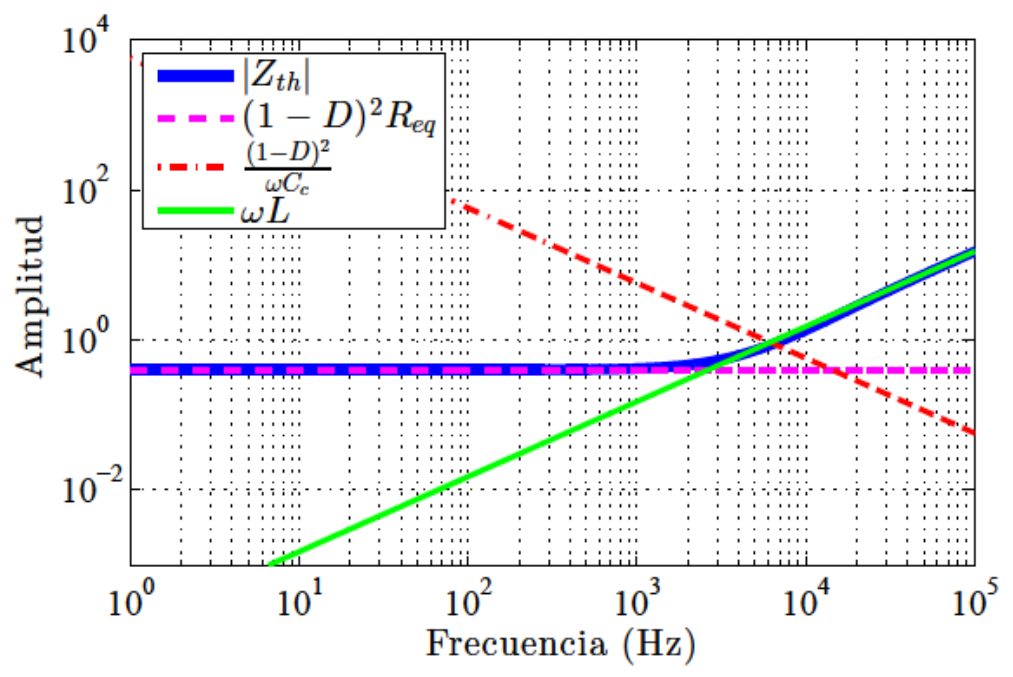

Figura A.28: Representación gráfica de $\left|Z_{t h}(s)\right|$ y los elementos que la forman.

un circuito abierto respectivamente. La fuente independiente de intensidad ahora es una fuente constante conectada en los terminales donde se va a medir esa resistencia. El circuito modificado está representado en la Figura A.29.

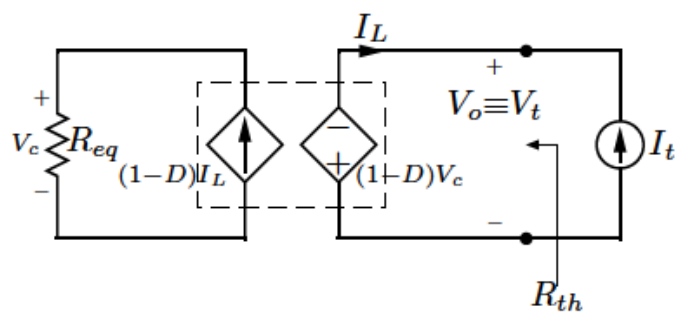

Figura A.29: Circuito empleado para el cálculo de $R_{t h}$ de un reductor ZVS.

Pasando la resistencia $R_{e q}$ al otro lado de las fuentes dependientes, la resistencia de salida es esa resistencia afectada de la relación de transformación, es decir:

$$
R_{t h}=(1-D)^{2} R_{e q}
$$

\section{A.7.2. Cálculo de $Z_{\text {out }}$.}

A la vista del circuito de la Figura A.26, la tensión en el condensador de clamp es igual a:

$$
\hat{v}_{C_{c}}(s)=(1-D) i_{L} Z_{e q}(s) .
$$

Pasando el conjunto $R_{e q}-C_{c}$, al otro lado de las fuentes dependientes se obtiene $(1-D)^{2} Z_{e q}(s)$ en serie con la bobina $L$, como está indicado en el 
circuito de la Figura A.30a, lo que da lugar a la igualdad de tensiones:

$$
-\hat{i}_{L}(s)\left[L s+(1-D)^{2} Z_{e q}(s)\right]=\hat{v}_{t}(s)
$$

donde $\hat{v}_{t}(s) \equiv \hat{v}_{o}(s)$.

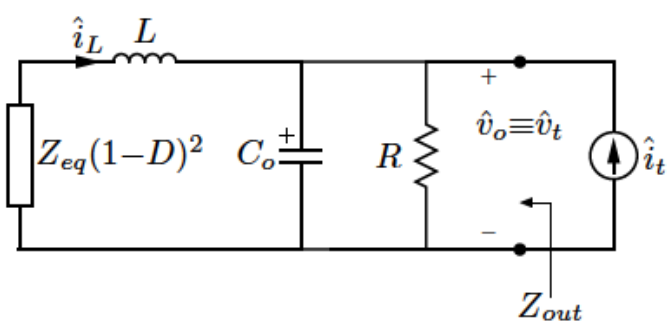

(a)

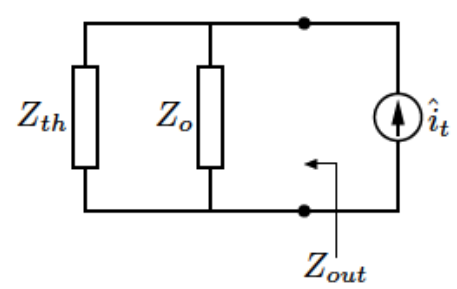

(b)

Figura A.30: Circuito empleado para el cálculo de $Z_{\text {out }}(s)$ de un reductor ZVS.

Al pasar todos los elementos hacia la salida, se observa que $Z_{\text {out }}$ es el paralelo de dos elementos: la impedancia $Z_{t h} ;$ y la impedancia $Z_{o}$, que agrupa a los elementos $C_{o}$ y $R$. Así se ha representado en la Figura A.30b.

$$
Z_{\text {out }}(s)=Z_{\text {th }}(s) \| Z_{o}(s)
$$

A esta expresión también se puede llegar desde las ecuaciones indicadas en (A.162), obtenidas a partir del modelo en pequeña señal indicado en (A.128) donde se anulan los términos independientes, $\hat{v}_{g}(t)$ y $\hat{d}(t)$. Después, hay que modificar la ecuación relativa a la tensión del condensador $C_{o}$ para introducir la intensidad $\hat{i}_{t}(t)$. Tras cambiar el término $\hat{v}_{C_{o}}(t)$ por $\hat{v}_{t}(t)$, se aplica Laplace a las ecuaciones modificadas y quedan:

$$
\begin{aligned}
s L \hat{i}_{L}(s) & =-\hat{v}_{t}(s)-(1-D) \hat{v}_{C_{c}}(s) \\
\hat{i}_{t}(s) & =-\hat{i}_{L}(s)+\hat{v}_{t}(s) \overbrace{\left[C_{o} s+\frac{1}{R}\right]}^{Z_{o}^{-1}(s)} \\
s C_{c} \hat{v}_{C_{c}}(s) & =(1-D) \hat{i}_{L}(s)-\frac{1}{R_{e q}} \hat{v}_{C_{c}}(s)
\end{aligned}
$$

Se despeja $\hat{v}_{C_{c}}$ de la tercera ecuación y se sustituye en la primera ecuación para despejar $\hat{i}_{L}(s)$ y utilizarla en la segunda ecuación. Ahora se puede conseguir una expresión de la impedancia de salida que es equivalente a la 
mostrada en (A.161):

$$
\begin{aligned}
\hat{v}_{C_{c}}(s) & =\frac{(1-D)}{s C_{c}+\frac{1}{R_{e q}}} \hat{i}_{L}(s)=(1-D) Z_{e q}(s) \hat{i}_{L}(s) \\
\hat{i}_{L}(s) & =-\frac{\hat{v}_{t}(s)}{L s+(1-D)^{2} Z_{e q}(s)}=-\frac{\hat{v}_{t}(s)}{Z_{t h}(s)} \\
\hat{i}_{t}(s) & =\frac{\hat{v}_{t}(s)}{Z_{t h}(s)}+\frac{\hat{v}_{t}(s)}{Z_{o}(s)}=\left[\frac{1}{Z_{t h}(s)}+\frac{1}{Z_{o}(s)}\right] \hat{v}_{t}(s)=\frac{\hat{v}_{t}(s)}{Z_{\text {out }}(s)}
\end{aligned}
$$

En la Figura A.31 se ha representado el módulo de la impedancia de salida para un Reductor ZVS, a partir de los parámetros indicados en la Tabla A.1, junto con el módulo de los términos que la forman, $\left|Z_{t h}(s)\right|$ y $\left|Z_{o}(s)\right|$.

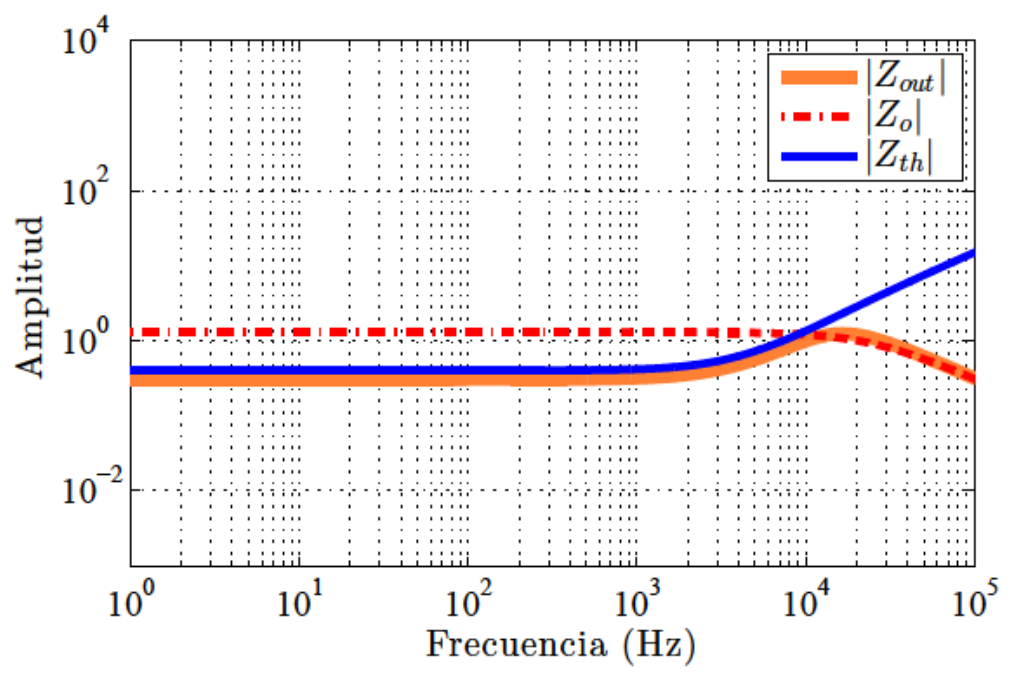

Figura A.31: Representación gráfica de $\left|Z_{\text {out }}(s)\right|$ y los elementos que la forman.

Al hacer $s=0$ se obtiene la impedancia en continua, que es paralelo de los términos $(1-D)^{2} R_{e q}$ y $R$, y se corresponde con la asíntota horizontal de $Z_{\text {out }}$ a bajas frecuencias.

$$
\begin{aligned}
Z_{\text {out }}(0) & =\frac{1}{\frac{1}{\left.Z_{\text {th }}\right)(0)}+\frac{1}{Z_{o}(0)}} \\
& =\frac{1}{\frac{1}{(1-D)^{2} R_{e q}}+\frac{1}{R}}
\end{aligned}
$$




\section{A.7.3. Validación de las expresiones obtenidas.}

La validación de la impedancia, se comprueba con la simulación de un convertidor conmutado sobre el que se mide la impedancia de salida. Con los parámetros de funcionamiento recogidos en la Tabla A.1 se añade a la salida una fuente de corriente de frecuencia variable y se mide la variación que experimenta la tensión de salida.

Como las expresiones de impedancia se han obtenido a partir del modelo promediado parece lógico medir la impedancia sobre este modelo y comparar los resultados simulados con la teoría empleando el ciclo de trabajo calculado para el modelo promediado. Si se emplea el convertidor conmutado para medir la impedancia, debería considerarse el valor del ciclo de trabajo obtenido para el circuito conmutado con la corrección propuesta en el apartado correspondiente.

En la Figura A.32 se han representado los valores medidos para el módulo de la impedancia de un Reductor ZVS simulado con los parámetros mencionados. También se ha representado el módulo de la impedancia de salida teórica, $\left|Z_{t o t}(s)\right|$. Como ya se conocía, presenta una asíntota horizontal, formada por el paralelo de $(1-D)^{2} R_{e q}$ y la carga $R$ y otra asíntota asociada al condensador $C_{o}$.

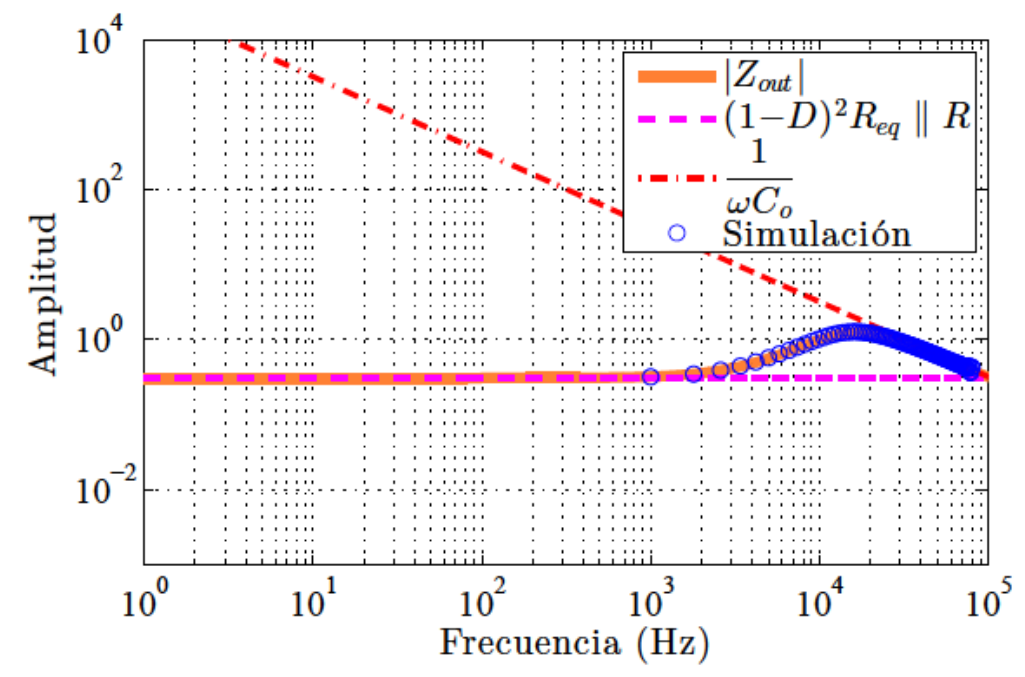

Figura A.32: Representación gráfica de $\left|Z_{\text {out }}(s)\right|$ y resultados de simulación.

A la vista de estas curvas, se observa la coincidencia de la impedancia teórica y los valores obtenidos en la simulación, por lo que se concluye la validez de las expresiones de impedancia obtenidas de forma teórica.

En el caso del reparto de corrientes entre varias etapas en paralelo para 
alimentar una carga común, la impedancia de salida de cada una de las etapas se debe determinar desde el punto común de conexión. Existiendo la posibilidad de considerar un único condensador de salida común a todas las etapas (aunque también podría tratarse el caso de un condensador en cada etapa). El análisis sería similar al realizado en el apartado anterior, con la precaución de no incluir ni $R$ ni $C_{o}$, (y por tanto, no incluir $Z_{o}$ ).

Por ejemplo, la impedancia multietapa sin el condensador de salida, $Z_{m e}(s)$, se corresponde con la impedancia $Z_{t h}(s)$, que ya se ha obtenido en apartados anteriores.

$$
Z_{m e}(s)=\left[\frac{1}{Z_{t h}(s)}\right]^{-1}=Z_{t h}(s)
$$

Para bajas frecuencias, se obtiene el término $2 L_{r} f_{s}$, mientras que cuando $s$ se hace muy grande, prevalece la bobina, siendo la asíntota para altas frecuencias.

Este término también coincide con la derivada de la característica de salida (obtenida en apartados anteriores), indicada en la expresión (A.51). Es la impedancia de salida en régimen permanente que presenta el convertidor. 


\section{Apéndice B}

\section{Elevador ZVS}

\section{B.1. Funcionamiento del Elevador ZVS}

El elevador ZVS es un reductor convencional al que se le ha añadido una red de enclavamiento activo que fija la tensión máxima en los extremos del transistor principal. Esta red facilita las conmutaciones a tensión cero, reduciendo las pérdidas que aparecen. Por contra, aumenta la complejidad del circuito y el número de componentes. En la figura A.1 está representado el circuito de este convertidor.

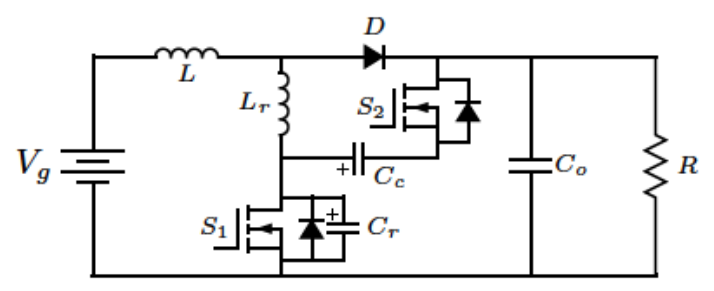

Figura B.1: Elevador ZVS.

La red de enclavamiento activo está formada por el condensador de clamp $C_{c}$ y el interruptor $S_{2}$ junto con su diodo en antiparalelo, $D_{2}$. Además se añade una pequeña inductancia, $L_{r}$, que incluye las inductancias parásitas y que actúa en resonancia con el condensador $C_{c}$ en determinados instantes del periodo. Se ha incluido el diodo $D_{1}$ al interruptor $S_{1}$ y un condensador $C_{r}$ que puede ser el propio del transistor o uno añadido que mejore sus características.

Para explicar el funcionamiento de este convertidor se plantea el estado de los diferentes semiconductores presentes en el circuito, que son los dos interruptores y los tres diodos. En el instante inicial, $t_{0}$, está cerrado $S_{1}$ y el condensador $C_{r}$ está descargado. Se considera el condensador de salida, $C_{o}$, de un valor grande para mantener la tensión $v_{C_{o}}$ constante. La bobina 
de entrada, $L$, es lo suficientemente grande para considerar una corriente constante, $I$. Para un elevador, la corriente de la bobina coincide con la corriente de entrada. De esta forma la fuente de entrada junto con esta bobina pueden sustituirse como una fuente de corriente constante de valor $I_{g}=I$. El condensador de clamp, $C_{c}$ presenta una tensión con la polaridad indicada en figura y se supone lo suficientemente grande como para considerar una tensión prácticamente constante, $V_{c}$ aunque haya intervalos donde éste se cargue y se descargue y la tensión de clamp pueda variar en torno a ese valor. Por último, indicar que el valor de $L_{r}$, aunque comparativamente mas pequeño que $L$, es lo suficientemente grande como para cumplir el criterio de energía que permite conmutaciones suaves en estas condiciones de carga.

\section{B.1.1. Etapa 1. $\left(t_{0}<t<t_{1}\right)$}

Se abre el interruptor $S_{1}$. Esta conmutación se produce a tensión cero puesto que el condensador $C_{r}$ está descargado. Este condensador se carga a corriente constante igual a $I_{g}$, hasta que alcanza el valor de $V_{o}$. La figura B.2a muestra los elementos que intervienen en esta etapa y el circuito equivalente, a partir de las suposiciones iniciales, donde la etapa de entrada se ha modelado como una fuente de corriente constante $I_{g}$.

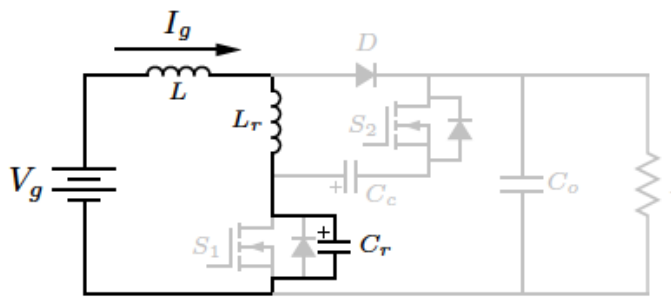

(a)

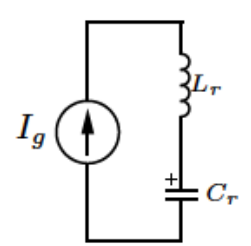

(b)

Figura B.2: Elevador ZVS: a)durante la etapa 1; b)circuito equivalente.

La tensión en el condensador crece de forma lineal, como se indica en (B.1), mientras que la tensión en la bobina $L_{r}$ es cero ya que la corriente que circula es constante.

$$
\begin{aligned}
& i_{C_{r}}(t)=I_{g} \\
& v_{C_{r}}(t)=\frac{1}{C_{r}} \int_{0}^{t} i_{C_{r}}(t) d t=\frac{I_{g}}{C_{r}} t
\end{aligned}
$$

Esta etapa terminará cuando $v_{C_{r}}(t)=V_{o}$, porque al alcanzar este valor, el diodo $D$ se polarizará en directa y empezará a conducir. La duración de esta etapa es igual a $d_{1} T$, indicado en (B.2):

$$
d_{1} T=\left(t_{1}-t_{0}\right)=\frac{V_{o} C_{r}}{I_{g}}
$$




\section{B.1.2. Etapa 2. $\left(t_{1}<t<t_{2}\right)$}

El diodo $D$ empieza a conducir. La entrada sigue suministrando una corriente igual a $I_{g}$. En la figura B.3a se muestran los elementos que intervienen y el circuito equivalente. El condensador $C_{r}$ sigue cargándose, pero ahora no es a corriente constante sino a una corriente variable, $i_{2}(t)$, obtenida por la resonancia del condensador y la bobina.

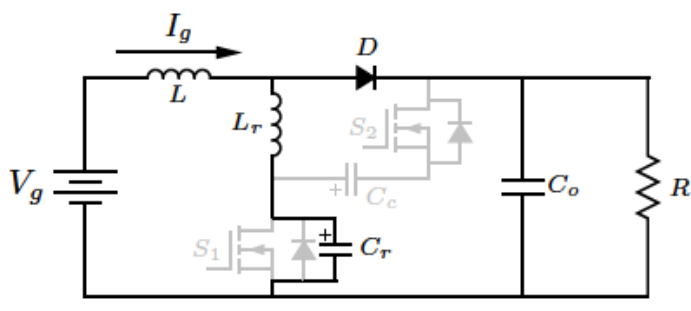

(a)

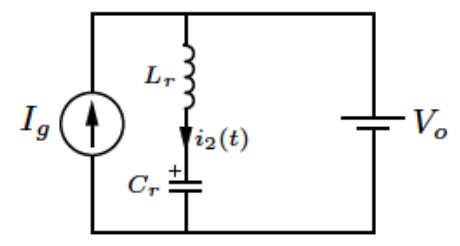

(b)

Figura B.3: Elevador ZVS a)durante la etapa 2; b)circuito equivalente.

Obteniendo la corriente $i_{2}(t)$, se conseguirá la evolución de la tensión en $C_{r}$, y cuando alcance el valor de $V_{o}+V_{c}$ terminará esta etapa, ya que se polarizará en directa el diodo del interruptor $S_{2}$, dando lugar a otro subcircuito. la ecuación para esta corriente se obtiene al plantear la tensión en la rama resonante, y está dada en (B.3), que es una ecuación integro-diferencial con coeficientes constantes.

$$
V_{o}=v_{C_{r}}(t)+v_{L_{r}}(t)=v_{C_{r}}(0)+\frac{1}{C_{r}} \int_{0}^{t} i_{2}(t) d t+L_{r} \frac{d i_{2}(t)}{d t}
$$

Las condiciones iniciales son (B.4):

$$
\begin{aligned}
& i_{2}(0)=I_{g} \\
& i_{2}^{\prime}(0)=\frac{1}{L_{r}} v_{L_{r}}(0)=\frac{1}{L_{r}}\left[V_{o}-v_{C_{r}}(0)\right]=0
\end{aligned}
$$

donde la intensidad debe cumplir la condición de continuidad, pero no la derivabilidad.

La resolución de esta ecuación diferencial se puede obtener de varias formas. Método A) derivar de nuevo la ecuación, y resolverla como una ecuación diferencial ordinaria de segundo orden con coeficientes constantes teniendo en cuenta las condiciones iniciales. Método B) Aplicar Laplace a la ecuación (B.3) teniendo en cuenta las condiciones iniciales al aplicar Laplace y despues obtener la transformada inversa. Método C): Aplicar Laplace al circuito y resolverlo en el dominio de Laplace, y una vez obtenida una expresión de la corriente hacer la transformada inversa.

METODO A) para obtener $i_{2}(t)$. 
Derivando la ecuación integro-diferencial, se obtiene (B.5), y se mantienen las concidiones inciales de (B.4):

$$
0=\frac{1}{C_{r}} i_{2}(t)+L_{r} \frac{d^{2} i_{2}(t)}{d t^{2}}
$$

Esta ecuación se resuelve a partir de la ecuación característica, cuya resolución ofrece dos raíces complejas conjugadas, dadas en (B.6):

$$
L_{r} D^{2}+\frac{1}{C_{r}}=0 \Rightarrow D= \pm \sqrt{\frac{-1}{L_{r} C_{r}}}= \pm j \omega_{r}
$$

donde $\omega_{r}=\left(\sqrt{L_{r} C_{r}}\right)^{-1}$ es la frecuencia de resonancia de la bobina y el condensador. Al ser dos raíces complejas conjugadas se prueba con una solución de la forma indicada en (B.7), a la que se le aplican las condiciones (B.4).

$$
i_{2}(t)=K_{1} \cos \left(\omega_{r} t\right)+K_{2} \sin \left(\omega_{r} t\right)
$$

$\mathrm{Al}$ aplicar la primera condicion, se deduce que $K_{1}=I_{g}$. Con la segunda condición se deduce que $K_{2}=0$.

$$
\begin{aligned}
i_{2}(0)=I_{g} & \Rightarrow I_{g}=K_{1} * 1+K_{2} * 0 \Rightarrow K_{1}=I_{g} \\
i_{2}^{\prime}(0)=0 & \Rightarrow K_{1} \sin \left(\omega_{r} t\right)\left(-\omega_{r}\right)+\left.K_{2} \cos \left(\omega_{r} t\right) \omega_{r}\right|_{t=0}=0 \Rightarrow K_{2}=0
\end{aligned}
$$

De esta forma la solución para la ecuación diferencial es la indicada en (B.9):

$$
i_{2}(t)=I_{g} \cos \left(\omega_{r} t\right)
$$

METODO B) para obtener $i_{2}(t)$.

Aplicando Laplace a la ecuación integro-diferencial (B.3), se obtiene una expresión como la indicada en (B.10) donde ahora entran en juego los valores iniciales de las variables implicadas, donde $I_{2}(s)=\mathscr{L}\left[i_{2}(t)\right]$.

$$
\begin{aligned}
\mathscr{L}\left[V_{o}\right] & =\mathscr{L}\left[v_{C_{r}}(0)+\frac{1}{C_{r}} \int_{0}^{t} i_{2}(t) d t+L_{r} \frac{d i_{2}(t)}{d t}\right] \\
\frac{V_{o}}{s} & =\frac{v_{C_{r}}(0)}{s}+\frac{1}{C_{r} s} I_{2}(s)+L_{r} s I_{2}(s)-L_{r} i_{2}(0)
\end{aligned}
$$

Recuerde que $v_{C_{r}}(0)=V_{o}$, y que $i_{2}(0)=I_{g}$. Una vez despejada $I_{2}(s)$, se obtiene, tras aplicar Laplace, el resultado (B.11):

$$
I_{2}(s)=I_{g} \frac{s}{s^{2}+\frac{1}{L_{r} C_{r}}} \Rightarrow i_{2}(t)=\mathscr{L}^{-}\left[I_{2}(s)\right]=I_{g} \cos \left(\omega_{r} t\right)
$$

METODO C) para obtener $i_{2}(t)$. 
Este método consiste en transformar el circuito al dominio de Laplace y después resolver la corriente transformada, es decir, $I_{2}(s)$ en el circuito resultante. Si se aplica Laplace al circuito de la figura B.4a (donde previamente se ha sustituido el condensador cargado a una tensión por un condensador vacio y una fuente de tensión en serie del mismo valor y polaridad que la que tenía inicialmente y la bobina se ha sustituido por una bobina descargada y una fuente de corriente en paralelo y del mismo valor y sentido que la que estaba circulando inicialmente), se obtiene el circuito en el dominio transformado como el de la figura B.4b. En este caso, la tensión inicial del condensador es $V_{o}$ y la corriente incial en la bobina es $I_{g}$.

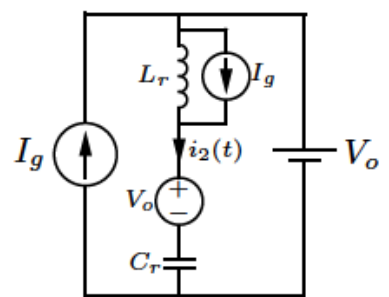

(a)

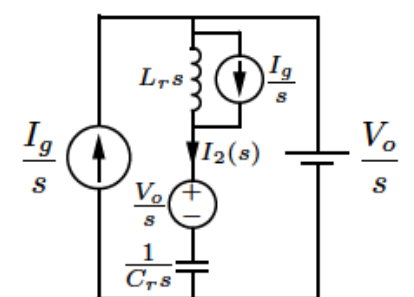

(b)

Figura B.4: Circuito equivalente de la Etapa 2: a) en el dominio del tiempo; y b) en el dominio de Laplace.

Para facilitar las operaciones, se sustituye el conjunto bobina-fuente de corriente en el circuito transformado por su equivalente Thévenin. en la figura B.5 se ha representado el circuito equivalente. De esta forma, se obtiene

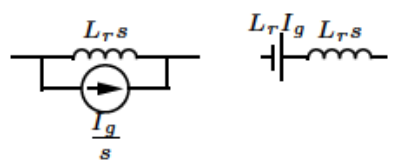

Figura B.5: Equivalente Thévenin del conjunto bobina-fuente de corriente.

fácilmente la expresión para $I_{2}(s)$, como se indica en (B.12), a partir de la ecuación de tensión en la rama resonante:

$$
\begin{aligned}
\frac{V_{o}}{s} & =\frac{V_{o}}{s}+I_{2}(s) \frac{1}{C_{r} s}-L_{r} I_{g}+I_{2}(s) L_{r} s \\
L_{r} I_{g} & =I_{2}(s)\left(\frac{1}{C_{r} s}+L_{r} s\right) \\
I_{2}(s) & =I_{g} \frac{s}{\frac{1}{L_{r} C_{r} s}+s^{2}} \Rightarrow i_{2}(t)=\mathscr{L}^{-}\left[I_{2}(s)\right]=I_{g} \cos \left(\omega_{r} t\right)
\end{aligned}
$$

Por cualquiera de los métodos se obtiene la misma expresión para la corriente $i_{2}(t)$. Una vez calculada, se obtiene la tensión en el condensador como (B.13): 


$$
\begin{aligned}
v_{C_{r}}(t) & =v_{C_{r}}(0)+\frac{1}{C_{r}} \int_{0}^{t} i_{2}(\lambda) d \lambda \\
& =V_{o}+\left[\frac{1}{C_{r}} I_{g} \sin \left(\omega_{r} \lambda\right) \omega_{r}^{-1}\right]_{0}^{t} \\
& =V_{o}+\frac{I_{g}}{\omega_{r} C_{r}} \sin \left(\omega_{r} t\right)
\end{aligned}
$$

la duración de esta etapa será el tiempo que tarda el condensador en alcanzar la tensión $V_{o}+V_{c}$, porque el diodo de $S 2$ se polariza en directa. Este tiempo se deduce de (B.13), y es igual a (B.14).

$$
d_{2} T=\left(t_{2}-t_{1}\right)=\omega_{r}^{-1} \arcsin \left(\frac{V_{c} \omega_{r} C_{r}}{I_{g}}\right)
$$

La corriente por el diodo principal, es igual a (B.15), mientras que la corriente $i_{2}(t)$ al final de esta etapa es $I^{\prime}$, y es ligeramente inferior a $I_{g}$.

$$
i_{D}(t)=I_{g}-i_{2}(t)=I_{g}\left(1-\cos \left(\omega_{r} t\right)\right)
$$

\section{B.1.3. Etapa 3. $\left(t_{2}<t<t_{3}\right)$}

El diodo del interruptor auxilar $S_{2}$ empieza a conducir. A partir de este instante, se puede cerrar este interruptor a tensión cero porque está circulando corriente por su diodo. En la figura B.6a se muestran los elementos que intervienen y el circuito equivalente, donde $i_{c}(t)$ representa la corriente que circula por el condensador de clamp.

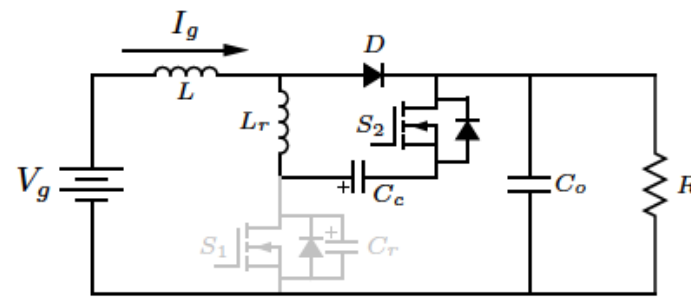

(a)

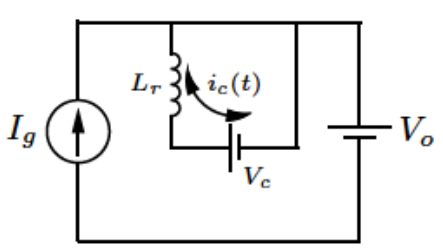

(b)

Figura B.6: Elevador ZVS a)durante la etapa 3; b)circuito equivalente.

Nótese que el condensador de clamp es grande para la energía que absorbe por lo que su tensión se mantiene práticamente constante durante todo este intervalo y de ahí que se represente como una fuente de tensión con la polaridad indicada. Además la corriente $i_{c}(t)$ circula inicialmente en un sentido y luego se invierte cuando la bobina se ha descargado completamente sobre $C_{c}$. Pero como el interruptor $S_{2}$ permanece cerrado, el condensador cederá esta energía extra de nuevo a la bobina, haciendo que la corriente 
circule en el sentido opuesto al inicial. En este caso, la corriente circulará por $S_{2}$ y no por su diodo.

La relación entre la tensión de clamp, y la duración de este intevalo se obtiene resolviendo el circuito equivalente. Para ello hay que resolver la malla cerrada de este circuito para obtener $i_{c}(t)$. En esta malla, la tensión es constante e igual a $V_{c}$. La bobina resonante se descarga a tensión constante y su expresión se obtiene resolviento la ec.diferencial de primer orden con condiciones iniciales de (B.16):

$$
\begin{aligned}
-V_{c} & =v_{L_{r}}(t)=L_{r} \frac{d i_{c}(t)}{d t} \\
i_{c}(0) & =I^{\prime}
\end{aligned}
$$

Haciendo la separación de variables en cada miembro de (B.16), e integrando se obtiene una solución general, en la que se despeja la constante de integración aplicando la condición inicial.

$$
\begin{aligned}
\frac{-V_{c}}{L_{r}} d t & =d i_{c}(t) \\
\frac{-V_{c}}{L_{r}} \int d t & =\int d i_{c}(t) \\
i_{c}(t) & =\frac{-V_{c}}{L_{r}} t+K \\
i_{c}(0) & =I^{\prime} \Rightarrow K=I^{\prime} \Rightarrow i_{c}(t)=I^{\prime}-\frac{V_{c}}{L_{r}} t
\end{aligned}
$$

En la solución dada en (B.17) se comprueba que $i_{c}(t)$ es decreciente y llega a ser negativa. La duración de esta etapaes $d_{3} T=\left(t_{3}-t_{2}\right)$.

Al final de este periodo, la corriente alcanza un valor igual a $I^{\prime}$ pero en sentido contrario. De esta relación se deduce el valor de la tensión de clamp en función de la bobina resonante y el ciclo de trabajo. Véase (B.18).

$$
i_{c}\left(t_{3}\right)=-I^{\prime} \Rightarrow-I^{\prime}=I^{\prime}-\frac{V_{c}}{L_{r}} d_{3} T \Rightarrow V_{c}=\frac{2 I^{\prime} L_{r}}{d_{3} T}
$$

También se puede decir lo siguiente: Si se habla de régimen permanente, la energía neta en el condensador de clamp durante un ciclo de trabajo, es decir, la energía que toma y que devuelve durante ese tiempo, debe ser nula. Como se ha supuesto un valor de $C_{c}$ suficientemente grande para que $V_{c}$ sea constante, al aplicar esta suposición se llega a que el valor medio de (B.17) es nulo. Dicho de otra forma, el balance de carga en $C_{c}$ deber se nulo. Siendo así, resulta que

$$
\begin{gathered}
\Delta E=0 \Rightarrow \int_{t_{0}}^{t_{0}+T} i_{c}(t) d t=\int_{t_{2}}^{t_{3}} i_{c}(t) d t=\int_{0}^{d_{3} T T}\left[I^{\prime}-\frac{V_{c}}{L_{r}} t\right] d t=0 \\
{\left[I^{\prime} t-\frac{V_{c}}{2 L_{r}} t^{2}\right]_{0}^{d_{3} T}=0 \Rightarrow V_{c}=\frac{2 I^{\prime} L_{r}}{d_{3} T}}
\end{gathered}
$$


Este resultado sustituido en (B.17) permite obtener el valor de $i_{c}\left(t_{3}\right)$ para el régimen permanente. Así se indica en (B.20).

$$
i_{c}\left(t_{3}\right)=I^{\prime}-\frac{2 I^{\prime} \mathscr{L}_{r}}{L_{r} d_{3} T} d_{3} T=-I^{\prime}
$$

Durante un transitorio, la tensión de clamp evolucionará en cada periodo que dure el transitorio de un valor a otro, y el balance de carga no será nulo, y por tanto, no se puede saber a priori el valor de $i_{c}\left(t_{3}\right)$ al final de cada periodo.

Ahora se abre el interruptor, y lo hace a tensión cero porque e condensador $C_{r}$ se había quedado cargado a una tensión $V_{o}+V_{c}$, de forma que el drenador de $S_{2}$ está a tensión cero.

La corriente que pasa por el diodo principal alcanza un valor máximo de (B.21): Véase (B.18).

$$
i_{D}(t)=I_{g}-i_{c}(t) \Rightarrow I_{D, \max }=i_{D}\left(t_{3}\right)=I_{g}-i_{c}\left(t_{3}\right)=I_{g}+I^{\prime}
$$

\section{B.1.4. Etapa 4. $\left(t_{3}<t<t_{4}\right)$}

Este intevalo se inicia con la apertura de $S_{2}$ a tensión nula. La corriente en $L_{r}$ tiene un sentido inicial opuesto al inicial de la etapa anterior, y ésta ya no puede circular ni por $S_{2}$ ni por su diodo, luego deberá circular a través de $C_{r}$. Dicho de otra forma, la bobina $L_{r}$ acumulará energía, pero esta vez de $C_{r}$ que se descarga de forma resonante sobre aquella. La corriente $i_{4}(t)$ experimenta durante este intervalo una evolución resonante, a la vez que la tensión $V_{C_{r}}(t)$ disminuye hasta hacerse cero, que es donde concluye esta etapa. A partir de este instante, la conducción de la corriente se realiza sobre el diodo $D_{1}$. En el circuito de la figura B.7a se muestran los elementos que están involucrados en esta etapa.

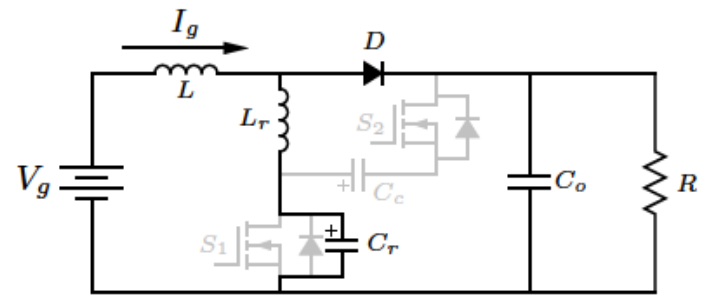

(a)

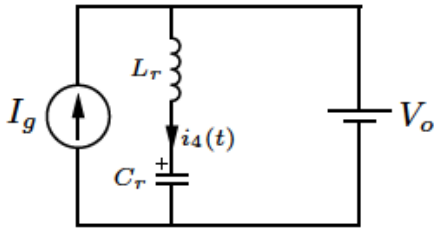

(b)

Figura B.7: Elevador ZVS a)durante la etapa 4; b)circuito equivalente.

Una condición necesaria, sobre la que volveremos mas adelante, es que la energía acumulada en $L_{r}$ debe ser como poco igual a la energía que acumula $C_{r}$ para que la bobina consiga descargar por completo al condensador antes de que se elimine. Para la obtención de $i_{4}(t)$ (donde se ha mantenido el 
mismo sentido que en los casos de las etapas anteriores), hay que plantear le ecuación de la tensión en la rama resonante del circuito equivalente y resolver la ecuacíón diferencial resultante utilizando las condiciones iniciales conocidas. La ecuación resultante es una ecuación integro-diferencial con coeficientes constantes dada por (B.22):

$$
V_{o}=v_{C_{r}}(t)+v_{L_{r}}(t)=v_{C_{r}}(0)+\frac{1}{C_{r}} \int_{0}^{t} i_{4}(t) d t+L_{r} \frac{d i_{4}(t)}{d t}
$$

Las condiciones iniciales son (B.23):

$$
\begin{aligned}
& i_{4}(0)=-I^{\prime} \\
& i_{4}^{\prime}(0)=\frac{1}{L_{r}} v_{L_{r}}(0)=\frac{1}{L_{r}}\left[V_{o}-v_{C_{r}}(0)\right]=-\frac{V_{c}}{L_{r}}
\end{aligned}
$$

donde la intensidad debe cumplir la condición de continuidad, pero no la derivabilidad.

La resolución de esta ecuación diferencial, tal y como se ha hecho en la ETAPA 2, se puede obtener de varias formas. Método A) derivar de nuevo la ecuación, y resolverla como una ecuación diferencial ordinaria de segundo orden con coeficientes constantes teniendo en cuenta las condiciones iniciales. Método B) Aplicar Laplace a la ecuación (B.22) teniendo en cuenta las condiciones iniciales al aplicar Laplace y despues obtener la transformada inversa. Método C): Aplicar Laplace al circuito y resolverlo en el dominio de Laplace, y una vez obtenida una expresión de la corriente hacer la transformada inversa.

METODO A) para obtener $i_{4}(t)$.

Derivando la ecuación integro-diferencial, se obtiene (B.24), y se mantienen las concidiones inciales de (B.23):

$$
0=\frac{1}{C_{r}} i_{4}(t)+L_{r} \frac{d^{2} i_{4}(t)}{d t^{2}}
$$

Esta ecuación se resuelve a partir de la ecuación característica, cuya resolución ofrece dos raices complejas conjugadas, dadas en (B.25):

$$
0=\frac{1}{C_{r}}+L_{r} D^{2} \Rightarrow D= \pm \sqrt{\frac{-1}{L_{r} C_{r}}}= \pm j \omega_{r}
$$

donde $\omega_{r}$ ya se había definido durante la ETAPA 2 como la frecuencia de resonancia bobina-condensador, y es igual a $\left(\sqrt{L_{r} C_{r}}\right)^{-1}$. Al ser dos raíces complejas conjugadas se prueba con una solución de la forma indicada en (B.26), a la que se le aplican las condiciones (B.23).

$$
i_{4}(t)=K_{3} \cos \left(\omega_{r} t\right)+K_{4} \sin \left(\omega_{r} t\right)
$$


$\mathrm{Al}$ aplicar la primera condición, se deduce que $K_{3}=-I^{\prime}$. Con la segunda condición se deduce el valor de $K_{4}$.

$$
\begin{aligned}
i_{4}(0) & =-I^{\prime} \Rightarrow-I^{\prime}=K_{3} * 1+K_{4} * 0 \Rightarrow K_{3}=-I^{\prime} . \\
i_{4}^{\prime}(0) & =-\frac{V_{c}}{L_{r}} \Rightarrow K_{3} \sin \left(\omega_{r} t\right)\left(-\omega_{r}\right)+\left.K_{4} \cos \left(\omega_{r} t\right) \omega_{r}\right|_{t=0}=0 \Rightarrow \\
& \Rightarrow K_{4}=-\frac{V_{c}}{\omega_{r} L_{r}}
\end{aligned}
$$

De esta forma la solución para la ecuación diferencial es la indicada en (B.28):

$$
i_{4}(t)=-I^{\prime} \cos \left(\omega_{r} t\right)-\frac{V_{c}}{\omega_{r} L_{r}} \sin \omega_{r} t
$$

METODO B) para obtener $i_{4}(t)$.

Aplicando Laplace a la ecuación integro-diferencial (B.22), se obtiene una expresión como la indicada en (B.29) donde ahora entran en juego los valores iniciales de las variables implicadas, donde $I_{4}(s)=\mathscr{L}\left[i_{4}(t)\right]$.

$$
\begin{aligned}
\mathscr{L}\left[V_{o}\right] & =\mathscr{L}\left[v_{C_{r}}(0)+\frac{1}{C_{r}} \int_{0}^{t} i_{4}(t) d t+L_{r} \frac{d i_{4}(t)}{d t}\right] \\
\frac{V_{o}}{s} & =\frac{V_{C_{r}}(0)}{s}+\frac{1}{C_{r} s} I_{4}(s)+L_{r} s I_{4}(s)-L_{r} i_{4}(0)
\end{aligned}
$$

Recuerde que $V_{C_{r}}(0)=V_{o}+V_{c}$, y que $i_{4}(0)=-I^{\prime}$. Una vez despejada $I_{4}(s)$, se obtiene, tras aplicar Laplace, el resultado (B.30):

$$
\begin{aligned}
& I_{4}(s)=-I^{\prime} \frac{s}{s^{2}+\frac{1}{L_{r} C_{r}}}-\frac{V_{c}}{L_{r}} \frac{1}{s^{2}+\frac{1}{L_{r} C_{r}}} \\
& i_{4}(t)=\mathscr{L}^{-}\left[I_{4}(s)\right]=-I^{\prime} \cos \left(\omega_{r} t\right)-\frac{V_{c}}{\omega_{r} L_{r}} \sin \omega_{r} t
\end{aligned}
$$

METODO C) para obtener $i_{4}(t)$.

Este método consiste en transformar el circuito al dominio de Laplace y después resolver la corriente transformada, es decir, $I_{4}(s)$ en el circuito resultante. Si se aplica Laplace al circuito equivalente de la figura B.8a, se obtiene el circuito transformado de la figura B.8b, (donde previamente se ha sustituido el condensador cargado a una tensión por un condensador vacio y una fuente de tensión en serie del mismo valor y polaridad que la que tenía inicialmente y la bobina se ha sustituido por una bobina descargada y una fuente de corriente en paralelo y del mismo valor y sentido que la que estaba circulando inicialmente). En este caso, la tensión inicial del condensador es $V_{o}+V_{c}$ y la corriente incial en la bobina es $-I^{\prime}$. 


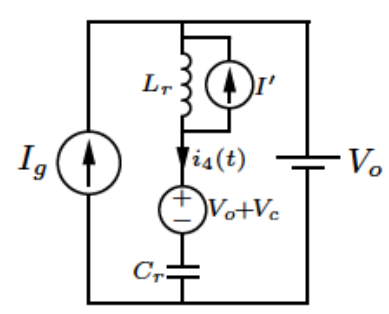

(a)

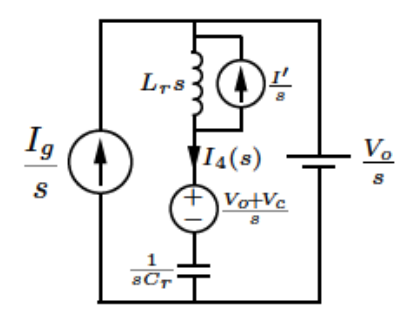

(b)

Figura B.8: Circuito equivalente en la Etapa 4: a) en el dominio del tiempo; y b) en el dominio de Laplace.

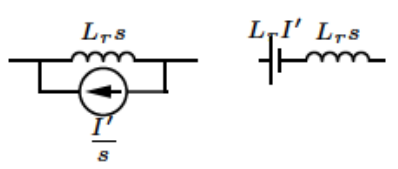

Figura B.9: Equivalente Thévenin del conjunto bobina-fuente de corriente.

Para facilitar las operaciones, se sustituirá el conjunto bobina-fuente de corriente en el circuito transformado por su equivalente Thévenin. En la figura B.9 se ha representado el circuito equivalente.

De esta forma, se obtiene fácilmente la expresión para $I_{4}(s)$, como se indica en (B.31), a partir de la ecuación de tensión en la rama resonante:

$$
\begin{aligned}
\frac{V_{o}}{s}-\frac{V_{o}+V_{c}}{s}-L_{r} I^{\prime} & =I_{4}(s) \frac{1}{C_{r} s}+I_{4}(s) L_{r} s \\
-\frac{V_{c}}{s}-L_{r} I^{\prime} & =I_{4}(s)\left(\frac{1+L_{r} C_{r} s^{2}}{C_{r} s}\right) \\
I_{4}(s) & =\left(-\frac{V_{c}}{s}-L_{r} I^{\prime}\right)\left(\frac{C_{r} s}{1+L_{r} C_{r} s^{2}}\right) \Rightarrow \\
i_{4}(t) & =\mathscr{L}^{-}\left[I_{4}(s)\right]=-\frac{V_{c}}{\omega_{r} L_{r}} \sin \omega_{r} t-I^{\prime} \cos \left(\omega_{r} t\right)
\end{aligned}
$$

Por cualquiera de los métodos se obtiene la misma expresión para la corriente $i_{4}(t)$. Una vez calculada, se obtiene la tensión en el condensador como (B.32):

$$
\begin{aligned}
v_{C_{r}}(t) & =v_{C_{r}}(0)+\frac{1}{C_{r}} \int_{0}^{t} i_{4}(\lambda) d \lambda \\
& =V_{o}+V_{c}-\left[\frac{1}{C_{r}} I^{\prime} \sin \left(\omega_{r} \lambda\right) \omega_{r}^{-1}\right]_{0}^{t}-\left[\frac{V_{c}}{C_{r} L_{r} \omega_{r}} \cos \left(\omega_{r} \lambda\right)\left(-\omega_{r}\right)^{-1}\right]_{0}^{t} \\
& =V_{o}+V_{c} \cos \omega_{r} t-\frac{I^{\prime}}{\omega_{r} C_{r}} \sin \left(\omega_{r} t\right)
\end{aligned}
$$


Esta expresión se puede simplificar, agrupando los dos términos de $\sin ()$ y $\cos ()$ en uno solo com amplitud y desfase. De esta forma la tensión en el condensador es (B.33):

$$
v_{C_{r}}(t)=V_{o}+V_{r} \cos \left(\omega_{r} t+\phi_{r}\right)
$$

siendo

$$
\begin{aligned}
& V_{r}=\sqrt{V_{c}^{2}+\left(\frac{I^{\prime}}{\omega_{r} C_{r}}\right)^{2}}=\sqrt{V_{c}^{2}+\frac{L_{r}}{C_{r}} I^{\prime 2}} \\
& \phi_{r}=\arctan \left(\frac{I^{\prime}}{V_{c} \omega_{r} C_{r}}\right)
\end{aligned}
$$

La duración de esta etapa será el tiempo que tarda el condensador en descargase por completo y su tensión es nula, instante en el cual la energía de $L_{r}$ empezará a circular a través del diodo de $S 1$. En este instante, la bobina se ha cargado a una corriente de valor negativo, $I^{\prime \prime}$, y mas pequeño que $-I^{\prime}$, puesto que la bobina toma la carga que le cede el condensador.

Para que la tensión pueda llegar a anularse se debe cumplir que la amplitud $V_{r}$ sea mayor, o al menos igual, que $V_{o}$, luego:

$$
\begin{aligned}
\sqrt{V_{c}^{2}+\frac{L_{r}}{C_{r}} I^{2}} & \geq V_{o} \\
V_{c}^{2}+\frac{L_{r}}{C_{r}} I^{\prime 2} & \geq V_{o}^{2} \\
C_{r} V_{c}^{2}+L_{r} I^{\prime 2} & \geq C_{r} V_{o}^{2}
\end{aligned}
$$

que indica que la bobina descarga al condensador con la energía que tiene acumulada. Lo que sucede es que antes de empezar a descargarse la bobina, esta aumenta su energía por una parte que ya le cede el propio condensador. Ésta, junto con la que tiene acumulada sirven para descargar el condensador. Se deberá cumplir por tanto la condición indicada en B.35.

La corriente por el diodo principal, es igual a (B.36).

$$
i_{D}(t)=I_{g}-i_{4}(t)
$$

B.1.5. Etapa 5. $\left(t_{4}<t<t_{5}\right)$

Esta etapa comienza en el instante en que $C_{r}$ se descarga totalmente y su tensión es nula. La corriente circulante por $L_{r}$ necesita un camino para seguir circulando, y lo hace a través del diodo de $S_{1}$, que pasa a estar poralizado en directa. Debido a esto, el interruptor $S_{1}$ puede entrar en conducción a tensión cero en cualquier instante de tiempo en el que circule corriente por su diodo, que será mientras la corriente por $L_{r}$ mantenga el mismo sentido de circulación. Una vez cerrado $S_{1}$, la bobina cede la energía a la fuente, y cuando está descargada, se carga de nuevo con otra polaridad, por lo que la 
corriente $i_{5}(t)$ terminará circulando en un sentido contrario al que tenía al iniciar esta etapa. En la figura B.10a se muestran los elementos que están involucrados en esta etapa y el circuito equivalente.

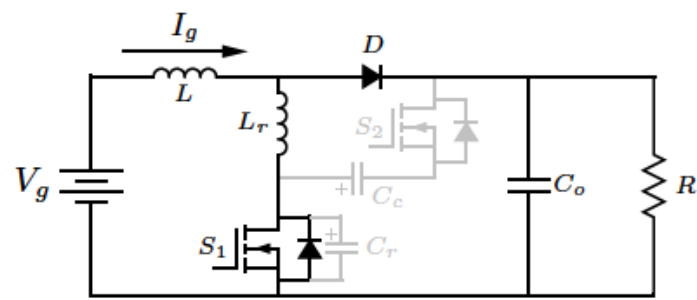

(a)

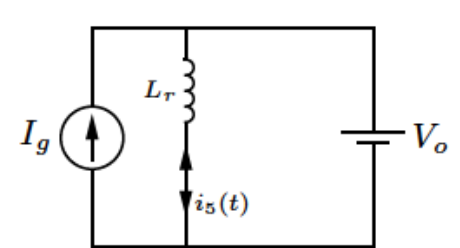

(b)

Figura B.10: Elevador ZVS a)durante la etapa 5; b)circuito equivalente.

Esta etapa terminará cuando el valor de $i_{5}(t)$ alcance el valor de $I_{g}$, instante en el cual, la corriente por el diodo principal, $i_{D}(t)$ se anulará y pasará a la siguiente etapa. Observando el circuito equivalente, la corriente circulante en la rama de bobina resonante evoluciona en función de una tensión constante. La relación entre esta tensión y la duración de este intevalo se obtiene resolviendo dicho circuito. En esta rama la tensión es igual a $V_{o}$. La bobina resonante se descarga a tensión constante y su expresión se obtiene resolviento la ec.diferencial de primer orden con condiciones iniciales de (B.37):

$$
\begin{aligned}
V_{o} & =v_{L_{r}}(t)=L_{r} \frac{d i_{5}(t)}{d t} \\
i_{5}(0) & =I^{\prime \prime}
\end{aligned}
$$

Haciendo la separación de variables en cada miembro de (B.37), e integrando se obtiene una solución general, en la que se despeja la constante de integración aplicando la condición inicial.

$$
\begin{aligned}
\frac{V_{o}}{L_{r}} d t & =d i_{5}(t) \\
\frac{V_{o}}{L_{r}} \int d t & =\int d i_{5}(t) \\
i_{5}(t) & =\frac{V_{o}}{L_{r}} t+K_{5} \\
i_{5}(0) & =I^{\prime \prime} \Rightarrow K_{5}=I^{\prime \prime} \Rightarrow i_{5}(t)=I^{\prime \prime}+\frac{V_{o}}{L_{r}} t
\end{aligned}
$$

De la expresión (B.38) se puede deducir la duración de este intervalo, que es el tiempo que tarda en alcanzarse el valor de $I_{g}$, que es igual a

$$
d_{5} T=\left(t_{5}-t_{4}\right)=\left(I_{g}-I^{\prime \prime}\right) \frac{L_{r}}{V_{o}}
$$




\section{B.1.6. Etapa 6. $\left(t_{5}<t<t_{6}\right)$}

Esta etapa comienza cuando el diodo $D$ deja de conducir y concluye cuando se abre el interruptor $S_{1}$, que conmuta a tensión cero puesto que el condensador $C_{r}$ está descargado. A partir de aquí comienza un nuevo ciclo. Los elementos que intervienen el circuito pasan a ser los indicados en la figura B.11a.

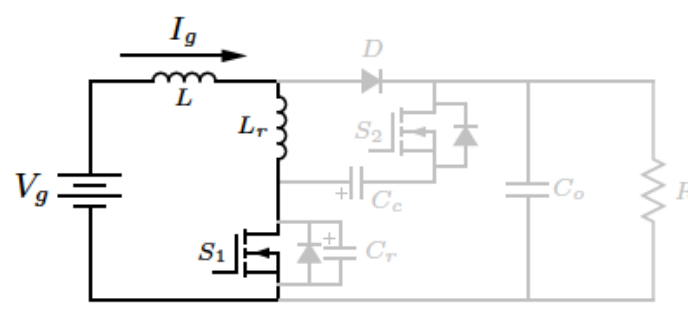

(a)

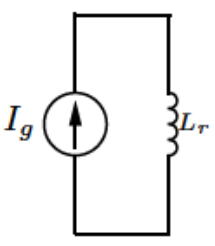

(b)

Figura B.11: Elevador ZVS durante la etapa 6.

Uno de los objetivos de la red ZVS es reducir las pérdidas, y se consigue puesto que todas las conmutaciones son a tensión cero. $S_{1}$ pasa a OFF al principio de la ETAPA 1 y lo hace a tensión cero puesto que el condensador $C_{r}$ está descargado. $S_{2}$ pasa a $\mathrm{ON}$ al principio de la ETAPA 3 y lo hace a tensión cero puesto que está circulando corriente por su diodo. $S_{2}$ pasa a OFF al final de la ETAPA 3 y lo hace a tensión cero puesto que el drenador está a $0 \mathrm{~V} . S_{1}$ pasa a $\mathrm{ON}$ en la ETAPA 5 mientras circula corriente por su diodo.

Nótese que se supone cumplida la ecuación (B.35) relativa que la energía almacenada en $L_{r}$ debida a la intensidad $I_{g}$, debe ser mayor que la energía del condensador del MOSFET, $C_{r}$, para descargarlo desde la tensión $V_{o}+V_{c}$ hasta cero. en esta ecuación hay que hacer notar que $I^{\prime}$ representa el valor medio de la corriente en ese instante. Utilizando los valores instantáneos, habría que sustituir ese valor por el valor de pico.

\section{B.2. Análisis en régimen permanente.}

\section{B.2.1. Sin considerar rizado.}

Se ha considerado una bobina $L$ lo suficientemente grande como para suponer corriente constante, de valor $I=I_{g}$, que es mucho mayor que la bobina resonante $L_{r}$. De forma similar, el valor de los condensadores $C_{o} \mathrm{y}$ $C_{c}$, es tal que la tensión en sus extremos se mantiene constante, de valores $V_{o}$ y $V_{c}$, durante todo el periodo $T$.

Después de estudiar el funcionamiento del circuito con estas mismas consideraciones, se ha representado en la figura B.12 las principales magnitudes 
a lo largo de las seis etapas por las que evoluciona esta topología durante un ciclo completo. La duración de cualquiera de estas etapas se indica con la letra $d$ y un subíndice que hace referencia al número de etapa. Por ejemplo, la duración de la etapa 5 es igual a $t_{5}-t_{4}=d_{5} T$.

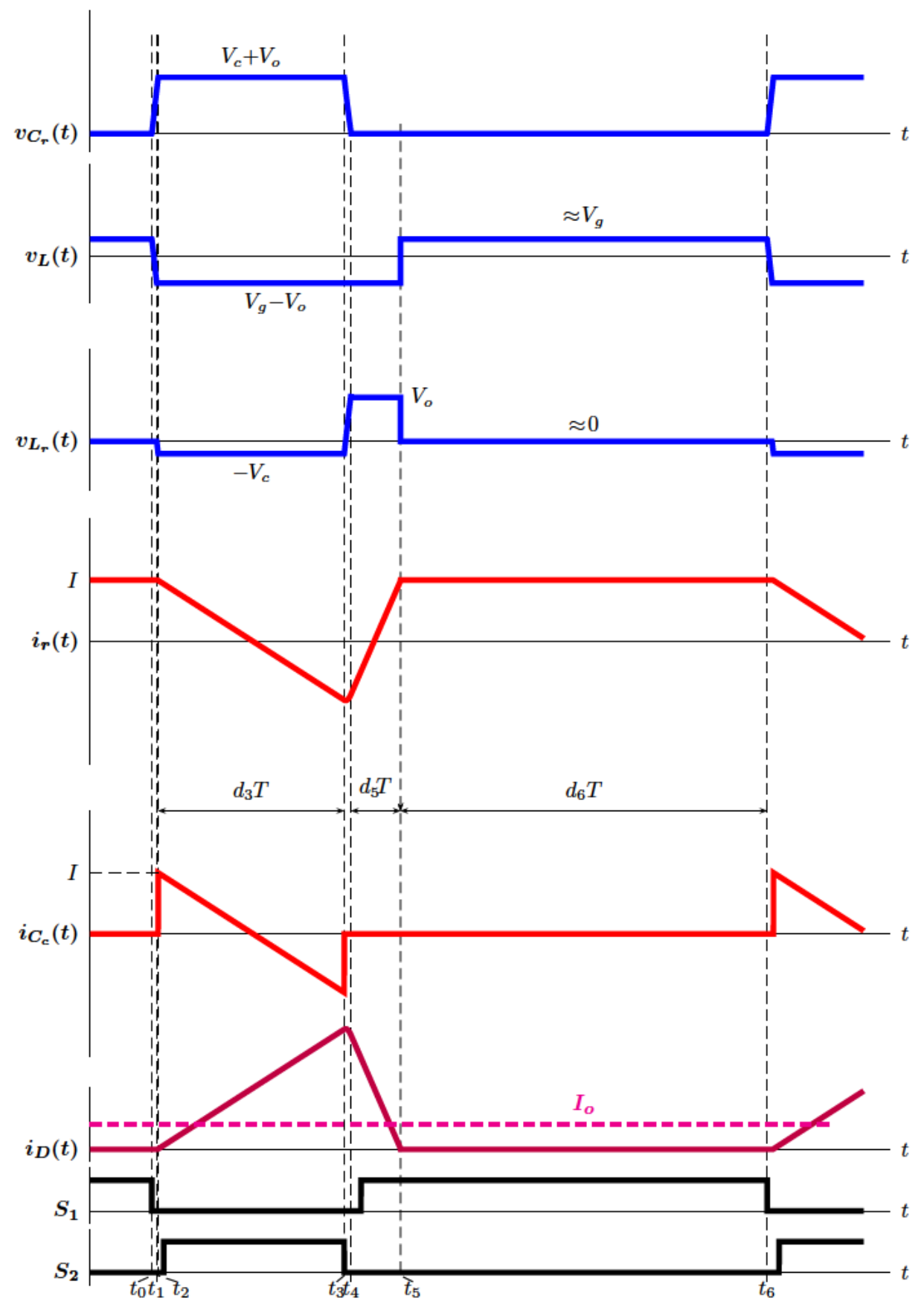

Figura B.12: Curvas principales de un elevador ZVS. 
Para el análisis se desprecian las etapas 1, 2 y 4, puesto que la frecuencia característica del conjunto $L_{r}-C_{r}$ es mucho mas grande que la frecuencia de conmutación y la carga-descarga de $C_{r}$ es prácticamente instantánea. De esta forma solo intervienen tres etapas en el análisis. En este caso se considera que $I^{\prime} \approx I$ y $I^{\prime \prime} \approx-I$. Y como la duración de la etapa 3 es el tiempo que no está cerrado el interruptor principal, resulta que

$$
\begin{aligned}
d_{3} T & =(1-D) T \\
d_{5} T+d_{6} T & =D T
\end{aligned}
$$

Las ecuaciones del régimen permanente se obtienen al considerar nulo tanto el balance voltios-segundo en las bobinas $L$ y $L_{r}$ como la carga neta en los condensadores $C_{o}$ y $C_{c}$.

\section{TENSION MEDIA EN BOBINA PRINCIPAL.}

La tensión media en la bobina $L$ es nula. De aqui se obtiene una expresión para la tensión de salida en función de la entrada:

$$
\frac{1}{\not{X}}\left[V_{g}-V_{o}\left(d_{3}+d_{5}\right) \not{X}+\left(V_{g}\right) d_{6} \not{X}\right]=0 \Rightarrow V_{o}=\frac{V_{g}}{\left(d_{3}+d_{5}\right)}=\frac{V_{g}}{\left(1-d_{6}\right)}
$$

A $d_{6}$ se le puede llamar ciclo efectivo de trabajo, $D_{e f}$, puesto que al identificar la expresión que relaciona la tensión de salida con la entrada en un elevador, aparece ese término en el denominador en lugar del término $D$ que identifica al ciclo de trabajo. También se puede identificar con el tiempo en el que la bobina principal del convertidor está absorbiendo energía. El valor del ciclo efectivo es menor que el valor del ciclo de trabajo empleado en las señales de disparo.

\section{TENSION MEDIA EN BOBINA RESONANTE.}

La tensión media en la bobina $L_{r}$ es nula. De aquí se obtiene una expresión para la tensión de $C_{c}$ en función de la tensión de entrada:

$$
\frac{1}{\not{X}}\left[-V_{c} d_{3} \not{X}+V_{o} d_{5} \not{T}\right]=0 \Rightarrow V_{c}=V_{o} \frac{d_{5}}{d_{3}}
$$

Sumando B.41 y B.42 también se puede despejar $V_{c}$ como:

$$
V_{c}=V_{g} \frac{1}{d_{3}}-V_{o}
$$

\section{CORRIENTE MEDIA EN EL CONDENSADOR DE CLAMP.}

La intensidad media en el condensador de clamp es nula. Entonces:

$$
\begin{aligned}
& \int_{t_{0}}^{t_{0}+T} i_{c}(t) d t=\int_{t_{2}}^{t_{3}} i_{c}(t) d t=\int_{0}^{d_{3} T}\left[I-\frac{V_{c}}{L_{r}} t\right] d t=0 \\
& {\left[I t-\frac{V_{c}}{2 L_{r}} t^{2}\right]_{0}^{d_{3} T}=0 \Rightarrow V_{c}=\frac{2 I L_{r}}{d_{3} T}}
\end{aligned}
$$


Sustituyendo este valor en la expresión empleada para $i_{c}(t)$ se puede obtener el valor de la intensidad del condensador transcurrido el tiempo $d_{3} T$. Entonces:

$$
i_{c}\left(d_{3} T\right)=I-\frac{V_{c}}{L_{r}} d_{3} T=I-\frac{\frac{2 I h_{r}}{d_{3} T}}{L_{r}} d_{3} T=-I
$$

\section{DURACIÓN DEL INTERVALO $d_{5}$.}

Se puede obtener a partir de igualar las expresiones de $V_{c}$ dadas en (B.42) y en (B.44). De esta forma:

$$
V_{c}=\frac{2 I L_{r}}{d_{3} T}=V_{g} \frac{d_{5}}{d_{3}} \Rightarrow d_{5}=\frac{2 I L_{r}}{V_{o} T}
$$

Expresión a la que se puede llegar también a partir de la tensión en la bobina $L r$ durante la etapa 5 , puesto que es conocida la variación que experimenta la intensidad circulante por esa bobina y la tensión a la que está sometida.

$$
\begin{aligned}
\Delta i_{r}(t) & =i_{r}\left(t_{5}\right)-i_{r}\left(t_{4}\right) \approx i_{r}\left(t_{5}\right)-i_{r}\left(t_{3}\right)=I-(-I)=2 I \\
\Delta i_{r}(t) & =\frac{1}{L_{r}} \Delta v_{L_{r}}(t) \Delta t \equiv \frac{1}{L_{r}} V_{o} d_{5} T \\
\Rightarrow d_{5} & =\frac{2 I L_{r}}{V_{o} T}
\end{aligned}
$$

Por otro lado, como se cumple la transmisión de potencia de la entrada a la salida, $P_{i n}=P_{\text {out }}$, entonces, se puede obtener otra expresión para $d_{5}$ en función de la corriente de salida, $I_{o}$ :

$$
P_{\text {out }}=P_{\text {in }} \Rightarrow V_{o} I_{o}=V_{g} I \Rightarrow \frac{I}{V_{o}}=\frac{V_{g}}{I_{o}} \Rightarrow d_{5}=\frac{2 I_{o} L_{r}}{V_{g} T}
$$

\section{TENSION DE SALIDA.}

Despejando $V_{g}$ de (B.41) y utilizando (B.46) junto con (B.40) se obtiene una expresión para la tensión de salida en régimen permanente:

$$
\begin{gathered}
V_{o}=\frac{V_{g}}{\left(1-d_{6}\right)} \\
\Rightarrow V_{g}=V_{o}\left(1-d_{6}\right)=V_{o}\left(1-\left(D-d_{5}\right)\right) \\
\quad=V_{o}(1-D)+V_{o} d_{5} \\
=V_{o}(1-D)+Y_{o} \frac{2 I L_{r}}{V_{o} T} \\
\Rightarrow V_{o}=\frac{V_{g}}{(1-D)}-I \frac{2 L_{r} f}{(1-D)}
\end{gathered}
$$


A esta expresión también se llega igualando (B.43) con (B.44) y despejando $V_{o}$. Como se puede observar, la tensión de salida de un elevador ZVS depende de la intensidad a la entrada.

Esta expresión pone de manifiesto un elemento, equivalente a una resistencia y por el que pasa la intensidad $I$, que se puede considerar en serie con la bobina principal puesto que, en un elevador, esta intensidad es la misma que pasa la bobina. Su expresión sería (B.50):

$$
R_{e s L}=2 L_{r} f
$$

\section{CARACTERÍSTICA DE SALIDA.}

Considerando la igualdad de potencia de entrada y salida junto con (B.49) se puede obtener la relación entre la tensión de salida y la intensidad de salida

$$
\begin{aligned}
& \left.\begin{array}{l}
V_{o}=\frac{V_{g}}{1-D}-I \frac{2 L_{r} f}{(1-D)} \\
\begin{array}{l}
I_{g}=\frac{I}{(1-D)} \\
V_{o} I_{o}=V_{g} I_{g}
\end{array}
\end{array}\right\} \Rightarrow V_{o}=\frac{V_{g}}{1-D}-\frac{V_{o} I_{o}}{V_{g}} 2 L_{r} f \Rightarrow \\
& \Rightarrow V_{o}=\frac{V_{g}}{1-D} \frac{1}{1+I_{o} \frac{2 L_{r} f}{(1-D) V_{g}}}
\end{aligned}
$$

La derivada de esta expresión respecto a la intensidad indica como varía la tensión de salida ante las variaciones de la intensidad y tiene dimensiones de resistencia. Esta derivada tiene la siguiente expresión:

$$
\frac{d V_{o}}{d I_{o}}=\frac{V_{g}}{(1-D)} \times \frac{-\frac{2 L_{r} f}{(1-D) V_{g}}}{\left[1+I_{o} \frac{2 L_{r} f}{(1-D) V_{g}}\right]^{2}}
$$

Multiplicando arriba y abajo por $V_{g}$, se puede conseguir la misma expresión de la característica de salida, por lo que se puede simplificar y queda como:

$$
\frac{d V_{o}}{d I_{o}}=-2 L_{r} f\left[\frac{V_{o}}{V_{g}}\right]^{2}
$$

\section{B.2.2. Considerando rizado.}

Si se considera que la bobina $L$ no es lo suficientemente grande como para suponer corriente constante, $I$, los circuitos equivalentes empleados para el análisis del circuito son diferentes y la relación entre la bobina principal y resonante aparece en las nuevas expresiones de las principales magnitudes. 
Los circuitos equivalentes están representados en la Figura B.13 donde se ha considerado la tensión constante en los condensadores $C_{o}$ y $C_{c}$, mientras que las principales magnitudes aparecen en la Figura B.14.

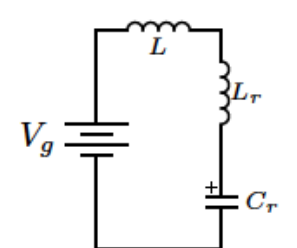

(a)

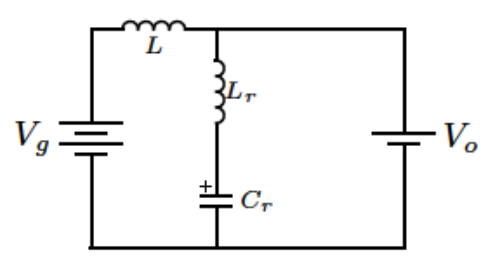

(b)

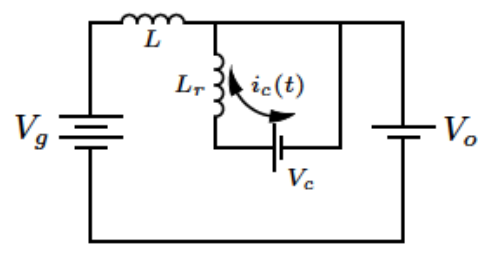

(c)

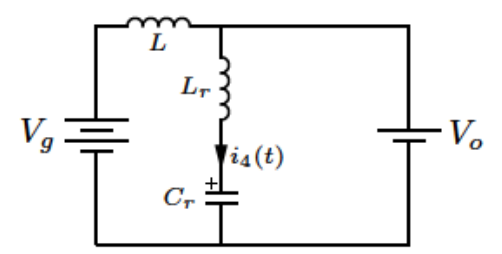

(d)

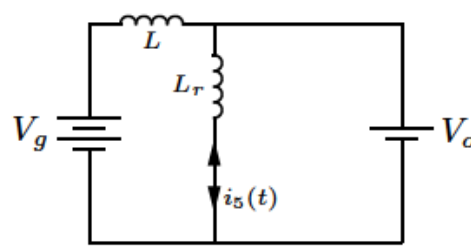

(e)

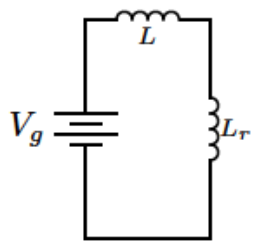

(f)

Figura B.13: Circuitos empleados para el análisis del elevador ZVS considerando rizado durante un ciclo completo: (a) Etapa 1. (b) Etapa 2. (c) Etapa 3. (d) Etapa 4. (e) Etapa 5. (f) Etapa 6.

Planteando el balance voltios-segundo en las bobinas $L$ y $L_{r}$ y la carga neta nula en los condensadores $C_{o}$ y $C_{c}$ con las nuevas curvas, se obtienen otras ecuaciones del régimen permanente. Se desprecian las etapas 1, 2 y 4 , y solo intervienen tres etapas.

\section{TENSION MEDIA EN BOBINA PRINCIPAL CON RIZADO.}

Como la tensión media en la bobina $L$ es nula, resulta que:

$$
\begin{aligned}
& \frac{1}{\mathscr{T}}\left[\left(V_{g}-V_{o}\right)\left(d_{3}+d_{5}\right) \not{T}+V_{g} \frac{L}{L+L_{r}} d_{6} X\right]=0 \\
& \Rightarrow V_{o}=V_{g} \frac{d_{3}+d_{5}+d_{6} \frac{L}{L+L_{r}}}{d_{3}+d_{5}}=V_{g} \frac{\left(1-d_{6} \frac{L_{r}}{L+L_{r}}\right)}{\left(1-d_{6}\right)}
\end{aligned}
$$

\section{TENSION MEDIA EN BOBINA RESONANTE CON RIZA-} DO.

Como la tensión media en la bobina $L_{r}$ es nula, entonces:

$$
\begin{aligned}
& \frac{1}{\mathscr{X}}\left[-V_{c} d_{3} \not{X}+V_{o} d_{5} \mathscr{T}+V_{g} d_{6} \frac{L_{r}}{L+L_{r}}\right]=0 \\
& \Rightarrow V_{c}=V_{o} \frac{d_{5}}{d_{3}}+V_{g} \frac{d_{6}}{d_{3}} \frac{L_{r}}{L+L_{r}}
\end{aligned}
$$




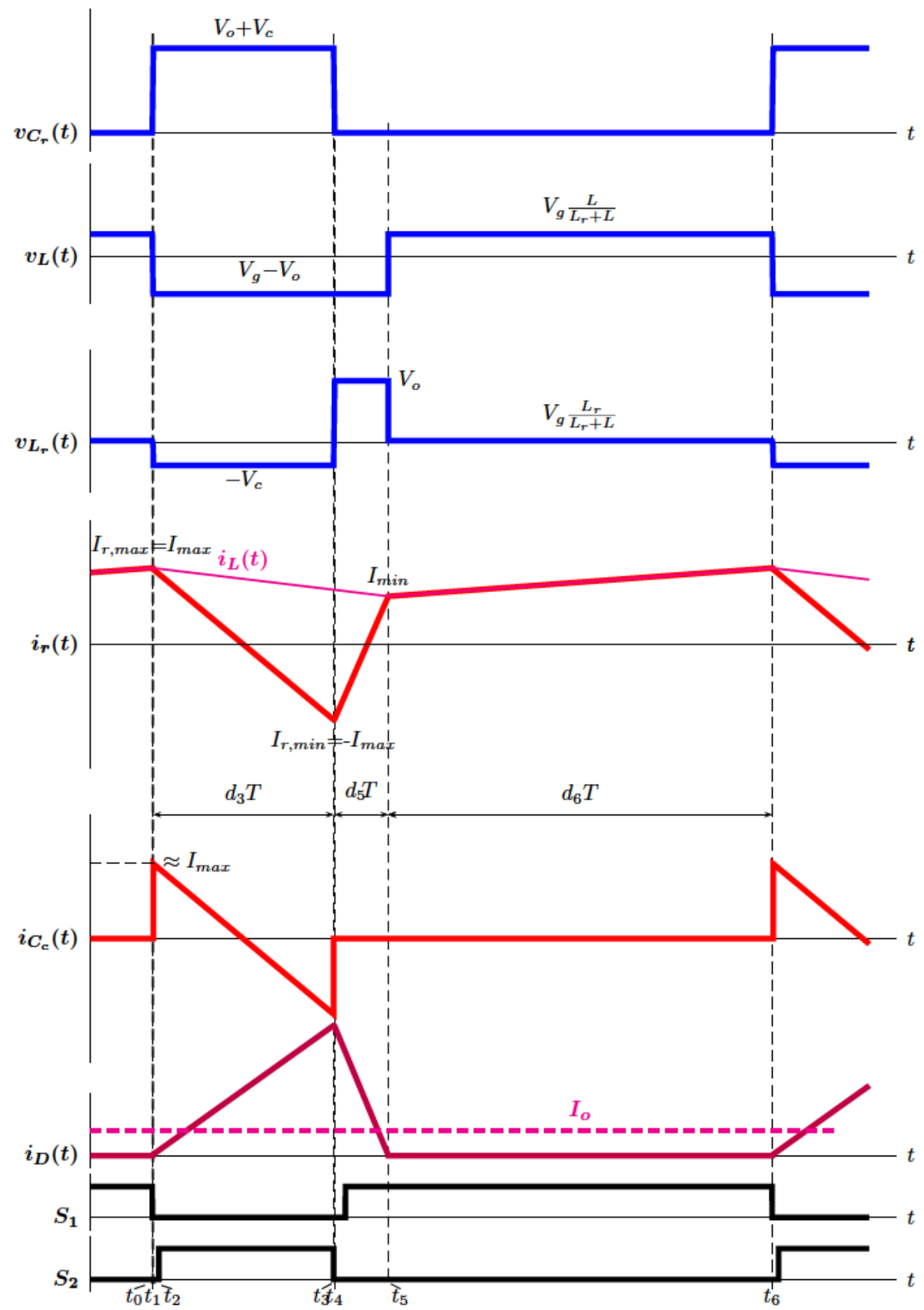

Figura B.14: Curvas principales de un elevador ZVS considerando rizado.

Sumando B.54 y B.55 también se puede despejar $V_{c}$ como:

$$
V_{c}=V_{g} \frac{1}{d_{3}}-V_{o}
$$




\section{CORRIENTE MEDIA EN EL CONDENSADOR DE CLAMP CON RIZADO.}

La intensidad media en el condensador de clamp es nula. Entonces:

$$
\begin{aligned}
& \int_{t_{0}}^{t_{0}+T} i_{c}(t) d t=\int_{t_{2}}^{t_{3}} i_{c}(t) d t=\int_{0}^{d_{3} T}\left[I_{\text {max }}-\frac{V_{c}}{L_{r}} t\right] d t=0 \\
& {\left[I_{\text {max }} t-\frac{V_{c}}{2 L_{r}} t^{2}\right]_{0}^{d_{3} T}=0 \Rightarrow V_{c}=\frac{2 I_{\text {max }} L_{r}}{d_{3} T}}
\end{aligned}
$$

Sustituyendo este valor en la expresión empleada para $i_{c}(t)$ se puede obtener el valor de la intensidad del condensador transcurrido el tiempo $d_{3} T$. Entonces:

$$
i_{c}\left(d_{3} T\right)=I_{\max }-\frac{V_{c}}{L_{r}} d_{3} T=I_{\max }-\frac{\frac{2 I_{\max } \not_{r}}{d_{3} T}}{L_{r}} d_{3} T=-I_{\max }
$$

\section{DURACIÓN DEL INTERVALO $d_{5}$ CON RIZADO.}

Se puede calcular a partir de la tensión en la bobina $\operatorname{Lr}$ durante la etapa 5 , puesto que es conocida la variación que experimenta la intensidad circulante por esa bobina y la tensión a la que está sometida.

$$
\begin{aligned}
\Delta i_{r}(t) & =i_{r}\left(t_{5}\right)-i_{r}\left(t_{4}\right) \approx i_{r}\left(t_{5}\right)-i_{r}\left(t_{3}\right)=I_{\text {min }}-\left(-I_{\text {max }}\right)=2 I \\
\Delta i_{r}(t) & =\frac{1}{L_{r}} \Delta v_{L_{r}}(t) \Delta t \equiv \frac{1}{L_{r}} V_{o} d_{5} T \\
\Rightarrow d_{5} & =\frac{2 I L_{r}}{V_{o} T}
\end{aligned}
$$

Una expresión diferente se obtiene al igualar (B.55) con (B.57) y empleando (B.40) para despejar $d_{5}$ :

$$
\begin{aligned}
& V_{o} \frac{d_{5}}{d_{3}}+V_{g} \frac{\left(D-d_{5}\right)}{\not / 3} \frac{L_{r}}{L+L_{r}}=\frac{2 I_{\max } L_{r} f}{\not / 3} \\
& \Rightarrow d_{5}=\frac{2 I_{\max } L_{r} f-V_{g} D \frac{L_{r}}{L+L_{r}}}{V_{o}-V_{g} \frac{L_{r}}{L+L_{r}}}
\end{aligned}
$$

\section{TENSION DE SALIDA CON RIZADO.}

Como $d_{6}=\left(D-d_{5}\right)$, resulta que:

$$
\begin{aligned}
d_{6} & =D-\frac{2 I_{\max } L_{r} f-V_{g} D \frac{L_{r}}{L+L_{r}}}{V_{o}-V_{g} \frac{L_{r}}{L+L_{r}}} \\
& =\frac{V_{o} D-2 I_{\max } L_{r} f}{V_{o}-V_{g} \frac{L_{r}}{L+L_{r}}}
\end{aligned}
$$


Por otro lado, despejando $d_{6}$ de (B.54) se obtiene:

$$
d_{6}=\frac{V_{o}-V_{g}}{V_{o}-V_{g} \frac{L_{r}}{L+L_{r}}}
$$

Igualando (B.61) y (B.62) se puede despejar $V_{o}$ como:

$$
V_{o}=\frac{V_{g}}{(1-D)}-I_{\max } \frac{2 L_{r} f}{(1-D)}
$$

También se podría conseguir si se iguala (B.56) con (B.57) y despejando $V_{o}$. En ambos casos, la expresión obtenida es parecida a (B.49) que se ha obtenido sin considerar rizado. Solo se ha cambiado $I$ por $I_{\max }$. Y el término que multiplica a la intensidad se puede considerar como una resistencia en serie con $L, R_{e s L}$, ya definida anteriormente en (B.50).

\section{RIZADO DE CORRIENTE EN $L$ CON RIZADO.}

Aplicando la definición de rizado de corriente en una bobina, se puede obtener el rizado de corriente en $L$ durante las etapas 3 a 5 o durante la etapa 6 siendo expresiones equivalentes en régimen permanente. Entonces:

$$
\begin{aligned}
\Delta i_{L, 3-5} & =\frac{1}{L}\left|V_{g}-V_{o}\right|\left(d_{3}+d_{5}\right) T=\frac{1}{L}\left(V_{o}-V_{g}\right)\left(1-d_{6}\right) T \\
\Delta i_{L, 6} & =\frac{1}{L}\left|V_{g}\right| \frac{L}{L+L_{r}} d_{6} T=\frac{V_{g}}{L+L_{r}} d_{6} T
\end{aligned}
$$

Empleando la ecuación (B.62) se obtiene la expresión para el rizado de corriente en $L$ como:

$$
\Delta i_{L}=\frac{\left(V_{g}-V_{o}\right) V_{g} T}{L_{r} V_{g}-\left(L+L_{r}\right) V_{o}}
$$

\section{CARACTERÍSTICA DE SALIDA CON RIZADO.}

La relación entre la tensión de salida y la intensidad de salida se obtiene a partir de la expresión (B.63). Primero se expresa $I_{\max }$ en función del valor medio y del rizado. Después se emplea la igualdad de potencia a la entrada y a la salida y la relación $I=I_{g}$, para obtener:

$$
\left.\begin{array}{l}
I=I_{g} \\
V_{g} I_{g}=V_{o} I_{o}
\end{array}\right\} \Rightarrow I=I_{o} \frac{V_{o}}{V_{g}}
$$

Para el rizado de corriente se emplea la segunda expresión de (B.64) y se considera $d_{6}=\left(D-d_{5}\right)$ :

$$
\Delta i_{L}=\frac{V_{g}}{L+L_{r}}\left(D-d_{5}\right) T=\frac{V_{g}}{L+L_{r}}\left(D-\frac{2 I L_{r}}{V_{o} T}\right) T
$$


Sólo queda sustituir (B.66) y (B.67) en (B.63) y despejar $V_{o}$ para obtener una función de la forma $V_{o}=f\left(I_{o}\right)$. Por tanto:

$$
\begin{aligned}
V_{o} & =\frac{V_{g}}{(1-D)}-I_{\max } \frac{2 L_{r} f}{(1-D)}=\frac{V_{g}}{(1-D)}-\left(I+\frac{1}{2} \Delta i_{L}\right) \frac{2 L_{r} f}{(1-D)} \\
& =\frac{V_{g}}{(1-D)}-I \frac{2 L_{r} f}{(1-D)}+\frac{1}{\not 2} \frac{V_{g}}{L+L_{r}}\left(D-\frac{2 I L_{r}}{V_{o} T}\right) \not \mathcal{X} \frac{\not L_{r} f}{(1-D)} \\
& =\frac{V_{g}}{(1-D)}-I_{o} \frac{V_{o}}{V_{g}} \times \frac{2 L_{r} f}{(1-D)}+\frac{V_{g}}{(1-D)} \times \frac{L_{r}}{L+L_{r}}\left(D-I_{o} \frac{Y_{o}}{V_{g}} \frac{2 L_{r} f}{Y_{o}}\right) \\
& =\frac{V_{g}}{(1-D)}\left[1-\frac{L_{r}}{L+L_{r}}\left(D-I_{o} \frac{2 L_{r} f}{V_{g}}\right)\right]-I_{o} \frac{V_{o}}{V_{g}} \times \frac{2 L_{r} f}{(1-D)} \\
& \Rightarrow V_{o}\left(I_{o}\right)=\frac{V_{g}}{1-D} \frac{1-\frac{L_{r}}{L_{r}+L}\left(D-I_{o} \frac{2 L_{r} f}{V_{g}}\right)}{1+I_{o} \frac{2 L_{r} f}{V_{g}(1-D)}}
\end{aligned}
$$

\section{B.2.3. Comparativa.}

Está claro que el análisis cuando se considera el rizado será de aplicación al circuito conmutado, donde se tienen en cuenta los valores de las bobinas, mientras que el análisis al no considerar rizado servirá para las expresiones derivadas del modelo promediado, dado que éste parte de la consideración de pequeño rizado.

Los resultados obtenidos para magnitudes tales como el ciclo de trabajo, la tensión de clamp y las variaciones de corriente, a partir de unos valores de diseño ponen de manifiesto estas diferencias. Por ejemplo, con los parámetros de funcionamiento recogidos en la Tabla B.1 se obtienen los valores indicados en la Tabla B.2, en el caso de no considerar el rizado de la corriente en la bobina principal y se compara con el obtenido cuando si se ha considerado.

\begin{tabular}{llll}
\hline \hline$V_{g}$ & $160 \mathrm{~V}$ & $C_{o}$ & $5 \mu \mathrm{F}$ \\
$P$ & $400 \mathrm{~W}$ & $C_{c}$ & $0,47 \mu \mathrm{F}$ \\
$V_{\text {out }}$ & $400 \mathrm{~V}$ & $L$ & $600 \mu \mathrm{H}$ \\
$f$ & $100 \mathrm{kHz}$ & $L_{r}$ & $70 \mu \mathrm{H}$ \\
$t_{m}$ & $100 \mathrm{~ns}$ & $C_{r}$ & $0,6 \mathrm{nF}$ \\
\hline
\end{tabular}

Tabla B.1: Condiciones de trabajo de un elevador ZVS.

En el elevador ZVS la intensidad en la bobina principal es igual a la intensidad en la entrada, $I_{g}$. Al considerar el rizado, el ciclo de trabajo obtenido es mayor. La tensión de clamp, $V_{c}$ también es diferente. Es lógico dado que depende del ciclo de trabajo. Si se calcula su valor a partir de la expresión que incluye la intensidad, también se obtendrá un valor diferente según se considere valor medio (sin rizado) o valor máximo (cuando hay rizado). 


\begin{tabular}{ccccccccc}
\hline & $I_{g}$ & $d_{5}$ & $d_{6}$ & $D$ & $\begin{array}{c}V_{c} \\
(\mathrm{~V})\end{array}$ & $\begin{array}{c}\Delta_{i_{L}} \\
(\mathrm{~A})\end{array}$ & $\begin{array}{c}I_{\max } \\
(\mathrm{A})\end{array}$ & $\begin{array}{c}I_{\min } \\
(\mathrm{A})\end{array}$ \\
\hline SIN RIZADO & \multirow{2}{*}{2,5} & \multirow{2}{*}{0,0875} & 0,6000 & 0,6875 & 112 & 0 & - & - \\
CON RIZADO & & & 0,6262 & 0,7137 & 158,85 & 1,495 & 3,248 & 1,752 \\
\hline \hline
\end{tabular}

Tabla B.2: Parámetros calculados cuando se considera o no el rizado.

En ambos análisis se han considerado despreciables algunas etapas por su pequeña duración en relación a las otras. Esta hipótesis se ha validado midiendo la duración de las seis etapas para los parámetros de funcionamiento de las dos tablas anteriores. Con ayuda de un programa de cálculo simbólico se han planteado las ecuaciones de estado durante las seis etapas y se han resuelto las ecuaciones y obtenido la fracción que ocupa cada etapa dentro de un periodo completo. Se han indicado en la Tabla B.3

\begin{tabular}{ccccccc}
\hline & $d_{1}$ & $d_{2}$ & $d_{3}$ & $d_{4}$ & $d_{5}$ & $d_{6}$ \\
\hline SIN RIZADO & $9,6 \times 10^{-3}$ & $2,7 \times 10^{-3}$ & 0,3098 & $12,7 \times 10^{-3}$ & $82,4 \times 10^{-3}$ & 0,5828 \\
CON RIZADO & $7,9 \times 10^{-3}$ & $2,4 \times 10^{-3}$ & 0,2759 & $11,1 \times 10^{-3}$ & $80,8 \times 10^{-3}$ & 0,6221 \\
\hline \hline
\end{tabular}

Tabla B.3: Fracción de tiempo para cada etapa por las que evoluciona el elevador ZVS.

A la vista de estos resultados, se puede comprobar que las etapas 1, 2 y 4 son las de menor duración por lo que, como se había previsto, se pueden despreciar.

En cada uno de los dos análisis realizados, la característica de salida obtenida muestra una relación diferente entre tensión e intensidad de salida. Cuando ambas se representan gráficamente se obtiene prácticamente la misma curva, como se pone de manifiesto al observar la Figura B.15 que contiene la característica de salida de un elevador ZVS con los datos de la Tabla B.1. En este caso, la curva representada sin considerar rizado emplea el valor de ciclo de trabajo obtenido con este mismo análisis, mientras que la curva representada al considerar el rizado está obtenida con el ciclo de trabajo calculado con rizado no despreciable.

\section{B.3. Modelo promediado}

La obtención del modelo promediado se puede conseguir mediante el promediado de las formas de onda, mediante el promediado de circuitos o mediante el promediado en el espacio de estados. En esa sección se va a obtener el modelo promediado del elevador ZVS descrito en la sección anterior, de la misma forma que se ha hecho para el reductor ZVS en capítulos anteriores. 


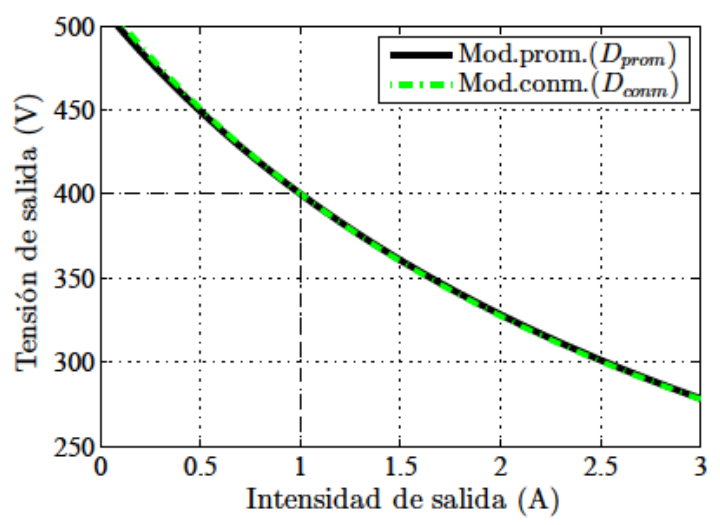

Figura B.15: Característica de salida de un Elevador ZVS.

\section{B.3.1. Modelo promediado para el elevador ZVS}

En la sección B.1 se ha explicado el funcionamiento en régimen permanente del elevador ZVS. Del análisis se deduce que la corriente en la bobina resonante $L_{r}$ y la tensión en el condensador resonante $C_{r}$ son las variables de estado rápidas y el resto son las variables de estado lentas. El procedimiento a seguir es obtener las expresiones para las variables rápidas $i_{L_{r}}(t)$ y $v_{C_{r}}(t)$, en un periodo de conmutación (comprobar que son periódicas). La variables lentas se suponen de valor constante para este cálculo. Las expresiones obtenidas, dependerán, por tanto, de las variables lentas y también del tiempo. Éstas se sustituyen en las ecuaciones de estado de las variables lentas de forma que queden desacopladas respecto a las variables rápidas. Se aplica ahora un promedio estándar en un periodo de conmutación para eliminar la dependencia con el tiempo, consiguiéndose un modelo promediado de tercer orden (orden reducido) para las tres variables de estado lentas, $v_{C_{c}}(t), v_{o}(t)$ y $i_{L}(t)$.

Aunque matemáticamente no es necesario, se suelen realizar algunas aproximaciones antes de empezar para simplificar los cálculos a realizar. En primer lugar, se ignoran los tiempos muertos entre las conmutaciones de $S_{1}$ y $S_{2}$ que normalmente son muy pequeños en comparación con el periodo. También se desprecian los intervalos resonantes, como se hace en (ATHALYE y otros, 2001) para obtener el modelo completo con el método del interruptor promediado. En este punto cabe indicar que el efecto debido a tener en cuenta los intervalos resonantes sería tan solo a frecuencias muy altas y fuera del rango de interés (que, generalmente, suele ser una fracción de la frecuencia de conmutación porque es donde se aplica el control en lazo cerrado). Además se supone que el condensador de clamp mantiene su tensión constante, lo que quiere decir que la frecuencia de resonancia entre $L_{r}$ y $C_{c}$ es bastante 
menor que la frecuencia de conmutación.

Considerando estas simplificaciones desaparecen los intervalos resonantes (Etapa 1, 2 y 4) y el tiempo muerto de la etapa 5, de forma que un periodo se puede escribir como:

$$
\begin{aligned}
T & \approx d_{3} T+d_{5} T+d_{6} T \\
(1-d) T & =d_{3} T \\
d T & =d_{5} T+d_{6} T
\end{aligned}
$$

puesto que $d_{1} T=d_{2} T=d_{4} T=0$.

Las curvas principales están indicadas en la figura B.12. Suponiendo valores constantes para las variables lentas, la tensión $v_{C_{r}}(t)$, tiene la siguiente expresión ${ }^{1}$ :

$$
v_{C_{r}}(t)= \begin{cases}v_{C_{o}}+v_{C_{c}} & t \in\left[t_{2}, t_{3}\right] \\ 0 & t \in\left[t_{4}, t_{5}\right] \\ 0 & t \in\left[t_{5}, t_{6}\right]\end{cases}
$$

mientras que la intensidad en la bobina resonante, $i_{L_{r}}(t)$ se expresa como

$$
i_{L_{r}}(t)= \begin{cases}i_{L}-\frac{v_{C_{c}}}{L_{r}}\left(t-t_{2}\right) & t \in\left[t_{2}, t_{3}\right] \\ i_{L}+\frac{v_{C_{o}}}{L_{r}}\left(t-t_{5}\right) & t \in\left[t_{4}, t_{5}\right] \\ i_{L} & t \in\left[t_{5}, t_{6}\right]\end{cases}
$$

Se obtiene $d_{5}$ a partir de la igualdad de valores de $i_{r}$ en $t_{3}=t_{4}$. Es decir:

$$
i_{L_{r}}\left(t_{3}\right)=i_{L_{r}}\left(t_{4}\right)
$$

Entonces, con (B.71) y (B.72) se obtiene una solución para $d_{5}$ :

$$
d_{5}=v_{C_{c}}(1-d) / v_{C_{o}}
$$

Para el resto de variables lentas si es necesario escribir las ecuaciones de estado. Necesitamos una ecuación para la intensidad en la bobina de entrada, $i_{L}(t)$; otra ecuación para la tensión en el condensador de salida, $v_{C_{o}}(t)$; y otra ecuación para la tensión en el condensador de clamp, $v_{C_{c}}(t)$. Estas ecuaciones

\footnotetext{
${ }^{1}$ Se mantiene la numeración de los intervalos, considerando que $t_{0}=t_{1}=t_{2}$ y que $t_{3}=t_{4}$.
} 
son:

$$
\begin{aligned}
& v_{L}(t)=L \frac{d i_{L}(t)}{d t}= \begin{cases}v_{g}(t)-v_{C_{o}}(t) & t \in\left[t_{2}, t_{3}\right] \\
v_{g}(t)-v_{C_{o}}(t) & t \in\left[t_{4}, t_{5}\right] \\
v_{g}(t) & t \in\left[t_{5}, t_{6}\right]\end{cases} \\
& i_{C_{o}}(t)=C_{o} \frac{d v_{C_{o}}(t)}{d t}= \begin{cases}i_{L}(t)-\frac{v_{C_{o}}(t)}{R} & t \in\left[t_{2}, t_{3}\right] \\
i_{L}(t)-i_{L_{r}}(t)-\frac{v_{C_{o}}(t)}{R} & t \in\left[t_{4}, t_{5}\right] \\
-\frac{v_{C_{o}}(t)}{R} & t \in\left[t_{5}, t_{6}\right]\end{cases} \\
& i_{C_{c}}(t)=C_{c} \frac{d v_{C_{c}}(t)}{d t}= \begin{cases}i_{L_{r}}(t) & t \in\left[t_{2}, t_{3}\right] \\
0 & t \in\left[t_{4}, t_{5}\right] \\
0 & t \in\left[t_{5}, t_{6}\right]\end{cases}
\end{aligned}
$$

La ecuación (B.74) es la ecuación de estado de un convertidor elevador sin enclavamiento activo, mientras que (B.75) y (B.76) son las ecuaciones del condensador de salida y del condensador de esta red añadida. Al igual que en el elevador convencional, se puede añadir una ecuación relacionada con la intensidad del diodo principal del convertidor, que es (B.77):

$$
i_{D}(t)= \begin{cases}i_{L}(t)-i_{L_{r}}(t) & t \in\left[t_{2}, t_{3}\right] \\ i_{L}(t)-i_{L_{r}}(t) & t \in\left[t_{4}, t_{5}\right] \\ 0 & t \in\left[t_{5}, t_{6}\right]\end{cases}
$$

Cuando se sustituyen las variables rápidas en las ecuaciones anteriores, se consigue tener un conjunto de ecuaciones de estado donde solo intervienen las variables de estado lentas, es decir, se han desacoplado de las variables de estado rápidas, que son

$$
\begin{aligned}
& L \frac{d i_{L}(t)}{d t}= \begin{cases}\left.v_{g}(t)-v_{C_{o}}(t)\right) & t \in\left[t_{2}, t_{3}\right] \\
v_{g}(t)-v_{C_{o}}(t) & t \in\left[t_{4}, t_{5}\right] \\
v_{g}(t) & t \in\left[t_{5}, t_{6}\right]\end{cases} \\
& C_{o} \frac{d v_{C_{o}}(t)}{d t}= \begin{cases}i_{L}(t)-\frac{v_{C_{o}}(t)}{R} & t \in\left[t_{2}, t_{3}\right] \\
-\frac{v_{C_{o}}}{L_{r}}\left(t-t_{5}\right)-\frac{v_{C_{o}}(t)}{R} & t \in\left[t_{4}, t_{5}\right] \\
-\frac{v_{C_{o}}(t)}{R} & t \in\left[t_{5}, t_{6}\right]\end{cases} \\
& C_{c} \frac{d v_{C_{c}}(t)}{d t}= \begin{cases}i_{L}(t)-\frac{v_{C_{c}}(t)}{L_{r}}\left(t-t_{2}\right) & t \in\left[t_{2}, t_{3}\right] \\
0 & t \in\left[t_{4}, t_{5}\right] \\
0 & t \in\left[t_{5}, t_{6}\right]\end{cases}
\end{aligned}
$$


junto con

$$
i_{D}(t)= \begin{cases}\frac{v_{C_{c}}}{L_{V_{C_{o}}}}\left(t-t_{2}\right) & t \in\left[t_{2}, t_{3}\right] \\ -\frac{v_{C_{0}}}{L_{r}}\left(t-t_{5}\right) & t \in\left[t_{4}, t_{5}\right] \\ 0 & t \in\left[t_{5}, t_{6}\right]\end{cases}
$$

Considerando la aproximación de pequeño rizado (valores instantáneos son muy parecidos a los valores promedio), es posible sustituir los valores instantáneos de las variables de estado por sus valores promedio, (por ejemplo, $i_{L}(t)$ se sustituye por $\bar{i}_{L}$, que es igual que $\left\langle i_{L}(t)\right\rangle_{T}$ donde se ha simplificado la notación, y así con el resto). De esta forma, las ecuaciones desacopladas se transforman en

$$
\begin{aligned}
L \frac{d i_{L}(t)}{d t} \approx \begin{cases}\bar{v}_{g}-\bar{v}_{C_{o}} & t \in\left[t_{2}, t_{3}\right] \\
\bar{v}_{g}-\bar{v}_{C_{o}} & t \in\left[t_{4}, t_{5}\right] \\
\bar{v}_{g} & t \in\left[t_{5}, t_{6}\right]\end{cases} \\
C_{o} \frac{d v_{C_{o}}(t)}{d t} \approx \begin{cases}\bar{i}_{L}-\frac{\bar{v}_{C_{o}}}{R} & t \in\left[t_{2}, t_{3}\right] \\
-\frac{\bar{v}_{C_{o}}}{L_{r}}\left(t-t_{5}\right)-\frac{\bar{v}_{C_{o}}}{R} & t \in\left[t_{4}, t_{5}\right] \\
-\frac{\bar{v}_{C_{o}}}{R} & t \in\left[t_{5}, t_{6}\right]\end{cases} \\
C_{c} \frac{d v_{C_{c}}(t)}{d t} \approx \begin{cases}\bar{i}_{L}-\frac{\bar{v}_{C_{c}}}{L_{r}}\left(t-t_{2}\right) & t \in\left[t_{2}, t_{3}\right] \\
0 & t \in\left[t_{4}, t_{5}\right] \\
0 & t \in\left[t_{5}, t_{6}\right]\end{cases} \\
i_{D}(t) \approx \begin{cases}\frac{\bar{v}_{C_{c}}}{L_{r_{r}}}\left(t-t_{2}\right) & t \in\left[t_{2}, t_{3}\right] \\
-\frac{v_{C_{o}}}{L_{r}}\left(t-t_{5}\right) & t \in\left[t_{4}, t_{5}\right] \\
0 & t \in\left[t_{5}, t_{6}\right]\end{cases}
\end{aligned}
$$

con

$$
d_{5} \approx \frac{\bar{v}_{C_{c}}}{\bar{v}_{C_{o}}}(1-d)
$$

Por último hay que eliminar la dependencia del tiempo realizando el promedio de las ecuaciones en un intervalo, de donde se obtiene el modelo promediado para el elevador ZVS, que es un modelo de orden tres, (es un modelo de orden reducido frente al modelo de orden completo que sería de 
orden cinco).

$$
\begin{aligned}
L \frac{d \bar{i}_{L}}{d t} & =\bar{v}_{g}-\left(1-d+d_{5}\right) \bar{v}_{C_{o}} \\
C_{o} \frac{d \bar{v}_{C_{o}}}{d t} & =(1-d) \bar{i}_{L}-\frac{\bar{v}_{C_{o}}}{R}+\frac{\bar{v}_{C_{o}} d_{5}^{2}}{2 L_{r} f_{s}} \\
C_{c} \frac{d \bar{v}_{C_{c}}}{d t} & =\bar{i}_{L}(1-d)-\frac{\bar{v}_{C_{c}}}{2 L_{r} f_{s}}(1-d)^{2} \\
\bar{i}_{D} & =\frac{\bar{v}_{C_{o}} d_{5}^{2}}{2 L_{r} f_{s}}+\frac{\bar{v}_{C_{c}}}{2 L_{r} f_{s}}(1-d)^{2}
\end{aligned}
$$

Con las ecuaciones anteriores se consigue obtener un circuito promediado para este convertidor. Previamente hay que sustituir el parámetro $d_{5}$ por su valor, y se obtienen las ecuaciones indicadas en (B.91).

$$
\begin{aligned}
L \frac{d \bar{i}_{L}}{d t} & =\bar{v}_{g}-(1-d) \bar{v}_{C_{o}}-(1-d) \bar{v}_{C_{c}} \\
C_{o} \frac{d \bar{v}_{C_{o}}}{d t} & =(1-d) \bar{i}_{L}-\frac{\bar{v}_{C_{o}}}{R}+\frac{\bar{v}_{C_{c}}^{2}}{\bar{v}_{C_{o}}} \frac{(1-d)^{2}}{2 L_{r} f_{s}} \\
C_{c} \frac{d \bar{v}_{C_{c}}}{d t} & =(1-d) \bar{i}_{L}-\frac{\bar{v}_{C_{c}}}{2 L_{r} f_{s}}(1-d)^{2} \\
\bar{i}_{D} & =\frac{\bar{v}_{C_{c}}^{2}}{\bar{v}_{C_{o}}} \frac{(1-d)^{2}}{2 L_{r} f_{s}}+\frac{\bar{v}_{C_{c}}}{2 L_{r} f_{s}}(1-d)^{2}
\end{aligned}
$$

El circuito obtenido es el indicado en la figura A.16, donde $r_{e q}$ es una resistencia equivalente sin pérdidas que consume la energía generada por la fuente dependiente de corriente de la etapa de entrada dada por $i_{e q}$.

$$
\begin{gathered}
r_{e q}=\frac{2 L_{r} f_{s}}{(1-d)^{2}} \\
i_{e q}=\frac{v_{C_{c}}^{2}}{v_{C_{o}} r_{e q}}
\end{gathered}
$$

\section{B.3.2. Balance energético}

En el modelo promediadohay dos elementos que generan energía, la fuente de tensión en la entrada y la fuente de corriente equivalente en la salida. La energía de la fuente de tensión se consume en la carga y se almacena o devuelve en los elementos reactivos, mientras que la energía generada en la fuente de corriente se consume toda en $r_{e q}$. Veámoslo.

En $r_{e q}$, la potencia consumida es igual a

$$
P_{r_{e q}}=\frac{v_{C_{c}}^{2}}{r_{e q}}
$$




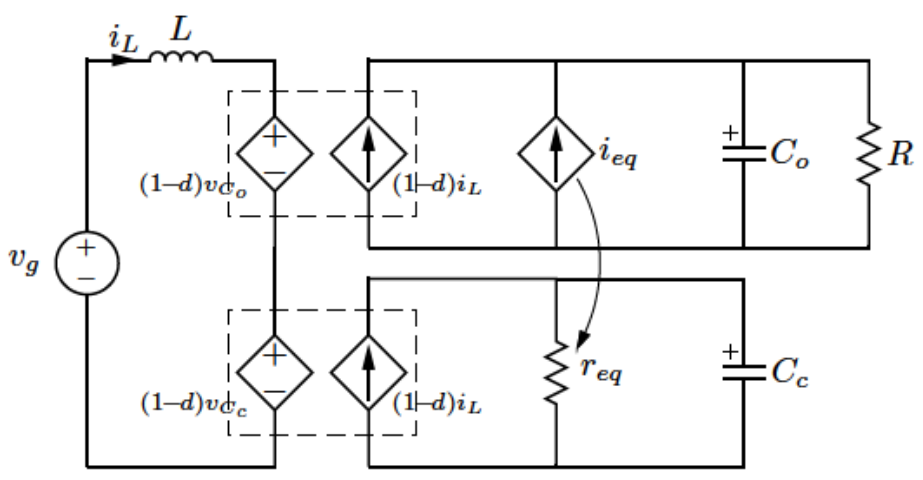

Figura B.16: Modelo promediado de un elevador ZVS.

Multiplicando arriba y abajo por $v_{C_{o}}$, se obtiene una expresión

$$
P_{r_{e q}}=\frac{v_{C_{c}}^{2}}{r_{e q}} \frac{v_{C_{o}}}{v_{C_{o}}}
$$

en la que, agrupando, se obtiene el producto de una fuente de tensión por otra de corriente cuyo valor es igual a $i_{e q}$

$$
P_{r_{e q}}=i_{e q} v_{C_{o}}
$$

Esta potencia coincide con la potencia generada por la fuente de corriente $i_{e q}$ en la salida.

\section{B.3.3. Validación del modelo promediado}

La validación del modelo promediado se realiza mediante simulación, y se compara su comportamiento, tanto en régimen permanente como en régimen dinámico, con un convertidor conmutado. Para ello se eligen de nuevo los parámetros de funcionamiento recogidos en la Tabla B.1, empleando en el convertidor conmutado el ciclo de trabajo calculado al considerar rizado. En el modelo promediado se ha considerado el ciclo de trabajo calculado cuando no se ha considerado el rizado.

Los resultados muestran una dinámica similar para el convertidor conmutado y el modelo promediado. En cuanto al régimen permanente, hay un error puesto que la tensión de salida no coincide. El motivo de esta diferencia tiene su causa en que en el modelo promediado no está incluido el tiempo muerto, $t_{m}$, que hay que dejar entre disparos de los transistores principal y de clamp, entre otras cosas, para facilitar las conmutaciones suaves. Además, parte de este tiempo muerto se emplea en cargar o descargar el condensador resonante, y corresponde a las tres etapas que se desprecian para obtener el modelo promediado. En concreto la etapa 4, siendo su duración igual a $d_{4} T$. 


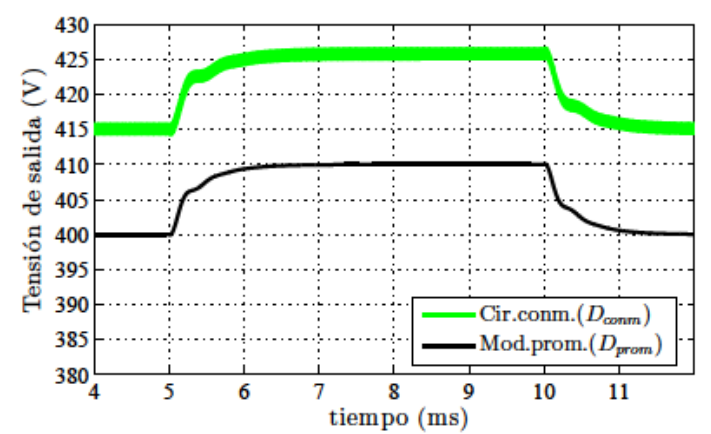

(a)

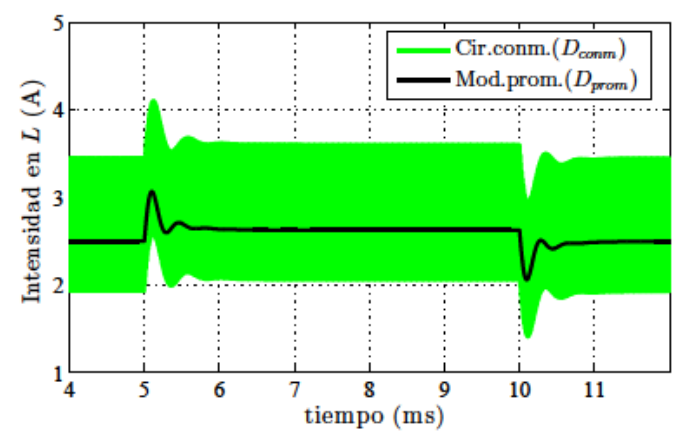

(b)

Figura B.17: Elevador ZVS. Respuesta a escalón del ciclo de trabajo: a) Tensión de salida; y b) Intensidad en la bobina.

Por tanto, hay que realizar una corrección en el ciclo de trabajo empleado para el circuito conmutado, en base a los tiempos mencionados, puesto que ya está incluido de forma implícita en la generación de disparos del propio circuito conmutado. De esta forma, se rehacen las simulaciones del circuito conmutado con el ciclo de trabajo corregido, $D_{\text {corr }}$, como:

$$
D_{\text {corr }}=D_{\text {conm }}-\frac{t_{m}-d_{4} T}{T}
$$

y se obtienen los resultados de la Figura B.18. La corrección equivalente pero para el modelo promediado consistiría en añadir este tiempo extra al ciclo de trabajo empleado en el modelo promediado.

Para apreciar la mejora en los resultados se representa el valor medio de la tensión de salida y la intensidad en la bobina para el circuito conmutado antes y después de la corrección y se comparan con el modelo promediado. En la Figura B.19 se pueden comprobar estas curvas.

\section{B.4. Modelo en régimen permanente}

El modelo en régimen permanente se puede obtener a partir del modelo promediado (o de gran señal) sustituyendo los valores promedio por valores constantes (por ejemplo, $\bar{i}_{L}$ se sustituye por $I_{g}$ ). De esta forma, las ecuaciones del modelo en régimen permanente serán las indicadas a continuación. Nótese que la derivada de una constante es cero, de ahí, que haya ecuaciones con un 


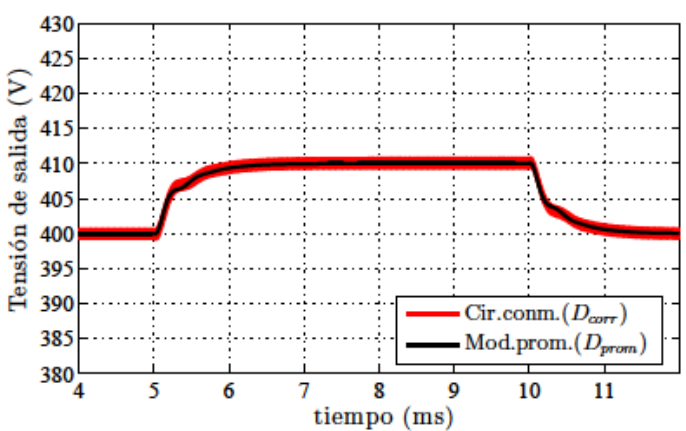

(a)

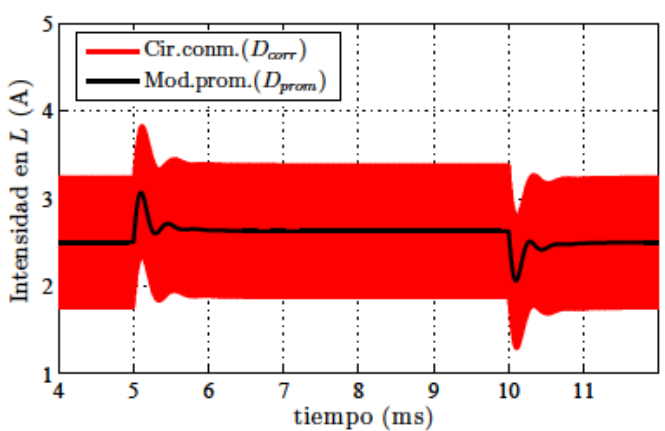

(b)

Figura B.18: Elevador ZVS. Respuesta a escalón en el ciclo de trabajo incluyendo corrección: a) Tensión de salida; y b) Intensidad en la bobina.

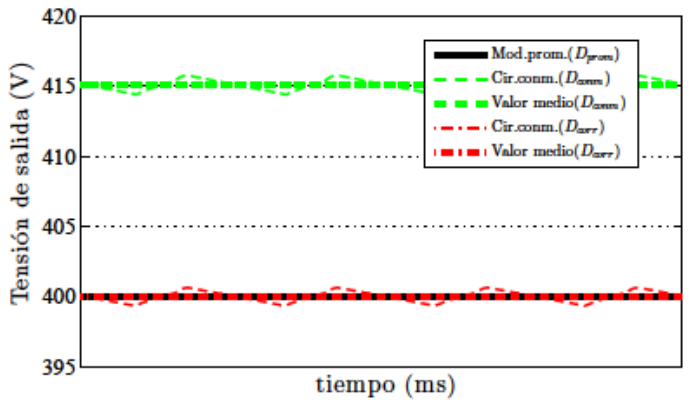

(a)

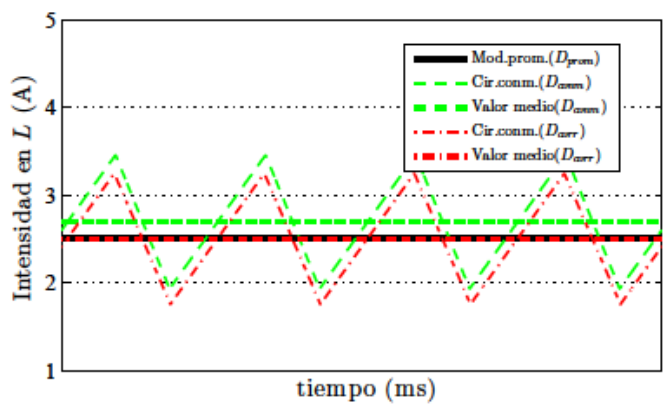

(b)

Figura B.19: Elevador ZVS en régimen permanente. Comparación entre el modelo promediado y los valores medios con y sin corrección en el ciclo de trabajo del circuito conmutado: a) Tensión de salida; y b) Intensidad en la bobina.

cero a la izquierda de la igualdad:

$$
\begin{aligned}
& 0=V_{g}-(1-D) V_{o}-(1-D) V_{c} \\
& 0=(1-D) I_{g}-\frac{V_{o}}{R}+I_{e q} \\
& 0=(1-D) I_{g}-\frac{V_{c}}{R_{e q}}
\end{aligned}
$$


donde

$$
R_{e q}=\frac{2 L_{r} f_{s}}{(1-D)^{2}} \quad I_{e q}=\frac{V_{C_{c}}^{2}}{V_{o} R_{e q}}
$$

A estas ecuaciones se puede llegar también a partir del circuito promediado de la figura B.16. Basta con sustituir los condensadores por un circuito abierto y las bobinas con un cortocircuito. De esta forma se obtienen los circuitos de las figuras B.20a y B.20b.

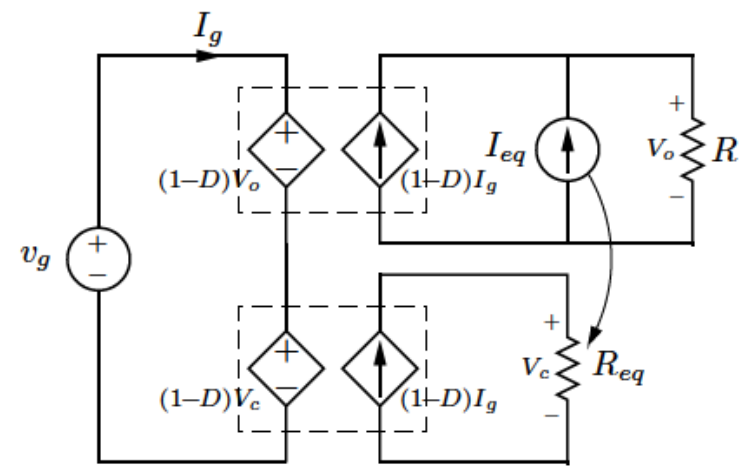

(a)

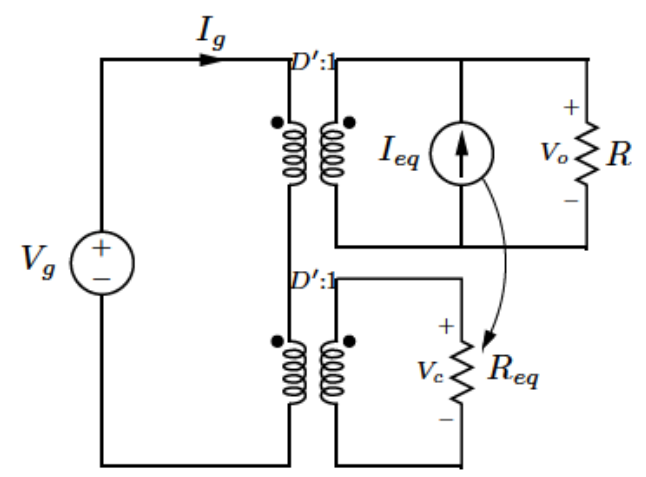

(b)

Figura B.20: Circuito en régimen permanente de un elevador ZVS en MCC: (a) con fuentes dependientes; (b) con transformador equivalente ideal.

Se obtienen las mismas expresiones para las principales magnitudes en régimen permanente cuando se deducen de (B.98) que a partir de los circuitos de la figura B.20.

$$
\begin{aligned}
V_{o} & =\frac{V_{g}}{1-D}-V_{c} \\
I_{g} & =\frac{V_{o}}{R(1-D)}-\frac{I_{e q}}{1-D} \\
V_{c} & =I_{g}(1-D) R_{e q}
\end{aligned}
$$

Si en estas expresiones se elimina $L_{r}$, (que significa que desaparece el circuito de enclavamiento activo), se obtienen las expresiones en régimen permanente para un reductor convencional y lo mismo sucede con los circuitos en régimen permanente de ambas topologías.

La expresión que relaciona la tensión de salida con la intensidad en la entrada se obtienen fácilmente a partir de (B.100) como:

$$
V_{o}=\frac{V_{g}}{1-D}-I_{g}(1-D) R_{e q}
$$

donde se observa que la tensión de salida de un elevador ZVS se puede asemejar a la de un elevador convencional que tiene a la salida una resistencia de valor $R_{e q}$ (con la particularidad de que es una resistencia equivalente sin 
pérdidas), y evoluciona con la corriente demandada por la carga (que en un elevador convencional es igual a $\left.I_{g}(1-D)\right)$.

\section{B.4.1. Balance energético}

En el modelo en régimen permanente hay dos elementos que generan energía, la fuente de tensión de la entrada, y la fuente de corriente dependiente en la etapa de salida. La energía generada en esta fuente de corriente se consume toda en $r_{e q}$. Veámoslo.

En $R_{e q}$, la potencia consumida es igual a

$$
P_{R_{e q}}=\frac{V_{C_{c}}^{2}}{R_{e q}}
$$

Multiplicando arriba y abajo por $V_{o}$, se obtiene una expresión

$$
P_{R_{e q}}=\frac{V_{C_{c}}^{2}}{R_{e q}} \frac{V_{o}}{V_{o}}
$$

en la que, agrupando, se obtiene el producto de una fuente de tensión por otra de corriente cuyo valor es igual a $I_{e q}$

$$
P_{R e q}=I_{e q} V_{o}
$$

Esta potencia coincide con la potencia generada por la fuente de corriente $I_{e q}$ en la entrada.

En $R$ la potencia consumida es igual a:

$$
\begin{aligned}
P_{R}=I_{R}^{2} R=V_{o} I_{R} & =V_{o}\left[(1-D) I_{g}+I_{e q}\right] \\
& =\left[\frac{V_{g}}{1-D}-V_{c}\right](1-D) I_{g}+V_{o} I_{e q} \\
& =V_{g} I_{g}-V_{c}(1-D) I_{g}+V_{o} I_{e q}=P_{G}
\end{aligned}
$$

que coincide, con la potencia generada en la fuente de tensión de la entrada. Esto es, la potencia que suministra la fuente de entrada se entrega a la carga en su totalidad.

\section{B.5. Modelo en pequeña señal}

El modelo en pequeña señal (modelo lineal) se consigue tras realizar el proceso de perturbación y linealización del modelo promediado (modelo no 
lineal) en torno a un punto de funcionamiento. Se asume que las entradas al modelo, $v_{g}$ y $d$ son iguales a un valor fijo, $V_{g}$ y $D$, mas una pequeña variación de alterna, $\hat{v}_{g}$ y $\hat{d}$. Es decir:

$$
\begin{aligned}
\bar{v}_{g} & =V_{g}+\hat{v}_{g} \\
d & =D+\hat{d}
\end{aligned}
$$

En respuesta a estas entradas, los valores promediados de las variables de estado sufrirán una variación similar, es decir, presentarán un término constante mas una pequeña variación de alterna. También sucede lo mismo con el resto de magnitudes estudiadas, de forma que:

$$
\begin{aligned}
\bar{i}_{L} & =I_{g}+\hat{i}_{L} \\
\bar{v}_{C_{o}} & =V_{o}+\hat{v}_{C_{o}} \\
\bar{v}_{C_{c}} & =V_{c}+\hat{v}_{C_{c}}
\end{aligned}
$$

Por supuesto, para que el modelo linealizado sea preciso debe cumplirse que las variaciones son pequeñas en comparación con el valor constante que indica el punto de operación del convertidor, es decir:

$$
\begin{aligned}
\left|\hat{v}_{g}\right| & \ll\left|V_{g}\right| \\
|\hat{d}| & \ll|D| \\
\left|\hat{i}_{L}\right| & \ll\left|I_{g}\right| \\
\left|\hat{v}_{C_{o}}\right| & \ll\left|V_{o}\right| \\
\left|\hat{v}_{C_{c}}\right| & \ll\left|V_{c}\right|
\end{aligned}
$$

Por lo que, introduciendo (B.106) y (B.107) en las ecuaciones del modelo promediado de orden reducido del elevador ZVS, reescritas de nuevo en (B.109) y desarrollando y despreciando los términos de segundo orden se consigue linealizar el modelo promediado.

$$
\begin{aligned}
L \frac{d \bar{i}_{L}}{d t} & =\bar{v}_{g}-(1-d) \bar{v}_{C_{o}}-(1-d) \bar{v}_{C_{c}} \\
C_{o} \frac{d \bar{v}_{C_{o}}}{d t} & =(1-d) \bar{i}_{L}-\frac{\bar{v}_{C_{o}}}{R}+\frac{\bar{v}_{C_{c}}^{2}}{\bar{v}_{C_{o}}} \frac{(1-d)^{2}}{2 L_{r} f_{s}} \\
C_{c} \frac{d \bar{v}_{C_{c}}}{d t} & =(1-d) \bar{i}_{L}-\frac{\bar{v}_{C_{c}}}{2 L_{r} f_{s}}(1-d)^{2}
\end{aligned}
$$

El proceso de linealización no es mas que un desarrollo de Taylor de una función, $f(x, y, z, \ldots)$ en torno a un punto determinado $\left(x_{0}, y_{0}, z_{0}, \ldots\right)$, considerando únicamente los términos hasta primer orden y despreciando el resto. Por tanto, aplicando el desarrollo de Taylor hasta primer orden a la parte derecha de cada una de las igualdades de las ecuaciones del modelo 
promediado se consigue llegar a una expresión que permite la obtención del modelo en pequeña señal y también, otra vez, el modelo en régimen permanente. Por ejemplo, la ecuación de la corriente en la bobina de entrada del modelo promediado dada en (B.109) se puede escribir como:

$$
L \frac{d \bar{i}_{L}}{d t}=f_{1}\left(\bar{v}_{g}, d, \bar{v}_{C_{o}}, \bar{v}_{C_{c}}\right)
$$

Linealizando la función $f_{1}$ con el desarrollo de Taylor en un punto de funcionamiento dado por $\left(V_{g}, D, I_{g}, V_{o}, V_{c}\right)$ se obtiene (B.111). Por supuesto, las ecuación obtenida no será una aproximación muy buena de la función no lineal cuando se trate de un punto alejado del punto de linealización.

$$
\begin{aligned}
& L\left(\frac{d I_{L}}{d t}+\frac{d \hat{i}_{L}}{d t}\right)=f_{1}\left(V_{g}, D, V_{o}, V_{c}\right) \\
& +\left.\hat{v}_{g}(t) \frac{\partial f_{1}\left(\bar{v}_{g}, D, V_{o}, V_{c}\right)}{\partial \bar{v}_{g}}\right|_{\bar{v}_{g}=V_{g}}+\left.\hat{v}_{C_{o}} \frac{\partial f_{1}\left(V_{g}, D, \bar{v}_{C_{o}}, V_{c}\right)}{\partial \bar{v}_{C_{o}}}\right|_{\bar{v}_{C_{o}}=V_{o}} \\
& +\left.\hat{v}_{C_{c}} \frac{\partial f_{1}\left(V_{g}, D, V_{o}, \bar{v}_{C_{c}}\right)}{\partial \bar{v}_{C_{c}}}\right|_{\bar{v}_{C_{c}}=V_{c}}+\left.\hat{d}(t) \frac{\partial f_{1}\left(V_{g}, d, V_{o}, V_{c}\right)}{\partial d}\right|_{d=D}
\end{aligned}
$$

+ términos de orden superior

A continuación se evalúan las derivadas parciales para la ecuación de la corriente en la bobina de entrada, $\bar{i}_{L}$. Las derivadas parciales son:

$$
\begin{gathered}
\left.\frac{\partial f_{1}\left(\bar{v}_{g}, D, V_{o}, V_{c}\right)}{\partial \bar{v}_{g}}\right|_{\bar{v}_{g}=V_{g}}=1 \\
\left.\frac{\partial f_{1}\left(V_{g}, D, \bar{v}_{C_{o}}, V_{c}\right)}{\partial \bar{v}_{C_{o}}}\right|_{\bar{v}_{C_{o}}=V_{o}}=-(1-D) \\
\left.\frac{\partial f_{1}\left(V_{g}, D, V_{o}, \bar{v}_{C_{c}}\right)}{\partial \bar{v}_{C_{c}}}\right|_{\bar{v}_{C_{c}=V_{c}}}=-(1-D) \\
\left.\frac{\partial f_{1}\left(V_{g}, d, V_{o}, V_{c}\right)}{\partial d}\right|_{d=D}=V_{o}+V_{c}
\end{gathered}
$$

La ecuación (B.111) queda ahora así:

$$
\begin{aligned}
L\left(0+\frac{d \hat{i}_{L}}{d t}\right) & =\left[V_{g}-(1-D) V_{o}-(1-D) V_{c}\right] \\
& +\hat{v}_{g}-(1-D) \hat{v}_{C_{o}}-(1-D) \hat{v}_{C_{c}}+\left(V_{o}+V_{c}\right) \hat{d} \\
& + \text { términos de orden superior }
\end{aligned}
$$


Los términos constantes de ambos miembros se igualan para conseguir una ecuación para el modelo en régimen permanente que coincide con la primera expresión de (B.98). Los términos de primer orden generan otra ecuación que forma parte del modelo en pequeña señal. El resto de términos de orden superior se desprecian.

Si se hace de forma similar para el restos de ecuaciones del modelo promediado, vistas como

$$
\begin{aligned}
C_{o} \frac{d \bar{v}_{C_{o}}}{d t} & =f_{2}\left(d, \bar{i}_{L}, \bar{v}_{C_{o}}, \bar{v}_{C_{c}}\right) \\
C_{c} \frac{d \bar{v}_{C_{c}}}{d t} & =f_{3}\left(d, \bar{i}_{L}, \bar{v}_{C_{c}}\right)
\end{aligned}
$$

será necesario evaluar las funciones $f_{2}$ y $f_{3}$ y sus derivadas parciales en torno al punto de funcionamiento elegido. Las funciones $f_{2}$ y $f_{3}$ evaluadas en el punto de funcionamiento son:

$$
\begin{aligned}
f_{2}\left(D, I_{g}, V_{o}, V_{c}\right) & =(1-D) I_{g}-\frac{V_{o}}{R}+I_{e q} \\
f_{3}\left(D, I_{L}, V_{c}\right) & =(1-D) I_{g}-\frac{V_{c}}{R_{e q}}
\end{aligned}
$$

Las derivadas parciales respecto a las variables de estado de las funciones anteriores, y evaluadas en torno al punto de funcionamiento están indicadas a continuación.

$$
\begin{gathered}
\left.\frac{\partial f_{2}(\ldots)}{\partial \bar{i}_{L}}\right|_{\bar{i}_{L}=I_{L}}=(1-D) \\
\left.\frac{\partial f_{2}(\ldots)}{\partial \bar{v}_{C_{o}}}\right|_{\bar{v}_{C_{o}=V_{o}}}=-\frac{1}{R}-\frac{I_{e q}}{V_{o}} \\
\left.\frac{\partial f_{2}(\ldots)}{\partial \bar{v}_{C_{c}}}\right|_{\bar{v}_{C_{c}=V_{c}}}=2 \frac{I_{e q}}{V_{c}} \\
\left.\frac{\partial f_{2}(\ldots)}{\partial d}\right|_{d=D}=-I_{g}+\frac{V_{C_{c}}^{2}}{V_{o}} \frac{2(1-D)}{2 L_{r} f_{s}}(-1)=-I_{g}-\frac{2}{1-D} I_{e q} \\
\left.\frac{\partial f_{3}(\ldots)}{\partial d}\right|_{d=D}=-I_{g}+2 \frac{V_{c}(1-D)}{2 L_{r} f_{s}}=I_{g} \\
\left.\frac{\partial f_{3}(\ldots)}{\partial \bar{i}_{L}}\right|_{\bar{i}_{L}=I_{L}}=(1-D) \\
\left.\frac{\partial f_{3}(\ldots)}{\partial \bar{v}_{C_{c}}}\right|_{\bar{v}_{C_{c}}=V_{c}}=-\frac{(1-D)^{2}}{2 L_{r} f_{s}}=-\frac{1}{R_{e q}}
\end{gathered}
$$


Entonces, cada una de las ecuaciones (B.114) quedan de la forma indicada en (B.118) y (B.119).

$$
\begin{aligned}
C_{o}\left(0+\frac{d \hat{v}_{C_{o}}}{d t}\right) & =\left[(1-D) I_{g}-\frac{V_{o}}{R}+I_{e q}\right] \\
& +(1-D) \hat{i}_{L}-\left[\frac{1}{R}+\frac{I_{e q}}{V_{o}}\right] \hat{v}_{C_{o}} \\
& +2 \frac{I_{e q}}{V_{c}} \hat{v}_{C_{c}}-\left[I_{g}+\frac{2}{1-D} I_{e q}\right] \hat{d} \\
& + \text { terminos de orden superior } \\
C_{c}\left(0+\frac{d \hat{v}_{C_{c}}}{d t}\right) & =\left[(1-D) I_{g}-\frac{V_{c}}{R_{e q}}\right] \\
& +I_{g} \hat{d}+(1-D) \hat{i}_{L}-\frac{1}{R_{e q}} \hat{v}_{C_{c}} \\
& + \text { términos de orden superior }
\end{aligned}
$$

Agrupando todos los términos constantes de las ecuaciones (B.113), (B.118) y (B.119) se obtiene el modelo en régimen permanente que coincide con el descrito en (B.100).

Tomando todos los términos de primer orden y despreciando los términos de orden superior, se consigue el modelo en pequeña señal para el elevador ZVS dado en (B.120).

$$
\begin{aligned}
L \frac{\hat{i}_{L}}{d t} & =\hat{v}_{g}-(1-D) \hat{v}_{C_{o}}-(1-D) \hat{v}_{C_{c}}+\left(V_{o}+V_{c}\right) \hat{d} \\
C_{o} \frac{d \hat{v}_{C_{o}}}{d t} & =(1-D) \hat{i}_{L}-\left[\frac{1}{R}+\frac{I_{e q}}{V_{o}}\right] \hat{v}_{C_{o}}+2 \frac{I_{e q}}{V_{c}} \hat{v}_{C_{c}}-\left[I_{g}+\frac{2}{1-D} I_{e q}\right] \hat{d} \\
C_{c} \frac{d \hat{v}_{C_{c}}}{d t} & =I_{g} \hat{d}+(1-D) \hat{i}_{L}-\frac{1}{R_{e q}} \hat{v}_{C_{c}}
\end{aligned}
$$

Con estas ecuaciones se construye el circuito en pequeña señal de la figura B.21. Los parámetros $j_{2}, r_{2}, g_{2}$ y $f_{2}$ están indicados en la tabla B.4.

\begin{tabular}{ccccc}
\hline Convertidor & $j_{2}$ & $r_{2}$ & $g_{2}$ & $f_{2}$ \\
\hline Elevador & $I_{g}+\frac{2 I_{e q}}{1-D}$ & $\frac{V_{o}}{I_{e q}}$ & $2 \frac{I_{e q}}{V_{c}}$ & $V_{c}+V_{o}$ \\
\hline
\end{tabular}

Tabla B.4: Parámetros del modelo en pequeña señal del reductor ZVS.

El modelo en pequeña señal, obtenido a partir de la linealización del modelo promediado de orden reducido, también se puede escribir en forma matricial. Así, se definen dos vectores, el vector $\hat{\mathbf{x}}$ para las variables de estado 


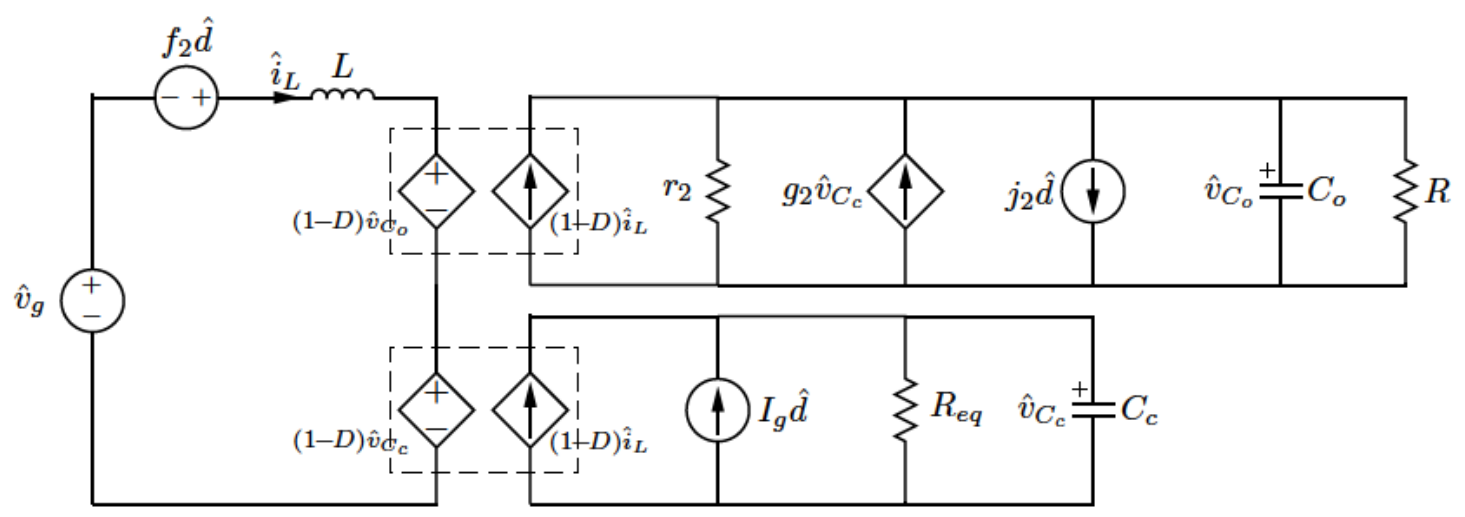

Figura B.21: Modelo en pequeña señal de un elevador ZVS.

y el vector $\hat{\mathbf{u}}$ para las entradas externas e independientes, y serán en este caso:

$$
\hat{\mathbf{x}}=\left[\begin{array}{c}
\hat{i}_{L} \\
\hat{v}_{C_{o}} \\
\hat{v}_{C_{c}}
\end{array}\right], \quad \hat{\mathbf{u}}=\left[\begin{array}{c}
\hat{v}_{g} \\
\hat{d}
\end{array}\right]
$$

de forma que el modelo en pequeña señal de (B.120) se puede escribir, de la forma siguiente:

$$
\mathbf{K} \frac{d}{d t}\left[\begin{array}{c}
\hat{i}_{L} \\
\hat{v}_{C_{o}} \\
\hat{v}_{C_{c}}
\end{array}\right]=\mathbf{A}\left[\begin{array}{c}
\hat{i}_{L} \\
\hat{v}_{C_{o}} \\
\hat{v}_{C_{c}}
\end{array}\right]+\mathbf{B}\left[\begin{array}{c}
\hat{v}_{g} \\
\hat{d}
\end{array}\right]
$$

donde

$$
\begin{aligned}
\mathbf{K} & =\left[\begin{array}{ccc}
L & 0 & 0 \\
0 & C_{o} & 0 \\
0 & 0 & C_{c}
\end{array}\right], \quad \mathbf{A}=\left[\begin{array}{ccc}
0 & D-1 & D-1 \\
1-D & -\frac{1}{R}-\frac{1}{r_{2}} & g_{2} \\
1-D & 0 & \frac{1}{R_{e q}}
\end{array}\right], \\
\mathbf{B} & =\left[\begin{array}{ll}
1 & f_{2} \\
0 & j_{2} \\
0 & I_{g}
\end{array}\right]
\end{aligned}
$$

\section{B.6. Funciones de transferencia}

Las diferentes funciones de transferencia se pueden obtener bien a partir del modelo en pequeña señal dado en las ecuaciones (B.120), bien a partir del circuito en pequeña señal de la figura B.21. Por ambos métodos hay que anular la variable de estado correspondiente y aplicar Laplace para obtener la relación deseada. Otra posibilidad es trabajar con el modelo matricial en pequeña señal, que se verá mas adelante. 


\section{B.6.1. Desde el modelo o el circuito de pequeña señal}

Para conseguir $G_{v g}(s)$, se hace nula la variación de la entrada independiente que no interviene en la función de transferencia, en este caso, $\hat{d}(t)=0$, y se aplica Laplace. De esta forma queda que:

$$
\begin{aligned}
s L \hat{i}_{L}(s) & =\hat{v}_{g}(s)-(1-D) \hat{v}_{C_{o}}(s)-(1-D) \hat{v}_{C_{c}}(s) \\
s C_{o} \hat{v}_{C_{o}}(s) & =(1-D) \hat{i}_{L}(s)-\left[\frac{1}{R}+\frac{1}{r_{2}}\right] \hat{v}_{C_{o}}(s)+g_{2} \hat{v}_{C_{c}} \\
s C_{c} \hat{v}_{C_{c}}(s) & =(1-D) \hat{i}_{L}(s)-\frac{1}{R_{e q}} \hat{v}_{C_{c}}(s)
\end{aligned}
$$

Restando la tercera ecuación a la segunda se consigue despejar $\hat{v}_{C_{c}}(s)$. Posteriormente se sustituye de nuevo en la tercera ecuación para obtener $\hat{i}_{L}(s)$. Ambas variables en función de $\hat{v}_{C_{o}}(s)$.

$$
\begin{aligned}
\hat{v}_{C_{c}}(s) & =\frac{\frac{1}{R}+s C_{o}+\frac{1}{r_{2}}}{\frac{1}{R_{e q}}+s C_{c}+g_{2}} \hat{v}_{C_{o}}(s)=\frac{Z_{e q}(s)}{Z_{o}(s)\left[1+g_{2} Z_{e q}(s)\right]} \hat{v}_{C_{o}}(s) \\
\hat{i}_{L}(s) & =\frac{1}{1-D}\left[s C_{c}+\frac{1}{R_{e q}}\right] \hat{v}_{C_{c}}(s)=\frac{1}{(1-D) Z_{o}(s)\left[1+g_{2} Z_{e q}(s)\right]} \hat{v}_{C_{o}}(s)
\end{aligned}
$$

en donde se ha definido

$$
\begin{aligned}
& Z_{o}(s)=\left(R\left\|C_{o}\right\| r_{2}\right)=\left[s C_{o}+\frac{1}{R}+\frac{1}{r_{2}}\right]^{-1} \\
& Z_{e q}(s)=\left(R_{e q} \| C_{c}\right)=\left[s C_{c}+\frac{1}{R_{e q}}\right]^{-1}
\end{aligned}
$$

$\mathrm{Al}$ introducir (B.125) en (B.124) se obtiene fácilmente la función de transferencia deseada.

$$
\begin{aligned}
& G_{v g}(s)=\left.\frac{\hat{v}_{C_{o}}(s)}{\hat{v}_{g}(s)}\right|_{\hat{d}=0}(1-D)\left[1+g_{2} Z_{e q}(s)\right] \\
& =\frac{(1-D)^{2} Z_{e q}(s) / Z_{o}(s)}{L s / Z_{o}(s)+(1-D)^{2}\left[1+g_{2} Z_{e q}(s)\right]+(1-}
\end{aligned}
$$

Para conseguir $G_{i g}(s)$, se despejan $\hat{v}_{C_{c}}(s)$ y $\hat{v}_{C_{o}}(s)$ en función de $\hat{i}_{L}(s)$ de la segunda y tercera ecuación, respectivamente, de (B.124).

$$
\begin{aligned}
& \hat{v}_{C_{c}}(s)=(1-D) \hat{i}_{L}(s) \frac{1}{s C_{c}+\frac{1}{R_{e q}}}=(1-D) Z_{e q}(s) \hat{i}_{L}(s) \\
& \hat{v}_{C_{o}}(s)=(1-D) Z_{o}(s)\left[1+g_{2} Z_{e q}(s)\right] \hat{i}_{L}(s)
\end{aligned}
$$


Introduciendo (B.128) en la primera ecuación se obtiene la función de transferencia deseada.

$$
\begin{aligned}
& G_{i g}(s)=\left.\frac{\hat{i}_{L}(s)}{\hat{v}_{g}(s)}\right|_{\hat{d}=0} \\
& =\frac{1 / Z_{o}(s)}{L s / Z_{o}(s)+(1-D)^{2}\left[1+g_{2} Z_{e q}(s)\right]+(1-D)^{2} Z_{e q}(s) / Z_{o}(s)}
\end{aligned}
$$

La mismas expresiones se obtienen si se utiliza el circuito equivalente en pequeña señal del elevador de la figura B.21. Inicialmente se anula la o las fuentes correspondientes a la entrada independiente que no entra en la función de transferencia y se hace Laplace. El circuito resultante para el cálculo de $G_{v g}(s)$ y $G_{i g}(s)$ está representado en la figura B.22 una vez anulado $\hat{d}$.

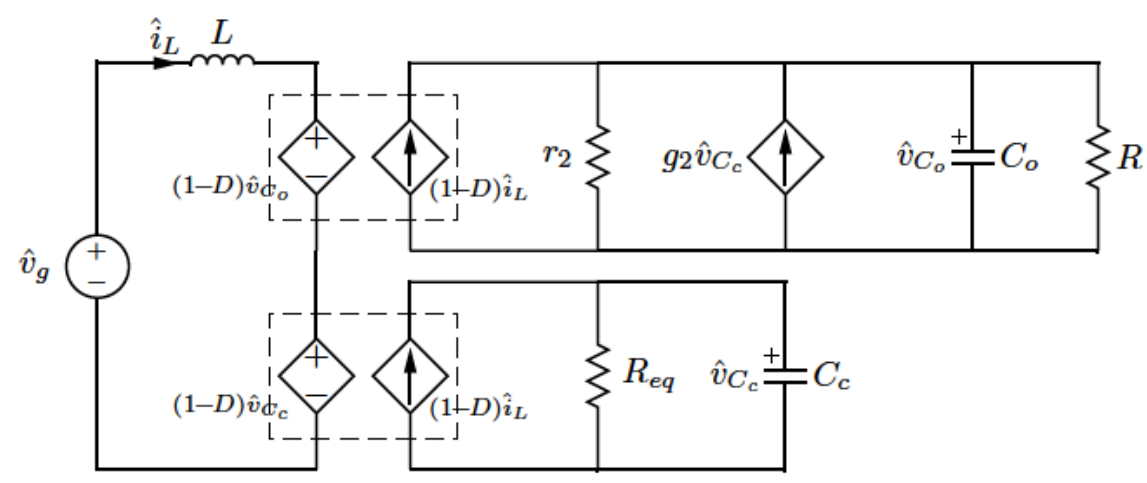

Figura B.22: Circuito equivalente de un elevador ZVS cuando $\hat{d}=0$

De la malla inferior derecha se deduce que

$$
\hat{v}_{C_{c}}(s)=(1-D) \hat{i}_{L}(s) Z_{e q}(s)
$$

Agrupando las impedancias de cada malla y pasando $Z_{e q}(s)$ al primario del transformador, queda un circuito como el indicado en la figura B.23. En la malla de la derecha de este circuito se cumple que

$$
\hat{v}_{C_{o}}(s)=\left[(1-D) \hat{i}_{L}(s)+g_{2}(1-D) \hat{i}_{L}(s) Z_{e q}(s)\right] Z_{o}(s)
$$

y en la malla de la izquierda se sabe que

$$
\hat{v}_{g}(s)=L s \hat{i}_{L}(s)+(1-D) \hat{v}_{C_{o}}(s)+(1-D)^{2} Z_{e q}(s) i_{L}(s)
$$

Despejando $\hat{i}_{L}(s)$ de (B.132)

$$
\hat{i}_{L}(s)=\frac{\hat{v}_{g}(s)-(1-D) \hat{v}_{C_{o}}(s)}{L s+(1-D)^{2} Z_{e q}(s)}
$$




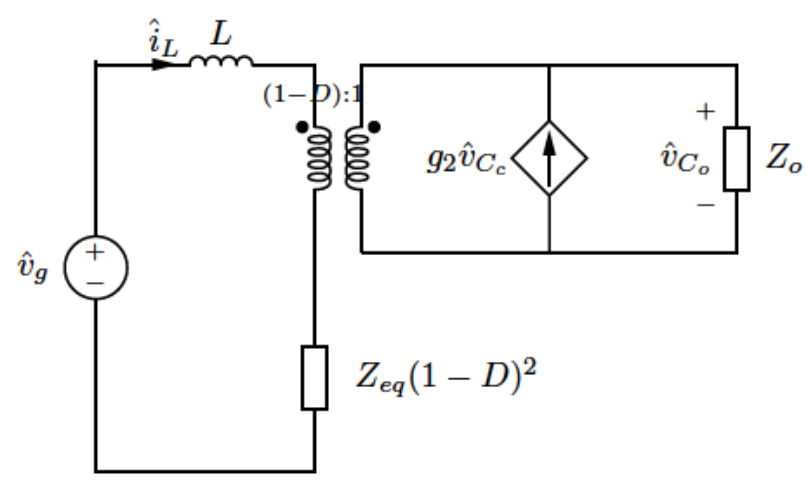

Figura B.23: Circuito resultante al simplificar la malla inferior derecha.

e introduciéndola en (B.131),

$$
\begin{aligned}
\hat{v}_{C_{o}}(s) & =(1-D) Z_{o}(s)\left[1+g_{2} Z_{e q}(s)\right] \hat{i}_{L}(s) \\
& =(1-D) Z_{o}(s)\left[1+g_{2} Z_{e q}(s)\right] \frac{\hat{v}_{g}(s)-(1-D) \hat{v}_{C_{o}}(s)}{L s+(1-D)^{2} Z_{e q}(s)}
\end{aligned}
$$

se puede obtener la expresión de $G_{v g}(s)$ idéntica a (B.127).

Cuando todas las impedancias de cada malla se pasan al primario del transformador respectivo el circuito que se obtiene está representado en la figura B.24.

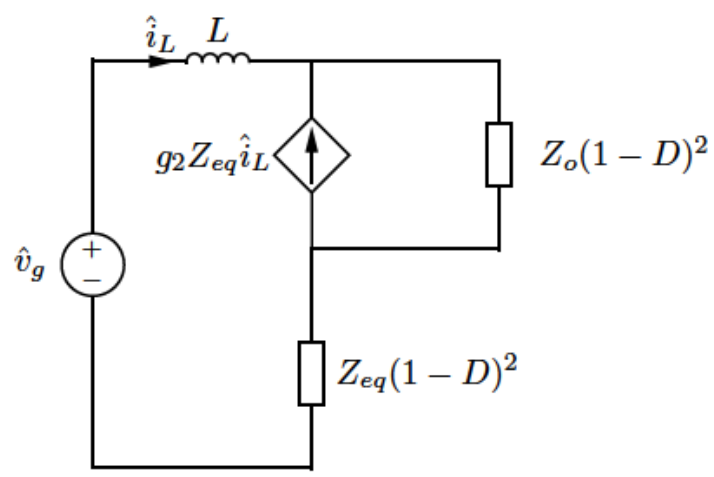

Figura B.24: Circuito resultante al pasar todo a la izquierda.

De la malla principal se obtiene la siguiente ecuación:

$$
\hat{v}_{g}(s)=\left[L s+(1-D)^{2} Z_{e q}(s)\right] \hat{i}_{L}(s)+\left[\hat{i}_{L}(s) g_{2} Z_{e q}(s)+\hat{i}_{L}(s)\right](1-D)^{2} Z_{o}(s)
$$

Es muy sencillo obtener la expresión para $G_{i g}(s)$, que es idéntica a (B.129).

Las dos funciones de transferencia coinciden con las de un elevador convencional cuando los términos relacionados con el enclavamiento activo, $C_{c}$ y $L_{r}$, desaparecen puesto que $V_{c}, R_{e q}$ y $Z_{e} q$ se anulan. 
En la Figura B.25 están representadas ambas funciones de transferencia con los parámetros de funcionamiento de la Tabla B.1, comparadas con las que tendría un elevador convencional con los mismos parámetros.

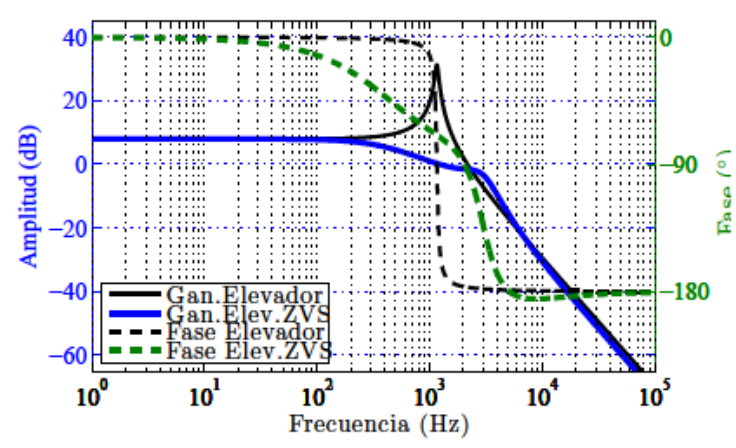

(a)

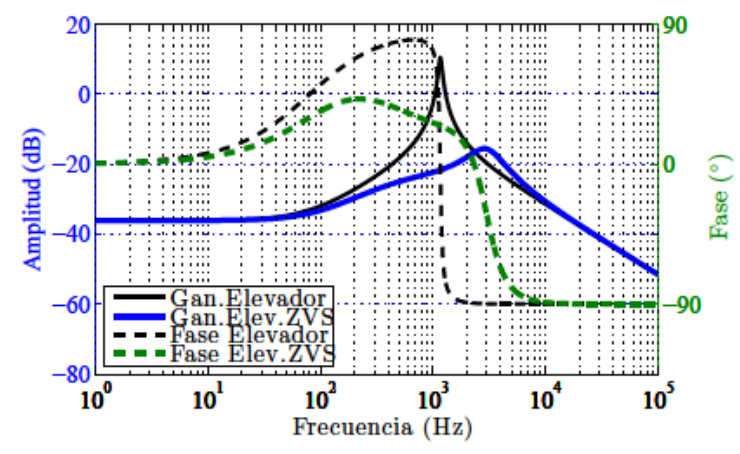

(b)

Figura B.25: Elevador ZVS. Func. de transferencia en función de la tensión de entrada para: a) Tensión de salida, $G_{v g}(s)$; y b) Intensidad en la bobina, $G_{i g}(s)$.

Igualmente, para conseguir $G_{v d}(s)$, se hace nula la variación de la entrada independiente que no interviene en la función de transferencia, en este caso, $\hat{v}_{g}(t)=0$, y se aplica Laplace. De esta forma queda que:

$$
\begin{aligned}
s L \hat{i}_{L}(s) & =-(1-D) \hat{v}_{C_{o}}(s)-(1-D) \hat{v}_{C_{c}}(s)+f_{2} \hat{d} \\
s C_{o} \hat{v}_{C_{o}}(s) & =(1-D) \hat{i}_{L}(s)-\left[\frac{1}{R}+\frac{1}{r_{2}}\right] \hat{v}_{C_{o}}(s)+g_{2} \hat{v}_{C_{c}}-j_{2} \hat{d} \\
s C_{c} \hat{v}_{C_{c}}(s) & =I_{g} \hat{d}+(1-D) \hat{i}_{L}(s)-\frac{1}{R_{e q}} \hat{v}_{C_{c}}(s)
\end{aligned}
$$

Primero se obtiene $\hat{i}_{L}(s)$ de la tercera ecuación como:

$$
\hat{i}_{L}(s)=\frac{1}{(1-D) Z_{e q}(s)} \hat{v}_{C_{c}}(s)-\frac{I_{g}}{1-D} \hat{d}(s)
$$

Después se despeja $\hat{v}_{C_{c}}(s)$ de la segunda ecuación como:

$$
\hat{v}_{C_{c}}(s)=\frac{Z_{e q}(s)}{Z_{o}(s)\left[1+g_{2} Z_{e q}(s)\right]} \hat{v}_{C_{o}}(s)+\frac{Z_{e q}(s)\left(I_{g}+j_{2}\right)}{1+g_{2} Z_{e q}(s)} \hat{d}(s)
$$

Ahora la primera ecuación, al utilizar (B.137), queda así:

$$
\frac{L s+(1-D)^{2} Z_{e q}(s)}{Z_{e q}(s)} \hat{v}_{C_{c}}(s)=-(1-D)^{2} \hat{v}_{C_{o}}(s)+\left[(1-D) f_{2}+I_{g} L s\right] \hat{d}(s)
$$


Y despues de utilizar (B.138) se obtiene la siguiente igualdad:

$$
\begin{gathered}
\frac{L s+(1-D)^{2} Z_{e q}(s)}{Z_{o}(s)\left[1+g_{2} Z_{e q}(s)\right]} \hat{v}_{C_{o}}(s)+\frac{\left(I_{g}+j_{2}\right)\left[L s+(1-D)^{2} Z_{e q}(s)\right]}{1+g_{2} Z_{e q}(s)} \hat{d}(s) \\
=-(1-D)^{2} \hat{v}_{C_{o}}(s)+\left[(1-D) f_{2}+I_{g} L s\right] \hat{d}(s)
\end{gathered}
$$

De otra forma, agrupando los términos similares:

$$
\begin{array}{r}
\frac{L s+(1-D)^{2} Z_{o}(s)\left[1+g_{2} Z_{e q}(s)\right]+(1-D)^{2} Z_{e q}(s)}{Z_{o}(s)\left[1+g_{2} Z_{e q}(s)\right]} \hat{v}_{C_{o}}(s) \\
=\frac{\left[(1-D) f_{2}+I_{g} L s\right]\left[1+g_{2} Z_{e q}(s)\right]-\left(I_{g}+j_{2}\right)\left[L s+(1-D)^{2} Z_{e q}(s)\right]}{1+g_{2} Z_{e q}(s)} \hat{d}(s)
\end{array}
$$

De esta ecuación se obtiene fácilmente la función de transferencia deseada.

$$
\begin{aligned}
& G_{v d}(s)=\left.\frac{\hat{v}_{C_{o}}(s)}{\hat{d}(s)}\right|_{\hat{v}_{g}=0} \\
& =\frac{\left[(1-D) f_{2}+I_{g} L s\right]\left[1+g_{2} Z_{e q}(s)\right]-\left(I_{g}+j_{2}\right)\left[L s+(1-D)^{2} Z_{e q}(s)\right]}{L s / Z_{o}(s)+(1-D)^{2}\left[1+g_{2} Z_{e q}(s)\right]+(1-D)^{2} Z_{e q}(s) / Z_{o}(s)} \\
& =\frac{(1-D) f_{2}\left[1+g_{2} Z_{e q}(s)\right]-(1-D)^{2} Z_{e q}(s)\left(I_{g}+j_{2}\right)-L s\left[j_{2}-I_{g} g_{2} Z_{e q}(s)\right]}{L s / Z_{o}(s)+(1-D)^{2}\left[1+g_{2} Z_{e q}(s)\right]+(1-D)^{2} Z_{e q}(s) / Z_{o}(s)}
\end{aligned}
$$

Para conseguir $G_{i d}(s)$, en la tercera ecuación de (B.136) se despeja $\hat{v}_{C_{c}}(s)$ en función de $\hat{i}_{L}(s)$ y $\hat{d}(s)$ :

$$
\hat{v}_{C_{c}}(s)=(1-D) Z_{e q}(s) \hat{i}_{L}(s)+I_{g} Z_{e q}(s) \hat{d}(s)
$$

Ahora, de la primera ecuación se despeja $\hat{v}_{C_{o}}(s)$ como:

$$
\begin{aligned}
\hat{v}_{C_{o}}(s) & =-\hat{v}_{C_{c}}(s)-\frac{L s}{1-D} \hat{i}_{L}(s)+\frac{f_{2}}{1-D} \hat{d}(s) \\
& =-\frac{(1-D)^{2} Z_{e q}(s)+L s}{1-D} \hat{i}_{L}(s)+\frac{f_{2}-(1-D) I_{g} Z_{e q}(s)}{1-D} \hat{d}(s)
\end{aligned}
$$

Introduciendo (B.143) y (B.144) en la segunda ecuación se obtiene la siguiente igualdad:

$$
\begin{aligned}
& -\frac{(1-D)^{2} Z_{e q}(s)+L s}{(1-D) Z_{o}(s)} \hat{i}_{L}(s)+\frac{f_{2}-(1-D) I_{g} Z_{e q}(s)}{(1-D) Z_{o}(s)} \hat{d}(s) \\
= & (1-D)\left[1+g_{2} Z_{e q}(s)\right] \hat{i}_{L}(s)+\left(I_{g} g_{2} Z_{e q}(s)-j_{2}\right) \hat{d}(s)
\end{aligned}
$$

Entonces, agrupando los términos similares, queda que:

$$
\begin{aligned}
& \frac{f_{2}-(1-D) I_{g} Z_{e q}(s)+(1-D) Z_{o}(s)\left[j_{2}-I_{g} g_{2} Z_{e q}(s)\right]}{Z_{o}(s)} \hat{d}(s) \\
& =\frac{L s+(1-D)^{2} Z_{o}(s)\left[1+g_{2} Z_{e q}(s)\right]+(1-D)^{2} Z_{e q}(s)}{Z_{o}(s)} \hat{i}_{L}(s)
\end{aligned}
$$


Y la función de transferencia deseada tiene la siguiente expresión:

$$
\begin{aligned}
G_{i d}(s) & =\left.\frac{\hat{i}_{L}(s)}{\hat{d}(s)}\right|_{\hat{v}_{g}=0} \\
& =\frac{1}{Z_{o}(s)} \frac{f_{2}-(1-D) I_{g} Z_{e q}(s)+(1-D) Z_{o}(s)\left[j_{2}-I_{g} g_{2} Z_{e q}(s)\right]}{L s / Z_{o}(s)+(1-D)^{2}\left[1+g_{2} Z_{e q}(s)\right]+(1-D)^{2} Z_{e q}(s) / Z_{o}(s)}
\end{aligned}
$$

Las mismas expresiones se obtienen si se utiliza el circuito equivalente en pequeña señal del elevador de la figura B.21. El circuito resultante para el cálculo de $G_{v d}(s)$ y $G_{i d}(s)$ está representado en la figura B.26 una vez anulado $\hat{v}_{g}$. De la malla inferior derecha se deduce que

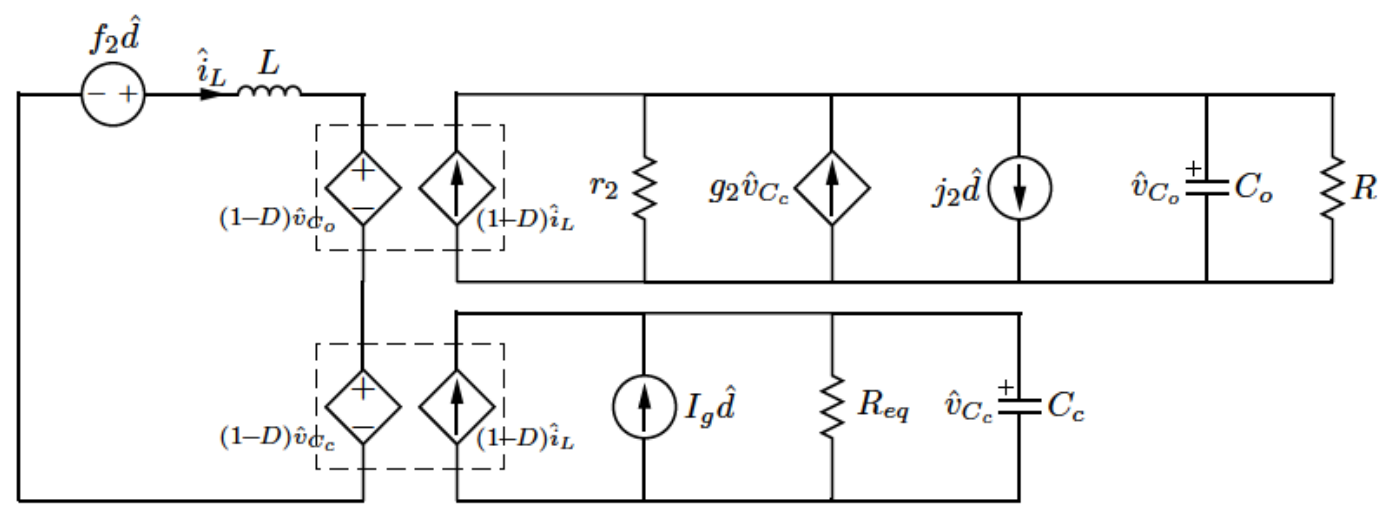

Figura B.26: Circuito equivalente de un elevador ZVS cuando $\hat{v}_{g}=0$

$$
\hat{v}_{C_{c}}(s)=(1-D) \hat{i}_{L}(s) Z_{e q}(s)+I_{g} \hat{d}(s) Z_{e q}(s)
$$

Agrupando las impedancias de cada malla y pasando $Z_{e q}(s)$ y la fuente independiente al primario del transformador, queda un circuito como el indicado en la figura B.27. En la malla de la derecha de este circuito se cumple que

$\hat{v}_{C_{o}}(s)=\left[(1-D) \hat{i}_{L}(s)+g_{2}(1-D) \hat{i}_{L}(s) Z_{e q}(s)+g_{2} I_{g} Z_{e q}(s) \hat{d}(s)-j_{2} \hat{d}(s)\right] Z_{o}(s)$

y en la malla de la izquierda se sabe que

$f_{2} \hat{d}(s)=L s \hat{i}_{L}(s)+(1-D) \hat{v}_{C_{o}}(s)+(1-D)^{2} Z_{e q}(s) i_{L}(s)+I_{g}(1-D) Z_{e q}(s) \hat{d}(s)$

Despejando $\hat{i}_{L}(s)$ de (B.150)

$$
\hat{i}_{L}(s)=\frac{\left[f_{2}-(1-D) I_{g} Z_{e q}(s)\right] \hat{d}(s)-(1-D) \hat{v}_{C_{o}}(s)}{L s+(1-D)^{2} Z_{e q}(s)}
$$




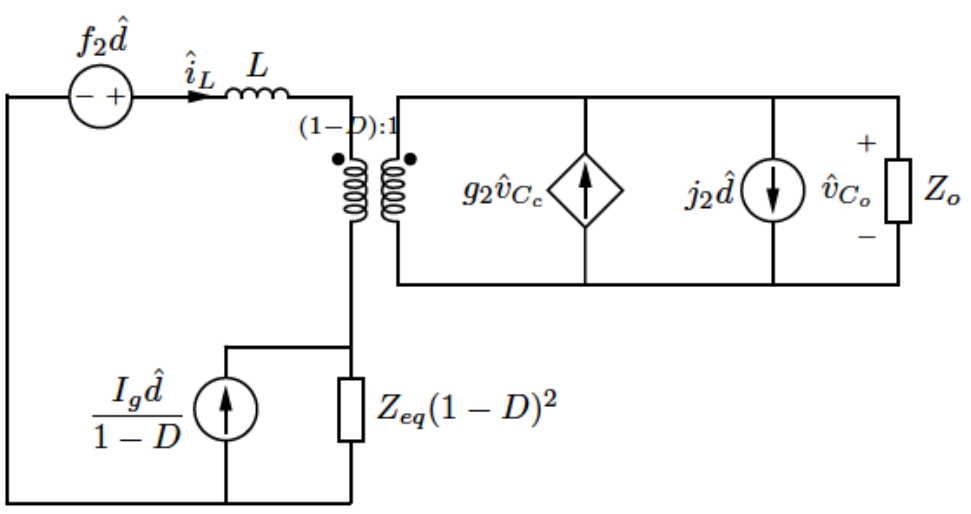

Figura B.27: Circuito resultante al simplificar la malla inferior derecha.

e introduciéndola en (B.149),

$$
\begin{aligned}
\frac{\hat{v}_{C_{o}}(s)}{Z_{o}(s)} & =(1-D)\left[1+g_{2} Z_{e q}(s)\right] \hat{i}_{L}(s)+\left[I_{g} g_{2} Z_{e q}(s)-j 2\right] \hat{d}(s) \\
& =(1-D)\left[1+g_{2} Z_{e q}(s)\right] \frac{\left[f_{2}-(1-D) I_{g} Z_{e q}(s)\right] \hat{d}(s)-(1-D) \hat{v}_{C_{o}}(s)}{L s+(1-D)^{2} Z_{e q}(s)} \\
& +\left[I_{g} g_{2} Z_{e q}(s)-j 2\right] \hat{d}(s)
\end{aligned}
$$

permite obtener la expresión de $G_{v d}(s)$. Para ello se expande la fracción a la derecha de la igualdad y se agrupan los términos similares en cada miembro.

$$
\begin{aligned}
& \hat{v}_{C_{o}}(s)\left\{L s / Z_{o}(s)+(1-D)^{2} Z_{e q}(s) / Z_{o}(s)+(1-D)^{2}\left[1+g_{2} Z_{e q}(s)\right]\right\} \\
= & \hat{d}(s)\left\{(1-D)\left[1+g_{2} Z_{e q}(s)\right]\left[f_{2}-(1-D) I_{g} Z_{e q}(s)\right]+\left[L s+(1-D)^{2} Z_{e q}(s)\right]\left[I_{g} g_{2} Z_{e q}(s)-j_{2}\right]\right\}
\end{aligned}
$$

Es sencillo comprobar que, expandiendo la parte derecha de(B.153), despejando $G_{v d}(s)$, se obtiene de nuevo (B.142).

$\mathrm{Si}$ ahora se pasan todas las impedancias y las fuentes de corriente, a la malla derecha, se obtiene el circuito de la figura B.28, de donde se puede operar para obtener de nuevo (B.147).

Las dos funciones de transferencia coinciden con las de un elevador convencional cuando los términos relacionados con el enclavamiento activo, $C_{c}$ y $L_{r}$, desaparecen puesto que $V_{c}, R_{e q}$ y $Z_{e q}$ se anulan. En la Figura B.29 están representadas ambas funciones de transferencia con los parámetros de funcionamiento de la Tabla B.1, comparadas con las que tendría un elevador convencional con los mismos parámetros. 


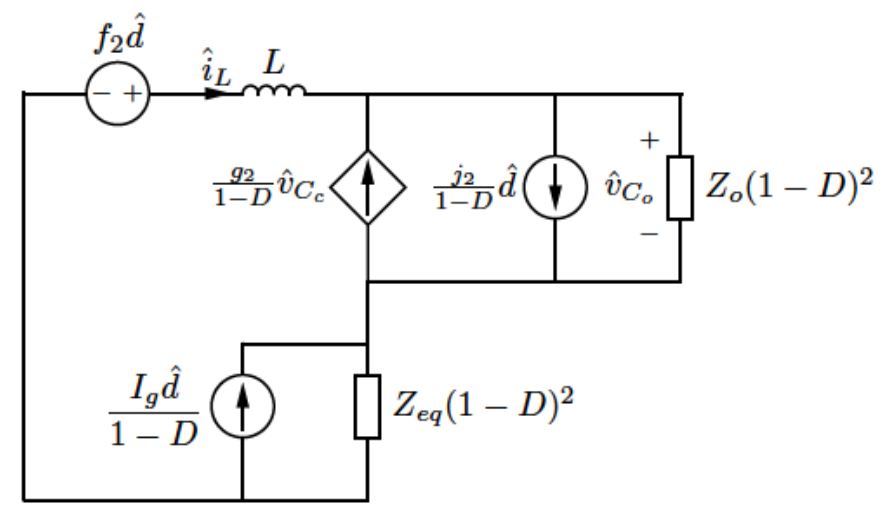

Figura B.28: Circuito resultante al pasar todo al lado derecho.

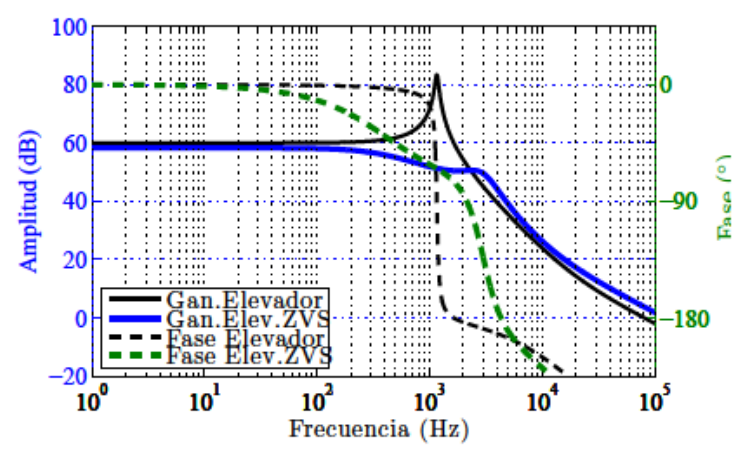

(a)

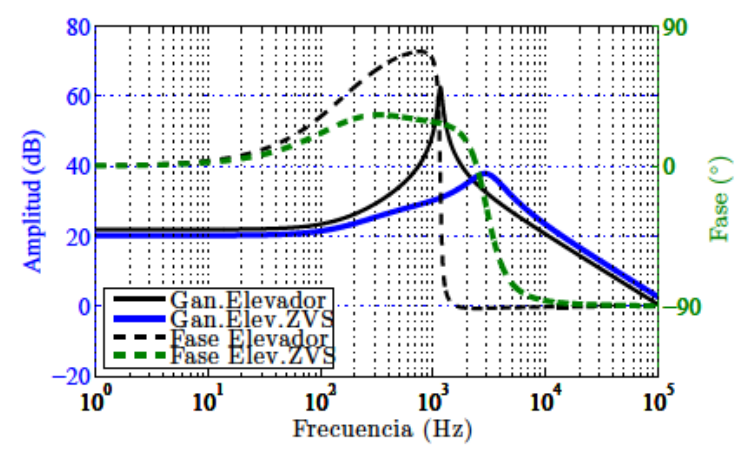

(b)

Figura B.29: Elevador ZVS. Func. de transferencia en función del ciclo de trabajo para: a) Tensión de salida, $G_{v d}(s)$; y b) Intensidad en la bobina, $G_{i g}(s)$.

\section{B.6.2. Desde el modelo matricial}

Para obtener las funciones de transferencia anteriores ${ }^{2}$, se aplica Laplace a las ecuaciones de estado en forma matricial (suponiendo que las condiciones iniciales con nulas).

$$
\begin{aligned}
\mathbf{K} s \hat{\mathbf{X}}(s) & =\mathbf{A} \hat{\mathbf{X}}(s)+\mathbf{B} \hat{\mathbf{U}}(s) \\
\hat{\mathbf{Y}}(s) & =\mathbf{C} \hat{\mathbf{X}}(s)+\mathbf{E} \hat{\mathbf{U}}(s)
\end{aligned}
$$

En la primera ecuación de (B.154) se despeja el vector de las variables de estado. Por tanto, se tiene que

$$
\mathbf{K} s \hat{\mathbf{X}}(s)-\mathbf{A} \hat{\mathbf{X}}(s)=\mathbf{B} \hat{\mathbf{U}}(s)
$$

\footnotetext{
${ }^{2}$ Ver (Ogata, 2003)
} 
o bien

$$
(\mathbf{K} s-\mathbf{A}) \hat{\mathbf{X}}(s)=\mathbf{B} \hat{\mathbf{U}}(s)
$$

Premultiplicando ambos miembros por $(\mathbf{K} s-\mathbf{A})^{-1}$ se obtiene:

$$
\hat{\mathbf{X}}(s)=(\mathbf{K} s-\mathbf{A})^{-1} \mathbf{B} \hat{\mathbf{U}}(s)
$$

Sustituyendo la ecuacion (B.155) en la segunda ecuación de (B.154) se llega a

$$
\hat{\mathbf{Y}}(s)=\left[\mathbf{C}(\mathbf{K} s-\mathbf{A})^{-1} \mathbf{B}+\mathbf{E}\right] \hat{\mathbf{U}}(s)
$$

Puesto, que la relación entre la entrada y la salida de un sistema es la función de transferencia, resulta que la matriz $\mathbf{G}(s)$ definida en (B.157) es la denominada matriz de transferencia que relaciona las diferentes salidas del sistema con las diferentes entradas. Tiene tantas filas como salidas y tantas columnas como entradas.

$$
\mathbf{G}(s)=\mathbf{C}(\mathbf{K} s-\mathbf{A})^{-1} \mathbf{B}+\mathbf{E}
$$

En el caso del elevador ZVS, se redefine el vector de salidas y las matrices C y $\mathbf{E}$, de la siguiente forma:

$$
\hat{\mathbf{y}}=\left[\begin{array}{l}
\hat{i}_{L} \\
\hat{v}_{o}
\end{array}\right], \quad \mathbf{C}=\left[\begin{array}{lll}
1 & 0 & 0 \\
0 & 1 & 0
\end{array}\right], \quad \mathbf{E}=\left[\begin{array}{ll}
0 & 0 \\
0 & 0
\end{array}\right]
$$

De esta forma, la matriz de transferencia, queda como:

$$
\mathbf{G}(s)=\left[\begin{array}{ll}
G_{i g}(s) & G_{i d}(s) \\
G_{v g}(s) & G_{v d}(s)
\end{array}\right]
$$

que es una matriz que recoge las funciones de transferencias obtenidas en el apartado anterior.

\section{B.7. Impedancia de salida.}

La impedancia de salida en pequeña señal se obtiene a partir del modelo en pequeña señal de la Figura B.21. Para ello hay que anular las entradas independientes, $\hat{v}_{g}$ y $\hat{d}$ y conectar una fuente independiente, $\hat{i}_{t}$, en los puntos donde se quiere medir la impedancia. La tensión que genera esta fuente sobre el sistema, $\hat{v}_{t}$, se emplea para obtener $Z_{\text {out }}(s)$ como:

$$
Z_{\text {out }}(s)=\left.\frac{\hat{v}_{t}(s)}{\hat{i}_{t}(s)}\right|_{\begin{array}{l}
\hat{v}_{g}=0 \\
\hat{d}=0
\end{array}}
$$


considerando que $\hat{i}_{t}(s)$ es la intensidad que entra por el terminal de mayor tensión de los dos puntos de medida considerados anteriormente. En el circuito de la Figura B.30 se indica gráficamente la conexión de la fuente independiente al modelo en pequeña señal una vez anuladas las fuentes independientes. Además se ha indicado la impedancia $Z_{t h}$, como la impedancia formada por el condensador del clamp, la resistencia equivalente y la bobina principal.

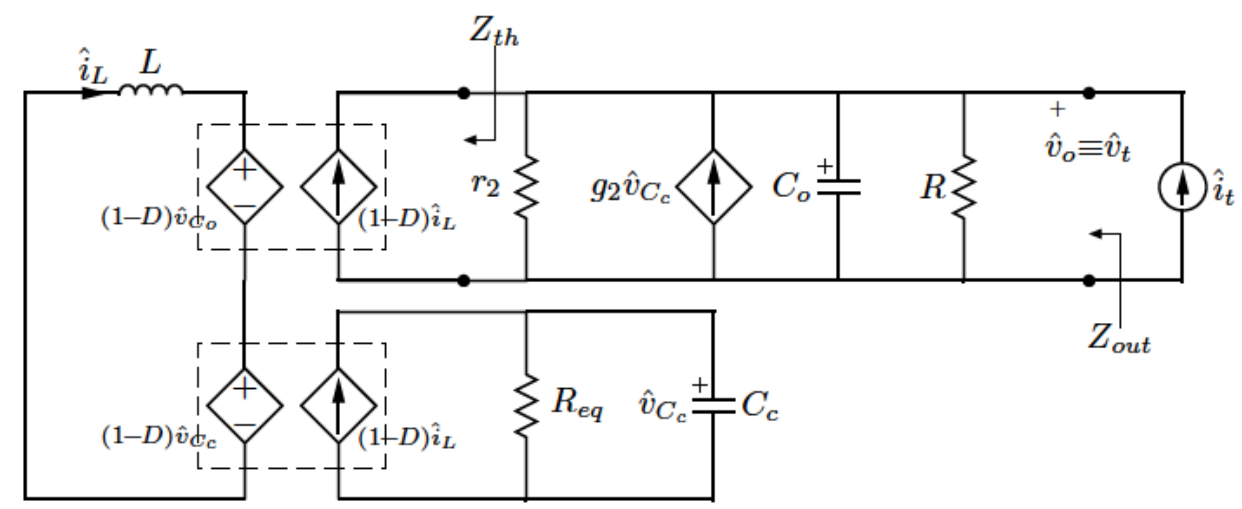

Figura B.30: Circuito para obtener la impedancia de salida de un elevador ZVS.

\section{B.7.1. Cálculo de $Z_{t h}$.}

Para obtener esta impedancia, se utiliza la parte del circuito anterior que concierne al punto de medida de $Z_{t h}$, en el que se conecta la fuente $\hat{i}_{t}$. Queda el circuito de la Figura B.31. Pasando el conjunto $R_{e q}-C_{c}$, al otro lado de las fuentes dependientes, se encontrará en serie con la bobina $L$. Estos elementos se pasan hacia la salida, obteniéndose la expresión:

$$
Z_{t h}(s)=\frac{L s+(1-D)^{2} Z_{e q}(s)}{(1-D)^{2}}=L^{\prime} s+Z_{e q}(s)
$$

A esta expresión también se puede llegar a partir del modelo en pequeña señal de (B.120). Basta con anular los términos independientes, $\hat{v}_{g}(t)$ y $\hat{d}(t)$ y cambiar la ecuación relativa a la tensión del condensador $C_{o}$, puesto que éste, junto con la carga $R$, quedan fuera y en su lugar se ha conectado una fuente de intensidad, $\hat{i}_{t}(t)$. La nueva ecuación relaciona la intensidad en la bobina, $\hat{i}_{L}(t)$, con la intensidad $\hat{i}_{t}(t)$. En el resto de ecuaciones hay que sustituir el término $\hat{v}_{C_{o}}(t)$ por la tensión $\hat{v}_{t}(t)$. Finalmente se aplica Laplace a las 


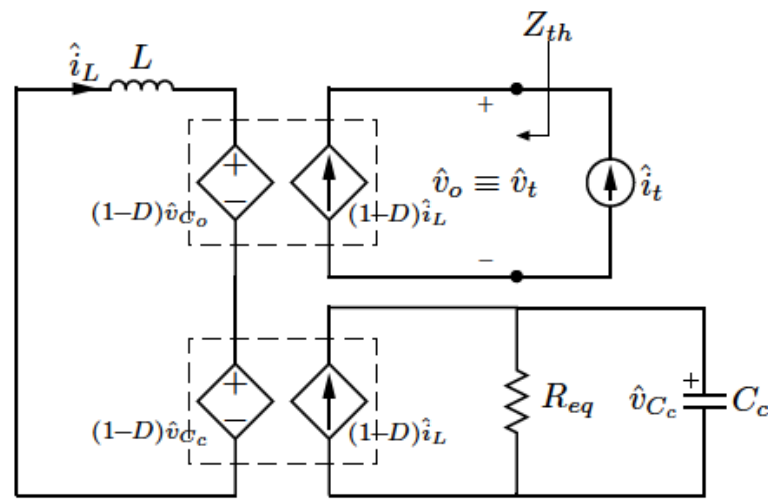

Figura B.31: Circuito empleado para obtener $Z_{t h}(s)$.

ecuaciones resultantes y quedan las ecuaciones indicadas en (B.162):

$$
\begin{aligned}
s L \hat{i}_{L}(s) & =-(1-D) \hat{v}_{t}(s)-(1-D) \hat{v}_{C_{c}}(s) \\
-\hat{i}_{t}(s) & =(1-D) \hat{i}_{L}(s) \\
s C_{c} \hat{v}_{C_{c}}(s) & =(1-D) \hat{i}_{L}(s)-\frac{1}{R_{e q}} \hat{v}_{C_{c}}(s)
\end{aligned}
$$

Empleando la igualdad de la segunda ecuación, se despeja $\hat{v}_{C_{c}}$ de la tercera ecuación y se sustituye en la primera, para obtener una expresión de la impedancia que es equivalente a la mostrada en (B.161):

$$
\begin{aligned}
\hat{v}_{C_{c}}(s) & =\frac{(1-D) \hat{i}_{L}(s)}{s C_{c}+\frac{1}{R_{e q}}}=-Z_{e q}(s) \hat{i}_{t}(s) \\
(1-D) \hat{v}_{t}(s) & =\left[\frac{L s}{(1-D)}+(1-D) Z_{e q}(s)\right] \hat{i}_{t}(s) \\
Z_{t h}(s) & =\frac{\hat{v}_{t}(s)}{\hat{i}_{t}(s)}=\frac{L s}{(1-D)^{2}}+Z_{e q}(s)
\end{aligned}
$$

En la Figura B.32 se ha representado $\left|Z_{t h}(s)\right|$ para un Elevador ZVS con los parámetros de la Tabla B.1. También están representadas los módulos de las impedancias de los elementos que forman parte de $Z_{t h}(s)$, esto es, la bobina $L$, el condensador $C_{c}$ y el término de resistencia equivalente $R_{e q}$, afectados por el factor correspondiente a su posición respecto al punto donde se obtiene la impedancia.

Al hacer $s=0$ se obtiene la impedancia en continua, que es igual al término $R_{e q}$, y se corresponde con la asíntota horizontal de la impedancia $Z_{t h}(s)$ a baja frecuencia.

$$
Z_{t h}(0)=Z_{e q}(0)=R_{e q}
$$




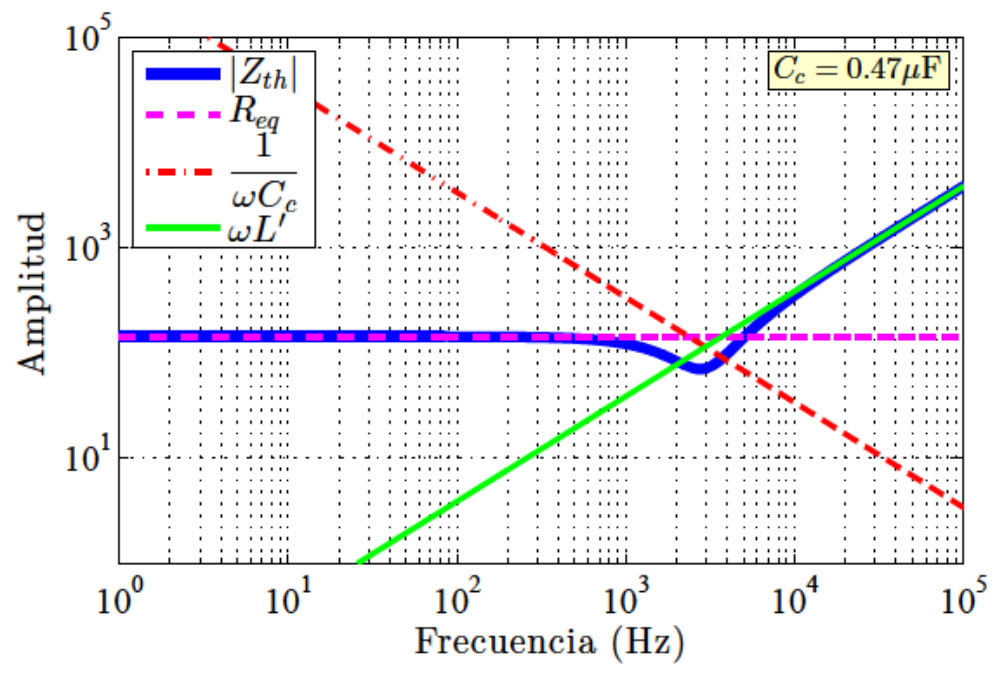

Figura B.32: Representación gráfica de $\left|Z_{t h}(s)\right|$ y los elementos que la forman.

Este mismo resultado se obtiene analizando el circuito de la Figura B.31 cuando la bobina y el condensador se convierten en un cortocircuito y en un circuito abierto respectivamente. La fuente independiente de intensidad ahora es una fuente constante conectada en los terminales donde se va a medir esa resistencia. El circuito modificado está representado en la Figura B.33.

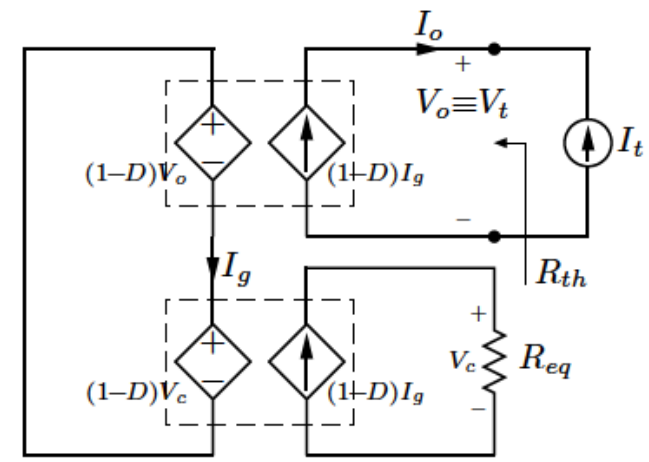

Figura B.33: Circuito empleado para el cálculo de $R_{t h}$ de un elevador ZVS.

Cambiando la posición de la resistencia $R_{e q}$ a través de los dos bloques de fuentes dependientes, se obtiene una expresión para la resistencia como:

$$
R_{t h}=R_{e q}
$$




\section{B.7.2. Cálculo de $Z_{\text {out }}$.}

A la vista del circuito de la Figura B.30, la tensión en el condensador de clamp es igual a:

$$
\hat{v}_{C_{c}}(s)=(1-D) i_{L} Z_{e q}(s)
$$

Pasando el conjunto $R_{e q}-C_{c}$, al otro lado de las fuentes dependientes, como está indicado en el circuito de la Figura B.34a, se obtiene $(1-D)^{2} Z_{e q}(s)$ en serie con la bobina $L$, lo que da lugar a la igualdad de tensiones:

$$
-\hat{i}_{L}(s)\left[L s+(1-D)^{2} Z_{e q}(s)\right]=(1-D) \hat{v}_{t}(s)
$$

donde $\hat{v}_{t}(s) \equiv \hat{v}_{o}(s)$.

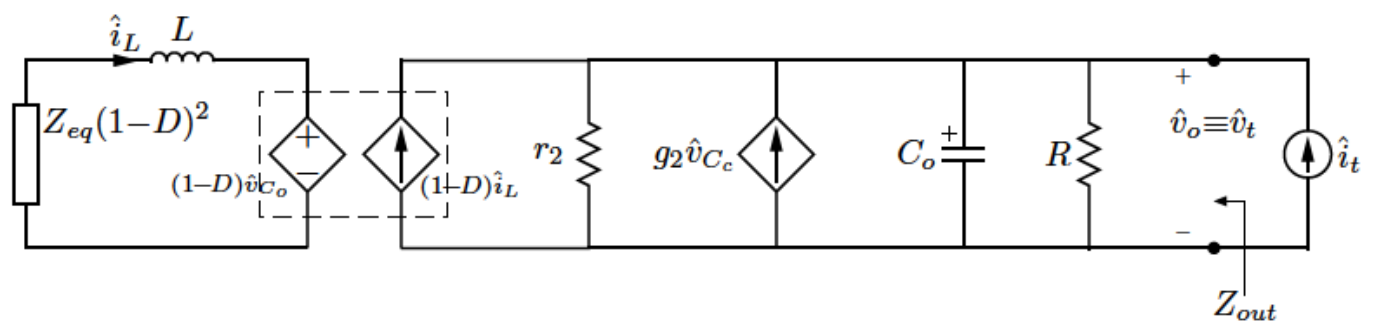

(a)

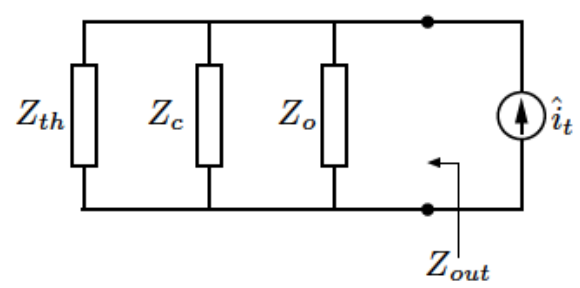

(b)

Figura B.34: Circuito empleado para el cálculo de $Z_{\text {out }}(s)$ de un elevador ZVS: a) desplazando elementos; y b) agrupando elementos.

Con esta última ecuación y con (B.166) se consigue una expresión para $\hat{v}_{C_{c}}$ en función de $\hat{v}_{t}$ como:

$$
\hat{v}_{C_{c}}(s)=-\frac{Z_{e q}(s)}{Z_{t h}(s)} \hat{v}_{t}(s)
$$

que dada la posición en la que se encuentra en el circuito, hace que la fuente de corriente $g_{2} \hat{v}_{C_{c}}$ (sobre la que se produce esa tensión $\hat{v}_{t}$ ) se pueda interpretar como una impedancia, $Z_{c}$, de expresión:

$$
Z_{c}(s)=\frac{Z_{t h}(s)}{g_{2} Z_{e q}(s)}
$$

Al pasar todos los elementos hacia la salida, se observa que $Z_{\text {out }}$ es el paralelo de tres elementos: la impedancia $Z_{t h}$; la impedancia $Z_{o}$, que agrupa 
a los elementos $r_{2}, C_{o}$ y $R$; y la impedancia $Z_{c}$. Así se ha representado en la Figura B.34b.

$$
Z_{\text {out }}(s)=Z_{\text {th }}(s)\left\|Z_{c}(s)\right\| Z_{o}(s)
$$

A esta expresión también se puede llegar desde las ecuaciones indicadas en (B.171), obtenidas a partir del modelo en pequeña señal indicado en (B.120) donde se anulan los términos independientes, $\hat{v}_{g}(t)$ y $\hat{d}(t)$. Después, hay que modificar la ecuación relativa a la tensión del condensador $C_{o}$ para introducir la intensidad $\hat{i}_{t}(t)$. Tras cambiar el término $\hat{v}_{C_{o}}(t)$ por $\hat{v}_{t}(t)$, se aplica Laplace a las ecuaciones modificadas y quedan:

$$
\begin{aligned}
s L \hat{i}_{L}(s) & =-(1-D) \hat{v}_{t}(s)-(1-D) \hat{v}_{C_{c}}(s) \\
-\hat{i}_{t}(s) & =(1-D) \hat{i}_{L}(s)-\hat{v}_{t}(s) \overbrace{\left[\frac{1}{r_{2}}+C_{o} s+\frac{1}{R}\right]}^{Z_{o}^{-1}(s)}+g_{2} \hat{v}_{C_{c}}(s) \\
s C_{c} \hat{v}_{C_{c}}(s) & =(1-D) \hat{i}_{L}(s)-\frac{1}{R_{e q}} \hat{v}_{C_{c}}(s)
\end{aligned}
$$

Se despeja $\hat{v}_{C_{c}}(s)$ de la tercera ecuación y se sustituye en la primera ecuación para despejar $\hat{i}_{L}(s)$ y utilizarla en la segunda ecuación. Ahora se puede conseguir una expresión de la impedancia de salida que es equivalente a la mostrada en (B.170):

$$
\begin{aligned}
\hat{v}_{C_{c}}(s) & =\frac{(1-D)}{s C_{c}+\frac{1}{R_{e q}}} \hat{i}_{L}(s)=(1-D) Z_{e q}(s) \hat{i}_{L}(s) \\
\hat{i}_{L}(s) & =-\frac{(1-D)}{L s+(1-D)^{2} Z_{e q}(s)} \hat{v}_{t}(s)=-\frac{\hat{v}_{t}(s)}{(1-D) Z_{t h}(s)} \\
\hat{i}_{t}(s) & =\frac{\hat{v}_{t}(s)}{Z_{t h}(s)}+\frac{\hat{v}_{t}(s)}{Z_{o}(s)}+\frac{g_{2} Z_{e q}(s) \hat{v}_{t}(s)}{Z_{t h}(s)} \\
& =\left[\frac{1}{Z_{t h}(s)}+\frac{g_{2} Z_{e q}(s)}{Z_{t h}(s)}+\frac{1}{Z_{o}(s)}\right] \hat{v}_{t}(s)=\frac{\hat{v}_{t}(s)}{Z_{\text {out }}(s)}
\end{aligned}
$$

En la Figura B.35 se ha representado el módulo de esta impedancia de salida para un Elevador ZVS, a partir de los parámetros indicados en la Tabla B.1, junto con el módulo de los términos que la forman, $\left|Z_{t h}(s)\right|,\left|Z_{c}(s)\right|$ $\mathrm{y}\left|Z_{o}(s)\right|$.

Al hacer $s=0$ se obtiene la impedancia en continua, que es el paralelo de los términos $R_{e q}, r_{2}, 1 / g_{2}$ y $R$. Y se corresponde con la asíntota horizontal 


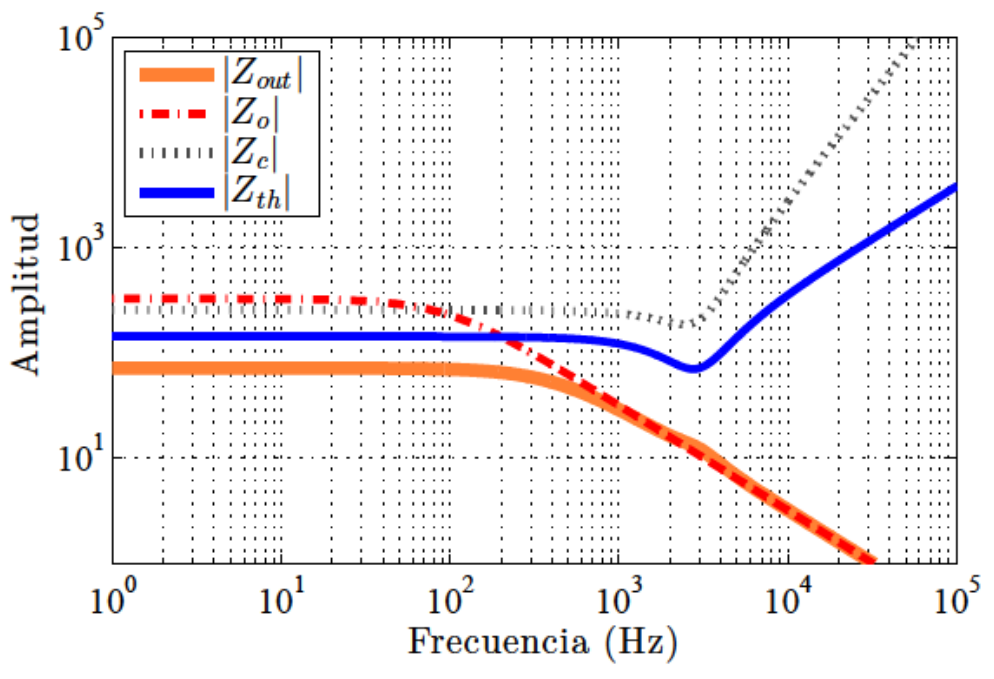

Figura B.35: Representación gráfica de $\left|Z_{\text {out }}(s)\right|$ y los elementos que la forman.

de $Z_{\text {out }}$ a bajas frecuencias.

$$
\begin{aligned}
Z_{\text {out }}(0) & =\frac{1}{\frac{g_{2} Z_{e q}(0)}{Z_{t h}(0)}+\frac{1}{Z_{t h}(0)}+\frac{1}{Z_{o}(0)}} \\
& =\frac{1}{\frac{g_{2} R_{e q}}{R_{e q}}+\frac{1}{R_{e q}}+\frac{1}{r_{2}}+\frac{1}{R}}=\left[g_{2}+\frac{1}{R_{e q}}+\frac{1}{r_{2}}+\frac{1}{R}\right]^{-1}
\end{aligned}
$$

\section{B.7.3. Validación de las expresiones obtenidas.}

La validación de la impedancia, se comprueba con la simulación de un convertidor conmutado sobre el que se mide la impedancia de salida. Con los parámetros de funcionamiento recogidos en la Tabla B.1 se añade a la salida una fuente de corriente de frecuencia variable y se mide la variación que experimenta la tensión de salida.

Como las expresiones de impedancia se han obtenido a partir del modelo promediado parece lógico medir la impedancia sobre este modelo y comparar los resultados simulados con la teoría empleando el ciclo de trabajo calculado para el modelo promediado. Si se emplea el convertidor conmutado para medir la impedancia, debería considerarse el valor del ciclo de trabajo obtenido para el circuito conmutado con la corrección propuesta en el apartado correspondiente. 
En la Figura B.36 se ha representado los valores obtenidos en la medida de la impedancia de salida de un Elevador ZVS simulado con los parámetros indicados en la Tabla B.1. También se ha representado el módulo de la impedancia de salida teórica, $\left|Z_{\text {out }}(s)\right|$. Como ya se conocía, presenta una asíntota horizontal, que es el paralelo de $R_{e q}, r_{2}, g_{2}$ y $R$, y otra asíntota asociada al condensador $C_{o}$. A la vista de esta gráfica, se observa la coincidencia

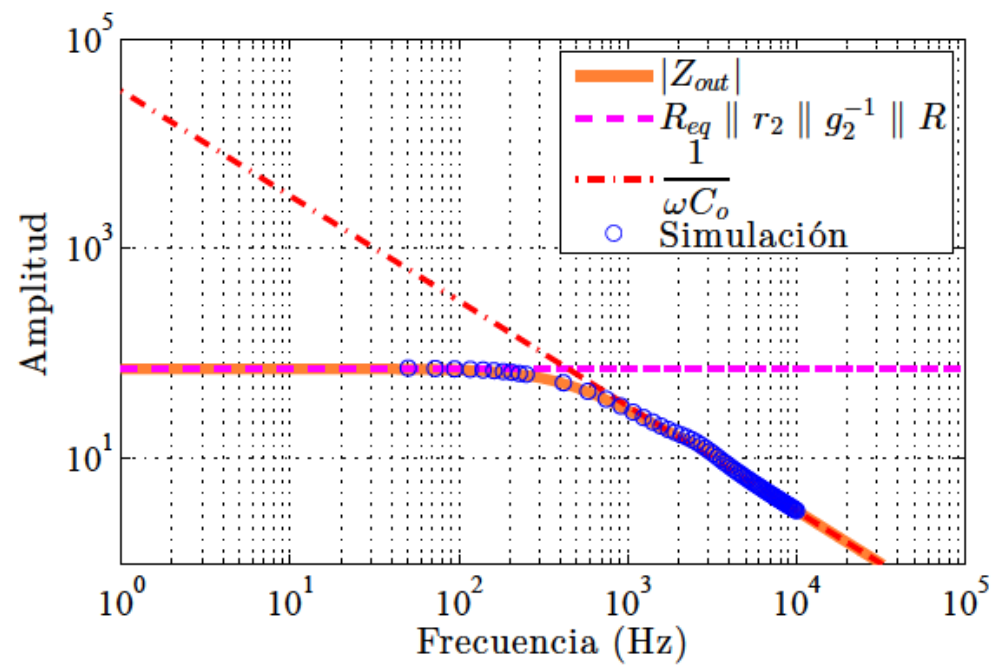

Figura B.36: Representación gráfica de $\left|Z_{\text {out }}(s)\right|$, y resultados de simulación.

de la impedancia teórica y los valores obtenidos en la simulación, por lo que se concluye la validez de las expresiones de impedancia obtenidas de forma teórica.

En el caso del reparto de corrientes entre varias etapas en paralelo para alimentar una carga común, la impedancia de salida de cada una de las etapas se debe determinar desde el punto común de conexión. Existiendo la posibilidad de considerar un único condensador de salida común a todas las etapas (aunque también podría tratarse el caso de un condensador en cada etapa). El análisis sería similar al realizado en el apartado anterior, con la precaución de no incluir ni $R$ ni $C_{o}$ dentro de $Z_{o}$.

Por ejemplo, la impedancia multietapa sin el condensador de salida, $Z_{m e}(s)$ se corresponde con la expresión:

$$
Z_{m e}(s)=\left[\frac{1}{Z_{t h}(s)}+\frac{g_{2} Z_{e q}}{Z_{t h}(s)}+\frac{1}{r_{2}}\right]^{-1}
$$

En la Figura B.37 se ha representado el módulo de esta impedancia y sus asíntotas de baja y alta frecuencia: 


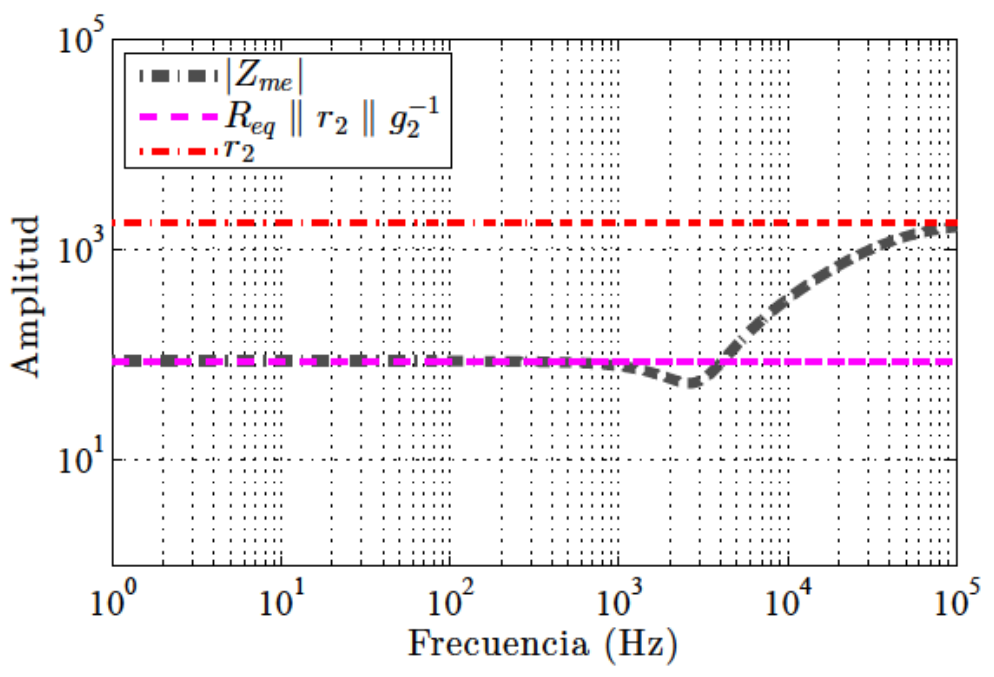

Figura B.37: Representación gráfica de $\left|Z_{m e}(s)\right|$.

Haciendo $s=0$, se consigue la asíntota de baja frecuencia, que es el paralelo de $R_{e q}, r_{2}$ y $1 / g_{2}$. Una expresión equivalente para esta asíntota se consigue al emplear sus expresiones indicadas en la tabla B.4. Entonces:

$$
\begin{aligned}
Z_{m e}(0) & =\left[\frac{2 I_{e q}}{V_{c}}+\frac{1}{R_{e q}}+\frac{I_{e q}}{V_{o}}\right]^{-1}=\left[\frac{2 V_{c}}{R_{e q} V_{o}}+\frac{1}{R_{e q}}+\frac{V_{C_{c}}^{2}}{R_{e q} V_{C_{o}}^{2}}\right]^{-1} \\
& =\frac{R_{e q}}{2 \frac{V_{c}}{V_{o}}+1+\left[\frac{V_{c}}{V_{o}}\right]^{2}}=R_{e q}\left[\frac{V_{o}}{V_{o}+V_{c}}\right]^{2}=R_{e q}\left[\frac{V_{o}}{V_{g} /(1-D)}\right]^{2} \\
& =2 L_{r} f\left[\frac{V_{o}}{V_{g}}\right]^{2}
\end{aligned}
$$

Esta última expresión coincide con la derivada de la característica de salida obtenida en apartados anteriores, y que está indicada en la expresión (B.53). Es la impedancia de salida en régimen permanente que presenta el convertidor.

Cuando $s$ se hace muy grande, los elementos que conforman $Z_{m e}(s)$ se hacen muy grandes, excepto $Z_{o}(s)$, que se transforma en $r_{2}$, y como están en paralelo, éste es el término que prevalece, siendo la asíntota para altas frecuencias. 


\section{Apéndice $\mathrm{C}$}

\section{Reductor Elevador ZVS}

\section{C.1. Funcionamiento del Reductor-Elevador ZVS}

El reductor-elevador ZVS es un reductor-elevador convencional al que se le ha añadido una red de enclavamiento activo que fija la tensión máxima en los extremos del transistor principal. Esta red facilita las conmutaciones a tensión cero, reduciendo las pérdidas que aparecen. Por contra, aumenta la complejidad del circuito y el número de componentes. En la figura C.1 está representado el circuito de este convertidor.

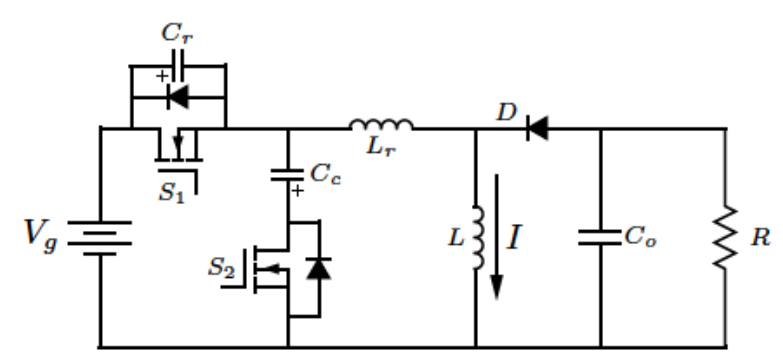

Figura C.1: Reductor-Elevador ZVS.

La red de enclavamiento activo está formada por el condensador de clamp $C_{c}$ y el interruptor $S_{2}$ junto con su diodo en antiparalelo, $D_{2}$. Además se añade una pequeña inductancia, $L_{r}$, que incluye las inductancias parásitas y que actúa en resonancia con el condensador $C_{c}$ en determinados instantes del periodo. Se ha incluido el diodo $D_{1}$ al interruptor $S_{1}$ y un condensador $C_{r}$ que puede ser el propio del transistor o uno añadido que mejore sus características.

Para explicar el funcionamiento de este convertidor se plantea el estado de los diferentes semiconductores presentes en el circuito, que son los dos interruptores y los tres diodos. En el instante inicial, $t_{0}$, está cerrado $S_{1}$ y el condensador $C_{r}$ está descargado. Se considera el condensador de salida, $C_{o}$, 
de un valor grande para mantener la tensión $v_{C_{o}}$ constante y se sustituirá el conjunto C-R por una fuente de tensión constante de valor $V_{o}$. La bobina principal, $L$, es lo suficientemente grande para considerar su corriente constante, $I$. El condensador de $c l a m p, C_{c}$ presenta una tensión con la polaridad indicada en figura y se considera lo suficientemente grande como para suponer una tensión prácticamente constante, $V_{c}$ aunque haya intervalos donde éste se cargue y se descargue y la tensión de clamp pueda variar en torno a ese valor. Por último, indicar que el valor de $L_{r}$, aunque comparativamente mas pequeño que $L$, es lo suficientemente grande como para cumplir el criterio de energía que permite conmutaciones suaves en estas condiciones de carga.

\section{C.1.1. Etapa 1. $\left(t_{0}<t<t_{1}\right)$}

Se abre el interruptor $S_{1}$. Esta conmutación se produce a tensión cero puesto que el condensador $C_{r}$ está descargado. Este condensador se carga a corriente constante igual a $I_{g}$, hasta que alcanza el valor de $V_{g}-V_{o}$. La figura C.2 muestra los elementos que intervienen en esta etapa y el circuito equivalente. A partir de las suposiciones iniciales, el circuito que queda planteado, se muestra en la figura C.2c donde la bobina se ha modelado como una fuente de corriente constante $I$.

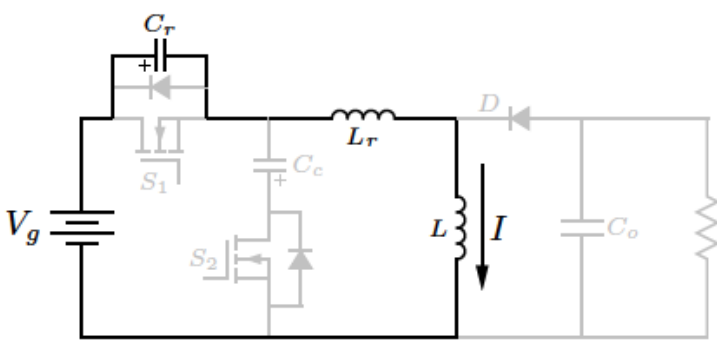

(a)

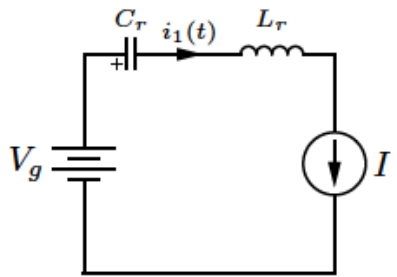

(b)

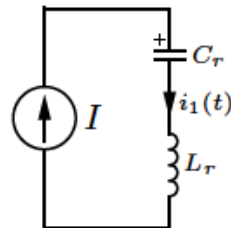

(c)

Figura C.2: Red-Elevador ZVS: a)durante la etapa 1; b)circuito equivalente; c) circuito para cálculos.

La tensión en el condensador crece de forma lineal, como se indica en (C.1), mientras que la tensión en la bobina $L_{r}$ es cero ya que la corriente que circula es constante.

$$
\begin{aligned}
i_{C_{r}}(t) & =I \\
v_{C_{r}}(t) & =\frac{1}{C_{r}} \int_{0}^{t} i_{C_{r}}(t) d t=\frac{I}{C_{r}} t
\end{aligned}
$$

Esta etapa terminará cuando $v_{C_{r}}(t)=V_{g}-V_{o}$, porque al alcanzar este valor, el diodo $D$ se polarizará en directa y empezará a conducir. La duración 
de esta etapa es igual a $d_{1} T$ indicada en (C.2):

$$
d_{1} T=\left(t_{1}-t_{0}\right)=\frac{\left(V_{g}-V_{o}\right) C_{r}}{I}
$$

\section{C.1.2. Etapa 2. $\left(t_{1}<t<t_{2}\right)$}

El diodo $D$ empieza a conducir. La entrada sigue suministrando una corriente igual a $I$. En la figura C.3 se muestran los elementos que intervienen y el circuito equivalente. El condensador $C_{r}$ sigue cargándose, pero ahora no es a corriente constante sino a una corriente variable, $i_{2}(t)$, obtenida por la resonancia del condensador y la bobina.

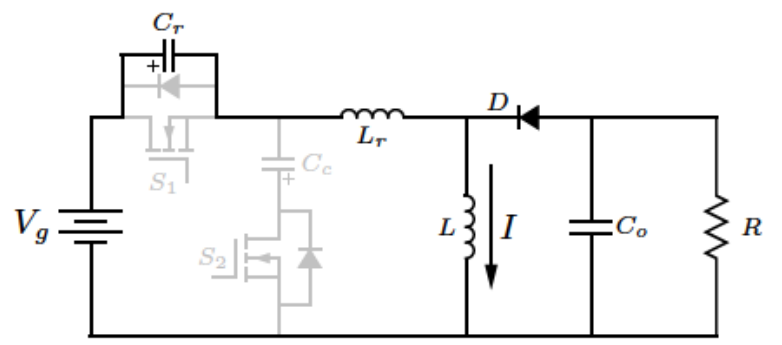

(a)

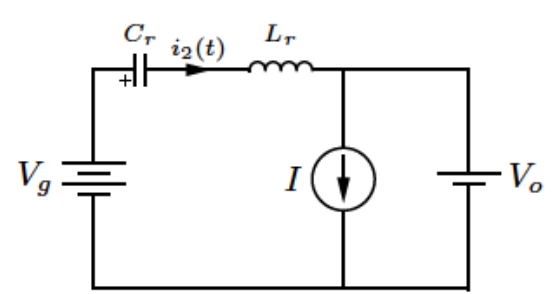

(b)

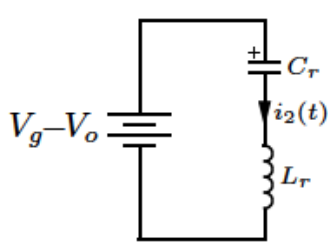

(c)

Figura C.3: Red-Elevador ZVS a)durante la etapa 2; b)circuito equivalente; c) circuito para cálculos.

Obteniendo la corriente $i_{2}(t)$, se conseguirá la evolución de la tensión en $C_{r}$, y cuando alcance el valor de $V_{g}+V_{c}$ terminará esta etapa, ya que se polarizará en directa el diodo del interruptor $S_{2}$, dando lugar a otro subcircuito. la ecuación para esta corriente se obtiene al plantear la tensión en la rama resonante, y está dada en (C.3), que es una ecuación integro-diferencial con coeficientes constantes.

$$
V_{g}-V_{o}=v_{C_{r}}(t)+v_{L_{r}}(t)=v_{C_{r}}(0)+\frac{1}{C_{r}} \int_{0}^{t} i_{2}(t) d t+L_{r} \frac{d i_{2}(t)}{d t}
$$

Las condiciones iniciales son (C.4):

$$
\begin{aligned}
& i_{2}(0)=I \\
& i_{2}^{\prime}(0)=\frac{1}{L_{r}} v_{L_{r}}(0)=\frac{1}{L_{r}}\left[V_{g}-v_{C_{r}}(0)-V_{o}\right]=0
\end{aligned}
$$

donde la intensidad debe cumplir la condición de continuidad, pero no la derivabilidad. 
La resolución de esta ecuación diferencial se puede obtener de varias formas. Método A) derivar de nuevo la ecuación, y resolverla como una ecuación diferencial ordinaria de segundo orden con coeficientes constantes teniendo en cuenta las condiciones iniciales. Método B) Aplicar Laplace a la ecuación (C.3) teniendo en cuenta las condiciones iniciales al aplicar Laplace y después obtener la transformada inversa. Método C): Aplicar Laplace al circuito y resolverlo en el dominio de Laplace, y una vez obtenida una expresión de la corriente hacer la transformada inversa.

METODO A) para obtener $i_{2}(t)$.

Derivando la ecuación integro-diferencial, se obtiene (C.5), y se mantienen las condiciones iniciales de (C.4):

$$
0=\frac{1}{C_{r}} i_{2}(t)+L_{r} \frac{d^{2} i_{2}(t)}{d t^{2}}
$$

Esta ecuación se resuelve a partir de la ecuación característica, cuya resolución ofrece dos raíces complejas conjugadas, dadas en (C.6):

$$
L_{r} D^{2}+\frac{1}{C_{r}}=0 \Rightarrow D= \pm \sqrt{\frac{-1}{L_{r} C_{r}}}= \pm j \omega_{r}
$$

donde $\omega_{r}=\left(\sqrt{L_{r} C_{r}}\right)^{-1}$ es la frecuencia de resonancia de la bobina y el condensador. Al ser dos raíces complejas conjugadas se prueba con una solución de la forma indicada en (C.7), a la que se le aplican las condiciones (C.4).

$$
i_{2}(t)=K_{1} \cos \left(\omega_{r} t\right)+K_{2} \sin \left(\omega_{r} t\right)
$$

$\mathrm{Al}$ aplicar la primera condición, se deduce que $K_{1}=I_{g}$. Con la segunda condición se deduce que $K_{2}=0$.

$$
\begin{aligned}
& i_{2}(0)=I \Rightarrow I=K_{1} * 1+K_{2} * 0 \Rightarrow K_{1}=I \\
& i_{2}^{\prime}(0)=0 \Rightarrow K_{1} \sin \left(\omega_{r} t\right)\left(-\omega_{r}\right)+\left.K_{2} \cos \left(\omega_{r} t\right) \omega_{r}\right|_{t=0}=0 \Rightarrow K_{2}=0
\end{aligned}
$$

De esta forma la solución para la ecuación diferencial es la indicada en (C.9):

$$
i_{2}(t)=I \cos \left(\omega_{r} t\right)
$$

METODO B) para obtener $i_{2}(t)$.

Aplicando Laplace a la ecuación integro-diferencial (C.3), se obtiene una expresión como la indicada en (C.10) donde ahora entran en juego los valores iniciales de las variables implicadas, donde $I_{2}(s)=\mathscr{L}\left[i_{2}(t)\right]$.

$$
\begin{aligned}
\mathscr{L}\left[V_{g}-V_{o}\right] & =\mathscr{L}\left[v_{C_{r}}(0)+\frac{1}{C_{r}} \int_{0}^{t} i_{2}(t) d t+L_{r} \frac{d i_{2}(t)}{d t}\right] \\
\frac{V_{g}-V_{o}}{s} & =\frac{v_{C_{r}}(0)}{s}+\frac{1}{C_{r} s} I_{2}(s)+L_{r} s I_{2}(s)-L_{r} i_{2}(0)
\end{aligned}
$$


Recuerde que $v_{C_{r}}(0)=V_{g}-V_{o}$, y que $i_{2}(0)=I$. Una vez despejada $I_{2}(s)$, se obtiene, tras aplicar Laplace, el resultado (C.11):

$$
I_{2}(s)=I \frac{s}{s^{2}+\frac{1}{L_{r} C_{r}}} \Rightarrow i_{2}(t)=\mathscr{L}^{-}\left[I_{2}(s)\right]=I \cos \left(\omega_{r} t\right)
$$

METODO C) para obtener $i_{2}(t)$.

Este método consiste en transformar el circuito al dominio de Laplace y después resolver la corriente transformada, es decir, $I_{2}(s)$ en el circuito resultante. Si se aplica Laplace al circuito de la figura C.4a (donde previamente se ha sustituido el condensador cargado a una tensión por un condensador vacío y una fuente de tensión en serie del mismo valor y polaridad que la que tenía inicialmente y la bobina se ha sustituido por una bobina descargada y una fuente de corriente en paralelo y del mismo valor y sentido que la que estaba circulando inicialmente), se obtiene el circuito en el dominio transformado como el de la figura C.4b. En este caso, la tensión inicial del condensador es $V_{g}-V_{o}$ y la corriente inicial en la bobina es $I$.

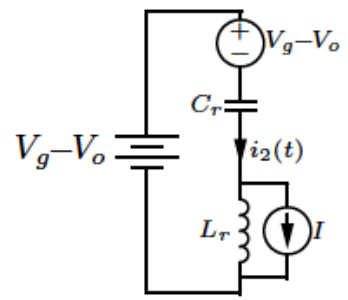

(a)

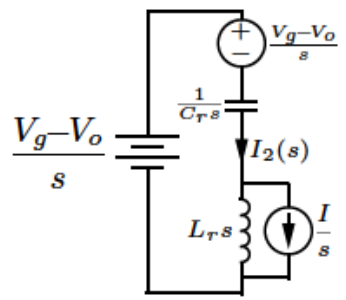

(b)

Figura C.4: Circuito equivalente de la Etapa 2: a) en el dominio del tiempo; y b) en el dominio de Laplace.

Para facilitar las operaciones, se sustituye el conjunto bobina-fuente de corriente en el circuito transformado por su equivalente Thévenin. en la figura C.5 se ha representado el circuito equivalente. De esta forma, se obtiene
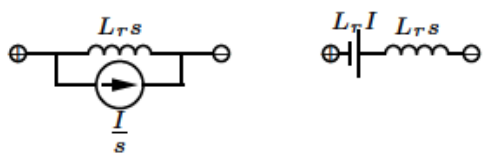

Figura C.5: Equivalente Thévenin del conjunto bobina-fuente de corriente.

fácilmente la expresión para $I_{2}(s)$, como se indica en (C.12), a partir de la 
ecuación de tensión en la rama resonante:

$$
\begin{aligned}
\frac{V_{g}-V_{o}}{s} & =\frac{V_{g}-V_{o}}{s}+I_{2}(s) \frac{1}{C_{r} s}-L_{r} I+I_{2}(s) L_{r} s \\
L_{r} I & =I_{2}(s)\left(\frac{1}{C_{r} s}+L_{r} s\right) \\
I_{2}(s) & =I \frac{s}{\frac{1}{L_{r} C_{r} s}+s^{2}} \Rightarrow i_{2}(t)=\mathscr{L}^{-}\left[I_{2}(s)\right]=I \cos \left(\omega_{r} t\right)
\end{aligned}
$$

Por cualquiera de los métodos se obtiene la misma expresión para la corriente $i_{2}(t)$. Una vez calculada, se obtiene la tensión en el condensador como (C.13):

$$
\begin{aligned}
v_{C_{r}}(t) & =v_{C_{r}}(0)+\frac{1}{C_{r}} \int_{0}^{t} i_{2}(\lambda) d \lambda \\
& =V_{g}-V_{o}+\left[\frac{1}{C_{r}} I \sin \left(\omega_{r} \lambda\right) \omega_{r}^{-1}\right]_{0}^{t} \\
& =V_{g}-V_{o}+\frac{I}{\omega_{r} C_{r}} \sin \left(\omega_{r} t\right)
\end{aligned}
$$

la duración de esta etapa será el tiempo que tarda el condensador en alcanzar la tensión $V_{g}+V_{c}$, porque el diodo de $S 2$ se polariza en directa. Este tiempo se deduce de (C.13), y es igual a (C.14).

$$
d_{2} T=\left(t_{2}-t_{1}\right)=\omega_{r}^{-1} \arcsin \left(\frac{\left(V_{c}+V_{o}\right) \omega_{r} C_{r}}{I}\right)
$$

La corriente por el diodo principal, es igual a (C.15), mientras que la corriente $i_{2}(t)$ al final de esta etapa es $I^{\prime}$, y tiene un valor ligeramente inferior a su valor inicial.

$$
i_{D}(t)=I-i_{2}(t)=I\left(1-\cos \left(\omega_{r} t\right)\right)
$$

\section{C.1.3. Etapa 3. $\left(t_{2}<t<t_{3}\right)$}

El diodo del interruptor auxilar $S_{2}$ empieza a conducir. A partir de este instante, se puede cerrar este interruptor a tensión cero porque está circulando corriente por su diodo. En la figura C.6 se muestran los elementos que intervienen y el circuito equivalente, donde $i_{c}(t)$ representa la corriente que circula por el condensador de clamp.

Nótese que el condensador de clamp es grande para la energía que absorbe por lo que su tensión se mantiene prácticamente constante durante todo este intervalo y de ahí que se represente como una fuente de tensión con la polaridad indicada. Además la corriente $i_{c}(t)$ circula inicialmente en un sentido y luego se invierte cuando la bobina se ha descargado completamente 


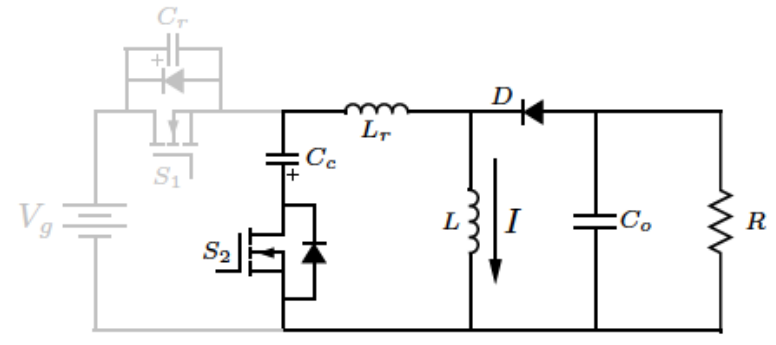

(a)

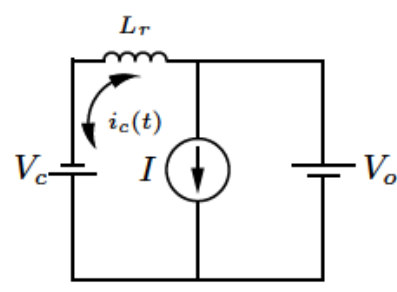

(b)

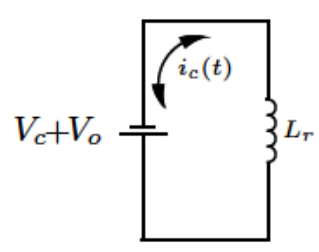

(c)

Figura C.6: Red-Elevador ZVS a)durante la etapa 3; b)circuito equivalente; c) circuito para cálculos.

sobre $C_{c}$. Pero como el interruptor $S_{2}$ permanece cerrado, el condensador cederá esta energía extra de nuevo a la bobina, haciendo que la corriente circule en el sentido opuesto al inicial. En este caso, la corriente circulará por $S_{2}$ y no por su diodo.

La relación entre la tensión de clamp, y la duración de este intervalo se obtiene resolviendo el circuito equivalente. Para ello hay que resolver el circuito de la figura C.6c para obtener $i_{c}(t)$. En esta malla, la tensión es constante e igual a $V_{c}$. La bobina resonante se descarga a tensión constante y su expresión se obtiene resolviendo la ec.diferencial de primer orden con condiciones iniciales de (C.16):

$$
\begin{aligned}
-V_{c}-V_{o} & =v_{L_{r}}(t)=L_{r} \frac{d i_{c}(t)}{d t} \\
i_{c}(0) & =I^{\prime}
\end{aligned}
$$

Haciendo la separación de variables en cada miembro de (C.16), e integrando se obtiene una solución general, en la que se despeja la constante de integración aplicando la condición inicial.

$$
\begin{aligned}
-\frac{V_{c}+V_{o}}{L_{r}} d t & =d i_{c}(t) \\
-\frac{V_{c}+V_{o}}{L_{r}} \int d t & =\int d i_{c}(t) \\
i_{c}(t) & =-\frac{V_{c}+V_{o}}{L_{r}} t+K \\
i_{c}(0) & =I^{\prime} \Rightarrow K=I^{\prime} \Rightarrow i_{c}(t)=I^{\prime}-\frac{V_{c}+V_{o}}{L_{r}} t
\end{aligned}
$$

En la solución dada en (C.17) se comprueba que $i_{c}(t)$ es decreciente y llega a ser negativa. La duración de esta etapa es $d_{3} T=\left(t_{3}-t_{2}\right)$.

Al final de este periodo, la corriente alcanza un valor igual a $I^{\prime}$ pero en sentido contrario. De esta relación se deduce el valor de la tensión de clamp 
en función de la bobina resonante y el ciclo de trabajo. Véase (C.18).

$$
i_{c}\left(t_{3}\right)=-I^{\prime} \Rightarrow-I^{\prime}=I^{\prime}-\frac{V_{c}+V_{o}}{L_{r}} d_{3} T \Rightarrow V_{c}=\frac{2 I^{\prime} L_{r}}{d_{3} T}-V_{o}
$$

También se puede decir lo siguiente: Si se habla de régimen permanente, la energía neta en el condensador de clamp durante un ciclo de trabajo, es decir, la energía que toma y que devuelve durante ese tiempo, debe ser nula. Como se ha supuesto un valor de $C_{c}$ suficientemente grande para que $V_{c}$ sea constante, al aplicar esta suposición se llega a que el valor medio de (C.17) es nulo. Dicho de otra forma, el balance de carga en $C_{c}$ deber se nulo. Siendo así, resulta que

$$
\begin{gathered}
\Delta E=0 \Rightarrow \int_{t_{0}}^{t_{0}+T} i_{c}(t) d t=\int_{t_{2}}^{t_{3}} i_{c}(t) d t=\int_{0}^{d_{3} T}\left[I^{\prime}-\frac{V_{c}+V_{o}}{L_{r}} t\right] d t=0 \\
{\left[I^{\prime} t-\frac{V_{c}+V_{o}}{2 L_{r}} t^{2}\right]_{0}^{d_{3} T}=0 \Rightarrow V_{c}=\frac{2 I^{\prime} L_{r}}{d_{3} T}-V_{o}}
\end{gathered}
$$

Este resultado sustituido en (C.17) permite obtener el valor de $i_{c}\left(t_{3}\right)$ para el régimen permanente. Así se indica en (C.20).

$$
i_{c}\left(t_{3}\right)=I^{\prime}-\frac{2 I \hbar_{r}}{L_{r} d_{3} T} d_{3} \Psi=-I^{\prime}
$$

Durante un transitorio, la tensión de clamp evolucionará en cada periodo que dure el transitorio de un valor a otro, y el balance de carga no será nulo, y por tanto, no se puede saber a priori el valor de $i_{c}\left(t_{3}\right)$ al final de cada periodo.

Ahora se abre el interruptor, y lo hace a tensión cero porque e condensador $C_{r}$ se había quedado cargado a una tensión $V_{g}+V_{c}$, de forma que el drenador de $S_{2}$ está a tensión cero.

La corriente que pasa por el diodo principal alcanza un valor máximo de (C.21): Véase (C.18).

$$
i_{D}(t)=I-i_{c}(t) \Rightarrow I_{D, \max }=i_{D}\left(t_{3}\right)=I-i_{c}\left(t_{3}\right)=I-I^{\prime}
$$

\section{C.1.4. Etapa 4. $\left(t_{3}<t<t_{4}\right)$}

Este intervalo se inicia con la apertura de $S_{2}$ a tensión nula. La corriente en $L_{r}$ tiene un sentido inicial opuesto al inicial de la etapa anterior, y ésta ya no puede circular ni por $S_{2}$ ni por su diodo, luego deberá circular a través de $C_{r}$. Dicho de otra forma, la bobina $L_{r}$ acumulará energía, pero esta vez de $C_{r}$ que se descarga de forma resonante sobre aquella. La corriente $i_{4}(t)$ 
experimenta durante este intervalo una evolución resonante, a la vez que la tensión $V_{C_{r}}(t)$ disminuye hasta hacerse cero, que es donde concluye esta etapa. A partir de este instante, la conducción de la corriente se realiza sobre el diodo $D_{1}$. En el circuito de la figura C.7 se muestran los elementos que están involucrados en esta etapa.

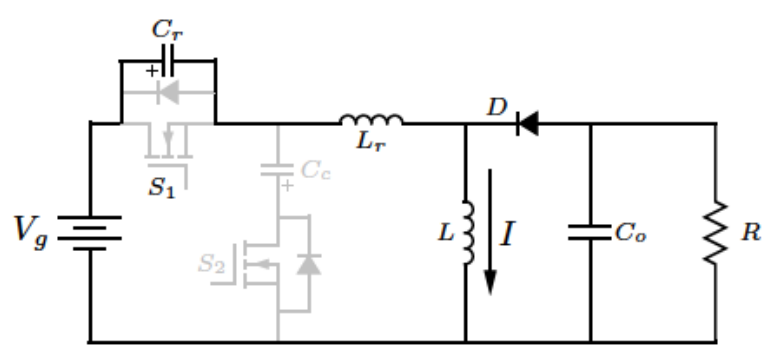

(a)

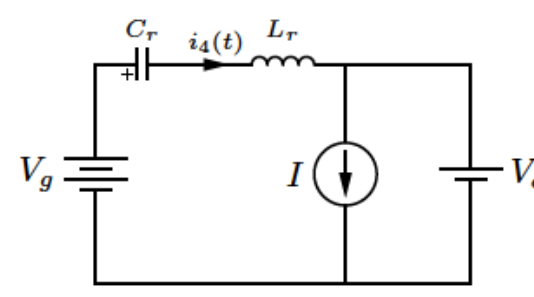

(b)

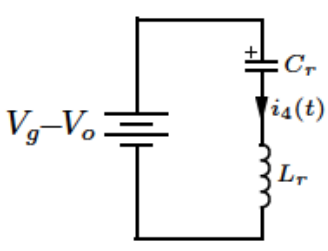

(c)

Figura C.7: Red-Elevador ZVS a)durante la etapa 4; b)circuito equivalente; c) circuito para cálculos.

Una condición necesaria, sobre la que volveremos mas adelante, es que la energía acumulada en $L_{r}$ debe ser como poco igual a la energía que acumula $C_{r}$ para que la bobina consiga descargar por completo al condensador antes de que se elimine. Para la obtención de $i_{4}(t)$ (donde se ha mantenido el mismo sentido que en los casos de las etapas anteriores), hay que plantear le ecuación de la tensión en la rama resonante del circuito equivalente y resolver la ecuación diferencial resultante utilizando las condiciones iniciales conocidas. La ecuación resultante es una ecuación integro-diferencial con coeficientes constantes dada por (C.22):

$$
V_{g}-V_{o}=v_{C_{r}}(t)+v_{L_{r}}(t)=v_{C_{r}}(0)+\frac{1}{C_{r}} \int_{0}^{t} i_{4}(t) d t+L_{r} \frac{d i_{4}(t)}{d t}
$$

Las condiciones iniciales son (C.23):

$$
\begin{aligned}
& i_{4}(0)=-I^{\prime} \\
& i_{4}^{\prime}(0)=\frac{1}{L_{r}} v_{L_{r}}(0)=\frac{1}{L_{r}}\left[V_{g}-v_{C_{r}}(0)-V_{o}\right]=-\frac{V_{c}+V_{o}}{L_{r}}
\end{aligned}
$$

donde la intensidad debe cumplir la condición de continuidad, pero no la derivabilidad.

La resolución de esta ecuación diferencial, tal y como se ha hecho en la ETAPA 2, se puede obtener de varias formas. Método A) derivar de nuevo la ecuación, y resolverla como una ecuación diferencial ordinaria de segundo orden con coeficientes constantes teniendo en cuenta las condiciones iniciales. Método B) Aplicar Laplace a la ecuación (C.22) teniendo en cuenta las condiciones iniciales al aplicar Laplace y después obtener la transformada 
inversa. Método C): Aplicar Laplace al circuito y resolverlo en el dominio de Laplace, y una vez obtenida una expresión de la corriente hacer la transformada inversa.

METODO A) para obtener $i_{4}(t)$.

Derivando la ecuación integro-diferencial, se obtiene (C.24), y se mantienen las condiciones inciales de (C.23):

$$
0=\frac{1}{C_{r}} i_{4}(t)+L_{r} \frac{d^{2} i_{4}(t)}{d t^{2}}
$$

Esta ecuación se resuelve a partir de la ecuación característica, cuya resolución ofrece dos raíces complejas conjugadas, dadas en (C.25):

$$
0=\frac{1}{C_{r}}+L_{r} D^{2} \Rightarrow D= \pm \sqrt{\frac{-1}{L_{r} C_{r}}}= \pm j \omega_{r}
$$

donde $\omega_{r}$ ya se había definido durante la ETAPA 2 como la frecuencia de resonancia bobina-condensador, y es igual a $\left(\sqrt{L_{r} C_{r}}\right)^{-1}$. Al ser dos raíces complejas conjugadas se prueba con una solución de la forma indicada en (C.26), a la que se le aplican las condiciones (C.23).

$$
i_{4}(t)=K_{3} \cos \left(\omega_{r} t\right)+K_{4} \sin \left(\omega_{r} t\right)
$$

$\mathrm{Al}$ aplicar la primera condición, se deduce que $K_{3}=-I^{\prime}$. Con la segunda condición se deduce el valor $\operatorname{de} K_{4}=0$.

$$
\begin{aligned}
i_{4}(0) & =-I^{\prime} \Rightarrow-I^{\prime}=K_{3} * 1+K_{4} * 0 \Rightarrow K_{3}=-I^{\prime} . \\
i_{4}^{\prime}(0) & =-\frac{V_{c}+V_{o}}{L_{r}} \Rightarrow K_{3} \sin \left(\omega_{r} t\right)\left(-\omega_{r}\right)+\left.K_{4} \cos \left(\omega_{r} t\right) \omega_{r}\right|_{t=0}=0 \Rightarrow \\
& \Rightarrow K_{4}=-\frac{V_{c}+V_{o}}{\omega_{r} L_{r}}
\end{aligned}
$$

De esta forma la solución para la ecuación diferencial es la indicada en (C.28):

$$
i_{4}(t)=-I^{\prime} \cos \left(\omega_{r} t\right)-\frac{V_{c}+V_{o}}{\omega_{r} L_{r}} \sin \omega_{r} t
$$

METODO B) para obtener $i_{4}(t)$.

Aplicando Laplace a la ecuación integro-diferencial (C.22), se obtiene una expresión como la indicada en (C.29) donde ahora entran en juego los valores iniciales de las variables implicadas, donde $I_{4}(s)=\mathscr{L}\left[i_{4}(t)\right]$.

$$
\begin{aligned}
\mathscr{L}\left[V_{g}-V_{o}\right] & =\mathscr{L}\left[v_{C_{r}}(0)+\frac{1}{C_{r}} \int_{0}^{t} i_{4}(t) d t+L_{r} \frac{d i_{4}(t)}{d t}\right] \\
\frac{V_{g}-V_{o}}{s} & =\frac{V_{C_{r}}(0)}{s}+\frac{1}{C_{r} s} I_{4}(s)+L_{r} s I_{4}(s)-L_{r} i_{4}(0)
\end{aligned}
$$


Recuerde que $V_{C_{r}}(0)=V_{g}+V_{c}$, y que $i_{4}(0)=-I^{\prime}$. Una vez despejada $I_{4}(s)$, se obtiene, tras aplicar Laplace, el resultado (C.30):

$$
\begin{aligned}
I_{4}(s) & =-I^{\prime} \frac{s}{s^{2}+\frac{1}{L_{r} C_{r}}}-\frac{V_{c}+V_{o}}{L_{r}} \frac{1}{s^{2}+\frac{1}{L_{r} C_{r}}} \\
i_{4}(t) & =\mathscr{L}^{-}\left[I_{4}(s)\right]=-I^{\prime} \cos \left(\omega_{r} t\right)-\frac{V_{c}+V_{o}}{\omega_{r} L_{r}} \sin \omega_{r} t
\end{aligned}
$$

METODO C) para obtener $i_{4}(t)$.

Este método consiste en transformar el circuito al dominio de Laplace y después resolver la corriente transformada, es decir, $I_{4}(s)$ en el circuito resultante. Si se aplica Laplace al circuito equivalente de la figura C.8a, se obtiene el circuito transformado de la figura C.8b, (donde previamente se ha sustituido el condensador cargado a una tensión por un condensador vacío y una fuente de tensión en serie del mismo valor y polaridad que la que tenía inicialmente y la bobina se ha sustituido por una bobina descargada y una fuente de corriente en paralelo y del mismo valor y sentido que la que estaba circulando inicialmente). En este caso, la tensión inicial del condensador es $V_{g}+V_{c}$ y la corriente inicial en la bobina es $-I^{\prime}$.

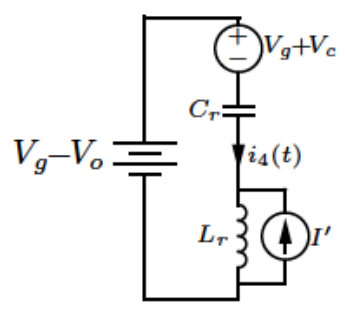

(a)

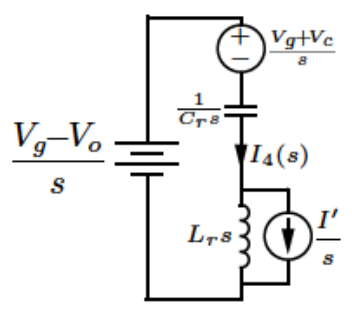

(b)

Figura C.8: Circuito equivalente en la Etapa 4: a) en el dominio del tiempo; y b) en el dominio de Laplace.

Para facilitar las operaciones, se sustituirá el conjunto bobina-fuente de corriente en el circuito transformado por su equivalente Thévenin. En la figura C.9 se ha representado el circuito equivalente.

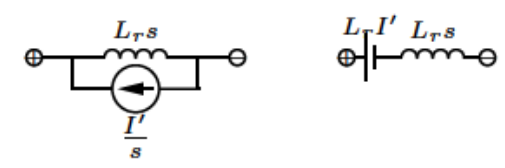

Figura C.9: Equivalente Thévenin del conjunto bobina-fuente de corriente.

De esta forma, se obtiene fácilmente la expresión para $I_{4}(s)$, como se 
indica en (C.31), a partir de la ecuación de tensión en la rama resonante:

$$
\begin{aligned}
\frac{V g-V_{o}}{s}-\frac{V_{g}+V_{c}}{s}-L_{r} I^{\prime} & =I_{4}(s) \frac{1}{C_{r} s}+I_{4}(s) L_{r} s \\
-\frac{V_{c}+V_{o}}{s}-L_{r} I^{\prime} & =I_{4}(s)\left(\frac{1+L_{r} C_{r} s^{2}}{C_{r} s}\right) \\
I_{4}(s) & =\left(-\frac{V_{c}+V_{o}}{s}-L_{r} I^{\prime}\right)\left(\frac{C_{r} s}{1+L_{r} C_{r} s^{2}}\right) \Rightarrow \\
i_{4}(t) & =\mathscr{L}^{-}\left[I_{4}(s)\right]=-\frac{V_{c}+V_{o}}{\omega_{r} L_{r}} \sin \omega_{r} t-I^{\prime} \cos \left(\omega_{r} t\right)
\end{aligned}
$$

Por cualquiera de los métodos se obtiene la misma expresión para la corriente $i_{4}(t)$. Una vez calculada, se obtiene la tensión en el condensador como (C.32):

$$
\begin{aligned}
v_{C_{r}}(t) & =v_{C_{r}}(0)+\frac{1}{C_{r}} \int_{0}^{t} i_{4}(\lambda) d \lambda \\
& =V_{g}+V_{c}-\left[\frac{1}{C_{r}} I^{\prime} \sin \left(\omega_{r} \lambda\right) \omega_{r}^{-1}\right]_{0}^{t}-\left[\frac{V_{c}+V_{o}}{C_{r} L_{r} \omega_{r}} \cos \left(\omega_{r} \lambda\right)\left(-\omega_{r}\right)^{-1}\right]_{0}^{t} \\
& =V_{g}-V_{o}+\left(V_{c}+V_{o}\right) \cos \omega_{r} t-\frac{I^{\prime}}{\omega_{r} C_{r}} \sin \left(\omega_{r} t\right)
\end{aligned}
$$

Esta expresión se puede simplificar, agrupando los dos términos de $\sin ()$ y $\cos ()$ en uno solo com amplitud y desfase. De esta forma la tensión en el condensador es (D.35):

$$
v_{C_{r}}(t)=V_{g}-V_{o}+V_{r} \cos \left(\omega_{r} t+\phi_{r}\right)
$$

siendo

$$
\begin{aligned}
& V_{r}=\sqrt{\left[V_{c}+V_{o}\right]^{2}+\left(\frac{I^{\prime}}{\omega_{r} C_{r}}\right)^{2}}=\sqrt{\left[V_{c}+V_{o}\right]^{2}+\frac{L_{r}}{C_{r}} I^{\prime 2}} \\
& \phi_{r}=\arctan \left(\frac{I^{\prime}}{\left(V_{c}+V_{o}\right) \omega_{r} C_{r}}\right)
\end{aligned}
$$

La duración de esta etapa será el tiempo que tarda el condensador en descargase por completo y su tensión es nula, instante en el cual la energía de $L_{r}$ empezará a circular a través del diodo de $S 1$. En este instante, la bobina se ha cargado a una corriente de valor negativo, $I^{\prime \prime}$, diferente a $-I^{\prime}$, puesto que la bobina toma la carga que le cede el condensador.

Para que la tensión pueda llegar a anularse se debe cumplir que la am- 
plitud $V_{r}$ sea mayor, o al menos igual, que $V_{g}-V_{o}$, luego:

$$
\begin{aligned}
\sqrt{\left[V_{c}+V_{o}\right]^{2}+\frac{L_{r}}{C_{r}} I^{\prime 2}} & \geq\left(V_{g}-V_{o}\right) \\
{\left[V_{c}+V_{o}\right]^{2}+\frac{L_{r}}{C_{r}} I^{\prime 2} } & \geq\left(V_{g}-V_{o}\right)^{2} \\
C_{r}\left[V_{c}+V_{o}\right]^{2}+L_{r} I^{\prime 2} & \geq C_{r}\left(V_{g}-V_{o}\right)^{2}
\end{aligned}
$$

que indica que la bobina descarga al condensador con la energía que tiene acumulada. Lo que sucede es que antes de empezar a descargarse la bobina, esta aumenta su energía por una parte que ya le cede el propio condensador. Ésta, junto con la que tiene acumulada sirven para descargar el condensador. Se deberá cumplir por tanto la condición indicada en C.35.

La corriente por el diodo principal, es igual a (C.36).

$$
i_{D}(t)=I-i_{4}(t)
$$

\section{C.1.5. Etapa 5. $\left(t_{4}<t<t_{5}\right)$}

Esta etapa comienza en el instante en que $C_{r}$ se descarga totalmente y su tensión es nula. La corriente circulante por $L_{r}$ necesita un camino para seguir circulando, y lo hace a través del diodo de $S_{1}$, que pasa a estar polarizado en directa. Debido a esto, el interruptor $S_{1}$ puede entrar en conducción a tensión cero en cualquier instante de tiempo en el que circule corriente por su diodo, que será mientras la corriente por $L_{r}$ mantenga el mismo sentido de circulación. Una vez cerrado $S_{1}$, la bobina cede la energía a la fuente, y cuando está descargada, se carga de nuevo con otra polaridad, por lo que la corriente $i_{5}(t)$ terminará circulando en un sentido contrario al que tenía al iniciar esta etapa. En la figura C.10a se muestran los elementos que están involucrados en esta etapa y el circuito equivalente.

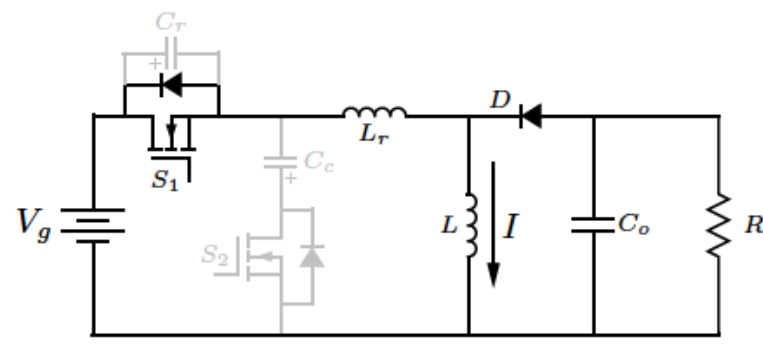

(a)

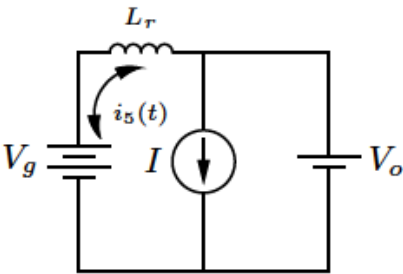

(b)

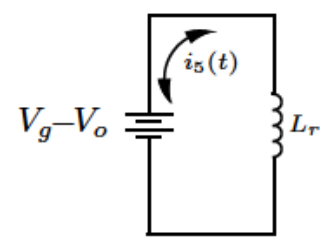

(c)

Figura C.10: Red-Elevador ZVS a)durante la etapa 5; b)circuito equivalente; c) circuito para cálculos. 
Esta etapa terminará cuando el valor de $i_{5}(t)$ alcance el valor de $I$, instante en el cual, la corriente por el diodo principal, $i_{D}(t)$ se anulará y pasará a la siguiente etapa. Observando el circuito equivalente, la corriente circulante en la rama de bobina resonante evoluciona en función de una tensión constante. La relación entre esta tensión y la duración de este intervalo se obtiene resolviendo dicho circuito. En esta rama la tensión es igual a $V_{g}-V_{o}$. La bobina resonante se descarga a tensión constante y su expresión se obtiene resolviendo la ec.diferencial de primer orden con condiciones iniciales de (C.37):

$$
\begin{aligned}
V_{g}-V_{o} & =v_{L_{r}}(t)=L_{r} \frac{d i_{5}(t)}{d t} \\
i_{5}(0) & =I^{\prime \prime}
\end{aligned}
$$

Haciendo la separación de variables en cada miembro de (C.37), e integrando se obtiene una solución general, en la que se despeja la constante de integración aplicando la condición inicial.

$$
\begin{aligned}
\frac{V_{g}-V_{o}}{L_{r}} d t & =d i_{5}(t) \\
\frac{V_{g}-V_{o}}{L_{r}} \int d t & =\int d i_{5}(t) \\
i_{5}(t) & =\frac{V_{g}-V_{o}}{L_{r}} t+K_{5} \\
i_{5}(0) & =I^{\prime \prime} \Rightarrow K_{5}=I^{\prime \prime} \Rightarrow i_{5}(t)=I^{\prime \prime}+\frac{V_{g}-V_{o}}{L_{r}} t
\end{aligned}
$$

De la expresión (C.38) se puede deducir la duración de este intervalo, que es el tiempo que tarda en alcanzarse el valor de $I$, que es igual a

$$
d_{5} T=\left(t_{5}-t_{4}\right)=\left(I-I^{\prime \prime}\right) \frac{L_{r}}{V_{g}-V_{O}}
$$

\section{C.1.6. Etapa 6. $\left(t_{5}<t<t_{6}\right)$}

Esta etapa comienza cuando el diodo $D$ deja de conducir y concluye cuando se abre el interruptor $S_{1}$, que conmuta a tensión cero puesto que el condensador $C_{r}$ está descargado. A partir de aquí comienza un nuevo ciclo. Los elementos que intervienen el circuito pasan a ser los indicados en la figura C.11a.

Uno de los objetivos de la red ZVS es reducir las pérdidas, y se consigue puesto que todas las conmutaciones son a tensión cero. $S_{1}$ pasa a OFF al principio de la ETAPA 1 y lo hace a tensión cero puesto que el condensador $C_{r}$ está descargado. $S_{2}$ pasa a $\mathrm{ON}$ al principio de la ETAPA 3 y lo hace a tensión cero puesto que está circulando corriente por su diodo. $S_{2}$ pasa a 


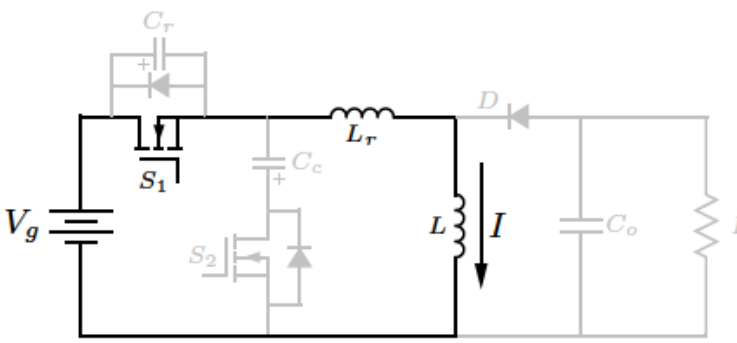

(a)

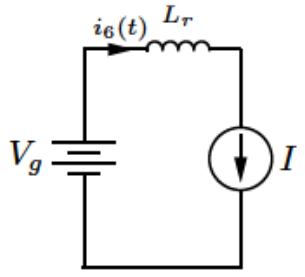

(b)

Figura C.11: Red-Elevador ZVS durante la etapa 6.

OFF al final de la ETAPA 3 y lo hace a tensión cero puesto que el drenador está a $0 \mathrm{~V}$. $S_{1}$ pasa a $\mathrm{ON}$ en la ETAPA 5 mientras circula corriente por su diodo.

Nótese que se supone cumplida la ecuación (C.35) relativa que la energía almacenada en $L_{r}$ debida a la intensidad $I$, debe ser mayor que la energía del condensador del MOSFET, $C_{r}$, para descargarlo desde la tensión $V_{g}-V_{o}+V_{c}$ hasta cero. en esta ecuación hay que hacer notar que $I$ representa el valor medio de la corriente en ese instante. Utilizando los valores instantáneos, habría que sustituir ese valor por el valor de pico, es decir por $I+\Delta I / 2$.

\section{C.2. Análisis en régimen permanente.}

\section{C.2.1. Sin considerar rizado.}

Se ha considerado una bobina $L$ lo suficientemente grande como para suponer corriente constante, de valor $I$, que es mucho mayor que la bobina resonante $L_{r}$. De forma similar, el valor de los condensadores $C_{o}$ y $C_{c}$, es tal que la tensión en sus extremos se mantiene constante, de valores $V_{C_{o}}$ y $V_{c}$, durante todo el periodo $T$.

Después de estudiar el funcionamiento del circuito con estas mismas consideraciones, se ha representado en la figura C.12 las principales magnitudes a lo largo de las seis etapas por las que evoluciona esta topología durante un ciclo completo. La duración de cualquiera de estas etapas se indica con la letra $d$ y un subíndice que hace referencia al número de etapa. Por ejemplo, la duración de la etapa 5 es igual a $t_{5}-t_{4}=d_{5} T$.

Para el análisis se desprecian las etapas 1, 2 y 4, puesto que la frecuencia característica del conjunto $L_{r}-C_{r}$ es mucho mas grande que la frecuencia de conmutación y la carga-descarga de $C_{r}$ es prácticamente instantánea. De esta forma solo intervienen tres etapas en el análisis. Y como la duración de la etapa 3, que es el tiempo que no está cerrado el interruptor principal, es $(1-D)$, resulta que

$$
d_{5} T+d_{6} T=D T
$$




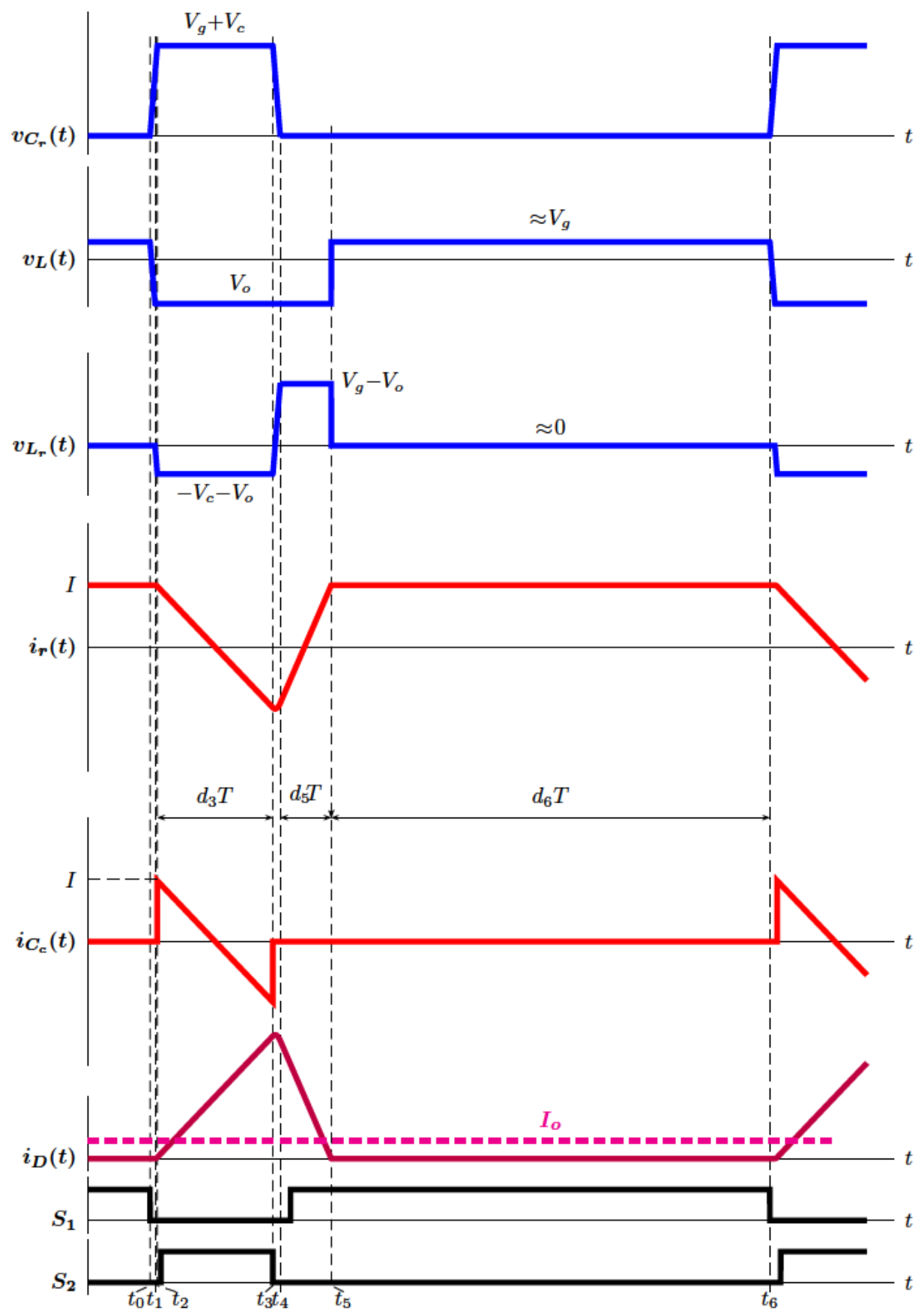

Figura C.12: Curvas principales de un reductor-elevador ZVS.

Las ecuaciones del régimen permanente se obtienen al considerar nulo tanto el balance voltios-segundo en las bobinas $L$ y $L_{r}$ como la carga neta en los condensadores $C_{o}$ y $C_{c}$. 


\section{TENSION MEDIA EN BOBINA PRINCIPAL.}

La tensión media en la bobina $L$ es nula. De aquí se obtiene una expresión para la tensión de salida en función de la entrada:

$$
\begin{aligned}
& \frac{1}{\mathscr{X}}\left[V_{C_{o}}\left(d_{3}+d_{5}\right) \not X+\left(V_{g}\right) d_{6} \not{X}\right]=0 \\
& \Rightarrow V_{C_{o}}=-V_{g} \frac{d_{6}}{\left(d_{3}+d_{5}\right)}=-V_{g} \frac{d_{6}}{\left(1-d_{6}\right)}
\end{aligned}
$$

A $d_{6}$ se le puede llamar ciclo efectivo de trabajo, $D_{e f}$, puesto que al identificar la expresión que relaciona la tensión de salida con la entrada en un reductorelevador, aparece ese término en lugar del término $D$ que identifica al ciclo de trabajo. También se puede identificar con el tiempo en el que la bobina principal del convertidor está absorbiendo energía. El valor del ciclo efectivo es menor que el valor del ciclo de trabajo empleado en las señales de disparo.

\section{TENSION MEDIA EN BOBINA RESONANTE.}

La tensión media en la bobina $L_{r}$ es nula. De aquí se obtiene una expresión para la tensión de $C_{c}$ en función de la tensión de entrada:

$$
\begin{aligned}
& \frac{1}{\mathscr{T}}\left[-\left(V_{c}+V_{C_{o}}\right) d_{3} \not{T}+\left(V_{g}-V_{C_{o}}\right) d_{5} \not\right]=0 \\
& \Rightarrow V_{c}=V_{g} \frac{d_{5}}{d_{3}}-V_{C_{o}} \frac{d_{3}+d_{5}}{d_{3}}=\left(V_{g}-V_{C_{o}}\right) \frac{d_{5}}{d_{3}}-V_{C_{o}}
\end{aligned}
$$

Sumando C.41 y C.42 también se puede despejar $V_{c}$ como:

$$
V_{c}=V_{g} \frac{d_{5}+d_{6}}{d_{3}}
$$

\section{CORRIENTE MEDIA EN EL CONDENSADOR DE CLAMP.}

La intensidad media en el condensador de clamp es nula. Entonces:

$$
\begin{aligned}
& \int_{t_{0}}^{t_{0}+T} i_{c}(t) d t=\int_{t_{2}}^{t_{3}} i_{c}(t) d t=\int_{0}^{d_{3} T}\left[I-\frac{V_{c}+V_{C_{o}}}{L_{r}} t\right] d t=0 \\
& {\left[I t-\frac{V_{c}+V_{C_{o}}}{2 L_{r}} t^{2}\right]_{0}^{d_{3} T}=0 \Rightarrow V_{c}=\frac{2 I L_{r}}{d_{3} T}-V_{C_{o}}}
\end{aligned}
$$

Sustituyendo este valor en la expresión empleada para $i_{c}(t)$ se puede obtener el valor de la intensidad del condensador transcurrido el tiempo $d_{3} T$. Entonces:

$$
i_{c}\left(d_{3} T\right)=I-\frac{V_{c}+V_{C_{o}}}{L_{r}} d_{3} T=I-\frac{\frac{2 I L_{r}}{d_{3} T}}{\not L_{r}} d_{3} T=-I
$$


DURACIÓN DEL INTERVALO $d_{5}$.

Se puede obtener a partir de igualar las expresiones de $V_{c}$ dadas en (C.42) y en (C.44). De esta forma:

$$
V_{c}=\frac{2 I L_{r}}{d / 3 T}-V_{C_{o}}=\left(V_{g}-V_{C_{o}}\right) \frac{d_{5}}{d_{3}}-V_{C_{o}} \Rightarrow d_{5}=\frac{2 I L_{r}}{\left(V_{g}-V_{C_{o}}\right) T}
$$

Expresión a la que se puede llegar también a partir de la tensión en la bobina $L r$ durante la etapa 5, puesto que es conocida la variación que experimenta la intensidad circulante por esa bobina y la tensión a la que está sometida.

$$
\begin{aligned}
\Delta i_{r}(t) & =i_{r}\left(t_{5}\right)-i_{r}\left(t_{4}\right) \approx i_{r}\left(t_{5}\right)-i_{r}\left(t_{3}\right)=I-(-I)=2 I \\
\Delta i_{r}(t) & =\frac{1}{L_{r}} \Delta v_{L_{r}}(t) \Delta t \equiv \frac{1}{L_{r}}\left(V_{g}-V_{C_{o}}\right) d_{5} T \\
\Rightarrow d_{5} & =\frac{2 I L_{r}}{\left(V_{g}-V_{C_{o}}\right) T}
\end{aligned}
$$

\section{TENSION DE SALIDA.}

Empleando (C.46) junto con (C.40) en (C.41) se obtiene una expresión para la tensión de salida en régimen permanente:

$$
\begin{aligned}
& V_{C_{o}}=-V_{g} \frac{d_{6}}{\left(1-d_{6}\right)}=-V_{g} \frac{\left(D-d_{5}\right)}{1-\left(D-d_{5}\right)} \\
& =\frac{-V_{g}\left(D-\frac{2 I L_{r}}{\left(V_{g}-V_{C_{o}}\right) T}\right)}{1-\left(D-\frac{2 I L_{r}}{\left(V_{g}-V_{C_{o}}\right) T}\right)}=\frac{-V_{g} \frac{\left(V_{g}-V_{C_{o}}\right) D-2 I L_{r} f}{\left(V_{g}-V_{C_{o}}\right)}}{\frac{\left(V_{g}-V_{C_{o}}\right)(1-D)+2 I L_{r} f}{\left(V_{g}-V_{C_{o}}\right)}} \\
& =\frac{-V_{g}\left(V_{g}-V_{C_{o}}\right) D-2 I L_{r} f}{\left(V_{g}-V_{C_{o}}\right)(1-D)+2 I L_{r} f} \\
& \Rightarrow V_{C_{o}}=-\left[\frac{V_{g} D}{(1-D)}-I \frac{2 L_{r} f}{(1-D)}\right]
\end{aligned}
$$

A esta expresión también se llega igualando (C.43) con (C.44) y despejando $V_{C_{o}}$. Como se puede observar, la tensión de salida de un reductor-elevador ZVS depende de la intensidad que circula por la bobina principal.

Esta expresión pone de manifiesto un elemento equivalente a una resistencia por la que pasa la intensidad $I$, que se puede considerar en serie con la bobina principal puesto que, en un elevador, esta intensidad es la misma que pasa la bobina. Su expresión sería (C.49).

$$
R_{e s L}=2 L_{r} f
$$

Respecto al signo negativo, aparece para indicar la inversión de la tensión respecto al criterio inicial marcado para realizar el análisis, por lo que 
podría eliminarse cuando se considere la tensión de salida invertida respecto al criterio inicial.

\section{CARACTERÍSTICA DE SALIDA.}

Considerando la igualdad de potencia de entrada y salida junto con (C.48) se puede obtener la relación entre la tensión de salida y la intensidad de salida

$$
\begin{aligned}
& \left.\begin{array}{l}
V_{o}=-\left[\frac{V_{g} D}{(1-D)}-I \frac{2 L_{r} f}{(1-D)}\right] \\
\begin{array}{l}
I=I_{g}-I_{o} \\
V_{o} I_{o}=V_{g} I_{g}
\end{array}
\end{array}\right\} \Rightarrow V_{o}=-\frac{V_{g} D}{1-D}+I_{o}\left(\frac{V_{o}}{V_{g}}-1\right) \frac{2 L_{r} f}{(1-D)} \Rightarrow \\
& \Rightarrow V_{o}=\frac{-\frac{V_{g} D}{1-D}-I_{o} \frac{2 L_{r} f}{1-D}}{1-I_{o} \frac{2 L_{r} f}{V_{g}(1-D)}}
\end{aligned}
$$

De nuevo el signo negativo aparece para indicar la inversión de la tensión respecto al criterio inicial marcado para realizar el análisis.

La derivada de esta expresión respecto a la intensidad indica como varía la tensión de salida ante las variaciones de la intensidad y tiene dimensiones de resistencia. La derivada tiene la siguiente expresión:

$$
\begin{aligned}
\frac{d V_{o}}{d I_{o}} & =\frac{-\frac{2 L_{r} f}{(1-D)}\left[1-I_{o} \frac{2 L_{r} f}{(1-D) V_{g}}\right]-\left[-\frac{V_{g} D}{(1-D)}-I_{o} \frac{2 L_{r} f}{(1-D)}\right]\left[-\frac{2 L_{r} f}{(1-D) V_{g}}\right]}{\left[1-I_{o} \frac{2 L_{r} f}{(1-D) V_{g}}\right]^{2}} \\
& =-2 L_{r} f\left[\frac{\frac{1}{(1-D)}}{1-I_{o} \frac{2 L_{r} f}{(1-D) V_{g}}}\right]^{2}
\end{aligned}
$$

Ahora, se sustituye $I_{o}$ después de despejarla de (C.50) y queda:

$$
\frac{d V_{o}}{d I_{o}}=-2 L_{r} f\left[\frac{V_{g}-V_{o}}{V_{g}}\right]^{2}
$$

\section{C.2.2. Considerando rizado.}

Si se considera que la bobina $L$ no es lo suficientemente grande como para suponer corriente constante, $I$, los circuitos equivalentes empleados para el análisis del circuito son diferentes y la relación entre la bobina principal y resonante aparece en las nuevas expresiones de las principales magnitudes. 
Los circuitos equivalentes están representados en la Figura C.13 donde se ha considerado la tensión constante en los condensadores $C_{o}$ y $C_{c}$, mientras que las principales magnitudes aparecen en la Figura C.14. Planteando el

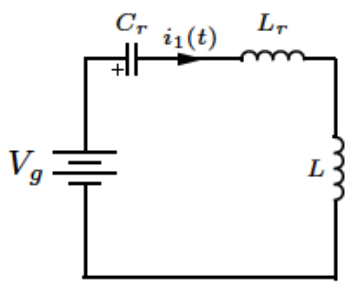

(a)

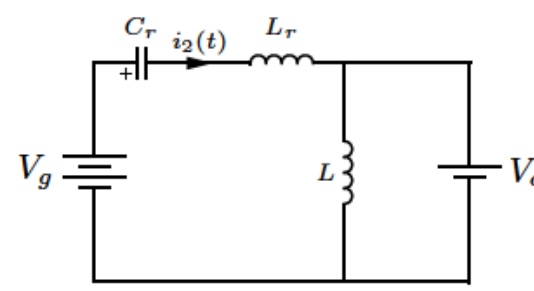

(b)

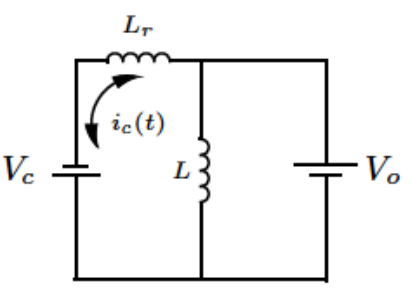

(c)

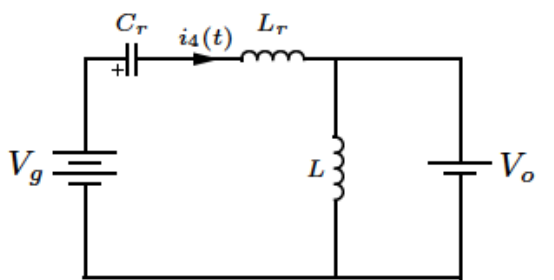

(d)

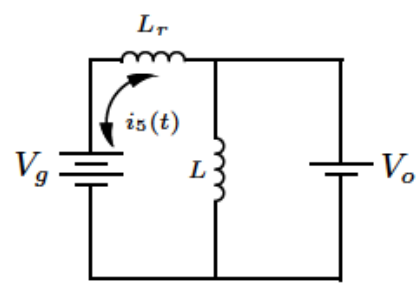

(e)

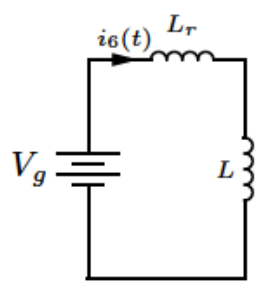

(f)

Figura C.13: Circuitos empleados para el análisis del reductor-elevador ZVS considerando rizado durante un ciclo completo: (a) Etapa 1. (b) Etapa 2. (c) Etapa 3. (d) Etapa 4. (e) Etapa 5. (f) Etapa 6.

balance voltios-segundo en las bobinas $L$ y $L_{r}$ y la carga neta nula en los condensadores $C_{o}$ y $C_{c}$ con las nuevas curvas, se obtienen otras ecuaciones del régimen permanente. Se desprecian las etapas 1,2 y 4 , y solo intervienen tres etapas.

TENSION MEDIA EN BOBINA PRINCIPAL CON RIZADO.

Como la tensión media en la bobina $L$ es nula, resulta que:

$$
\begin{gathered}
\frac{1}{\bar{T}}\left[V_{C_{o}}\left(d_{3}+d_{5}\right) \mathcal{T}+V_{g} \frac{L}{L+L_{r}} d_{6} T\right]=0 \\
\Rightarrow V_{C_{o}}=-V_{g} \frac{d_{6} \frac{L}{L+L_{r}}}{d_{3}+d_{5}}=-V_{g} \frac{d_{6} \frac{L}{L+L_{r}}}{\left(1-d_{6}\right)}
\end{gathered}
$$

TENSION MEDIA EN BOBINA RESONANTE CON RIZADO.

Como la tensión media en la bobina $L_{r}$ es nula, entonces:

$$
\begin{aligned}
& \frac{1}{\mathscr{T}}\left[-\left(V_{c}+V_{C_{o}}\right) d_{3} \mathscr{T}+\left(V_{g}-V_{C_{o}}\right) d_{5} \not{T}+V_{g} d_{6} \frac{L_{r}}{L+L_{r}}\right]=0 \\
& \Rightarrow V_{c}=-V_{C_{o}}-V_{C_{o}} \frac{d_{5}}{d_{3}}+V_{g} \frac{\left(d_{5}+d_{6} \frac{L_{r}}{L+L_{r}}\right)}{d_{3}}
\end{aligned}
$$




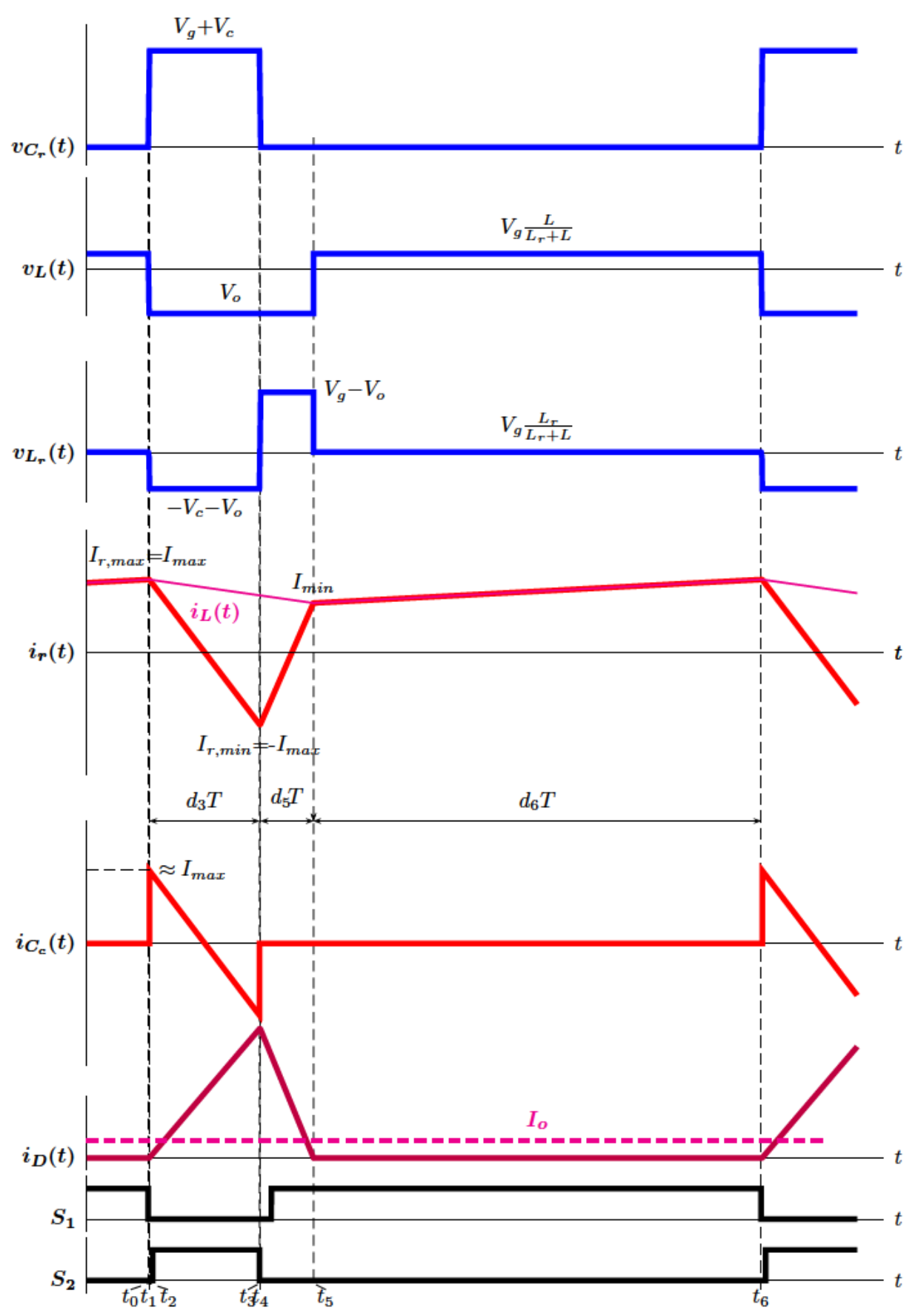

Figura C.14: Curvas principales de un reductor-elevador ZVS considerando rizado.

Sumando C.53 y C.54 también se puede despejar $V_{c}$ como:

$$
V_{c}=V_{g} \frac{d_{5}+d_{6}}{d_{3}}
$$


CORRIENTE MEDIA EN EL CONDENSADOR DE CLAMP CON RIZADO.

La intensidad media en el condensador de clamp es nula. Entonces:

$$
\begin{aligned}
& \int_{t_{0}}^{t_{0}+T} i_{c}(t) d t=\int_{t_{2}}^{t_{3}} i_{c}(t) d t=\int_{0}^{d_{3} T}\left[I_{\max }-\frac{V_{c}+V_{C_{o}}}{L_{r}} t\right] d t=0 \\
& {\left[I_{\text {max }} t-\frac{\left(V_{c}+V_{C_{o}}\right)}{2 L_{r}} t^{2}\right]_{0}^{d_{3} T}=0 \Rightarrow V_{c}=\frac{2 I_{\max } L_{r}}{d_{3} T}-V_{C_{o}}}
\end{aligned}
$$

Sustituyendo este valor en la expresión empleada para $i_{c}(t)$ se puede obtener el valor de la intensidad del condensador transcurrido el tiempo $d_{3} T$. Entonces:

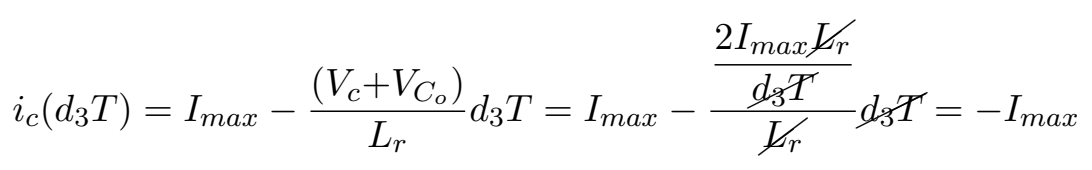

\section{DURACIÓN DEL INTERVALO $d_{5}$ CON RIZADO.}

Se puede calcular a partir de la tensión en la bobina $L r$ durante la etapa 5 , puesto que es conocida la variación que experimenta la intensidad circulante por esa bobina y la tensión a la que está sometida.

$$
\begin{aligned}
& \Delta i_{r, 5}=i_{r}\left(t_{5}\right)-i_{r}\left(t_{4}\right) \approx i_{r}\left(t_{5}\right)-i_{r}\left(t_{3}\right)=I_{\min }-\left(-I_{\max }\right)=2 I \\
& \Delta i_{r, 5}=\frac{1}{L_{r}} v_{L_{r}, 5} \Delta t \equiv \frac{1}{L_{r}}\left(V_{g}-V_{C_{o}}\right) d_{5} T \\
& \Rightarrow d_{5}=\frac{2 I L_{r}}{\left(V_{g}-V_{C_{o}}\right) T}
\end{aligned}
$$

Una expresión diferente se obtiene al igualar (C.54) con (C.56) y empleando (C.40) para despejar $d_{5}$ :

$$
\begin{aligned}
& =V_{C_{o}}-V_{C_{o}} \frac{d_{5}}{d_{3}}+V_{g} \frac{\left(d_{5}+\left(D-d_{5}\right) \frac{L_{r}}{L+L_{r}}\right)}{d_{3}}=\frac{2 I_{\max } L_{r}}{d_{3} T}=V_{C_{o}} \\
& \Rightarrow d_{5}=\frac{2 I_{\max } L_{r} f-V_{g} D \frac{L_{r}}{L+L_{r}}}{V_{g} \frac{L}{L+L_{r}}-V_{C_{o}}}
\end{aligned}
$$

\section{TENSION DE SALIDA CON RIZADO.}

Como $d_{6}=\left(D-d_{5}\right)$, resulta que:

$$
\begin{aligned}
d_{6} & =D-\frac{2 I_{\max } L_{r} f-V_{g} D \frac{L_{r}}{L+L_{r}}}{V_{g} \frac{L}{L+L_{r}}-V_{C_{o}}} \\
& =\frac{\left(V_{g}-V_{C_{o}}\right) D-2 I_{\max } L_{r} f}{V_{g} \frac{L}{L+L_{r}}-V_{C_{o}}}
\end{aligned}
$$


Por otro lado, despejando $d_{6}$ de (C.53) se obtiene:

$$
d_{6}=\frac{V_{C_{o}}}{V_{C_{o}}-V_{g} \frac{L}{L+L_{r}}}
$$

Igualando (C.60) y (C.61) se puede despejar $V_{C_{o}}$ como:

$$
V_{C_{o}}=-\left[\frac{V_{g} D}{(1-D)}-I_{\max } \frac{2 L_{r} f}{(1-D)}\right]
$$

También se podría conseguir si se iguala (C.55) con (C.56) y se despeja $V_{C_{o}}$. En ambos casos, la expresión obtenida es parecida a (C.48) que se ha obtenido sin considerar rizado. Solo se ha cambiado $I$ por $I_{\max }$. Y el término que multiplica a la intensidad se puede considerar como una resistencia en serie con $L, R_{e s L}$, ya definida anteriormente en (C.49).

Respecto al signo negativo, aparece para indicar la inversión de la tensión respecto al criterio seguido para el análisis, por lo que puede eliminarse cuando se considera la tensión de salida invertida respecto al criterio marcado inicialmente.

\section{RIZADO DE CORRIENTE EN $L$ CON RIZADO.}

Aplicando la definición de rizado de corriente en una bobina, se puede obtener el rizado de corriente en $L$ durante las etapas 3 a 5 o durante la etapa 6 siendo expresiones equivalentes en régimen permanente. Entonces:

$$
\begin{aligned}
\Delta i_{L, 3-5} & =\frac{1}{L}\left|-V_{o}\right|\left(d_{3}+d_{5}\right) T=\frac{1}{L} V_{o}\left(1-d_{6}\right) T \\
\Delta i_{L, 6} & =\frac{1}{L}\left|V_{g}\right| \frac{L}{L+L_{r}} d_{6} T=\frac{V_{g}}{L+L_{r}} d_{6} T
\end{aligned}
$$

Empleando la ecuación (C.61) se obtiene la expresión para el rizado de corriente en $L$ como:

$$
\Delta i_{L}=\frac{V_{o} V_{g} T}{\left(L+L_{r}\right) V_{o}-L V_{g}}
$$

\section{CARACTERÍSTICA DE SALIDA CON RIZADO.}

La relación entre la tensión de salida y la intensidad de salida se obtiene a partir de la expresión (C.62). Primero se expresa $I_{\max }$ por su expresión en función del rizado. Después se emplea la igualdad de potencia a la entrada y a la salida y la relación $I=I_{g}-I_{o}$, para obtener:

$$
\left.\begin{array}{l}
I=I_{g}-I_{o} \\
V_{g} I_{g}=V_{o} I_{o}
\end{array}\right\} \Rightarrow I=I_{o}\left(\frac{V_{o}}{V_{g}}-1\right)
$$

Para el rizado de corriente se emplea la segunda expresión de (C.63) y se considera $d_{6}=\left(D-d_{5}\right)$ :

$$
\Delta i_{L}=\frac{V_{g}}{L+L_{r}}\left(D-d_{5}\right) T=\frac{V_{g}}{L+L_{r}}\left(D-\frac{2 I L_{r}}{\left(V_{g}-V_{o}\right) T}\right) T
$$


Sólo queda sustituir (C.65) y (C.66) en (C.62) y despejar $V_{o}$ para obtener una función de la forma $V_{o}=f\left(I_{o}\right)$. Por tanto:

$$
\begin{aligned}
V_{o} & =-\left[\frac{V_{g} D}{(1-D)}-I_{\max } \frac{2 L_{r} f}{(1-D)}\right]=-\frac{V_{g} D}{(1-D)}+\left(I+\frac{1}{2} \Delta i_{L}\right) \frac{2 L_{r} f}{(1-D)} \\
& =-\frac{V_{g} D}{(1-D)}+I \frac{2 L_{r} f}{(1-D)}+\frac{1}{\not 2} \frac{V_{g}}{L+L_{r}}\left(D-\frac{2 I L_{r}}{\left(V_{g}-V_{o}\right) T}\right) \not \frac{2 L_{r} f}{(1-D)} \\
& =-\frac{V_{g} D}{(1-D)}\left[1-\frac{L_{r}}{L+L_{r}}\right]+I_{o} \frac{V_{o}}{V_{g}} \times \frac{2 L_{r} f}{(1-D)}-I_{o} \frac{2 L_{r} f}{(1-D)}-\frac{Y_{g}}{L+L_{r}} \times \frac{2 I_{o}\left(V_{o}-V_{g}\right) L_{r}^{2}}{V_{g}\left(V_{g}-V_{o}\right) T(1-D)} \\
& =-\frac{V_{g} D}{1-D}\left[1-\frac{L_{r}}{L+L_{r}}\right]-I_{o} \frac{2 L_{r} f}{1-D}\left[1-\frac{L_{r}}{L+L_{r}}\right]+I_{o} \frac{V_{o}}{V_{g}} \times \frac{2 L_{r} f}{(1-D)} \\
& \Rightarrow V_{o}\left(I_{o}\right)=\frac{\left[-\frac{V_{g} D}{1-D}-I_{o} \frac{2 L_{r} f}{1-D}\right] \frac{L}{L+L_{r}}}{1-I_{o} \frac{2 L_{r} f}{V_{g}(1-D)}}
\end{aligned}
$$

\section{C.2.3. Comparativa.}

Está claro que el análisis cuando se considera el rizado será de aplicación al circuito conmutado, donde se tienen en cuenta los valores de las bobinas, mientras que el análisis al no considerar rizado servirá para las expresiones derivadas del modelo promediado, dado que éste parte de la consideración de pequeño rizado.

Los resultados obtenidos para magnitudes tales como el ciclo de trabajo, la tensión de clamp y las variaciones de corriente, a partir de unos valores de diseño ponen de manifiesto estas diferencias. Por ejemplo, con los parámetros de funcionamiento recogidos en la Tabla C.1 se obtienen los valores indicados en la Tabla C.2, en el caso de no considerar el rizado de la corriente en la bobina principal y se compara con el obtenido cuando si se ha considerado.

\begin{tabular}{llll}
\hline \hline$V_{g}$ & $160 \mathrm{~V}$ & $C_{o}$ & $5 \mu \mathrm{F}$ \\
$P$ & $400 \mathrm{~W}$ & $C_{c}$ & $0,47 \mu \mathrm{F}$ \\
$V_{\text {out }}$ & $400 \mathrm{~V}$ & $L$ & $800 \mu \mathrm{H}$ \\
$f$ & $100 \mathrm{kHz}$ & $L_{r}$ & $30 \mu \mathrm{H}$ \\
$t_{m}$ & $200 \mathrm{~ns}$ & $C_{r}$ & $0,6 \mathrm{nF}$ \\
\hline
\end{tabular}

Tabla C.1: Condiciones de trabajo de un Red-Elev. ZVS.

En el Reductor-elevador ZVS la intensidad en la bobina principal es igual a la intensidad en la entrada mas de salida, $I_{g}+I_{o}$. Al considerar el rizado, el ciclo de trabajo obtenido es mayor. La tensión de clamp, $V_{c}$ también es diferente. Es lógico dado que depende del ciclo de trabajo. Si se calcula su valor a partir de la expresión que incluye la intensidad, también se obtendrá 


\begin{tabular}{ccccccccc}
\hline & $I$ & $d_{5}$ & $d_{6}$ & $D$ & $V_{c}$ & $\Delta_{i_{L}}$ & $I_{\max }$ & $\begin{array}{c}I_{\min } \\
(\mathrm{A})\end{array}$ \\
& $(\mathrm{A})$ & - & - & - & $(\mathrm{V})$ & $(\mathrm{A})$ & $(\mathrm{A})$ \\
\hline SIN RIZADO & \multirow{2}{*}{3,5} & \multirow{2}{*}{0,0375} & 0,7143 & 0,7518 & 647,2 & 0 & - & - \\
CON RIZADO & & & 0,7217 & 0,7592 & 504,45 & 1,391 & 4,196 & 2,805 \\
\hline \hline
\end{tabular}

Tabla C.2: Parámetros calculados cuando se considera o no el rizado.

un valor diferente según se considere valor medio (sin rizado) o valor máximo (cuando hay rizado).

En ambos análisis se han considerado despreciables algunas etapas por su pequeña duración en relación a las otras. Esta hipótesis se ha validado midiendo la duración de las seis etapas para los parámetros de funcionamiento de las dos tablas anteriores. Con ayuda de un programa de cálculo simbólico se han planteado las ecuaciones de estado durante las seis etapas y se han resuelto las ecuaciones y obtenido la fracción que ocupa cada etapa dentro de un periodo completo. Se han indicado en la Tabla C.3

\begin{tabular}{ccccccc}
\hline & $d_{1}$ & $d_{2}$ & $d_{3}$ & $d_{4}$ & $d_{5}$ & $d_{6}$ \\
\hline SIN RIZADO & $9,6 \times 10^{-3}$ & $1,5 \times 10^{-3}$ & 0,2467 & $12,2 \times 10^{-3}$ & $31,8 \times 10^{-3}$ & 0,6983 \\
CON RIZADO & $8,2 \times 10^{-3}$ & $1,28 \times 10^{-3}$ & 0,2388 & $10,1 \times 10^{-3}$ & $32,8 \times 10^{-3}$ & 0,7088 \\
\hline \hline
\end{tabular}

Tabla C.3: Fracción de tiempo para cada etapa por las que evoluciona el Red-Elev. ZVS.

A la vista de estos resultados, se puede comprobar que las etapas 1, 2 y 4 son las de menor duración por lo que, como se había previsto, se pueden despreciar.

En cada uno de los dos análisis realizados, la característica de salida obtenida muestra una relación diferente entre tensión e intensidad de salida. Cuando ambas se representan gráficamente se obtiene prácticamente la misma curva, como se pone de manifiesto al observar la Figura C.15 que contiene la característica de salida de un reductor-elevador ZVS con los datos de la Tabla C.1. En este caso, la curva representada sin considerar rizado emplea el valor de ciclo de trabajo obtenido con este mismo análisis, mientras que la curva representada al considerar el rizado está obtenida con el ciclo de trabajo calculado con rizado no despreciable.

\section{C.3. Modelo promediado}

La obtención del modelo promediado se puede conseguir mediante el promediado de las formas de onda, mediante el promediado de circuitos o mediante el promediado en el espacio de estados. En esa sección se va a obtener el modelo promediado del reductor-elevador ZVS descrito en la 


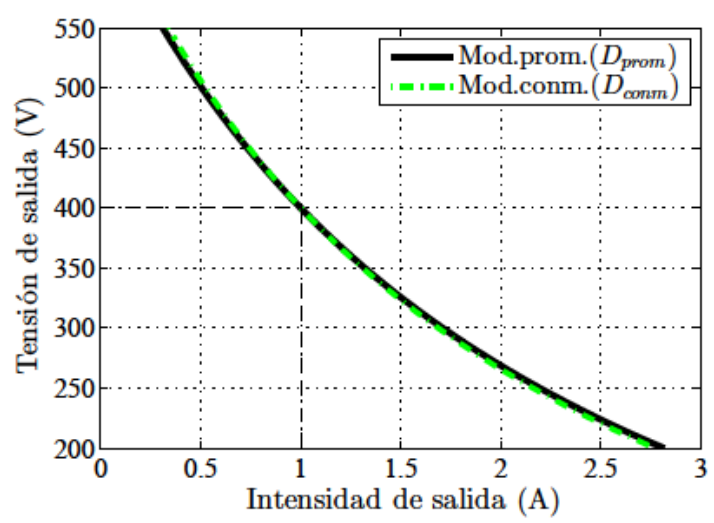

Figura C.15: Característica de salida de un Red.-Elev. ZVS.

sección anterior, de la misma forma que se ha hecho para el reductor ZVS en capítulos anteriores.

\section{C.3.1. Modelo promediado para el reductor-elevador ZVS}

En la sección C.1 se ha explicado el funcionamiento en régimen permanente del reductor-elevador ZVS. Del análisis se deduce que la corriente en la bobina resonante $L_{r}$ y la tensión en el condensador resonante $C_{r}$ son las variables de estado rápidas y el resto son las variables de estado lentas. El procedimiento a seguir es obtener las expresiones para las variables rápidas $i_{L_{r}}(t)$ y $v_{C_{r}}(t)$, en un periodo de conmutación (comprobar que son periódicas). La variables lentas se suponen de valor constante para este cálculo. Las expresiones obtenidas, dependerán, por tanto, de las variables lentas y también del tiempo. Éstas se sustituyen en las ecuaciones de estado de las variables lentas de forma que queden desacopladas respecto a las variables rápidas. Se aplica ahora un promedio estándar en un periodo de conmutación para eliminar la dependencia con el tiempo, consiguiéndose un modelo promediado de tercer orden (orden reducido) para las tres variables de estado lentas, $v_{C_{c}}(t), v_{C_{o}}(t)$ y $i_{L}(t)$.

Aunque matemáticamente no es necesario, se suelen realizar algunas aproximaciones antes de empezar para simplificar los cálculos a realizar. En primer lugar, se ignoran los tiempos muertos entre las conmutaciones de $S_{1}$ y $S_{2}$ que normalmente son muy pequeños en comparación con el periodo. También se desprecian los intervalos resonantes, como se hace en (ATHALYE y otros, 2001) para obtener el modelo completo con el método del interruptor promediado. En este punto cabe indicar que el efecto debido a tener en cuenta los intervalos resonantes sería tan solo a frecuencias muy altas y fuera del rango de interés (que, generalmente, suele ser una fracción de la frecuencia 
de conmutación porque es donde se aplica el control en lazo cerrado). Además se supone que el condensador de clamp mantiene su tensión constante, lo que quiere decir que la frecuencia de resonancia entre $L_{r}$ y $C_{c}$ es bastante menor que la frecuencia de conmutación.

Considerando estas simplificaciones desaparecen los intervalos resonantes (Etapa 1, 2 y 4) y el tiempo muerto de la etapa 5, de forma que un periodo se puede escribir como:

$$
\begin{aligned}
T & \approx d_{3} T+d_{5} T+d_{6} T \\
(1-d) T & =d_{3} T \\
d T & =d_{5} T+d_{6} T
\end{aligned}
$$

puesto que $d_{1} T=d_{2} T=d_{4} T=0$.

Las curvas principales están representadas en la Figura C.12. Considerando los valores constantes para las variables lentas, la tensión $v_{C_{r}}(t)$, tiene la siguiente expresión ${ }^{1}$ :

$$
v_{C_{r}}(t)= \begin{cases}v_{g}+v_{C_{c}} & t \in\left[t_{2}, t_{3}\right] \\ 0 & t \in\left[t_{4}, t_{5}\right] \\ 0 & t \in\left[t_{5}, t_{6}\right]\end{cases}
$$

mientras que la intensidad en la bobina resonante, $i_{L_{r}}(t)$ se expresa como

$$
i_{L_{r}}(t)= \begin{cases}i_{L}-\frac{v_{C_{c}}+v_{C_{o}}}{L_{r}}\left(t-t_{2}\right) & t \in\left[t_{2}, t_{3}\right] \\ i_{L}+\frac{v_{g}-v_{C_{o}}}{L_{r}}\left(t-t_{5}\right) & t \in\left[t_{4}, t_{5}\right] \\ i_{L} & t \in\left[t_{5}, t_{6}\right]\end{cases}
$$

Se obtiene el parámetrod $d_{5}$ a partir de la igualdad de valores de $i_{L_{r}}$ en $t_{3}=t_{4}$. Es decir:

$$
i_{L_{r}}\left(t_{3}\right)=i_{L_{r}}\left(t_{4}\right)
$$

Entonces, con (C.70) y (C.71) se obtiene una solución para $d_{5}$

$$
d_{5}=\frac{v_{C_{c}}+v_{C_{o}}}{v_{g}-v_{C_{o}}}(1-d)
$$

Para el resto de variables lentas si es necesario escribir las ecuaciones de estado. Necesitamos una ecuación para la intensidad en la bobina principal, $i_{L}(t)$; otra ecuación para la tensión en el condensador de salida, $v_{C_{o}}(t)$; y otra ecuación para la tensión en el condensador de clamp, $v_{C_{c}}(t)$. Estas ecuaciones

\footnotetext{
${ }^{1}$ Se mantiene la numeración de los intervalos, considerando que $t_{0}=t_{1}=t_{2}$ y que $t_{3}=t_{4}$.
} 
son:

$$
\begin{aligned}
& v_{L}(t)=L \frac{d i_{L}(t)}{d t}= \begin{cases}v_{C_{o}}(t) & t \in\left[t_{2}, t_{3}\right] \\
v_{C_{o}}(t) & t \in\left[t_{4}, t_{5}\right] \\
v_{g}(t) & t \in\left[t_{5}, t_{6}\right]\end{cases} \\
& i_{C_{o}}(t)=C_{o} \frac{d v_{C_{o}}(t)}{d t}= \begin{cases}i_{L_{r}}(t)-i_{L}(t)-\frac{v_{C_{o}}(t)}{R} & t \in\left[t_{2}, t_{3}\right] \\
i_{L_{r}}(t)-i_{L}(t)-\frac{v_{C_{o}}(t)}{R} & t \in\left[t_{4}, t_{5}\right] \\
-\frac{v_{C_{o}}(t)}{R} & t \in\left[t_{5}, t_{6}\right]\end{cases} \\
& i_{C_{c}}(t)=C_{c} \frac{d v_{C_{c}}(t)}{d t}= \begin{cases}i_{L_{r}}(t) & t \in\left[t_{2}, t_{3}\right] \\
0 & t \in\left[t_{4}, t_{5}\right] \\
0 & t \in\left[t_{5}, t_{6}\right]\end{cases}
\end{aligned}
$$

La ecuación (C.73) es la ecuación de estado de un convertidor reductorelevador sin enclavamiento activo, mientras que (C.74) y (C.75) son las ecuaciones del condensador de salida y del condensador de esta red añadida. $\mathrm{Al}$ igual que en el reductor-elevador convencional, se puede añadir una ecuación relacionada con la intensidad de entrada al convertidor, que es (C.76):

$$
i_{g}(t)= \begin{cases}0 & t \in\left[t_{2}, t_{3}\right] \\ i_{L_{r}}(t) & t \in\left[t_{4}, t_{5}\right] \\ i_{L_{r}}(t) & t \in\left[t_{5}, t_{6}\right]\end{cases}
$$

Cuando se sustituyen las variables rápidas en las ecuaciones anteriores, se consigue tener un conjunto de ecuaciones de estado donde solo intervienen las variables de estado lentas, es decir, se han desacoplado de las variables de estado rápidas, que son

$$
\begin{aligned}
& L \frac{d i_{L}(t)}{d t}= \begin{cases}v_{C_{o}}(t) & t \in\left[t_{2}, t_{3}\right] \\
v_{C_{o}}(t) & t \in\left[t_{4}, t_{5}\right] \\
v_{g}(t) & t \in\left[t_{5}, t_{6}\right]\end{cases} \\
& C_{o} \frac{d v_{C_{o}}(t)}{d t}= \begin{cases}-\frac{v_{C_{c}}(t)+v_{C_{o}}(t)}{L_{r}}\left(t-t_{2}\right)-\frac{v_{C_{o}}(t)}{R} & t \in\left[t_{2}, t_{3}\right] \\
\frac{v_{g}(t)-v_{C_{o}}(t)}{L_{r}}\left(t-t_{5}\right)-\frac{v_{C_{o}}(t)}{R} & t \in\left[t_{4}, t_{5}\right] \\
-\frac{v_{C_{o}}(t)}{R} & t \in\left[t_{5}, t_{6}\right]\end{cases} \\
& C_{c} \frac{d v_{C_{c}}(t)}{d t}= \begin{cases}i_{L}(t)-\frac{v_{C_{c}}(t)+v_{C_{o}}(t)}{L_{r}}\left(t-t_{2}\right) & t \in\left[t_{2}, t_{3}\right] \\
0 & t \in\left[t_{4}, t_{5}\right] \\
0 & t \in\left[t_{5}, t_{6}\right]\end{cases}
\end{aligned}
$$


junto con

$$
i_{g}(t)= \begin{cases}0 & t \in\left[t_{2}, t_{3}\right] \\ i_{L}(t)+\frac{v_{g}(t)-v_{C_{o}}(t)}{L_{r}}\left(t-t_{5}\right) & t \in\left[t_{4}, t_{5}\right] \\ i_{L}(t) & t \in\left[t_{5}, t_{6}\right]\end{cases}
$$

Considerando la aproximación de pequeño rizado (valores instantáneos son muy parecidos a los valores promedio), es posible sustituir los valores instantáneos de las variables de estado por sus valores promedio, (por ejemplo, $i_{L}(t)$ se sustituye por $\bar{i}_{L}$, que es igual que $\left\langle i_{L}(t)\right\rangle_{T}$ donde se ha simplificado la notación, y así con el resto). De esta forma, las ecuaciones desacopladas se transforman en

$$
\begin{gathered}
L \frac{d i_{L}(t)}{d t} \approx \begin{cases}\bar{v}_{C_{o}} & t \in\left[t_{2}, t_{3}\right] \\
\bar{v}_{C_{o}} & t \in\left[t_{4}, t_{5}\right] \\
\bar{v}_{g} & t \in\left[t_{5}, t_{6}\right]\end{cases} \\
C_{o} \frac{d v_{C_{o}}(t)}{d t} \approx \begin{cases}-\frac{\bar{v}_{C_{c}}+\bar{v}_{C_{o}}}{L_{r}}\left(t-t_{2}\right)-\frac{\bar{v}_{C_{o}}}{R} & t \in\left[t_{2}, t_{3}\right] \\
\frac{\bar{v}_{g}-\bar{v}_{C_{o}}}{L_{r}}\left(t-t_{5}\right)-\frac{\bar{v}_{C_{o}}}{R} & t \in\left[t_{4}, t_{5}\right] \\
-\frac{\bar{v}_{C_{o}}}{R} & t\left[t_{5}, t_{6}\right]\end{cases} \\
C_{c} \frac{d v_{C_{c}}(t)}{d t} \approx \begin{cases}\bar{i}_{L}-\frac{\bar{v}_{C_{c}}+\bar{v}_{C_{o}}}{L_{r}}\left(t-t_{2}\right) & t \in\left[t_{2}, t_{3}\right] \\
0 & t \in\left[t_{4}, t_{5}\right] \\
0 & t \in\left[t_{5}, t_{6}\right]\end{cases} \\
i_{g}(t) \approx \begin{cases}0 & t \in\left[t_{2}, t_{3}\right] \\
\bar{i}_{L}+\frac{\bar{v}_{g}-\bar{v}_{C_{o}}}{L_{r}}\left(t-t_{5}\right) & t \in\left[t_{4}, t_{5}\right] \\
\bar{i}_{L} & t \in\left[t_{5}, t_{6}\right]\end{cases}
\end{gathered}
$$

con

$$
d_{5} \approx \frac{\bar{v}_{C_{c}}+\bar{v}_{C_{o}}}{\bar{v}_{g}-\bar{v}_{C_{o}}}(1-d)
$$

Por último, hay que eliminar la dependencia del tiempo realizando el promedio de las ecuaciones en un intervalo, de donde se obtiene el modelo promediado para el red.-elevador ZVS, que es un modelo de orden tres, (es un modelo de orden reducido frente al modelo de orden completo que sería 
de orden cinco).

$$
\begin{aligned}
L \frac{d \bar{i}_{L}}{d t} & =\left(d-d_{5}\right) \bar{v}_{g}+\left(1-d+d_{5}\right) \bar{v}_{C_{o}} \\
C_{o} \frac{d \bar{v}_{C_{o}}}{d t} & =-\frac{\bar{v}_{g}-\bar{v}_{C_{o}}}{2 L_{r} f_{s}} d_{5}^{2}-\frac{\bar{v}_{C_{c}}+\bar{v}_{C_{o}}}{2 L_{r} f_{s}}(1-d)^{2}-\frac{\bar{v}_{C_{o}}}{R} \\
C_{c} \frac{d \bar{v}_{C_{c}}}{d t} & =\bar{i}_{L}(1-d)-\frac{\bar{v}_{C_{c}}+\bar{v}_{C_{o}}}{2 L_{r} f_{s}}(1-d)^{2} \\
\bar{i}_{g} & =\bar{i}_{L} d-\frac{\bar{v}_{g}-\bar{v}_{C_{o}}}{2 L_{r} f_{s}} d_{5}^{2}
\end{aligned}
$$

Con las ecuaciones anteriores se consigue obtener un circuito promediado para este convertidor. Previamente hay que sustituir el parámetro $d_{5}$ por su valor, y se obtienen las ecuaciones indicadas en (C.90).

$$
\begin{aligned}
L \frac{d \bar{i}_{L}}{d t} & =\bar{v}_{g} d-\bar{v}_{C_{c}}(1-d) \\
C_{o} \frac{d \bar{v}_{C_{o}}}{d t} & =-\frac{\bar{v}_{C_{o}}}{R}-\frac{\left(\bar{v}_{C_{c}}+\bar{v}_{C_{o}}\right)^{2}}{\bar{v}_{g}-\bar{v}_{C_{o}}} \frac{(1-d)^{2}}{2 L_{r} f_{s}}-\frac{\bar{v}_{C_{c}}+\bar{v}_{C_{o}}}{2 L_{r} f_{s}}(1-d)^{2} \\
C_{c} \frac{d \bar{v}_{C_{c}}}{d t} & =\bar{i}_{L}(1-d)-\frac{\bar{v}_{C_{c}}+\bar{v}_{C_{o}}}{2 L_{r} f_{s}}(1-d)^{2} \\
\bar{i}_{g} & =\bar{i}_{L} d-\frac{\left(\bar{v}_{C_{c}}+\bar{v}_{C_{o}}\right)^{2}}{\bar{v}_{g}-\bar{v}_{C_{o}}} \frac{(1-d)^{2}}{2 L_{r} f_{s}}
\end{aligned}
$$

El circuito obtenido es el indicado en la figura C.16, donde $r_{e q}$ es una resistencia equivalente sin pérdidas que consume la energía generada por la dos fuentes dependientes de corriente de valor $i_{e q}$.

$$
\begin{gathered}
r_{e q}=\frac{2 L_{r} f_{s}}{(1-d)^{2}} \\
i_{e q}=\frac{\left(v_{C_{c}}+v_{C_{o}}\right)^{2}}{\left(v_{g}-v_{C_{o}}\right) r_{e q}}
\end{gathered}
$$

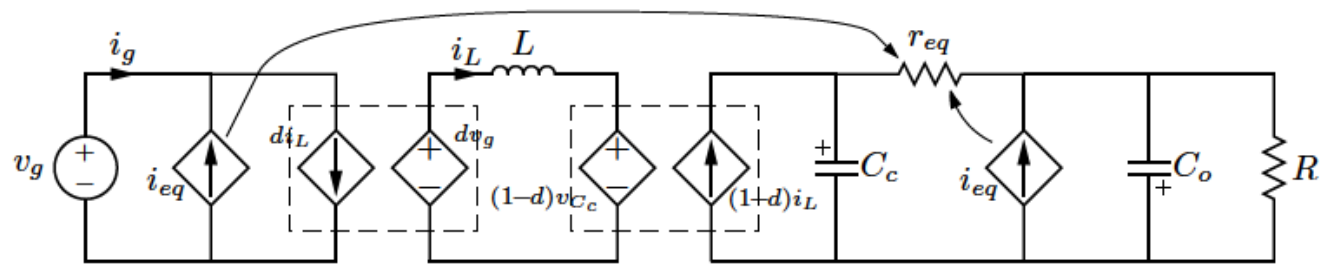

Figura C.16: Modelo promediado de un reductor elevador ZVS. 


\section{C.3.2. Balance energético}

En el modelo promediadohay tres elementos involucrados, la fuente de tensión en la entrada las fuente de corriente equivalente en la entrada y la fuente de corriente equivalente en la salida. La energía de la fuente de tensión se consume en la carga y se almacena o devuelve en los elementos reactivos, mientras que la energía generada en la fuente dependiente de la entrada se consume en $r_{e q}$, al igual que sucede con la energía generada en la fuente dependiente de la salida. Veámoslo:

En $r_{e q}$, la potencia consumida es igual a

$$
P_{r_{e q}}=\frac{\left(v_{C_{c}}+v_{C_{o}}\right)^{2}}{r_{e q}}
$$

Multiplicando arriba y abajo por $\left(v_{g}-v_{C_{o}}\right)$, se obtiene una expresión

$$
P_{r_{e q}}=\frac{\left(v_{C_{c}}+v_{C_{o}}\right)^{2}}{r_{e q}\left(v_{g}-v_{C_{o}}\right)}\left(v_{g}-v_{C_{o}}\right)
$$

en la que, agrupando, se obtienen dos productos de una intensidad $i_{e q}$ por una tensión,

$$
P_{r_{e q}}=i_{e q} v_{g}-i_{e q} v_{C_{o}}=P_{i_{e q 1}}+P_{i_{e q_{2}}}
$$

por tanto, esta potencia coincide con la potencia generada por las fuentes de corriente dependientes.

\section{C.3.3. Validación del modelo promediado}

La validación del modelo promediado se realiza mediante simulación, y se compara su comportamiento, tanto en régimen permanente como en régimen dinámico, con un convertidor conmutado. Para ello se eligen de nuevo los parámetros de funcionamiento recogidos en la Tabla C.1, empleando en el convertidor conmutado el ciclo de trabajo calculado al considerar rizado. En el modelo promediado se ha considerado el ciclo de trabajo calculado cuando no se ha considerado el rizado.

Los resultados muestran una dinámica similar para el convertidor conmutado y el modelo promediado. En cuanto al régimen permanente, hay un error puesto que la tensión de salida no coincide. El motivo de esta diferencia tiene su causa en que en el modelo promediado no está incluido el tiempo muerto, $t_{m}$, que hay que dejar entre disparos de los transistores principal y de clamp, entre otras cosas, para facilitar las conmutaciones suaves. Además, parte de este tiempo muerto se emplea en cargar o descargar el condensador 


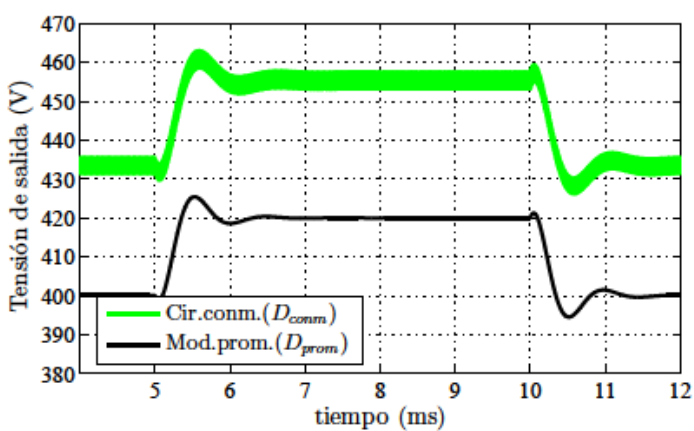

(a)

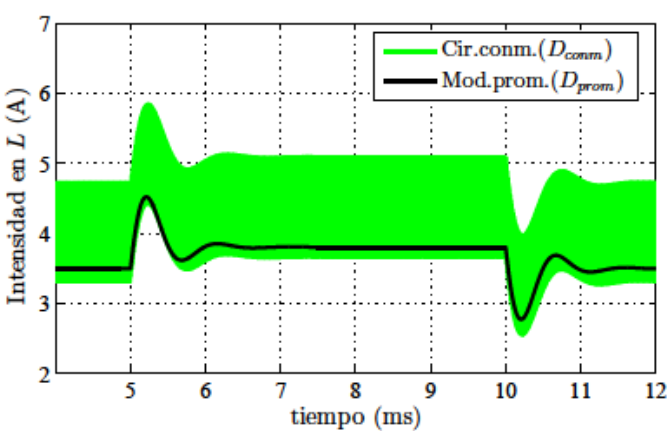

(b)

Figura C.17: Red.-Elevador ZVS. Respuesta a escalón del ciclo de trabajo: a) Tensión de salida; y b) Intensidad en la bobina.

resonante, y corresponde a las tres etapas que se desprecian para obtener el modelo promediado. En concreto la etapa 4, siendo su duración igual a $d_{4} T$.

Por tanto, hay que realizar una corrección en el ciclo de trabajo empleado para el circuito conmutado, en base a los tiempos mencionados, puesto que ya está incluido de forma implícita en la generación de disparos del propio circuito conmutado. De esta forma, se rehacen las simulaciones del circuito conmutado con el ciclo de trabajo corregido, $D_{\text {corr }}$, como:

$$
D_{\text {corr }}=D_{\text {conm }}-\frac{t_{m}-d_{4} T}{T}
$$

y se obtienen los resultados de la Figura C.18. La corrección equivalente pero para el modelo promediado consistiría en añadir este tiempo extra al ciclo de trabajo empleado en dicho modelo.

Para apreciar la mejora en los resultados se representa el valor medio de la tensión de salida y la intensidad en la bobina para el circuito conmutado antes y después de la corrección y se comparan con el modelo promediado. En la Figura C.19 se pueden comprobar estas curvas.

\section{C.4. Modelo en régimen permanente}

El modelo en régimen permanente se puede obtener a partir del modelo promediado (o de gran señal) sustituyendo los valores promedio por valores constantes (por ejemplo, $\bar{i}_{L}$ se sustituye por $I$ ). De esta forma, las ecuaciones del modelo en régimen permanente serán las indicadas a continuación. Nótese que la derivada de una constante es cero, de ahí, que haya ecuaciones con un 


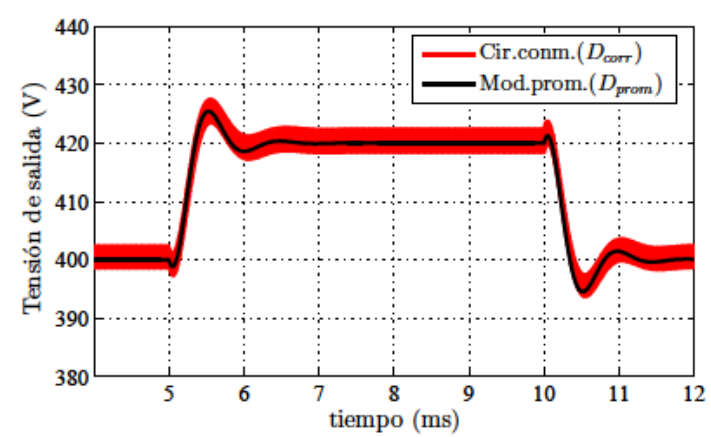

(a)

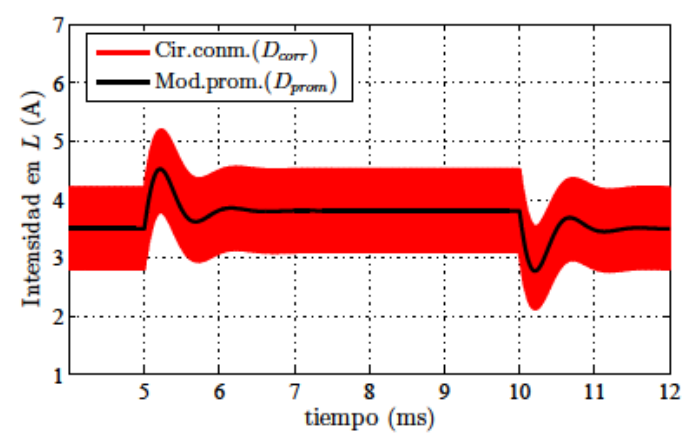

(b)

Figura C.18: Red.-Elevador ZVS. Respuesta a escalón en el ciclo de trabajo incluyendo corrección: a) Tensión de salida; y b) Intensidad en la bobina.

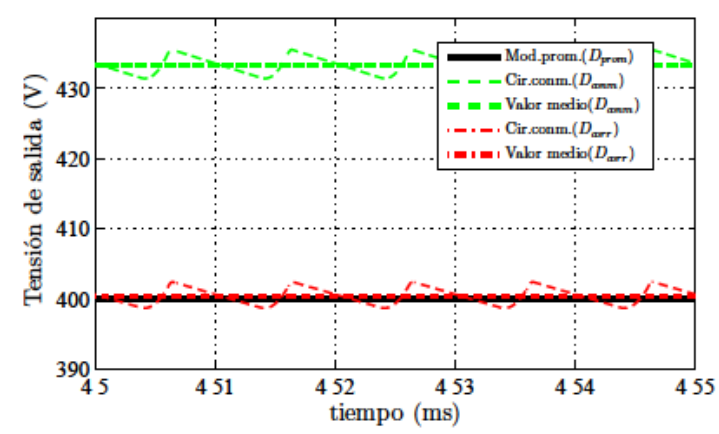

(a)

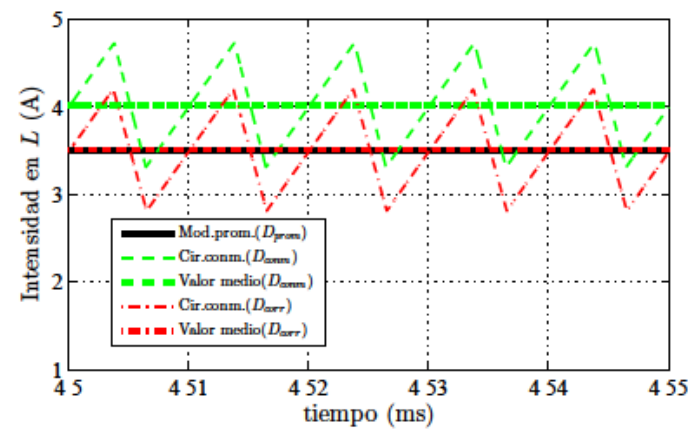

(b)

Figura C.19: Red.-Elevador ZVS en régimen permanente. Comparación entre el modelo promediado y los valores medios con y sin corrección en el ciclo de trabajo del circuito conmutado: a) Tensión de salida; y b) Intensidad en la bobina.

cero a la izquierda de la igualdad:

$$
\begin{aligned}
0 & =D V_{g}-(1-D) V_{c} \\
0 & =-I_{e q}-\frac{V_{c}+V_{C_{o}}}{R_{e q}}-\frac{V_{C_{o}}}{R} \\
0 & =(1-D) I-\frac{V_{c}+V_{C_{o}}}{R_{e q}} \\
I_{g} & =D I-I_{e q}
\end{aligned}
$$


donde

$$
R_{e q}=\frac{2 L_{r} f_{s}}{(1-D)^{2}} \quad I_{e q}=\frac{\left(V_{c}+V_{C_{o}}\right)^{2}}{\left(V_{g}-V_{C_{o}}\right) R_{e q}}
$$

A estas ecuaciones se puede llegar también a partir del circuito promediado de la figura C.16. Basta con sustituir los condensadores por un circuito abierto y las bobinas con un cortocircuito. De esta forma se obtienen los circuitos de las figuras C.20a y C.20b.

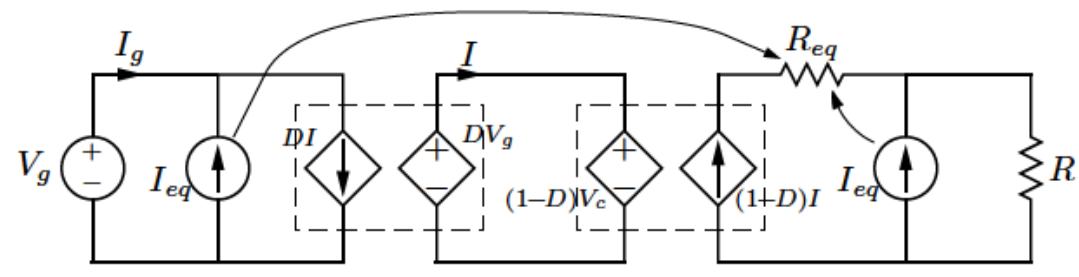

(a)

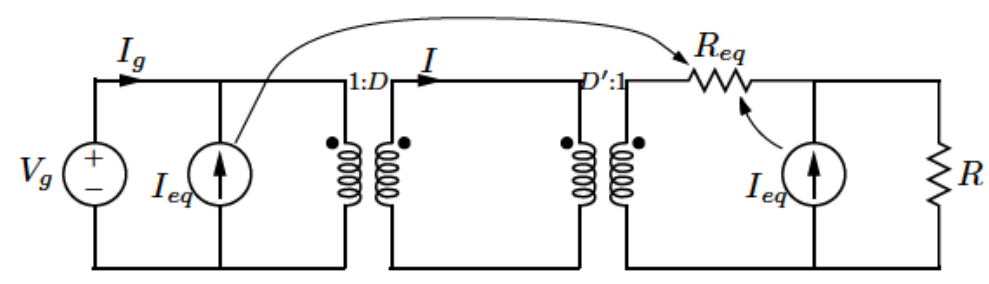

(b)

Figura C.20: Circuito en régimen permanente de un reductor elevador ZVS en MCC: (a) con fuentes dependientes; (b) con transformadores ideales.

Se obtienen las mismas expresiones para las principales magnitudes en régimen permanente cuando se deducen de (C.97) que a partir de los circuitos de la figura C.20.

$$
\begin{aligned}
V_{c} & =\frac{D V_{g}}{(1-D)} \\
I_{o} & =\frac{V_{C_{o}}}{R}-(1-D) I-I_{e q} \\
V_{C_{o}} & =(1-D) I R_{e q}-V_{c} \\
I_{g} & =D I-I_{e q}
\end{aligned}
$$

$\mathrm{Si}$ en estas expresiones se elimina $L_{r}$, (que significa que desaparece el circuito de enclavamiento activo), se obtienen las expresiones en régimen permanente para un reductor convencional y lo mismo sucede con los circuitos en régimen permanente de ambas topologías.

La expresión que relaciona la tensión de salida con la intensidad se obtiene fácilmente a partir de (C.99) como:

$$
V_{C_{o}}=-\frac{D V_{g}}{1-D}+(1-D) I R_{e q}
$$


donde se observa que la tensión de salida de un reductor-elevador ZVS se puede asimilar a un reductor-elevador convencional que tiene a la salida una resistencia de valor $R_{e q}$, con la particularidad de que es una resistencia equivalente sin pérdidas, y evoluciona con la corriente demandada por la carga (que en un reductor elevador convencional es igual a $I(1-D)$ ).

Por otro lado, la relación entre intensidades de entrada y salida se obtiene a partir de la resta de la cuarta y segunda expresión de (C.99), de donde:

$$
I_{g}-I_{o}=I
$$

\section{C.4.1. Balance energético}

En el modelo en régimen permanente hay tres elementos que generan energía, la fuente de tensión de la entrada, y la dos fuentes dependientes de corriente en la etapa de entrada y de salida. La energía generada en estas fuente de corriente se consume toda en $R_{e q}$. Veámoslo.

En $R_{e q}$, la potencia consumida es igual a

$$
P_{R_{e q}}=\frac{\left(V_{c}+V_{C_{o}}\right)^{2}}{R_{e q}}
$$

Multiplicando arriba y abajo por $\left(V_{g}-V_{C_{o}}\right)$, se obtiene una expresión

$$
P_{R_{e q}}=\frac{\left(V_{c}+V_{C_{o}}\right)^{2}}{R_{e q}\left(V_{g}-V_{C_{o}}\right)}\left(V_{g}-V_{C_{o}}\right)
$$

en la que, agrupando, se obtienen dos productos de una intensidad $I_{e q}$ por una tensión,

$$
P_{R_{e q}}=I_{e q} V_{g}-I_{e q} V_{C_{o}}=P_{I_{e q_{1}}}+P_{I_{e q_{2}}}
$$

por tanto, esta potencia coincide con la potencia generada por las fuentes de corriente dependientes.

Por otro lado, toda la potencia generada en $V_{g}$ se consume en la carga $R$ a la salida. La potencia que se genera es:

$$
\begin{aligned}
& P_{g}=V_{g} I_{g}=V_{g}\left(D I-I_{e q}\right)=V_{g} D I-V g I_{e q} \\
& =V_{c}(1-D) I-V_{g} I_{e q}=\left[(1-D) I R_{e q}-V_{C_{o}}\right](1-D) I-V_{g} I_{e q} \\
& =(1-D)^{2} R_{e q}-V_{C_{o}}(1-D) I-V_{g} I_{e q} \\
& =\frac{\left(V_{c}+V_{C_{o}}\right)^{2}}{R_{e q}}-V_{C_{o}}\left[-I_{o}-I_{e q}\right]-V_{g} I_{e q} \\
& =\frac{\left(V_{C_{c}}+V_{C_{o}}\right)^{2}}{R_{e q}}+V_{C_{o}} I_{o}+V_{C_{o}} I_{e q}-V_{g} I e q=P_{R}
\end{aligned}
$$

que coincide, con la potencia consumida por $R$. Esto es, la potencia que suministra la fuente de entrada se entrega a la carga en su totalidad. 


\section{C.5. Modelo en pequeña señal}

El modelo en pequeña señal (modelo lineal) se consigue tras realizar el proceso de perturbación y linealización del modelo promediado (modelo no lineal) en torno a un punto de funcionamiento. Se asume que las entradas al modelo, $v_{g}$ y $d$ son iguales a un valor fijo, $V_{g}$ y $D$, mas una pequeña variación de alterna, $\hat{v}_{g}$ y $\hat{d}$. Es decir:

$$
\begin{aligned}
\bar{v}_{g} & =V_{g}+\hat{v}_{g} \\
d & =D+\hat{d}
\end{aligned}
$$

En respuesta a estas entradas, los valores promediados de las variables de estado sufriran una variación similar, es decir, presentarán un término constante mas una pequeña variación de alterna. También sucede lo mismo con el resto de magnitudes estudiadas, de forma que:

$$
\begin{aligned}
\bar{i}_{L} & =I+\hat{i}_{L} \\
\bar{v}_{C_{o}} & =V_{C_{o}}+\hat{v}_{C_{o}} \\
\bar{v}_{C_{c}} & =V_{c}+\hat{v}_{C_{c}} \\
\bar{i}_{g} & =I_{g}+\hat{i}_{g}
\end{aligned}
$$

Por supuesto, para que el modelo linealizado sea preciso debe cumplirse que las variaciones son pequeñas en comparación con el valor constante que indica el punto de operación del convertidor, es decir:

$$
\begin{aligned}
\left|\hat{v}_{g}\right| & \ll\left|V_{g}\right| \\
|\hat{d}| & \ll|D| \\
\left|\hat{i}_{L}\right| & \ll|I| \\
\left|\hat{v}_{C_{o}}\right| & \ll\left|V_{C_{o}}\right| \\
\left|\hat{v}_{C_{c}}\right| & \ll\left|V_{c}\right| \\
\left|\hat{i}_{g}\right| & \ll\left|I_{g}\right|
\end{aligned}
$$

Por lo que, introduciendo (C.106) y (C.107) en las ecuaciones del modelo promediado de orden reducido del reductor elevador ZVS, reescritas de nuevo en (C.109), y desarrollando y despreciando los términos de segundo orden se 
consigue linealizar el modelo promediado.

$$
\begin{aligned}
L \frac{d \bar{i}_{L}}{d t} & =\bar{v}_{g} d-\bar{v}_{C_{c}}(1-d) \\
C_{o} \frac{d \bar{v}_{C_{o}}}{d t} & =-\frac{\bar{v}_{C_{o}}}{R}-\frac{\left(\bar{v}_{C_{c}}+\bar{v}_{C_{o}}\right)^{2}}{\bar{v}_{g}-\bar{v}_{C_{o}}} \frac{(1-d)^{2}}{2 L_{r} f_{s}}-\frac{\bar{v}_{C_{c}}+\bar{v}_{C_{o}}}{2 L_{r} f_{s}}(1-d)^{2} \\
C_{c} \frac{d \bar{v}_{C_{c}}}{d t} & =\bar{i}_{L}(1-d)-\frac{\bar{v}_{C_{c}}+\bar{v}_{C_{o}}}{2 L_{r} f_{s}}(1-d)^{2} \\
\bar{i}_{g} & =\bar{i}_{L} d-\frac{\left(\bar{v}_{C_{c}}+\bar{v}_{C_{o}}\right)^{2}}{\bar{v}_{g}-\bar{v}_{C_{o}}} \frac{(1-d)^{2}}{2 L_{r} f_{s}}
\end{aligned}
$$

El proceso de linealización no es mas que un desarrollo de Taylor de una función, $f(x, y, z, \ldots)$ en torno a un punto determinado $\left(x_{0}, y_{0}, z_{0}, \ldots\right)$, considerando únicamente los términos hasta primer orden y despreciando el resto. Por tanto, aplicando el desarrollo de Taylor hasta primer orden a la parte derecha de cada una de las igualdades de las ecuaciones del modelo promediado se consigue llegar a una expresión que permite la obtención del modelo en pequeña señal y también, otra vez, el modelo en régimen permanente. Por ejemplo, la ecuación de la corriente en la bobina de entrada del modelo promediado dada en (C.109) se puede escribir como:

$$
L \frac{d \bar{i}_{L}}{d t}=f_{1}\left(\bar{v}_{g}, d, \bar{v}_{C_{c}}\right)
$$

Linealizando la función $f_{1}$ con el desarrollo de Taylor en un punto de funcionamiento dado por $\left(V_{g}, D, I, V_{c}\right)$ se obtiene (C.111). Por supuesto, la ecuación obtenida no será una aproximación muy buena de la función no lineal cuando se trate de un punto alejado del punto de linealización.

$$
\begin{aligned}
& L\left(\frac{d I}{d t}+\frac{d \hat{i}_{L}}{d t}\right)=f_{1}\left(V_{g}, D, V_{c}\right) \\
& +\left.\hat{v}_{g}(t) \frac{\partial f_{1}\left(\bar{v}_{g}, D, V_{c}\right)}{\partial \bar{v}_{g}}\right|_{\bar{v}_{g}=V_{g}}+\left.\hat{v}_{C_{c}} \frac{\partial f_{1}\left(V_{g}, D, \bar{v}_{C_{c}}\right)}{\partial \bar{v}_{C_{c}}}\right|_{\bar{v}_{C_{c}}=V_{c}} \\
& +\left.\hat{d}(t) \frac{\partial f_{1}\left(V_{g}, d, V_{c}\right)}{\partial d}\right|_{d=D}+\text { términos orden superior }
\end{aligned}
$$

A continuación se evalúan las derivadas parciales para la ecuación de la 
corriente en la bobina de entrada, $\bar{i}_{L}$. Las derivadas parciales son:

$$
\begin{aligned}
& \left.\frac{\partial f_{1}\left(\bar{v}_{g}, D, V_{c}\right)}{\partial \bar{v}_{g}}\right|_{\bar{v}_{g}=V_{g}}=D \\
& \left.\frac{\partial f_{1}\left(V_{g}, D, \bar{v}_{C_{c}}\right)}{\partial \bar{v}_{C_{c}}}\right|_{\bar{v}_{C_{c}=V_{c}}}=-(1-D) \\
& \left.\frac{\partial f_{1}\left(V_{g}, d, V_{c}\right)}{\partial d}\right|_{d=D}=V_{g}+V_{c}
\end{aligned}
$$

La ecuación (C.111) queda ahora así:

$$
\begin{aligned}
L\left(0+\frac{d \hat{i}_{L}}{d t}\right) & =\left[D V_{g}-(1-D) V_{c}\right] \\
& +D \hat{v}_{g}-(1-D) \hat{v}_{C_{c}}+\left(V_{g}+V_{c}\right) \hat{d} \\
& + \text { términos de orden superior }
\end{aligned}
$$

Los términos constantes de ambos miembros se igualan para conseguir una ecuación para el modelo en régimen permanente que coincide con la primera expresión de (C.97). Los términos de primer orden generan otra ecuación que forma parte del modelo en pequeña señal. El resto de términos de orden superior se desprecian.

Si se hace de forma similar para el restos de ecuaciones del modelo promediado, vistas como

$$
\begin{aligned}
C_{o} \frac{d \bar{v}_{C_{o}}}{d t} & =f_{2}\left(\bar{v}_{g}, d, \bar{v}_{C_{o}}, \bar{v}_{C_{c}}\right) \\
C_{c} \frac{d \bar{v}_{C_{c}}}{d t} & =f_{3}\left(d, \bar{i}_{L}, \bar{v}_{C_{o}}, \bar{v}_{C_{c}}\right) \\
\bar{i}_{g} & =f_{4}\left(\bar{v}_{g}, d, \bar{i}_{L}, \bar{v}_{C_{o}}, \bar{v}_{C_{c}}\right)
\end{aligned}
$$

será necesario evaluar las funciones $f_{2}, f_{3} \mathrm{y} f_{4}$ y sus derivadas parciales en torno al punto de funcionamiento elegido. Las funciones $f_{2}, f_{3}$ y $f_{4}$ evaluadas en el punto de funcionamiento son:

$$
\begin{aligned}
f_{2}\left(V_{g}, D, V_{C_{o}}, V_{c}\right) & =-\frac{V_{C_{o}}}{R}-I_{e q}-\frac{V_{c}+V_{C_{o}}}{R_{e q}} \\
f_{3}\left(D, I, V_{C_{o}} V_{c}\right) & =(1-D) I-\frac{V_{c}+V_{C_{o}}}{R_{e q}} \\
f_{4}\left(V_{g}, D, I, V_{C_{o}}, V_{c}\right) & =D I-I_{e q}
\end{aligned}
$$

Las derivadas parciales respecto a las variables de estado de las funciones anteriores, y evaluadas en torno al punto de funcionamiento están indicadas 
a continuación.

$$
\begin{aligned}
& \left.\frac{\partial f_{2}(\ldots)}{\partial \bar{v}_{g}}\right|_{\bar{v}_{g}=V_{g}}=\frac{I_{e q}}{V_{g}-V_{C_{o}}} \\
& \left.\frac{\partial f_{2}(\ldots)}{\partial d}\right|_{d=D}=2\left(\frac{I_{e q}}{1-D}+I\right) \\
& \left.\frac{\partial f_{2}(\ldots)}{\partial \bar{v}_{C_{o}}}\right|_{\bar{v}_{C_{o}}=V_{C_{o}}}=-\frac{1}{R}-\frac{2(1-D) I+I_{e q}}{V_{g}-V_{C_{o}}}-\frac{1}{R_{e q}} \\
& \left.\frac{\partial f_{2}(\ldots)}{\partial \bar{v}_{C_{c}}}\right|_{\bar{v}_{C_{c}=V_{c}}}=-\frac{2 I_{e q}}{V_{c}+V_{C_{o}}}-\frac{1}{R_{e q}} \\
& \left.\frac{\partial f_{3}(\ldots)}{\partial d}\right|_{d=D}=-I+2(1-D) \frac{V_{c}+V_{C_{o}}}{2 L_{r} f_{s}}=I \\
& \left.\frac{\partial f_{3}(\ldots)}{\partial \bar{i}_{L}}\right|_{\bar{i}_{L}=I}=(1-D) \\
& \left.\frac{\partial f_{3}(\ldots)}{\partial \bar{v}_{C_{o}}}\right|_{\bar{v}_{C_{o}}=V_{C_{o}}}=-\frac{(1-D)^{2}}{2 L_{r} f_{s}}=-\frac{1}{R_{e q}} \\
& \left.\frac{\partial f_{3}(\ldots)}{\partial \bar{v}_{C_{c}}}\right|_{\bar{v}_{C_{c}}=V_{c}}=-\frac{(1-D)^{2}}{2 L_{r} f_{s}}=-\frac{1}{R_{e q}} \\
& \left.\frac{\partial f_{4}(\ldots)}{\partial \bar{v}_{g}}\right|_{\bar{v}_{g}=V_{g}}=\frac{I_{e q}}{V_{g}-V_{C_{o}}} \\
& \left.\frac{\partial f_{4}(\ldots)}{\partial d}\right|_{d=D}=I+2 \frac{I_{e q}}{1-D} \\
& \left.\frac{\partial f_{4}(\ldots)}{\partial \bar{i}_{L}}\right|_{\bar{i}_{L}=I}=D \\
& \left.\frac{\partial f_{4}(\ldots)}{\partial \bar{v}_{C_{o}}}\right|_{\bar{v}_{C_{o}}=V_{C_{o}}}=-\frac{2(1-D) I+I_{e q}}{V_{g}-V_{C_{o}}} \\
& \left.\frac{\partial f_{4}(\ldots)}{\partial \bar{v}_{C_{c}}}\right|_{\bar{v}_{C_{c}}=V_{c}}=-\frac{2 I_{e q}}{V_{c}+V_{C_{o}}}
\end{aligned}
$$

Entonces, cada una de las ecuaciones (C.114) quedan de la forma indicada 
en (C.119), (C.120) y (C.121)

$$
\begin{aligned}
C_{o}\left(0+\frac{d \hat{v}_{C_{o}}}{d t}\right) & =\left[-\frac{V_{C_{o}}}{R}-I_{e q}-\frac{V_{c}+V_{C_{o}}}{R_{e q}}\right] \\
& +\frac{I_{e q}}{V_{g}-V_{C_{o}}} \hat{v}_{g}+\left[2\left(\frac{I_{e q}}{1-D}+I\right)\right] \hat{d} \\
& +\left[-\frac{1}{R}-\frac{2(1-D) I+I_{e q}}{V_{g}-V_{C_{o}}}-\frac{1}{R_{e q}}\right] \hat{v}_{C_{o}}+\left[-\frac{2 I_{e q}}{V_{c}+V_{C_{o}}}-\frac{1}{R_{e q}}\right] \hat{v}_{C_{c}} \\
& + \text { términos de orden superior }
\end{aligned}
$$

$$
\begin{aligned}
C_{c}\left(0+\frac{d \hat{v}_{C_{c}}}{d t}\right) & =\left[(1-D) I-\frac{V_{c}+V_{C_{o}}}{R_{e q}}\right] \\
& +I \hat{d}+(1-D) \hat{i}_{L}-\frac{1}{R_{e q}} \hat{v}_{C_{o}}-\frac{1}{R_{e q}} \hat{v}_{C_{c}} \\
& + \text { términos de orden superior }
\end{aligned}
$$

$$
\begin{aligned}
I_{g}+\hat{i}_{g} & =\left[D I-I_{e q}\right] \\
& +\frac{I_{e q}}{V_{g}-V_{C_{o}}} \hat{v}_{g}+\left[I+\frac{2}{1-D} I_{e q}+\right] \hat{d} \\
& +D \hat{i}_{L}+\left[-\frac{2(1-D) I+I_{e q}}{V_{g}-V_{C_{o}}}\right] \hat{v}_{C_{o}}-\frac{2 I_{e q}}{V_{c}+V_{C_{o}}} \hat{v}_{C_{c}} \\
& + \text { términos de orden superior }
\end{aligned}
$$

Agrupando todos los términos constantes de las ecuaciones (C.113), (C.119), (C.120) y (C.121) se obtiene el modelo en régimen permanente que coincide con el descrito en (C.99).

Tomando todos los términos de primer orden y despreciando los términos de orden superior, se consigue el modelo en pequeña señal para el reductor 
elevador ZVS dado en (C.122).

$$
\begin{aligned}
L \frac{\hat{i}_{L}}{d t} & =D \hat{v}_{g}-(1-D) \hat{v}_{C_{c}}+\left(V_{g}+V_{c}\right) \hat{d} \\
C_{o} \frac{d \hat{v}_{C_{o}}}{d t} & =\frac{I_{e q}}{V_{g}-V_{C_{o}}} \hat{v}_{g}+\left[2\left(\frac{I_{e q}}{1-D}+I\right)\right] \hat{d} \\
& -\frac{\hat{v}_{C_{o}}}{R}-\left[\frac{2(1-D) I+I_{e q}}{V_{g}-V_{C_{o}}}\right] \hat{v}_{C_{o}}-\left[\frac{2 I_{e q}}{V_{c}+V_{C_{o}}}\right] \hat{v}_{C_{c}}-\frac{\hat{v}_{C_{c}}+\hat{v}_{C_{o}}}{R_{e q}} \\
C_{c} \frac{d \hat{v}_{C_{c}}}{d t} & =I \hat{d}+(1-D) \hat{i}_{L}-\frac{\hat{v}_{C_{c}}+\hat{v}_{C_{o}}}{R_{e q}} \\
\hat{i}_{g} & =\frac{I_{e q}}{V_{g}-V_{C_{o}}} \hat{v}_{g}+\left[I+\frac{2 I_{e q}}{1-D}\right] \hat{d} \\
& +D \hat{i}_{L}-\frac{2(1-D) I+I_{e q}}{V_{g}-V_{C_{o}}} \hat{v}_{C_{o}}-\frac{2 I_{e q}}{V_{c}+V_{C_{o}}} \hat{v}_{C_{c}}
\end{aligned}
$$

Con estas ecuaciones se construye el circuito en pequeña señal de la figura B.21. Los parámetros $f_{3}, j_{3}, g_{3}, j_{4}, g_{4}$ y $g_{c}$ están indicados en la tabla C.4. Además, $r_{3}=1 / g_{3}$ y $r_{4}=1 / g_{4}$.

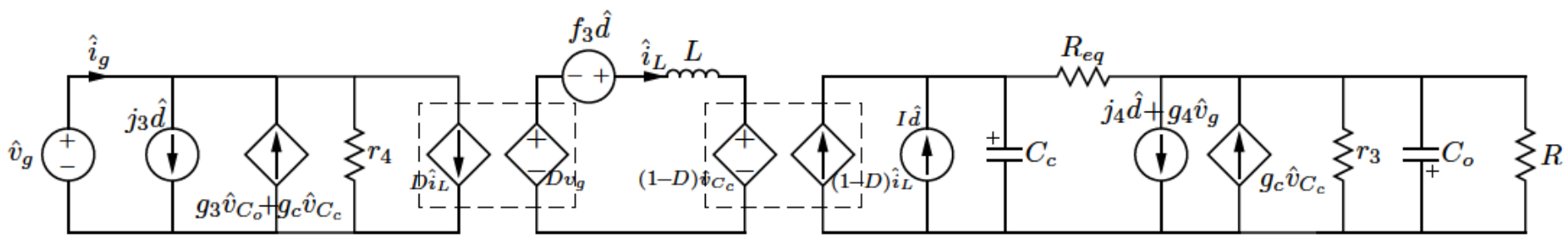

Figura C.21: Modelo en pequeña señal de un reductor elevador ZVS.

\begin{tabular}{ccccccc}
\hline Convertidor & $f_{3}$ & $j_{3}$ & $g_{3}$ & $j_{4}$ & $g_{4}$ & $g_{c}$ \\
\hline Red-Elev & $V_{g}+V_{c}$ & $I+2 \frac{I_{e q}}{1-D}$ & $\frac{2(1-D) I+I_{e q}}{V_{g}-V_{C_{o}}}$ & $2\left(I+\frac{I_{e q}}{1-D}\right)$ & $\frac{I_{e q}}{V_{g}-V_{C_{o}}}$ & $\frac{2 I_{e q}}{V_{c}+V_{C_{o}}}$ \\
\hline
\end{tabular}

Tabla C.4: Parámetros del modelo en pequeña señal del Red-Elev.ZVS.

El modelo en pequeña señal, obtenido a partir de la linealización del modelo promediado de orden reducido, también se puede escribir en forma matricial. Así, se definen dos vectores, el vector $\hat{\mathbf{x}}$ para las variables de estado y el vector $\hat{u}$ para las entradas externas e independientes, y serán en este caso:

$$
\hat{\mathbf{x}}=\left[\begin{array}{c}
\hat{i}_{L} \\
\hat{v}_{C_{o}} \\
\hat{v}_{C_{c}}
\end{array}\right], \quad \hat{\mathbf{u}}=\left[\begin{array}{c}
\hat{v}_{g} \\
\hat{d}
\end{array}\right]
$$


de forma que el modelo en pequeña señal de (C.122) se puede escribir, de la forma siguiente:

$$
\begin{array}{r}
\mathbf{K} \frac{d}{d t}\left[\begin{array}{c}
\hat{i}_{L} \\
\hat{v}_{C_{o}} \\
\hat{v}_{C_{c}}
\end{array}\right]=\mathbf{A}\left[\begin{array}{c}
\hat{i}_{L} \\
\hat{v}_{C_{o}} \\
\hat{v}_{C_{c}}
\end{array}\right]+\mathbf{B}\left[\begin{array}{c}
\hat{v}_{g} \\
\hat{d}
\end{array}\right] \\
{\left[\hat{i}_{g}\right]=\mathbf{C}\left[\begin{array}{c}
\hat{i}_{L} \\
\hat{v}_{C_{o}} \\
\hat{v}_{C_{c}}
\end{array}\right]+\mathbf{E}\left[\begin{array}{c}
\hat{v}_{g} \\
\hat{d}
\end{array}\right]}
\end{array}
$$

donde

$$
\begin{aligned}
& \mathbf{K}=\left[\begin{array}{ccc}
L & 0 & 0 \\
0 & C_{o} & 0 \\
0 & 0 & C_{c}
\end{array}\right], \quad \mathbf{A}=\left[\begin{array}{ccc}
0 & 0 & D-1 \\
0 & -\frac{1}{R}-\frac{1}{R_{e q}}-g_{3} & -\frac{1}{R_{e q}}-g_{c} \\
1-D & -\frac{1}{R_{e q}} & -\frac{1}{R_{e q}}
\end{array}\right], \\
& \mathbf{B}=\left[\begin{array}{cc}
D & f_{3} \\
g_{4} & j_{4} \\
0 & I
\end{array}\right], \quad \mathbf{C}=\left[\begin{array}{lll}
D & -g_{3} & -g_{c}
\end{array}\right], \quad \mathbf{E}=\left[\begin{array}{ll}
g_{4} & j_{3}
\end{array}\right]
\end{aligned}
$$

\section{C.6. Funciones de transferencia}

Las diferentes funciones de transferencia se pueden obtener bien a partir del modelo en pequeña señal dado en las ecuaciones (C.122), bien a partir del circuito en pequeña señal de la figura B.21. Por ambos métodos hay que anular la variable de estado correspondiente y aplicar Laplace para obtener la relación deseada. Otra posibilidad es trabajar con el modelo matricial en pequeña señal, que se verá mas adelante.

\section{C.6.1. Desde el modelo o el circuito de pequeña señal}

Para conseguir $G_{v g}(s)$, se hace nula la variación de la entrada independiente que no interviene en la función de transferencia, en este caso, $\hat{d}(t)=0$, y se aplica Laplace. De esta forma queda que:

$$
\begin{aligned}
s L \hat{i}_{L}(s) & =D \hat{v}_{g}(s)-(1-D) \hat{v}_{C_{c}}(s) \\
s C_{o} \hat{v}_{C_{o}}(s) & =\frac{1}{r_{4}} \hat{v}_{g}(s)-\left[\frac{1}{R}+\frac{1}{r_{3}}+\frac{1}{R_{e q}}\right] \hat{v}_{C_{o}}(s)-\left[g_{c}+\frac{1}{R_{e q}}\right] \hat{v}_{C_{c}}(s) \\
s C_{c} \hat{v}_{C_{c}}(s) & =(1-D) \hat{i}_{L}(s)-\frac{1}{R_{e q}} \hat{v}_{C_{o}}(s)-\frac{1}{R_{e q}} \hat{v}_{C_{c}}(s) \\
\hat{i}_{g}(s) & =\frac{1}{r_{4}} \hat{v}_{g}(s)+D \hat{i}_{L}(s)+\frac{1}{r_{3}} \hat{v}_{C_{o}}(s)+g_{c} \hat{v}_{C_{c}}(s)
\end{aligned}
$$


Despejando en la tercera ecuación una expresión para $\hat{v}_{C_{c}}(s)$, como:

$$
\hat{v}_{C_{c}}(s)=(1-D) Z_{e q}(s) \hat{i}_{L}(s)-Z_{e q}(s) / R_{e q} \hat{v}_{C_{o}}(s)
$$

y sustituyendo en la primera ecuación, se consigue obtener la expresión de $\hat{i}_{L}(s)$ en función de $\hat{v}_{g}(s)$ y $\hat{v}_{C_{o}}(s)$, dada por:

$$
\hat{i}_{L}(s)=\frac{D \hat{v}_{g}(s)+(1-D) Z_{e q}(s) / R_{e q} \hat{v}_{C_{o}}(s)}{L s+(1-D)^{2} Z_{e q}(s)}
$$

donde se han definido las siguientes expresiones:

$$
\begin{aligned}
Z_{o}(s) & =\left(R\left\|C_{o}\right\| r_{3}\right)=\left[s C_{o}+\frac{1}{R}+\frac{1}{r_{3}}\right]^{-1} \\
Z_{e q}(s) & =\left(R_{e q} \| C_{c}\right)=\left[s C_{c}+\frac{1}{R_{e q}}\right]^{-1}
\end{aligned}
$$

Utilizando (C.128) en (C.127), se obtiene una expresión de $\hat{v}_{C_{c}}(s)$, dada por:

$$
\hat{v}_{C_{c}}(s)=\frac{(1-D) D Z_{e q}(s) \hat{v}_{g}(s)-L s Z_{e q}(s) / R_{e q} \hat{v}_{C_{o}}(s)}{L s+(1-D)^{2} Z_{e q}(s)}
$$

Introduciendo (C.130) en la segunda expresión de (C.126), se consigue una ecuación, dada por (C.131), donde únicamente aparecen $\hat{v}_{g}(s)$ y $\hat{v}_{C_{c}}(s)$ que permite obtener fácilmente la función de transferencia deseada, $G_{v g}(s)$, que está indicada en (C.132).

$$
\begin{gathered}
{\left[\frac{1}{Z_{o}(s)}+\frac{1}{R_{e q}}-\frac{\left[g_{c} R_{e q}+1\right] L s Z_{e q}(s)}{R_{e q}^{2}\left[L s+(1-D)^{2} Z_{e q}(s)\right]}\right] \hat{v}_{C_{o}}(s)} \\
=\left[\frac{1}{r_{4}}-\frac{\left[g_{c} R_{e q}+1\right](1-D) D Z_{e q}(s)}{R_{e q}\left[L s+(1-D)^{2} Z_{e q}(s)\right]}\right] \hat{v}_{g}(s) \\
G_{v g}(s)=\left.\frac{\hat{v}_{C_{o}}(s)}{\hat{v}_{g}(s)}\right|_{\hat{d}=0} \\
=\frac{Z_{o}(s)}{r_{4}} \frac{R_{e q}\left[L s+(1-D)^{2} Z_{e q}(s)\right]-r_{4}\left[1+g_{c} R_{e q}(s)\right](1-D) D Z_{e q}(s)}{\left[Z_{o}(s)+R_{e q}\right]\left[L s+(1-D)^{2} Z_{e q}(s)\right]-Z_{o}(s)\left[1+g_{c} R_{e q}(s)\right] L s Z_{e q}(s) / R_{e q}}
\end{gathered}
$$

Para conseguir $G_{i g}(s)$, se utiliza (C.127), para obtener para obtener $\hat{v}_{C_{o}}(s)$ en función de $\hat{i}_{L}(s)$ y $\hat{v}_{g}(s)$ en la segunda ecuacion de (C.126). Por tanto,

$$
\left[\frac{1}{Z_{o}(s)}+\frac{1}{R_{e q}}\right] \hat{v}_{C_{o}}(s)=\frac{1}{r_{4}} \hat{v}_{g}(s)-\left[g_{c}+\frac{1}{R_{e q}}\right]\left[(1-D) Z_{e q}(s) \hat{i}_{L}(s)-\frac{Z_{e q}(s)}{R_{e q}} \hat{v}_{C_{o}}(s)\right]
$$


Entonces, $\hat{v}_{C_{o}}(s)$ es igual a la expresión dada por

$$
\hat{v}_{C_{o}}(s)=\frac{\frac{1}{r_{4}} \hat{v}_{g}(s)-\left[1+g_{c} R_{e q}\right](1-D) \frac{Z_{e q}(s)}{R_{e q}} \hat{i}_{L}(s)}{\left[\frac{1}{Z_{o}(s)}+\frac{1}{R_{e q}}-\left[1+g_{c} R_{e q}\right] \frac{Z_{e q}(s)}{R_{e q}^{2}}\right]}
$$

que, introducida en la primera ecuación de (C.126) resulta,

$$
\begin{gathered}
s L \hat{i}_{L}(s)=D \hat{v}_{g}(s)-(1-D)^{2} Z_{e q}(s) \hat{i}_{L}(s)+(1-D) \frac{Z_{e q}(s)}{R_{e q}} \hat{v}_{C_{o}}(s) \\
{\left[L s+(1-D)^{2} Z_{e q}(s)\right] \hat{i}_{L}(s)=D \hat{v}_{g}(s)+\frac{\frac{(1-D) Z_{e q}(s)}{r_{4}} \hat{v}_{g}(s)-\left[1+g_{c} R_{e q}\right](1-D)^{2} \frac{Z_{e q}^{2}(s)}{R_{e q}} \hat{i}_{L}(s)}{\left[\frac{R_{e q}}{Z_{o}(s)}+1-\left[1+g_{c} R_{e q}\right] \frac{Z_{e q}(s)}{R_{e q}}\right]}}
\end{gathered}
$$

Agrupando los términos en cada miembro de la igualdad:

$$
\begin{array}{r}
{\left[L s+(1-D)^{2} Z_{e q}(s)+\frac{\left[1+g_{c} R_{e q}\right](1-D)^{2} \frac{Z_{e q}^{2}(s)}{R_{e q}}}{\left[\frac{R_{e q}}{Z_{o}(s)}+1-\left[1+g_{c} R_{e q}\right] \frac{Z_{e q}(s)}{R_{e q}}\right]}\right] \hat{i}_{L}(s)} \\
=\left[D+\frac{\frac{(1-D) Z_{e q}(s)}{r_{4}}}{\frac{R_{e q}}{Z_{o}(s)}+1-\left[1+g_{c} R_{e q}\right] \frac{Z_{e q}(s)}{R_{e q}}}\right] \hat{v}_{g}(s)
\end{array}
$$

Desarrollando la suma de fracciones de cada miembro,

$$
\begin{aligned}
& {\left[\frac{\left[L s+(1-D)^{2} Z_{e q}(s)\right]\left[Z_{o}(s)+R_{e q}\right] / Z_{o}(s)-\left[1+g_{c} R_{e q}\right] L s Z_{e q}(s) / R_{e q}}{\left[R_{e q}+Z_{o}(s)\right] / Z_{o}(s)-\left[1+g_{c} R_{e q}\right] Z_{e q}(s) / R_{e q}}\right] \hat{i}_{L}(s)} \\
& =\left[\frac{D\left[Z_{o}(s)+R_{e q}\right] / Z_{o}(s)-D\left[1+g_{c} R_{e q}\right] Z_{e q}(s) / R_{e q}+(1-D) Z_{e q} / r_{4}}{\left[R_{e q}+Z_{o}(s)\right] / Z_{o}(s)-\left[1+g_{c} R_{e q}\right] Z_{e q}(s) / R_{e q}}\right] \hat{v}_{g}(s)
\end{aligned}
$$

y haciendo el cociente entre las variables de estado, se obtiene la función de transferencia deseada.

$$
\begin{aligned}
& G_{i g}(s)=\left.\frac{\hat{i}_{L}(s)}{\hat{v}_{g}(s)}\right|_{\hat{d}=0} \\
& =Z_{o}(s) \frac{D\left[Z_{o}(s)+R_{e q}\right] / Z_{o}(s)-D\left[1+g_{c} R_{e q}\right] Z_{e q}(s) / R_{e q}+(1-D) Z_{e q}(s) / r_{4}}{\left[L s+(1-D)^{2} Z_{e q}(s)\right]\left[Z_{o}(s)+R_{e q}\right]-Z_{o}(s)\left[1+g_{c} R_{e q}\right] L s Z_{e q}(s) / R_{e q}}
\end{aligned}
$$


Las dos funciones de transferencia coinciden con las de un reductorelevador convencional cuando los términos relacionados con el enclavamiento activo, $C_{c}$ y $L_{r}$, desaparecen puesto que $V_{c}, R_{e q}$ y $Z_{e} q$ se anulan.

En la Figura C.22 están representadas ambas funciones de transferencia con los parámetros de funcionamiento de la Tabla C.1, comparadas con las que tendría un red.-elev. convencional con los mismos parámetros.

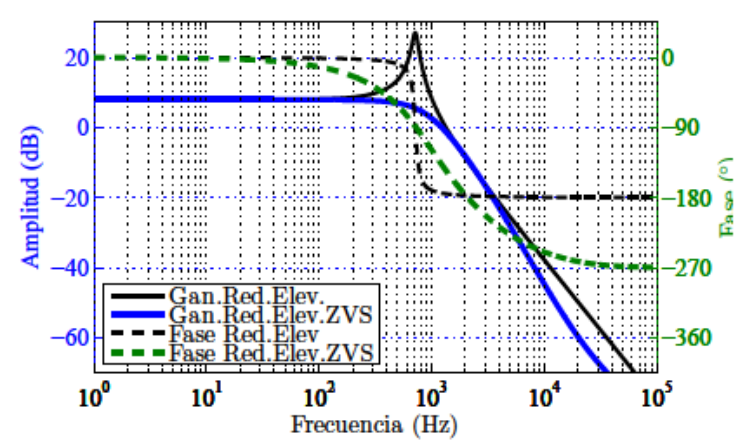

(a)

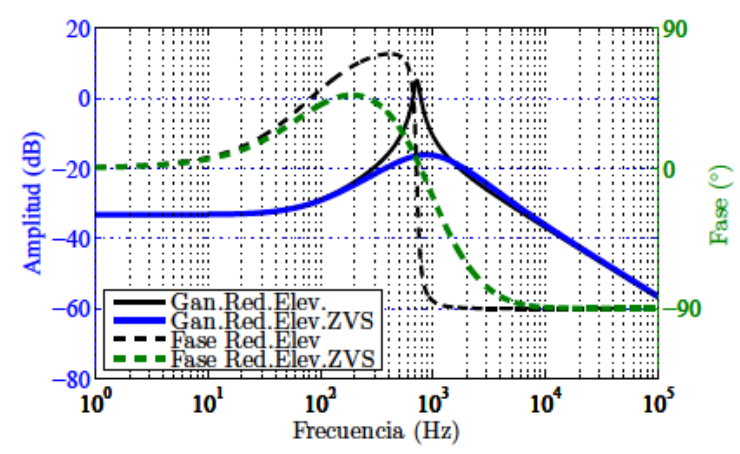

(b)

Figura C.22: Reductor-Elevador ZVS. Func. de transferencia en función de la tensión de entrada para: a) Tensión de salida, $G_{v g}(s)$; y b) Intensidad en la bobina, $G_{i g}(s)$.

Igualmente, para conseguir $G_{v d}(s)$, se hace nula la variación de la entrada independiente que no interviene en la función de transferencia, en este caso, $\hat{v}_{g}(t)=0$, y se aplica Laplace. De esta forma queda que:

$$
\begin{aligned}
s L \hat{i}_{L}(s) & =f_{3} \hat{d}-(1-D) \hat{v}_{C_{c}}(s) \\
s C_{o} \hat{v}_{C_{o}}(s) & =j_{4} \hat{d}-\left[\frac{1}{R}+\frac{1}{r_{3}}+\frac{1}{R_{e q}}\right] \hat{v}_{C_{o}}(s)-\left[g_{c}+\frac{1}{R_{e q}}\right] \hat{v}_{C_{c}}(s) \\
s C_{c} \hat{v}_{C_{c}}(s) & =I \hat{d}+(1-D) \hat{i}_{L}(s)-\frac{1}{R_{e q}} \hat{v}_{C_{o}}(s)-\frac{1}{R_{e q}} \hat{v}_{C_{c}}(s) \\
\hat{i}_{g}(s) & =j_{3} \hat{d}+D \hat{i}_{L}(s)+\frac{1}{r_{3}} \hat{v}_{C_{o}}(s)+g_{c} \hat{v}_{C_{c}}(s)
\end{aligned}
$$

Primero se obtiene $\hat{i}_{L}(s)$ con la primera y la tercera ecuación. Para ello despejamos $\hat{v}_{C_{c}}(s)$ de la tercera ecuación como:

$$
\hat{v}_{C_{c}}(s)=Z_{e q}(s)\left[I \hat{d}+(1-D) \hat{i}_{L}(s)-\frac{1}{R_{e q}} \hat{v}_{C_{o}}(s)\right]
$$

Esta expresión se introduce en la primera ecuación para obtener $\hat{i}_{L}(s)$ :

$$
\hat{i}_{L}(s)=\frac{\left[f_{3}-I(1-D) Z_{e q}(s)\right] \hat{d}+(1-D) Z_{e q}(s) / R_{e q} \hat{v}_{C_{o}}(s)}{L s+(1-D)^{2} Z_{e q}(s)}
$$


De esta forma, se consigue una expresión de $\hat{v}_{C_{c}}(s)$ en función de las variables $\hat{d} \mathrm{y} \hat{v}_{C_{o}}(s)$.

$$
\begin{aligned}
& \hat{v}_{C_{c}}(s)= \\
& =Z_{e q}(s)\left[I \hat{d}+(1-D) \frac{\left[f_{3}-I(1-D) Z_{e q}(s)\right] \hat{d}+(1-D) \frac{Z_{e q}(s)}{R_{e q}} \hat{v}_{C_{o}}(s)}{L s+(1-D)^{2} Z_{e q}(s)}-\frac{\hat{v}_{C_{o}}(s)}{R_{e q}}\right] \\
& =Z_{e q}(s)\left[\frac{\left[I L s+f_{3}(1-D)\right] \hat{d}+(1-D)^{2} \frac{Z_{e q}(s)}{R_{e q}} \hat{v}_{C_{o}}(s)}{L s+(1-D)^{2} Z_{e q}(s)}-\frac{\hat{v}_{C_{o}}(s)}{R_{e q}}\right]
\end{aligned}
$$

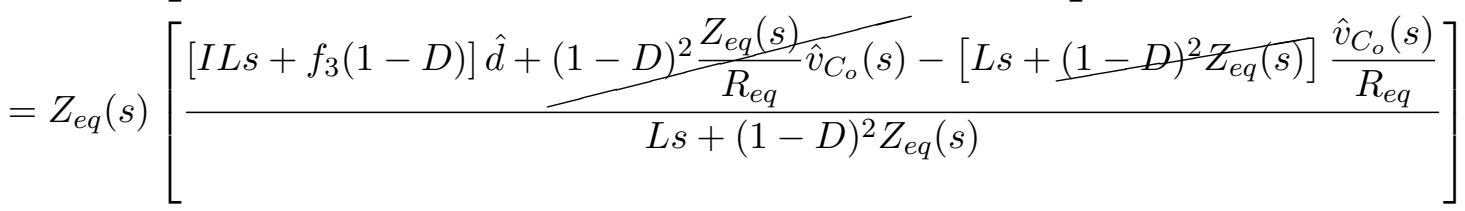

$$
\begin{aligned}
& =\frac{\left[I L s+f_{3}(1-D)\right] Z_{e q}(s) \hat{d}-L s Z_{e q}(s) / R_{e q} \hat{v}_{C_{o}}(s)}{L s+(1-D)^{2} Z_{e q}(s)}
\end{aligned}
$$

Utilizando (C.141) y (C.142) en la segunda ecuación de (C.139) se puede conseguir una expresión en la que únicamente aparecen $\hat{v}_{C_{o}}(s)$ y $\hat{d}$, y donde es fácil obtener $G_{v d}(s)$.

$$
\begin{aligned}
& {\left[\frac{1}{Z_{o}(s)}+\frac{1}{R_{e q}}-\frac{L s\left[g_{c}+\frac{1}{R_{e q}}\right] Z_{e q}(s) / R e q}{L s+(1-D)^{2} Z_{e q}(s)}\right] \hat{v}_{C_{o}}(s) } \\
= & {\left[j_{4}-\left[g_{c}+\frac{1}{R_{e q}}\right] \frac{\left[I L s+f_{3}(1-D)\right] Z_{e q}(s)}{L s+(1-D)^{2} Z_{e q}(s)}\right] \hat{d} }
\end{aligned}
$$

De la ecuación (C.143) se consigue la función de transferencia deseada:

$$
\begin{aligned}
& G_{v d}(s)=\left.\frac{\hat{v}_{C_{o}}(s)}{\hat{d}(s)}\right|_{\hat{v}_{g}=0} \\
& =Z_{o}(s) \frac{j_{4}\left[L s+(1-D)^{2} Z_{e q}(s)\right] R_{e q}-\left[g_{c} R_{e q}+1\right]\left[f_{3}(1-D) Z_{e q}(s)+I L s Z_{e q}(s)\right]}{\left[Z_{o}(s)+R_{e q}(s)\right]\left[L s+(1-D)^{2} Z_{e q}(s)\right]-Z_{o}(s)\left[g_{c} R_{e q}+1\right] L s Z_{e q}(s) / R_{e q}}
\end{aligned}
$$

Para conseguir $G_{i d}(s)$, se utiliza (C.140) en la segunda ecuación de (C.139) 
para obtener $\hat{v}_{C_{o}}(s)$ en función de $\hat{i}_{L}(s)$ y $\hat{d}(s)$ :

$\hat{v}_{C_{o}}(s)=Z_{o}(s) R_{e q} \frac{\left[j_{4}-I\left[1+g_{c} R_{e q}\right] \frac{Z_{e q}(s)}{R_{e q}}\right] \hat{d}-\left[1+g_{c} R_{e q}\right](1-D) \frac{Z_{e q}(s)}{R_{e q}} \hat{i}_{L}(s)}{\left[Z_{o}(s)+R_{e q}\right]-Z_{o}(s)\left[1+g_{c} R_{e q}\right] \frac{Z_{e q}(s)}{R_{e q}}}$

entonces, $\hat{v}_{C_{c}}(s)$ tendrá la siguiente expresión:

$$
\begin{aligned}
\hat{v}_{C_{c}}(s)=Z_{e q}(s)\left[I-Z_{o}(s) \frac{j_{4}-I\left[1+g_{c} R_{e q}\right] \frac{Z_{e q}(s)}{R_{e q}}}{\left[Z_{o}(s)+R_{e q}\right]-Z_{o}(s)\left[1+g_{c} R_{e q}\right] \frac{Z_{e q}(s)}{R_{e q}}}\right] \hat{d} \\
+Z_{e q}(s)\left[(1-D)+Z_{o}(s) \frac{\left[1+g_{c} R_{e q}\right](1-D) \frac{Z_{e q}(s)}{R_{e q}}}{\left[Z_{o}(s)+R_{e q}\right]-Z_{o}(s)\left[1+g_{c} R_{e q}\right] \frac{Z_{e q}(s)}{R_{e q}}}\right] \hat{i}_{L}(s) \\
=Z_{e q}(s) \frac{\left[I\left[Z_{o}(s)+R_{e q}\right]-j_{4} Z_{o}(s)\right] \hat{d}+(1-D)\left[Z_{o}(s)+R_{e q}\right] \hat{i}_{L}(s)}{\left[Z_{o}(s)+R_{e q}\right]-Z_{o}(s)\left[1+g_{c} R_{e q}\right] Z_{e q}(s) / R_{e q}}
\end{aligned}
$$

Introduciendo (C.146) en la primera ecuación de (C.139), se obtiene la siguiente igualdad:

$$
\begin{aligned}
& {\left[L s+\frac{(1-D)^{2}\left[Z_{o}(s)+R_{e q}\right] Z_{e q}(s)}{\left[Z_{o}(s)+R_{e q}\right]-Z_{o}(s)\left[1+g_{c} R_{e q}\right] Z_{e q}(s) / R_{e q}}\right] \hat{i}_{L}(s) } \\
= & {\left[f_{3}-\frac{(1-D)\left[I\left[Z_{o}(s)+R_{e q}\right]-j_{4} Z_{o}(s)\right] Z_{e q}(s)}{\left[Z_{o}(s)+R_{e q}\right]-Z_{o}(s)\left[1+g_{c} R_{e q}\right] Z_{e q}(s) / R_{e q}}\right] \hat{d} }
\end{aligned}
$$

A partir de esta última ecuación se obtiene la función de transferencia deseada:

$$
\begin{aligned}
& G_{i d}(s)=\left.\frac{\hat{i}_{L}(s)}{\hat{d}(s)}\right|_{\hat{v}_{g}=0} \\
& =\frac{\left[Z_{o}(s)+R_{e q}\right]\left[f_{3}-(1-D) I Z_{e q}(s)\right]-j_{4} Z_{o}(s)(1-D) Z_{e q}(s)-f_{3} Z_{o}(s)\left[1+g_{c} R_{e q}\right] Z_{e q}(s) / R_{e q}}{\left[Z_{o}(s)+R_{e q}\right]\left[L s+(1-D)^{2} Z_{e q}(s)\right]-Z_{o}(s)\left[1+g_{c} R_{e q}\right] L s Z_{e q}(s) / R_{e q}}
\end{aligned}
$$

Las dos funciones de transferencia coinciden con las de un red.-elev. convencional cuando los términos relacionados con el enclavamiento activo, $C_{c}$ y $L_{r}$, desaparecen puesto que $V_{c}, R_{e q}$ y $Z_{e} q$ se anulan. En la Figura C.23 están representadas ambas funciones de transferencia con los parámetros de funcionamiento de la Tabla C.1, comparadas con las que tendría un red.-elev. convencional con los mismos parámetros. 


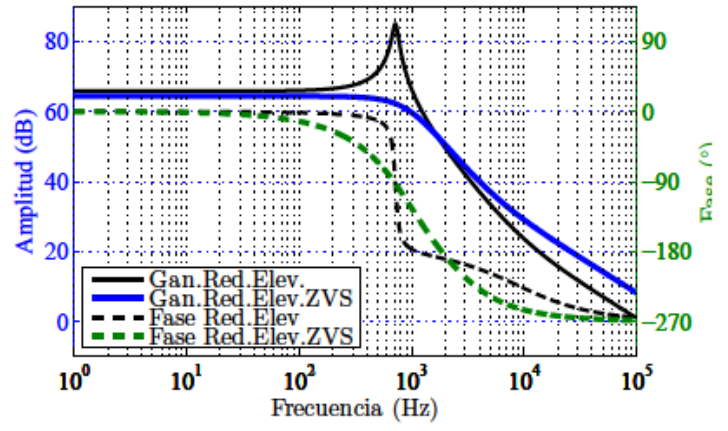

(a)

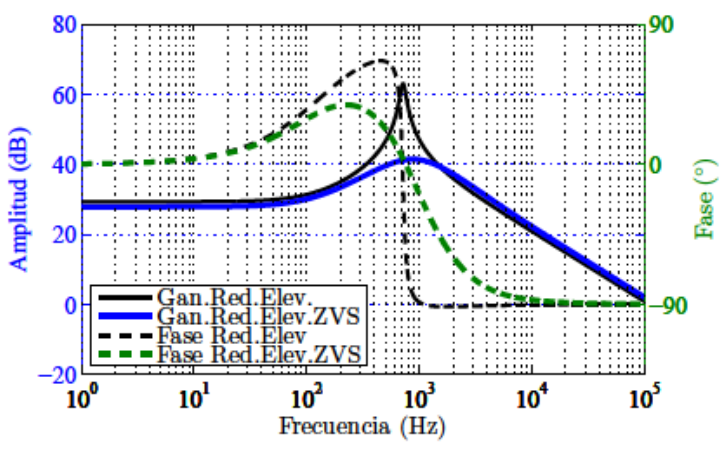

(b)

Figura C.23: Red.-Elev. ZVS. Func. de transferencia en función del ciclo de trabajo para: a) Tensión de salida, $G_{v d}(s)$; y b) Intensidad en la bobina, $G_{i g}(s)$.

\section{C.6.2. Desde el modelo matricial}

Para obtener las funciones de transferencia anteriores se aplica Laplace a las ecuaciones de estado en forma matricial (suponiendo que las condiciones iniciales con nulas).

$$
\begin{aligned}
\mathbf{K} s \hat{\mathbf{X}}(s) & =\mathbf{A} \hat{\mathbf{X}}(s)+\mathbf{B} \hat{\mathbf{U}}(s) \\
\hat{\mathbf{Y}}(s) & =\mathbf{C} \hat{\mathbf{X}}(s)+\mathbf{E} \hat{\mathbf{U}}(s)
\end{aligned}
$$

En la primera ecuación de (C.149) se despeja el vector de las variables de estado. Por tanto, se tiene que

$$
\mathbf{K} s \hat{\mathbf{X}}(s)-\mathbf{A} \hat{\mathbf{X}}(s)=\mathbf{B} \hat{\mathbf{U}}(s)
$$

o bien

$$
(\mathbf{K} s-\mathbf{A}) \hat{\mathbf{X}}(s)=\mathbf{B} \hat{\mathbf{U}}(s)
$$

Premultiplicando ambos miembros por $(\mathbf{K} s-\mathbf{A})^{-1}$ se obtiene:

$$
\hat{\mathbf{X}}(s)=(\mathbf{K} s-\mathbf{A})^{-1} \mathbf{B} \hat{\mathbf{U}}(s)
$$

Sustituyendo la ecuación (C.150) en la segunda ecuación de (C.149) se llega a

$$
\hat{\mathbf{Y}}(s)=\left[\mathbf{C}(\mathbf{K} s-\mathbf{A})^{-1} \mathbf{B}+\mathbf{E}\right] \hat{\mathbf{U}}(s)
$$

Puesto, que la relación entre la entrada y la salida de un sistema es la función de transferencia, resulta que la matriz $\mathbf{G}(s)$ definida en (C.152) es la 
denominada matriz de transferencia que relaciona las diferentes salidas del sistema con las diferentes entradas. Tiene tantas filas como salidas y tantas columnas como entradas.

$$
\mathbf{G}(s)=\mathbf{C}(\mathbf{K} s-\mathbf{A})^{-1} \mathbf{B}+\mathbf{E}
$$

En el caso del elevador ZVS, se redefine el vector de salidas y las matrices

C y $\mathbf{E}$, de la siguiente forma:

$$
\hat{\mathbf{y}}=\left[\begin{array}{l}
\hat{i}_{L} \\
\hat{v}_{o}
\end{array}\right], \quad \mathbf{C}=\left[\begin{array}{lll}
1 & 0 & 0 \\
0 & 1 & 0
\end{array}\right], \quad \mathbf{E}=\left[\begin{array}{ll}
0 & 0 \\
0 & 0
\end{array}\right]
$$

De esta forma, la matriz de transferencia, queda como:

$$
\mathbf{G}(s)=\left[\begin{array}{ll}
G_{i g}(s) & G_{i d}(s) \\
G_{v g}(s) & G_{v d}(s)
\end{array}\right]
$$

que es una matriz que recoge las funciones de transferencias obtenidas en el apartado anterior.

\section{C.7. Impedancia de salida}

La impedancia de salida en pequeña señal se obtiene a partir del modelo en pequeña señal de la Figura C.21. Para ello hay que anular las entradas independientes, $\hat{v}_{g}$ y $\hat{d}$, y conectar una fuente independiente, $\hat{i}_{t}(s)$, en los puntos donde se quiere medir la impedancia. La tensión que genera esta fuente sobre el sistema, $\hat{v}_{t}$, se emplea para obtener $Z_{\text {out }}(s)$ como:

$$
Z_{\text {out }}(s)=\frac{\hat{v}_{t}(s)}{\hat{i}_{t}(s)} \mid \begin{aligned}
& \hat{v}_{g}=0 \\
& \hat{d}=0
\end{aligned}
$$

considerando que $\hat{i}_{t}(s)$ es la intensidad que entra por el terminal de mayor tensión de los dos terminales considerados anteriormente. En el circuito de la Figura C.24 se indica gráficamente la conexión de la fuente independiente al modelo de pequeña señal una vez anuladas las fuentes independientes. Además, se ha indicado la impedancia $Z_{t h}$ como la impedancia formada por el condensador del clamp, la resistencia equivalente y la bobina principal.

\section{C.7.1. Cálculo de $Z_{t h}$.}

Para obtener esta impedancia se utiliza la parte del circuito anterior que concierne al punto de medida de $Z_{t h}$, en el que se conecta la fuente $\hat{i}_{t}$. Queda el circuito de la Figura C.25. Pasando la bobina $L$ al otro lado de las fuentes 


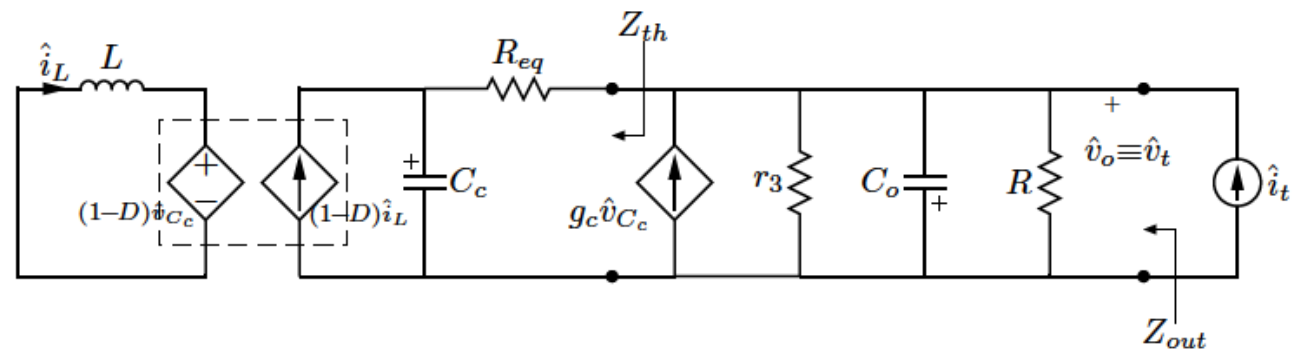

Figura C.24: Circuito para el cálculo de la impedancia de salida de un red.elev. ZVS.

dependientes, aparece con otro valor en paralelo con $C_{c}$. Este conjunto se encuentra en serie con $R_{e q}$. Por tanto la expresión de esta impedancia es:

$$
Z_{t h}(s)=\left[\frac{L}{(1-D)^{2}} \| C_{c}\right]+R_{e q} \equiv \frac{L^{\prime} s}{L^{\prime} C s^{2}+1}+R_{e q}
$$

donde $L^{\prime}=L /(1-D)^{2}$.

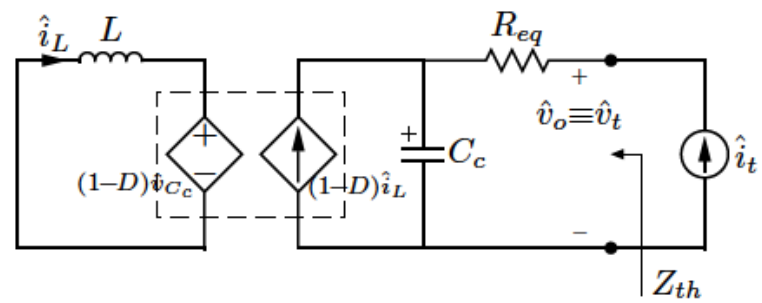

Figura C.25: Circuito empleado para el cálculo de $Z_{t h}(s)$ de un reductorelevador ZVS.

A esta expresión también se puede llegar desde las ecuaciones del modelo en pequeña señal indicado en (C.122). Basta con anular los términos independientes, $\hat{v}_{g}(t)$ y $\hat{d}(t)$ y cambiar la ecuación relativa a la tensión del condensador $C_{o}$, puesto que éste,junto con la carga $R$ quedan fuera y en su lugar se ha conectado una fuente de intensidad, $\hat{i}_{t}(t)$. La nueva ecuación relaciona la intensidad en $R_{e q}$ con la intensidad que entrega esa fuente. También hay que sustituir el término $\hat{v}_{C_{o}}(t)$ por $\hat{v}_{t}(t)$ en el resto de ecuaciones. Finalmente, tras aplicar Laplace, las ecuaciones resultantes quedan indicadas en (C.157):

$$
\begin{aligned}
s L \hat{i}_{L}(s) & =-(1-D) \hat{v}_{C_{c}}(s) \\
-R_{e q} \hat{i}_{t}(s) & =\hat{v}_{C_{c}}(s)-\hat{v}_{t}(s) \\
s C_{c} \hat{v}_{C_{c}}(s) & =(1-D) \hat{i}_{L}(s)+\hat{i}_{t}(s)
\end{aligned}
$$

Se despeja $\hat{i}_{L}$ de la primera ecuación y se sustituye en la tercera ecuación 
para despejar $\hat{v}_{C_{c}}(s)$ y utilizarla en la segunda ecuación.

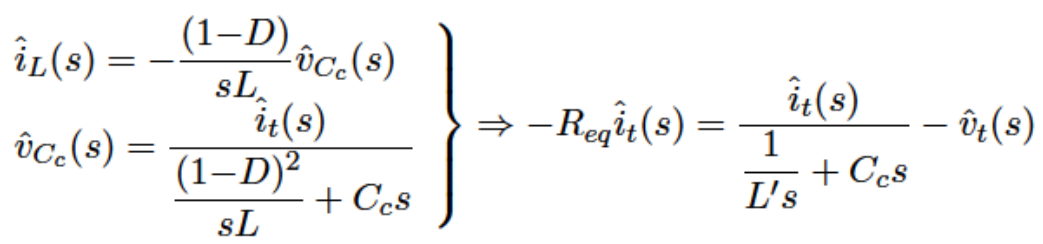

Ahora se puede conseguir una expresión de la impedancia buscada. El resultado obtenido coincide con el mostrado en (C.156).

$$
\hat{v}_{t}(s)=\overbrace{\left[R_{e q}+\frac{1}{\frac{1}{L^{\prime} s}+C_{c} s}\right]}^{Z_{t h}(s)} \hat{i}_{t}(s)
$$

En la Figura C.26 se ha representado esta impedancia para un Red.-Elevador ZVS con los parámetros de la Tabla C.1. También están representados los módulos de las impedancias que forman parte de $Z_{t h}(s)$, esto es, la bobina $L^{\prime}$, el condensador $C_{c}$ y el término de resistencia equivalente $R_{e q}$.

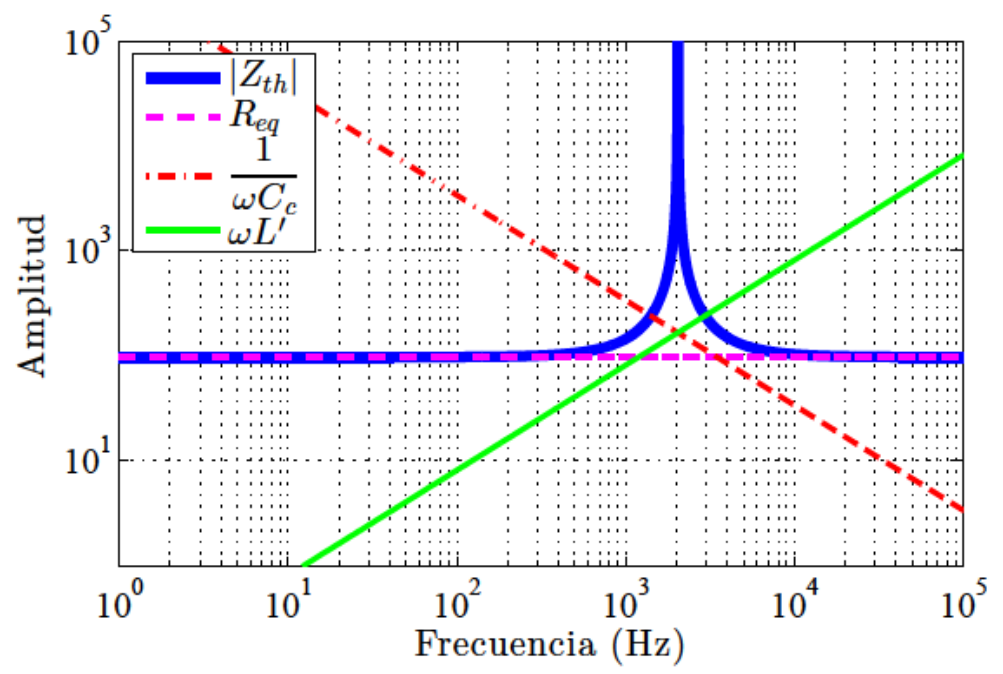

Figura C.26: Representación gráfica de $\left|Z_{t h}(s)\right|$ y los elementos que la forman.

Al hacer $s=0$ se obtiene la impedancia en continua que es igual al término $R_{e q}$, y se corresponde con la asíntota horizontal de la impedancia $Z_{t h}(s)$ a baja frecuencia:

$$
Z_{t h}(0)=Z_{e q}(0)=R_{e q}
$$

Este mismo resultado se obtiene analizando el circuito de la Figura C.25 cuando la bobina y el condensador se convierten en un cortocircuito y en 
un circuito abierto respectivamente. La fuente independiente de intensidad ahora es una fuente constante conectada en los terminales donde se va a medir esa resistencia. El circuito modificado está representado en la Figura C.27:

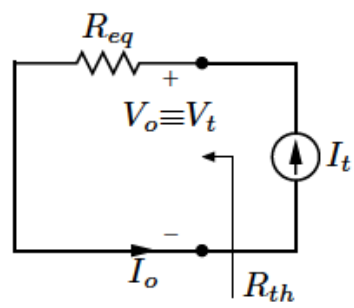

Figura C.27: Circuito empleado para el cálculo de $R_{t h}$ de un reductorelevador ZVS.

Se obtiene una resistencia igual a:

$$
R_{t h}=R_{e q}
$$

\section{C.7.2. Cálculo de $Z_{\text {out }}$.}

A la vista del circuito de la Figura C.24, se establecen la siguiente relación de tensiones:

$$
-L s \hat{i}_{L}(s)=(1-D) \hat{v}_{C_{c}}(s)
$$

Por otro lado, se establece la relación de corrientes:

$$
(1-D) \hat{i}_{L}(s)=C_{c} s \hat{v}_{C_{c}}(s)+\frac{\hat{v}_{C_{c}}(s)-\hat{v}_{t}(s)}{R_{e q}}
$$

Pasndo la bobina $L$ al otro lado de las fuentes dependientes, aparece una bobina equivalente , $L^{\prime}=L /(1-D)^{2}$, que está en paralelo con $C_{e q}$, como está indicado en el circuito de la Figura C.28a.

Despejando $\hat{v}_{C_{c}}$ de (C.163), y sustituyendo la expresión de $\hat{i}_{L}$ que se deduce de (C.162) se obtiene una expresión en función de $\hat{v}_{t}$ como:

$$
\begin{aligned}
\hat{i}_{L}(s) & =-\frac{(1-D) \hat{v}_{C_{c}}(s)}{L s} \\
\hat{v}_{C_{c}}(s) & =\frac{\hat{v}_{t}(s)}{R_{e q}} \underbrace{\left[C_{C} s+\frac{1}{R_{e q}}+\frac{1}{L^{\prime} s}\right]^{-1}}_{Z_{1}(s)}
\end{aligned}
$$

donde se han agrupado diferentes elementos en $Z_{1}(s)$. Dada la posición de la fuente de corriente $g_{c} \hat{v}_{C_{c}}$, sobre la que aparece la tensión $\hat{v}_{t}$, se puede interpretar como una impedancia, $Z_{c}$, de expresión:

$$
Z_{c}(s)=-\frac{R_{e q}}{g_{c} Z_{1}(s)}
$$




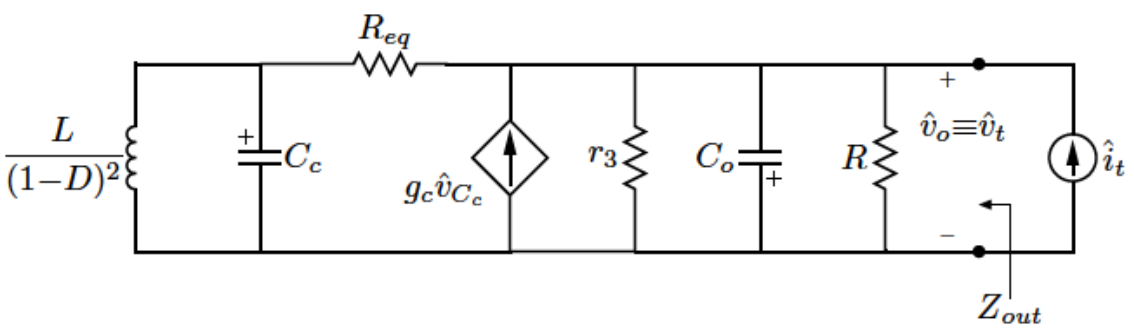

(a)

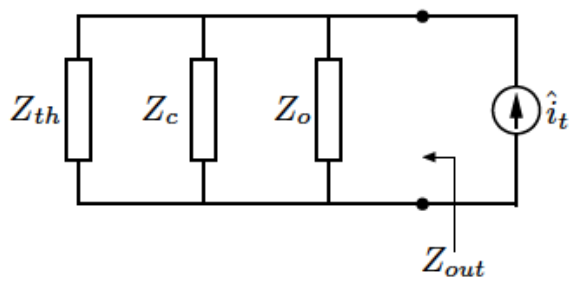

(b)

Figura C.28: Circuito de la Figura C.24 a) tras cambiar L de posición; y b) agrupando elementos.

Agrupando elementos, se puede observar que $Z_{\text {out }}$ es el paralelo de tres impedancias: la impedancia $Z_{t h}$, que engloba los términos $R_{e q}, C_{c}$ y $L^{\prime}$; la impedancia $Z_{o}$, que agrupa a los elementos $r_{3}, C_{o}$ y $R$; y la impedancia $Z_{c}$. Así se ha representado en la Figura C.28b.

$$
Z_{\text {out }}(s)=Z_{o}(s)\left\|Z_{c}(s)\right\| Z_{\text {th }}(s)
$$

A esta expresión también se puede llegar desde las ecuaciones indicadas en (C.167), obtenidas a partir del modelo en pequeña señal indicado en (C.122) donde se anulan los términos independientes, $\hat{v}_{g}(t)$ y $\hat{d}(t)$. Después, hay que cambiar la ecuación relativa a la tensión del condensador $C_{o}$ para introducir la intensidad $\hat{i}_{t}(t)$. Tras cambiar el término $\hat{v}_{C_{o}}(t)$ por $\hat{v}_{t}(t)$, se aplica Laplace a las ecuaciones modificadas, y quedan:

$$
\begin{aligned}
s L \hat{i}_{L}(s) & =-(1-D) \hat{v}_{C_{c}}(s) \\
\hat{i}_{t}(s) & =\hat{v}_{t}(s) \overbrace{\left[s C_{o}+\frac{1}{r_{3}}+\frac{1}{R}\right]}^{Z_{o}^{-1}(s)}-g_{c} \hat{v}_{C_{c}}(s)-\frac{\hat{v}_{C_{c}}(s)-\hat{v}_{t}(s)}{R_{e q}} \\
s C_{c} \hat{v}_{C_{c}}(s) & =(1-D) \hat{i}_{L}(s)-\frac{\hat{v}_{C_{c}}(s)-\hat{v}_{t}(s)}{R_{e q}}
\end{aligned}
$$

De la primera y tercera ecuación se obtiene una expresión para $\hat{v}_{C_{c}}(s)$ igual a la indicada en (C.164), que sustituida en la segunda ecuación permite 
obtener $\hat{i}_{t}(s)$ en función de $\hat{v}_{t}(s)$ como:

$$
\hat{i}_{t}(s)=\frac{\hat{v}_{t}(s)}{Z_{o}(s)}-\hat{v}_{t}(s) \frac{g_{c} Z_{1}(s)}{R_{e q}}-\hat{v}_{t}(s) \frac{\frac{Z_{1}(s)}{R_{e q}}-1}{R_{e q}}
$$

La impedancia de salida tiene la expresión:

$$
Z_{\text {out }}(s)=\left[\frac{1}{Z_{o}(s)}-g_{c} \frac{Z_{1}(s)}{R_{e q}}+\frac{R_{e q}-Z_{1}(s)}{R_{e q}^{2}}\right]^{-1}
$$

que es equivalente a la obtenida anteriormente en (C.166), puesto que:

$$
\begin{aligned}
Z_{1}(s) & =\left[\frac{1}{R_{e q}}+\frac{1}{L^{\prime} s}+C_{c} s\right]^{-1}=\frac{R_{e q} L^{\prime} s}{R_{e q}+L^{\prime} s+R_{e q} L^{\prime} C_{c} s^{2}} \\
R_{e q}-Z_{1}(s) & =\frac{R_{e q}^{2}+\underline{R}_{e q} L^{\prime} s}{R_{e q}+R_{e q}^{2} L^{\prime} C_{c} s^{2}-R_{e q} L^{\prime} s}=\frac{R_{e q}^{2}}{Z_{e q}(s)}
\end{aligned}
$$

Entonces, la impedancia de salida, indicada en (C.166) y en (C.169), queda finalmente como:

$$
Z_{\text {out }}(s)=\left[\frac{1}{Z_{o}(s)}-\frac{1}{Z_{c}(s)}+\frac{1}{Z_{\text {th }}(s)}\right]^{-1}
$$

En la Figura C.29 se ha representado el módulo de esta impedancia para un Red.-Elev. ZVS, a partir de los parámetros indicados en la Tabla C.1 junto con el módulo de los términos que la forman, $\left|Z_{t h}\right|,\left|Z_{c}(s)\right|$ y $\left|Z_{o}(s)\right|$.

Al hacer $s=0$ se obtiene la impedancia de salida en continua, que es el paralelo de los términos $R_{e q}, r_{3}$ y $R$. Y se corresponde con la asíntota horizontal de $Z_{\text {out }}$ a bajas frecuencias.

$$
Z_{\text {out }}(0)=\frac{1}{\frac{1}{Z_{o}(0)}-\frac{g_{C} Z_{1}(0)}{R_{e q}}+\frac{1}{R_{e q}}}=\left[\frac{1}{r_{3}}+\frac{1}{R}-0+\frac{1}{R_{e q}}\right]^{-1}
$$

\section{C.7.3. Validación de las expresiones obtenidas.}

La validación de la expresión de la impedancia, se comprueba con la simulación de un convertidor conmutado al que se le mide la impedancia de salida. Con los parámetros de funcionamiento recogidos en la Tabla C.1 se añade a la salida una fuente de corriente de frecuencia variable y se mide la variación que experimenta la tensión de salida.

Como las expresiones de impedancia se han obtenido a partir del modelo promediado parece lógico medir la impedancia sobre la simulación de este modelo y comparar los resultados obtenidos con la teoría. En este caso, se 


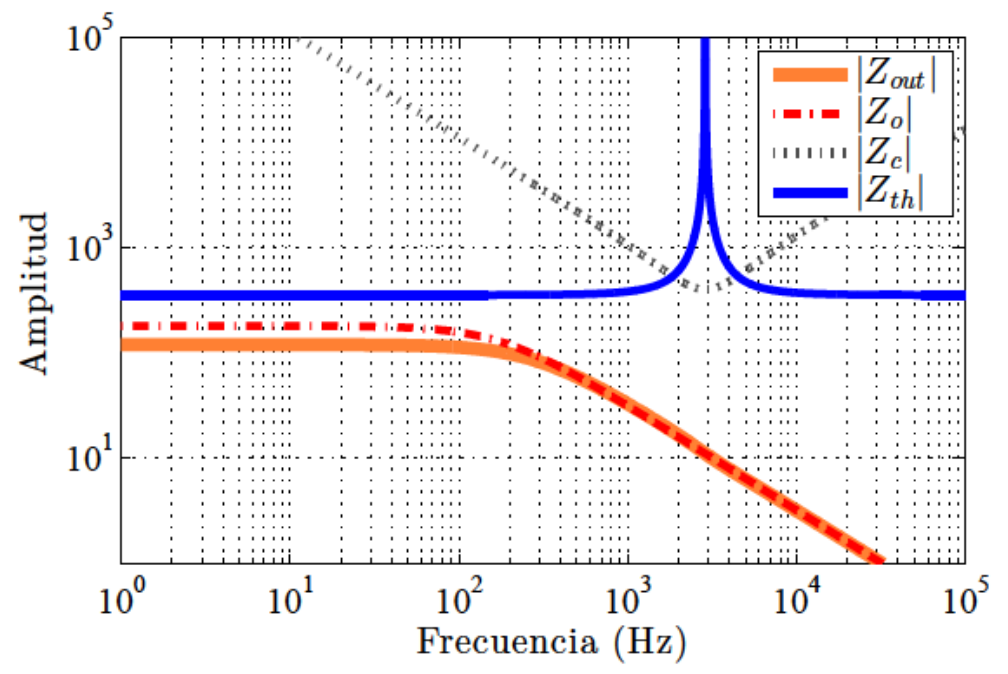

Figura C.29: Representación gráfica de $\left|Z_{\text {out }}(s)\right|$ y los elementos que la forman.

emplearía el ciclo de trabajo calculado para el modelo promediado. Si se emplea el convertidor conmutado para medir la impedancia, debería considerarse el valor del ciclo de trabajo obtenido para el circuito conmutado con la corrección propuesta en el apartado correspondiente.

En la Figura C.30 se han representado los valores obtenidos en la medida de la impedancia de salida de un Red.-Elev. ZVS simulado con los parámetros indicados en la Tabla C.1. También se ha representado el módulo de la impedancia de salida teórica, $\left|Z_{\text {out }}(s)\right|$.

Como ya se conocía, presenta una asíntota horizontal que es el paralelo de $R_{e q}, r_{3}$ y $R$, otra asíntota asociada al condensador $C_{o}$. A la vista de esta gráfica, se observa la coincidencia de la impedancia teórica y los valores obtenidos en la simulación, por lo que se concluye la validez de las expresiones de impedancia obtenidas de forma teórica.

Por ejemplo, la impedancia multietapa sin el condensador de salida, $Z_{m e}(s)$ se corresponde con la expresión:

$$
Z_{m e}(s)=\left[\frac{1}{r_{3}}-\frac{g_{c} Z_{1}(s)}{R_{e q}}+\frac{1}{Z_{t h}(s)}\right]^{-1}
$$

En la Figura C.31 se ha representado el módulo de esta impedancia y las asíntotas correspondientes:

Haciendo $s=0$ se consigue la asíntota de baja frecuencia, que es el paralelo de $R_{e q}$ y de $r_{3}$. Una expresión equivalente se consigue al emplear sus 


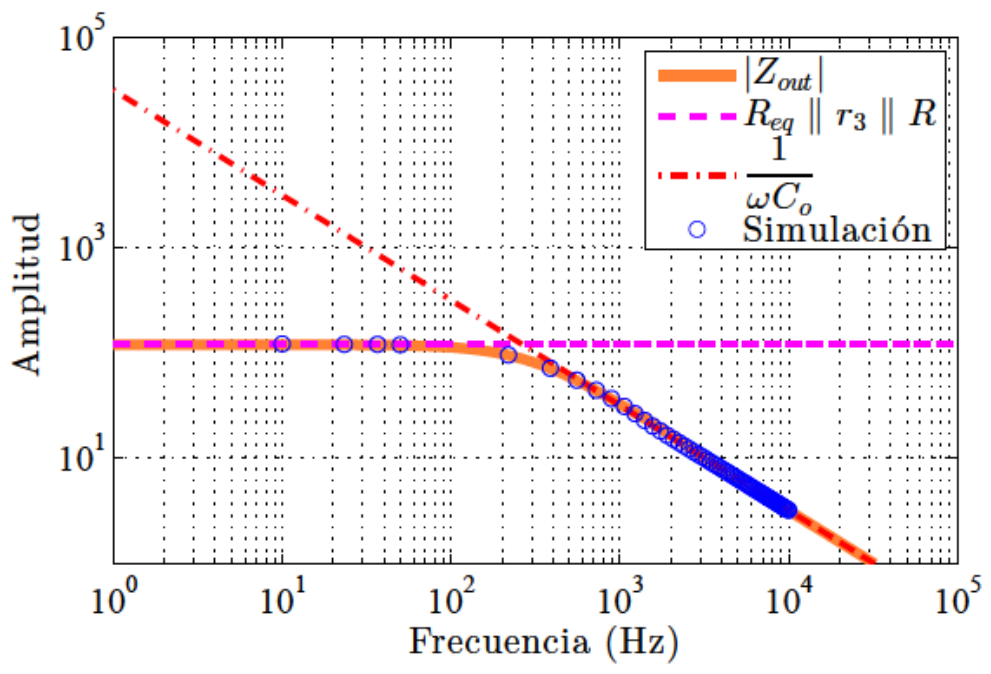

Figura C.30: Representación gráfica de $\left|Z_{\text {out }}(s)\right|$ y resultados de simulación.

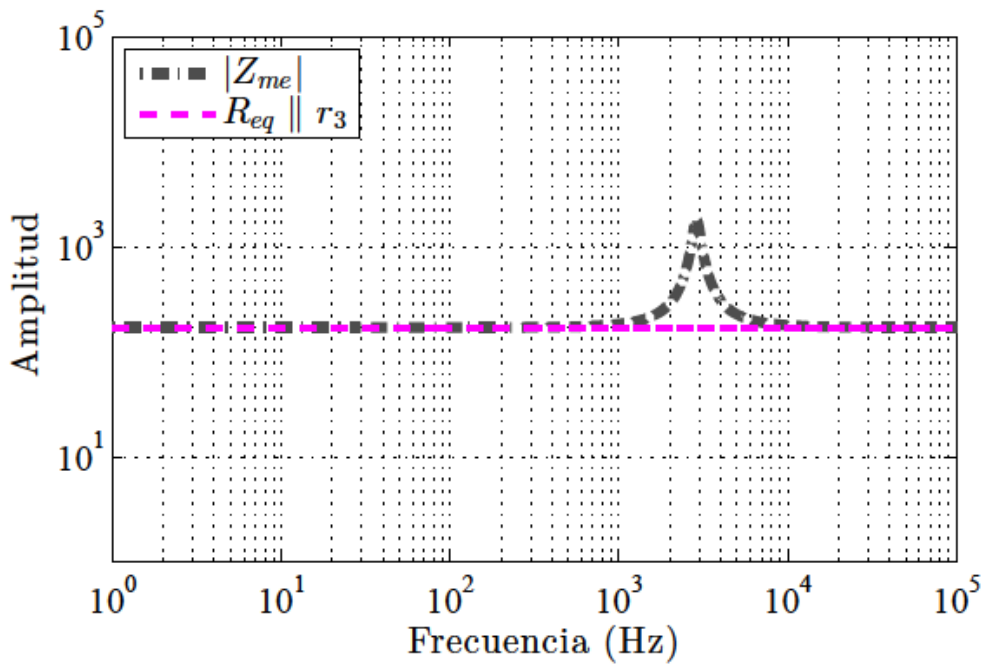

Figura C.31: Representación gráfica de $\left|Z_{m e}(s)\right|$.

expresiones indicadas en la tabla C.4, y ayudándose de la primera y tercera 
ecuación del modelo en régimen permanente indicado en (C.99). Entonces:

$$
\begin{aligned}
Z_{m e}(0) & =\left[\frac{2(1-D) I+I_{e q}}{V_{g}-V_{C_{o}}}+\frac{1}{R_{e q}}\right]^{-1}=\left[\frac{\frac{2\left(V_{c}+V_{C_{o}}\right)}{R_{e q}}+\frac{\left(V_{c}+V_{C_{o}}\right)^{2}}{\left(V_{g}-V_{C_{o}}\right) R_{e q}}}{V_{g}-V_{C_{o}}}+\frac{1}{R_{e q}}\right]^{-1} \\
& =\left[\frac{1}{R_{e q}}+\frac{2\left(V_{c}+V_{C_{o}}\right)}{\left(V_{g}-V_{C_{o}}\right) R_{e q}}+\frac{\left(V_{c}+V_{C_{o}}\right)^{2}}{\left(V_{g}-V_{C_{o}}\right)^{2} R_{e q}}\right]^{-1}=R_{e q}\left[1+\frac{V_{c}+V_{C_{o}}}{V_{g}-V_{C_{o}}}\right]^{-2} \\
& =R_{e q}\left[\frac{V_{g}-V_{C_{o}}}{V_{g}+V_{c}}\right]^{2}=2 \operatorname{Lrf}\left[\frac{V_{g}-V_{C_{o}}}{V_{g}}\right]^{2}
\end{aligned}
$$

Esta expresión coincide con la derivada de la característica de salida, y que está indicada en la expresión (C.52). Es la impedancia de salida en régimen permanente que presenta el convertidor.

Cuando $s$ se hace muy grande, $Z_{1}(s)$ tiende a cero, mientras que $Z_{t h}(s)$ y $Z_{o}$ se transforman en $R_{e q}$ y $r_{3}$ respectivamente. El paralelo de estos dos términos es la asíntota para altas frecuencias, y coincide con la asíntota de bajas frecuencias. 



\section{Apéndice D}

\section{Flyback ZVS tipo buck-boost}

\section{D.1. Descripción y funcionamiento.}

El flyback ZVS es un flyback convencional al que se le ha añadido una red de enclavamiento activo que fija la tensión máxima en los extremos del transistor principal. Esta red facilita las conmutaciones a tensión cero, reduciendo las pérdidas que aparecen. Por contra, aumenta la complejidad del circuito y el número de componentes. En la figura D.1 está representado el circuito de este convertidor.

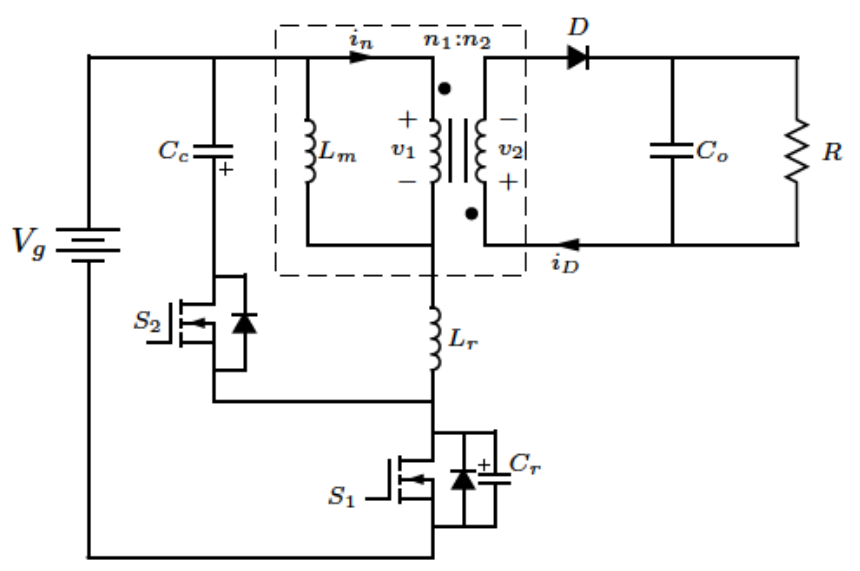

Figura D.1: Convertidor flyback ZVS.

El flyback convencional se construye a partir de un convertidor reductorelevador al que se añade aislamiento a la salida con unas bobinas acopladas (que en conjunto equivale a un transformador y por eso se ha representado así) antes del condensador, y presenta una inductancia de magnetización, $L_{m}$, incluida en el primario que almacena la energía durante un tiempo y luego la devuelve cuando lo permiten los componentes del circuito. El diodo que aparece en el secundario hace la misma función que en el red-elevador y 
solo permite circulación de la corriente en un sentido.

Gracias a las bobinas acopladas es posible adaptar la polaridad de la tensión de salida para que no aparezca con polaridad opuesta a la esperada, que es lo que ocurre en el reductor-elevador. Basta con construirlas y conectarlas de forma que los terminales correspondientes sean los que se indican en el circuito representado en la figura. Los puntos se emplean para marcar dichos terminales e indican que la tensión de primario tiene la misma polaridad que la de secundario en esos terminales. Respecto a las corrientes, si se considera positiva una de ellas cuando entra al trafo por un terminal marcado por un punto, en el otro terminal correspondiente, la corriente será considerada como positiva si tiene el sentido de salida por el punto. Por ejemplo, si se considera positiva la corriente que entra por el punto del primario, representada como $i_{n}(t)$, la corriente en el diodo del secundario, $i_{D}(t)$, será igual, pero de signo contrario a la corriente que sale por el terminal marcado con el punto en el secundario puesto que tiene sentido contrario al considerado positivo.

La red de enclavamiento activo está formada por el condensador de clamp $C_{c}$ y el interruptor $S_{2}$ junto con su diodo en antiparalelo, $D_{2}$. Además se añade una pequeña inductancia, $L_{r}$, que incluye las inductancias parásitas y que actúa en resonancia con el condensador $C_{c}$ en determinados instantes del periodo. Se ha incluido el diodo $D_{1}$ al interruptor $S_{1}$ y un condensador $C_{r}$ que puede ser el propio del transistor o uno añadido que mejore sus características. Se considerará la inductancia de magnetización del transformador, $L_{m}$, como el elemento reactivo que acumula y devuelve energía durante un ciclo de funcionamiento.

Para explicar el funcionamiento de este convertidor se plantea el estado de los diferentes semiconductores presentes en el circuito, que son los dos interruptores y los tres diodos. En el instante inicial, $t_{0}$, está cerrado $S_{1}$ y el condensador $C_{r}$ está descargado. Se considera el condensador de salida, $C_{o}$, de un valor grande para mantener la tensión $v_{C_{o}}$ constante y se sustituirá el conjunto C-R por una fuente de tensión constante de valor $V_{o}$. La bobina de magnetización, $L_{m}$, es lo suficientemente grande para considerar que pasa una corriente constante, $I$, por ella. El condensador de clamp, $C_{c}$ presenta una tensión con la polaridad indicada en figura y se supone lo suficientemente grande como para suponer una tensión prácticamente constante, $V_{c}$, aunque haya intervalos donde éste se cargue y se descargue y la tensión de clamp pueda variar en torno a ese valor. Por último, indicar que el valor de $L_{r}$, aunque comparativamente mas pequeño que $L_{m}$, es lo suficientemente grande como para cumplir el criterio de energía que permite conmutaciones suaves en estas condiciones de carga. 


\section{D.1.1. Etapa 1. $\left(t_{0}<t<t_{1}\right)$}

Se abre el interruptor $S_{1}$. Esta conmutación se produce a tensión cero puesto que el condensador $C_{r}$ está descargado. Este condensador se carga a corriente constante igual a $I$, hasta que alcanza el valor de $V g+n V_{o}$, siendo $n$ la relación de transformación dada por $n=n_{1} / n_{2}$. La figura D.2 muestra los elementos que intervienen en esta etapa y el circuito equivalente. El diodo principal está polarizado en inversa y no pasa ninguna corriente por él. A partir de las suposiciones iniciales, el circuito que queda planteado, se muestra en la figura D.2c donde la bobina se ha modelado como una fuente de corriente constante $I$.

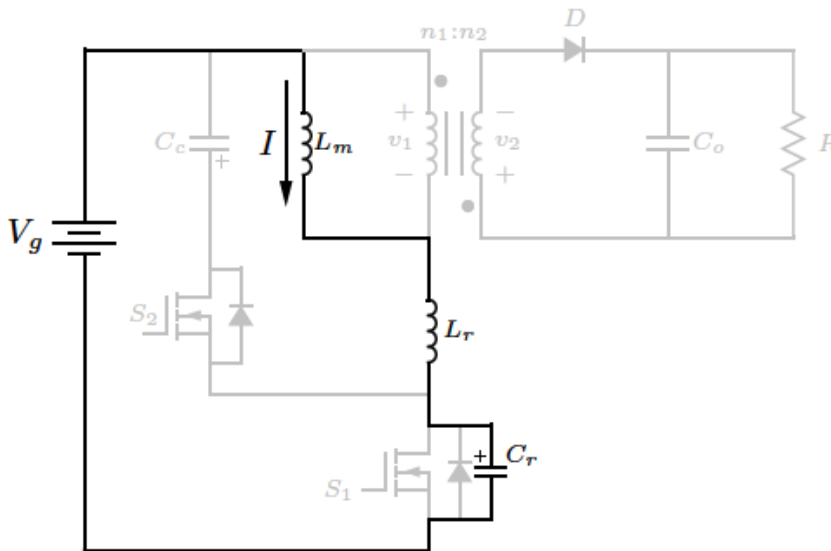

(a)

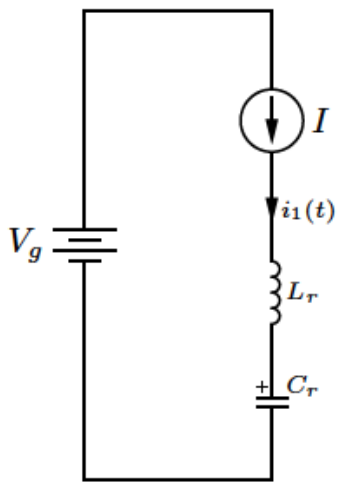

(b)

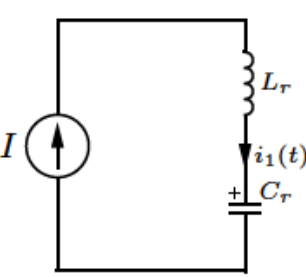

(c)

Figura D.2: Convertidor flyback ZVS: a)durante la etapa 1; b)circuito equivalente; c) circuito para cálculos.

La tensión en el condensador crece de forma lineal, como se indica en (D.1), mientras que la tensión en la bobina $L_{r}$ es cero ya que la corriente que circula es constante.

$$
\begin{aligned}
i_{C_{r}}(t) & =I \\
v_{C_{r}}(t) & =\frac{1}{C_{r}} \int_{0}^{t} i_{C_{r}}(t) d t=\frac{I}{C_{r}} t
\end{aligned}
$$

Esta etapa terminará cuando $v_{C_{r}}(t)=V_{g}+n V_{o}$, porque al alcanzar este valor, el diodo $D$ se polarizará en directa y empezará a conducir. La duración de esta etapa es igual $d_{1} T$, y está indicada en a (D.2):

$$
d_{1} T=\left(t_{1}-t_{0}\right)=\frac{\left(V_{g}+n V_{o}\right) C_{r}}{I}
$$




\section{D.1.2. Etapa 2. $\left(t_{1}<t<t_{2}\right)$}

El diodo $D$ empieza a conducir porque se polariza en directa al igualar su tensión del ánodo iguala a la tensión del cátodo que es $V_{o}$. Veámoslo:

$$
v_{2}=\frac{1}{n} v_{1}=\frac{V_{g}-\left(V_{g}+n V_{o}\right)}{n}=-V_{o}
$$

Ahora ya hay una intensidad circulando por ambas bobinas acopladas, y la corriente que pasa por $L_{r}$ es la suma de la corriente en la bobina de magnetización, que es igual a $I$, y la corriente que pasa por la bobina acoplada de primario, $i_{n}(t)$. El condensador $C_{r}$ sigue cargándose, pero ahora no es a corriente constante sino a una corriente variable, $i_{2}(t)$, obtenida por la resonancia del condensador y la bobina. En la figura D.3 se muestran los elementos que intervienen y el circuito equivalente.

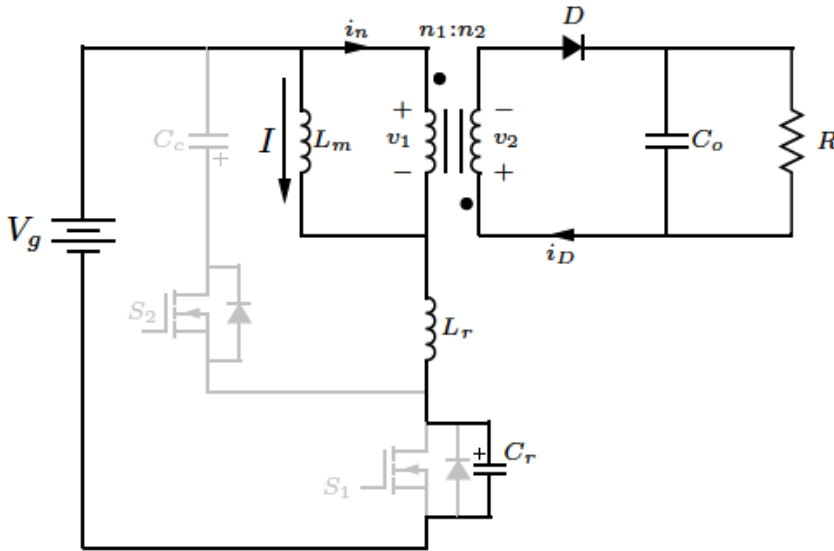

(a)

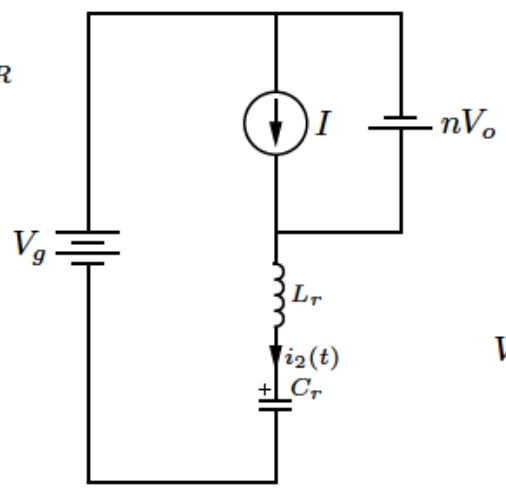

(b)

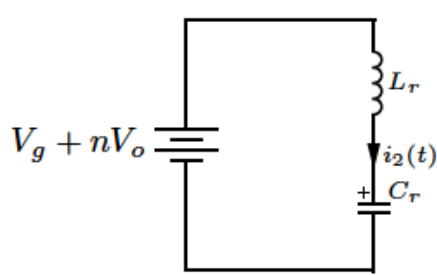

(c)

Figura D.3: Convertidor flyback ZVS a)durante la etapa 2; b)circuito equivalente; c) circuito para cálculos.

Obteniendo la corriente $i_{2}(t)$, se conseguirá la evolución de la tensión en $C_{r}$, y cuando alcance el valor de $V_{g}+V_{c}$ terminará esta etapa, ya que se polarizará en directa el diodo del interruptor $S_{2}$, dando lugar a otro subcircuito. la ecuación para esta corriente se obtiene al plantear la tensión en la rama resonante, y está dada en (D.4), que es una ecuación integro-diferencial con coeficientes constantes.

$$
V_{g}+n V_{o}=v_{L_{r}}(t)+v_{C_{r}}(t)=L_{r} \frac{d i_{2}(t)}{d t}+v_{C_{r}}(0)+\frac{1}{C_{r}} \int_{0}^{t} i_{2}(t) d t
$$

Las condiciones iniciales son (D.5):

$$
\begin{aligned}
& i_{2}(0)=I \\
& i_{2}^{\prime}(0)=\frac{1}{L_{r}} v_{L_{r}}(0)=\frac{1}{L_{r}}\left[V_{g}+n V_{o}-v_{C_{r}}(0)\right]=0
\end{aligned}
$$


donde la intensidad debe cumplir la condición de continuidad, pero no la derivabilidad.

La resolución de esta ecuación diferencial se puede obtener de varias formas. Método A) derivar de nuevo la ecuación, y resolverla como una ecuación diferencial ordinaria de segundo orden con coeficientes constantes teniendo en cuenta las condiciones iniciales. Método B) Aplicar Laplace a la ecuación (D.4) teniendo en cuenta las condiciones iniciales al aplicar Laplace y después obtener la transformada inversa. Método C): Aplicar Laplace al circuito y resolverlo en el dominio de Laplace, y una vez obtenida una expresión de la corriente hacer la transformada inversa.

METODO A) para obtener $i_{2}(t)$.

Derivando la ecuación integro-diferencial, se obtiene (D.6), y se mantienen las condiciones iniciales de (D.5):

$$
0=L_{r} \frac{d^{2} i_{2}(t)}{d t^{2}}+\frac{1}{C_{r}} i_{2}(t)
$$

Esta ecuación se resuelve a partir de la ecuación característica, cuya resolución ofrece dos raíces complejas conjugadas, dadas en (D.7):

$$
L_{r} D^{2}+\frac{1}{C_{r}}=0 \Rightarrow D= \pm \sqrt{\frac{-1}{L_{r} C_{r}}}= \pm j \omega_{r}
$$

donde $\omega_{r}=\left(\sqrt{L_{r} C_{r}}\right)^{-1}$ es la frecuencia de resonancia de la bobina y el condensador. Al ser dos raíces complejas conjugadas se prueba con una solución de la forma indicada en (D.8), a la que se le aplican las condiciones (D.5).

$$
i_{2}(t)=K_{1} \cos \left(\omega_{r} t\right)+K_{2} \sin \left(\omega_{r} t\right)
$$

$\mathrm{Al}$ aplicar la primera condición, se deduce que $K_{1}=I_{g}$. Con la segunda condición se deduce que $K_{2}=0$.

$$
\begin{aligned}
& i_{2}(0)=I \Rightarrow I=K_{1} * 1+K_{2} * 0 \Rightarrow K_{1}=I \\
& i_{2}^{\prime}(0)=0 \Rightarrow K_{1} \sin \left(\omega_{r} t\right)\left(-\omega_{r}\right)+\left.K_{2} \cos \left(\omega_{r} t\right) \omega_{r}\right|_{t=0}=0 \Rightarrow K_{2}=0
\end{aligned}
$$

De esta forma la solución para la ecuación diferencial es la indicada en (D.10):

$$
i_{2}(t)=I \cos \left(\omega_{r} t\right)
$$

METODO B) para obtener $i_{2}(t)$.

Aplicando Laplace a la ecuación integro-diferencial (D.4), se obtiene una expresión como la indicada en (D.11) donde ahora entran en juego los valores iniciales de las variables implicadas, donde $I_{2}(s)=\mathscr{L}\left[i_{2}(t)\right]$.

$$
\begin{gathered}
\mathscr{L}\left[V_{g}+n V_{o}\right]=\mathscr{L}\left[L_{r} \frac{d i_{2}(t)}{d t}+v_{C_{r}}(0)+\frac{1}{C_{r}} \int_{0}^{t} i_{2}(t) d t\right] \\
\frac{V_{g}+n V_{o}}{s}=L_{r} s I_{2}(s)-L_{r} i_{2}(0)+\frac{v_{C_{r}}(0)}{s}+\frac{1}{C_{r} s} I_{2}(s)
\end{gathered}
$$


Recuerde que $v_{C_{r}}(0)=V_{g}+n V_{o}$, y que $i_{2}(0)=I$. Una vez despejada $I_{2}(s)$, se obtiene, tras aplicar Laplace, el resultado (D.12):

$$
I_{2}(s)=I \frac{s}{s^{2}+\frac{1}{L_{r} C_{r}}} \Rightarrow i_{2}(t)=\mathscr{L}^{-}\left[I_{2}(s)\right]=I \cos \left(\omega_{r} t\right)
$$

METODO C) para obtener $i_{2}(t)$.

Este método consiste en transformar el circuito al dominio de Laplace y después resolver la corriente transformada, es decir, $I_{2}(s)$ en el circuito resultante. Si se aplica Laplace al circuito de la figura D.4a (donde previamente se ha sustituido el condensador cargado a una tensión por un condensador vacío y una fuente de tensión en serie del mismo valor y polaridad que la que tenía inicialmente y la bobina se ha sustituido por una bobina descargada y una fuente de corriente en paralelo y del mismo valor y sentido que la que estaba circulando inicialmente), se obtiene el circuito en el dominio transformado como el de la figura D.4b. En este caso, la tensión inicial del condensador es $V_{g}+n V_{o}$ y la corriente inicial en la bobina es $I$.

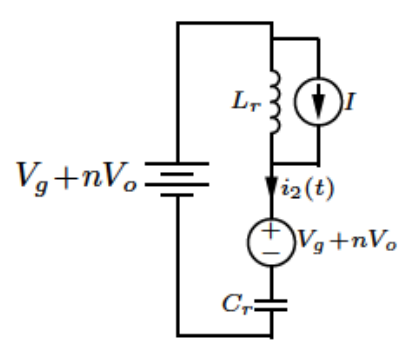

(a)

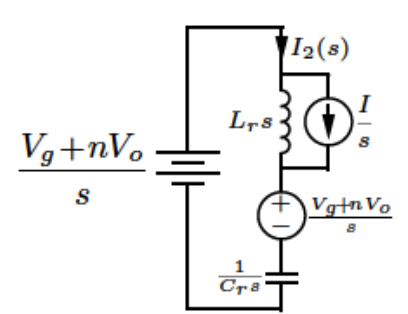

(b)

Figura D.4: Circuito equivalente de la Etapa 2: a) en el dominio del tiempo; y b) en el dominio de Laplace.

Para facilitar las operaciones, se sustituye el conjunto bobina-fuente de corriente en el circuito transformado por su equivalente Thévenin. en la figura D.5 se ha representado el circuito equivalente. De esta forma, se obtiene
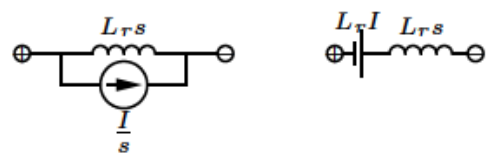

Figura D.5: Equivalente Thévenin del conjunto bobina-fuente de corriente.

fácilmente la expresión para $I_{2}(s)$, como se indica en (D.13), a partir de la 
ecuación de tensión en la rama resonante:

$$
\begin{aligned}
\frac{V_{g}+n V_{o}}{s} & =-L_{r} I+I_{2}(s) L_{r} s+\frac{V_{g}+n V_{o}}{s}+I_{2}(s) \frac{1}{C_{r} s} \\
L_{r} I & =I_{2}(s)\left(\frac{1}{C_{r} s}+L_{r} s\right) \\
I_{2}(s) & =I \frac{s}{\frac{1}{L_{r} C_{r} s}+s^{2}} \Rightarrow i_{2}(t)=\mathscr{L}^{-}\left[I_{2}(s)\right]=I \cos \left(\omega_{r} t\right)
\end{aligned}
$$

Por cualquiera de los métodos se obtiene la misma expresión para la corriente $i_{2}(t)$. Una vez calculada, se obtiene la tensión en el condensador como (D.14):

$$
\begin{aligned}
v_{C_{r}}(t) & =v_{C_{r}}(0)+\frac{1}{C_{r}} \int_{0}^{t} i_{2}(\lambda) d \lambda \\
& =V_{g}+n V_{o}+\left[\frac{1}{C_{r}} I \sin \left(\omega_{r} \lambda\right) \omega_{r}^{-1}\right]_{0}^{t} \\
& =V_{g}+n V_{o}+\frac{I}{\omega_{r} C_{r}} \sin \left(\omega_{r} t\right)
\end{aligned}
$$

La duración de esta etapa será el tiempo que tarda el condensador en alcanzar la tensión $V_{g}+V_{c}$, porque el diodo de $S 2$ se polariza en directa. Este tiempo se deduce de (D.14), y es igual a (D.15).

$$
d_{2} T=\left(t_{2}-t_{1}\right)=\omega_{r}^{-1} \arcsin \left(\frac{\left(V_{c}-n V_{o}\right) \omega_{r} C_{r}}{I}\right)
$$

La corriente que pasa por el diodo principal, $i_{D}(t)$, es igual a la corriente de la bobina acoplada de secundario. Según el criterio de los puntos, al entrar por el punto en el secundario, la intensidad en el primario debe salir por el punto, es decir, debe tener un sentido contrario al marcado para $i_{n}(t)$. Ésta es igual a la diferencia entre la que pasa por la bobina de magnetización menos la que circula por la bobina resonante y afectada por la relación de transformación. Está indicada en (D.16). Resulta que la corriente $i_{2}(t)$ al final de esta etapa es igual a $I^{\prime}$ y tiene un valor ligeramente inferior a su valor inicial.

$$
i_{D}(t)=-n i_{n}(t)=-n\left(i_{2}(t)-I\right)=n I\left(1-\cos \left(\omega_{r} t\right)\right)
$$

\section{D.1.3. Etapa 3. $\left(t_{2}<t<t_{3}\right)$}

El diodo del interruptor auxiliar $S_{2}$ empieza a conducir. A partir de este instante, se puede cerrar este interruptor a tensión cero porque está circulando corriente por su diodo. En la figura D.6 se muestran los elementos que intervienen y el circuito equivalente, donde $i_{c}(t)$ representa la corriente que circula por el condensador de clamp. 


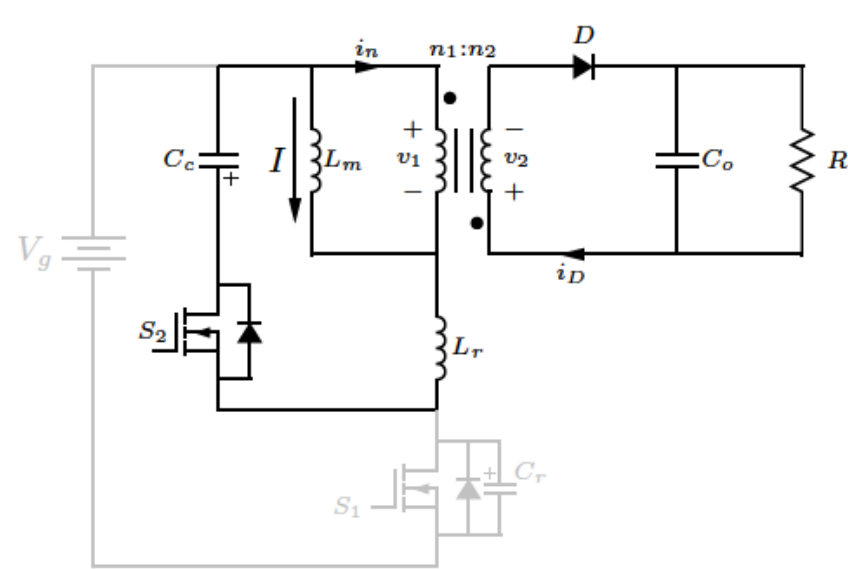

(a)

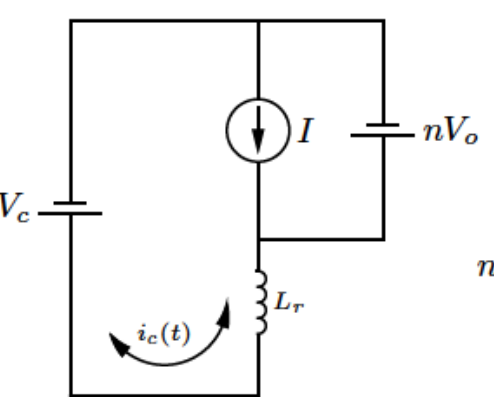

(b)

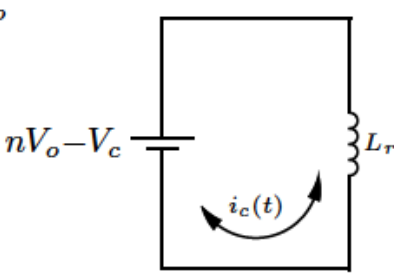

(c)

Figura D.6: Convertidor flyback ZVS a)durante la etapa 3; b)circuito equivalente; c) circuito para cálculos.

Nótese que el condensador de clamp es grande para la energía que absorbe por lo que su tensión se mantiene prácticamente constante durante todo este intervalo y de ahí que se represente como una fuente de tensión con la polaridad indicada. Además la corriente $i_{c}(t)$ circula inicialmente en un sentido y luego se invierte cuando la bobina se ha descargado completamente sobre $C_{c}$. Pero como el interruptor $S_{2}$ permanece cerrado, el condensador cederá esta energía extra de nuevo a la bobina, haciendo que la corriente circule en el sentido opuesto al inicial. En este caso, la corriente circulará por $S_{2}$ y no por su diodo.

La relación entre la tensión de clamp, y la duración de este intervalo se obtiene resolviendo el circuito equivalente. Para ello hay que resolver el circuito de la figura C.6c para obtener $i_{c}(t)$. En esta malla, la tensión es constante e igual a $V_{c}$. La bobina resonante se descarga a tensión constante y su expresión se obtiene resolviendo la ec.diferencial de primer orden con condiciones iniciales de (D.17):

$$
\begin{aligned}
-V_{c}+n V_{o} & =v_{L_{r}}(t)=L_{r} \frac{d i_{c}(t)}{d t} \\
i_{c}(0) & =I^{\prime}
\end{aligned}
$$

Haciendo la separación de variables en cada miembro de (D.17), e integrando se obtiene una solución general, en la que se despeja la constante de 
integración aplicando la condición inicial.

$$
\begin{aligned}
-\frac{V_{c}-n V_{o}}{L_{r}} d t & =d i_{c}(t) \\
-\frac{V_{c}-n V_{o}}{L_{r}} \int d t & =\int d i_{c}(t) \\
i_{c}(t) & =-\frac{V_{c}-n V_{o}}{L_{r}} t+K \\
i_{c}(0) & =I^{\prime} \Rightarrow K=I^{\prime} \Rightarrow i_{c}(t)=I^{\prime}-\frac{V_{c}-n V_{o}}{L_{r}} t
\end{aligned}
$$

En la solución dada en (D.18) se comprueba que $i_{c}(t)$ es decreciente y llega a ser negativa. De hecho, la duración de esta etapa es $d_{3} T=\left(t_{3}-t_{2}\right)$.

Al final de este periodo, la corriente alcanza un valor igual a $I^{\prime}$ pero en sentido contrario. De esta relación se deduce el valor de la tensión de clamp en función de la bobina resonante y el ciclo de trabajo. Véase (D.19).

$$
i_{c}\left(t_{3}\right)=-I^{\prime} \Rightarrow-I^{\prime}=I^{\prime}-\frac{V_{c}-n V o}{L_{r}} d_{3} T \Rightarrow V_{c}=\frac{2 I^{\prime} L_{r}}{d_{3} T}+n V_{o}
$$

También se puede decir lo siguiente: Si se habla de régimen permanente, la energía neta en el condensador de clamp durante un ciclo de trabajo, es decir, la energía que toma y que devuelve durante ese tiempo, debe ser nula. Como se ha supuesto un valor de $C_{c}$ suficientemente grande para que $V_{c}$ sea constante, al aplicar esta suposición se llega a que el valor medio de (D.18) es nulo. Dicho de otra forma, el balance de carga en $C_{c}$ deber se nulo. Siendo así, resulta que

$$
\begin{gathered}
\Delta E=0 \Rightarrow \int_{t_{0}}^{t_{0}+T} i_{c}(t) d t=\int_{t_{2}}^{t_{3}} i_{c}(t) d t=\int_{0}^{d_{3} T}\left[I^{\prime}-\frac{V_{c}-n V_{o}}{L_{r}} t\right] d t=0 \\
{\left[I^{\prime} t-\frac{V_{c}-n V_{o}}{2 L_{r}} t^{2}\right]_{0}^{d_{3} T}=0 \Rightarrow V_{c}=\frac{2 I^{\prime} L_{r}}{d_{3} T}+n V_{o}}
\end{gathered}
$$

Este resultado sustituido en (D.18) permite obtener el valor de $i_{c}\left(t_{3}\right)$ para el régimen permanente. Así se indica en (D.21).

$$
i_{c}\left(t_{3}\right)=I^{\prime}-\frac{2 I^{\prime} h_{r}}{L_{r} d_{3} T} d_{3} T=-I^{\prime}
$$

Durante un transitorio, la tensión de clamp evolucionará en cada periodo que dure el transitorio de un valor a otro, y el balance de carga no será nulo, y por tanto, no se puede saber a priori el valor de $i_{c}\left(t_{3}\right)$ al final de cada periodo.

Ahora se abre el interruptor, y lo hace a tensión cero porque e condensador $C_{r}$ se había quedado cargado a una tensión $V_{g}+V_{c}$, de forma que el drenador de $S_{2}$ está a tensión cero. 
La corriente que pasa por el diodo principal vuelve a ser, como en la etapa anterior, igual a la corriente de la bobina acoplada de secundario. Según el criterio de los puntos, al entrar por el punto en el secundario, la intensidad en el primario debe salir por el punto, es decir, debe tener un sentido contrario al marcado para $i_{n}(t)$. Ésta es igual a la diferencia entre la que pasa por la bobina de magnetización menos la que circula por la bobina resonante y afectada por la relación de transformación. Está indicada en (D.22).

$$
\begin{aligned}
i_{D}(t) & =-n i_{n}(t)=-n\left(i_{c}(t)-I\right)=n\left[I-i_{c}(t)\right] \\
& =n\left[I-\left(I^{\prime}-\frac{V_{c}-n V_{o}}{L_{r}} t\right)\right]=n\left(I-I^{\prime}\right)+n \frac{V_{c}-n V_{o}}{L_{r}} t
\end{aligned}
$$

La corriente máxima que pasa por el diodo principal alcanza el valor máximo de (D.23): Véase (D.19).

$$
I_{D, \max }=n\left(I-i_{c, \min }\right)=n\left(I-i_{c}\left(t_{3}\right)\right)=n\left(I+I^{\prime}\right)
$$

D.1.4. Etapa 4. $\left(t_{3}<t<t_{4}\right)$

Este intervalo se inicia con la apertura de $S_{2}$ a tensión nula. La corriente en $L_{r}$ tiene un sentido inicial opuesto al inicial de la etapa anterior, y ésta ya no puede circular ni por $S_{2}$ ni por su diodo, luego deberá circular a través de $C_{r}$. Dicho de otra forma, la bobina $L_{r}$ acumulará energía, pero esta vez de $C_{r}$ que se descarga de forma resonante sobre aquella. La corriente $i_{4}(t)$ experimenta durante este intervalo una evolución resonante, a la vez que la tensión $V_{C_{r}}(t)$ disminuye hasta hacerse cero, que es donde concluye esta etapa. A partir de este instante, la conducción de la corriente se realiza sobre el diodo $D_{1}$. En el circuito de la figura D.7 se muestran los elementos que están involucrados en esta etapa.

Una condición necesaria, sobre la que volveremos mas adelante, es que la energía acumulada en $L_{r}$ debe ser como poco igual a la energía que acumula $C_{r}$ para que la bobina consiga descargar por completo al condensador antes de que agote. Para la obtención de $i_{4}(t)$ (donde se ha mantenido el mismo sentido que en los casos de las etapas anteriores), hay que plantear le ecuación de la tensión en la rama resonante del circuito equivalente y resolver la ecuación diferencial resultante utilizando las condiciones iniciales conocidas. La ecuación resultante es una ecuación integro-diferencial con coeficientes constantes dada por (D.24):

$$
V_{g}+n V_{o}=v_{L_{r}}(t)+v_{C_{r}}(t)=L_{r} \frac{d i_{4}(t)}{d t}+v_{C_{r}}(0)+\frac{1}{C_{r}} \int_{0}^{t} i_{4}(t) d t
$$




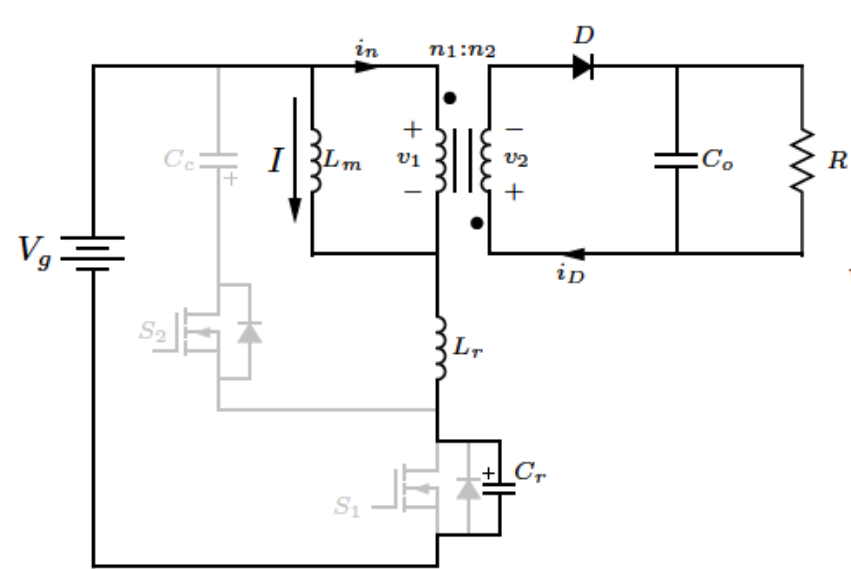

(a)

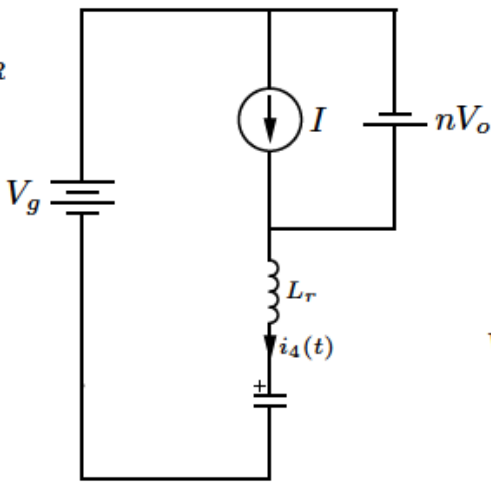

(b)

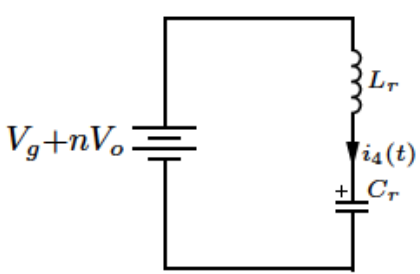

(c)

Figura D.7: Convertidor flyback ZVS a)durante la etapa 4; b)circuito equivalente; c) circuito para cálculos.

Las condiciones iniciales son (D.25):

$$
\begin{aligned}
& i_{4}(0)=-I^{\prime} \\
& i_{4}^{\prime}(0)=\frac{1}{L_{r}} v_{L_{r}}(0)=\frac{1}{L_{r}}\left[V_{g}-v_{C_{r}}(0)+n V_{o}\right]=-\frac{V_{c}-n V_{o}}{L_{r}}
\end{aligned}
$$

donde la intensidad debe cumplir la condición de continuidad, pero no la derivabilidad.

La resolución de esta ecuación diferencial, tal y como se ha hecho en la ETAPA 2, se puede obtener de varias formas. Método A) derivar de nuevo la ecuación, y resolverla como una ecuación diferencial ordinaria de segundo orden con coeficientes constantes teniendo en cuenta las condiciones iniciales. Método B) Aplicar Laplace a la ecuación (D.24) teniendo en cuenta las condiciones iniciales al aplicar Laplace y después obtener la transformada inversa. Método C): Aplicar Laplace al circuito y resolverlo en el dominio de Laplace, y una vez obtenida una expresión de la corriente hacer la transformada inversa.

METODO A) para obtener $i_{4}(t)$.

Derivando la ecuación integro-diferencial, se obtiene (D.26), y se mantienen las condiciones iniciales de (D.25):

$$
0=\frac{1}{C_{r}} i_{4}(t)+L_{r} \frac{d^{2} i_{4}(t)}{d t^{2}}
$$

Esta ecuación se resuelve a partir de la ecuación característica, cuya resolución ofrece dos raíces complejas conjugadas, dadas en (D.27):

$$
0=\frac{1}{C_{r}}+L_{r} D^{2} \Rightarrow D= \pm \sqrt{\frac{-1}{L_{r} C_{r}}}= \pm j \omega_{r}
$$


donde $\omega_{r}$ ya se había definido durante la ETAPA 2 como la frecuencia de resonancia bobina-condensador, y es igual a $\left(\sqrt{L_{r} C_{r}}\right)^{-1}$. Al ser dos raíces complejas conjugadas se prueba con una solución de la forma indicada en (D.28), a la que se le aplican las condiciones (D.25).

$$
i_{4}(t)=K_{3} \cos \left(\omega_{r} t\right)+K_{4} \sin \left(\omega_{r} t\right)
$$

$\mathrm{Al}$ aplicar la primera condición, se deduce que $K_{3}=-I^{\prime}$. Con la segunda condición se deduce el valor de $K_{4}$.

$$
\begin{aligned}
i_{4}(0) & =-I^{\prime} \Rightarrow-I^{\prime}=K_{3} * 1+K_{4} * 0 \Rightarrow K_{3}=-I^{\prime} \\
i_{4}^{\prime}(0) & =-\frac{V_{c}-n V_{o}}{L_{r}} \Rightarrow K_{3} \sin \left(\omega_{r} t\right)\left(-\omega_{r}\right)+\left.K_{4} \cos \left(\omega_{r} t\right) \omega_{r}\right|_{t=0}=0 \Rightarrow \\
& \Rightarrow K_{4}=-\frac{V_{c}-n V_{o}}{\omega_{r} L_{r}}
\end{aligned}
$$

De esta forma la solución para la ecuación diferencial es la indicada en (D.30):

$$
i_{4}(t)=-I^{\prime} \cos \left(\omega_{r} t\right)-\frac{V_{c}-n V_{o}}{\omega_{r} L_{r}} \sin \omega_{r} t
$$

METODO B) para obtener $i_{4}(t)$.

Aplicando Laplace a la ecuación integro-diferencial (D.24), se obtiene una expresión como la indicada en (D.31) donde ahora entran en juego los valores iniciales de las variables implicadas, donde $I_{4}(s)=\mathscr{L}\left[i_{4}(t)\right]$.

$$
\begin{gathered}
\mathscr{L}\left[V_{g}+n V_{o}\right]=\mathscr{L}\left[L_{r} \frac{d i_{4}(t)}{d t}+v_{C_{r}}(0)+\frac{1}{C_{r}} \int_{0}^{t} i_{4}(t) d t\right] \\
\frac{V_{g}+n V_{o}}{s}=L_{r} s I_{4}(s)-L_{r} i_{4}(0)+\frac{V_{C_{r}}(0)}{s}+\frac{1}{C_{r} s} I_{4}(s)
\end{gathered}
$$

Recuerde que $V_{C_{r}}(0)=V_{g}+V_{c}$, y que $i_{4}(0)=-I^{\prime}$. Una vez despejada $I_{4}(s)$, se obtiene, tras aplicar Laplace, el resultado (D.32):

$$
\begin{aligned}
& I_{4}(s)=-I^{\prime} \frac{s}{s^{2}+\frac{1}{L_{r} C_{r}}}-\frac{V_{c}-n V_{o}}{L_{r}} \frac{1}{s^{2}+\frac{1}{L_{r} C_{r}}} \\
& i_{4}(t)=\mathscr{L}^{-}\left[I_{4}(s)\right]=-I^{\prime} \cos \left(\omega_{r} t\right)-\frac{V_{c}-n V_{o}}{\omega_{r} L_{r}} \sin \omega_{r} t
\end{aligned}
$$

METODO C) para obtener $i_{4}(t)$.

Este método consiste en transformar el circuito al dominio de Laplace y después resolver la corriente transformada, es decir, $I_{4}(s)$ en el circuito resultante. Si se aplica Laplace al circuito equivalente de la figura D.8a, se obtiene el circuito transformado de la figura D.8b, (donde previamente se ha 
sustituido el condensador cargado a una tensión por un condensador vacío y una fuente de tensión en serie del mismo valor y polaridad que la que tenía inicialmente y la bobina se ha sustituido por una bobina sin corriente y una fuente de corriente en paralelo y del mismo valor y sentido que la que estaba circulando inicialmente). En este caso, la tensión inicial del condensador es $V_{g}+V_{c}$ y la corriente inicial en la bobina es $-I^{\prime}$.

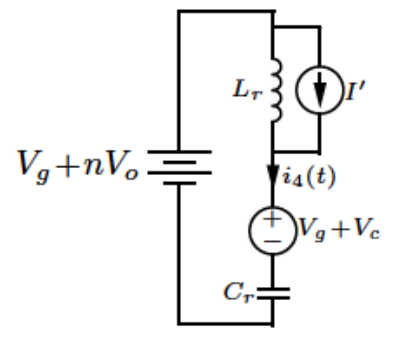

(a)

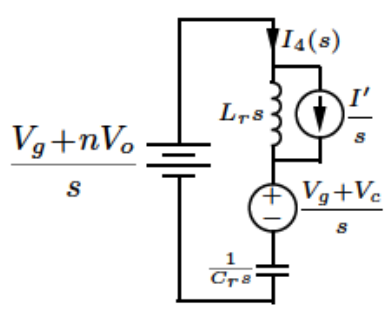

(b)

Figura D.8: Circuito equivalente en la Etapa 4: a) en el dominio del tiempo; y b) en el dominio de Laplace.

Para facilitar las operaciones, se sustituirá el conjunto bobina-fuente de corriente en el circuito transformado por su equivalente Thévenin. En la figura D.9 se ha representado el circuito equivalente.

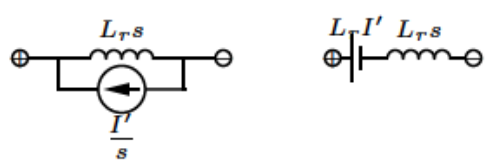

Figura D.9: Equivalente Thévenin del conjunto bobina-fuente de corriente.

De esta forma, se obtiene fácilmente la expresión para $I_{4}(s)$, como se indica en (D.33), a partir de la ecuación de tensión en la rama resonante:

$$
\begin{aligned}
\frac{V g+n V_{o}}{s}-\frac{V_{g}+V_{c}}{s}-L_{r} I^{\prime} & =I_{4}(s) \frac{1}{C_{r} s}+I_{4}(s) L_{r} s \\
-\frac{V_{c}-n V_{o}}{s}-L_{r} I & =I_{4}(s)\left(\frac{1+L_{r} C_{r} s^{2}}{C_{r} s}\right) \\
I_{4}(s) & =\left(-\frac{V_{c}-n V_{o}}{s}-L_{r} I^{\prime}\right)\left(\frac{C_{r} s}{1+L_{r} C_{r} s^{2}}\right) \Rightarrow \\
i_{4}(t) & =\mathscr{L}^{-}\left[I_{4}(s)\right]=-\frac{V_{c}-n V_{o}}{\omega_{r} L_{r}} \sin \omega_{r} t-I^{\prime} \cos \left(\omega_{r} t\right)
\end{aligned}
$$

Por cualquiera de los métodos se obtiene la misma expresión para la corriente $i_{4}(t)$. Una vez calculada, se obtiene la tensión en el condensador 
como (D.34) :

$$
\begin{aligned}
v_{C_{r}}(t) & =v_{C_{r}}(0)+\frac{1}{C_{r}} \int_{0}^{t} i_{4}(\lambda) d \lambda \\
& =V_{g}+V_{c}-\left[\frac{1}{C_{r}} I^{\prime} \sin \left(\omega_{r} \lambda\right) \omega_{r}^{-1}\right]_{0}^{t}-\left[\frac{V_{c}-n V_{o}}{C_{r} L_{r} \omega_{r}} \cos \left(\omega_{r} \lambda\right)\left(-\omega_{r}\right)^{-1}\right]_{0}^{t} \\
& =V_{g}+n V_{o}+\left(V_{c}-n V_{o}\right) \cos \omega_{r} t-\frac{I^{\prime}}{\omega_{r} C_{r}} \sin \left(\omega_{r} t\right)
\end{aligned}
$$

Esta expresión se puede simplificar, agrupando los dos términos de $\sin ()$ $\mathrm{y} \cos ()$ en uno solo com amplitud y desfase. De esta forma la tensión en el condensador es (D.35):

$$
v_{C_{r}}(t)=V_{g}+n V_{o}+V_{r} \cos \left(\omega_{r} t+\phi_{r}\right)
$$

siendo

$$
\begin{aligned}
& V_{r}=\sqrt{\left[V_{c}-n V_{o}\right]^{2}+\left(\frac{I^{\prime}}{\omega_{r} C_{r}}\right)^{2}}=\sqrt{\left[V_{c}-n V_{o}\right]^{2}+\frac{L_{r}}{C_{r}} I^{\prime 2}} \\
& \phi_{r}=\arctan \left(\frac{I^{\prime}}{\left(V_{c}-n V_{o}\right) \omega_{r} C_{r}}\right)
\end{aligned}
$$

La duración de esta etapa será el tiempo que tarda el condensador en descargase por completo y su tensión es nula, instante en el cual la energía de $L_{r}$ empezará a circular a través del diodo de $S 1$. En este instante, la bobina se ha cargado a una corriente de valor negativo, $I^{\prime \prime}$, diferente a $-I^{\prime}$, puesto que la bobina toma la carga que le cede el condensador.

Para que la tensión pueda llegar a anularse se debe cumplir que la amplitud $V_{r}$ sea mayor, o al menos igual, que $V_{g}+n V_{o}$, luego:

$$
\begin{gathered}
\sqrt{\left[V_{c}-n V_{o}\right]^{2}+\frac{L_{r}}{C_{r}} I^{\prime 2}} \geq\left(V_{g}+n V_{o}\right) \\
{\left[V_{c}-n V_{o}\right]^{2}+\frac{L_{r}}{C_{r}} I^{\prime 2} \geq\left(V_{g}+n V_{o}\right)^{2}} \\
C_{r}\left[V_{c}-n V_{o}\right]^{2}+L_{r} I^{\prime 2} \geq C_{r}\left(V_{g}+n V_{o}\right)^{2}
\end{gathered}
$$

que indica que la bobina descarga al condensador con la energía que tiene acumulada. Lo que sucede es que antes de empezar a descargarse la bobina, esta aumenta su energía por una parte que ya le cede el propio condensador. Ésta, junto con la que tiene acumulada sirven para descargar el condensador. Se deberá cumplir por tanto la condición indicada en (D.37).

La corriente por el diodo principal está indicada en (D.38), y vuelve a ser, como en las etapas anteriores, igual a la corriente de la bobina acoplada de secundario. Según el criterio de los puntos, como entra por el punto en 
el secundario (y la intensidad en el primario se considera positiva si sale por el punto), es igual a la intensidad $i_{n}(t)$ cambiada de signo y afectada por la relación de transformación. Este intervalo de tiempo es muy pequeño, lo que permite suponer que la corriente se mantiene prácticamente constante durante la descarga de $C_{r}$.

$$
i_{D}(t)=-n i_{n}(t)=-n\left(i_{4}(t)-I\right)
$$

\section{D.1.5. Etapa 5. $\left(t_{4}<t<t_{5}\right)$}

Esta etapa comienza en el instante en que $C_{r}$ se descarga totalmente y su tensión es nula. La corriente circulante por $L_{r}$ necesita un camino para seguir circulando, y lo hace a través del diodo de $S_{1}$, que pasa a estar polarizado en directa. Debido a esto, el interruptor $S_{1}$ puede entrar en conducción a tensión cero en cualquier instante de tiempo en el que circule corriente por su diodo, que será mientras la corriente por $L_{r}$ mantenga el mismo sentido de circulación . Una vez cerrado $S_{1}$, la bobina cede la energía a la fuente, y cuando está descargada, se carga de nuevo con otra polaridad, por lo que la corriente $i_{5}(t)$ terminará circulando en un sentido contrario al que tenía al iniciar esta etapa. En la figura D.10a se muestran los elementos que están involucrados en esta etapa y el circuito equivalente.

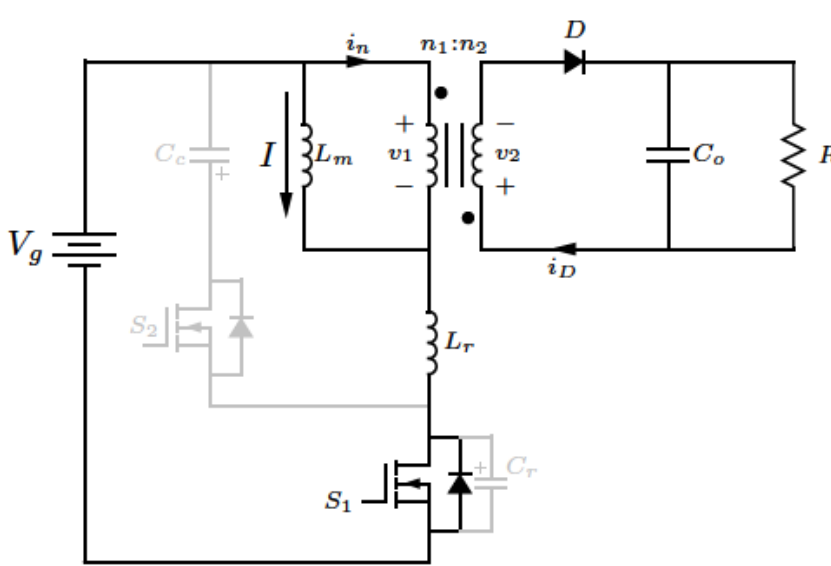

(a)

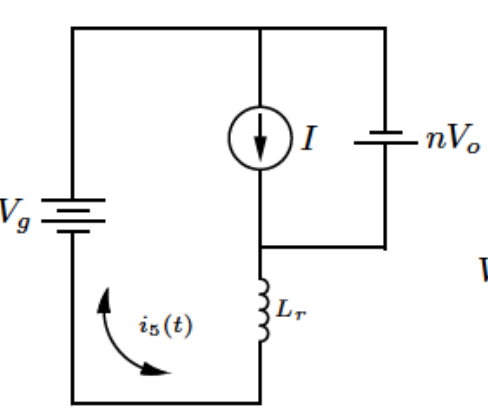

(b)

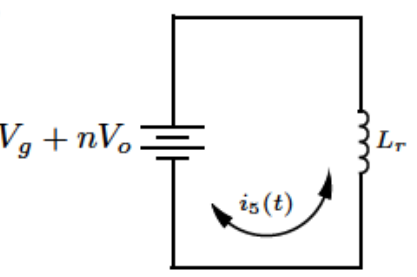

(c)

Figura D.10: Convertidor flyback ZVS a)durante la etapa 5; b)circuito equivalente; c) circuito para cálculos.

Esta etapa terminará cuando el valor de $i_{5}(t)$ alcance el valor de $I$, instante en el cual, la corriente por el diodo principal, $i_{D}(t)$ se anulará y pasará a la siguiente etapa. Observando el circuito equivalente, la corriente circulante en la rama de bobina resonante evoluciona en función de una tensión 
constante. La relación entre esta tensión y la duración de este intervalo se obtiene resolviendo dicho circuito. En esta rama la tensión es igual a $V_{g}+n V_{o}$. La bobina resonante se descarga a tensión constante y su expresión se obtiene resolviendo la ec.diferencial de primer orden con condiciones iniciales de (D.39):

$$
\begin{aligned}
V_{g}+n V_{o} & =v_{L_{r}}(t)=L_{r} \frac{d i_{5}(t)}{d t} \\
i_{5}(0) & =I^{\prime \prime}
\end{aligned}
$$

Haciendo la separación de variables en cada miembro de (D.39), e integrando se obtiene una solución general, en la que se despeja la constante de integración aplicando la condición inicial.

$$
\begin{aligned}
\frac{V_{g}+n V_{o}}{L_{r}} d t & =d i_{5}(t) \\
\frac{V_{g}+n V_{o}}{L_{r}} \int d t & =\int d i_{5}(t) \\
i_{5}(t) & =\frac{V_{g}+n V_{o}}{L_{r}} t+K_{5} \\
i_{5}(0) & =I^{\prime \prime} \Rightarrow K_{5}=I^{\prime \prime} \Rightarrow i_{5}(t)=I^{\prime \prime}+\frac{V_{g}+n V_{o}}{L_{r}} t
\end{aligned}
$$

De la expresión (D.40) se puede deducir la duración de este intervalo, que es el tiempo que tarda en alcanzarse el valor de $I$, que es igual a

$$
d_{5} T=\left(t_{5}-t_{4}\right)=\left(I-I^{\prime \prime}\right) \frac{L_{r}}{V_{g}+n V o}
$$

La corriente por el diodo principal está indicada en (D.42), y está relacionada, como en las etapas anteriores, con la intensidad $i_{n}(t)$ cambiada de signo y afectada por la relación de transformación.

$$
\left.i_{D}(t)\right)=-n i_{n}(t)=-n\left(i_{5}(t)-I\right)
$$

\section{D.1.6. Etapa 6. $\left(t_{5}<t<t_{6}\right)$}

Esta etapa comienza cuando el diodo $D$ deja de conducir y concluye cuando se abre el interruptor $S_{1}$, que conmuta a tensión cero puesto que el condensador $C_{r}$ está descargado. A partir de aquí comienza un nuevo ciclo. Los elementos que intervienen el circuito pasan a ser los indicados en la figura D.11a.

Uno de los objetivos de la red ZVS es reducir las pérdidas, y se consigue puesto que todas las conmutaciones son a tensión cero. $S_{1}$ pasa a OFF al principio de la ETAPA 1 y lo hace a tensión cero puesto que el condensador 


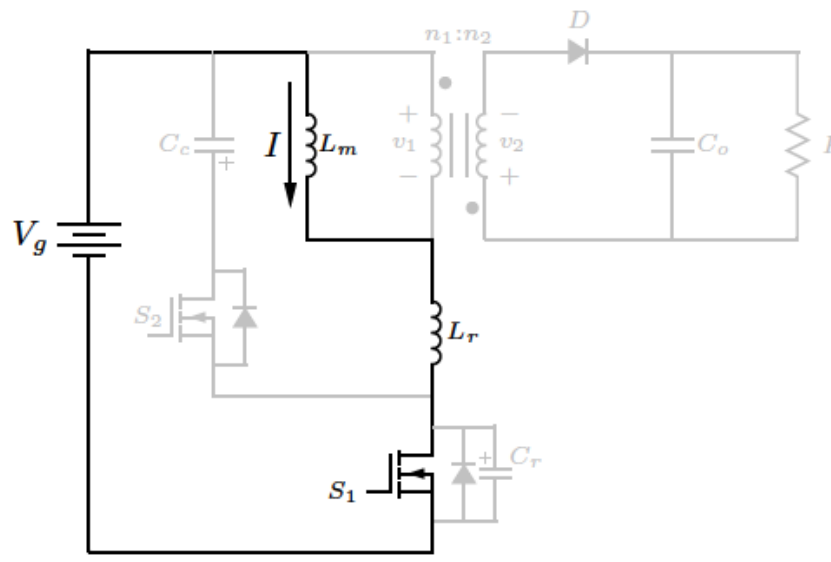

(a)

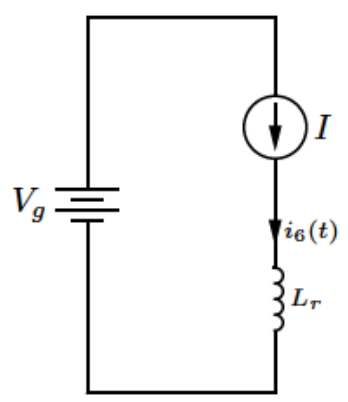

(b)

Figura D.11: Convertidor flyback ZVS durante la etapa 6.

$C_{r}$ está descargado. $S_{2}$ pasa a ON al principio de la ETAPA 3 y lo hace a tensión cero puesto que está circulando corriente por su diodo. $S_{2}$ pasa a OFF al final de la ETAPA 3 y lo hace a tensión cero puesto que el drenador está a $0 \mathrm{~V}$. $S_{1}$ pasa a $\mathrm{ON}$ en la ETAPA 5 mientras circula corriente por su diodo.

Nótese que se supone cumplida la ecuación (D.37) relativa que la energía almacenada en $L_{r}$ debida a la intensidad $I$, debe ser mayor que la energía del condensador del MOSFET, $C_{r}$, para descargarlo desde la tensión $V_{g}+V_{c}$ hasta cero. en esta ecuación hay que hacer notar que $I$ representa el valor medio de la corriente en ese instante. Utilizando los valores instantáneos, habría que sustituir ese valor por el valor de pico, es decir por $I+\Delta I / 2$.

\section{D.2. Análisis en régimen permanente.}

\section{D.2.1. Sin considerar rizado.}

Se ha considerado una bobina $L$ lo suficientemente grande como para suponer corriente constante, de valor $I$, que es mucho mayor que la bobina resonante $L_{r}$. De forma similar, el valor de los condensadores $C_{o}$ y $C_{c}$, es tal que la tensión en sus extremos se mantiene constante, de valores $V_{o}$ y $V_{c}$, durante todo el periodo $T$.

Después de estudiar el funcionamiento del circuito con estas mismas consideraciones, se ha representado en la figura D.12 las principales magnitudes a lo largo de las seis etapas por las que evoluciona esta topología durante un ciclo completo. La duración de cualquiera de estas etapas se indica con la letra $d$ y un subíndice que hace referencia al número de etapa. Por ejemplo, la duración de la etapa 5 es igual a $t_{5}-t_{4}=d_{5} T$. 


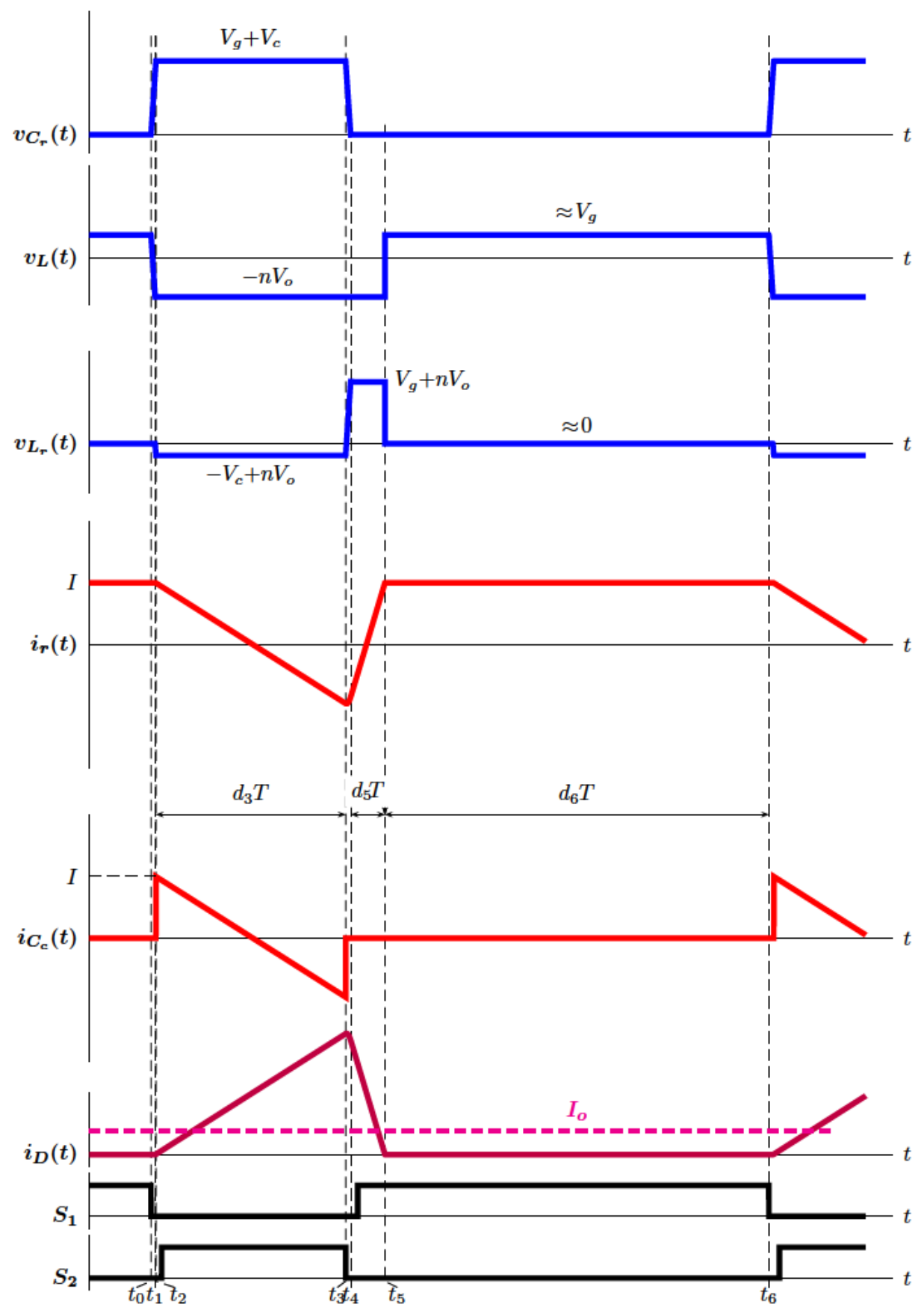

Figura D.12: Curvas principales de un flyback ZVS.

Para el análisis se desprecian las etapas 1, 2 y 4, puesto que la frecuencia característica del conjunto $L_{r}-C_{r}$ es mucho mas grande que la frecuencia de conmutación y la carga-descarga de $C_{r}$ es prácticamente instantánea. De esta 
forma solo intervienen tres etapas en el análisis. En este caso se considera que $I^{\prime} \approx I$ y $I^{\prime \prime} \approx-I$. Y como la duración de la etapa 3 es el tiempo que no está cerrado el interruptor principal resulta que

$$
\begin{aligned}
d_{3} T & =(1-D) T \\
d_{5} T+d_{6} T & =D T
\end{aligned}
$$

Las ecuaciones del régimen permanente se obtienen al considerar nulo tanto el balance voltios-segundo en las bobinas $L$ y $L_{r}$ como la carga neta en los condensadores $C_{o}$ y $C_{c}$.

\section{TENSION MEDIA EN BOBINA DE MAGNETIZACIÓN.}

La tensión media en la bobina $L$ es nula. De aquí se obtiene una expresión para la tensión de salida en función de la entrada:

$$
\begin{aligned}
& \frac{1}{\not{X}}\left[-n V_{o}\left(d_{3}+d_{5}\right) \not{X}+\left(V_{g}\right) d_{6} \not X\right]=0 \\
& \Rightarrow V_{o}=V_{g} \frac{d_{6}}{\left(d_{3}+d_{5}\right) n}=V_{g} \frac{d_{6}}{\left(1-d_{6}\right) n}
\end{aligned}
$$

A $d_{6}$ se le puede llamar ciclo efectivo de trabajo, $D_{e f}$, puesto que al identificar la expresión que relaciona la tensión de salida con la entrada en un flyback, aparece ese término en lugar del término $D$ que identifica al ciclo de trabajo. También se puede identificar con el tiempo en el que la bobina principal del convertidor está absorbiendo energía. El valor del ciclo efectivo es menor que el valor del ciclo de trabajo empleado en las señales de disparo.

\section{TENSION MEDIA EN BOBINA RESONANTE.}

La tensión media en la bobina $L_{r}$ es nula. De aquí se obtiene una expresión para la tensión de $C_{c}$ en función de la tensión de entrada:

$$
\begin{aligned}
& \frac{1}{\not{X}}\left[\left(V_{c}-n V_{o}\right) d_{3} \not{X}+\left(V_{g}+n V_{o}\right) d_{5} \not{X}\right]=0 \\
& \Rightarrow V_{c}=\left(V_{g}+n V_{o}\right) \frac{d_{5}}{d_{3}}+n V_{o}
\end{aligned}
$$

Sumando D.44 y D.45 también se puede despejar $V_{c}$ como:

$$
V_{c}=V_{g} \frac{d_{5}+d_{6}}{d_{3}}
$$

\section{CORRIENTE MEDIA EN EL CONDENSADOR DE CLAMP.}

La intensidad media en el condensador de clamp es nula. Entonces:

$$
\begin{aligned}
& \int_{t_{0}}^{t_{0}+T} i_{c}(t) d t=\int_{t_{2}}^{t_{3}} i_{c}(t) d t=\int_{0}^{d_{3} T}\left[I-\frac{V_{c}-n V_{o}}{L_{r}} t\right] d t=0 \\
& {\left[I t-\frac{V_{c}-n V_{o}}{2 L_{r}} t^{2}\right]_{0}^{d_{3} T}=0 \Rightarrow V_{c}=\frac{2 I L_{r}}{d_{3} T}+n V_{o}}
\end{aligned}
$$


Sustituyendo este valor en la expresión empleada para $i_{c}(t)$ se puede obtener el valor de la intensidad del condensador transcurrido el tiempo $d_{3} T$. Entonces:

$$
i_{c}\left(d_{3} T\right)=I-\frac{V_{c}-n V_{o}}{L_{r}} d_{3} T=I-\frac{\frac{2 I L_{r}}{d_{3} T}}{L_{r}} d_{3} T=-I
$$

\section{DURACIÓN DEL INTERVALO $d_{5}$.}

Se puede obtener a partir de igualar las expresiones de $V_{c}$ dadas en (D.45) y en (D.47). De esta forma:

$$
V_{c}=\frac{2 I L_{r}}{d_{3} T}+n V_{o}=\left(V_{g}+n V_{o}\right) \frac{d_{5}}{d_{3}}+n V_{o} \Rightarrow d_{5}=\frac{2 I L_{r}}{\left(V_{g}+n V_{o}\right) T}
$$

Expresión a la que se puede llegar también a partir de la tensión en la bobina $L r$ durante la etapa 5 , puesto que es conocida la variación que experimenta la intensidad circulante por esa bobina y la tensión a la que está sometida.

$$
\begin{aligned}
& \Delta i_{r}(t)=i_{r}\left(t_{5}\right)-i_{r}\left(t_{4}\right) \approx i_{r}\left(t_{5}\right)-i_{r}\left(t_{3}\right)=I-(-I)=2 I \\
& \Delta i_{r}(t)=\frac{1}{L_{r}} \Delta v_{L_{r}}(t) \Delta t \equiv \frac{1}{L_{r}}\left(V_{g}+n V_{o}\right) d_{5} T \\
& \Rightarrow d_{5}=\frac{2 I L_{r}}{\left(V_{g}+n V_{o}\right) T}
\end{aligned}
$$

\section{TENSION DE SALIDA.}

Empleando (D.49) junto con (D.43) y (D.44) se obtiene una expresión para la tensión de salida en régimen permanente. Como $d_{6}=\left(D-d_{5}\right)$, resulta que:

$$
d_{6}=D-\frac{2 I L_{r} f}{V_{g}+n V_{o}}=\frac{\left(V_{g}+n V_{o}\right) D-2 I L_{r} f}{V_{g}+n V_{o}}
$$

Por otro lado, despejando $d_{6}$ de (D.44) se obtiene:

$$
d_{6}=\frac{n V_{o}}{V_{g}+n V_{o}}
$$

Igualando (D.51) y (D.52) se puede despejar $V_{o}$ como:

$$
V_{o}=\frac{V_{g} D}{(1-D) n}-I \frac{2 L_{r} f}{(1-D) n}
$$

A esta expresión también se llega igualando (D.46) con (D.47) y despejando $V_{o}$. Como se puede observar, la tensión de salida de un flyback ZVS depende de la intensidad que circula por la bobina de magnetización. Esta 
expresión muestra que hay un elemento equivalente a una resistencia por la que pasa la intensidad $I$, que se puede considerar en serie con la bobina de magnetización puesto que, en un flyback, esta intensidad es la misma que pasa la bobina. Su expresión sería (D.54).

$$
R_{e s L_{m}}=2 L_{r} f
$$

\section{CARACTERÍSTICA DE SALIDA.}

Considerando la igualdad de potencia de entrada y salida junto con (D.53) se puede obtener la relación entre la tensión de salida y la intensidad de salida:

$$
\begin{aligned}
& \begin{array}{l}
V_{o}=\frac{V_{g} D}{(1-D) n}-I \frac{2 L_{r} f}{(1-D) n} \\
\begin{array}{l}
\begin{array}{l}
I=I_{g}+I_{o} / n \\
V_{o} I_{o}=V_{g} I_{g}
\end{array} \\
I
\end{array}
\end{array} \\
& \Rightarrow V_{o}=\frac{V_{g} D}{(1-D) n}-\frac{I_{o}}{n}\left(\frac{n V_{o}}{V_{g}}+1\right) \frac{2 L_{r} f}{(1-D) n} \Rightarrow \\
& \Rightarrow V_{o}=\frac{\frac{V_{g} D}{(1-D) n}-I_{o} \frac{2 L_{r} f}{(1-D) n^{2}}}{1+I_{o} \frac{2 L_{r} f}{V_{g}(1-D) n}}
\end{aligned}
$$

La derivada de esta expresión respecto a la intensidad indica como varía la tensión de salida ante las variaciones de la intensidad y tiene dimensiones de resistencia. La derivada tiene la siguiente expresión:

$$
\begin{aligned}
\frac{d V_{o}}{d I_{o}} & =\frac{-\frac{2 L_{r} f}{(1-D) n^{2}}\left[1+I_{o} \frac{2 L_{r} f}{V_{g}(1-D) n}\right]-\left[\frac{V_{g} D}{(1-D) n}-I_{o} \frac{2 L_{r} f}{(1-D) n^{2}}\right]\left[\frac{2 L_{r} f}{V_{g}(1-D) n}\right]}{\left[1+I_{o} \frac{2 L_{r} f}{V_{g}(1-D) n}\right]^{2}} \\
& =-2 L_{r} f\left[\frac{\frac{1}{(1-D) n^{2}}}{1+I_{o} \frac{2 L_{r} f}{V_{g}(1-D) n}}\right]^{2}
\end{aligned}
$$

Despejando $I_{o}$ en la relación (D.55) y sustituyendo, se obtiene la expresión final:

$$
\frac{d V_{o}}{d I_{o}}=-2 L_{r} f\left[\frac{V_{g}+n V_{o}}{n V_{g}}\right]^{2}
$$

\section{D.2.2. Considerando rizado.}

Si se considera que la bobina $L$ no es lo suficientemente grande como para suponer corriente constante, $I$, los circuitos equivalentes empleados para el 
análisis del circuito son diferentes y la relación entre la bobina principal y resonante aparece en las nuevas expresiones de las principales magnitudes.

Los circuitos equivalentes están representados en la Figura D.13 donde se ha considerado la tensión constante en los condensadores $C_{o}$ y $C_{c}$, mientras que las principales magnitudes aparecen en la Figura D.14. Planteando el

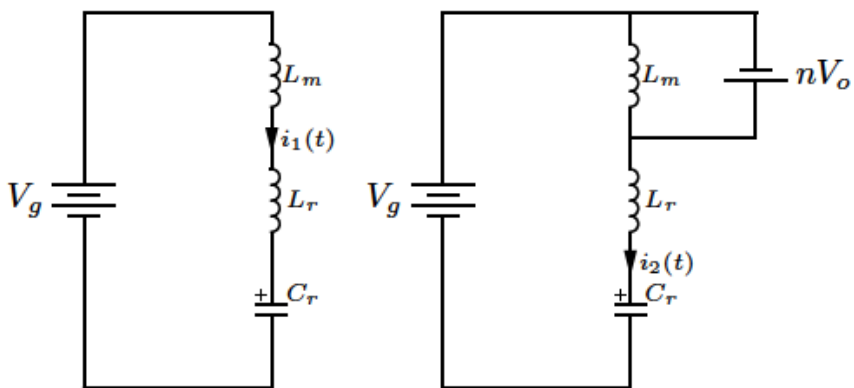

(a) (b)

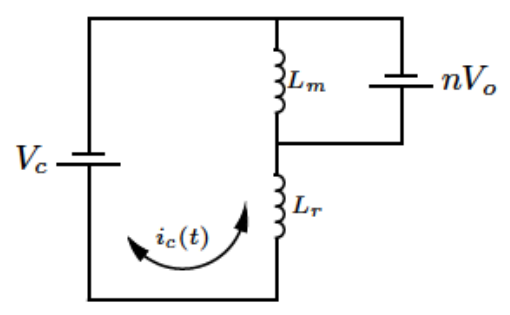

(c)

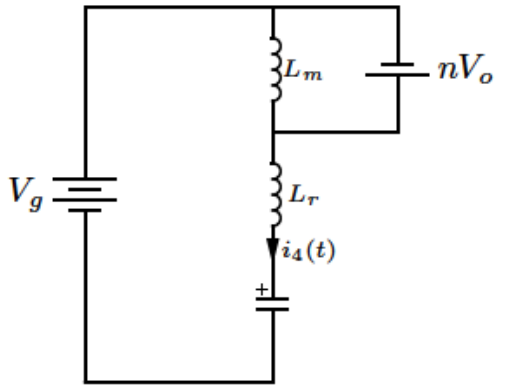

(d)

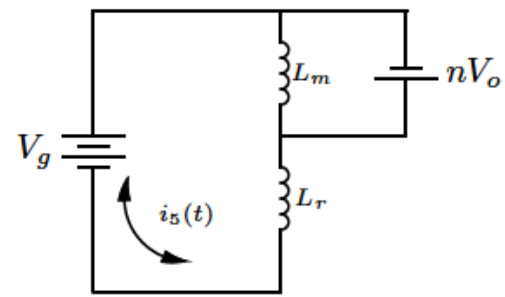

(e)

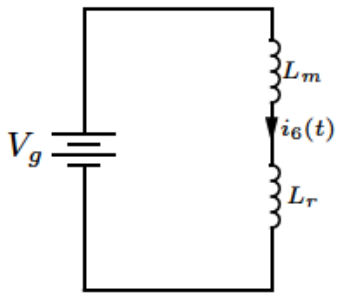

(f)

Figura D.13: Circuitos empleados para el análisis del flyback ZVS considerando rizado durante un ciclo completo: (a) Etapa 1. (b) Etapa 2. (c) Etapa 3. (d) Etapa 4. (e) Etapa 5. (f) Etapa 6.

balance voltios-segundo en las bobinas $L$ y $L_{r}$ y la carga neta nula en los condensadores $C_{o}$ y $C_{c}$ con las nuevas curvas, se obtienen otras ecuaciones del régimen permanente. Se desprecian las etapas 1, 2 y 4, y solo intervienen tres etapas.

\section{TENSION MEDIA EN BOBINA DE MAGNETIZACIÓN CON RIZADO.}

Como la tensión media en la bobina $L_{m}$ es nula, resulta que:

$$
\begin{aligned}
& \frac{1}{\mathscr{T}}\left[-n V_{o}\left(d_{3}+d_{5}\right) \not{T}+V_{g} \frac{L_{m}}{L_{r}+L_{m}} d_{6} \not{T}\right]=0 \\
& \Rightarrow V_{o}=V_{g} \frac{d_{6} \frac{L_{m}}{L_{r}+L_{m}}}{\left(d_{3}+d_{5}\right) n}=V_{g} \frac{d_{6} \frac{L_{m}}{L_{r}+L_{m}}}{\left(1-d_{6}\right) n}
\end{aligned}
$$




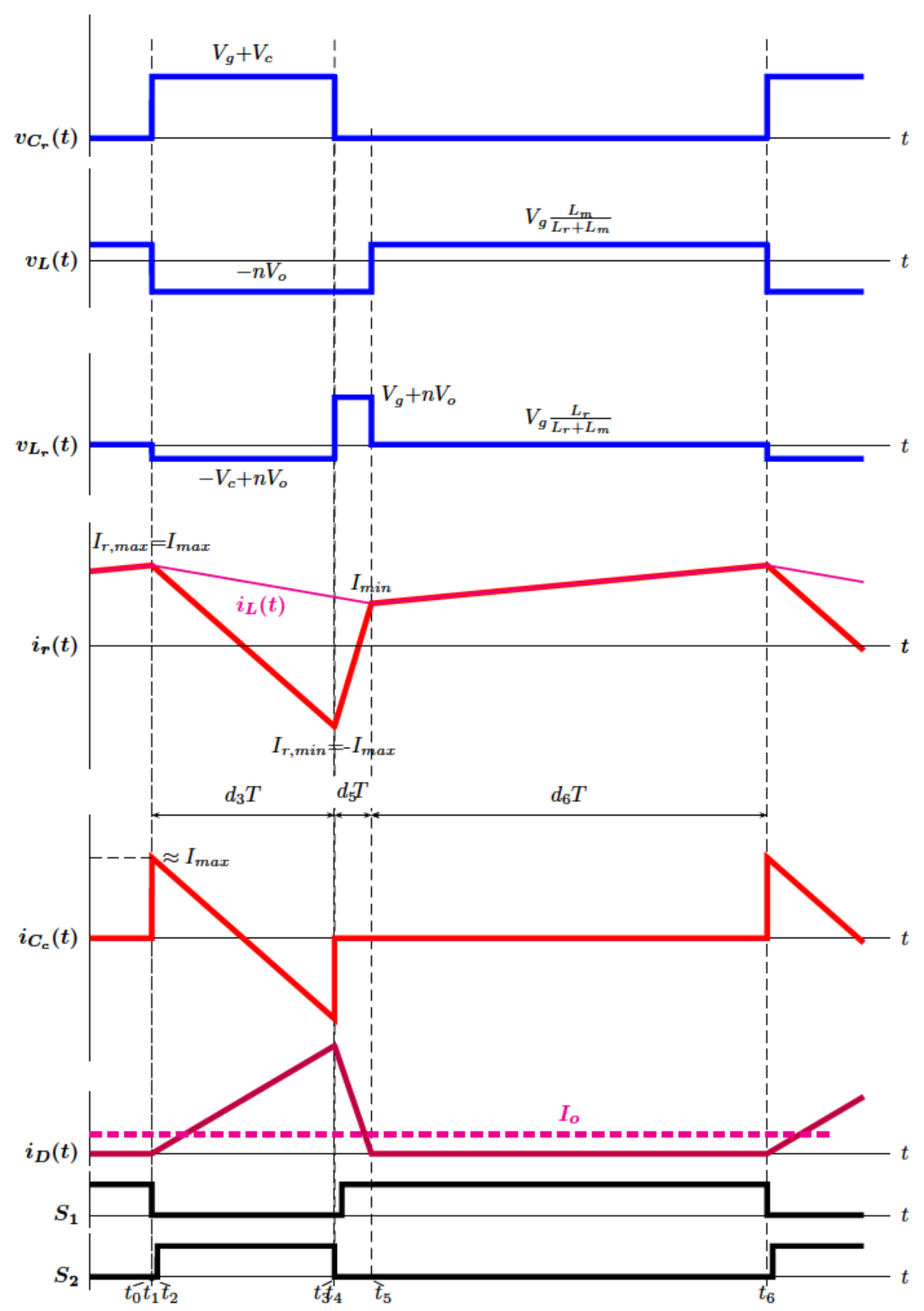

Figura D.14: Curvas principales de un flyback ZVS considerando rizado.

TENSION MEDIA EN BOBINA RESONANTE CON RIZADO. 
Como la tensión media en la bobina $L_{r}$ es nula, entonces:

$$
\begin{aligned}
& \frac{1}{\mathscr{X}}\left[-\left(V_{c}-n V_{o}\right) d_{3} \not{X}+\left(V_{g}+n V_{o}\right) d_{5} \not{X}+V_{g} d_{6} \frac{L_{r}}{L_{r}+L_{m}}\right]=0 \\
& \Rightarrow V_{c}=n V_{o}+n V_{o} \frac{d_{5}}{d_{3}}+V_{g} \frac{\left(d_{5}+d_{6} \frac{L_{r}}{L_{r}+L_{m}}\right)}{d_{3}}
\end{aligned}
$$

Sumando D.58 y D.59 también se puede despejar $V_{c}$ como:

$$
V_{c}=V_{g} \frac{d_{5}+d_{6}}{d_{3}}
$$

\section{CORRIENTE MEDIA EN EL CONDENSADOR DE CLAMP CON RIZADO.}

La intensidad media en el condensador de clamp es nula. Entonces:

$$
\begin{aligned}
& \int_{t_{0}}^{t_{0}+T} i_{c}(t) d t=\int_{t_{2}}^{t_{3}} i_{c}(t) d t=\int_{0}^{d_{3} T}\left[I_{\text {max }}-\frac{V_{c}-n V_{o}}{L_{r}} t\right] d t=0 \\
& {\left[I_{\text {max }} t-\frac{\left(V_{c}-n V_{C_{o}}\right)}{2 L_{r}} t^{2}\right]_{0}^{d_{3} T}=0 \Rightarrow V_{c}=\frac{2 I_{\text {max }} L_{r}}{d_{3} T}+n V_{o}}
\end{aligned}
$$

Sustituyendo este valor en la expresión empleada para $i_{c}(t)$ se puede obtener el valor de la intensidad del condensador transcurrido el tiempo $d_{3} T$. Entonces:

$$
i_{c}\left(d_{3} T\right)=I_{\max }-\frac{\left(V_{c}-n V_{o}\right)}{L_{r}} d_{3} T=I_{\max }-\frac{\frac{2 I_{\max } L_{r}}{d_{3} T}}{\not L_{r}} d_{3} T=-I_{\max }
$$

\section{DURACIÓN DEL INTERVALO $d_{5}$ CON RIZADO.}

Se puede calcular a partir de la tensión en la bobina $L r$ durante la etapa 5 , puesto que es conocida la variación que experimenta la intensidad circulante por esa bobina y la tensión a la que está sometida.

$$
\begin{aligned}
& \Delta i_{r}(t)=i_{r}\left(t_{5}\right)-i_{r}\left(t_{4}\right) \approx i_{r}\left(t_{5}\right)-i_{r}\left(t_{3}\right)=I_{\min }-\left(-I_{\max }\right)=2 I \\
& \Delta i_{r}(t)=\frac{1}{L_{r}} \Delta v_{L_{r}}(t) \Delta t \equiv \frac{1}{L_{r}}\left(V_{g}+n V_{o}\right) d_{5} T \\
& \Rightarrow d_{5}=\frac{2 I L_{r}}{\left(V_{g}+n V_{o}\right) T}
\end{aligned}
$$

Una expresión diferente se obtiene al igualar (D.59) con (D.61) y empleando (D.43) para despejar $d_{5}$ :

$$
\begin{aligned}
& n V_{o}+n V_{o} \frac{d_{5}}{d_{3}}+V_{g} \frac{\left(d_{5}+\left(D-d_{5}\right) \frac{L_{r}}{L_{r}+L_{m}}\right)}{d_{3}}=\frac{2 I_{\max } L_{r}}{d_{3} T} \neq n V_{o} \\
& \Rightarrow d_{5}=\frac{2 I_{\max } L_{r} f-V_{g} D \frac{L_{r}}{L_{r}+L_{m}}}{V_{g} \frac{L_{m}}{L_{r}+L_{m}}+n V_{o}}
\end{aligned}
$$




\section{TENSION DE SALIDA CON RIZADO.}

Como $d_{6}=\left(D-d_{5}\right)$, resulta que:

$$
\begin{aligned}
d_{6} & =D-\frac{2 I_{\max } L_{r} f-V_{g} D \frac{L_{r}}{L_{r}+L_{m}}}{V_{g} \frac{L_{m}}{L_{r}+L_{m}}+n V_{o}} \\
& =\frac{\left(V_{g}+n V_{o}\right) D-2 I_{\max } L_{r} f}{V_{g} \frac{L_{m}}{L_{r}+L_{m}}+n V_{o}}
\end{aligned}
$$

Por otro lado, despejando $d_{6}$ de (D.58) se obtiene:

$$
d_{6}=\frac{n V_{o}}{V_{g} \frac{L_{m}}{L_{m}+L_{r}}+n V_{o}}
$$

Igualando (D.65) y (D.66) se puede despejar $V_{o}$ como:

$$
V_{o}=\frac{V_{g} D}{(1-D) n}-I_{\max } \frac{2 L_{r} f}{(1-D) n}
$$

También se podría conseguir si se iguala (D.60) con (D.61) y se despeja $V_{o}$. En ambos casos, la expresión obtenida es parecida a (D.53) que se ha obtenido sin considerar rizado. Solo se ha cambiado $I$ por $I_{\max }$.Y el término que multiplica a la intensidad es la resistencia equivalente en serie con $L_{m}$, $R_{e s L_{m}}$, definida anteriormente en (D.54).

\section{RIZADO DE CORRIENTE EN BOBINA DE MAGNETIZACIÓN CON RIZADO.}

Aplicando la definición de rizado de corriente en una bobina, se puede obtener el rizado de corriente en $L_{m}$ durante las etapas 3 a 5 o durante la etapa 6 siendo expresiones equivalentes en régimen permanente. Entonces:

$$
\begin{aligned}
\Delta i_{L_{m}, 3-5} & =\frac{1}{L_{m}}\left|-n V_{o}\right|\left(d_{3}+d_{5}\right) T=\frac{1}{L_{m}} n V_{o}\left(1-d_{6}\right) T \\
\Delta i_{L_{m}, 6} & =\frac{1}{L_{m}}\left|V_{g}\right| \frac{L_{m}}{L_{m}+L_{r}} d_{6} T=\frac{V_{g}}{L_{m}+L_{r}} d_{6} T
\end{aligned}
$$

Empleando la ecuación (D.66) se obtiene la expresión para el rizado de corriente en $L_{m}$ como:

$$
\Delta i_{L_{m}}=\frac{n V_{o} V_{g} T}{L_{m} V_{g}+\left(L_{m}+L_{r}\right) n V_{o}}
$$

\section{CARACTERÍSTICA DE SALIDA CON RIZADO.}

La relación entre la tensión de salida y la intensidad de salida se obtiene a partir de la expresión (D.67). Primero se expresa $I_{\max }$ por su expresión en 
función del rizado. Después se emplea la igualdad de potencia a la entrada y a la salida y la relación $I=I_{g}+I_{o} / n$, para obtener:

$$
\left.\begin{array}{l}
I=I_{g}+I_{o} / n \\
V_{g} I_{g}=V_{o} I_{o}
\end{array}\right\} \Rightarrow I=\frac{I_{o}}{n}\left(\frac{n V_{o}}{V_{g}}+1\right)
$$

Para el rizado de corriente se emplea la segunda expresión de (D.68) y se considera $d_{6}=\left(D-d_{5}\right)$ :

$$
\Delta i_{L_{m}}=\frac{V_{g}}{L_{m}+L_{r}}\left(D-d_{5}\right) T=\frac{V_{g}}{L+L_{r}}\left(D-\frac{2 I L_{r}}{\left(V_{g}+n V_{o}\right) T}\right) T
$$

Sólo queda sustituir (D.70) y (D.71) en (D.67) y despejar $V_{o}$ para obtener una función de la forma $V_{o}=f\left(I_{o}\right)$. Por tanto:

$$
\begin{aligned}
V_{o} & =\frac{V_{g} D}{(1-D) n}-I_{\max } \frac{2 L_{r} f}{(1-D) n}=\frac{V_{g} D}{(1-D) n}-\left(I+\frac{1}{2} \Delta i_{L_{m}}\right) \frac{2 L_{r} f}{(1-D) n} \\
& =\frac{V_{g} D}{(1-D) n}-I \frac{2 L_{r} f}{(1-D) n}-\frac{1}{\not 2} \frac{V_{g}}{L_{m}+L_{r}}\left(D-\frac{2 I L_{r}}{\left(V_{g}+n V_{o}\right) T}\right) \not{X} \frac{2 L_{r} f}{(1-D) n} \\
& =-\frac{V_{g} D}{(1-D) n}\left[1-\frac{L_{r}}{L_{m}+L_{r}}\right]-I_{o} \frac{V_{o} 2 L_{r} f}{V_{g}(1-D) n}-I_{o} \frac{2 L_{r} f}{(1-D) n^{2}}+\frac{1}{\left(L_{m}+L_{r}\right)} \frac{2 I_{o} L_{r}^{2}}{T(1-D) n^{2}} \\
& =-\frac{V_{g} D}{(1-D) n}\left[1-\frac{L_{r}}{L_{m}+L_{r}}\right]-I_{o} \frac{2 L_{r} f}{(1-D) n^{2}}\left[1-\frac{L_{r}}{L_{m}+L_{r}}\right]-I_{o} \frac{V_{o}}{V_{g}} \frac{2 L_{r} f}{(1-D) n} \\
& \Rightarrow V_{o}\left(I_{o}\right)=\frac{\left[\frac{V_{g} D}{(1-D) n}-I_{o} \frac{2 L_{r} f}{(1-D) n^{2}}\right] \frac{L_{m}}{L_{m}+L_{r}}}{1+I_{o} \frac{2 L_{r} f}{V_{g}(1-D) n}}
\end{aligned}
$$

\section{D.2.3. Comparativa.}

Está claro que el análisis cuando se considera el rizado será de aplicación al circuito conmutado, donde se tienen en cuenta los valores de las bobinas, mientras que el análisis al no considerar rizado servirá para las expresiones derivadas del modelo promediado, dado que éste parte de la consideración de pequeño rizado.

Los resultados obtenidos para magnitudes tales como el ciclo de trabajo, la tensión de clamp y las variaciones de corriente, a partir de unos valores de diseño ponen de manifiesto estas diferencias. Por ejemplo, con los parámetros de funcionamiento recogidos en la Tabla D.1 se obtienen los valores indicados en la Tabla D.2, en el caso de no considerar el rizado de la corriente en la bobina principal y se compara con el obtenido cuando si se ha considerado.

En el flyback ZVS la intensidad en la bobina principal es igual a la intensidad en la entrada mas de salida, $I_{g}+I_{o} / n$. Al considerar el rizado, el ciclo 


\begin{tabular}{llll}
\hline \hline$V_{g}$ & $20 \mathrm{~V}$ & $C_{o}$ & $5 \mu \mathrm{F}$ \\
$P$ & $100 \mathrm{~W}$ & $C_{c}$ & $2,2 \mu \mathrm{F}$ \\
$V_{\text {out }}$ & $400 \mathrm{~V}$ & $L_{m}$ & $24 \mu \mathrm{H}$ \\
$f$ & $100 \mathrm{kHz}$ & $L_{r}$ & $2 \mu \mathrm{H}$ \\
$n_{1}: n_{2}$ & $1: 12$ & & \\
$t_{m}$ & $300 \mathrm{~ns}$ & $C_{r}$ & $1 \mathrm{nF}$ \\
\hline
\end{tabular}

Tabla D.1: Condiciones de trabajo de un flyback. ZVS.

\begin{tabular}{ccccccccc}
\hline & $\begin{array}{c}I \\
(\mathrm{~A})\end{array}$ & $\begin{array}{c}d_{5} \\
-\end{array}$ & $\begin{array}{c}d_{6} \\
-\end{array}$ & $\begin{array}{c}D \\
-\end{array}$ & $\begin{array}{c}V_{c} \\
(\mathrm{~V})\end{array}$ & $\begin{array}{c}\Delta_{i_{L}} \\
(\mathrm{~A})\end{array}$ & $\begin{array}{c}I_{\max } \\
(\mathrm{A})\end{array}$ & $\begin{array}{c}I_{\min } \\
(\mathrm{A})\end{array}$ \\
\hline SIN RIZADO & \multirow{2}{*}{8} & \multirow{2}{*}{0,06} & 0,625 & 0,685 & 43,49 & 0 & - & - \\
CON RIZADO & & & 0,6436 & 0,7036 & 47,48 & 4,95 & 10,475 & 5,525 \\
\hline \hline
\end{tabular}

Tabla D.2: Parámetros calculados cuando se considera o no el rizado.

de trabajo obtenido es mayor. La tensión de clamp, $V_{c}$ también es diferente. Es lógico dado que depende del ciclo de trabajo. Si se calcula su valor a partir de la expresión que incluye la intensidad, también se obtendrá un valor diferente según se considere valor medio (sin rizado) o valor máximo (cuando hay rizado).

En ambos análisis se han considerado despreciables algunas etapas por su pequeña duración en relación a las otras. Esta hipótesis se ha validado midiendo la duración de las seis etapas para los parámetros de funcionamiento de las dos tablas anteriores. Con ayuda de un programa de cálculo simbólico se han planteado las ecuaciones de estado durante las seis etapas y se han resuelto las ecuaciones y obtenido la fracción que ocupa cada etapa dentro de un periodo completo. Se han indicado en la Tabla D.3

\begin{tabular}{ccccccc}
\hline & $d_{1}$ & $d_{2}$ & $d_{3}$ & $d_{4}$ & $d_{5}$ & $d_{6}$ \\
\hline SIN RIZADO & $667 \times 10^{-6}$ & $127 \times 10^{-6}$ & 0,3149 & $796 \times 10^{-6}$ & $59,7 \times 10^{-3}$ & 0,6238 \\
CON RIZADO & $536 \times 10^{-6}$ & $109 \times 10^{-6}$ & 0,2960 & $645 \times 10^{-6}$ & $59,7 \times 10^{-3}$ & 0,6430 \\
\hline \hline
\end{tabular}

Tabla D.3: Fracción de tiempo para cada etapa por las que evoluciona el flyback ZVS.

A la vista de estos resultados, se puede comprobar que las etapas 1, 2 y 4 son las de menor duración por lo que, como se había previsto, se pueden despreciar.

En cada uno de los dos análisis realizados, la característica de salida obtenida muestra una relación diferente entre tensión e intensidad de salida. Cuando ambas se representan gráficamente se obtiene prácticamente la misma curva, como se pone de manifiesto al observar la Figura D.15 que contiene la característica de salida de un reductor ZVS con los datos de la Tabla D.1. En este caso, se ha representado la curva sin considerar rizado, que emplea 


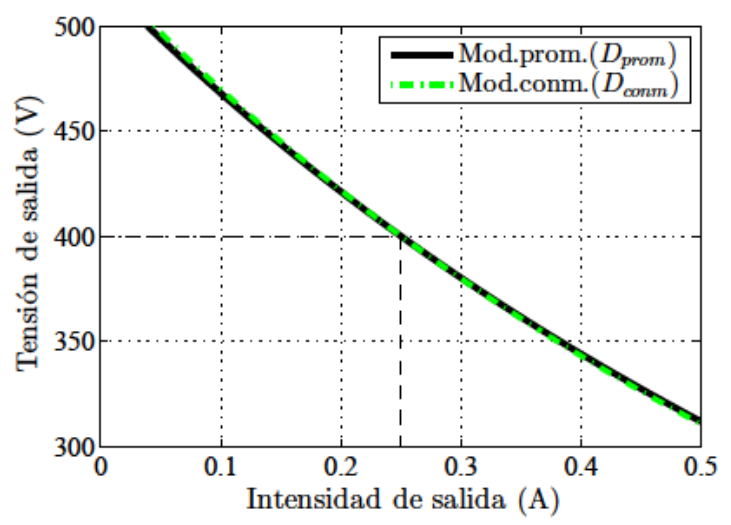

Figura D.15: Característica de salida de un flyback ZVS.

el valor de ciclo de trabajo obtenido con este mismo análisis, mientras que la misma curva cuando se considera el rizado está obtenida con el ciclo de trabajo calculado con rizado no despreciable.

\section{D.3. Modelo promediado}

La obtención del modelo promediado se puede conseguir mediante el promediado de las formas de onda, mediante el promediado de circuitos o mediante el promediado en el espacio de estados. En esta sección se va a obtener el modelo promediado del flyback ZVS descrito en la sección anterior, de la misma forma que se ha hecho para el reductor ZVS en capítulos anteriores.

\section{D.3.1. Modelo promediado para el flyback ZVS}

En la sección D.1 se ha explicado el funcionamiento en régimen permanente del flyback ZVS. Del análisis se deduce que la corriente en la bobina resonante $L_{r}$ y la tensión en el condensador resonante $C_{r}$ son las variables de estado rápidas y el resto son las variables de estado lentas. El procedimiento a seguir es obtener las expresiones para las variables rápidas $i_{L_{r}}(t)$ y $v_{C_{r}}(t)$, en un periodo de conmutación (comprobar que son periódicas). La variables lentas se suponen de valor constante para este cálculo. Las expresiones obtenidas, dependerán, por tanto, de las variables lentas y también del tiempo. Dichas expresiones se sustituyen en las ecuaciones de estado de las variables lentas de forma que queden desacopladas respecto a las variables rápidas. Se aplica ahora un promedio estándar en un periodo de conmutación para eliminar la dependencia con el tiempo, consiguiéndose un modelo promediado 
de tercer orden (orden reducido) para esas tres variables de estado lentas, $v_{C_{c}}(t), v_{C_{o}}(t)$ y $i_{m}(t)$.

Aunque matemáticamente no es necesario, se suelen realizar algunas aproximaciones antes de empezar para simplificar los cálculos a realizar. En primer lugar, se ignoran los tiempos muertos entre las conmutaciones de $S_{1}$ y $S_{2}$ que normalmente son muy pequeños en comparación con el periodo. También se desprecian los intervalos resonantes, como se hace en (ATHALYE y otros, 2001) para obtener el modelo completo con el método del interruptor promediado. En este punto cabe indicar que el efecto debido a tener en cuenta los intervalos resonantes sería tan solo a frecuencias muy altas y fuera del rango de interés (que, generalmente, suele ser una fracción de la frecuencia de conmutación porque es donde se aplica el control en lazo cerrado). Otra suposición es considerar que el condensador de clamp mantiene su tensión constante, lo que quiere decir que la frecuencia de resonancia entre $L_{r}$ y $C_{c}$ es bastante menor que la frecuencia de conmutación.

Considerando estas simplificaciones desaparecen los intervalos resonantes (Etapa 1, 2 y 4) y el tiempo muerto de la etapa 5, de forma que un periodo se puede escribir como:

$$
\begin{aligned}
T & \approx d_{3} T+d_{5} T+d_{6} T \\
(1-d) T & =d_{3} T \\
d T & =d_{5} T+d_{6} T
\end{aligned}
$$

puesto que $d_{1} T=d_{2} T=d_{4} T=0$. Las curvas principales están indicadas en la Figura D.12. Considerando los valores constantes para las variables lentas, la tensión $v_{C_{r}}(t)$, tiene la siguiente expresión ${ }^{1}$ :

$$
v_{C_{r}}(t)= \begin{cases}v_{g}+v_{C_{c}} & t \in\left[t_{2}, t_{3}\right] \\ 0 & t \in\left[t_{4}, t_{5}\right] \\ 0 & t \in\left[t_{5}, t_{6}\right]\end{cases}
$$

mientras que la intensidad en la bobina resonante, $i_{L_{r}}(t)$ se expresa como

$$
i_{L_{r}}(t)= \begin{cases}i_{m}-\frac{v_{C_{c}}-n v_{C_{o}}}{L_{r}}\left(t-t_{2}\right) & t \in\left[t_{2}, t_{3}\right] \\ i_{m}+\frac{v_{g}+n v_{C_{o}}}{L_{r}}\left(t-t_{5}\right) & t \in\left[t_{4}, t_{5}\right] \\ i_{m} & t \in\left[t_{5}, t_{6}\right]\end{cases}
$$

Se obtiene $d_{5}$ a partir de la igualdad de valores de $i_{L_{r}}$ en $t_{3}=t_{4}$. Es decir:

$$
i_{L_{r}}\left(t_{3}\right)=i_{L_{r}}\left(t_{4}\right)
$$

Entonces, con (D.75) y (D.76) se obtiene una solución para $d_{5}$

$$
d_{5}=\frac{v_{C_{c}}-n v_{C_{o}}}{v_{g}+n v_{C_{o}}}(1-d)
$$

\footnotetext{
${ }^{1}$ Se mantiene la numeración de los intervalos, considerando que $t_{0}=t_{1}=t_{2}$ y que $t_{3}=t_{4}$.
} 
Para el resto de variables lentas si es necesario escribir las ecuaciones de estado. Se construye una ecuación para la intensidad en la bobina de magnetización, $i_{m}(t)$; otra ecuación para la tensión en el condensador de salida, $v_{C_{o}}(t)$; y otra ecuación para la tensión en el condensador de clamp, $v_{C_{c}}(t)$. Estas ecuaciones son:

$$
\begin{aligned}
& v_{L_{m}}(t)=L_{m} \frac{d i_{m}(t)}{d t}= \begin{cases}-n v_{C_{o}}(t) & t \in\left[t_{2}, t_{3}\right] \\
-n v_{C_{o}}(t) & t \in\left[t_{4}, t_{5}\right] \\
v_{g}(t) & t \in\left[t_{5}, t_{6}\right]\end{cases} \\
& i_{C_{o}}(t)=C_{o} \frac{d v_{C_{o}}(t)}{d t}= \begin{cases}-n \underbrace{\left(i_{L_{r}}(t)-i_{m}(t)\right)}_{i_{n}(t)}-\frac{v_{C_{o}}(t)}{R} & t \in\left[t_{2}, t_{3}\right] \\
-n \underbrace{\left(i_{L_{r}}(t)-i_{m}(t)\right)}_{i_{n}(t)}-\frac{v_{C_{o}}(t)}{R} & t \in\left[t_{4}, t_{5}\right] \\
-\frac{v_{C_{o}}(t)}{R} & t \in\left[t_{5}, t_{6}\right]\end{cases} \\
& i_{C_{c}}(t)=C_{c} \frac{d v_{C_{c}}(t)}{d t}= \begin{cases}i_{L_{r}}(t) & t \in\left[t_{2}, t_{3}\right] \\
0 & t \in\left[t_{4}, t_{5}\right] \\
0 & t \in\left[t_{5}, t_{6}\right]\end{cases}
\end{aligned}
$$

La ecuación (D.78) es la ecuación de estado de un convertidor flyback sin enclavamiento activo, mientras que (D.79) y (D.80) son las ecuaciones del condensador de salida y del condensador de la red añadida. Al igual que en el flyback convencional, se puede añadir una ecuación para con la intensidad de entrada al convertidor y otra ecuación para la intensidad en el diodo principal, que son (D.81) y (D.82):

$$
\begin{gathered}
i_{g}(t)= \begin{cases}0 & t \in\left[t_{2}, t_{3}\right] \\
i_{L_{r}}(t) & t \in\left[t_{4}, t_{5}\right] \\
i_{L_{r}}(t) & t \in\left[t_{5}, t_{6}\right]\end{cases} \\
i_{D}(t)= \begin{cases}n\left(i_{m}(t)-i_{L_{r}}(t)\right) & t \in\left[t_{2}, t_{3}\right] \\
n\left(i_{m}(t)-i_{L_{r}}(t)\right) & t \in\left[t_{4}, t_{5}\right] \\
0 & t \in\left[t_{5}, t_{6}\right]\end{cases}
\end{gathered}
$$

Cuando se sustituyen las variables rápidas en las ecuaciones anteriores, se consigue tener un conjunto de ecuaciones de estado donde solo intervienen las variables de estado lentas, es decir, se han desacoplado de las variables 
de estado rápidas, que son

$$
\begin{aligned}
L_{m} \frac{d i_{m}(t)}{d t}= \begin{cases}-n v_{C_{o}}(t) & t \in\left[t_{2}, t_{3}\right] \\
-n v_{C_{o}}(t) & t \in\left[t_{4}, t_{5}\right] \\
v_{g}(t) & t \in\left[t_{5}, t_{6}\right]\end{cases} \\
C_{o} \frac{d v_{C_{o}}(t)}{d t}= \begin{cases}n \frac{v_{C_{c}}(t)-n v_{C_{o}}(t)}{L_{r}}\left(t-t_{2}\right)-\frac{v_{C_{o}}(t)}{R} & t \in\left[t_{2}, t_{3}\right] \\
-n \frac{v_{g}(t)+n v_{C_{o}}(t)}{L_{r}}\left(t-t_{5}\right)-\frac{v_{C_{o}}(t)}{R} & t \in\left[t_{4}, t_{5}\right] \\
-\frac{v_{C_{o}}(t)}{R} & t \in\left[t_{5}, t_{6}\right]\end{cases} \\
C_{c} \frac{d v_{C_{c}}(t)}{d t}= \begin{cases}i_{m}(t)-\frac{v_{C_{c}}(t)-n v_{C_{o}}(t)}{L_{r}}\left(t-t_{2}\right) & t \in\left[t_{2}, t_{3}\right] \\
0 & t \in\left[t_{4}, t_{5}\right] \\
0 & t \in\left[t_{5}, t_{6}\right]\end{cases}
\end{aligned}
$$

junto con

$$
\begin{array}{r}
i_{g}(t)= \begin{cases}0 & t \in\left[t_{2}, t_{3}\right] \\
i_{m}(t)+\frac{v_{g}(t)+n v_{C_{o}}(t)}{L_{r}}\left(t-t_{5}\right) & t \in\left[t_{4}, t_{5}\right] \\
i_{m}(t) & t \in\left[t_{5}, t_{6}\right]\end{cases} \\
i_{D}(t)= \begin{cases}n \frac{v_{C_{c}}(t)-n v_{C_{o}}(t)}{L_{r}}\left(t-t_{2}\right) & t \in\left[t_{2}, t_{3}\right] \\
-n \frac{v_{g}(t)+n v_{C_{o}}(t)}{L_{r}}\left(t-t_{5}\right) & t \in\left[t_{4}, t_{5}\right] \\
0 & t \in\left[t_{5}, t_{6}\right]\end{cases}
\end{array}
$$

Considerando la aproximación de pequeño rizado (valores instantáneos son muy parecidos a los valores promedio), es posible sustituir los valores instantáneos de las variables de estado por sus valores promedio, (por ejemplo, $i_{m}(t)$ se sustituye por $\bar{i}_{L_{m}}$, que es igual que $\left\langle i_{m}(t)\right\rangle_{T}$ donde se ha simplificado la notación, y así con el resto). De esta forma, las ecuaciones desacopladas se transforman en

$$
\begin{gathered}
L_{m} \frac{d i_{m}(t)}{d t} \approx \begin{cases}-n \bar{v}_{C_{o}} & t \in\left[t_{2}, t_{3}\right] \\
-n \bar{v}_{C_{o}} & t \in\left[t_{4}, t_{5}\right] \\
\bar{v}_{g} & t \in\left[t_{5}, t_{6}\right]\end{cases} \\
C_{o} \frac{d v_{C_{o}}(t)}{d t} \approx \begin{cases}n \frac{\bar{v}_{C_{c}}-n \bar{v}_{C_{o}}}{L_{r}}\left(t-t_{2}\right)-\frac{\bar{v}_{C_{o}}}{\bar{R}_{C}} & t \in\left[t_{2}, t_{3}\right] \\
-n \frac{\bar{v}_{g}+n \bar{v}_{C_{o}}}{L_{r}}\left(t-t_{5}\right)-\frac{\bar{v}_{C_{o}}}{R} & t \in\left[t_{4}, t_{5}\right] \\
-\frac{\bar{v}_{C_{o}}}{R} & t \in\left[t_{5}, t_{6}\right]\end{cases}
\end{gathered}
$$




$$
\begin{array}{r}
C_{c} \frac{d v_{C_{c}}(t)}{d t} \approx \begin{cases}\bar{i}_{L_{m}}-n \frac{\bar{v}_{C_{c}}-n \bar{v}_{C_{o}}}{L_{r}}\left(t-t_{2}\right) & t \in\left[t_{2}, t_{3}\right] \\
0 & t \in\left[t_{4}, t_{5}\right] \\
0 & t \in\left[t_{5}, t_{6}\right]\end{cases} \\
i_{g}(t) \approx \begin{cases}0 & t \in\left[t_{2}, t_{3}\right] \\
\bar{i}_{L_{m}}+\frac{\bar{v}_{g}+n \bar{v}_{C_{o}}}{L_{r}}\left(t-t_{5}\right) & t \in\left[t_{4}, t_{5}\right] \\
\bar{i}_{L_{m}} & t \in\left[t_{5}, t_{6}\right]\end{cases} \\
i_{D}(t) \approx \begin{cases}n \frac{\bar{v}_{C_{c}}-n \bar{v}_{C_{o}}\left(t-t_{2}\right)}{L_{r}}(t)\left[t_{2}, t_{3}\right] \\
-n \frac{\bar{v}_{g}+n \bar{v}_{C_{o}}}{L_{r}}\left(t-t_{5}\right) & t \in\left[t_{4}, t_{5}\right] \\
0 & t \in\left[t_{5}, t_{6}\right]\end{cases} \\
d_{5} \approx \frac{\bar{v}_{C_{c}}-n \bar{v}_{C_{o}}}{\bar{v}_{g}+n \bar{v}_{C_{o}}}(1-d)
\end{array}
$$

Por último hay que eliminar la dependencia del tiempo realizando el promedio de las ecuaciones en un intervalo, de donde se obtiene el modelo promediado para el flyback ZVS, que es un modelo de orden tres, (es un modelo de orden reducido frente al modelo de orden completo que sería de orden cinco).

$$
\begin{aligned}
L_{m} \frac{d \bar{i}_{m}}{d t} & =\left(d-d_{5}\right) \bar{v}_{g}-\left(1-d+d_{5}\right) n \bar{v}_{C_{o}} \\
C_{o} \frac{d \bar{v}_{C_{o}}}{d t} & =n \frac{\bar{v}_{g}+n \bar{v}_{C_{o}}}{2 L_{r} f_{s}} d_{5}^{2}+n \frac{\bar{v}_{C_{c}}-n \bar{v}_{C_{o}}}{2 L_{r} f_{s}}(1-d)^{2}-\frac{\bar{v}_{C_{o}}}{R} \\
C_{c} \frac{d \bar{v}_{C_{c}}}{d t} & =\bar{i}_{L_{m}}(1-d)-\frac{\bar{v}_{C_{c}}-n \bar{v}_{C_{o}}}{2 L_{r} f_{s}}(1-d)^{2} \\
\bar{i}_{g} & =\bar{i}_{L} d-\frac{\bar{v}_{g}+n \bar{v}_{C_{o}}}{2 L_{r} f_{s}} d_{5}^{2} \\
\bar{i}_{D} & =n \frac{\bar{v}_{g}+n \bar{v}_{C_{o}}}{2 L_{r} f_{s}} d_{5}^{2}+n \frac{\bar{v}_{C_{c}}-n \bar{v}_{C_{o}}}{2 L_{r} f_{s}}(1-d)^{2}
\end{aligned}
$$

Con las ecuaciones anteriores se consigue obtener un circuito promediado para este convertidor. Previamente hay que sustituir el parámetro $d_{5}$ por su 
valor, y se obtienen las ecuaciones indicadas en (D.99).

$$
\begin{aligned}
L_{m} \frac{d \bar{i}_{m}}{d t} & =\bar{v}_{g} d-\bar{v}_{C_{c}}(1-d) \\
C_{o} \frac{d \bar{v}_{C_{o}}}{d t} & =-\frac{\bar{v}_{C_{o}}}{R}+n \frac{\left(\bar{v}_{C_{c}}-n \bar{v}_{C_{o}}\right)^{2}}{\bar{v}_{g}+n \bar{v}_{C_{o}}} \frac{(1-d)^{2}}{2 L_{r} f_{s}}+n \frac{\bar{v}_{C_{c}}-n \bar{v}_{C_{o}}}{2 L_{r} f_{s}}(1-d)^{2} \\
C_{c} \frac{d \bar{v}_{C_{c}}}{d t} & =\bar{i}_{m}(1-d)-\frac{\bar{v}_{C_{c}}-n \bar{v}_{C_{o}}}{2 L_{r} f_{s}}(1-d)^{2} \\
\bar{i}_{g} & =\bar{i}_{m} d-\frac{\left(\bar{v}_{C_{c}}-n \bar{v}_{C_{o}}\right)^{2}}{\bar{v}_{g}+n \bar{v}_{C_{o}}} \frac{(1-d)^{2}}{2 L_{r} f_{s}} \\
\bar{i}_{D} & =n \frac{\left(\bar{v}_{C_{c}}-n \bar{v}_{C_{o}}\right)^{2}}{\bar{v}_{g}+n \bar{v}_{C_{o}}} \frac{(1-d)^{2}}{2 L_{r} f_{s}}+n \frac{\bar{v}_{C_{c}}-n \bar{v}_{C_{o}}}{2 L_{r} f_{s}}(1-d)^{2}
\end{aligned}
$$

El circuito obtenido es el indicado en la figura D.16, donde $r_{e q}$ es una resistencia equivalente sin pérdidas que consume la energía generada por la dos fuentes dependientes de corriente de valor $i_{e q}$.

$$
\begin{gathered}
r_{e q}=\frac{2 L_{r} f_{s}}{(1-d)^{2}} \\
i_{e q}=\frac{\left(v_{C_{c}}-n v_{C_{o}}\right)^{2}}{\left(v_{g}+n v_{C_{o}}\right) r_{e q}}
\end{gathered}
$$

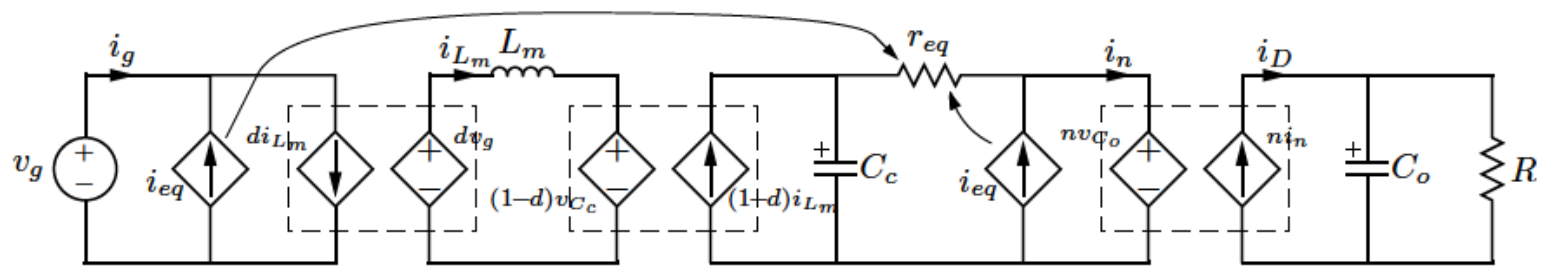

Figura D.16: Modelo promediado de un flyback ZVS.

\section{D.3.2. Balance energético}

En el modelo promediado hay tres elementos involucrados, la fuente de tensión en la entrada las fuente de corriente equivalente en la entrada y la fuente de corriente equivalente en la salida. La energía de la fuente de tensión se consume en la carga y se almacena o devuelve en los elementos reactivos, mientras que la energía generada en la fuente dependiente de la entrada se consume en $r_{e q}$, al igual que sucede con la energía generada en la fuente dependiente de la salida. 
En $r_{e q}$, la potencia consumida es igual a

$$
P_{r_{e q}}=\frac{\left(v_{C_{c}}-n v_{C_{o}}\right)^{2}}{r_{e q}}
$$

Multiplicando arriba y abajo por $\left(v_{g}+n v_{C_{o}}\right)$, se obtiene una expresión

$$
P_{r_{e q}}=\frac{\left(v_{C_{c}}-n v_{C_{o}}\right)^{2}}{r_{e q}\left(v_{g}+n v_{C_{o}}\right)}\left(v_{g}+n v_{C_{o}}\right)
$$

en la que, agrupando, se obtienen dos productos de una intensidad $i_{e q}$ por una tensión,

$$
P_{r_{e q}}=i_{e q} v_{g}+i_{e q} n v_{C_{o}}=P_{i_{e q_{1}}}+P_{i_{e q_{2}}}
$$

por tanto, esta potencia coincide con la potencia generada por las dos fuentes de corriente dependientes.

\section{D.3.3. Validación del modelo promediado}

La validación del modelo promediado se realiza mediante simulación, y se compara su comportamiento, tanto en régimen permanente como en régimen dinámico, con un convertidor conmutado. Para ello se eligen de nuevo los parámetros de funcionamiento recogidos en la Tabla D.1, empleando en el convertidor conmutado el ciclo de trabajo calculado al considerar rizado. En el modelo promediado se ha considerado el ciclo de trabajo calculado cuando no se ha considerado el rizado.

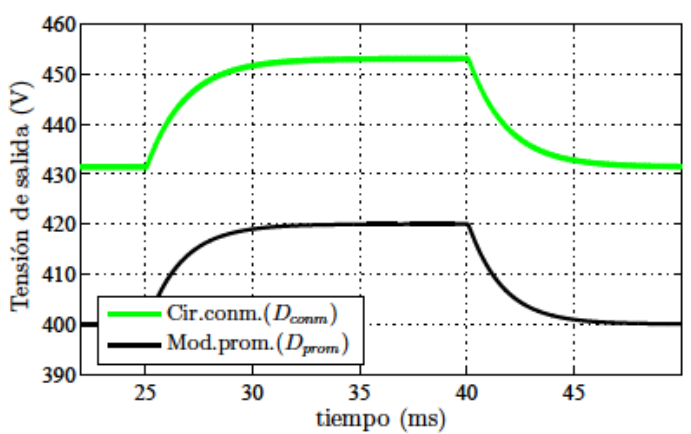

(a)

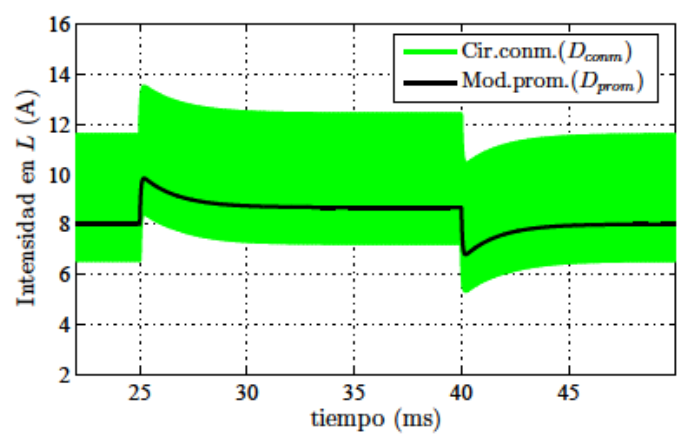

(b)

Figura D.17: Flyback ZVS. Respuesta a escalón del ciclo de trabajo: a) Tensión de salida; y b) Intensidad en la bobina. 
Los resultados muestran una dinámica similar para el convertidor conmutado y el modelo promediado. En cuanto al régimen permanente, hay un error puesto que la tensión de salida no coincide. El motivo de esta diferencia tiene su causa en que en el modelo promediado no está incluido el tiempo muerto, $t_{m}$, que hay que dejar entre disparos de los transistores principal y de clamp, entre otras cosas, para facilitar las conmutaciones suaves. Además, parte de este tiempo muerto se emplea en cargar o descargar el condensador resonante, y corresponde a las tres etapas que se desprecian para obtener el modelo promediado. En concreto la etapa 4 , siendo su duración igual a $d_{4} T$.

Por tanto, hay que realizar una corrección en el ciclo de trabajo empleado para el circuito conmutado, en base a los tiempos mencionados, puesto que ya está incluido de forma implícita en la generación de disparos del propio circuito conmutado. De esta forma, se rehacen las simulaciones del circuito conmutado con el ciclo de trabajo corregido, $D_{\text {corr }}$, como:

$$
D_{\text {corr }}=D_{\text {conm }}-\frac{t_{m}-d_{4} T}{T}
$$

y se obtienen los resultados de la Figura D.18. La corrección equivalente pero para el modelo promediado consistiría en añadir este tiempo extra al ciclo de trabajo empleado en el modelo promediado.

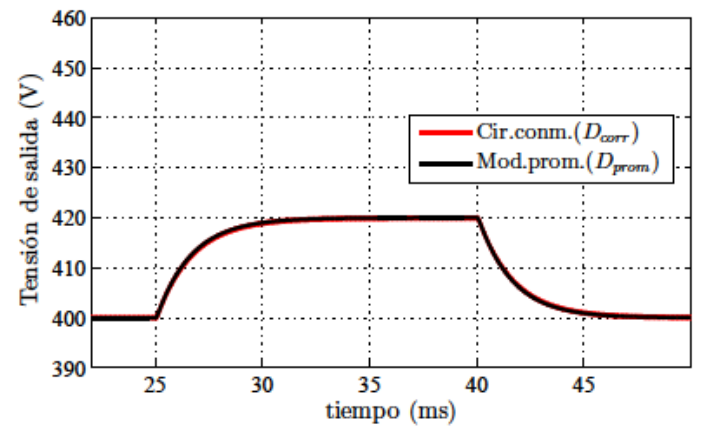

(a)

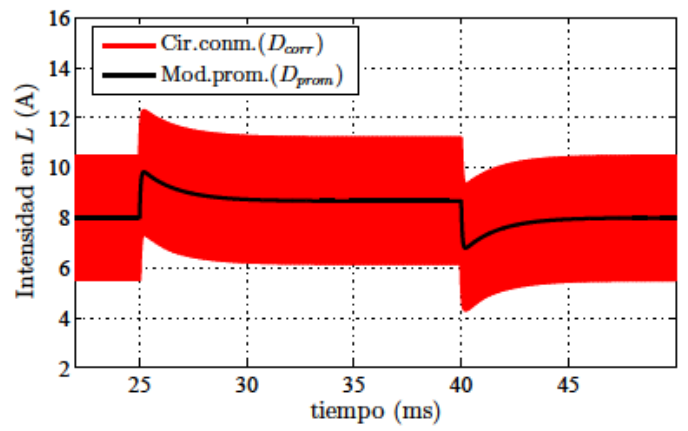

(b)

Figura D.18: Flyback ZVS. Respuesta a escalón en el ciclo de trabajo incluyendo corrección: a) Tensión de salida; y b) Intensidad en la bobina.

Para apreciar la mejora en los resultados se representa el valor medio de la tensión de salida y la intensidad en la bobina para el circuito conmutado antes y después de la corrección y se comparan con el modelo promediado. En la Figura D.19 se pueden comprobar estas curvas. 


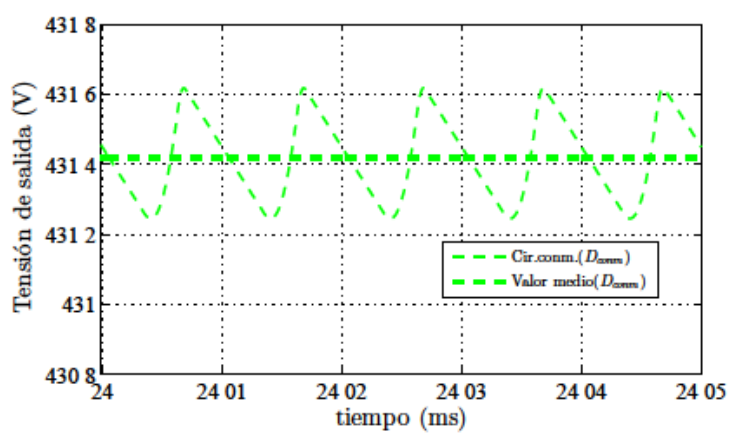

(a)

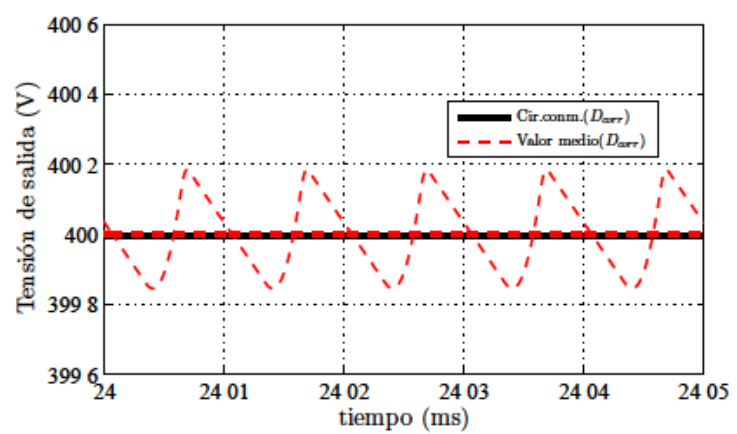

(b)

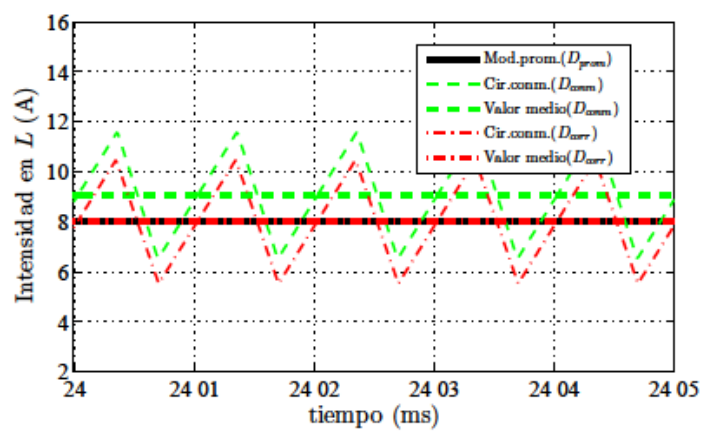

(c)

Figura D.19: Flyback ZVS en régimen permanente. Curvas de tensión e intensidad con y sin corrección en el ciclo de trabajo del circuito conmutado: a) Tensión de salida sin corrección e; b) Idem aplicando corrección; y c) Intensidad en la bobina.

\section{D.4. Modelo en régimen permanente}

El modelo en régimen permanente se puede obtener a partir del modelo promediado (o de gran señal) sustituyendo los valores promedio por valores constantes (por ejemplo, $\bar{i}_{m}$ se sustituye por $I$ ). De esta forma, las ecuaciones del modelo en régimen permanente serán las indicadas a continuación. Nótese que la derivada de una constante es cero, de ahí, que haya ecuaciones con un 
cero a la izquierda de la igualdad:

$$
\begin{aligned}
& 0=D V_{g}-(1-D) V_{c} \\
& 0=n I_{e q}+n \frac{V_{c}-n V_{o}}{R_{e q}}-\frac{V_{o}}{R} \\
& 0=(1-D) I-\frac{V_{c}-n V_{o}}{R_{e q}} \\
& I_{g}=D I-I_{e q} \\
& I_{D}=n\left(I_{e q}+\frac{V_{c}-n V_{o}}{R_{e q}}\right)
\end{aligned}
$$

donde

$$
R_{e q}=\frac{2 L_{r} f_{s}}{(1-D)^{2}} \quad I_{e q}=\frac{\left(V_{c}-n V_{o}\right)^{2}}{\left(V_{g}+n V_{o}\right) R_{e q}}
$$

A estas ecuaciones se puede llegar también a partir del circuito promediado de la figura D.16. Basta con sustituir los condensadores por un circuito abierto y las bobinas con un cortocircuito. De esta forma se obtienen los circuitos de las figuras D.20a y D.20b.

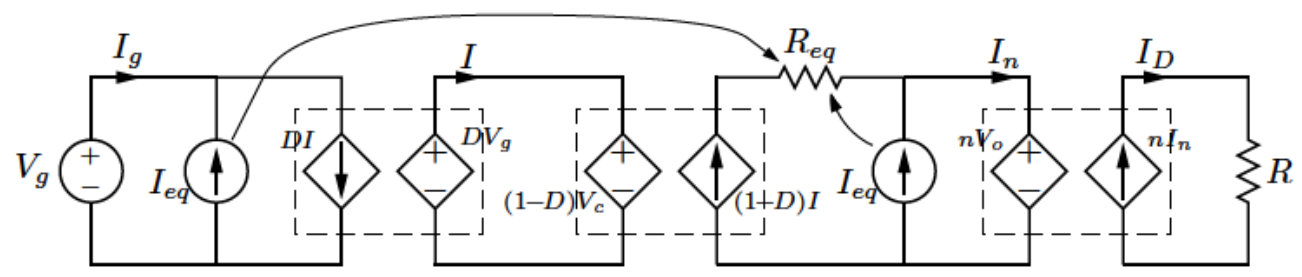

(a)

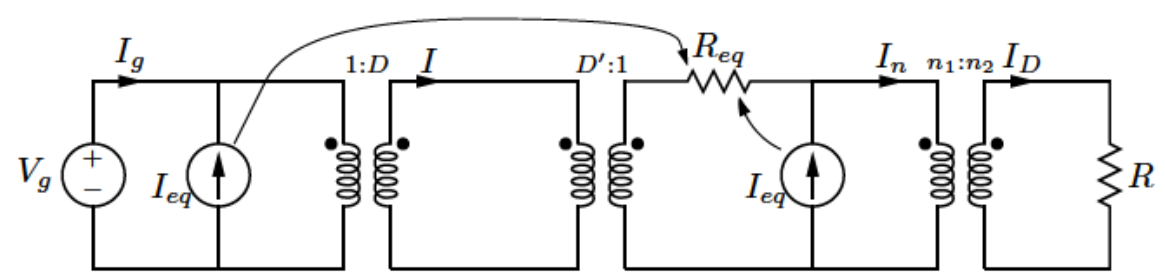

(b)

Figura D.20: Circuito en régimen permanente de un flyback ZVS en MCC: (a) con fuentes dependientes; (b) con transformadores ideales.

Se obtienen las mismas expresiones para las principales magnitudes en régimen permanente cuando se deducen de (D.106) que a partir de los circuitos 
de la figura D.20.

$$
\begin{aligned}
V_{o} & =\frac{1}{n}\left(V_{c}-(1-D) I R_{e q}\right) \\
I_{g} & =D I-I_{e q} \\
V_{c} & =\frac{D V_{g}}{(1-D)} \\
I_{D} & =\frac{V_{o}}{R}=n\left(I_{e q}+(1-D) I\right)
\end{aligned}
$$

Si en estas expresiones se elimina $L_{r}$, (que significa que desaparece el circuito de enclavamiento activo), se obtienen las expresiones en régimen permanente para un reductor convencional y lo mismo sucede con los circuitos en régimen permanente de ambas topologías.

La expresión que relaciona la tensión de salida con la intensidad en la carga se obtiene fácilmente a partir de la primera expresión de (D.108) donde se sustituye $R_{e q}$ y $V_{c}$.

$$
V_{o}=\frac{1}{n} V_{g} \frac{D}{1-D}-\frac{1}{n}(1-D) I R_{e q}
$$

Se puede observar que la tensión de salida de flyback ZVS se asimila a la de un flyback convencional que tiene a la salida una resistencia de valor $R_{e q} / n^{2}$ (con la particularidad de que es una resistencia equivalente sin pérdidas), y evoluciona con la corriente demandada por la carga (que en un flyback convencional es igual a $n I(1-D))$.

Por otro lado, la relación entre intensidades de entrada y salida y bobina se obtiene empleando la segunda y cuarta expresión de (D.108), sabiendo que $I_{D}=I_{o}$. Entonces:

$$
I_{g}+\frac{I_{o}}{n}=I
$$

\section{D.4.1. Balance energético}

En el modelo en régimen permanente hay tres elementos que generan energía, la fuente de tensión de la entrada, y la dos fuentes dependientes de corriente en la etapa de entrada y de salida. La energía generada en estas fuente de corriente se consume toda en $R_{e q}$. Veámoslo.

En $R_{e q}$, la potencia consumida es igual a

$$
P_{R_{e q}}=\frac{\left(V_{c}-n V_{o}\right)^{2}}{R_{e q}}
$$

Multiplicando arriba y abajo por $\left(V_{g}+n V_{o}\right)$, se obtiene una expresión

$$
P_{R_{e q}}=\frac{\left(V_{c}-n V_{o}\right)^{2}}{R_{e q}\left(V_{g}+n V_{o}\right)}\left(V_{g}+n V_{o}\right)
$$


en la que, agrupando, se obtienen dos productos de una intensidad $I_{e q}$ por una tensión,

$$
P_{R_{e q}}=I_{e q} V_{g}+I_{e q} n V_{o}=P_{I_{e q_{1}}}+P_{I_{e q_{2}}}
$$

por tanto, esta potencia coincide con la potencia generada por las fuentes de corriente dependientes.

Por otro lado, toda la potencia generada en $V_{g}$ se consume en la carga $R$ a la salida. La potencia que se genera es:

$$
\begin{aligned}
P_{g} & =V_{g} I_{g}=V_{g}\left(D I-I_{e q}\right)=V_{g} D I-V g I_{e q}=V_{c}(1-D) I-V_{g} I_{e q} \\
& =\left(n V_{o}-(1-D) I R_{e q}\right)(1-D) I-V_{g} I_{e q}=n V_{o}(1-D) I+(1-D)^{2} I^{2} R_{e q}-V_{g} I_{e q} \\
& =\not V_{o} \frac{V_{o}}{\not R}-n V_{o} I_{e q}+\frac{\left(V_{c}-n V_{o}\right)^{2}}{R_{e q}^{2}} R_{e q}-V_{g} I_{e q} \\
& =\frac{V_{o}^{2}}{R}+\frac{\left(V_{c}-n V_{o}\right)^{2}}{R_{e q}}-\left(n V_{o}+V_{g}\right) I_{e q} \\
& =\frac{V_{o}^{2}}{R}+\frac{\left(V_{c}-n V_{o}\right)^{2}}{R_{e q}}-\frac{\left(V_{c}-n V_{o}\right)^{2}}{R_{e q}}=P_{R}
\end{aligned}
$$

que coincide, con la potencia consumida por $R$. Esto es, la potencia que suministra la fuente de entrada se entrega a la carga en su totalidad.

\section{D.5. Modelo en pequeña señal}

El modelo en pequeña señal (modelo lineal) se consigue tras realizar el proceso de perturbación y linealización del modelo promediado (modelo no lineal) en torno a un punto de funcionamiento. Se asume que las entradas al modelo, $v_{g}$ y $d$ son iguales a un valor fijo, $V_{g}$ y $D$, mas una pequeña variación de alterna, $\hat{v}_{g}$ y $\hat{d}$. Es decir:

$$
\begin{aligned}
\bar{v}_{g} & =V_{g}+\hat{v}_{g} \\
d & =D+\hat{d}
\end{aligned}
$$

En respuesta a estas entradas, los valores promediados de las variables de estado sufrirán una variación similar, es decir, presentarán un término constante mas una pequeña variación de alterna. También sucede lo mismo con 
el resto de magnitudes estudiadas, de forma que:

$$
\begin{gathered}
\bar{i}_{m}=I+\hat{i}_{m} \\
\bar{v}_{C_{o}}=V_{o}+\hat{v}_{C_{o}} \\
\bar{v}_{C_{c}}=V_{c}+\hat{v}_{C_{c}} \\
\bar{i}_{g}=I_{g}+\hat{i}_{g} \\
\bar{i}_{D}=I_{D}+\hat{i}_{D}
\end{gathered}
$$

Por supuesto, para que el modelo linealizado sea preciso debe cumplirse que las variaciones son pequeñas en comparación con el valor constante que indica el punto de operación del convertidor, es decir:

$$
\begin{aligned}
&\left|\hat{v}_{g}\right| \ll\left|V_{g}\right| \\
&|\hat{d}| \ll|D| \\
&\left|\hat{i}_{L_{m}}\right| \ll|I| \\
&\left|\hat{v}_{C_{o}}\right| \ll\left|V_{o}\right| \\
&\left|\hat{v}_{C_{c}}\right| \ll\left|V_{c}\right| \\
&\left|\hat{i}_{g}\right| \ll\left|I_{g}\right| \\
&\left|\hat{i}_{D}\right| \ll\left|I_{D}\right|
\end{aligned}
$$

Por lo que, introduciendo (D.115) y (D.116) en las ecuaciones del modelo promediado de orden reducido del flyback ZVS, reescritas de nuevo en (D.118) y desarrollando y despreciando los términos de segundo orden se consigue linealizar el modelo promediado.

$$
\begin{aligned}
L_{m} \frac{d \bar{i}_{m}}{d t} & =\bar{v}_{g} d-\bar{v}_{C_{c}}(1-d) \\
C_{o} \frac{d \bar{v}_{C_{o}}}{d t} & =-\frac{\bar{v}_{C_{o}}}{R}+n \frac{\left(\bar{v}_{C_{c}}-n \bar{v}_{C_{o}}\right)^{2}}{\bar{v}_{g}+n \bar{v}_{C_{o}}} \frac{(1-d)^{2}}{2 L_{r} f_{s}}+n \frac{\bar{v}_{C_{c}}-n \bar{v}_{C_{o}}}{2 L_{r} f_{s}}(1-d)^{2} \\
C_{c} \frac{d \bar{v}_{C_{c}}}{d t} & =\bar{i}_{m}(1-d)-\frac{\bar{v}_{C_{c}}-n \bar{v}_{C_{o}}}{2 L_{r} f_{s}}(1-d)^{2} \\
\bar{i}_{g} & =\bar{i}_{m} d-\frac{\left(\bar{v}_{C_{c}}-n \bar{v}_{C_{o}}\right)^{2}}{\bar{v}_{g}+n \bar{v}_{C_{o}}} \frac{(1-d)^{2}}{2 L_{r} f_{s}} \\
\bar{i}_{D} & =n \frac{\left(\bar{v}_{C_{c}}-n \bar{v}_{C_{o}}\right)^{2}}{\bar{v}_{g}+n \bar{v}_{C_{o}}} \frac{(1-d)^{2}}{2 L_{r} f_{s}}+n \frac{\bar{v}_{C_{c}}-n \bar{v}_{C_{o}}}{2 L_{r} f_{s}}(1-d)^{2}
\end{aligned}
$$

El proceso de linealización no es mas que un desarrollo de Taylor de una función, $f(x, y, z, \ldots)$ en torno a un punto determinado $\left(x_{0}, y_{0}, z_{0}, \ldots\right)$, considerando únicamente los términos hasta primer orden y despreciando el resto. Por tanto, aplicando el desarrollo de Taylor hasta primer orden a la 
parte derecha de cada una de las igualdades de las ecuaciones del modelo promediado se consigue llegar a una expresión que permite la obtención del modelo en pequeña señal y también, otra vez, el modelo en régimen permanente. Por ejemplo, la ecuación de la corriente en la bobina de entrada del modelo promediado dada en (D.118) se puede escribir como:

$$
L \frac{d \bar{i}_{m}}{d t}=f_{1}\left(\bar{v}_{g}, d, \bar{v}_{C_{c}}\right)
$$

Linealizando la función $f_{1}$ con el desarrollo de Taylor en un punto de funcionamiento dado por $\left(V_{g}, D, I, V_{c}\right)$ se obtiene (D.120). Por supuesto, las ecuación obtenida no será una aproximación muy buena de la función no lineal cuando se trate de un punto alejado del punto de linealización.

$$
\begin{aligned}
& L_{m}\left(\frac{d I}{d t}+\frac{d \hat{i}_{L_{m}}}{d t}\right)=f_{1}\left(V_{g}, D, V_{c}\right) \\
& +\left.\hat{v}_{g}(t) \frac{\partial f_{1}\left(\bar{v}_{g}, D, V_{c}\right)}{\partial \bar{v}_{g}}\right|_{\bar{v}_{g}=V_{g}}+\left.\hat{v}_{C_{c}} \frac{\partial f_{1}\left(V_{g}, D, \bar{v}_{C_{c}}\right)}{\partial \bar{v}_{C_{c}}}\right|_{\bar{v}_{C_{c}}=V_{c}} \\
& +\left.\hat{d}(t) \frac{\partial f_{1}\left(V_{g}, d, V_{c}\right)}{\partial d}\right|_{d=D}+\text { términos orden superior }
\end{aligned}
$$

A continuación se evalúan las derivadas parciales para la ecuación de la corriente en la bobina de magnetización, $\bar{i}_{m}$. Las derivadas parciales son:

$$
\begin{gathered}
\left.\frac{\partial f_{1}\left(\bar{v}_{g}, D, V_{c}\right)}{\partial \bar{v}_{g}}\right|_{\bar{v}_{g}=V_{g}}=D \\
\left.\frac{\partial f_{1}\left(V_{g}, D, \bar{v}_{C_{c}}\right)}{\partial \bar{v}_{C_{c}}}\right|_{\bar{v}_{C_{c}=V_{c}}}=-(1-D) \\
\left.\frac{\partial f_{1}\left(V_{g}, d, V_{c}\right)}{\partial d}\right|_{d=D}=V_{g}+V_{c}
\end{gathered}
$$

La ecuación (D.120) queda ahora así:

$$
\begin{aligned}
L_{m}\left(0+\frac{d \hat{i}_{L_{m}}}{d t}\right) & =\left[D V_{g}-(1-D) V_{c}\right] \\
& +D \hat{v}_{g}-(1-D) \hat{v}_{C_{c}}+\left(V_{g}+V_{c}\right) \hat{d} \\
& + \text { términos de orden superior }
\end{aligned}
$$

Los términos constantes de ambos miembros se igualan para conseguir una ecuación para el modelo en régimen permanente que coincide con la primera expresión de (D.106). Los términos de primer orden generan otra ecuación 
que forma parte del modelo en pequeña señal. El resto de términos de orden superior se desprecian.

Si se hace de forma similar para el restos de ecuaciones del modelo promediado, vistas como

$$
\begin{aligned}
C_{o} \frac{d \bar{v}_{C_{o}}}{d t} & =f_{2}\left(\bar{v}_{g}, d, \bar{v}_{C_{o}}, \bar{v}_{C_{c}}\right) \\
C_{c} \frac{d \bar{v}_{C_{c}}}{d t} & =f_{3}\left(d, \bar{i}_{m}, \bar{v}_{C_{o}}, \bar{v}_{C_{c}}\right) \\
\bar{i}_{g} & =f_{4}\left(\bar{v}_{g}, d, \bar{i}_{m}, \bar{v}_{C_{o}}, \bar{v}_{C_{c}}\right) \\
\bar{i}_{D} & =f_{5}\left(\bar{v}_{g}, d, \bar{i}_{m}, \bar{v}_{C_{o}}, \bar{v}_{C_{c}}\right)
\end{aligned}
$$

será necesario evaluar las funciones $f_{2}, f_{3}, f_{4}$ y $f_{5}$ y sus derivadas parciales en torno al punto de funcionamiento elegido. Las funciones $f_{2}, f_{3}, f_{4}$ y $f_{5}$ evaluadas en el punto de funcionamiento son:

$$
\begin{aligned}
& f_{2}\left(V_{g}, D, V_{o}, V_{c}\right)=-\frac{V_{o}}{R}+n I_{e q}+n \frac{V_{c}-n V_{o}}{R_{e q}} \\
& f_{3}\left(D, I, V_{o} V_{c}\right)=(1-D) I-\frac{V_{c}-n V_{o}}{R_{e q}} \\
& f_{4}\left(V_{g}, D, I, V_{o}, V_{c}\right)=D I-I_{e q} \\
& f_{5}\left(D, I, V_{o} V_{c}\right)=n\left(I_{e q}+\frac{V_{c}-n V_{o}}{R_{e q}}\right)
\end{aligned}
$$

Las derivadas parciales respecto a las variables de estado de las funciones anteriores, y evaluadas en torno al punto de funcionamiento están indicadas a continuación.

$$
\begin{aligned}
& \left.\frac{\partial f_{2}(\ldots)}{\partial \bar{v}_{g}}\right|_{\bar{v}_{g}=V_{g}}=-n \frac{I_{e q}}{V_{g}+n V_{o}} \\
& \left.\frac{\partial f_{2}(\ldots)}{\partial d}\right|_{d=D}=-2 n\left(I+\frac{I_{e q}}{1-D}\right) \\
& \left.\frac{\partial f_{2}(\ldots)}{\partial \bar{v}_{C_{o}}}\right|_{\bar{v}_{C_{o}}=V_{o}}=-\frac{1}{R}-n^{2} \frac{2(1-D) I+I_{e q}}{V_{g}+n V_{o}}-n^{2} \frac{1}{R_{e q}} \\
& \left.\frac{\partial f_{2}(\ldots)}{\partial \bar{v}_{C_{c}}}\right|_{\bar{v}_{C_{c}}=V_{c}}=n \frac{2 I_{e q}}{V_{c}-n V_{o}}+\frac{n}{R_{e q}}
\end{aligned}
$$




$$
\begin{aligned}
& \left.\frac{\partial f_{3}(\ldots)}{\partial d}\right|_{d=D}=-I+2(1-D) \frac{V_{c}-n V_{o}}{2 L_{r} f_{s}}=I \\
& \left.\frac{\partial f_{3}(\ldots)}{\partial \bar{i}_{m}}\right|_{\bar{i}_{m}=I}=(1-D) \\
& \left.\frac{\partial f_{3}(\ldots)}{\partial \bar{v}_{C_{o}}}\right|_{\bar{v}_{C_{o}=V_{o}}}=\frac{(1-D)^{2}}{2 L_{r} f_{s}}=\frac{n}{R_{e q}} \\
& \left.\frac{\partial f_{3}(\ldots)}{\partial \bar{v}_{C_{c}}}\right|_{\bar{v}_{C_{c}}=V_{c}}=-\frac{(1-D)^{2}}{2 L_{r} f_{s}}=-\frac{1}{R_{e q}} \\
& \left.\frac{\partial f_{4}(\ldots)}{\partial \bar{v}_{g}}\right|_{\bar{v}_{g}=V_{g}}=\frac{I_{e q}}{V_{g}+n V_{o}} \\
& \left.\frac{\partial f_{4}(\ldots)}{\partial d}\right|_{d=D}=I+2 \frac{I_{e q}}{1-D} \\
& \left.\frac{\partial f_{4}(\ldots)}{\partial \bar{i}_{m}}\right|_{\bar{i}_{L_{m}}=I}=D \\
& \left.\frac{\partial f_{4}(\ldots)}{\partial \bar{v}_{C_{o}}}\right|_{\bar{v}_{C_{o}}=V_{o}}=n \frac{2(1-D) I+I_{e q}}{V_{g}+n V_{o}} \\
& \left.\frac{\partial f_{4}(\ldots)}{\partial \bar{v}_{C_{c}}}\right|_{\bar{v}_{C_{c}}=V_{c}}=-\frac{2 I_{e q}}{V_{c}-n V_{o}} \\
& \left.\frac{\partial f_{5}(\ldots)}{\partial \bar{v}_{g}}\right|_{\bar{v}_{g}=V_{g}}=-n \frac{I_{e q}}{V_{g}+n V_{o}} \\
& \left.\frac{\partial f_{5}(\ldots)}{\partial d}\right|_{d=D}=-2 n\left(I+\frac{I_{e q}}{1-D}\right) \\
& \left.\frac{\partial f_{5}(\ldots)}{\partial \bar{v}_{C_{o}}}\right|_{\bar{v}_{C_{o}}=V_{o}}=-n^{2} \frac{2(1-D) I+I_{e q}}{V_{g}+n V_{o}}-n^{2} \frac{1}{R_{e q}} \\
& \left.\frac{\partial f_{5}(\ldots)}{\partial \bar{v}_{C_{c}}}\right|_{\bar{v}_{C_{c}}=V_{c}}=n \frac{2 I_{e q}}{V_{c}-n V_{o}}+\frac{n}{R_{e q}}
\end{aligned}
$$

Entonces, cada una de las ecuaciones (D.123) quedan de la forma indicada 
en (D.129), (D.130), (D.131) y (D.132).

$$
\begin{aligned}
C_{o}\left(0+\frac{d \hat{v}_{C_{o}}}{d t}\right) & =\left[-\frac{V_{o}}{R}+n I_{e q}+n \frac{V_{c}-n V_{o}}{R_{e q}}\right] \\
& -n \frac{I_{e q}}{V_{g}+n V_{o}} \hat{v}_{g}-2 n\left(I+\frac{I_{e q}}{1-D}\right) \hat{d} \\
& -\left[\frac{1}{R}+n^{2} \frac{2(1-D) I+I_{e q}}{V_{g}+n V_{o}}+n^{2} \frac{1}{R_{e q}}\right] \hat{v}_{C_{o}}+\left[n \frac{2 I_{e q}}{V_{c}-n V_{o}}+n \frac{1}{R_{e q}}\right] \hat{v}_{C_{c}} \\
& + \text { términos de orden superior }
\end{aligned}
$$

$$
\begin{aligned}
C_{c}\left(0+\frac{d \hat{v}_{C_{c}}}{d t}\right) & =\left[(1-D) I-\frac{V_{c}-n V_{o}}{R_{e q}}\right] \\
& +I \hat{d}+(1-D) \hat{i}_{L_{m}}+\frac{n}{R_{e q}} \hat{v}_{C_{o}}-\frac{1}{R_{e q}} \hat{v}_{C_{c}}
\end{aligned}
$$

+ términos de orden superior

$$
\begin{aligned}
I_{g}+\hat{i}_{g}= & {\left[D I-I_{e q}\right] } \\
& +\frac{I_{e q}}{V_{g}+n V_{o}} \hat{v}_{g}+\left[I+2 \frac{I_{e q}}{1-D}\right] \hat{d} \\
& +D \hat{i}_{L_{m}}+\left[n \frac{2(1-D) I+I_{e q}}{V_{g}+n V_{o}}\right] \hat{v}_{C_{o}}-\frac{2 I_{e q}}{V_{c}-n V_{o}} \hat{v}_{C_{c}} \\
& + \text { términos de orden superior } \\
I_{D}+\hat{i}_{D}= & {\left[n I_{e q}+n \frac{V_{c}-n V_{o}}{R_{e q}}\right] } \\
- & n \frac{I_{e q}}{V_{g}+n V_{o}} \hat{v}_{g}-2 n\left(I+\frac{I_{e q}}{1-D}\right) \hat{d} \\
- & {\left[n^{2} \frac{2(1-D) I+I_{e q}}{V_{g}+n V_{o}}+n^{2} \frac{1}{R_{e q}}\right] \hat{v}_{C_{o}}+\left[n \frac{2 I_{e q}}{V_{c}-n V_{o}}+n \frac{1}{R_{e q}}\right] \hat{v}_{C_{c}} } \\
+ & \text { términos de orden superior }
\end{aligned}
$$

Agrupando todos los términos constantes de las ecuaciones (D.122), (D.129), (D.130), (D.131) y (D.132) se obtiene el modelo en régimen permanente que coincide con el descrito en (D.108).

Tomando todos los términos de primer orden y despreciando los términos de orden superior, se consigue el modelo en pequeña señal para el flyback ZVS dado en (D.133). 


$$
\begin{aligned}
& L_{m} \frac{\hat{i}_{L_{m}}}{d t}=D \hat{v}_{g}-(1-D) \hat{v}_{C_{c}}+\left(V_{g}+V_{c}\right) \hat{d} \\
& C_{o} \frac{d \hat{v}_{C_{o}}}{d t}=-n \frac{I_{e q}}{V_{g}+n V_{o}} \hat{v}_{g}-2 n\left(I+\frac{I_{e q}}{1-D}\right) \hat{d} \\
& -\left[\frac{1}{R}+n^{2} \frac{2(1-D) I+I_{e q}}{V_{g}+n V_{o}}+n^{2} \frac{1}{R_{e q}}\right] \hat{v}_{C_{o}} \\
& +\left[n \frac{2 I_{e q}}{V_{c}-n V_{o}}+n \frac{1}{R_{e q}}\right] \hat{v}_{C_{c}} \\
& C_{c} \frac{d \hat{v}_{C_{c}}}{d t}=I \hat{d}+(1-D) \hat{i}_{L_{m}}+\frac{n}{R_{e q}} \hat{v}_{C_{o}}-\frac{1}{R_{e q}} \hat{v}_{C_{c}} \\
& \hat{i}_{g}=\frac{I_{e q}}{V_{g}+n V_{o}} \hat{v}_{g}+\left[I+2 \frac{I_{e q}}{1-D}\right] \hat{d} \\
& +D \hat{i}_{L_{m}}+\left[n \frac{2(1-D) I+I_{e q}}{V_{g}+n V_{o}}\right] \hat{v}_{C_{o}}-\frac{2 I_{e q}}{V_{c}-n V_{o}} \hat{v}_{C_{c}} \\
& \hat{i}_{D}=-n \frac{I_{e q}}{V_{g}+n V_{o}} \hat{v}_{g}-2 n\left(I+\frac{I_{e q}}{1-D}\right) \hat{d} \\
& -\left[n^{2} \frac{2(1-D) I+I_{e q}}{V_{g}+n V_{o}}+n^{2} \frac{1}{R_{e q}}\right] \hat{v}_{C_{o}}+\left[n \frac{2 I_{e q}}{V_{c}-n V_{o}}+n \frac{1}{R_{e q}}\right] \hat{v}_{C_{c}}
\end{aligned}
$$

Con estas expresiones se construye el circuito en pequeña señal de la figura D.21. Los parámetros $f_{5}, j_{5}, g_{5}, j_{6}, g_{6}$ y $g_{f}$ están indicados en la tabla D.4. Además, $r_{5}=1 / g_{5}$ y $r_{6}=1 / g_{6}$.

\begin{tabular}{ccccccc}
\hline Convertidor & $f_{5}$ & $j_{5}$ & $g_{5}$ & $j_{6}$ & $g_{6}$ & $g_{f}$ \\
\hline \multirow{2}{*}{ Flyback } & $V_{g}+V_{c}$ & $I+\frac{2 I_{e q}}{1-D}$ & $\frac{2(1-D) I+I_{e q}}{V_{g}+n V_{o}}$ & $2\left(I+\frac{I_{e q}}{1-D}\right)$ & $\frac{I_{e q}}{V_{g}+n V_{o}}$ & $\frac{2 I_{e q}}{V_{c}-n V_{o}}$ \\
\hline
\end{tabular}

Tabla D.4: Parámetros del modelo en pequeña señal del flyback ZVS.

El modelo en pequeña señal, obtenido a partir de la linealización del modelo promediado de orden reducido, también se puede escribir en forma matricial. Así, se definen dos vectores, el vector $\hat{\mathbf{x}}$ para las variables de estado y el vector $\hat{\mathbf{u}}$ para las entradas externas e independientes, y serán en este caso:

$$
\hat{\mathbf{x}}=\left[\begin{array}{l}
\hat{i}_{L_{m}} \\
\hat{v}_{C_{o}} \\
\hat{v}_{C_{c}}
\end{array}\right], \quad \hat{\mathbf{u}}=\left[\begin{array}{c}
\hat{v}_{g} \\
\hat{d}
\end{array}\right], \quad \hat{\mathbf{y}}=\left[\begin{array}{c}
\hat{i}_{g} \\
\hat{i}_{D}
\end{array}\right]
$$

de forma que el modelo en pequeña señal de (D.133) se puede escribir, de la 


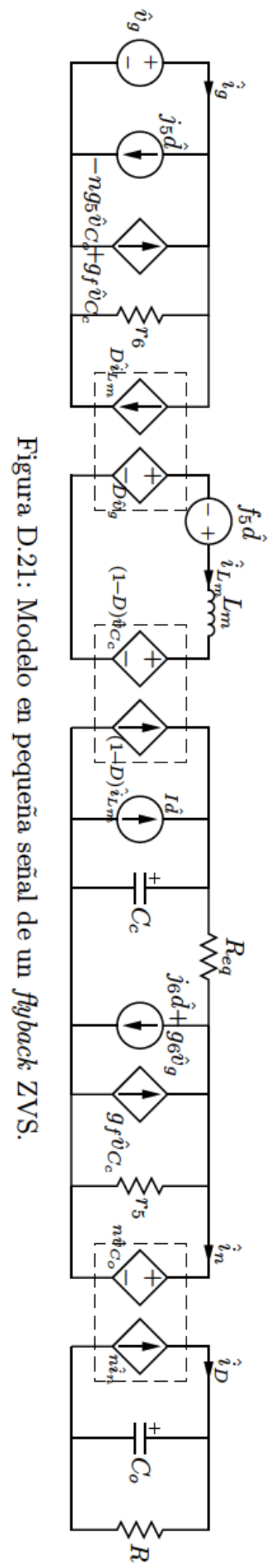


forma siguiente:

$$
\begin{aligned}
\mathbf{K} \frac{d}{d t}\left[\begin{array}{l}
\hat{i}_{L_{m}} \\
\hat{v}_{C_{o}} \\
\hat{v}_{C_{c}}
\end{array}\right] & =\mathbf{A}\left[\begin{array}{l}
\hat{i}_{L_{m}} \\
\hat{v}_{C_{o}} \\
\hat{v}_{C_{c}}
\end{array}\right]+\mathbf{B}\left[\begin{array}{c}
\hat{v}_{g} \\
\hat{d}
\end{array}\right] \\
{\left[\begin{array}{l}
\hat{i}_{g} \\
\hat{i}_{D}
\end{array}\right] } & =\mathbf{C}\left[\begin{array}{l}
\hat{i}_{L_{m}} \\
\hat{v}_{C_{o}} \\
\hat{v}_{C_{c}}
\end{array}\right]+\mathbf{E}\left[\begin{array}{l}
\hat{v}_{g} \\
\hat{d}
\end{array}\right]
\end{aligned}
$$

donde

$\mathbf{K}=\left[\begin{array}{ccc}L_{m} & 0 & 0 \\ 0 & C_{o} & 0 \\ 0 & 0 & C_{c}\end{array}\right]$

$\mathbf{A}=\left[\begin{array}{ccc}0 & 0 & D-1 \\ 0 & -\frac{1}{R}-n^{2}\left(\frac{1}{R_{e q}}+g_{5}\right) & n\left(\frac{1}{R_{e q}}+g_{f}\right) \\ 1-D & n \frac{1}{R_{e q}} & -\frac{1}{R_{e q}}\end{array}\right], \quad \mathbf{B}=\left[\begin{array}{cc}D & f_{5} \\ -n g_{6} & -n j_{6} \\ 0 & I\end{array}\right]$,

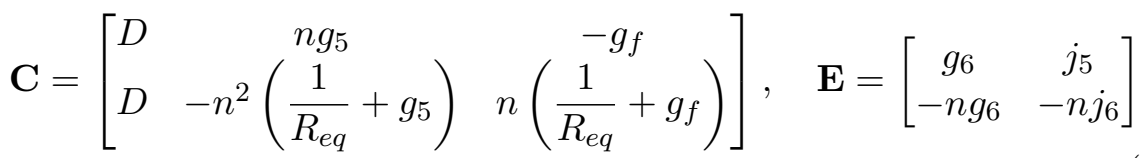

\section{D.6. Funciones de transferencia}

Las diferentes funciones de transferencia se pueden obtener bien a partir del modelo en pequeña señal dado en las ecuaciones (D.133), bien a partir del circuito en pequeña señal de la figura D.21. Por ambos métodos hay que anular la variable de estado correspondiente y aplicar Laplace para obtener la relación deseada. Otra posibilidad es trabajar con el modelo matricial en pequeña señal, que se verá mas adelante.

\section{D.6.1. Desde el modelo o el circuito de pequeña señal}

Para conseguir $G_{v g}(s)$, se hace nula la variación de la entrada independiente que no interviene en la función de transferencia, en este caso, $\hat{d}(t)=0$, 
y se aplica Laplace. De esta forma queda que:

$$
\begin{aligned}
& s L \hat{i}_{m}(s)=D \hat{v}_{g}(s)-(1-D) \hat{v}_{C_{c}}(s) \\
& s C_{o} \hat{v}_{C_{o}}(s)=-n \frac{1}{r_{6}} \hat{v}_{g}(s)-\left[\frac{1}{R}+n^{2} \frac{1}{r_{5}}+n^{2} \frac{1}{R_{e q}}\right] \hat{v}_{C_{o}}(s)+n\left[g_{f}+\frac{1}{R_{e q}}\right] \hat{v}_{C_{c}}(s) \\
& s C_{c} \hat{v}_{C_{c}}(s)=(1-D) \hat{i}_{m}(s)+n \frac{1}{R_{e q}} \hat{v}_{C_{o}}(s)-\frac{1}{R_{e q}} \hat{v}_{C_{c}}(s) \\
& \hat{i}_{g}(s)=\frac{1}{r_{6}} \hat{v}_{g}(s)+D \hat{i}_{L_{m}}(s)+n \frac{1}{r_{5}} \hat{v}_{C_{o}}(s)-g_{f} \hat{v}_{C_{c}}(s) \\
& \hat{i}_{D}(s)=-n \frac{1}{r_{6}} \hat{v}_{g}(s)-n^{2}\left[\frac{1}{r_{5}}+\frac{1}{R_{e q}}\right] \hat{v}_{C_{o}}(s)+n\left[g_{f}+\frac{1}{R_{e q}}\right] \hat{v}_{C_{c}}(s)
\end{aligned}
$$

Despejando en la tercera ecuación una expresión para $\hat{v}_{C_{c}}(s)$, como:

$$
\hat{v}_{C_{c}}(s)=(1-D) Z_{e q}(s) \hat{i}_{m}(s)+n Z_{e q}(s) / R_{e q} \hat{v}_{C_{o}}(s)
$$

y sustituyendo en la primera ecuación, se consigue obtener la expresión de $\hat{i}_{L}(s)$ en función de $\hat{v}_{g}(s)$ y $\hat{v}_{C_{o}}(s)$, dada por:

$$
\hat{i}_{m}(s)=\frac{D \hat{v}_{g}(s)-n(1-D) Z_{e q}(s) / R_{e q} \hat{v}_{C_{o}}(s)}{L s+(1-D)^{2} Z_{e q}(s)}
$$

donde se han definido las siguientes expresiones:

$$
\begin{aligned}
& Z_{o}(s)=\left(R\left\|C_{o}\right\| n^{2} r_{5}\right)=\left[s C_{o}+\frac{1}{R}+n^{2} \frac{1}{r_{5}}\right]^{-1} \\
& Z_{e q}(s)=\left(R_{e q} \| C_{c}\right)=\left[s C_{c}+\frac{1}{R_{e q}}\right]^{-1}
\end{aligned}
$$

Utilizando (D.139) en (D.138), se obtiene una expresión de $\hat{v}_{C_{c}}(s)$, dada por:

$$
\hat{v}_{C_{c}}(s)=\frac{(1-D) D Z_{e q}(s) \hat{v}_{g}(s)+n L s Z_{e q}(s) / R_{e q} \hat{v}_{C_{o}}(s)}{L s+(1-D)^{2} Z_{e q}(s)}
$$

Introduciendo (D.141) en la segunda expresión de (D.137), se consigue una ecuación, dada por (D.142), donde únicamente aparecen $\hat{v}_{g}(s)$ y $\hat{v}_{C_{c}}(s)$ que permite obtener fácilmente la función de transferencia deseada, $G_{v g}(s)$, que está indicada en (C.132).

$$
\begin{aligned}
& {\left[\frac{1}{Z_{o}(s)}+\frac{n^{2}}{R_{e q}}-\frac{n^{2}\left[g_{f} R_{e q}+1\right] L s Z_{e q}(s)}{R_{e q}^{2}\left[L s+(1-D)^{2} Z_{e q}(s)\right]}\right] \hat{v}_{C_{o}}(s)} \\
& \quad=\left[-\frac{n}{r_{6}}-\frac{n\left[g_{c} R_{e q}+1\right](1-D) D Z_{e q}(s)}{R_{e q}\left[L s+(1-D)^{2} Z_{e q}(s)\right]}\right] \hat{v}_{g}(s)
\end{aligned}
$$




$$
\begin{aligned}
& G_{v g}(s)=\left.\frac{\hat{v}_{C_{o}}(s)}{\hat{v}_{g}(s)}\right|_{\hat{d}=0} \\
& =\frac{Z_{o}(s)}{r_{6}} \frac{-n R_{e q}\left[L s+(1-D)^{2} Z_{e q}(s)\right]+n r_{6}\left[1+g_{f} R_{e q}(s)\right](1-D) D Z_{e q}(s)}{\left[n^{2} Z_{o}(s)+R_{e q}\right]\left[L s+(1-D)^{2} Z_{e q}(s)\right]-n^{2} Z_{o}(s)\left[1+g_{f} R_{e q}(s)\right] L s Z_{e q}(s) / R_{e q}}
\end{aligned}
$$

Para conseguir $G_{i g}(s)$, se utiliza (D.138), y se obtiene $\hat{v}_{C_{o}}(s)$ en función de $\hat{i}_{m}(s)$ y $\hat{v}_{g}(s)$ en la segunda ecuación de (D.137). Por tanto,

$$
\left[\frac{1}{Z_{o}(s)}+n^{2} \frac{1}{R_{e q}}\right] \hat{v}_{C_{o}}(s)=-n \frac{1}{r_{6}} \hat{v}_{g}(s)+\left[g_{f}+\frac{1}{R_{e q}}\right]\left[(1-D) Z_{e q}(s) \hat{i}_{m}(s)+n \frac{Z_{e q}(s)}{R_{e q}} \hat{v}_{C_{o}}(s)\right]
$$

Entonces, $\hat{v}_{C_{o}}(s)$ es igual a la expresión dada por

$$
\hat{v}_{C_{o}}(s)=\frac{-\frac{n}{r_{6}} \hat{v}_{g}(s)+n\left[1+g_{f} R_{e q}\right](1-D) \frac{Z_{e q}(s)}{R_{e q}} \hat{i}_{m}(s)}{\left[\frac{1}{Z_{o}(s)}+\frac{n^{2}}{R_{e q}}-n^{2}\left[1+g_{c} R_{e q}\right] \frac{Z_{e q}(s)}{R_{e q}^{2}}\right]}
$$

que, introducida en la primera ecuación de (D.137) resulta,

$$
\begin{aligned}
& s L \hat{i}_{m}(s)=D \hat{v}_{g}(s)-(1-D)^{2} Z_{e q}(s) \hat{i}_{L_{m}}(s)-n(1-D) \frac{Z_{e q}(s)}{R_{e q}} \hat{v}_{C_{o}}(s) \\
& {\left[L s+(1-D)^{2} Z_{e q}(s)\right] \hat{i}_{L_{m}}(s)=D \hat{v}_{g}(s)+\frac{\not x \frac{(1-D) Z_{e q}(s)}{r_{6}} \hat{v}_{g}(s)-\not \not\left[1+g_{f} R_{e q}\right](1-D)^{2} \frac{Z_{e q}^{2}(s)}{R_{e q}} \hat{i}_{m}(s)}{\left[\frac{R_{e q}}{n^{2} Z_{o}(s)}+\not \chi-\not x\left[1+g_{f} R_{e q}\right] \frac{Z_{e q}(s)}{R_{e q}}\right]}}
\end{aligned}
$$

Agrupando los términos en cada miembro de la igualdad:

$$
\begin{gathered}
{\left[L s+(1-D)^{2} Z_{e q}(s)+\frac{\left[1+g_{f} R_{e q}\right](1-D)^{2} \frac{Z_{e q}^{2}(s)}{R_{e q}}}{\left[\frac{R_{e q}}{n^{2} Z_{o}(s)}+1-\left[1+g_{f} R_{e q}\right] \frac{Z_{e q}(s)}{R_{e q}}\right]}\right] \hat{i}_{L_{m}}(s)} \\
=\left[D+\frac{\frac{(1-D) Z_{e q}(s)}{r_{6}}}{\frac{R_{e q}}{n^{2} Z_{o}(s)}+1-\left[1+g_{f} R_{e q}\right] \frac{Z_{e q}(s)}{R_{e q}}}\right] \hat{v}_{g}(s)
\end{gathered}
$$


Desarrollando la suma de fracciones de cada miembro,

$$
\begin{gathered}
{\left[\frac{\left[L s+(1-D)^{2} Z_{e q}(s)\right]\left[n^{2} Z_{o}(s)+R_{e q}\right] /\left(n^{2} Z_{o}(s)\right)-\left[1+g_{f} R_{e q}\right] L s Z_{e q}(s) / R_{e q}}{\left[R_{e q}+n^{2} Z_{o}(s)\right] /\left(n^{2} Z_{o}(s)\right)-\left[1+g_{f} R_{e q}\right] Z_{e q}(s) / R_{e q}}\right] \hat{i}_{L_{m}}(s)} \\
=\left[\frac{D\left[n^{2} Z_{o}(s)+R_{e q}\right] /\left(n^{2} Z_{o}(s)\right)-D\left[1+g_{f} R_{e q}\right] Z_{e q}(s) / R_{e q}+(1-D) Z_{e q} / r_{6}}{\left[R_{e q}+n^{2} Z_{o}(s)\right] /\left(n^{2} Z_{o}(s)\right)-\left[1+g_{f} R_{e q}\right] Z_{e q}(s) / R_{e q}}\right] \hat{v}_{g}(s)
\end{gathered}
$$

y haciendo el cociente entre las variables de estado, se obtiene la función de transferencia deseada.

$$
\begin{aligned}
& G_{i g}(s)=\left.\frac{\hat{i}_{m}(s)}{\hat{v}_{g}(s)}\right|_{\hat{d}=0} \\
& =n^{2} Z_{o}(s) \frac{D\left[n^{2} Z_{o}(s)+R_{e q}\right] /\left(n^{2} Z_{o}(s)\right)-D\left[1+g_{f} R_{e q}\right] Z_{e q}(s) / R_{e q}+(1-D) Z_{e q}(s) / r_{6}}{\left[L s+(1-D)^{2} Z_{e q}(s)\right]\left[n^{2} Z_{o}(s)+R_{e q}\right]-n^{2} Z_{o}(s)\left[1+g_{f} R_{e q}\right] L s Z_{e q}(s) / R_{e q}}
\end{aligned}
$$

La mismas expresiones se obtienen si se utiliza el circuito equivalente en pequeña señal del flyback de la figura D.21. Inicialmente se anula la o las fuentes correspondientes a la entrada independiente que no entra en la función de transferencia y se hace Laplace. El circuito resultante para el cálculo de $G_{v g}(s)$ y $G_{i g}(s)$ está representado en la figura D.22 una vez anulado $\hat{d}$.

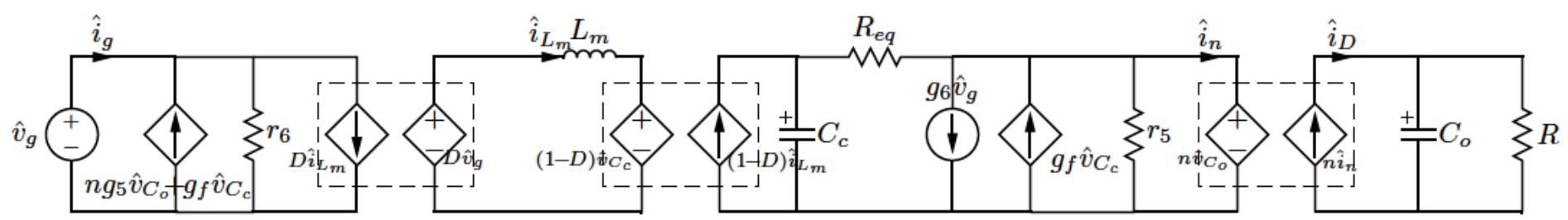

Figura D.22: Circuito equivalente de un flyback ZVS cuando $\hat{d}=0$

Las dos funciones de transferencia coinciden con las de un flyback convencional cuando los términos relacionados con el enclavamiento activo, $C_{c}$ y $L_{r}$, desaparecen puesto que $V_{c}, R_{e q}$ y $Z_{e} q$ se anulan.

En la Figura D.23 están representadas ambas funciones de transferencia con los parámetros de funcionamiento de la Tabla D.1, comparadas con las que tendría un flyback convencional con los mismos parámetros.

Igualmente, para conseguir $G_{v d}(s)$, se hace nula la variación de la entrada independiente que no interviene en la función de transferencia, en este caso, 


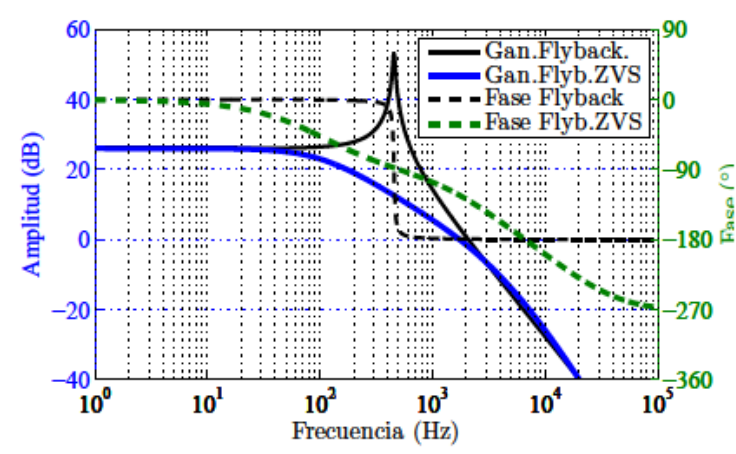

(a)

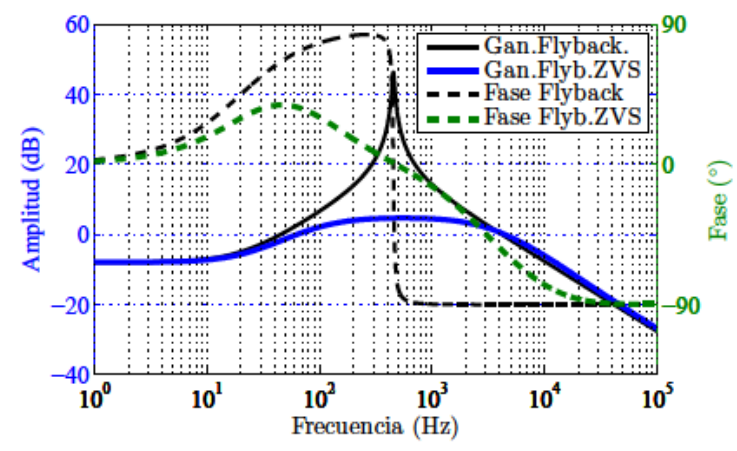

(b)

Figura D.23: Flyback ZVS. Func. de transferencia en función de la tensión de entrada para: a) Tensión de salida, $G_{v g}(s)$; y b) Intensidad en la bobina, $G_{i g}(s)$.

$\hat{v}_{g}(t)=0$, y se aplica Laplace. De esta forma queda que:

$$
\begin{aligned}
& s L \hat{i}_{m}(s)=f_{5} \hat{d}-(1-D) \hat{v}_{C_{c}}(s) \\
& s C_{o} \hat{v}_{C_{o}}(s)=-n j_{6} \hat{d}-\left[\frac{1}{R}+n^{2} \frac{1}{r_{5}}+n^{2} \frac{1}{R_{e q}}\right] \hat{v}_{C_{o}}(s)+n\left[g_{f}+\frac{1}{R_{e q}}\right] \hat{v}_{C_{c}}(s) \\
& s C_{c} \hat{v}_{C_{c}}(s)=I \hat{d}+(1-D) \hat{i}_{m}(s)+n \frac{1}{R_{e q}} \hat{v}_{C_{o}}(s)-\frac{1}{R_{e q}} \hat{v}_{C_{c}}(s) \\
& \hat{i}_{g}(s)=D \hat{i}_{L_{m}}(s)+n \frac{1}{r_{5}} \hat{v}_{C_{o}}(s)-g_{f} \hat{v}_{C_{c}}(s) \\
& \hat{i}_{D}(s)=-n j_{6} \hat{d}-n^{2}\left[\frac{1}{r_{5}}+\frac{1}{R_{e q}}\right] \hat{v}_{C_{o}}(s)+n\left[g_{f}+\frac{1}{R_{e q}}\right] \hat{v}_{C_{c}}(s)
\end{aligned}
$$

Primero se obtiene $\hat{i}_{L_{m}}(s)$ con la primera y la tercera ecuación. Para ello despejamos $\hat{v}_{C_{c}}(s)$ de la tercera ecuación como:

$$
\hat{v}_{C_{c}}(s)=Z_{e q}(s)\left[I \hat{d}+(1-D) \hat{i}_{L_{m}}(s)+n \frac{1}{R_{e q}} \hat{v}_{C_{o}}(s)\right]
$$

Esta expresión se introduce en la primera ecuación para obtener $\hat{i}_{L_{m}}(s)$ :

$$
\hat{i}_{m}(s)=\frac{\left[f_{5}-I(1-D) Z_{e q}(s)\right] \hat{d}-n(1-D) Z_{e q}(s) / R_{e q} \hat{v}_{C_{o}}(s)}{L s+(1-D)^{2} Z_{e q}(s)}
$$

De esta forma, se consigue una expresión de $\hat{v}_{C_{c}}(s)$ en función de las 
variables $\hat{d}$ y $\hat{v}_{C_{o}}(s)$.

$\hat{v}_{C_{c}}(s)=$

$$
\begin{aligned}
& =Z_{e q}(s)\left[I \hat{d}+(1-D) \frac{\left[f_{5}-I(1-D) Z_{e q}(s)\right] \hat{d}-n(1-D) \frac{Z_{e q}(s)}{R_{e q}} \hat{v}_{C_{o}}(s)}{L s+(1-D)^{2} Z_{e q}(s)}+n \frac{\hat{v}_{C_{o}}(s)}{R_{e q}}\right] \\
& =Z_{e q}(s)\left[\frac{\left[I L s+f_{5}(1-D)\right] \hat{d}-n(1-D)^{2} \frac{Z_{e q}(s)}{R_{e q}} \hat{v}_{C_{o}}(s)}{L s+(1-D)^{2} Z_{e q}(s)}+n \frac{\hat{v}_{C_{o}}(s)}{R_{e q}}\right] \\
& =Z_{e q}(s)\left[\frac{\left[I L s+f_{5}(1-D)\right] \hat{d}+n(1-D)^{2} \frac{Z_{e q}(s)}{R_{e q}} \hat{v}_{C_{o}}(s)+n\left[L s+(1-D)^{2} Z_{e q}(s)\right] \frac{\hat{v}_{C_{o}}(s)}{R_{e q}}}{L s+(1-D)^{2} Z_{e q}(s)}\right] \\
& =Z_{e q}(s) \frac{\left[I L s+f_{5}(1-D)\right] \hat{d}+n L s / R_{e q} \hat{v}_{C_{o}}(s)}{L s+(1-D)^{2} Z_{e q}(s)}
\end{aligned}
$$

Utilizando (D.152) y (D.153) en la segunda ecuación de (D.150) se puede conseguir una expresión en la que únicamente aparecen $\hat{v}_{C_{o}}(s)$ y $\hat{d}$, y donde es fácil obtener $G_{v d}(s)$.

$$
\begin{aligned}
& {\left[\frac{1}{Z_{o}(s)}+\frac{n^{2}}{R_{e q}}-n^{2}\left[g_{f}+\frac{1}{R_{e q}}\right] \frac{L s Z_{e q}(s) / R e q}{L s+(1-D)^{2} Z_{e q}(s)}\right] \hat{v}_{C_{o}}(s)} \\
& \quad=\left[-n j 6+n\left[g_{f}+\frac{1}{R_{e q}}\right] \frac{\left[I L s+f_{5}(1-D)\right] Z_{e q}(s)}{L s+(1-D)^{2} Z_{e q}(s)}\right] \hat{d}
\end{aligned}
$$

De la ecuación (D.154) se consigue la función de transferencia deseada:

$$
\begin{aligned}
& G_{v d}(s)=\left.\frac{\hat{v}_{C_{o}}(s)}{\hat{d}(s)}\right|_{\hat{v}_{g}=0} \\
& =Z_{o}(s) \frac{-n j_{6} R_{e q}\left[L s+(1-D)^{2} Z_{e q}(s)\right]+n\left[g_{f} R_{e q}+1\right]\left[f_{5}(1-D)+I L s\right] Z_{e q}(s)}{\left[n^{2} Z_{o}(s)+R_{e q}(s)\right]\left[L s+(1-D)^{2} Z_{e q}(s)\right]-n^{2} Z_{o}(s)\left[g_{f} R_{e q}+1\right] L s Z_{e q}(s) / R_{e q}}
\end{aligned}
$$

Para conseguir $G_{i d}(s)$, se utiliza (D.151) en la segunda ecuación de (D.150) para obtener $\hat{v}_{C_{o}}(s)$ en función de $\hat{i}_{m}(s)$ y $\hat{d}(s)$ :

$$
\hat{v}_{C_{o}}(s)=Z_{o}(s) R_{e q} \frac{-n\left[j_{6}+n I\left[1+g_{f} R_{e q}\right] \frac{Z_{e q}(s)}{R_{e q}}\right] \hat{d}+n\left[1+g_{f} R_{e q}\right](1-D) \frac{Z_{e q}(s)}{R_{e q}} \hat{i}_{L_{m}}(s)}{\left[n^{2} Z_{o}(s)+R_{e q}\right]-n^{2} Z_{o}(s)\left[1+g_{f} R_{e q}\right] \frac{Z_{e q}(s)}{R_{e q}}}
$$


entonces, $\hat{v}_{C_{c}}(s)$ tendrá la siguiente expresión:

$$
\begin{aligned}
\hat{v}_{C_{c}}(s) & =Z_{e q}(s)\left[\begin{array}{c}
-j_{6}+I\left[1+g_{f} R_{e q}\right] \frac{Z_{e q}(s)}{R_{e q}} \\
{\left[n^{2} Z_{o}(s)+R_{e q}\right]-n^{2} Z_{o}(s)\left[1+g_{f} R_{e q}\right] \frac{Z_{e q}(s)}{R_{e q}}}
\end{array}\right] \hat{d} \\
& +Z_{e q}(s)\left[(1-D)+n^{2} Z_{o}(s) \frac{\left[1+g_{f} R_{e q}\right](1-D) \frac{Z_{e q}(s)}{R_{e q}}}{\left[n^{2} Z_{o}(s)+R_{e q}\right]-n^{2} Z_{o}(s)\left[1+g_{f} R_{e q}\right] \frac{Z_{e q}(s)}{R_{e q}}}\right] \hat{i}_{L_{m}}(s) \\
& =Z_{e q}(s) \frac{\left[I\left[n^{2} Z_{o}(s)+R_{e q}\right]-n^{2} j_{6} Z_{o}(s)\right] \hat{d}+(1-D)\left[n^{2} Z_{o}(s)+R_{e q}\right] \hat{i}_{L_{m}}(s)}{\left[n^{2} Z_{o}(s)+R_{e q}\right]-n^{2} Z_{o}(s)\left[1+g_{f} R_{e q}\right] Z_{e q}(s) / R_{e q}}
\end{aligned}
$$

Introduciendo (D.157) en la primera ecuación de (D.150), se obtiene la siguiente igualdad:

$$
\begin{aligned}
& {\left[L s+\frac{(1-D)^{2}\left[n^{2} Z_{o}(s)+R_{e q}\right] Z_{e q}(s)}{\left[n^{2} Z_{o}(s)+R_{e q}\right]-n^{2} Z_{o}(s)\left[1+g_{f} R_{e q}\right] Z_{e q}(s) / R_{e q}}\right] \hat{i}_{L_{m}}(s) } \\
= & {\left[f_{5}-\frac{(1-D)\left[I\left[n^{2} Z_{o}(s)+R_{e q}\right]-n^{2} j_{6} Z_{o}(s)\right] Z_{e q}(s)}{\left[n^{2} Z_{o}(s)+R_{e q}\right]-n^{2} Z_{o}(s)\left[1+g_{f} R_{e q}\right] Z_{e q}(s) / R_{e q}}\right] \hat{d} }
\end{aligned}
$$

A partir de esta última ecuación se obtiene la función de transferencia deseada:

$$
\begin{aligned}
& G_{i d}(s)=\left.\frac{\hat{i}_{L}(s)}{\hat{d}(s)}\right|_{\hat{v}_{g}=0} \\
& =\frac{\left[n^{2} Z_{o}(s)+R_{e q}\right]\left[f_{5}-(1-D) I Z_{e q}(s)\right]-n^{2} j_{6} Z_{o}(s)(1-D) Z_{e q}(s)-n^{2} f_{5} Z_{o}(s)\left[1+g_{f} R_{e q}\right] Z_{e q}(s) / R_{e q}}{\left[n^{2} Z_{o}(s)+R_{e q}\right]\left[L s+(1-D)^{2} Z_{e q}(s)\right]-n^{2} Z_{o}(s)\left[1+g_{f} R_{e q}\right] L s Z_{e q}(s) / R_{e q}}
\end{aligned}
$$

Las mismas expresiones se obtienen si se utiliza el circuito equivalente en pequeña señal del elevador de la figura B.21. El circuito resultante para el cálculo de $G_{v d}(s)$ y $G_{i d}(s)$ está representado en la figura B.26 una vez anulado $\hat{v}_{g}$.

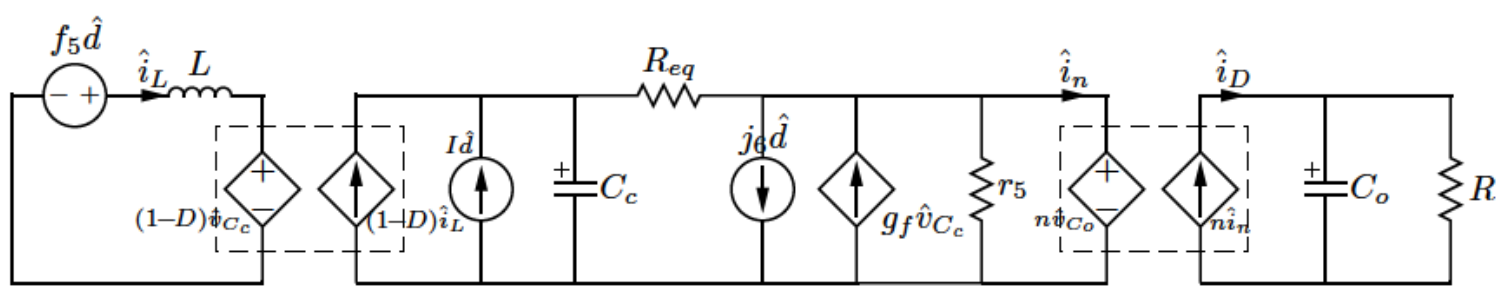

Figura D.24: Circuito equivalente de un flyback ZVS cuando $\hat{v}_{g}=0$ 
Las dos funciones de transferencia coinciden con las de un flyback convencional cuando los términos relacionados con el enclavamiento activo, $C_{c}$ y $L_{r}$, desaparecen puesto que $V_{c}, R_{e q}$ y $Z_{e} q$ se anulan. En la Figura D.25 están representadas ambas funciones de transferencia con los parámetros de funcionamiento de la Tabla D.1, comparadas con las que tendría un flyback convencional con los mismos parámetros.

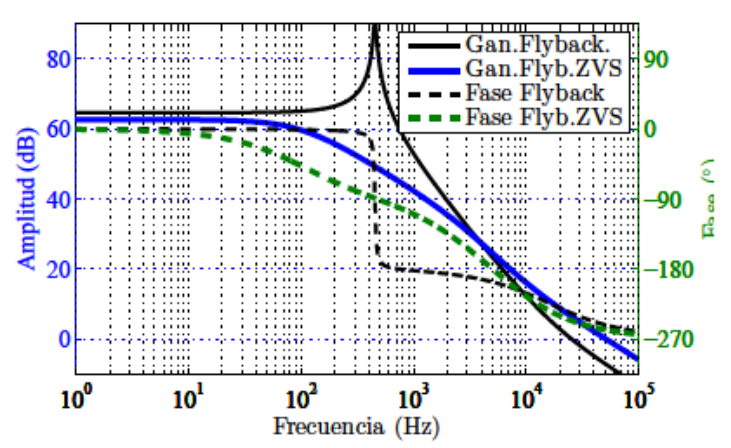

(a)

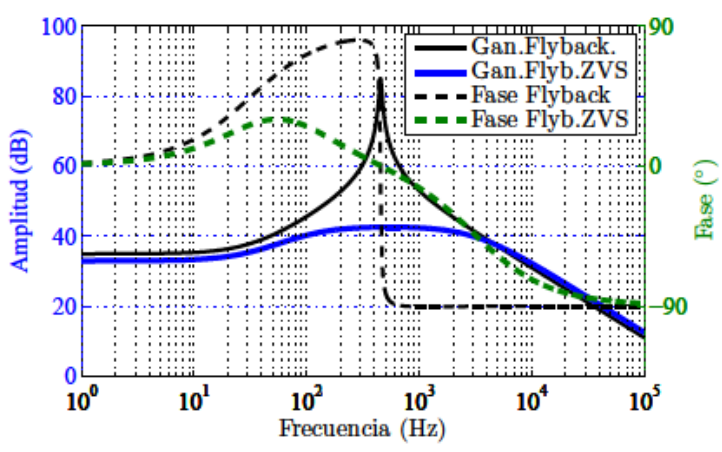

(b)

Figura D.25: Flyback ZVS. Func. de transferencia en función del ciclo de trabajo para: a) Tensión de salida, $G_{v d}(s)$; y b) Intensidad en la bobina, $G_{i g}(s)$.

\section{D.6.2. Desde el modelo matricial}

Para obtener las funciones de transferencia anteriores se aplica Laplace a las ecuaciones de estado en forma matricial (suponiendo que las condiciones iniciales con nulas).

$$
\begin{aligned}
\mathbf{K} s \hat{\mathbf{X}}(s) & =\mathbf{A} \hat{\mathbf{X}}(s)+\mathbf{B} \hat{\mathbf{U}}(s) \\
\hat{\mathbf{Y}}(s) & =\mathbf{C} \hat{\mathbf{X}}(s)+\mathbf{E} \hat{\mathbf{U}}(s)
\end{aligned}
$$

En la primera ecuación de (D.160) se despeja el vector de las variables de estado. Por tanto, se tiene que

$$
\mathbf{K} s \hat{\mathbf{X}}(s)-\mathbf{A} \hat{\mathbf{X}}(s)=\mathbf{B} \hat{\mathbf{U}}(s)
$$

o bien

$$
(\mathbf{K} s-\mathbf{A}) \hat{\mathbf{X}}(s)=\mathbf{B} \hat{\mathbf{U}}(s)
$$

Premultiplicando ambos miembros por $(\mathbf{K} s-\mathbf{A})^{-1}$ se obtiene:

$$
\hat{\mathbf{X}}(s)=(\mathbf{K} s-\mathbf{A})^{-1} \mathbf{B} \hat{\mathbf{U}}(s)
$$


Sustituyendo la ecuacion (D.161) en la segunda ecuación de (D.160) se llega a

$$
\hat{\mathbf{Y}}(s)=\left[\mathbf{C}(\mathbf{K} s-\mathbf{A})^{-1} \mathbf{B}+\mathbf{E}\right] \hat{\mathbf{U}}(s)
$$

Puesto, que la relación entre la entrada y la salida de un sistema es la función de transferencia, resulta que la matriz $\mathbf{G}(s)$ definida en (D.163) es la denominada matriz de transferencia que relaciona las diferentes salidas del sistema con las diferentes entradas. Tiene tantas filas como salidas y tantas columnas como entradas.

$$
\mathbf{G}(s)=\mathbf{C}(\mathbf{K} s-\mathbf{A})^{-1} \mathbf{B}+\mathbf{E}
$$

En el caso del flyback ZVS, se redefine el vector de salidas y las matrices $\mathbf{C}$ y $\mathbf{E}$, de la siguiente forma:

$$
\hat{\mathbf{y}}=\left[\begin{array}{c}
\hat{i}_{L_{m}} \\
\hat{v}_{o}
\end{array}\right], \quad \mathbf{C}=\left[\begin{array}{lll}
1 & 0 & 0 \\
0 & 1 & 0
\end{array}\right], \quad \mathbf{E}=\left[\begin{array}{ll}
0 & 0 \\
0 & 0
\end{array}\right]
$$

De esta forma, la matriz de transferencia, queda como:

$$
\mathbf{G}(s)=\left[\begin{array}{ll}
G_{i g}(s) & G_{i d}(s) \\
G_{v g}(s) & G_{v d}(s)
\end{array}\right]
$$

que es una matriz que recoge las funciones de transferencias obtenidas en el apartado anterior.

\section{D.7. Impedancia de salida}

La impedancia de salida en pequeña señal se obtiene a partir del modelo en pequeña señal de la Figura D.21. Para ello hay que anular las entradas independientes $\hat{v}_{g}$ y $\hat{d}$ y conectar una fuente independiente, $\hat{i}_{t}(s)$, en los puntos donde se quiere medir la impedancia. El resto de elementos no se tienen en cuenta. La tensión que genera esta fuente sobre el sistema, $\hat{v}_{t}$, se emplea para obtener $Z_{\text {out }}(s)$ como:

$$
Z_{\text {out }}(s)=\frac{\hat{v}_{t}(s)}{\hat{i}_{t}(s)} \mid \begin{aligned}
& \hat{v}_{g}=0 \\
& \hat{d}=0
\end{aligned}
$$

considerando que $\hat{i}_{t}(s)$ es la intensidad que entra por el terminal de mayor tensión de los dos terminales considerados anteriormente. En el circuito de la Figura D.26 se indica gráficamente la conexión de la fuente independiente al modelo de pequeña señal. Además, se ha indicado como $Z_{t h}$ a la impedancia formada por el condensador del clamp, la resistencia equivalente y la bobina principal. 


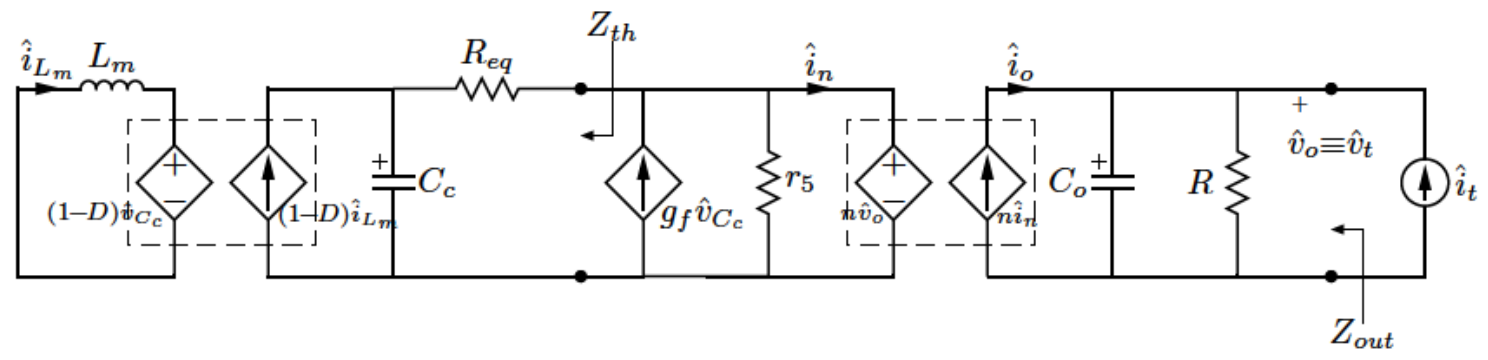

Figura D.26: Circuito para obtener $Z_{\text {out }}(s)$ de un flyback ZVS.

\section{D.7.1. Cálculo de $Z_{t h}$.}

Para obtener esa impedancia se utiliza la parte del circuito anterior que concierne al punto de medida de $Z_{t h}$, en el que se conecta la fuente $\hat{i}_{t}$. Queda el circuito de la Figura D.27. Pasando la bobina $L_{m}$, al otro lado de las fuentes dependientes, aparece con otro valor y en paralelo con $C_{c}$. Este conjunto se encuentra en serie con $R_{e q}$. Por tanto la expresión de esta impedancia es:

$$
Z_{t h}(s)=\frac{1}{\frac{1}{s L_{m}^{\prime}}+s C_{c}}+R_{e q}=R_{e q}+\frac{s L_{m}^{\prime}}{L_{m}^{\prime} C_{c} s^{2}+1}
$$

donde $L_{m}^{\prime}=L_{m} /(1-D)^{2}$.

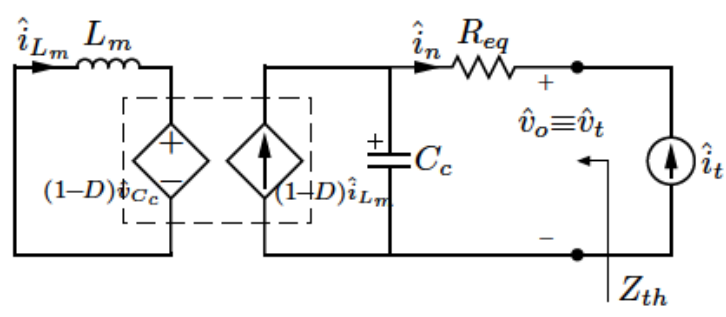

Figura D.27: Circuito empleado para el cálculo de $Z_{t h}(s)$ de un flyback ZVS.

A esta expresión también se puede llegar desde las ecuaciones del modelo en pequeña señal indicado en (D.133). Basta con anular los términos independientes, $\hat{v}_{g}(t)$ y $\hat{d}(t)$ y cambiar la ecuación relativa a la tensión del condensador $C_{o}$, puesto que éste junto con la carga $R$ quedan fuera y en su lugar se ha conectado una fuente de intensidad, $\hat{i}_{t}(t)$. La nueva ecuación relaciona la intensidad en $R_{e q}$ con la intensidad que entrega esa fuente. También hay que sustituir el término $\hat{v}_{C_{o}}(t)$ por $\hat{v}_{t}(t)$ en el resto de ecuaciones. Finalmente, tras aplicar Laplace, las ecuaciones resultantes quedan indicadas 
en (D.168):

$$
\begin{aligned}
s L \hat{i}_{L_{m}}(s) & =-(1-D) \hat{v}_{C_{c}}(s) \\
-R_{e q} \hat{i}_{t}(s) & =\hat{v}_{C_{c}}(s)-\hat{v}_{t}(s) \\
s C_{c} \hat{v}_{C_{c}}(s) & =(1-D) \hat{i}_{L_{m}}(s)+\hat{i}_{t}(s)
\end{aligned}
$$

Despejando $\hat{i}_{L_{m}}$ de la primera ecuación y sustituyéndola en la tercera ecuación se despeja $\hat{v}_{C_{c}}(s)$ para poder emplearla en la segunda ecuación.

$$
\left.\begin{array}{l}
\hat{i}_{L_{m}}(s)=-\frac{(1-D)}{s L_{m}} \hat{v}_{C_{c}}(s) \\
\hat{v}_{C_{c}}(s)=\frac{\hat{i}_{t}(s)}{\frac{1}{s L_{m}^{\prime}}+s C}
\end{array}\right\} \Rightarrow-R_{e q} \hat{i}_{t}(s)=\frac{\hat{i}_{t}(s)}{\frac{1}{s L_{m}^{\prime}}+s C}-\hat{v}_{t}(s)
$$

Ahora se puede conseguir una expresión de la impedancia de salida que coincide con el mostrado en (D.167).

$$
\hat{v}_{t}(s)=\hat{i}_{t}(s)\left[R_{e q}+\frac{s L_{m}^{\prime}}{L_{m}^{\prime} C_{c} s^{2}+1}\right] \Rightarrow Z_{t h}(s)=R_{e q}+\frac{s L_{m}^{\prime}}{L_{m}^{\prime} C_{c} s^{2}+1}
$$

En la Figura D.28 se ha representado esta impedancia para un flyback ZVS con los parámetros de la Tabla D.1. También están representados los módulos de las impedancias que forman parte de $Z_{t h}(s)$, esto es, la bobina $L_{m}^{\prime}$, el condensador $C_{c}$ y el término de resistencia equivalente $R_{e q}$.

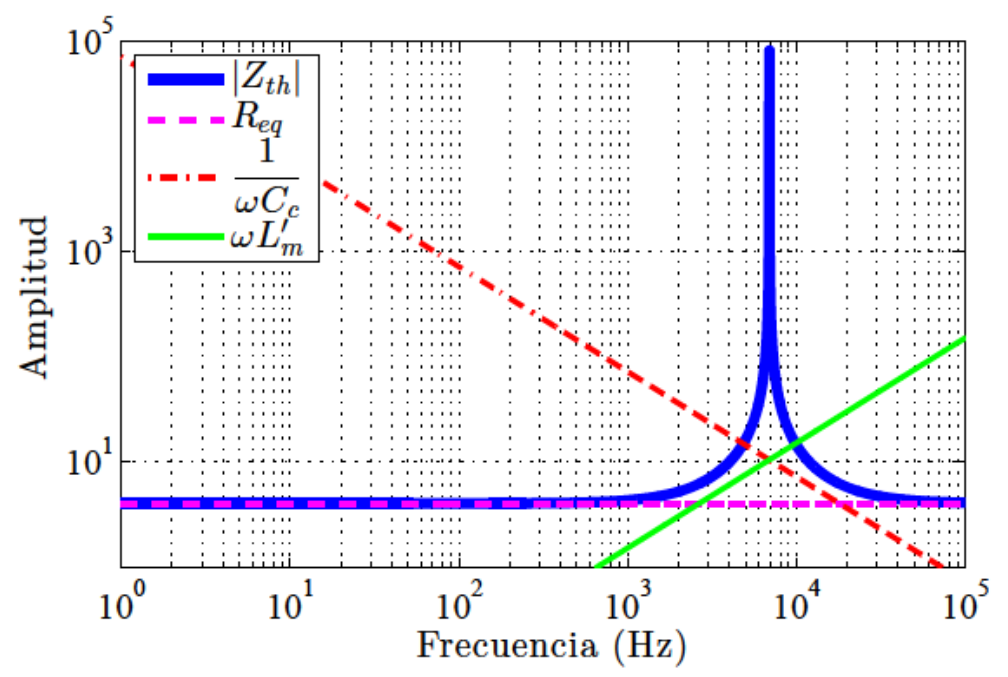

Figura D.28: Representación gráfica de $\left|Z_{t h}(s)\right|$ y los elementos que la forman. 
Al hacer $s=0$ se obtiene la impedancia en continua que es igual al término $R_{e q}$ :

$$
Z_{t h}(0)=Z_{e q}(0)=R_{e q}
$$

Este mismo resultado se obtiene analizando el circuito de la Figura D.27 cuando la bobina y el condensador se convierten en un cortocircuito y en un circuito abierto respectivamente. La fuente independiente de intensidad ahora es una fuente constante conectada en los terminales donde se va a medir esa resistencia. El circuito modificado está representado en la Figura D.29:

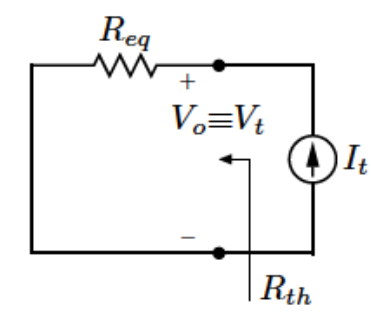

Figura D.29: Circuito empleado para el cálculo de $R_{t h}$ de un flyback ZVS.

Se obtiene una resistencia de salida igual a:

$$
R_{t h}(s)=\frac{R_{e q}}{n^{2}}
$$

\section{D.7.2. Cálculo de $Z_{\text {out }}$.}

A la vista del circuito de la Figura D.26, se establece la siguiente relación de tensiones:

$$
-(1-D) \hat{v}_{C_{c}}(s)=s L_{m} \hat{i}_{L_{m}}(s)
$$

Por otro lado, se establece la relación de corrientes:

$$
(1-D) \hat{i}_{L}(s)=s C_{c} \hat{v}_{C_{c}}(s)+\frac{\hat{v}_{C_{c}}(s)-n \hat{v}_{t}(s)}{R_{e q}}
$$

Pasando la bobina $L_{m}$ al otro lado de las fuentes dependientes, aparece una bobina equivalente $L_{m}^{\prime}$ que está en paralelo con $C_{e q}$, como está indicado en el circuito de la Figura D.30a.

Despejando $\hat{v}_{C_{c}}$ de (D.174), y sustituyendo la expresión de $\hat{i}_{L_{m}}$ que se deduce de (D.173) se obtiene una expresión en función de $\hat{v}_{t}$ como:

$$
\begin{aligned}
& \hat{i}_{L_{m}}(s)=-\frac{(1-D) \hat{v}_{C_{c}}(s)}{s L_{m}} \\
& \hat{v}_{C_{c}}(s)=\frac{n \hat{v}_{t}(s)}{R_{e q}} \underbrace{\left[s C_{c}+\frac{1}{R_{e q}}+\frac{1}{s L_{m}^{\prime}}\right]^{-1}}_{Z_{1}(s)}
\end{aligned}
$$




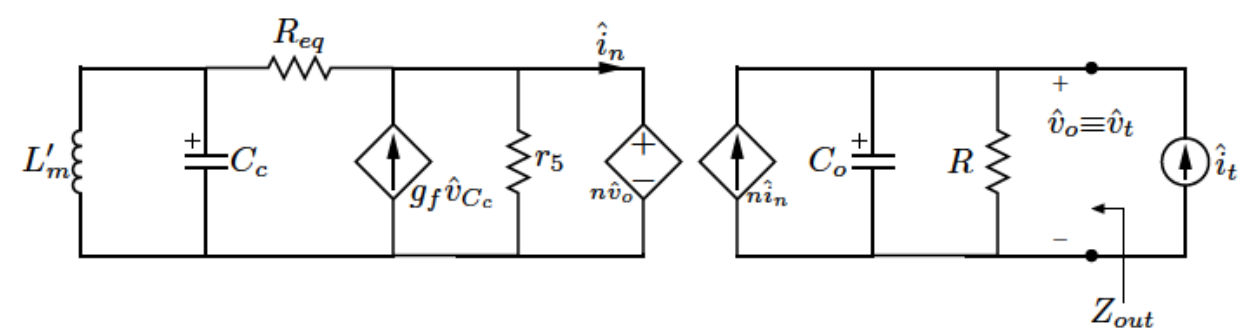

(a)

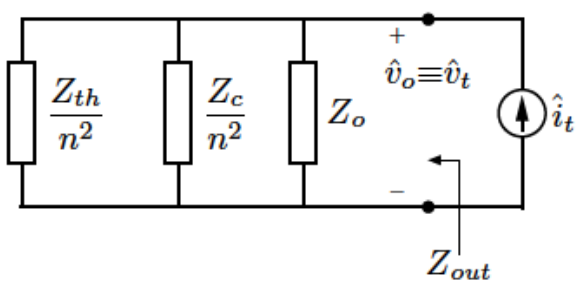

(b)

Figura D.30: Circuito de la Figura D.26 a) tras cambiar $L_{m}$ de posición; b) y agrupando elementos.

donde se han agrupado diferentes términos en $Z_{1}(s)$. Dada la posición de la fuente de corriente $g_{f} \hat{v}_{C_{c}}$, sobre la que aparece la tensión $n \hat{v}_{t}$, se puede interpretar como una impedancia, $Z_{c}$, de expresión:

$$
Z_{c}(s)=-\frac{R_{e q}}{g_{f} Z_{1}(s)}
$$

Agrupando, elementos, se puede observar que $Z_{\text {out }}$ es el paralelo de tres impedancias: la impedancia $Z_{t h}$ con un factor de $n^{2}$ cuando se pasa al otro lado de las bobinas acopladas, y que engloba los elementos $R_{e q}, C_{c}$ y $L_{m}^{\prime}$; la impedancia $Z_{c}$ también pasada al otro lado de las bobinas acopladas; y la impedancia $Z_{o}$, que es el paralelo de $C_{o}, R$ y $r_{5}$ previamente ha pasado al otro lado. Así se ha representado en la Figura D.30b.

$$
Z_{\text {out }}(s)=Z_{o}(s)\left\|\frac{Z_{c}(s)}{n^{2}}\right\| \frac{Z_{t h}(s)}{n^{2}}
$$

A esta expresión también se puede llegar desde las ecuaciones indicadas en (D.178), obtenidas a partir del modelo en pequeña señal indicado en (D.133) donde se anulan los términos independientes, $\hat{v}_{g}(t)$ y $\hat{d}(t)$. Después, hay que cambiar la ecuación relativa a la tensión del condensador $C_{o}$,para introducir la intensidad, $\hat{i}_{t}(t)$. Tras cambiar el término $\hat{v}_{C_{o}}(t)$ por 
$\hat{v}_{t}(t)$ se aplica Laplace a las ecuaciones modificadas, y quedan:

$$
\begin{aligned}
s L_{m} \hat{i}_{L_{m}}(s) & =-(1-D) \hat{v}_{C_{c}}(s) \\
\hat{i}_{t}(s) & =\hat{v}_{t}(s) \overbrace{\left[s C_{o}+\frac{1}{R}+\frac{n^{2}}{r_{5}}\right]}^{Z_{o}^{-1}(s)}-n g_{f} \hat{v}_{C_{c}}(s)-n \frac{\hat{v}_{C_{c}}(s)-n \hat{v}_{t}(s)}{R_{e q}} \\
s C_{c} \hat{v}_{C_{c}}(s) & =(1-D) \hat{i}_{L_{m}}(s)-\frac{\hat{v}_{C_{c}}(s)-n \hat{v}_{t}(s)}{R_{e q}}
\end{aligned}
$$

De la primera y tercera ecuación se obtiene una expresión para $\hat{v}_{C_{c}}(s)$ igual a la indicada en (D.175), que sustituida en la segunda ecuación permite obtener $\hat{i}_{t}(s)$ en función de $\hat{v}_{t}(s)$ como:

$$
\hat{i}_{t}(s)=\frac{\hat{v}_{t}(s)}{Z_{o}(s)}-\hat{v}_{t}(s) \frac{n^{2} g_{f} Z_{1}(s)}{R_{e q}}-n^{2} \hat{v}_{t}(s) \frac{\frac{Z_{1}(s)}{R_{e q}}-1}{R_{e q}}
$$

La impedancia de salida tiene la expresión:

$$
Z_{\text {out }}(s)=\left[\frac{1}{Z_{o}(s)}-n^{2} g_{f} \frac{Z_{1}(s)}{R_{e q}}+n^{2} \frac{R_{e q}-Z_{1}(s)}{R_{e q}^{2}}\right]^{-1}
$$

que es equivalente a la obtenida anteriormente en (D.177) puesto que:

$$
\begin{aligned}
& Z_{1}(s)=\left[\frac{1}{R_{e q}}+\frac{1}{s L_{m}^{\prime}}+s C_{c}\right]^{-1}=\frac{R_{e q} s L_{m}^{\prime}}{R_{e q}+s L_{m}^{\prime}+R_{e q} L_{m}^{\prime} C_{c} s^{2}} \\
& R_{e q}-Z_{1}(s)=\frac{R_{e q}^{2}+\underline{R}_{e q} s E_{m}^{\prime}+R_{e q}^{2} L_{m}^{\prime} C_{c} s^{2}-\underline{R}_{e q} s E_{m}^{\prime}}{R_{e q}+s L_{m}^{\prime}+R_{e q} L^{\prime} C_{c} s^{2}}=\frac{R_{e}^{2} q}{Z_{t h}}
\end{aligned}
$$

Entonces, la impedancia de salida indicada en (D.177) y en (D.180) queda finalmente como:

$$
Z_{\text {out }}(s)=\left[\frac{1}{Z_{o}(s)}-\frac{n^{2}}{Z_{c}}+\frac{n^{2}}{Z_{t h}(s)}\right]^{-1}
$$

En la Figura D.31 se ha representado el módulo de esta impedancia para un flyback ZVS, a partir de los parámetros indicados en la Tabla D.1 junto con el módulo de los términos que la forman, $\left|Z_{t h} / n^{2}\right|,\left|Z_{c}(s) / n^{2}\right|$ y $\left|Z_{o}(s)\right|$.

Al hacer $s=0$ se obtiene la impedancia de salida en continua, que es el paralelo de los términos $R_{e q} / n^{2}, r_{5} / n^{2}$ y $R$. Y se corresponde con la asíntota horizontal de Zout(s) a bajas frecuencias.

$$
Z_{\text {out }}(0)=\frac{1}{\frac{1}{Z_{o}(0)}-\frac{n^{2}}{Z_{c}(0)}+\frac{n^{2}}{Z_{t h}(0)}}=\left[\frac{n^{2}}{r_{5}}+\frac{1}{R}+\frac{n^{2}}{R_{e q}}\right]^{-1}
$$




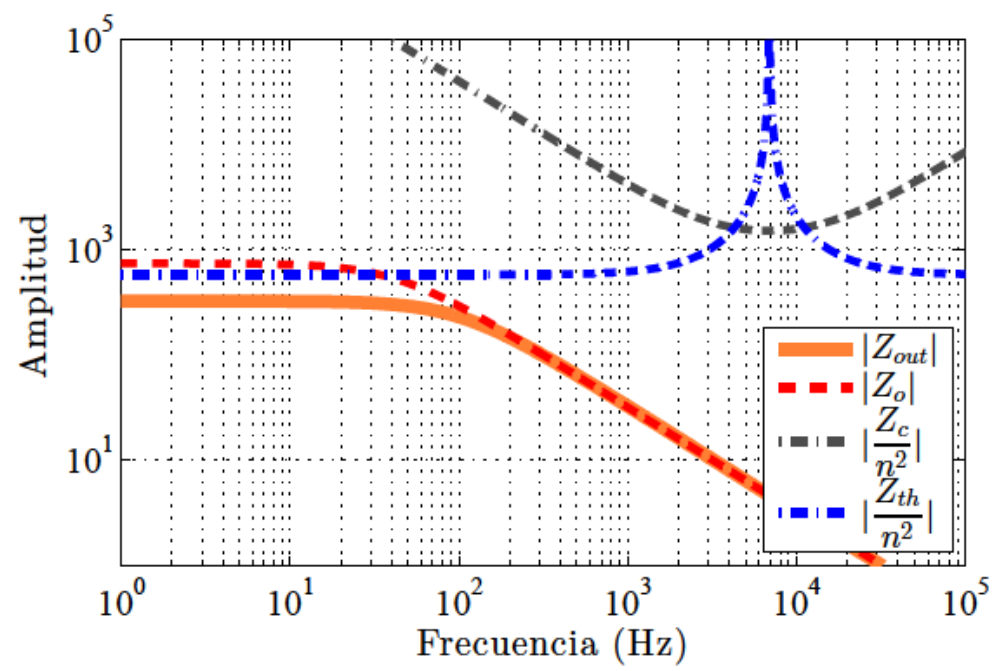

Figura D.31: Representación gráfica de $\left|Z_{\text {out }}(s)\right|$ y los elementos que la forman.

\section{D.7.3. Validación de las expresiones obtenidas.}

La validación de la expresión de la impedancia, se comprueban con la simulación de un convertidor conmutado al que se le mide la impedancia de salida. Con los parámetros de funcionamiento recogidos en la Tabla D.1 se añade a la salida una fuente de corriente de frecuencia variable y se mide la variación que experimenta la tensión de salida.

Como las expresiones de impedancia se han obtenido a partir del modelo promediado, parece lógico medir la impedancia sobre la simulación de este modelo y, comparar los resultados obtenidos con la teoría. En este caso, se emplearía el ciclo de trabajo obtenido para el modelo promediado. Si se emplea el convertidor conmutado para medir la impedancia, debería considerarse el valor del ciclo de trabajo obtenido para el circuito conmutado con la corrección propuesta en el apartado correspondiente.

En la Figura D.32 se ha representado los valores obtenidos en la medida de la impedancia de un flyback ZVS simulado con los parámetros indicados en la Tabla D.1. También se ha representado el módulo de la impedancia teórica, $\left|Z_{\text {out }}(s)\right|$.

Como ya se conocía, presenta una asíntota horizontal, que es el paralelo de $R, R_{e q} / n^{2}, r_{5} / n^{2}$. A la vista de estas curvas, se observa la coincidencia de la impedancia teórica y los valores obtenidos en la simulación, por lo que se concluye la validez de las expresiones de impedancia obtenidas de forma teórica para el modelo promediado y el circuito conmutado. 


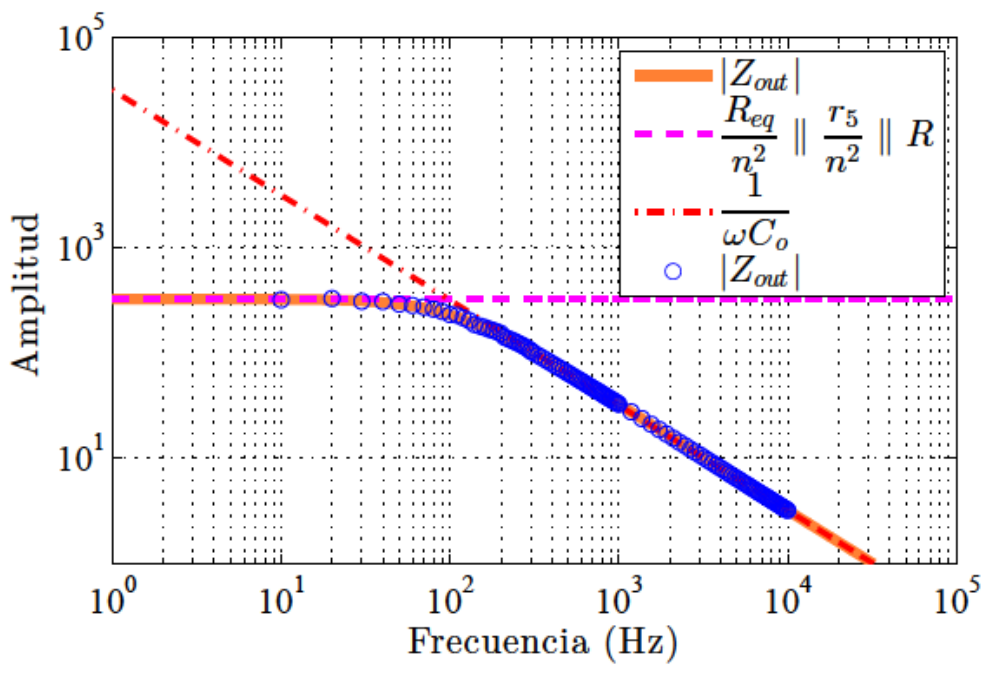

Figura D.32: Representación gráfica de $\left|Z_{\text {tot }}(s)\right|,\left|Z_{\text {out }}(s)\right|$ y resultados de simulación.

En el caso del reparto de corrientes entre varias etapas en paralelo para alimentar una carga común, la impedancia de salida de cada una de las etapas se debe determinar desde el punto común de conexión. Existiendo la posibilidad de considerar un único condensador de salida común a todas las etapas (aunque también podría tratarse el caso de un condensador en cada etapa). El análisis sería similar al realizado en el apartado anterior, con la precaución de no incluir ni $R$ ni $C_{o}$ dentro de $Z_{o}$.

Por ejemplo, la impedancia multietapa sin el condensador de salida, $Z_{m e}(s)$ se corresponde con la expresión:

$$
Z_{m e}(s)=\frac{1}{n^{2}}\left[\frac{1}{r_{5}}-\frac{g_{f} Z_{1}(s)}{R_{e q}}+\frac{1}{Z_{t h}(s)}\right]^{-1}
$$

En la Figura D.33 se ha representado el módulo de esta impedancia y las asíntotas correspondientes:

Haciendo $s=0$ se consigue la asíntota de baja frecuencia, que es el paralelo de $R_{e q}$ y de $r_{3}$ afectados del factor $n^{2}$. Una expresión equivalente se consigue al emplear sus expresiones indicadas en la tabla D.4, y ayudándose de la primera y tercera ecuación del modelo en régimen permanente indicado 


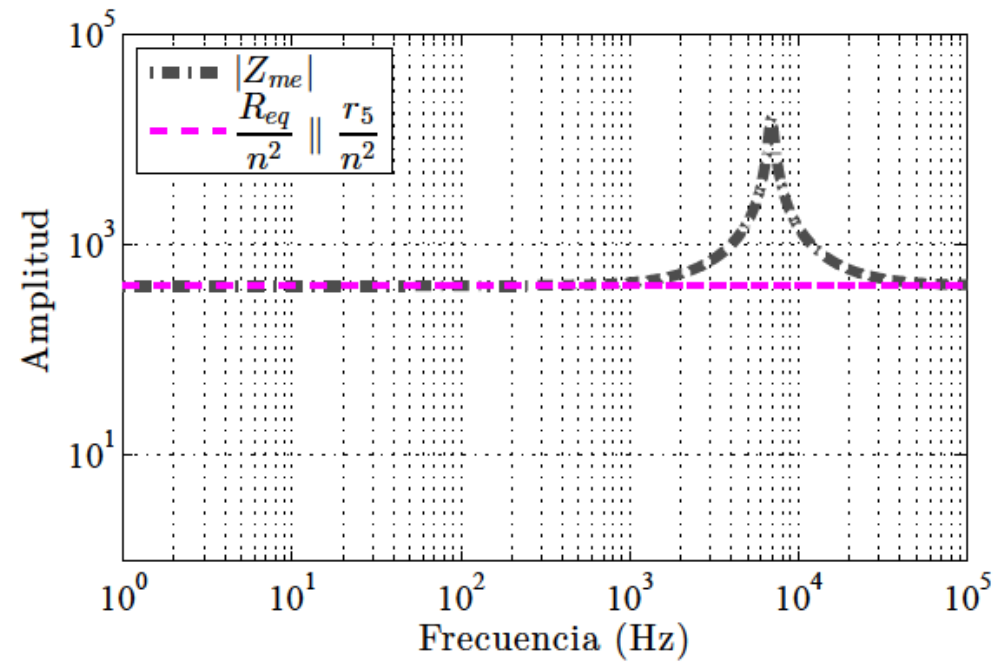

Figura D.33: Representación gráfica de $\left|Z_{m e}(s)\right|$.

en (D.108). Entonces:

$$
\begin{aligned}
Z_{m e}(0) & =\frac{1}{n^{2}}\left[\frac{2(1-D) I+I_{e q}}{V_{g}+n V_{o}}+\frac{1}{R_{e q}}\right]^{-1}=\frac{1}{n^{2}}\left[\frac{\frac{2\left(V_{c}-n V_{o}\right)}{R_{e q}}+\frac{\left(V_{c}-n V_{o}\right)^{2}}{\left(V_{g}+n V_{o}\right) R_{e q}}}{V_{g}-n V_{o}}+\frac{1}{R_{e q}}\right]^{-1} \\
& =\frac{1}{n^{2}}\left[\frac{1}{R_{e q}}+\frac{2\left(V_{c}-n V_{o}\right)}{\left(V_{g}+n V_{o}\right) R_{e q}}+\frac{\left(V_{c}-n V_{o}\right)^{2}}{\left(V_{g}+n V_{o}\right)^{2} R_{e q}}\right]^{-1}=\frac{R_{e q}}{n^{2}}\left[1+\frac{V_{c}-n V_{o}}{V_{g}+n V_{o}}\right]^{-2} \\
& =\frac{R_{e q}}{n^{2}}\left[\frac{V_{g}+n V_{o}}{V_{g}+V_{c}}\right]^{2}=\frac{2 L r f}{n^{2}}\left[\frac{V_{g}+n V_{o}}{V_{g}}\right]^{2}
\end{aligned}
$$

Cuando $s$ se hace muy grande, $Z_{1}(s)$ tiende a cero, mientras que $Z_{t h}(s)$ y $Z_{o}$ se transforman en $R_{e q}$ y $r_{5} / n^{2}$ respectivamente, de forma que la asíntota para altas frecuencias coincide con la asíntota de bajas frecuencias. 



\section{Apéndice E}

\section{Flyback ZVS tipo boost}

\section{E.1. Descripción y funcionamiento.}

En la Figura E.1 ha representado un convertidor flyback con enclavamiento activo tipo boost (de ahora en adelante flyback ZVS-boost, para distinguirlo del convertidor flyback ZVS con enclavamiento activo tipo buck-boost del Anexo D). La diferencia entre ambos circuitos radica en la disposición de la red de enclavamiento activo empleada para proteger al transistor principal, $S_{1}$. Está formada por un condensador, $C_{c}$, y un elemento activo, $S_{2}$.

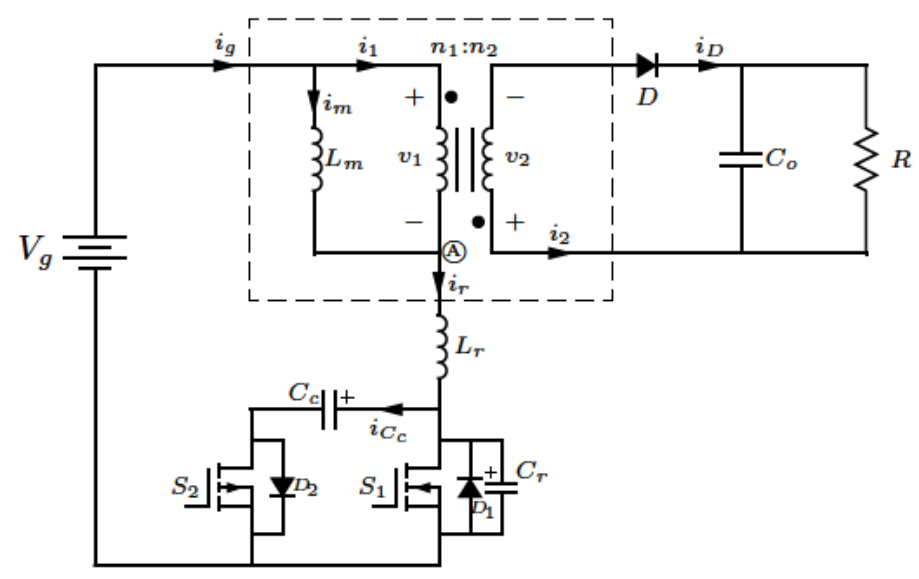

Figura E.1: Convertidor flyback con enclavamiento activo-boost.

Sobre este circuito se puede comprobar que la corriente del diodo de salida, $i_{D}(t)$, que tiene sentido opuesto a la corriente de secundario, $i_{2}(t)$, está relacionada con la corriente de primario, $i_{1}(t)$ de la siguiente forma:

$$
n_{1} i_{1}(t)=n_{2} i_{2}(t)=-n_{2} i_{D}(t)
$$

En este caso se ha considerado el sentido positivo de $i_{1}(t)$ entrando por el punto de primario y de $i_{2}(t)$ saliente por el punto de secundario. 
Cuando se aplica la ley de nodos de Kirchhoff en (A, se comprueba que:

$$
i_{m}(t)+i_{1}(t)=i_{r}(t)
$$

siendo $i_{m}(t)$ la corriente que circula por la bobina de magnetización $L_{m}$, e $i_{r}(t)$ la corriente circulante por la bobina resonante $L_{r}$. Combinando las expresiones (E.1) y (E.2) se consigue (E.3), donde $n=n_{1} / n_{2}$.

$$
i_{m}(t)=i_{r}(t)+\frac{1}{n} i_{D}(t)
$$

Por otro lado la corriente de entrada, $i_{g}(t)$, es igual a la corriente $i_{r}(t)$, y puede relacionarse con la corriente de salida $i_{D}$ de la siguiente forma:

$$
i_{g}(t)+\frac{1}{n} i_{D}(t)=i_{m}(t)
$$

\section{E.1.1. Etapas.}

El convertidor pasa por seis etapas a lo largo de un periodo de conmutación, $T$. Estas seis etapas y los elementos que intervienen en cada una de ellas están representadas en la Figura E.2. Se considera $L_{m}$ lo suficientemente grande como para considerar una corriente constante de valor $I$. También se considera $L_{r}$ pequeña en comparación con $L_{m}$. El valor de la tensión en los condensadores $C_{o}$ y $C_{c}$ se mantiene constante, de valores $V_{o}$ y $V_{c}$, durante todo el periodo $T$.

Etapa $1\left[t_{0}-t_{1}\right]$ : Antes de $t_{0}$, el interruptor principal $S_{1}$ está conduciendo, y el interruptor auxiliar $S_{2}$ está abierto. La corriente que circula es constante y de valor $I$, debida a $L_{m}$. En $t=t_{0}, S_{1}$ pasa a OFF y la corriente que circula por $L_{r}$, es decir, $i_{r}$, es constante, y carga el condensador $C_{r}$ hasta llegar a la tensión $V_{g}+n V_{o}$, que es cuando acaba esta etapa.

Etapa $2\left[t_{1}-t_{2}\right]$ : Cuando $v_{C_{r}}=V_{g}+n V_{o}$ en $t_{1}$, el diodo de salida $D$ empieza a conducir al quedar polarizado en directa. Ahora, el conjunto $L_{r}-C_{r}$ es resonante y está sometido a una tensión de valor $V_{g}+n V_{o}$. El condensador $C_{r}$ se sigue cargando hasta alcanzar la tensión $v_{C_{r}}=V_{c}$. La corriente alcanza un valor igual a $I^{\prime}$, que es ligeramente distinto a $I$.

Etapa $3\left[t_{2}-t_{3}\right]$ : Cuando $v_{C_{r}}$ alcanza la tensión $V_{c}$, el diodo $D_{2}$ empieza a conducir porque queda polarizado en directa. Desde este momento, la tensión en la bobina resonante es igual a $V_{g}+n V_{o}-V_{c}$. La corriente en $L_{r}$ evoluciona de forma lineal, al igual que, $i_{D}$, la corriente en $D$. En algún momento de este intervalo, y antes de que $i_{r}$ cambie su sentido, se puede conmutar $S_{2} \sin$ pérdidas porque no tiene tensión en su extremos al estar conduciendo $D_{2}$. Esta etapa acaba cuando $S_{2}$ pase a OFF. La corriente resonante alcanza un valor igual a $-I^{\prime}$.

Etapa $4\left[t_{3}-t_{4}\right]$ : Ahora la corriente de la bobina resonante se emplea en descargar la energía almacenada en $C_{r}$ hasta hacer su tensión nula y alcanza un valor igual a $I^{\prime \prime}$. En $t_{5}$ se hace $v_{C_{r}}=0$. 


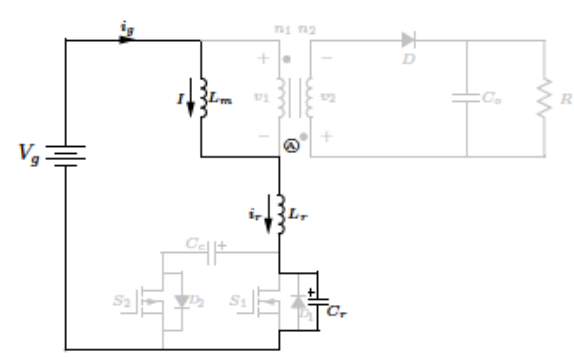

(a)

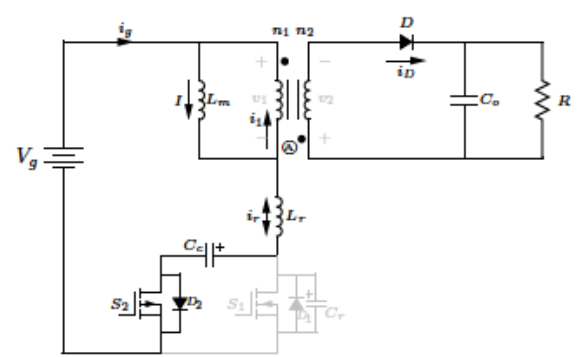

(c)

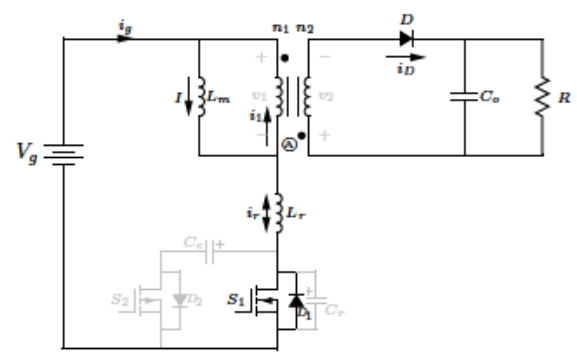

(e)

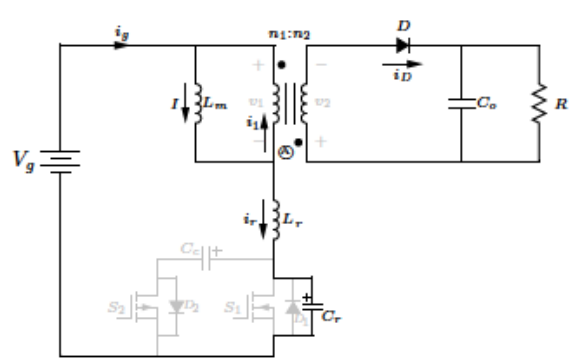

(b)

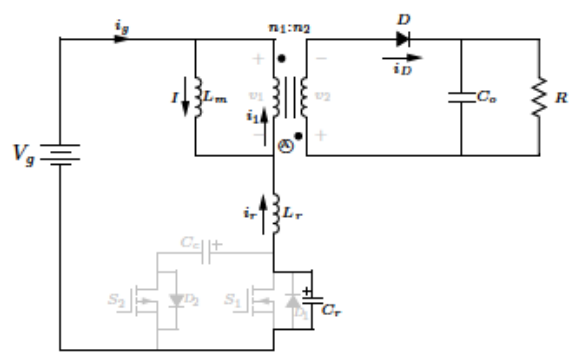

(d)

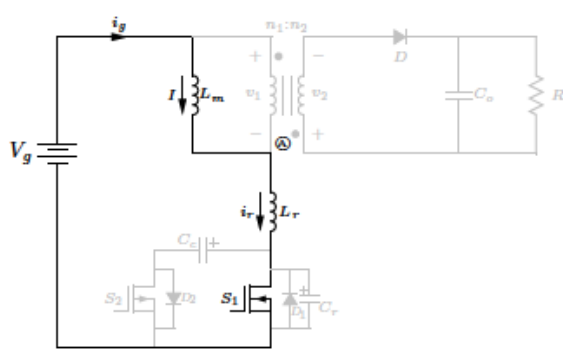

(f)

Figura E.2: Etapas por las que evoluciona el convertidor en un periodo: (a) Etapa 1; (b) Etapa 2; (c) Etapa 3; (d) Etapa 4; (e) Etapa 5; y (f) Etapa 6.

Etapa $5\left[t_{4}-t_{5}\right]$ : En el instante que la tensión de $C_{r}$ es nula, empieza a circular corriente por el diodo $D_{1}$, y es posible la conmutación de $S_{1}$ con tensión nula. La conmutación debería realizarse antes de que $i_{r}$ cambie de nuevo su sentido de circulación al inicial. Su evolución es lineal porque $L_{r}$ está sometida a una tensión $V_{g}+n V_{o}$. También $i_{D}$ evoluciona de forma lineal. Al final de esta etapa, ambas corrientes alcanzan los valores de $I$ y 0 respectivamente. En este momento, $D$ deja de conducir.

Etapa $6\left[t_{5}-t_{6}\right]$ : Esta etapa comienza cuando el diodo $D$ ha dejado de conducir, manteniéndose constante la intensidad circulante por $L_{r}$. Esta etapa termina cuando $S_{1}$ pasa a OFF y volvería a la primera etapa.

Los circuitos equivalentes ${ }^{1}$ que facilitan el análisis del circuito con estas

\footnotetext{
${ }^{1}$ Son los mismos circuitos que en el caso del clamp tipo buck-boost, excepto en la etapa
} 
consideraciones están representados en la Figura E.3.

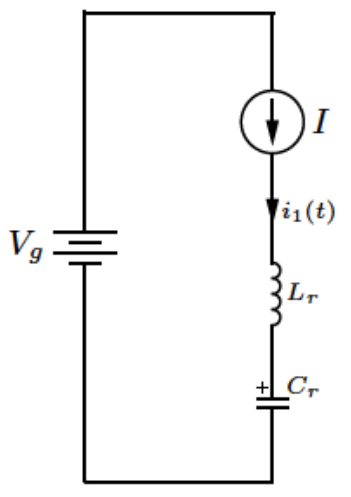

(a)

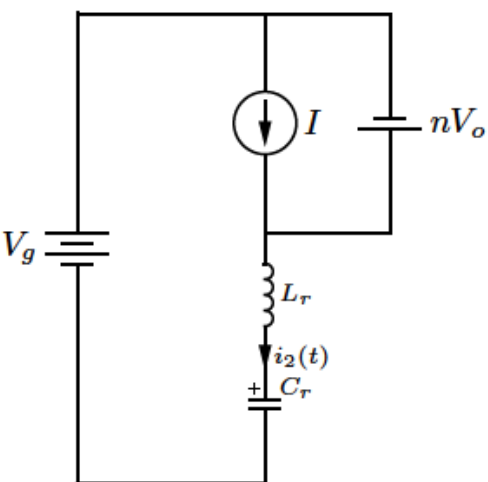

(b)

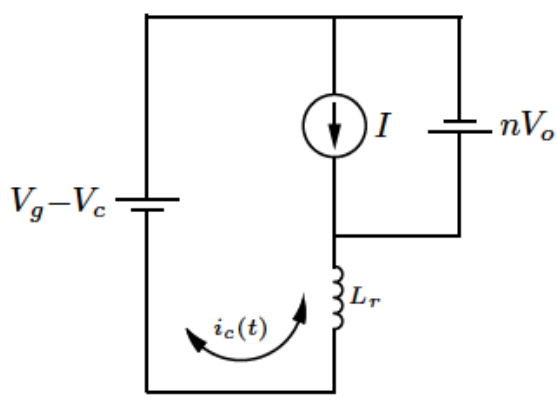

(c)

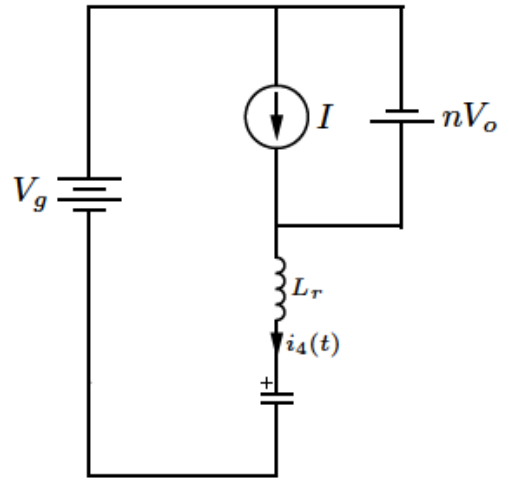

(d)

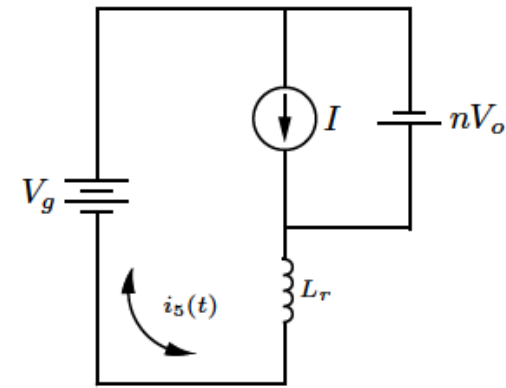

(e)

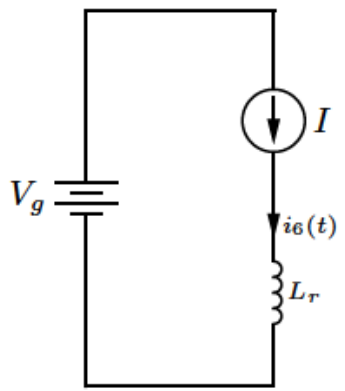

(f)

Figura E.3: Circuitos equivalentes a los de la Figura E.2 empleados para el análisis: (a) Etapa 1; (b) Etapa 2; (c) Etapa 3; (d) Etapa 4; (e) Etapa 5; y (f) Etapa 6. (La corriente $i_{x}$ indica a $i_{r}$ durante la etapa $x$ )

Las expresiones de la tensión en $C_{r}$ y la intensidad en $L_{r}$ son:

$$
v_{C_{r}}(t)=\left\{\begin{array}{lc}
\frac{I}{C_{r}}\left(t-t_{0}\right) & t \in\left[t_{0}, t_{1}\right] \\
V_{g}+n V_{o}+I Z_{r} \sin \left[\omega_{r}\left(t-t_{1}\right)\right] & t \in\left[t_{1}, t_{2}\right] \\
V_{c} & t \in\left[t_{2}, t_{3}\right] \\
V_{g}+n V_{o}+I_{r 3} Z_{r} \sin \left[\omega_{r}\left(t-t_{3}\right)\right] & t \in\left[t_{3}, t_{4}\right] \\
\quad-\left(V_{g}+n V_{o}-V_{c}\right) \cos \left[\omega_{r}\left(t-t_{3}\right)\right] & \text { resto }
\end{array}\right.
$$

3, puesto que el condensador tiene otra disposición diferente. 


$$
i_{r}(t)= \begin{cases}I & t \in\left[t_{0}, t_{1}\right] \\ I \cos \left[\omega_{r}\left(t-t_{1}\right)\right] & t \in\left[t_{1}, t_{2}\right] \\ I_{r 2}+\frac{\left(V_{g}+n V_{o}-V_{c}\right)}{L_{r}}\left(t-t_{2}\right) & t \in\left[t_{2}, t_{3}\right] \\ I_{r 3} \cos \left[\omega_{r}\left(t-t_{3}\right)\right]+\frac{\left(V_{g}+n V_{o}-V_{c}\right)}{Z_{r}} \sin \left[\omega_{r}\left(t-t_{3}\right)\right] & t \in\left[t_{3}, t_{4}\right] \\ I_{r 4}+\frac{\left(V_{g}+n V_{o}\right)}{L_{r}}\left(t-t_{4}\right) & t \in\left[t_{4}, t_{5}\right] \\ I & t \in\left[t_{5}, t_{6}\right]\end{cases}
$$

donde se han considerado los siguientes términos:

$$
\begin{aligned}
& Z_{r}=\sqrt{L_{r} / C_{r}} \\
& \omega_{r}=1 / \sqrt{L_{r} C_{r}} \\
& I_{r 2} \equiv i_{r}\left(t_{2}\right)=I \cos \omega_{r} d_{2} T \equiv I^{\prime} \\
& I_{r 3} \equiv i_{r}\left(t_{3}\right)=I_{r 2}+\frac{\left(V_{g}+n V_{o}-V_{c}\right)}{L_{r}} d_{3} T \equiv-I^{\prime} \\
& I_{r 4} \equiv i_{r}\left(t_{4}\right)=I_{r 3} \cos \omega_{r} d_{4} T+\frac{\left(V_{g}+n V_{o}-V_{c}\right)}{Z_{r}} \sin \omega_{r} d_{4} T \equiv I^{\prime \prime}
\end{aligned}
$$

La intensidad que circula por el condensador de $\operatorname{clamp} C_{c}$, es la misma que por $L_{r}$ durante la etapa 3, entonces:

$$
i_{C_{c}}(t)= \begin{cases}I_{r 2}+\frac{\left(V_{g}+n V_{o}-V_{c}\right)}{L_{r}}\left(t-t_{2}\right) & t \in\left[t_{2}, t_{3}\right] \\ 0 & \text { resto }\end{cases}
$$

La tensión en la bobina de magnetización está relacionada con la tensión de salida en las etapas 2, 3 y 4 . Durante la etapa 5 y 6 es prácticamente toda la tensión de entrada, $V_{g}$, porque es mucho mas grande que la bobina resonante.

$$
v_{L_{m}}(t)= \begin{cases}V_{g}-\frac{I}{C_{r}}\left(t-t_{0}\right) & t \in\left[t_{0}, t_{1}\right] \\ -n V_{o} & t \in\left[t_{1}, t_{5}\right] \\ \approx V_{g} & t \in\left[t_{5}, t_{6}\right]\end{cases}
$$

La tensión en la bobina resonante se puede escribir en relación a la tensión $v_{C_{r}}(t)$ como:

$$
v_{L_{r}}(t)= \begin{cases}\approx 0 & t \in\left[t_{0}, t_{1}\right] \\ V_{g}+n V_{o}-v_{C_{r}}(t) & t \in\left[t_{1}, t_{2}\right] \\ V_{g}+n V_{o}-V_{c} & t \in\left[t_{2}, t_{3}\right] \\ V_{g}+n V_{o}-v_{C_{r}}(t) & t \in\left[t_{3}, t_{4}\right] \\ V_{g}+n V_{o} & t \in\left[t_{4}, t_{5}\right] \\ \approx 0 & t \in\left[t_{5}, t_{6}\right]\end{cases}
$$


Cuando se introducen las expresiones correspondientes de la tensión en $C_{r}$ queda como (E.11):

$$
v_{L_{r}}(t)=\left\{\begin{array}{lc}
\approx 0 & t \in\left[t_{0}, t_{1}\right] \\
-I Z_{r} \sin \left[\omega_{r}\left(t-t_{1}\right)\right] & t \in\left[t_{1}, t_{2}\right] \\
V_{g}+n V_{o}-V_{c} & t \in\left[t_{2}, t_{3}\right] \\
-I_{r 3} Z_{r} \sin \left[\omega_{r}\left(t-t_{3}\right)\right] & t \in\left[t_{3}, t_{4}\right] \\
\multicolumn{1}{c}{+\left(V_{g}+n V_{o}-V_{c}\right) \cos \left[\omega_{r}\left(t-t_{3}\right)\right]} & t \in\left[t_{4}, t_{5}\right] \\
V_{g}+n V_{o} & t \in\left[t_{5}, t_{6}\right] \\
\approx 0 &
\end{array}\right.
$$

Se ha representado en la figura E.4 las principales magnitudes a lo largo de las seis etapas por las que evoluciona esta topología durante un ciclo completo. La duración de cualquiera de estas etapas se indica con la letra $d$ y un subíndice que hace referencia al número de etapa. Por ejemplo, la duración de la etapa 5 es igual a $t_{5}-t_{4}=d_{5} T$.

\section{E.2. Análisis en régimen permanente.}

\section{E.2.1. Sin considerar rizado.}

Para este análisis se mantienen las consideraciones mencionadas anteriormente, respecto a $L_{m}$ para suponer corriente constante, de valor $I$, respecto a $L_{r}$ que es mucho mas pequeña qe $L_{m}$. También se mantiene constante la tensión en $C_{o}$ y en $C_{c}$, de valores $V_{o}$ y $V_{c}$ respectivamente.

Además, se desprecian las etapas 1, 2 y 4, puesto que la frecuencia característica del conjunto $L_{r}-C_{r}$ es mucho mas grande que la frecuencia de conmutación y la carga-descarga de $C_{r}$ es prácticamente instantánea. De esta forma solo intervienen tres etapas en el análisis. En lo que respecta a las corrientes, se puede decir que $I^{\prime} \approx I$ y $I^{\prime \prime} \approx-I$. Y como la duración de la etapa 3 es el tiempo que no está cerrado el interruptor principal, resulta que

$$
\begin{aligned}
d_{3} T & =(1-D) T \\
d_{5} T+d_{6} T & =D T
\end{aligned}
$$

Las ecuaciones del régimen permanente se obtienen al considerar nulo tanto el balance voltios-segundo en las bobinas $L$ y $L_{r}$ como la carga neta en los condensadores $C_{o}$ y $C_{c}$.

\section{TENSION MEDIA EN BOBINA DE MAGNETIZACIÓN.}

La tensión media en la bobina $L$ es nula. De aquí se obtiene una expresión 


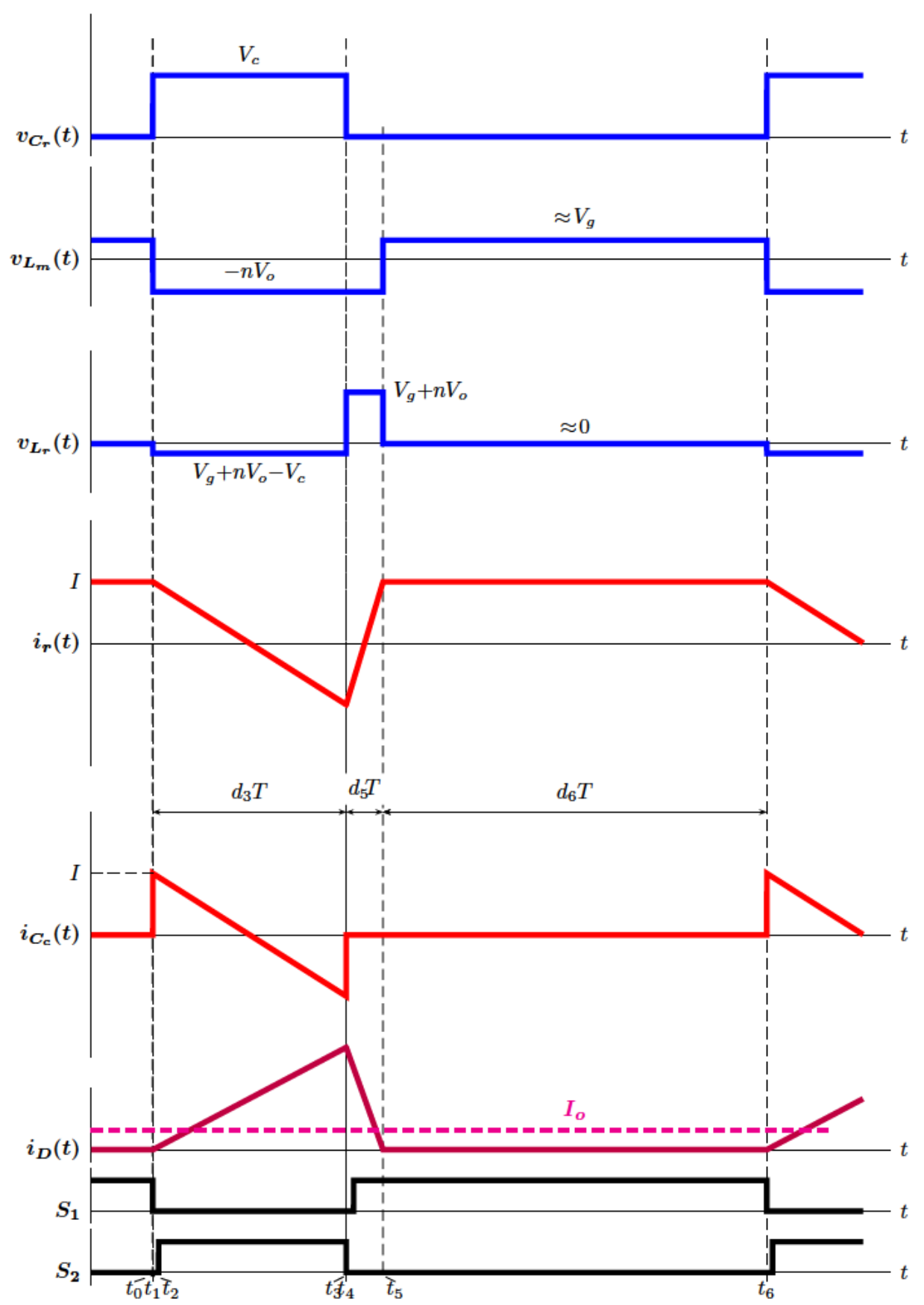

Figura E.4: Curvas principales de un flyback ZVS-boost.

para la tensión de salida en función de la entrada:

$$
\begin{aligned}
& \frac{1}{\not}\left[-n V_{o}\left(d_{3}+d_{5}\right) \not+\left(V_{g}\right) d_{6} X\right]=0 \\
& \Rightarrow V_{o}=V_{g} \frac{d_{6}}{\left(d_{3}+d_{5}\right) n}=V_{g} \frac{d_{6}}{\left(1-d_{6}\right) n}
\end{aligned}
$$


A $d_{6}$ se le puede llamar ciclo efectivo de trabajo, $D_{e f}$, puesto que al identificar la expresión que relaciona la tensión de salida con la entrada en un flyback, aparece ese término en lugar del término $D$ que identifica al ciclo de trabajo. También se puede identificar con el tiempo en el que la bobina principal del convertidor está absorbiendo energía. El valor del ciclo efectivo es menor que el valor del ciclo de trabajo empleado en las señales de disparo.

\section{TENSION MEDIA EN BOBINA RESONANTE.}

La tensión media en la bobina $L_{r}$ es nula. De aquí se obtiene una expresión para la tensión de $C_{c}$ en función de la tensión de entrada:

$$
\begin{aligned}
& \frac{1}{\mathscr{X}}\left[\left(V_{g}+n V_{o}-V_{c}\right) d_{3} \not{X}+\left(V_{g}+n V_{o}\right) d_{5} X\right]=0 \\
& \Rightarrow V_{c}=\left(V_{g}+n V_{o}\right) \frac{d_{3}+d_{5}}{d_{3}}
\end{aligned}
$$

Sustituyendo (E.13) en (E.14) también se puede despejar $V_{c}$ como:

$$
V_{c}=V_{g} \frac{1}{d_{3}}
$$

CORRIENTE MEDIA EN LA BOBINA RESONANTE, $I_{r}$, Y EN LA ENTRADA $I_{g}$.

Haciendo el valor medio a la expresión (E.4) resulta que

$$
I_{g}+\frac{I_{o}}{n}=I
$$

Ademas, como $V_{g} I_{g}=V_{o} I_{o}$, cuando se sustituye $I_{g}$ en (E.17) se obtiene $I_{r}$ en función de $I$ :

$$
I_{r} \equiv I_{g}=I \frac{n V_{o}}{n V_{o}+V_{g}}
$$

CORRIENTE MEDIA EN EL CONDENSADOR DE CLAMP.

La intensidad media en el condensador de clamp es nula. Entonces:

$$
\begin{aligned}
& \int_{t_{0}}^{t_{0}+T} i_{c}(t) d t=\int_{t_{2}}^{t_{3}} i_{c}(t) d t=\int_{0}^{d_{3} T}\left[I+\frac{V_{g}+n V_{o}-V_{c}}{L_{r}} t\right] d t=0 \\
& {\left[I t+\frac{V_{g}+n V_{o}-V_{c}}{2 L_{r}} t^{2}\right]_{0}^{d_{3} T}=0 \Rightarrow V_{c}=\frac{2 I L_{r}}{d_{3} T}+\left(V_{g}+n V_{o}\right)}
\end{aligned}
$$

Sustituyendo este valor en la expresión empleada para $i_{c}(t)$ se puede obtener el valor de la intensidad del condensador transcurrido el tiempo $d_{3} T$. Entonces:

$$
i_{c}\left(d_{3} T\right)=I+\frac{V_{g}+n V_{o}-V_{c}}{L_{r}} d_{3} T=I-\frac{\frac{2 I h_{r}}{d_{3} T}}{L_{r}} d_{3} T=-I
$$




\section{DURACIÓN DEL INTERVALO $d_{5}$.}

Se puede obtener a partir de igualar las expresiones de $V_{c}$ dadas en (E.14) y en (E.18). De esta forma:

$$
V_{c}=\frac{2 I L_{r}}{d_{3} T}+\left(V_{g}+n V_{o}\right)=\left(V_{g}+n V_{o}\right) \frac{d_{3}+d_{5}}{d_{3}} \Rightarrow d_{5}=\frac{2 I L_{r}}{\left(V_{g}+n V_{o}\right) T}
$$

Expresión a la que se puede llegar también a partir de la tensión en la bobina $L r$ durante la etapa 5, puesto que es conocida la variación que experimenta la intensidad circulante por esa bobina y la tensión a la que está sometida.

$$
\begin{aligned}
\Delta i_{r}(t) & =i_{r}\left(t_{5}\right)-i_{r}\left(t_{4}\right) \approx i_{r}\left(t_{5}\right)-i_{r}\left(t_{3}\right)=I-(-I)=2 I \\
\Delta i_{r}(t) & =\frac{1}{L_{r}} \Delta v_{L_{r}}(t) \Delta t \equiv \frac{1}{L_{r}}\left(V_{g}+n V_{o}\right) d_{5} T \\
\Rightarrow d_{5} & =\frac{2 I L_{r}}{\left(V_{g}+n V_{o}\right) T}
\end{aligned}
$$

\section{TENSION DE SALIDA.}

Empleando (E.20) junto con (E.12) y (E.13) se obtiene una expresión para la tensión de salida en régimen permanente. Como $d_{6}=\left(D-d_{5}\right)$, resulta que:

$$
d_{6}=D-\frac{2 I L_{r} f}{V_{g}+n V_{o}}=\frac{\left(V_{g}+n V_{o}\right) D-2 I L_{r} f}{V_{g}+n V_{o}}
$$

Por otro lado, despejando $d_{6}$ de (E.13) se obtiene:

$$
d_{6}=\frac{n V_{o}}{V_{g}+n V_{o}}
$$

Igualando (E.22) y (E.23) se puede despejar $V_{o}$ como:

$$
V_{o}=\frac{V_{g} D}{(1-D) n}-I \frac{2 L_{r} f}{(1-D) n}
$$

A esta expresión también se llega igualando (E.15) con (E.18) y despejando $V_{o}$. Como se puede observar, la tensión de salida de un flyback ZVS-boost depende de la intensidad que circula por la bobina principal. Esta expresión indica que hay un término equivalente a una resistencia, por la que pasa la intensidad $I$, qe se puede considerar en serie con la bobina de magnetización puesto que, en un flyback, esta intensidad es la misma que pasa por la bobina. Su expresión sería (E.25):

$$
R_{e s L_{m}}=2 L_{r} f
$$

\section{CARACTERÍSTICA DE SALIDA.}


Considerando la igualdad de potencia de entrada y salida junto a la expresión resultante de hacer el valor medio a (E.4) se puede obtener la siguiente relación:

$$
\left.\begin{array}{l}
I=I_{g}+\frac{I_{o}}{n} \\
V_{o} I_{o}=V_{g} I_{g}
\end{array}\right\} \Rightarrow I=\frac{I_{o}}{n}\left(\frac{n V_{o}}{V_{g}}+1\right)
$$

Empleando ahora este resultado en (E.24) se puede obtener la relación entre la tensión de salida y la intensidad de salida:

$$
\begin{aligned}
V_{o}= & \frac{V_{g} D}{(1-D) n}-\frac{I_{o}}{I}\left(\frac{n V_{o}}{V_{g}}-1\right) \frac{2 L_{r} f}{(1-D) n} \\
= & \frac{\frac{V_{g} D}{(1-D) n}-I_{o} \frac{2 L_{r} f}{(1-D) n^{2}}}{1+I_{o} \frac{2 L_{r} f}{V_{g}(1-D) n}}
\end{aligned}
$$

La derivada de esta expresión respecto a la intensidad indica como varía la tensión de salida ante las variaciones de la intensidad y tiene dimensiones de resistencia. La derivada tiene la siguiente expresión:

$$
\begin{aligned}
\frac{d V_{o}}{d I_{o}} & =\frac{-\frac{2 L_{r} f}{(1-D) n^{2}}\left[1+I_{o} \frac{2 L_{r} f}{V_{g}(1-D) n}\right]-\left[\frac{V_{g} D}{(1-D) n}-I_{o} \frac{2 L_{r} f}{(1-D) n^{2}}\right]\left[\frac{2 L_{r} f}{V_{g}(1-D) n}\right]}{\left[1+I_{o} \frac{2 L_{r} f}{V_{g}(1-D) n}\right]^{2}} \\
& =-2 L_{r} f\left[\frac{\frac{1}{(1-D) n^{2}}}{1+I_{o} \frac{2 L_{r} f}{V_{g}(1-D) n}}\right]^{2}
\end{aligned}
$$

Despejando $I_{o}$ en la relación (E.27) y sustituyendo, se obtiene la expresión final:

$$
\frac{d V_{o}}{d I_{o}}=-2 L_{r} f\left[\frac{V_{g}+n V_{o}}{n V_{g}}\right]^{2}
$$

\section{E.2.2. Considerando rizado.}

Si se considera que la bobina $L$ no es lo suficientemente grande como para suponer corriente constante, $I$, los circuitos equivalentes empleados para el análisis del circuito son diferentes y la relación entre la bobina principal y resonante aparece en las nuevas expresiones de las principales magnitudes.

Los circuitos equivalentes ${ }^{2}$ están representados en la Figura E.5 donde se

\footnotetext{
${ }^{2}$ Son los mismos circuitos que en el caso del clamp tipo buck-boost, excepto en la etapa 3 , puesto que el condensador tiene otra disposición diferente.
} 
ha considerado la tensión constante en los condensadores $C_{o}$ y $C_{c}$, mientras que las principales magnitudes aparecen en la Figura E.6.

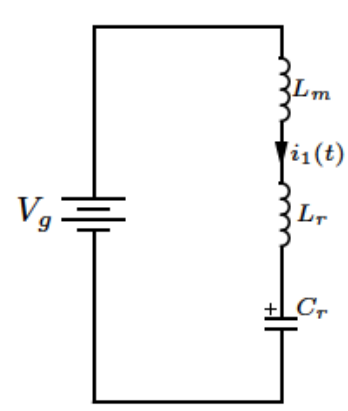

(a)

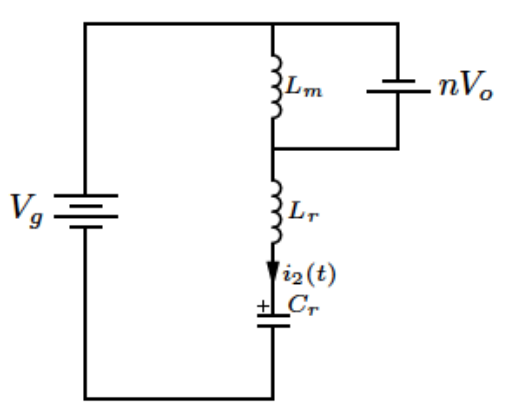

(b)

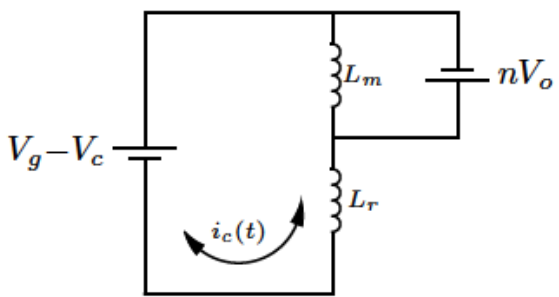

(c)

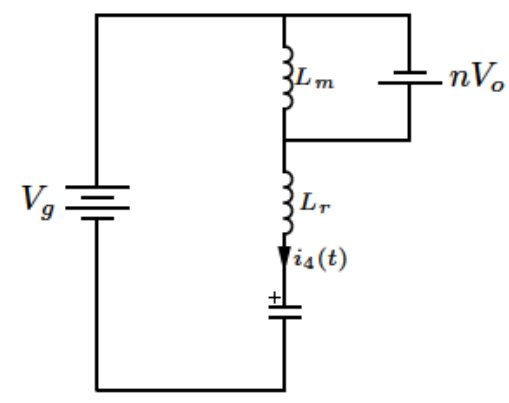

(d)

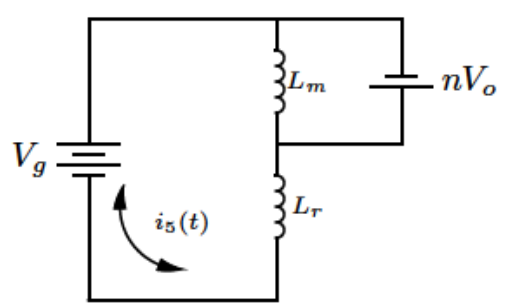

(e)

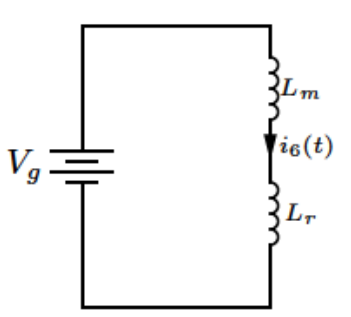

(f)

Figura E.5: Circuitos empleados para el análisis del flyback ZVS considerando rizado durante un ciclo completo: (a) Etapa 1. (b) Etapa 2. (c) Etapa 3. (d) Etapa 4. (e) Etapa 5. (f) Etapa 6.

Planteando el balance voltios-segundo en las bobinas $L$ y $L_{r}$ y la carga neta nula en los condensadores $C_{o}$ y $C_{c}$ con las nuevas curvas, se obtienen otras ecuaciones del régimen permanente. Se desprecian las etapas 1, 2 y 4, y solo intervienen tres etapas.

TENSION MEDIA EN BOBINA DE MAGNETIZACIÓN CON RIZADO.

Como la tensión media en la bobina $L_{m}$ es nula, resulta que:

$$
\begin{aligned}
& \frac{1}{\mathscr{T}}\left[-n V_{o}\left(d_{3}+d_{5}\right) \not{T}+V_{g} \frac{L_{m}}{L_{r}+L_{m}} d_{6} \not{X}\right]=0 \\
& \Rightarrow V_{o}=V_{g} \frac{d_{6} \frac{L_{m}}{L_{r}+L_{m}}}{\left(d_{3}+d_{5}\right) n}=V_{g} \frac{d_{6} \frac{L_{m}}{L_{r}+L_{m}}}{\left(1-d_{6}\right) n}
\end{aligned}
$$




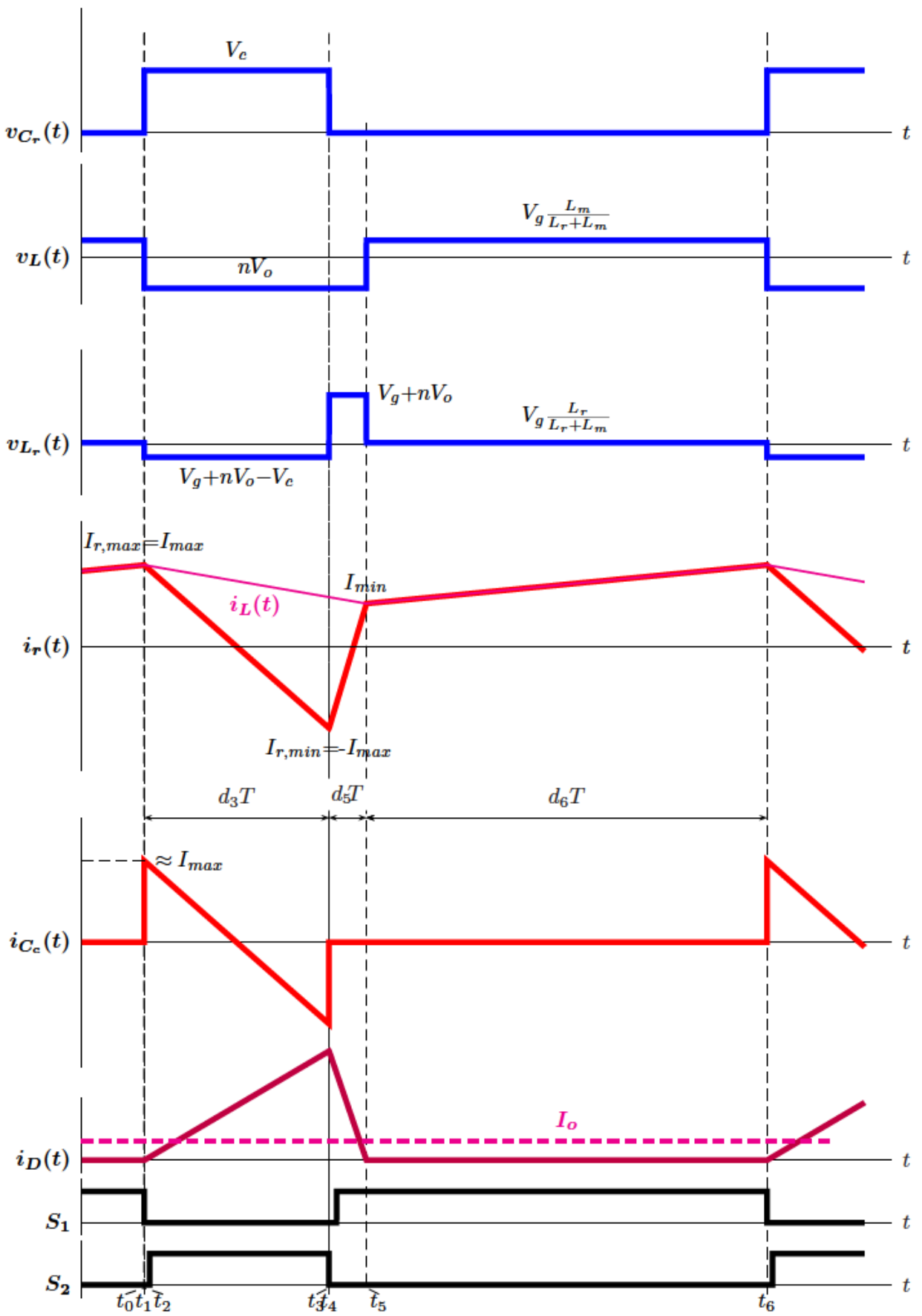

Figura E.6: Curvas principales de un flyback ZVS-boost considerando rizado.

Como la tensión media en la bobina $L_{r}$ es nula, entonces:

$$
\begin{aligned}
& \frac{1}{\mathcal{T}}\left[\left(V_{g}+n V_{o}-V_{c}\right) d_{3} \not{X}+\left(V_{g}+n V_{o}\right) d_{5} \not{T}+V_{g} d_{6} \frac{L_{r}}{L_{r}+L_{m}}\right]=0 \\
& \Rightarrow V_{c}=\left(V_{g}+n V_{o}\right) \frac{d_{3}+d_{5}}{d_{3}}+V_{g} \frac{d_{6}}{d_{3}} \frac{L_{r}}{L_{r}+L_{m}}
\end{aligned}
$$


Utilizando (E.30) en (E.31) se puede despejar $V_{c}$ como:

$$
V_{c}=V_{g} \frac{1}{d_{3}}
$$

\section{CORRIENTE MEDIA EN EL CONDENSADOR DE CLAMP CON RIZADO.}

La intensidad media en el condensador de clamp es nula. Entonces:

$$
\begin{aligned}
& \int_{t_{0}}^{t_{0}+T} i_{c}(t) d t=\int_{t_{2}}^{t_{3}} i_{c}(t) d t=\int_{0}^{d_{3} T}\left[I_{\max }+\frac{V_{g}+n V_{o}-V_{c}}{L_{r}} t\right] d t=0 \\
& {\left[I_{\text {max }} t+\frac{\left(V_{g}+n V_{o}-V_{C_{c}}\right)}{2 L_{r}} t^{2}\right]_{0}^{d_{3} T}=0 \Rightarrow V_{c}=\frac{2 I_{\max } L_{r}}{d_{3} T}+\left(V_{g}+n V_{o}\right)}
\end{aligned}
$$

Sustituyendo este valor en la expresión empleada para $i_{c}(t)$ se puede obtener el valor de la intensidad del condensador transcurrido el tiempo $d_{3} T$. Entonces:

$$
i_{c}\left(d_{3} T\right)=I_{\max }+\frac{\left(V_{g}+n V_{o}-V_{c}\right)}{L_{r}} d_{3} T=I_{\max }-\frac{\frac{2 I_{\max } L_{r}}{d_{3} T}}{L_{r}} d_{3} T=-I_{\max }
$$

\section{DURACIÓN DEL INTERVALO $d_{5}$ CON RIZADO.}

Se puede calcular a partir de la tensión en la bobina $L r$ durante la etapa 5 , puesto que es conocida la variación que experimenta la intensidad circulante por esa bobina y la tensión a la que está sometida.

$$
\begin{aligned}
\Delta i_{r}(t) & =i_{r}\left(t_{5}\right)-i_{r}\left(t_{4}\right) \approx i_{r}\left(t_{5}\right)-i_{r}\left(t_{3}\right)=I_{\text {min }}-\left(-I_{\text {max }}\right)=2 I \\
\Delta i_{r}(t) & =\frac{1}{L_{r}} \Delta v_{L_{r}}(t) \Delta t \equiv \frac{1}{L_{r}}\left(V_{g}+n V_{o}\right) d_{5} T \\
\Rightarrow d_{5} & =\frac{2 I L_{r}}{\left(V_{g}+n V_{o}\right) T}
\end{aligned}
$$

Una expresión diferente se obtiene al igualar (E.31) con (E.33) y empleando (E.12) para despejar $d_{5}$ :

$$
\begin{aligned}
& \left(V_{g}+n V_{o}\right) \frac{d_{3}+d_{5}}{d_{3}}+V_{g} \frac{d_{6}}{d_{3}} \frac{L_{r}}{L_{r}+L_{m}}=\frac{2 I_{\max } L_{r}}{d_{3} T}+\left(V_{g}+n V_{o}\right) \\
& \left(V_{g}+n V_{o}\right) \frac{d_{5}}{d_{3}}+V_{g} \frac{\left(D-d_{5}\right)}{\not / 3} \frac{L_{r}}{L_{r}+L_{m}}=\frac{2 I_{\max } L_{r}}{\phi_{3} T} \\
& \Rightarrow d_{5}=\frac{2 I_{\text {max }} L_{r} f-V_{g} D \frac{L_{r}}{L_{r}+L_{m}}}{V_{g} \frac{L_{m}}{L_{r}+L_{m}}+n V_{o}}
\end{aligned}
$$

\section{TENSION DE SALIDA CON RIZADO.}


Como $d_{6}=\left(D-d_{5}\right)$, resulta que:

$$
\begin{aligned}
d_{6} & =D-\frac{2 I_{\max } L_{r} f-V_{g} D \frac{L_{r}}{L_{r}+L_{m}}}{V_{g} \frac{L_{m}}{L_{r}+L_{m}}+n V_{o}} \\
& =\frac{\left(V_{g}+n V_{o}\right) D-2 I_{\max } L_{r} f}{V_{g} \frac{L_{m}}{L_{r}+L_{m}}+n V_{o}}
\end{aligned}
$$

Por otro lado, despejando $d_{6}$ de (E.30) se obtiene:

$$
d_{6}=\frac{n V_{o}}{V_{g} \frac{L_{m}}{L_{m}+L_{r}}+n V_{o}}
$$

Igualando (E.37) y (E.38) se puede despejar $V_{o}$ como:

$$
V_{o}=\frac{V_{g} D}{(1-D) n}-I_{\max } \frac{2 L_{r} f}{(1-D) n}
$$

También se podría conseguir si se iguala (E.32) con (E.33). Esta expresión es parecida a (E.24) que se ha obtenido sin considerar rizado. Solo se ha cambiado $I$ por $I_{\max }$. Y el término que multiplica a la intensidad es la resistencia equivalente en serie con $L_{m}$ definida anteriormente en (E.25).

\section{RIZADO DE CORRIENTE EN BOBINA DE MAGNETIZACIÓN CON RIZADO.}

Aplicando la definición de rizado de corriente en una bobina, se puede obtener el rizado de corriente en $L_{m}$ durante las etapas 3 a 5 o durante la etapa 6 , siendo expresiones equivalentes en régimen permanente. Entonces:

$$
\begin{aligned}
\Delta i_{L_{m}, 3-5} & =\frac{1}{L_{m}}\left|-n V_{o}\right|\left(d_{3}+d_{5}\right) T=\frac{1}{L_{m}} n V_{o}\left(1-d_{6}\right) T \\
\Delta i_{L_{m}, 6} & =\frac{1}{L_{m}}\left|V_{g}\right| \frac{L_{m}}{L_{m}+L_{r}} d_{6} T=\frac{V_{g}}{L_{m}+L_{r}} d_{6} T
\end{aligned}
$$

Empleando la ecuación (E.38) se obtiene la expresión para el rizado de corriente en $L_{m}$ como:

$$
\Delta i_{L_{m}}=\frac{n V_{o} V_{g} T}{L_{m} V_{g}+\left(L_{m}+L_{r}\right) n V_{o}}
$$

\section{CARACTERÍSTICA DE SALIDA CON RIZADO.}

La relación entre la tensión de salida y la intensidad de salida se obtiene a partir de la expresión (E.39). Primero se expresa $I_{\max }$ por su expresión en función del rizado. Después se emplea la igualdad de potencia a la entrada y a la salida y la relación $I=I_{g}+I_{o} / n$, para obtener:

$$
\left.\begin{array}{l}
I=I_{g}+I_{o} / n \\
V_{g} I_{g}=V_{o} I_{o}
\end{array}\right\} \Rightarrow I=\frac{I_{o}}{n}\left(\frac{n V_{o}}{V_{g}}+1\right)
$$


Para el rizado de corriente se emplea la segunda expresión de (E.40) y se considera $d_{6}=\left(D-d_{5}\right)$

$$
\Delta i_{L_{m}}=\frac{V_{g}}{L_{m}+L_{r}}\left(D-d_{5}\right) T=\frac{V_{g}}{L+L_{r}}\left(D-\frac{2 I L_{r}}{\left(V_{g}+n V_{o}\right) T}\right) T
$$

Sólo queda sustituir (E.42) y (E.43) en (E.39) y despejar $V_{o}$ para obtener una función de la forma $V_{o}=f\left(I_{o}\right)$. Por tanto:

$$
\begin{aligned}
V_{o} & =\frac{V_{g} D}{(1-D) n}-I_{\max } \frac{2 L_{r} f}{(1-D) n}=\frac{V_{g} D}{(1-D) n}-\left(I+\frac{1}{2} \Delta i_{L_{m}}\right) \frac{2 L_{r} f}{(1-D) n} \\
& =\frac{V_{g} D}{(1-D) n}-I \frac{2 L_{r} f}{(1-D) n}-\frac{1}{\not 2} \frac{V_{g}}{L_{m}+L_{r}}\left(D-\frac{2 I L_{r}}{\left(V_{g}+n V_{o}\right) T}\right) \not 2 \frac{\not L_{r} f}{(1-D) n} \\
& =-\frac{V_{g} D}{(1-D) n}\left[1-\frac{L_{r}}{L_{m}+L_{r}}\right]-\left[I_{o} \frac{V_{o}}{V_{g}}+\frac{I_{o}}{n}\right] \frac{2 L_{r} f}{(1-D) n} \\
& \quad+\frac{V_{g}}{L_{m}+L_{r}} \times \frac{2 I_{o}\left(n V_{o}+V_{g}\right) L_{r}^{2}}{V_{g}\left(V_{g}+n V_{o}\right) T(1-D) n^{2}} \\
& =-\frac{V_{g} D}{(1-D) n}\left[1-\frac{L_{r}}{L_{m}+L_{r}}\right]-I_{o} \frac{V_{o}}{V_{g}} \frac{2 L_{r} f}{(1-D) n}-I_{o} \frac{2 L_{r} f}{(1-D) n^{2}}\left[1-\frac{L_{r}}{L_{m}+L_{r}}\right] \\
& \Rightarrow V_{o}\left(I_{o}\right)=\frac{\left[\frac{V_{g} D}{(1-D) n}-I_{o} \frac{2 L_{r} f}{(1-D) n^{2}}\right] \frac{L_{m}}{L_{m}+L_{r}}}{1+I_{o} \frac{2 L_{r} f}{V_{g}(1-D) n}}
\end{aligned}
$$

\section{E.2.3. Comparativa.}

El análisis cuando se considera el rizado será de aplicación al circuito conmutado, donde se deben tener en cuenta los valores de las bobinas, mientras que el análisis al no considerar rizado servirá para las expresiones derivadas del modelo promediado, dado que éste parte de la consideración de pequeño rizado.

Los resultados obtenidos para magnitudes tales como el ciclo de trabajo, la tensión de clamp y las variaciones de corriente, a partir de unos valores de diseño ponen de manifiesto estas diferencias. Por ejemplo, empleando los mismos parámetros de funcionamiento de convertidor flyback ZVS del Anexo anterior, y que se indican en la Tabla E.1 se obtienen los valores indicados en la Tabla E.2, tanto para el caso de no considerar el rizado de la corriente en la bobina principal como para el que si se tiene en cuenta.

En este convertidor la intensidad en la bobina principal es igual a la intensidad en la entrada mas la de salida afectada de la relación de transformación, $I_{g}+I_{o} / n$. Al considerar el rizado, el ciclo de trabajo obtenido es mayor. La tensión de clamp, $V_{c}$ también es diferente. Es lógico dado que 


\begin{tabular}{llll}
\hline \hline$V_{g}$ & $20 \mathrm{~V}$ & $C_{o}$ & $5 \mu \mathrm{F}$ \\
$P$ & $100 \mathrm{~W}$ & $C_{c}$ & $2,2 \mu \mathrm{F}$ \\
$V_{\text {out }}$ & $400 \mathrm{~V}$ & $L_{m}$ & $24 \mu \mathrm{H}$ \\
$f$ & $100 \mathrm{kHz}$ & $L_{r}$ & $2 \mu \mathrm{H}$ \\
$n_{1}: n_{2}$ & $1: 12$ & & \\
$t_{m}$ & $300 \mathrm{~ns}$ & $C_{r}$ & $1 \mathrm{nF}$ \\
\hline
\end{tabular}

Tabla E.1: Condiciones de trabajo de un flyback ZVS-boost.

\begin{tabular}{ccccccccc}
\hline & $I$ & $d_{5}$ & $d_{6}$ & $D$ & $V_{c}$ & $\Delta_{i_{L}}$ & $I_{\max }$ & $\begin{array}{c}I_{\min } \\
(\mathrm{A})\end{array}$ \\
& $(\mathrm{A})$ & - & - & - & $(\mathrm{V})$ & $(\mathrm{A})$ & $(\mathrm{A})$ \\
\hline SIN RIZADO & \multirow{2}{*}{0} & \multirow{2}{*}{0,06} & 0,625 & 0,685 & 63,49 & 0 & - & - \\
CON RIZADO & & & 0,6436 & 0,7036 & 67,48 & 4,95 & 10,475 & 5,525 \\
\hline \hline
\end{tabular}

Tabla E.2: Parámetros calculados cuando se considera o no el rizado.

depende del ciclo de trabajo. Si se calcula su valor a partir de la expresión que incluye la intensidad, también se obtendrá un valor diferente según se considere valor medio (sin rizado) o valor máximo (cuando hay rizado).

En ambos análisis se han considerado despreciables algunas etapas por su pequeña duración en relación a las otras. Esta hipótesis se ha validado midiendo la duración de las seis etapas para los parámetros de funcionamiento de las dos tablas anteriores. Con ayuda de un programa de cálculo simbólico se han planteado las ecuaciones de estado durante las seis etapas y se han resuelto las ecuaciones y obtenido la fracción que ocupa cada etapa dentro de un periodo completo. Se han indicado en la Tabla E.3.

\begin{tabular}{ccccccc}
\hline & $d_{1}$ & $d_{2}$ & $d_{3}$ & $d_{4}$ & $d_{5}$ & $d_{6}$ \\
\hline SIN RIZADO & $667 \times 10^{-6}$ & $127 \times 10^{-6}$ & 0,3149 & $796 \times 10^{-6}$ & $59,7 \times 10^{-3}$ & 0,6238 \\
CON RIZADO & $536 \times 10^{-6}$ & $109 \times 10^{-6}$ & 0,2960 & $645 \times 10^{-6}$ & $59,7 \times 10^{-3}$ & 0,6430 \\
\hline \hline
\end{tabular}

Tabla E.3: Fracción de tiempo para cada etapa por las que evoluciona el convertidor.

A la vista de estos resultados, se puede comprobar que las etapas 1, 2 y 4 son las de menor duración por lo que, como se había previsto, se pueden despreciar. También se comprueba que coinciden con los valores obtenidos para el convertidor flyback ZVS en el Anexo anterior. De hecho, excepto la expresión de la tensión en el condensador de clamp, el resto de expresiones desarrolladas aquí son coincidentes con sus equivalentes para el otro convertidor.

Lo mismo se puede decir de la característica de salida, cuya representación se muestra en la Figura E.7 con y sin rizado.

La curva representada sin considerar rizado emplea el valor de ciclo de trabajo obtenido en este mismo tipo de análisis, mientras que la curva cuando 


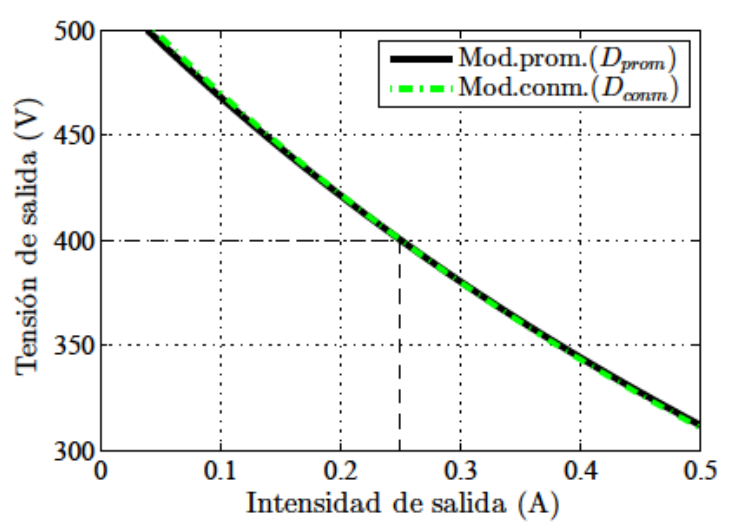

Figura E.7: Característica de salida de un flyback ZVS-boost.

se considera el rizado está conseguida a partir del ciclo de trabajo calculado con rizado no despreciable. Los rótulos indican modelo promediado para el caso sin rizado y modelo conmutado para el caso con rizado.

\section{E.3. Modelo promediado.}

Para hacer el modelo promediado se toman como variables de estado las intensidades en bobinas y las tensiones en condensadores. En concreto, las intensidades $i_{r}$ e $i_{m}$ en las bobinas resonante $L_{r}$ y de magnetización $L_{m}$ respectivamente, y las tensiones $v_{C_{r}}, v_{C_{c}}$ y $v_{C_{o}}$ en los condensadores resonante, $C_{r}$, de $\operatorname{clamp} C_{c}$ y de salida $C_{o}$. El número de variables da lugar a un modelo de orden cinco, pero puede reducirse a un modelo de orden tres si se agrupan las variables de estado en variables rápidas y variables lentas. En un primer paso, se escriben las variables rápidas en función de las lentas (que se suponen constantes), a lo largo de un periodo. Las expresiones obtenidas dependerán, por tanto, de las variables lentas y del tiempo. Esta expresiones se sustituyen en las ecuaciones de estado de las variables lentas que quedan desacopladas respecto a las variables rápidas. Se aplica un promedio estandar para eliminar la dependencia con el tiempo, y se obtiene un modelo promediado de tercer orden (reducido) para las tres variables de estado lentas.

Aunque matemáticamente no es necesario, se suelen realizar algunas aproximaciones antes de empezar para simplificar los cálculos a realizar. En primer lugar, se ignoran los tiempos muertos entre las conmutaciones de $S_{1}$ y $S_{2}$ que normalmente son muy pequeños en comparación con el periodo. También se desprecian los intervalos resonantes, como se hace en (ATHALYE y otros, 2001) para obtener el modelo completo con el método del interruptor 
promediado. En este punto cabe indicar que el efecto debido a tener en cuenta los intervalos resonantes sería tan solo a frecuencias muy altas y fuera del rango de interés (que, generalmente, suele ser una fracción de la frecuencia de conmutación porque es donde se aplica el control en lazo cerrado). Otra suposición es considerar que el condensador de clamp mantiene su tensión constante, lo que quiere decir que la frecuencia de resonancia entre $L_{r}$ y $C_{c}$ es bastante menor que la frecuencia de conmutación.

Considerando estas simplificaciones desaparecen los intervalos resonantes (Etapa 1, 2 y 4) y el tiempo muerto de la etapa 5, de forma que un periodo se puede escribir como:

$$
\begin{aligned}
T & \approx d_{3} T+d_{5} T+d_{6} T \\
(1-d) T & =d_{3} T \\
d T & =d_{5} T+d_{6} T
\end{aligned}
$$

puesto que $d_{1} T=d_{2} T=d_{4} T=0$.

La tensión en $C_{r}$ y la intensidad en $L_{r}$ se pueden escribir en función de las variables lentas que se consideran constantes ${ }^{3}$ :

$$
\begin{gathered}
v_{C_{r}}(t)= \begin{cases}v_{C_{c}} & t \in\left[t_{2}, t_{3}\right] \\
0 & t \in\left[t_{4}, t_{5}\right] \\
0 & t \in\left[t_{5}, t_{6}\right]\end{cases} \\
i_{r}(t)= \begin{cases}i_{m}+\frac{\left(v_{g}+n v_{C_{o}}-v_{C_{c}}\right)}{L_{r}}\left(t-t_{2}\right) & t \in\left[t_{2}, t_{3}\right] \\
i_{m}+\frac{\left(v_{g}+n v_{C_{o}}\right)}{L_{r}}\left(t-t_{5}\right) & t \in\left[t_{4}, t_{5}\right] \\
i_{m} & t \in\left[t_{5}, t_{6}\right]\end{cases}
\end{gathered}
$$

donde se conoce que $d_{3}=(1-d)$. Se puede despejar $d_{5}$ a partir de la igualdad de valores de $i_{r}$ en $t_{3}=t_{4}$. Es decir:

$$
\begin{aligned}
i_{r}\left(t_{3}\right) & =i_{r}\left(t_{4}\right) \\
i_{m}+\frac{\left(v_{g}+n v_{C_{o}}-v_{C_{c}}\right)}{L_{r}} d_{3} T & =i_{m}-\frac{\left(v_{g}+n v_{C_{o}}\right)}{L_{r}} d_{5} T
\end{aligned}
$$

de donde:

$$
d_{5}=\frac{\left(v_{C_{c}}-v_{g}-n v_{C_{o}}\right)}{\left(v_{g}+n v_{C_{o}}\right)}(1-d)
$$

Para el resto de variables lentas si es necesario escribir las ecuaciones de estado. Se construye una ecuación para la intensidad en la bobina de magnetización,$i_{m}(t)$; otra ecuación para la tensión en el condensador de

\footnotetext{
${ }^{3}$ Se ha mantenido la numeración de los intervalos, considerando que $t_{0}=t_{1}=t_{2}$ y que $t_{3}=t_{4}$
} 
clamp, $v_{C_{c}}(t)$; y otra ecuación para la tensión en el condensador de salida , $v_{C_{o}}(t)$. Estas ecuaciones son:

$$
\begin{gathered}
v_{L_{m}}(t)=L_{m} \frac{d i_{m}(t)}{d t}= \begin{cases}-n v_{C_{o}}(t) & t \in\left[t_{2}, t_{3}\right] \\
-n v_{C_{o}}(t) & t \in\left[t_{4}, t_{5}\right] \\
v_{g}(t) & t \in\left[t_{5}, t_{6}\right]\end{cases} \\
i_{C_{c}}(t)=C_{c} \frac{d v_{C_{c}}(t)}{d t}= \begin{cases}i_{r}(t) & t \in\left[t_{2}, t_{3}\right] \\
0 & t \in\left[t_{4}, t_{5}\right] \\
0 & t \in\left[t_{5}, t_{6}\right]\end{cases} \\
i_{C_{o}}(t)=C_{o} \frac{d v_{C_{o}}(t)}{d t}= \begin{cases}-n \underbrace{\left(i_{r}(t)-i_{m}(t)\right)}_{i_{1}(t)}-\frac{v_{C_{o}}(t)}{R} & t \in\left[t_{2}, t_{3}\right] \\
-n \underbrace{\left(i_{r}(t)-i_{m}(t)\right)}_{i_{1}(t)}-\frac{v_{C_{o}}(t)}{R} & t \in\left[t_{4}, t_{5}\right] \\
-\frac{v_{C_{o}}(t)}{R} & t \in\left[t_{5}, t_{6}\right]\end{cases}
\end{gathered}
$$

Se añaden dos ecuaciones mas, para la intensidad de entrada, $i_{g}$, y para la intensidad del diodo de salida, $i_{D}$.

$$
\begin{gathered}
i_{g}(t)= \begin{cases}i_{r}(t) & t \in\left[t_{2}, t_{3}\right] \\
i_{r}(t) & t \in\left[t_{4}, t_{5}\right] \\
i_{r}(t) & t \in\left[t_{5}, t_{6}\right]\end{cases} \\
i_{D}(t)= \begin{cases}-n\left(i_{r}(t)-i_{m}(t)\right) & t \in\left[t_{2}, t_{3}\right] \\
-n\left(i_{r}(t)-i_{m}(t)\right) & t \in\left[t_{4}, t_{5}\right] \\
0 & t \in\left[t_{5}, t_{6}\right]\end{cases}
\end{gathered}
$$

Cuando se sustituyen las variables rápidas en las ecuaciones anteriores, se consigue tener un conjunto de ecuaciones de estado donde solo intervienen las variables de estado lentas, es decir, se han desacoplado de las variables de estado rápidas.

$$
\begin{gathered}
L_{m} \frac{d i_{m}(t)}{d t}= \begin{cases}-n v_{C_{o}}(t) & t \in\left[t_{2}, t_{3}\right] \\
-n v_{C_{o}}(t) & t \in\left[t_{4}, t_{5}\right] \\
v_{g}(t) & t \in\left[t_{5}, t_{6}\right]\end{cases} \\
C_{c} \frac{d v_{C_{c}}(t)}{d t}= \begin{cases}i_{m}(t)+\frac{\left(v_{g}(t)+n v_{C_{o}}(t)-v_{C_{c}}(t)\right)}{L_{r}}\left(t-t_{2}\right) & t \in\left[t_{2}, t_{3}\right] \\
0 & t \in\left[t_{4}, t_{5}\right] \\
0 & t \in\left[t_{5}, t_{6}\right]\end{cases}
\end{gathered}
$$




$$
C_{o} \frac{d v_{C_{o}}(t)}{d t}= \begin{cases}-n \frac{\left(v_{g}(t)+n v_{C_{o}}(t)-v_{C_{c}}(t)\right)}{L_{r}}\left(t-t_{2}\right)-\frac{v_{C_{o}}(t)}{R} & t \in\left[t_{2}, t_{3}\right] \\ -n \frac{\left(v_{g}(t)+n v_{C_{o}}(t)\right)}{L_{r}}\left(t-t_{5}\right)-\frac{v_{C_{o}}(t)}{R} & t \in\left[t_{4}, t_{5}\right] \\ -\frac{v_{C_{o}}(t)}{R} & t \in\left[t_{5}, t_{6}\right]\end{cases}
$$

junto con:

$$
\begin{array}{r}
i_{g}(t)= \begin{cases}i_{m}(t)+\frac{\left(v_{g}(t)+n v_{C_{o}}(t)-v_{C_{c}}(t)\right)}{L_{r}}\left(t-t_{2}\right) & t \in\left[t_{2}, t_{3}\right] \\
i_{m}(t)+\frac{\left(v_{g}(t)+n v_{C_{o}}(t)\right)}{L_{r}}\left(t-t_{5}\right) & t \in\left[t_{4}, t_{5}\right] \\
i_{m}(t) & t \in\left[t_{5}, t_{6}\right]\end{cases} \\
i_{D}(t)= \begin{cases}-n \frac{\left(v_{g}(t)+n v_{C_{o}}(t)-v_{C_{c}}(t)\right)}{L_{r}}\left(t-t_{2}\right) & t \in\left[t_{2}, t_{3}\right] \\
-n \frac{\left(v_{g}(t)+n v_{C_{o}}(t)\right)}{L_{r}}\left(t-t_{5}\right) & t \in\left[t_{4}, t_{5}\right] \\
0 & t \in\left[t_{5}, t_{6}\right]\end{cases}
\end{array}
$$

siendo:

$$
d_{5}=\frac{\left(v_{C_{c}}(t)-v_{g}(t)-n v_{C_{o}}(t)\right)}{\left(v_{g}(t)+n v_{C_{o}}(t)\right)}(1-d)
$$

Considerando la aproximación de pequeño rizado (valores instantáneos son muy parecidos a los valores promedio), es posible sustituir los valores instantáneos de las variables de estado por sus valores promedio, (por ejemplo, $i_{m}(t)$ se sustituye por $\bar{i}_{m}$, que es igual que $\left\langle i_{m}(t)\right\rangle_{T}$ donde se ha simplificado la notación, y así con el resto). De esta forma, las ecuaciones desacopladas se transforman en:

$$
\begin{gathered}
L_{m} \frac{d i_{m}(t)}{d t} \approx \begin{cases}-n \bar{v}_{C_{o}} & t \in\left[t_{2}, t_{3}\right] \\
-n \bar{v}_{C_{o}} & t \in\left[t_{4}, t_{5}\right] \\
\bar{v}_{g} & t \in\left[t_{5}, t_{6}\right]\end{cases} \\
C_{c} \frac{d v_{C_{c}}(t)}{d t} \approx \begin{cases}\bar{i}_{m}+\frac{\left(\bar{v}_{g}+n \bar{v}_{C_{o}}-\bar{v}_{C_{c}}\right)}{L_{r}}\left(t-t_{2}\right) & t \in\left[t_{2}, t_{3}\right] \\
0 & t \in\left[t_{4}, t_{5}\right] \\
0 & t \in\left[t_{5}, t_{6}\right]\end{cases}
\end{gathered}
$$




$$
C_{o} \frac{d v_{C_{o}}(t)}{d t} \approx \begin{cases}-n \frac{\left(\bar{v}_{g}+n \bar{v}_{C_{o}}-\bar{v}_{C_{c}}\right)}{L_{r}}\left(t-t_{2}\right)-\frac{\bar{v}_{C_{o}}}{R} & t \in\left[t_{2}, t_{3}\right] \\ -n \frac{\left(\bar{v}_{g}+n \bar{v}_{C_{o}}\right)}{L_{r}}\left(t-t_{5}\right)-\frac{\bar{v}_{C_{o}}}{R} & t \in\left[t_{4}, t_{5}\right] \\ -\frac{\bar{v}_{C_{o}}}{R} & t \in\left[t_{5}, t_{6}\right]\end{cases}
$$

junto con:

$$
\begin{array}{r}
i_{g}(t) \approx \begin{cases}\bar{i}_{m}+\frac{\left(\bar{v}_{g}+n \bar{v} C_{o}-\bar{v}_{C_{c}}\right)}{L_{r}}\left(t-t_{2}\right) & t \in\left[t_{2}, t_{3}\right] \\
\bar{i}_{m}+\frac{\left(\bar{v}_{g}+n \bar{v}_{C_{o}}\right)}{L_{r}}\left(t-t_{5}\right) & t \in\left[t_{4}, t_{5}\right] \\
\bar{i}_{m} & t \in\left[t_{5}, t_{6}\right]\end{cases} \\
i_{D}(t) \approx \begin{cases}-n \frac{\left(\bar{v}_{g}+n \bar{v}_{C_{o}}-\bar{v}_{C_{c}}\right)}{L_{r}}\left(t-t_{2}\right) & t \in\left[t_{2}, t_{3}\right] \\
-n \frac{\left(\bar{v}_{g}+n \bar{v}_{C_{o}}\right)}{L_{r}}\left(t-t_{5}\right) & t \in\left[t_{4}, t_{5}\right] \\
0 & t \in\left[t_{5}, t_{6}\right]\end{cases}
\end{array}
$$

con:

$$
d_{5} \approx \frac{\left(\bar{v}_{C_{c}}-\bar{v}_{g}-n \bar{v}_{C_{o}}\right)}{\left(\bar{v}_{g}+n \bar{v}_{C_{o}}\right)}(1-d)
$$

\section{ECUACION DE ESTADO PARA $\bar{i}_{m}$.}

Haciendo el promedio a lo largo de un periodo de la ecuación (E.60) queda que:

$$
\begin{aligned}
L_{m} \frac{d \bar{i}_{m}}{d t} & =-n \bar{v}_{C_{o}}\left(d_{3}+d_{5}\right)+\bar{v}_{g} d_{6} \\
& =-n \bar{v}_{C_{o}}\left[(1-d)+d_{5}\right]+\bar{v}_{g}\left(d-d_{5}\right) \\
& =-n \bar{v}_{C_{o}}(1-d)-\left(n \bar{v}_{C_{o}}+\bar{v}_{g}\right) d_{5}+\bar{v}_{g} d \\
& =-n \bar{v}_{C_{o}}(1-d)-\left(\bar{v}_{C_{c}}-\bar{v}_{g}-n \bar{v}_{C_{o}}\right)(1-d)+\bar{v}_{g} d \\
& =\bar{v}_{g}-\bar{v}_{C_{c}}(1-d)
\end{aligned}
$$

\section{ECUACION DE ESTADO PARA $\bar{v}_{C_{c}}$.}

El promedio de la ecuación de tensión en $C_{c}$ se obtiene a partir de (E.61). Entonces:

$$
\begin{aligned}
C_{c} \frac{d \bar{v}_{C_{c}}}{d t} & =\bar{i}_{m} d_{3}+\left(\bar{v}_{g}+n \bar{v}_{C_{o}}-\bar{v}_{C_{c}}\right) \frac{d_{3}^{2} T}{2 L_{r}} \\
& =\bar{i}_{m}(1-d)-\frac{\bar{v}_{C_{c}}-\left(\bar{v}_{g}+n \bar{v}_{C_{o}}\right)}{2 L_{r} f_{s}}(1-d)^{2} \\
& =\bar{i}_{m}(1-d)-\frac{\bar{v}_{C_{c}}-\left(\bar{v}_{g}+n \bar{v}_{C_{o}}\right)}{r_{e q}}
\end{aligned}
$$


donde se define una resistencia equivalente, $r_{e q}$, que depende de $d$ y tiene la siguiente expresión:

$$
r_{e q}=\frac{2 L_{r} f_{s}}{(1-d)^{2}}
$$

ECUACION DE ESTADO PARA $\bar{v}_{C_{o}}$.

Para la tensión de salida, su ecuación de estado es:

$$
\begin{aligned}
C_{o} \frac{d \bar{v}_{C_{o}}}{d t}= & -n\left(\bar{v}_{g}+n \bar{v}_{C_{o}}-\bar{v}_{C_{c}}\right) \frac{d_{3}^{2} T}{2 L_{r}}+n\left(\bar{v}_{g}+n \bar{v}_{C_{o}}\right) \frac{d_{5}^{2} T}{2 L_{r}} \\
& -\frac{\bar{v}_{C_{o}}}{R}\left(d_{3}+d_{5}+d_{6}\right) \\
= & n\left[\frac{\bar{v}_{C_{c}}-\left(\bar{v}_{g}+n \bar{v}_{C_{o}}\right)}{r_{e q}}+\frac{\left(\bar{v}_{C_{c}}-\bar{v}_{g}-n \bar{v}_{C_{o}}\right)^{2}}{\left(\bar{v}_{g}+n \bar{v}_{C_{o}}\right) r_{e q}}\right]-\frac{\bar{v}_{C_{o}}}{R} \\
= & n\left[\frac{\bar{v}_{C_{c}}-\left(\bar{v}_{g}+n \bar{v}_{C_{o}}\right)}{r_{e q}}+i_{e q}\right]-\frac{\bar{v}_{C_{o}}}{R}
\end{aligned}
$$

donde se define una fuente de corriente equivalente, $i_{e q}$, como:

$$
i_{e q}=\frac{\left(\bar{v}_{C_{c}}-\bar{v}_{g}-n \bar{v}_{C_{o}}\right)^{2}}{\left(\bar{v}_{g}+n \bar{v}_{C_{o}}\right) r_{e q}}
$$

ECUACION DE ESTADO PARA $\bar{i}_{g}$.

También se puede obtener una ecuación para la intensidad de entrada a partir del promedio de la ecuación (E.63):

$$
\begin{aligned}
\bar{i}_{g}= & \bar{i}_{m}\left(d_{3}+d_{5}+d_{6}\right) \\
& +\left(\bar{v}_{g}+n \bar{v}_{C_{o}}-\bar{v}_{C_{c}}\right) \frac{d_{3}^{2} T}{2 L_{r}}-\left(\bar{v}_{g}+n \bar{v}_{C_{o}}\right) \frac{d_{5}^{2} T}{2 L_{r}} \\
= & \bar{i}_{m}-\frac{\bar{v}_{C_{c}}-\left(\bar{v}_{g}+n \bar{v}_{C_{o}}\right)}{r_{e q}}-\frac{\left(\bar{v}_{C_{c}}-\bar{v}_{g}-n \bar{v}_{C_{o}}\right)^{2}}{\left(\bar{v}_{g}+n \bar{v}_{C_{o}}\right) r_{e q}} \\
= & \bar{i}_{m}-\frac{\bar{v}_{C_{c}}-\left(\bar{v}_{g}+n \bar{v}_{C_{o}}\right)}{r_{e q}}-i_{e q}
\end{aligned}
$$

\section{ECUACION DE ESTADO PARA $\bar{i}_{D}$.}

La intensidad del diodo $D$ tiene también su ecuación para el modelo promediado:

$$
\begin{aligned}
\bar{i}_{D} & =-n\left(\bar{v}_{g}+n \bar{v}_{C_{o}}-\bar{v}_{C_{c}}\right) \frac{d_{3}^{2} T}{2 L_{r}}+n\left(\bar{v}_{g}+n \bar{v}_{C_{o}}\right) \frac{d_{5}^{2} T}{2 L_{r}} \\
& =n\left[\frac{\bar{v}_{C_{c}}-\left(\bar{v}_{g}+n \bar{v}_{C_{o}}\right)}{r_{e q}}+\frac{\left(\bar{v}_{C_{c}}-\bar{v}_{g}-n \bar{v}_{C_{o}}\right)^{2}}{\left(\bar{v}_{g}+n \bar{v}_{C_{o}}\right) r_{e q}}\right] \\
& =n\left[\frac{\bar{v}_{C_{c}}-\left(\bar{v}_{g}+n \bar{v}_{C_{o}}\right)}{r_{e q}}+i_{e q}\right]
\end{aligned}
$$


El circuito obtenido con las ecuaciones del modelo promediado está indicado en la Figura E.8.

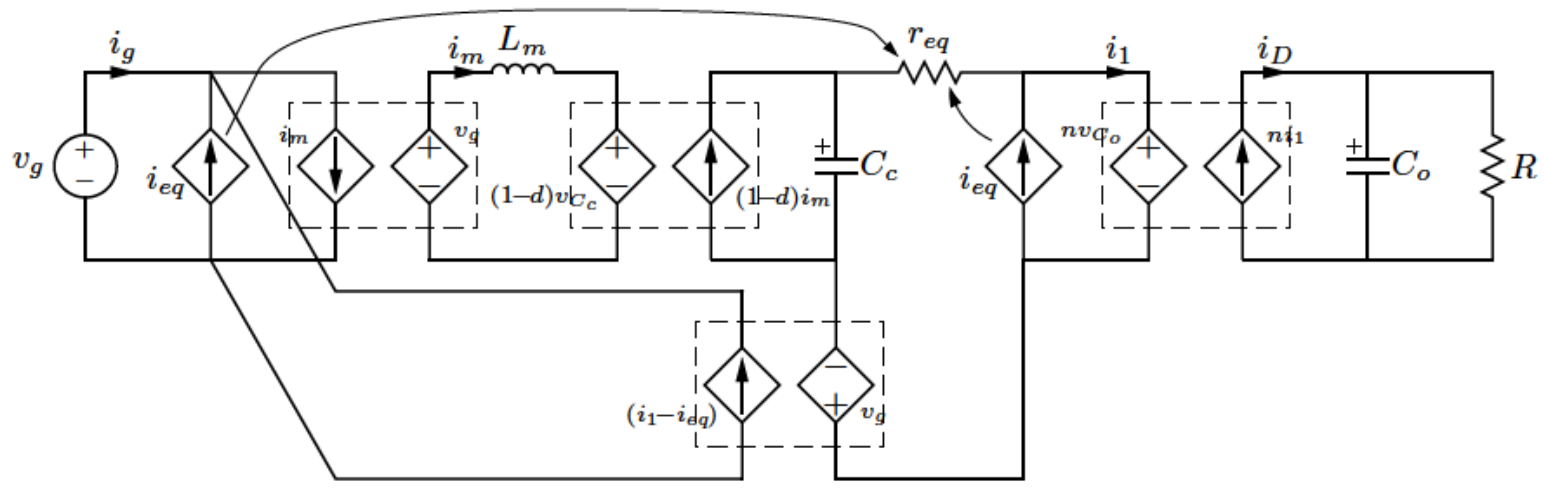

(a)

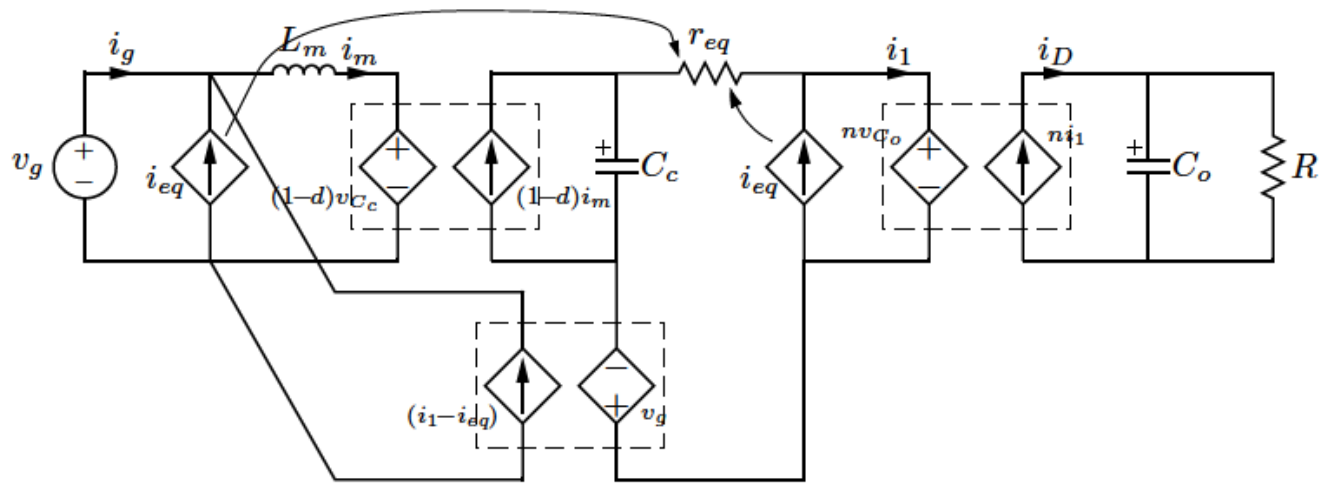

(b)

Figura E.8: Modelo promediado de un flyback ZVS ideal: a) obtenido a partir de las ecuaciones; b) después de simplificar. Son magnitudes promediadas.

\section{E.3.1. Balance energético}

En el modelo promediadohay tres elementos involucrados, la fuente de tensión en la entrada las fuente de corriente equivalente en la entrada y la fuente de corriente equivalente en la salida. La energía de la fuente de tensión se consume en la carga y se almacena o devuelve en los elementos reactivos, mientras que la energía generada en la fuente dependiente de la entrada se consume en $r_{e q}$, al igual que sucede con la energía generada en la fuente dependiente de la salida. Veámoslo: 
En $r_{e q}$, la potencia consumida es igual a

$$
P_{r_{e q}}=\frac{\left[\bar{v}_{C_{c}}-\left(\bar{v}_{g}+n \bar{v}_{C_{o}}\right)\right]^{2}}{r_{e q}}
$$

Multiplicando arriba y abajo por $\left(\bar{v}_{g}+n \bar{v}_{C_{o}}\right)$, se obtiene una expresión

$$
P_{r_{e q}}=\frac{\left[\bar{v}_{C_{c}}-\left(\bar{v}_{g}+n \bar{v}_{C_{o}}\right)\right]^{2}}{r_{e q}\left(\bar{v}_{g}+n \bar{v}_{C_{o}}\right)}\left(\bar{v}_{g}+n \bar{v}_{C_{o}}\right)
$$

en la que, agrupando, se obtienen dos productos de una intensidad $\bar{i}_{e q}$ por una tensión,

$$
P_{r_{e q}}=\bar{i}_{e q} \bar{v}_{g}+\bar{i}_{e q} n \bar{v}_{C_{o}}=P_{i_{e q_{1}}}+P_{i_{e q_{2}}}
$$

por tanto, esta potencia coincide con la potencia generada por las dos fuentes de corriente dependientes.

\section{E.3.2. Validación del modelo promediado}

La validación del modelo promediado se realiza mediante simulación, y se compara su comportamiento, tanto en régimen permanente como en régimen dinámico, con un convertidor conmutado. Para ello se eligen de nuevo los parámetros de funcionamiento recogidos en la Tabla E.1, empleando en el convertidor conmutado el ciclo de trabajo calculado al considerar rizado. En el modelo promediado se ha considerado el ciclo de trabajo calculado cuando no se ha considerado el rizado.

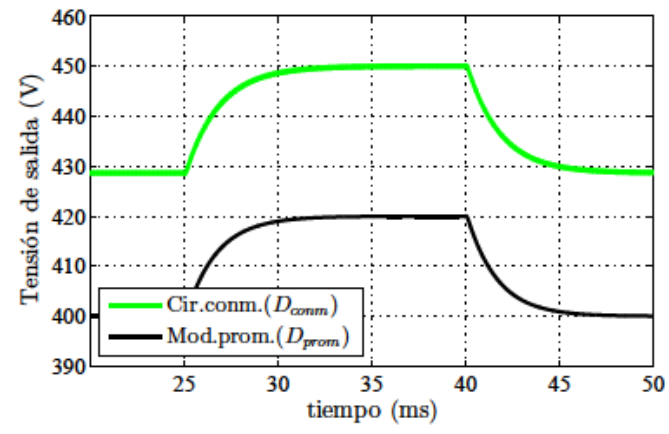

(a)

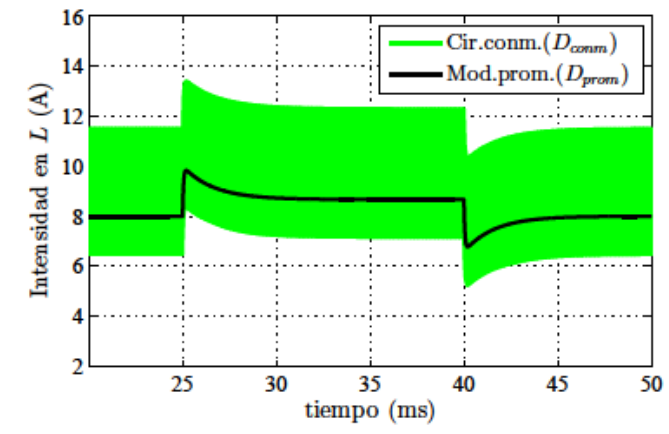

(b)

Figura E.9: Flyback ZVS. Respuesta a escalón del ciclo de trabajo: a) Tensión de salida; y b) Intensidad en la bobina.

Los resultados muestran una dinámica similar para el convertidor conmutado y el modelo promediado. En cuanto al régimen permanente, hay un 
error puesto que la tensión de salida no coincide. El motivo de esta diferencia tiene su causa en que en el modelo promediado no está incluido el tiempo muerto, $t_{m}$, que hay que dejar entre disparos de los transistores principal y de clamp, entre otras cosas, para facilitar las conmutaciones suaves. Además, parte de este tiempo muerto se emplea en cargar o descargar el condensador resonante, y corresponde a las tres etapas que se desprecian para obtener el modelo promediado. En concreto la etapa 4, siendo su duración igual a $d_{4} T$.

Por tanto, hay que realizar una corrección en el ciclo de trabajo empleado para el circuito conmutado, en base a los tiempos mencionados, puesto que ya está incluido de forma implícita en la generación de disparos del propio circuito conmutado. De esta forma, se rehacen las simulaciones del circuito conmutado con el ciclo de trabajo corregido, $D_{\text {corr }}$, como:

$$
D_{\text {corr }}=D_{\text {conm }}-\frac{t_{m}-d_{4} T}{T}
$$

y se obtienen los resultados de la Figura E.10. La corrección equivalente pero para el modelo promediado consistiría en añadir este tiempo extra al ciclo de trabajo empleado en el modelo promediado.

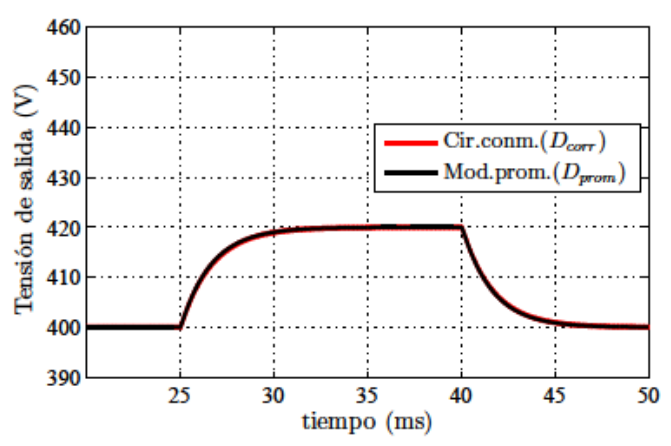

(a)

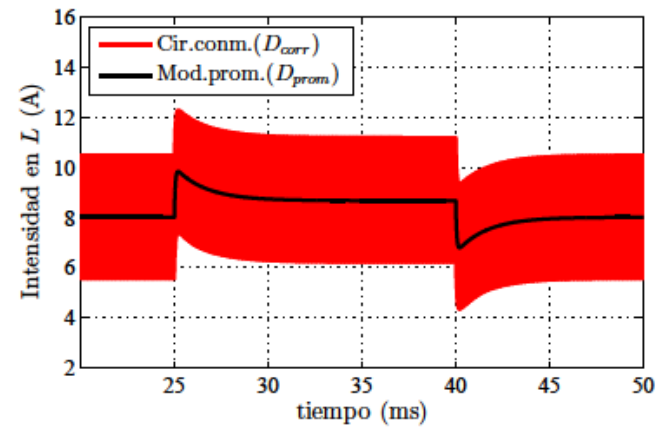

(b)

Figura E.10: Respuesta a escalón en el ciclo de trabajo incluyendo corrección: a) Tensión de salida; y b) Intensidad en la bobina.

Para apreciar la mejora en los resultados se representa el valor medio de la tensión de salida y la intensidad en la bobina para el circuito conmutado antes y después de la corrección y se comparan con el modelo promediado. En la Figura E.11 se pueden comprobar estas curvas.

\section{E.4. Modelo en régimen permanente}

El modelo en regimen permanente se puede obtener a partir del modelo promediado sustituyendo los valores promedio por valores constantes (por 


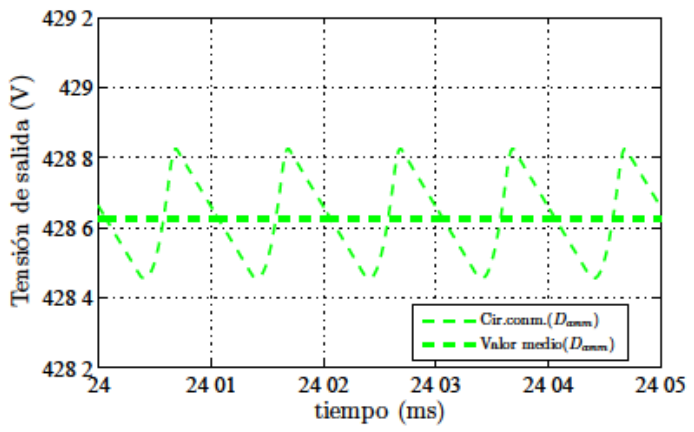

(a)

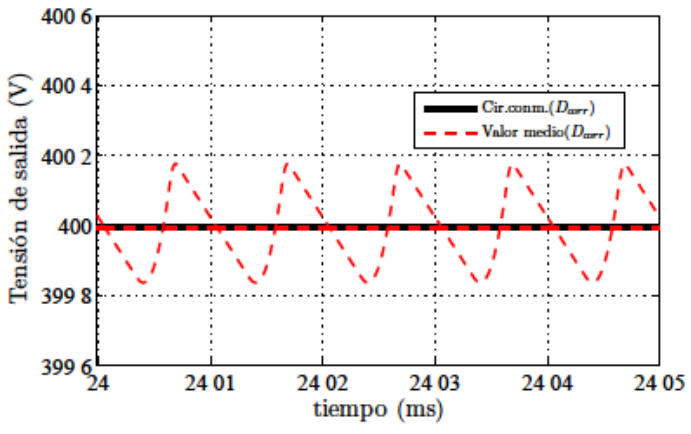

(b)

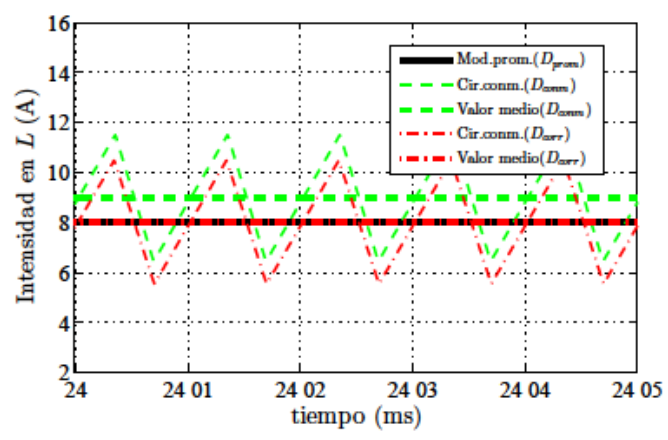

(c)

Figura E.11: Curvas de tensión e intensidad con y sin corrección en el ciclo de trabajo del circuito conmutado: a) Tensión de salida sin corrección e; b) Ídem aplicando corrección; y c) Intensidad en la bobina.

ejemplo, $\bar{i}_{m}$ se sustituye por $I$ ). De esta forma, las ecuaciones del modelo en régimen permanente serán las siguientes.

$$
\begin{aligned}
0 & =V_{g}-(1-D) V_{c} \\
0 & =n I_{e q}+n \frac{V_{c}-\left(V_{g}+n V_{o}\right)}{R_{e q}}-\frac{V_{o}}{R} \\
0 & =I(1-D)-\frac{V_{c}-\left(V_{g}+n V_{o}\right)}{R_{e q}} \\
I_{g} & =I-\frac{V_{c}-\left(V_{g}+n V_{o}\right)}{R_{e q}}-I_{e q} \\
I_{D} & =n\left[\frac{V_{c}-\left(V_{g}+n V_{o}\right)}{R_{e q}}+I_{e q}\right]
\end{aligned}
$$

donde

$$
R_{e q}=\frac{2 L_{r} f_{s}}{(1-D)^{2}} \quad I_{e q}=\frac{\left[V_{c}-\left(V_{g}+n V_{o}\right)\right]^{2}}{\left(V_{g}+n V_{o}\right) R_{e q}}
$$


Nótese que la derivada de una constante es cero, de ahí, que haya ecuaciones con un cero a la izquierda de la igualdad. El parámetro $d_{5}$ se expresa con valores constantes como:

$$
d_{5}=\frac{V_{c}-\left(V_{g}+n V_{o}\right)}{\left(V_{g}+n V_{o}\right)}(1-D)
$$

A estas ecuaciones se puede llegar también a partir del circuito promediado de la Figura E.8. Basta con sustituir los condensadores por un circuito abierto y las bobinas con un cortocircuito. De esta forma se obtienen los circuitos de la Figura E.12a y E.12b.

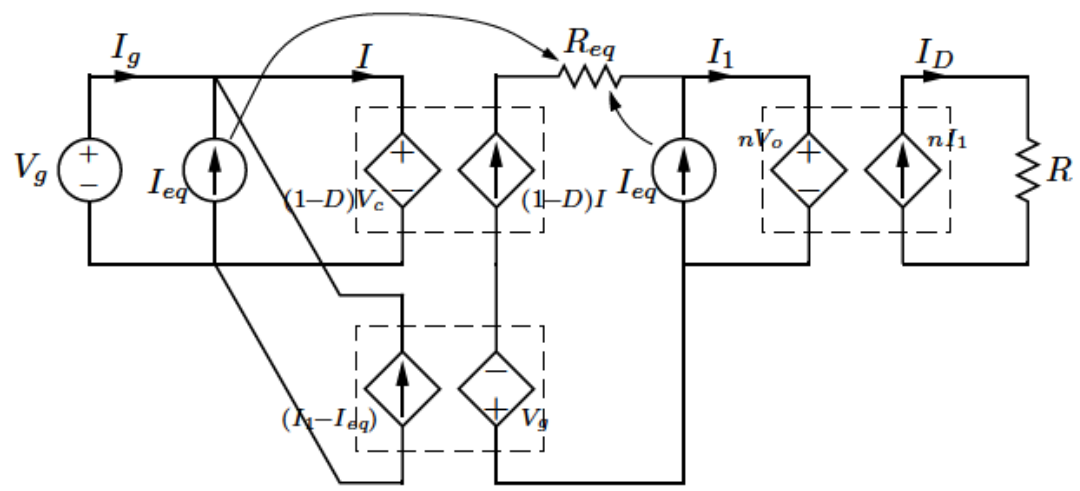

(a)

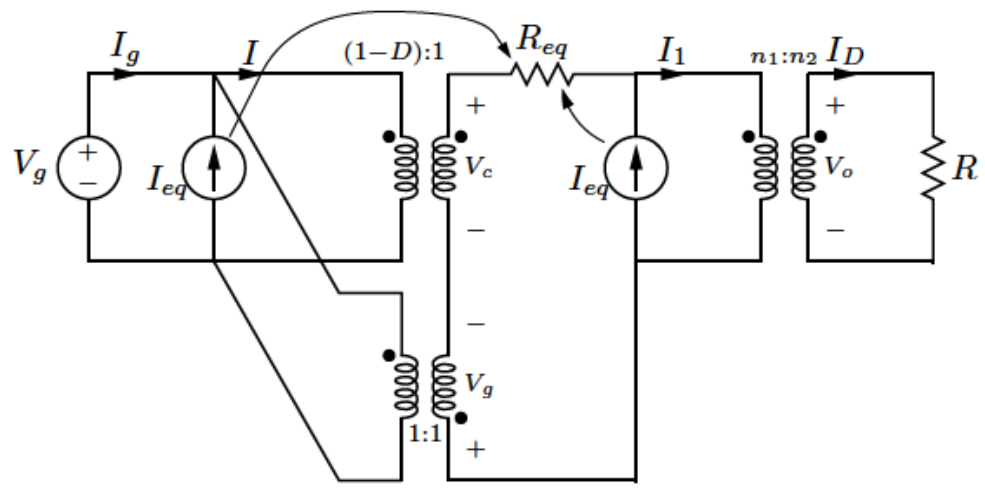

(b)

Figura E.12: Circuito en régimen permanente de un flyback ZVS en MCC: (a) con fuentes dependientes; (b) con transformadores ideales.

Se obtienen las mismas expresiones para las principales magnitudes en régimen permanente cuando se deducen de (E.77) que a partir de los circuitos 
de la Figura E.12.

$$
\begin{aligned}
V_{o} & =\frac{1}{n}\left(V_{c}-V_{g}-(1-D) I R_{e q}\right) \\
I_{g} & =I-\frac{V_{c}-\left(V_{g}+n V_{o}\right)}{R_{e q}}-I_{e q} \\
V_{c} & =\frac{V_{g}}{(1-D)} \\
I_{D} & =\frac{V_{o}}{R}=n\left[I_{e q}+\frac{V_{c}-\left(V_{g}+n V_{o}\right)}{R_{e q}}\right]
\end{aligned}
$$

Si en estas expresiones se elimina $L_{r}$, (que significa que desaparece el circuito de enclavamiento activo), se obtienen las expresiones en régimen permanente para un flyback convencional y lo mismo sucede con los circuitos en régimen permanente de ambas topologías.

La expresión que relaciona la tensión de salida con la intensidad en la carga se obtiene fácilmente a partir de la primera expresión de (E.80) donde se sustituye $R_{e q}$ y $V_{c}$.

$$
V_{o}=\frac{1}{n} \frac{V_{g} D}{1-D}-\frac{1}{n}(1-D) I R_{e q}
$$

Se puede observar que la tensión de salida de flyback ZVS se asimila a la de un flyback convencional que tiene a la salida una resistencia de valor $R_{e q} / n^{2}$ (con la particularidad de que es una resistencia equivalente sin pérdidas), y evoluciona con la corriente demandada por la carga (que en un flyback convencional es igual a $n I(1-D)$ ).

Por otro lado, la relación entre intensidades de entrada y salida y bobina se obtiene empleando la segunda y cuarta expresión de (E.80), sabiendo que $I_{D}=I_{o}$. Entonces:

$$
I_{g}+\frac{I_{o}}{n}=I
$$

\section{E.4.1. Balance energético}

En el modelo en régimen permanente hay tres elementos que generan energía, la fuente de tensión de la entrada, y la dos fuentes dependientes de corriente en la etapa de entrada y de salida. La energía generada en estas fuente de corriente se consume toda en $R_{e q}$. Veámoslo.

En $R_{e q}$, la potencia consumida es igual a

$$
P_{R_{e q}}=\frac{\left[V_{c}-\left(V_{g}-n V_{o}\right)\right]^{2}}{R_{e q}}
$$


Multiplicando arriba y abajo por $\left(V_{g}+n V_{o}\right)$, se obtiene una expresión

$$
P_{R_{e q}}=\frac{\left[V_{c}-\left(V_{g}-n V_{o}\right)\right]^{2}}{R_{e q}\left(V_{g}+n V_{o}\right)}\left(V_{g}+n V_{o}\right)
$$

en la que, agrupando, se obtienen dos productos de una intensidad $I_{e q}$ por una tensión,

$$
P_{R_{e q}}=I_{e q} V_{g}-I_{e q} n V_{o}=P_{I_{e q_{1}}}+P_{I_{e q_{2}}}
$$

por tanto, esta potencia coincide con la potencia generada por las fuentes de corriente dependientes.

Por otro lado, toda la potencia generada en $V_{g}$ se consume en la carga $R$ a la salida. La potencia que se genera es:

$$
\begin{aligned}
P_{g} & =V_{g} I_{g}=V_{g}\left(I-\frac{V_{c}-\left(V_{g}+n V_{o}\right)}{R_{e q}}-I_{e q}\right)=V_{g} I-V_{g} \frac{V_{c}-\left(V_{g}+n V_{o}\right)}{R_{e q}} I_{e q}-V_{g} I_{e q} \\
& =V_{c}(1-D) I-V_{g} I_{1}=\left[V_{g}+n V_{o}+(1-D) I R_{e q}\right](1-D) I-V_{g} I_{1} \\
& =\left(V_{g}+n V_{o}\right)(1-D) I+(1-D)^{2} I^{2} R_{e q}-V_{g} I_{1} \\
& =\left(V_{g}+n V_{o}\right)\left(I_{1}-I_{e q}\right)+\frac{\left[V_{c}-\left(V_{g}+n V_{o}\right)\right]^{2}}{R_{e q}^{q}} B e q-V_{g} I_{1} \\
& =V_{g} I_{1}+n V_{o} I_{1}-\left(V_{g} \neq n V_{o}\right) I_{e q}+\frac{\left[V_{c}-\left(V_{g}+n V_{o}\right)\right]^{2}}{R_{e q}}-V_{g} I_{1} \\
& =n V_{o} \frac{I_{D}}{n}=V_{o} \frac{V_{o}}{R}=\frac{V_{o}^{2}}{R}=V_{o} I_{o} \equiv P_{o}
\end{aligned}
$$

que coincide, con la potencia consumida en $R$. Esto es, la potencia que suministra la fuente de entrada se entrega a la carga en su totalidad.

\section{E.5. Modelo en pequeña señal}

El modelo en pequeña señal (modelo lineal) se consigue tras realizar el proceso de perturbación y linealización del modelo promediado (modelo no lineal) en torno a un punto de funcionamiento. Se asume que las entradas al modelo, $v_{g}$ y $d$ son iguales a un valor fijo, $V_{g}$ y $D$, mas una pequeña variación

de alterna, $\hat{v}_{g}$ y $\hat{d}$. Es decir:

$$
\begin{aligned}
\bar{v}_{g} & =V_{g}+\hat{v}_{g} \\
d & =D+\hat{d}
\end{aligned}
$$


En respuesta a estas entradas, los valores promediados de las variables de estado sufrirán una variación similar, es decir, presentarán un término constante mas una pequeña variación de alterna. También sucede lo mismo con el resto de magnitudes estudiadas, de forma que:

$$
\begin{gathered}
\bar{i}_{m}=I+\hat{i}_{m} \\
\bar{v}_{C_{o}}=V_{o}+\hat{v}_{C_{o}} \\
\bar{v}_{C_{c}}=V_{c}+\hat{v}_{C_{c}} \\
\bar{i}_{g}=I_{g}+\hat{i}_{g} \\
\bar{i}_{D}=I_{D}+\hat{i}_{D}
\end{gathered}
$$

Por supuesto, para que el modelo linealizado sea preciso debe cumplirse que las variaciones son pequeñas en comparación con el valor constante que indica el punto de operación del convertidor, es decir:

$$
\begin{aligned}
&\left|\hat{v}_{g}\right| \ll\left|V_{g}\right| \\
&|\hat{d}| \ll|D| \\
&\left|\hat{i}_{L_{m}}\right| \ll|I| \\
&\left|\hat{v}_{C_{o}}\right| \ll\left|V_{o}\right| \\
&\left|\hat{v}_{C_{c}}\right| \ll\left|V_{c}\right| \\
&\left|\hat{i}_{g}\right| \ll\left|I_{g}\right| \\
&\left|\hat{i}_{D}\right| \ll\left|I_{D}\right|
\end{aligned}
$$

Por lo que, introduciendo (E.87) y (E.88) en las ecuaciones del modelo promediado de orden reducido del flyback ZVS-boost, reescritas de nuevo en (E.90) y desarrollando y despreciando los términos de segundo orden se consigue linealizar el modelo promediado.

$$
\begin{aligned}
L_{m} \frac{d \bar{i}_{m}}{d t} & =\bar{v}_{g}-\bar{v}_{C_{c}}(1-d) \\
C_{o} \frac{d \bar{v}_{C_{o}}}{d t} & =-\frac{\bar{v}_{C_{o}}}{R}+n\left[\frac{\bar{v}_{C_{c}}-\left(\bar{v}_{g}+n \bar{v}_{C_{o}}\right)}{r_{e q}}+i_{e q}\right] \\
C_{c} \frac{d \bar{v}_{C_{c}}}{d t} & =\bar{i}_{m}(1-d)-\frac{\bar{v}_{C_{c}}-\left(\bar{v}_{g}+n \bar{v}_{C_{o}}\right)}{r_{e q}} \\
\bar{i}_{g} & =\bar{i}_{m}-\left[\frac{\bar{v}_{C_{c}}-\left(\bar{v}_{g}+n \bar{v}_{C_{o}}\right)}{r_{e q}}+i_{e q}\right] \\
\bar{i}_{D} & =n\left[\frac{\bar{v}_{C_{c}}-\left(\bar{v}_{g}+n \bar{v}_{C_{o}}\right)}{r_{e q}}+i_{e q}\right]
\end{aligned}
$$

El proceso de linealización no es mas que un desarrollo de Taylor de una función, $f(x, y, z, \ldots)$ en torno a un punto determinado $\left(x_{0}, y_{0}, z_{0}, \ldots\right)$, 
considerando únicamente los términos hasta primer orden y despreciando el resto. Por tanto, aplicando el desarrollo de Taylor hasta primer orden a la parte derecha de cada una de las igualdades de las ecuaciones del modelo promediado se consigue llegar a una expresión que permite la obtención del modelo en pequeña señal y también, otra vez, el modelo en régimen permanente. Por ejemplo, la ecuación de la corriente en la bobina de entrada del modelo promediado dada en (E.90) se puede escribir como:

$$
L \frac{d \bar{i}_{m}}{d t}=f_{1}\left(\bar{v}_{g}, d, \bar{v}_{C_{c}}\right)
$$

Linealizando la función $f_{1}$ con el desarrolo de Taylor en un punto de funcionamiento dado por $\left(V_{g}, D, I, V_{c}\right)$ se obtiene (E.92). Por supuesto, las ecuación obtenida no será una aproximación muy buena de la función no lineal cuando se trate de un punto alejado del punto de linealización.

$$
\begin{aligned}
& L_{m}\left(\frac{d I}{d t}+\frac{d \hat{i}_{L_{m}}}{d t}\right)=f_{1}\left(V_{g}, D, V_{c}\right) \\
& +\left.\hat{v}_{g}(t) \frac{\partial f_{1}\left(\bar{v}_{g}, D, V_{c}\right)}{\partial \bar{v}_{g}}\right|_{\bar{v}_{g}=V_{g}}+\left.\hat{v}_{C_{c}} \frac{\partial f_{1}\left(V_{g}, D, \bar{v}_{C_{c}}\right)}{\partial \bar{v}_{C_{c}}}\right|_{\bar{v}_{C_{c}}=V_{c}} \\
& +\left.\hat{d}(t) \frac{\partial f_{1}\left(V_{g}, d, V_{c}\right)}{\partial d}\right|_{d=D}+\text { términos orden superior }
\end{aligned}
$$

A continuación se evalúan las derivadas parciales para la ecuación de la corriente en la bobina de magnetización, $\bar{i}_{m}$. Las derivadas parciales son:

$$
\begin{gathered}
\left.\frac{\partial f_{1}\left(\bar{v}_{g}, D, V_{c}\right)}{\partial \bar{v}_{g}}\right|_{\bar{v}_{g}=V_{g}}=1 \\
\left.\frac{\partial f_{1}\left(V_{g}, D, \bar{v}_{C_{c}}\right)}{\partial \bar{v}_{C_{c}}}\right|_{\bar{v}_{C_{c}=V_{c}}}=-(1-D) \\
\left.\frac{\partial f_{1}\left(V_{g}, d, V_{c}\right)}{\partial d}\right|_{d=D}=V_{c}
\end{gathered}
$$

La ecuación (E.92) queda ahora así:

$$
\begin{aligned}
L_{m}\left(0+\frac{d \hat{i}_{L_{m}}}{d t}\right) & =\left[V_{g}-(1-D) V_{c}\right] \\
& +\hat{v}_{g}-(1-D) \hat{v}_{C_{c}}+V_{c} \hat{d} \\
& + \text { términos de orden superior }
\end{aligned}
$$

Los términos constantes de ambos miembros se igualan para conseguir una ecuación para el modelo en régimen permanente que coincide con la primera 
expresión de (E.77). Los términos de primer orden generan otra ecuación que forma parte del modelo en pequeña señal. El resto de términos de orden superior se desprecian.

Si se hace de forma similar para el restos de ecuaciones del modelo promediado, vistas como

$$
\begin{aligned}
C_{o} \frac{d \bar{v}_{C_{o}}}{d t} & =f_{2}\left(\bar{v}_{g}, d, \bar{v}_{C_{o}}, \bar{v}_{C_{c}}\right) \\
C_{c} \frac{d \bar{v}_{C_{c}}}{d t} & =f_{3}\left(d, \bar{i}_{m}, \bar{v}_{C_{o}}, \bar{v}_{C_{c}}\right) \\
\bar{i}_{g} & =f_{4}\left(\bar{v}_{g}, d, \bar{i}_{m}, \bar{v}_{C_{o}}, \bar{v}_{C_{c}}\right) \\
\bar{i}_{D} & =f_{5}\left(\bar{v}_{g}, d, \bar{i}_{m}, \bar{v}_{C_{o}}, \bar{v}_{C_{c}}\right)
\end{aligned}
$$

será necesario evaluar las funciones $f_{2}, f_{3}, f_{4}$ y $f_{5}$ y sus derivadas parciales en torno al punto de funcionamiento elegido. Las funciones $f_{2}, f_{3}, f_{4}$ y $f_{5}$ evaluadas en el punto de funcionamiento son:

$$
\begin{aligned}
f_{2}\left(V_{g}, D, V_{o}, V_{c}\right) & =-\frac{V_{o}}{R}+n\left[I_{e q}+\frac{V_{c}-\left(V_{g}+n V_{o}\right)}{R_{e q}}\right] \\
f_{3}\left(D, I, V_{o} V_{c}\right) & =(1-D) I-\frac{V_{c}-\left(V_{g}+n V_{o}\right)}{R_{e q}} \\
f_{4}\left(V_{g}, D, I, V_{o}, V_{c}\right) & =I-\left[I_{e q}+\frac{V_{c}-\left(V_{g}+n V_{o}\right)}{R_{e q}}\right] \\
f_{5}\left(D, I, V_{o} V_{c}\right) & =n\left[I_{e q}+\frac{V_{c}-\left(V_{g}+n V_{o}\right)}{R_{e q}}\right]
\end{aligned}
$$

Las derivadas parciales respecto a las variables de estado de las funciones anteriores, y evaluadas en torno al punto de funcionamiento están indicadas a continuación.

$$
\begin{aligned}
& \left.\frac{\partial f_{2}(\ldots)}{\partial \bar{v}_{g}}\right|_{\bar{v}_{g}=V_{g}}=-n \frac{V_{C_{c}}^{2}}{R_{e q}\left(V_{g}+n V_{o}\right)^{2}} \\
& \left.\frac{\partial f_{2}(\ldots)}{\partial d}\right|_{d=D}=-2 n\left[I+\frac{I_{e q}}{1-D}\right] \\
& \left.\frac{\partial f_{2}(\ldots)}{\partial \bar{v}_{C_{o}}}\right|_{\bar{v}_{C_{o}}=V_{o}}=-\frac{1}{R}-n^{2} \frac{V_{C_{c}}^{2}}{R_{e q}\left(V_{g}+n V_{o}\right)^{2}} \\
& \left.\frac{\partial f_{2}(\ldots)}{\partial \bar{v}_{C_{c}}}\right|_{\bar{v}_{C_{c}=V_{c}}}=n \frac{2 I_{e q}}{V_{c}-\left(V_{g}+n V_{o}\right)}+\frac{n}{R_{e q}}
\end{aligned}
$$




$$
\begin{aligned}
& \left.\frac{\partial f_{3}(\ldots)}{\partial \bar{v}_{g}}\right|_{\bar{v}_{g}=V_{g}}=\frac{1}{R_{e q}} \\
& \left.\frac{\partial f_{3}(\ldots)}{\partial d}\right|_{d=D}=-I+2(1-D) \frac{V_{c}-\left(V_{g}+n V_{o}\right)}{2 L_{r} f_{s}}=I \\
& \left.\frac{\partial f_{3}(\ldots)}{\partial \bar{i}_{m}}\right|_{\bar{i}_{m}=I}=(1-D) \\
& \left.\frac{\partial f_{3}(\ldots)}{\partial \bar{v}_{C_{o}}}\right|_{\bar{v}_{C_{o}}=V_{o}}=\frac{(1-D)^{2}}{2 L_{r} f_{s}}=\frac{n}{R_{e q}} \\
& \left.\frac{\partial f_{3}(\ldots)}{\partial \bar{v}_{C_{c}}}\right|_{\bar{v}_{C_{c}}=V_{c}}=-\frac{(1-D)^{2}}{2 L_{r} f_{s}}=-\frac{1}{R_{e q}} \\
& \left.\frac{\partial f_{4}(\ldots)}{\partial \bar{v}_{g}}\right|_{\bar{v}_{g}=V_{g}}=\frac{V_{C_{c}}^{2}}{R_{e q}\left(V_{g}+n V_{o}\right)^{2}} \\
& \left.\frac{\partial f_{4}(\ldots)}{\partial d}\right|_{d=D}=2\left[I+\frac{I_{e q}}{1-D}\right] \\
& \left.\frac{\partial f_{4}(\ldots)}{\partial \bar{i}_{m}}\right|_{\bar{i}_{L_{m}}=I}=1 \\
& \left.\frac{\partial f_{4}(\ldots)}{\partial \bar{v}_{C_{o}}}\right|_{\bar{v}_{C_{o}}=V_{o}}=n \frac{V_{C_{c}}^{2}}{R_{e q}\left(V_{g}+n V_{o}\right)^{2}} \\
& \left.\frac{\partial f_{4}(\ldots)}{\partial \bar{v}_{C_{c}}}\right|_{\bar{v}_{C_{c}=V_{c}}}=-\frac{2 I_{e q}}{V_{c}-\left(V_{g}+n V_{o}\right)}-\frac{1}{R_{e q}} \\
& \left.\frac{\partial f_{5}(\ldots)}{\partial \bar{v}_{g}}\right|_{\bar{v}_{g}=V_{g}}=-n \frac{V_{C_{c}}^{2}}{R_{e q}\left(V_{g}+n V_{o}\right)^{2}} \\
& \left.\frac{\partial f_{5}(\ldots)}{\partial d}\right|_{d=D}=-2 n\left[I+\frac{I_{e q}}{1-D}\right] \\
& \left.\frac{\partial f_{5}(\ldots)}{\partial \bar{v}_{C_{o}}}\right|_{\bar{v}_{C_{o}}=V_{o}}=-n^{2} \frac{V_{C_{c}}^{2}}{R_{e q}\left(V_{g}+n V_{o}\right)^{2}} \\
& \left.\frac{\partial f_{5}(\ldots)}{\partial \bar{v}_{C_{c}}}\right|_{\bar{v}_{C_{c}}=V_{c}}=n \frac{2 I_{e q}}{V_{c}-\left(V_{g}+n V_{o}\right)}+\frac{n}{R_{e q}}
\end{aligned}
$$


Entonces, cada una de las ecuaciones (E.95) quedan de la forma indicada en (E.101), (E.102), (E.103) y (E.104).

$$
\begin{aligned}
& C_{o}\left(0+\frac{d \hat{v}_{C_{o}}}{d t}\right)=-\frac{V_{o}}{R}+n\left[I_{e q}+\frac{V_{c}-\left(V_{g}+n V_{o}\right)}{R_{e q}}\right] \\
& -n \frac{V_{C_{c}}^{2}}{R_{e q}\left(V_{g}+n V_{o}\right)^{2}} \hat{v}_{g}-2 n\left[I+\frac{I_{e q}}{1-D}\right] \hat{d} \\
& -\left[\frac{1}{R}+n^{2} \frac{V_{C_{c}}^{2}}{R_{e q}\left(V_{g}+n V_{o}\right)^{2}}\right] \hat{v}_{C_{o}} \\
& +\left[n \frac{2 I_{e q}}{V_{c}-\left(V_{g}+n V_{o}\right)}+\frac{n}{R_{e q}}\right] \hat{v}_{C_{c}} \\
& + \text { términos de orden superior } \\
& C_{c}\left(0+\frac{d \hat{v}_{C_{c}}}{d t}\right)=(1-D) I-\frac{V_{c}-\left(V_{g}+n V_{o}\right)}{R_{e q}} \\
& +\frac{1}{R_{e q}} \hat{v}_{g}+I \hat{d}+(1-D) \hat{i}_{L_{m}}+\frac{n}{R_{e q}} \hat{v}_{C_{o}}-\frac{1}{R_{e q}} \hat{v}_{C_{c}} \\
& + \text { términos de orden superior } \\
& I_{g}+\hat{i}_{g}=\left[D I-I_{e q}\right] \\
& +\frac{V_{C_{c}}^{2}}{R_{e q}\left(V_{g}+n V_{o}\right)^{2}} \hat{v}_{g}+2\left[I+\frac{I_{e q}}{1-D}\right] \hat{d} \\
& +\hat{i}_{L_{m}}-n \frac{V_{C_{c}}^{2}}{R_{e q}\left(V_{g}+n V_{o}\right)^{2}} \hat{v}_{C_{o}} \\
& -\left[\frac{2 I_{e q}}{V_{c}-\left(V_{g}+n V_{o}\right)}+\frac{1}{R_{e q}}\right] \hat{v}_{C_{c}} \\
& + \text { términos de orden superior } \\
& I_{D}+\hat{i}_{D}=n\left[I_{e q}+\frac{V_{c}-\left(V_{g}+n V_{o}\right)}{R_{e q}}\right] \\
& -n \frac{V_{C_{c}}^{2}}{R_{e q}\left(V_{g}+n V_{o}\right)^{2}} \hat{v}_{g}-2 n\left[I+\frac{I_{e q}}{1-D}\right] \hat{d} \\
& -n^{2} \frac{V_{C_{c}}^{2}}{R_{e q}\left(V_{g}+n V_{o}\right)^{2}} \hat{v}_{C_{o}} \\
& +\left[n \frac{2 I_{e q}}{V_{c}-\left(V_{g}+n V_{o}\right)}+\frac{n}{R_{e q}}\right] \hat{v}_{C_{c}} \\
& + \text { términos de orden superior }
\end{aligned}
$$

Agrupando todos los términos constantes de las ecuaciones (E.94), (E.101), (E.102), (E.103) y (E.104) se obtiene el modelo en régimen permanente que coincide con el descrito en (E.80). 
Tomando todos los términos de primer orden y despreciando los términos de orden superior, se consigue el modelo en pequeña señal para el flyback ZVS dado en (D.133).

$$
\begin{aligned}
L_{m} \frac{d \hat{i}_{L_{m}}}{d t} & =\hat{v}_{g}-(1-D) \hat{v}_{C_{c}}+V_{c} \hat{d} \\
C_{o} \frac{d \hat{v}_{C_{o}}}{d t} & =-\left[\frac{n}{R_{e q}}+n \frac{V_{C_{c}}^{2}-\left(V_{g}+n V_{o}\right)^{2}}{R_{e q}\left(V_{g}+n V_{o}\right)^{2}}\right] \hat{v}_{g}-2 n\left[I+\frac{I_{e q}}{1-D}\right] \hat{d} \\
& -\left[\frac{1}{R}+\frac{n^{2}}{R_{e q}}+n^{2} \frac{V_{C_{c}}^{2}-\left(V_{g}+n V_{o}\right)^{2}}{R_{e q}\left(V_{g}+n V_{o}\right)^{2}}\right] \hat{v}_{C_{o}}+\left[n \frac{2 I_{e q}}{V_{c}-\left(V_{g}+n V_{o}\right)}+\frac{n}{R_{e q}}\right] \hat{v}_{C_{c}} \\
C_{c} \frac{d \hat{v}_{C_{c}}}{d t}= & \frac{1}{R_{e q}} \hat{v}_{g}+I \hat{d}+(1-D) \hat{i}_{L_{m}}+\frac{n}{R_{e q}} \hat{v}_{C_{o}}-\frac{1}{R_{e q}} \hat{v}_{C_{c}} \\
\hat{i}_{g}= & {\left[\frac{1}{R_{e q}}+\frac{V_{C_{c}}^{2}-\left(V_{g}+n V_{o}\right)^{2}}{R_{e q}\left(V_{g}+n V_{o}\right)^{2}}\right] \hat{v}_{g}+2\left[I+\frac{I_{e q}}{1-D}\right] \hat{d} .105 } \\
+ & \hat{i}_{L_{m}}+\left[\frac{n}{R_{e q}}+n \frac{V_{C_{c}}^{2}-\left(V_{g}+n V_{o}\right)^{2}}{R_{e q}\left(V_{g}+n V_{o}\right)^{2}}\right] \hat{v}_{C_{o}}-\left[\frac{2 I_{e q}}{V_{c}-\left(V_{g}+n V_{o}\right)}+\frac{1}{R_{e q}}\right] \hat{v}_{C_{c}} \\
\hat{i}_{D}= & -\left[\frac{n}{R_{e q}}+n \frac{V_{C_{c}}^{2}-\left(V_{g}+n V_{o}\right)^{2}}{R_{e q}\left(V_{g}+n V_{o}\right)^{2}}\right] \hat{v}_{g}-2 n\left[I+\frac{I_{e q}}{1-D}\right] \hat{d} \\
& -\left[\frac{n^{2}}{R_{e q}}+n^{2} \frac{V_{C_{c}}^{2}-\left(V_{g}+n V_{o}\right)^{2}}{R_{e q}\left(V_{g}+n V_{o}\right)^{2}}\right] \hat{v}_{C_{o}}+\left[n \frac{2 I_{e q}}{V_{c}-\left(V_{g}+n V_{o}\right)}+\frac{n}{R_{e q}}\right] \hat{v}_{C_{c}}
\end{aligned}
$$

Con estas expresiones se construye el circuito en pequeña señal de la figura E.13. Los parámetros $f_{6}, j_{7}, g_{7}$, y $g_{g}$ están indicados en la tabla E.4. Además, $r_{7}=1 / g_{7}$.

\begin{tabular}{ccccc}
\hline Convertidor & $f_{6}$ & $j_{7}$ & $g_{7}$ & $g_{g}$ \\
\hline Flyback (boost) & $V_{c}$ & $2\left[I+\frac{I_{e q}}{1-D}\right]$ & $\frac{V_{C_{c}}^{2}-\left(V_{g}+n V_{o}\right)^{2}}{R_{e q}\left(V_{g}+n V_{o}\right)^{2}}$ & $\frac{2 I_{e q}}{V_{c}-\left(V_{g}+n V_{o}\right)}$ \\
\hline
\end{tabular}

Tabla E.4: Parámetros del modelo en pequeña señal del flyback ZVS-boost.

El modelo en pequeña señal, obtenido a partir de la linealización del modelo promediado de orden reducido, también se puede escribir en forma matricial. Así, se definen dos vectores, el vector $\hat{\mathbf{x}}$ para las variables de estado y el vector $\hat{\mathbf{u}}$ para las entradas externas e independientes, y serán en este caso:

$$
\hat{\mathbf{x}}=\left[\begin{array}{l}
\hat{i}_{L_{m}} \\
\hat{v}_{C_{o}} \\
\hat{v}_{C_{c}}
\end{array}\right], \quad \hat{\mathbf{u}}=\left[\begin{array}{c}
\hat{v}_{g} \\
\hat{d}
\end{array}\right], \quad \hat{\mathbf{y}}=\left[\begin{array}{c}
\hat{i}_{g} \\
\hat{i}_{D}
\end{array}\right]
$$




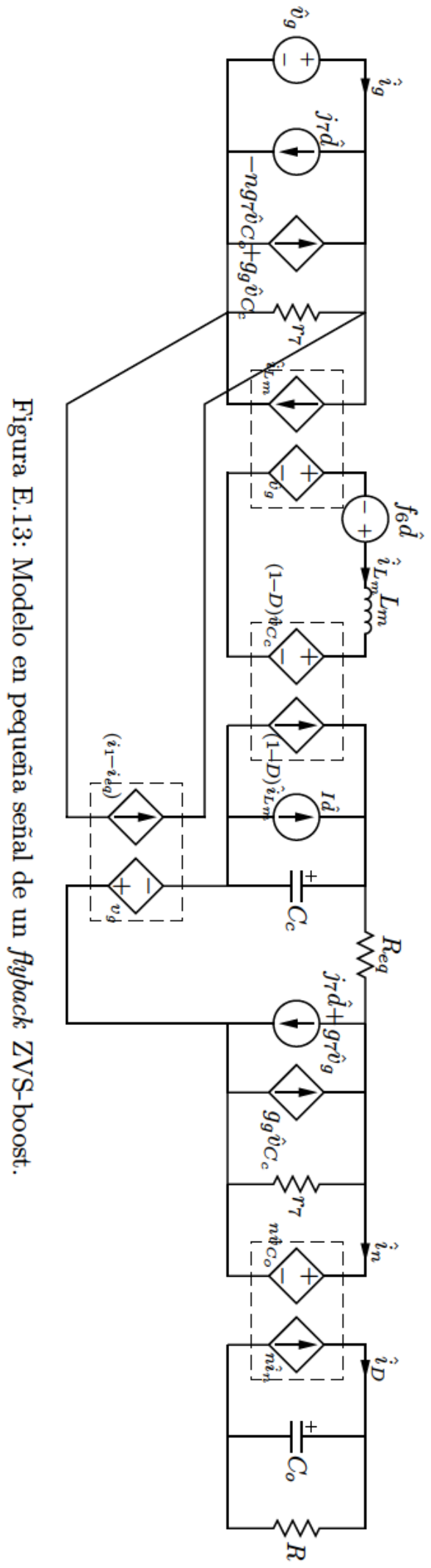


de forma que el modelo en pequeña señal de (E.105) se puede escribir, de la forma siguiente:

$$
\begin{array}{r}
\mathbf{K} \frac{d}{d t}\left[\begin{array}{l}
\hat{i}_{L_{m}} \\
\hat{v}_{C_{o}} \\
\hat{v}_{C_{c}}
\end{array}\right]=\mathbf{A}\left[\begin{array}{l}
\hat{i}_{L_{m}} \\
\hat{v}_{C_{o}} \\
\hat{v}_{C_{c}}
\end{array}\right]+\mathbf{B}\left[\begin{array}{l}
\hat{v}_{g} \\
\hat{d}
\end{array}\right] \\
{\left[\begin{array}{l}
\hat{i}_{g} \\
\hat{i}_{D}
\end{array}\right]=\mathbf{C}\left[\begin{array}{l}
\hat{i}_{L_{m}} \\
\hat{v}_{C_{o}} \\
\hat{v}_{C_{c}}
\end{array}\right]+\mathbf{E}\left[\begin{array}{c}
\hat{v}_{g} \\
\hat{d}
\end{array}\right]}
\end{array}
$$

donde

$$
\begin{aligned}
\mathbf{K} & =\left[\begin{array}{ccc}
L_{m} & 0 & 0 \\
0 & C_{o} & 0 \\
0 & 0 & C_{c}
\end{array}\right], \\
\mathbf{A} & =\left[\begin{array}{ccc}
0 & 0 & D-1 \\
0 & -\frac{1}{R}-\frac{n^{2}}{R_{e q}}-n^{2} g_{7} & \frac{n}{R_{e q}}+n g_{g} \\
1-D & \frac{n}{R_{e q}} & -\frac{1}{R_{e q}}
\end{array}\right], \quad \mathbf{B}=\left[\begin{array}{cc}
-\frac{n}{R_{e q}}-n g_{7} & -n j_{7} \\
\frac{1}{R_{e q}} & I
\end{array}\right], \\
\mathbf{C} & =\left[\begin{array}{ccc}
1 & \frac{n}{R_{e q}}+n g_{7} & -\frac{1}{R_{e q}}-g_{g} \\
0 & -\frac{n^{2}}{R_{e q}}-n^{2} g_{7} & \frac{n}{R_{e q}}+n g_{g}
\end{array}\right], \quad \mathbf{E}=\left[\begin{array}{cc}
\frac{1}{R_{e q}}+g_{7} & j_{7} \\
-\frac{n}{R_{e q}}-n g_{7} & -n j_{7}
\end{array}\right]
\end{aligned}
$$

\section{E.6. Impedancia de salida}

La impedancia de salida en pequeña señal se obtiene a partir del modelo en pequeña señal de la Figura E.13. Para ello hay que anular las entradas independientes $\hat{v}_{g}$ y $\hat{d}$ y conectar una fuente independiente, $\hat{i}_{t}(s)$, en los puntos donde se quiere medir la impedancia. El resto de elementos no se tienen en cuenta. La tensión que genera esta fuente sobre el sistema, $\hat{v}_{t}$, se emplea para obtener $Z_{\text {out }}(s)$ como:

$$
Z_{\text {out }}(s)=\frac{\hat{v}_{t}(s)}{\hat{i}_{t}(s)} \mid \begin{aligned}
& \hat{v}_{g}=0 \\
& \hat{d}=0
\end{aligned}
$$

considerando que $\hat{i}_{t}(s)$ es la intensidad que entra por el terminal de mayor tensión de los dos terminales considerados anteriormente. En el circuito de la Figura E.14 se indica gráficamente la conexión de la fuente independiente al modelo de pequeña señal. Además, se ha indicado como $Z_{t h}$ a la impedancia formada por el condensador del clamp, la resistencia equivalente y la 
bobina principal. Nótese la similitud de este circuito con el equivalente para el flyback ZVS del Anexo anterior, en el que ha cambiado $r_{5} \mathrm{y} g_{f}$ por $r_{7} \mathrm{y}$ $g_{g}$ respectivamente.

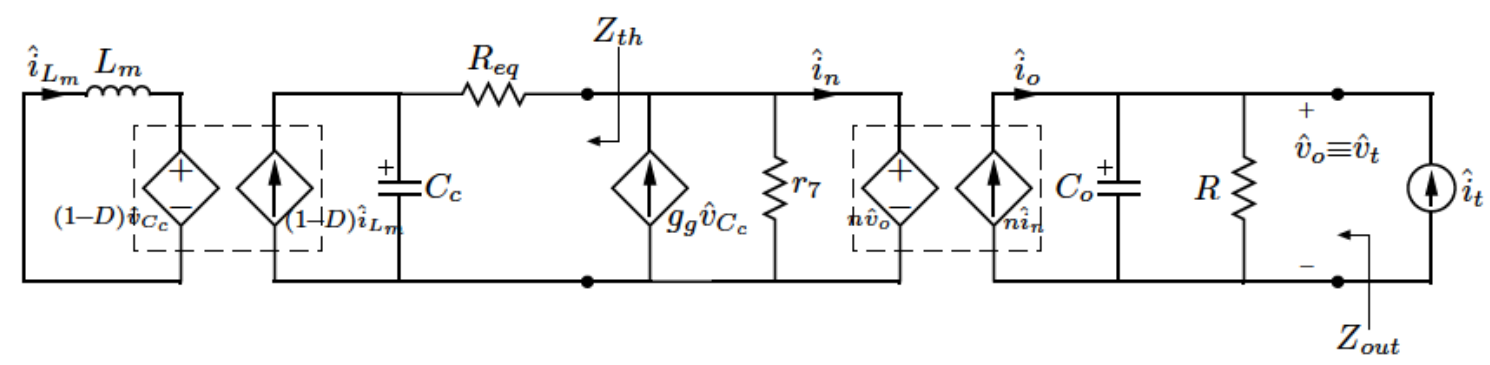

Figura E.14: Circuito para obtener $Z_{\text {out }}(s)$ en un flyback ZVS-boost.

\section{E.6.1. Cálculo de $Z_{t h}$.}

Para obtener esa impedancia se utiliza la parte del circuito anterior que concierne al punto de medida de $Z_{t h}$, en el que se conecta la fuente $\hat{i}_{t}$. Queda el circuito de la Figura E.15. Pasando la bobina $L_{m}$, al otro lado de las fuentes dependientes, aparece con otro valor y en paralelo con $C_{c}$. Este conjunto se encuentra en serie con $R_{e q}$. Por tanto la expresión de esta impedancia es:

$$
Z_{t h}(s)=\frac{1}{\frac{1}{s L_{m}^{\prime}}+s C_{c}}+R_{e q}=R_{e q}+\frac{s L_{m}^{\prime}}{L_{m}^{\prime} C_{c} s^{2}+1}
$$

donde $L_{m}^{\prime}=L_{m} /(1-D)^{2}$.

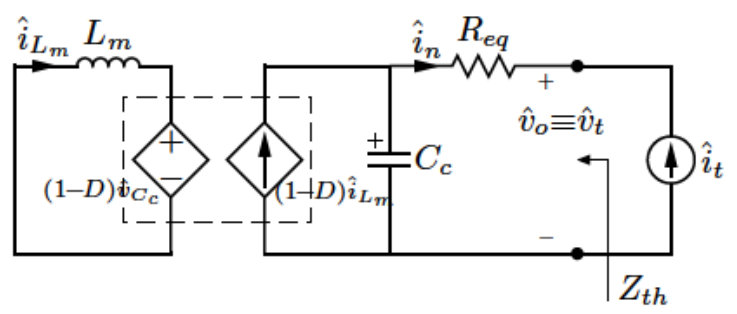

Figura E.15: Circuito para calcular $Z_{t h}(s)$ en un flyback ZVS-boost.

A esta expresión también se puede llegar desde las ecuaciones del modelo en pequeña señal indicado en (E.105). Basta con anular los términos independientes, $\hat{v}_{g}(t)$ y $\hat{d}(t)$ y cambiar la ecuación relativa a la tensión del condensador $C_{o}$, puesto que éste junto con la carga $R$ quedan fuera y en su lugar se ha conectado una fuente de intensidad, $\hat{i}_{t}(t)$. La nueva ecuación relaciona la intensidad en $R_{e q}$ con la intensidad que entrega esa fuente. También 
hay que sustituir el término $\hat{v}_{C_{o}}(t)$ por $\hat{v}_{t}(t)$ en el resto de ecuaciones. Finalmente, tras aplicar Laplace, las ecuaciones resultantes quedan indicadas en (E.111):

$$
\begin{aligned}
s L \hat{i}_{L_{m}}(s) & =-(1-D) \hat{v}_{C_{c}}(s) \\
-R_{e q} \hat{i}_{t}(s) & =\hat{v}_{C_{c}}(s)-\hat{v}_{t}(s) \\
s C_{c} \hat{v}_{C_{c}}(s) & =(1-D) \hat{i}_{L_{m}}(s)+\hat{i}_{t}(s)
\end{aligned}
$$

Despejando $\hat{i}_{L_{m}}$ de la primera ecuación y sustituyéndola en la tercera ecuación se despeja $\hat{v}_{C_{c}}(s)$ para poder emplearla en la segunda ecuación.

$$
\left.\begin{array}{l}
\hat{i}_{L_{m}}(s)=-\frac{(1-D)}{s L_{m}} \hat{v}_{C_{c}}(s) \\
\hat{v}_{C_{c}}(s)=\frac{\hat{i}_{t}(s)}{\frac{1}{s L_{m}^{\prime}}+s C}
\end{array}\right\} \Rightarrow-R_{e q} \hat{i}_{t}(s)=\frac{\hat{i}_{t}(s)}{\frac{1}{s L_{m}^{\prime}}+s C}-\hat{v}_{t}(s)
$$

Ahora se puede conseguir una expresión de la impedancia de salida que coincide con el mostrado en (E.110).

$$
\hat{v}_{t}(s)=\hat{i}_{t}(s)\left[R_{e q}+\frac{s L_{m}^{\prime}}{L_{m}^{\prime} C_{c} s^{2}+1}\right] \Rightarrow Z_{t h}(s)=R_{e q}+\frac{s L_{m}^{\prime}}{L_{m}^{\prime} C_{c} s^{2}+1}
$$

En la Figura E.16 se ha representado esta impedancia para un flyback ZVS-boost con los parámetros de la Tabla E.1. También están representados los módulos de las impedancias que forman parte de $Z_{t h}(s)$, esto es, la bobina $L_{m}^{\prime}$, el condensador $C_{c}$ y el término de resistencia equivalente $R_{e q}$.

$\mathrm{Al}$ hacer $s=0$ se obtiene la impedancia en continua que es igual al término $R_{e q}$ :

$$
Z_{t h}(0)=Z_{e q}(0)=R_{e q}
$$

Este mismo resultado se obtiene analizando el circuito de la Figura E.15 cuando la bobina y el condensador se convierten en un cortocircuito y en un circuito abierto respectivamente. La fuente independiente de intensidad ahora es una fuente constante conectada en los terminales donde se va a medir esa resistencia. El circuito modificado está representado en la Figura E.17:

Se obtiene una resistencia de salida igual a:

$$
R_{t h}(s)=\frac{R_{e q}}{n^{2}}
$$

\section{E.6.2. Cálculo de $Z_{\text {out }}$.}

A la vista del circuito de la Figura E.14, se establece la siguiente relación de tensiones:

$$
-(1-D) \hat{v}_{C_{c}}(s)=s L_{m} \hat{i}_{L_{m}}(s)
$$




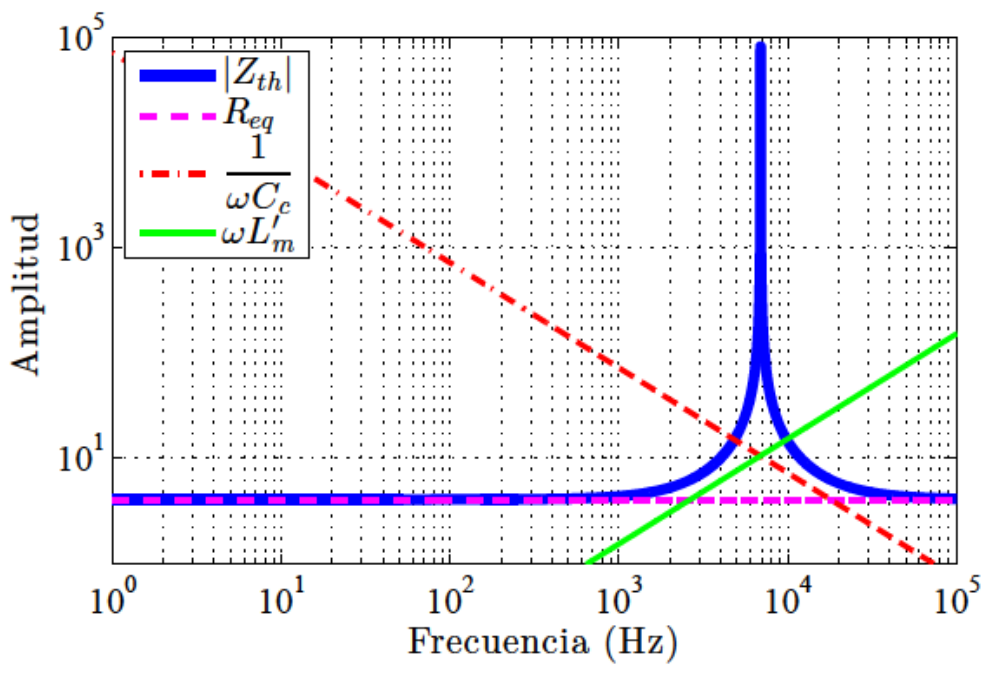

Figura E.16: Representación gráfica de $\left|Z_{t h}(s)\right|$ y los elementos que la forman.

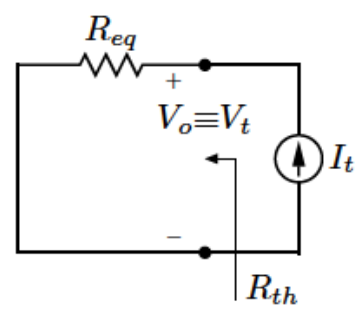

Figura E.17: Circuito empleado para el cálculo de $R_{t h}$ de un flyback ZVS.

Por otro lado, se establece la relación de corrientes:

$$
(1-D) \hat{i}_{L_{m}}(s)=s C_{c} \hat{v}_{C_{c}}(s)+\frac{\hat{v}_{C_{c}}(s)-n \hat{v}_{t}(s)}{R_{e q}}
$$

Pasando la bobina $L_{m}$ al otro lado de las fuentes dependientes, aparece una bobina equivalente $L_{m}^{\prime}$ que está en paralelo con $C_{e q}$, como está indicado en el circuito de la Figura E.18a.

Despejando $\hat{v}_{C_{c}}$ de (E.117), y sustituyendo la expresión de $\hat{i}_{L_{m}}$ que se deduce de (E.116) se obtiene una expresión en función de $\hat{v}_{t}$ como:

$$
\begin{aligned}
& \hat{i}_{L_{m}}(s)=-\frac{(1-D) \hat{v}_{C_{c}}(s)}{s L_{m}} \\
& \hat{v}_{C_{c}}(s)=\frac{n \hat{v}_{t}(s)}{R_{e q}} \underbrace{\left[s C_{c}+\frac{1}{R_{e q}}+\frac{1}{s L_{m}^{\prime}}\right]^{-1}}_{Z_{1}(s)}
\end{aligned}
$$




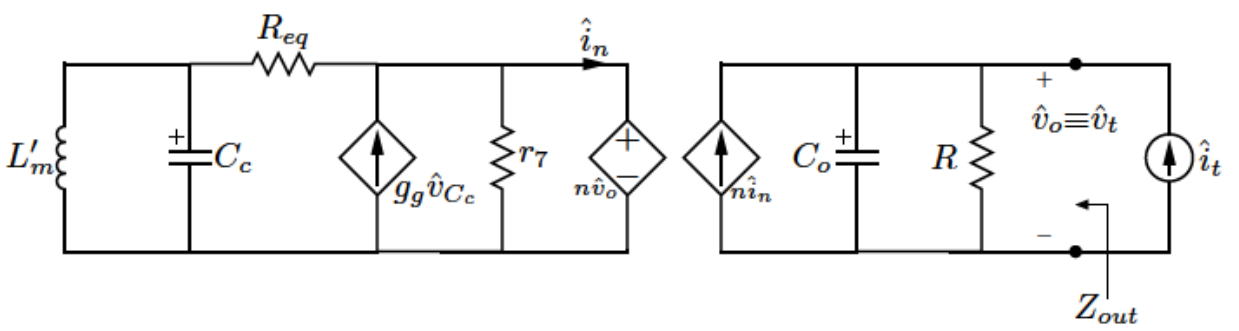

(a)

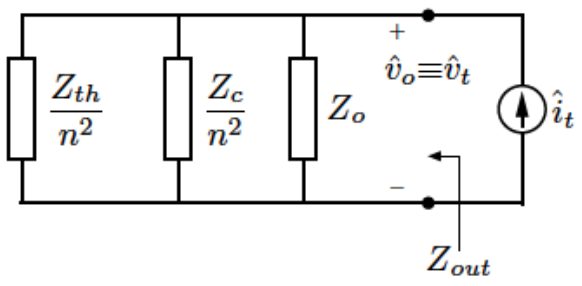

(b)

Figura E.18: Circuito de la Figura E.14 a) tras cambiar $L_{m}$ de posición; b) y agrupando elementos.

donde se han agrupado diferentes términos en $Z_{1}(s)$. Dada la posición de la fuente de corriente $g_{g} \hat{v}_{C_{c}}$, sobre la que aparece la tensión $n \hat{v}_{t}$, se puede interpretar como una impedancia, $Z_{c}$, de expresión:

$$
Z_{c}(s)=-\frac{R_{e q}}{g_{g} Z_{1}(s)}
$$

Agrupando, elementos, se puede observar que $Z_{\text {out }}$ es el paralelo de tres impedancias: la impedancia $Z_{t h}$ con un factor de $n^{2}$ cuando se pasa al otro lado de las bobinas acopladas, y que engloba los elementos $R_{e q}, C_{c}$ y $L_{m}^{\prime}$; la impedancia $Z_{c}$ también pasada al otro lado de las bobinas acopladas; y la impedancia $Z_{o}$, que es el paralelo de $C_{o}, R$ y $r_{7}$ previamente ha pasado al otro lado. Así se ha representado en la Figura E.18b.

$$
Z_{\text {out }}(s)=Z_{o}(s)\left\|\frac{Z_{c}(s)}{n^{2}}\right\| \frac{Z_{t h}(s)}{n^{2}}
$$

A esta expresión también se puede llegar desde las ecuaciones indicadas en (E.121), obtenidas a partir del modelo en pequeña señal indicado en (E.105) donde se anulan los términos independientes, $\hat{v}_{g}(t)$ y $\hat{d}(t)$. Después, hay que cambiar la ecuación relativa a la tensión del condensador $C_{o}$, para introducir la intensidad, $\hat{i}_{t}(t)$. Tras cambiar el término $\hat{v}_{C_{o}}(t)$ por 
$\hat{v}_{t}(t)$ se aplica Laplace a las ecuaciones modificadas, y quedan:

$$
\begin{aligned}
s L_{m} \hat{i}_{L_{m}}(s) & =-(1-D) \hat{v}_{C_{c}}(s) \\
\hat{i}_{t}(s) & =\hat{v}_{t}(s) \overbrace{\left[s C_{o}+\frac{1}{R}+\frac{n^{2}}{r_{7}}\right]}^{Z_{o}^{-1}(s)}-n g_{g} \hat{v}_{C_{c}}(s)-n \frac{\hat{v}_{C_{c}}(s)-n \hat{v}_{t}(s)}{R_{e q}} \\
s C_{c} \hat{v}_{C_{c}}(s) & =(1-D) \hat{i}_{L_{m}}(s)-\frac{\hat{v}_{C_{c}}(s)-n \hat{v}_{t}(s)}{R_{e q}}
\end{aligned}
$$

De la primera y tercera ecuación se obtiene una expresión para $\hat{v}_{C_{c}}(s)$ igual a la indicada en (E.118), que sustituida en la segunda ecuación permite obtener $\hat{i}_{t}(s)$ en función de $\hat{v}_{t}(s)$ como:

$$
\hat{i}_{t}(s)=\frac{\hat{v}_{t}(s)}{Z_{o}(s)}-\hat{v}_{t}(s) \frac{n^{2} g_{g} Z_{1}(s)}{R_{e q}}-n^{2} \hat{v}_{t}(s) \frac{\frac{Z_{1}(s)}{R_{e q}}-1}{R_{e q}}
$$

La impedancia de salida tiene la expresión:

$$
Z_{\text {out }}(s)=\left[\frac{1}{Z_{o}(s)}-n^{2} g_{g} \frac{Z_{1}(s)}{R_{e q}}+n^{2} \frac{R_{e q}-Z_{1}(s)}{R_{e q}^{2}}\right]^{-1}
$$

que es equivalente a la obtenida anteriormente en (E.120) puesto que:

$$
\begin{aligned}
& Z_{1}(s)=\left[\frac{1}{R_{e q}}+\frac{1}{s L_{m}^{\prime}}+s C_{c}\right]^{-1}=\frac{R_{e q} s L_{m}^{\prime}}{R_{e q}+s L_{m}^{\prime}+R_{e q} L_{m}^{\prime} C_{c} s^{2}} \\
& R_{e q}-Z_{1}(s)=\frac{R_{e q}^{2}+\underline{R}_{e q} s E_{m}^{\prime}+R_{e q}^{2} L_{m}^{\prime} C_{c} s^{2}-\underline{R}_{e q} s E_{m}^{\prime}}{R_{e q}+s L_{m}^{\prime}+R_{e q} L^{\prime} C_{c} s^{2}}=\frac{R_{e q}^{2}}{Z_{t h}(s)}
\end{aligned}
$$

Entonces, la impedancia de salida indicada en (E.120) y en (E.123) queda finalmente como:

$$
Z_{\text {out }}(s)=\left[\frac{1}{Z_{o}(s)}-\frac{n^{2}}{Z_{c}(s)}+\frac{n^{2}}{Z_{t h}(s)}\right]^{-1}
$$

En la Figura E.19 se ha representado el módulo de esta impedancia para un flyback ZVS-boost, a partir de los parámetros indicados en la Tabla E.1 junto con el módulo de los términos que la forman, $\left|Z_{t h} / n^{2}\right|,\left|Z_{c}(s) / n^{2}\right|$ y $\left|Z_{o}(s)\right|$.

Al hacer $s=0$ se obtiene la impedancia de salida en continua, que es el paralelo de los términos $R_{e q} / n^{2}, r_{7} / n^{2}$ y $R$. Y se corresponde con la asíntota horizontal de $Z$ out $(s)$ a bajas frecuencias.

$$
Z_{\text {out }}(0)=\frac{1}{\frac{1}{Z_{o}(0)}-\frac{n^{2}}{Z_{c}(0)}+\frac{n^{2}}{Z_{t h}(0)}}=\left[\frac{n^{2}}{r_{7}}+\frac{1}{R}+\frac{n^{2}}{R_{e q}}\right]^{-1}
$$




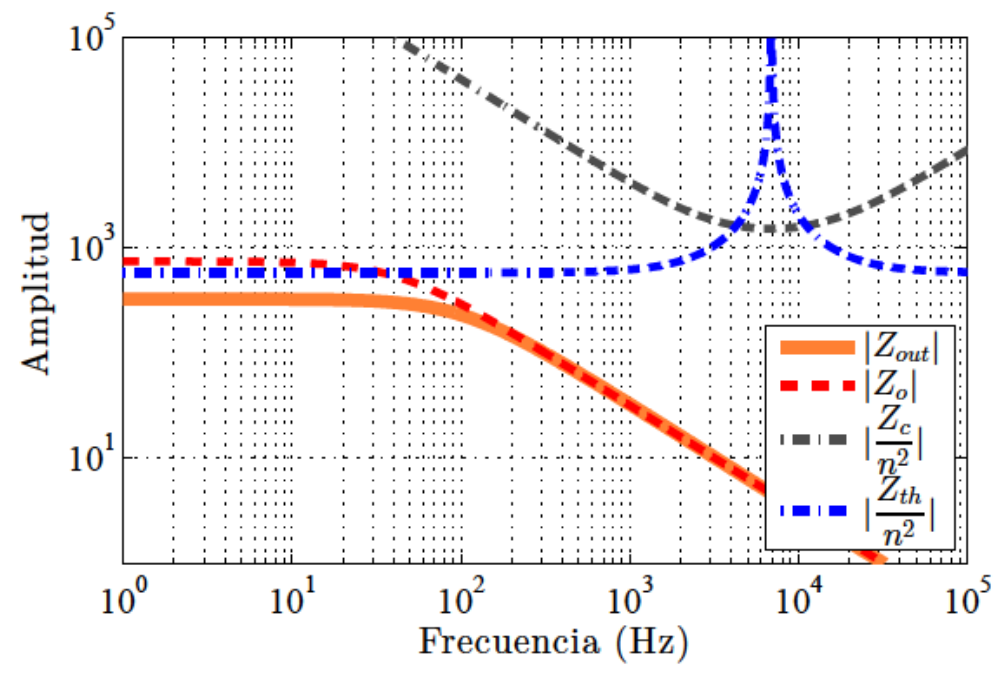

Figura E.19: Representación gráfica de $\left|Z_{\text {out }}(s)\right|$ y los elementos que la forman.

\section{E.6.3. Validación de las expresiones obtenidas.}

La validación de la expresión de la impedancia, se comprueban con la simulación de un convertidor conmutado al que se le mide la impedancia de salida. Con los parámetros de funcionamiento recogidos en la Tabla E.1 se añade a la salida una fuente de corriente de frecuencia variable y se mide la variación que experimenta la tensión de salida.

Como las expresiones de impedancia se han obtenido a partir del modelo promediado, parece lógico obtener la impedancia sobre la simulación de este modelo y, comparar los resultados obtenidos con la teoría. En este caso, se emplearía el ciclo de trabajo obtenido para el modelo promediado. Si se emplea la simulación del convertidor conmutado para medir la impedancia, debería considerarse un valor del ciclo de trabajo para el circuito conmutado que contenga la corrección propuesta en el apartado correspondiente.

En la Figura D.32 se ha representado los valores obtenidos en la medida de la impedancia de un flyback ZVS simulado con los parámetros indicados en la Tabla E.1. También se ha representado el módulo de la impedancia teórica, $\left|Z_{\text {out }}(s)\right|$.

Como ya se conocía, presenta una asíntota horizontal, que es el paralelo de $R, R_{e q} / n^{2}, r_{7} / n^{2}$. A la vista de estas curvas, se observa la coincidencia de la impedancia teórica y los valores obtenidos en la simulación, por lo que se concluye la validez de las expresiones de impedancia obtenidas de forma teórica para el modelo promediado y el circuito conmutado. 


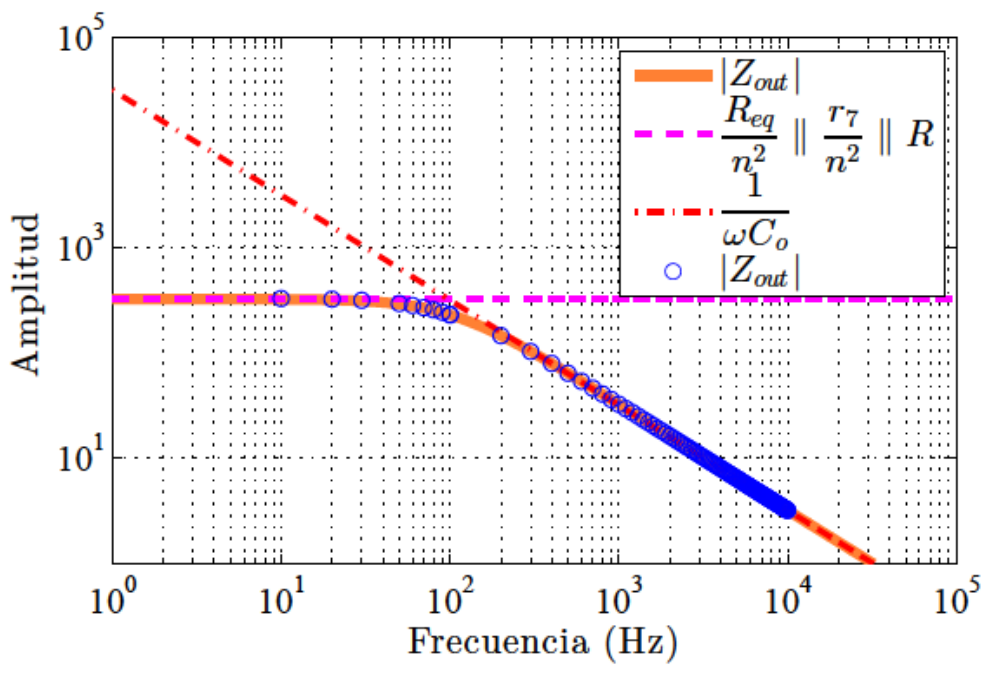

Figura E.20: Representación gráfica de $\left|Z_{\text {tot }}(s)\right|,\left|Z_{\text {out }}(s)\right|$ y resultados de simulación.

En el caso del reparto de corrientes entre varias etapas en paralelo para alimentar una carga común, la impedancia de salida de cada una de las etapas se debe determinar desde el punto común de conexión. Existiendo la posibilidad de considerar un único condensador de salida común a todas las etapas (aunque también podría tratarse el caso de un condensador en cada etapa). El análisis sería similar al realizado en el apartado anterior, con la precaución de no incluir ni $R$ ni $C_{o}$ dentro de $Z_{o}$.

Por ejemplo, la impedancia multietapa sin el condensador de salida, $Z_{m e}(s)$ se corresponde con la expresión:

$$
Z_{m e}(s)=\frac{1}{n^{2}}\left[\frac{1}{r_{7}}-\frac{g_{f} Z_{1}(s)}{R_{e q}}+\frac{1}{Z_{t h}(s)}\right]^{-1}
$$

En la Figura E.21 se ha representado el módulo de esta impedancia y las asíntotas correspondientes:

Haciendo $s=0$ se consigue la asíntota de baja frecuencia, que es el paralelo de $R_{e q}$ y de $r_{7}$ afectados del factor $n^{2}$. Una expresión equivalente se consigue al emplear sus expresiones indicadas en la tabla E.4, y ayudándose de la primera y tercera ecuación del modelo en régimen permanente indicado 


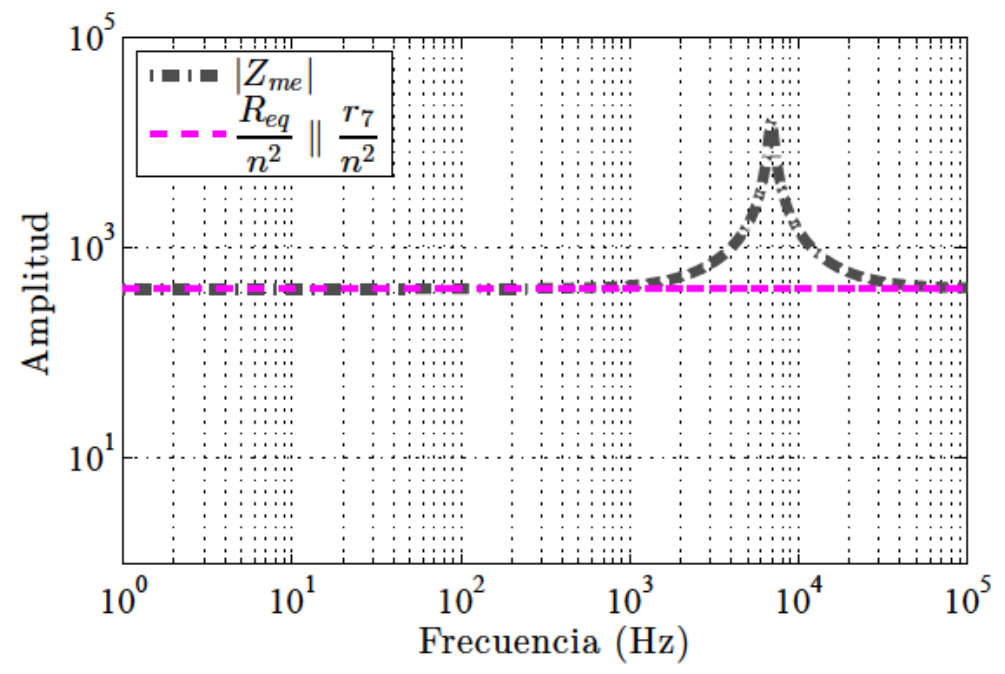

Figura E.21: Representación gráfica de $\left|Z_{m e}(s)\right|$.

en (E.80). Entonces:

$$
\begin{aligned}
Z_{m e}(0) & =\frac{1}{n^{2}}\left[\frac{V_{C_{c}}^{2}-\left(V_{g}+n V_{o}\right)^{2}}{R_{e q}\left(V_{g}+n V_{o}\right)^{2}}+\frac{1}{R_{e q}}\right]^{-1} \\
& =\frac{R_{e q}}{n^{2}}\left[\frac{V_{C_{c}}^{2}-\left(V_{g}+n V_{o}\right)^{2}+\left(V_{g}+n V_{o}\right)^{2}}{\left(V_{g}+n V_{o}\right)^{2}}\right]^{-1}=\frac{R_{e q}}{n^{2}}\left[\frac{V_{g}+n V_{o}}{V_{c}}\right]^{2} \\
& =\frac{R_{e q}}{n^{2}}\left[\frac{\left(V_{g}+n V_{o}\right)(1-D)}{V_{g}}\right]^{2}=\frac{2 L r f}{n^{2}}\left[\frac{V_{g}+n V_{o}}{V_{g}}\right]^{2}
\end{aligned}
$$

Cuando $s$ se hace muy grande, $Z_{1}(s)$ tiende a cero, mientras que $Z_{t h}(s)$ y $Z_{o}$ se transforman en $R_{e q}$ y $r_{7} / n^{2}$ respectivamente, de forma que la asíntota para altas frecuencias coincide con la asíntota de bajas frecuencias. 



\section{Apéndice $\mathrm{F}$}

\section{Convertidor flyback ZVS modificado}

\section{F.1. Descripción y funcionamiento.}

En la Figura F.1 ha representado un convertidor flyback con enclavamiento activo tipo boost y real, al que se añade un diodo, $D_{c}$, entre el terminal de masa y el nodo $A$. De ahora en adelante, se citará esta topología como flyback ZVS-modificado. En la misma figura están presentes las capacidades parásitas existentes en el flyback ZVS-real, que son la capacidad del secundario de las bobinas acopladas, $C_{t}$, la capacidad del diodo de salida, $C_{f} ; \mathrm{y}$ una nueva capacidad parásita debida al nuevo diodo de clamp, $C_{d}$.

La novedad de esta topología consiste en eliminar la resonancia que hay en el convertidor flyback ZVS-real debida a la interacción de la bobina resonante, $L_{r}$ con las capacidades parásitas presentes en la topología inicial. A través del diodo $D_{c}$ se fija la tensión del nodo $A$ a un valor constante, quedando la capacidad $C_{e q}$ sometida a una tensión constante en la que no hay ninguna oscilación. Esta topología se va a denominar flyback ZVS-modificado. Durante parte del funcionamiento del circuito todas las capacidades quedan fijadas a una tensión constante por lo que no tendrán interacción con la inductancia parásita del circuito.

Desde el punto de vista del análisis del circuito, las capacidades parásitas se pueden agrupar en una única capacidad, $C_{c l}$, en paralelo con el nuevo diodo añadido al circuito. Así está indicado en la Figura F.2. Su valor es:

$$
C_{c l}=C_{d}+\left(\frac{n_{2}}{n_{1}}\right)^{2}\left(C_{t}+C_{f}\right)
$$




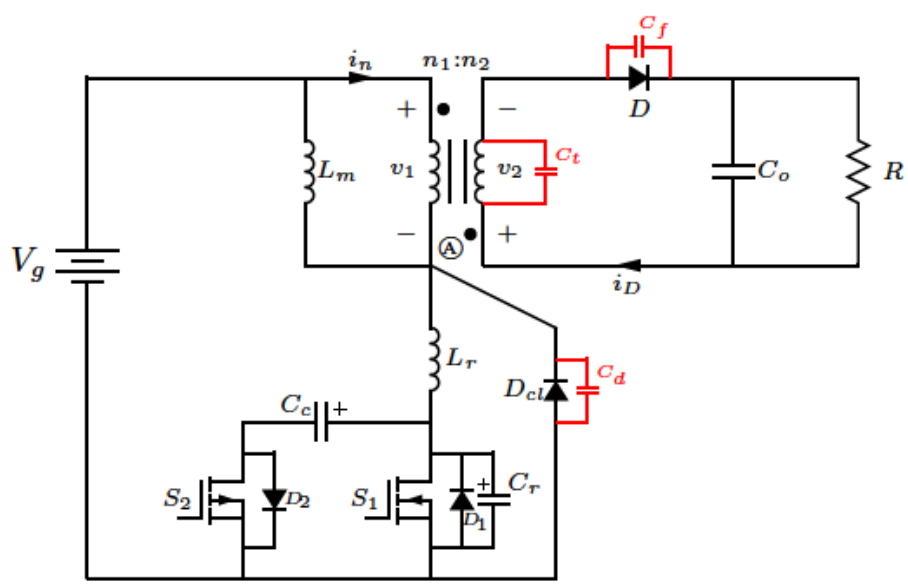

Figura F.1: Flyback ZVS-modificado. Se añade un diodo $D_{c}$ entre A y masa al flyback ZVS-real.

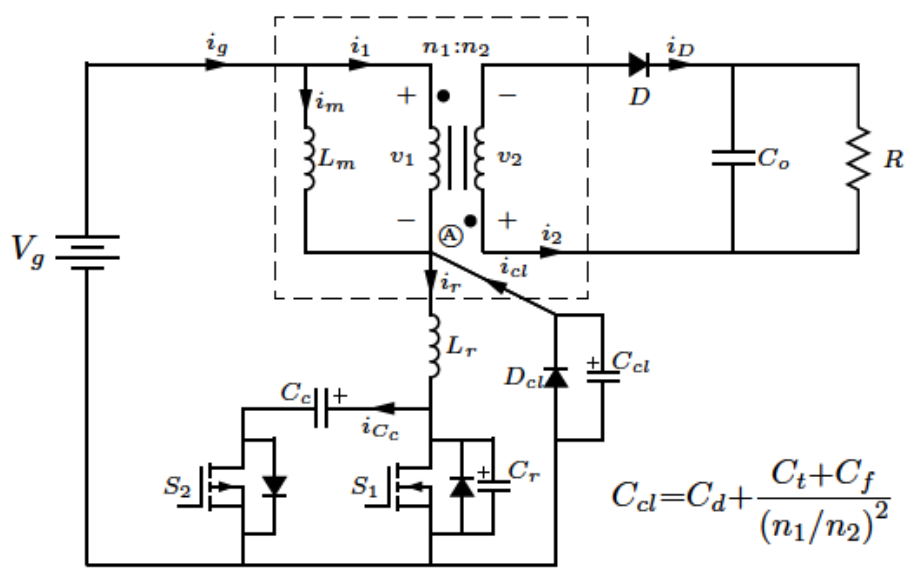

Figura F.2: Flyback ZVS-modificado tras agrupar las capacidades parásitas.

En las bobinas acopladas, se puede comprobar la corriente del diodo de salida, $i_{D}(t)$, está relacionada con la corriente de primario, $i_{1}(t)$ de la siguiente forma:

$$
n_{1} i_{1}(t)=n_{2} i_{2}(t)=-n_{2} i_{D}(t)
$$

donde se ha considerado el sentido positivo de $i_{1}(t)$ entrando por el punto de primario y de $i_{2}(t)$ saliente por el punto de secundario.

Considerando $i_{c l}(t)$ como la corriente que circula por la rama del diodo de clamp, se aplica la ley de nodos de Kirchhoff en (A):

$$
i_{m}(t)+i_{1}(t)=i_{r}(t)-i_{c l}(t)=i_{r}(t)-\left(i_{D_{c l}}(t)-i_{C_{c l}}(t)\right)
$$

Siendo $i_{m}(t)$ la corriente que circula por la bobina de magnetización, $i_{r}(t)$ la corriente circulante por la bobina resonante. Las intensidades en el diodo $D_{c}$ 
y en el condensador $C_{c l}$ se denotan como $i_{D_{c l}}(t)$ e $i_{C_{c l}}(t)$ respectivamente. Combinando las expresiones (F.2) y (F.3) se consigue:

$$
i_{m}(t)+i_{c l}(t)=i_{r}(t)+\frac{1}{n} i_{D}(t)
$$

siendo $n=n_{1} / n_{2}$.

Por otro lado la corriente de entrada, $i_{g}(t)$ cumple la relación (F.5):

$$
i_{g}(t)=i_{1}+i_{m}(t)
$$

que puede relacionarse con la corriente de salida $i_{D}$ de la siguiente forma:

$$
i_{g}(t)+\frac{1}{n} i_{D}(t)=i_{m}(t)
$$

También se puede relacionar con $i_{c l}(t)$ a partir de (F.3) como:

$$
i_{g}(t)+i_{c l}(t)=i_{r}(t)
$$

La corriente en el diodo de salida se obtiene fácilmente a partir de estas dos últimas ecuaciones como:

$$
i_{D}(t)=n\left[i_{m}(t)-i_{r}(t)+i_{c l}(t)\right]
$$

\section{F.1.1. Etapas.}

El convertidor evoluciona por ocho etapas a lo largo de un periodo de conmutación. Estas ocho etapas y los elementos que intervienen en cada una de ellas está representada en la Figura F.3. Se ha tomado $L_{m}$ lo suficientemente grande como para que su corriente sea constante, y de valor $I$, mientras que se considera $L r$ pequeña en comparación con $L_{m}$. El valor de los condensadores $C_{o}$ y $C_{c}$, es tal que la tensión en sus extremos se mantiene constante, de valores $V_{o}$ y $V_{c}$.

Etapa $1\left[t_{0}-t_{1}\right]$ : Antes de $t_{0}$, el diodo de clamp, $D_{c l}$ y el interruptor principal $S_{1}$ están conduciendo, y el interruptor auxiliar $S_{2}$ está abierto. La corriente que está pasando por la bobina $L_{r}$ es $I+I_{D c}$. En $t=t_{0}, S_{1}$ pasa a OFF y la corriente $i_{r}$ disminuye mientras se carga el condensador $C_{r}$ de forma resonante. Esta etapa acaba cuando el condensador se ha cargado a la tensión $V_{c}$. La corriente por $D_{c l}$ también disminuye de igual forma que $i_{r}$.

Etapa $2\left[t_{1}-t_{2}\right]$ : Cuando $v_{C_{r}}$ alcanza la tensión $V_{c}$, el diodo de $S_{2}$ empieza a conducir, mientras que $i_{r}$ evoluciona linealmente hasta alcanzar el valor de $I$, al estar sometida a una tensión constante de valor $-V_{c}$. La corriente de $D_{c}$ disminuye linealmente hasta anularse.

Etapa $3\left[t_{2}-t_{3}\right]$ : El diodo $D_{c l}$ deja de conducir. Como $i_{r}$ sigue disminuyendo, la capacidad $C_{c l}$, aumenta su tensión desde cero hasta $V_{g}+n V_{o}$, de 


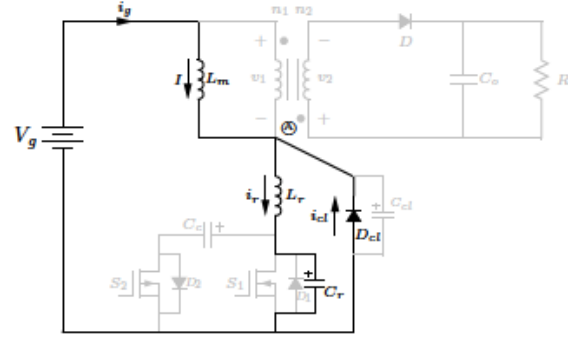

(a)

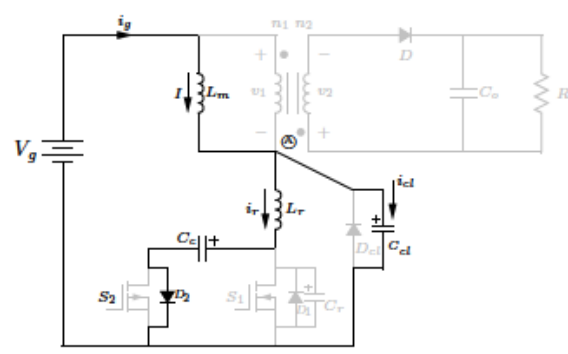

(c)

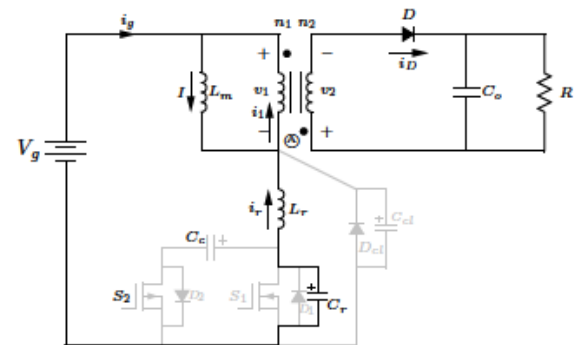

(e)

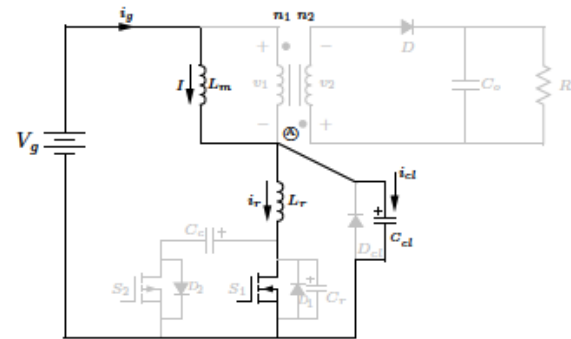

(g)

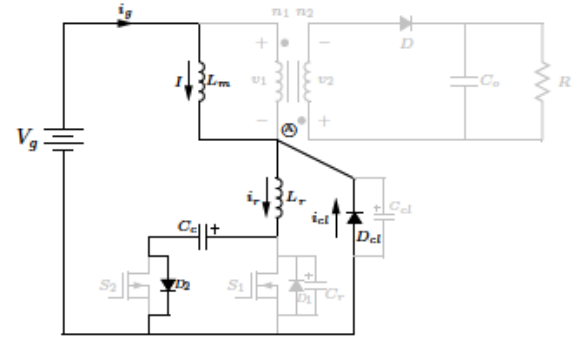

(b)

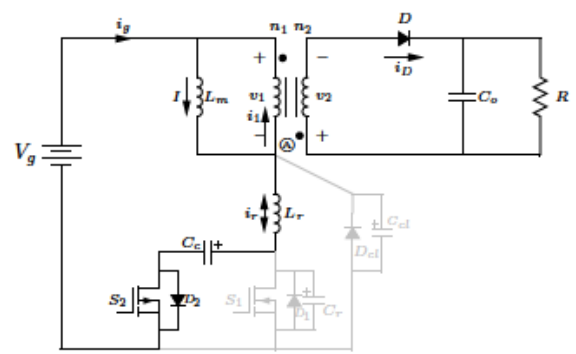

(d)

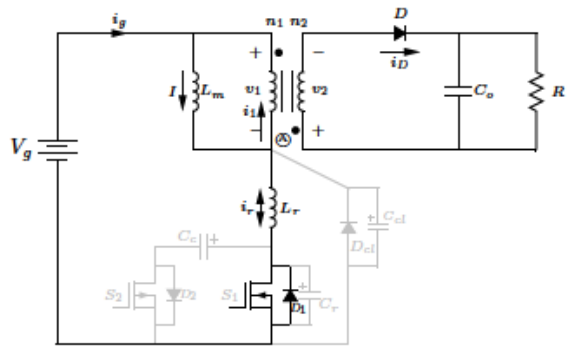

(f)

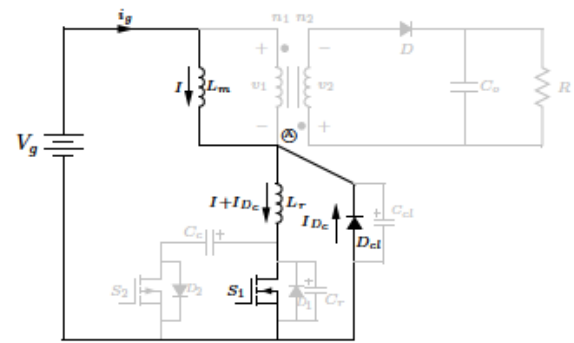

(h)

Figura F.3: Etapas por las que evoluciona el flyback ZVS modificado durante un periodo: (a) Etapa 1. (b) Etapa 2. (c) Etapa 3. (d) Etapa 4. (e) Etapa 5. (f) Etapa 6. (g) Etapa 7. (h) Etapa 8.

forma resonante con $L_{r}$. Esta tensión se alcanza en $t_{3}$.en este instante el diodo de salida $D$ queda polarizado en directa y empieza a conducir. 
Etapa $4\left[t_{3}-t_{4}\right]$ : A partir de $t_{3}$, la corriente $i_{r}$ evoluciona de forma lineal, porque la bobina está sometida a una tensión constante de valor $V_{g}+n V_{o}-V_{c}$. En algún momento de este intervalo, y antes de que $i_{r}$ cambie su sentido, se puede conmutar $S_{2}$ sin pérdidas porque no tiene tensión en su extremos al estar conduciendo su diodo. Esta etapa acaba cuando $S_{2}$ pase a OFF.

Etapa $5\left[t_{4}-t_{5}\right]$ : Ahora la corriente de la bobina resonante descarga la energía almacenada en $C_{r}$ hasta hacer su tensión nula. En $t_{5}$ se hace $v_{C_{r}}=0$.

Etapa $6\left[t_{5}-t_{6}\right]$ : En el instante que la tensión de $C_{r}$ es nula, la corriente pasa a circular por el diodo que hay en paralelo con $S_{1}$ y es posible su conmutación con tensión nula. La conmutación debe realizarse antes de que la corriente $i_{r}$ cambie otra vez de polaridad. Su evolución es lineal porque $L_{r}$ está sometida a una tensión $V_{g}+n V_{o}$. También evoluciona de forma lineal la corriente en $D$, al final de esta etapa alcanzan los valores de $I$ y 0 respectivamente. En este momento, $D$ deja de conducir.

Etapa $7\left[t_{6}-t_{7}\right]$ : Esta etapa comienza cuando el diodo $D$ ha dejado de conducir, y la capacidad $C_{c l}$ cargada a un valor constante, se descarga de forma resonante sobre $L_{r}$ aumentando su corriente desde $I$ hasta un valor $I+I_{D c}$ que coincide con la descarga todal de la capacidad mencionada.

Etapa $8\left[t_{7}-t_{8}\right]$ : La corriente por $L_{r}$ es constante, de valor $I+I_{D c}$. Esta etapa termina cuando $S_{1}$ pasa a OFF.

Para analizar el funcionamiento del circuito se recurre a los circuitos equivalentes que están representados en la Figura F.4.

Tras analizar los diferentes circuitos equivalentes, se obtienen las expresiones de las principales magnitudes, que están representadas gráficamente en la Figura F.5. La duración de cualquiera de las ocho etapas está indicada con la letra $d$ y un subíndice que indica su número. Por ejemplo, la duración del intervalo 7 es igual a $t_{7}-t_{6}=d_{7} T$.

$$
v_{C_{r}}(t)=\left\{\begin{array}{lc}
\left(I+I_{D_{c}}\right) Z_{r} \sin \left(\omega_{r}\left(t-t_{0}\right)\right) & t \in\left[t_{0}, t_{1}\right] \\
V_{c} & t \in\left[t_{1}, t_{4}\right] \\
V_{g}+n V_{o}-\left(V_{g}+n V_{o}-V_{c}\right) \cos \left(\omega_{r}\left(t-t_{4}\right)\right) & t \in\left[t_{4}, t_{5}\right] \\
\quad+I_{r 4} Z_{r} \sin \left(\omega_{r}\left(t-t_{4}\right)\right) & \\
0 & \text { resto }
\end{array}\right.
$$




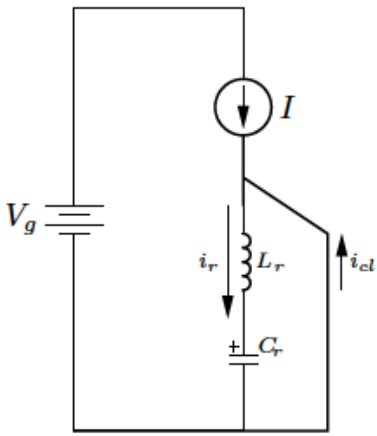

(a)

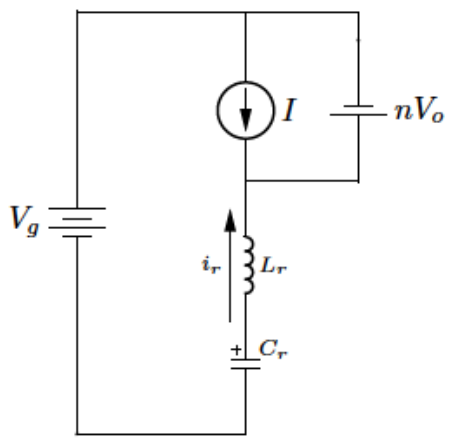

(e)

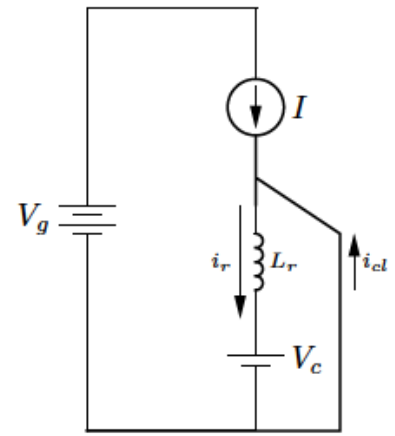

(b)

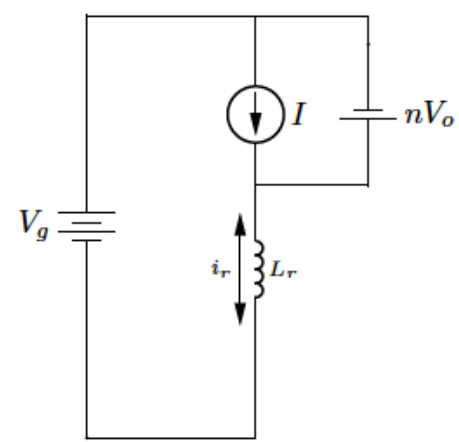

(f)

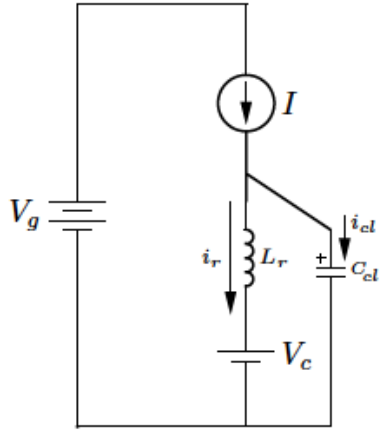

(c)

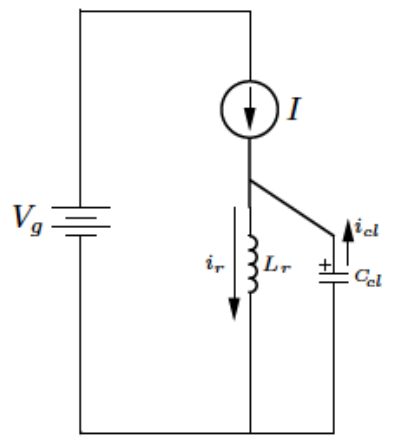

(g)

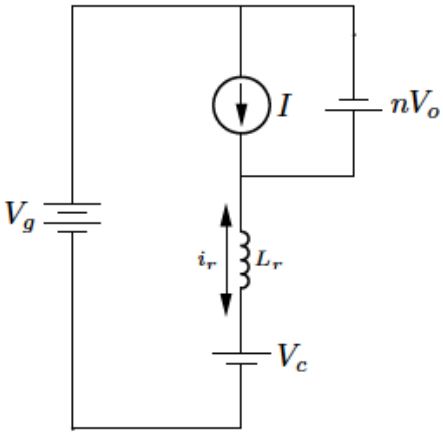

(d)

Figura F.4: Circuitos empleados para el análisis del flyback ZVS-modif. durante un periodo completo: (a) Etapa 1. (b) Etapa 2. (c) Etapa 3. (d) Etapa 4. (e) Etapa 5. (f) Etapa 6. (g) Etapa 7. (h) Etapa 8.

$$
i_{r}(t)=\left\{\begin{array}{l}
\left(I+I_{D_{c}}\right) \cos \left(\omega_{r}(t-t 0)\right) \\
I_{r 1}-\frac{V_{c}}{L_{r}}\left(t-t_{1}\right) \\
I-\frac{V_{c}}{Z_{D_{c}}} \sin \left(\omega_{D_{c}}(t-t 2)\right) \\
I_{r 3}+\frac{\left(V_{g}+n V_{o}-V_{c}\right)}{L_{r}}\left(t-t_{3}\right) \\
I_{r 4} \cos \left(\omega_{r}\left(t-t_{4}\right)\right)+\frac{\left(V_{g}+n V_{o}-V_{c}\right)}{Z_{r}} \sin \left(\omega_{r}\left(t-t_{4}\right)\right) \\
I_{r 5}+\frac{\left(V_{g}+n V_{o}\right)}{L_{r}}\left(t-t_{5}\right) \\
I+\frac{\left(V_{g}+n V_{o}\right)}{Z_{D_{c}}} \sin \left(\omega_{D_{c}}\left(t-t_{6}\right)\right) \\
\left(I+I_{D_{c}}\right)
\end{array}\right.
$$$$
t \in\left[t_{0}, t_{1}\right]
$$$$
t \in\left[t_{1}, t_{2}\right]
$$$$
t \in\left[t_{2}, t 3\right]
$$$$
t \in\left[t_{3}, t_{4}\right]
$$$$
t \in\left[t_{4}, t_{5}\right]
$$$$
t \in\left[t_{5}, t_{6}\right]
$$$$
t \in\left[t_{6}, t_{7}\right]
$$$$
t \in\left[t_{7}, T_{8}\right]
$$ 
donde se han utilizado los siguientes términos:

$$
\begin{gathered}
I_{D_{c}}=\frac{V_{g}+n V_{o}}{Z_{D_{c}}} \\
I_{r 1} \equiv i_{r}\left(t_{1}\right)=\left(I+I_{D_{c}}\right) \cos \omega_{r} d_{1} T \\
I_{r 3} \equiv i_{r}\left(t_{3}\right)=I-\frac{V_{c}}{Z_{D_{c}} \sin \omega_{D_{c}} d_{3} T} \\
I_{r 4} \equiv i_{r}\left(t_{4}\right)=I_{r 3}+\frac{\left(V_{g}+n V_{o}-V_{c}\right)}{L_{r}} d_{4} T \\
I_{r 5} \equiv i_{r}\left(t_{5}\right)=I_{r 4} \cos \omega_{r} d_{4} T+\frac{\left(V_{g}+n V_{o}-V_{c}\right)}{Z_{r}} \sin \omega_{r} d_{4} T
\end{gathered}
$$




$$
v_{L_{r}}(t)= \begin{cases}-\left(I+I_{D_{c}}\right) Z_{r} \sin \left(\omega_{r}(t-t 0)\right) & t \in\left[t_{0}, t_{1}\right] \\ -V_{c} & t \in\left[t_{1}, t_{2}\right] \\ -V_{c} \cos \left(\omega_{D_{c}}\left(t-t_{2}\right)\right) & t \in\left[t_{2}, t_{3}\right] \\ \left(V_{g}+n V_{o}-V_{c}\right) & t \in\left[t_{3}, t_{4}\right] \\ \left(V_{g}+n V_{o}-V_{c}\right) \cos \left(\omega_{r}\left(t-t_{4}\right)\right)-I_{r 4} Z_{r} \sin \left(\omega_{r}\left(t-t_{4}\right)\right) & t \in\left[t_{4}, t_{5}\right] \\ \left(V_{g}+n V_{o}\right) & t \in\left[t_{5}, t_{6}\right] \\ \left(V_{g}+n V_{o}\right) \cos \left(\omega_{D_{c}}\left(t-t_{6}\right)\right) & t \in\left[t_{6}, t_{7}\right] \\ 0 & t \in\left[t_{7}, T_{8}\right]\end{cases}
$$

\section{F.2. Análisis en régimen permanente.}

\section{F.2.1. Sin considerar rizado.}

Para este caso, se va a suponer corriente constante, $I$, en la bobina de magnetización $L_{m}$, que es mucho mas grande que $L_{r}$. El condensador de clamp, $C_{c}$ va a mantener su tensión constante a un valor $V_{c}$, igual que el condensador $C_{o}$, que presenta una tensión constante $V_{o}$.

También se supone despreciables tanto la etapa 1 como la etapa 5 puesto que el intercambio resonante de energía del conjunto $L_{r}-C_{r}$ se realiza a una frecuencia mucho mas grande que la frecuencia de conmutación. La cargadescarga de $C_{r}$ será prácticamente instantánea. Por tanto, $d_{1} \approx 0$ y $d_{5} \approx 0$, por lo que se considerarán seis etapas en ese análisis. Entonces:

$$
\begin{aligned}
& d_{2} T+d_{3} T+d_{4} T=(1-D) T \\
& d_{6} T+d_{7} T+d_{8} T=D T
\end{aligned}
$$

\section{TENSION MEDIA EN BOBINA DE MAGNETIZACIÓN}

La tensión media en la bobina $L_{m}$ es nula. Se puede escribir la siguiente ecuación:

$$
\begin{aligned}
& V_{g}\left(d_{2}+d_{8}\right)+\underbrace{\frac{1}{T} \int_{0}^{d_{3} T}\left(V_{g}-V_{c}\left(1-\cos w_{D_{c}} t\right)\right) d t}_{I_{1}}-n V_{o}\left(d_{4}+d_{6}\right) \\
& \underbrace{\frac{1}{T} \int_{0}^{d_{7} T}\left(V_{g}-\left(V_{g}+n V_{o}\right) \cos w_{D_{c}} t\right) d t}_{I_{2}}=0
\end{aligned}
$$

Cuando se resuelven las integrales $I_{1}$ e $I_{2}$ se llega a la siguiente ecuación: 


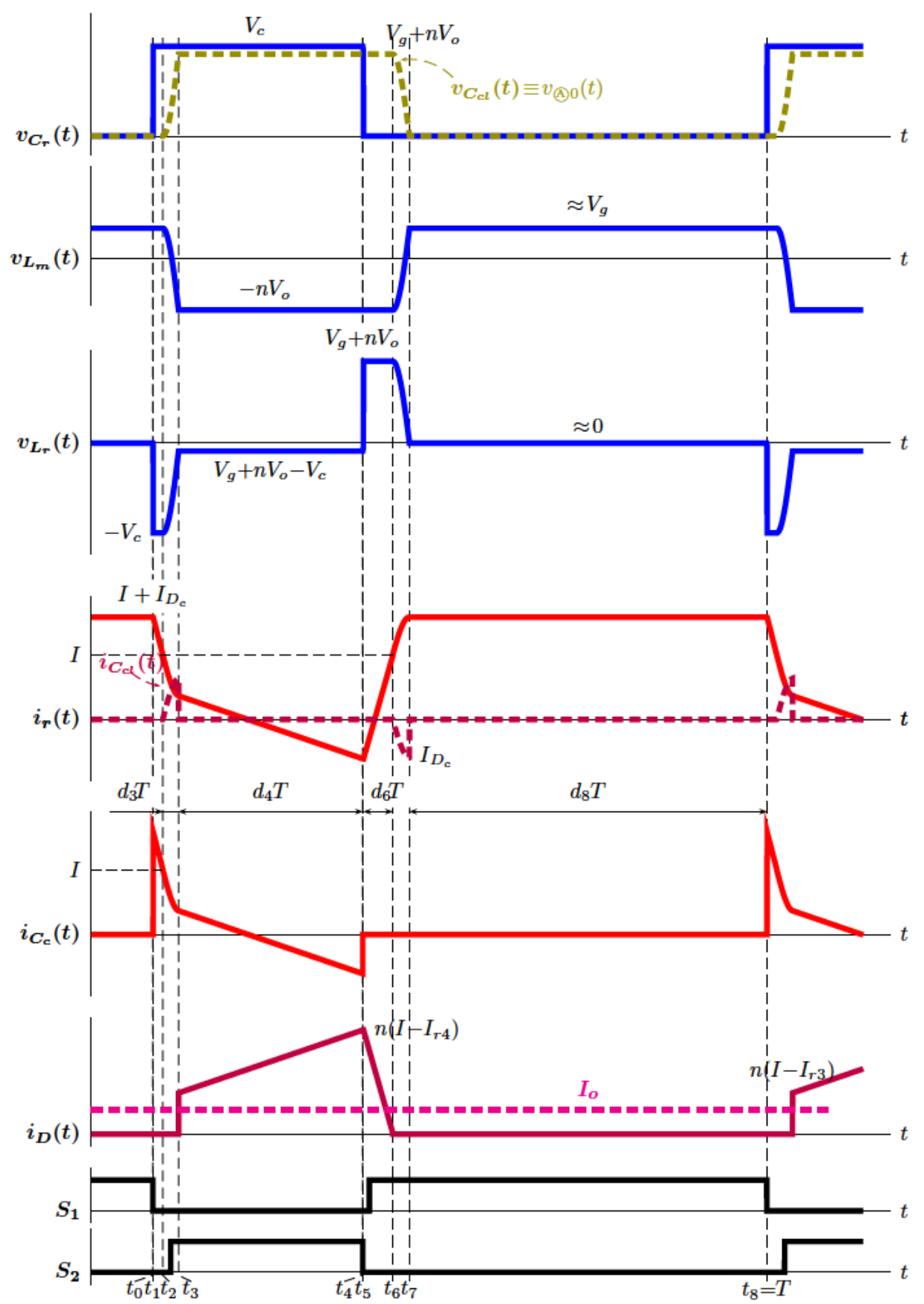

Figura F.5: Principales curvas para el flyback ZVS-modif.

$$
V_{g}\left(d_{2}+d_{3}+d_{7}+d_{8}\right)-V_{c} d_{3}-n V_{o}\left(d_{4}+d_{6}\right)+\frac{V_{c}}{w_{D_{c}} T} \sin \left(w_{D_{c}} d_{3} T\right)-\frac{\left(V_{g}+n V_{o}\right)}{w_{D_{c}} T} \sin \left(w_{D_{c}} d_{7} T\right)=0
$$




\section{TENSION MEDIA EN BOBINA RESONANTE}

La tensión media en la bobina $L_{r}$ es nula. Se puede escribir la siguiente ecuación:

$$
\begin{aligned}
& -V_{c} d_{2}+\underbrace{\frac{1}{T} \int_{0}^{d_{3} T}\left(-V_{c} \cos w_{D_{c}} t\right) d t}_{I_{3}}-\left(V_{g}+n V_{o}-V_{c}\right) d_{4} \\
& +\left(V_{g}+n V_{o}\right) d_{6}+\underbrace{\frac{1}{T} \int_{0}^{d_{7} T}\left(\left(V_{g}+n V_{o}\right) \cos w_{D_{c}} t\right) d t}_{I_{4}}=0
\end{aligned}
$$

Cuando se resuelven las integrales $I_{3}$ e $I_{4}$ se llega a la siguiente ecuación:

$-V_{c}\left(d_{2}+d_{4}\right)+\left(V_{g}+n V_{o}\right)\left(d_{4}+d_{6}\right)-\frac{V_{c}}{w_{D_{c}} T} \sin \left(w_{D_{c}} d_{3} T\right)+\frac{\left(V_{g}+n V_{o}\right)}{w_{D_{c}} T} \sin \left(w_{D_{c}} d_{7} T\right)=0$

Sumando las ecuaciones (F.20) y (F.22) desaparecen los términos trigonométricos y se llega a la ecuación (F.23)

$V_{g}\left(d_{2}+d_{3}+d_{7}+d_{8}\right)-V_{c} d_{3}-n V_{o}\left(d_{4}+d_{6}\right)-V_{c}\left(d_{2}+d_{4}\right)+\left(V_{g}+n V_{o}\right)\left(d_{4}+d_{6}\right)=0$

en donde se sabe que $d_{2}+d_{3}+d_{4}+d_{6}+d_{7}+d_{8}=1$. Entonces se despeja $V_{c}$ que queda como:

$$
V_{c}=\frac{V_{g}}{d_{2}+d_{3}+d_{4}}=\frac{V_{g}}{(1-D)}
$$

siendo $d_{2}+d_{3}+d_{4}=(1-D)$. Es necesario calcular la duración de los intervalos para obtener una expresión aplicable a partir de la tensión entrada y salida y potencia.

\section{DESCARGA DE $C_{c l}$. CÁLCULO DE $I_{D_{c}}$ y $d_{7}$}

La duración de la etapa 7 está relacionada con la descarga del condensador $C_{c l}$ desde $V_{g}+n V_{o}$ hasta cero. La evolución durante esta etapa está indicada en (F.12). y en $t_{7}$, resulta que $v_{C_{c l}}\left(t_{7}\right)=0$, por lo que:

$$
0=\left(V_{g}+n V_{o}\right) \cos \omega_{D_{c}} d_{7} T \quad \Rightarrow \quad d_{7}=\frac{\pi}{2 T \omega_{D_{c}}}
$$

Se puede calcular una expresión para la corriente al final de esta etapa, que tendrá un valor igual a $-I_{D_{c}}$. Usando (F.13):

$$
\begin{aligned}
-I_{D_{c}}=i_{C_{c l}}\left(t_{7}\right) & \Rightarrow-I_{D_{c}}=-\frac{\left(V_{g}+n V_{o}\right)}{Z_{D_{c}}} \sin \omega_{D_{c}} d_{7} T \Rightarrow \\
& \Rightarrow I_{D_{c}}=\frac{\left(V_{g}+n V_{o}\right)}{Z_{D_{c}}}
\end{aligned}
$$

VARIACIÓN DE $i_{r}(t)$ EN ETAPA 2. CÁLCULO DE $d_{2}$ 
La corriente resonante, $i_{r}(t)$ evoluciona linealmente disminuyendo su valor desde $I+I_{D_{c}}$ hasta $I$ durante la etapa 2. La bobina $L_{r}$ está sometida a una tensión $V_{c}$, que es la tensión del condensador de clamp, $C_{c}$.

Resulta que, la variación experimentada por la corriente es igual a $I_{D_{c}}$, luego:

$$
\begin{aligned}
\Delta i_{r}= & \frac{1}{L_{r}} V_{c} d_{2} T=I_{D_{c}} \\
& \Rightarrow d_{2}=\frac{I_{D_{c}}}{V_{c}} L_{r} f_{s}=\frac{\left(V_{g}+n V_{o}\right)}{Z_{D_{c}} V_{c}} L_{r} f_{s}
\end{aligned}
$$

\section{CORRIENTE MEDIA EN EL CONDENSADOR EQUIVALEN-} TE $C_{c l}$. CÁLCULO DE $d_{3}$

Haciendo $I_{c l, m e d}=0$ se obtiene la siguiente ecuación:

$$
\frac{1}{T} \int_{0}^{d_{3} T}\left(\frac{V_{c}}{Z_{D_{c}}} \sin w_{D_{c}} t\right) d t+\frac{1}{T} \int_{0}^{d_{7} T}\left(-\frac{V_{g}+n V_{o}}{Z_{D_{c}}} \sin w_{D_{c}} t\right) d t=0
$$

Resolviento las integrales y simplificando queda que:

$$
\frac{V_{c}}{I Z_{D_{c}}}\left(\cos w_{D_{c}} d_{3} T-1\right)=\frac{V_{g}+n V_{o}}{I Z_{D_{c}}}\left(\cos w_{D_{c}} d_{7} T-1\right)
$$

Utilizando el resultado (F.25) en (F.29) y despejando el término trigonométrico que engloba a $d_{3}$ se obtiene:

$$
\cos w_{D_{c}} d_{3} T=1-\frac{V_{g}+n V_{o}}{V_{c}}
$$

\section{CORRIENTE MEDIA EN EL DIODO $D_{c l}$}

Haciendo el valor medio a (F.14) se obtiene la corriente media en el diodo $D_{c l}$, representada como $I_{D_{c l}, \text { med }}$ :

$$
\begin{aligned}
I_{D_{c l}, \text { med }} & =\frac{1}{T}\left[\int_{0}^{d_{2} T}\left(I_{D_{c}}-\frac{V_{c}}{L_{r}} t\right) d t+\int_{0}^{d_{8} T} I_{D_{c}} d t\right] \\
& =\frac{1}{T}\left[I_{D_{c}}\left(d_{2}+d_{8}\right) \not t-\frac{V_{c}}{L_{r}} \frac{d_{2}^{2} T^{\not 2}}{2}\right]
\end{aligned}
$$

Empleando la expresión de $I_{D_{c}}$ obtenida al calcular $d_{7}$, y su expresión en función de $d_{2}$, es decir, (F.26) y (F.27) respectivamente, se obtiene que:

$$
I_{D_{c l}, \text { med }}=\frac{V_{c}}{L_{r}} \frac{d_{2}^{2} T}{2}+\frac{V_{g}+n V_{o}}{Z_{D_{c}}} d_{8}
$$

CORRIENTE MEDIA EN EL CONDENSADOR DE CLAMP $C_{c}$. 
Como se sabe que $I_{c, \text { med }}=0$, se obtiene:

$$
\begin{aligned}
& \frac{1}{T}[\underbrace{\int_{0}^{d_{2} T}\left(I+I_{D_{c}}-\frac{V_{c}}{L_{r}} t\right) d t}_{I_{5}}+\underbrace{\int_{0}^{d_{3} T}\left(I-\frac{V_{c}}{Z_{D_{c}}} \sin w_{D_{c}} t\right) d t}_{I_{6}} \\
& +\underbrace{\int_{0}^{d_{4} T}\left(I_{r 3}+\frac{\left(V_{g}+n V_{o}-V_{c}\right)}{L_{r}} t\right) d t}_{I_{7}}]=0
\end{aligned}
$$

donde $I_{r 3} \equiv i_{r}\left(t_{3}\right)$.

Desarrollando las integrales $I_{5}, I_{6}$ e $I_{7}$ se obtiene que:

$$
\begin{gathered}
I_{5}=\left[\left(I+I_{D_{c}}\right) t-\frac{V_{c}}{L_{r}} \frac{t^{2}}{2}\right]_{0}^{d_{2} T} \\
=I d_{2} T+\frac{V_{c}}{L_{r}} d_{2}^{2} T^{2}-\frac{V_{c}}{L_{r}} \frac{d_{2}^{2} T^{2}}{2}=I d_{2} T+\frac{V_{c}}{L_{r}} \frac{d_{2}^{2} T^{2}}{2} \\
I_{6}=\left[I t-\frac{V_{c}}{Z_{D_{c}}} \frac{1}{\left(-\omega_{D_{c}}\right)} \cos \omega_{D_{c}} t\right]_{0}^{d_{3} T} \\
=I d_{3} T+\frac{V_{c}}{\omega_{D_{c}} Z_{D_{c}}}\left[\cos \omega_{D_{c}} d_{3} T-1\right] \\
=I d_{3} T+\frac{V_{g}+n V_{o}}{\omega_{D_{c}} Z_{D_{c}}}\left[\cos \omega_{D_{c}} d_{7} T-1\right]=I d_{3} T-\frac{V_{g}+n V_{o}}{\omega_{D_{c}} Z_{D_{c}}} \\
I_{7}=\left[I_{r 3} t+\frac{\left(V_{g}+n V_{o}-V_{c}\right)}{L_{r}} \frac{t^{2}}{2}\right]_{0}^{d_{4} T}=I_{r 3} d_{4} T+\frac{\left(V_{g}+n V_{o}-V_{c}\right)}{L_{r}} \frac{d_{4}^{2} T^{2}}{2} \\
=\left[I-\frac{V_{c}}{Z_{D_{c}}} \sin \omega_{D_{c}} d_{3} T\right] d_{4} T+\frac{\left(V_{g}+n V_{o}-V_{c}\right)}{L_{r}} \frac{d_{4}^{2} T^{2}}{2}
\end{gathered}
$$

La suma será:

$$
\begin{aligned}
0=I_{C_{c}, \text { med }} & =I\left(d_{2}+d_{3}+d_{4}\right)+\frac{V_{c}}{L_{r}} \frac{d_{2}^{2} T}{2}-\frac{V_{g}+n V_{o}}{\omega_{D_{c}} Z_{D_{c}} T} \\
& -d_{4} \frac{V_{c}}{Z_{D_{c}}} \sin \omega_{D_{c}} d_{3} T+\frac{\left(V_{g}+n V_{o}-V_{c}\right)}{L_{r}} \frac{d_{4}^{2} T}{2}
\end{aligned}
$$

Y se consigue una expresión para $I$, que queda indicada en (F.38):

$$
\begin{aligned}
I= & \frac{1}{d_{2}+d_{3}+d_{4}}\left[-\frac{V_{c}}{L_{r}} \frac{d_{2}^{2} T}{2}+\frac{V_{g}+n V_{o}}{\omega_{D_{c}} Z_{D_{c}} T}+\right. \\
& \left.+d_{4} \frac{V_{c}}{Z_{D_{c}}} \sin \omega_{D_{c}} d_{3} T-\frac{\left(V_{g}+n V_{o}-V_{c}\right)}{L_{r}} \frac{d_{4}^{2} T}{2}\right]
\end{aligned}
$$




\section{CORRIENTE MEDIA EN LA BOBINA RESONANTE, $I_{r}$, Y EN}

\section{LA ENTRADA, $I_{g}$}

La relación entre $I$ y la corriente media en $L_{r}$, expresada como $I_{r}$, se puede obtener haciendo el valor medio a las expresiones (F.6) y (F.7), que se han reescrito a continuación:

$$
\left.\begin{array}{l}
i_{g}(t)+\frac{1}{n} i_{D}(t)=i_{m}(t) \\
i_{g}(t)+i_{c l}(t)=i_{r}(t)
\end{array}\right\} \stackrel{\text { (V.med.) }}{\Longrightarrow}\left\{\begin{array}{l}
I_{g}+\frac{I_{o}}{n}=I \\
I_{g}+I_{D_{c l}, \text { med }}=I_{r}
\end{array}\right.
$$

y como $P_{g}=P_{o}$, entonces:

$$
I_{o} \frac{V_{o}}{V_{g}}+\frac{I_{o}}{n}=I
$$

Por otro lado, la corriente media en la bobina resonante es igual a:

$$
\begin{aligned}
& I_{r}=\frac{1}{T}[\underbrace{\int_{0}^{d_{2} T}\left(I+I_{D_{c}}-\frac{V_{c}}{L_{r}} t\right) d t}_{I_{8}}+\underbrace{\int_{0}^{d_{3} T}\left(I-\frac{V_{c}}{Z_{D_{c}}} \sin w_{D_{c}} t\right) d t}_{I_{9}} \\
& +\underbrace{\int_{0}^{d_{4} T}\left(I_{r 5}+\frac{\left(V_{g}+n V_{o}-V_{c}\right)}{L_{r}} t\right) d t}_{I_{1} 0}+\underbrace{\int_{0}^{d_{6} T}\left(I_{r 5}+\frac{V_{g}+n V_{o}}{L_{r}} t\right) d t}_{I_{11}} \\
& +\underbrace{\int_{0}^{d_{7} T}\left(I+\frac{V_{g}+n V_{o}}{Z_{D_{c}}} \sin \omega_{D_{c}} t\right) d t}_{I_{12}}+\underbrace{\int_{0}^{d_{8} T}\left(I+I_{D_{c}}\right) d t}_{I_{1} 3}]
\end{aligned}
$$

donde $I_{r 5} \equiv i_{r}\left(t_{5}\right) \approx i_{r}\left(t_{4}\right) \equiv I_{r 4}$. Las tres primeras integrales son nulas porque corresponden al valor medio de la corriente en el condensador $C_{c}$. Desarrollando las otras tres integrales, $I_{11}, I_{12}$ e $I_{13}$ :

$$
\begin{aligned}
I_{11}= & {\left[I_{r 5} t+\frac{V_{g}+n V_{o}}{L_{r}} \frac{t^{2}}{2}\right]_{0}^{d_{6} T} } \\
= & {\left[I-\frac{V_{c}}{Z_{D_{c}}} \sin \omega_{D_{c}} d_{3} T+\frac{V_{g}+n V_{o}-V_{c}}{L_{r}} d_{4} T\right] d_{6} T+\frac{\left(V_{g}+n V_{o}\right)}{L_{r}} \frac{d_{6}^{2} T^{2}}{2} } \\
I_{12} & =\left[I t+\frac{V_{g}+n V_{o}}{Z_{D_{c}}} \frac{1}{\left(-\omega_{D_{c}}\right)} \cos \omega_{D_{c}} t\right]_{0}^{d_{7} T} \\
& =I d_{7} T-\frac{V_{g}+n V_{o}}{Z_{D_{c}} \omega_{D_{c}}}\left[\cos \omega_{D_{c}} d_{7} T-1\right]=I d_{7} T+\frac{V_{g}+n V_{o}}{Z_{D_{c}} \omega_{D_{c}}}
\end{aligned}
$$




$$
I_{13}=\left(I+I_{D_{c}}\right) d_{8} T=I d_{8} T+\frac{V_{g}+n V_{o}}{Z_{D_{c}}} d_{8} T
$$

Y se obtiene:

$$
\begin{aligned}
I_{r} & =I\left(d_{6}+d_{7}+d_{8}\right)+\frac{V_{g}+n V_{o}}{Z_{D_{c}}} d_{8}+\frac{V_{g}+n V_{o}}{\omega_{D_{c}} Z_{D_{c}} T} \\
& -\left[\frac{V_{c}}{Z_{D_{c}}} \sin \omega_{D_{c}} d_{3} T-\frac{\left(V_{g}+n V_{o}-V_{c}\right)}{L_{r}} d_{4} T\right] d_{6}+\frac{\left(V_{g}+n V_{o}\right)}{L_{r}} \frac{d_{6}^{2} T}{2}
\end{aligned}
$$

Como $i_{r}\left(t_{6}\right)=I$ :

$$
\begin{aligned}
& I_{r 5}=I-\frac{V_{c}}{Z_{D_{c}}} \sin \omega_{D_{c}} d_{3} T+\frac{V_{g}+n V_{o}-V_{c}}{L_{r}} d_{4} T \\
& I=I_{r 5}+\frac{V_{g}+n V_{o}}{L_{r}} d_{6} T \\
& \Rightarrow \frac{V_{c}}{Z_{D_{c}}} \sin \omega_{D_{c}} d_{3} T=\frac{V_{g}+n V_{o}-V_{c}}{L_{r}} d_{4} T+\frac{V_{g}+n V_{o}}{L_{r}} d_{6} T
\end{aligned} \Rightarrow
$$

Esta última expresión sustituida en (F.45) da lugar a que:

$I_{r}=I\left(d_{6}+d_{7}+d_{8}\right)+\frac{V_{g}+n V_{o}}{Z_{D_{c}}} d_{8}+\frac{V_{g}+n V_{o}}{\omega_{D_{c}} Z_{D_{c}} T}-\frac{\left(V_{g}+n V_{o}\right)}{L_{r}} \frac{d_{6}^{2} T}{2}$

Sumando esta última expresión con (F.37) se obtiene que:

$$
\begin{aligned}
I_{r} & =I(\overbrace{d_{2}+d_{3}+d_{4}+d_{6}+d_{7}+d_{8}}^{1})+\overbrace{\frac{V_{c}}{L_{r}} \frac{d_{2}^{2} T}{2}+\frac{V_{g}+n V_{o}}{Z_{D_{c}}} d_{8}}^{I_{D_{c l}, \text { med }}} \\
& -\frac{\left(V_{g}+n V_{o}\right)}{L_{r}} \frac{d_{6}^{2} T}{2}-d_{4} \frac{V_{c}}{Z_{D_{c}}} \sin \omega_{D_{c}} d_{3} T+\frac{\left(V_{g}+n V_{o}-V_{c}\right)}{L_{r}} \frac{d_{4}^{2} T}{2}
\end{aligned}
$$

Finalmente, se obtiene la corriente media en la entrada, $I_{g}$ :

$$
I_{g}=I-\frac{\left(V_{g}+n V_{o}\right)}{L_{r}} \frac{d_{6}^{2} T}{2}-d_{4} \frac{V_{c}}{Z_{D_{c}}} \sin \omega_{D_{c}} d_{3} T+\frac{\left(V_{g}+n V_{o}-V_{c}\right)}{L_{r}} \frac{d_{4}^{2} T}{2}
$$

donde se ha utilizado la igualdad (F.104).

\section{CARACTERÍSTICA DE SALIDA}

La expresión que relaciona la tensión de salida con la intensidad de salida se puede obtener a partir de la expresión (F.38), y la relación entre intensidades de magnetización y salida dada en (F.40). 
Despejar $I_{o}$ en esas expresiones se obtiene una expresión $I_{o}\left(V_{o}\right)$ como la siguiente:

$I_{o}=\frac{n V_{g}\left[-\frac{V_{c}}{L_{r}} \frac{d_{2}^{2} T}{2}+\frac{V_{g}+n V_{o}}{\omega_{D_{c}} Z_{D_{c}} T}+d_{4} \frac{V_{c}}{Z_{D_{c}}} \sin \omega_{D_{c}} d_{3} T-\frac{\left(V_{g}+n V_{o}-V_{c}\right)}{L_{r}} \frac{d_{4}^{2} T}{2}\right]}{(1-D)\left(V_{g}+n V_{o}\right)}$

Del análisis realizado se han encontrado ciertas relaciones, como el valor máximo de la corriente que pasar por el diodo $D_{c}$, (véase (F.26)) o la duración de la la etapa 7, (véase (F.25)), que no dependen del ciclo de trabajo.

Los términos $V_{c}, d_{2}$ y $d_{3}$ se pueden expresar como función de $D$ (véase (F.24), (F.27), y (F.30)). También $d_{4}$ se puede expresar como función del ciclo de trabajo puesto que $d_{4}=(1-D)-d_{2}-d_{3}$.

Introduciendo ahora todos los términos en la ecuación de la característica de salida dada en (F.50), se consigue una expresión que relaciona $I_{o}$ con $D$, de la que se obtiene uno de los dos parámetros, en función del otro.

\section{F.2.2. Considerando rizado.}

Si se considera que la bobina $L_{m}$ no es lo suficientemente grande como para suponer corriente constante, $I$, los circuitos equivalentes empleados para el análisis del circuito son diferentes y la relación entre la bobina principal y resonante aparece en las nuevas expresiones de las principales magnitudes.

Los circuitos equivalentes están representados en la Figura F.6 donde se ha considerado la tensión constante en los condensadores $C_{o}$ y $C_{c}$, mientras que las expresiones de las principales magnitudes se indican mas adelante, y se han representado gráficamente en la Figura F.7.

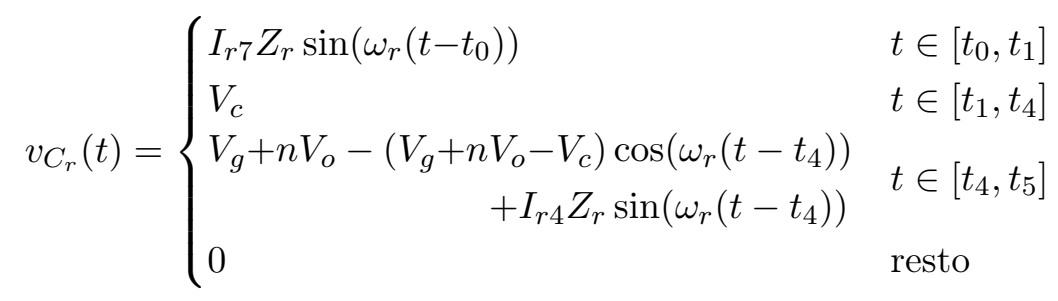




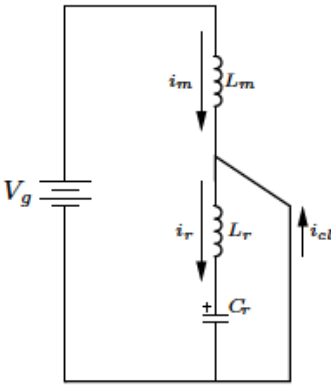

(a)

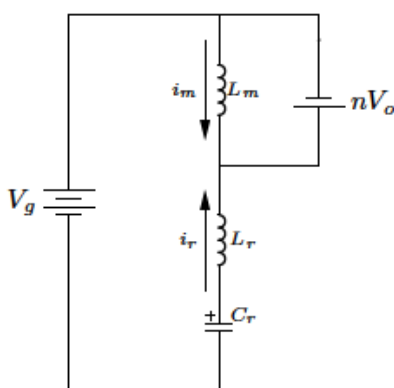

(e)

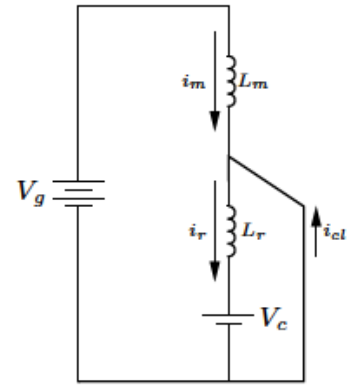

(b)

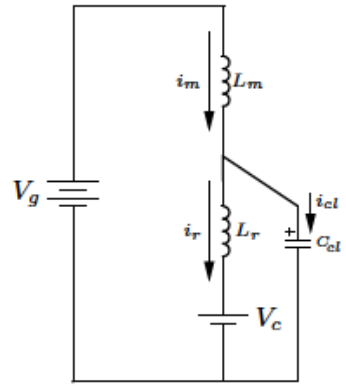

(c)

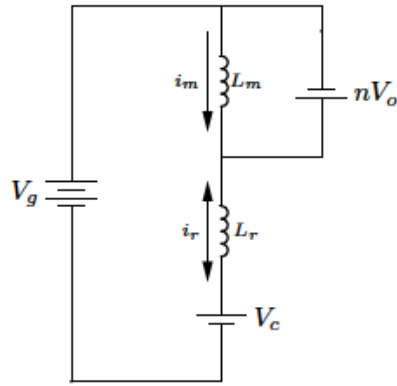

(d)

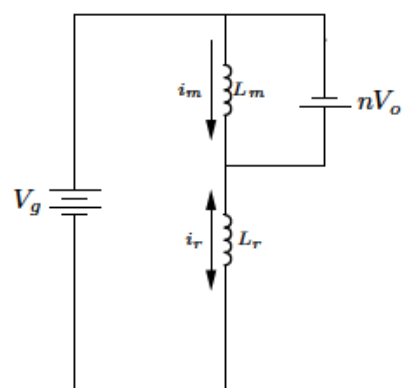

(f)

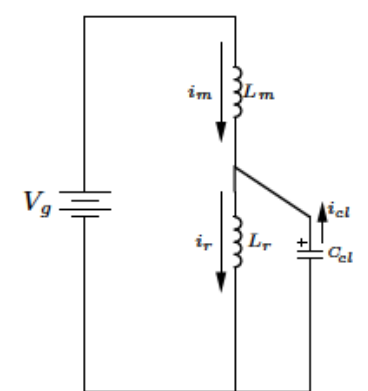

(g)

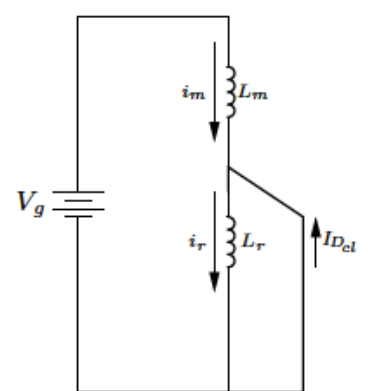

(h)

Figura F.6: Circuitos empleados para el análisis del flyback ZVS-modificado, durante un periodo completo considerando rizado: (a) Etapa 1. (b) Etapa 2. (c) Etapa 3. (d) Etapa 4. (e) Etapa 5. (f) Etapa 6. (g) Etapa 7. (h) Etapa 8. 


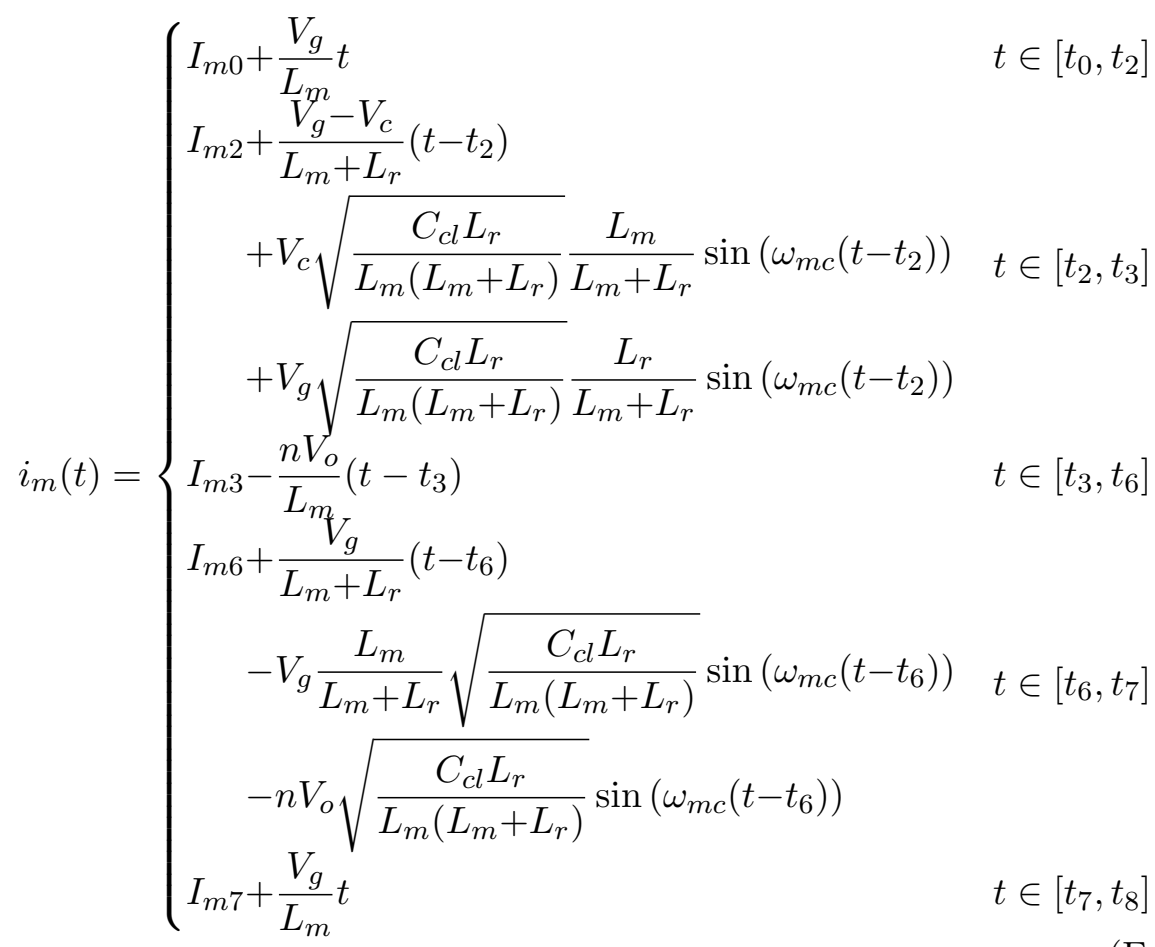

Donde se han definido los siguientes términos para $i_{m}(t)$ :

$$
\begin{aligned}
& I_{m 0} \equiv i_{m}\left(t_{0}\right)=I_{m 7}+\frac{V_{g}}{L_{m}} d_{8} T \\
& I_{m 2} \equiv i_{m}\left(t_{2}\right)=I_{m 0}+\frac{V_{g}}{L_{m}}\left(d_{1}+d_{2}\right) T \\
& I_{m 3} \equiv i_{m}\left(t_{3}\right)=I_{m 2}+\frac{V_{g}-V_{c}}{L_{m}+L_{r}} d_{3} T+\frac{V_{c} L_{m}+V_{g} L_{r}}{\omega_{m c} L_{m}\left(L_{m}+L_{r}\right)} \sin \omega_{m c} d_{3} T \\
& I_{m 6} \equiv i_{m}\left(t_{6}\right)=I_{m 3}-\frac{n V_{o}}{L_{m}}\left(d_{4}+d_{5}+d_{6}\right) T \\
& I_{m 7} \equiv i_{m}\left(t_{7}\right)=I_{m 6}+\frac{V_{g}}{L_{m}+L_{r}} d_{7} T-\frac{V_{g} L_{m}+n V_{o}\left(L_{m}+L_{r}\right)}{\omega_{m c} L_{m}\left(L_{m}+L_{r}\right)} \sin \omega_{m c} d_{7} T \\
& \text { donde } L_{m r}=L_{m} \| L_{r}=\frac{L_{m} L_{r}}{L_{m}+L_{r}} \mathrm{y} \\
& \omega_{m c}=\frac{1}{\sqrt{L_{m r} C_{c l}}}=\sqrt{\frac{L_{m}+L_{r}}{L_{m} L_{r} C_{c l}}} \mathrm{y} Z_{m c}=\sqrt{\frac{L_{m r}}{C_{c l}}}=\sqrt{\frac{L_{m} L_{r}}{\left(L_{m}+L_{r}\right) C_{c l}}}
\end{aligned}
$$




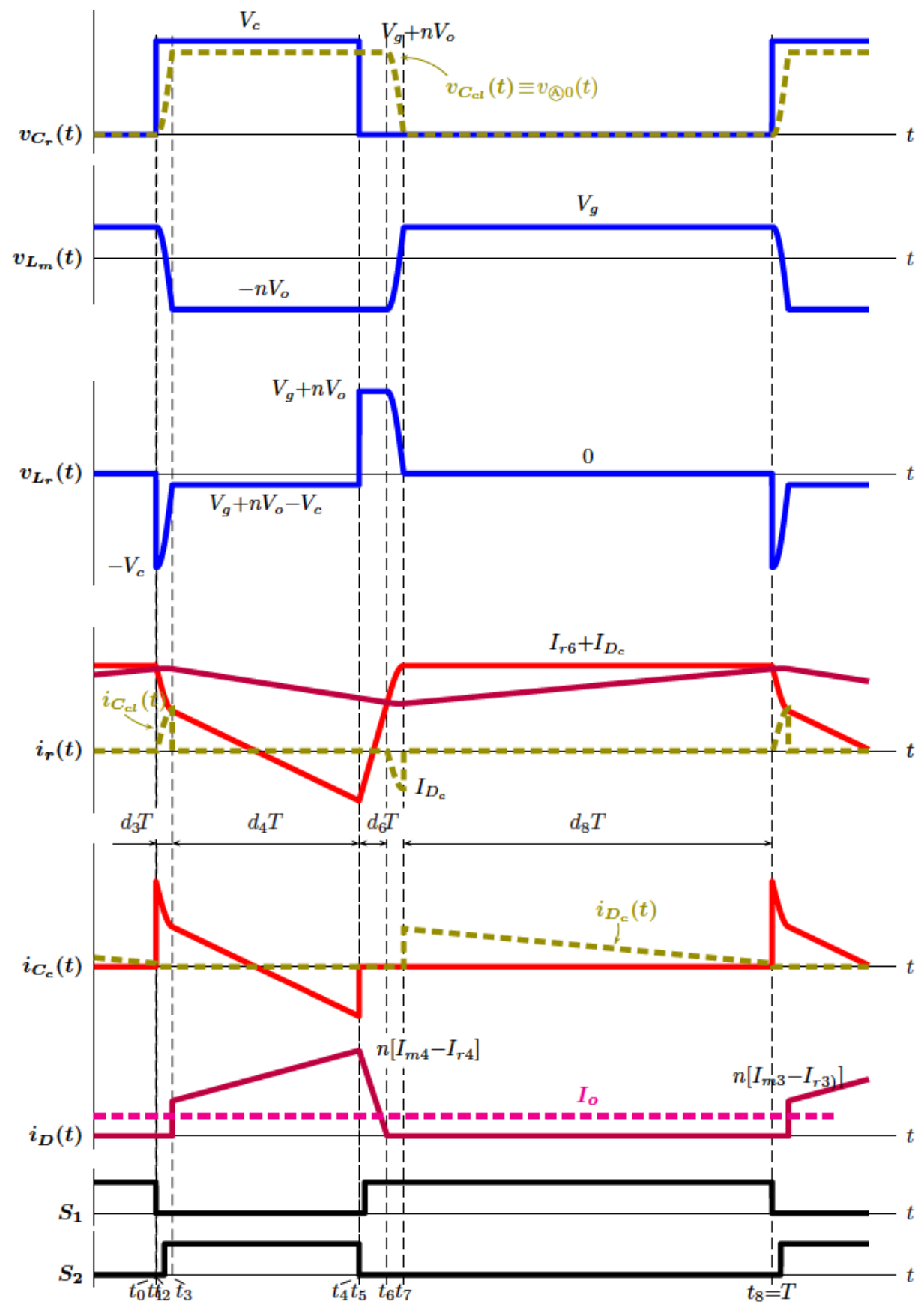

Figura F.7: Principales curvas de un flyback ZVS-modif. considerando rizado. 


$$
i_{r}(t)= \begin{cases}I_{r 0} \cos \left(\omega_{r}(t-t 0)\right) & t \in\left[t_{0}, t_{1}\right] \\ I_{r 1}-\frac{V_{c}}{L_{r}}\left(t-t_{1}\right) & t \in\left[t_{1}, t_{2}\right] \\ I_{r 2}+\frac{V_{g}-V_{c}}{L_{m}+L_{r}\left(t-t_{2}\right)} & \\ \quad-V_{c} \sqrt{\frac{C_{c l} L_{m}}{L_{r}\left(L_{m}+L_{r}\right)} \frac{L_{m}}{L_{m}+L_{r}} \sin \left(\omega_{m c}\left(t-t_{2}\right)\right)} & t \in\left[t_{2}, t_{3}\right] \\ \quad-V_{g} \sqrt{\frac{C_{c l} L_{m}}{L_{r}\left(L_{m}+L_{r}\right)} \frac{L_{r}}{L_{m}+L_{r}} \sin \left(\omega_{m c}\left(t-t_{2}\right)\right)} & \\ I_{r 3}+\frac{\left(V_{g}+n V_{o}-V_{c}\right)}{L_{r}}\left(t-t_{3}\right) & t \in\left[t_{3}, t_{4}\right] \\ I_{r 4} \cos \left(\omega_{r}\left(t-t_{4}\right)\right)+\frac{\left(V_{g}+n V_{o}-V_{c}\right)}{Z_{r}} \sin \left(\omega_{r}\left(t-t_{4}\right)\right) & t \in\left[t_{4}, t_{5}\right] \\ I_{r 5}+\frac{\left(V_{g}+n V_{o}\right)}{L_{r}}\left(t-t_{5}\right) & t \in\left[t_{5}, t_{6}\right] \\ I_{r 6}+\frac{V_{g}}{L_{m}+L_{r}}\left(t-t_{6}\right) & t \in\left[t_{6}, t_{7}\right] \\ \quad+V_{g} \frac{L_{m}}{L_{m}+L_{r}} \sqrt{\frac{C_{c l} L_{m}}{L_{r}\left(L_{m}+L_{r}\right)}} \sin \left(\omega_{m c}\left(t-t_{6}\right)\right) & t \in\left[t_{7}, t_{8}\right] \\ \quad+n V_{o} \sqrt{\frac{C_{c l} L_{m}}{L_{r}\left(L_{m}+L_{r}\right)}} \sin \left(\omega_{m c}\left(t-t_{6}\right)\right) & \\ I_{r 7} \quad & \end{cases}
$$

donde se han definido los siguientes términos para $i_{r}(t)$ :

$$
\begin{aligned}
& I_{r 1} \equiv i_{r}\left(t_{1}\right)=I_{r 0} \cos \omega_{r} d_{1} T \\
& I_{r 2} \equiv i_{r}\left(t_{2}\right)=I_{r 1}-\frac{V_{c}}{L_{r}} d_{2} T \\
& I_{r 3} \equiv i_{r}\left(t_{3}\right)=I_{r 2}+\frac{V_{g}-V_{c}}{L_{m}+L_{r}} d_{3} T-\frac{V_{c} L_{m}+V_{g} L_{r}}{\omega_{m c} L_{r}\left(L_{m}+L_{r}\right)} \sin \omega_{m c} d_{3} T \\
& I_{r 4} \equiv i_{r}\left(t_{4}\right)=I_{r 3}+\frac{\left(V_{g}+n V_{o}-V_{c}\right)}{L_{r}} d_{4} T \\
& I_{r 5} \equiv i_{r}\left(t_{5}\right)=I_{r 4} \cos \omega_{r} d_{4} T+\frac{\left(V_{g}+n V_{o}-V_{c}\right)}{Z_{r}} \sin \omega_{r} d_{4} T \\
& I_{r 6} \equiv i_{r}\left(t_{6}\right)=I_{r 5}+\frac{V_{g}+n V_{o}}{L_{r}} d_{6} T \\
& I_{r 7} \equiv i_{r}\left(t_{7}\right)=I_{r 6}+\frac{V_{g}}{L_{m}+L_{r}} d_{7} T+\frac{V_{g} L_{m}+n V_{o}\left(L_{m}+L_{r}\right)}{\omega_{m c} L_{r}\left(L_{m}+L_{r}\right)} \sin \omega_{m c} d_{7} T \\
& \operatorname{con} Z_{r}=\sqrt{L_{r} / C_{r}} \mathrm{y} \omega_{r}=1 / \sqrt{L_{r} C_{r}}
\end{aligned}
$$$$
\text { considerando que: }
$$ 


$$
\begin{aligned}
\omega_{D_{c}} & =\lim _{L_{m} \rightarrow \infty} \omega_{m c} \\
Z_{D_{c}} & =\lim _{L_{m} \rightarrow \infty} Z_{m c}
\end{aligned}
$$

Sustituyendo las definiciones anteriores:

$i_{r}(t)=\left\{\begin{array}{lr}I_{r 0} \cos \left(\omega_{r}\left(t-t_{0}\right)\right) & t \in\left[t_{0}, t_{1}\right] \\ I_{r 1}-\frac{V_{c}}{L_{r}}\left(t-t_{1}\right) & t \in\left[t_{1}, t_{2}\right] \\ I_{r 2}+\frac{V_{g}-V_{c}}{L_{m}+L_{r}}\left(t-t_{2}\right)-\frac{V_{c} L_{m}+V_{g} L_{r}}{\omega_{m c} L_{r}\left(L_{m}+L_{r}\right)} \sin \left(\omega_{m c}\left(t-t_{2}\right)\right) & t \in\left[t_{2}, t_{3}\right] \\ I_{r 3}+\frac{\left(V_{g}+n V_{o}-V_{c}\right)}{L_{r}}\left(t-t_{3}\right) & t \in\left[t_{3}, t_{4}\right] \\ I_{r 4} \cos \left(\omega_{r}\left(t-t_{4}\right)\right)+\frac{\left(V_{g}+n V_{o}-V_{c}\right)}{Z_{r}} \sin \left(\omega_{r}\left(t-t_{4}\right)\right) & t \in\left[t_{4}, t_{5}\right] \\ I_{r 5}+\frac{\left(V_{g}+n V_{o}\right)}{L_{r}}\left(t-t_{5}\right) & t \in\left[t_{5}, t_{6}\right] \\ I_{r 6}+\frac{V_{g}}{L_{m}+L_{r}}\left(t-t_{6}\right)+\frac{V_{g} L_{m}+n V_{o}\left(L_{m}+L_{r}\right)}{\omega_{m c} L_{r}\left(L_{m}+L_{r}\right)} \sin \left(\omega_{m c}\left(t-t_{6}\right)\right) & t \in\left[t_{6}, t_{7}\right] \\ I_{r 7} & t \in\left[t_{7}, t_{8}\right]\end{array}\right.$

$i_{m}(t)= \begin{cases}I_{m 0}+\frac{V_{g}}{L_{m}}\left(t-t_{0}\right) & t \in\left[t_{0}, t_{2}\right] \\ I_{m 2}+\frac{V_{g}-V_{c}}{L_{m}+L_{r}}\left(t-t_{2}\right)+\frac{V_{c} L_{m}+V_{g} L_{r}}{\omega_{m c} L_{m}\left(L_{m}+L_{r}\right)} \sin \left(\omega_{m c}\left(t-t_{2}\right)\right) & t \in\left[t_{2}, t_{3}\right] \\ I_{m 3}-\frac{n V_{o}}{L_{m}}\left(t-t_{3}\right) & t \in\left[t_{3}, t_{6}\right] \\ I_{m 6}+\frac{V_{g}}{L_{m}+L_{r}}\left(t-t_{6}\right)-\frac{V_{g} L_{m}+n V_{o}\left(L_{m}+L_{r}\right)}{\omega_{m c} L_{m}\left(L_{m}+L_{r}\right)} \sin \left(\omega_{m c}\left(t-t_{6}\right)\right) & t \in\left[t_{6}, t_{7}\right] \\ I_{m 7}+\frac{V_{g}}{L_{m}}\left(t-t_{7}\right) & t \in\left[t_{7}, t_{8}\right]\end{cases}$

$v_{C_{c l}}(t)= \begin{cases}\frac{V_{c} L_{m}+V_{g} L_{r}}{L_{m}+L_{r}}\left[1-\cos \left(\omega_{m c}\left(t-t_{2}\right)\right)\right] & t \in\left[t_{2}, t_{3}\right] \\ \left(V_{g}+n V_{o}\right) & t \in\left[t_{3}, t_{6}\right] \\ V_{g} \frac{L_{r}}{L_{m}+L_{r}}+\left[V_{g} \frac{L_{m}}{L_{m}+L_{r}}+n V_{o}\right] \cos \left(\omega_{m c}\left(t-t_{6}\right)\right) & t \in\left[t_{6}, t_{7}\right] \\ 0 & \text { resto }\end{cases}$ 


$$
i_{C_{c l}}(t)= \begin{cases}\frac{V_{c} L_{m}+V_{g} L_{r}}{\left(L_{m}+L_{r}\right) Z_{m c}} \sin \left(\omega_{m c}\left(t-t_{2}\right)\right) & t \in\left[t_{2}, t_{3}\right] \\ -\frac{V_{g} L_{m}+n V_{o}\left(L_{m}+L_{r}\right)}{\left(L_{m}+L_{r}\right) Z_{m c}} \sin \left(\omega_{m c}\left(t-t_{6}\right)\right) & t \in\left[t_{6}, t_{7}\right] \\ 0 & \text { resto }\end{cases}
$$

$i_{D_{c l}}= \begin{cases}I_{r 0} \cos \left(\omega_{r}(t-t 0)\right)-I_{m 0}-\frac{V_{g}}{L_{m}}\left(t-t_{0}\right) & t \in\left[t_{0}, t_{1}\right] \\ \underbrace{\frac{V_{g} L_{m}+n V_{o}\left(L_{m}+L_{r}\right)}{\left(L_{m}+L_{r}\right) Z_{m c}} \sin \omega_{m c} d_{7} T-\frac{V_{g}}{L_{m}}\left(t-t_{7}\right)}_{I_{D_{c}}-\frac{V_{c}}{L_{r}}\left(t-t_{1}\right)-I_{m 0}-\frac{V_{g}}{L_{m}}\left(t-t_{0}\right)} & t \in\left[t_{7}, t_{2}\right] \\ 0 & \text { resto }\end{cases}$

$$
\begin{aligned}
& i_{C_{c}}(t)= \begin{cases}i_{r}(t) & t \in\left[t_{1}, t_{2}\right] \\
i_{r}(t) & t \in\left[t_{2}, t 3\right] \\
i_{r}(t) & t \in\left[t_{3}, t_{4}\right] \\
0 & \text { resto }\end{cases} \\
& i_{D}(t)= \begin{cases}n\left[i_{m}(t)-i_{r}(t)\right] & t \in\left[t_{3}, t_{6}\right] \\
0 & \text { resto }\end{cases}
\end{aligned}
$$

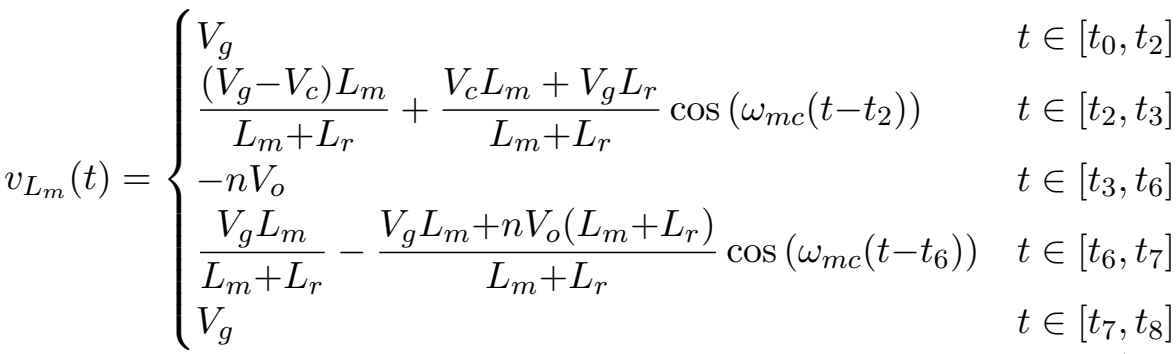




$$
v_{L_{r}}(t)= \begin{cases}-I_{r 0} Z_{r} \operatorname{sen}\left(\omega_{r}\left(t-t_{0}\right)\right) & t \in\left[t_{0}, t_{1}\right] \\ -V_{c} & t \in\left[t_{1}, t_{2}\right] \\ \frac{\left(V_{g}-V_{c}\right) L_{r}}{L_{m}+L_{r}}-\frac{V_{c} L_{m}+V_{g} L_{r}}{L_{m}+L_{r}} \cos \left(\omega_{m c}\left(t-t_{2}\right)\right) & t \in\left[t_{2}, t_{3}\right] \\ V_{g}+n V_{o}-V_{c} & t \in\left[t_{3}, t_{4}\right] \\ -I_{r 4} Z_{r} \sin \left(\omega_{r}\left(t-t_{4}\right)\right)+\left(V_{g}+n V_{o}-V_{c}\right) \cos \left(\omega_{r}\left(t-t_{4}\right)\right) & t \in\left[t_{4}, t_{5}\right] \\ V_{g}+n V_{o} & t \in\left[t_{5}, t_{6}\right] \\ \frac{V_{g} L_{r}}{L_{m}+L_{r}}+\frac{V_{g} L_{m}+n V_{o}\left(L_{m}+L_{r}\right)}{L_{m}+L_{r}} \cos \left(\omega_{m c}\left(t-t_{6}\right)\right) & t \in\left[t_{6}, t_{7}\right] \\ 0 & t \in\left[t_{7}, t_{8}\right]\end{cases}
$$

Planteando el balance voltios-segundo en las bobinas $L$ y $L_{r}$ y la carga neta nula en los condensadores $C_{o}$ y $C_{c}$ con las nuevas curvas, se obtienen otras ecuaciones del régimen permanente. Se desprecian las etapas 1 y 5 , quedando seis etapas

\section{TENSIÓN MEDIA EN BOBINA DE MAGNETIZACIÓN CON RIZADO}

La tensión media en la bobina $L_{m}$ es nula. Se puede escribir la siguiente ecuación:

$$
\begin{aligned}
& V_{g}\left(d_{2}+d_{8}\right)+\underbrace{\frac{1}{T} \int_{0}^{d_{3} T}\left[\left(V_{g}-V_{c}\right) \frac{L_{m}}{L_{m}+L_{r}}+\frac{V_{c} L_{m}+V_{g} L_{r}}{L_{m}+L_{r}} \cos w_{m c} t\right] d t}_{I_{1}} \\
& -n V_{o}\left(d_{4}+d_{6}\right)+\underbrace{\frac{1}{T} \int_{0}^{d_{7} T}\left[V_{g} \frac{L_{m}}{L_{m}+L_{r}}-\frac{V_{g} L_{m}+n V_{o}\left(L_{m}+L_{r}\right)}{L_{m}+L_{r}} \cos w_{m c} t\right] d t}_{I_{2}}=0
\end{aligned}
$$

Cuando se resuelven las integrales $I_{1}$ e $I_{2}$ se llega a la siguiente ecuación:

$$
\begin{aligned}
& V_{g}\left(d_{2}+d_{8}\right)+\left(V_{g}-V_{c}\right) \frac{L_{m}}{L_{m}+L_{r}} d_{3}-n V_{o}\left(d_{4}+d_{6}\right)+V_{g} \frac{L_{m}}{L_{m}+L_{r}} d_{7} \\
& +\frac{V_{c} L_{m}+V_{g} L_{r}}{\omega_{m c}\left(L_{m}+L_{r}\right) T} \operatorname{sen} w_{m c} d_{3} T-\frac{V_{g} L_{m}+n V_{o}\left(L_{m}+L_{r}\right)}{\omega_{m c}\left(L_{m}+L_{r}\right) T} \sin w_{m c} d_{7} T=0
\end{aligned}
$$

\section{TENSIÓN MEDIA EN BOBINA RESONANTE CON RIZADO}

La tensión media en la bobina $L_{R}$ es nula. Se puede escribir la siguiente 
ecuación:

$$
\begin{aligned}
& -V_{c} d_{2}+\underbrace{\frac{1}{T} \int_{0}^{d_{3} T}\left[\left(V_{g}-V_{c}\right) \frac{L_{r}}{L_{m}+L_{r}}-\frac{V_{c} L_{m}+V_{g} L_{r}}{L_{m}+L_{r}} \cos w_{m c} t\right] d t}_{I_{3}} \\
& +\left(V_{g}+n V_{o}-V_{c}\right) d_{4}+\left(V_{g}+n V_{o}\right) d_{6} \\
& +\underbrace{\frac{1}{T} \int_{0}^{d_{7} T}\left[V_{g} \frac{L_{r}}{L_{m}+L_{r}}+\frac{V_{g} L_{m}+n V_{o}\left(L_{m}+L_{r}\right)}{L_{m}+L_{r}} \cos w_{m c} t\right] d t}_{I_{2}}=0
\end{aligned}
$$

Cuando se resuelven las integrales $I_{3}$ e $I_{4}$, se llega a la siguiente ecuación:

$$
\begin{aligned}
& -V_{c}\left(d_{2}+d_{4}\right)+\left(V_{g}+n V_{o}\right)\left(d_{4}+d_{6}\right)+\left(V_{g}-V_{c}\right) \frac{L_{r}}{L_{m}+L_{r}} d_{3}+V_{g} \frac{L_{r}}{L_{m}+L_{r}} d_{7} \\
& -\frac{V_{c} L_{m}+V_{g} L_{r}}{\omega_{m c}\left(L_{m}+L_{r}\right) T} \operatorname{sen} w_{m c} d_{3} T+\frac{V_{g} L_{m}+n V_{o}\left(L_{m}+L_{r}\right)}{\omega_{m c}\left(L_{m}+L_{r}\right) T} \sin w_{m c} d_{7} T=0
\end{aligned}
$$

Sumando las ecuaciones (F.67) y (F.68) desaparecen los términos trigonométricos, y se llega a la ecuación (F.69), una vez agrupados y simplificados todos los términos.

$$
V_{g}\left(d_{2}+d_{3}+d_{7}+d_{4}+d_{6}+d_{8}\right)-V_{c}\left(d_{2}+d_{3}+d_{4}\right)=0
$$

en donde se sabe que $d_{2}+d_{3}+d_{4}+d_{6}+d_{7}+d_{8}=1$. Entonces se despeja $V_{c}$ que queda como:

$$
V_{c}=\frac{V_{g}}{d_{2}+d_{3}+d_{4}}=\frac{V_{g}}{(1-D)}
$$

siendo $d_{2}+d_{3}+d_{4}=(1-D)$.

\section{DESCARGA DE $C_{c l}$. CÁLCULO DE $I_{D_{c}}$ y $d_{7}$ CON RIZADO}

La duración de la etapa 7 está relacionada con la descarga del condensador $C_{c l}$ desde $V_{g}+n V_{o}$ hasta cero. La evolución durante esta etapa está indicada en (F.58). y en $t_{7}$, resulta que $v_{C_{c l}}\left(t_{7}\right)=0$, por lo que:

$$
\begin{gathered}
0=V_{g} \frac{L_{r}}{L_{m}+L_{r}}+\left[V_{g} \frac{L_{m}}{L_{m}+L_{r}}+n V_{o}\right] \cos \left(\omega_{m c} d_{7} T\right) \Rightarrow \\
\Rightarrow \cos \left(\omega_{m c} d_{7} T\right)=\frac{-V_{g} \frac{L_{r}}{L_{m}+L_{r}}}{V_{g} \frac{L_{m}}{L_{m}+L_{r}}+n V_{o}}=\frac{-V_{g} L_{r}}{V_{g} L_{m}+n V_{o}\left(L_{m}+L_{r}\right)} \Rightarrow \\
\Rightarrow d_{7}=\frac{1}{\omega_{m c}} \operatorname{arc} \cos \left(\frac{-V_{g} L_{r}}{V_{g} L_{m}+n V_{o}\left(L_{m}+L_{r}\right)}\right)
\end{gathered}
$$


Durante la descarga del condensador, su intensidad alcanza el valor $I_{c l 7}$, dado por:

$$
I_{c l 7} \equiv=i_{C_{c l}}\left(t_{7}\right)=-\frac{V_{g} L_{m}+n V_{o}\left(L_{m}+L_{r}\right)}{\left(L_{m}+L_{r}\right) Z_{m c}} \sin \omega_{m c} d_{7} T
$$

Este término, pero en valor absoluto, indica el incremento que experimenta $i_{r}(t)$ durante la etapa 7 . Por tanto, $I_{D_{c}} \equiv\left|I_{c l 7}\right|$. Empleando la relación (F.71) se puede obtener una expresión para $I_{D_{c}}$ que no depende de ningún parámetro temporal.

$$
\begin{aligned}
I_{D_{c}} & =\frac{V_{g} L_{m}+n V_{o}\left(L_{m}+L_{r}\right)}{\left(L_{m}+L_{r}\right) Z_{m c}} \sqrt{1-\left[\frac{-V_{g} L_{r}}{V_{g} L_{m}+n V_{o}\left(L_{m}+L_{r}\right)}\right]^{2}} \\
& =\sqrt{\frac{\left[V_{g} L_{m}+n V_{o}\left(L_{m}+L_{r}\right)\right]^{2}-\left[V_{g} L_{r}\right]^{2}}{\left(L_{m}+L_{r}\right)^{2} Z_{m c}^{2}}} \\
& =\sqrt{\frac{\left(V_{g} L_{m}+n V_{o}\left(L_{m}+L_{r}\right)+V_{g} L_{r}\right)\left(V_{g} L_{m}+n V_{o}\left(L_{m}+L_{r}\right)-V_{g} L_{r}\right)}{\left(L_{m}+L_{r}\right)^{2} Z_{m c}^{2}}} \\
& =\frac{1}{Z_{m c}} \sqrt{\left(V_{g}+n V_{o}\right)\left[V_{g} \frac{L_{m}-L_{r}}{L_{m}+L_{r}}+n V_{o}\right]}
\end{aligned}
$$

\section{CORRIENTE MEDIA EN EL CONDENSADOR EQUIVALEN-} TE $C_{c l}$. CÁLCULO DE $d_{3}$ CON RIZADO

Haciendo $I_{c l, m e d}=0$ se obtiene la siguiente ecuación:

$\frac{1}{T} \int_{0}^{d_{3} T}\left(\frac{V_{c} L_{m}+V_{g} L_{r}}{\left(L_{m}+L_{r}\right) Z_{m c}} \sin \omega_{m c} t\right) d t-\frac{1}{T} \int_{0}^{d_{7} T}\left(\frac{V_{g} L_{m}+n V_{o}\left(L_{m}+L_{r}\right)}{\left(L_{m}+L_{r}\right) Z_{m c}} \sin \omega_{m c} t\right) d t=0$

Resolviendo las integrales y simplificando, queda que:

$\frac{V_{c} L_{m}+V_{g} L_{r}}{\left(L_{m}+L_{r}\right) T Z_{m c}}\left(1-\cos \omega_{m c} d_{3} T\right)=-\frac{V_{g} L_{m}+n V_{o}\left(L_{m}+L_{r}\right)}{\left(L_{m}+L_{r}\right) T Z_{m c}}\left(\cos \omega_{m c} d_{7} T-1\right)$

Utilizando el resultado (F.71) en (F.75) y despejando el término trigonométrico que engloba a $d_{3}$ se obtiene:

$$
\cos \omega_{m c} d_{3} T=1-\frac{\left(V_{g}+n V_{o}\right)\left(L_{m}+L_{r}\right)}{V_{c} L_{m}+V_{g} L_{r}}
$$

CORRIENTE MEDIA EN EL DIODO $D_{c l}$ CON RIZADO 
Haciendo el valor medio a (F.60), se obtiene la corriente media el diodo $D_{c l}$, representada como $I_{D_{c l}, \text { med }}$ :

$$
\begin{aligned}
I_{D_{c l}, \text { med }} & =\frac{1}{T}\left[\int_{0}^{d_{2} T}\left(I_{r 0}-\frac{V_{c}}{L_{r}} t-I_{m 0}-\frac{V_{g}}{L_{m}} t\right) d t+\int_{0}^{d_{8} T} I_{D_{c}}-\frac{V_{g}}{L_{m}} t d t\right] \\
& =\frac{1}{T}\left[I_{D_{c}}\left(d_{2}+d_{8}\right) X-\frac{V_{c}}{L_{r}} \frac{d_{2}^{2} T^{\not 2}}{2}--\frac{V_{g}}{L_{m}} \frac{\left(d_{2}+d_{8}\right)^{2} T^{\not 2}}{2}\right]
\end{aligned}
$$

Para la resolución se ha resuelto previamente la diferencia $I_{r 0}-I_{m 0}$ :

$$
\begin{aligned}
& I_{r 0}-I_{m 0}=\left[I_{r 6}+\frac{V_{g}}{L_{m}+L_{r}} d_{7} T+\frac{V_{g} L_{m}+n V_{o}\left(L_{m}+L_{r}\right)}{\omega_{m c} L_{r}\left(L_{m}+L_{r}\right)} \sin \omega_{m c} d_{7} T\right] \\
& \quad-\left[I_{m 6}+\frac{V_{g}}{L_{m}+L_{r}} d_{7} T-\frac{V_{g} L_{m}+n V_{o}\left(L_{m}+L_{r}\right)}{\omega_{m c} L_{m}\left(L_{m}+L_{r}\right)} \sin \omega_{m c} d_{7} T+\frac{V_{g}}{L_{m}} d_{8} T\right] \\
& =\frac{V_{g} L_{m}+n V_{o}\left(L_{m}+L_{r}\right)}{Z_{m c}\left(L_{m}+L_{r}\right)} \sin \omega_{m c} d_{7} T=I_{D_{c}}-\frac{V_{g}}{L_{m}} d_{8} T
\end{aligned}
$$

CORRIENTE MEDIA EN EL CONDENSADOR DE CLAMP $C_{c}$ CON RIZADO

Como $I_{c, \text { med }}=0$ :

$$
\begin{aligned}
& \frac{1}{T}[\underbrace{\int_{0}^{d_{2} T}\left(I_{r 0}-\frac{V_{c}}{L_{r}} t\right) d t}_{I_{5}}+\underbrace{\int_{0}^{d_{3} T}\left(I_{r 2}+\frac{V_{g}-V_{c}}{L_{m}+L_{r}} t-\frac{V_{c} L_{m}+V_{g} L_{r}}{\omega_{m c} L_{r}\left(L_{m}+L_{r}\right)} \sin w_{m c} t\right) d t}_{I_{6}} \\
& +\underbrace{\int_{0}^{d_{4} T}\left(I_{r 3}+\frac{\left(V_{g}+n V_{o}-V_{c}\right)}{L_{r}} t\right) d t}_{I_{7}}]=0
\end{aligned}
$$

Si se expresa $I_{r 0}$ como función de $I_{r 2}$ se puede obtener una expresión en función del resto de parámetros. Entonces, atendiendo la expresión de $i_{r}(t)$ en el intervalo $\left[t_{1}, t_{2}\right]$ :

$$
I_{r 2} \equiv i_{r}\left(t_{2}\right)=I_{r 0}-\frac{V_{c}}{L_{r}} d_{2} T \Rightarrow I_{r 0}=I_{r 2}+\frac{V_{c}}{L_{r}} d_{2} T
$$

Resolviendo cada una de las integrales $I_{5}, I_{6}$ e $I_{7}$ :

$$
\begin{aligned}
I_{5}= & {\left[\left(I_{r 2}+\frac{V_{c}}{L_{r}} d_{2} T\right) t-\frac{V_{c}}{L_{r}} \frac{t^{2}}{2}\right]_{0}^{d_{2} T} } \\
& =I_{r 2} d_{2} T+\frac{V_{c}}{L_{r}} d_{2}^{2} T^{2}-\frac{V_{c}}{L_{r}} \frac{d_{2}^{2} T^{2}}{2}=I_{r 2} d_{2} T+\frac{V_{c}}{L_{r}} \frac{d_{2}^{2} T^{2}}{2}
\end{aligned}
$$




$$
\begin{aligned}
I_{6}= & {\left[I_{r 2} t+\frac{V_{g}-V_{c}}{L_{m}+L_{r}} \frac{t^{2}}{2}-\frac{V_{c} L_{m}+V_{g} L_{r}}{\omega_{m c} L_{r}\left(L_{m}+L_{r}\right)} \frac{1}{\left(-\omega_{m c}\right)} \cos \omega_{m c} t\right]_{0}^{d_{3} T} } \\
& =I_{r 2} d_{3} T+\frac{V_{g}-V_{c}}{L_{m}+L_{r}} \frac{d_{3}^{2} T^{2}}{2}+\frac{V_{c} L_{m}+V_{g} L_{r}}{\omega_{m c}^{2} L_{r}\left(L_{m}+L_{r}\right)}\left[\cos \omega_{m c} d_{3} T-1\right] \\
& =I_{r 2} d_{3} T+\frac{V_{g}-V_{c}}{L_{m}+L_{r}} \frac{d_{3}^{2} T^{2}}{2}-\frac{V_{g}+n V_{o}}{\omega_{m c}^{2} L_{r}} \\
I_{7}= & {\left[I_{r 3} t+\frac{\left(V_{g}+n V_{o}-V_{c}\right)}{L_{r}} \frac{t^{2}}{2}\right]_{0}^{d_{4} T}=I_{r 3} d_{4} T+\frac{\left(V_{g}+n V_{o}-V_{c}\right)}{L_{r}} \frac{d_{4}^{2} T^{2}}{2} } \\
& =I_{r 2} d_{4} T+\frac{V_{g}-V_{c}}{L_{m}+L_{r}} d_{3} d_{4} T^{2}-\frac{V_{c} L_{m}+V_{g} L_{r}}{\omega_{m c} L_{r}\left(L_{m}+L_{r}\right)} d_{4} T \sin \omega_{m c} d_{3} T \\
& +\frac{\left(V_{g}+n V_{o}-V_{c}\right)}{L_{r}} \frac{d_{4}^{2} T^{2}}{2}
\end{aligned}
$$

Finalmente:

$$
\begin{aligned}
0 & =I_{C_{c}, \text { med }}=\frac{1}{T}\left(I_{5}+I_{6}+I_{7}\right) \\
& =I_{r 2}\left(d_{2}+d_{3}+d_{4}\right)+\frac{V_{c}}{L_{r}} \frac{d_{2}^{2} T}{2}+\frac{V_{g}-V_{c}}{L_{m}+L_{r}}\left(\frac{d_{3}^{2} T}{2}+d_{3} d_{4} T\right) \\
& -\frac{V_{c} L_{m}+V_{g} L_{r}}{\omega_{m c} L_{r}\left(L_{m}+L_{r}\right)} d_{4} \sin \omega_{m c} d_{3} T+\frac{\left(V_{g}+n V_{o}-V_{c}\right)}{L_{r}} \frac{d_{4}^{2} T}{2}-\frac{V_{g}+n V_{o}}{\omega_{m c}^{2} L_{r} T}
\end{aligned}
$$

Despejando $I_{r 2}$ se obtiene:

$$
\begin{aligned}
I_{r 2} & =\frac{1}{\left(d_{2}+d_{3}+d_{4}\right)}\left[\frac{V_{g}+n V_{o}}{\omega_{m c}^{2} L_{r} T}-\frac{V_{c}}{L_{r}} \frac{d_{2}^{2} T}{2}-\frac{V_{g}-V_{c}}{L_{m}+L_{r}}\left(\frac{d_{3}^{2} T}{2}+d_{3} d_{4} T\right)+\right. \\
& \left.+\frac{V_{c} L_{m}+V_{g} L_{r}}{\omega_{m c} L_{r}\left(L_{m}+L_{r}\right)} d_{4} \sin \omega_{m c} d_{3} T-\frac{\left(V_{g}+n V_{o}-V_{c}\right)}{L_{r}} \frac{d_{4}^{2} T}{2}\right]
\end{aligned}
$$

\section{CORRIENTE MEDIA EN LA BOBINA DE MAGNETIZACIÓN CON RIZADO}

Haciendo el valor medio a la expresión de $i_{m}(t)$ dada en (F.57), se obtendrá una expresión en función de la duración de los intervalos. Si se emplean las relaciones anteriores, también se puede conseguir en función de $I_{m 2}$.

$$
I \equiv I_{m, m e d}=\sum_{k=1}^{8}\left[\frac{1}{T} \int_{t_{k-1}}^{t_{k}} i_{m}(t) d t\right]=\frac{1}{T}\left[I_{8}+I_{9}+I_{10}+I_{11}+I_{12}\right]
$$


Donde se calculan las integrales $I_{8}$ a $I_{12}$ como:

$$
\begin{gathered}
I_{8}=\int_{0}^{d_{2} T}\left(I_{m 0}+\frac{V_{g}}{L_{m}} t\right) d t=I_{m 2} d_{2} T-\frac{V_{g}}{L_{m}} \frac{d_{2}^{2} T^{2}}{2} \\
I_{9}=\int_{0}^{d_{3} T}\left(I_{m 2}+\frac{V_{g}-V_{c}}{L_{m}+L_{r}} t+\frac{V_{c} L_{m}+V_{g} L_{r}}{\omega_{m c} L_{m}\left(L_{m}+L_{r}\right)} \sin \omega_{m c} t\right) d t \\
=I_{m 2} d_{3} T+\frac{V_{g}-V_{c}}{L_{m}+L_{r}} \frac{d_{3}^{2} T^{2}}{2}-\frac{V_{c} L_{m}+V_{g} L_{r}}{\omega_{m c}^{2} L_{m}\left(L_{m}+L_{r}\right)}\left[\cos \left(\omega_{m c} d_{3} T\right)-1\right] \\
I_{10}=\int_{0}^{\left(d_{4}+d_{6}\right) T}\left(I_{m 3}-\frac{n V_{o}}{L_{m}} t\right) d t \\
=\left[I_{m 2}-\frac{V_{g}}{L_{m}}\left(d_{2}+d_{8}\right) T-\frac{V_{g}}{L_{m}+L_{r}} d_{7} T+I_{D_{c}} \frac{L_{r}}{L_{m}+L_{r}}+\frac{n V_{o}}{L_{m}}\left(d_{4}+d_{6}\right) T\right]\left(d_{4}+d_{6}\right) T \\
-\frac{n V_{o}}{L_{m}} \frac{\left(d_{4}+d_{6}\right)^{2} T^{2}}{2} \\
I_{11}=\int_{0}^{d_{7} T}\left(I_{m 6}+\frac{V_{g}}{L_{m}+L_{r}} t-\frac{V_{g} L_{m}+n V_{o}\left(L_{m}+L_{r}\right)}{\omega_{m c} L_{m}\left(L_{m}+L_{r}\right)} \sin \omega_{m c} t\right) d t \\
=\left[I_{m 2}-\frac{V_{g}}{L_{m}}\left(d_{2}+d_{8}\right) T-\frac{V_{g}}{L_{m}+L_{r}} d_{7} T+I_{D_{c}} \frac{L_{r}}{L_{m}+L_{r}}\right] d_{7} T \\
\quad+\frac{V_{g}}{L_{m}+L_{r}} \frac{d_{7}^{2} T^{2}}{2}+\frac{V_{g} L_{m}+n V_{o}\left(L_{m}+L_{r}\right)}{\omega_{m c}^{2} L_{m}\left(L_{m}+L_{r}\right)}\left[\cos \omega_{m c} d_{7} T-1\right] \\
I_{12}=\int_{0}^{d_{8} T}\left(I_{m 7}+\frac{V_{g}}{L_{m}} t\right)=I_{m 2} d_{8} T-\frac{V_{g}}{L_{m}}\left[d_{2} d_{8} T^{2}+\frac{d_{8}^{2} T^{2}}{2}\right]
\end{gathered}
$$

Para el cálculo de las integrales se han empleado las relaciones dadas en (F.53) y en (F.55) que permiten relacionar $I_{m 0}$, e $I_{m 7}$ con $I_{m 2}$ :

$$
\begin{aligned}
& I_{m 0}=I_{m 2}-\frac{V_{g}}{L_{m}} d_{2} T \\
& I_{m 7}=I_{m 2}-\frac{V_{g}}{L_{m}}\left(d_{8}+d_{2}\right) T
\end{aligned}
$$

y también las expresiones de $I_{m 6}$ en función de $I_{m 2}$ y de $I_{r 6}$ en función de $I_{r 2}$. Se indican a continuación:

$$
\begin{aligned}
I_{m 6, a} & =I_{m 2}-\frac{V_{g}}{L_{m}}\left(d_{2}+d_{8}\right) T-\frac{V_{g}}{L_{m}+L_{r}} d_{7} T+I_{D_{c}} \frac{L_{r}}{L_{m}+L_{r}} \\
I_{m 6, b} & =I_{m 2}+\frac{V_{g}-V_{c}}{L_{m}+L_{r}} d_{3} T+\frac{V_{c} L_{m}+V_{g} L_{r}}{\omega_{m c} L_{m}\left(L_{m}+L_{r}\right)} \sin \omega_{m c} d_{3} T \\
& -\frac{n V_{o}}{L_{m}}\left(d_{4}+d_{6}\right) T
\end{aligned}
$$


Las expresiones de los términos $I_{r x}$ dados en (F.55) también permiten relacionar $I_{r 6}$ en función de $I_{r 2}$ mediante dos expresiones equivalentes:

$$
\begin{aligned}
I_{r 6, a} & =I_{r 2}-\frac{V_{g}}{L_{m}+L_{r}} d_{7} T-I_{D_{c}} \frac{L_{m}}{L_{m}+L_{r}}+\frac{V_{c}}{L_{r}} d_{2} T \\
I_{r 6, b} & =I_{r 2}+\frac{V_{g}-V_{c}}{L_{m}+L_{r}} d_{3} T-\frac{V_{c} L_{m}+V_{g} L_{r}}{\omega_{m c} L_{r}\left(L_{m}+L_{r}\right)} \sin \omega_{m c} d_{3} T \\
& +\frac{\left(V_{g}+n V_{o}-V_{c}\right)}{L_{r}} d_{4} T+\frac{V_{g}+n V_{o}}{L_{r}} d_{6} T
\end{aligned}
$$

Dado que $i_{r}(t)$ e $i_{m}(t)$ coinciden en los instantes $t_{2}$ y $t_{6}$ se pueden deducir dos relaciones partir de igualar las expresiones (F.93) y (F.94):

$$
\begin{aligned}
I_{r 6, a} & =I_{m 6, a} \Rightarrow I_{D_{c}}=\frac{V_{g}}{L_{m}}\left(d_{8}+d_{2}\right) T+\frac{V_{c}}{L_{r}} d_{2} T \\
I_{r 6, b} & =I_{r 6, b} \Rightarrow \frac{V_{c} L_{m}+V_{g} L_{r}}{Z_{m c}\left(L_{m}+L_{r}\right)} \sin \omega_{m c} d_{3} T \\
& =\frac{V_{g}+n V_{o}}{L_{r}}\left(d_{4}+d_{6}\right) T+\frac{n V_{o}}{L_{m}}\left(d_{4}+d_{6}\right) T-\frac{V_{c}}{L_{r}} d_{4} T
\end{aligned}
$$

El valor medio de la corriente en la bobina es:

$$
\begin{aligned}
I= & \frac{1}{T}\left[I_{m 2}\left(d_{2}+d_{3}+d_{4}+d_{6}+d_{7}+d_{8}\right) T-\frac{V_{g}}{L_{m}} \frac{d_{2}^{2} T^{2}}{2}\right. \\
& +\frac{V_{g}-V_{c}}{L_{m}+L_{r}} \frac{d_{3}^{2} T^{2}}{2}-\frac{V_{c} L_{m}+V_{g} L_{r}}{\omega_{m c}^{2} L_{m}\left(L_{m}+L_{r}\right)}\left[\cos \left(\omega_{m c} d_{3} T\right)-1\right] \\
& -\frac{V_{g}}{L_{m}}\left(d_{2}+d_{8}\right)\left(d_{4}+d_{6}\right) T^{2}-\frac{V_{g}}{L_{m}+L_{r}}\left(d_{4}+d_{6}\right) d_{7} T^{2}+I_{D_{c}} \frac{L_{r}}{L_{m}+L_{r}}\left(d_{4}+d_{6}\right) T \\
& +\frac{n V_{o}}{L_{m}} \frac{\left(d_{4}+d_{6}\right)^{2} T^{2}}{2} \\
& -\frac{V_{g}}{L_{m}}\left(d_{2}+d_{8}\right) d_{7} T^{2}-\frac{V_{g}}{L_{m}+L_{r}} \frac{d_{7}^{2} T^{2}}{2}+I_{D_{c}} \frac{L_{r}}{L_{m}+L_{r}} d_{7} T \\
+ & \frac{V_{g}}{L_{m}+L_{r}} \frac{d_{7}^{2} T^{2}}{2}+\frac{V_{g} L_{m}+n V_{o}\left(L_{m}+L_{r}\right)}{\omega_{m c}^{2} L_{m}\left(L_{m}+L_{r}\right)}\left[\cos \omega_{m c} d_{7} T-1\right] \\
& \left.-\frac{V_{g}}{L_{m}} d_{2} d_{8} T^{2}-\frac{V_{g}}{L_{m}} \frac{d_{8}^{2} T^{2}}{2}\right]
\end{aligned}
$$


Finalmente se obtiene una expresión en función de $I_{m_{2}}$ como:

$$
\begin{aligned}
I= & \frac{1}{T}\left[I_{m 2}\left(d_{2}+d_{3}+d_{4}+d_{6}+d_{7}+d_{8}\right) T-\frac{V_{g}}{L_{m}} \frac{\left(d_{2}+d_{8}\right)^{2} T^{2}}{2}+\frac{V_{g}-V_{c}}{L_{m}+L_{r}} \frac{d_{3}^{2} T^{2}}{2}\right. \\
& -\frac{V_{g}}{L_{m}}\left(d_{2}+d_{8}\right)\left(d_{4}+d_{6}\right) T^{2}-\frac{V_{g}}{L_{m}+L_{r}}\left(d_{4}+d_{6}\right) d_{7} T^{2}+I_{D_{c}} \frac{L_{r}}{L_{m}+L_{r}}\left(d_{4}+d_{6}+d_{7}\right) T \\
& \left.+\frac{n V_{o}}{L_{m}} \frac{\left(d_{4}+d_{6}\right)^{2} T^{2}}{2}-\frac{V_{g}}{L_{m}}\left(d_{2}+d_{8}\right) d_{7} T^{2}\right] \\
= & I_{m 2}-\frac{V_{g}}{L_{m}}\left(d_{2}+d_{8}\right) T\left[\frac{\left(d_{2}+d_{8}\right)}{2}+d_{4}+d_{6}+d_{7}\right]+\frac{V_{g}-V_{c}}{L_{m}+L_{r}} \frac{d_{3}^{2} T}{2} \\
& -\frac{V_{g}}{L_{m}+L_{r}}\left(d_{4}+d_{6}\right) d_{7} T+I_{D_{c}} \frac{L_{r}}{L_{m}+L_{r}}\left(d_{4}+d_{6}+d_{7}\right)+\frac{n V_{o}}{L_{m}} \frac{\left(d_{4}+d_{6}\right)^{2} T}{2}
\end{aligned}
$$

Y como, ya se ha indicado, $I_{m 2} \equiv I_{r 2}$, por lo que empleando la expresión (F.85) se consigue una expresión de $I$, que queda indicada en (F.98):

$$
\begin{aligned}
I= & \frac{1}{\left.d_{2}+d_{3}+d_{4}\right)}\left[\frac{V_{g}+n V_{o}}{\omega_{m c}^{2} L_{r} T}-\frac{V_{c}}{L_{r}} \frac{d_{2}^{2} T}{2}-\frac{V_{g}-V_{c}}{L_{m}+L_{r}}\left(\frac{d_{3}^{2} T}{2}+d_{3} d_{4} T\right)+\right. \\
& \left.+\frac{V_{c} L_{m}+V_{g} L_{r}}{\omega_{m c} L_{r}\left(L_{m}+L_{r}\right)} d_{4} \sin \omega_{m c} d_{3} T-\frac{\left(V_{g}+n V_{o}-V_{c}\right)}{L_{r}} \frac{d_{4}^{2} T}{2}\right] \\
& -\frac{V_{g}}{L_{m}}\left(d_{2}+d_{8}\right) T\left[\frac{\left(d_{2}+d_{8}\right)}{2}+d_{4}+d_{6}+d_{7}\right]+\frac{V_{g}-V_{c}}{L_{m}+L_{r}} \frac{d_{3}^{2} T}{2} \\
& -\frac{V_{g}}{L_{m}+L_{r}}\left(d_{4}+d_{6}\right) d_{7} T+I_{D_{c}} \frac{L_{r}}{L_{m}+L_{r}}\left(d_{4}+d_{6}+d_{7}\right)+\frac{n V_{o}}{L_{m}} \frac{\left(d_{4}+d_{6}\right)^{2} T}{2}
\end{aligned}
$$

\section{RIZADO DE CORRIENTE EN BOBINA DE MAGNETIZA-}

\section{CIÓN}

El rizado de corriente en $L_{m}$, se obtiene como la diferencia entre el valor máximo y el valor mínimo de $i_{m}(t)$.

El valor máximo, $I_{\max }$ se produce durante la etapa 3 , en el instante $t_{\text {max }}$. Derivando la expresión de $i_{m}(t)$ en ambas etapas e igualando a cero se obtiene el instante temporal en el que se produce el valor máximo. Definiendo $d_{\max } T=\left(t_{\max }-t_{2}\right)$ se llega a:

$$
\begin{gathered}
\left.\begin{array}{c}
0=\left.\frac{d i_{m}(t)}{d t}\right|_{t=t_{\max }} \\
t_{\text {max }} \in\left[t_{2}, t_{3}\right]
\end{array}\right\} \Rightarrow \frac{V_{g}-V_{c}}{L_{m}+L_{r}}+\frac{V c L_{m}+V_{g} L_{r}}{L_{m}\left(L_{m}+L_{r}\right)} \cos \left(\omega_{m c} d_{\text {max }} T\right)=0 \\
\Rightarrow d_{\max } T=\frac{\operatorname{arc~} \cos \left[\frac{L_{m}\left(V_{g}-V_{c}\right)}{V_{c} L_{m}+V_{g} L_{r}}\right]}{\omega_{m c}}
\end{gathered}
$$




$$
\begin{aligned}
I_{\max } \equiv i_{m}\left(t_{\text {max }}\right)= & I_{m 2}+\frac{V_{g}-V_{c}}{L_{m}+L_{r}} d_{\max } T \\
& +\frac{V_{c} L_{m}+V_{g} L_{r}}{\omega_{m c} L_{m}\left(L_{m}+L_{r}\right)} \sin \left(\omega_{m c} d_{\text {max }} T\right)
\end{aligned}
$$

El valor mínimo, $I_{\min }$ se produce durante la etapa 7 , en el instante $t_{\min }$. Derivando la expresión de $i_{m}(t)$ en ambas etapas e igualando a cero se obtiene el instante temporal en el que se produce el valor mínimo. Definiendo $d_{\min } T=\left(t_{\min }-t_{6}\right)$ se llega a:

$$
\begin{aligned}
\left.\begin{array}{l}
0=\left.\frac{d i_{m}(t)}{d t}\right|_{t=t_{\text {min }}} \\
t_{\text {min }} \in\left[t_{6}, t_{7}\right]
\end{array}\right\} & \Rightarrow \frac{V_{g}}{L_{m}+L_{r}}+\frac{V_{g} L_{m}+n V_{o}\left(L_{m}+L_{r}\right)}{L_{m}\left(L_{m}+L_{r}\right)} \cos \left(\omega_{m c}\right. \\
& \Rightarrow d_{m i n} T=\frac{\operatorname{arc~cos}\left[\frac{V_{g} L_{m}}{V_{g} L_{m}+n V_{o}\left(L_{m}+L_{r}\right)}\right]}{\omega_{m c}} \\
I_{\text {min }} \equiv i_{m}\left(t_{\text {min }}\right)= & I_{m 6}+\frac{V_{g}}{L_{m}+L_{r}} d_{m i n} T \\
& +\frac{V_{g} L_{m}+n V_{o}\left(L_{m}+L_{r}\right)}{\omega_{m c} L_{m}\left(L_{m}+L_{r}\right)} \sin \left(\omega_{m c} d_{m i n} T\right)
\end{aligned}
$$

El rizado se obtiene como la diferencia entre el valor máximo y el mínimo de corriente, sabiendo que $I_{m 6}$ se puede poner en función $I_{m 2}$ :

$$
\begin{aligned}
& \Delta_{m}=I_{\max }-I_{\min } \\
& =\frac{V_{g}-V_{c}}{L_{m}+L_{r}}\left(d_{m a x} T-d_{3} T\right)+\frac{V_{c} L_{m}+V_{g} L_{r}}{\omega_{m c} L_{m}\left(L_{m}+L_{r}\right)}\left[\sin \left(\omega_{m c} d_{m a x} T\right)-\sin \left(\omega_{m c} d_{3} T\right)\right] \\
& +\frac{n V_{o}}{L_{m}}\left(d_{4} T+d_{6} T\right)-\frac{V_{g}}{L_{m}+L_{r}} d_{m i n} T-\frac{V_{g} L_{m}+n V_{o}\left(L_{m}+L_{r}\right)}{\omega_{m c} L_{m}\left(L_{m}+L_{r}\right)} \sin \left(\omega_{m c} d_{m i n} T\right)
\end{aligned}
$$

CORRIENTE MEDIA EN LA BOBINA RESONANTE, $I_{r}$, Y EN LA ENTRADA, $I_{g}$.

La relación entre la corriente media en la bobina de magnetización y la corriente media en $L_{r}$, expresada como $I_{r}$ se puede obtener haciendo el valor medio a las expresiones (F.6) y (F.7), que se han reescrito a continuación:

$$
\left.\begin{array}{l}
i_{g}(t)+\frac{1}{n} i_{D}(t)=i_{m}(t) \\
i_{g}(t)+i_{c l}(t)=i_{r}(t)
\end{array}\right\} \stackrel{\text { (V.med.) }}{\Longrightarrow}\left\{\begin{array}{l}
I_{g}+\frac{I_{o}}{n}=I \\
I_{g}+I_{D_{c l}, \text { med }}=I_{r}
\end{array}\right.
$$


y como $P_{g}=P_{o}$, entonces:

$$
I_{o} \frac{V_{o}}{V_{g}}+\frac{I_{o}}{n}=I
$$

Entonces, $I_{r}$ se expresa como:

$$
I_{r}=I_{o} \frac{V_{o}}{V_{g}}+I_{D_{c l}, \text { med }}=I \frac{n V_{o}}{V_{g}+n V_{o}}+I_{D_{c l}, \text { med }}
$$

\section{CARACTERÍSTICA DE SALIDA CON RIZADO}

La expresión que relaciona la tensión de salida con la intensidad de salida de puede obtener a partir de la expresión (F.98) y la relación entre intensidades de magnetización y salida dada en (F.105). Tras despejar $I_{o}$ en esas expresiones se obtiene una expresión $I_{o}\left(V_{o}\right)$ como la siguiente:

$$
\begin{aligned}
I_{o}= & \frac{n V_{g}}{(1-D)\left(V_{g}+n V_{o}\right)}\left[\frac{V_{g}+n V_{o}}{\omega_{m c}^{2} L_{r} T}-\frac{V_{c}}{L_{r}} \frac{d_{2}^{2} T}{2}-\frac{V_{g}-V_{c}}{L_{m}+L_{r}}\left(\frac{d_{3}^{2} T}{2}+d_{3} d_{4} T\right)+\right. \\
& \left.+\frac{V_{c} L_{m}+V_{g} L_{r}}{\omega_{m c} L_{r}\left(L_{m}+L_{r}\right)} d_{4} \sin \omega_{m c} d_{3} T-\frac{\left(V_{g}+n V_{o}-V_{c}\right)}{L_{r}} \frac{d_{4}^{2} T}{2}\right] \\
& -\frac{V_{g}}{L_{m}}\left(d_{2}+d_{8}\right) T\left[\frac{\left(d_{2}+d_{8}\right)}{2}+d_{4}+d_{6}+d_{7}\right]+\frac{V_{g}-V_{c}}{L_{m}+L_{r}} \frac{d_{3}^{2} T}{2} \\
& -\frac{V_{g}}{L_{m}+L_{r}}\left(d_{4}+d_{6}\right) d_{7} T+I_{D_{c}} \frac{L_{r}}{L_{m}+L_{r}}\left(d_{4}+d_{6}+d_{7}\right)+\frac{n V_{o}}{L_{m}} \frac{\left(d_{4}+d_{6}\right)^{2} T}{2}
\end{aligned}
$$

Del análisis realizado al tener en cuenta el rizado, se han encontrado ciertas relaciones, como el valor máximo de la corriente que pasar por el diodo $D_{c}$, (véase (F.73)) o la duración de la la etapa 7, (véase (F.71)), que no dependen del ciclo de trabajo. Los términos $V_{c}$ y $d_{3}$ se pueden expresar como función de $D$ (véase (F.70) y (F.76)).

La duración del resto de intervalos, $d_{2}, d_{4}, d_{6}$ y $d_{8}$ se obtiene al resolver un sistema de ecuaciones que incluyen los términos indicados en el párrafo anterior, tomando $D$ como parámetro. Las ecuaciones que forman este sistema ya se han obtenido anteriormente: la primera ecuación es la que se obtiene al igualar las corrientes de magnetización y resonante al final de la etapa 2 y al final de la etapa 6 ; las otras ecuaciones se han obtenido al hacer $V_{L_{m}, \text { med }}=0 \mathrm{y}$ al hacer $V_{L_{r}, \text { med }}=0$; por último, se sabe que la suma de todos los intervalos es igual a la unidad.

La resolución de este sistema, que es lineal si se toma $D$ como un parámetro, ofrece expresiones para la duración de los intervalos en función de ese 
parámetro. El sistema descrito de forma matricial se indica a continuación:

$$
\left[\begin{array}{cccc}
V_{g} & -n V_{o} & -n V_{o} & V_{g} \\
-V_{c} & \left(V_{g}+n V_{o}-V_{c}\right) & \left(V_{g}+n V_{o}\right) & 0 \\
\frac{V_{c}}{L_{r}}+\frac{V_{g}}{L_{m}} & 0 & 0 & \frac{V_{g}}{L_{m}} \\
1 & 1 & 1 & 1
\end{array}\right]\left[\begin{array}{l}
d_{2} \\
d_{4} \\
d_{6} \\
d_{8}
\end{array}\right]=\left[\begin{array}{l}
b_{1} \\
b_{2} \\
b_{3} \\
b_{4}
\end{array}\right]
$$

Los términos independientes, $b_{x}$, de las ecuaciones mencionadas son:

$$
\begin{aligned}
b_{1}= & -\frac{V_{c} L_{m}+V_{g} L_{r}}{\omega_{m c}\left(L_{m}+L_{r}\right) T} \operatorname{sen} w_{m c} d_{3} T-\left(V_{g}-V_{c}\right) \frac{L_{m}}{L_{m}+L_{r}} d_{3} \\
& +\frac{V_{g} L_{m}+n V_{o}\left(L_{m}+L_{r}\right)}{\omega_{m c}\left(L_{m}+L_{r}\right) T} \sin w_{m c} d_{7} T-V_{g} \frac{L_{m}}{L_{m}+L_{r}} d_{7} \\
b_{2}= & +\frac{V_{c} L_{m}+V_{g} L_{r}}{\omega_{m c}\left(L_{m}+L_{r}\right) T} \operatorname{sen} w_{m c} d_{3} T-\left(V_{g}-V_{c}\right) \frac{L_{r}}{L_{m}+L_{r}} d_{3} \\
& -\frac{V_{g} L_{m}+n V_{o}\left(L_{m}+L_{r}\right)}{\omega_{m c}\left(L_{m}+L_{r}\right) T} \sin w_{m c} d_{7} T-V_{g} \frac{L_{r}}{L_{m}+L_{r}} d_{7} \\
b_{3}= & +\frac{V_{g} L_{m}+n V_{o}\left(L_{m}+L_{r}\right)}{Z_{m c}\left(L_{m}+L_{r}\right) T} \sin \omega_{m c} d_{7} T \\
b_{4}= & 1-d_{3}-d_{7}
\end{aligned}
$$

Introduciendo ahora todos los términos en la ecuación de la característica de salida dada en (F.98), se consigue una expresión que relaciona $I_{o}$ con $D$, de la que se obtiene una de los dos parámetros en función del otro.

\section{F.2.3. Comparativa.}

El análisis cuando se considera el rizado será de aplicación al circuito conmutado, donde se deben tener en cuenta los valores de las bobinas, mientras que el análisis al no considerar rizado servirá para las expresiones derivadas del modelo promediado, dado que éste parte de la consideración de pequeño rizado.

Los resultados obtenidos para magnitudes tales como el ciclo de trabajo, la tensión de clamp y las variaciones de corriente, a partir de unos valores de diseño ponen de manifiesto estas diferencias. Por ejemplo, empleando los parámetros de funcionamiento de la Tabla F.1 se obtienen los valores indicados en la Tabla F.2, tanto para el caso de no considerar el rizado de la corriente en la bobina principal como para el que si se tiene en cuenta.

En este convertidor la intensidad en la bobina principal es igual a la intensidad en la entrada mas la de salida afectada de la relación de transformación, $I_{g}+I_{o} / n$. La tensión de clamp, $V_{c}$ también es diferente, dado que depende del ciclo de trabajo. 


\begin{tabular}{llll}
\hline \hline$V_{g}$ & $20 \mathrm{~V}$ & $C_{o}$ & $5 \mu \mathrm{F}$ \\
$P$ & $100 \mathrm{~W}$ & $C_{c}$ & $2,2 \mu \mathrm{F}$ \\
$V_{\text {out }}$ & $400 \mathrm{~V}$ & $L_{m}$ & $28 \mu \mathrm{H}$ \\
$f$ & $100 \mathrm{kHz}$ & $L_{r}$ & $2 \mu \mathrm{H}$ \\
$n_{1}: n_{2}$ & $1: 12$ & & \\
$t_{m}$ & $300 \mathrm{~ns}$ & $C_{r}$ & $1 \mathrm{nF}$ \\
\hline
\end{tabular}

Tabla F.1: Condiciones de trabajo de un flyback ZVS-modif.

\begin{tabular}{ccccccc}
\hline & $\begin{array}{c}I \\
(\mathrm{~A})\end{array}$ & $\begin{array}{c}D \\
-\end{array}$ & $\begin{array}{c}V_{c} \\
(\mathrm{~V})\end{array}$ & $\begin{array}{c}\Delta_{i_{L}} \\
(\mathrm{~A})\end{array}$ & $\begin{array}{c}I_{\max } \\
(\mathrm{A})\end{array}$ & $\begin{array}{c}I_{\min } \\
(\mathrm{A})\end{array}$ \\
\hline SIN RIZADO & & 0,6582 & 58,514 & 0 & - & - \\
CON RIZADO & 8 & 0,6701 & 60,632 & 4,371 & 10,183 & 5,812 \\
\hline \hline
\end{tabular}

Tabla F.2: Parámetros calculados cuando se considera o no el rizado.

En ambos análisis se han considerado despreciables algunas etapas por su pequeña duración en relación a las otras. Esta hipótesis se ha validado midiendo la duración de las seis etapas para los parámetros de funcionamiento de las dos tablas anteriores. Con ayuda de un programa de cálculo simbólico se han planteado las ecuaciones de estado durante las ocho etapas y se han resuelto las ecuaciones y obtenido la fracción que ocupa cada etapa dentro de un periodo completo. Se han indicado en la Tabla F.3.

\begin{tabular}{ccccccccc}
\hline & $d_{1}$ & $d_{2}$ & $d_{3}$ & $d_{4}$ & $d_{5}$ & $d_{6}$ & $d_{7}$ & $d_{8}$ \\
\hline SIN RIZADO & $464 \times 10^{-6}$ & 0,0156 & 0,0257 & 0,2999 & 0,0012 & 0,0476 & 0,0273 & 0,5820 \\
CON RIZADO & $577 \times 10^{-6}$ & 0,0010 & 0,0259 & 0,3031 & $991 \times 10^{-6}$ & 0,0450 & 0,0269 & 0,5973 \\
\hline \hline
\end{tabular}

Tabla F.3: Fracción de tiempo para cada etapa por las que evoluciona el convertidor.

A la vista de estos resultados, se puede comprobar que las etapas 1 y 5 son las de menor duración por lo que, como se había previsto, se pueden despreciar.

La característica de salida que se ha obtenido en los dos análisis realizados, tiene una expresión diferente en cada caso. Cuando se representan gráficamente en la Figura F.8 se obtienen curvas muy similares.

La curva representada sin considerar rizado emplea el valor de ciclo de trabajo que se obtiene con las expresiones de este mismo tipo de análisis, mientras que la curva cuando se considera el rizado está conseguida a partir del ciclo de trabajo calculado con rizado no despreciable. El caso sin rizado se considera equivalente al circuito promediado, mientras que el modelo con rizado es apropiado para un circuito conmutado. 


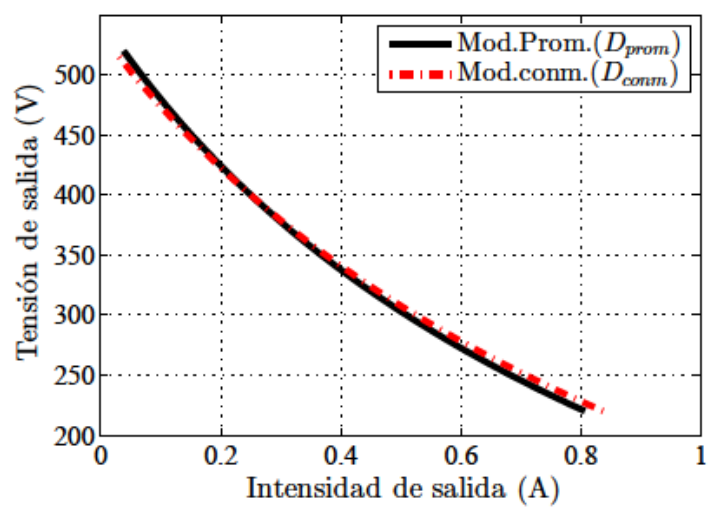

Figura F.8: Característica de salida de un flyback ZVS-modif.

\section{F.3. Modelo promediado.}

Para hacer el modelo promediado se toman como variables de estado las intensidades en bobinas y las tensiones en condensadores. En concreto, las intensidades $i_{r}$ e $i_{m}$ en las bobinas resonante $L_{r}$ y de magnetización $L_{m}$ respectivamente, y las tensiones $v_{C_{r}}, v_{C_{c}}, v_{C_{o}}$ y $v_{C_{c l}}$ en los condensadores resonante, $C_{r}$, de clamp $C_{c}$, de salida $C_{o}$ y el del diodo del clamp $C_{c l}$. El número de variables da lugar a un modelo de orden seis, pero puede reducirse a un modelo de orden cuatro si se agrupan las variables de estado en variables rápidas y variables lentas. En un primer paso, se escriben las variables rápidas en función de las lentas (que se suponen constantes), a lo largo de un periodo. Las expresiones obtenidas dependerán, por tanto, de las variables lentas y del tiempo. Esta expresiones se sustituyen en las ecuaciones de estado de las variables lentas que quedan desacopladas respecto a las variables rápidas. Se aplica un promedio estándar para eliminar la dependencia con el tiempo, y se obtiene un modelo promediado de cuarto orden (reducido) para las cuatro variables de estado lentas.

Aunque matemáticamente no es necesario, se suelen realizar algunas aproximaciones antes de empezar para simplificar los cálculos a realizar. En primer lugar, se ignoran los tiempos muertos entre las conmutaciones de $S_{1}$ y $S_{2}$ que normalmente son muy pequeños en comparación con el periodo. También se desprecian los intervalos resonantes, como se hace en (ATHALYE y otros, 2001) para obtener el modelo completo con el método del interruptor promediado. En este punto cabe indicar que el efecto debido a tener en cuenta los intervalos resonantes sería tan solo a frecuencias muy altas y fuera del rango de interés (que, generalmente, suele ser una fracción de la frecuencia de conmutación porque es donde se aplica el control en lazo cerrado). Otra 
suposición es considerar que el condensador de clamp mantiene su tensión constante, lo que quiere decir que la frecuencia de resonancia entre $L_{r}$ y $C_{c}$ es bastante menor que la frecuencia de conmutación.

Considerando estas simplificaciones desaparecen los intervalos resonantes (Etapa 1 y 5) y el tiempo muerto de la etapa 6 , de forma que un periodo se puede escribir como:

$$
T \approx d_{2} T+d_{3} T+d_{4} T+d_{6} T+d_{7} T+d_{8} T
$$

La tensión en $C_{r}$ y la intensidad en $L_{r}$ se pueden escribir en función de las variables lentas que se consideran constantes.

$$
\begin{gathered}
v_{C_{r}}(t)= \begin{cases}v_{C_{c}} & t \in\left[t_{1}, t_{4}\right] \\
0 & \text { resto }\end{cases} \\
i_{r}(t)= \begin{cases}i_{m}-\frac{v_{C_{c}}}{L_{r}}\left(t-t_{2}\right) & t \in\left[t_{1}, t_{2}\right] \\
i_{m}-\frac{v_{C_{c}}}{Z_{D_{c}}} \sin \left[\omega_{D_{c}}(t-t 2)\right] & t \in\left[t_{2}, t_{3}\right] \\
i_{r 3}+\frac{\left(v_{g}+n v_{C_{o}}-v_{C_{c}}\right)}{L_{r}}\left(t-t_{3}\right) & t \in\left[t_{3}, t_{4}\right] \\
i_{m}+\frac{\left(v_{g}+n v_{C_{o}}\right)}{L_{r}}\left(t-t_{6}\right) & t \in\left[t_{5}, t_{6}\right] \\
i_{m}+\frac{\left(v_{g}+n v_{C_{o}}\right)}{Z_{D_{c}}} \sin \left[\omega_{D_{c}}\left(t-t_{6}\right)\right] & t \in\left[t_{6}, t_{7}\right] \\
\left(i_{m}+i_{D_{c}}\right) & t \in\left[t_{7}, t_{8}\right]\end{cases}
\end{gathered}
$$

Además, a partir de la tensión en $C_{c l}$ dada por:

$$
v_{C_{c l}}(t)= \begin{cases}v_{C_{c}}-v_{C_{c}} \cos \left(\omega_{D_{c}}(t-t 2)\right) & t \in\left[t_{2}, t_{3}\right] \\ v_{g}+n v_{C_{o}} & t \in\left[t_{3}, t_{4}\right] \\ v_{g}+n v_{C_{o}} & t \in\left[t_{5}, t_{6}\right] \\ \left(v_{g}+n v_{C_{o}}\right) \cos \left(\omega_{D_{c}}\left(t-t_{6}\right)\right) & t \in\left[t_{6}, t_{7}\right] \\ 0 & \text { resto }\end{cases}
$$

se obtiene la expresión para $d_{7}$, puesto que este condensador se descarga desde su tensión hasta cero durante la etapa 7.

$$
\left(v_{g}+n v_{C_{o}}\right) \cos \left[\omega_{D_{c}}\left(t-t_{6}\right)\right]=0 \Rightarrow d_{7}=\frac{\pi}{2 T \omega_{D c}}
$$

Por otro lado, en torno a $t_{3}$ se debe cumplir que:

$$
v_{C_{c}}-v_{C_{c}} \cos \left(\omega_{D_{c}} d_{3} T\right)=\left(v_{g}+n v_{C_{o}}\right) \Rightarrow \cos \left(\omega_{D_{c}} d_{3} T\right)=\frac{v_{C_{c}}-\left(v_{g}+n v_{C_{o}}\right)}{v_{C_{c}}}
$$


Igualando los valores de $i_{r}(t)$ en torno a $t_{3}$ se pueden obtener la expresión para $i_{r 3}$.

$$
i_{m}-\frac{v_{C_{c}}}{Z_{D_{c}}} \sin \left(\omega_{D_{c}} d_{3} T\right)=i_{r 3}
$$

Igualando los valores de $i_{r}(t)$ en torno a $t_{7}$ se puede obtener la expresión para $i_{D_{c}}$, utilizando (F.115).

$$
i / m+\frac{\left(v_{g}+n v_{C_{o}}\right)}{Z_{D_{c}}} \sin \left(\omega_{D_{c}} d_{7} T\right)=i / m+i_{D_{c}} \Rightarrow i_{D_{c}}=\frac{\left(v_{g}+n v_{C_{o}}\right)}{Z_{D_{c}}}
$$

La expresión para $d_{2}$ se obtiene de la periodicidad de $i_{r}(t)$, es decir, $i_{r}\left(t_{1}\right)=i_{r}\left(t_{8}\right)$ :

$$
i / m-\frac{v_{C_{c}}}{L_{r}}\left(-d_{2} T\right)=i / m+i_{D_{c}} \Rightarrow d_{2} T=\frac{i_{D_{c}} L_{r}}{v_{C_{c}}}
$$

Como $t_{4}=t_{5}$, se puede obtener una relación entre $d_{4} \mathrm{y} d_{6}$ a partir de la identidad $i_{r}\left(t_{4}\right)=i_{r}\left(t_{5}\right)$. Entonces:

$$
i / m-\frac{v_{C_{c}}}{Z_{D_{c}}} \sin \left(\omega_{D_{c}} d_{3} T\right)+\frac{\left(v_{g}+n v_{C_{o}}-v_{C_{c}}\right)}{L_{r}} d_{4} T=i / m+\frac{v_{g}+n v_{C_{o}}}{L_{r}}\left(-d_{6} T\right)
$$

Y por tanto:

$$
\frac{v_{C_{c}}}{Z_{D_{c}}} \sin \left(\omega_{D_{c}} d_{3} T\right)=\frac{\left(v_{g}+n v_{C_{o}}-v_{C_{c}}\right)}{L_{r}} d_{4} T+\frac{v_{g}+n v_{C_{o}}}{L_{r}} d_{6} T
$$

Para el resto de variables lentas si es necesario escribir las ecuaciones de estado. Se construye una ecuación para la intensidad en la bobina de magnetización, $i_{m}(t)$; otra ecuación para la tensión en el condensador de salida, $v_{C_{o}}(t)$; otra ecuación para la tensión en el condensador de clamp, $v_{C_{c}}(t)$; y otra ecuación para el condensador $C_{c l}$. Estas ecuaciones son:

$$
\begin{gathered}
v_{L_{m}}(t)=L_{m} \frac{d i_{m}(t)}{d t}=\left\{\begin{array}{lr}
v_{g}(t) & t \in\left[t_{1}, t_{2}\right] \\
v_{g}(t)-v_{C_{c}}(t)+v_{C_{c}}(t) \cos \left[\omega_{D_{c}}\left(t-t_{2}\right)\right] & t \in\left[t_{2}, t_{3}\right] \\
-n v_{C_{o}}(t) & t \in\left[t_{3}, t_{4}\right] \\
-n v_{C_{o}}(t) & t \in\left[t_{5}, t_{6}\right] \\
v_{g}(t)-\left(v_{g}(t)+n v_{C_{o}}(t)\right) \cos \left[\omega_{D_{c}}\left(t-t_{6}\right)\right] & t \in\left[t_{6}, t_{7}\right] \\
v_{g}(t) & t \in\left[t_{7}, t_{8}\right]
\end{array}\right) \\
(\mathrm{F} .121)
\end{gathered}
$$




$$
i_{C_{o}}(t)=C_{o} \frac{d v_{C_{o}}(t)}{d t}= \begin{cases}-\frac{v_{C_{o}}(t)}{R} & t \in\left[t_{1}, t_{2}\right] \\ -\frac{v_{C_{o}}(t)}{R} & t \in\left[t_{2}, t_{3}\right] \\ -n \underbrace{\left(i_{r}(t)-i_{m}(t)\right)}_{i_{1}(t)}-\frac{v_{C_{o}}(t)}{R} & t \in\left[t_{3}, t_{4}\right] \\ -n \underbrace{\left(i_{r}(t)-i_{m}(t)\right)}_{i_{1}(t)}-\frac{v_{C_{o}}(t)}{R} & t \in\left[t_{5}, t_{6}\right] \\ -\frac{v_{C_{o}}(t)}{R} & t \in\left[t_{6}, t_{7}\right] \\ -\frac{v_{C_{o}}(t)}{R} & t \in\left[t_{7}, t_{8}\right] \\ i_{C_{c l}}(t)=C_{c l} \frac{d v_{C_{c l}}(t)}{d t}= \begin{cases}0 & t \in\left[t_{1}, t_{2}\right] \\ i_{m}(t)-i_{r}(t) & t \in\left[t_{2}, t_{3}\right] \\ 0 & t \in\left[t_{3}, t_{4}\right] \\ 0 & t \in\left[t_{5}, t_{6}\right] \\ i_{m}(t)-i_{r}(t) & t \in\left[t_{6}, t_{7}\right] \\ 0 & t \in\left[t_{7}, t_{8}\right]\end{cases} \end{cases}
$$

Se añaden dos ecuaciones mas, para la intensidad de entrada, $i_{g}$, y para la intensidad del diodo de salida, $i_{D}$.

$$
\begin{gathered}
i_{g}(t)= \begin{cases}i_{m}(t) & t \in\left[t_{1}, t_{2}\right] \\
i_{m}(t) & t \in\left[t_{2}, t_{3}\right] \\
i_{r}(t) & t \in\left[t_{3}, t_{4}\right] \\
i_{r}(t) & t \in\left[t_{5}, t_{6}\right] \\
i_{m}(t) & t \in\left[t_{6}, t_{7}\right] \\
i_{m}(t) & t \in\left[t_{7}, t_{8}\right]\end{cases} \\
i_{D}(t)= \begin{cases}0 & t \in\left[t_{1}, t_{2}\right] \\
0 & t \in\left[t_{2}, t_{3}\right] \\
-n\left(i_{r}(t)-i_{m}(t)\right) & t \in\left[t_{3}, t_{4}\right] \\
-n\left(i_{r}(t)-i_{m}(t)\right) & t \in\left[t_{5}, t_{6}\right] \\
0 & t \in\left[t_{6}, t_{7}\right] \\
0 & t \in\left[t_{7}, t_{8}\right]\end{cases}
\end{gathered}
$$

Cuando se sustituyen las variables rápidas en las ecuaciones anteriores, se consigue tener un conjunto de ecuaciones de estado donde solo intervienen 
las variables de estado lentas, es decir, se han desacoplado de las variables de estado rápidas.

$L_{m} \frac{d i_{m}(t)}{d t}= \begin{cases}v_{g}(t) & t \in\left[t_{1}, t_{2}\right] \\ v_{g}(t)-v_{C_{c}}(t)+v_{C_{c}}(t) \cos \left[\omega_{D_{c}}\left(t-t_{2}\right)\right] & t \in\left[t_{2}, t_{3}\right] \\ -n v_{C_{o}}(t) & t \in\left[t_{3}, t_{4}\right] \\ -n v_{C_{o}}(t) & t \in\left[t_{5}, t_{6}\right] \\ v_{g}(t)-\left(v_{g}(t)+n v_{C_{o}}(t)\right) \cos \left[\omega_{D_{c}}\left(t-t_{6}\right)\right] & t \in\left[t_{6}, t_{7}\right] \\ v_{g}(t) & t \in\left[t_{7}, t_{8}\right]\end{cases}$

$C_{c} \frac{d v_{C_{c}}(t)}{d t}= \begin{cases}i_{m}(t)-\frac{v_{C_{c}}(t)}{L_{r}}\left(t-t_{2}\right) & t \in\left[t_{1}, t_{2}\right] \\ i_{m}(t)-\frac{v_{C_{c}}(t)}{Z_{D_{c}}} \sin \left[\omega_{D_{c}}(t-t 2)\right] & t \in\left[t_{2}, t_{3}\right] \\ i_{r 3}(t)+\frac{\left(v_{g}(t)+n v_{C_{o}}(t)-v_{C_{c}}(t)\right)}{L_{r}}\left(t-t_{3}\right) & t \in\left[t_{3}, t_{4}\right] \\ 0 & t \in\left[t_{5}, t_{8}\right]\end{cases}$

$C_{o} \frac{d v_{C_{o}}(t)}{d t}= \begin{cases}-\frac{v_{C_{o}}(t)}{R} & t \in\left[t_{1}, t_{3}\right] \\ -n\left[i_{r 3}(t)-i_{m}(t)+\frac{\left(v_{g}(t)+n v_{C_{o}}(t)-v_{C_{c}}(t)\right)}{L_{r}}\left(t-t_{3}\right)\right]-\frac{v_{C_{o}}(t)}{R} & t \in\left[t_{3}, t_{4}\right] \\ -n \frac{\left(v_{g}(t)+n v_{C_{o}}(t)\right)}{L_{r}}\left(t-t_{6}\right)-\frac{v_{C_{o}}(t)}{R} & t \in\left[t_{5}, t_{6}\right] \\ -\frac{v_{C_{o}}(t)}{R} & t \in\left[t_{6}, t_{7}\right] \\ -\frac{v_{C_{o}}(t)}{R} & t \in\left[t_{7}, t_{8}\right]\end{cases}$

$C_{c l} \frac{d v_{C_{c l}}(t)}{d t}= \begin{cases}\frac{v_{C_{c}}(t)}{Z_{D_{c}}} \sin \left[\omega_{D_{c}}\left(t-t_{2}\right)\right] & t \in\left[t_{2}, t_{3}\right] \\ -\frac{\left(v_{g}(t)+n v_{C_{o}}(t)\right)}{Z_{D_{c}}} \sin \left[\omega_{D_{c}}\left(t-t_{6}\right)\right] & t \in\left[t_{6}, t_{7}\right] \\ 0 & \text { resto }\end{cases}$ 
junto con:

$$
i_{g}(t)= \begin{cases}i_{m}(t) & t \in\left[t_{1}, t_{2}\right] \\ i_{m}(t) & t \in\left[t_{2}, t_{3}\right] \\ i_{r 3}(t)+\frac{\left(v_{g}(t)+n v_{C_{o}}(t)-v_{C_{c}}(t)\right)}{L_{r}}\left(t-t_{3}\right) & t \in\left[t_{3}, t_{4}\right] \\ i_{m}(t)+\frac{\left(v_{g}(t)+n v_{C_{o}}(t)\right)}{L_{r}}\left(t-t_{6}\right) & t \in\left[t_{5}, t_{6}\right] \\ i_{m}(t) & t \in\left[t_{6}, t_{7}\right] \\ i_{m}(t) & t \in\left[t_{7}, t_{8}\right]\end{cases}
$$

$$
i_{D}(t)= \begin{cases}0 & t \in\left[t_{1}, t_{2}\right] \\ 0 & t \in\left[t_{3}, t_{3}\right] \\ -n\left[i_{r 3}(t)-i_{m}(t)+\frac{\left(v_{g}(t)+n v_{C_{o}}(t)-v_{C_{c}}(t)\right)}{L_{r}}\left(t-t_{3}\right)\right] & t \in\left[t_{3}, t_{4}\right] \\ n\left[i_{m}(t)+\frac{\left(v_{g}(t)+n v_{C_{o}}(t)\right)}{L_{r}}\left(t-t_{6}\right)\right] & t \in\left[t_{5}, t_{6}\right] \\ 0 & t \in\left[t_{6}, t_{7}\right] \\ 0 & t \in\left[t_{7}, t_{8}\right]\end{cases}
$$

siendo:

$$
\begin{aligned}
& d_{2} T=\frac{\left(v_{g}(t)+n v_{C_{o}}(t)\right)}{v_{C_{c}}(t) \omega_{D_{c}}} \\
& \cos \left(\omega_{D_{c}} d_{3} T\right)=\frac{v_{C_{c}}(t)-v_{g}(t)-n v_{C_{o}}(t)}{v_{C_{c}}(t)} \\
& d_{4} T=(1-d) T-d_{2} T-d_{3} T \\
& \frac{v_{C_{c}}}{Z_{D_{c}}} \sin \left(\omega_{D_{c}} d_{3} T\right)=\frac{\left(v_{g}+n v_{C_{o}}-v_{C_{c}}\right)}{L_{r}} d_{4} T+\frac{v_{g}+n v_{C_{o}}}{L_{r}} d_{6} T \\
& d_{7} T=\frac{\pi}{2 \omega_{D_{c}}} \\
& d_{8} T=d T-d_{6} T-d_{7} T
\end{aligned}
$$

$\mathrm{y}$

$$
\begin{aligned}
i_{D_{c}}(t) & =\frac{\left(v_{g}(t)+n v_{C_{o}}(t)\right)}{Z_{D_{c}}} \\
i_{r 3}(t) & =i_{m}(t)-\frac{v_{C_{c}}(t)}{Z_{D_{c}}} \sin \left(\omega_{D_{c}} d_{3} T\right)
\end{aligned}
$$

Considerando la aproximación de pequeño rizado, es posible sustituir los valores instantáneos de las variables de estado por sus valores promedio, (por ejemplo, $i_{m}(t)$ se sustituye por $\bar{i}_{m}$, que es igual que $\left\langle i_{m}(t)\right\rangle_{T}$ donde se 
ha simplificado la notación, y así con el resto). De esta forma, las ecuaciones desacopladas se transforman en:

$$
\begin{aligned}
& L_{m} \frac{d i_{m}(t)}{d t} \approx \begin{cases}\bar{v}_{g} & t \in\left[t_{1}, t_{2}\right] \\
\bar{v}_{g}-\bar{v}_{C_{c}}+\bar{v}_{C_{c}} \cos \left[\omega_{D_{c}}\left(t-t_{2}\right)\right] & t \in\left[t_{2}, t_{3}\right] \\
-n \bar{v}_{C_{o}} & t \in\left[t_{3}, t_{4}\right] \\
-n \bar{v}_{C_{o}} & t \in\left[t_{5}, t_{6}\right] \\
\bar{v}_{g}-\left(\bar{v}_{g}+n \bar{v}_{C_{o}}\right) \cos \left[\omega_{D_{c}}\left(t-t_{6}\right)\right] & t \in\left[t_{6}, t_{7}\right] \\
\bar{v}_{g} & t \in\left[t_{7}, t_{8}\right]\end{cases} \\
& C_{c} \frac{d v_{C_{c}}(t)}{d t} \approx \begin{cases}\bar{i}_{m}-\frac{\bar{v}_{C_{c}}}{\underline{L}_{r}}\left(t-t_{2}\right) & t \in\left[t_{1}, t_{2}\right] \\
\bar{i}_{m}-\frac{\bar{v}_{C_{c}}}{Z_{D_{c}}} \sin \left[\omega_{D_{c}}\left(t-t_{2}\right)\right] & t \in\left[t_{2}, t_{3}\right] \\
\bar{i}_{r 3}+\frac{\left(\bar{v}_{g}+n \bar{v}_{C_{o}}-\bar{v}_{C_{c}}\right)}{L_{r}}\left(t-t_{3}\right) & t \in\left[t_{3}, t_{4}\right] \\
0 & t \in\left[t_{5}, t_{6}\right] \\
0 & t \in\left[t_{6}, t_{7}\right] \\
0 & t \in\left[t_{7}, t_{8}\right]\end{cases} \\
& C_{o} \frac{d v_{C_{o}}(t)}{d t} \approx\left\{\begin{array}{lr}
-\frac{\bar{v}_{C_{o}}}{R} & t \in\left[t_{1}, t_{2}\right] \\
-\frac{\bar{v}_{C_{o}}}{R} & t \in\left[t_{2}, t_{3}\right] \\
\left.-n \bar{i}_{r_{3}}-\bar{i}_{m}+\frac{\left(\bar{v}_{g}+n \bar{v}_{C_{o}}-\bar{v}_{C_{c}}\right)}{L_{r}}\left(t-t_{3}\right)\right]-\frac{\bar{v}_{C_{o}}}{R} & t \in\left[t_{3}, t_{4}\right] \\
-n \frac{\left(\bar{v}_{g}+n \bar{v}_{C_{o}}\right)}{L_{r}}\left(t-t_{6}\right)-\frac{\bar{v}_{C_{o}}}{R} & t \in\left[t_{5}, t_{6}\right] \\
-\frac{\bar{v}_{C_{o}}}{\frac{R}{v_{C_{o}}}} & t \in\left[t_{6}, t_{7}\right] \\
-\frac{v^{R}}{R} & t \in\left[t_{7}, t_{8}\right]
\end{array}\right. \\
& C_{c l} \frac{d v_{C_{c l}}(t)}{d t} \approx \begin{cases}0 & t \in\left[t_{1}, t_{2}\right] \\
\frac{\bar{v}_{C_{c}}}{Z_{D_{c}}} \sin \left[\omega_{D_{c}}\left(t-t_{2}\right)\right] & t \in\left[t_{2}, t_{3}\right] \\
0 & t \in\left[t_{3}, t_{4}\right] \\
0 & t \in\left[t_{5}, t_{6}\right] \\
-\frac{\left(\bar{v}_{g}+n \bar{v}_{C_{o}}\right)}{Z_{D_{c}}} \sin \left[\omega_{D_{c}}\left(t-t_{6}\right)\right] & t \in\left[t_{6}, t_{7}\right] \\
0 & t \in\left[t_{7}, t_{8}\right]\end{cases}
\end{aligned}
$$


junto con:

$$
\begin{aligned}
& i_{g}(t)= \begin{cases}\bar{i}_{m} & t \in\left[t_{1}, t_{2}\right] \\
\bar{i}_{m} & t \in\left[t_{2}, t_{3}\right] \\
\bar{i}_{r 3}+\frac{\left(\bar{v}_{g}+n \bar{v}_{C_{o}}-\bar{v}_{C_{c}}\right)}{L_{r}}\left(t-t_{3}\right) & t \in\left[t_{3}, t_{4}\right] \\
\bar{i}_{m}+\frac{\left(\bar{v}_{g}+n \bar{v}_{C_{o}}\right)}{L_{r}}\left(t-t_{6}\right) & t \in\left[t_{5}, t_{6}\right] \\
\bar{i}_{m} & t \in\left[t_{6}, t_{7}\right] \\
\bar{i}_{m} & t \in\left[t_{7}, t_{8}\right]\end{cases} \\
& i_{D}(t)=\left\{\begin{array}{lr}
0 & t \in\left[t_{1}, t_{2}\right] \\
0 & t \in\left[t_{2}, t_{3}\right] \\
-n\left[\bar{i}_{r 3}-\bar{i}_{m}+\frac{\left(\bar{v}_{g}+n \bar{v}_{C_{o}}-\bar{v}_{C_{c}}\right)}{L_{r}}\left(t-t_{3}\right)\right] & t \in\left[t_{3}, t_{4}\right] \\
-n \frac{\left(\bar{v}_{g}+n \bar{v}_{C_{o}}\right)}{L_{r}}\left(t-t_{6}\right) & t \in\left[t_{5}, t_{6}\right] \\
0 & t \in\left[t_{6}, t_{7}\right] \\
0 & t \in\left[t_{7}, t_{8}\right]
\end{array}\right.
\end{aligned}
$$

con:

$$
\begin{aligned}
& d_{2} T=\frac{\left(\bar{v}_{g}+n \bar{v}_{C_{o}}\right)}{\bar{v}_{C_{c}} \omega_{D_{c}}} \\
& \cos \left(\omega_{D c} d_{3} T\right)=\frac{\bar{v}_{C_{c}}-\bar{v}_{g}-n \bar{v}_{C_{o}}}{\bar{v}_{C_{c}}} \\
& d_{4} T=(1-d) T-d_{2} T-d_{3} T \\
& \frac{\bar{v}_{C_{c}}}{Z_{D c}} \sin \left(\omega_{D_{c}} d_{3} T\right)=\frac{\left(\bar{v}_{g}+n \bar{v}_{C_{o}}-\bar{v}_{C_{c}}\right)}{L_{r}} d_{4} T+\frac{\bar{v}_{g}+n \bar{v}_{C_{o}}}{L_{r}} d_{6} T \\
& d_{7} T=\frac{\pi}{2 \omega_{D_{c}}} \\
& d_{8} T=d T-d_{6} T-d_{7} T
\end{aligned}
$$

y

$$
\begin{aligned}
& \bar{i}_{D_{c}}=\frac{\left(\bar{v}_{g}+n \bar{v}_{C_{o}}\right)}{Z_{D_{c}}} \\
& \bar{i}_{r 3}=\bar{i}_{m}-\frac{\bar{v}_{C_{c}}}{Z_{D_{c}}} \sin \left(\omega_{D_{c}} d_{3} T\right)
\end{aligned}
$$

ECUACION DE ESTADO PROMEDIADA PARA $\bar{i}_{m}$. 
Haciendo el promedio a lo largo de un periodo de la ecuación (F.135) queda que:

$$
\begin{aligned}
L_{m} \frac{d \bar{i}_{m}}{d t} & =\bar{v}_{g}\left(d_{2}+d_{3}+d_{7}+d_{8}\right)-\bar{v}_{C_{c}} d_{3}+\frac{\bar{v}_{C_{c}}}{\omega_{D_{c}} T} \sin \left(\omega_{D_{c}} d_{3} T\right) \\
& -n \bar{v}_{C_{o}}\left(d_{4}+d_{6}\right)-\frac{\bar{v}_{g}+n \bar{v}_{C_{o}}}{\omega_{D_{c}} T} \sin \left(\omega_{D_{c}} d_{7} T\right)
\end{aligned}
$$

y se transforma en (F.144) cuando se emplean los resultados para $d_{2} T$, $d_{6} T$ y $d_{7} T$ de (F.141).

$$
\begin{aligned}
L_{m} \frac{d \bar{i}_{m}}{d t}= & \bar{v}_{g}\left(d_{2}+d_{3}+d_{7}+d_{8}\right)-\bar{v}_{C_{c}} d_{3}+\frac{\bar{v}_{C_{c}}}{\omega_{D_{c}} T} \sin \left(\omega_{D_{c}} d_{3} T\right) \\
& -n \bar{v}_{C_{o}}\left(d_{4}+d_{6}\right)-\frac{\bar{v}_{g}+n \bar{v}_{C_{o}}}{\omega_{D_{c}} T} \\
= & \bar{v}_{g}\left(d_{2}+d_{3}+d_{7}+d_{8}\right)-\bar{v}_{C_{c}} d_{3}+\left(\bar{v}_{g}+n \bar{v}_{C_{o}}-\bar{v}_{C_{c}}\right) d_{4} T+\left(\bar{v}_{g}+n \bar{v}_{C_{o}}\right) d_{6} T \\
& -n \bar{v}_{C_{o}}\left(d_{4}+d_{6}\right)-\bar{v}_{C_{c}} d_{2} \\
= & \bar{v}_{g}\left(d_{2}+d_{3}+d_{4}+d_{6}+d_{7}+d_{8}\right)-\bar{v}_{C_{c}}\left(d-2+d_{3}+d_{4}\right. \\
= & \bar{v}_{g}-\bar{v}_{C_{c}}(1-d)
\end{aligned}
$$

ECUACION DE ESTADO PROMEDIADA PARA $\bar{v}_{C_{c}}$.

Haciendo el promedio a lo largo de un periodo de la ecuación (F.136) queda que:

$$
\begin{aligned}
C_{c} \frac{d \bar{v}_{C_{c}}}{d t}= & \bar{i}_{m}\left(d_{2}+d_{3}+d_{4}\right)+\frac{\bar{v}_{C_{c}}}{L_{r}} \frac{d_{2}^{2} T}{2}+\frac{\bar{v}_{C_{c}}}{Z_{D_{c}} \omega_{D_{c}} T}\left[\cos \left(\omega_{D_{c}} d_{3} T\right)-1\right] \\
& -\frac{\bar{v}_{C_{c}}}{Z_{D_{c}}} \sin \left(\omega_{D_{c}} d_{3} T\right) d_{4}+\frac{\left(\bar{v}_{g}+n \bar{v}_{C_{o}}-\bar{v}_{C_{c}}\right)}{L_{r}} \frac{d_{4}^{2} T}{2} \\
= & \bar{i}_{m}(1-d)+i_{e q, 2}+i_{e q, 3}-i_{e q, 4}-\frac{\bar{v}_{C_{c}}-\left(\bar{v}_{g}+n \bar{v}_{C_{o}}\right)}{r_{e q}}
\end{aligned}
$$

donde se definen varias fuentes de corriente equivalentes y una resistencia equivalente sin pérdidas, que depende de $d_{4}$, como:

$$
\begin{aligned}
r_{e q} & =\frac{2 L_{r} f_{s}}{d_{4}^{2}} \\
i_{e q, 2} & =\frac{\bar{v}_{C_{c}}}{L_{r}} \frac{d_{2}^{2} T}{2} \\
i_{e q, 3} & =\frac{\bar{v}_{C_{c}}}{Z_{D_{c}} \omega_{D_{c}} T}\left[\cos \left(\omega_{D_{c}} d_{3} T\right)-1\right] \\
i_{e q, 4} & =\frac{\bar{v}_{C_{c}}}{Z_{D_{c}}} \sin \left(\omega_{D_{c}} d_{3} T\right) d_{4}
\end{aligned}
$$


ECUACION DE ESTADO PROMEDIADA PARA $\bar{v}_{C_{o}}$.

Haciendo el promedio a lo largo de un periodo de la ecuación (F.137) queda que:

$$
\begin{aligned}
C_{o} \frac{d \bar{v}_{C_{o}}}{d t}= & -\frac{\bar{v}_{C_{o}}}{R}\left(d_{2}+d_{3}+d_{4}+d_{6}+d_{7}+d_{8}\right)+n \frac{\bar{v}_{C_{c}}}{Z_{D_{c}}} \sin \left(\omega_{D_{c}} d_{3} T\right) d_{4} \\
& -n \frac{\left(\bar{v}_{g}+n \bar{v}_{C_{o}}-\bar{v}_{C_{c}}\right)}{L_{r}} \frac{d_{4}^{2} T}{2}+n \frac{\left(\bar{v}_{g}+n \bar{v}_{C_{o}}\right)}{L_{r}} \frac{d_{6}^{2} T}{2} \\
= & n\left[i_{e q, 4}+\frac{\bar{v}_{C_{c}}-\left(\bar{v}_{g}+n \bar{v}_{C_{o}}\right)}{r_{e q}}+i_{e q}\right]-\frac{\bar{v}_{C_{o}}}{R}
\end{aligned}
$$

donde se define otra fuente de corriente equivalente como:

$$
i_{e q}=\frac{\left(\bar{v}_{g}+n \bar{v}_{C_{o}}\right)}{2 L_{r} f_{s}} d_{6}^{2}
$$

ECUACION DE ESTADO PROMEDIADA PARA $\bar{v}_{C_{c l}}$.

El promedio de la ecuación (F.137) da lugar al resultado indicado en (F.149), cuando se utilizan los resultados de $d_{2} T$ y $d_{7} T$ de (F.141):

$$
\begin{aligned}
C_{c l} \frac{d \bar{v}_{C_{c l}}}{d t} & =-\frac{\bar{v}_{C_{c}}}{Z_{D_{c}} \omega_{D_{c} T} T}\left[\cos \left(\omega_{D_{c}} d_{3} T\right)-1\right]+\frac{\bar{v}_{g}+n \bar{v}_{C_{o}}}{Z_{D_{c}} \omega_{D_{c}} T}\left[\cos \left(\omega_{D_{c}} d_{7} T\right)-1\right] \\
& =\frac{\bar{v} C_{c}}{Z_{D_{c}} \omega_{D_{c}} T} \frac{\bar{v}_{g}+n \bar{v}_{C_{o}}}{\bar{y} C_{c}}-\frac{\bar{v}_{g}+n \bar{v}_{C_{o}}}{Z_{D_{c}} \omega_{D_{c}} T}=0
\end{aligned}
$$

Este resultado indica que el condensador $C_{c l}$ como tal no aparece en el modelo promediado, aunque su valor estará presente en determinados parámetros de dicho modelo.

\section{ECUACION DE ESTADO PROMEDIADA PARA $\bar{i}_{g}$.}

También se puede obtener una ecuación para la intensidad de entrada a partir del promedio de la ecuación (F.139):

$$
\begin{aligned}
\bar{i}_{g} & =\bar{i}_{m}\left(d_{2}+d_{3}+d_{4}+d_{6}+d_{7}+d_{8}\right)-\frac{\bar{v}_{C_{c}}}{Z_{D_{c}}} \sin \left(\omega_{D_{c}} d_{3} T\right) d_{4} \\
& +\frac{\left(\bar{v}_{g}+n \bar{v}_{C_{o}}-\bar{v}_{C_{c}}\right)}{L_{r}} \frac{d_{4}^{2} T}{2}-\frac{\left(\bar{v}_{g}+n \bar{v}_{C_{o}}\right)}{L_{r}} \frac{d_{6}^{2} T}{2} \\
& =\bar{i}_{m}-i_{e q, 4}-\frac{\bar{v}_{C_{c}}-\left(\bar{v}_{g}+n \bar{v}_{C_{o}}\right)}{r_{e q}}-i_{e q}
\end{aligned}
$$

\section{ECUACION DE ESTADO PARA $\bar{i}_{D}$.}


La intensidad del diodo $D$ tiene también su ecuación para el modelo promediado:

$$
\begin{aligned}
& \bar{i}_{D}=n \frac{\bar{v}_{C_{c}}}{Z_{D_{c}}} \sin \left(\omega_{D_{c}} d_{3} T\right) d_{4}-n \frac{\left(\bar{v}_{g}+n \bar{v}_{C_{o}}-\bar{v}_{C_{c}}\right)}{L_{r}} \frac{d_{4}^{2} T}{2}+n \frac{\left(\bar{v}_{g}+n \bar{v}_{C_{o}}\right)}{L_{r}} \frac{d_{6}^{2} T}{2} \\
& =n\left[i_{e q, 4}+\frac{\bar{v}_{C_{c}}-\left(\bar{v}_{g}+n \bar{v}_{C_{o}}\right)}{r_{e q}}+i_{e q}\right]
\end{aligned}
$$

El circuito obtenido con las ecuaciones del modelo promediado está indicado en la Figura F.9

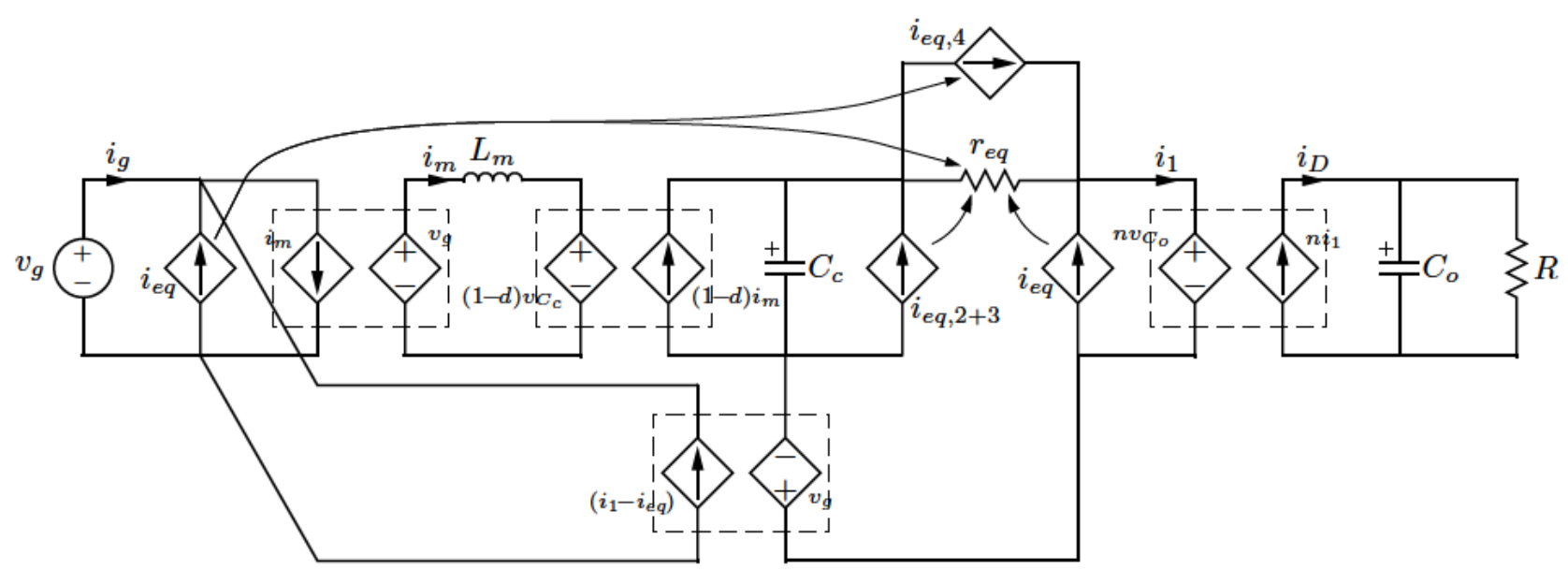

(a)

Figura F.9: Modelo promediado de un flyback ZVS-modificado. Son magnitudes promediadas. La fuente dependiente $i_{e q, 2+3}$ equivale a la suma de las fuentes $i_{e q, 2}$ con $i_{e q, 3}$.

\section{F.3.1. Balance energético}

En el modelo promediadohay varios elementos involucrados, la fuente de tensión en la entrada y las fuente de corriente dependientes de la entrada, de la salida y las que están conectadas a $C_{c}$. La energía de la fuente de tensión se consume en la carga y se almacena o devuelve en los elementos reactivos, mientras que la energía consumida por $r_{e q}$ y la fuente $i_{e q, 4}$ se genera en el resto de fuentes dependientes. Veámoslo:

En $r_{e q}$, la potencia consumida es igual a

$$
P_{r_{e q}}=\frac{\left[\bar{v}_{C_{c}}-\left(\bar{v}_{g}+n \bar{v}_{C_{o}}\right)\right]^{2}}{2 L_{r} f_{s}} d_{4}^{2}
$$


En $i_{e q, 4}$ la potencia consumida es:

$$
\begin{aligned}
P_{i_{e q, 4}} & =\left[\bar{v}_{C_{c}}-\left(\bar{v}_{g}+n \bar{v}_{C_{o}}\right)\right] \frac{\bar{v}_{C_{c}}}{Z_{D_{c}}} \sin \left(\omega_{D_{c}} d_{3} T\right) d_{4}= \\
& =-\frac{\left[\bar{v}_{C_{c}}-\left(\bar{v}_{g}+n \bar{v}_{C_{o}}\right)\right]^{2}}{L_{r} f_{s}} d_{4}^{2}+\frac{\left[\bar{v}_{C_{c}}-\left(\bar{v}_{g}+n \bar{v}_{C_{o}}\right)\right]\left(\bar{v}_{g}+n \bar{v}_{C_{o}}\right)}{L_{r} f_{s}} d_{6} d_{4}
\end{aligned}
$$

donde se han utilizado los resultados de (F.141).

En las dos fuentes de corriente de valor $i_{e q}$, la potencia generada es igual a

$$
P_{i_{e q}}=\bar{v}_{g} i_{e q}+n \bar{v}_{C_{o}} i_{e q}=\frac{\left(\bar{v}_{g}+n \bar{v}_{C_{o}}\right)^{2}}{2 L_{r} f_{s}} d_{6}^{2}
$$

mientras que en las dos fuentes de corriente $i_{e q, 2}$ e $i_{e q, 3}$ la potencia generada es igual a:

$$
\begin{aligned}
P_{i_{e q, 2+3}} & =\bar{v}_{C_{c}}\left[\frac{\bar{v}_{C_{c}}}{L_{r}} \frac{d_{2}^{2} T}{2}+\frac{\bar{v}_{C_{c}}}{Z_{D_{c}} \omega_{D_{c}} T}\left[\cos \left(\omega_{D_{c}} d_{3} T\right)-1\right]\right] \\
& =\frac{\left(\bar{v}_{g}+n \bar{v}_{C_{o}}\right)^{2}}{2 Z_{D_{c}} \omega_{D_{c}} T}-\frac{\bar{v}_{C_{c}}\left(\bar{v}_{g}+n \bar{v}_{C_{o}}\right)}{Z_{D_{c}} \omega_{D_{c}} T}
\end{aligned}
$$

La potencia total consumida debe ser igual a la generada, es decir, que

$$
P_{r_{e q}}+P_{i_{e q, 4}}=P_{i_{e q}}+P_{i_{e q, 2+3}}
$$

luego:

$$
\begin{aligned}
& -\frac{\left[\bar{v}_{C_{c}}-\left(\bar{v}_{g}+n \bar{v}_{C_{o}}\right)\right]^{2}}{2 L_{r} f_{s}} d_{4}^{2}+\frac{\left[\bar{v}_{C_{c}}-\left(\bar{v}_{g}+n \bar{v}_{C_{o}}\right)\right]\left(\bar{v}_{g}+n \bar{v}_{C_{o}}\right)}{L_{r} f_{s}} d_{6} d_{4} \\
= & \frac{\left(\bar{v}_{g}+n \bar{v}_{C_{o}}\right)^{2}}{2 L_{r} f_{s}} d_{6}^{2}+\frac{\left(\bar{v}_{g}+n \bar{v}_{C_{o}}\right)^{2}}{2 Z_{D_{c}} \omega_{D_{c}} T}-\frac{\bar{v}_{C_{c}}\left(\bar{v}_{g}+n \bar{v}_{C_{o}}\right)}{Z_{D_{c}} \omega_{D_{c}} T}
\end{aligned}
$$

Pasando el primer termino de la derecha al lado izquierdo aparece el cuadrado de una suma de dos términos en el numerador de una fracción, que está relacionado con los resultados de (F.141):

$$
\begin{aligned}
-\frac{\left[\left(\bar{v}_{g}+n \bar{v}_{C_{o}}\right) d_{6}-\left[\bar{v}_{C_{c}}-\left(\bar{v}_{g}+n \bar{v}_{C_{o}}\right)\right] d_{4}\right]^{2}}{2 L_{r} f_{s}}=\frac{\left(\bar{v}_{g}+n \bar{v}_{C_{o}}\right)^{2}}{2 Z_{D_{c}} \omega_{D_{c}} T}-\frac{\bar{v}_{C_{c}}\left(\bar{v}_{g}+n \bar{v}_{C_{o}}\right)}{Z_{D_{c}} \omega_{D_{c}} T} \\
-\frac{\left[\frac{\bar{v}_{C_{c}}}{Z_{D_{c}}} \sin \left(\omega_{D_{c}} d_{3} T\right) L_{r} f_{s}\right]^{2}}{2 L_{r} f_{s}}=\frac{\left(\bar{v}_{g}+n \bar{v}_{C_{o}}\right)^{2}-2 \bar{v}_{C_{c}}\left(\bar{v}_{g}+n \bar{v}_{C_{o}}\right)}{2 Z_{D_{c}} \omega_{D_{c}} T} \\
\frac{\left[\frac{\bar{v}_{C_{c}}}{Z_{D_{c}}} \sin \left(\omega_{D_{c}} d_{3} T\right) L_{r} f_{s}\right]^{2}}{2 L_{r} f_{s}}=\frac{2 \bar{v}_{C_{c}}\left(\bar{v}_{g}+n \bar{v}_{C_{o}}\right)-\left(\bar{v}_{g}+n \bar{v}_{C_{o}}\right)^{2}}{2 Z_{D_{c}} \omega_{D_{c}} T}
\end{aligned}
$$


Cuando se desarrolla el primer término de la izquierda,

$$
\begin{aligned}
\frac{\left[\frac{\bar{v}_{C_{c}}}{Z_{D_{c}}} \sin \left(\omega_{D_{c}} d_{3} T\right) L_{r} f_{s}\right]^{2}}{2 L_{r} f_{s}} & =\frac{\bar{v}_{C_{c}}^{2} \sin ^{2}\left(\omega_{D_{c}} d_{3} T\right)}{2 Z_{D_{c}} \omega_{D_{c}} T} \\
& =\frac{\bar{v}_{C_{c}}^{2}}{2 Z_{D_{c}} \omega_{D_{c}} T}\left[1-\left(\frac{\bar{v}_{C_{c}}-\bar{v}_{g}-n \bar{v}_{C_{o}}}{\bar{v}_{C_{c}}}\right)^{2}\right] \\
& =\frac{\bar{v}_{C_{c}}^{2}-\left[\bar{v}_{C_{c}}-\left(\bar{v}_{g}+n \bar{v}_{C_{o}}\right)\right]^{2}}{2 Z_{D_{c}} \omega_{D_{c}} T} \\
& =\frac{-\left(\bar{v}_{g}+n \bar{v}_{C_{o}}\right)^{2}+2 \bar{v}_{C_{c}}\left(\bar{v}_{g}+n \bar{v}_{C_{o}}\right)}{2 Z_{D_{c}} \omega_{D_{c}} T}
\end{aligned}
$$

se llega al segundo término de (F.157), por lo que queda comprobado el balance energético del modelo promediado.

\section{F.3.2. Validación del modelo promediado}

La validación del modelo promediado se realiza mediante simulación, y se compara su comportamiento, tanto en régimen permanente como en régimen dinámico, con un convertidor conmutado. Para ello se eligen de nuevo los parámetros de funcionamiento recogidos en la Tabla F.1, empleando en el convertidor conmutado el ciclo de trabajo calculado al considerar rizado. En el modelo promediado se ha considerado el ciclo de trabajo calculado cuando no se ha considerado el rizado.

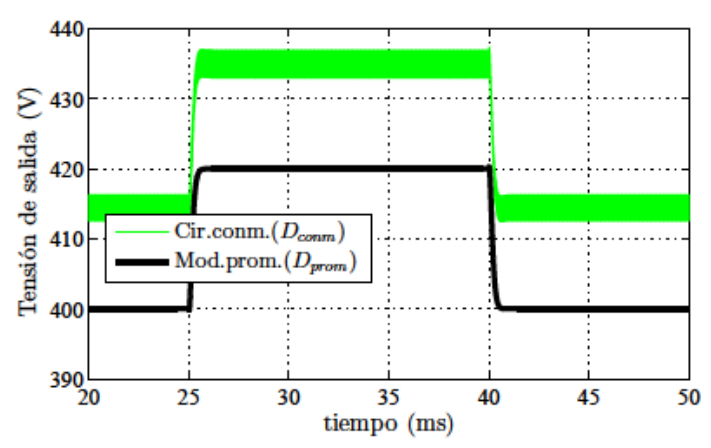

(a)

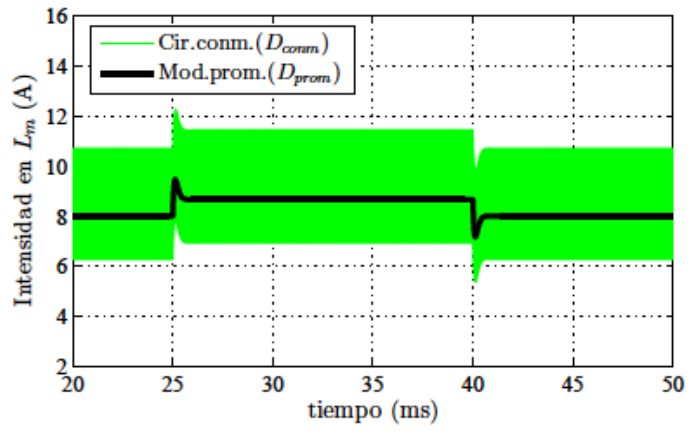

(b)

Figura F.10: Flyback ZVS-modif. Respuesta a escalón del ciclo de trabajo: a) Tensión de salida; y b) Intensidad en la bobina.

Los resultados muestran una dinámica similar para el convertidor conmutado y el modelo promediado. En cuanto al régimen permanente, hay un error puesto que la tensión de salida no coincide. El motivo de esta diferencia tiene su causa en que en el modelo promediado no está incluido el tiempo 
muerto, $t_{m}$, que hay que dejar entre disparos de los transistores principal y de clamp, entre otras cosas, para facilitar las conmutaciones suaves. Además, parte de este tiempo muerto se emplea en descargar el condensador resonante, y corresponde a las dos etapas que se desprecian para obtener el modelo promediado. En concreto la etapa 5, siendo su duración igual a $d_{5} T$.

Por tanto, hay que realizar una corrección en el ciclo de trabajo empleado para el circuito conmutado, en base a los tiempos mencionados, puesto que ya está incluido de forma implícita en la generación de disparos del propio circuito conmutado. De esta forma, se rehacen las simulaciones del circuito conmutado con el ciclo de trabajo corregido, $D_{\text {corr }}$, como:

$$
D_{\text {corr }}=D_{\text {conm }}-\frac{t_{m}-d_{5} T}{T}
$$

y se obtienen los resultados de la Figura F.11. La corrección equivalente pero para el modelo promediado consistiría en añadir este tiempo extra al ciclo de trabajo empleado en el modelo promediado.

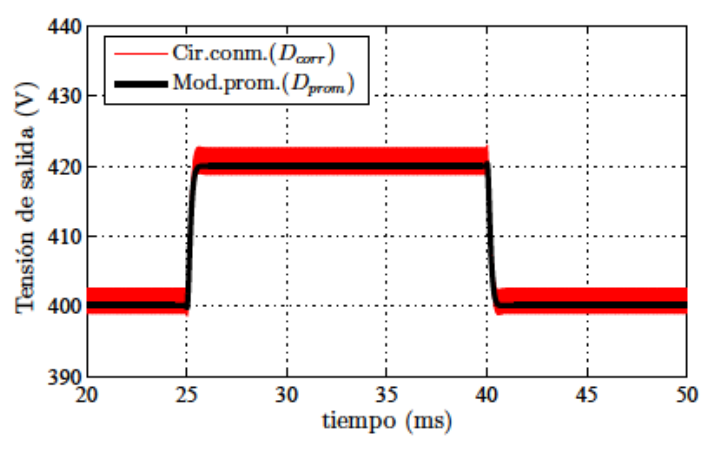

(a)

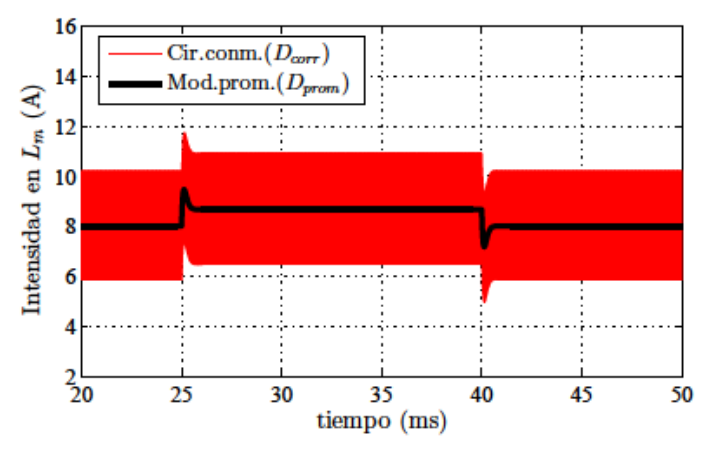

(b)

Figura F.11: Respuesta a escalón en el ciclo de trabajo incluyendo corrección: a) Tensión de salida; y b) Intensidad en la bobina.

Para apreciar la mejora en los resultados se representa el valor medio de la tensión de salida y la intensidad en la bobina para el circuito conmutado antes y después de la corrección y se comparan con el modelo promediado. En la Figura F.12 se pueden comprobar estas curvas.

\section{F.4. Modelo en régimen permanente}

El modelo en regimen permanente se puede obtener a partir del modelo promediado sustituyendo los valores promedio por valores constantes (por ejemplo, $\bar{i}_{m}$ se sustituye por $I$ ). De esta forma, las ecuaciones del modelo en régimen permanente serán las indicadas en (F.160), en las que hay ecuaciones 


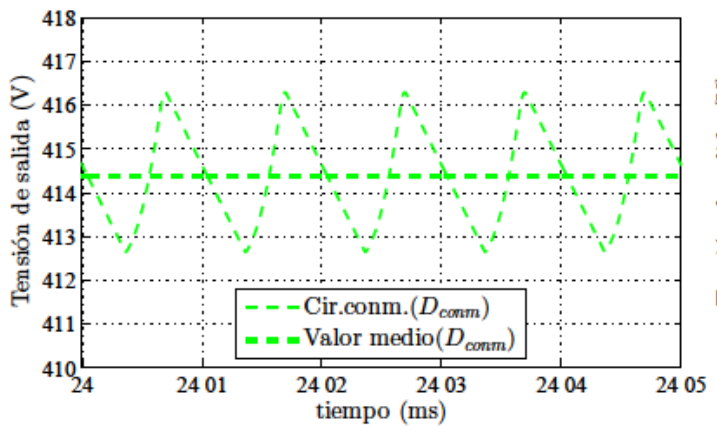

(a)

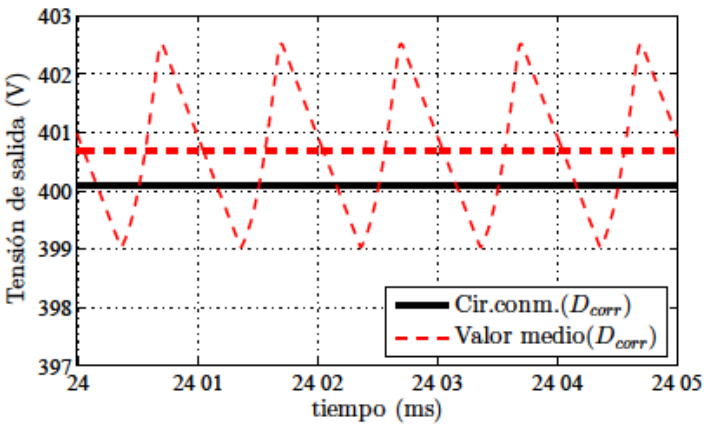

(b)

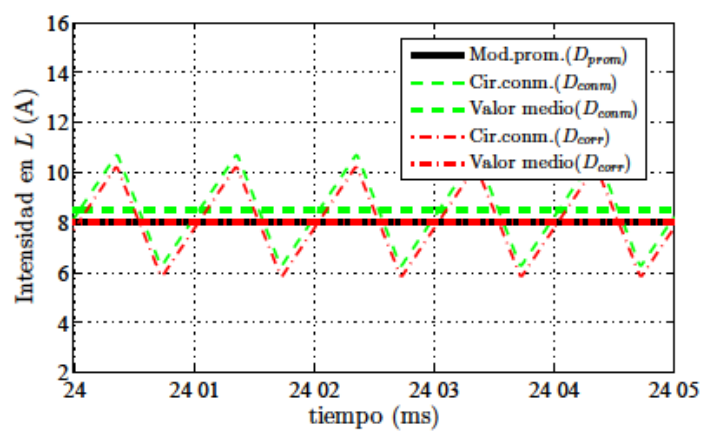

(c)

Figura F.12: Curvas de tensión e intensidad con y sin corrección en el ciclo de trabajo del circuito conmutado: a) Tensión de salida sin corrección e; b) Ídem aplicando corrección; y c) Intensidad en la bobina.

con un cero a la izquierda de la igualdad, porque la derivada de una constante es nula.

$$
\begin{aligned}
0 & =V_{g}-(1-D) V_{c} \\
0 & =n I_{e q, 4}+n \frac{V_{c}-\left(V_{g}+n V_{o}\right)}{R_{e q}}+n I_{e q}-\frac{V_{o}}{R} \\
0 & =I(1-D)+I_{e q, 2}+I_{e q, 3}-I_{e q, 4}-\frac{V_{c}-\left(V_{g}+n V_{o}\right)}{R_{e q}} \\
I_{g} & =I-I_{e q, 4}-\frac{V_{c}-\left(V_{g}+n V_{o}\right)}{R_{e q}}-I_{e q} \\
I_{D} & =n\left[I_{e q, 4}+\frac{V_{c}-\left(V_{g}+n V_{o}\right)}{R_{e q}}+I_{e q}\right]
\end{aligned}
$$


donde

$$
\begin{aligned}
R_{e q} & =\frac{2 L_{r} f_{s}}{D_{4}^{2}} \\
I_{e q} & =\frac{\left(V_{g}+n V_{o}\right)}{2 L_{r} f_{s}} D_{6}^{2} \\
I_{e q, 2} & =\frac{V_{c}}{L_{r}} \frac{D_{2}^{2} T}{2} \\
I_{e q, 3} & =\frac{V_{c}}{Z_{D_{c}} \omega_{D_{c}} T}\left[\cos \left(\omega_{D_{c}} D_{3} T\right)-1\right] \\
I_{e q, 4} & =\frac{V_{c}}{Z_{D_{c}}} \sin \left(\omega_{D_{c}} D_{3} T\right) D_{4}
\end{aligned}
$$

y:

$$
\begin{aligned}
& D_{2} T=\frac{\left(V_{g}+n V_{o}\right)}{V_{c} \omega_{D_{c}}} \\
& \cos \left(\omega_{D c} D_{3} T\right)=\frac{V_{c}-V_{g}-n V_{o}}{V_{c}} \\
& D_{4} T=(1-D) T-D_{2} T-D_{3} T \\
& \frac{V_{c}}{Z_{D c}} \sin \left(\omega_{D_{c}} D_{3} T\right)=\frac{\left(V_{g}+n V_{o}-V_{c}\right)}{L_{r}} D_{4} T+\frac{V_{g}+n V_{o}}{L_{r}} D_{6} T \\
& D_{7} T=\frac{\pi}{2 \omega_{D_{c}}} \\
& D_{8} T=D T-D_{6} T-D_{7} T
\end{aligned}
$$

A estas ecuaciones se puede llegar también a partir del circuito promediado de la Figura F.9. Basta con sustituir los condensadores por un circuito abierto y las bobinas con un cortocircuito. De esta forma se obtienen los circuitos de la Figura F.13a.

Se obtienen las mismas expresiones para las principales magnitudes en régimen permanente cuando se deducen de (F.160) que a partir de los circuitos de la Figura F.13. El término $V_{o} / R$ es la intensidad a la salida y coincide con la intensidad del diodo $I_{D}$.

$$
\begin{aligned}
V_{c} & =\frac{V_{g}}{(1-D)} \\
\frac{V_{o}}{R} & =n I_{e q, 4}+n \frac{V_{c}-\left(V_{g}+n V_{o}\right)}{R_{e q}}+n I_{e q} \equiv I_{o} \\
V_{o} & =\frac{1}{n}\left(V_{c}-V_{g}-(1-D) I R_{e q}\right)-\frac{1}{n}\left[I_{e q, 2}+I_{e q, 3}-I_{e q, 4}\right] R_{e q} \\
I_{g} & =I-I_{e q, 4}-\frac{V_{c}-\left(V_{g}+n V_{o}\right)}{R_{e q}}-I_{e q} \\
I_{D} & =n\left[I_{e q, 4}+\frac{V_{c}-\left(V_{g}+n V_{o}\right)}{R_{e q}}+I_{e q}\right] \equiv I_{o}
\end{aligned}
$$




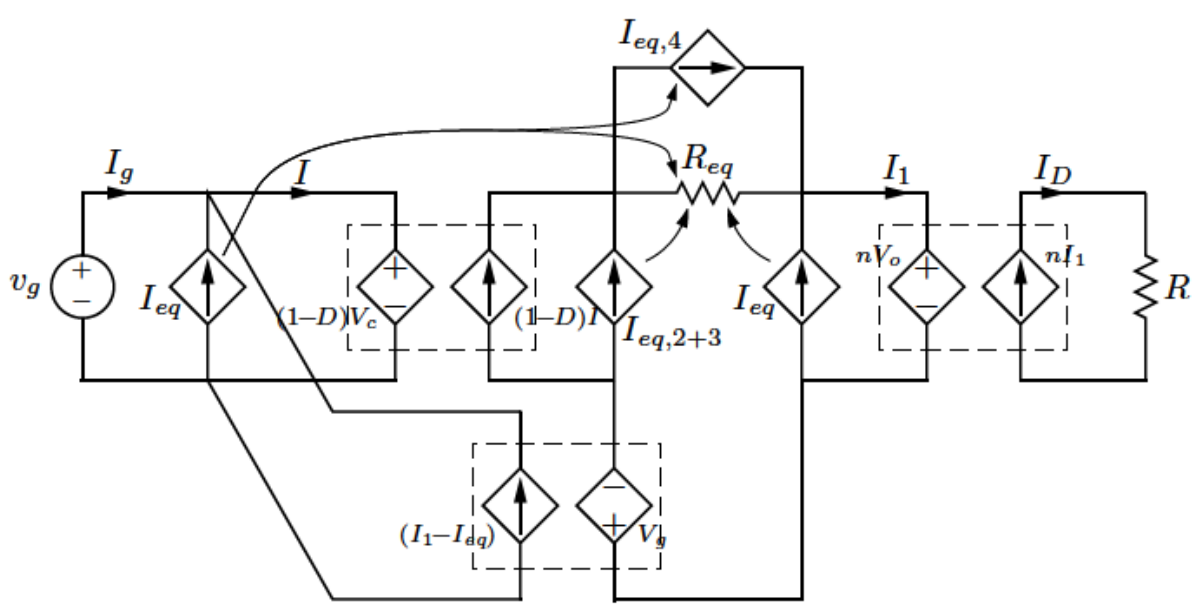

(a)

Figura F.13: Circuito en régimen permanente de un flyback ZVS-modificado. La fuente dependiente $I_{e q, 2+3}$ equivale a la suma de las fuentes $I_{e q, 2} \operatorname{con} I_{e q, 3}$.

\section{F.4.1. Balance energético}

En el modelo en régimen permanente hay varios elementos involucrados, la fuente de tensión en la entrada y las fuente de corriente dependientes de la entrada, de la salida y las que están conectadas a $C_{c}$. La energía de la fuente de tensión se consume en la carga y se almacena o devuelve en los elementos reactivos, mientras que la energía consumida por $R_{e q}$ y la fuente $I_{e q, 4}$ se genera en el resto de fuentes dependientes. Veámoslo:

En $R_{e q}$, la potencia consumida es igual a:

$$
P_{R_{e q}}=\frac{\left[V_{c}-\left(V_{g}+n V_{o}\right)\right]^{2}}{2 L_{r} f_{s}} D_{4}^{2}
$$

En $I_{e q, 4}$ la potencia consumida es:

$$
\begin{aligned}
P_{I_{e q, 4}} & =\left[V_{c}-\left(V_{g}+n V_{o}\right)\right] \frac{V_{c}}{Z_{D_{c}}} \sin \left(\omega_{D_{c}} D_{3} T\right) D_{4} \\
& =-\frac{\left[V_{c}-\left(V_{g}+n V_{o}\right)\right]^{2}}{L_{r} f_{s}} D_{4}^{2}+\frac{\left[V_{c}-\left(V_{g}+n V_{o}\right)\right]\left(V_{g}+n V_{o}\right)}{L_{r} f_{s}} D_{6} D_{4}
\end{aligned}
$$

donde se han utilizado los resultados de (F.162).

En las fuentes de corriente de valor $I_{e q}$ la potencia generada es igual a:

$$
P_{I_{e q}}=V_{g} I_{e q}+n V_{o} I_{e q}=\frac{\left(V_{g}+n V_{o}\right)^{2}}{2 L_{r} f_{s}} D_{6}^{2}
$$


mientras que en las dos fuentes de corriente $I_{e q, 2}$ e $I_{e q, 3}$ la potencia generada es igual a:

$$
\begin{aligned}
P_{I_{e q, 2+3}} & =V_{c}\left[\frac{V_{c}}{L_{r}} \frac{D_{2}^{2} T}{2}+\frac{V_{c}}{Z_{D_{c}} \omega_{D_{c}} T}\left[\cos \left(\omega_{D_{c}} D_{3} T\right)-1\right]\right] \\
& =\frac{\left(V_{g}+n V_{o}\right)^{2}}{2 Z_{D_{c}} \omega_{D_{c}} T}-\frac{V_{c}\left(V_{g}+n V_{o}\right)}{Z_{D_{c}} \omega_{D_{c}} T}
\end{aligned}
$$

La potencia total consumida debe ser igual a la generada, es decir, que

$$
P_{R_{e q}}+P_{I_{e q, 4}}=P_{I_{e q}}+P_{I_{e q, 2+3}}
$$

luego:

$$
\begin{aligned}
& -\frac{\left[V_{c}-\left(V_{g}+n V_{o}\right)\right]^{2}}{2 L_{r} f_{s}} D_{4}^{2}+\frac{\left[V_{C}-\left(V_{g}+n V_{o}\right)\right]\left(V_{g}+n V_{o}\right)}{L_{r} f_{s}} D_{6} D_{4} \\
= & \frac{\left(V_{g}+n V_{o}\right)^{2}}{2 L_{r} f_{s}} D_{6}^{2}+\frac{\left(V_{g}+n V_{o}\right)^{2}}{2 Z_{D_{c}} \omega_{D_{c}} T}-\frac{V_{c}\left(V_{g}+n V_{o}\right)}{Z_{D_{c}} \omega_{D_{c}} T}
\end{aligned}
$$

Pasando el primer termino de la derecha al lado izquierdo aparece el cuadrado de una suma de dos términos en el numerador de una fracción, que está relacionado con los resultados de (F.162):

$$
\begin{aligned}
-\frac{\left.\left[V_{g}+n V_{o}\right) D_{6}-\left[V_{c}-\left(V_{g}+n V_{o}\right)\right] D_{4}\right]^{2}}{2 L_{r} f_{s}} & =\frac{\left(V_{g}+n V_{o}\right)^{2}}{2 Z_{D_{c}} \omega_{D_{c}} T}-\frac{V_{c}\left(V_{g}+n V_{o}\right)}{Z_{D_{c}} \omega_{D_{c}} T} \\
- & \frac{\left[\frac{V_{c}}{Z_{D_{c}}} \sin \left(\omega_{D_{c}} D_{3} T\right) L_{r} f_{s}\right]^{2}}{2 L_{r} f_{s}}=\frac{\left(V_{g}+n V_{o}\right)^{2}-2 V_{c}\left(V_{g}+n V_{o}\right)}{2 Z_{D_{c}} \omega_{D_{c}} T} \\
& \frac{\left[\frac{V_{c}}{Z_{D_{c}}} \sin \left(\omega_{D_{c}} D_{3} T\right) L_{r} f_{s}\right]^{2}}{2 L_{r} f_{s}}=\frac{2 V_{c}\left(V_{g}+n V_{o}\right)-\left(V_{g}+n V_{o}\right)^{2}}{2 Z_{D_{c}} \omega_{D_{c}} T}
\end{aligned}
$$

Cuando se desarrolla el primer término de la izquierda,

$$
\begin{aligned}
\frac{\left[\frac{V_{c}}{Z_{D_{c}}} \sin \left(\omega_{D_{c}} D_{3} T\right) L_{r} f_{s}\right]^{2}}{2 L_{r} f_{s}} & =\frac{V_{c}^{2} \sin ^{2}\left(\omega_{D_{c}} D_{3} T\right)}{2 Z_{D_{c}} \omega_{D_{c}} T} \\
& =\frac{V_{c}^{2}}{2 Z_{D_{c}} \omega_{D_{c}} T}\left[1-\left(\frac{V c-V_{g}-n V_{o}}{V_{c}}\right)^{2}\right] \\
& =\frac{V_{c}^{2}-\left[V_{c}-\left(V_{g}+n V_{o}\right)\right]^{2}}{2 Z_{D_{c}} \omega_{D_{c}} T} \\
& =\frac{-\left(V_{g}+n V_{o}\right)^{2}+2 V_{c}\left(V_{g}+n V_{o}\right)}{2 Z_{D_{c}} \omega_{D_{c}} T}
\end{aligned}
$$


se llega al segundo término de (F.169).

Por otro lado, toda la potencia generada en $V_{g}$ se consume en la carga $\mathrm{R}$ a la salida, como se comprueba en (F.171):

$$
\begin{aligned}
P_{g}= & V_{g} I_{g}=V_{g}\left[I-I_{e q}-\left(I_{1}-I_{e q}\right)\right]=V_{g} I-V_{g} I_{1}=V_{c}(1-D) I-V_{g} I_{1} \\
= & {\left[V_{g}+n V_{o}+B_{e q} \frac{V_{c}-\left(V_{g}+V_{o}\right)}{R_{e q}}\right](1-D) I-V_{g} I_{1} } \\
= & \left(V_{g}+n V_{o}\right)(1-D) I+\left[V_{c}-\left(V_{g}+V_{o}\right)\right](1-D) I-V_{g} I_{1} \\
= & \left(V_{g}+n V_{o}\right)\left(I_{1}-I_{e q}-I_{e q, 2+3}\right)+\left[V c-\left(V_{g}-n V_{o}\right)\right]\left[\frac{V c-\left(V_{g}-n V_{o}\right)}{R_{e q}}+I_{e q, 4}-I_{e q, 23}\right]-V_{g} I_{1} \\
= & V_{g} I_{1}+n V_{o} I_{1}-\left(V_{g}+n V_{o}\right) I_{e q}-\left(V_{g}+n V_{o}\right) I_{e q, 2+3} \\
& +\frac{\left[V c-\left(V_{g}+n V_{o}\right)\right]^{2}}{R_{e q}}+\left[V_{c}-\left(V_{g}+n V_{o}\right)\right] I_{e q, 4}-\left[V_{c}-\left(V_{g}+n V_{o}\right)\right] I_{e q, 2+3}-V_{g} I_{1}= \\
= & \not n V_{o} \frac{I_{D}}{\not x} \underbrace{-P_{I_{e q}}+P_{R_{e q}}+P_{I_{e q, 4}}-P_{I_{e q, 2+3}}}_{\text {resultado neto }=0}=V_{o} \frac{V_{o}}{R}=V_{o} I_{o} \equiv P_{o}
\end{aligned}
$$




\section{Bibliografía}

Abu-QAhouq, J.; MaO, Hong y Batarseh, I (2004). «Multiphase voltagemode hysteretic controlled DC-DC converter with novel current sharing». Power Electronics, IEEE Transactions on, 19(6), pp. 1397-1407. ISSN 0885-8993. doi: 10.1109/TPEL.2004.836639.

ABu-QAhouq, J.A. (2010). «Analysis and Design of N-Phase CurrentSharing Autotuning Controller». Power Electronics, IEEE Transactions on, 25(6), pp. 1641-1651. ISSN 0885-8993. doi: 10.1109/TPEL.2009. 2037892 .

Abu-Qhhouq, J.A.; Huang, L. y Huard, D. (2008a). «Efficiency-Based Auto-Tuning of Current Sensing and Sharing Loops in Multiphase Converters». Power Electronics, IEEE Transactions on, 23(2), pp. 1009-1013. ISSN 0885-8993. doi: 10.1109/TPEL.2008.917808.

(2008b). «Sensorless Current Sharing Analysis and Scheme For Multiphase Converters». Power Electronics, IEEE Transactions on, 23(5), pp. 2237-2247. ISSN 0885-8993. doi: 10.1109/TPEL.2008.2001897.

Anand, S. y Fernandes, B.G. (2012). «Modified droop controller for paralleling of dc-dc converters in standalone dc system». Power Electronics, IET, 5(6), pp. 782-789. ISSN 1755-4535. doi: 10.1049/iet-pel.2011.0346.

Athalye, P.; Maksimovic, D. y Erickson, R. (2001). «Averaged switch modeling of active-clamped converters». En: Industrial Electronics Society, 2001. IECON '01. The 27th Annual Conference of the IEEE, volumen 2, pp. $1078-1083$ vol.2. doi: 10.1109/IECON.2001.975930.

(2003). «High-Performance Front-End Converter for Avionics Applications». Aerospace and Electronic Systems, IEEE Transactions on, 39(2), pp. $462-470$. 
Bae, Hyunsu; Lee, Jaeho; Yang, Jeonghwan y Cho, Bo Hyung (2008). «Digital Resistive Current (DRC) Control for the Parallel Interleaved DC-DC Converters». Power Electronics, IEEE Transactions on, 23(5), pp. 2465-2476. ISSN 0885-8993. doi: 10.1109/TPEL.2008.2002059.

Balogh, Laszlo (2002). «Paralleling Power-Choosing and Applying the Best Technique for Load Sharing». Texas Instruments/UNITRODE Power Design Seminar SLUP207, pp.6-1-to 6-30, Texas Instruments.

Basso, Christophe P. (2008). Switch-Mode Power Supplies. SPICE Simulations and Practical Designs. McGraw-Hill, New York NY, USA, third ${ }^{\mathrm{a}}$ edición.

Batarseh, I; Siri, K. y Lee, H. (1994). «Investigation of the output droop characteristics of parallel-connnected DC-DC converters». En: Power Electronics Specialists Conference, PESC '94 Record., 25th Annual IEEE, pp. 1342-1351 vol.2. doi: 10.1109/PESC.1994.373859.

Behjati, H.; Davoudi, A y Lewis, F. (2014). «Modular DC/DC Converters on Graphs: Cooperative Control». Power Electronics, IEEE Transactions on, 29(12), pp. 6725-6741. ISSN 0885-8993. doi: 10.1109/TPEL. 2014.2303192 .

Calkin, E. T. y Hamilton, B. H. (1976). «Circuit Techniques for Improving the Switching Loci of Transistor Switches in Switching Regulators》. Industry Applications, IEEE Transactions on, IA12(4), pp. 364 -369. ISSN 0093-9994. doi: 10.1109/TIA.1976.349438.

Carsten, B. (1990). «Design techniques for transformer active reset circuits at high frequencies and power levels». En: Proc. High Frequency Power Conversion Conf., 1990, pp. 235-246.

Carsten, Bruce (1978). «Epic Data Multi-Output Switched Mode Power Supply». Epic data intermal memo, Epic Data Industries.

Chen, Min y Sun, Jian (2006). «Reduced-order averaged modeling of active-clamp converters». Power Electronics, IEEE Transactions on, 21(2), pp. 487 - 494. ISSN 0885-8993. doi: 10.1109/TPEL.2005.869761.

Chen, Wu; Ruan, Xinbo; Yan, Hong y Tse, C.K. (2009). «DC/DC Conversion Systems Consisting of Multiple Converter Modules: Stability, Control, and Experimental Verifications». Power Electronics, IEEE Transactions on, 24(6), pp. 1463-1474. ISSN 0885-8993. doi: 10.1109/TPEL. 2009.2012406 .

Chen, Yi; Cheng, D.K.-W. y Lee, Y. S (2004). «A simulation-based comparison of direct and indirect current-sharing methods». En: Power 
Electronics Specialists Conference, 2004. PESC 04. 2004 IEEE 35th Annual, volumen 4, pp. 2746-2752 Vol.4. ISSN 0275-9306. doi: 10.1109/ PESC.2004.1355267.

Cheng, D.K.-W.; Lee, Y. S y Chen, Yi (2005). «A current-sharing interface circuit with new current-sharing technique». Power Electronics, IEEE Transactions on, 20(1), pp. 35-43. ISSN 0885-8993. doi: 10.1109/TPEL.2004.839875(410)20.

Choi, Byungcho (1992). Dynamic and control of switchmode power conversion in distributed power systems. Tesis doctoral, Virgina Polytechnic Institute and State University.

(1998). «Comparative study on paralleling schemes of converter modules for distributed power applications». Industrial Electronics, IEEE Transactions on, 45(2), pp. 194-199. ISSN 0278-0046. doi: 10.1109/41.681217.

Dalal, Dhaval (1996). «Desing Considerations for Active Clamp and Reset Technique». Texas Instruments/UNITRODE Seminar SLUP112, pp.3-1 to 3-23, Texas Instruments.

De Jodar, E.; Villarejo, J. y Jimenez, J.M. (2013). «Multiphase ZVS Active Clamp Boost Converter: DC and Dynamic Current Sharing». Industrial Electronics, IEEE Transactions on, 60(11), pp. 4947-4959. ISSN 0278-0046. doi: 10.1109/TIE.2012.2227896.

De Jodar, E.; Villarejo, J.; Moreno, M. y Jimenez, J. (2012). «Current sharing in multiphase zero-voltage transition boost converter». Power Electronics, IET, 5(3), pp. 376-384. ISSN 1755-4535. doi: 10.1049/iet-pel.2011.0115.

De Jodar, E.; Villarejo, J.A; Jimenez, J. y Moreno, M. (2010). «Current sharing in multiphase ZVT boost converter». En: Energy Conversion Congress and Exposition (ECCE), 2010 IEEE, pp. 3022-3029. doi: 10.1109/ECCE.2010.5618372.

De Jodar, E.; Villarejo, J.A; Soto, F. y Muro, J.S. (2008). «Effect of the Output Impedance in Multiphase Active Clamp Buck Converters». Industrial Electronics, IEEE Transactions on, 55(9), pp. 3231-3238. ISSN 0278-0046. doi: 10.1109/TIE.2008.928124.

De JodAR, Esther (2010). Convertidores multifase con conmutacion suave: modelado y ecualizado de carga pasivo. Tesis doctoral, Universidad Politecnica de Cartagena.

DuArTe, C.M.C. y BARBI, I. (1995). «A family of ZVS-PWM activeclamping DC-to-DC converters: synthesis, analysis, and experimentation». 
En: Proc. th Int. Telecommunications Energy Conf. INTELEC '95, pp. 502-509.

(1997a). «A family of ZVS-PWM active-clamping DC-to-DC converters: synthesis, analysis, design, and experimentation». Circuits and Systems I: Fundamental Theory and Applications, IEEE Transactions on, 44(8), pp. 698 -704. ISSN 1057-7122. doi: 10.1109/81.611265.

(1997b). «A new family of ZVS-PWM active-clamping DC-to-DC boost converters: analysis, design, and experimentation». Power Electronics, IEEE Transactions on, 12(5), pp. 824 -831. ISSN 0885-8993. doi: 10. $1109 / 63.623000$.

(2002). «An improved family of ZVS-PWM active-clamping DC-to-DC converters». Power Electronics, IEEE Transactions on, 17(1), pp. $1-7$. ISSN 0885-8993. doi: 10.1109/63.988661.

Erdogan, A.D. y Aydemir, Mehmet Timur (2009a). «Use of input power information for load sharing in parallel connected boost converters». Electrical Engineering, 91(4-5), pp. 229-250. ISSN 0948-7921. doi: 10. 1007/s00202-009-0138-6.

http://dx.doi.org/10.1007/s00202-009-0138-6

Erdogan, AD. y Aydemir, M.T. (2009b). «Application of adaptive droop method to boost converters operating at the output of fuel cells». En: Electrical and Electronics Engineering, 2009. ELECO 2009. International Conference on, pp. I-321-I-325.

Erickson, Robert W. y Maksimovic, Dragan (2005). Fundamental of Power Electronics. Kluwer Academic Publishers, Norwell MA, USA, second ${ }^{\mathrm{a}}$ edición.

Glaser, J.S. y Witulski, AF. (1994). «Output plane analysis of loadsharing in multiple-module converter systems». Power Electronics, IEEE Transactions on, 9(1), pp. 43-50. ISSN 0885-8993. doi: 10.1109/63. 285492.

Grbovic, P.J. (2009). «Master/Slave Control of Input-Series- and OutputParallel-Connected Converters: Concept for Low-Cost High-Voltage Auxiliary Power Supplies». Power Electronics, IEEE Transactions on, 24(2), pp. 316-328. ISSN 0885-8993. doi: 10.1109/TPEL.2008.2006975.

Hakoda, Y.; Ninomiya, T.; Shoyama, M. y Hashimoto, T. (1998). «Effect of clamp capacitor on the stability of active-clamp DC-DC converters». En: Power Electronics Specialists Conference, 1998. PESC 98 Record. 29th Annual IEEE, volumen 1, pp. 355-361 vol.1. ISSN 0275-9306. doi: 10.1109/PESC.1998.701923. 
HAmar, J. (2009). «Decentralized, agent-based control of low and moderate power DC-DC converters». En: Power Electronics Conference, 2009. COBEP '09. Brazilian, pp. 410-416. ISSN 2175-8603. doi: 10.1109/COBEP.2009.5347699.

Hamar, J. y Tотн, A. (2009). «Agent-based control of parallel dc-dc converters». En: Power Electronics and Applications, 2009. EPE '09. 13th European Conference on, pp. 1-10.

Harada, K. y Sakamoto, H. (1990). «Switched snubber for high frequency switching». En: Power Electronics Specialists Conference, 1990. PESC'90 Record., 21st Annual IEEE, pp. 181 -188. doi: 10.1109/PESC.1990.131187.

Hedel, K.K. (1980). «High-Density Avionic Power Supply». Aerospace and Electronic Systems, IEEE Transactions on, AES-16(5), pp. 615-619. ISSN 0018-9251. doi: 10.1109/TAES.1980.308927.

Heng, Phua Chee y Oruganti, R. (1994). «Family of two-switch softswitched asymmetrical PWM DC/DC converters». En: Power Electronics Specialists Conference, PESC '94 Record., 25th Annual IEEE, pp. 85-94 vol.1. doi: 10.1109/PESC.1994.349745.

Henze, C.P.; Martin, H.C. y Parsley, D.W. (1988). «Zero-voltage switching in high frequency power converters using pulse width modulation». En: Applied Power Electronics Conference and Exposition, 1988. APEC '88. Conference Proceedings 1988., Third Annual IEEE, pp. 33 -40. doi: 10.1109/APEC.1988.10548.

HirshberG, W.J. (1985). «Current sharing of paralleled power supplies». En: Power Electronics Specialists Conference, 1985. PESC 1985 IEEE, pp. 76-83.

Hren, A.; Korelic, J. y Milanovic, M. (2006). «RC-RCD clamp circuit for ringing losses reduction in a flyback converter». Circuits and Systems II: Express Briefs, IEEE Transactions on, 53(5), pp. 369-373. ISSN 15497747. doi: 10.1109/TCSII.2006.870547.

Hua, Guichao (1994). Soft-switching techniques for PWM converters. Tesis doctoral, Virginia Polytechnic Institute and State University, Backsburg, VA.

Huang, Yuehi (2008). Circuit Theoretic Analysis and Design of Interconnected $D C / D C$ Converters. Tesis doctoral, The Hong Kong Polytechnic University.

Huang, Yuehui y Tse, Chi K. (2009). «Circuit theory of paralleling switching converters». International Journal of Circuit Theory and Applications, 37(1), pp. 109-135. ISSN 1097-007X. doi: 10.1002/cta.529. 
Huang, Yuehui y Tse, C.K.0 (2007). «Circuit Theoretic Classification of Parallel Connected DC-DC Converters». Circuits and Systems I: Regular Papers, IEEE Transactions on, 54(5), pp. 1099-1108. ISSN 1549-8328. doi: 10.1109/TCSI.2007.890631.

Huth, S. (1996). «DC/DC-converters in parallel operation with digital load distribution control». En: Industrial Electronics, 1996. ISIE '96., Proceedings of the IEEE International Symposium on, volumen 2, pp. 808-813 vol.2. doi: 10.1109/ISIE.1996.551047.

Inoue, S. y AkAGi, H. (2007). «A Bidirectional Isolated DC ndash;DC Converter as a Core Circuit of the Next-Generation Medium-Voltage Power Conversion System». Power Electronics, IEEE Transactions on, 22(2), pp. 535-542. ISSN 0885-8993. doi: 10.1109/TPEL.2006.889939.

Irving, B.T. y Jovanovic, M.M. (2000). «Analysis, design, and performance evaluation of droop current-sharing method». En: Applied Power Electronics Conference and Exposition, 2000. APEC 2000. Fifteenth Annual IEEE, volumen 1, pp. 235-241 vol.1. doi: 10.1109/APEC.2000. 826110.

Jamerson, C.; Long, T. y Mullett, C. (1993). «Seven ways to parallel a magamp». En: Applied Power Electronics Conference and Exposition, 1993. APEC '93. Conference Proceedings 1993., Eighth Annual, pp. 469474. doi: 10.1109/APEC.1993.290703.

JiTARU, I.D. (1992). «A new high frequency, zero-voltage switched, PWM converter». En: Applied Power Electronics Conference and Exposition, 1992. APEC '92. Conference Proceedings 1992., Seventh Annual, pp. 657664. doi: 10.1109/APEC.1992.228349.

Jitaru, I.D. y Birca-Galateanu, Serban (1998). «Small-signal Characterization of the Forward-Flyback Converters with Active Clamp». En: Applied Power Electronics Conference and Exposition, 1988. APEC '88. Conference Proceedings 1988., Third Annual IEEE, pp. 626-632.

Jordan, Mark (1991). «UC3907 Load Share IC Simplifies Parallel Power Supply Design». UNITRODE Application Note U-129, Texas Instruments.

Jovanovic, M.M. (1998). «A technique for reducing rectifier reverserecovery-related losses in high-power boost converters». Power Electronics, IEEE Transactions on, 13(5), pp. 932 -941. ISSN 0885-8993. doi: $10.1109 / 63.712314$.

Jovanovic, M.M.; Crow, D.E. y FAng-Yi, Lieu (1996). «A novel, lowcost implementation of democratic load-current sharing of paralleled converter modules». Power Electronics, IEEE Transactions on, 11(4), pp. 604-611. ISSN 0885-8993. doi: 10.1109/63.506126. 
Kassakian, John G.; Schlecht, Martin F. y Verghese, George C. (1991). Principles of Power Electronics. Addison-Wesley, Reading MA, USA, first ${ }^{\text {a }}$ edición.

Kim, Jung-Won; Choi, Hang-Seok y Cho, Bo Hyung (2002). «A novel droop method for converter parallel operation». Power Electronics, IEEE Transactions on, 17(1), pp. 25-32. ISSN 0885-8993. doi: 10.1109/63. 988666 .

Kim, Jung-Won; Yon, Jung-Sik y Cho, B. H (2001). «Modeling, control, and design of input-series-output-parallel-connected converter for highspeed-train power system». Industrial Electronics, IEEE Transactions on, 48(3), pp. 536-544. ISSN 0278-0046. doi: 10.1109/41.925580.

King, Brian y Strasser, David (2010). «Incorporating Active-Clamp Technology to Maximize Efficiencyin Flyback and Forward Designs». Texas Instruments Power Supply Design Seminar SLUP262, pp.1-23, Texas Instruments.

KISLOVSKI, A.S. (1983). «On parallel operation of regulated dc powersupplies with feedback-controlled current sharing». international Journal Of Circuit Theory And Applications, 11(1), pp. 97-98. ISSN 0098-9886. doi: $10.1002 /$ cta.4490110108.

LAI, Y.M.; TAN, S.-C. y Tsang, Y.M. (2009). «Wireless control of load current sharing information for parallel-connected DC/DC power converters». Power Electronics, IET, 2(1), pp. 14-21. ISSN 1755-4535. doi: 10.1049/iet-pel:20070311.

LAKShminarasamma, N.; Swaminathan, B. y Ramanarayanan, V. (2004). «A unified model for the ZVS DC-DC converters with active clamp». En: Power Electronics Specialists Conference, 2004. PESC 04. 2004 IEEE 35th Annual, volumen 3, pp. 2441 - 2447. ISSN 0275-9306. doi: 10.1109/PESC.2004.1355504.

Lee, F.C.; Barbona, P.; Xu, Peng; Zhang, Jindong; Yang, B. y CaNALES, Francisco (2001). «Topologies and design considerations for distributed power system applications». Proceedings of the IEEE, 89(6), pp. 939-950. ISSN 0018-9219. doi: 10.1109/5.931492.

LI, Q.; Lee, F.C. y Jovanovic, M.M. (2002). «Large-signal transient Analisys of Forward Converter with Active-Clamp Reset». Power Electronics, IEEE Transactions on, 17(1), pp. 15-24.

Li, Wuhua; Li, Weichen; He, Xiangning; Xu, D. y Wu, Bin (2012). «General Derivation Law of Nonisolated High-Step-Up Interleaved Converters With Built-In Transformer». Industrial Electronics, IEEE Tran- 
sactions on, 59(3), pp. 1650-1661. ISSN 0278-0046. doi: 10.1109/TIE. 2011.2163375 .

Lin, Bor-Ren; Chiang, Huann-Keng; Chen, KaO-Cheng y Wang, DaVID (2005). «Analysis, design and implementation of an active clamp flyback converter». En: Power Electronics and Drives Systems, 2005. PEDS 2005. International Conference on, volumen 1, pp. 424-429. doi: 10.1109/PEDS.2005.1619724.

Lin, Chang-Shiarn y Chen, Chern-Lin (2000). «Single-wire currentshare paralleling of current-mode-controlled DC power supplies». Industrial Electronics, IEEE Transactions on, 47(4), pp. 780-786. ISSN 02780046. doi: 10.1109/41.857958.

Luo, Shiguo y Batarseh, I (2005). «A review of distributed power systems part I: DC distributed power system». Aerospace and Electronic Systems Magazine, IEEE, 20(8), pp. 5-16. ISSN 0885-8985. doi: 10.1109/MAES. 2005.1499272 .

Luo, Shiguo; Ye, Zhihong; Lin, RaY-Lee y Lee, F.C. (1999). «A classification and evaluation of paralleling methods for power supply modules». En: Power Electronics Specialists Conference, 1999. PESC 99. 30th Annual IEEE, volumen 2, pp. 901-908 vol.2. ISSN 0275-9306. doi: 10.1109/PESC.1999.785618.

Mammano, Bob (1993). «Distributed Power Systems». Texas Instruments/UNITRODE Seminar SLUP099, pp.1-1 to 1-11, Texas Instruments.

Mammano, Bob y Jordan, Mark (1991). «Load Sharing with Paralleled Power Supplies.» Texas Instruments/UNITRODE Seminar SLUP094, pp 2-1 to 2-14, Texas Instruments.

Manias, S.N. y Kostakis, G. (1993). «Modular DC-DC convertor for high-output voltage applications». Electric Power Applications, IEE Proceedings $B$, 140(2), pp. 97-102. ISSN 0143-7038.

Mao, Hong; Yao, Liangbin; Wang, Caisheng y Batarseh, I (2007). «Analysis of Inductor Current Sharing in Nonisolated and Isolated Multiphase dc/dc Converters». Industrial Electronics, IEEE Transactions on, 54(6), pp. 3379-3388. ISSN 0278-0046. doi: 10.1109/TIE.2007.905966.

Mappus, Steve (2004). «Active Clamp Transformer Reset: High or Low Side?» Texas Instruments/UNITRODE Seminar SLUA322, pp.1 to 2, Texas Instruments.

Masihuzzaman, M. y Lakshminarasamma, N. (2010). «An improved ZVS-PWM, active clamp/reset forward converter: Analysis, design and 
dynamic model». En: Control and Modeling for Power Electronics (COMPEL), 2010 IEEE 12th Workshop on, pp. 1 -8. doi: 10.1109/COMPEL. 2010.5562442 .

Masihuzzaman, M.; Lakshminarasamma, N. y Ramanarayanan, V. (2010). «Steady-State Stability of Current Mode Active-Clamp ZVS DCDC Converters». Power Electronics, IEEE Transactions on, 25(6), pp. 1546 -1555. ISSN 0885-8993. doi: 10.1109/TPEL.2009.2031226.

Mazumder, S.K.; TAhiR, M. y Acharya, K. (2008). «Master-Slave Current-Sharing Control of a Parallel DC-DC Converter System Over an RF Communication Interface». Industrial Electronics, IEEE Transactions on, 55(1), pp. 59-66. ISSN 0278-0046. doi: 10.1109/TIE.2007.896138.

Mazumder, S.K.; Tahir, M. y Kamisetty, S.L. (2005). «Wireless PWM control of a parallel DC-DC buck converter». Power Electronics, IEEE Transactions on, 20(6), pp. 1280-1286. ISSN 0885-8993. doi: 10.1109/ TPEL.2005.857527.

McMurray, William (1972). «Optimum Snubbers for Power Semiconductors». Industry Applications, IEEE Transactions on, IA-8(5), pp. 593 -600. ISSN 0093-9994. doi: 10.1109/TIA.1972.349788.

(1980). «Selection of Snubbers and Clamps to Optimize the Design of Transistor Switching Converters». Industry Applications, IEEE Transactions on, IA-16(4), pp. 513 -523. ISSN 0093-9994. doi: 10.1109/TIA. 1980.4503823 .

Miwa, Brett A.; Otten, D.M. y Schlecht, M.F. (1992). «High efficiency power factor correction using interleaving techniques». En: Applied Power Electronics Conference and Exposition, 1992. APEC '92. Conference Proceedings 1992., Seventh Annual, pp. 557-568. doi: 10.1109/APEC. 1992.228361 .

MiwA, Brett ANDREw (1992). Interleaved conversion techniques for high density power supplies. Tesis doctoral, Massachusetts Insitute of Technology.

Mohan, Ned; Undeland, Tore M. y Robbins, William P. (2003). Power Electronics. Converters, Applications, and Design. John Wiley and Sons, Hoboken, NJ, USA, third ${ }^{\mathrm{a}}$ edición.

Ogata, Katsduhiko (2003). Ingeniería de Control Moderna. Prentice Hall, $4^{\mathrm{a}}$ edición.

PAnov, Y. y Jovanovic, M.M. (2002). «Stability and dynamic performance of current-sharing control for paralleled voltage regulator modu- 
les». Power Electronics, IEEE Transactions on, 17(2), pp. 172-179. ISSN 0885-8993. doi: 10.1109/63.988827.

(2008). «Loop Gain Measurement of Paralleled DC-DC Converters With Average-Current-Sharing Control». Power Electronics, IEEE Transactions on, 23(6), pp. 2942-2948. ISSN 0885-8993. doi: 10.1109/TPEL. 2008.2002955 .

Panov, Y.; Rajagopalan, J. y Lee, F.C. (1997). «Analysis and design of N paralleled DC-DC converters with master-slave current-sharing control». En: Applied Power Electronics Conference and Exposition, 199\%. APEC '97 Conference Proceedings 1997., Twelfth Annual, volumen 1, pp. 436442 vol.1. doi: 10.1109/APEC.1997.581489.

PaPAnikolaou, N.P. y Tatakis, E.C. (2004). «Active voltage clamp in flyback converters operating in CCM mode under wide load variation». Industrial Electronics, IEEE Transactions on, 51(3), pp. 632-640. ISSN 0278-0046. doi: 10.1109/TIE.2004.825342.

Perreault, D.J. y Kassakian, J.G. (1997). «Distributed interleaving of paralleled power converters». Circuits and Systems I: Fundamental Theory and Applications, IEEE Transactions on, 44(8), pp. 728-734. ISSN 10577122. doi: 10.1109/81.611269.

Peterchev, A.V.; Xiao, J. y Sanders, S.R. (2003). «Architecture and IC implementation of a digital VRM controller». Power Electronics, IEEE Transactions on, 18(1), pp. 356-364. ISSN 0885-8993. doi: 10.1109/ TPEL.2002.807099.

Petruzziello, F.; Ziogas, P.D. y Joos, G. (1990). «A novel approach to paralleling of power converter units with true redundancy». En: Power Electronics Specialists Conference, 1990. PESC '90 Record., 21st Annual IEEE, pp. 808-813. doi: 10.1109/PESC.1990.131272.

Renaudineau, H.; Houari, A.; Shahin, A.; Martin, J.-P.; PierfederiCi, S.; Meibody-Tabar, F. y Gerardin, B. (2014). «Efficiency Optimization Through Current-Sharing for Paralleled DC-DC Boost Converters With Parameter Estimation». Power Electronics, IEEE Transactions on, 29(2), pp. 759-767. ISSN 0885-8993. doi: 10.1109/TPEL.2013.2256369.

Saggini, S.; Ghioni, M. y Geraci, A. (2004). «An innovative digital control architecture for low-Voltage, high-current DC-DC converters with tight voltage regulation». Power Electronics, IEEE Transactions on, 19(1), pp. 210-218. ISSN 0885-8993. doi: 10.1109/TPEL.2003.820543.

Sepsi, D.T.; Hamar, J. y JÁrdÁn, R.K. (2010). «Sliding mode agentbased control of parallel buck converters». En: Power Electronics and 
Motion Control Conference (EPE/PEMC), 2010 14th International, pp. S13-20-S13-27. doi: 10.1109/EPEPEMC.2010.5606661.

Shi, J.; Liu, T.; Cheng, J. y He, X. (2014). «Automatic CurrentSharing of an Input-Parallel Output-Parallel (IPOP) Connected DCDC Converter System with Chain-connected Rectifiers». Power Electronics, IEEE Transactions on, PP(99), pp. 1-1. ISSN 0885-8993. doi: 10.1109/TPEL.2014.2334896.

Shi, Jianjiang; Zhou, Lingbing y He, Xiangning (2012). «CommonDuty-Ratio Control of Input-Parallel Output-Parallel (IPOP) Connected DC-DC Converter Modules With Automatic Sharing of Currents». Power Electronics, IEEE Transactions on, 27(7), pp. 3277-3291. ISSN 08858993. doi: 10.1109/TPEL.2011.2180541.

Shortt, D. J.; Michael, W. T.; Avant, R. L. y Palma, R. E. (1987). «A 600-W Four-Stage Phase-Shifted-Parallel DC-to-DC Converter». IEEE Transactions on Power Electronics, PE-2(2), pp. 101-108. ISSN 08858993. doi: 10.1109/TPEL.1987.4766344.

SIRI, K.; LEE, C. Q. y WU, T. F (1992a). «Current distribution control for parallel connected converters. I». Aerospace and Electronic Systems, IEEE Transactions on, 28(3), pp. 829-840. ISSN 0018-9251. doi: 10. $1109 / 7.256303$.

- (1992b). «Current distribution control for parallel connected converters. II». Aerospace and Electronic Systems, IEEE Transactions on, 28(3), pp. 841-851. ISSN 0018-9251. doi: 10.1109/7.256304.

Small, Kenneth T. (1988a). «Single wire current share paralleling of power supplies». US Patent. US 4717833 A.

Small, K.T. (1988b). «Single wire current share paralleling of power supplies».

Spiazzi, G.; Mattavelli, P. y Costabeber, A. (2009). «Effect of parasitic components in the integrated boost-flyback high step-up converter». En: Industrial Electronics, 2009. IECON '09. 35th Annual Conference of IEEE, pp. 420-425. ISSN 1553-572X. doi: 10.1109/IECON.2009.5414959.

Spiazzi, G.; Mattavelli, P.; Gazoli, J.R.; Magalhaes, R. y Frattini, G. (2010). «Improved integrated boost-flyback high step-up converter». En: Industrial Technology (ICIT), 2010 IEEE International Conference on, pp. 1169-1174. doi: 10.1109/ICIT.2010.5472596.

Su, Jen-Ta y Liu, Chin-Wen (2013). «A Novel Phase-Shedding Control Scheme for Improved Light Load Efficiency of Multiphase Interleaved DC- 
DC Converters». Power Electronics, IEEE Transactions on, 28(10), pp. 4742-4752. ISSN 0885-8993. doi: 10.1109/TPEL.2012.2233220.

Sun, J. y Grotstollen, H. (1992). «Averaged modelling of switching power converters: reformulation and theoretical basis». En: Power Electronics Specialists Conference, 1992. PESC '92 Record., 23rd Annual IEEE, pp. 1165 -1172 vol.2. doi: 10.1109/PESC.1992.254742.

Sun, Jian y Grotstollen, H. (1997). «Symbolic analysis methods for averaged modeling of switching power converters». Power Electronics, IEEE Transactions on, 12(3), pp. 537 -546. ISSN 0885-8993. doi: 10. $1109 / 63.575681$.

Sun, Juanjuan (2007). Dynamic Performance Analyses of CCurrent Sharing Control for $D C / D C$ Converters. Tesis doctoral, Virginia Polytechnic Institute and State University, Blacksburg, Virginia.

Tabisz, W.A; Jovanovic, M.M. y Lee, F.C. (1992). «Present and future of distributed power systems». En: Applied Power Electronics Conference and Exposition, 1992. APEC '92. Conference Proceedings 1992., Seventh Annual, pp. 11-18. doi: 10.1109/APEC.1992.228437.

Tanaka, H.; Kobayashi, K.; Ihara, F.; Asahi, K. y Motoyama, M. (1988). «Method for centralized voltage control and current balancing for parallel operation of power supply equipment». En: Telecommunications Energy Conference, 1988. INTELEC '88., 10th International, pp. 434-440. doi: 10.1109/INTLEC.1988.22388.

Thorsell, L. y Lindman, P. (1988). «Reliability analysis of a direct parallel connected $\mathrm{n}+1$ redundant power system based on highly reliable DC/DC modules». En: Telecommunications Energy Conference, 1988. INTELEC '88., 10th International, pp. 551-556. doi: 10.1109/INTLEC.1988. 22407.

Thottuvelil, V.J. y Verghese, George C. (1998). «Analysis and control design of paralleled DC/DC converters with current sharing». Power Electronics, IEEE Transactions on, 13(4), pp. 635-644. ISSN 0885-8993. doi: $10.1109 / 63.704129$.

Tseng, K.C. y Liang, T.J. (2004). «Novel high-efficiency step-up converter». Electric Power Applications, IEE Proceedings -, 151(2), pp. 182-190. ISSN 1350-2352. doi: 10.1049/ip-epa:20040022.

Venable, H. Dean (1983). «The K Factor: A New Mathematical Tool for Stability Analisys and Synthesis». Informe técnico, Linear Technology. In Proceedings POWERCON Vol. 10.. 
Villarejo, J.; De Jodar, E.; Mateo, A y Suardiaz, J. (2009). «Dynamic current sharing in multiphase active-clamping converters». En: Power Electronics and Applications, 2009. EPE '09. 13th European Conference on, pp. 1-9.

Vinciarelli, PATRIZIO (1984). «Optimal resetting of the transformer's core in single ended forward converters». US Patent. US 4441146.

WANG, J.B. (2011). «Design a parallel buck derived converter system using the primary current droop sharing control». Power Electronics, IET , 4(5), pp. 491-502. ISSN 1755-4535. doi: 10.1049/iet-pel.2010.0042.

(2012). «Parallel DC/DC converters system with a novel primary droop current sharing control». Power Electronics, IET, 5(8), pp. 1569-1580. ISSN 1755-4535. doi: 10.1049/iet-pel.2012.0216.

Watson, R.; Hua, G.C. y Lee, F.C. (1996a). «Characterization of an active clamp flyback topology for power factor correction applications». Power Electronics, IEEE Transactions on, 11(1), pp. 191 -198. ISSN 0885-8993. doi: 10.1109/63.484432.

Watson, R.; Lee, F.C. y HuA, G.C. (1994). «Utilization of an activeclamp circuit to achieve soft switching in flyback converters». En: Power Electronics Specialists Conference, PESC '94 Record., 25th Annual IEEE, pp. 909-916 vol.2. doi: 10.1109/PESC.1994.373787.

(1996b). «Utilization of an active-clamp circuit to achieve soft switching in flyback converters». Power Electronics, IEEE Transactions on, 11(1), pp. 162 -169. ISSN 0885-8993. doi: 10.1109/63.484429.

Watson, Robert (1998). New techniques in the design of distributed power systems. Tesis doctoral, Virginia Polytechnic Institue and State University, Blacksburg, Virginia.

XI, Youhao y Jain, P.K. (2003). «A forward converter topology employing a resonant auxiliary circuit to achieve soft switching and power transformer resetting». Industrial Electronics, IEEE Transactions on, 50(1), pp. 132 - 140. ISSN 0278-0046. doi: 10.1109/TIE.2002.804982.

Yoshida, K.; Ishi, T. y NAGAgata, N. (1992). «Zero voltage switching approach for flyback converter». En: Telecommunications Energy Conference, 1992. INTELEC '92., 14th International, pp. 324-329. doi: 10.1109/INTLEC.1992.268424.

Zhang, J.M.; Xie, X.G.; Wu, X.K. y Qian, Zhaoming (2004). «Stability study for paralleled DC/DC converters». En: Power Electronics Specialists Conference, 2004. PESC 04. 2004 IEEE 35th Annual, volumen 2, pp. 1569-1575 Vol.2. ISSN 0275-9306. doi: 10.1109/PESC.2004.1355659. 
Zhang, X.; Corradini, L. y Maksimovic, D. (2009). «Digitally controlled distributed multiphase DC-DC converters». En: Energy Conversion Congress and Exposition, 2009. ECCE 2009. IEEE, pp. 36-42. doi: 10.1109/ECCE.2009.5316151.

Zhang, Y.; Zane, R. y Maksimovic, D. (2005). «Current Sharing in Digitally Controlled Masterless Multi-phase DC-DC Converters». En: Power Electronics Specialists Conference, 2005. PESC '05. IEEE 36th, pp. 2722-2728. doi: 10.1109/PESC.2005.1582018. 